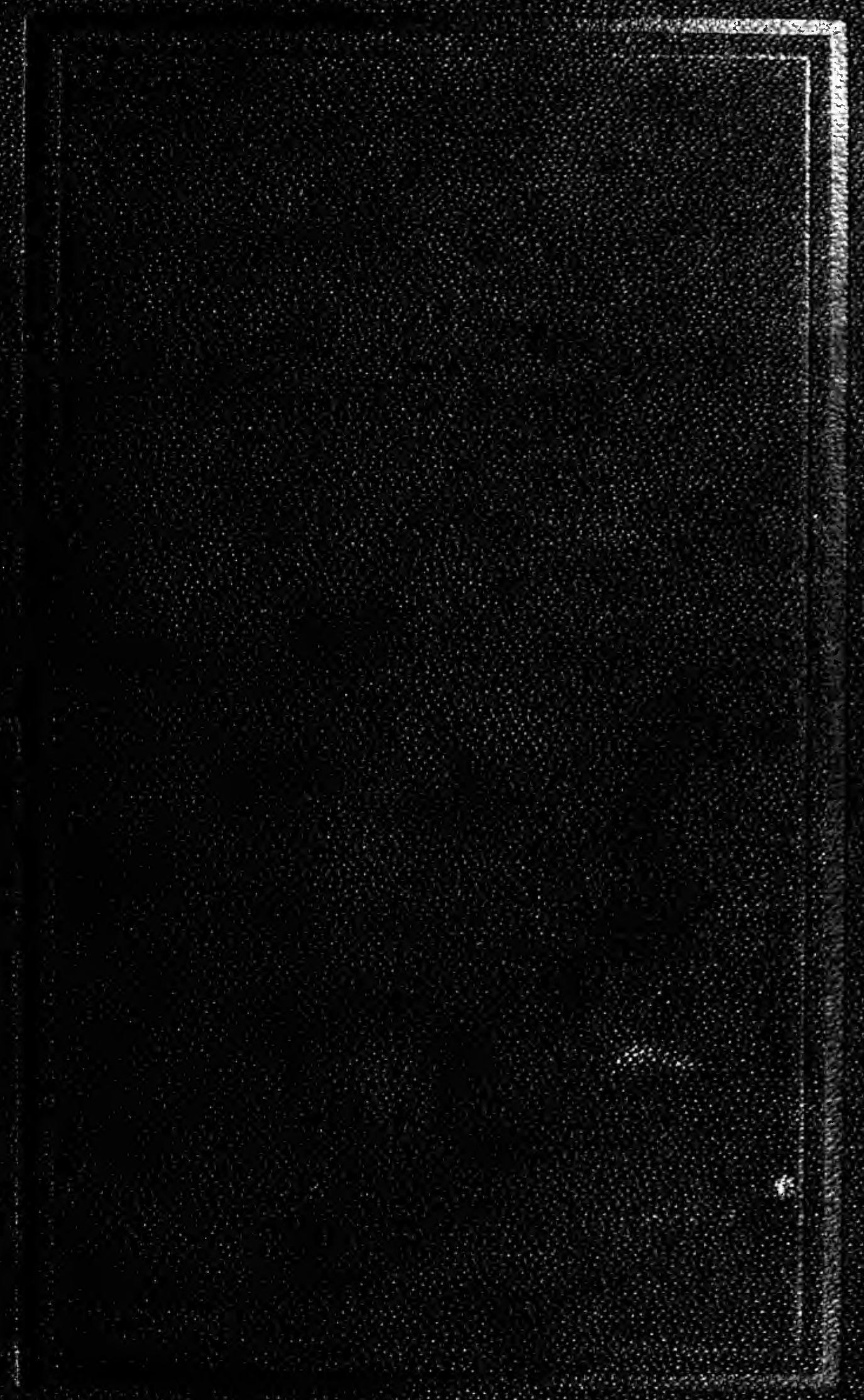



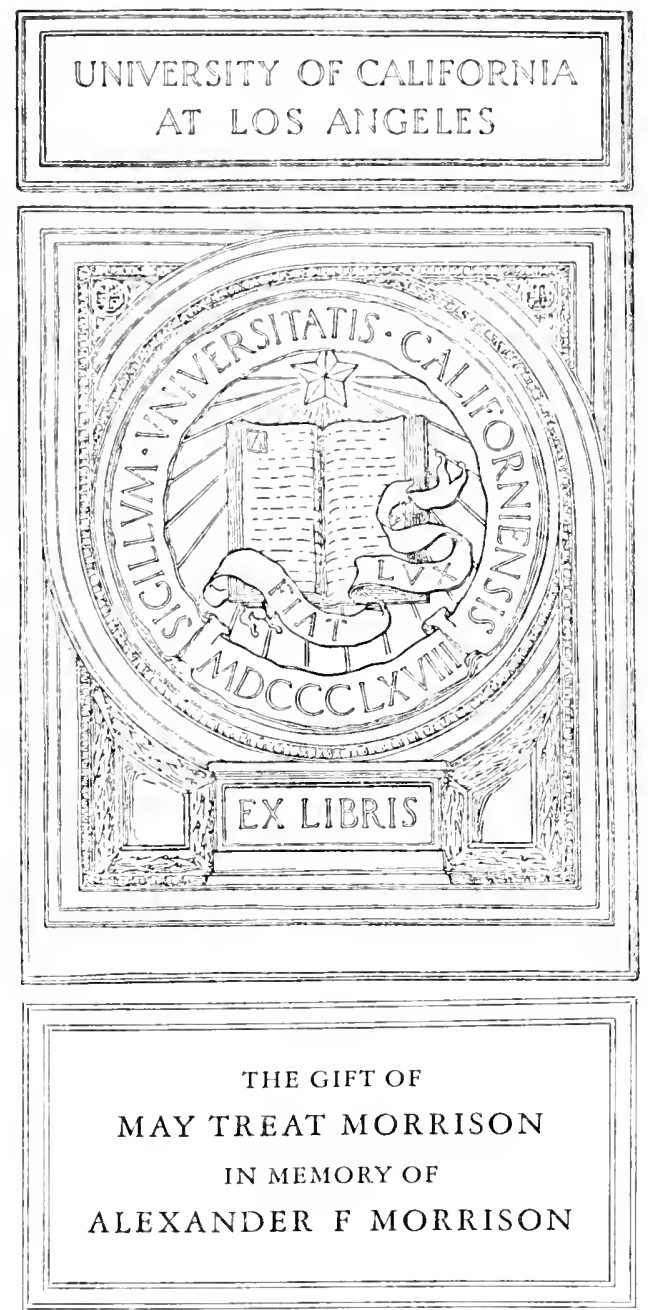

T H E

\section{AMERICAN GOVERNMENT}

ORGANIZATION AND OFFICIALS

WITH THE

DUTIES AND POWERS OF FEDERAL OFFICE HOLDERS

Second Edition

An Original, Summarization

BY

H. C. GAUSS

With a Compilation of Data from Official Sources

NHW YORK, IgOS

L. R. HAMERSLY \& CO. 
Copyright, 1907, by

L. R. HAMERSLY \& COMPANY

New York 


\section{PREFACE.}

In rotnd numbers there are a half a million persons holding commissions and appointments from the United States, and cxercising, in all parts of the world, functions which cannot be exercised by a citizen of any State as such. The persons referrel to are, however, the servants of every citizen of every State, and their business comes to be, in an increasing measture with each year, the business of every community and of every person in cvery community.

A vague distrust that by some means the Federal Government is trenching upon the rights and prerogatives of the independent States may be created by persons who have become, properly enough, subject to Federal control. Lest any citizens of the United States should be led to an unreasoning hostility to the regulation of matters which cannot be controlled by the individual State, it is a duty of citizenship, not only to read, in its original form, the Constitution of the United States, but to be informed as well as to the developments resulting from the natural growth of the Fecleral powers; whether such developments have in any sense proved to be injurious and wherein they have proved beneficial. As an introduction to the study of the very many phases of Federal activity, this compilation has the merit merely of assembling in a single book details of the Federal system of government from many and not always accessible sources. An accurate definition of every detail of the subject is possible only as a governmental work, and its accuracy would be wholly that of the moment, for the reason that growth and change are both constantly going on. At the same time much can and should be done officially in the way of digesting departmental law and regulations, so that Federal practice may be less of a mystery to the millions who are at once the sovereigns of the respective States and of the Federal nation.

January I, Igo8.

H. C. Gauss.

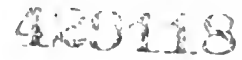


• 


\section{INDEX.}

Abbreviations used in Diplomatic and Consular Lists, table of. . 265 Accounts against Government, audit and approval of .........440 suspension and rejection of. 441 Acting Stcretaries, order of precedence ................. 135

Adjutant General's Department... 455 Agriculture, Department of, bureaus in ..............752

Bureau of Animal Industry.. $7 \pm 0$

Bureau of Biological Survey.. 753

Burcau of Chemistry....... 745

Bureau of Entomology...... 752

Bureau of Plant Industry.... 755

Bureau of Foils.......... 754

Eurealu of Statistics........ 748

revelopment and jurisdietion of

Onfice of Good Roads....... 756

Presidential appointments in. 712

Publications of ......... 757

solicitor of ............. 39

Agriculture, Secretary of, Appointment. salary and duties...... 161

Eighth in Cabinet rank...... 162

Has charge of Quarantine matters. ............ 164

Reports of $\ldots \ldots \ldots \ldots \ldots \ldots 163$

Assistant .................... 181

Agricultural Experiment Stations. 163

Air brakes on railroads......... $\$ 16$

Alaska, Territorial organization and officials ............. 734

Alaskan Boundary Commission... 275

Aicohol, denatured .......... 377

Alien who has declared intention of citizenship may have passport, when ............. 247

Ambassadors, foreign, received by President ................ 19

Ambassadors of U. S.- See Embassidors.

Amounts due Government Departments, in charge of Auditors. .

Animals, rules for land transportation of ............ 742 ports designated for importation of ........... 743 prevention of contagious diseaste among .......... 742 quarantine against infeeted.. 743

Anti-Trust Law, prosecutions under .................... 521

Appeals and ivrits of Error in Su-

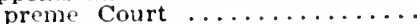
Appointments, by president, requiring confirmation .......6-12 not requiring confirmation...16-18 during recess of Senate..... 15 Appointments by Speaker....... 102 by Vice-President of United States .............. 101

Appraisers, general, appointment and qualifications ...........
Appraisers of merchandise, locations and bonds, IIst......361-362 assistant, locatious and bonds,

list ..................

Appraisers must certify appralse-

ment to collector .......... 318

Appraisement and inspection, of inuported goods............. 318 appeals from ............. 319 experts tmployed on ...... 320

Appraisement-see also "Reappraisement."

Architect of Treasury, Supervising 446 Arizona, organization, personnel and limitations ........ 733

Armed fores of $\mathcal{U}$. S.-President commander-in-chicf of ........

May be used by president for exeeution of criminal laws.

Army, Adjutant General's Department ................... 455

Banners of ........... 509

Clice of staff of .........451

Commissioned ofteers in different grades .........4 457 Composition of ..........450

Contract Surgeons in.......4463

Dintal surgeons in........ 463 Enlistments and discharges. 515 Gineral staff Corps, duties of 450 Gineral Staff Corps, duties of 453 Insignia of ........... 501

Ifedical bepartment ..... 462 Ton-commissioned officers, rial and duties ......... 455

Nurse corps of .........4463

Orgunization of regiments and compinies ............ 4 ss

Pay of ...........490, 497

Pay Department, organization ind luties ........4 465

Peace organization ........ 453

President is commander-inchief of ............. romotion and retirement of officers a...........49, 501

Punisinents in ......... 515

Quarternasters Department. 459

Schools

Stroneth of ........... 487

Subsistence Demartment......4 461

Surgon General of........462

War organization of ........ 453

See also "Viar Department."

Artillery, const and fleld....... 453

Assayer of Assay Otlice. New Fork, ex-officio member of $\mathrm{Com}$ mission to lixamine and Test

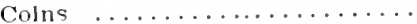

Assistant Secretaries of Departments and thelr futles (see also the viulous Departments)...134, 167 Assistant treasurers of the Unlted states ................420 
Assnciate Justices-see "supreme Court."

Attachés, militiry and naval, of (mbassies or iogations, duties, rank, te. . . . . . . . . 271

Atticlıés, eivilian, probibited.... 271

Attorn - General, appointment, salaty and lutios.......... 147 Tourth in Cabinct......... 147

Lezal adviser of President ind hears of departments.. 147 Arrues more important matters in supreme Court.... 520

orieial visit to President.... $9 \mathrm{~s}$

Oninions of their force..... 147

Palsses on titles to land purchased by Gorrmment.... 534

Attorne General - Issistant to the appointmont, sirlary and dutios. 521

Attomey General, Assistant, appointment, salaries and duties. . 52t

Attrrory Ginceal, Assistants in Court of Claims, Indian deprerations, Snanish Trratr, Claims Commission ................

Attomers, Assistant, in Department of Justice, salaries and

Attorreys. Snocial Assistant (Denartment nf Justice) .......... Tnited Stites Attorners.

Aubiting offeris of Government.. 851 Auting ixpenses of Government nficials and emploves 852 samnl cxponse account..... 853

Auriter for Post ofice Departmrnt. mail route contracts to be cirnifien to ............. 616

Aulitors of the Treasury six...44 438 $\therefore$ nnnintmont, salaries and du-

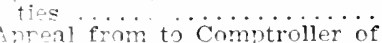
the Treasury. Bauk in United States Courts. 551 Bark pletes and paper, how guardert ................4 429

Bankrunter, referees in, duties ant fos.............. 240 trusteps in dutios and fees.... 241

Bill nf Health, given by U. S. Consul .................4 416

Bills, the colurse through Senato and House. . . . . . . . . 53, 117

Board of visitors to Military Acarlemy, anpointed by VicePresirent and Sneaker.....101, 102

Board of Visjtors to Naval Academr, annointed by Vice-Presidont and speaker........101, 102

Boare on Construction, organizatinn and duties............ 644

Pond, Transportation in ........... 312

Bond issues, premared under direction of Rigistor of Troasury....4423 proceeds mid to Treasurer. 423

Bonrled warehouses (customs), withr? rawal of goods from..... 321 ran be nnenerl, when....... 321 List of norts where established 327
Bonded warehouses in revenue distriets ............... 374

Bookktening and Warrants, Division of .............. 444

Botanic Garden-under jurisdiction of Congress ........... 130

Butlling in bond ............ 872

Bureau of Accounts (State Depart$m+n t s) \quad \ldots \ldots \ldots \ldots \ldots \ldots \ldots 244$

Burcau of American Republics, In-

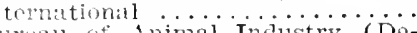
Burat of Animal Industry (DeBulvall of Appointments (State Department) $\ldots \ldots \ldots \ldots \ldots \ldots$

Eurtau of Blological Survey (Department of Agrieuture) .....753

Burrau of Census (Denartment of Commeree and libbor)...... 782

Burtau of Chemistiy (Department ot Agriculture) ........... 745

Burcau of Citizenship (State Derartment) .............24

Burtau of Construction and Repair (Nary Department) .........6631

Burau, Consular (State Depart$m n t) \ldots \ldots \ldots \ldots \ldots \ldots \ldots \ldots \ldots \ldots$

Bureau of Corporations (Department of Commere and Labor ) $\ldots \ldots \ldots \ldots \ldots \ldots \ldots 165$, 73

Bureatr, Diblomatic (state Department, ................243

Burat of Engraving and Printing (Treasury Department) .......

Sutmau of Entomolngy (Department of dericulture) .......752

Bureau of Eifuiment (Navy Department) $\ldots \ldots \ldots \ldots \ldots \ldots$ Furfall of Fisheries (Department of Commeree and Labor) ......

Burean of Immigration and Naturalization (Deparment of Commer and Lahol').........166, 803

Bureau of Indexes and Archives (state Department) ........

Bureau of Labor (Department of Commerce and Labor).......775 Publieations of ............7 766

Bureau of Manufaetures (Department of Commerce arri Labor) . . 773

Purfau of Medicine and Surgery (Nary Donartment)..........66

Turaus of Nary Department.... 630

Bureau of Narigation (Department of Commerce and Labor) ......796

Buratu of Narigation (Nary De-

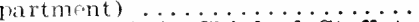
Chiof of, is chief of Staff to Se cretiry of Nav ......6 644

Bureau of Ordnance (Navy Department) ..............6634 Bureau of Plant Industry (Department of Agriculture) ........ Introfluction of new agricultural nroruets bv.........756

Bureau of Rolls (State Department) ................ 245 Eureau if Soils (Denartment of Agriculture) ............ 754

Burfau of Standards......... 809 Advisorv and Inspecting visiting Committen .......... 
1.NDEX.

PAGE

Rules for verification of apparatus ............ 810

Bureau of Steam Engineering (Navy Department) .........6 634 Bureau of Statistics (Department of Agriculture) $\ldots \ldots \ldots \ldots \ldots \ldots$

Crop information ........ 748

Forigin crops reported by consuls to ...........748

Publications .............. 74

Bureau of Steam Inspection (Department of Commerce and Latbor).
Bureau of Supplies and Accounts (Navy Department) ........6667

Burtau of Trade Rulations (State Department, .............

Bureau of Iards and Doclis (Navy

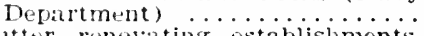

Butter renoviating establishments. supervised by Luriau of Animal Inbustry ............... $7+4$

Cabinet directed by President.... 23

Cabinet Derartments, administered by sfeletaties moler control of

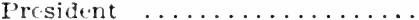

California Deliris Commission... ss s

Canal Zone, Governor of...... 835

Capitol nolice ............. 125

Capitol, superintentent of ....... 124

Captain of vessul-see "Master of Vessel."

Cargo, time for unlading....... 317

Census Bureau ..............

Director of, appointment, salary and duties.......... ist

Enumerators of ........ 785

Special agents of ........ TS Special, of Indian irritory and Olilahoma......... 786

Supervisors of ......... $\mathrm{i} s 4$

Claplains, Army ............

Cheplains, Nav ........... fir

Chaplains of IJouse and Senate.. 115

Chief Cle?k of Troasury Depart-

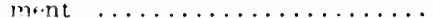

Chief of Engineers of Army.... 465

Chief Justief-osep Supreme Court.

Chicf Justice of Supreme Court, presides in cases of Impeachmont of President...........

Chiets of Bureaus. their appointment and duties-sce under each Bureaix.

Child born abroad becomes citizen by fathor's naturalization..... 247

Chinese Exclusion .......... 805

Cigars and tobacen, revenue pro-

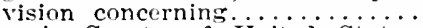

Circuit Comrts of United States, places and terms of......... 216

Jurisdletion of ........... 204

Circuit Court of Apneals, organization and furisdiction ........227

Times and places of holding.. 229

Circuit Judges, important functions ot ................. 204

Clvil rights, jurisdiction.......... 211

Civil rlghts, restoration of after imprisonment ...........532

Clvil Service Appointments .. $\$ \$ 1$, $8 \$ 5$ defects which debar from .. 842
Pusitions ampted from classification ............. 823

Civil sirvice Commisston $\mathrm{em}$ liluyes and appropriation for. $8 \geq 0$

Civil Sirvice Cummlssioners, aijpointment, salarites antl dutios. 818 Clvil Service Examlnations, place and requir.ments of ....... 819

qualifications of applicants. s: s

for lnsulir fovernment l'osltions in l'hilippines....... \$21

Examinations of Midshipmen for Naly and for litrenue Cuttro service.......... 821

Civil Service persons may be re moved by Presldent ......... 19

Civil service preons, minimum and maximum sitldriı........ $\$ 46$

Dictricts . . . . . . . . . s 21

Prefrence to disibler Army and Navy Veterans in..\$20, \$26

Promotions in .......... 649

Rules of ............... Sti

Rogister of unstilled and unclassified linorers ....... 821

Salary classitication in ...... $x^{2} 2$

Sepalation from ........ s.

Transfer of Lmployes ...... S4s

Civil War Fonsions. ........ To-710

Cluk at Speakir"s Table expert onl rulis of llouse ........ 117

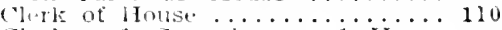

Clarks of Committees of liouse aind Sinat ............ 120

Clerks, Gov rnment ........ 539

Coast and Geodetic survey (bepaltrent of Commerce and lat bor $\ldots \ldots \ldots \ldots \ldots \ldots \ldots \ldots$. Superintenisent of appointment. sitlary and duti..s... iss

Coins reserved for cxamination and tust ............ 434

Coins, serutary of Trustiry directive officer in prouluetion of.. 142 Also see "Mint."

Cuinagr, gold standard for, established $\ldots \ldots \ldots \ldots \ldots \ldots \ldots \ldots 41$ based on gold dollar as unlt. 431 procentings from bullion to coin ............ 433 silver only subsidiary ..... 432

Coiner of ilint.............. 433

Collector of Customs-sece "Customs."

Collector of Intermal Revente-sue "Internal! Revenum."

Commane? r-in-chinf of Army and

- Nilvs. president is ..........

Commerce and labor, Department of ............. Bureau of Corporations . .145, 773 Bureau of Immisration and Natlaralization ........tib, $s, 3$ Bureau of I abor ........ 755 Burean of Manuficturers... i 73 Bureau of Navigation ..... Tis Bur.aul of Statistics......... Tss Burcall of Stam Inspection.. 792 Prosiluntial appointments in 11.17 Assistant seretary of ......182 
PAGE

Commerce and Labor, Secretary of 164 has dircet control of salmon and seal fisheries ....... 166

Commerce between the Stateslatitude of legislation concerning ..................... 27

Commissioner of Corporations Commissioner of Education (Department of Interior) $\because \cdots$ Land

Commissioner of General Land $15 \mathrm{~s}$ Office …......... in Revenue 366 Commissloner of Internal Revenue 366

Commissioner of Navigation.....7796

Commissioner of Labor........... 157

Commissioner of Pensions $\ldots \ldots$ of

Commissioners of the District of 876 Columbia ... Ü.... Stäe....... 236

Commissioner General of Immigration and Naturalization....... 804

Commissions produced by Bureau of Engraving and Printing.....4 428

Commissions, not assigned to any main Department under direction of President ...............

Commissions (Administrative) under the American Government.. 812 der the Americanse of Representatives ................ (standing Committees of Senate (standing and select) $\ldots \ldots \ldots \ldots \ldots \ldots \ldots .3$ Employes of, list and salaries. 121 Compounding and rectifying 11 quors ...................... 372

comptroller of for five-year term.. 426 Apnointed for five-year term... to examine and test coins... 434 In charge of Currency issues and National Bank Circula-

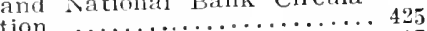

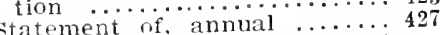
Takes charge of Insolvent banks ...............4 427

Comptroller of the Treasury ..... 442 Confirmation of Presidential apnointees by senate ...........

Confirmation of Treaties by Senate $\ldots \ldots \ldots \ldots \ldots \ldots \ldots \ldots$ congress counts the Electoral vote, how .............. Has exclusive control of District of Columbia...........

Way choose time of appointing Presidential electors .......

Nembers may not practice before Court of Claims......

powers liberally construed by Courts $\ldots \ldots \ldots \ldots$ o.......

Sessions, dates of beginning and ending from foundation of Government ..........

Sessions, long and short.... Subordinate officials, how chosen .............. 109

See also "Senate" and "House of Representatives."

Congressional Districts, list of, with boundaries ...........55-83

Constitution of the United States, proposing amendments to.....
Consular Bureau (State Department) $\ldots \ldots \ldots \ldots \ldots \ldots \ldots 243$ Consular Clerks, appointment, duties and salaries........... 301

Consular Courts, organization, procedure, sentences and appeals. . 260

Consular fees, tariff of ........ 30.6

Consular officers, depositions before and procedure in case of crime on ligh seas............ 293

Consular officers, diplomatic representatives have general supervision over ............ 262

Diplomatic officers may perform duties when........ 262

Duties in extradition cases... 297

Duties in regard to citizens not seafaring men....... 286

Duties in relation to shipping and seamen of merchant marine ............. 286

Duties under Chinese Exclusion Act .............294

Entitled to salute from naval vessel, when ...........2 285

Gifts not to be accepted without consent of Congress.... 297

Have charge as custodians of property of citizens which has been wrecked....... 292

Invoice of goods shipped to U. S. authenticated by..... 299

Judicial power of .......... 298

Marriages solemnized in presence of, effect of ........290

May appoint associates to try case, when ........... 299

May become custodian of consular duties of foreign nation, when ........256, 286

May request services of Navy. 285

Must give bonds........... 280

Not to become endorsers for citizen ...............299

Privilege and protection of... 283

Protection of native subordinates ...............286

Rank and precedence.......2 284

Rent of offices............ 281

Rights under "favored nation" clause of treaties.........285

Reports of ........... 297

Sunervise sales and transfers of vessels .......... 293

To administer estates of citizens dying abroad or on high seas, when.........295

To give bill of health to vessels ................ 294

To prevent transportation of improper and excluded classes to United States.... 294

To visit vessel of Navy on invitation of commanding officer ................285

Use of dispatch bags by.... 257

Verification of powers of at torney and pension vouchers by ................... 297

Wearing of uniforms by..... 297
Consular officers, subordinate.... 300 
PAGE

Consular agents ............ 300

Deputy consuls .................. 300

Consular service, grades of ..... 27 supervision of .......18, 302

Consular Service, promotions and

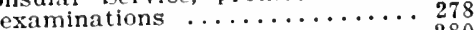

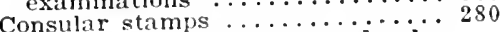

Consulates, classification and sala-

Consuls, appointed by examination above sth and 9 th grades..... 27

Appointed by promotion..... 278

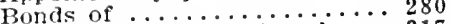

Certification of invoices by .. 317

Exequatur procured through diplomatic officer........2S

Qualifications, examinations and compensation .......2 279

Must turn in fees to Treasury. 307

Consuls-general at large, appointment, duties and salary.......

Consuls-general, classification and salaries in adidion to ordinary duties of consuls ....... 277

how appointed and promoted. 277 may make trips of inspection. 277

Contracts, postal ...........6 609

Copyright, general provisions in

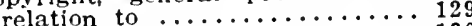

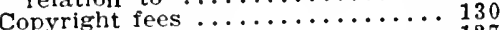

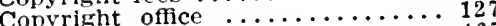

Corps of Engineers ..........465

Counterfeiting. detection and prevention of, in charge of Secret

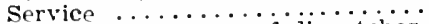

Couriers and bearers of dismatches, privileges and immunities.....2 254

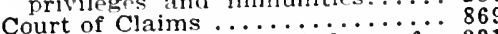
Chief justice and judges of. . 231 Organization and jurisdiction. 230

Courts "Supreme Court" "Circuit court," "Inferlor Courts," "United" States Court for China," "District Courts," etc.

Court dress, dipiomatic offlcers

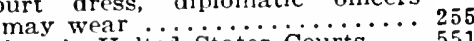

Crlers in United States Courts.... 551

Currency notes, redeemed or dam-

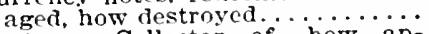

Customs, Collector of, how appointed, terms and duties..... 313

Customs, Collector of, may make special arrangements with regu-

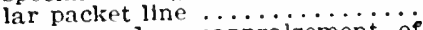
may order reappralsement of goods ................... 319

permit of, to unload vessel or to enter other than port of

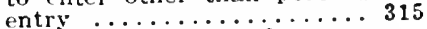

redelivers manlfest of cargo,

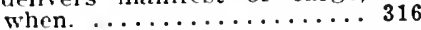

ports, boards and compensation 341

Customs collections, cost of ....... 314 Customs, deputy collectors of, how

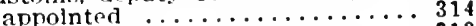

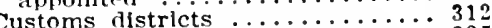

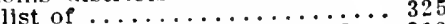
Customs duties, where coliccted... 312
Customs, Inspectors of .................. 323

Customs, Naval oflletrs of...... 355

Customs ofllcers must account to

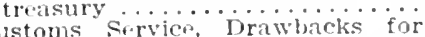

Customs Srrvice. Drawbacks for

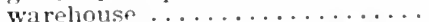

Dutlabie gonds, weighing and

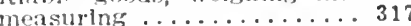

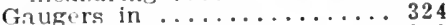

General appralsers .........319

Ports where custom-bouse prenises are used for storage of imported goods in

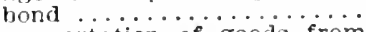

Re-exportation of goods from public or bonded warnhouses 321

Sale of goods. for non-payment of duties and charges, from nublic or bond.(1 war wouses 321

Secretary of Treasury is head

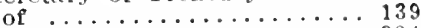

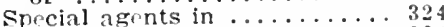

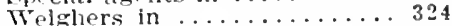

Customs. Solicitor of, at $\mathrm{New}$ York $32 n$

Survevor of, dutles of....... 323

Surveyors of, location, bonds

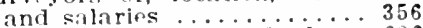

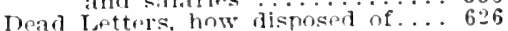

Depositary offices for receipts of nost-offices ...............613

Department of Agriculturn-see "Agriculture. Department of."

Department of Commeree and Lahor-see "Commerce and Labor, Department of."

Department of the Intorior-see "Interior. Donartmont of."

Department of . Justicn-see "Justice. Denartment of."

Department. Secretarics of, are allowed carriages and appurte-

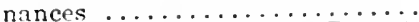

Diplomatic Burnau (State Depart-

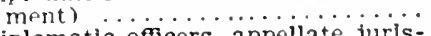

Diplomatic officers, anpellate jurlsaction over consular courts...
class sent to each nation, how

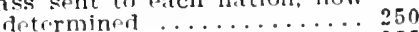
cannot accopt gifts ........... 255 communications and correspondents .............. 556 Court dress niy he vorn hy.. 255

Criminal jurisiliction of, in certain countries ............. 260 do not use open malls....... 25? dutles of ............. 55 ? duties in connection with clains asainst forelgn governimonts duties in extridition cases.... 264 duties in relation to naritime affirs ..............251

dutles in rolation to treatles, acreements and unilorstand-

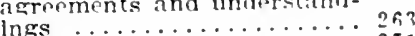

eree entrir for gonds if...... 251

formal communications to forelon covernments must be in English ............... 255 
PAGE

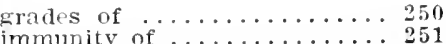

immunity does not extend fully to natives of country where located ..............253

issuance of passports by ....2 258

leave of absence.........2 273

letters directed to his care... $25 \mathrm{~s}$

may represent foreign nation in a case of war......... 259

must be persona grata...... 251

must not correspond with newspapers or magazines. . 255

must not endorse bills or become pecuniarily responsible for American citizens ..... 262

procure exequaturs for consuls ................ 262 protection to American citizens

by .................. $25 s$

nublic speeches by.......... 255

ranks and salary, list of.... 265

recalled when hecome obnoxious to country where 10arted ................ 251

resignation or recall of ..... 273

reticence required of ........ 255 safe conduct to frontier in case of war ............ 274 servants of, entitled to immu-

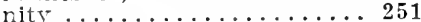

subordinate .......... 271 war does not always terminate office..............274

wearing of uniforms by......2 254

Director of the Nint.......... 429

Disbursing officers furnished with funds, how and when........4 421

Discrimination in freight and passenger rates $\ldots \ldots \ldots \ldots \ldots \ldots$.

Dispatch bags, official, used by diplomatic officers ............. 257 consular officers may use.... 297

Dispatchos diplomatic, bearers of, compensation and special passport for ............... 257

Distillation, restrictions on business 369

Distillation of fruits ......... 369

Distillers must provide warehouses 370

Distrnss signnls ............. 799

District of Columbia, Congress has sole legislative control over.... Commissioners of ....... 870 Schonl Board of........... 870

Distriet Courts, jurisdiction....... 187 places and terms of list..216, 226

District judge anpoints United States Commissioners ......... 189

District judge may aet as circuit judge or in Circuit Court of Appeals. when ............ 192 Must resirle in his district 66188 Salary of ............ 187

Districts, Congressional, List of, with boundaries ...........55-83

Dixision of Bookkeeping and Warrants (Treasury Department)...444

Divisions in Post Office Department - see Post Office Department.

Domestic Manufactures, taxes on which are a sourcf of revenue. 309
Dormments. Public, how distributed and sold ................... 861 superintendent of ....... 860

Duties on imports a main source of National refenue ........... 309 How paid .............. 313

Education, in charge of the Secretary of the Interior.......... 158

Elueition. Commissioner of ..... 729

Elucation, Department of-see Interior Department.

Election of the President........ 19-24

Electoral Cotint, Declarcd by Pres-

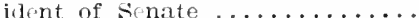
Disputes about. how settled.. Writnessed by Congress ......

Electors, presidential, Election of how certified................
No Senator, Representative or other ederal officer can be. Number, how fixed, how and when chosen, and qualifica-

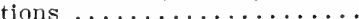

Vacancy in list, how filled...

When they vote...........

Embassadors, extraordinary and plenipotentiary, rank and func-

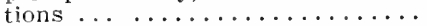

Embassy, right of asylum in American ................ 253 clerlss of and native assistants

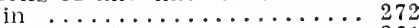
marriages in ................. 261

Embassy, Secretary of, appointment, exarnination and duties. . 270 Second Secretary of, duties... 271 Student interpreters ........ 272

Engineers, chief of (War Departmont). anpointment and duties. . 465

Engineers, Corps of, military and civil duties ............4 465

Engineer officers appointed from West Point .............4434 Engraver for the Mints, duties of. 466

Engraving and Printing, Bureau of 428 Produces all bonds and notes of U. S. pension certificates, bank certificates, commissions, disbursing officers' checks, portraits, etc., postage, customs and revenue stamns ..................

Manufactory maintained by Government ........... 428

Engraving and Printing, Director of appointment and salary.....4428

Envoys Extraordinary and Ministers Plenipotentiary, rank and functions .............. 250

Examiner of Titles to real estate acquired by Government....... 534

Examination of deck officers and engineers of steam vessels..... 792

Examinations, Civil Service..... 819

Exclusion of Chinese.......... 805

Exclusion of Undesirable Immigrants ................ 803 Expatriation of American citizens 246 Expenses of Post Office Depart-

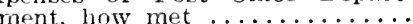


Experiment stations, onice of, scope and functions......... $7 \$ 9$

Federal judicial districts, how divided ................. Is

Fees, consular ............ 306

Copyright ........... 130

Marshili's ............... 5 .1s

Patent offic............. 520

Regist"r in liankruptey . . . . 2 40

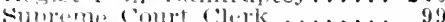

Filled cheese, internal revenue provislons conerning .......... 375

I'lag forecists ........... 763

Flour, mixed, intral revenue provisions eme ruing ......... 375

Fog sismals .............77, $79 \mathrm{~s}$

Folling Roum of llouse and Senate 114

Fonds, arlulteratid and mishmanded, rules and proedure against... T 45

Foreign Governments communicated with directly by President or through Sferetary of State....

Foreign mails governed by Con-

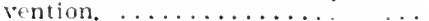

Foreion money at mint ......... 436

Forecasts and warnings issued by Vitither Burtilu ............ 76.1

Forc cast crntres, list of . . . . . 765

Fon'st res.rvations .......... 751

Forrst service, scope and prersonnel

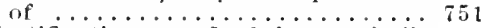

Fortifications and defenses built

by Colps of Engineers..........

Franked mail mattre........6.6. 624

Franking privilege, who has. . . . 6. 6.

Free delivery of mail........... $58^{2}$

Free delivrr. rural .......... 624

Gaugers in Customs Service..... 324

Gaugers in Intrenal Revenue Ser-

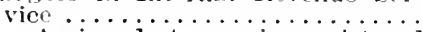
Assigned to each registered nistillerv .................36s

Gencral Arnraisers, how appointed and qualifications ...........

General Iand Otres in charge of Secretary of Interior.......... Assistant Commissioner and Rrenrder of ...........6.6. 693 Commissioner of ....... Clerk of, signs Presldent's name to patents............

General Welfare claus: of Consti. tution. hot construed......27, Gcolngical Survey ............ director of appointiment salary and dutics.......... i2f cconomic and scientific value of work ..............

emnloyes sojected ixclusively for quallfications as professional experts ...........

Reclamation service ........ 729

under Secretary of the interior 159

Gerrymanders ............ 46

Gold bullion of standiard fineness coined without charge...... 435

Gold deposited for colns at mints and assay offices............

Gold Standard for coinage established ................ 431 Good Roiads, Oflice of criport- mint of Arricultur. ..... PArE

Governmernt, its general policis framed by congress ........ 23

Governmental systers of the Uniterl stilles

Governnint clerks, ifpolutinent is $8: 39+1$

correspondence and indexing by .............. \$ 51 distribution of ........... $s+1$.

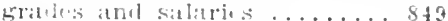
holiulass and vaciations ..... 854 liours and rextric hours. ...... but

prespuets of frometion and re-

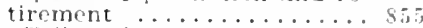
Jague 'ribunal .............275

llawait, omanimetion and ofledals uf Perriterial Gow rnmont..... 735

Irealth, bill of, siven by U. S. Consul . . . . . . . . . 416 Italth, inspection card griven to

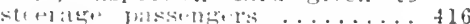

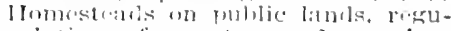
litims for entry and acqulrement ............6.65-69s

Insrital Corps (irmy) organiza-

tion ....................... seliool of Instruction for..... 46,3 llospitils. Naval, of Unit+a states beo Ilouse docments, how distriluuterl. 114 llonse of Yourtsentatives, absence of menilurs ............ \$6 Adjoumments of ........ 117

Arjointive power of members. 52

Basis and apportionment of menbers in ........... 41 .

Clork of election silary, and dutios ................. 110

Clark calls roll on election of speakir ............ 111

Cl.?l holds over from mritolus Congress .......... 111

Cherk of is parmaster of hure emploves

cluk at simalier's talble.... 117

Committees ........... 55

Constitutional powers of and how excreised ......... 49

Doorkener of ............ $11: 3$

Employes of list and sillaries 122 Enrolling Clork of dutius.... 112

File Clork ot, dutifa......... d1

Forrign relitions, its share in. 51

Inotehment is in sole iuwer of

Jomrnal clitlis, dutés. . . . . 112

lembers exomit from arrest. 48

In mbors may he jumished $\mathrm{or}^{\circ}$

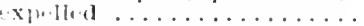

Mremberes privilege of recommenulation of linteritl of tie $r$. . . . . . . . . . .

Irombers molifications ....

Mlilatire, compensation and al. hiwiners of members.... ti

Palr clerk, dutles......... 119

Plivileges and prerogatives of memburs ........... 49

Procendines romerted how... 116

Jrocedelings of legislathe day

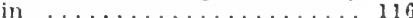


PAGE

Reading clerks, duties....... 112

Reporters of ............ 116

Representation of State may be reduced, when........ 44

Revenue bills must originate in ..............35, 48

Rule clerk, duties........... 117

Rules and preliminary procedures ................ 117

House of Representatives, Sergeant-at-Arms of ......... 112

Speaker of, how chosen......4 $4 \mathrm{~s}$

Vacancies in, how filled..... 46

Tote of, how taken.......... 117

Who may vote for members of. 44

Hydrograpbic Office and $j$ ts publications $\ldots . . . \ldots . . .641$

Illegal distilling ...........

Immigrants, Exclusion of Undesir-

able ................ 803

Impeachiment, charges presented by

House of Representatives...... 36

Chief Justice presides at impeachment of President.....

Grounds for .............

List of cases and results.....

Procedure in cases of. ....30,

Senators sit as judges in cases

of ................... presides, except in case against president ....... 100

Income of United States, main

Sources of .............. 309

Indian Affairs, Commissioner of, appointment and duties....... 715

Indian Agencies............. 715

Indian Commissioners, Board of. 716

Indians, Government in relation to. 713

Payments to ..............716

Lands of .............. 718

Sale of liquor to, prohibited.. 717

Inferior Courts, duties and jurisdiction ................ 183

Injunctions in Federal Courts..... 185

Inspection of boilers and appurtenances of steam vessels ..... 792

Inspection Service, Steamboat... 789

Inspector General's Department. . . 457

Interior, Department of the ....6 692 Assistant Attorney General for ............... $52 \mathrm{~s}$

Assistant Secretary of ........ 180

First Assistant Secretary of.. 178

General Land Office in......693

Presidential appointments in $10, \quad 17$

Interior, Secretary of ......... 156

Guardian of financial affairs of Indians $\ldots \ldots \ldots \ldots \ldots \ldots$

Has supervision of operation of laws relating to pensions, public lands and surveys and Indian affairs........... 157

Has supervision of Government assisted colleges......... 160

Has general supervision of Territorial Governments 161

Internal Revenue, cases in Federal

Courts ................... 367

chief sources of ......... 367
PAGE

collection of taxes, how enforeed ............... 364 collectors, appointment, bonds and duties............... 363

collectors must make monthly statrments ................ 368

collectors, locations, bonds and salaries, list of ........ 382

districts, list of ............ 382

districts, may be consolidated. 363

licenses, to whom issued.... 367

provisions concerning filled cheese and mixed flour.... 375

provisions concerning eleomargarine ...............375

provisions concerning smoking opium.............. 374 service, subordinate officers of. 364

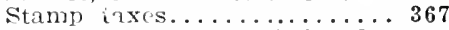

Storekeepers and their duties ............. 364, 369

Storekeepers assigned to each distillery ..............368

Internal revenue tax on liquor, how

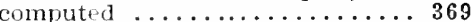

taxation, articles subject to.. 379

taxation, occupations subject to .................... 378

Internal Revenue, Commissioner of, Court of Appeal on internal revenue assessments and collections. 366

has charge of real estate held as security for jnternal revenue taxes.............

Interpreters, student, in embassies and legations..........272, 301

qualifications and examinations of .........278, 279

Interpreters to Consuls......... 301

Internal Boards and Commissions. 275

International Institute of Agriculture ................ 275

International Lake Levels Commission ................ 275

International Prison Commission. 275

Interstate Commerce, not fully defined ............... 813

Interstate Commerce Commission, its membership, terms, qualifications and compensation...... 813 jurisdiction and powers..... 814 chairman of, how selected... 817 duties under Arbitration Act.. 817 jurisdiction over telegraph lines ............... 817

Interstate commerce Law, provisions of .............. 814 Long and short haul clause of 816

Isthmian Canal Commission, qualification, salaries and status of members ............... 834 Chief Engineer, powers and duties ............. 835

Governor of Canal Zone..... 835

Secretary of ........... 835

Judge of U. S. District Court for the Eastern District of Pennsylvania is ex-officio member of Commission to Examine and Test Coins ...............4 434 
Jutge difocate General (Xiavg PAGE Department) ..........6629 Judge Advoente Creval (iva) Department) .............447

Judge Adrocates, duties of ..... 458 appointed by President, with confirmation by senate...

Judges, take onth of impartiality.. $14 \mathrm{~S}$ lemoval only by impeachmint ............19, 184 salary for lifo, when........ 184 wits issiber ly .......... 184

Judicial bistricts, lederal, how divirled ........................ 189 list and boumditios.... 192,203

Judiciary of th. Lnit.d States.... 1s3 See also "Supreme Court."

Jurisdiction of lowr Courts of the United Strites............... 208

Jurors in Trited States Courts, qualifications and pay........ 550 Jury Commissioners in Unitred States, $12: 7 y$ of .............. 552

Justice, D) partmint of (see also "Attorney General") ........ 147

Develnment and present functinns ............... 519

Docket Clark of ......... 539

Passes on titles to sites for public bubinge ........ $4+6$

Ialke Surveys, madt by Corps of Enginerers ............. 460 Land districts, low established... T00

Land office, register and receivers,

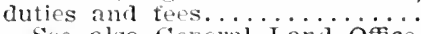
Sue also Genrral Iand Office.

Lands, arid, reclamation of .... 702 Government irrisation of .... 70 :

Land grants to railroads and States ................ 704 Lanrs, pulitic, patrintins of "by

United states ...............69t prices of .............. 703 "Soonce"s" and theip methods in procuring........... 703 Survey of in west.......... 694 Surveyors general, their duties 695

Legation, American, right of asy-

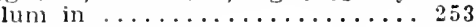
marriaces in ............ 261

Legation, clerlis of.......... 272 Native assistants in......... 272 Secretary and second secretary of $\ldots \ldots \ldots \ldots \ldots \ldots \ldots$ Secretary of acts as charge d'affaires, when..........271 student interpreters.......... 272

Legislative day, proceedings of in House and Senate.......... 116 Letters of soldiers and sailors transmitted without prepayment. 624

Librarian of Congress, appointment, salary and duties...... 126

Library of Congress......... 125, 130

Life-saving Service, board on lifesaving appliances............413 District and assistant district superintendents .........4 408

districts of .............4 407 districts of, section divislons. 411 Superintendent of........ 407
Surervisory boare of ....... 40 s

Surtmen, appointmint, exam-

Ination and (lutios...... $\$ 111,412$

I,ife Silving stations, Korevers of . 409 sulerintionel*nts of constructicin of.............. 413

Lishthoug. Buatre, its personnel : 1 1] functions.............. 75

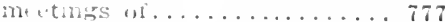

Lienthoust bistricts, administration of $\ldots \ldots \ldots \ldots \ldots \ldots \ldots \ldots$

list and imminerie..........

Ifichthutis kefpers ant assistants, futifs and uniformio

Lighthomses, classification of lights. $7 \mathrm{SL}$ Light vegenls on shouls........ 781

Liglits on stean and siling ves, ls 70 t Mail matt r, alplabetical classificiltion of .................. classification and rates of postage ............. 615 froudu! nt, how disposed of. . 622 fres ............... secomel class, paid in limp sum ............. 623 tilinl class, phid in lump sum, when ................... 623

Mails, cost of carrying. . . . . . 59

Mall reute contracts to be certified to Amlitor for rost Uified Depirtmont ..............6.610

Nalt borrages, porenue provisions concening .................. 373 Marine Lind................ 656

Marine Corms, Enited states. . . . Gst Discinline, mantenance and duties of ................6ss

Eximinations of .......... 685 Oflicers appointed from Nival Acalemy or civil lite...... $6 \$ 5$ Lin. otficers of ...........6.685 Pay tiable of enlisted man... $67 \pi$ I'romotion of non-commissionel officers in ............. $6 s s$ Staff officers of ........... 65 Stations and duty of niarines. 659

Marine IJosnitals, admission to...415 established at various points. H14 frep tratment in to stamen of Antericin Mercliant diarine, $\$ 15$ foreign seaman chirg. d for treatment in........... $\$ 15$

Marine llospital Service; see Public IIealth and Marine IIospital service.

Master of vessels, bond for delivery

of balance of cargo......... 316

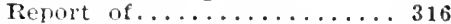

jecenses of ................ 792

Statement and certified involce to be sent to collector by... 313

Masters of merchant vessels engaging seamen abroad must procure consul's sanction........2s7 must procure consul's sanction to discharge seaman in forcign port...............287

must deposit recister and ship's papers with consul... 286

Marshal of Supreme Court....... 97 
PAGE

Marshals of Consular Courts, appointment, duties and salaries.. 301

Marshals, United States, appointment hond and duties....... 542

Chief office dleputies........ 546 deputies of ........... 546 Expenses of............ 547 citrardinary expenditures by. 553 fo list............. 548 C.el deputy........... 546 principal disbursing officer of U. : Courts ......... 550 salaries of ............ 543 s.rice of processes by..... 547 Temporary, how appointed... 545 Mcasurcment and registration of ressels ............... Medical Corps of Army, examination and admission to ....... 463

duties and work of ........ 464

Medical officers of $U$. S. at foreign infected ports, duties of .......

Mexican Water Boundary Commission ...................

Militia of States, organized streligth of ............4 489

President is commander-inchief of, when.........

Frovided by Quartermaster's General's Department with clothing and equipment, when ...................

Military Academy, United States, see under "United States Military Academy.'

Ministers, foreign, received by President $\ldots \ldots \ldots \ldots \ldots \ldots \ldots$.

Minister plenipotentiary, rank and

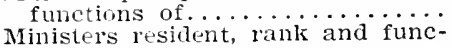
tions ............... 250 may also be consul general... 252

Mint, director of the, duties, term, compensation and reports...429-436

Mints of United States.........430 Coiner of .............. 433

Engraver for............ 4si Torcign moncy may be carried b.

Iricition of ........... 450 Officers of, bonds and saiaries .............430,437

Medals may be struck by mint. 436

Melter and refiner, duties of.. 433

Reserves coins for examination and test............. 434

Superintendent receives and cares for coins........... ün Money, foreign, received by United States must be recoined before 436 being reissued............... partment).

National Bank Notes, how issued and upon what security....... printed on distinctive paper by Bureau of Engraving and Printing ................. received in Treasury, how disposed of.............4423

reserve requirements.......4426
Sent to U. S. Treasury for redemption ............4426

National Cemeteries in charge of Quartermaster Genoral....... 460 National Home for Disabled Volunter. suldiers............866 rectuisites of admission to.... 868

Nitional Parks, reservations of under control of Secretary of the Intrior ................ 731

Naturalization, requircments and certilicates of ............ 809 Naturalized citizens, protection of, by diplomatic olficers.........259

Naval Academy, appointment to and course in ............6 658 Naval liospitals of United States. 666

Naval Observatory ..........640

Naval officers of customs, appointment and juties............ compensation not to exceed $\$ 5,000$ a rear.......... 315

locations, bonds and salaries,

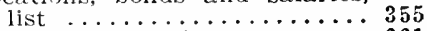

Naval training stations........ 661

Navigation rules for inland waters. 800

Navy, Admiral, flag and insignia. 647

Bureaus of ...........6 630

Bureaus represented on each ship ............6650

Cartains and commanders in. 649

Chiefs of bureaus, term and rank .............6631

Commanding officer of vessel on detached service on foreign station, responsibility of ..............6651

Commanding officers of ships, duties and powers........643

Conflicts of jurisdiction in bureaus of ............6 632

Corps of Civil Engineers in, duties and rank of......671

Courts of Inquiry and courtmartial in ............661 651

Distinctive naval marks, by corps and rank.........657

Engineer officer's on naval vesscls ...............6. 655

Fnlisted men in Navy.......66 661

Enlisted men, additional allowances and nayments to: 679

Enlisted men, distinguishing marlis of ..........6662

Ensigns, duties of ........6 656

Examination of officers for promotion ..........6 680

Examinations for Medical Corps .............665

Executive officer of vessel, duties of .............6.653

General officers, rank of. . . . 645

Honors to be paid to distinguished visitors.........6 648

Lieutenant - commanders, sea duties of ............653

Lieutenants and lieutenants (junior grade), duties of.. 656

List of ships according to

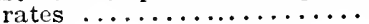

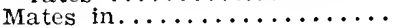


Naviguting ullicer of vessel, duties $1 . \ldots \ldots \ldots \ldots \ldots \ldots .654$

Officers la low rank of commander maly be given commands ................ Oflicers of tlu decek on naval resseds, dutics of ......... 655 Ordnance oflicer, duties of .. 655 Pay uflicers of bonds of . . . 670 Par ollicers, elerks and subordinntes ............ 670

Pay otlicers rank......... 609

Pay, hase schedule ........ 674

pay system............ 672

pay, quarter's allowance.... 674

Pay scherlule of enlisted men. 677

Pay sehedule of officers appointed from eivil life.....675

Pay sclitelule of other officers. 676

Personnel of Medical Corps... 675

Petty otlicers in..........66 601

Petty ollicers, distinguishing marks of ............662

Presid.nt is commander-lnchis of ............ 5

Profesor of mathematies... 671

Promotions in ........645, 6so

Punishment of oflicers and $\min \ldots \ldots \ldots \ldots \ldots \ldots \ldots 6 . \ldots \ldots$

Rank of general ollicers..... 645

Rank of merlical officers..... 665

Ranking officer..........645

Rear admiral, llag and insignia .............6647

Rear admiral, thanked by Congriss, may be restored to

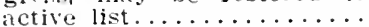

Relative rank of army and nidv officers..........6. 657

Retirel officer may be employed ..............6.6. 684

Retirement compulsory at 62 , except the admiral........ $6 \mathrm{~S} 1$

Retiruments of otlicers in....6 6so

Petirements voluntary and compulsory ...........6 682

Warrant and enlisted personnol under medical corps.

Fetirement of enlisted men... 682

Sea service division of day into watehes ............6. 650

Ships of, nam.d by President. 18

Shore and sua commands in, how disposed of . . . . . . . 646

Vessels of classified ....... G4t

Warrant officers, devices of . 660

Warrant oflicers, gritdes and duties ...............65s

Warrant and enlisted personnel under Medical Corps.... 666 Thatehes on vessels of ..... 655

Sie also "Armed Forces."

Navy Department (see also "Navy,

Secretary of the").......... 154

Assistant Secretary of Navy. 177

Board on Construction, organization and duties...... 644

Bureau system prevails in... $62 \mathrm{~s}$

Bureau of Construction and

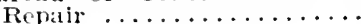

Bureau of Equipment........ burtidu of Medicine atrd sur-

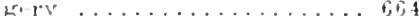

Buratu of Novigation...... tis

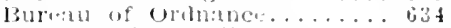
Harnall of Fupplies and $A C-$ counts ............... Buratu we Yarls aml Duclis. ifi

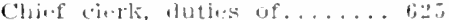
Dislursine paty uflicer, dutits. Gto Gentral butrel of, its functiuns .............64t

Gneral Boiral not orbanizud by statute but by naral repulations ........... 643 Gemeral storekeever of Naby Yatri, duties........... 6f;s

Iydrograthic Office ....... ist

Juilgt Arrocat Ge neral, l.gal atristr of secretary......623

Judge Aavocat, General, powrrs and duties..........629

Judg, Adrocate General, relition to Marine Corps.... 629 Naval Observatory ........ 640 jay ofticers contract for and jurchise sutplies........667 Pay ollicers, dutics of ......667

pily otlicers, cial duties of... Ges

Paymaster (itneral, how appointed ............ 669 Prisidential appointments in 10,17 Solicitor of ........... 636

sincial lyaining scinools for orelnance. for enlisted $m \cdot n$. 635 Nary, sicretary of tle, alproint-

ment, salary and duties...... 154

Directs Naval Militia...... 155

Jurisuletion ofer Island of Guitm and American territory in s.moa......... 155

Reports annually to Congress. 150

New Mexico, government of..... 733

Nurse Conps U. S. Army, composed

of lemales............. 163

Qualitications and appointment of nursus in ............ $4 \mathrm{G}$

Suparintundent of ......... 4b4

Occurations stoject to internal reverte taxition........... 378

othictrs mily be remorid by l'resi-

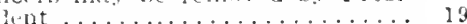

Otticials, miscellanoons, l'pesiden(ia) appintments of......12, 17

Olenentariane, internal revenue provisions concelning. . . . . . 375 Opium (smoking), internal repenue provisions concerning........ 374

Packing houses, sinitary resulrements and insmetion of ...... it?

Pages of semate and House..... 114

yinann Camal in charge of Corps of Engineers.............460

Panama Cimil, ste "lsthmlan Canal Commission."

Pardonine power of Presiant.... 12

Pardons in Army and Nily..... 531 in general.............. 53I itturney in charge of ...... 531 procedure on applicatlons for. 532 rules relating to applications for ................ 533 
Party selection of candidates, not controlled by Federal statutes.. 25 Pässenger and freight rates, discrimination in ........... $\$ 14$

Passengers pelsonal effects and bacroce customs regulations., 338

Passports, amplications for...... 247 wis wom josued........... 245

issuted when ............ 246 embassadors and ministers may issue only on emergrner ............. 258

qualifications entitling to.... 246

requlaly issued may be verified lis diplomatic or consular rificers ......... 25 s

for nersons in Porto Rico and philipines ..........24s

for persons visiting Russia and liverey should be viséed 249

Patent Office, Arpeals from Commissioner's' decisions ........723

Commissioner, assistant commiscioner and examiners-inchific silaries and duties... $71 \mathrm{~s}$

Conditions and requirements for patents ..........718

Fres and prices of publications 720

Interferences and claims of priority ............. 722

Ender enntrol of Secretary of the Interior ........... 159

Pay" Departments, see "Army," "Navy."

Payments from Treasury, only in accordance with appropriation by Congress .............4 438

Payments, see Treasury.

Penalty envelope for official business ..................

Pension agents and payment of ponsions ............. 713

Pension Bureau (Interior Depart-

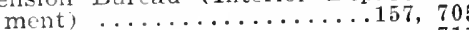

Dirisions of $\ldots \ldots \ldots \ldots \ldots \ldots 711$

Division of special examination ............... 712

Procedure in ............ 712

Pension certificates, produced by Bureau of Engraving and Print-

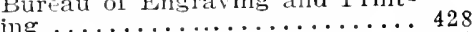

ing $\ldots$. .

Pensions, Commissioner of, subordinate to Secretary of the Interior ................ 157

Commissioner and Deputy Commissioner, appointment and salaries .......... 706

Civil IVar Pensions, amount of at various periods..... 707

Civil War Pensions for superior officers ......... 710

for various wars.......... 706

invalid and service.........705

vouchers for, fourth-class postmasters may authenticate. . 574

Philippine Commission, organiza-

tion and powers...........829

members of, appointed and compensation ........8830
PAGE

Philippine Islands, Rill of Rights

of $\ldots \ldots \ldots \ldots \ldots \ldots \ldots \ldots . \ldots 30$

character of govermment in... 833

judicial system of ........ 832

Philipuins Assembly, nemberslipre terms and sessions...

Rosidint Commissioners to

United States............ 832

School system in.........8833

Porto Rico, officers of report to

Secrotary of State.......275, 736

orginization and oflicials... 736

Salaris of officers of......737

Ports at which merchandise may be intered for transportation to other ports without appraise-

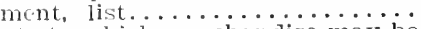

Ports to which michandise ma be transported without appraise-

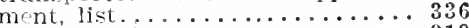

Ports of Delivery ........... 312

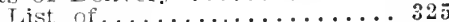

Ports of Entry............ 312

List of ............. 325

Only, may be entered by vessel cirryins dutiable goods.... 315

Postal contracts, certified to Au-

ditor .........600 600

regulations concerning ...... 609

Sections and terms of con-

tracts .............6 609

Postal conrentions, Postmaster

Genonal may conclude ....... 598

must be approved by President 598

Postal rates, domestic......615, 619

Postal rexenufs, sources of .....6 612

Postal subventions and treaties, payment of to carriers of foreign mail ..............611 conditions of payment........611

Postmaster General, appointment, Salary and duties.......... 150 Contracts for mail supplies.. 152 Exclusive appointment of fourth-class postmasters.... 152

Fith in cabinet rank....... 154

Exclusive right to establish post roads ............ 152

Postal conventions and treaties concluded by .......... 398

Removable only by consent of Senate .............

Term extends one month beyond that of President appointing him.......... 150

Fostmasters, appointment and eligibility ..............568 assistant, appointed by postmaster ............. 579

Classification and compensation ............. 570 commission on money order business ............ 572

fourth class, compensation of. 572

fourth class, how appolnted.. 569

fourth class may authenticate pension vouchers.........574

may transact other business.. 574 subordinates of, under civil service rules...........574 temporary, compensation of.. 572 
PAGE

Postoflice D.purtment, assistant attornfy general for......... 528

Auditor for, contracts and orders affecting contract nall routes to be certified to....

Assistant Postmastir Generai, their duties ..............

First Assistant Postmaster General, duties of ....... 566

Second Assistant Postmaster General, dutles........... 590

Second Assistant has charge of Railway liail service..... 591

Second Assistint has charge of Division of Foreign ialls.

Second Assistant has charge of Division of Railway Accountants .............

Sccond Assistant bas charge of Division of Contracts..... 609

Second Assistant has charge of Division of Inspection..... 610

Second Assistant in charge of marment of Postal subventions ............6. 611

Thirel Assistant postmaster Generai has charge of mat ters of postal finance..... 612

Third Assistant has charge of Division of Finance.......614

Third Assistant has charge of Division of Stamps.......

Third Assistant has charge of Division of Money Orders. .

Third Assistant inas cliarge of Division of Registered Mails. 615

Third Assistant has charge of Division of Classification . 615

Fourth Assistant Postmaster General controis Rural Free Delivery ...............

Fourth Assistant controls $\mathrm{Di}$ vision of Supplies.........

Fourth Assistant controls $\ddot{D i}_{-}$ vision of Dead Letters.....

Fourth Assistant controis Division of Redemption......627

Post Office Department, Chief Clerk

of ................... 562

Post Office Department, Divisions of :

Division of Contracts has charge of "star route" lettings and contracts for steamboat mail service. .609, 610

Division of Classification.....6.615

Division of Dead Letters.... 626

Divislon of Equipment......6 611

Division of rinance........6.614

Division of Foreign Mails.... 598

Division of Inspection.......611

Division of Money Orders....6 614

Division of Railway Adjustment .............6.607

Division of Railway Mail service ................ 591

Divlsion of Redemption .......66 626

Division of Registered Mail.. 615

Division of Stamps........6.614

Division of Supplies..........6.625
Post Office Department, exponses of, how m.t..............612 Foreign matis governed by emventions ........... 598

Forfign mails, rogulations concoming ............ 605

Fortisn malis, soo al a "unirersal Postal Lnion."

P'ost Untief inspretors, dutirs, salary and bonds......565, 566

Monry Ortier Accounts separately kfpt............ 613

Ion .y Orders, how paid ...... 613

Purcilasing Arent, duties of. 562

Reverutes of, under control of Postmister Gineral ...... 560

Pural Free Inlirery, its origin, growth and scope.........613

Post Office, Assistant postmasters

and casinirs, bonds of ....... 5.81

Enx-rents in ........... 599

Post Offices, Carriers, wamination, qualification and appointment... 5\$4 bonds, duties and uniforms... 585

leave ot absence......... $5 s 6$ overtime not allowed............ 584 rules of work and conduct.... $5 \$ 7$ substitute carriers ......... $5 \$ 6$

Post Oflece, Civil Servic exaninations of postal distributing clerks .................. $5 s 0$

Clerical assistance to postmastors and allowances for same.............. 580

to emninyees of, salary iist...5 574

Promotions of cierts and carriers ............... 578

Regulations eoncerning ...... 573

Fifnt allowance for......... 552

Special delivery service...... 5ss

Substitute postal cleris and carriers ................. 578

Subrintendent of deivery... 586

Post roads, how estabilished...... 627 President of Senate, Tice-President

is $\ldots \ldots \ldots \ldots \ldots \ldots \ldots . \ldots \ldots$

President pro tempore of Senate,

elected liy Senate............. 101

Acts in cases of absence or deatin of Vice-President, or when latter becomes President ................. 101

Has emoluments but not title of Vice-President ........ 101

President of the United States, appointments of requiring confirmation, list of ............6-12

Appointments of, not requiring confirmation, llst of ......16-18

Appointments during recess of Senate...$\ldots \ldots \ldots \ldots \ldots$

Armed forces may be used by on request of Governor of State

Armed forces used by to enforce criminal laws.......

Bills signed by before adjournment of Congress.........

Bills signed by within ten days after passage $\ldots \ldots \ldots . . .14$ 
sulumited to $\ldots . . . . .14$

Ciril service rules may be pro-

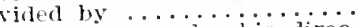

Coast survey under his direc-

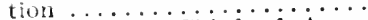
Commandir-in-Chief of Army and Nary ... Cinief of Mili-

Commander-in-Cinef of Militial, when ..............

compunte for traveling.......

Consular selvice under his su-

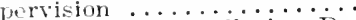

Designates who shall sign Department mail in absence of

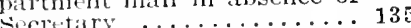

Directs Cibinet oflicers..... 23

Duties with relation to domestic aministration ........

Duties with relation to foreign governments ............

Foreign Governments communicated with directly by him or through Sccretary of State $\ldots \ldots \ldots \ldots \ldots \ldots$

Impeachment of $\ldots \ldots \ldots \ldots$.

May direct details of officers of Army and Navy.......

Iay discharge debtors from debts due U. S., when......

May discharge soldiers and sailors $\ldots \ldots \ldots \ldots \ldots \ldots$.

May select commandants of

Messages of, to Congress.....

Ministers, foreign, received by.

Noninating power ..........

Not bound by set rules in niethod of administration of constitutional duties ......

Obligation of $\ldots \ldots \ldots \ldots \ldots$

Officers may be removed by..

Pardoning power of . ........

Pardoring power, limitation

patents to public lands issued

belation to his political party.

Removal of ...........

Reprieves granted by.......

Safety of, in charge of secret Service ..............

Sale of public lands authorized by Congress, directed by....

Ships of Navy named by.....

Term of $\ldots \ldots \ldots \ldots \ldots \ldots$

Transportation on Government

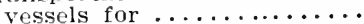

Treaty power of .........6-19

Teto power of ........... 13

Teto passage of bills over.... 14

Presidential candidate, low nomlnation of is made............ Presidential electors, dispute as to

legality of election.........20-21

Vote of, how certified and counted $\ldots \ldots \ldots \ldots \ldots$

Presidential succession, order of. . Prisons and prisoners, superintendent of, appointments, salary and duties ............553, 554
PAGE

Prize jurisdiction of U. S. Courts. 210 Protective tariff, theory and practice of .................. 310,31

Public I Tealth and Marine Hospital Service collects and dissemiliates health statistics........4 417 Compensation of officers of. . 419 deports immigrants having disabling disease ........4 417

Employes of .......... 415

Examines immigrants for infictious diseases constituting disability .........

Examinations and qualifications for assistant surgeon in ............. 418

officer of may be detailed to office of consul.......... 294

Official seal of ............ 418

Quaral tine matters in charge of ..............4 416

Rank and precedence in ...... 417

Secretary of Treasury is head of $\ldots \ldots \ldots \ldots \ldots \ldots \ldots \ldots \ldots$

Surgeon General of, appointment and duties.........4 414

See also "Marine Hospitals."

Public lands, patents to, issued by

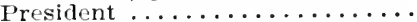

Patents to, clerk signs name of President ...........

Sales of, authorized by Congress and directed by President ................ 18

Unsold railroad grants....... 705

Public printer, appointment, salary, bond and duties........... 859

Public printing office controlled by

Jolnt Committee of Congress... 858

Chief clerk of, qualifications and salary ........... 859

Docunients issued by........ 861

Foreman of printing and binding ............... 859

TVorkmi in ................ 860

Quarantine, coördination of $\mathrm{Na}$ tional and State authorities in. 417 regulations for, made by Secretary of the Treasury .... 416

State and National........4 416

Time of detention of vesseis in. 417

Vessels from foreign infected ports are all in........... Quartermaster General's Department of Army............459 Has charge of National cemeteries .............4460

Militia provided with clothing and equipage by .........

organization, personnel and duties ............... 459

Supplies camp equipage and transportation to sailors and marines on shore duty....

Transportation, clothing and equipment furnished to Army by .............4 459

21 Railway mail pay, how adjusted.. 607

Railway Mail Service under Second Assistant Postmaster Gen-

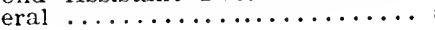


PAGE

Boare of Promotion and rules Soverning promotion ...... Bunls of cluks, and how appointint ............ 593 C!rks ot, rxaminations and dutios 595

Clerlis, salatries alul classes... 592

Divisions of ............ $59 \overline{3}$

Expenses of ollice l's and clerks in ................ 592

general ascistint, division and assistant division superintendont of, salaties...5591, 592

Pallway mail tratssportation, in clrarge of Division of Railway Adjustments Post Ollice Jebart-

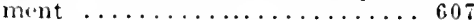

Railway post offices, what are... 595

Reappraisement of gools, Collector of Customs may order, and importers may demind. . . . . . . Reftrees in binkruptes, duties and $\mathrm{f}+\mathrm{es} \ldots \ldots \ldots \ldots \ldots \ldots \ldots \ldots$

Registers and receivers of publie

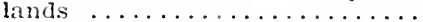

Register of the Treasury, appointnont and salary............

Currency and national bank notes for rodomption recived, exanined, arranged and registered by.........

Plepares, signs and issues bonds of U. S. . . . . . 423,

Signs all U. S. notes and pa-

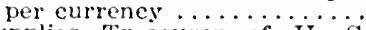

Supplies Treasurer of U. $\mathrm{S}$. with list of registered bonds. 427

Transactions in registered lonts recorted by........

Transfers of money from U. S. Treasury to sub-trasuries or depositors are sinned by.

Registered inail system..........6. 615

Regristered vessel, sale of .......... 32.

Registration of foreigm-born children contitled to citizenship.....2 247

Registration of vessels............321

Reintier impurtod to Alaska in charge of Commissioner of Edu-

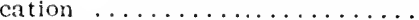

Removal of Causes from state to Federal Courts .............

Reprieves, Presilent may grant.. Revenue of United States, chief sources of ...............

Revenue Cutter Service, under Secretary of Troasury ...........

Cadets of, apprintment, qualification, cximination, training and salar...........

Comparative rank of officers and uniform regulations of.

Discipline and equipment....

Wulisted men of . .

Llst of commissioned, warrant and petty ofleers and enlisted men .............4404

Oficials, scope and functions

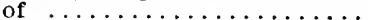

Pay of offlcers, petty officers

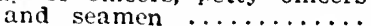

Transfurred to Navy in time

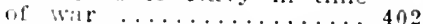

its wark in Aliskil.............. 403

Revinue futur stations.......39s, 400

Piver atml linlur jmprovements,

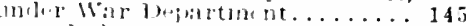
marl. ly Groms of Enginiers. 460

Siffuly aluliancts on rableads... 816

sililing rulus for vossuls........ T9S

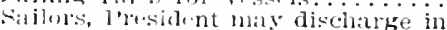

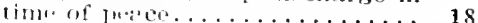

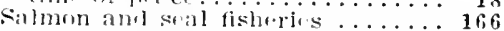

Sor storis. 1.xwmpt from duty..... 316

Sitilskin ginmouts, rogistrition of. 340

Seanmen, Imiriean, cannot be dis-

clinlwori in forejirn port without

intrervation of consular offeer.. 2SS

Consul may ciluse arrest of

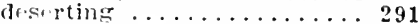

Consul may care for ship. wrelied or destitut.....2 290

Entitled to juotetion of con-

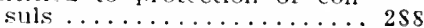

Sicret service, chifof of, salary and duties ...................439

Secretaries of Exicutive jopartments, calinet and general du-

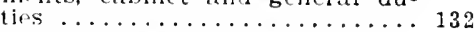

Sceretary of Agriculture, see "Asriculture, Sceretary of."

Sceretary uf Commeree and Labor, see "Cummerce and Labor, Secretary of."

Secretiry of the Interior, see "Interior, Scerctary of the."

Secretary of the Navy, see "Xavy, Sincretary of."

Secretary of State of each State must ecrtify election of Presi-

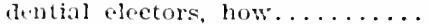

Secrutiry of state, apjointment, siliry and dutits........... 135

Custodian of seal of United

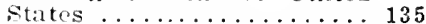

Otlicers of Porto Rico report to ...................... has diretion of affairs in Porto kico ................ 135 mankins member of eibinot... 135 730 Secretiry of sitit", suc also "stite, Dejurtment of.

Secretary of Treasiry, see "Treasury, Secretary of."

Seevetiry of War, see "Mar, Seeretary of."

Sergints of lrms of Somate and Jouse of Renresentatives..... 112

Senate, origin and purpose of .... 29 Camnot originaterevenue bilis. 35

Commitees of (stanting and

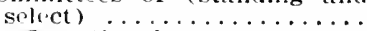

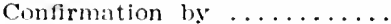

Fmployes of list and salaries.

Exclusive power of contirmation of litrge number of prosidential nominations ...

Exclusive prerogative of acting on treaties with foreign

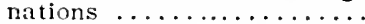

Nembers, their legislative 
riglits. privileges and duties (see also "Senators") ..... Proible Tictoresident of [nited states is.......... Presidtent's pro tenpore from foundation of Corrmment. Prne edinss of legislative day 110 rocerings ponted how.... 116 Sertiv of, election, salary and dities .................... 110 Sersint at Arms of....... 112 - vial sessions of (list).....

Viee-plesident of United States

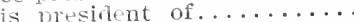

Senator exempt from arrest.....

how appoint d to fill vacancies mole of election of......... nomination of military and

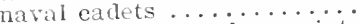
reommanos lederal appointments in his state, if of samis narty as Prosident...

sit as judges in cases of im-

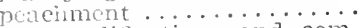
telm, qualifications and comnensation .............. Sematolial courtess, its extent and

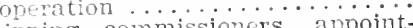
Shipping eommissioners, appointment, bonds, ruties and fees....

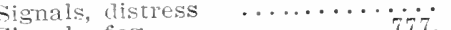
signals, fog ............77, 798 Signals, warning (weatier bureau) 764 silver, notv only subsidiary coin... 432 umefined, may be made into bo ${ }^{*}$ and ingots by nints. . 433

Smithsonian Institution, its organization . Astropirsical Observatory of. 863

Pnard of Regents of........ 861

Collections of............ 862

Zölogieal Park .......... 863

Smithsonian Institution, Secretary of ............................. 869

director of National iisuseum. $\$ 62$

Soldiers, President may discharge in time of peace............

Solicitor General, appointment, salary and duties........... 520 becomes Acting Attorney Gen-

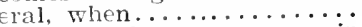
prepares and argues cases in Supreme Court .......... Solicitor for Department of Commerce and Labor........... 529

Solicitor for Department of State. $\mathbf{5 2 9}$ Solicitor of Internal Revenue.... 376 Solicitor of the Treasury.......5 526 Spanish Treaty Claims Commis-

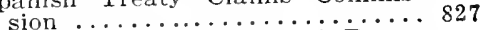
Speaker of the House of Repre-

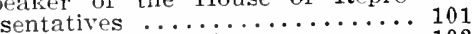
Appointments by .......... 102 Assignment of committees by. 108 Assignment of commitrol over legislation...... 102 Counting quorum ................. 104 Disciplinary power of.......... 107 Fills vacancies on committees. 109 How and when elected....46, 107
Increase of power of ....... 101

Hay appoint speaker pro tem-

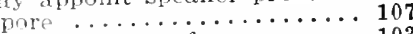

Jorning levee of ........ 103

Powars and prerogatives conferred by rules......... 102

Pecomition of members by ... 103 Signs all acts and resolutions of House ............. 104

Statutory duties and salary.. 102

Spealier pro tempore, appointed by Speaker or by House, when..... 107 Spealiers from foundation of the Government, list.......... 39

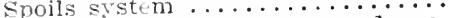

Stamns, postage, customs and revenue, produeed by Bureau of Engraving and Printing.........428

State, Department of (see also "Sceretiry of State") ........ 132 Ascistant Secretary, appointment, salary and duties.... 169

Second Assistant Secretary, duties of ............. 170

Third Assistant Secretary, dutifs of ............. 170

Eureau of Accounts ........ 244

Eureau of Appointments ..... 244

Eureau of Citizenship ...... 244

Bureau of Indexes and Archives

Purea Polls ............. 245

Eureau of Trade Relations, duties of chief of.......249

Chief of Bureau of Trade Relations is intermediary between State Department and Department of Commerce

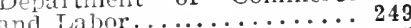

Consular Bureau ........... 245

Contingent Fund of, expenditures from ${ }^{\text {thich }}$ may be tures from report...... 137 made $\pi$ ithout report.j. 243

Diplomatic Bureau, its duties. 243

Presidential appointments in 6, 529

Solicitor for $\ldots \ldots \ldots \ldots \ldots$ raised by

State revenues chiefly raised by 310

direct taxes $\ldots \ldots \ldots$........ $\ldots$ lights

Steam and sailing vessels, lights 797

on .................... Exspection

functions of $\ldots \ldots \ldots \ldots \ldots \ldots 78$

Inspector general, appointment, salary and duties.... 789

Steamboat inspection districts. 790

Supervising and assistant in-

Supervising and assistant 790, 791

Steam vessels, inspection of boilers and appurtenances and examination of deck officers and engineers of .............. 792

neer of ...................... 420

Sub-treasury at New York, depot of deposit and withdrawal of bullion .................

Superintendent of Mints, duties, powers, reports and statements. 430

supervising architect of the Treas144 ury Carries on negotiations for sites of public buildings.... 446 
Powers, duties and responsibilities of ...........447

Supreme Court of the United States 89 Assembles on second Monday

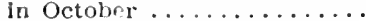

Calls on President to pay re-

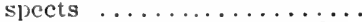

Cases before, precedence of...

Chiep and associate justices of how apyointed..........

Chlef justice presides in case of impeichment of President ................... 100

Chiefly occupied with appeals. 93

Clerk of, duties and compen-

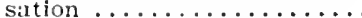

Clerk of, table of fees.......

Decisions prepared by single

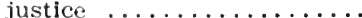

Dissenting opinion of justices of $\ldots \ldots \ldots \ldots \ldots \ldots \ldots$

Independent of jegislative and executive powers .........

Julicial power of . . . . . . . . .

Justices assigned to circults. .

Justices to loold at least one term in their respective circuits in each two years....

Marslial of, duties and com-

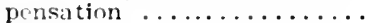

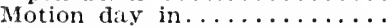

Original jurisdiction, when it

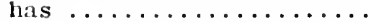

Proeflure upon appeals and writs of error...........

Procedure for removal of causes from highest State

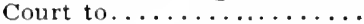

Reporter of, duties and com-

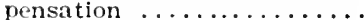

Salary of justices of .......

Tenure of justices..........

Writs of mandamus, certiorari and injunction from. Surgeon General of Public Health and Marine Hospital Service.... 414

Surveyors General ...........695

Tally clerks of House, duties and

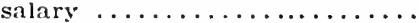

Tariff, protective theory of, maintained since Civil War......... 311

theory of low.......... 310

theory of protective........ 311

Territorial Governments, under Department of Interior........733

Territorial judges, apnointed by President for four-year term...231

Territorial judges, federal jurisdiction of $\ldots \ldots \ldots \ldots \ldots \ldots \ldots$

Tobaceo and cigars, revenue provisions concerning........... 374

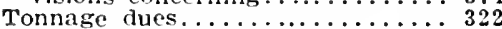

Trade-mark cases, fees in...... 725

Trade-mark registration, how pro-

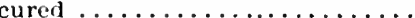

Transportation of dutiable goods in bond.................. 312

Treasurer of the United States.419, 424

Appointment, bond and salary. 420

custodian of precious metals coined into money........422 fiscal agent for bond issues of dependrneies ...........4424 issurs all laper currency.... 422 national bank notes sealed by. 422 national bank notes redeemed

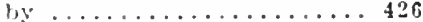

recuipts for all moneys....... 426

reecivs fines, forfeitures and proceeds of sales of public linnds ..................

regulitor of small change of country ............... subsiary coin to polnts where needed ........... rustee of funds undrr care of

Unitrd States .........4 424

see also "sub-treasuries."

Treasury, Comptroller of, chief of auditing systom of Treasury Department .............. 442

Appointment, salary and dutios ................4442

decides ruestions relating to apropriations ......... 140

decidis on lerality of expenditure ............... 443

decisions of, published in series of volumes............. 444

may be called on by departments for advance decision on legality of expenditures. . 444

Treasury Department (see also "Treasury, Seretary of the," and "rreasury")........138, 309

Assistant seeretaries.......... 171

Cbief clerk of Treasury Department, duti+s of ......448

has charge of furnishing remirs and personnel of Gorfrmment buildings all over

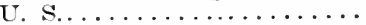

Division of Bookkeeping and Warrants ............ 444

Payments fron Treasury made only in accordance with appropriation by Congress....

Payments on judgments by Federal Courts..........4 438

Presidential appointees in....

Public moneys collected must be paid into Treasury....4421

Solicitor of the Treasury..... 526

Supervising Architect of the Treasury .............. 144

Treasury, Secretary of the, appointment, silary and dutles: second in Cabinet..........137, 144

custodian of bonds.......... 144 directive officer in production of coins............ 143

has charge of erection of public buildings of U. S. outside of Washington........... 144 has direction of bond issues.. 144 head of Customs Service.... 139 head of Lifc-saving Service.. 143 head of monetary system.... 141 jurisdiction over Bureau of Engraving and Printing.... 143 
may compromise and settle claims against debtors of U. S................ 140 nower to relieve tight-money maret ............. 142

Quarantine regulations made by ..............440

Renorts directly to Conunress ...........23, 141

Treaties, hois prepared......... 264

Extradition ..............264

how ratified............ 33

Trustees in Bankruptey, duties and

fees

Uniforms, $\pi$ raring of, $b y$ consular oficers ............. 237

Wearing of by diplomatic oflers ............. 254

Lnited States Attorness, appoint-

mont ot..................... 535 emplorment of stenographers, experts and interpreters... 540

fixed salarios of ......... 536

may engase in private law practice ............ 540

may kerp records..........5 54 personal expfnses, how paid. 423 special reports in appeal cases. 541 temnorary appointments..... 585

United States Commissioners appointed by District Court, powers and jurisdiction.......... 236

foe list ............. 238

United States Courts, clerks of. their appointment, qualifications, bonds an.l duties........555, $55 \mathrm{f}$ fees and compensation of clerks of ..........557, $55 \mathrm{~s}$

venue of criminal actions in. 215

United States Circuit Court, places and terms of ........216, 226

United states Court for China... 260 entir ly und ror jusdiction of State Demartment........2 276

United States Military Academy (see "Military Academy," "War Department.")

Pay table of nfficers at.....493

Universal Postal Union, agreement

in $f f \in c t \ldots \ldots \ldots . . \ldots 98$

comtries in, list of ........ 593

comutries not in, but reached through Postal Union countr...............602

mail matter within, classification of ............. 003 rates of postage within...... 603

Vessel arriving must report to col-

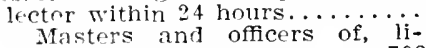
censed how..........792-795 must special report on distilled spirits and wine......... notice of merchandise to be renorted on ...............315

vesel putting into $\dot{U} . \dot{S}$ port in distress, unlading of goods is nermitted when ........3 317

Vessels, registration and measuremant of $\ldots \ldots \ldots \ldots \ldots \ldots 79,797$ Vicr-president of the United States, lt ction, quilifications and sal-

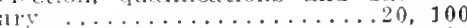
Aprointments by........... 101

Fremnt flom militia duty.... 100

lix-officin menther Board of Rrurints of Smithsonian Institution, and apjoints three numbers of sam for Senate. 101

Presides in cases of impeachment, Excert of President. 36

['rsifint of Senate duties as moi . 100

sims all bills which have masor both houses........ 101

suce eds to presidency on death of President........ 100

War Departm nt (see also "Trar,

st cretary of," also "Army"... 17t

Assistant Secretary of Tivar, dutios of ............ 174

Chance of system in since $1903 \ldots \ldots \ldots \ldots \ldots \ldots . . \ldots 49$

Chies of Enginefrs, appointmfrt and dutiss.........4455

rivil dirfetive officials....... 451

Corps of Engineers, military and civil ruties.......... 465

has control of National Guard and other State forces..... 450

Insncetor General's Departmint, duties and organization ............. 457

Judge Advoncate General's Denartment, duties and organization ........... 457 Judge Adrocates, duties of ... 458

Pay tahle of officers....... 492

Pay table of officers at Military Amdemr........... 493

Presidential appointments in. 8,16

Signal Service of ......... 146

Varving theories of military administration ......... 449

War Scretary of, appointment, salary and duties (third in cabinet in order of precedence) $\ldots 144,147$

Has custody and control of the parks of Washington...... 146

has dirfetion of arministration of Philippine Islands...... 146

has general oversight of Canal Zone mitters and the building of the Panama Canal.. 146

has supervision of the Corps of Army Engineers....... 145

Reports to Congress........ 145

War taxes levied and collected through Internal Revenue...... 367 TVar veteran employes of House of Renresentatives ............ 114 Warehouses, bonded and public... 321 withcirawal of goods from.... 321 Weather Bureau organization, sta-

tions and forecasts..........759 Chief of, appointment and duties ................. 758 Flag forecasts .........763 Forcensts and warnings issued $\mathbf{7 6 4}$ how weather reports are collected ......759

list of forecast centers.......7765 
INDEX.

xxiii

list of Weather Bureau sta. tions ............ 766 management anil equipment. 759

Warning signals ........7 764 Weights and Measures-jurisdic-

tion of Bureau of Standarls over 809

Whiskey, straight anrl biended.... 371

Witnesses in United States Courts,

pay and mileage........... 550

Woman marying foreigner tak PAGE nutionality of her hustong takes may resume citizenship by registration or residence..... 246

Woman of for ign birth may acquire citizenslip by marrying American ..............246

Yhllow fever, work for suppres$\operatorname{sion}$ of $\ldots \ldots \ldots \ldots \ldots \ldots \ldots \ldots 417$ 


\section{CHAPTER I.}

INTRODUCTION-PRINCIPLES OF THE GOVERNMENTAL SYSTEM OF THE UNITED STATES.

In compiling this volume it has been the purpose to summarize the duties and powers of the officials of the Federal Government in a statement in regard to each, which includes such information as will be needed by the general reader. There has been little attempt to include verbatim citations, or to give the very many references on which the statements are based, since it is desired to simplify the presentation as much as possible. The sources are the Constitution of the United States, the Revised Statutes and regulations promulgated by the heads of the different Departments. In addition to these authorities there is also a considerable amount of unwritten custom and usage, which has been stated in as definite tcrms as possible in connection with the specific provisions as to the duties of Federal officials.

The body of law made up of provisions relating to the public officers of the government is widely scattered and not easily attainable by the layman and general reader. It is believed therefore that this compilation will be found not only a source of interesting general information, but a valuable book of reference as well.

\section{THE GOVERNMENT OF THE UNITED STATES.}

The Federal form of the government of the independent States, composing the United States of America, has been described as a government of laws and not of men. How accurate a description this is will be seen in the following pages, which will deal chiefly with the restrictions and requirements imposed by statute upon the agents of the Federal government. Such a book as is here proposed would not naturally, and in the usual order of 
governmerital aftairs, so iargely deal with restrictive enactments. In most of the governmental systems of the world, scope, in some cases comparatively wide scope, is given to the discretion of the official functionary, and his powers and duties are stated in wide terms or exist in a condition of amplitude, because of the traditions and lack of restrictions which surround his office. It is, however, entirely different with the incumbents of Federal offices in the United States, from the highest to the lowest. The power to do all things necessary for the proper conduct of an administrative position is seldon or never granted in the Fuleral system. Sn much is this the fact that the language of a recent act creating the Board of Exposition Commissioners with "power to do all things necessary" is almost unique in the history of Federal iegislation.

The tangible evidences of the existence of a Federal government, which were represented in a former generation only by the iocal postmaster or the local collector of customs or internal revenue, become more definite as the United States is forced, through the increase of population and the decrease of state distinctiveness, to enter more closely into the social and commercial relations of the people. The fabric of the United States courts, formerly to the popular mind a nebulous and little understood system of jurisprudence, comes with the increase of Federal penal legislation to occupy a larger and larger share of the public attention, since it is in this manifestation of the Federal power that the increased activities of the Federal government come to popular notice. The manifestations of the Federal government can, however, only be understood by bearing in mind that there is no single Federal power as such, but that the operations referred to are those of three co-equal branches, the distinction between which and the powers of which were carefully prescribed by the Constitutional Convention. The provision for each branch was carefully framed to meet a particular need which presented itself to the founders of the government. The conflicts of the formatory period of the present British governmental system, the influences of the school of thought contemporary with 
the beginnings of the French Revolution and the ardent and passionate discussions which preceded and accompanied the Convention which formed the Constitution of the United States, together with the experience of the colonists under the first Federation, made it very definite and clear to the framers of the Constitution iust why each of these separate branches was established and just where and why their several powers were limited. Thus they provided a dual legislative body whose functions were established $b y$ the fact that it was composed of representatives of all the people of all the states and whose powers vere definitcly linited by a distinct grant and a distinct reservation. Secondly, the execntive branch, with no discretionary power to cxtend provisions made by the legislative branch was also given a means of check upon the legislative iunction, and for the purpose of adjusting not only the relation between the legislative and the executive powers, but for the purpose as well of conserving the rights of the independent State and the individual citizen from the possible eneroachment of the other two branches of the government, there was established what has been described as the crowning effort of the Constitution makers, a judicial power which is independ(nt of both the legislative and the executive, and whose action constitutes the final step necessary to formulate the will of the people into a portion of the governing law of the nation. The executive may propose but cannot execute until the legislative has approved. The lecrislative nuy both propose and aprove. but is subject to a qualifed veto by the executive, and in neither case can there be final approval of a measure affecting the right of any citizen until the question has been passed npon by the highest court of what is in effect the highest power, namely, the judiciary. Understanding from their previous experience the tests which the new nation wonld be called upon to undergo, the framers of the Constitution deliberately and with a premonition of some, at lenst, of the later developments, conceived and executed a plan of government which seems to have surpassed in its practical benefit that of any nation, ancient or modern. 


\section{CHAPTER II. \\ The President of the United States.}

The characteristics of the executive power of the United States was the most serious problem relating to the personnel of the sovernment undertaken by the Constitutional Convention. Of all the provisions it was only matched in importance by that fixing the relations of the States themselves. The Articles of Confederation made no provision for an executive head of the sovernment. Two well-defined schools of thought, whose influences were felt for n110re than half a century, were already established among the delegates to the Convention. There were, on the one hand, those who doubted if a successful government could be maintained, unless the executive head had some kingly prerogatives. On the other hand, there were those who vehemently denied the right of personal prerogative, either inherent or conferred, and who could comprehend nothing but the destruction of a representative form of government whose executive officer should be more than a superior clerk. As a mutual concession, there was an entire lack of definition of what constituted the executive power. No provision is made in the Constitution prescribing or limiting the executive powers vested "in a President of the United States of America." Definite provision is made as to the President's duties with relation to foreign governments, but as between the States themselves, the duties of the President are left to be determined by the course of events, subject to the checks which Congress may from time to time place upon them.

The President of the United States must have been born within the United States. He must have attained to the age of thirty-five years, and he must have been fourteen years a resident within the United States. In practice the President is always chosen from another State, and usually from another section 
of the country, than the Vice-President. This is to an extent, founded upon the provision of the r 2 th Amendment of the Constitution, which directs that when clectors vote for President and Vice-President, one of the persons so voted for shall not be an inhabitant of the same State with the electors. The President may not receive during the period of his office any emolument from the United States or from any of them, other than the compensation fixed by Congress, and which may neither be increased nor diminished during the period for which he has been elected. This compensation at the present time is fixed at fifty thousand dollars, and he is allowed in addition the use of the official residence with the services of the persons attached thereto, as prescribed by Congress, and has as the supervisory official of all departments, free transportation on all vessels owned by the government of the United States, but receives no allowance for subsistence or entertainment, except that by a recent provision of Congress an annual appropriation it made to pay his expenses when travelling.

While the powers of the President with respect to domestic administration are left largely to the discretion of the Congress, and to the definition of the Supreme Court as to what constitutes executive power, distinct provision is made for the powers which he shall excrcise; first, with relation to foreign countries; second, with relation to the appointment of the subordinate officers of the government; and, third, with reference to the exercise of the supreme power of clemency. Under the first head he is the Commander-in-Chief of the Army and Navy of the United States, and is placed in control of the militia of the varions States, when it has been called into the actual service of the United States. With the details of the organization and direction of the militia in time of peace he has nothing to do, except so far as Congress may direct Federal aid to be placed at the disposal of the States for the improvement of the efficiency of the militia upon compliance with certain requirements. But when the respective quotas of the States have been tendered to and enrolled by the United States, they come under the command of the President 
as the Commander-in-Chief of the armed forces of the country. These powers of the President have, largely, in practice been put into commission under the direction of the respective Secretaries of the Departments of IVar and the Navy, but the condition has been the fact, in all the wars of the United States, of a civilian in supreme control of the military operations of the United States. The reasons for this provision were undoubtedly two-fold. First, - because of the independent powers of the President in dealing with foreign affairs; and, second, because the framers of the Constitution undoubtedly had in mind the impending Presidency of General Washington. and the fact that many of the prominent men of the immediate time were successful military leaders of the Revolution. The provision by which the President is made the Commander-in-Chief of the Army and Navy places upon him the burden of responsibility for the successful outcome of the military operations of the country, and while, as has been stated, the details have been for the most part entrusted to subordinates, - the President himself is actual director of the military power of the Republic. The President has power to make treaties with , foreign countries, subject to the advice and consent of the Senate, two-thirds of the Senators present at the time of the vote concurring: The treaty power thus bestowed carries with it the necessity of military power, which is conferred in the first clause of the section conferring the treaty making power.

- The second in importance, of the defined powers conferred upon the President, is that of nominating all officers whose appointment is not otherwise provided for under the Constitution. The list of these classes of Presidential nominations, requiring the confirmation of the Senate, is as follows:

STATE DEPARTMENT.

Secretary of State. Assistant Secretary of State.

Second and Third Assistant Secretaries of State. Ambassadors. 
Ministers.

Consuls General

Consuls.

Ministers Resident.

Charges and Secretaries receiving compensation.

Secretaries of Embassies.

Secretaries of Legations.

Diplomatic Agents.

TREASURY DEPARTMENT.

Secretary of the Treasury.

Three Assistant Secretaries of the Treasury.

Comptroller of the Treasury.

Assistant Comptroller of the Treasury.

Auditor for the Treasury Department.

Deputy Auditor for the Treasury Department.

Auditor for the Var Department.

Deputy Auditor for the War Department.

Auditor for the Interior Department.

Deputy Auditor for the Interior Department.

Auditor for the Navy Department.

Deputy Auditor for the Navy Department.

Auditor for State and other Departments.

Deputy Auditor for State and other Departments.

Auditor for the Post Office Department.

Two Deputy Auditors for the Post Office Department.

Treasurer of the United States.

Assistant Treasurer of the United States.

Register of the Treasury.

Assistant Register of the Treasury.

Commissioner of Internal Revenue.

Comptroller of the Currency.

Director of the Mint.

Commissioned Officers of the Revenue Cutter Service. Collectors of Customs. 
Assistant Collectors of Customs.

Naval Officers of Customs.

Surveyors of Customs.

General Appraisers of Merchandise.

Appraisers of Merchandise.

Assistant Appraisers of Merchandise.

Collectors of Internal Revenue.

Superintendents of Mints.

Assayers of Mints.

Melters and Refiners at Mints.

Coiners at Mints.

Engraver at the Philadelphia Mint.

Assistant Treasurers of the United States at various points.

Surgeon General of the Marine Hospital Service.

Medical Officers of the Marine Hospital Service.

General Superintendent of Life Saving Service.

Deputy Assistant Treasurer.

Treasurer of the Isle of Porto Rico.

Auditor for the Isle of Porto Rico.

General Inspector of the Treasury.

Examiners of Drugs and Chemicals.

Superintendent of Assay Office at New York.

Assistant at Assay Office in New York

Melter and Refiner at Assay Office in New York.

Assayers in Charge of Assay Office at Charlotte, N. C. Assistant and Melter of Assay Office at Charlotte, N. C. Officers of the Revenue Cutter service.

WAR DEPARTMENT.

Secretary of War.

Assistant Secretary of War.

Inspector General.

Quartermaster General.

Commissary General.

Surgeon General. 
Paymaster General.

Chief Signal Officer.

Professors at the Military Academy.

Chief of Artillery.

Civilian Member Board of Ordnance and Fortification.

All Officers of the Line and Staff of the Army.

Officers below the rank of Major, Porto Rico (Provisional) Regiment.

Members of the Mississippi River Commission.

Members of the California Debris Commission.

DEPARTMENT OF JUSTICE.

Attorney General.

Solicitor General.

Assistant to the Attorney General.

Assistant Attorneys General.

Solicitor for the Treasury.

Assistant Solicitor for the Treasury.

Solicitor of Internal Revenue.

Solicitor for the Department of State.

Solicitor for the Department of Commerce and Labor.

Nembers of the Spanish Treaty Claims Commission.

JUDICIARY.

Chief Justice of the Supreme Court of the United States. Associate Justices of the Supreme Court of the United States. United States Circuit Judges.

United States District Judges.

United States Attorneys.

United States Marshals.

Chief Justice Court of Claims.

Associate Justices of the Court of Claims.

Territorial Judges.

Chief Justice of the Court of Appeals of District of Columbia. 
Associate Justices of the Court of Appeals of District of Columbia. Chief Justice of the Supreme Court of District of Columbia. Judges of the Police Courts of District of Columbia.

\section{POST OFFICE DEPARTMENT.}

Postmaster General.

First Assistant Postmaster General.

Second Assistant Postmaster General.

Third Assistant Postmaster General.

Fourth Assistant Postmaster General.

First, second and third classes of Postmasters.

Purchasing Agent of the Post Office Department.

\section{NAYY DEPARTMENT.}

Secretary of the Navy.

Assistant Secretary of the Navy.

Admiral of the Navy.

Chief of the Bureau of Navigation.

Chief Constructor of the Navy.

Chief of the Bureau of Ordnance.

Chief of the Bureau of Equipment.

Engineer-in-Chief of the Navy.

Surgeon General of the Navy.

Paymaster General of the Navy.

Chief of the Bureau of Yards and Docks.

Judge Advocate General of the Navy.

Brigadier General. Commandant Marine Corps.

Visitors to the Naval Observatory.

All Officers of the Line and Staff of the Navy and Marine Corps, except the Warrant Officers of the Navy.

INTERIOR DEPARTMENT.

Secretary of the Interior.

First Assistant Secretary of the Interior. 
Assistant Secretary.

Assistant Attorney General.

Commissioner of the General Land Office.

Assistant Commissioner of the Land Office.

Commissioner of Patents.

Assistant Commissioner of Patents.

Commissioner of Pensions.

First Deputy Commissioner.

Second Deputy Commissioner.

Commissioner of Indian Affairs.

Assistant Commissioner of Indian Affairs.

Commissioner of Education.

Director of the Geological Survey.

Examiners in Chief of Patents.

Recorder of the Land Office.

Registers of the Land Office.

Receivers of Public Moneys.

Surveyors General.

Indian Agents.

Indian Inspectors.

Superintendent of Indian Schools.

Pension Agents.

Territorial Governors.

Territorial Secretaries.

Commissioner of the Interior for Porto Rico.

Commissioner of Education for Porto Rico.

Recorder of Deeds.

Register of Wills.

Inspector of Gas and Meters.

Rock Creek Park Commissioners.

DEPARTMENT OF COMMERCE AND LABOR.

Secretary of Commerce and Labor.

Assistant Secretary of Commerce and Labor.

Commissioner General of Immigration. 
Director of the Census.

Not to exceed 300 Supervisors of the i 3 th Census.

Commissioner of Navigation.

Commissioner of Fisheries.

Supervising Inspector General of Steam-ressels.

Ten Supervising Inspectors.

Superintendent of the Coast and Geodetic Survey.

Director of the Bureau of Standards.

Commissioner of Labor.

Commissioners of Immigration at various points. Agent for the Salmon Fisheries of Alaska.

Assistant Agent for the Salmon Fisheries of Alaska.

$$
\text { DEPARTMENT OF AGRICULTURE. }
$$

Secretary of Agriculture.

Assistant Secretary of Agriculture.

Chief of the Weather Bureau.

\section{MISCELLANEOUS OFFICIALS.}

Interstate Commerce Commissioners.

Public Printer.

Civil Servce Commissioners.

Chief Examiner of the Civil Service Commission.

Commissioners of the District of Columbia.

Members of the Board of Charities of D. C.

Governor of Porto Rico.

Secretary of Porto Rico.

Attorney General of Porto Rico.

Treasurer of Porto Rico.

Auditor for Porto Rico.

Nembers of the Executive Council of Porto Rico.

- The third power granted to the President, is that of granting reprieves and pardons for offenses against the United States, 
except in case of Impeachment. This, of course, confers no power of reprieve or pardon for ofienses against any State, punishable by the laws of such State.

The duties of the President with reference to Congress are: That he is to give to the Congress from time to time, iniomation as to the state of the Union, and this includes not only messages of a voluntary character, such as the annual message of the President upon the convening of Congress in December, and such other messages as he may deem to be cxpedient from time to time, but it also includes the sending, upon the request of Congress, of such information upon matters relating to public affairs as Congress may desire. He has the privilege of convening either House or both Houses, at any time, either before the assembling of the regular session, or after it has adjourned If the Houses of Congress fail to agree upon the date of adjournment, he may adjourn then to such time as he may deem proper. Every bill before it becomes a law, and every order, resolution, or vote, requiring the concurrence of the Scnate and the House of Representatives, except resolutions on the question of adjournment, must be presented to the President of the United States. The terms of the Constitution on this point are specific, but there is, nevertheless, a class of concurrent action, which it has been the practice not to submit to the President. Such actions in concurrence, generally known under the term of concurrent resolutions, which refer to the affairs of Congress itself, or which are essentially temporary in their nature, have not, under the practice of Congress, been submitted to the Exccutive for approval. The dividing line between concurrent votes presented and not presented, is generally marked by terming the former "joint resolutions," and the latter, "concurrent resolutions," though the precedents in this matter are not uniform. When a bill, resolution, or vote is presented to the President, it must be approved within ten days. Sundays excepted, after it shall have been presented to him. If it remains unacted upon, it becomes a law as if he had signed it, unless Congress has by adjournment prevented its return. During the period of the session of Con- 
gress, the President in exercising the veto power, must return a bill or resolution to the House of the Congress in which it originated, without his approval, stating his objections. When he does this, the House to which it is returned may proceed to reconsider it, and if the measure is passed again, two-thirds of the members voting for it, it is then sent to the other House, and if approved by two-thirds of that House, it becomes a law notwithstanding the Presidential objections. It is the practice of the President at the close of each session of Congress to attend upon the Congress at the Capitol for the purpose of signing the bills passed in the closing hours. The neglect or refusal of the President to sign a bill at that time, acts as a complete veto of the legislation in question. The language of the Constitution relative to the treatment of bills after the adjournment of Congress is not altogether clear. It is, of course, clear as applied to the second or short session of each Congress when that body has adjourned without day on the fourth of March. Just what the procedure might legally be with reference to a bill which has been passed by both houses, but not presented to the President, is not altogether certain, but the consideration is merely an academic one, as it is the practice, as above stated, for the President to attend upon Congress on the last day of both the iong and the short sessions, and to sign all bills whch have been passed up to the last moment. This procedure was probably adopted in order that there might be no question in regard to the matter. The point lies in the construction of whai constitutes an adjournment of Congress for this purpose. There would be no practical difficulty in the retention of a law during the recess of Congress and the presentation to the President of such a bill upon the reassembling of Congress in December. It seems quite apparent, however, if the bill were presented to the Fresident on the last day of the long session, it could not become a law unless he signed it before the adjournment of Congress on that day, since there would be no way in which the President, being in possession of a bill, could bridge over the Congressional recess. For this reason Congress remains in session until it is 
advised that the President has signed every bill which has been passed by both Houses, and this, in the short session of Congress, which must adjourn at twelve o clock on March fourth, brings about the turning back of the hands of the clocks of the two houses during such period as may be necessary to complete the legislation and present it for the Presidential signature.

The nominating power of the President before referred to, is supplemented by an appointing power, limited by the requirement that the appointment shall be with the advice and consent of the Senate. The nominations made by the President for the classes of officers before listed, are considered by the Senate, and upon approval appointments are made by the President. The President has the power of direct and uncontrolled temporary appointment to all positions which are otherwise filled with the advice and consent of the Senate but which become vacant during a recess of the Senate. Such appointments, however, are strictly temporary, and terminate at the end of the next Senate session. This power of the President being granted by the Constitution, cannot be limited as to the actual appointment of persons to the positions which nay be vacant. Congress, has, however, put a rather effective check upon this unlimited power by a provision that no salary shall be paid to any persons appointed under a recess commission if the vacancy to which he was appointed existed during the session of the Senate. Thus it would not be advisable for the President to allow a position to remain vacant without nomination during a session of the Senate, so that he might make a recess appointment after the Scnate session had expired, as the incumbent, under such conditions, could draw no salary until he had been confirmed by the Senate, and if he was not confirmed by the Senate, could draw no salary at all. This provision would secm to apply only to positions which become vacant during the session of the Senate and to which no recess appointment has already been made. In case of a recess appointment antedating the session of the Senate, and for which the Senate has failed to confirm a nominee, another recess appointment can be made immediately after the adjournment of 
the Senate, a second vacancy thus occurring by virtue of the expiration of the first recess appointment at the end of the session of the Senate held next after the date on which the recess appointment was made.

The President obligates himself faithfully to execute the duties of his office, to preserve and protect the Constitution, and is required to take care that the laws are faithfully executed by all persons under his direction. He may be removed from office by impeachment process, on conviction of treason, bribery, and breach of his obligation to preserve the Constitution of the United States, or for manifest negligence in securing the execution of the laws, the last two offenses being included under the general description, "other high crimes and misdemeanors."

Congress is empowered to add to the appointing power of the President, the appointment of such inferior officers as is thought proper. Under this power as delegated, the President has the exclusive power of appointment of such inferior officers, and it is not necessary to nominate them to the Senate for confirmation by that body. The various classes of public officials thus appointed by the President are as follows:

\section{STATE DEPARTMENT.}

Vice-Consuls.

Deputy Consuls.

Commercial Agents.

Vice Commercial Agents.

Consular Agents.

Consular Clerks.

Student Interpreters in China.

Interpreters to Legations.

Interpreters to Consulates.

Marshals of Consular Courts.

WAR DEPARTMENT.

Chief of Staff (from Officers of the Army). All Military Cadets. 
Foard of Visitors to the Military Acarlemy.

Superintendent of the Military Academy.

Commandant of Carlets at the Military Academy.

DEP.ARTMENT OF J CSTICE.

Commissioners of 1)ecls for D. C.

Notaries Public in D. C.

Trustees of the Two Reform Schools in D. C.

Justices of the I'ace in the District of Columbia.

\section{NAYY DEP.MRTAEXT.}

Viarrant Officers of the Nary except Pharmacists.

Midshipmen at large.

Mitlshipmen from the District of Columbia.

Board of Tisitors to the Naval Acadeny.

Acting Assistant Surgeous in the Navy.

INTERIOR DEPARTMENT.

Board of Indian Commissioners.

Allotting Agents for Lands.

Coal and Asphalt Nine Trustees.

Superintendent of the Capitol Building and Grounds.

Board of Visitors to Government Hospital for the Insane.

Clerk in the Interior Department to sign Land Patents.

DEPARTMENT OF COMMERCE AND LABOR.

Members of the Light-House Board.

Commissioner of Corporations.

Deputy Commissioner of Corporations.

Chief of the Bureau of Nanufactures.

MISCELLANEOUS OFFICIALS.

Secretary to the President.

Assistant Secretary to the President. 
Two Executive Clerks to the President.

One Steward at the IVhite House.

One Messenger at the White House.

Librarian of Congress.

Superintendent of Library Building and Grounds.

Engineer Commissioner of the District of Columbia

Purchasing Agent, Post Office Department.

The pardoning power of the President is comprehensive and without Constitutional restriction, except in cases of impeachment. The scope of this power includes not only criminal offenses under the civil statutes of the United States, but offenses against military law and discipline, including even those of the gravest character. Where disabilities have been imposed by Congress upon conviction of certain offenses, the exercise of the pardoning power by the President removes such disability. The pardoning power of the President includes cases which have not been tried or in which punishment has not been awarded.

The President has from time to time received authorization from Congress for the exercise of power under general legislation effected by Congress. Among these powers is that of a supervision of the Consular Service, and the direction of expenditures for necessary means of carrying on the foreign business of the nation. He is given the power to use the armed forces of the country for the purpose of carrying out the penalties imposed for crimes against the United States, issues patents for public lands, a clerk being provided to sign his name to such patents, and may discharge debtors from debts due the United States, in cases where provision has not been made to empower the Secretary of the Treasury to issue such discharge. The direction of the sale of public lands, when authorized by Congress, is also placed in the hands of the President, and he is given discretionary power to permit the discharge of soldiers and sailors in time of peace, under such regulations as he may fix.

The President is given by Congress the direction of the operations of the Coast Survey. He may name the ships of the Navy 
within the classes fixcd by Congress, and may provide rules for the admission of persons into the Civil Service, under the restrictions of the act establishing the Civil Service Commission and providing means for the improvement of the Civil Service. He also by virtue of his position as Commander in Chief of the Army and Navy, has power to direct any details of officers from the military branches of the government, and may select Commandants of Navy Yards and Stations, and all persons from the appropriate line or stafi of the military forces, for such positions as may be established by Congress. He has the absolute power of removal of all persons whether appointed by himself alone or with the consent of the Senate, and of persons in the Civil Scrvice, except members of the judiciary who may only be removed by impeachment. He may also employ the armed forces of the United States for the assistance of the Governor of any of the States, when requested to do so by the Executive of the State in question.

The President receives as the representative of the United States, the Ambassadors and Ministers of foreign countries, and conducts directly, or through the Secretary of State, all negotiations with the heads of foreign governments, as represented by their Ambassadors and Ministers, which do not call for a formal conclusion or written treaty stipulations. All communications to foreign governments are sent in his name, and all conmunications from foreign governments are transmitted to him, and by him, when necessary, forwarded to the Congress. For the purposes of foreign intercourse he is the head of the Government of the United States, subject only to restriction by the Senate when it shall have become necessary to conclude a formal treaty. The general power lies in Congress to indicate its disapproval of the relations of the President with foreign powers, by a resolution stating the objections held, but such resolution has no valid force on the Executive, unless it is made the basis of impeachment proceedings.

The popilar conception of the election of the President of the United States becomes confused so frequently by the impor- 
tance attached to the entumeration of the popular vote, that a careful statement of the machinery by which the President is elected is essential. Although the announcement made quadriennially, within a few days after the Presidential election, of the number of rotes cast for each Presidential nominee is usually regarded as conclusive, no actual election takes place until the second Vednesday of the February next following. The Constitution provides that each State shall appoint, in the manner to be determined by the legislature of the State, the Presidential Electors, who shall be equal in number to the whole number of Senators and Representatives to which the State is entitled in Congress. This number is composed of two, representing the number of Senators, and a sufficient number in addition to give one Elector for each Representative in Congress, proportioned on the apportionment of Representatives provided by law, or if the apportionment has not been made on the last decennial census, the number provided under the old apportionment remains in effect. No Senator, Representative, or other Federal officeholder can be appointed as an Elector. It is the universal custom to appoint Electors by popular ballot, though it was formerly the practice in some of the States, for the legislature to select the Electors. The power is given to Congress to choose the time of appointing the Electors, and the day on which they shall give their votes, which day must be uniform throughout the United States. The day of choosing the Electors has been fixed as the first Tuesday after the first Monday in November. The States are empowered to fill any vacancies which may occur in the list of Electors, and if no election occurs the legislature may set a later date for the election. In case of a dispute as to the legality of an election of any Elector or Electors, the determination of the person properly selected may be fixed by a provision of the State statutes, providing such a statute has been passed by the State Legislature six days prior to the second Monday in January, on which day the Electors are required to meet and cast their votes. The Secretary of State of each State must provide three copies of a list certifying the names of the persons who have been 
properly elected as Presidential Electors. On the second Monday in January, the Electors must meet and cast their ballots for President and Vice-President respectively, making three copie: of the number of ballots cast, and annexing to each cupy the certified list of Electors, furnished them by the Secretary of State. These three certificates must be sealed up and a certification placed upon each of its contents, that it contains the result of the election. One of the copies so sealed up is cutrusted to a person who is appointed by the Electors in writing to be delivered by him to the President of the Senate of the Lnited States, before the Wednesday next ensuing. The second copy is forwarded by the Post Office to the President of the Senate, and the third copy is deposited with the Judge of the United States District Court of the district in which the election has been held.

The two Houses of the Congress meet in the House of Representatives Hall, at one o'clock in the afternoon of the second Wednesday in February, following the meeting of the Electors, to witness the count of the number of ballots thus cast. The President of the Senate presides and two members of each House are appointed as tellers, previously to the meeting in assemblage. The President of the Senate opens the ballots returned by the States in the alphabetical order of the States, beginning with the letter " $A$," and the result of each ballot is announced, and later the total result is declared. Should there be any objection to the ballots as returned, challenging their legality, such objection must be presented in writing and signed by at least one Senator and one. Nember of the House of Representatives. If objections are presented to the rote of any State, the opening of the ballots is suspended, and the Senate withdraws to consider the objections in separate session. The House also goes into separate session to consider the objections presented. If only one set of ballots is returned from a State, no vote may be rejected muless the two Houses concur that the Electors had not voted regularly. If more than one set of ballots are presented, those are to be counted which are certified 
as regular by the machinery provided by the State to determine the regularity of such ballots, but if it appcars that there are two atthoritics claiming to pass on the regularity of the votes, the matter is settled by concurrent vote of both Houses in separate session. If 110 machinery has been provided by the State for the determination of the regularity of the electoral ballot, the choice between contesting ballots is to be by concurrent action, and in case of disagreement between the two Houses, the certificate of the Executive of the State in question, as to the regularity of either of the ballots, is to be accepted.

During the pendency of the question as to the regularity of the ballot of any State, no action can be taken on the returns of any other States. No recess of Congress can be taken except from day to day, minless over Sunday, and after five days, no recess at all can be taken. Upon the completion of the opening of the ballots and the tabulation of the results, the vote is declared by the President of the Senate as the official announcement of the election of the President of the United States. If the returns from any State are not received by the President of the Senate before the tourth Monday in January, the Secretary of State of the United States must send a special messenger to the Judge with whom the third copy of the election returns has been deposited, to secure that copy. When there is no President of the Senate in Vashington to receive the returns, the Secretary of State is empowered to receive them.

The term of the President of the United States begins on the fourth of March and ends on the fourth of March four years later. He can only refuse to serve or resign by a written statement under his own hand, addressed to the Secretary of State. In case of either death, resignation or removal of the President, the Vice-President becomes the President of the United States, and successively in case of failures to serve, the Secretary of State, the Secretary of the Treasury, the Secretary of War, the Attorney General, the Postmaster General, the Secretary of the Navy, and the Secretary of the Interior are to serve, provided they are eligible under the Constitution and are not under im- 
peachment proceedings. Such officials, serving as I'resident of the United States, are to complete the term in which the vacancy exists, but if Congress is not in session or will not be in session within twenty days, after the time they assmme the Presidential duties, they munt convene Congress, griving twenty das notice of the date of assembling.

The administration of the executive duties imposed upon the President by the Constitution, and in the detals of which he is to a considerable extent independent of the Congress, is nowhere defined or set down in any set of rules binding upon the Chief Executive. The general policies of the government are framed by Congress, and means are provided for carrying them out through the various appropriation bills. It is the duty of the President to direct the machinery by which these expenditures are made and these operations are checked by the Treasury Department, the Secretary of which reports directly to Congress. The President is provided with a board of assistants, one of whom is at the head of each department, and all of whom constitute the President's advisory calbinet in the general administration of affairs. With the exeeption of reports directed by Congress on public expenditures, the members of the cabinet are under the direction of the President as to duties which are not otherwise regulated by statute. The result of this centering of the responsibility of the conduct of the executive departments in the President, is to make him the final arbiter on all departmental matters. He is more or less, according to his disposition, the directive force in each department. It is in his discretion how far he shall leave the determination of matters relating to any particular department wholly to the Secretary of that Department, or how far he shall exercise personal direction and influence in such departmental affairs. He must inevitably exercise the deciding influence, not only in matters of general public policy, not regulated by Congress, but in affairs in which the interests of more than one department are concerned. Much of the President's participation in the affairs of any department, depends upon the strength and ability of the cabinet officer charged 
with the particular administration of those affairs. Much also depends upon the personal interest of the Chief Executive in the work of any given department. It is the general practice for all directions regarding departmental business, which may come from the Executive to be transmitted through the Departmental Head. There is nothing in law, however, to prevent the President from giving personal directions to any official of any department, or to prevent him from taking personal charge of any detail of the governmental work.

Congress has at different times established commissions independent of the main executive departments, for the purpose of carrying on specific lines of work, such commissions as the Interstate Commerce Commission, the Civil Service Commission, and the Panama Canal Commission, together with other commissions of less importance, having to do with more or less transitory duties, and being appointed by the President, are under his direction, sometimes by direct provision of statute, and sometimes by implication, and are at least subject to his advice and admonition, even when they are not susceptible of his individual direction.

The nomination of a candidate for President of the United States, occupies the Spring and Summer of the year in which the Presidential election takes place. It was the practice in the earlier years of the Republic, for each State to present the name of a candidate. It would, of course, appear that certain of the candidates promised to command the greater strength than the body of the nominees, and it was the practice to solicit for them the support of the States of the weaker candidates. But as political parties became well defined and especially in the last half of the century, machinery for procuring the nomination of a man representing the views of a given political party, has come into usage. The inception of such nomination is the mass assemblage of the party voters of the smallest subdivision of a municipality, that is to say, in the ward, or precinct, as the case may be. These voters elect certain delegates to a convention which represents the whole body of voters of their political faith in the State, and this conven- 
tion in turn elects a given number of delegates to the national convention, the number of delegates in each case being determines by the strength oi the party vote cast in the State in the precerling election. At the national convention of the party, mecting in some great city in June of the Presidential year, the general sentiment of the party has so far crystallized that the choice of availability lies generally between three or four men, each representing some particular section, or some particular shade of the party tenets. If the current administration is of the political party then holding the convention, and if the President retains the confidence of his party, a renomination is marle by acclamation. Should the convention be that of the opposition, or should the President in office be unavailable, ballots are taken by states, each state roting $i$ a body for the choice of candidates respectively, for President and for Vice-President.

While national prominence is sought in a candidate it has been most generally the fact that the residence of an available man in a politically doubtful section, which would be influenced by the sclection of a resident of that section, has been the chief determining factor in the Presidential nominations of modern political history. The Vice-I'residential candiclate has been largely selected either for advantage of location in a similar way, or for his infuence in connection with campaign contributions. No Federal statute covers the selection of the candiclates of a party, though in some States laws have been enacted prescribing the methor of prinary elections for the selection of lesser candidates and of delegates to conventions.

The President of the United States is senerally regarded as the head of the national organization of his political party. Upon the nomination of a Presidential candiclate the choice is accorded him of the Chairman of the National Committec having charge of the conduct of the campaign. Upon the induction of a new President into office, he is expected in his annual message to Congress to lay stress upon the policies which have heen advocated in the compaign of the election, and he is supposed to consider party affiliations in the selection of at least his cabinet. and 
to a large extent in the nomination of officers for confirmation by the Senate. With the Speaker of the House of Representatives, and the leader of his party membership in the Senate, he is supposed in a general way to shape legislation to conform to the promises of the party platform, subject to the expression of opinion made by the party members of Congress in caucuses held by Senators and Represcntatives respectively. When differences have arisen between the President and other prominent representatives of the party which nominated him, Presidents in the past have been accused of treachery in not carrying out the majority expression of party sentiment. The situation herein described is entirely traditional and has no basis in constitutional or statutory provision. Legally the sentiment of a political party has no binding force whatever upon the President, who has sworn to enforce the laws impartially, and withont regard to affiliation with any section of his constituency.

The President may or may not be the directive power in the general management of the party which elected him, outside of matters of legislation. This will largely depend on the circumstances pertaining to current polities. It has sometimes been the fact that the President has been the actual power in his party, but it is more often that the real power is exercised by those who constitute the inner circle of the National Committee, an organization whose members are elected by the State Conventions and whose membership offers an opportunity for securing and wielding real power by a man skilled in making combinations. Such a man would be and has been able to utilize the Federal patronage for the advancement of his plans and can make the President subordinate in party affairs. Many political contests in the United States have grown out of the resistance of Presidents to party emulators of the great Warwick. 
CHIIPTER III.

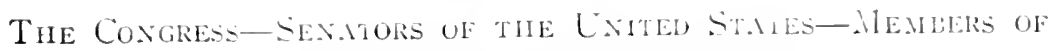
HH: HUC'SE OF REPRESENTATIES.

The national legislature of the Linited States of America, which is the Congress of the representatives of the sovereign States, is, apparently, strictiy limited as to its powers by the terms of the Constitution. It may be remarled, by the way, that although this body is usually spoken of as Congress, its proper title is "The Congress," and it is so termed in law except when through inalvertence a slip is made into the popular iurm. When an examination is made of the legislation effected by Congress and supported by the courts, it becomes apparent that the Congressional powers are by no means as limited as they would seen to be upon a first reading of the Constitution. is a matter of fact, the Congress, as it is toclay with respect to its powers, is almost as much a self-creation as it is a creation of the Constitution. The terms of the latter document, which are summarized at a further point in this chapter. delegate to the Congress certain powers inherent in sovereignty: Certain powers, also equally attributes of sovereignty, have been reserved to the independent States, but between the two there is a field of general power and of possibility for legislation which can he and which has been construed in favor of the Congress not only by the Congress itself, but by the judicial power of the United States culminating in the Supreme Court. Among the powers delegated to the Congress which have been given a wide latitude and which may be still further extended, is that which gives the Congress the power to provide for the general welfare of the United States. A strict construction would refer this to the matter of laying and collecting taxes only, yet propositions, in no way germane to the subject of taxation, have been argued out under this clause. The power to borrow money on the 
credit of the United States and to coin money and regulate the value thereof, has been argued into a power to establish a currency system for the country. The further power to make all laws which shall be necessary and proper for carrying into execution the other powers delegated, is a general provision under which the burden of proof is on the other side to show the invalidity of any measure that may be deemed by Congress to be within the grant of its powers. In support of a wide measure of construction of this clause, there are opinions dating from the earliest days of the Supreme Court that that body will not interfere with the work of the Congress in carrying out its legislative functions, unless it can be clearly shown that the measures taken are contrary to the terms of the Constitution or subversive of the intent of that document.

The earliest departure from the strict construction of the powers of Congress was under the general welfare clause in the adoption of the policy of a system of internal improvements. This policy of securing a ready intercourse between the seaboard and the then undeveloped interior was supplemented by an improvement of the harbors of the sea coasts, which resulted in the tremendous system of river and harbor improvements, which have continued until the present day. Subsequent extensions of the power of the Congress to legislate for the general good have followed upon real public needs, and their accompanying public demands, but no more striking example of the manner in which the powers of Congress are extended in accordance with a general demand exists than is presented by the legislation under the clause which empowers Congress to regulate commerce with foreign nations and among the several States. In consequence of the grant contained in this power, the Congress has not only been able to inspect certain products produced within the States of the Union and to prescribe the conditions under which they shall be produced, but it has passed legislation intended to regulate mercantile competition, ostensibly in interstate commerce, but in reality within the States themselves, and has further effected legislation which practically 
prescribes the price at which transportation carried on by companies which are wholly and solely the creations of the inllividual States, shall be sold. In view of the extent to which the letter of the Constitution has been extended, it is useless to describe as fixed and limited those things which the Congress can and cannot do. Certain matters are set down with definiteness, but beyond and above them it would seem that the only limit to the construction that will be given to the general clauses of the Constitution is the sufficiently ardent desire of a sufficient number of people of the United States to add to the Congressional prerogative.

Each Congress lasts for two years, beginning on the first Monday in December in the odd year, and concluding on the fourth of March in the following odd year. The first session of each Congress lasts until the completion of the business of the Congress, or rather until the hot weather makes Vashington uncomfortable, and there is then no Congressional business which must absolutely be completed; that is to say, from the first of June to the first of July. This is known as the long session, and efforts are made during this session to complete the general legislation, for which the majority party has pleclged itself at a previous election. The second session, ending at noon on the fourth of March, is mainly devoted to passing the necessary appropriation bills with as little general legislation as possible. In case matters of importance arise in the recess between the two sessions, or the business has not been completed, the Congress may be called together again by the President during the summer or early fall. This, however, is seldom done, and has been done in recent years only to effect tariff legislation, while tariff legislation and the exigencies of war have extended the regular sessions of Congress far beyond the limits in certain years.

\section{SENATORS OF TIIE UNITED STATES.}

The establishment of the position of Senator of the United States is an example of the common sense in govermental mat- 
ters which has distinguished the Anglo-Saxon above all the other nations of the world. Theoretically, the creation of an artistocratic body in connection with the democratic form of government proposed by the Constitutional Convention was almost an absurdity. As an adjunct to the strictly representative character of the lower House of the Congress, it represents so diametrically opposite a theory of government that it might well have been argued that the Senate was an anomaly and wholly foreign to the purpose and project of a representative government. While, however, the delegates to the Constitutional Convention talked and wrote at length in the style of the French philosophers of the period, they were none the less keenly alive to the practical side of the problem which lay before them and were more concerned in creating a form of government that would "march" than in making its details conform to the philosophic principles upon which it was alleged to be based. The idea upon which the Constitutional Convention provided the legislative power of the United States has been tested by time and found to be sound. Repeated experiments have been made with the single-chambered legislative body, and the results have shown that the fears of the conventionists were well founded, that is to say, that hasty and ill-considered legislation, stimulated by the expediency of the moment, is promoted by a single legislative body, and is checked by a dual legislative body. While the revolutionists in America ardently rejected the title and prerogative which was a part of the English House of Lords, they could not reject and ignore the substantial value of the weight of conservative opinion as a balance-wheel for the legislative machine. They were wholly familiar with the value of such checks in their own colonial affairs, since councils to the Governor and similar bodies had been constituted under the various charters to steady and dignify the popular branches of the law-framing bodies. It is fair to assume that the delegates to the convention foresaw what has since been seen to be the fact, that a numerous body of immediate representatives of the people would be likely to be swayed by currents of public opinion 
and hurried on to the enactment of opportunist legislation, only to be driven in the other direction and to the necessity of repeal when it appeared that an ill-considered remedy had failed of the desired effect. It is evident, from the Constitutional provisionsthat Senators should be thirty years of age, that is, five years older than the minimum age of Representatives; and should be nine years a citizen of the country as against seven years, the minimum for Representatives, - that it was proposed for the Senate the selection of older, more conservative, and, from the method of their election, more carefully selected men than might be sent immediately from the people through the medium of direct popular representation. It is shown, of course, by the history of the Constitutional Convention, that the smaller and weaker States insisted upon equal representation in the upper branch as a defense against encroachment from the larger and more populous. This, however, it may be held, was not the main purpose of the establishment of the Senate, but rather that it was deliberately chosen as a check and conservator in the legislative enactments. The term of a Senator is six years. and his compensation is $\$ 7,500$ per year. In order to further carry out the idea of a conservative body as the upper branch of the national legislature, it was provided by the Constitution that, upon the election of the first body of Senators, they should be divided as equally as might be into three classes; the first class to serve for two years, the second for four years, and the third for six years. By means of this provision one-third of the membership of the Senate passes out of office or comes up for re-election every two years. Senators are elected by the legislatures of their respective States, and must be, as has been stated, thirty years of age, nine years citizens of the United States, and residents of States from which they are elected.

The body which elects the United States Senator is similar in its constitution to the national legislature in having two separate and distinct chambers with a mutual check on legislative propositions It has, however, this important difference in most of the States, that both of the branches of the State legislative body are directly rep- 
resentative, the members of the upper branch being elected by the ballot of the whole number of electors in the State, although representing larger districts than the members of the lower house. This difference from the original pattern has lent some color of logic to the proposition that the Senators should be elected by the ballot of the whole people of the States they represent. Objection has been made that influences which may be used more effectively on a comparatively small body of men than on the whole body of electors, has made it possible for certain interests to secure representation in the upper house of the national legislature to the detriment of legislation for the interests of the whole people. It cannot, however, be successfully established as a general proposition that deliberations of the Senate and the consequent checlss and delays of hasty action, with the changes that have been made in proposed legislation as it comes from the House of Representatives, have been materially and designedly injurious to the general welfare, in any case, while on the other hand it is apparent in every session of Congress that these checks and delays are of inestimable value in securing the consideration not only by the Congress itself, but by the people at large, of legislative propositions. At the same time there is nothing in the movement for the election of Senators by direct ballot of the people of the State which would interfere with the real purpose and efficiency of Senators. The crux of the theory is that of a select and more carefully chosen representation than in the lower house, and the means by which the special representative shall be chosen by the people do not seem to be material. Popular representation in the State Senate has not proved to be a failure, but, on the other hand, it nust be remembered that State Senates have been made the subject of similar clarges as to the representation of special interests as have been against the Senate of the United States.

In case of a vacancy in the senatorial delegation from a state during the recess of the Legislature the Governor makes a temporary appointment intil the Legislature can choose a person to fill out the unexpired term. The Senate of the United States is one of the few legislative bodies in the world which does not choose its own presiding officer. During the incumbency of the Vice-President of 
the Uniterl States, that official is ex-oficio the I'resident of the Senate. Senators, however, elect a president pro tempore and also all of the other officers. The legislative rights, privileges and duties of a member of the Senate are described under the head of the members of the House of Representatives. These in botl cases are very similar, but in adclition the members of the Senate have the exclusive prerogative of acting upon treaties with foreign nations. Matters relating to the foreign affairs of the United States do not come beiore the House of Representatives except so far as it may be necessary to pass appropriations to carry on proposed measures. Ali agreements with foreign nations which have the nature of formal treaties must be presented to the Senate for ratification. Unless two-thirds of the Senators present on the occasion of a vote on a treaty or convention vote in favor of the proposition, it is lost and the treaty, although it may have been solemnly concluded by the representatives of the two nations and approved by the President, connot be ratified and becomes null and void. This power constitutes the rather unusual condition of a practical veto power granted to the legislative body over a branch of public negotiations which is otherwise entrusted to the President and his advisors. The Senate also has a rule of holding secret or executive sessions, which is not paralleled by anything in the practice of the other house. Such sessions are supposed to be for the transaction of business relative to treaties and nominations, but are used as well for conferences between the Senators on matters in regard to which pubiicity is deemed inadvisable.

In addition to the general prerogatives named, the members of the Senate hold the confirming power with reference to a large number of the Federal officers. Nominations made by the President must be confirmed by the Senate before an appointment can be made, and it has frequently happened in the history of the United States, that a Presidential nomination has been negatived by, or has been withdrawn in the face of the opposition of, the confirming power. The members of the Senate also sit as judges in cases of impeachment. and when so sitting are under special oath or affirmation. The votes of two-thirds of the members present are required 
for a conviction upon an impeaclment. Owing to the continuous character of the organization of the Senate, it has its own peeuliar code of rules and traditions, differing in many respects from those of the House of Representatives. The chief of these is the principle of Senatorial courtesy', under which business can only be transacted by consent of the minority, no method of closure such as is maintained by the lower house, being in effect in the Senate. Senatorial courtesy refers also to the matter of nonninations or patronage under a system in which the President and members of his political party in the Senate are frequently curiously entangled. A considerable number of the Presidential appointments refer to local places and constitute especially the Federal patronage with which it has been the tradition to reward political adherents. Each Senator of the same political party as the President expects to be consulted with regard to the persons from his State whom the Fresident shall offer to the Senate for confirmation. In case the President names a person obnoxious to a Senator, the latter will, probably, through the operation of Senatorial courtesy, be able to secure unfavorable action on the nomination, if the place is in the Senator's orin State, and he is stipported by his colleague, while an obnoxious nomination in a State not represented in the Senate by persons of the same political party as the Administration may also frequently be defeated. That is to say, that, except for excellent reasons, the other Senators will not stand with the President in ignoring the claims of a fellowpartisan on the patronage in his own State, and will give the Senator a hearing and as favorable consideration as possible on other matters relating to his political prosperity; this arrangement being, of course, mutual, and extending also to Senatorial nembers of the opposition when strong personal reasons exist. Senatorial courtesy is also exemplified in the matter of getting business before the Senate, the day's proceedings being arranged by agreement instead of by the presiding officer, as in the House, and Senators are careful not to interfere with each other's business or plans and measures except upon unusual occasion. The most notable aspect of Senatorial courtesy, however, is that which enables a small minority to prevent final action upon a measure by continuing the debate and refusing to ac- 
cede to a compromise for the purpose of getting to a vote on the matter. This personal prerogative of the individual has been carried so far that a member has been able, especially during the closing hours of a session, to engage continuously in speaking upon a question, until the hour of adjournment arrived, thereby preventing a vote upon the measure to which he was opposed and in regard to which he was aware that he was in the minority. While this absence of what is technically known as a closure, that is, a provision for ending a lebate and taking a vote at the will of a majority, has been held to obstruct the transaction of public business, there is no question that it is of the greatest value in securing mature consideration of measures, while the severe closure rules in effect in the House have more than once been used to force the passage of ill-digested law's, on which the Senate has exereised the valuable function of amendment or disapproval.

In addition to the compensation allowed Senators, \$7500 and mileage at ro cents a mile which is the same as that allowed memhers of the House, the smaller size of the Senate makes it possible for each Senator of the majority and many of the minority Senators, to have the chairmanship of a committee, with the accompanying perquisites of a Committce Room and clerk hire. Rooms are also provided for Senators not having committee chairmanships. The stationery and contingent fund of the Senate is also more liberal in the matter of personal perquisites than would be possible in the larger membership of the House.

With a single exception, the legislative functions of a Senator are not materially different from those of a Member of the House of Representatives. That exception is, however, an extremely important one and consists of the lack of the power to originate revenue bills. It is held that the exclusive jurisdiction conferred by the Constitution on the House of Representatives to c.irinate all bills for raising revenue extends also to the origin of appropriation bills, and it is a point on which the House of Representatives is tenacious that not only shall the Senate not begin legislation for the annual appropriations, but that it shall not put provisions in appropriation bills that are entirely novel propositions and that have not been ap- 
proved by the lower body. The Senate has, however, the right of amendment of bills for raising revenue and frequently substitutes its own measure for a House measure, but this under the color of an amendment which strikes out all after the enacting clause and substitutes the Senate bill. Thus in bills imposing a tariff on imports, while the bill is originally framed in the Honse, it is subject to so considerable amendment in the Senate that at least one tariff bill has returned to the lower House almost unrecognizable. The exclusive authority over the inception of appropriation bills seems to be something in the nature of an arrogated authority by the House of Representatives, but it is substantially maintained by the refusal of the House to agree to obnoxious amendments, and it is challenged by the Senate most frequently for the purpose of securing a vantage ground from which to secure concessions in conferences between the two Houses.

Besides the recommendatory function before-mentioned exercised by a Senator with relation to the Federal appointments in his State, he recommends persons to fill one of the two cadetships at West Point provided for each State, the appointments being made by the President, and nominates under the provisions of law candidates to keep two midshipmen continuously at the Naval Academy at Annapolis.

When charges presented against the President, a member of the judiciary or other public official subject to impeachment are formulated by the House of Representatives and presented to the Senate, the latter sits as a judicial body for the trial of the case, presided over by the Vice-President, except in cases of impeachment of the President, when, for obvious reasons, the Chief Justice of the United States presides. The case is prosecuted by a committee of the House and defended by private attorneys. On completion of the testimony and arguments, the decision is effected by vote of the Senators, and it is necessary that two-thirds of those present shall vote in support of the charges in order to secure an impeachment with consequent removal from office and disqualification for subsequent public employment. 
COMMITTEES OF TIE SENATE.

STANDING COMMITTEES.

Agriculture and Forestry; Appropriations; Audic and Control the Contingent Expenses of the Senate; Canalian Relations; Census; Civil Service and Retrenchment; Claims: Coast and Insular Survey; Coast Defenses; Commerce; Corporations Organized in the District of Columbia; Cuban Relations; District of Columbia; Education and Labor; Engrossed Bills; Enrolled Bills; Examme the Sevcral Branches of the Civil Service; Finance; Iisheries; Foreign Relations; Forest Reservations and the Protection of Game; Geological Survey; Immigration; Indian Affairs; Indian Depredations; Interoceanic Canals; Interstate Commerce; Irrigation; Judiciary ; Library; Manufacturers; Military Affairs; Mines and Mining; Mississippi River and Its Tributaries; Naval Affairs; Organization, Conduct and Expenditures of the Executive Departments; Pacific Islands and Porto Rico; Pacific Railroads; Patents; Pensions; Philippines; Post Offices and Post Roads; Printing; Private Land Claims; Privileges and Elections; Public Buildings and Grounds; Public Health and National Quarantine; Public Lands; Railroads; Revision of the Laws of the United States; Revolutionary Clains; Rules; Territories; Transportation Routes to the Scaboard; Cniversity of the United States.

\section{SELECT COMMITTEES.}

Additional Accommodations for the Library of Congress; Disposition of Useless Papers in the Executive Departments; Examination and Disposition of Documents; Five Civilized Tribes of Indians; Industrial Expositions; Investigation of the Condition of the Potomac River Front at Washington; Investigate Trespassers upon Indian Lands; National Banks; Standards, Weights and Measures; Transportation and Sale of Meat Products; Ventilation and Acoustics; Voman Suffrage.

\section{SPECIAL SESSIONS OF THE SENATE.}

Year. Date of beginning. Date of adjourmment. I791......... Friday, March 4........ Friday, March 4 . $1793 \ldots \ldots \ldots \ldots$ Monday, March $4 \ldots \ldots \ldots$ Monday, March 4 .

$1795 \ldots \ldots \ldots \ldots$ Monday, June $8 \ldots \ldots \ldots$ Friday, June 26.

$1797 \ldots . . \ldots$. Saturday, March $4 \ldots .$. Saturday, March 4.

I798.......... Tuesday, July I7........ Thursday, July 19.

I801........... Vednesday, March 4..... Thursday, March 5 .

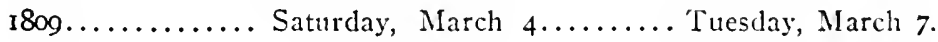

$1825 \ldots \ldots \ldots$ Friday, March $4 \ldots \ldots \ldots$. Wednesday, March 9 . 
SPECIAL SESSIONS OF THE SENATE-Continued.

Year.

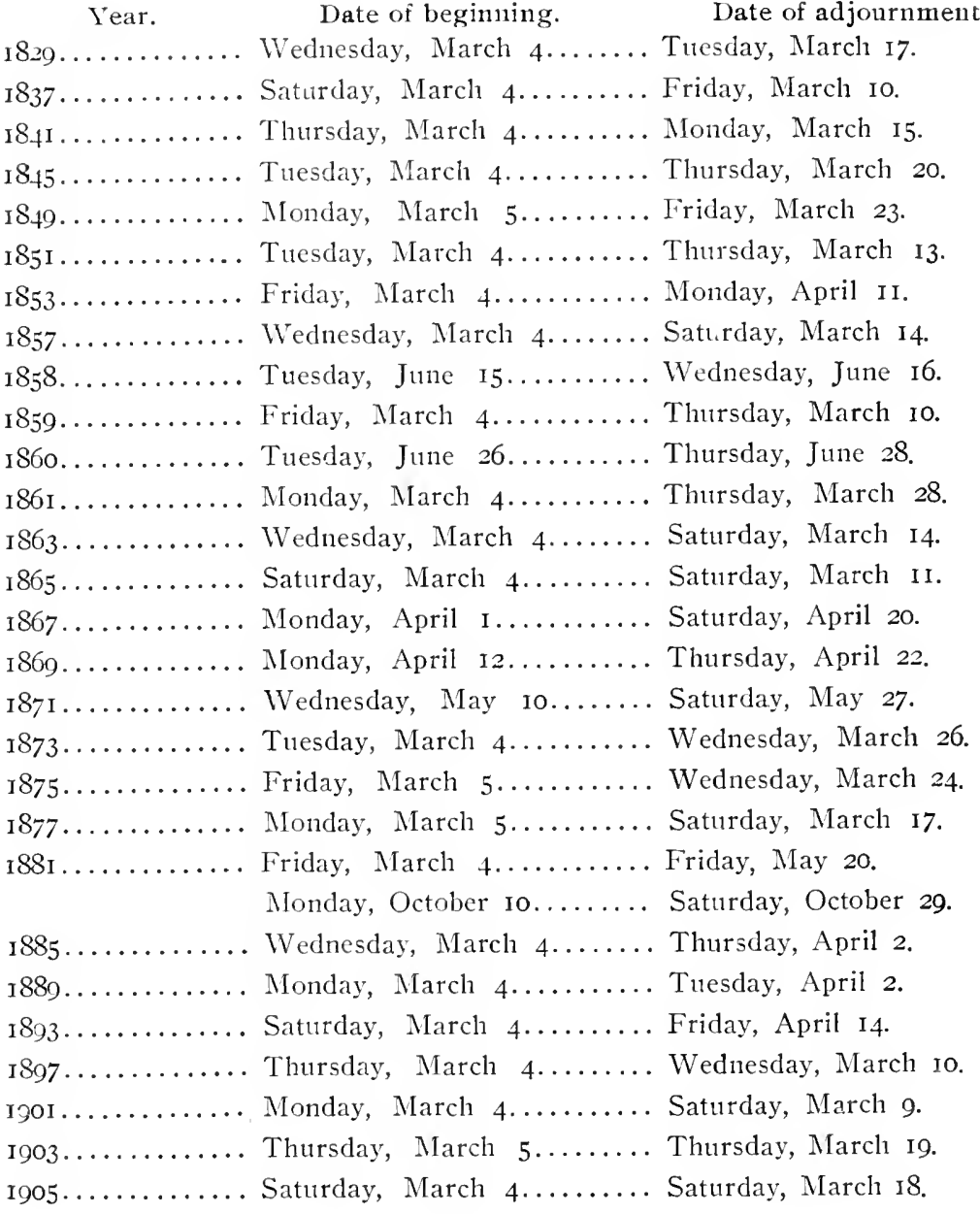

\section{COURT OF IMPEACHMEN'T.}

The Senate has sat as a Court of Impeachment in the cases of the following accused officials, with the result stated and for the periods named:

WILLIAM BLOUNT, a Senator of the United States from Georgia; charges dismissed for want of jurisdiction; Monday, December I7, 1798, to Monday, January I4, I799. 
JOHN PICKIERING, Judge of the Lnited States District Court ior the District of New limpshire; remored from office; Thursday, March 3, I8o3. to Monday, March I2, I So4.

SAMUEL. CHASE, Associate Justice of the Suprome Court of the United States; acquitted; liriday, November 30, 1 So4, to March I, ISO5.

JAMES H. PECK, Judge of the United States I)istrict Court ior the District of Missouri; acquitted; Monday, April 26, 1830 , to Monday, January 31, 1831 .

WEST H. HUMPIREYS, Judge of the United States District Court for the Middle, Eastern, and Western Districts of Tennessee; removed from office: Wednesday, May 7, i862, to Thursday, June 26, I 862.

ANDREW JOHNSON, President of the United States; acquitted; Tuesday, February 25, 1868 , to Tuesday, May 26, 1868.

WILLIAM IV. BEI.KNAP, Secretary of War; acquitted; Friday, March 3, 18;6, to Tuesday, August I, 18;6.

CHARLES SWAYNE, Judge of the United States District Court for the Northern District of Florida; accluitted; Mednesday, December I4, Igof, to Monday, February 27, 1905.

\section{MEMBERS OF THE HOUSE OF REPRESENTATIVES.}

Whatever may have been the indecision of the members of the Constitutional Convention as to the powers and duties to be ascribed to other portions of the governmental machinery they were constructing, there is no reason to suppose that there was any doubt among them as to the powers and prerogatives of the members of the lower House of the legislative body of the new nation. Many, if not most of the delegates, had been members of the Continental Congress and of the legislative body of the short lived Confederation and the members of the Constitutional Convention could well anticipate that they wonld themselves constitute the bulk of the lower House of the Congress for which they were to provide. The theory upon which the Revolution had been fonght was that of the establishment of a government by a representative body. It was the legitimate outcome of the Anglo-Saxon idea represented successively by the primitive folk-mete, the English Parliament, the Colonial town meeting, and the general courts of the governments which had been self-erected by the independent colonies. There was some question and discussion of the powers which the States might confer upon the general assembly, but no question as to 
the nature of the national assembly itself. As the earlier representative bodies had been composed of delegates from the great body of the people themselves, there was no other provision to be made but that the popular branch of the government should be similarly chosen.

The first provision with relation to a member of the House of Representatives of the United States, is that he shall be chosen by electors having the same qualifications as the electors who choose the most numerous branch of the State Legislature. In this provision, bringing the House of Representatives into the closest touch with a people choosing its own government, there is warrant in ranking the member of the House of Representatives as second only to the President in importance in the list of the officers of the Federal organization. While by courtesy divers other officers are given higher rank, the power and importance of the member of the House of Representatives is superior to that of any other official except the Executive, and in some respects it is co-equal with that of the President himself.

A member of the House of Representatives of the Congress of the United States must have attained to the age of twenty-five years and have been a citizen of the United States for seven years. He must be an inhabitant of the State in which he is chosen, but need not necessarily be a resident of the particular Congressional district he represents, although it is usual to restrict the selection of Representatives to the actual inhabitants of the respective districts. In the original Constitution citizenship of the United States was not considered in the qualifications of the electors of Representatives. There is, however, in the I 4 th amendment of the Constitution, ratified in I868, a provision penalizing such States as shall deny the right to vote for Representatives in Congress to citizens of the United States.

A reduction in the basis of representation in the House of Representatives, equal to the proportionate number of citizens of the United States so denied the right to vote is provided for, but, it may be added, this provision is not enforced. The basis of representation, fixing the number of members of the House of Representatives is one Representative for every thirty thou- 
sand, but each State is to have at least one Representative. The whole number of persons in each State, excluding Indians not taxed, is to be counted in making up the result on this basis of representation, unless the penalizing clause above stated becomes effective. A census is required by the Constitution to be taken every ten years, and the apportionments under the decennial censuses have been as follows:

\begin{tabular}{|c|c|c|c|c|c|c|c|c|c|c|c|c|c|}
\hline States. & 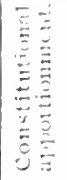 & 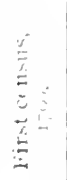 & 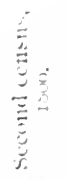 & 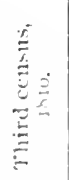 & 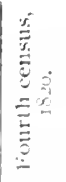 & 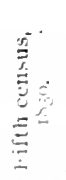 & 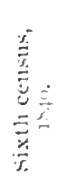 & 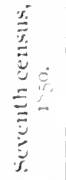 & 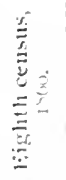 & 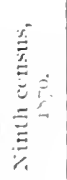 & 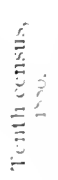 & 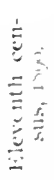 & 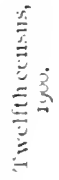 \\
\hline Alalnana. & & & $\cdots$ & I & 3 & 5 & 7 & 7 & 6 & si & i & 2 & 9 \\
\hline Atrantas. & & & & $\cdots$ & ..... & I & 1 & 2 & 3 & 4 & 5 & c. & 7 \\
\hline Californial......... & $i$ & $\ldots \ldots$ & .. & $\ldots \ldots$ & ...... & 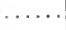 & 2 & 2 & 3 & 4 & c & 7 & $s$ \\
\hline oloratlo............ & & & & & & & & & & I & $i$ & 2 & 3 \\
\hline Connecticut........ & 5 & 7 & 7 & 7 & 6 & 0 & it & 4 & 4 & 4 & t & 1 & 5 \\
\hline Delawarc.......... & i & I & I & 2 & I & I & I & i & I & t & I & $i$ & 1 \\
\hline I lorida............ & $\ldots \ldots$ & .... & & & . & ..... & I & 1 & I & 2 & 2 & 2 & 3 \\
\hline Georgria............ & 3 & 2 & 4 & 0 & 7 & 9 & 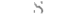 & $s$ & 7 & 9 & 10 & i1 & $1 \mathrm{t}$ \\
\hline 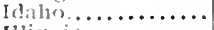 & $\cdots$ & . & & & & & & & & & I & I & $\mathrm{t}$ \\
\hline nllinus........... & & .... & ....... & 1 & 1 & 3 & 7 & ) & 1.4 & Iy & 24) & 22 & 25 \\
\hline Indinna.......... & ... & . & ....... & I & 3 & 7 & Ii) & it & II & $I_{3}$ & 1.3 & $1 ;$ & 13 \\
\hline Iowa............. & & & & 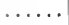 & ...... & .. & 2 & 2 & 6 & 9 & It & It & $1 \mathrm{l}$ \\
\hline$\ldots$. & & & & & ...... & & ...... & $\ldots$ & I & 3 & $i$ & i & 3 \\
\hline Kentucky......... & & 2 & 6 & 10 & 12 & I3 & I) & I0 & 9 & (ii) & ti & II & $1 \mathrm{I}$ \\
\hline lonisiana ......... & & $\ldots \ldots$ & $\ldots \ldots$ & 1 & 3 & 3 & 4 & + & 5 & i) & 0 & 6 & 7 \\
\hline$\ldots \ldots \ldots \ldots$ & $\ldots \ldots$ & $\ldots \ldots$ & $\ldots \ldots$ & 7 & 7 & 8 & 7 & 6 & 5 & 5 & 4 & 4 & it \\
\hline Maryland......... & 6 & is & 9 & 9 & 9 & 8 & 6 & 6 & 5 & 6 & 6 & 6 & 6 \\
\hline Matsiaclunsetts..... & s & If & 17 & 13 & 13 & 12 & 10 & II & 11) & 11 & 12 & 13 & I) \\
\hline Michisan .......... & $\ldots \ldots$ & ....... & ...... & $\ldots \ldots$ & $\ldots \ldots$ & 1 & 3 & 4 & 6 & y & 11 & 12 & 12 \\
\hline$\ldots$ & & ....... & ...... & ..... & $\cdots$ & .. & $\ldots \ldots$ & 2 & 2 & 3 & 5 & 7 & 9 \\
\hline ippi......... & $\ldots \ldots$ & $\ldots \ldots$ & $\ldots \ldots$ & 1 & I & 2 & 4 & 5 & 5 & i) & 7 & 7 & 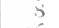 \\
\hline$\ldots$ & & $\ldots \ldots$ & $\ldots \ldots$ & $\ldots \ldots$ & 1 & 2 & 5 & $i$ & 9 & 13 & I. 1 & 13 & 16 \\
\hline ... & & $\ldots \ldots$ & $\ldots \ldots$ & $\ldots \ldots$ & . . & 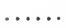 & ..... & .. & ... & $\ldots$ & I & 1 & I \\
\hline Nebr & & & & $\ldots$ & .... & & ....... & ...... & 1 & 1 & 3 & 6 & 6 \\
\hline$\cdots \cdots$ & & & & & & ... & $\ldots \ldots$ & ... & I & 1 & 1 & I & $\mathrm{I}$ \\
\hline New lIampshire... & 3 & if & 5 & 6 & 6 & 5 & if & 3 & 3 & 3 & 2 & 2 & 2 \\
\hline$y \ldots \ldots$ & 4 & 5 & 6 & 6 & 6 & 0 & 5 & 5 & 5 & 7 & 7 & s & 10 \\
\hline New York.... & 6 & io & 17 & 27 & 3.4 & to & 34 & 3,3 & $3 \mathrm{I}$ & 33 & 3.4 & 34 & 37 \\
\hline na... & 5 & 10 & 12 & 13 & 13 & $I_{3}$ & 9 & $s$ & 7 & $s$ & (1) & 9 & 10 \\
\hline Nortlı Dakota..... & & $\ldots$. & & .... & ..... & $\ldots$. & $\ldots \ldots$ & $\cdots$ & & ... & $i$ & I & 2 \\
\hline Ohi & & $\ldots \ldots$ & I & 6 & 1.4 & J) & 21 & 21 & Iy & 20 & $2 \mathrm{I}$ & $2 \mathrm{I}$ & 21 \\
\hline & & $\ldots \ldots$ & $\ldots$. & ... & $\ldots$ & .... & $\ldots \ldots$ & I & I & I & I & 2 & 2 \\
\hline ....... & 8 & ${ }_{13}$ & is & 23 & 26 & 24 & 24 & 25 & 24 & 27 & 24 & $5^{\circ}$ & 32 \\
\hline$\ldots \ldots$ & 1 & 2 & 2 & 2 & 2 & 2 & 2 & 2 & 2 & 2 & 2 & 2 & 2 \\
\hline Soutli Cárolina.... & 5 & 6 & $s$ & 9 & 9 & 9 & 7 & 6 & 4 & 5 & 7 & 7 & 7 \\
\hline ....... & & $\cdots$ & & & & & & $\cdots$ & & & & & 2 \\
\hline 'Tennessee. & ... & 1 & 3 & 6 & y & 13 & II & 10 & S & 10 & 10 & 10 & 10 \\
\hline 'Texas. . & ... & $\ldots \ldots$ & $\ldots \ldots$ & $\cdots$ & ... & ....... & 2 & 2 & 4 & 6 & $\amalg$ & I 3 & Io \\
\hline Utah. & 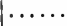 & $\ldots \ldots$ & & & ... & $\ldots$ & & & & & & I & I \\
\hline Vermo & 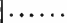 & 2 & 4 & 6 & 5 & 5 & 4 & 3 & 3 & 3 & 2 & 2 & 2 \\
\hline lirginia... & Io & 19 & 22 & 23 & $\therefore 2$ & 21 & 15 & 13 & 11 & 9 & 10 & 10 & 10 \\
\hline & . & $\ldots \ldots$ & & & ... & $\ldots \ldots$ & $\ldots \ldots$ & ..... & $\cdots$ & $\cdots$ & 1 & 2 & 3 \\
\hline & $\cdots$ & & & $\ldots$ & & $\ldots \ldots$ & $\cdots$ & & & 3 & 4 & 4 & 5 \\
\hline Wisconsin......... & 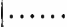 & ..... & ..... & $\cdots$ & & ...... & 2 & 3 & 6 & 3 & 9 & to & 11 \\
\hline Wyn:ming . & & $\cdots$ & $\cdots \cdots$ & & & $\cdots \cdots$ & $\ldots \ldots$ & $\ldots \ldots$ & $\cdots \cdots$ & $\ldots \ldots$ & 1 & I & 1 \\
\hline Tota1 .. & 65 & 106 & 142 & 1 in 6 & 213 & $24^{2}$ & 232 & 237 & 243 & 293 & 332 & 357 & 30 \\
\hline
\end{tabular}


Where but a single Representative falls to a State, such Representative is clected from the State at large. Where there is more than one Representative the State is divided into Representative districts by action of the State Legislature, and it sometimes occurs that one or more Congressmen at large are elected by the entire body of State electors in States having more than one Representative, this action being due to the failure of the legislature to divide the State into the requisite number of Representative districts. It is generally the purpose of the majority of a State Legislature in creating Representative districts, to afford as far as possible within the limits of public policy, the advantage to the political party dominant in the State at the time of the adjustment of district limits. An excessive distortion of district lines so as to procure political advantage, is called a gerrymander, a term originating in the construction of a Congressional district in Massachusetts, which when mapped out, had the general outlines of a salamander. The name of Elbridge Gerry, who was supposed to have been the moving spirit in the arrangement, was combined with the name of the fabled animal to coin the now celebrated political cognomen.

Vacancies occurring in the representation in any State are filled by a special election, and no person has any appointive power with regard to a member of the House of Representatives. The members of the House choose the Speaker and other officers of the House by ballot. The sole power of impeachment lies in the members of the House of Representatives. The President, Vice-President, and all civil officers of the United States may be removed upon impeachment. Treason and bribery are specifically named as ground for impeachment, and other high crimes and misdemeanors are left to definition by the impeaching power. Upon the presentation of charges forming ground for impeachment, the subject is referred to a Committee of the House, and upon a report of the Committee favoring impeachment, the matter is considered by the whole House, which decides upon the manner in which the managers of the impeachment shall be selected. Proceedings having been decided upon, the whole House proceeds in theory 
before the Senate, which acts as the court of impeachment. In practice, however, the House is represented and the case conducted by the members of the House of Representatives appointed as managers.

The members of the House of Representatives are judges of the clection and qualifications of the members of the House. It is necessary that the majority of the members of the House be present in order that business may be conducted, but a smaller number than a majority of the members may meet and may compel the attendance of absent members under such conditions as may have been provided by the rules adopted by the House, or established by precedent. A member may not be absent from the sessions of the House, cxcept he is excused, and a member absent without excuse may be brought to the bar of the House by the Sergeant-at-Arms, and renders himself, by such absence, liable to such penalties as the House may impose. Members may also be punished for disorderly behavior, and a member may be expelled by a coneurrence of two-thirds of the House. The House of Representatives cannot adjourn for more than three days during the session of Congress without the consent of the Senate, and cannot adjourn to any other place than that in which the two Houses may be sitting. Provision is made in the Constitution for an open ballot, in the requirement that yeas and nays on any rote shall be entered upon the journal upon the desire of one-fifth of the members present. Rules have been adopted with the purpose of enabling the majority of a quorum to transact business, notwithstanding the obstruction of a minority, and the rights of the minority are to some degree safeguarded by the same code of laws and rules.

A menber of the House of Representatives receives a compensation which is now fixed by law, at seven thousand five hundred dollars a year, and mileage at the rate of ten cents a mile for attending and returning from sessions. He also receives an allowance for stationery, amounting to one hundred and fifty clollars per year, and is allowed clerical assistance to the amount of one thousand five hundred dollars per year. Members who are chairmen of committees are allowed the services 
of clerks of committees for their own legislative business as well as for the business of the committees. Committee clerks are of two classes, annual clerks, and session clerks, the former receiving generally two thousand dollars per year, though the range of salary is from twelve hundred to fourteen hundred dollars per year, and the latter six dollars per diem.

Members of the House of Representatives as well as Senators are highly privileged and are exempt from arrest during their attendance at the session of the House, and during the time of their going to and returning from the session, this being construed to mean, not only the time actual appearance in the House, and of going to and from their residences in Washington, but as well the entire time of their residence in Washington during the session of Congress, and the time which many elapse from their departure from their legal residences to their return thereto. They may not be questioned for any speech or debate in the House, at any place, and it is also held that interference or protest on the part of the Executive, or of a member of either House of Congress, against the speech, vote, or motion of a member of the lower House, constitutes a breach of his privilege and of the privileges of the House of Representatives. Any question affecting the privilege of a member, or of the House, takes precedence over other business.

Members of the House of Representatives may not be appointed to any civil office under the United States which has been created, or whose compensation has been increased, during the time for which he is elected, and no person holding office under the United States can be a member of the House.

A prerogative of the highest power is given exclusively to the members of the House of Representatives in that all bills for raising revenue must originate in that House. This covers that very important class of legislation relating to the imposition of taxes on imports, as well as internal revenue provisions and other enactments from which a revenue is derived for the support of the general government. The House of Representatives also claims the initiative in preparing measures appropriating money for the expenditures of the general government, and 
regards as an encroachment upon the privileges of the House, original propositions by the Senate for the expenditures of public moneys, While the revenue bills are subject to amendments in the Senate and frequent and extensive amendments of appropriation bills are also made in the upper House, it is the theory that the House of Representatives has the control of the means of governmental expenditures, which is the foundation of real governmental power. The provision that bills for raising revenue shall originate in the House of Representatives, is an echo of the historic contests of the English Kings with their parliaments, and the prerogative of directing national expenditures which had been achicred by the English House of Commons as the lasis of the similar claim to the power which has been continued in the popular branch of the newer AngloSaxon government.

The powers delegated by the States to the Congress, and in which the House of Representatives participates, are as follows:

To raise moneys by taxes, impositions, duties, and excises which shall be uniform throughout the United States, for the purpose of paying the debts of the government and to provide for the general welfare of the country, and in anticipation of revenues to be thus raised, to borrow money on the credit of the United States and to direct the disposition of the public land and other public propertics.

It is empowered, also to regulate commerce with foreign nations, and with Indian tribes, and among the several States. The latter provision has been given perhaps the widest construction of any of the provisions of the Constitutions, since from it has been derived the power to regulate the rates of transportation of persons and commodities, and to provide for inspection and regulation of trade in many lines.

A uniform rule of naturalization by means of courts of record was authorized and has been established.

The authorization of a bankruptcy law, general throughout the United States, was given in the Constitution, but was not taken advantage of until i 867 . 
Congress may, and does, establish means for coining money and determines the values of the coins, and fixes the value of foreign coins, and is the authoritative source of standards of weights and measures throughout the United States. Upon the authority to coin money and the provision which grants power to borrow money on the credit of the United States, it bases its action in directing the issue of treasury notes and of legal tender certificates representing money so coined. In connection with this power it is given authority to provide for the punishment of counterfeiting the securities and coins of the United States.

It is empowered to establish postoffices and post roads. The latter provision was chiefly useful during the earlier days of lack of communication with remote sections of the Union, not provided with road facilities. It has, however, been utilized for sstablishing rights of way over bridges and upon the railroad systems of the country. Whether it may be utilized as a Constitutional provision on which to establish a system of national railroads, may be a nice question for the future to decide.

Congress is empowered to promote science and the useful arts, in a manner which is represented by the patent and copyright system of the Federal Government.

It may constitute courts inferior to the Supreme Court, the latter being established by the Constitution.

The foregoing provisions are those which appertain to sovereignty, and the following especial attributes of the sovereign are bestowed upon the Congress: to declare war, raise and support armies, with the proviso that no continuing appropriation shall be made for this purpose for a longer term than two years. As an auxiliary power Congress may grant letters to private persons to cmpower them to seize any vessels of a nation against which war is being waged, and the power is specified to make rules with regard to both land and water captures. As a further auxiliary to the Federal armies, the militia of the various States may be called out for the purpose of supporting the laws and to repel invasion. In order that the auxiliary may be made 
an effective one, Congress may provide for organizing and arming the militia, and may prescribe the discipline under which they shall be trained, but the States themselves reserve the authority of training the militia under ordinary circumstances, and of the appointment of their officers. Such part of the militia, howerer, as may be employed in the service of the United States, is to be governed by provisions made by Congress.

The Honse of Representatives also shares in a certain portion of the foreign relations of the United States, namely, in the power to define and punish piracy and other felonies committed upon the high seas, and all offenses against the law of nations. The major portion of the foreign relations, the power to declare war excepted, is vested in other than the House of Representatives.

The House of Representatives with the Senate the gorerning power having exclusive legislative rights over the District of Columbia and over the places purclased from and ceded by the several States, for the location of necessary structures belonging to the Federal Government, such as forts, arsenals, navy stations, lighthouse installations, etc. In the way of general powers, the House of Representatives shares in the authorization of Congress to make laws necessary for the carrying into effect of the powers granted to it, and of powers vested by the Constitution in the general government, or in departments, or officers of it.

In the way of limitation, there is a distinct provision contained in the roth amendment of the Constitution, being the final provision of those demanded by certain States as a condition of entering into the grant of powers contained in the original Constitution, that the powers not specifically delegated to the United States, are reserved to the respective States or to the people. The limitations specifically set forth are, that the privilege of the right of habeas corpus shall not be suspended, unless public safety requires it: that no direct tax shall be laid except in proportion to the general census; that no export tax shall be applied to any State: that no preference shall be given 
ports of one State over another; that duties shall not be imposed upon resseis going from one State to another; that no money shall be taken from the Treasury without an appropriation: That no title of nobility shall be granted by the United States; that the right of speedy and public trial by jury in criminal cases, and jury trial in common lawsuits for a value of more than twenty dollars, shall be preserved; that excessive bail, excessive fines, and cruel and unusual punishments, shall be prohibited. The States, on the other hand, agree with Congress, that no State shall undertake to cxercise the rights and prerogatives of sovereignty above described, and which have been conferred upon the Congress, with the sole proviso covering actual invasion or imminent danger which will not permit of delay in the use of armed forces.

The direct appointive power of members of the House of Representatives is limited to that of personal and committee clerks and of midshipmen at the Naval Academy, representing their districts. They are allowed to maintain two midshipmen continuously at the Academy, candidates being subject to examination by the Navy Department. The former theory of patronage by which positions in the civil service were allotted to Congressional districts has been abolished by the Civil Service Act, but members of the House of Representatives are supposed to have a certain privilege of recommendation to Federal offices filled by the President in their districts if such members are of the same political party as the Administration. The underlying theory is that the Representative is able to officially vouch for the character of an appointee, but the recommendatory privilege is the last remnant of a former powerful patronage exercised by the Representatives in Congress of the dominant party. The full extent of such patronage consists of places as postmaster, internal revenue and customs officials and a small number of places under the control of the officers of the House of Representatives, the employees of the House still being appointed in accordance with a spoils system similar to that which was abolished when the merit system was adopted 
in the civil service of the govermment for other than direct Presidential appointments.

Finally, among the prerogatives accruing to a menber of the House of Representatives is that which he holds jointly with the member of the Senate, that of proposing amendments to the Constitution, the fundamental law of the lancl. The concurrence of twothirds of the membership is necessary to propose an amendment to the States for ratification.

Formerly only those nembers of Congress who were chairmen of comnittees were furnished with office rooms in the Capitol, the remainder of the membership being expected to use their desks in the House of Representatives hall for the transaction of public business, and if this did not suffice to frequent the committee rooms of others or hire quarters outside. The new building of the House of Representatives has been designed to furnish each Representative with one office, while a corresponding building on the other side of the Capitol will provide rooms, and, in some cases, suites, for the Senators.

The rclations between the House of Representatives and the Senate are carefully adjusted with a riew of preventing friction, which, nevertheless, sometimes arises. On all measures except those relating to raising revenue and appropriations, propositions for legislation may be introluced either in the House or the Senate; as a matter of fact a very large number of identical bills are introduced, both in the House and the Senate, for the purpose of facilitating their enactment.

All bills are referred to appropriate committees for action, and the first effort of the advocates of a measure is to get a favorable report from the committec. This favorable report gives the bill a position on the calendar of the House or Senate, and either by arrangement witb the Speaker or with the Senate "steering" committee, it comes before the session for discussion and action. Appropriation and certain other bills in the House are referred on report by the committee to a Committee of the Whole House, which is the entire membership of the House, but with some one other than the Speaker in 
the chair. When perfected such bills are reported to the House itself for final passage. Bills which have been passed by one House are sent to the other for action. If they are passed without amendment they go at once to the President for signature. If, however, they are passed with one or more amendments they are "messaged" back to the House in which they originated for its action on the amendments.

A message having been received that the other House has passed a bill with amendments, the House in which the bill originated either concurs in the amendments or disagrees to them. In the latter case the bill is returined to the other House, stating a disagreement with the amendments of that House. The other House may either recede from its amendments or decline to recede therefrom. In the first case the bill passes in its original form; in the second, the bill is returned to the House where it originated, with the message that the second House insists on its amendments and requests a committee of conference. Members of such a committee being appointed by each of the two Houses, the committee confers, and if it can arrange the points of disagreement between the Houses, a report is made to either House in which the recommendation may be for the amending House to recede from its amendment, the originating House to recede from its disagreement with the amendment, or either or both to recede with an amendment. If the conferees fail to agree, the originating House notifies the other that it still disagrees, and may "still further" disagree until the point of no possibility of an agreement exists, when one House may notify the other that it "adheres," and the second House must then either recede or the measure fails. It is seldom that this final point is reached, and even when notice of adherence is given it can be recalled and a less decided message which would permit of a conference substituted.

In addition to members of the House from the States, there is a delegate from each Territory, including Porto Rico, elected by the people of the Territories, who have seats on the floor of the House, membership on committees, and the pay and emoluments of members. They can only speak, however, on matters relating to the 
affairs of their respective Territories and cannot vote on any measure.

COMMITTEES OF TIL HOUSE.

Accounts; Agriculture: Alcoholic Liquor Traffic; Appropriations; Banking and Currency; Census; Claims: Coinage, Neights and Measures; Disposition of Uscless Executive Papers; District of Columbia; Erlucation; Election of President, Vice-President, and Representatives in Congress; Election No. 1; Elcction No. 2; Election No. 3; Enrolled Bills; Expenditures in the Department of Agricnlture; Expenditures in the Department of Commerce and Laber; Expenditures in the Departinent of Justice; Expenditures in the Interior Department; Expenditures in the Navy Department; Expenditures in the Post Office Department; Expenditures in the State Department; Expenditures in the Treasury Department; Expenditures in the War Department; Expenditures on Public Buildings; Foreign Affairs; Immigration and Naturalization; Indian Affairs; Industrial Arts and Expositions; Insular Affairs; Interstate and Forcion Connerce; Invalid Pensions; Irrigation of Arid Lands; Judiciary; Labor; Levees and Improvements of the Mississippi River; Library; Manufactures; Merchant Marine and Fisheries; Mileage; Military Affairs; Militia: Mines and Mining: Naval Affairs; Pacific Railroads; Patents; Pensions, Post Office and Post-Roads; Printing; Private Land Claims; Public Buildings and Grounds; Public Lands; Railways and Canals; Reform in the Civil Service; Revision of the Laws; Rivers and Harbors; Rules; Territories: Ventilation and Aconstics; War Claims; Ways and Means.

\section{Alabama: $\quad$ REPRESENTATIVE DISTRICTS.}

First District: Counties.-Chocktaw, Clarke, Marengo, Mobile, Monroe and Washington.

Second District: Connties.-Baldwin, Butler, Conccul, Covington, Crenshaw, Escanbia, Mintgomery, Pike and Wilcox.

Third District: Counties.-Barbour, Bullock, Coffec, Dale, Geneva, Henry, Houston, Lee and Russell.

Fourth District: Counties.-Cahhom, Chilton, Cleburne, Dallas, Shelby and Talladega.

Fifth District: Counties.-Antanga, Chambers, Clay, Coosa, Elmore, Loundes, Macon, Rardolph and Tallapoosa.

Sixth District: Counties.-Fayette, Greene, Hale, Lamar, Marion, Pickens, Sumter, Tuscaloosa and Walker.

Seventh District: Counties.-Cherokec, Cullman, Dekalb, Etowal, Franklin, Marshall, St. Clair and Winston.

Eighth District: Counties.-Co!bert, Jackson, Lauderdale, Lawrence, Limestone, Madison and Morgan.

Ninth District: Counties.-Bibb, Blount, Jefferson and Perry. 


\section{Arkansas :}

Firse District: Comnties.-Clay, Craighead, Crittenden, Cross, Greene, Lec, Mississippi, Phillips, Poinsett, St. Francis and Woodruff.

Second District: Counties.-Cleburne, Fulton, Independence, Izard, Jackson, Lawrence, Monroe, Prairie, Randolph, Sharp, Stone and White.

Third District: Countics.-Baxter, Benton, Boone, Carrol, Madison, Marion, Newton, Searcy, Van Buren and Washington.

Fourth District: Counties.-Crawford, Howard, Little River, Logan, Miller, Montgomery, Pike, Polk, Scott, Sebastian and Sevier.

Fifth District: Counties.-Conway, Faulkner, Franklin, Jolnnson, Perry, Pope, Pulaski and Yell.

Sixth District: Counties.-Arkansas, Cleveland, Dallas, Desha, Drew, Garland, Grant, Hot Springs, Jefferson, Lincoln, Lonoke and Saline.

Seventh District: Counties.-Ashley, Bradley, Calhoun, Chicot, Clark, Columbia, Hempstead, Lafayette, Nevada, Ouachita and Union.

\section{Califorisia :}

First District: Counties.-Alpine, Amador, Calaveras, Del Norte, Eldorado, Humboldt, Lassen, Mariposa, Modoc, Mono, Placer, Plumas, Shasta, Sierra, Nevada, Siskiyou, Tehama, Trinity and Tuolumne.

Second District: Counties.-Butte, Colusa, Gleen, Lake, Marin, Mendocino, Napa, Sacramento, Sonoma, Sutter, Yolo and Yuba.

Third District: Counties.-Alameda, Contra Costa and Salano.

Fourth District: City of San Francisco.-Twenty-eighth, Twenty-ninth, Thirticth, Thirty-first, Fortieth, Forty-first, Forty-second, Forty-third, Forty-fourth and Forty-filth assembly districts.

Fifti, District: Counties.-San Mateo, Santa Clara, and the Thirty-second, Thirty-third, Thirty-fourth, Thirty-fifth, Thirty-sixth, Thirtyseventh, Thirty-eighth and Thirty-ninth assembly districts of San Francisco.

Sixth District: Counties.-Fresno, Kings, Madera, Merced, Monterey, San Benito, San Joaquin, Santa Cruz and Stanislans.

Seventh District: Counties.-Los Angeles.

Eighth District: Counties.-Inyo, Kern, Orange, Riverside, San Bernardino, San Diego, San Luis Obispo, Santa Barbara, Tulare and Ventura.

\section{Colorado:}

First District: Counties.-Adams, Boulder, Denver, Jefferson, Lake, Larimer, Logan, Morgan, Park, Phillips, Sedgwick, South Arapahoe, Washington, Weld and Yuma.

Second District: Counties.-Archuleta, Baca, Bent, Chaffee, Cheyenne, Clear Creek. Conejos, Costilla, Custer, Delta, Dolores, Douglas, Eagle, Elbert, El Paso, Fremont, Garfield, Gilpin, Grand, Gunnison, Hinsdale, Huerfano, Kiowa, Kit Carson, La Plata, Las Animas, Lincoln, Mesa, 
Mineral, Montezuma, Montrose, Otero, Ouray, Pitkin, Prowers, Pucblo, Rio Blanco, Rio Grande, Routt, Saguache, San Juan, San Miguel, Summit and Teller.

\section{Connecticut :}

First District: Counties.-Hartford and Tolland, including the cities of Hartford, New Britain and Rockville.

Sccond District: Counties._Middlesex and New Haven, including the cities of New Haven, Meriden, Waterbury, Ansonia, Derby and Middletown.

Third District: Countics.-New London and Windhan, including the cities of New London and Norwich.

Fourth District: Counties.-Fairfield and Litchfield.

DelawARE:

Representative at large.

FLORIDA :

First District: Counties.-Citrus, De Soto, Hernando, Hillsboro, Lafayette, Lake, Lee, Levy, Manatce, Marion, Monroe, Pasco, Polk, Sumter and Taylor.

Second District: Counties.-Alachua, Baker, Bradford, Brevard, Clay, Columbia, Dade, Duval, Hamilton, Nassau, Orange, Osceola, Putnam, St. Johns, Suwanee and Volusia.

Third District: Counties.-Calhoun, Escambia, Franklin, Gadsden, Holmes, Jackson, Jefferson, Lcon, Liberty, Madison, Santa Rosa, Wakulla, Walton and Washington.

\section{Georgia :}

First District: Counties.-Bryan, Bulloch, Burke, Chatham, Effingham, Emanuel, Jenkins, Liberty, McIntosh, Screven, Tattuall and Toombs.

Second District: Counties.-Baker, Berrien, Calhoun, Clay, Colquitt, Decatur, Dougherty, Early, Grady, Miller, Mitchell, Quitman, Randolph, Terrell, Tift, Thomas, Twrner and Worth.

Third District: Counties.--Craw ford, Crisp, Dooly, Houston, Lee, Macon, Pulaski, Schley, Stewart, Sumter, Taylor, Twiggs, Webster and Wilcox. Fourth District: Counties.-Carroll, Chattahoochec, Coweta, Harriss, Heard, Marion, Meriwether, Muscogee, Talbot and Troup.

Fifth District: Counties.-Campbell, Clayton, Dekalb, Douglas, Fulton, Newton, Rockdale and IValton.

Sixth District: Counties.-Baldwin, Bibb, Butts, Fayette, Henry, Jones, Monroe, Pike, Spalding and Upson.

Seventh District: Counties.-Bartow, Catoosa, Chatooga, Cobb, Dade, Floyd, Gordon, Haralson, Murray, Paulding, Polk, Walker and Whitfield. 
Eighth District: Counties.-Clarke, Elbert, Franklin, Greene, Hart, Jasper, Madison, NIorgan, Oconee, Oglethorpe, Putnam and Wilkes.

Ninth District: Counties.-Banks, Cherokee, Dawson, Fannin, Forsyth, Gilmer, Gwinnett, Habersham, Hall, Jackson, Lumpkin, Milton, Pickens, Rabun, Stephens, Towns, Union and White.

Tenth District: Counties._Columbia, Glascock, Jefferson, Hancock, Lincoln, NicDuffie, Richmond, Taliaferro, Warren, Washington and Wilkinson.

Eleventh District: Counties.-Appling, Brooks, Camden, Charlton, Clinch, Coffee, Dodge, Echols, Glynn, Irwin, Jeff Davis, Johnson, Laurens, Lowndes, Montgomery, Pierce, Telfair, Vare and Wayne.

IDAHO:

Pepresentative at Large.

\section{IllinoIS :}

Firs ${ }^{2}$ District: City of Chicago.-First and Second wards; part of the Third ward east of Halstead Street; part of the Sixth ward north of Forty-third Street.

Second District: City of Chicago.--Seventh, Eighth and Thirty-third wards; part of the Sixth ward south of Forty-third Street.

Third District: Cook County.--Towns of Bloom, Bremen, Calumet, Lemont, Orland, Palos, Rich, Thornton and Worth.

City of Chicago-Thirty-first and Thirty-second wards; parts of the Twenty-ninth and Thirtieth wards south of Fifty-first Street.

Fourth District: City of Chicago._Fifth ward; part of the Third ward west of Stuart Avenue; part of the Fourth ward west of Halstead Street; part of the Eleventh and Twelfth wards south of Twenty-second Street; part of the Twenty-ninth and Thirtieth wards north of Fiftyfirst Street.

Fifth District: City of Chicago.--Ninth and Tenth wards; part of the Eleventh and Twelfth wards north of Twenty-second Street.

Sixth District: Cook County.-Towns of Cicero, Lyons, Proviso, Riverside and Stickney.

City of Chicago.-Thirteenth, Twentieth and Thirty-fourth wards; part of the Thirty-fifth ward south of the Chicago and Northwestern Railway right of way.

Seventh District: Cook County.-Towns of Barrington, Elk Grove, Hanover, Leyden, Maine, Norwood Park, Palatine, Schaumberg and Wheeling.

City of Chicago.-Twenty-seventh and Twenty-eighth wards; part of the Fourteenth and Fifteenth wards west of Robey Street; part of the Thirty-fifth ward north of the Chicago and Northwestern Railway right of way. 
Eiglath District: City of Chicago-_Sixtecnth. Serenteenth, Eighteentli and Nincteenth warls; part of the lifteentl ward east of Robey Street. Nintl District: City of Chicago.-Twenty-first and Twenty-second wards; part of the Twenty-thirel ward east of llalstead Strect; part of the Twenty-fifth ward south of Graccland Arenue.

Tenth District: Cook Comnty.-Towns of Eranston, Niles, New Prior and Northificl.

City of Chicago.-Twenty-fourth and Twenty-sixth wards; part of the Twenty-third ward west of Hlalstead Street; part of the Twenty-fifth ward north of Graccland Avenue.

Lake County.

Eleventh District: Counties.-Dupage, Kane, Mcllenry and Will.

Twelfth District: Counties.-Boone, Dekalh, Grundy, Kendall, Lasalle and Winnebago.

Thirtenth District: Countics.-Carroll, Jo-Daviess, Lee, Ogle, Stephenson and Whiteside.

Fourteenth District: Counties.-Hancock, Henderson, McDonough, Mercer, Rock Island and Warren.

Fifteenth District: Counties.-Adams, Fulton, Henry, Knox and Schuyler.

Sixteenth District: Counties.-Burcan, Marshall, Pcoria, Putnam, Stark and Tazewell.

Seventeenth District: Conntics.-Ford, Livingston, Logan, McLean and Woodford.

Eighteenth District: Comnties.-Clark, Cumberland, Edgar, Iroquois, Kankakee and Vermillion.

Nineteenth District: Counties.-Champaign, Coles, Dewitt, Douglas, Macon, Nonltrie, Piatt and Sheiby.

Twentieth District: Counties.-Brown, Calhoun, Cass, Greene, Jersey, Mason, Menard, Morgan, Pike and Scott.

Twenty-first District: Connties.-Christian, Macoupin, Montgomery and Sangamon.

Twenty-sccond District: Counties.-Bond, Madison, Monroe, St. Clair and Washington.

Twenty-third District: Connties-Clinton, Crawford, Effinglam, Fayette, Jasper, Jefferson, Lawrence. Marion, Richland and Wabash.

Twenty-fourth: Counties-Clay, Edwards, Gallatin, Hamilton, Hardin, Johnson, Massac, Pope, Saline, Wayne and White.

Twenty-fifth District: Counties.-Alexander, Franklin, Jackson, Perry, Pulaski, Randolph, Union and Williamson.

\section{INDIANA :}

First District: Counties.-Gibson, Pike, Posey, Spencer, Vanderburg and Warrick. 
Second District: Counties.-Daviess, Greene, Knox, Lawrence, Martin, Monroe, Owen and Sullivan.

Third District: Counties.-Clark, Crawford, Dubois, Floyd, Harrison, Orange, Perry, Scott and Washington.

Fourth District: Counties.-Bartholomew, Brown, Dearborn, Jackson, Jefferson, Jennings, Jolinson, Ohio, Ripley and Switzerland.

Fiftl District: Counties.-Clay, Hendricks, Morgan, Parke, Putnam, Vermillion and Vigo.

Sixth District: Counties.-Decatur, Fayette, Franklin, Hancock, Henry, Rush, Shelby, Union and Mayne.

Seventh District: County.--Marion.

Eighth District: Counties.-Adans, Delaware, Jay, Madison, Randolph and Wells.

Ninth District: Counties.-Boone, Carroll, Clinton, Fountain, Hamilton, Howard, Montgomery and Tipton.

Tenth District: Counties.-Benton, Jasper, Lake Laporte, Newton, Porter, Tippecanoe, Warren and White.

Eleventh District: Counties.-Blackford, Cass, Grant, Huntington, Miami and Wabash.

Twelfth District: Counties.--Allen, Dekalb, Lagrange, Noble, Steuben and Whitley.

Thirteenth District: Counties.-Elkhart, Fulton, Kosciusko, Marshall, Pulaski, St. Joseph and Starke.

IowA :

First District: Ccunties.-Des Moines, Henry, Jefferson, Lee, Louisa, Van Buren and Washington.

Second District: Counties.-Clinton, Iowa, Jackson, Johnson, Muscatine and Scott.

Third District: Counties.-Blackhawk, Bremer, Buchanan, Butler, Delaware, Dubuque, Franklin, Hardin and Wright.

Fourth District: Counties.--Allamakee, Cerro Gordo, Chickasaw, Clayton, Fayette, Floyd, Howard, Nitchell, Winneshiek and Worth.

Fifth District: Counties.-Benton, Cedar, Grundy, Jones, Linn, Marshall and Tama.

Sixth District: Counties.-Davis, Jasper, Keokuk, Mahaska, Monroe, Powshiek and Wapello.

Seventh District: Counties.-Dallas, Madison, Marion, Polk, Story and Warren.

Eighth District: Courties.-Adams, Appanoose, Clarke, Decatur, Fremont, Lucas, Page, Ringgold, Taylor, Union and Wayne.

Ninth District: Counties.-Adair, Audubon, Cass, Guthrie, Harrison, Mills, Montgomery, Pottawattamie and Shelby.

Tenth District: Counties.-Boone, Calhoun, Carroll, Crawford, Emmett, 
Greene, Hanilton, Ilancock, Humboldt, Kussutl, Palo Alto, Pocahontas, Vebster and Vinuebago.

Eleventh District: Ccunties.-Buena Vista, Cherokee, Clay, Dickinson, Ida, Lyon, Monona, O'Brien, Osceola, Plymouth, Sac, Sioux and Woodbury.

Kansas:

First District: Comntics.-Atchison, Brown, Duniphan, Jackson, Jefferson, Leavenworth, Nemaha and Slawnec.

Second District: Comnties.-Allen, Anderson, Bourbon, Douglas, Franklin, Jolnson, Linn, Niami and Wyandotte.

Third District: Counties.-Chauttauqua, Cherokec, Cowley, Crawford, Elk, Labette, Montgomery, Nicosho and Wilson.

Fourth District: Counties,-Butler, Chase, Coffec, Greenwood, Lyon, Marion, Morris, Osage, Pottawatomic, Wabaunse and Woodson.

Fifth District: Counties.-Clay, Cloud, Dickinson, Geary, Marshall, Ottawa, Republic, Riley, Saline and Washington.

Sixth District: Counties.-Cheyenne, Decatur. Ellis, Ellsworth, Gove, Graham, Jewell, Lincoln, Logan, Mitchell, Norton, Osborne, Phillips, Rawlins, Rooks, Russell, Sheridan, Sherman, Smith, Thomas, Trego and Wallace.

Seventh District: Counties.-Barber, Barton, Clark, Comanche, Edwards, Finney, Ford, Grant, Gray, Greeley, Hamilton, Harper, Harvey, Haskell, Hodgeman, Kearney, Kingman, Kiowa, Lane, McPherson, Meade, Morton, Ness, Pawnec, Pratt, Reno, Rice, Rush, Scott, Seward, Sedgwick, Stafford, Stanton, Sterens, Sumner, Wichita.

\section{KenteCKY:}

First District: Counties.-Ballard, Caldwell, Calloway, Carlisle, Crittenden, Fulton, Graves, Hickman, Livingston, Lyon, McCracken, Marshall and Trigg.

Second District: Comnties.-Christian, Daviess, IIancock, Henderson, Hopkins, McLean, Union and Vebster.

Third District: Counties.-Allen, Barren, Butler, Edmonson, Logan, Metcalf, Muhlenberg, Simpson, Todd and Warren.

Fourth District: Countics.-Breckinridge, Bullitt, Grayson, Green, Hardin, Hart, Larue, Marion, Meade, Nelson, Ohio, Taylor and Washington.

Fifth District: County.-Jefferson.

Sixth District: Counties.-Boone, Campbell, Carroll, Gallatin, Grant, Kenton, Pendleton and Trimble.

Seventh District: Counties.-Bourbon, Fayette, Franklin, Henry, Oldham, Owen, Scott and Woodford. 
Eighth District: Counties.-Anderson, Boyle, Garrard, Jessamine, Lincoln, Madison, Mercer, Rockcastle, Shelby and Spencer.

Ninth District: Counties.-Bath, Boyd, Bracken, Carter, Fleming, Greenup, Harrison, Lawrence, Lewis, Mason, Nicholas, Robertson and Rowan. Tenth District: Counties.-Breathitt, Clark, Elliot, Estill, Floyd, Johnson, Knott, Lee, Magoffin, Martin, Mennifee, Montgomery, Morgan, Pike, Powell and Wolf.

Eleventh District: Counties,-Adair, Bell, Casey, Clay, Clinton, Cumberland, Harlan, Jackson, Knox, Letcher, Leslie, Laurel, Monroe, Owsley, Perry, Pulaski, Russell, Wayne and Whitley.

\section{LOUISIANA :}

First District: Counties.-City of New Orleans, Third, Fourth, Fifth, Sixth, Seventh, Eighth, Ninth and Fifteenth wards.

Parishes.-St. Bermard and Plaquemines.

Second District: Counties.-City of New Orleans, First, Second, Tenth, Eleventh, Twelfth, Thirteenth, Fourteenth, Sixteenth and Seventeenth wards.

Parishes.-Jefferson, St. Charles, St. James and St. John the Baptist.

Third District: Counties.-Parishes.-Assumption, Iberia, Lafayette, Lafourche, St. Martin, St. Mary, Terrebone and Vermillion.

Fourth District: Parishes.-Bienville, Bossier, Caddo, De Soto, Natchitoches, Red River, Sabine, Webster and Winn.

Fifth District: Parishes.-Caldwell, Catahoula, Claiborne, Concordia, East Carroll, Franklin, Jackson, Lincoln, Madison, Morehouse, Ouachita, Richland, Tensas, Union and West Carroll.

Sixth District: Parishes.-Ascension, Iberville, East Baton Rouge, East Feliciana, Livingston, Pointe, Coupee, St. Helena, St. Tammany, Tangipahoa, Washington, West Baton Rouge and West Felicina.

Seventh District: Parishes.-Acadia, Avoyelles, Calcasieu, Cameron, Grant, Rapides, St. Landry and Vernon.

MAINe:

First District: Counties.-Cumberland and York.

Second District: Counties.-Androscoggin, Franklin, Knox, Lincoln, Oxford and Sagadahoc.

Third District: Counties.-Hancock, Kennebec, Somerset and Waldo.

Fourth District: Counties.-Aroostook, Penobscot, Piscataquis and Washington.

MARYland :

First District: Counties.-Caroline, Cecil, Dorchester, Kent, Qeen Anne, Somerset, Talbot, IVicomico and Worcester.

Second District: Counties.-Baltimore, Carroll and Hartford; and Fifteenth and Sixteenth wards of Baltimore City. 
Third District: City of Baltimore-CIirst, Sccond, Third, Fourth, l.ifth, Sixtin, Seventh, Eighth and Twenty-second wards, and the Ninth, Tenth, Eleventh and Thirteenth precincts of the Eighteenth ward.

Fourth District: City of Baltimore.-Ninth, Tenth, Eleventh, Twelfth, Thirteenth, ["ourteenth, Seventecnth, Nineteenth and Twenticth wards, and the First, Second, Third and Twelfth precincts of the Eighteenth ward.

Fifth District: Counties.-Anne Arundel, Calvert, Charles, Howard, Prince George and St. Mary, and the Fourth, Fifth, Sixth, Seventh and Eighth precincts of the Eighteenth ward, and the Twenty-first, Twenty-third and Twenty-fourth wards of Baltimore City.

Sixth District: Counties.-Allegheny, Frederick, Garrett, Mlontgomery and Washington.

\section{Massachusetts :}

First District: Berkshire County.

Franklin County.-Towns of Aslfield, Bernardston, Buckland, Charlemont, Colerain, Conway, Decrfield, Gill, Greenfield, Hawley, Heath, Leyden, Rowe, Shelburne and Whatley.

Hampshire County: Towns of Chesterfield, Cummington, Goshen, Ilatfield, Huntington, Middlefield, Plainfield, Southampton, Westhampton, Williamsburg, and Worthington.

Hampden County: City of Holyoke and town of Agawam, Blandford, Chester, Granville, Montgomery, Russell, Southwick, Tolland, Westfield and West Springfield.

Second District: Franklin Connty.-Towns of Erving, Leverett, Montague, New Salem, Northfield, Orange, Shutesbury, Sunderland, Warwick and Wendell.

Hampshire County: City of Northampton: towns of Amherst, Belchertown, Easthampton. Enfield, Granby, Greenwich. Hadley, Pelham, Prescott, South Hadley and Ware.

Hampden County: Citics of Chicopee and Springfield; towns of Brimfield, East Longmeadow, Hampden, Holland, Long Mleadow, Ludlow. Monson, Palmer, Wales and Wilbraham.

Worcester County: Towns of Athol, Barre, Brookfield, Dana, Hardwick, New Braintree. North Brookfreld, Oakhan, Petersham, Phillipston, Warren and West Brookfield.

Third District: Worcester County.-City of Worcester; towns of Auburn, Charlton, Douglass, Dudley, Grafton, Holden, Leicester, Millbury, Northbridge, Oxford, Paxton, Rutland, Shrewsbury, Southbridge, Spencer, Sturbridge, Sutton, Uxbridge, Webster, Westboro and West Boylston.

Fourth District: Worcester County.-City of Fitchburg; towns of Ashburnham, Berlin, Bolton, Boylston, Clinton, Gardiner, Harvard, Hub- 
bardston, Lancaster, Lunenburg, Leominster, Northboro, Princeton, Southboro, Sterling, Templeton, IVestminster and Winchenden.

Middlesex County.-Cities of Marlboro and Waltham; towns of Acton, Asliby, Ashland, Ayer, Bedford, Boxboro, Concord, Framingham, Groton, Hudson, Lexington, Lincoln, Littleton, Maynard, Natick, Pepperell, Shirley, Stow, Sudbury, Townsend, Wayland, Westford and Weston.

Fifth District: Middlesex County.-City of Lowell; towns of Billerica, Burlington, Carlisle, Chelmsford, Dracut, Dunstable, North Reading, Reading, Tewksbury, Tyngsboro and Vilmington.

Essex County.-City of Lawrence; towns of Andover, Lynnfield, Methuen and North Aindover.

Sixth District: Essex County-Cities of Beverly, Gloncester, Haverhill, Newburyport, and Salem, and towns of Amesbury, Boxford, Danvers, Essex, Georgetown, Groveland, Hamilton, Ipswich, Manchester, Marblehead, Merrimac, Middleton, Newbury, Peabody, Rockport, Rowley, Salisbury, Swampscott, Topsfield, Wenham and West Newbury.

Seventh District: Essex County.-City of Lynn; towns of Nahant and Saugus.

Middlesex County: Cities of Everett, Malden and Melrose; Suffolk County: City of Chelsea; town of Revere.

Eighth District: Middlesex County.--Cities of Cambridge, Medford, Somerville and IVoburn; towns of Arlington, Belmont and Winchester.

Ninth District: Suffolk County.-Wards numbered One, Two, Three, Four, Five, Six, Seven, Eiglit, Nine, and precincts Six and Seven of Ward numbered Twelve, in the City of Boston; the town of Winthrop. Tenth District: Suffolk County.-Wards numbered Thirteen, Fourteen, Fifteen, Sixteen, Seventeen, Twenty and Twenty-four, in the city of Boston.

Norfolk County: City of Quincy and the town of Milton.

Eleventh District: Suffolk County.-Wards numbered Ten, Eleven, and precincts one, two three, four and five of ward numbered twelve, and wards numbered Eighteen, Nineteen, Twenty-one, Twenty-two, Twenty-three, and Twenty-five in the city of Boston.

Twelfth District: Norfolk County.-Towns of Avon, Bellingham, Braintree, Brookline, Canton, Dedham, Dover, Foxboro, Franklin, Holbrook, Hyde Park, Medfield, Medway, Millis, Needham, Norfolk, Norwood, Randolph, Sharon, Stoughton, Walpole, Wellesley, Westwood, Weymouth, and IVrentlan.

Middlesex County.-City of Newton, towns of Holliston, Hopkinton, Sherborn and Watertown.

Worcester County.-Towns of Blackstone, Hopedale, Mendon, Milford and Upton.

Bristol County.--Town of North Attleboro.

Thirteenth District: Bristol County.-Cities of Fall River and New Bed- 
ford; towns of Acushuct, Berkley; Dartmouth, Dighton, Fairhaven, Freetown, Rehoboth, Scekonk, Somerset, Swansea and Westport.

Plymouth County.-Towns of Marion, Mattapoisett and Rochester.

Dukes and Nantucket Counties.

Fourteenth District: Plymontl County.-City of Brockton, towns of Abington, Bridgewater, Carver, Duxbury, East Bridgewater, Ilalifax, Hanover, Hanson, Hingham, Hull, Kingston, Lakeville, Marshfield, Middleboro, Norwell, Penuloroke, Plymonth, Rockland, Scituate, Wareham, West Bridgewater and Whitman.

Bristol County.-City of Taunton; towns of Attleboro, Easton, Mansfield, Norton and Raynham.

Norfolk County.-Town of Cohasset.

Barnstable County.

\section{Michighi: :}

First District: City of Detroit.-First, Second, Third, Fourth, Fifth, Sixth, Serenth, Eighth, Ninth, Tenth, Eleventl, Thirteenth, Fifteenth and Seventeentli wards.

Second District: Comnties.-Jackson, Lenawee, Monroe and Washtenaw. Wayne County--Townships of Brownstone, Canton, Ecorse, Huron, Montguagon, Nankin, Northrille, Plymouth, Romulus, Sumpter, Taylor, and Van Buren and Wyandotte City.

Third District: Countics.-Branch, Calhoun. Eaton, Hillsdale and Kalamazoo.

Fourth District: Counties.-Allegan, Barry, Berrien, Cass, St. Joseph and Van Buren.

Fifth District: Counties.-Ionia, Kent and Ottowa.

Sixth District: Counties.-Genesee, Ingham, Livingston, Oakland; townships of Lavonia, Redford, Greenfield, Dearborn, Nankin and springwells, of the county of Wayne, and the Twelfth, Fourteenth and Sixteenth wards of the city of Detroit.

Seventh District: Countics.-Huron, Lapeer, Macomb, Sanila and St. Clair, and Grosse Pointc, Gratiot and Hamtranck: townships of IVayne county.

Eighth District: Counties.-Clinton, Saginaw, Shiawassee and Tuscola. Ninth District: Counties-Benzie, Lake, Leelanaw, Manistee, Mason, Muskegon, Newaygo, Oceana and Wexford.

Tenth District: Counties.-Alcona, Alphena, Arenac, Bay, Cheboygan, Crawford, Emmett, Gladwin, Iosco, Midland, Montmorency, Ogemaw, Oscoda, Ostego and Fresque Isle.

Eleventh District: Counties.-Antrim, Charlevoix, Clare, Grand Traverse. Gratiot, Isabe!la, Kalkaska, Mecosta, Missaukee, Montcalm, Osceola and Roscommon. 
Twelfth District: Connties.-Aiger, Baraga, Chippewa, Delta, Dickinson, Gogebic, Houghton, Iron, Keweenaw, Luce, Mackinac, Marquette, Menominee, Ontonagon and Schoolcraft.

Minnesota:

First District: Counties.-Dodge, Fillmore, Freeborn, Houston, Mower, Olmsted, Steele, Wabasha, Waseca, and Winona.

Second District: Counties.-Blue Earth, Brown, Cottonwood, Faribault, Jackson, Martin, Murray, Nobles, Pipestone, Rock and Watonwan. Third District: Counties.-Carver, Dakota, Goodhue, Lesueur, McLeod, Nicollet, Rice, Scott, and Sibley.

Fourth District: Counties.-Chisago, Ramsey and Washington.

Fiftin District: County.-Hennepin.

Sixth District: Counties.-Benton, Cass, Crow IVing, Douglas, Hubbard, Meeker, Morrison, Sherburne, Stearns, Todd, Wadena and Wright. Seventh District: Counties: Bigstone, Chippewa, Grant, Kandiyohi, Lacqui Parle, Lincoln, Lyon, Pope, Redwood, Renville, Stevens, Swift, I raverse and Yellow Medicine.

Eighth District: Counties.-Aitkin, Anoka, Carlton, Cook, Isanti, Itasca, Kanabcc, Lake, Millelacs, Pine and St. Louis.

Ninth District: Connties.-Becker, Beltrami, Clay, Clearwater, Kittson, Marshall, Norman, Ottertail, Polk, Red Lake, Rosean and Wilkin.

\section{Mississippi :}

First District: Counties.-Alcorn, Itawamba, Lee, Lowndes, Monroe, Noxubee, Oktibbelna, Prentiss and Tishomingo.

Second District: Counties.-Benton, Lafaycte, Marshall, Panola, Tallahatchie, Tate, Tippah and Union.

Third District: Counties.-Bolivar, Coahoma, Holmes, Issaquena, Leflore, Quitman, Sharkey, Sunflower, Tunica, and Washington.

Fourth District: Counties.-Attala, Calhoun, Carroll, Chickasaw, Choctaw, Clay, Grenada, Montgomery, Pontotoc, Webster and Yalobusha. Fiftl District: Counties.-Clarke, Jasper, Kemper, Lauderdale, Leake, Neshoba, Newton, Scott, Smith and Winston.

Sixth District: Counties.-Covington, Greene, Hancock, Harrison, Jackson, Jeff Davis, Jones, Lawrence, Marion, Lamar, Pearl River, Perry, Simpson and Wayne.

Seventh District: Counties.-Adans, Amite, Claiborne, Copia, Franklin, Jefferson, Lincoln, Pike and WVilkinson.

Eighth District: Counties.-Hinds, Madison, Rankin, Warren and Yazoo. 
Missouri:

First District: Comntics.-Adair, Clark, Knox, Lewis, Macon, Marion, Putman, Schuyler, Scotland and Shclly.

Second District: Connties.-Cartoll, Chariton, Grundy, Linn, Livingstone, Monroe, Randolph and Sullivan.

Third District: Connties.-Caldweil. Cliy, Clinton, Dariess, Dekalb, Gentry, Harrison, Mereer, Ray and Worth.

Fourth District: Counties._Andrew, Atchinson, Buchanan, Ilolt. Dodaway and Platte.

Fifth District: Connty-Jackson.

Sixth District: Connties.-Bates, Cass, Cedar, Dade, Henry, Johnson and St. Clair.

Seventh District: Connties.-Benton, Greene, Hickery, Howard, Lafayette, Petis. Polk and Saline.

Eighth District: Connties.-Boone, Canden, Cole, Cooper, Miller, Moniteau, Morgan and Osage.

Ninth District: Counties.-Audrain. Callaway, Franklin, Gasconda, Lincoln, Montgomery, Pike, Ralls, St. Charles and Marren.

Tenth District: Counties: St. Louis County and the First, Seventh, Eighth, Ninth, Tenth, Eleventh, Twelfth, Ninetecuth, Twenty-fonrth, Twenty-eighth wards, and eleventh precinct of the Twenty-seventh ward of the city of St. Louis.

Eleventh District: City of St. Louis.-Second, Third, Sixteenth, Serenfeenth, Eighteenth. Twentieth, Twonty-first and Twenty-sixth wards, and precincts one to ten inclusive of the Twenty-seventh ward.

Twelfth District: City of St. Louis: Fourth, Fifth, Sixth, Thirteenth, Fonteenth, Fiftecnth, Twenty-sccond, Twenty-third and Twenty-fifth wards.

Thirteenth District: Counties.-Boilinger, Carter, Iron, Jefferson, Madison, Perry, Reynolds, St. Francis, Ste. Genevicve, Washington, and Wayne.

Fourteenth District: Counties.-Butler, Cape Girardeat, Christian, Donglas, Dunklin, Howell, Mississippi, New Madrid, Oregon, Ozark, Pemiscot, Ripley, Scott, Stoddard, Stone, and Taney.

Fifteenth District: Counties.-Barry, Barton, Jasper, Lawrence, McDonald, Newton and Vernon.

Sixteenth District: Comnties.-Crawford, Dallas, Dent, Laclede, Maries, Phelps, Pulaski, Shannon, Texas, Webster and Wright.

\section{Montana :}

Representative at large. 


\section{Nebraska :}

First District: Counties.--Cass, Jolnson, Lancaster, Nemaha, Otoe, Pawnce and Richardson.

Second District: Countics.--Donglas, Sarpy and Washington.

Third District: Counties.-Antelope, Boonc, Burt, Cedar, Colfax, Cuming, Dakota, Dixon, Dodge, Knox, Madison, Merrick, Nance, Pierce, Platte, Stanton, Thurston and Wayne.

Fourth District: Counties.-Butler, Fillmore, Gage, Hamilton, Jefferson, Polk. Saline, Saunders, Seward, Thayer and York.

Fifth District: Countics.-Adams, Chasc, Clay, Dundy, Franklin, Fronticr, Furnas, Gosper, Hall, Harlan, Haycs, Hitchcock, Kearney, Nuckolls, Perlins, Phelps, Redwillow and Vebster.

Sixth District: Counties.-Banner, Blaine, Boxbutte, Boyd, Brown, Buffalo, Cherry, Cheyenne, Custer, Dawes. Dawson, Deuel, Garfield, Grant, Greeley, Holt, Hooker, Howard, Keith, Keyapaha, Kimball, Lincoln, Logan, Loup, McPherson, Rock, Scotts Bluffs, Sheridar, Sherman, Sioux, Thomas, Valley and Wheeler.

Nevada :

Representative at Large.

New Hampshire:

First District: Counties.-_Belknap, Carroll, Rockingham and Strafford. Hillsboro County.-City of Manchester; towns of Bedford, Goffstown, Merrimack, Hudson, Litchfield and Pelham.

Merrimack County. - Towns of Allenstown, Canterbury, Chichester, Epsom, Hooksett, Loudon, Northfield, Pembroke and Pittsfield.

Sccond District: Counties.-Cheshire, Coos, Grafton and Sullivan.

Hillsboro County.-City of Nashua; towns of Amherst, Antrim, Bennington, Brookline, Deering, Francestown, Greenfield, Greenville, Hancock, Hillsboro, Hollis, Lyndeboro, Mason, Milford, Mount Vernon, New Boston, New Ipswich, Peterboro, Sharon, Temple, Wcare, Wilton and Windsor.

Merrimack County.-Cities of Concord and Franklin; towns of Andover, Boscawen, Bow, Bradiford, Danbury, Dunbarton, Henniker, Hill, Hopkinton, Newbury, New London, Salisbury, Sutton, Warner, Webster and Wilmot.

New Jersey:

First District: Counties.-Camden, Gloucester and Salem.

Second District: Counties.-Atlantic, Burlington, Cumberiand and Cape May.

Third District: Counties.-Middlesex, Monmouth and Ocean.

Fourth District: Counties.-Hunterdon, Mercer and Somerset. 
Fifth District: Counties.-Morris, U:mon and Warren.

Sixth District: Countics.-Bergen, Passaic and Sussex.

Seventh District: Essex County.-First, Fourth, Sixth, Seventh, Eighth, Eleventh and Fifteenth wards of the city of Newark; city oi Orange; towns of Bloomfield, West Orange and Montelair; the boroughs of Caldwell, Gien Ridge and North Caldwell, and the townships of Belleville, Caldwell, Frankl:n, Livingston and Verona.

Eighth District: Essex County.-Second, Third, Fifth, Ninth, Tenth, Twelfth, Thirtcentis and Fourteentin wards of the city of Newark; city of East Orange; town of Irvington; the borough of Vailsburgh; the village and township of South Orange, and the townships of Clinton and Millburn.

Ninth District: IIudson County-City of Bayonne; Seventh, Eighth, Ninth, Tenth, Eleventh and Twelfth wards and part of the Sixth ward of Jersey City; the towns of Harrison and kearny, and the borough of E:st Newark.

Tenth District: Hudson County.-First, Sccond, Third, Fourth and Fifth wards and part of the Sixth ward of Jersey City; city of Hoboken; towns of West Hoboken, Union. West New York and Guttenberg; the townships of North Bergen and Weehawken, and the borough of Secaucus.

NEW YORK:

First District: Counties.-Nassau and Suffolk.

Borough of Queens (County of Qucens).-Third, Fourth and Fifth wards.

Second District: Borough of Brooklyn (County of Kings).-The Fourtenth, Fifteenth, Sixtcenth, Seventeenth and Eighteenth wards, and also that portion of the Twenty-seventh ward bounded on the north by the line dividing Kings and Queens counties from Flushing arcnue to Jefferso:1 street, Jefferson street south to Evergreen avenue, west to Noll street, south to Bushwick avenue, east to Arion place, south to Broadway, west to Flushing avenue, and north to point of beginning.

Third District: Borough of Brooklyn (County of Kings).-The Thirteenth, Nineteenth and Twenty-first wards, anj also that portion of the Twenty-seventh ward bounded on the north by the line dividing Kings and Qucens counties, from Jefferson street to Stockholm strect, sonth to Bushwick avenue, east to Kosciusko street, sonth to Broadway, west to Arion place, north to Bushwick avenue, west to Noll street, north to Evergreen avemue, east to Jefferson street, and north to point of beginning; and also that part of the Twenty-third ward bounded on the north by Lafayette avenue, from Bedford avenue to Stuyvesant avenue, south to Bainbridge street, west to Sumner avenue, north to McDonough street, west to Tompkins avenue, south to Fulton street, west to New 
York avenue, south to Atlantic avenue, west to Franklin avenue, north to Brevoort place, east to Bedford avenue, and north to point of beginning.

Fotrth District: Borongh of Brooklyn (Connty of Kings).-The Twenty-sixth, Twenty-eighth, Thirty-first and Thirty-second wards, and also that portion of the Twenty-fifth ward bounded on the north by Broadway, from Howard avente to boundary line of the Twenty-sixth ward, south to Atlantic avenue, west to Howard avenue, north to Fulton street, east to Howard avenue, and north to point of beginning.

Fifth District: Borough of Brooklyn (County of Kings).-The Eighth, Twenty-fourth, Twenty-ninth and Thirticth wards, and also that portion of the Twenty-third ward bounded on the north by Lafayette avenue, from Stuyvesant avenue east to Reid avenue, south to Fulton street, west to Utica avenue, south to Atlantic avenue, west to New York avenue, north to Fulton street, east to Tompkins avemue, north to McDonough street, east to Stmmer avente, south to Bainbridge street, east to Stuyvesant avente, and north to the point of beginning; and also that portion of the Twenty-fifth ward bounded on the north by Lafayette avenue, from Reid avente east to Broadway, southeast to Howard avenue, south to Fulton street, east to Howard avenue, south to Atlantic avenue, west to Utica avenue, north to Fultcn strect, east to Reid avenue, and north to the point of beginning.

Sixth District: Borough of Brooliyn (County of Kings).-The Seventh, Ninth, Twentieth and Twenty-second wards, and also that portion of the Eleventh ward bounded on the north by Jolnson street, from Bridge strect east to Hudson avenue, sonth to Myrtle avenue, cast to Navy street, south to Bolivar street, west to Hudson avenue, south to Willoughby street, east to Navy street, south to De Kalb avenue, east to South Portland avenue, south to Atlantic aventre. west to Flatbush avenue, northwest to Fulton street, west to Bridge street, and north to point of beginning.

Seventh District: Borough of Brooklyn (County of Kings).-The First, Second, Third, Fourth, Fifth, Sixth, Tenth and Twelfth wards, and also that portion of the Eleventh ward bounded on the north by Flushing avenue, from Nary strect east to North Portland avenue, across Fort Greene Park to De Kalb avenue, opposite South Portland avenue, west to Navy street, north to Willoughby street, west to Hudson avenue, north to Bolivar street, east to Navy street, north to Myrtle avenue, west to Hudson avenue, north to Johnson street, east to Navy street, and north to point of beginning.

Eighth District: Richmond County.

New York County.-That portion bounded as follows: Beginning at Battery place and North River, north to West street, north to Barrow street and Hudson River, east to Hudson street, north to Grove street, 
northeast to Blecelier strect, soulcast to Cornelia strect, northeast to Sixth avente, south to West Third strect, cast to Rroadway, north to East Fourth street, cast to the Bowery, north to Third avenue, to St. Mark's place, cast to Second avenue, south to Second strect, east to First avenue, sonth to East Houston sirect, west to Eldridge street, south to Stanton strect, west to Chrystic strcet, south to Divisi n strect, west to northeast corner of Division street and Bowery, to the northeast corner of Chatham Square and Catherine strcet, southeasterly to Nlonroe street, east to Mechanic alley, and south to Cherry street, west to Market slip, south to the East River.

Ninth District: New York Connty--Parts of the Scond, Fourth, Eighth, Tenth, Twelith and Sixteenth asscmbly districts, as follows: Beginning at the East River and Market slip, north to Cherry strect, east to Mechanic alley, north to Monroe street, west to Catharine street, north to Division strect, east to Chrystie strect, north to Stanton strect, east to Canmon strcet, south to Proome street, west to Sheriff strect, south to Grand street, west to Pitt strect, south to Division strect, to Montgomery strect. south to southwest conner of Henry street and Montgomery street, diagonally throngh the midtle of said block to the northeast corner of Madison strect and Clinton street, south to South street at East River, thence along the East River to the point or place of begiming.

Tenth District: New York County--That part bounded as follows: Beginning at East Fonsteenth strect and the East River, west to Third avenuc, soutl to St. Niark's place, cast to Second arenuc, south to Second strect, east to First avenue, south to East Houston strect, west to Eldridge street, south to Stanton strect, east to Cannon street, south to Broome street, west to Sheriff strect, south to Grand street, west on the south side of Grand street to Pitt street, south to Divisinn street, west to Montgomery strect, to northeast corner of Henry sireet and Montgomery street, diagonally through said block to the southwest corner of Madison strect and Clinton street, south to the East River, thence along the Fast River to the peint or place of beginning.

Eleventh District: New York County--That part bonnded as follows: Beginning at Iludson River and Parrow street, east to Ifulson street, north to Grove street, casterly to Bleceker strect. easterly nn Blecker street to Cornelia street, easterly on Cornelia strect to Sixth aremue, south to West Third strect, east to Broadway, north to East Fourth strect, east to Third avenue, north to East Fourteenth street, west to University place, south to East Tenth street, west to Fifth avenue, south to West Ninth strect, West to Christopher street, Westerly to West Fourth street, northerly to Eighth avenue, to Hudson street, sontherly a)ong Hudson street to West Eleventh street, west to Grcenwich street, north to Horatio street, east to IIudson street, north to West Fourteenth 
street, cast to Eighth avenue, north to IVest Nineteenth street, east to Seventh avenue, nortl 1 to West Twenty-first street, west to Eighth avenue, north to West Twenty-third street, east to Seventh avenue, north to West Fortieth street, west to Eighth avenue, north to West Forty-third street, west to Ninth avenue, north to West Sixtieth street, west to Hudson River to point of beginning at Hudson River and Barrow street.

Twelfth District: New York County.-That part bounded as follows: Beginning at the East River and East Fourteenth street, west to Second avenue, north to East Eighteenth strect, west to Third avenue, north to East Twenty-third street, west to Lexington avenue, north to East Twenty-ninth street, east to Second Avenue, north to East Thirtyseventh street, west to Third avenue, north to East Thirty-ninth street, west to Lexington avenue, north to East Forty-second street, east to Third avenue, north to East Fifty-third street, west to Lexington avenue, north to East Fifty-ninth street, east to Third avenue, north to East Sixty-fourth street, west to Lexington avenue, north to East Seventysecond street, to the East River to the point of beginning at the East River and East Fourteenth street, inciuding Blackwell's Island.

Thirteenth District: New York County.-That part bounded as follows: Beginning at the northwest corner of Hudson street and West Eleventh street, north to Eighth avenue, to West Fourth street, south to Christopher street, east to West Ninth street, east to Fifth avenue, north to East Tenth street, east to University place, north to East Fourteenth street, east to Second aventie, north to East Eighteenth street, west to Third avenue, north to East Twenty-third street, west to Lexington avenue, north to East Twenty-ninth street, east to Second avenue, north to East Thirty-seventh street, west to Third avenue, north to East Thirty-ninth street, west to Lexington avenue, north to East Fortysecond street, east to Third avenue, north to East Fifty-third street, west to Lexington arenue, north to East Fifty-ninth street, east to Third avenue, north to Sixty-fourth street, west to Lexington avenue, north to East Eighty-ninth street, west to Park avenue, north to East Ninetythird street, west to Fifth avente, south along Fifth avenue to Eightysixth street, west across Central Park to West Eighty-sixth street and Central Park West, south to West Fifty-ninth street, east to Sixth avenue, south to West Fifty-fifth street, west to Seventh avenue, south to West Fifty-third street, west to Eighth avenue, south to West Fortieth street, east to Seventh avenue, south to West Twenty-third street, west to Eighth avenue, south to West Twenty-first street, east to Seventh avenue, south to West Nineteenth street, west to Eighth avenue, south to West Fourteenth street, west to Hudson street, south to Horatio street, west to Greenwich street, south to West Eleventh street, east 
to point of beginning at the northwest corner of West Eleventh street and Hudson street.

Fourteenth District: New York County.-That part bounded as follows: Beginning at the East River and East Seventy-second strect, west to Lexington avenue, north to East Eighty-1inth strect, east to Third avenue, south to East Eighty-eighth strect, east to the East River, to point of begiming at East River and East Seventy-second street.

Queens County.-That part known as the First and Second wards of Queens County, whose boundaries are as follows: Beginning at Newtown Crcek and the East River to Flushing Creek, south to Ward street, Richmond Hill, vest to Forest Park, along the southern boundary of Forest Park through Cypress Hill Cemetery, to the Kings County line, northwest to Newtown Creek, to peint of beginning at Newtown Creek and the East River.

Fiftecnth District: New York County.-That part bounded as follows: Begiming at the IIudson River and West Sixtieth street, east to Columbus avenue, south along Columbus avenue and Ninth avenue to West Forty-third street, east to Eighth avenue, north to West Fifty-third street, east to Seventh avenue, north to West Fifty-fifth street, east to Sixth avenue, north to West Fify-ninth strcet, west to Central Park West, north to West Eighty-sixth street, cast across Central Park to Eighty-sixth street and Fifth avenue, north along Fifth arenue to Nincty-third strect, cast to Park arenne, south to East Eighty-ninth strect, cast to Lexington avenue, north to East Nincty-sixth strect, west to Fifth avenue, north to cast Nincty-seventh street, west across Central Park transverse road to West Nincty-seventh street and Central Park West, north to West One hundred and Second street, west to Columbus avenue, south to West One hundred and First street, west to Hudson River to the point of beginning at lludson River and West Sixticth street.

Sixteenth District: New York County.-That part bounded as follows: Beginning at the East River and East Eighty-eighth street, west to Third avenue, nortin to East Eighty-ninth street, west to Lexington avenue, north to East Nincty-sixth street, west to Fifth avenue, north to East One hundred and Twentieth strect, east to Park avenue, south to East One hundred and Nineteenth street, east to the East River to point of beginning at the East River and East Eighty-eighth street, including Randalls and Wards lslands.

Seventeenth District: New York County.-That part bounded as follows: Begimning at the Hudson River and West One hundred and First street, east to Columbus avente, north to West One Hundred and Second street, east to Central Park Wcst, south to West Ninety-seventh street, east across the Central Park transverse road to Fifth avenue and East Ninetyseventh street, north to East One hundred and Twenty-ninth street, 
west to Fifth avenue, north to the Harlem River, to the Hudson River, to the point of beginning at the Hudson River and West One hundred and First street.

Eighteenth District: New Iork Connty.--The Thirty-fourth and Thirtyfifth assembly districts and the annexed district bounded as follows: Beginning at the East River and East One hundred and Nineteenth street, west to Park avenue, nortl to East One hundred and Twentyninth sireet, west to Fifth avenue, north to the Harlem River, to the Hudson River, to the Yonkers city line, to Long Island Sound, to the East River, to the point of beginning at the East River and East One hundred and Nineteenth street, including islands in Long Island Sound and Harlem River attached to the said Thirty-fourth and Thirty-fifth assembly districts and the annexed district.

Nineteenth District: County.-Westchester.

Twentieth District: Counties.-Orange, Rockland and Sullivan.

Twenty-first District: Counties.-Columbia, Dutchess, Greene and Putnam. Twenty-second District: Counties--Rensselaer and Washington.

Twenty-third District: Counties.-Albany and Schenectady.

Twenty-fourth District: Counties.-Delaware, Otsego, Schoharie and Ulster.

Trventy-fifth District: Counties.-Fulton, Hamilton, Montgomery, Saratoga and Warren.

Twenty-sixth District: Counties.-Clinton, Essex, Franklin and St. Lawrence.

Twenty-seventh District: Counties.-Herkimer and Oneida.

Twenty-eighth District: Counties.-Jefferson, Lewis and Oswego.

Twenty-ninth District: Counties.-Onondaga and Madison.

Thirtieth District: Connties.-Broome, Chenango, Cortland, Tioga and Tompkins.

Thirty-first District: Counties.-Cayuga, Ontario, Wayne and Yates.

Thirty-second District: County.-Monroe.

Thirty-third District: Counties.-Chemung, Schuyler, Seneca and Steuben.

Thirty-fourth District: Counties.-Genesee, Livingston, Niagara, Orleans and Wyoming.

Thirty-fifth District: City of Buffalo.-The First, Second, Third, Fourth, Fifth, Sixth, Seventh, Eighth, Ninth, Tenth, Eleventh, Twelfth, Thirteenth, Fourteenth, Fifteenth, Sixteenth and Eighteenth wards.

Thirty-sixth District: Erie County.-Seventh and Eighth assembly districts.

City of Buffalo. - The Seventeenth, Nineteenth, Twentieth, Twenty-first, Twenty-second, Twenty-third, Twenty-fourth and Twenty-fifth wards. Thirty-seventh District: Counties.-Allegany, Cattaraugus and Chautauqua. 
North Carolina:

First District: Connties.-Beaufort, Canden, Clowan, Currituck, Dare, Gates, Hertiord, IIyde, Martin, Pasquotank, Perquimans, Pitt, Tyrell and Washington.

Second District: Counties.-Bertie, Edgecombe, Greene, Halifax, Lenoir, Northhampton, Warren and Wilson.

Third District: Connties.-Carteret, Craven, Duplin, Jones, Onslow, Pamlico, Pender, Sampson and Wayne.

Fourth District: Counties.-Chatham, Franklin, Johnston, Nash, Vance and Wake.

Fifth District: Counties._Alamance, Caswell, Durhan, Forsyth, Granville, Guilford, Orange, Person, Rockingham and Stokes.

Sixth District: Counties.-Bladen, Brunswick, Columbus, Cumberland, Harnett, New Hanover and Robeson.

Seventh District: Counties-Anson, Davidson, Davie, Nontgomery, Moore, Randolph, Richmond, Scotland, Union and Yadkin.

Eighth District: Counties.-Alexander, Allegheny, Ashe, Cabarrus, Caldwell, Iredell, Rowan, Stanley, Surray, Watanga and Wilkes.

Nintli District: Counties.-Burke, Catawba, Cleveland, Gaston, Lincoln, Madison, Mecklenlurg, Mitchell and Yancey.

Tenth District: Counties-Buncombe, Cherokec, Clay, Graham, Haywood Henderson, Iackson, McDowell, Macon, Polk, Rutherford, Swain and Transylvania.

\section{North Dakota:}

Representatives at large.

\section{Оніо :}

First District: Hamilton County-First, Second, Third, Fourth, Fifth, Sixth, Serenth, Eighth, Ninth, Tenth, Eleventh, Eighteenth, Twentysixth, Twenty-seventh and Thirty-first wards of the city of Cincinnati; Anderson, Columbia, Sycamore, Symmes and Spencer townships, and precincts of Mill Creek township, as follows: Bond Hill, Carthage, Norwood, St. Bernard and Evanston.

Second District: Ianilton County.-Twelfth, Thirteenth, Fourteenth, Fifteenth, Sixtecnth, Seventeenth, Nineteenth, Twentieth, Twenty-first, Twenty-second, Twenty-third, Twnty-fourth, Twenty-fifth, Twentyeighth, Twenty-ninth and Thirtieth wards of the city of Cincinnati, the towns of Colcrain, Crosby, Delhi, Green, Harrison, Miami, Springfield and Whitewater, and Elmwood, College Hill, Western and Winton Place precincts of Mill Creek township.

Third District: Counties.-Butler, Montgomery and Preble.

Fourth District: Counties.-Allen, Auglaize, Darke, Mercer and Shelby. 
Fifth District: Counties.-Defance, Henry, Paulding, Putnam, Van Wert and Williams.

Sixth District: Connties.-Brown, Clermont, Clinton, Greene, Highlands and Warren.

Seventh District: Counties.-Clark, Fayette, Madison, Miami and Pickaway.

Eighth District: Counties.-Champaign, Delaware, Hancock, Hardin, Logan and Union.

Ninth District: Counties.-Fulton, Lucas, Ottawa and Vood.

Tenth District: Counties.-Adams, Gallia, Jackson, Lawrence, Pike and Scioto.

Eleventh District: Comnties.-Athens, Fairfield, Hocking, Meigs, Perry, Ross and Vinton.

Twelfth District: County.-Franklin.

1 hirteenth District: Counties.-Crawford, Erie, Marion, Sandusky, Seneca and Wyandot.

Fourteenth District: Counties.--Ashland, Huron, Knox, Lorain, Morrow and Richland.

Fifteenth District: Counties.-Guernsey, Morgan, Muskingum, Noble and Washington.

Sixteenth District: Counties.-Belmont, Carroll, Harrison, Jefferson and Monroe.

Seventeenth District: Counties.-Coshocton, Holmes, Licking, Tuscarawas and Wayne.

Eighteenth District: Counties.-Columbiana, Mahoning and Stark.

Ninetcenth District: Counties.-Ashtabula, Geauga, Portage, Summitt and Trumbull.

Twentieth District: Counties.-Lake, Medenia and the townships of Bedford, Brecksville, Jrooklyn, Chagrin Falls, Collinwood, Dover, East Cleveland, Euclid, Glenville, Independence, Mayfield, Middleburg, Newburg, Olmstead, Orange, Palma, Rockport, Royalton, Solon, Strongsville, Warrensville and West Park, of Cuyahoga County, and the Twentysixth, Twenty-eighth, Twenty-ninth, Thirtieth, Thirty-first. Thirtysecond, Thirty-third, Thirty-fourth, Thirty-fifth, Thirty-sixth, Thirtyseventh, Thirty-eighth, Thirty-ninth, Fortieth, Forty-first and Fortysecond wards of the city of Cleveland.

Twenty-first District: City of Cleveland.-First, Second, Third, Fourth, Fifth, Sixth, Seventh, Eighth, Ninth, Tenth, Eleventh, Twelfth, Thirteenth, Fourteenth, Fifteenth, Sixteenth, Seventeenth, Eighteenth, Nineteenth, Twentieth, Iwenty-first, Twenty-second, Twenty-third, Twentyfourth, Twenty-fifth and Twenty-seventh wards. 


\section{OKLAHOMA :}

First District: The counties of Grant, Kay, Garfield, Noble, Pawnee, Kingfisher, Logan, Payne, Lincoln, and the territory comprising the Osage and Kansas Indian reservations.

Second District: The counties of Oklahoma, Canadian, Blaine, Caddo, Custer, Dewey, Day, Woods, Woodward and Beaver.

Third District: (With the exception of that part of recording district numbered twelve, which is in the Cherokee and Creek nations) all the territory now constituting the Cherokee, Creek and Seminole nations and the Indian reservations lying northeast of the Cherokee Nation, with said State.

Fourth District: All the territory now constituting the Choctaw Nation, that part of recording district numbered twelve which is in the Cherokee and Creek nations, that part of recording district numbered twenty-five which is in the Chickasaw Nation, and the territory comprising recording districts numbered sixteen, twenty-one, twenty-two and twenty-six, in the Indian Territory.

Fifth District: The connties of Greer, Roger Mills, Kiowa, Washita, Comanche, Cleveland and Pottawatomie, and the territory comprising recording districts numbered seventeen, eighteen, nineteen and twenty, in the Chickasaw Nation, Indian Territory.

\section{OREGON:}

First District: Connties.-Benton, Clackamas, Cons, Curry, Douglas, Jackson, Josephine, Klamath, Lake, Lane, Lincoln, Linn, Marion, Polk, Tillamook, Washington and Yamhill.

Second District: Counties.-Baker, Clatsop, Columbia, Crook, Gilliam, Grant, Harney, Malheur, Norrow, Multnomah, Sherman, Umatilla, Union, Wallowa, Wasco and Wheeler.

\section{Pennsyluania:}

First District: City of Philadelphia.-First, Seventh, Twenty-sixth, Thirtieth, Thirty-sixth and Thirty-ninth wards.

Second District: City of Philadelphia--Eighth, Ninth, Tenth, Thirteenth, Fourteenth, Fifteenth, Twentieth and Thirty-seventh wards.

Tlird District: City of Philadelphia.-Second, Third, Fourth, Fifth, Sixth, Eleventh, Twelfth, Sixteenth, Seventeenth, Eighteenth and Nineteenth wards.

Fourth District: City of Philadelphia.-Twenty-eighth, Twenty-ninth, Thirty-second and Thirty-eighth wards.

Sixth District: City of Philadelphia.-Twenty-first, Twenty-second, Twenty-fourth, Twenty-seventh, Thirty-fourth, Fortieth and Fortysecond wards.

Seventh District: Counties.-Chester and Delaware. 
Eighth District: Counties.-Bucks and Montgomery.

Ninth District: County.-Lancaster.

Tenth District: County.-Lackawanna.

Eleventh District: County.-Luzerne.

Twelfth District: County.-Schuylkill.

Thirteenth District: Counties.-Berks and Lehigh.

Fourteenth District: Counties.-Bradford, Susquehanna, Wayne and Wyoming.

Fifteenth District: Counties.-Clinton, Lycoming, Potter and Tioga.

Sixteenth District: Counties.-Columbia, Montour, Northumberland and Sullivan.

Seventeenth District: Counties.-Franklin, Fulton, Huntingdon, Juniata, Mifflin, Perry, Snyder and Union.

Eighteenth District: Counties.-Cumberland, Dauphin and Lebanon.

Nineteenth District: Counties.-Bedford, Blair and Cambria.

Twentieth District: Counties.-Adams and York.

Twenty-first District: Counties.-Cameron, Center, Clearfield and McKean.

Twenty-second District: Counties.-Butler and Westmoreland.

Twenty-third District: Counties.-Fayette, Greene and Somerset.

Twenty-fourth District: Counties.-Beaver, Lawrence and Washington.

Twenty-fifth District: Counties.-Crawford and Erie.

Twenty-sixth District: Counties.-Carbon, Monroe, Northampton and Pike.

Twenty-seventh District: Counties.-Armstrong, Clarion, Indiana and Jefferson.

Twenty-eighth District: Counties.-Elk, Forest, Mercer, Venango and Warren.

Twenty-ninth District: Counties.-Allegheny County; City of Allegheny and the townships and boroughs north of the Ohio River.

Thirtieth District: Counties.-Allegheny County; Twentieth, Twentyfirst and Thirty-serenth wards of the city of Pittsburg, the city of McKeesport, boroughs of Braddock, East McKeesport, East Pittsburg, Edgwood, Elizabeth, North Braddock, Oakmont, Pitcairn, Port Vue, Rankin, Swissrale, Turtle Creek, Verona, Versailles, Wilkinsburg and Vilmerding, townships of Braddock, Elizabeth, Forward, Lincoln, North Versailles, Patton, Penn, Plum, South Versailles, Sterrett, Versailles and Wilkins.

Thirty-first District: City of Pittsburg.-First, Second, Third, Fourth, Fifth, Sixth, Seventh, Eighth, Ninth, Tenth, Eleventh, Twelfth, Thirteenth, Fourteenth, Fifteenth, Sixteenth, Seventeenth, Eighteenth, Nineteenth and Twenty-third wards.

Thirty-second District: Allegheny County.-Twenty-second, Twentyfourth, Twenty-fifth, Twenty-sixth, Twenty-seventh, Twenty-eighth, 
Twenty-ninth, Thirtieth, Thirty-first, Thirty-second, Thirty-third, Thirtyfourth, Thirty-fifth, Thirty-sixth and Thirty-eighth wards of the city of Pittsburg; boroughs of Caraopolis, Crafton, Carnegie, Duquesne, Esplen, Elliott, Greentree, Homestcad, Knoxville, Montooth, Mount Oliver, McKees Rocks, Oakdale, Sheraden, West Liberty and West Elizabeth; townships of Baldwin, Bethel, Crescent, Chartiers, Collier, Findley, Jefferson, Lowes, Mifflin, Moon, North Fayette, Neville, Robinson, St. Clair, Scott, Snowden, South Fayette, Stowe, Union and Upper St. Clair.

RHODE ISLAND:

First District: Counties.-Bristol and Newport, and the city of Providence.

Second District: Counties.-Kent and Washington, and all of Providence outside the city of Providence.

South Carolina:

First District: Counties.-Berkeley, Charleston, Clarendon, Colleton and Dorchester.

Second District: Countics.-Aiken, Bamberg, Barnwell, Beaufort, Edgefield, Hampion and Saluda.

Third District: Counties._Abbeville, Anderson, Greenwood, Newberry, Oconee and Pickens.

Fourth District: Counties.-Greenville, Laurens, Spartanburg and Union. Fifth District: Counties.-Cherokee, Chester, Chesterfield, Fairfield, Kershaw, Lancaster and York.

Sixth District: Counties.-Darlington, Florence, Georgetown, Horry, Marion, Marlboro and Williamsburg.

Seventh District: Counties.-Lee, Lexington, Orangeburg, Richland and Sumter.

South Dakota:

Representatives at large.

TenNessee:

First District: Countics-Carter, Claiborne, Cocke, Grainger, Greene, Hancock, Hawkins, Johnson, Sevier, Sullivan, Unicoi and Washington.

Second District: Counties.-Anderson, Blount, Campbell, Hamblen, Jefferson, Knox, Loudon, Roane, Scott and Union.

Third District: Counties.-Bledsoe, Bradley, Franklin, Grundy, Hamilton, Marion, McMinn, Mcigs, Monroe, Polk, Sequatchie, Van Buren, Warren and White.

Fourth District: Counties.-Clay, Cumberland, Fentress, Jackson, Macon, Morgan, Overton, Pickett, Putnam, Rhea, Smith, Sumner, Trousdale and Wilson. 
Fifth District: Counties.-Bedford, Cannon, Coffee, DeKalb, Lincoln, Marshall, Moore and Rutherford.

Sixtli District: Counties,-Cheatham, Davidson, Montgomery, Robertson and Stewart.

Seventh District: Counties.-Dickinson, Giles, Hickman, Houston, Humphreys, Lawrence, Lcwis, Maury, Wayne and Williamson.

Eighth District: Countics.-Benton, Carroll, Chester, Decatur, Hardin, Henderson, Henry, McNairy, Madison and Perry.

Ninth District: Counties.-Crockett, Dyer, Gibson, Haywood, Lake, Lauderdale, Obion and Weakley.

Tenth District: Cotnties.-Fayctte, Hardeman, Shelby and Tipton.

TExas :

First District: Counties.-Bowic, Camp, Cass, Delta, Franklin, Hopkins, Lamar, Marion, Morris, Red River and Titus.

Second District: Counties.-Angelina, Cherokee, Hardin, Harrison, Jasper, Jefferson, Nacogdoches, Newton, Orange, Panola, Sabine, San Augustine, Shelby and Tyler.

Third District: Counties.-Gregg, Henderson, Kaufman, Rusk, Smith, Upsluur, Van Zandt and Wood.

Fourth District: Counties.-Collin, Fannin, Grayson, Hunt and Rains.

Fifth District: Counties.-Bosque, Dallas, Ellis, Hill and Rockwall.

Sixth District: Counties.-Brazos, Freestone, Limestone, Milam, Navarro and Robertson.

Seventh District: Counties.-Anderson, Chambers, Galveston, Houston, Liberty, Polk, San Jacinto and Trinity.

Eighth District: Counties.-Austin, Fort Bend, Grimes, Harris, Leon, Madison, Montgomery, Walker and Waller.

Ninth District: Counties.-Arkansas, Bee, Brazoria, Calhoun, Colorado, DeWitt, Fayette, Goliad, Gonzales, Jackson, Karnes, Lavaca, Matagorda, Refugio, Victoria and Wharton.

Tenth District: Counties.-Bastrop, Burleston, Caldwell, Hays, Lee, Travis, Washington and Williamson.

Eleventh District: Counties.-Bell, Coryell, Falls, Hamilton and McLennan.

Twelfth Disirict: Counties.-Comanche, Erath, Hood, Johnson, Parker, Somerville and Tarrant.

Thirteenth District: Counties.-Archer, Armstrong, Bailey, Bailor, Briscoe, Carson, Castro, Cliildless, Clay, Collingsworth, Cook, Cottle, Dallam, Deaf Smith, Denton, Dickens, Donley, Floyd, Foard, Gray, Hale, Hall, Hansford, Hardeman, Hartley, Hemphill, Hutchinson, Jack, Knox, Lamb, Lipscomb, Montague, Moore, Motley, Ochiltree, Oldham, Parmer, Potter, Randall, Roberts, Sherman, Swisher, Throckmorton, Wheeler, Wichita, Wilbarger, Wise and Young. 
Fourteenth District: Counties.-Bandera, Bexar, Blance, Brown, Burnett, Coleman, Comal, Gillespie, Kendall, Kerr, Lampassas, Llano, McCulloch, Mason, Mills and San Saba.

Fifteenth District: Counties.-Atacosa, Cameron, Dimmitt, Duval, Frio, Guadalupe, Ilidalgo, Kinney, Lasalle, Live Oak, Maverick, McMullen, Medina, Nueces, San Patricio, Starr Uvalde, Valverde, Webh, Wilson, Zapata and Zavalla.

Sixteentl 1)istrict: Counties.-Andrews, Borden, Brewster, Callahan Cochran, Coke, Concho, Crane, Crockitt, Crosby, Dawson, Eastland, Ector, Edwards, El Paso, Fisher, Gaines, Garza, Glasscock, Haskell, Hockley, Howard, Irion, Jeff Davis, Jones, Kent, Kimble, King, Loving, Lubbock, Lynn, Martin, Menard, Midland, Mitchell, Nolan, Palo, Pinto, Pecos, Presidio, Reagan, Reeres, Rumnels, Schleicher, Scurry, Shakleford, Stephens, Sterling, Stonewall, Sutton, Taylor, Tirrell, Terry, Tom Green, Upton, Ward, Walker and Yoakmm.

\section{UTAIF :}

Representative at lasge.

\section{VerMoNT:}

First District: Comnties.-Addison, Bennington, Chittenden, Franklin, Grand Isle, Lamoille and Rutiand.

Second District: Counties.-Caledonia, Essex, Orange, Orleans, Wasiington, Windham and llindsor.

\section{Virginia :}

First District: Counties.-Accomac, Caroline, Essex, Gloncester, Ling and Queen, Lancaster, Matthews, Midllesex, Northampton, Northmberland, Richmond, Spottsylvania and Westmoreland.

Second District: Counties.-Charles City, Elizabeth City, Isle of Wight, James City, Nansemond, Norfolk, Princess Anne, Sonthumpton, Surry, Warwick and York, and the cities of Norfolk, Portsmouth, Williamsburg and Newport News.

Third District: Comnties.-Chesterficld. Goochland, Hanover, Ienrico, King William and New Kent, and the cities of Richmond and Man. chester.

Fourth District: Counties.-Amelia, Brunswick, Dinwiddie, Greensville, Lunenburg, Mecklenburg, Nottoway, Powhatan, Prince Edward, Prince George and Sussex, and the city of Petersburg.

Fifth District: Counties-Carroll, Floyd, Franklin, Grayson, Itenry, Patrick and Pittsylyania, and the city of Dansville.

Sixth District: Counties.-Bedford, Campbell, Charlotte, Halifax, Montgomery and Roanoke, and the cities of Lynchburg, Radford and Roanoke. Seventh District: Counties.-Albemarle, Clarke, Frederick, Greene, Mad- 
ison, Page, Rappahannock, Rockingham, Shenandoah and Warten, and the cities of Charlottesville and IVinchester.

Eighth District: Counties.-Alexandria, Culpeper, Fairfax, Fauquier, King George, Loudon, Louisa, Orange, Prince William and Stafford, and the city of Alexandria.

Ninth District: Counties.-Bland, Buchanan, Craig, Dickenson, Giles, Lee, Pulaski, Russell, Scott, Smyth, Tazewell, Washington, Wise and Wythe, and the city of Bristol.

Tenth District: Counties.-Alleghany, Amherst, Appomattox, Augusta, Bath, Botetourt, Buckingham, Cumberland, Fluvanna, Highland, Nelson and Rockbridge, and the cities of Buena Vista and Staunton.

\section{WASHINGTON :}

Representatives at large.

West Virginia :

First District: Counties.-Brooke, Hancock, Harrison, Lewis, Marion, Marshall, Ohio and Wetzel.

Second District: Counties.-Barbour, Berkeley, Grant, Hampshire, Hardy, Jefferson, Mineral, Monongalia, Morgan, Pendleton, Preston, Randolph, Taylor and Tucker.

Third District: Counties.-Clay, Fayette, Greenbrier, Kanawha, Monroe, Nicholas, Pocahontas, Summers, Upshur and Webster.

Fourth District: Counties.-Braxton, Calhoun, Doddridge, Gilmer, Jackson. Pleasants, Ritchie, Roane, Tyler, Wirt and Wood.

Fifth District: Counties.-Boone, Cabell, Lincoln, Logan, McDowell, Mason, Mercer, Mingo, Putnam, Raleigh, Wayne and Wyoming.

\section{Wisconsin :}

First District: Counties.-Green, Kenosha, Lafayette, Racine, Rock and Walworth.

Second District: Counties.-Adams, Columbia, Dane, Green Lake, Jefferson and Marquette.

Third District: Counties.-Crawford, Grant, Iowa, Juneau, Richland, Sauk and Vernon.

Fourth District: Nilwankee County.-Second, Third, Fourth, Fifth, Seventh, Eightl, Eleventh, Twelfth, Fourteenth, Fifteenth, Sixteenth, Seventeenth and Twenty-third wards of the city of Milwaukee; cities of South Milwaukee and Wauwatosa; towns of Franklin, Greenfield, Lake, Oak Creek and Wauwatosa.

Fifth District: Milwaukee Connty.-First, Sixth, Ninth, Tenth, Thirteenth, Eighteenth, Nineteenth, Twentieth, Twenty-first and Twentysecond wards of the city of Milwaukee; towns of Granville and Milwaukee; villages of North Milwaukee and Whitefish Bay. 
Sixth District: Countics.-Dodge, Fond du Lac, Ozaukec, Sheboygan and Washington.

Seventh District: Countics.-Buffalo, Clark, Eau Claire, Jackson, La Crosse, Monroe, Pepin and Trempealeau.

Eighth District: Countics.-Calumet Manitcwoc, Portage, Waupaca, Waushara and Winnebago.

Ninth District: Counties.-Brown, Door, Kewaunce, Marinette, Oconto and Outagamic.

Tenth District: Counties.-Ashland, Florence, Forest, Iron, Langlade, Lincoln, Marathon, Oneida, Price, Shawano, Taylor, Vilas and Wood.

Eleventh District: Countics.-Barron, Bayfield, Burnett, Chippewa, Douglas, Dunn, Pierce, Polk, Rusk, St. Croix, Sawyer and Washburn.

\section{Wyoming:}

Representative at lares'. 


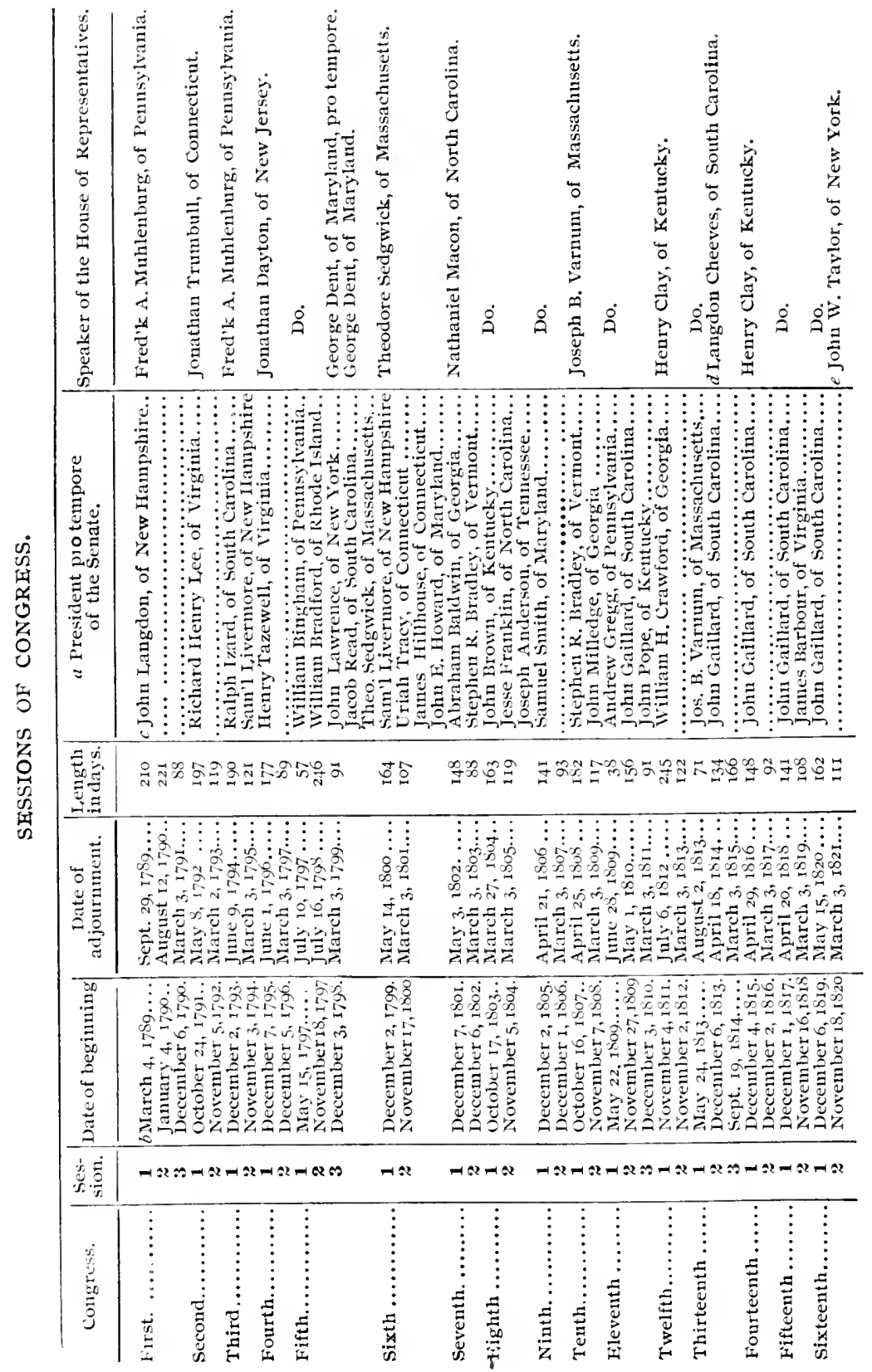




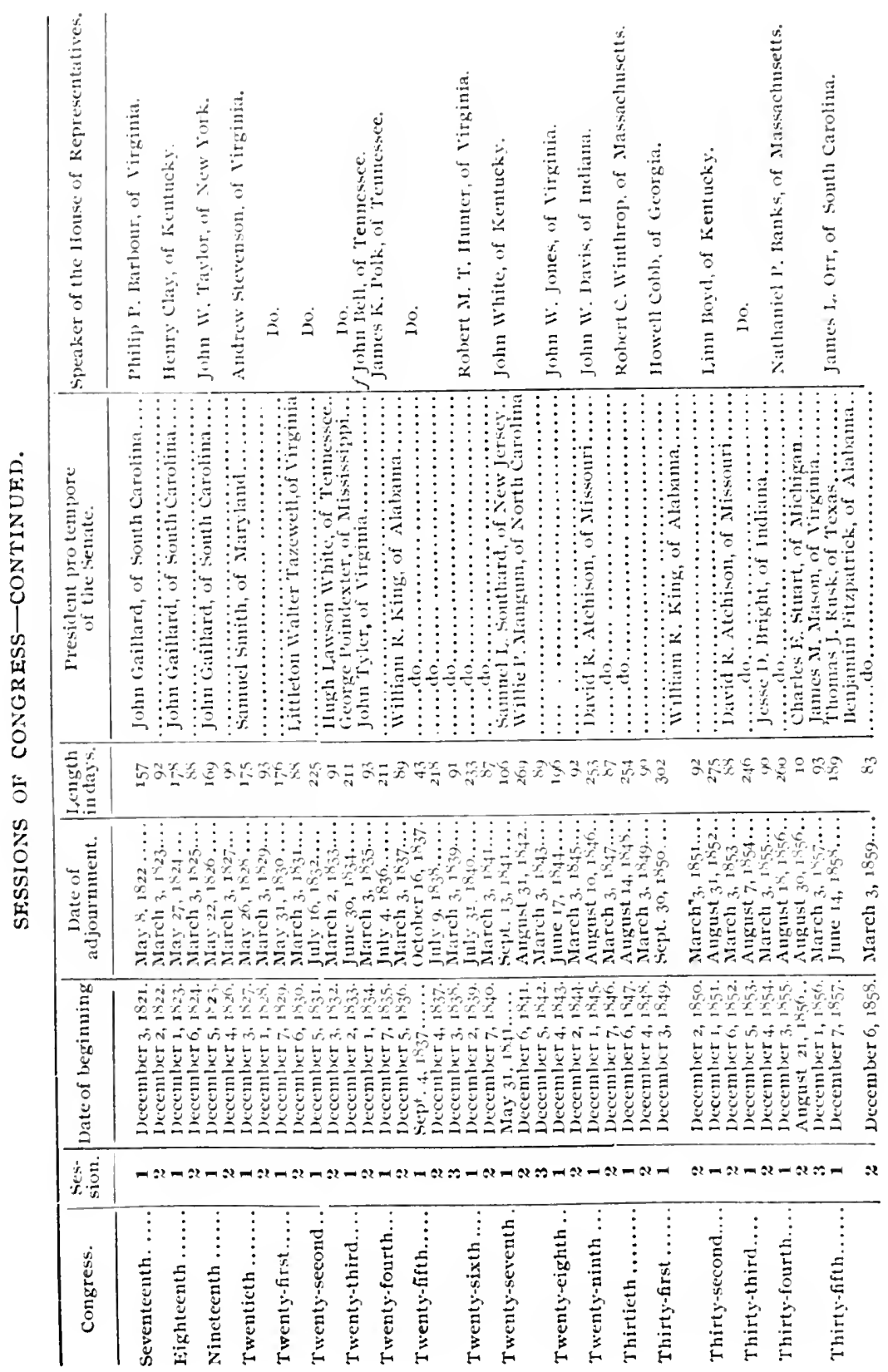




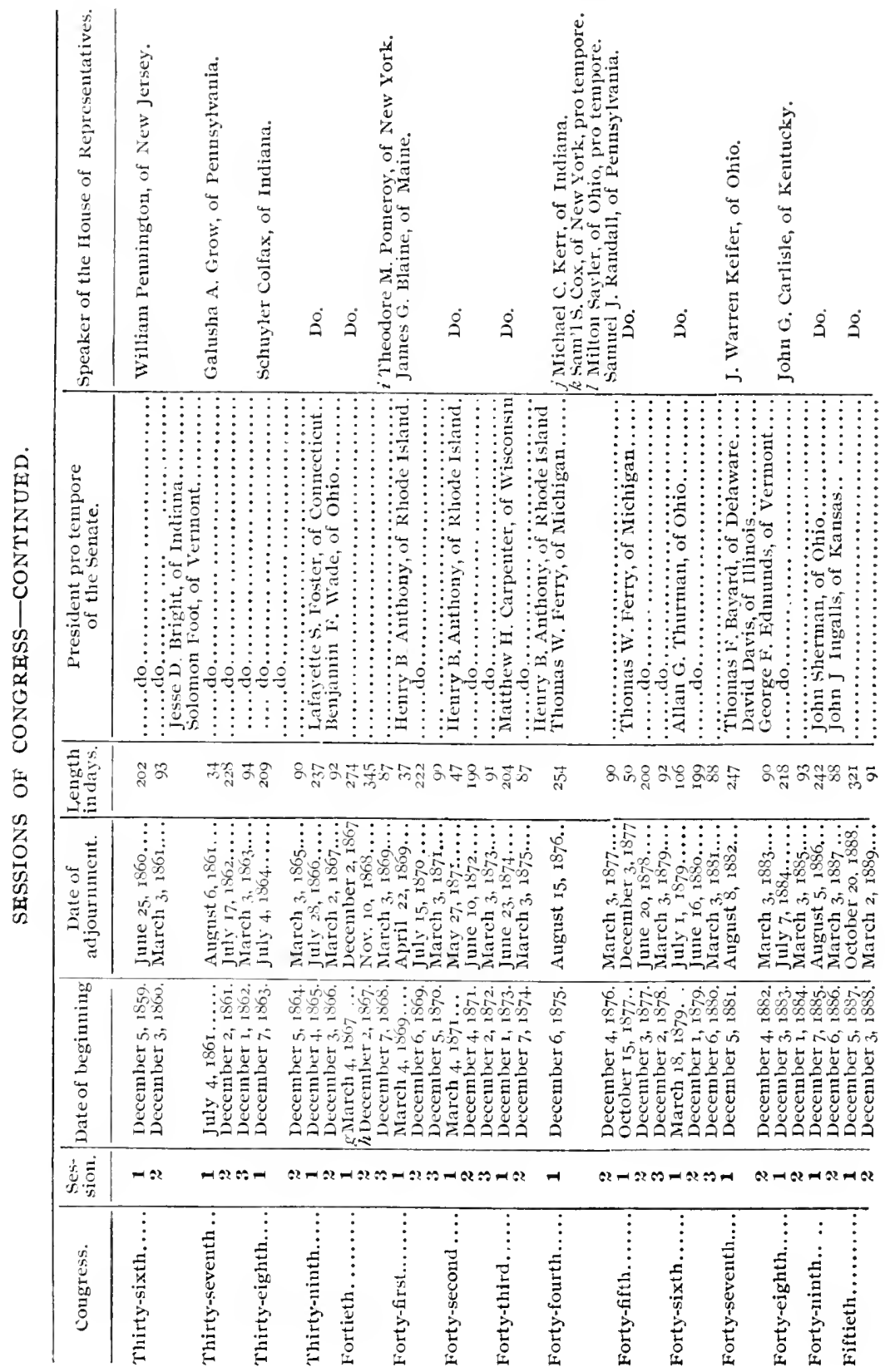


THE AMERIC.N GOVERMMENT.

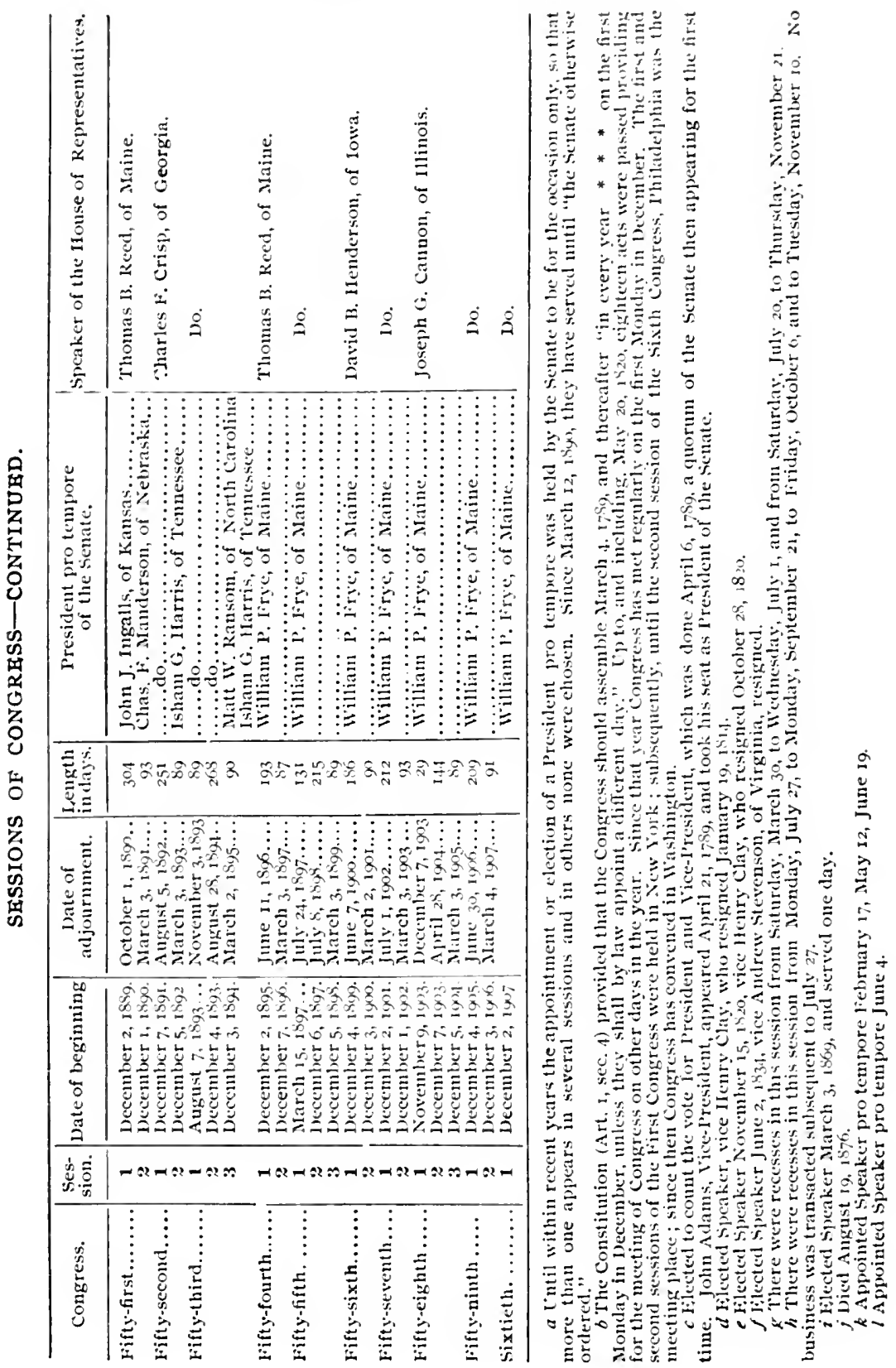


CHAPTER IV.

THE SUPREME COURT OF THE UNITED STATES.

Fully to detail the powers and prerogatives of members of the Supreme Court of the United States would require entrance upon the consideration of the jurisdiction of that body which would extend the limits of this chapter far beyond those proposed for the original size of the entire book. The English courts of law, in determining upon and establishing the common law of England have, throughout the centuries, treated the English jurisprudence at a constantly growing entity, of which, at no time, could any man say that thus stood the law in finality. The same is true of the Supreme Court of the United States. So long as questions arise affecting the economic and political conditions of this country, the limtis of the jurisdiction of the Supreme Court will not be defined, and any description of that body will necessarily be incomplete, for the reason that no one can tell what part of the future will be influenced and developed by the views of the Supreme Court as to its functions with relation to the progress of this country in those directions which may be reached by judicial action.

For all practical purposes and considerations, the Supreme Court, as it stands to-day, may be regarded, at least by laymen, as the Supreme Court which was intended by the founders of the Constitution. The conclusions on matters relating to the characteristics of the Supreme Court which were arrived at by Marshall and Story and have been confirmed by a long line of brilliant and able jurists as the result of nearly a century of most painstaking care, study and deliberation, while the favorite subject of discussion of learned doctors of constitutional law, may well be taken as sufficiently authentic, at least, for popularly describing the Supreme Court as it is to-day, as the Supreme Court which was contemplated by the founders of the Constitution. 
The chief subject in the consideration and description of the Supreme Court is the question of jurisdiction. In the matters of procedure, it follows the usual conrse of American jurisprudence, that is to say, its procedure is founded upon that of the English courts, with such modifications as have seemed to be wise under the conditions obtaining in the United States. With the exception of the State of Louisiana, which adopted the French or civil law, all of the States of the American Union have adopted, as a basis of their jurisprudence, the English common law as it existed at the time of the Revolution, and have amended and affirmed it as has seemed to be best suited to the purposes of the various States. In the application of the Federal law, however, there is this marked difference: that while the State courts apply the principles of law and equity with a view wholly to determine whether there has been a wrong which should be righted, or whether there is a right that should be affirmed, the Judges of the Federal courts have to determine whether the cause presented to them comes within the terms of the constitutional grant of jurisdiction. It would seem that the judical power of the United States, and especially the Supreme Court, was contemplated in a three-fold capacity by the framers of the Constitution; as a means for the settlement of disputes between the Statcs; as a guarantec to the citizens of each State that their rights under the Federal form of government should not be encroached upon by their own State government; and as a means for the adjudication of cases arising under the administration of the Federal Government, and on the ligh seas and in such other places as no process of a State court could run. It has been questioned if the framers of the Constitution contemplated the fourth function cxercised by the Supreme Court of the United States, namely, that of determining the validity or nonvalidity of a law passed by Congress. It has been said that the Supreme Court of the United States was the first court in the history of the world to exercise the power of so passing upon a law duly cnacted by a formally constituted legislative body. Since the judicial power of the United States is, by the Constitution, cxtended to all cases in law and equity arising under the Constitution, and since the Congress, in the exercise of 
the legislative power, is strictly limited by the terms of the Constitution, it has been held to follow that a case which challenges the constitutionality of an enactment of Congress, and which is decided against the terms of the act by the Supreme Court, secures, by virtue of such decision, the nullification of the law although passed in due form by both Houses of Congress and approved by the President. It is not to be misunderstood that the Justices of the Supreme Court of themselves have this power of nullification. They are, however, made by the Constitution interpreters of its terms, and their decision adverse to a law passed as suggested, is simply the notification to the Congress and to the President that the interpretation of the last two was at fault. Thus, while Congress was entrusted with making the laws of the country, and the President was given the responsibility of their execution, a third power was, even if not actually intended, put into effect which should stand between both of the other powers and the people. The judicial power was vested with all the majesty of the law for the purpose of punishing offenders while, at the same time, it constituted a great defensive force by which the people, without the necessity of resort to overt acts, could be defended against encroachment upon their rights whether by the other two powers of the Federal Government, or by the act of the independent States themselves. Finally, there was established, by the device of the Supreme Court, the machinery for the peaceful settlement of disputes between the independent States which had come together to form the Federal Union.

That the judiciary was established as such a safeguard is evident by the care with which its membership is made independent of both legislative and executive power. Alone, among the constitutional functionaries, the judges are to hold their offices during good behavior, and specific provision is made for a compensation to be paid at stated times, and which shall not be diminished during the continuance of the judges in office, that is to say, no judge may receive at any time a smaller salary than that which he receives at the date of his appointment.

In the constitution of the Supreme Court as a solemn tri- 
bunal of original jurisdiction for the most important classes of cases, and of final resort for contested questions, there appears the agreement of the pcople of the various colonies, differing so widely in characteristics, to submit their many controversies, apparent and unforeseen, to an arbitration founded on the structure of the English law and built with such wisdom that the sueceeding amplifications have fallen into their places with harmony, so that the Supreme Court of the United States is to-day, as it always has been, one of the most satisfactory contemplations of the American citizen.

The Supreme Court consists of a presiding justice, whose title is that of the Chicf Justice of the United States, and eight associate justices. All of these are appointed by the President, by and with the advice and consent of the Senate, and hold their offices during good behavior, and can only be removed upon impeachment. They receive the annual salary of $\$ 12,500$, and $\$ 500$ additional is paid to the Chief Justice, making his salary $\$ 13,000$.

Sessions of the Supreme Court of the United States are held during one term commencing on the sccond Monday of October in each year. The Justices may also provide adjourned or special terms. Six members constitute a quorum, and if less than six members are present at any session, those present may adjourn from day to day for twenty days, at the expiration of which if a quorum does not attend, the court is adjourned to the next appointed session. During the twenty days, however, in spite of the lack of a quorum, necessary orders may be made for various proceedings preparatory to the trial of cases. The precedence of the associate members of the Supreme Court of the United States is in accordance with the dates of their commissions, but if two commissions bear the same date the older Justice has the precedence. If there is a vacancy in the office of the Chief Justice or the Chief Justice is unable to perform his duties for any reason, the Associate Justice first in the order of precedence acts as Chief Justice until the lack of a Chief Justice is supplied. It is not usual to appoint a Chief Justice from among the Associate Justices. Members of the Supreme Court appoint a clerk, a marshal, and a reportet 
of Decisions. Sessions of the court which were formerly held in a room on the ground floor of the Capitol of the United States in Washington, are now held in the dignified chamber occupied by the Senate of the United States before the erection of the present Senate wing of the Capitol. In the original constitution of the court, the Justices of the Supreme Court were to act as presiding justices of the circuit courts, which were arranged by the original judiciary act to include the various district courts of the country, in accordance with their geographical positions. The practice of Justices of the Supreme Court going on the circuits, however, has been almost obsolete for years on account of the pressure of the business of the court, but each Justice is still assigned to a circuit as the titular head of the courts of that division of the judiciary, and may, when there is no judge of the circuit court available, issue certain orders as presiding justice of the circuit. Unless, however, necessity exists because of the absence of the circuit judge or of the district judge qualified to act in the case, such an order could not be signed in Washington, but the Justice would have to proceed to the circuit. It may also occur that an important case might need the presence of the Supreme Court Justice assigned to the circuit, at a sitting of the circuit court, or circuit court of appeals, and in general the business relative to the supervision of the judicial affairs of the United States circuit courts comes respectively under the Justices in accordance with their circuit assignments. The statutes require that each Justice of the Supreme Court shall hold at least one term of court in the Circuit to which he is assigned in each two years. On account of the pressure of other business these sittings are necessarily brief.

As has been previously suggested, in addition to the questions arising under the practice of the modified common law in the Supreme Court of the United States, the additional questions arise in Federal courts as to the jurisdiction of such courts under the Constitution. Problems relating to the jurisdiction of the Supreme Court are especially difficult, and have not only been considered frequently and at length in previous decisions of the court itself, 
but are being presented daily for consideration and decision. The Constitution provides that in all cases affecting ambassadors, other public ministers and consuls, and those in which a State shall be a party, the Supreme Court shall have original jurisdiction; that is to say, that any case involving or affecting the classes of litigants narned may be brought directly into the Supreme Court. The principle of the inferior and the superior courts is a familiar one, namely, that causes of lesser importance shall be determined in the lower and more numerous courts, leaving the higher courts, which are fewer in number, free to devote their time to other than what may be called the routine matters of jurisprudence. The necessary oversight and direction of the procedure of the lower courts by the higher is exercised through the right of appeal, and litigants, dissatisfied with the decree of the lower court, may take the case to a higher court for review and reconsideration. The Supreme Court of the United States being the court of last resort in the Federal branch of American jurisprudence, it is evident that only cases of the highest importance should be brought into the Supreme Court as original or initiatory procecdings. Under the power given to Congress to establish and ordain inferior courts, the former has, for the purpose of discouraging, so far as possible, original suits in the Supreme Court, limited its exclusive jurisdiction, that is, that shared with no other court, to three classes of cases, namely, those in which two States are the parties, or the United States and a State and those brought against ambassadors or menbers of their houschold. In cach of these cases the principle of sovereignty is involved in that the States, as being in themselves independent sovereigns, and ministers as representatives of sovereigns, ouglit not to be brought before an inferior court to that of the highest, and ought not to be subjected to any delay in the settlement of a controversy, unless in case of the ambassador, such controversy should be initiated by the foreign diplomatic representative himself. When, however, in a controversy in which a State is a party, the opposing party is not another State or the United States, while the case may be brought in the Supreme Court, it may also be brought in the inferior court. and when an ambassador 
or other public minister in a foreign country brings an action, or a consul or vice-consul is a party in any case, the action may be brought either in the Supreme Court or in the inferior court, as may be deemed advisable. The situation, then, as to cases which may be begun in the Supreme Court, is that controversies between sovereign States and against ambassadors and members of their households must be brought in the Supreme Court. Controversies in which a State is a party with citizens on the other side, may be brought either in the Supreme Court or in a lower court, and the same is true of a suit brought by an ambassador or minister or in which a consul or vice-consul is a party.

It is evident that the foregoing classes of cases, even if advantage were taken of the option to appear in the Supreme Court in every case of possible original jurisdiction, would not account for the great number of cases now before the Supreme Court of the United States. As a matter of fact, the original docket, or list of cases in which the court takes original jurisdiction, is comparatively insignificant. The greater part of the work of the court comes from the classes of cases in which the court is given appellate jurisdiction, and questions so arising present the many and novel propositions which make the Supreme Court of the United States a living entity and a perpetual fountain of law and jurisprudence. Appeals to the Supreme Court may be taken in certain cases from the district courts, in certain other cases from the circuit courts, and in another class of cases by appeal to the Supreme Court itself for certain writs, the issuance of which is provided for by the statutes in cases which come within the Constitutional jurisdiction of the Supreme Court. Thus while the Supreme Court cannot issue all of the prerogative writs as can a court proceeding under the common law, unlimited by Constitution, it can issue writs of prohibition to district courts when such courts are proceeding as courts of admiralty and with maritime jurisdiction, that is to say, it can direct a district court not to do certain things relating to the operation of vessels. By like provisions of law the Supreme Court can issue writs of mandamus to any courts under the authority of the United States or to 
any persons holding office under the United States, commanding them to do certain things when a State or a foreign representative is a party in the case in which the mandanus is framed; otherwise the issuance of writs out of the Supreme Court consists in the issuance of writs of certiorari in certain cases, and of injunction in cases in which the Supreme Court would have jurisdiction if brought as proceedings in that court. Appcals or writs of error, however, may be taken to the Supreme Court in a considerable number of classes of cases from the district and circuit courts. If, in sny case, the jurisdiction of the district court is in question, an appeal or writ of error may be taken direct to the Supreme Court instead of through the circuit court and circuit court of appeal. In this case, however, the facts and merits of the controversy, except so far as they affect the jurisdiction of the court, are not to be considered by the Supreme Court, which would take cognizance of the question of jurisdiction alone. Similar actions in appeal from final decisions in prize cases in the district court, or in cases of conviction of a capital crime, can be taken. In any case brought in the district court which involves the construction or application of the Constitution of the United States, or any case in which the constitutionality of any law or the validity and construction of any treaty is drawn into question, or any case in which the constitution or law of a State is claimed to be in contravention of the Constitution of the United States, an appeal or writ of error may be taken directly to the Supreme Court, without the necessity of going through the lower appellate court. This latter provision is made in order that there shall be as little delay as possible in detcrmining on the questions of constitutional law, such law including within its scope all of the matters suggested in the foregoing ground for direct appeal.

It is contemplated that all the classes of cases not belonging to the foregoing and which constitute the bulk of the litigation in the Federal courts, shall proceed from the lower courts to the circuit court of appeals and to be as far as possible finally determined upon without the necessity of action by the Supreme Court. In case, however, any controversy duly be- 
fore the circuit court judges presents questions of law concerning which the court desires the instruction of the Supreme Court, it may be certified by the judges of the circuit court upon the law points and the Supreme Court may either decide upon the point raised, in which case the instructions given are binding upon the judges of the circuit court, or the Supreme Court may direct that the whole record shall be sent up for its consideration and review, in which case the controversy would be heard and adjudicated as though it had been brought to the court for review by a writ of error or an appeal. Moreover, it is made competent for and in some cases the duty of the Supreme Court to issue a writ of certiorari or certification to the circuit court in order that a case which, but for this action on the part of the Supreme Court, would be finally decided in the circuit court or court of appeals may be brought up for consideration and adjudication by the Supreme Court. This action would be especially taken in the case of a decision by the lower courts which would have a tendency to confuse or complicate the administration of the law by a decision differing from those previously given on the same point of law. In certain classes of cases stated in the chapter on "The Judiciary," the decisions of the lower courts are made final, but in others than these cases, a right is established by statute to an appeal, writ of error or review. The United States may also appeal in cases in the Court of Claims where the judgments are adverse to the United States, and claimants in the Court of Claims may appeal to the Supreme Court where the amount in controversy is in excess of $\$ 3,000$, or where an order of forfeiture of the claim to the United States has been made by reason of alleged fraud in the claim. By recent legislation, the United States has also been given the right of appeal in criminal cases from the district or circuit courts when the invalidity or construction of a statute is in question, or when there has been a decision or judgment sustaining a plea in bar, so that the defendant has not been put in jeopardy.

A further and much discussed right is that of the removal of cases from State courts to the courts of the United States and to 
the Supreme Court. The following described prerogative of the Supreme Court is in addition to the right of removal from a State court to an inferior court of the Federal judiciary before the proceedings have been completed in the courts of a State. When a case has been prosecuted to a decree in the highest court of any State, and it appears that the decision is against the validity of a treaty, statute or other evidence of the authority of the United States, or where it appears that complaint is made that a law of the State is repugnant to the Constitution and statutes of the United States, and the validity of the law has been sustained, or where it appears that there has been a decision against any right or title set up by any person under the laws and authority of the United States, that is to say, when any question has been raised and decided adversely to the Federal authorities, power is given to the Supreme Court to examine the case upon a writ of error, the writ having the same effect as if it had been sued out in a court of the United States. The Supreme Court is given authority to reverse, modify or affirm the judgment or decree of the Statc courts, and may award execution, or remand the case to the court in which it had its former decision, for execution of the decree as determined upon by the Supreme Court.

Similar provisions to those in effect with regard to the Federal courts in the United States are made applicable as to appeals from the supreme courts of the various territories and with Porto Rico, and the Supreme Court of the United States has jurisdiction to review and take appropriate action on the final decrees of the supreme court of the Philippine Islands involving constitutional questions, or in which real estate is involved exceeding in value the sum of $\$ 25,000$. Certain provisions which are common to the Supreme Court, as well as to the other Federal courts, will be summarized under the heading of "The Judiciary," the aim of the present chapter being to ontline the particulars in which the Supreme Court differs from the inferior courts of the Federal system. 
SUBORDINATE OFFICERS OF THE SUPREME COURT.

Subordinate officers of the Supreme Court who are appointed by the court but whose compensation is fixed by law, are the clerk, the marshal and the reporter of the decisions of the Supreme Court. The clerk, who must give a bond which may be from $\$ 5,000$ to $\$ 20,000$, is compensated by fees in accordance with a fee table which is fixed by the court, his compensation, however, not to exceed $\$ 6,000$ per year. He is allowed deputies according to the pleasure of the court, and provision is made by which he may recover for the malfeasance in office of any deputy for whose conduct he is responsible. The marshal, who attends upon the court and serves all processes, who takes charge of the property of the United States and used by the court and its members, and who disburses the official expense money of the court, receives $\$ 3,500$ per year. He is required to attend all the sessions of the court, and is provided with such assistance and messengers as the Chief Justice may approve, including a bailiff or crier for the court, their compensation to be fixed by the court. The reporter of the Supreme Court of the United States occupies an important and, it is believed, a lucrative office. He is allowed by law a salary of $\$ 4,500$ a year, for the publication of one volume of the reports of the decisions of the Supreme Court annually, and \$I,200 more if he publishes a second volume annually by direction of the court. He is also allowed \$I,200 per annum for clerk hire, and $\$ 600$ for office rent and stationery. For these appropriations he is required to furnish the Government with three hundred copies of each volume of the decisions published by him, and he is allowed to sell other volumes to the public at a fixed price of $\$ 2$ per volume. The decisions of the court are furnished to the reporter in manuscript by the justices making the decisions, and his work consists of making the syllabus or synopsis of the case which heads the text of the decision, and of arranging for the printing of the volumes, and performing the editorial work thereon.

Upon the assembling of the Supreme Court on the second 
Monday in October, it is the practice for the court to adjourn after the opening ceremonies, for the purpose of calling upon the President in a body, accompanied by the Attorney General and the officers of the court, in order to pay the court's respects to the Executive. On the following day the business of the session begins with the hearing of motions, admission of new members to the bar, and the calling of the docket. The court exercises considerable discretion in the matter of forwarding cases upon the list, as without such discretion cases of importance might be required to remain without decision for a long period, while comparatively unimportant cases were being argued. It is provided by law that criminal cases on writs of error from State courts shall be given precedence over other cases, except such as the Supreme Court may deem to be of public importance. The latter class of cases, including those in which the government of the United States is espccially anxious for a speedr determination, and cases on which depend the adjudication of a considerable number of controversies in the lower courts, may be adranced, at the discretion of the court, for hearing before other cases which have been entered on the list at an earlier date. Monday is generally utilized as motion day and for rendering decisions in the Supreme Court, and arguments are heard on the following days of the week except on Saturday, when no arguments are heard except in unusual cases. While the arguments in cases are generally heard by the whole bench, the work of preparing decisions is assigned in each case to one member, who submits his conclusion to the full bench in their consultation room for assent or dissent. In the majority of cases, perhaps, the opinion read by the individual justice is that of the entire court. It is by no means uncommon, however, for notice to be given that one or more of the judges dissent, either expressing their unwillingness to agree to the view of the majority, or reading a dissenting opinion, giving their views of the case at length. The decisions are read in full in court from carefully prepared manuscripts, but generally in so low a tone that few outside the first circle of the attorney's within the bar profit 
materially from the decision as it is read, a fact which is of little consequence, as the decisions are available in printed form very shortly after they have been delivered.

TABLE OF FEES TO BE CHARGED BY THE CLERK OF THE SUPREME COURT OF TIE UNITED STATES.

For docketing a case and filing and indorsing the transcript of the record, five dollars.

For entering an appearance, twenty-five cents.

For entering a continuance, twenty-five cents.

For filing a motion, order, or other paper, twenty-five cents.

For entering any rule, or for making or copying any record or other paper, twenty cents per folio of each one hundred words.

For transferring each case to a subsequent docket and indexing the same, one dollar.

For cntering a judgment or decree, one dollar.

For every search of the records of the court, one dollar.

For a certificate and seal, two dollars.

For receiving, keeping, and paying money in pursuance of any statute or order of court, two per cent. on the amount so received, kept, and paid.

For an admission to the bar and certificate under seal, ten dollars.

For preparing the record or a transcript thereof for the printer, indexing the same, supervising the printing and distributing the printed copics to the justices, the reporter, the law library, and the parties or their counsel, fifteen cents per folio.

For making a manuscript copy of the record, when required under Rule Io, twenty cents per folio, but nothing in addition for supervising the printing.

For issuing a writ of error and accompanying papers, five dollars.

For a mandate or other process, five dollars.

For filing briefs, five dollars for each party appearing.

For every copy of any opinion of the court or any justice thereof, certified under seal, one dollar for every printed page, but not to exceed five dollars in the whole for any copy. 


\section{CHAPTER I:}

THE OFFICERS OF THE CONGRESS-THE VICE PRESIDENT OF TIIE UNITED STATES-SPE.IKER OF THE HOL'SE OF REPRESENTATIVES-SUBORDINATE OFFICI.ILS.

The Vice-President of the United States is elected at the same time and in the same manner as the President of the United States and must have similar qualifications. He would not be a resident of the same State as the person elected President for the reason explained in the chapter on the President. He receives a salary of $\$_{12,000}$ a year and cannot resign or refuse to serve except in writing over his own name, the paper to be filed with the Secretary of State.

Upon the death or inability to perform his official duties of the President, the Vice-President becomes President upon taking the oath of office. While Vice-President, the person holding that office is also President of the Senatc. He is sworn in by a member of the Senate, the function being assigned as a matter of courtesy to the oldest member of the Senate or to the retiring President pro tempore. The Vice-President as President of the Senate may administer oaths of office to Senators and to witnesses in Senate proceedings of inquiry. He presides orer the sessions of the Senate, but has no vote unless in case of a tie vote of the Senate, when he casts the deciding vote. He presides over impeachment proceedings except in the event of the impeachment of a President, when, as a party interested in the result, the Vice-President is replaced by the Chief Justice of the United States.

The Vice-President heads the list of those officials, including members of Congress and Tnited States officials generally, who are exempt from militia duty and is specifically named as being entitled to the privileges of the Congressional library. $\mathrm{He}$ is by 
virtue of his office a member of the Board of Regents of the Smithsonian Institution, appoints three members on behalf of the Senate on the Board of Regents and presides at the meetings of the Board when the President of the United States is absent. The Vice-Presilent also appoints two members of the Senate as members of the Board of Visitors of the Military Academy, two as members of the similar board at the Naval Academy and such other representation of the Senate as may be directed by the Senate or by law. He does not, however, exercise any power in the organization and direction of the Senate, being in the position simply of an impartial functionary carrying out rules which have been determined by the membership of the Senate of which he is not a part. The Vice-President signs all bills which have passed both houses and in the final form as engrossed on parchment, his signature appearing before that of the Speaker of the House on Senate bills and vice versa in the case of bills originating in the House, the signatures of the two presiding officers being warrant to the President that the bill as engrossed has actually passed both houses of the Congress.

THE PRESIDENT PRO TEMPORE OF THE SENATE.

In case of the absence or death of the Vice-President acting as President of the Senate, or in case of his assumption of the office of President, the Senate elects from its membership a President pro tempore who presides over the sessions of the Senate and who then has the emoluments of the Vice-President, but no other functions than those of the President of the Senate, except that he retains his powers as a member of the Senate and may speak from the floor, having temporarily relinquished the chair.

THE SPEAKER OF THE HOUSE OF REPRESENTATIVES.

One of the most interesting studies in the government of the United States is that contained in the contemplation of the increase of the power of the Speaker of the House of Representatives through its delegation by the majority of the members of 
the House, representing the dominating political mode of thought. Provision is made in the Constitution that the House of Representatives shall choose its Speaker. The official in mind was, undoubtedly, a presiding officer, simply, charged with the impartial administration of the rules which should be adopted by the House to direct its course of procedure.

The statutory provisions relating to the Speaker are meagre and are those stated in the sentences of the following paragraph. He may administer oaths of office to members and officers after having himself been sworn in by any nember of the House. $\mathrm{He}$ is to receive a salary of $\$ 12,000$ a year and in addition to a clerk at his desk on the floor of the House, he is allowed a private secretary besides the usual emoluments of a member of mileage and stationery. The reporters of the debates in the House are appointed on his approval and he may administer oaths to witnesses before the House. He makes up the Committees of the House, certifies to the salary and mileage accounts of members and appoints three members of the House each to the Boards of Visitors of the military and naval academies, respectively, three members of the Board of Regents of the Smithsonian Institution and such members of the House as may be provided by law in making up the membership of supervising bodies over institutions in whole or in part supported by governmental appropriations. In addition to the foregoing, the Speaker of the House appoints a committee on accounts, consisting of three members who have the direction of the expenditures on the part of the House during the months between the close of one Congress and the assembling of the next.

The chief of the powers and prerogatives of the Speaker of the House are those conferred upon him by the rules of that body. He has control of the hall and corridors of the House wing of the Capitol and of such rooms in that wing as may not be assigned. He is charged with maintaining order in the galleries and may order them cleared when disorder occurs. Originally regarded only as the impartial presiding officer of the body and with a function similar to that of the President of the Senate, he 
has become in later years one of the strongest factors in determining the character of legislation effected. He secures this strength through the operation of two prerogatives which have been delegated to him by the House under the Constitutional provision that each House may adopt rules to govern its procedure. The first is that covering recognition of members on the floor of the House. It is apparent that no measure can secure a learing before the House unless its mover is able to attract the attention and secure the recognition of the Speaker. There is a well-established code by which the order of the recognition of the majority and minority members of a committee is established with respect to bills that have been reported, and the Speaker, after having arranged for the consideration of any measure is bound by precedent and rule to first recognize the reporting member of a committee in favor of a bill and to grant certain rights to the dissenting member or members. But it is within the discretion of the Speaker to say with a considerable degree of finality whether a measure shall come before the House at all. The number of legislative propositions before each session of Congress is so great that it is impossible that every one of them should be fully heard and considered during the session. Some one must weed out from the mass and reject those whose importance is not sufficient to warrant especial consideration and the Speaker having the responsibility of securing the termination of the important business of a session within the limits of time set for it, has assumed the power and control over matters to be debated and acted upon, subject to the restriction only that his discrimination must not be so oppressive that a majority of the members of the House can be allied against him in an appeal from a decision of the chair.

Growing out of this power of recognition on the floor by the Speaker there has developed a morning levee in the Speaker's room, where members attend for the purpose of securing the promise of the presiding officer that they will be recognized at a given moment to make a motion in regard to a particular bill in which they may be interested. The important measures to be 
acted upon during the week have already been arranged for by conference between the Speaker and the heads of committees, and other time available during the week is then apportioned, out by the Speaker to individuals according to.ilie.importanee of the measure or the importunity of the momber. I Iists are nade.of the moments at which members are to be'recognized for particular purposes and it has sometimes happened that a member himself has forgotten that he was to be recognized and when called upon by the presiding officer has been at loss, for the moment, to state the motion for which he had been recognized.

This condition, of course, renders the course of business of a day in the House of Representatives practically a prcarranged ceremony and offers but little opportunity for individual initiative and digression. So much is this the fact that although the rules of the House require that a member shall restrict himself in debate to the subject under discussion, an ingenious subterfuge is in effect to enable members to state their views on remote subjects. In the preliminary discussion of important measures, the House goes into Committee of the Whole and arrangement is then made for a general debate in which the utmost latitude as to subjects of discussion is allowed and members are able to state the arguments for measurcs which are not likely to find time for consideration during the session. Intervals are also accorded in the sessions of the House itself when by unanimous consent a few moments of speaking time are accorded to a member, and, with such devices as these, the thcory of free debate is made compatible with the necessity of a strict limitation of time for the actually important matters of a session.

Besides determining the nature of the business presented to the House of Representatives, the Speaker wields the weapon of the majority in cnforcing affirmative action. This weapon is forged from the Constitutional provision that a smaller number than a quorum of the House may compel the attendance of absent members. Under the rules of the House fifteen members may exercise this power, and the Speaker is vested with the authority of the fundamental law of the land to enable him, on 
vote of such number of members, to bring the absent members to the bar of the House and to compel their attendance under penalty of such punishment as the sitting members may prescribe. Under such,condirious; when the actual attendance of the members in the hall of assernbly can be enforced, there are only two means by wlich a minority can defeat legislation. One is that of unlimited debate, such as obtains in the Senate of the United States. This means is rendered impossible in the House by reason of the provision for moving the previous question, the "cloture," so-called, which when approved by a majority of a quorum of the House, terminates debate and enforces a vote upon the main question under discussion. The cloture has existed in some form for a century in the history of the House of Representatives, but up to 1890 , the second device at the command of a minority was possible. The Constitution provides that a quorum of the House of Representatives must be present in order that business may be transacted. A quorum consists, according to recent ruling of the Speaker of a majority of the persons elected and living who have actually taken the oath of office. Prior to 1890 , it was held that the presence of a quorum in the House of Representatives could only be determined by the enumeration of a vote; that if the members who had been actually brought into presence of the House refused to vote and the presence of a quorum could not be shown by the number of members voting, there was no way in which the House could transact business.

The holding was overset by Speaker Reed in his famous ruling of January, I8go, in which he held that any member who could he seen and counted by the chair or by an employe of the House under his direction within the meeting place of the House, could be counted in the enumeration of a quorum and thus make it possible for the business of the House to continue. The Reed ruling has been fortified by the course of the House since I890, including a period of Democratic practice in which the difference was made of having two members of the House count the members present instead of the Speaker. 
Under this practice, the majority is able, with only slight delays due to the length of time required for roll calls, to force the transaction of business according to the will of the majority of the House. The limitation of free debate and parliamentary tactics has been drawn still closer by the operation of a system of special rules which nay be presented to the House by the Committee on Rules and carried by the majority vote and which are given the effect of stopping or preventing debate by moving the previous question thus requiring an immediate vote on the proposition involved. These are colloquially known as "gag" rules and the power of the Speaker and of the managing majority of the majority party is only limited by the possibility of serious revolt against any portion of the procedure.

When the Speaker of the House is a strong man, well versed in the procedure of the House and fortified by its confidence, the measures before described operate to give him almost autocratic control of the details of legislation. If, however, he is dominated by any men or set of men on the floor of the House, such a coterie would be able to exercise the power over the acts of the lower branch of the national legislature which is somewhat loosely flying about, subject to seizure by the strongest force in its membership. The conditions which have been described, not being based on statute law, and, indeed, being practically based on sufferance and the supposed party necessities of the present majority party in the House, are subject to change, although it is cloubtful if business could now be transacted in the volume required without a similar system.

The Speaker of the House signs all acts, resolutions and other evidences of the action of the House as well as all subpoenas issued by order of the House. As presiding officer, he, of course, decides all points of order arising in the sessions of the House and is required to rise when putting a question. It is regarded as a breach of order if he refuses to put a question when it is in order. The Speaker cannot be deprived of his Constitutional right to vote in the proceedings of the House as the representative of a district, but he is not required to vote in the ordinary 
legislative proceedings except when his vote would be a decisive one when the House is voting by ballot. The decisive effects of a vote by the Speaker would be, in an ordinary vote, to break a tie, or to make a tie should the majority be a single vote. In the latter case the effect of the Speaker's vote would be to decide the question in the negative as in case of a tie vote it is provided that the question shall be lost. In such extraordinary votes as those which require a two-thirds vote, the Speaker's vote might complete the two-thirds required. When, formerly, the determination of a quorum depended on the enumeration of a vote, the Speaker's vote was sometimes required to complete the quorum, but this is no longer the case.

The Speaker of the House is elected by the spoken vote of the members of the House immediately after it has been called to order by the clerk. He may appoint a member to perform the duties of Speaker temporarily, but the appointment cannot extend beyond the adjournment for the day, except it is made in writing with the approval of the House. In such case an appointment may be made by the Speaker for not exceeding ten days. When the House goes into Committee of the Whole the Speaker names the member who shall take the chair, and if the Speaker desires to speak from the floor during sessions of the House he names a member to take his place. The prerogative of the Speaker of speaking from the floor is, however, seldom exercised. In case no speaker pro tempore is named by the Speaker, one is elected by the House, generally by affirmative vote on a resolution, to serve until the Speaker is able to resume his duties.

The disciplinary power of the Speaker over the members of the House refers to any proceeding that may be necessary to obtain order. If a member persist in disorderly conduct, the Speaker is empowered to call the member by his name, force being given to the unusual proceeding by the fact that the Speaker generally calls on members en masse for order or distinguishes a member by the name of his State. If the member is named in full by the Speaker, he may be required by the House to withdraw and the Speaker then stating the nature of the member's offense, the 
House prescribes his punishment. In case of confusion and persistent disorder anong a group of nembers or in case it becomes necessary for the House to enforce the withdrawal of a member, the sergeant-at-arms takes the mace and proceeds to the scene of confusion. The Speaker must call a member using unparliamentary language to order in connection with his general duty of preserving order and decorum of debate, but the matter of calling offensive language to the attention of the House is not exclusively that of the Speaker, but any member nay take the initiative in proceedings of this sort.

The Speaker of the House of Representatives, as is the case with the presiding officers of similar legislative bodies in the States, has exclusive powers in connection with the composition of the various committees of the House. It is the practice at the opening of a Congress for the Speaker to receive applications from the less important members and intimations from the more prominent ones as to congenial committee assignments. Beginning with the Committee on Ways and Means and the Committce on Appropriations, the importance of the committees dwindles with considerable rapidity after those committees are passed which have to do with making up appropriation bills and at the tail end are a considerable number of committees which are desirable only as affording chairmanships and committee rooms. To assign not only the chairmen, but the members of the committees in their appropriate ranks is a difficult and delicate matter. Althongh each Congress is a new proposition, as to committec memberships, members who are re-elected expect to hold their places on committees and to be advanced when ranking members retire from Congress. The task of the Speaker is to select men who can carry on the work of the important committees successfully and at the same time satisfy the political conditions and the demands of members based on their political importance and their service in previous Congresses. The representation of the minority on the different committees, being the minority of each committee, is generally suggested by the minority leader in the House, but in case of a disagreement between the 
minority leader and his supporters, it is the task of the Speaker to decide the difference. The coveted positions are either chairmanships or membership on the Committee on Ways and Means or Appropriations, and members having one are not generally assigned to the other. The work of making up the committees, although started by the Speaker at the opening of a new Congress, is generally not completed until after the holiday recess and the first days of the session are extremely busy ones for the presiding officer of the House and frequently constitute a time of extreme perplexity. When vacancics occur in committee assignments during a Congress the Speaker generally makes a series of promotions, moving up the ranking member in each case, but it sometimes occurs that selections are made outside of the committee in which the vacancy occurs, in which case, also, the Speaker has his troubles.

\section{SUBORDINATE OFFICIALS OF THE CONGRESS.}

The Capitol of the United States is like a self-contained and selfsupporting little village. Within its walls a small army of employees conduct operations necessary for the conduct of legislative business and for the comfort of the members of the two Houses. There is, also, nothing within the walls of the Capitol which answers to the restrictive system under which other employees of the United States are selected and retained in office. That is to say, there is no civil service reform legislation affecting the employees of the Senate and House of Representatives. The principal officers are elected by the vote of the respective Houses, and the subordinates are selected in accordance with the legislative importance of the various members. It is the theory that the appointments are distributed as equally as may be among the members of the Congress, but in practice the more assiduous of the place-hunters, especially if they have secured a chairmanship or a membership on an important committee, are the recipients of the largest share of the patronage favors which are dispensed by the elective officers of the two houses. This is more especially true of the House of Representatives for 
the reason that arrangements are made so that Senators within their rank and with due regard to whether they belong to the majority or to the minority party are given about equally proportionate consideration in the appointments. On the House side, however, the legislative and political importance of a member has much to do with the number of persons he has secured to be placed on the various rolls.

The chief subordinate official of the Senate is the Secretary, elected by the members of that body, but holding his position in practical permanence since it is the tradition of the Senate to retain employees practically during good behavior and satisfactory performance of their duties, although a change in the majority party might break some from their anchorages. The contrary is true in the House, where the tentire of office depends strictly upon the continuance of the membership of the Representative at whose request the appointment was made. No formalities are used in connection with the separation from the service of the House of Representatives of an official whose member has retired from Congress. He is expected to consider himself by reason of the non-existence of his "influence" as officially dead and to retire from his position and the pay-roll as promptly as may be practicable. The fact that he may have been an efficient public servant is of no moment, and unless he can secure the backing of equally influential membership, he has no alternative but to retire to the joys and privileges of private life.

The Secretary of the Senate. who is also the disbursing officer of the Senate and of the salaries of Senators, receives $\$ 5,000.00$ per year, with $\$ 396.00$ additional in the last named capacity. He supervises all matters relative to the conduct of the legislative operations of the Senate and also pays all bills and disburses all salaries. As the Senate is a continuing body, the Secretary has no function similar to that of the Clerk of the House, who calls the House of Representatives to order at the beginning of each Congress. The corresponding officer to the Secretary of the Senate, who performs similar functions for the House is the Clerk of the House of Representatives. This official is elected by the riza woce vote of the House and receives a salary of $\$ 5,000.00$ per year. As he has the 
most important office in the election of the House, controlling a large proportion of the patronage, the election of a new Clerk is accompanied by a considerable amount of electioneering, and is usually accomplished by a combination of certain members of the majority having for its purpose to partition out the subordinate places in the service of the House of Representatives. Upon the convening of a new Congress the Clerk calls the members to order and serves as a temporary presiding officer until the election of the Speaker pro tempore who presides until and at the election of the Speaker of the House. He calls the roll of the States in alphabetical order, for the election of the latter official, who is usually the oldest nember of the House in point of service. The Clerk performs this office, of course, as a holdover from the previous Congress, serving until his successor shall be elected. The Clerk is also required at the opening of the sessions of Congress to send to each member a list of the reports required by law from heads of departiments, and in case of a contest for a seat in the House makes up the record containing the respective claims of the contending parties to be seated.

When the house has been organized, it is the duty of the Clerk through his subordinates, when directed by the Speaker, to read all motions that may be put before the House, including the bills in the course of passage, and to note in the journal all the steps of parliamentary procedure. $\mathrm{He}$ is required also to note all points of order, and to have these, together with the journal, printed and indexed and sent to the members of the House as soon as possible after the close of the session. Two copies of each of the documents and books printed for Congress are retained in the library formerly under the care of the Clerk, but now directed from the Library of Congress. The Clerk also is required to see that one copy of the reports and documents printed during the session of Congress are properly bound and sent to the members of the House at the close of the session. The Clerk attests all actions of the House on bills and resolutions, and his certification is necessary to their validity. His signature is affixed to all bills which have passed the House certifying that they have passed in due form. The contingent 
funds of the House are under his charge, as well as the stationery room, and the fund from which the members of the House receive their annual allowance of stationery amounting to approximately $\$ 150$ in value. The Clerk is also required to furnish all materials and supplies for the furnishing of the House of Representatives and its Committee Rooms, and also has charge of the labor performed for the comfort of members aside of the care of the capitol building itself. which is under the charge of the Superintenclent of the Capitol.

The matters which are herein detailed with regard to the Speaker are similarly periormed for the Senate by the Secretary of that body, and the subordinates of both officers have similar duties in each house respectively. Among the subordinates are the Journal Clerks and their assistants, who attend at the desk of the presiding officers and keep a record of the legislative transactions. The Reading Clerks take turns in reading the bills and resolutions under consideration. The Enrolling Clerks receive bills as they come from the respective houses to prepare them for printing, read the proofs and sce that all amendments are made in accordance with the vote of the House as indicated by the entries in the Journal. The File Clcrks have the custody of all papers relating to legislative matters pending before the House and the appropriate documents relating thereto.

In addition to the duties previously named, the Clerk of the House is paymaster for the House employees of all classes, but does not pay the members, that duty being performed by the Sergeant at Arms.

The Scrgeant at Arms of the Senate performs the duties of the Doorkecper as well, and is also charged with disbursements and provision for the comfort of the Senators, similar to the functions discharged under the direction of the Clerk of the House of Representatives. The Sergeant at Arms of the House of Representatives is required to maintain order in the House under the direction of the Speaker, and is the custodian, as the badge of his office, of the mace of the House, the symbol of the Speaker's authority. He is charged with removing all persons who may create disturbances and if the 
members of the House refuse to come to order at the call of the Speaker, the latter may call upon the Sergeant at Arms, who thereupon takes the mace from its position at the Speaker's desk and proceeds to the scene of the disturbance upon the door of the House. Should the presence of the symbol of authority fail to restore order, he has power, under the direction of the Speaker, to exercise such force by himself and his assistants as may be necessary to restore such order, even to the point of ejecting a member from the floor. Such a contingency, however, is not expected to arise as the members, or a sufficient number of them, would recognize the authority of the officer with his official symbol and terminate any disturbance which might arise. The Sergeant at Arms is the marshal or sheriff of the House and serves all process ordered by it. On the call of the House it is his duty to visit such places as he may on information ascertain to be those at which members absent themselves from the House, and require such absent members to accompany him before the Speaker. When it appears that a quorum is not present in the House of Representatives and the Speaker directs a call of the House and that the Sergeant at Arms shall summon absent members, it is his duty and that of his assistants to proceed at once to secure the presence of as many members as possible in order that a quorum may be filled as soon as practicable. In addition to his other duties he is the paymaster of the members of the House of Representatives and keeps their pay and mileage accounts, making disbursements to them as required by law. In the case of a funeral of a deceased member, the arrangements for the attendance by a committee of the House are made by the Sergeant at Arms, and upon occasion of state funerals he is the master of ceremonies.

The Doorkeeper of the House has charge of the messengers at the doors and entrance-ways to the halls of the House of Representatives and is required to clear the House and its lobbies I 5 minutes before the beginning of a session, and to allow no persons to enter upon the floor of the House except those who are members or who are entitled to the privilege of the floor by reason of previous membership, or because they have received the thanks of Congress. This restriction from the floor of the House of Representatives 
is strictly carried out, and to a degree not the fact in the various State legislative bodies. It is, howerer, a necessary provision for the reason that the public lobbies and halls of the Capitol in the House of Representatives wing are crowded with people during the session who desire to see members of Congress. If any system of special privileged entry should he in effect the demands which could not be ignored for the exereise of such privileges would be so great that the halls of the House would be over crowded at all times.

Included among the employes of the Doorkeeper's department are a number of veterans of the wars of the United States who had specially meritorious services and whose names have been placed on what is known as the soldiers' roll, and who are regarded as permanent employes. Most of these are placed at the doors of the galleries of the Ilouse, and where their duties are not inconsistent with their crippled condition.

Employes of the Doorkeper at the main doors in the lobbies of the House of Representatives not only serve to keep unathorized persons from the floor of the House, but also act as messengers between visitors and the representatives. If it is clesired to see a member of Congress, the visitor must end in his name on a card, and the celerity witl which the member answers the call gives the visitor an opportunity to gauge his own political importance. When the messenger returns with the information that the nember is not on the floor, the visitor has also the pleasing office of determining whether the information is based on the truth or whether his own political importance is nil.

In addition to the Doorkeeper's messengers, the Doorkeeper is in charge of the staft of pages, boys of from i 2 to 16 , employed during the session at $\$ 75$ a month to run errands for the members, bring them documents, and perform other services. The positions as pages are eagerly sought by the knowing youths of Washington, although it may be said that the position of page in either the House or Senate is not without its drawbacks and dangers to a boy of the age named.

The Doorkeeper of the House controls one of the large sources of House patronage, namely, the folding room, where all the doc- 
uments printed for the House are sent to be retained until sent out by orders of members. The business of the folding room occupies a large portion of the basement rooms and cellars of the House of Representatives, and tons of printed matter are added yearly to the stock kept therein, while tons are sent out yearly to be disposed of as waste paper, without having been of use to any person. Editions prescribed by law or by the resolution calling for the printing of particular books and documents are sent in bulk to the care of the Doorkeeper of the House, and by him allotted proportionately to the members. On the order of the members they are sent free of postage to such persons as may care to have them, or as may be designated by the members, and a large force of employes is kept busy during the session of the House in enclosing these documents in wrappers for mailing. In addition to the organization for mailing these documents, the Doorkeeper has a document room on the House floor of the Capitol to which copies of each document are sent, to supply the current needs and demands of the members and of officials of the Government. The number of employes engaged in work on printed documents under the direction of the Doorkeeper is a considerable and an elastic one and it is comparatively easy for a representative to place a specially favored constituent in at least a temporary place on this roll.

Chaplains of the House and Senate are elected respectively by each body and receive a salary of $\$ \mathrm{r}, 000$ a year. They are expected to attend upon the opening of each session of the House and to offer prayer. They are also the official pastors of the members of the two Houses and conduct the funeral services which may take place in Washington of deceased members, but it may be assumed are not held to strict accountability for the morals of the legislators under their respective charges.

The duties of the subordinates of the Congress may best be explained by detailing the course of procedure of a legislative day. The employes whose duties are not regulated by the sessions of the House and Senate include the Chief Clerk and his staff, the Disbursing Clerk and his subordinates, the Superin- 
tendent of the Folding and Document rooms and those under him, together with those who have charge of the committee rooms or employed in work therein.

Fifteen minutes before the beginning of the legislative day, which is at twelve o'clock, the messengers under the charge of the Doorkeeper, clear the lobbies of the House and Senate of the sightseers and visitors who have been passing through them. The description which follows applies to the House of Representatives, but is substantially the same for the Senate, similar officers in most of the cases performing similar duties.

Promptly on the stroke of twelve the Speaker of the House strikes with his gavel upon the marble block in front of him and calls the House to order. The chaplain offers prayer, after which the Speaker announces the business of the day. The Journal Clerk or his assistant on duty at the desk in front of the Speaker makes a minute of the various steps of parliamentary procedure, but not of the terms of the debate. The service of verbatim reporting of the proceedings of the House is performed by a corps of official reporters, six in number, who receive $\$ 5,000$ each, with an assistant reporter at $\$ I, 500$. These reporters take their positions as near as possible to the member who is speaking, and work in relays of fifteen minutes, the reporter who has been taking the debate in shorthand for the preceding fifteen minutes, giving way to another of the members of the corps, and retiring to the reporters' rooms where he dictates from his notes into a phonograph, the matter being transcribed by typewriter operators and sent at once to the Government printing office, where it is put into type so that a proof of what is said during the day is furnished to each member participating in the debate during the evening for his correction, the corrected matter being printed in the Congressional Record of the following morning.

The reporting of the Senate is carried on in a similar manner except that the compensation of the reporters is not the subject of annual appropriation in salary. A lump sum of $\$ 30.000$ is provided in the annual appropriation bill payable in equal monthiy installments, and the reporting is undertaken by one person 
who provides and pays his assistants. In addition to the foregoing, stenographers are provided to take the reports of proceedings in committee when hearings are held on any subject. Four stenographers to committees are provided in the House at an annual salary of $\$ 5.000$ each. In addition to this service it is also necessary during the session of Congress to employ outside stenographic services to a considerable degree.

The Clerk of the House of Representatives is himself very seldom upon the floor of the House, and that only upon special occasions. His duties are performed by the several officers herein described, and the duty of the Clerk to state all propositions which may be before the House is performed by two reading clerks who receive a compensation of $\$ 3.600$ each, and who are neccssarily employed only while the House is in session. Their duties are, however, of a most exacting character, as they are compelled during the passage of long bills to read continuously in half-hour periods sometimes for nearly the entire legislative day.

In the Senate in addition to the regular reading of the bills it is customary to have other matters read from the Clerk's desk, and during the course of a speech, a Senator will have considerable extracts from publications read, especially when the speech is being made for the purpose of delaying a vote on a particular measure.

The rules of the House of Representatives and the parliamentary procedure are based upon the compilation made by Thomas Jefferson of the practice and procedure of the English Parliament. This has, of course, been extended and enlarged by the precedents established by the action of the House on disputed questions, and in the course of nearly every day's legislative session some parliamentary matter arises which requires the decision of the Speaker. In order that he may readily rule upon the questions as they arise, an official is provided who is an expert on the rules of the House and on the decisions made thereunder, and who is termed the Clerk at the Speaker's table. He receives $\$ 3,600$ a year, with $\$ \mathbf{r}, 000$ additional for preparing the digest of 
the rules, a publication which contains memoranda of the decisions which have been made on varions points. This official prompts the Speaker on parliamentary points, and when a new proposition arises the Spealier may reserve his decision, referring the matter to the Clerk for the purpose of securing information for the Speaker's guidance in his ultimate decision. Informal votes of the House are taken by yea and nay, in which case the Speaker decides as to the preponderance of the affirmative or the negative by the volume of sonnd. In case he is not able to determine by this means, the Speaker announces that he is unable to determine the rote and requests the members to rise in accordance with their rote on the matter. count being made by the subordinates at the Clerk's desk. A rote by tellers may be demanded, however, and representatives of the affirmative and of the negative, being members of the House, count the members as they pass between them and announce the vote to the Speaker. If a ballot is demanded and ordered by the House, members answer as their names are called in alphabetical order, the tally of the result being kept by the Tally Clerk, an official receiving $\$ 3.000$ a year. who in addition to recording and announcing the results of all formal ballots assists the Speaker in determining the results of other forms of rotes of the House.

In addition to the foregoing employes on the floor of the House, clerks are assigned to keeping the pairs of members; that is to say, they record the agreements made between members of the minority and the majority, respectively, not to vote either on some particular measure, or not to rote generally on measures coming before the House during a specified time. It is usual for a member desiring to be absent to make an arrangement with a member of the opposite party who is also to be absent, so that the one vote will balance the other, and neither be recorded on any fomal ballot which may come up during the time of the absence. In order that there shall be no misunderstanding, such agreements are recorded with the Pair Clerk and all pairs of this nature are announced following each ballot.

Uncier a general rule all bills introduced into the House are 
simply filed at the Clerk's desk, where they are recorded in the journal and then forwarded to the File and Printing Clerks and by the latter put in print, each bill being given a serial number in accordance with the order in which it is introduced. According to the subject, reference is at once made to an appropriate committee of the House, all matters relating to reception, reference and distribution of bills being the subject of general rules, so that bills do not actually come up in the House until they are called up for legislative action. It is the duty of various subordinates to see that the bills so introduced are forwarded and reach the proper committee, and that upon the report of the same by committee they are placed on the appropriate calendars of the House for action in their turn, although spccial bills may be advanced for consideration upon request, either by unanimous consent, or upon the passing of a rule from the Committee on Rules. Bills have ostensibly three readings, but the first is the entirely formal one of reference to a committee, the second is the stage of consideration and amendment by the House, and the third is the reading of the completed bill. Certain classes of bills are, as previously stated, considered in Committee of the Whole, and have their reading for purposes of amendment in that committee. Upon the conclusion of the action of the House, the bill in its original printed form, together with the amendments attached thereto, is sent to the Enrolling Clerk, who inserts the amendments, has the bill reprinted, secures the certification of the Clerk of the House that it has duly passed that body, and forwards it to the Senate.

Upon the completion of the Senate's action and of the concurrent action of the House in the Senate amendments, if any, the bill, as a whole, is passed again to be engrossed, being the final stage preliminary to the approval of the act by the President. The stages in a bill are, therefore, introduction, first reading and reference to committee, report by committee, placing on the calendar of the House, second reading, passage to be enacted,

A very important staff of the employees of the House of passage to be engrossed, signature by the President. 
Representatives is made up by the clerks of committees, such clerkship being either annual at $\$ 2,000$ a year or for the session at $\$ 6.00$ a day. The appointments are made exclusively by the Chairman of the committees, and the clerks act as secretaries to the Chairmen besides performing their duties in connection with the clerical work of the committee. In the case of a committee clerk of long service, very much of the actual work relating to the legislation under the charge of that committee is performed by the clerk, who has become, by virtue of his service, an expert in that particular line, and whose conclusions are frequently coincided with by the members of the committee, many of whom know less about the details of the matter involved than the clerk. This is especially true with regard to the clerks of the committees on appropriations of the two houses and is also more apt to be true with regard to the Senate clerks, because of their longer service.

The compensation mentioned in the following list of officers, especially on the House side, for a considerable number of the officials is in consideration of work done while the Congress is actually in session. In addition, an extra month's pay, theoretically in lieu of mileage is generally roted in each session, so that the majority of annual empleyes under the House actually receive twenty-six months' pay for about ten months' work every two years, the other fourteen months being devoted to their own affairs and those of the members of whom they owe their employment. Some of the House employes are required by the nature of their duties to be present through most of the year and the Senate employees, besides their own work have to take part in the general work so that they have to be in Washington the greater part of the year. Those who work only during sessions scatter very shortly after the gavel drops for the last time, and the Capitol knows them no more until late in the next Fall. Under the circumstances the compensation paid is a liberal one.

The principal positions under the Congress and their salaries are as follows: 
IN THE SENATE.

Secretary of the Vice-President, four thousand dollars.

Chaplain of the Senate, one thousand dollars.

Secretary of the Senate, including compensation as disbursing officer of the contingent fund of the Senate, five thousand dollars, and for compensation as disbursing officer of salaries of Senators, three hundred and ninety-six dollars: assistant Secretary, five thousand dollars: chief clerk, three thousand two hundred and fifty dollars; financial clerk, four thousand dollars; ninute and journal clerk, and enrolling clerk, three thousand dollars each; principal clerk, two thousand seven hundred and fifty dollars; reading clerk, two thousand five hundred dollars; assistant financial clerk, two thousand four hundred dollars; librarian, two thousand two hundred and twenty dollars; assistant librarian, one thousand eight hundred dollars; assistant librarian, one thousand six hundred dollars; keeper of stationary, two thotsand two hundred and twenty dollars; superintendent of the document room, tinree thousand dollars; clerk of printing records, two thousand two hundred and twenty dollars; clerk to the Committee on Appropriations, four thousand dollars; clerk to the Committee on Finance, two thousand five hundred dollars; clerk to the Committee on Claims, two thousand two hundred and twenty dollars; clerk to the Committee on Commerce, two thousand two hundred and twenty dollars; clerk to the Committee on Pensions, two thousand two hundred and twenty dollars; clerk to the Committee on the Judiciary, two thousand two hundred and twenty dollars; clerk to the Committee on Military Affairs, two thousand two hundred and twenty dollars; clerk to the Committee on Post-Office and Post-Roads, two thousand two liundred and twenty dollars: clerk to the Committee on the District of Columbia, two thousand two hundred and twenty dollars; clerk to the Committee on Foreign Relations, two thousand two hundred and twenty dollars; clerk to the Committee on Engrossed Bills, two thousand two hundred and twenty dollars; clerk to the Joint Committee on the Library, two thousand two hundred and twenty dollars; 36 other clerks, at $\$ 2,220$ 
each and two at \$2, I00 each. Twenty clerks to committees, at one thousand eight hundred dollars each.

Sergeant-at-Arms and Doorkeeper, five thousand dollars; clerk to Sergeant-at-Arms, two thousand dollars; assistant doorkeeper, two thousand five hundred and ninety-two dollars; acting assistant doorkeeper, two thousand five hundred and ninety-two dollars; three messengers, acting as assistant doorkeepers, at one thousand eight hundred dollars eacli; forty-seven messengers, at one thousand four hundred and forty dollars each; besides assistant messengers, artificers ansd laborers ansd sixteen pages for the Senate Chamber, at the rate of two dollars and fifty cents per day each during the session.

Postmaster, two thousand two hundred and fifty dollars; besides assistants and four riding pages, at nine hundred and twelve dollars and fifty cents each.

For Superintendent of Folding Room, two thousand one hundred and sixty dollars; foreman in folding room, one thousand four hundred dollars; folders, at one thousand dollars each, and at eight hundred and forty dollars each. Thirty-two annual clerks to Senators who are not chairmen of committees, at one thonsand eight hundred dollars each.

Capitol Police.-Captain, one thousand six liundred dollars, and three lientenants, at one thousand two hundred dollars each, two special officers, at one thousand two hundred dollars each, sixty-seren privates, at one thonsand and fifty dollars each.

UNDER TIIE IIOUSE OF REPRESENTATIVES.

Secretary to the Speaker, four thousand dollars; clerk to the Speaker's table, three thousand six hundred dollars, and for preparing Digest of the Rules, one thousand dollars per annum; clerk to the Speaker, one thousand six hundred dollars: messenger to the Speaker, one thousand two hundred dollars: Chaplain of the House, one thousand two hundred dollars. Clerk of the House of Representatives, including compensation as disbursing officer of the contingent fund, five thousand dollars; Chief Clerk, four 
thousand dollars; Journal Clerk, four thousand dollars; two Reading Clerks, at three thousand six hundred dollars each; Tally Clerk, three thousand dollars; File Clerk, two thousand seven hundred and fifty dollars; Printing and Bill Clerk, Disbursing Clerk, and Enrolling Clerk, two thousand five hundred dollars each; Distributing Clerk, two thousand two hundred and fifty dollars; Docket Clerk, Resolution and Petition Clerk, Printing and Document Clerk, Index Clerk, Stationery Clerk, two thousand dollars each; Librarian, and Superintendent Clerk's Document Room, one thousand eight hundred dollars each. Clerk to the Committee on Ways and Means, three thousand dollars; Clerk to the Committee on Appropriations, four thousand dollars. Other clerks to committees at salaries ranging from $\$ \mathrm{I}, 400$ to $\$ 2,000$, chiefly at the latter figure, with assistant clerks from $\$ 1,200$ upwards. Ten clerks to committees at six dollars a day.

Sergeant-at-Arms, five thousand dollars; Deputy Sergeant-atArms, two thousand dollars; Cashier, three thousand dollars; Paying Teller, two thousand five hundred dollars; Bookkeeper, two thousand two hundred dollars; Deputy Sergeant-at-Arms in charge of pairs, one thousand six hundred dollars; one Clerk in charge of pairs, one thousand six hundred dollars; Inspector of Cabs and Other Vehicles, seven hundred and twenty dollars.

Doorkeeper, four thousand five hundred dollars; Assistant Doorkeeper, and Department Messenger, at two thousand dollars each; Superintendent of Reporters' Gallery, one thousand two hundred dollars; twenty-five messengers, at one thousand one hundred dollars each; Messenger to the Speaker's Table, one thousand dollars; fourteen messengers on the soldiers' roll, at one thousand two hundred dollars each; ten laborers, known as Cloakroom Men, two at seventy dollars per month each and eight at fifty dollars per month each; Female Attendant in Ladies' Retiring Room, seven hundred and twenty dollars; Superintendent of Folding Room, two thousand dollars; Foreman, one thousand five hundred dollars; thirty-two Folders, at eight hundred collars each; two Chief Pages, at nine hundred dollars each; forty-four Pages, during the session, including two Riding Pages, 
two Telephone Payes, Press-Gallery Page, and ten Pages for duty at the entrances to the Hall of the House, at two dollars and fifty cents per day each: Superintendent of Document Room, two thousand five hindred dollars; Assistant Superintendent of Document Room, one thousand eight hundred dollars.

Postmaster, three thousand dollars; Assistant Postmaster, $\$ 2,000$.

\section{SUPERINTENDENT OF THE CAPITOL.}

The care of the Capitol building of the United States is committed to an official selected by joint action of the Senate and House with the title of Superintendent of the Capitol. He has charge of all matters relating to the heating, lighting and ventilating of the Capitol with the exception of purchasing certain supplies. His jurisdiction extends to the halls and corridors of the building, and any fittings which are permanently affixed to the building itself and he has charge of the cleaning and repair of the portions of the building outside of the interior furnishings of the committee rooms. Responsibility for the furnishing and care of the Capitol is rather oddly divided, the Superintendent of the Capitol having those portions already mentioned; the Clerk of the House of Representatives and the Sergeant-at-Arms of the Senate supervise the furnishing of the committee rooms, and the Doorkeeper of the House of Representatives has charge of the janitor work within the House committee rooms, and is responsible for the furniture, fittings, typewriters, and so on, after they have been supplied by the office of the Clerk.

There is also a dual superintendency over the Superintendent of the building, in that there is a strict division of the Capitol space between the House and Senate. North of the Supreme Court corridor the Superintendent of the Capitol is the official of the Senate. When he crosses the line to the south he becomes an official of the House of Representatives. As separate appropriations are made for the care of the separate wings of the Capitol, he is in charge of two distinct plants, one furnishing light, heat and ventilation for the Senate and the other performing a 
similar service for the House. The Superintendent of the Capitol is also in charge of the two new buildings now under construction, one to the south and east of the Capitol, for office rooms for the House of Representatives, and the other to the north and east, for rooms for Senators who cannot be accommodated in the Senate wing; these buildings containing all modern improvements, and connected with the Capitol by subways, in which run miniature railways so that the new buildings are practically a part of the Capitol building without interfering with the architectural proportions of the present structure. It has been proposed, in accordance with the plan of the former architect of the Capitol, to extend the front of the Capitol materially, but in view of the newly constructed buildings, and the opposition to any change in the present appearance of the Capitol, it is doubtful if this extension will be carried out.

The policing of the Capitol and of the Capitol grounds is separated from the similar work in the city of Washington, and is done by a force of policemen appointed respectively by the Sergeant-at-Arms of the Senate and Sergeant-at-Arms of the House of Representatives, an equal division in employment of men being arranged, and the supervision of the board being undertaken iointly by the two chief officials named. The Capitol policemen maintain order in the building itself during the day and at night act as watchmen within the building and as a patrol in the grounds. The appointment being vested in the Sergeants-atArms, entirely, the tenure of office is wholly political and appointment is secured by members of either House in accordance with their influence as brought to bear upon the appointing offi$\therefore$ er.

THE LIBRARY OF CONGRESS.

From the nucleus of the Library, created by the collection of the books from Thomas Jefferson to the present collection of volumes which long ago reached the million mark is, in time, not much more than three-quarters of that of the history of the United States. In rapidity of growth the Library of Congress 
eclipses all other similar institutions and it promises to reach proportions in the near future equaled by no other library on the face of the globe. It was originally housed in a portion of the Capitol building, but before the crection of the present library butlding it had crowded every inch of available space, and would have crowded out eventually the House and Senate themselves had it been allowed to grow in its original location. The present magnificent building, erected at a cost of $\$ 6,000,000$, is, however, already too small for the accumulation of the ycars following its completion, and the clicf concern of those having it in charge is the Library's constant growth and its ultimate unwieldiness, which is inevitable, owing to the relations which it sustains to the publishing business of the country.

The Librarian of Congress, appointed by the President, receives a salary of $\$ 6,000$, and is also the supervising head of the copyright system of the United States. The right of exclusive publication of books, music, maps, etc., is reserved to the authors or producers and their assigns upon complying with the provisions of the copyright laws. One of such provisions is the deposit in the Library of Congress of two copies of the work of which the rights are reserved. The tremendous volume of publication made possible by the improvements in printing machinery and the cheapening of printing paper will alone, in time, tax the resources of any library provision that can be made. In addition, however, in order that the library may be well balanced and an effective working institution, it is necessary to accumulate by purchase, additional volumes, either of current foreign publications or of older editions and works relating to literature, art and history, so that the limit of the possible collections of the Congressional Library cannot be foreseen.

Large acquisitions are received from collectors, both by gift and demise, while historical manuscripts and other historical material have been turned over to the Library of Congress from the Exccutive departments, making that institution a working library where historical research can be carried on under better 
auspices and with better results than has ever been possible in the United States.

The details of the copyright office are carried on under direction of the Librarian by a register of copyrights, who receives $\$ 3,000$ per year. The regulations for securing copyrights are attached hereto. As a recent outgrowth of the copyright office and the Library, provision has been made for the assistance of libraries throughout the country in the preparation of their card indexes, by duplicating the original index cards of new publications which may be purchased for other libraries at a slight cost compared with the cost of making a separate index for each library.

The Library of Congress is primarily for the use of the members of the House and Senate, but the use of the Library building itself is cliiefly by others than Representatives and Senators. For the convenience of the latter a subway runs between the Library and the Capitol building, in which an endless chain conveys books to the Capitol, so that the members may have the volumes they desire in their committee-room without the necessity of going to the Library.

The reading room of the Library of Congress with its vast collection of indexed cards is at the disposal of the public and arrangements are made for the comfort and convenience of those who desire to do special work in the Library.

The building itself, which is unequaled in its interior decoration in the United States, is under the charge of the Superintendent, who has charge also of the grounds surrounding the building, and who has under his direction the necessary force of watchmen and attendants for the proper care of the building and its fixtures, not directly connected with the library work.

The staff of the Library of Congress necessarily includes for the purposes of an institution of this magnitude subordinate officials in considerable number and having a wide range of duty. The Reading Room is in charge of a Superintendent with a corps of assistants and attendants, who, besides keeping up the catalogues and attending to the wants of the visitors, arrange for 
sending out the books required by members of Congress and others who are on the list of those entitled to remove books from the Library. Any person who goes to the Library to read, upon consulting the catalogue and making out a list of the books desired, will be fumished at the desk or reading place which he or she has selected with the volumes asked for, which will be removed and replaced by the attendants. Within the walls of the Library building, and are not accessible to or seen by the visitors, are a considerable number of departments each occupied with the arrangement or care of the books of the Library. The division of bibliography is charged with the preparation of lists of books relating to given subjects, and may be perhaps regarded as a division of subject analysis and classification. The catalogue division provides the places of the books in the Library and makes u:p the various finding and reference catalogues. A separate division is devoted to public documents, and another division to the care and custody of manuscripts, including very difficult and delicate work in repairing and restoring old manuscripts which have become partially obliterated, and which are of historic value. In addition to these there are divisions devoted to the care and arrangement of maps and charts, of music, and of periodicals and prints. A special reading room for the blind, containing the books published in raised letters, is provided, and in connection with this, during the winter season arrangements are made for musical and literary entertainments to which blind people are invited, artists residing in or temporarily visitors to Washington volunteering for this service. There is also connected with the Library of Congress an extensive law library under the charge of a special custodian, and a separate division has charge of the desails relating to ordering books. Included within the activities of the Library are a complete printing office and book bindery, the latter of especial importance for the purpose of repairing the wear and tear of time and nse on the collections of the Library and in restoring volumes which are added to the collection by means of purchase or gift.

In addition to the main reading room, heretofore referred to, 
a periodical reading room is maintained where newspapers and magazines are displayed and are available to the general reader, and in the other display rooms of the Library exhibitions are made trom time to time of prints and other works of art, of rare books, and of charts and of various publications relative to the history of the United States, and to subjects that may be for the moment prominently in the public attention.

\section{GENERAL PROIISIONS RELATING TO COPYRIGHTS.}

The author, inventor, designer, or proprietor of any book, map, chart, dramatic or musical composition, engraving, cut, print, or pliotograph or negative thereof, or of a painting, drawing, chromo, statie, statuary, and of models or designs intended to be perfected as works of the fine arts, and the executors, administrators, or assigns of any such person shall, upon complying with the provisions of law, have the sole liberty of printing, reprinting, publishing, completing, copying, executing, finishing, and vending the same; and, in the case of a dramatic composition, of publicly performing or representing it, or causing it to be performed or represented by others; and aththors or their assigns shall have exclusive right to dramatize and translate any of their works for which copyright shall have becn obtained under the laws of the United States.

Copyrights are granted for the term of twenty-eight years from the time of recording the title thereof, in the manner hereinafter directed.

The author, inventor, or designer, if he be still living, or his widow or children, if he be dead, may have the same exclusive right continued for the further term of fourteen years, ipon recording the title of the work or description of the article so secured a sccond time, and complying with all other regulations in regard to original copyrights, within six months before the expiration of the first term.

Copyrights are assignable in law, by any instrument of writing, and such assignnent sliall be recorded in the office of the Librarian of Congress within sixty days after its execution; in default of which it shall be void as against any subsequent purchaser or mortgagee for a valuable consideration, withont notice.

No person is entitled to a copyright unless he shall, on or before the day of publication in this or any foreign country, deliver at the office of the Librarian of Congress, or deposit in the mail within the United States, addressed to the Librarian of Congress, at Washington, District of Columbia, a printed copy of the title of the book, map, chart, dramatic or musical composition. engraving, cut, print, photograph, or chromo, or a description of the painting, drawing, statue, statuary, or a model or design for a work 
of the fine arts for which he desires a copyright, nor unless he shall also, not later than the day of the publication thercof in this or any foreign country, deliver at the office of the Librarian of Congress, at Washingtun, District of Colmmbia, or deposit in the mail within the United States, addressed to the Librarian of Congress, Washington, District of Columbia, two copies of such copyright look, map, chart, dramatic or musical composition, engraving, chromo, cult, print, or photograph, or in case of a painting, drawing, statue, statuary, model, or design for a work of the fine arts, a photograph of the same.

The Librarian of Congress shall receive from the persons to whom the services designated are rendered, the following fees: I. For recording the title or description of any copyricht book or othes article, fifty cents. 2. For cvery copy under seal of such record actually given to the person claiming the copyright, or his assigns, fifty cents. 3. For recording and certifying any instrument of writing for the assignment of a copyright, one dollar. 4. For every copy of an assignment, che dollar. All fees so received are paid into the trcasury of the United States. The charge for recording the title or description of any article entered for copyright, the production of a person not a citizen or resident of the United States, shall be one dollar, to be paid as above into the treasury of the United States, to defray the cxpenses of lists of copyrightcel articles as hereinafter provided for.

No person can maintain an action for the infringement of his copyriglit unless he shall give notice thereof by inserting in the several copics of erery edition published, on the title-fage or the page immediatcly following, if it be a book; or if a map, chart, musical composition, print, cut, cngraring, photograph, painting, drawing. chromn, statue, statuary, or model or design intended to be perfected and completed as a worls of the fine arts, by inscribing upon some visible portion thercof, or of the substance on which the same shall be mounted, the following words, viz.: "Entered according to act of Congress, in the year - - by A. B., in the oftice of the Librarian of Congress, at Washington;" or, at his option the word "Cepyrialat," together with the year the copyright was entered, and the name of the party by whom it was taken ont: thus: "Copyrisht, I8-, hy A. B."

A penalty is providel for wrongful use of words denoting a conyright, and infringements may be enjerined and civil damages and penalties recovered.

TIIE BOTANIC GARDEN.

Another governmental activity directly under jurisdiction of the Congress is the Botanic Garden, which is located at the foot of the Capitol hill and directly west of one of the entrances to the Capitol grounds. It consists of an installation of green- 
houses, surrounded by a circular garden in which most of the ornamental and flowering plants introduced into the United States are maintained for the entertainment and instruction of visitors. The Botanic Garden also contains the Bartholdi fountain presented to the United States at the close of the Centennial Exposition. The garden is at the head of the system of parks which continue from the Capitol to the Potomac River west of the White House. A comprehensive plan has been proposed under which all of the private buildings on the south side of Pennsylvania Avenue are to be removed, the land acquired by the Government and used for parks and for the location of public buildings. To carry out this plan, it probably would be necessary to remove or abolish the Botanic Garden, which is now simply a show place of more or less interest to visitors, but entirely disconnected with any general plan of beautification of the city, and as a matter of fact a detriment to the proper treatment of the territory from the Capitol to the White House, which intelligently and discriminatingly laid ont would be a source of enjoyment and of pride to the thousands of annual excursionists to Washington. 


\section{CHAPTER VI.}

THE EXECUTIVE OFFICIALS-SECRETARIES AND ASSISTANT SECRETAR-

IES OF DEPARTMENTS.

It is sometimes intimated that the business of the various $d \epsilon-$ partments under the direction of subordinate officials and clerks of long experience in the government service, could be transacted even should the absences of cabinet officers from Washington be materially prolonged. Much has been said of this supposed ability of subordinates as compared with the smaller experience and alleged ignorance on the part of cabinet officers of the affairs of their departments. A slight experience in the departments in Washington, however, makes it plain that while something of the sort might be possible if departmental affairs went through a uniform course of routine, it is when new policies are to be marked out and old policies are to be applied that the advantage and value of the Secretary becomes apparent, and the old and experienced employe, very often in proportion to his age and experience hastens to take advantage of the decision, and ability to cope with new situations of the Secretary and the possession of these qualities enables the latter to listen with a tolerant smile to the suggestions that the department could get along just as well without its head. No other one man in the department sees every aspect of the departmental activities. Every subordinate official is concerned with the affairs under his immediate charge, and regards them of superior importance to the affairs of every other branch. No other man in the department can see and apply the outside view to the situations which daily arise in departmental matters and while a Secretary may be ignorant of the details of the work of the department when he first takes his portfolio, his difficulty later is not lack of information, but to discriminate between the kinds of information with which he is furnished by his 
subordinates. Nearly every Secretary of a department in addition to his administrative duties, is required to act in a semijudicial capacity constantly in deciding upon the merits of contentions, in adjusting conflicting claims between different officers, or divisions of the department under his charge. It is, therefore, of greater value that a Secretary should have a clear understanding and a lucid mind and a knowledge of the methods by which adjustments are made in the outside world, than that he should have an intimate knowledge of the affairs and workings of the department of which he has become the head.

The heads of departments of the Federal Government forming the cabinet or advisory body of the President, are interested, not alone in the affairs of their own departments, but in those of other departments of the Government, in the general policy of the administration and in the foreign affairs of the United States. While each question which may arise and which is submitted to the President is presented by the Secretary having immediate jurisdiction over the subject matter, questions of importance are made the subject of a general consultation and it is quite possible that the head of a department might be overruled by the rest of the cabinet officials on a matter relating to the affairs of his own denartment. The President is, of course, the arbitrator of the relations between the different departments, and his decision in a mooted question is final. As between the heads of the departments in matters arising outside of the cabinet meetings there is the most scrupulous care taken not to interfere in the affairs of one department by the head of another. This is especially the case with regard to the matter of recommendations for employment and promotions, and it is the rule that such suggestions shall not be made for the very obvious reasons that they would tend to embarrass the administration of the personnel of the departments. Frequent and friendly conferences are held between the different cabinet officers for the purpose of adjusting matters relative to business which affects two departments. These cordial official relations are also carried into social life, and in view of the necessarily constant intercourse, official and social, the cab- 
inet official who entertains an unfavorable opinion of another member of the cabinet is prevented from at least the expression of such opinion until one of them has terminated official life.

Assistant Secretaries of departments have no part in the calsinet duties when they are serving as Acting Secretarics during the absence of the heads of their departments. They do not attend cabinet meetings, nor go to the White House, unless upon special business or when summoned by the President. The duties of Assistant Secretaries vary in accordance with the manner of the organization of the different departments, but generally consist in an oversight and direction of one section of the departmental owrk in a semi-independeit manner, although at all times subject to the criticism of and perhaps reversal of action by the Secretary himself. An appeal lies at all times on the action of an Assistant Secretary to the Secretary, and it depends upon the latter whether he will support his Assistant without question or whether he will grant a review of the matter appealed.

One of the least agreeable of the duties of the Secretary is the signing of the department mail. In some departments a portion of this work is permanently assigned to the Assistant and in all the Assistant Secretaries sign as Acting Secretaries when the head of the department is absent, or when from pressure of other business he relegates the duty to his Assistant. The signing of the departmental mail gives control over the details of the work of the department, so that it frequently happens that the Assistant Secretary while acting in the capacity of Acting Secretary has an opportunity to change or modify the policies of the department in accorlance with his own personal views. Should such modifications later come to the attention of the Secretary, a further modification or return to the original policy may be directed, and it is in this direction that the greatest possibilities exist of friction between the head and the second in authority of a department.

In case both the Secretary and the senior Assistant Secretary are absent the duty of acting as Secretary devolves upon the Assistant Secretary, if any, next in line, otherwise upon the chief 
bureau officer or rather the officer in the department who is regarded as next in power and authority to the Assistant Secretary. As there would be frequent differences of opinion as to who was entitled to the honor, the matter has, in departments where there is but one Assistant Secretary, been placed in the hands of the President, who designates in writing the official who shall sign the departmental mail in case of the absence of both the Secretary and Assistant Secretary.

THE SECRETARY OF STATE.

The Secretary of State is appointed by the President by and with the advice and consent of the Senate, and receives a salary of $\$$ I2,000 per year. While he is the head of the smallest Executive Department, in point of numbers, counting those employed at Washington, he is the ranking member of the cabinet, both because of priority in the statuics of the State Department and because of his function of attesting the acts of the President. The office of the Secretary of State is frequently called the Premiership in the American system of govermment, for the reason that the offce has been filled many times by prominent memhers of the dominant political party, and sometimes by a man more powerful in the councils of the party than the President kimself. The office of Secretary of State has at times contained the real power in an Administration. At other times the occupant of the position has had less power than some other member of the cabinet ranking lower in the list. In the hands of a strong man the office has been made a most potential one, while when the contrary was the fact, it has become largely a clerkship for the administration of the forcign business of the United States. With respect to domestic affairs the Secretary of State has the custody of the seal of the United States, and there are deposited under his charge all laws, orders and resolutions of a concurrent nature passed by Congress, which have been signed by the President or which have become law after ten days, or which have been passed over the President's reto. The laws of the United States are enrolled by being printed 
on parchment, and in this form after having been signed by the presiding officers of the two Houses of Congress and the President, are deposited in the Burcau of Rolls of the State Department, and the Secretary of State furnishes an accurate copy of the law so enrolled to the public printer for the purpose of publication. When the Secretary of State is notified that an amendment to the Constitution of the United States has been adopted according to the provisions of the Constitution, he must cause a publication to be made of the amendment in the newspapers in the various States, at the same time certifying that the amendment has been adopted and that it has become a part of the Constitution of the United States. He also issues proclamations declaring the admission of new States into the Union.

The Executive directions are also promulgated by the Secretary of State in attesting the signature of the President to any Exccutive order or proclamation, the Secretary of State thus having the function of the official Secretary to the President in matters in which the President takes action as representing the general government. The other function of the Secretary of State consists in the administration under the direction of the Presicent of the foreign relations of the United States. These duties include the intercourse with the diplomatic and consular representatives sent by foreign governments to the United States, and the intercourse of the United States with foreign governments by means of Ambassadors or Ministers, and with foreign commcreial interests by means of various consular officers. Although it is not uncommon for the President himself to communicate direcily with the diplonatic representatives of foreign governments, the major portion of such intercourse is carried on by the Secretary of State, who receives upon a designated day in each week visits from the various accredited Ambassadors and Ministers.

The Secretary of State has the preparation of the various State papers addressed to forcign governments, either through their representatives or through the representatives of the United States abroad, and, as well, has ficquently taken prominent part in the preparation of domestic papers. Besides the diplomatic representa- 
tives, the Secretary of State administers the large force of consular officers who represent the United States in each of the larger foreign shipping points and manufacturing centres. All matters relating to the clains of American citizens against foreign countries, and of the citizens and subjects of foreign countries against the United States, are sent through the Department of State, and the Secretary of State acts in a large number of cases as mediator in securing the settlement of stich claims, as may be based on the principles of international law and are not within the jurisdiction of courts. American citizens travelling abroad are given passports under the direction of the Secretary of State, and foreign royalty visiting the United States in an official capacity are generally entertained by the Secretary of State or his representative.

In the early history of the United States the Secretary of State was made the vehicle of report to Congress of the number of impressments from the vessels of the Merchant Marine of the United States by the naval vessels of Great Britain. This requirement has been repealed, though not until $\mathbf{1} 897$, but a provision still exists requiring the Secretary of State to make a statement to Congress of the returns made by the customs collectors in the various seaports of the United States, of the number of seamen registered by them, a practice which was put into effect in order to prevent impressment, but which has now been long obsolete.

The Secretary of State must also report to Congress annually the changes in the commercial systems of other nations; a synopsis of the information forwarded by the diplomatic and consular officers cluring the year, together with various items of information with regard to the fees and salaries of officers. He was formerly charged with the duty of disseminating the reports of Consuls with regard to commercial conditions, but these are now transmitted to the Department of Commerce and Labor, by whom the publication is now carried on. The contingent fund of the State Department is the only fund appropriated under the Government of the United States, from which expenditures may be made without the requirement of public statement as to the objects for which the amounts were expended. While certain portions of this fund are to be re- 
ported upon, exception is made of such expenditures as may be settled upon the certificate of the President, which is taken as sufficient juriscliction of the expenditure made without special authorization of law or particular report as to the objects to which the amounts were applied.

The Secretary of State has, in a sense, under his direction the affairs of the Island of Porto Rico, since the government of that island is not under the general insular administration vested in the Insular Bureau of the War Department, but is a separate administrative entity, the officers of which are commissioned through the Secretary of State, and the reports of which are trasmitted through the Secretary of State to the President.

The Secretary of State formerly had under lis jurisdiction the registration and issuance of commissions to officers under the various executive departments, but this function has been by law vested in the appropriate departments and the former functions of the Secretary of State witl reference to the government of the territories are now vested in the Secretary of the Interior.

THE SECRETARY OF THE TREASURY.

The Secretary of the Treasury, appointed, as are the rest of the Cabinet officers, by and with the advice and consent of the Senate, and receiving a salary of $\$ 12,000$ per year, bears to the general government the character of a treasurer, with the well-known duties appertaining to sucl an office. He is the custodian of the public moneys and the paymaster for all expenditures. At the same time, the opcration of these functions las developed one of the most widereaching branches of the activities of the Exccutive. Roughly divided, the duties of the Secretary of the Treasury are under three heads. The collection of the revenue of the United States; the disbursement of all moneys for the expenses of the country; and the maintenance of the stability of the monetary system of the nation. The position of the Secretary of the Treasury is more independent than that of any other member of the Cabinet. While he is, of course, subject to the direction of the President, and to removal 
in case he does not agree with the Presidential policies, at the same time he is vested by law with great discretionary powers and with wide direction for report to Congress. $\mathrm{He}$ is supposed to be the ultimate authority in the United States on all questions of finance, and while the President may go counter to the views of the Secretary of the Treasury, he does so at the risk of making a mistake in non-compliance with what is assumed to be expert opinion.

The Secretary of the Treasury in his capacity as chief of the machinery for collecting the revenues of the country, is at the head of the Customs Service, which is charged with the collection of taxes imposed by law upon goods imported into the United States. He is also the head and the directive force of the Internal Revenue establishment, by which the taxes on intoxicating liquors and tobacco and certain other commodities produced and manufactured in the United States are collected. In addition to these two main sources of revenue, the Secretary of the Treasury receives and accounts for all the miscellaneous receipts of the United States, from taxes not above enumerated and from postal revenues, and from sales of public property, and from any other sources of income whatever. So minute is the provision for the collection of the public revenues by the Secretary of the Treasury, that without special authorization of law no proceeds of even the sale of waste material under another department can be retained and utilized by that Department, but the entire proceeds must be turned into the Treasury of the United States to be applied to the general funds of the United States. As chief disburser of the public funds, the Secretary of the Treasury, upon the authorization of expenditure by an appropriation by Congress, causes warrants to be issued placing at the disposal of the proper disbursing officer the amount and in the manner prescribed by Congress in making the appropriation. Under a Constitutional provision no money can be drawn from the Treasury except in accordance with an appropriation made by law, and the Secretary of the Treasury must keep within the exact terms of the law in issuing warrants and permitting expenditures. To secure this result, the machinery consists of a number of Auditors, each one of whom is charged with settling the accounts of a particular De- 
partment, while the general authority and procedure is regulated by a Comptroller whose decisions on a question relating to an appropriation has the effect of finality, subject to review by the Court of Claims within its jurisdiction. For the proper accounting of moneys received and clisbursed, the Sccretary of the Treasury is charged with the maintenance of a system of bookkecping, not only cnvering the transactions of his own department, but the transactions of other departments as well, and he has the power to prescribe the forms and methods to be used by the different departments in making subsidiary accounts.

All claims and demands made by or against the United States are finally settled under the direction of the Secretary of the Treasury, and directly, both as to lebts owed to and by the United States, when the intervention of the Court of Claims or other judicial authority is not found to be necessary.

The Secretary of the Treasury is authorized to compromise and settle claims against debtors of the United States when the full amounts of claims are not collectible, except those arising under the postal laws, and where poor debtor proceedings have been taken he is authorized to direct the discharge of the debtor except where fines or penalties have been imposed under the criminal law or where officials or agents of the United States have received moneys for the use of the United States, which cases must be referred to the President.

The Secretary of the Treasury has the power to construe the laws imposing customs duties, and is charged with carrying out such laws to the end of securing the largest amount of just dues to the United States. Having once made a ruling, the Secretary of the Treasury may not reverse or modify it adversely to the United States, except in concurrence with the Attorney General or in accordance with a competent judicial decision not appealed. In case the Secretary of the Treasury deems the decision of an inferior court to be a proper subject for appeal to the highest court of adjudication, he may ignore such decision until the final appeal has been acted upon.

The Secretary of the Treasury is charged with the duty of pre- 
paring plans for the improvement and management of the revenue, and under this provision must keep Congress advised of the state of the revenues and of an impending deficiency should one be in prospect. The several Departments make up annual estimates of the amounts which it is anticipated will be needed for expenses during the coming fiscal year which begins on July ist, and these estimates are transmitted to Congress together with the proper comparisons of appropriations and expenditures for the preceding year. This compilation, known as the book of estimates, is the basis on which Congress makes appropriations for the expenses of the government for the fiscal year following that in which the session of Congress is held. In addition the Secretary of the Treasury is required to make quarterly a statement of the whole receipts and expenditures of the quarter, and also to publish the weekly statement of the Treasurer of the United States, showing the amounts of money on hand and the outstanding drafts.

The Secretary of the Treasury is the head of the monetary system of the United States and is the direct regulating power of the volume of the currency. The various conditions relating to trade, both domestic and international, are held to require the action of the United States in increasing the volume of the circulating medium, when for any reason there is an extra demand for such means of commercial intercourse. The basis of such a circulating medium is the amount of gold in the possession of the country as a whole. Provision is made by which any person may deposit gold, either as bullion or as coin in the United States Treasury or in one of its Sub-Treasuries, receiving therefor the certificates of the United States. The gold thus deposited becomes the basis of interchange not only between the various sections of the United States, but between the United States and foreign countries as well, and the certificates of the government showing an equivalent amount actually held in reserve, are of equal value in facilitating the interchange of commodities with the payment of the coin itself.

In order to meet the expenses of the government at a time when the expenditures are largely in excess of the receipts, Congress has authorized from time to time the issue of bonds, being the promise 
of the United States to pay the amount stated on a given date, and, in the meantime, to pay a stipulated amount of interest for the use of the money. These bond issues are made by the Secretary of the Treasury, and the processes incidental to the interest paying and final redemption are carried out by his direction, under the provision of law which imposes upon him the care of the public credit. With the securities thus issued and guaranteed by the credit of the United States as a basis, a national banking system is maintained, by which individuals form themselves into stock companies, and having placed a sufficient amount of money, represented by bonds and similar securities under the control of the Secretary of the Treasury, are permitted to issue notes of hand which are secured by such deposits and which form a portion of the circulating medium of the country. This system of banks covering the United States, is controlled under the direction of the Secretary of the Treasury, who is responsible for their continued solvency, and who in case of loss to depositors must require the stockholders to reimburse the losers as provided by law. Another portion of the circulating medium, under the direction of the Secretary of the Treasury, consists of notes issued by the United States, based upon the stock of coin, either gold or silver, which has been paid into the Treasury of the United States, and which is there held as a reserve for the retirement of such notes, this form of circulating medium being a more convenient means of transacting business than the payment of the actual coin.

The whole of the circulating medium thus made available for the operations of commerce is regulated through the machinery of the office of the Comptroller of the Currency, and by virtue of accumulations in the Treasury of the United States, which under certain conditions of trade are laiger than at other times, can be made flexible through the action of the Secretary of the Treasury in placing considerable sums from such accumulations on deposit in the various national banks of the country, where the money so deposited becomes available for increasing the ready stock of cash, by which commercial transactions may be carried on. The effects of a tight money market may in this manner be ameliorated by the 
Secretary of the Treasury, when in his judgment it would be for the common good to increase the volume of the circulating medium readily accessible for commercial purposes. When, on the other hand, it appears that no general good would be subserved by such action on the part of the Secretary of the Treasury, and that the apparent stringency is speculative only, his refusal to participate in the situation has the tendency to bring about an earlier liquidation and of restricting the volume of trade within safe limits. A tremendous power is thus placed in the hands of the Secretary of the Treasury, upon whose conservatism and accurate appreciation of commercial conditions may sometimes depend the safety of the country from a serious financial panic.

The Secretary of the Treasury is also the directive officer in the production of the coins of the United States and of the paper currency certificates and securities utilized as mediums of exchange. The operations of the various mints of the United States assay offices are under his jurisdiction, as well as the Bureau of Engraving and Printing in Washington, where all paper money and securities, as well as postage stamps, are printed.

For the purpose of maintaining a proper supervision along the coast and for the purpose of preventing smuggling, he is at the head of a semi-military organization, the Revenue Cutter Service, which besides is also utilized for assisting the Merchant Marine in event of disaster, and for the enforcement of maritime laws. An allied service under the direction of the Secretary of the Treasury is the Public Health and Marine Hospital Service, which has charge of the hospitals for the care of the sailors of the Merchant Marine, and also of questions of public health which do not come under the jurisdiction of the States of the Union. For the purpose of preventing frauds in the Customs and Internal Revenue Services, and also for the protection of the coin and currency of the United States, the Secretary of the Treasury has under his jurisdiction a Secret Service Bureau, comprising special agents, whose duty it is to collect evidence for the prevention and punishment of such offenses. As an adjunct of the Revenue Cutter Service, the Secretary of the Treasury has under his jurisdiction a Life-Saving Service intended 
to ameliorate the disastrous effects of wrecks along the seacoast and on the shores of the internal waters of the United States.

In many of the cities and towns of the United States it has been found to be economical for the govermment to erect its own buildings rather than to pay rent to private persons. The erection of public buildings outside of Washington, and their care and maintenance after completion is under the jurisdiction of the Secretary of the Treasury, through the office of the Supervising Architect of the Treasury.

The Secretary of the Treasury is charged with the custody of the bonds oi the officials of the United States receiving and disbursing moneys, including those of the officials of the Congress having to do with fmancial matters, and is charged with the examination of bonds, their approval, and of carrying out the provisions of law relating to the review and renewal of bonds biennially.

\section{TIIE SECRETARY OF WAR.}

The Secretary of Var is appointed by the President by and with the advice and consent of the Senate, and receives a compensation of \$12,000 dollars a year. The Secretary of War ranks third in the order of precelence in the Cabinet and performs such duties as may be intrusted to him by the President. Relative to military affairs, he has the custody of the authority of the President as Commanderin-Chief of the Army, and may exercise such power in accordance with the President's direction to do all things relating to the command and control of the Army and its munitions, including the transportation of troops, their storcs, and all munitions of war. In the administration of this branch of the functions of the War Department the Secretary of War is assisted by the General Staff, which under the current statutory provisions practically administers all the details of military operation and administration. There are, however, some semi-independent bureau organizations still maintained, although subordinate to the administration of the General Staff, and which have important functions in the manufacture and purchase of arms, clothes, food and other provisions for the mainte- 
nance of the Army. Over the whole of this organization the Secretary of War maintains a directive and controlling power in matters of policy, even though many matters of detail carried out in his name are not originated by him. The strictly military side of the War Department is but a portion of its total activities. Since the inception of the system of seacoast and internal improvements in the first quarter of the nineteenth century, the War Department, through the Corps of Army Engineers, has supervised and directed the operations for the improvement of harbors on the seacoast, of facilities for navigation on the great lakes, and for the deepening of the channels of navigable rivers. All contracts for such work are carried out under the direction of the War Department, and in connection with this work the Secretary of War is given the custody of the Federal authority over certain portions of navigable waters, so that bridges or other obstructions to free navigation cannot be installed or maintained except under rules prescribed by the Secretary of War. When special authorization is given for a bridge or similar construction affecting the navigation of a body of water, it is generally coupled with the proviso of inspection by the War Department.

The Secretary of War is also charged with the duty of stating to Congress facts relating to the promotion of the general commerce of the country by improvements proposed, and has the duty not only of reporting on the practicability and cost of various river and harbor improvements, but must also report as to the practical value and benefit to commerce of the expenditure desired. As a part of the work on the great lakes and in connection with surveys made for the purpose of improving conditions of navigation, the War Department maintains a permanent survey force on the lakes, and publishes charts for the use of lake commerce. It is charged as well with the maintenance of improvements, and the operation by Federal employes of undertakings of this character requiring manipulation.

A large part of the force under the jurisdiction of the Secretary of War is engaged in maintaining the records of the soldiers in the wars of the United States for the use of the Pension Office, which is under the jurisdiction of the Interior Department. The various 
records covering the personnel of the Army in the wars of the United States from the Revolution up to the present day, have been collected in one section of the War Department, and progressive work has gone on for nearly twenty years in making a complete record of the service of every person who has served in the land forces of the United States at any time.

While the Signal Service of the War Department, which formerly carried on the forecasting work now being done by the Weather Bureau of the Asricultural Department, has been reduced to its original status of military communication only, very large additions have been made from time to time to the civilian activities of the War Department. Following the military occupation of the Philippines the civil administrative details were allowed to remain under the War Department on account of the sequence of events, so that the Secretary of War now has under his direction the details relating to the administration of the Philippine Islands, together with the general oversight of matters relating to the Panama Canal zone. The military forces maintained in the Philippine Islands are, of course, under the dircction of the Var Department proper, while the civil administration is carried on by a semi-independent bureau. All matters relating to the administration of the Philippine Islands reach the Executive through this bureau, which is the collating and dircctive force of the civil officers engaged in the work in the Philippines.

For want also of a morc appropriate location, and because a bureau of external administration had already been established in the War Department, the direction of the work of building the Panama Canal has been placed under the direction of the Secretary of War. Although the actual operations are being carried on by an independently organized commission, the commission as a whole, together with its work, is under the direction of the Secretary of War, who, under the direction of the President, prescribes the general policies to be carried out.

In addition to the functions above described, the Secretary of War has the custody and control of the parks in Washington, and their care and maintenance, and also such of the public buildings as are 
not otherwise provided with superintendents. He is also generally a member of commissions appointed by Congress for the purpose of locating staties in Washington and of determining upon their designs.

THE ATTORNEY GENERAL.

In addition to the other assistants provided for the President in the original enactments establishing a form of government, there was provision marle for an Attorney General, an official ranking fourth in the cabinet succession. The position was first established as that of a legal adviser to the President and heads of departments only, and not as the head of a department, and the creation of the Department of Justice was not effected until r870. The position, as with all other cabinet positions, has a compensation of $\$ 12,000$ a year, and the apointment is made by the President by and with the consent of the Senate. The Attorney General has the other prerogatives of cabinet officers, countersigns all commissions of officers under his direction and of the judiciary, and signs requisitions on the Treasury for moneys to be paid on accounts or to be advanced. He designates the disbursing officer of his department and performs other duties pertaining to an executive head. Formerly commissions equivalent to those under the Department of Justice and before its establishment were issued by the State Department, whose functions in this respect have been materially curtailed by legislation separating the commissioning of officers under the different departments from the State Department and placing the work of issuing and recording the commissions in the jurisdiction of the departments to which the appointments refer.

The Attorney General of the United States is primarily the adviser of the President on matters of law. He is required to give his advice and opinion on any matter of law on which the President may desire an opinion. He is, in practice, not only the source of the Presidential information on the construction of legislation and on the principles of law. but he is frequently required to pass upon the form and purport of proposed legislation recommended by the Execu- 
tive. While, strictly speaking, the Executive is supposed to be ignorant of legislation until it is presented to the President in accordance with the vote of the Congress, the views of the Executive are frequently presented either in the form of bills or of amendments to bills in an informal manner to the members of Congress engaged in the preparation of legislation.

The opinion of the Attorney General is also taken by the President on the form of laws presented for his signature, and for a general opinion on their character when a material change is made in reference. to judicial procedure, jurisdiction or other matters germane to the Department of Justice or affecting a general policy of the government. In addition the Attorney General is the representative of the President in matters relating to pardons; the records of such cases are kept under his direction and action is taken by the President largely on his recommendation.

The Attorney General is also the legal adviser of the heads of the Executive departments who may require his opinion on questions of law connected with the business of their departments, and especial provision is made for the submission of law questions by the IVar and Navy Departments, it being intended to make especial provision for furnishing these departments with facilities for determining points of civil law, nilitary law being regarded as more particularly appertaining to them. The Attorney General is charged with the duty of giving a written opinion on the validity of the title of any land to be purchased by or granted to the United States, but the preparation of the evidence is required of the department securing the opinion, and in cases of grants of building sites the grantors must furnish such evidence without cost to the government.

The Attorney General is not required to give a personal opinion in every case, as except on matters requiring a construction of the terms of the Constitution, he may delegate the question to a subordinate whose opinion, if approved by the Attorney General, becomes that of the Attorney General and has the effect of binding the acts of public officials unless set aside by the courts. Thus an opinion of the Attoney General becomes of equivalent weight in determining practice in the Executive departments with that of a 
court, unless the matter is carried into court and the opinion is not sustained.

Besides his duty of directing the rendering of opinions, the Attorney General is required to oversee the progress of suits at law in which the United States is a party in prosecuting or defending the same in behalf of the government. He has placed at his command not only a Solicitor General and Assistant Attorneys General, but a corps of District Attorneys and in special cases, may employ special counsel, fix their compensation and direct their conduct of cases. He generally appears himself in the Supreme Court of the United States in the argument of important causes, but may be represented by the Solicitor General, and in the lower courts is so represented by the subordinate counsel.

The Attorney General is required to supply legal assistance to the Executive Departments when required to conduct the examination of witnesses and nominates the special counsel provided by law to represent the interests of the United States before the Board of General Appraisers. He is given the direction and control of United States marshals and supervises the accounts of marshals, clerks, and other court officers, besides directing the district attorneys and receiving their reports. $\mathrm{He}$ also controls the supplies for the various United States courts and directs the disbursement of compensations of the court officials and settles expense accounts.

The Attorney General is authorized to publish annually a vo'ume of his opinions and those of his subordinates to an extent which he may regulate, selecting those deemed important, and must report to Congress annually on the business of his department, the amount expended to defray the expenses of the United States courts, the statistics of crime under the laws of the United States, the number of causes settled and pending during the year and the number of persons and their names retained as special attorneys during the year and the roster of assistant district attorneys.

There is no function exercised by the Attorney General with 
reference to the perficting or criticism of bills pending before Congress before they have been passed. Certain bills which relate to the affairs of the Department of Justice or to changes in the judicial proceedings of the Federal courts are scnt to the Attorney General for consideration and report by committees of Congress, but these references are no different in effect from the usual arlministrative references made by committees to heads of other departments of bills affecting departmental affairs, the members of Congress constituting themselves the juclges of the form of the bills, of their constitutionality and of their sufficiency to carry out the purpose proposed.

\section{THE POSTMASTER GENERAL.}

One of the first concerted acts of the colonics which later formed the United States of America was the establishment of a postal system. In the schemes of government which followed, provision was made for the continuance of the office of Postmaster General, and he was one of the original members of the Cabinct. The position of Postmaster General, which is filled by the President by and with the advice and consent of the Senate and carries a compensation of eight thousand dollars a year, is unique among the Cabinct positions by virtue of the provisions made for tenure of office by the incumbent. He can only be removed by and with the consent of the Scnate and mnless so remored retains his office during the term of the President appointing him and for one month after the term of the President has expired.

This provision is due to the character of the duties of the Postmaster General with respect to the postal revenues and expenditures, as well as to the importance of maintaining mail communication without interruption. The postal revenues, while turned into the general treasury, are maintained as a separate fund, added to, as deficiencies are apparent by appropriation from the general funds, and the control and direction of the financial matters relating to these funds may be the basis of the exceptional consideration given this Cabinet office 
as well as the consideration of the supposed expert service in maintaining intercommunication by the Postmaster General which would make it undesirable to make an immediate change in this position immediately on the incoming of a new President. The seeming independence given the Postmaster General has not, however, been marked by action on his part materially differentiating him from other Cabinet members, nor is the office regarded as especially desirable on account of the more secure tenure of office provided by law.

The United States statutes provide that every public highway, all railroads and canals and the navigable waterways of the United States shall be post roads. The Postmaster General is empowered to establish post routes over such post roads wherever in his judgment such routes are needed and to establish such post offices as he may deem to be essential for the purpose of carrying on mail communication. He is only restricted by the provision that county seats must have postal facilties, both in the matter of establishment of post routes communicating to them if they are not on post roads and in the establishment of post offices at such county seats and their maintenance, even thongh the business transacted is not sufficient to otherwise secure such establishment or maintenance. The Postmaster General may also temporarily establish a post route where a post road does not exist, but only until the following session of Congress, when if a post road is not established, the route must be discontinued. Generally speaking, the Postmaster General must do all things necessary for the efficient operation of the postal service, and his specific duties as established by law are as follows:

To establish and discontinue post offices.

To instruct all persons in the postal service with reference to their duties.

To decide on the forms of all official papers.

To prescribe the manner of keeping and stating accounts.

To enforce the prompt rendition of returns relative to accounts. 
To control, according to law, and subject to the settlement of the Auditor for the Post Office Department, all expenses incident to the service of the department.

To superintend the disposal of the moneys of the department.

To dircet the manner in which balances shall be paid over, issue warrants to cover money into the Treasury and to pay out the same.

To superintend generally the business of the department and to execute all laws relative to the postal service.

It will be seen that the Postmaster General has, with reference to the postal revenues, functions in a way equivalent to those of the Secretary of the Treasury with regard to the general funds. He is given a wide range of powers and prerogatives in exccuting these requirements, some of which are as follows: No other person may establish a post office under penalty of a fine of $\$ 500$. He has exclusive appointment of fourth class postmasters and keeps the records with respect to the appointments of other classes of postmaters by the President, advises all candidates of their appointments and all postmasters of their removal and must certify these changes to the Auditor for the Post Office Department. He has supervision over the bonds of postmasters and other postal officials in deciding upon their sufficiency. When a vacancy occurs in a post office which is not filled within 60 days, he must upon the demand of the bondsmen of the former postmaster designate a special agent to take charge of the office and release the bonds from liability.

The statutes provide that all letters posted in a post office half an hour before the time set for the departure of a mail must be included in that mail. but the Postmaster General has the option in special cases of extending this time to one hour.

The compensation of postmasters is determined on the busis of the business done by their respective offices, except in a few of the largest offices. The Postmaster General requires under the law that there shall be full statements filed as to the business done and the moneys received, and if he is satisfied that a false or erroneous return has been made he can set it aside 
and allow such compensation as he may deem to be reasonable. The rating of post offices under the various classes and grades established by Congress is made by the Postmaster General on the basis of business done, the new ratings to go into effect on the first day of the succeeding quarter to that in which the rerating is effected.

The Postmaster General is empowered to adjust any losses that may be sustained by postmasters in stamps or other postal supplies or in money from fires, burglaries or other causes not due to the official's neglect, provided the amount does not exceed \$ro,ooo. He must, however, report the action so taken to Congress. He may designate Presidential postmasters as disbursing officers and may direct expenditures from the postal revenues in any office, subject to the usual settlements. $\mathrm{He}$ may discontinue any post office or post route where the safety or efficiency of the service is threatened or impaired, subject to the restriction that he may not deprive a county seat of its postal facilities. He may also consider the cases of persons who may be indebted to the United States on account of the postal revenues either by reason of fines, penalties of other debts, and his approval gives effect to settlements which seem to be advantageous when the full amount of such indebtedness cannot be collected. He is also empowered to direct the discharge of any person in jail on a civil process growing out of indebtedness to the postal funds when the person is unable to pay the amount due.

Provision is made by statute giving the Postmaster General the right to make contracts for mail supplies running for four years; he is given the custody of the seal of the department, may provide substitutes for postal employees when subpoenaed as witnesses in United States courts; may designate a clerk to act as superintendent of the free delivery service. The Postmaster General also appoints or employs the superintendents and agents of the different branches of the postal service under general and special provisions in the statutes and supervises the operations of persons appointed for postal work under the 
classified service. He is required to have proper inventories made of the property of the government at the various places under his jurisdiction and may direct the sale of useless documents as waste papear. He must see that amounts receired from these sources as well as miscellaneous amounts received by postal employecs and officials from incidental sources, such as money found in the mails and incidental receipts are turned into the Treasury.

The Postmaster General may with the advice and consent of the President conclude postal treaties with foreign countries and must transmit a copy of each treaty so made to the Secretary of State and furnish copies to the public printer for publication. The Postmaster General makes contracts for carrying the mails, both in the United States and to foreign countries, and appoints agents and makes provision for the share of the United States in the ocean postal service. He is by law charged with the duty of making a rate for the transmission of government telegrams and annually estabiish a rate applicable to all government business, together with a schedule of uniform points for telegraph charges stating the rates to be charged at each in accordance with the distance charges which have been decided upon under agreement with the telegraph companies. He is required to keep the Auditor for the Post Office Department advised of all contracts and all orders of the department which may effect claims or accounts.

He appoints the Assistant Attorney General for the Post Office Department, and directs the operations of certain special Assistant Attorneys.

He may not be interested in any contract for carrying the mail and must report to Congress annually on the finances of the department.

THE SECRETARY OF THE NAVY.

The office of the Secretary of the Navy was established in I798, and the Secretary is appointed by the President, by and with the advice and consent of the Senate, and receives a compensation of $\$ 12,000$ per year. He ranks sixth in the order of precedence in the 
cabinet. He administers the powers of the President as Commander-in-Chief of the Navy of the United States, and under such delegated powers can do all things pertaining to such superior command. He is required by law to execute such orders as he shall receive from the President relative to the procurement of naval stores and materials and the construction, armament, equipment and employment of vessels of war. He also is empowered to furnish naval equipments and vessels to nautical and other schools and to the naval militia of the various States. He is required to distribute the work of his department among eight bureaus, which, with the office of the Judge Advocate General, constitute the department. The general duties of the Secretary of the Navy consist in the oversight and direction of the operations of the bureaus, to which the various functions of the department are assigned. A very large amount of the routine work of the department is thus carried on without the direct intervention of the Secretary, who, however, constitutes a court of appeal in case of difference between the various bureaus, or of complaint of a private person having business with any of the departmental subdivisions. In time of peace the Secretary of the Navy has the general direction of the movements of the vessels of the Navy, as well as of the assignment of officers and men. $\mathrm{He}$ is also the medium by which the needs of the department are presented to Congress, and controls within the limits of Congressional requirement and of the necessities of the service the expenditure for building new vessels, and for repairing those already built. The Secretary of the Navy also has jurisdiction over a portion of the colonial system of the United States, as the Island of Guam, as part of the former possessions of Spain, and that portion of the Samoan Islands alloted to the United States, are governed by naval officers who come under the jurisdiction of the Secretary of the Navy. The operations of the naval militia established by the several States is to a certain extent under the direction of the Secretary of the Navy, who disburses the appropriation made by Congress for the assistance of such bodies of militia, and who has the authority to loan such vessels and equipment as may be available for the purposes of the organizations. The 
Secretary of the Navy must make an annual report to Congress, of appropriations and expenditures with the probable demands upon such balances as may remain. A statement of all offers of contracts for supplies must be made annually, indicating those accepted and those rejected, and a statement of all acts relating to the sale of vessels and materials during the year.

In connection with the hydrographic office maintained in the Bureau of Equipment, the Secretary of the Navy is authorized to issue a Nautical Almanac, and maps, charts, and certain nautical books, to be sold at the price necessary to cover the cost of paper and binding, such sums to be placed to the credit of the Department for further publications. The Secretary of the Navy also has the supervision of the Naval Observatory in Washington, the head of which is a naval officer of the rank of Rear Admiral or Captain.

Certain amounts accruing to the United States from prize money have been devoted by Congress to the accumulation of a pension fund for persons who have served twenty years in the Navy, the surplus to be applicable to certain cases after ten years' service. The merits of pension applications are passed upon by the Secretary of the Navy and, when favorably, acted upon, are warranted by him to the Commissioner of Pensions for payments from the Navy pension fund in accordance with law.

TIIE SECRETARY OF THE INTERIOR.

The office of the Secretary of the Interior was not established until the Federal Government had been in operation for more than half a century. The original organization, consisting of the Secretaries of State, Treasury and War, the Attorney General and the Postmaster Gencral, had been added to shortly after the inauguration of the new government, by the separation of naval affairs from the War office, and in ${ }_{1} S_{49}$ the operations relating to the sale and other disposal of public lands, and the affairs relating to Indian Tribes, had reached such proportions as to warrant a collection of the independent Bureaus and Commissions variously pro- 
vided by law, into a separate Department which should have the administration of these and allied subjects, the new Department being termed the Department of the Interior, although all matters relating to interior affairs were not then, nor have they been since, exclusively under this Department.

The chief duties of the Secretary of the Interior are to supervise the operations of the laws relating to pensions, to public lands and surveys, and to Indian affairs:. Until the establishment of the Department of Commerce and Labor, the taking of the decennial census was under the direction of the Secretary of the Interior. The administration of the pension business of the government, including not only the pensions resulting from the Civil War, but the Revolutionary, Mexican and Spanish Wars as well, that is to say the pensions of the volunteers and of persons of the regular service, discharged on account of wounds or disabilities received while in the service, is carried on through the medium of a Commissioner of Pensions, who is directly subordinate to the Secretary of the Interior, and who pays all pensions and adjudicates all claims except those to be paid for the Navy Pension. In addition to the pensions payable in money, the Secretary of the Interior has the administration of the bounty lands provided by Congress as a reward for persons who had service in the wars of the United States previous to the Civil War. The Secretary of the Interior acts a final court of appeal in cases of pension applications which have been disallowed by the Pension Bureau. In connection with the practice before him, he has the power to regulate the professional conduct of persons practicing in the Department, and may for cause bar such persons from practicing in the Department.

The affairs relating to the public lands, are administered through a Commissioner of the General Land Office, and the work of assigning land to settlers under the homesstead law, of the sale of the lands, of the allottment of lands granted to soldiers and sailors, to states for educational purposes, and to railroads, comes under the direction of the Secretary of the Interior, who in a similar manner to his function with relation to pensions is a court of final appeal from acts of the Land Office adverse to the claimant or petitioner 
The Secretary of the Interior directs all matters relating to the management of Indian Affairs, and all matters arising from Indian relations. This portion of the duties of the Secretary of the Interior constitutes one of the most important of the functions of the Government. Throughout the history of the United States successive treaties have been made with the Indian tribes, most of them involving cessions of territory which then became a portion of the public lands of the United States, and were parcelled out among settlers in accordance with the general laws. The successive treaties contain various stipulations as to the payment of indemnities and bounties both in money and commoditics. As certain of these payments which were held for the benefit of the tribe as a whole, have accumulated, and with the increasing value of the lands still retained by the tribes, the administration of the affairs of the Indians has become one involving considerable amounts of money. Within the past few ycars there has been the development of a policy of segregating the tribal amounts, and of the payment to the individuals of their shares of the accumulations. The Secretary of the Interior until the completion of such scgregation, is the guardian of the financial affairs of all the Indians, and after the individuals of a tribe have sccured the lands due to them, the guardianship of the Sccretary of the Interior, is continued for a certain period in order that the Indians of the country may not be dispossessed of their holdings.

The Secretary of the Interior is also clarged with the direction of the activities of the general government in reference to education. Through a Commissioner of Education he provides for the instruction of Indian children and of the children of the natives of Alaska, and is also charged with the collection and dissemination of general information on educational topics, and the details of the educational systems maintaincd by the several States. No provision for a national educational system having been made in the Constitution of the United States, the Federal Government is without power to include such work among its activities and can only exercise an advisory function in this respect, except among those 
persons who are entirely under the control of the Federal Government.

Since 1870 the Patent Office of the United States, formerly an independent Bureau, has been under the direction of the Secretary of the Interior, and all matters relating to patents and trade marks, but not to copyrights, are under the jurisdiction of the head of this Department. All contracts which are made by the Secretary of War, the Secretary of the Navy, and the Secretary of the Interior, must be made the subject of a return to the Secretary of the Interior, where such returns are filed and indexed so that they may be readily examined by any person desiring access thereto, and copies of the returns are to be furnished to any person on demand, upon the payment of a fee for the work of copying, which fee is fixed by law.

In connection with the disposal of the public lands, there is the necessity for an accurate survey and definition of boundaries of lands to be allotted. In addition to this definition of the boundaries of lands to be allotted, elaborate surveys are carried on under the direction of the Commissioner of the General Land Office above referred to. Besides these surveys there is a necessity for a thorough inspection of public lands, in order to secure a proper classification of them in accordance with their value, especially in reference to the mineral lands and timber areas. This work is carried out under the direction of the Secretary of the Interior by the Geological Survey, which in addition to the functions noted, has the measurement of streams and the determination of the water supply of the United States, and especially the duty of reclaiming the arid lands of the United States by means of irrigation projects now being undertaken. A provision was made in 1902, by which a fund was created for the work of reclaiming arid lands, the amounts expended to be returned to the fund by the proceeds of the sales of the lands made valuable by irrigation. The administration of this fund and the prosecution of the successive works of reclamation, are under the direction of the Secretary of the Interior. From time to time Congress has set aside certain sections of the public lands containing remarkable natural curiosities, to be 
maintained perpetually as public parks. In addition, for the purpose of preventing the denudation of the lands of the IVest, with the consequent damage to subsidiary farming lands, forest reservations of large extent are made by Congress. All of these public reservations were originally assigned to the jurisdiction of the Secretary of the Interior, but recently the forest reservations and the work of the Forest Service have been transferred to the Department of Agriculture, the Secretary of the Interior still retaining the supervision of the other national parks.

For many years the United States has becn appropriating to the several States lands and funds, for the assistance of mechanical and agricultural colleges. The appropriations are disbursed by the Secretary of the Interior, who by virtue of such disbursement, has a general supervision over the operations of the colleges, to see that they come within the terms of the laws granting assistance. The Secretary of the Interior also has the supervision of the Government Hospital for the Insane in the District of Columbia, and certain other asylums and hospitals maintained in part or wholly by appropriations made by Congress. The Secretary of the Interior was formerly charged with the distribution of public documents, but this function has been superseded by the appointment of a Superintendent of Public Documents under the direction of the Public Printer. An important class of public business coming under the direction of the Secretary of the Interior, is the consideration Indian depredation claims. Under ccrtain conditions in which the United States was held to be responsible for the destruction of property by the members of Indian tribes who were under treaty with United States, the Government has paid considerable amounts to persons suffering losses from this cause. The Secretary of the Interior was formerly charged with making findings on such claims but this function has recently been conferred upon the Court of Clains leaving to the Secretary of the Interior the details of preparing the preliminaries and preparing the evidence upon the part of the United States, the cases being conducted by the Department of Justice.

The Official Register of the United States, containing the names 
of every federal officer and employee, together with his compensation and information as to his appointment, was formerly compiled by the Chief Clerk of the Interior Department under the direction of the Secretary of the Interior, but is now transferred to the Census Bureau of the Department of Commerce and Labor. The Secretary of the Interior signs requisitions for his Department for the money necessary for advances in anticipation of necessary payments, or for the settlement of accounts subject to adjustment and control by the officers of the Treasury, and, of course, within the limits of the appropriations made by Congress, and countersigns and records commissions of his subordinates; is required to make annual reports to Congress, showing the amounts of all claims for damages on account of Indian depredations, and also an itemized statement of the expenditures and payments on the account of the Indian affairs, for each fiscal year, and must also make detailed reports as to the expenditures for educational purposes. He is also required to furnish copies of papers relating to titles of lands granted or patented by the United States, and may sell the surplus of the various plats and maps printed in the course of the work of the General Land Office, at stated prices.

The Secretary of the Interior has the authority to put into effect such rules as may be necessary to prohibit the sale of arms and ammunition in any district or county within the jurisdiction of the United States occupied by uncivilized or hostile Indians.

The Secretary of the Interior has also, under the direction of the President, general supervision over the affairs of the territorial governments. The appointments of governors and other territorial officers are recorded in the Interior Department, commissions issued and countersigned by the Secretary, this function having been transferred from the State Department in 1873 .

THE SECRETARY OF AGRICULTURE.

While it is not strictly true that the paternal operations of the Federal government began with the activities of the Department of Agriculture, a wider construction of the Constitution than that 
which prevailed during the first half century of the history of the country is necessary to account for the many Federal activities that have been carried on under this Department. The first steps in paternalism were undoubtedly taken in the extension of the system of internal improvements in the early days of the development of the West, but the chicf examples of clirect legislation and appropriation for a special interest on the plea of the general welfare have been for the benefit of the Department whose Secretary was for years the junior member of the Cabinet. The Secretary of Agriculture is the successor and succeeds to the duties of a Commissioner of Agriculture, which position was authorized in 1862. The Department of Agriculture as organized under a Commissioner was established as an Exccutive Department in 1889 and the new Secretary was charged with the duties of the former Commissioner and other duties have been added from time to time until they include something with relation to nearly every matter of food supply.

The original provision of the duties of the Secretary was as follows: "to acquire and to diffuse among the people of the United States useful information on subjects connected with agriculture in the most general and comprehensive sense of that word, and to procure, propagate and distribute among the people new and valuable seeds and plants." The Secretary of Agriculture is appointed by the President by and with the advice and consent of the Senate, and receives a salary of twelve thousaid dollars a year. $\mathrm{He}$ is eighth in rank and has the usual powers and prerogatives besides those specifically granted by law, having jurisdiction over the departmental seal, commissions of officials, buildings, property, etc.

Under subseqent acts of Congress to that before cited, the Secretary of Agriculture has been given jurisdiction over entomological investigations as the result of the inquiry into the grasshopper devastations; over the preservation of game birds and their restoration in districts where they had become extinct; is authorized to make monthly reports on the crop conditions of the country; to investigate and report upon the condition of the domestic animals of the United States; is authorized to tests seeds offered for sale in the open market and to report on adulterations 
and substitutions; to provide a large quantity of seeds, bulbs and cuttings for distribution to the constituents of Senators and Representatives in Congress; to carry on the inspection and regulation of oleomargarine and dairy products and to inspect meat destined for export, and for the interstate trade and to enforce the pure food law.

In the administration of his department he is given a wider discretion in the matter of employment of services than any of the other Secretaries. While most of the Departments are restricted to employment of persons specifically appropriated for, and in only a few of them are lump sum appropriations made available for personal services, the Secretary of Agriculture has under his control a number of lump sum appropriations from which he may employ scientists and field workers, although there is a tendency to restrict this privilege and provide employment only for persons actually estimated and appropriated for.

Special provision is made for the publication of the annual report of the Secretary of Agriculture which is made up to a considerable degree of the results of the investigations of the Department during the year covered. A large edition, frequently handsomely illustrated is authorized by Congress, the copies of which are distributed by allotment to members and in turn sent out to their constituents.

Under the personal supervision of the Secretary in addition to the administration of the general departmental affairs are matters relating to the agricultural experiment stations. These stations are supported by annual appropriations by Congress from the proceeds of the sale of public lands and the Secretary of Agriculture is charged with general advisory duties relating to them, with directing the course of their experiments within the lines laid down by statute law and with compiling and tabulating the results of their experiments and observations and with disseminating useful information derived from this source. He has also control of the quarantine laws relating to the importation and transportation of diseased cattle and is charged with the duty of making rules and developing means for preventing infection and for stamping out disease. When made possible by the laws of any state, the Secretary 
of Agriculture is authorized by the general government to carry on operations within that state for the extinction of a contagious disease and is charged by law with the responsibility of preventing by quarantine rules the transportation of animals affected by the epidemic into neighboring states. The Secretary of Agriculture has also under his personal charge the execution of the laws which prohibit the importation of noxious animals whose appearance and increase in the United States would be prejudical to agriculture and to native birds and animals of value. In addition he cxecutes the law prohibiting the interstate transportation of birds and animals killed in violation of state game laws.

THE SECRETARY OF THE DEPARTMENT OF COMMERCE AND LABOR.

The theory of the establishment of the Agricultural Department, devoted to the interests of a particular class of the citizens of the United States was that agriculture was the basis of all national prosperity and that the legislation which provided for the dissemination of agricultural knowledge was in fact general legislation in an indirect form, even thougl a particular interest might be the direct beneficiary. Other interests have regarded agriculture as especially favored and agitation has resulted first in a IJepartment of Labor, under the direction of a commissioner and devoted to the interests of wage workers, and then in an Executive department having duties in relation to many different interests of manufacturing and commcrce, and with the title of the Department of Commerce and Labor.

The purpose of the constitution of the new department as stated by the law under which it was formed in 1903 is to "foster, promote and develop foreign and domestic commercc, mining, manufacturing and slipping industries, labor interests and transportation facilities."

Under this law the Secertary of the Department of Commerce and Labor is provided at a salary of $\$ 12,000$ a year, to be appointed by the President by and with the advice and consent of the Senate, and having the usual prerogatives of a secretary, including the establishment and custody of a seal, control of the property and records of the department and such other powers 
as may be expressed, implied or customary with reference to a Secretary. The body of law referring to the department consists mainly of the original act under which the powers exercised by other Secretaries and by Commissioners were transferred to the Secretary of the new department. One specific authority is given which is rather novel, namely, the right to employ special agents at a compensation of ten dollars a day and travelling expenses to make reports on trade conditions at home and abroad on general and roving commission.

Specific duties of the Secretary of Commerce and Labor are largely such as he himself may care to undertake. Various bureaus were transferred from other departments of the Government, especially from the Treasury Department, and various independent offices were consolidated under the direction of the new Secretary upon the constitution of the Department of Commerce and Labor in 1903. Among the bureaus thus laid under his direction are so many of different functions and classes of work that any Secretary of this department may, according to his particular and personal taste, take up one of several lines. Thus, for example, the Secretary may personally direct the work of the Bureau of Corporations in investigating and laying the foundation for a system of regulating the corporations doing interstate business under charters from the individual State. Or he may pursue the line which was laid down as that on which the department was to progress when its institution was adrocated in Congress; namely, the co-ordination of the products of the American manufacturers with the demands of the market of the world. As it was stated that the position was intended, the Secretary of Commerce and Labor was to be the great promoter of the introduction of American manufactures and products in competition with those of other nations. If, homever, neither of these appeal to the taste of the Secretary of Commerce and Labor and he is not fond of the opportunities of excursions which the vessels of the light-house service afford, he may become the chief directive activity in relation to immigration affairs, and may constitute himself either the 
bulwark of the counti"y against an unlue influx of foreign population or may ameliorate the severity of the immigration laws so that large numbers of ambitious residents of forcign lands may secure the advantage of homes within the Lnited States. In all of these matters he may suit his own tastes and convenience for the reason that his especial interest in one of the branches of the activities of the department will not in any wise interfere with the successful conduct of the others. Each of the subdivisions of the Department of Commerce and I abor has its own responsible experieneed and expert head who is able to continue the successful operation of the burean under his charge with a minimum of attention from the Secretary. It is even quite possible that the head of such a burcau would welcome the interest and activity of the Secretary in regard to the affairs of another burean than his own, provided always such lack of interest was not permitted to have its effect in diminishing the Secretary's ammal estimates for appropriations.

For the foregoing reasons, the activities of the Department of Conmerce and Labor may be approximately described under the heading of the departmental organization than in connection with the duties of the Secretary. There is, however, one aspect of the work of the department which is not under a particular burcau, but comes dircetly under the Secretary himsclf. It is the care of the Alaskan salmon and seal fisheries. In order to prevent serious waste and the resulting exhanstion of these two sources of national wealth stringent laws have been made by Congress including the provision for licensing certain persons to take the product of these fisheries. The Secretary of Commerce and Labor, through special agents, whose dutics are elsewhere described, exercises direct control over these matters and is charged with the responsibility of seeing that these fisheries are properly conserved for the benefit of future generations. The Secretary of Commerce and Labor is charged with the supervision of the various reports and compilations required by law from the bureaus under his direction, and may also direct such special reports and investigations as he may deem to 
be desirable or which may be directed by the President or the Congress.

ASSISTANT SECRETARIES OF DEPARTMENTS.

At the point of the Assistant Secretaryships of the departments, the phenomena of the personai equation, the influence of the individual in administration begins and continues through the list of directive officials. The grand divisions of the governmental functions have their limits fixed by the logical scope of each or by the direct action of the legislative branch of the government, but within these divisions, the many forms of activity are divided and directed by means which nowhere appear in the statute books and by no means completely and comprehensively in the departmental regulations. Personal initiative, time of service, tradition, chance, a dozen causes dictate the location of the details of the business of the department in certain hands and as circumstances change, so frequent and considerable changes are made in the relative importance of the various directive positions.

It would be almost impossible to state closely the nature of the power and functions of the Assistant Secretaries of the departments in an impersonal way because they depend so largely upon the personality of the individual occupying the position for the time being. While in most of the departments certain sections of the work are assigned to the Assistant Secretary or Secretarics, the line of demarcation is constantly changing and the fact that in the absence of the Secretary the Assistant acts as the Secretary, has a tendency to cause a gradual accumulation of details toward the functionary who is the longer in office of the two so alternating or who is more frequently called upon to take the formal action on details that constitute the chief portion of the secretarial routine. In other words, a strong man of close application in the position of Assistant Secretary tends to gather about him a considerable portion of the actual work and power of the department, while the contrary is true of the more or less transient incumbent who does not seek 
additional responsibility to that directly imposed upon him by regulation or by direcion of the Secretary.

The general description of the duties of the Assistant Secretary in any department would be that he is to take such portion of the work of the Secretary as the latter may deem to be for his best assistance and in the absence of the Secretary to act as the head of the department. There is, in the practical working, a considerable difference, however, in the relative position of an Assistant Secretary in the respective departments, and, while in some instances, the Assistant is in close touch with general departmental details, in others the Assistant is practically the head of a more or less detached section without close affiliation with the department itself.

There are no perquisites and privileges attached to the positions of assistant secretary similar and subordinate to those which attach to the position of the head of the department. While the latter is allowed a carriage and its appurtenances, this perquisite is specifically denied to assistants through a provision of law which forbids the use of public money for such purpose, and the prohibition is strengthened by a requirement that all public vehicles of every kind except the Secretaries' carriages, shall be marked with the name of the department to which they are attached, with the idea of preventing all but absolutely official use of the government's horses and vehicles.

The assistants to the Postmaster General and the Solicitor Gencral and other assistants in the Department of Justice, have similar duties to those of the titular Assistant Secretaries, but as they are more closely connected with the details of sulb-organization their duties will be described under the heads of the respective departments.

\section{TIIE ASSISTANT SECRETARY OF STATE.}

Althongh there are three Assistant Secreiaries in the Statc Department, the ranking officer of the three is not styled the First Assistant, but is the Assistant Secretary solely, the distinction marking the subsidiary nature of the Second and Third 
Assistants, apparently, as it is strictly insisted upon. The Assistant Secretary of State is regarded as a sccondary Secretary of State, and the actual Assistant of the head of the department. The other Assistant Secretaries, though they may on occasion become Acting Secretaries, are not regarded as having the powers and discretion with relation to the more important matters of the department that is vested in the Assistant. The Assistant Secretary of State is appointed by the President, by and with the advice and consent of the Senate and receives a compensation of $\$ 4,500$ a year. In the absence of the Secretary he assumes the full powers of the office of Secretary of State and at other times receives by delegation the administration of such of the Secretary's duties as may be allotted to him. A portion of his duties relate to the consular services and he has charge of the details of selection of consular appointees, of change in their assignments and of questions relating to their discipline and the conduct of their offices. He also has similar functions with relation to transfers and discipline in the diplomatic branch of the service, subject, however, to the closer scrutiny of the Secretary himself than in the case of the consular service. As it is not frequent that both the Secretary and Assistant Secretary are away from the department at the same time, a considerable share of the determination of important matters comes to the Assistant Secretary, and if he should be absent from Washington and one of the Secretaries of lower rank should be acting, the definition of policy on any important matter in the absence of the Secretary of State from easy communication, would be referred to him. Many matters relating to the diplomatic negotiations are referred to him also, and he takes an active part in the foreign negotiations.

In addition to the above duties, the Assistant Secretary of State has also general supervision over the various offices and bureaus of the department under the direction of the Secretary since both of the other Assistant Secretaries have specific duties assigned to them which have no bearing on the miscellaneous work of the department, so that the Assistant is, in reality, the 
executive officer and right hand man of the Secretary of State.

All of the Assistant Secretaries of the State Department receive equal salarics, $\$ 4,500$ per year, and are appointed by the President by and with the advice and consent of the Senate and have the same legal powers as the Secretary when called upon to act as the head of the department.

\section{SECOND ASSISTANT SECRETARY OF STATE.}

The truth of the general statement that the character of the duties assigned to Assistant Secretaries is largely dependent on personality, on length of service and upon particular experience, is especially borne out by the character of the duties of the Second Assistant Secretary of State. The present incumbent. who, subject to human limitations, is a permanent occupant of the office, stands in the position of diplomatic expert, though no provision has been made by law for such an official and none of such duties have been prescribed. Those functions of the Secretary of State which relate to diplomatic correspondence, to drafting of treaties, conventions and other similar State papers, have, because of the entire familiarity of the present incumbent with the whole of the diplomatic traditions and precedents of the United States come to be cntrusted to him for preparation, subject, of course, to the direction of the Secretary as to policy.

TIIIRD ASSISTANT SECRETARY OF STATE.

The Third Assistant Secretary of State has specific duties assigned him in the direction of the details of the Consular Service outside of the appointment and disciplinary details. These duties include the immediate supervision of the consular correspondence and of correspondence relating to consular affairs, and the direction of the auditing system of consular accounts and of such other accounts as may be submitted to the Third Assistant Secretary by the Secretary of State The annual cstimates for the expenses of the Consular Service are made up by the Third Assistant and he is also empowered to sign the rouchers for the expenditures of the department generally. 


\section{ASSISTANT SECRETARIES OF THE TREASURY.}

There are three Assistant Secretaries of the Treasury who are, so far as statutory provision goes, on an equality as to their importance, their compensation and the nature of their duties. No distinction was made in the law which established the positions, except that at first two Assistant Secretaries were provided for and that a third was added to the number at a later date. The method of appointment is identical, by the President by and with the consent of the Senate, and the compensation of the office in each case is the same, $\$_{4}, 500$ a year. The duties of Assistant Secretaries of the Executive Departments are in most cases defined in very general terms, namely, that they shall be such as the Secretary of the Department or the law may prescribe. Few or no provisions are made as to specific duties. The statute is more explicit as to the Assistant Secretaries of the Treasury Department, who are required to examine the letters, contracts, and warrants which have been prepared for the signature of the Secretary of the Treasury. The official mail of the latter is voluminous and this provision is intended to obviate the necessity on his part of doing more than affixing the official signature to a large portion of the mail. A certain amount of relief is, however, afforded the Secretary in a further provision that he may designate one of the Assistant Secretaries to sign warrants for the payment of money into and from the Treasury of the United States and it is provided that such a signature shall have the same validity as that of the Secretary himself.

While no order of rank is assigned by law to the several Assistant Secretaries of the Treasury Department, the Assistant Secretary who is charged with the details relating to the currency is understood to be the ranking official, followed by the Assistant Secretary having charge of the customs matters, while the Third Assistant in point of rank has charge of the miscellaneous bureaus. The assignment of the various branches of the departmental activities is made entirely by the Secretary, but the arrangement herein given is generally followed with 
minor re-arrangements, and while an incoming Assistant Secretary would generally take up the work of his predecessor, the superior political importance of the newcomer might lead to his installation in a higher relative rank, or the superior experience of a lower Assistant might lead to his promotion to the higher place and a new appointment to the lower position.

The arrangement of dutics at the present writing, is as follows, it being understood that the classification before-mentioned is followed: In the first division are included matters relating to the preparation and issue of currency, including those under the office of the Dircctor of the Mint and the Burcau of Engraving and Printing. As an incident to this, the Secret Service division comes under the control of this Assistant Secretary. The issue of currency and the administration of the loans to the United States are combined under one division of the Treasury Department and come under this Assistant, who is also charged with the direction of the general bookkeeping of the department and of the issue of warrants through the Division of Bookkeeping and Warrants, this being incidental to the jurisdiction of the administration of public moneys by the appropriate division, which is also included under the authority of this Assistant. In general, this Assistant has to do with the administration of the functions of the Treasury Department which are germane to the science of banking or of the conservation and payment of money as opposed to the collection of income. In addition he is charged with the administration of the personncl of the department in the District of Columbia, that is, the service of the department proper, and has charge of all official communications rclating to or making appointment, removals, or clianges in compensation of persons scrving the department within the limits of the capital, and has charge of the supplies of the department in the line of printing and stationery.

The second division comprises one of the two chief methods of securing an income for the support of the government of the United States. All matters relating to the customs service are placed under the direction of this Assistant and he has the ad- 
ministration of the laws passed primarily for the discouragement of the importation of foreign articles which would compete with articles of domestic manufacture, but which secondarily provide for raising a large proportion of the sum necessary to meet the expenses of the government from a tax on imported articles. In the administration of the customs laws, this Assistant has jurisdiction over the Division of Customs and the Division of Special Agents. The former relates to the details of collection of import duties, including the direction of the large number of collection officials at the various ports and on the frontiers, and also to the determination of the many and vexed questions which are constantly arising as to the amounts due on various classes of imports. While there is machinery devised by law for the equitable determination of customs disputes, a very large class of decisions come up for the decision of this Assistant who finds the administration of the customs an ample occupation without arlditional charges.

To the other Assistant Secretary is assigned a diversity of interests having to do with the immediate department and also with the collection of a part of the national income. For the department he has supervision of the office of the Chief Clerk and Superintendent who has charge of the personnel of the care takers of not only the department building in Washington, but of the Federal buildings throughout the United States. For the department also he has the supervision of the Division of Mail and Files which attends to the outgoing and incoming mail of the department and makes the proper record and distribution. While directing the administration of buildings after completion he has also direction through the office of the Supervising Architect of the plans and erection of public buildings and of the work of keeping them in repair. He also supervises a portion of the income-collection in having direction of the Office of Internal Revenue and has charge of the military side of the Treasury Department, which consists of the Revenue Cutter Service, which is the force employed for the suppression of smuggling and for the orderly keeping of the immediate waters of the United States and 
which has as co-ordinate services the Public Health and Marine Hospital Service and the Life Saving Service, both of the latter being designed for the preservation of the welfare and life of the personnel of the mercliant marine.

Specific directions as to the method of executing their duties are given by the Secretary to the Assistants under the following requirements which are here summarized. All official communications on subjects relating to the divisions assigned to them except those relating to personnel outside of Washington and to modification of methorls and those which are required by the law to be signed by the Secretary are to be signed by the Assistants. This means that they are to transact business relating to the divisions under them at their own discretion with the exceptions named. Natters relating to personnel and to modifications of methods of conducting business must be forwarded to the Secretary for signature, but no other person can prepare letters for the Secretary's signature on matiers relating to personnel coming within an Assistant Secretary's jurisdiction without referring such letters to the Assistant or forwarding them through him to the Secretary. Matters for which the Secretary's signature is required by law must by statute be examined by one of the Assistant Secretaries, but no distinct delegation of power to sign warrants appears in the current circular except that the Assistant Secretaries are given power to sign orclers and approve vouchers for purchases payable from appropriations for contingent expenses. The only restriction as to the control of general business, however, not otherwise specifically provided for, is contained in the reservation by the Secretary of the prerogative of signing all letters adilessed to the President, the Vice-President, the members of the Cabinet and to the Congress or its committees, as well as all regulations and general instructions affecting the public service.

THE ASSISTANT SECRETARY OF WAR.

Possibly no Assistant Secretary in Washington has assigned to him as his regular duties affairs of a similar magnitude to 
those assigned to the Assistant Secretary of War. The considerable and diverse interests which have in the past few years been added to the purely military functions of the War Department have caused a re-division of the duties of the Secretary of War in which the purely military details have largely been assigned to the Chief of the General Staff, while to the Assistant Secretary of War has been assigned those matters in which the civil and military aspects are united and which do not relate solely to departmental matters.

Under the division of the duties of the office of the Secretary of VVar, while final action with regard to policies and which would change precedents and established regulations is reserved by the Secretary of War, the Assistant Secretary is given jurisdiction over the following matters:

Details relating to the recruiting of enlisted men and to their discharge from the service and those relating to courts-martial and discipline of enlisted men, to commutation of quarters and of rations and of matters generally relating to the relations of enlisted men with the department, including clemency for military prisoners and medals of honor and certificates of merit for service.

Matters relating to boards of survey convened under the regulations for the disposition of government property come under the jurisdiction of the Assistant Secretary, who also directs the sales of quartermasters' stores and articles of subsistence to civilians, such sales being authorized by law to employees of the department and other governmental officials at a distance from sources of usual commercial supply.

The large and growing details of the establishment, maintenance and increase of the different military posts of the army in the United States and island possessions come under the Assistant Secretary, who has the regulation of the expenditure for such purposes, and has also the authority to grant permits for the occupation of land under the control of the War Department, whether in connection with such posts, or in connection with the reservations belonging to the United States in the District of 
Columbia or elsewhere which have been placed by law under the direction of the War Department.

The power vested in the War Department to control the navigable waters of the United States, so that no obstruction shall be placed in the path of commerce and the details of this supervision are also placed under the control of the Assistant Secretary, who decides upon the location of bridges and their construction, as to whether they come within the limitations established by Congress in any empowering acts and upon the location and effect of retaining walls, wharves, piers, or other structures which may encroach upon navigable waters or are likely to change the character of navigable channels in rivers, tideways or lakes.

All matters relating to the militia and national guard of the United States, including equipping and training of the volunteer forces so that they may be an effective part of the army of the United States in case of war are uncler the jurisdiction of the Assistant Secretary, and he has also the inventory and inspection reports of the regular army under his direction.

The very considerable provisions made by the Congress for marking the sites of battlefields of the Civil War, including the operations of the several commissions established for this purpose, are under the direction of the Assistant Secretary, who has also the charge of the various national cemeterics.

With relation to the operations of the department, the Assistant Secretary has jurisdiction over open market purchases for the army, miscellaneous claims and accounts, calls for information or for papers from the files of the department and authentication of the signatures of bureau chiefs. He has joint direction with the Secretary of War over the office of the Chief Clerk of the Department and the details of the immediate departmental organization and signs all requisitions on the Secretary of the Treasury, letters to Congress, transmitting routine reports and letters to heads of Executive Departments relating to subjects within his own jurisdiction. 


\section{THE ASSISTANT SECRETARY OF THE NAVY.}

It has been the theory during considerable periods of the history of the United States that the assistance afforded to the Secretary of the Navy could best take the form of advice by technically trained officers serving under the department, generally officers of the line of the navy. Under this theory there have been at intervals single naval assistants or boards of officers, and during at least one period the functions of the Secretariat were in commission under a board of officers. During the Civil War there was provision for an Assistant Secretary and a gentleman was appointed who had formerly been a naval officer and who served with signal success. The present position of Assistant Secretary of the Navy was established by the act of July II, I890. His duties were fixed in I89I as being such as might be prescribed by the Secretary of the Navy or required by law. $\mathrm{He}$ is appointed from civil life by the President by and with the advice and consent of the President and receives a compensation of $\$ 4,500$ per annum.

His duties as prescribed by the Secretary of the Navy are as follows:

He has charge of matters relating to the naval militia of the various States, under which function he allots and distributes the appropriation made by Congress for the support of these bodies, and also has charge of the allotment of vessels of the navy to such organizations for practice purposes. He receives the required reports and takes such steps as he may deem to be advisable for the promotion of the efficiency of the organizations.

He acts upon all applications from private parties for the gift or loan of public property under the control of the department, but has no power, of course, to permit such gift or loan except under the requirements of law, so that the matter is largely restricted to the loans made for exposition purposes under the acts of Congress, to the loan of condemned ordnance and to the determination of the propriety of the assignment of ships and men of the navy to be present at celebrations or other public occasions on request of States or municipalities. 
He has the very important function of passing on all repairs to be made to ships of the navy and of prescribing where and how they shall be made. In furtherance of this function he is required to inspect vessels in commission and the navy yards and naval stations as far as may be practicable and to make reports thereon to the Sccretary of the Navy.

He authorizes all advertisements inserted on behalf of the department and in a preliminary way determines on the qualifications of candidates for commissions in the staff corps of the navy and in the Marine Corps when appointments are to be made from civil life. He does not finally pass upon their qualifications, but picks out from the list of applicants those who seem to be best calculated to pass the required examinations, the favorable recommendation of an examining board being required in every case.

The duties prescribed by law are that in the absence of the Secretary of the Navy he shall perform the duties of the Secretary, and while occupying such a position he has all the powers and prerogatives which are conferred on the Secretary by law or by his delegated authority from the Commander-in-Chief, the President of the United States. The specific functions above mentioned are carried out over the signature of the Assistant Secretary, as such, but other functions relating to the department are exercised in the absence of the Secretary not as Assistant Secretary but as Acting Secretary.

\section{FIRST ASSISTANT SECRETARY OF THE INTERIOR.}

In direct opposition to the practice in the State Department, the ranking Assistant Secretary of the Interior is given a number, namely, First Assistant Secretary, while the junior officer is styled the Assistant Secretary. The salary of the office was for years $\$ 6,000$, but the additional $\$ I, 500$ provided, was only to be paid during the term of the First Assistant who retired in the fall of I907, so that the salary becomes the same as that of other Assistant Secretaries. The First Assistant is appointed by the President by and with the advice and consent of the Senate and is required to 
perform such duties as may be prescribed by the Secretary or may be required by law. Under the old law the President was empowered to appoint an Assistant Secretary of the Interior to sign land patents, but this has been superseded by the provision of an Executive Clerk for this purpose.

It is probably true that individual cases in the routine of the Interior Department come more closely to the Secretary and his immediate representatives than is the case in other departments. A large proportion of the business of the department consists of the definition of the provisions of law and the Secretariat of the Interior constitutes a court of appeal to which a very large number of cases are carried. The duties of the First Assistant Secretary relate, broadly, to the administrative details and decisions on appeal of territorial and Indian affairs. All matters relating to the government and control of the territories coming under the department are assigned to him and under Indian affairs he has charge of allotments in severalty, being the distribution of tribal lands and moneys; review of contracts for attorneys for the Indians to safeguard their interests against unreasonable claims for legal services in prosecuting claims before the department; matters relating to claims for damages on account of destruction of property by Indians in forays from reservations and on account of other Indian depredations recognized by Congress as establishing a liability on the part of the United States; the supervision of the amounts paid by the government as annuities to Indians in accordance with treaty stipulation and the investments resulting from unused surplus. He has charge also of contracts for Indian supplies and of the details of open market purchases and renting warehouse accommodations outside of Washington.

The First Assistant Secretary has supervision of matters relating to the administration of Indian lands, such as the removal of intruders, the review of deeds and leases, the rights of railroad companies through and on such lands, the survey of the lands and the rights of Indians thereto, including the construction of the terms of Indian treaties. The First Assistant Secretary has 
also the details relating to the management of the National Park reservations, the Yellowstone, Yosemite, Grant, Sequoia, Mt. Rainier, Crater Lake and IVind Cave parks, for the purpose of retaining the natural curiosities thus impounded in their pristine condition and for preventing depredations thereon.

He also takes a considerable portion of the management of the immediate department, besides acting as Secretary in the absence of the Secretary of the Interior and has charge of leasing buildings in the District of Columbia for the use of the department, making contracts for supplies and service; signs requisitions for treasury warrants, passes on the correctness of official bonds and bonds for the fulfilling of contracts and handles all matters relating to leaves of absence except in regard to the thirty days' annual leave provided for by law.

With reference to the public lands he has charge of the details of repayment of money for lands erroneously sold to individuals out of specific allotments made by law and of payments of cash indemnity for swamp lands diverted from grants made to States. He has also charge of matters relating to land scrip or evidence of right to an allotment of the public lands on account of military service and in accordance with the general provisions of law, such scrip being accepted in lieu of cash in payment of amounts duc for land finally located.

\section{THE ASSISTANT SECRETARY OF TIIE INTERIOR.}

The position of Assistant Secretary of the Interior was first established by appropriation in I 886 and is continued by annual appropriation without specific establishment of the position. No distinct provision is made as to the manner of his appointment and his salary is fixed at $\$ 4.500$ per annum. He is, however, appointed and confirmed in a similar manner to other assistant secretaries and has the usual functions, acting as Secretary of the Interior in the absence of the Secretary and Assistant Secretary. He exercises a considerable part of the functions of the Secretary with reference to appeals, having charge of appeals in pension and bounty land cases, and appeals from administrative action of 
the Commissioner of Patents. He is also charged with the supervision of the Pension Appeal Board and of correspondence relating to the business of the Patent Office. In connection with hearings before the department he has charge of matters relating to admission to practice and disbarment of attorneys before the department and of the regulation of fees. He is also charged with inaugurating and conducting proceedings for the prosecution of violations of the pension laws and has charge of the quarters of pension agencies outside of Washington.

The Assistant Secretary has charge of the institutions in the District of Columbia directed by the department, including the Columbia Institution for the Deaf and Dumb, the Freedmen's Hospital, the Government Hospital for the Insane and has charge of the education of the blind in the District of Columbia. He also administers the departmental matters relating to agricultural colleges in the different States which have received grants of public lands and supervises the business of the Bureau of Education. For the department he has the supervision and approval of expense accounts of inspectors, special agents, special examiners, pension agents and other officers and of all transportation accounts and approves request and vouchers for advertising.

ASSISTANT SECRETARY OF AGRICULTURE.

The position of Assistant Secretary of Agriculture was established by the law of 1889 , which constituted the department, then under a commissioner, as one of the Executive Departments. Definite division of a portion of the duties of the execution of the affairs of the department have not been made by law, or by departmental regulation. The general requirement that the Assistant Secretary shall perform such duties as may be prescribed by law or required by the Secretary is strictly carried out, and as there are no duties as Assistant Secretary required of the functionary by law, his duties in the presence of the Secretary are those which are delegated to him from day to day by the head of the department. In the absence of the Secretary he becomes 
Acting Secretary and exercises all the powers and prerogatives of the Secretary, subject, of course, to the Secretary's known wishes in matters of policy. His direct subordination in the matter of departmental dutics was emphasized by a recent appropriation act, which re-enacted the general terms of the former act. The Assistant Secretary of Agriculture occupies an office adjoining that of the Secretary and all matters are referred to the Secretary, who transfers such matters as he deems best to the Assistant Secretary for consideration. The Assistant Secretary of Agriculture is appointed by the President, by and with the advice and consent of the Senate and receives $\$+, 500$ per annum as compensation.

ASSISTANT SECRETARY OF TIE DEPARTMENT OF COMMERCE AND LABOR.

The Assistant Secretaryship of the Department of Commerce and Labor resembles that of the Agricultural Department and differs from most of the similar positions in the Executive Departments in that no specific part of the departmental duties are assigned to it for independent or semi-independent action. The position was established by the act creating the department in I903 and the duties are defined to be such as may be prescribed by the Secretary or required by law. No departmental regulation has been made fixing the duties of the Assistant Secretary, who shares with the Secretary the work of administering the executive details of the department and who, in the absence of the Secretary acts as Secretary under the usual provisions as to power and the usual limitations as to general departmental policy. The position pays more money than any of the others, namely, $\$ 5,000$ a year. The appointment is made by the President and there is no requirement that it shall be by and with the advice and consent of the Senate. Although no definite declaration of policy on the subject is made, the Assistant Secretary is generally named as chairman and directive head of investigating and other similar boards on matters relative to the work of the department, and conducts the greater part of the work relating to the routine administration of the department and the supervision of the various bureaus. 


\section{CHAPTER VII.}

THE JUDICIARY.

The remarks which have been made in the chapter upon the Supreme Court apply as well to the other branches of the judiciary so far as they affect matters of limitation of jurisdiction. The function of the Supreme Court as the determining power of the validity or constitutionality of legislation does not extend to the inferior courts established by Congress as such a determination can only be made by a court of last resort. The inferior courts have, however, important duties in adjusting the relations between citizens of different States and between aliens and citi. zens of the United States, and the former adjustments are yearly becoming of more importance for the reason that the more general intercourse between all parts of the country tends to bring with it an increasing number of controversies between persons who are citizens of one State and natural and artificial persons who are citizens of another. This is especially true with relation to corporations created by the different States and whose field of operation not only is not limited to the States by which they were created, but includes, in a large number of instances, the entire territory of the United States and its possessions. As will be further shown, the Federal statutes have provided for a large number of contingencies in which persons or corporations foreign to a given State are protected against discriminative legislation or practice which would favor the citizen of the State in whose courts the litigation was commenced. It has come to be a very general practice for corporate interests to seek the courts of the United States when practicable, and in certain of the States an attempt has been made to remove this recourse by legislation providing that when a foreign corporation shall seek to remove an action brought by a citizen of the State to the Federal Court that corporation shall be debarred from further exercise 
of the privileges which have been accorded to it to carry on business in the State on the same footing as corporations created by the State in question.

There are ccrtain provisions of law which are common to all the members of the Federal judiciary, the principal of which are those intended to secure an absolutely independent and unbiased status on the part of the Judge. A Judge of an United States Court, having been appointed by the President, by and with the advice and consent of the Senate can only be removed upon impeachment proceedings initiated by the House of Representatives and consummated by a vote of two-thirds of the members present of the Senate that the impeachment charges have been sustained. The salary which is assigned to a Judge upon his appointment cannot be diminished during his term of office, and when he reaches the age of serenty years, if he has been in office for ten years he is entitled during the residue of his natural life to receive the same salary which he has had paid to him during his term of office.

Each Judge is required to take an oath that he will "administer justice without respect to persons, and do equal right to the poor and to the rich," and that he will faithfully and impartially discharge and perform all the duties incumbent upon him according to the best of his abilities and understanding. It is unlawful for any Judge to excrcise the profession or employment of counsel or attorney or to be engaged in any form of the practicc of the law. The infraction of this provision is deemed a high misdemeanor and subjects the offender to the penalty of a successful impeachment.

Federal Judges, as has been said in the chapter on the Supreme Court, cannot issue all the common law writs, but only such as appertain to their jurisdiction. Sucl writs as they may issue include those to prevent a person from leaving the country, but only when a suit in equity has been brought within the jurisdictional powers of the court. The Federal Courts also have the power to issue the necessary writs to compel persons to appear and plead in cases brought within their jurisdiction, and may 
also issue wtits of injunction in certain cases. The issuance of writs of injunction by which the power of the United States is exercised to prevent the doing of certain acts has been the subject of much agitation during the past few years, and legislation has been sought to limit or to entirely abolish this power in instances in which its exercise has been claimed to be an abuse. Primarily the injunction is a decree of a court sitting in equity to stop some procedure of a person or persons which is either repugnant to the orderly determination of a question submitted or to be submitted to the court for adjudication, or where some wrong is alleged to be abont to be committed which will produce irremediable injury and one for which an adequate amount in damages eannot be recovered. Injunctions ean only be issued by Judges of Federal District Courts in certain specified instances. A wider power of injunction, so long as the matter lies within the subject of Federal jurisdiction is given to Circuit Judges and to Justices of the Supreme Court. The latter can only issue such injunctions if they are actually in the circuit for which the injunction is issued, unless there is an express stipulation of both parties that the order may be issued outside the circuit, or unless it cannot be issued by the Circuit Judge or by a District Judge sitting on behalf of the Circuit Court. The cases in which complaint has been made of the use of the writ of injunction or other restraining order have been those relating to strikes and labor troubles, especially with reference to the means of transportation. The aspect in which the interest of the United States has been found has been chiefly with reference to the carriage of United States mail. In prohibiting the interference of strikers with this transportation, the courts have directed the Federal power towards maintaining the running of trains which the associations of railroad employees who have been on strike have endeavored to stop. Interference with interstate commerce has also been advanced as a reason for the passage of a restraining order by a Federal Court, and such action has been referred to by the opponents of the exercise of the power as government by injunction. The opposition in this matter is stated to be on the 
ground that an application for an injunction is an ex parte proceeding and that it is possible for a prejucliced Judge in acceding to the view of only one party to trench upon the rights of the other party under the color of having taken an equitable action. The remedy which has been proposed is that no injunction should be issued, except upon the hearing of both parties and such a provision has been incorporated in the constitution of the new State of Oklahoma.

While provision is made by law for the due transfer of suits begun in the courts of the different States to United States courts under appropriate circumstances, the Federal Courts are forbidden to interfere with the courts of the States by injunction proceedings, except in bankruptcy cases, the exception arising out of the fact that the Constitution provides for bankruptcy jurisdiction in the Federal Government.

The statutes contain the usual provisions for the support of the authority of the Federal courts and for due procedure in cases which come within their jurisdiction. Among such provisions is that empowering the court to grant new trials in cases of jury trial under the usual condition obtaining in courts of law and to hold to good behavior and security of the peace as well as to direct the arrest and custody of persons who may appropriately come before the court. The usual provision is made that suits in equity shall not be entertained unless there is not a plain, adequate and complete remedy to be had at law and the Federal courts are given power to punish for contempt, the latter, however, being strictly defined as misbehavior in the presence of the contrt or so near thereto as to obstruct the administration of justice, the misbehavior of any of the officers of the court in their official transactions, and the disobedience or resistance by any officer, party, juror or witness to a lawful writ of process or command of the court. The punishment for such contempt may be by fine or imprisonment, and the action is not appealable or to be reviewed by another court, although it is maintained that the President has the power to pardon for a contempt. The exercise of such power, if it exists, would, however, be undertaken only in an extraordinary case. 


\section{THE DISTRICT COURT.}

The most numerous body of the Federal Judiciary, and that which comes into closest communication with the great body of the people of the United States, of the Federal Judges, is that of the District Judges of the United States. In each of the districts, a list of which is given hereafter, there is, generally, one judge. In some districts, however, the amount of business requires the services of two judges, while there are other districts for which one judge holds court in two districts. Sometimes, also, because of the infirmities or age of the district judge, an additional judge is named for his district, the total number of judges to be reduced to one when the first judge dies or retires.

The salaries of the district judges are $\$ 6,000$ per year each, paid in monthly installments. District judges also receive reimbursement for expenses incurred when they are designated to hold court outside of their district, the expenses allowed not to exceed \$1o per day. The district judge has the appointment of the clerk in his district, and also the deputies of the clerk, although the latter are generally nominated by the clerk himself who is responsible for the acts of his deputy. Where there is more than one place of holding court in a district, the judge may designate the point at which the records of the court shall be kept, unless such place is designated by law. The district judge is deemed to be always sitting as a court for hearings in various motions, orders and rules in admiralty and in equity cases. He may in such cases issue necessary orders either in his chambers or in the clerk's office as if he had been sitting in the usual place of holding court. The session of district courts may be held continuously if the business at a given point requires it, notwithstanding another term of court may have been set by law at another place, and notwithstanding another term of court at the same place may have been set for a time when business of a former term is still going on. In the latter case the old term of court may be continued, and in the former case, the new term of court provided by law be adjourned, and the business of the former session completed. Provision is, however, made so that criminal sittings may not be unduly postponed by reason of 
civil business. It is required that monthly adjournments of civil sittings shall be made when necessary to take up criminal business in order that the transaction of the latter may not be delayed or rendered unduly expensive. District judges have also the power to order special sittings of district courts at such times and places as they may deem nccessary. If the judge is unable to attend at the beginning of any term of court, he may direct the clerk or marshal to adjourn the same. It frequently occurs that because of the death or disability of a judge, or because of his interest in some suit then pending, or because of the pressure of the business of the court that it becomes necessary to appoint some other judge to hold court in that particular district. In such event upon notice of the condition to the Circuit Court, the senior Judge of that court designates some other judge within the circuit to hold the court, or, if there is no district judge in the circuit who can be so assigned, or there is no circuit judge available to make the assignment, the facts are certified to the Clief Justice of the United States, who may designate any district judge from any circuit to hold the court. It is sometimes also arranged as a matter of convenience that district judges shall exchange sittings of court, and in all these exchanges the powers and duties of one judge are made applicable to him when sitting in another district than his own.

It is a comment in a standard work on Federal practice that when the system of United States courts was established no one anticipated the growth, numcrically and in scope, of the cases before the Federal courts. The original districts were established on the theory that a State should constitute a Federal judicial district. Geographical considerations and the increase of business brought about numerous subsequent enactments and the process of subdividing the judicial districts is still going on.

The fundamental requirement of a judge of a Federal district court is that he shall reside permanently in the district for which he is appointed. It is not required that a resident of a district shall be appointed to be the judge for the district, but on his appointment he must take up his residence within the district and the penalty attaching to high misdemeanor is threatened against a judge who 
shall offend against the requirement. That this is not an idle threat is evidenced by the fact that one of the seriously regarded charges in a recent impeachment case before the Senate of the United States was that a district judge had established an ostensible residence within his district but had actually resided for considerable periods at a remote point. The reason for this requirement is that the judge shall be reasonably accessible for the purpose of such motions, orders, etc., as may be required in legal process in the interim of court terms or sittings, and that the legal power of the United States shall not be absent from any of its legally constituted districts for considerable periods of time.

The Congress has made a close definition of the place and time of holding district courts and in each act establishing a district court, the place of its holding, of its time of sitting, of the location of its records and the residence of its clerk are all definitely set forth. Care has been taken that litigants shall not be put to the trouble, delay and expense of going to remote points at different times for the purpose of securing adjudication under the Federal laws, and that the power of the central government need not be sought at Washington, but that within reach of the most distant parts of the nation, a Federal representative is seated to administer the Federal laws.

The district judge directs the method of selecting names for drawing of grand and petty juries of his court and of the selection of jurors from the names thus selected. The District Judge also appoints the United States Commissioners in his district, but the clerk of the court cannot be so appointed without the approval of the Attorney General.

For reasons that will be more fully set forth hereafter, the description of the jurisdiction of a Judge of the District Court to hear and determine causes is by no means a simple matter. Federal procedure is most complex because of the overlapping of lines of jurisdiction and often confusing because of the merging, in so many cases, of the personality of the District Judge, the Judge of the Circuit Court and of the Judge of the Circuit Court of Appeals in one person. Generally speaking, however, a Dis- 
trict Judge has jurisdiction over all crimes and offenses cognizable under the authority of the United States committed within his district or upon the high seas, except in cases where the offense is such as to render the offender liable to capital punishment An cxception to this general statute is, however, that growing out of the land title condition in California, where it became necessary to make it punishable to secretly or fraudulently place any document among the archives of the Surveyor-General's office in California. This means of preventing land frauds is placed in the jurisdiction of the Circuit Court.

The jurisdiction of the District Court over offenses on the high seas, except in capital cases, is exercised in the district in which an offender is found, or to which he may first be brought after the offense has been committed. This, of course, relates to offenses committed under the American flag. Of offenses committed against the laws of nations, the District Court is given jurisdiction over cases of alleged piracy when no Circuit Court is held within the district.

The Judge of the District Court has jurisdiction in all suits for penalties and forfeitures incurred under any law of the United States and of suits at common law brought by the United States or an officer authorized to sue, in suits in equity to enforce the payment of internal-revenue taxes and suits for the recovery of the penalties imposed against those who make or present false claims against the United States. He has also concurrent jurisdiction with the Circuit Court Judge in all cases arising under the postal laws of the United States.

In the carlier Federal jurisprudence, one of its most important aspects was that relating to the determination of prize cases, and in case of war, the District Courts have important functions in determining the lawfulness of a capture and the disposition of the prize and the procceds thercfrom. The District Court is given original and exclusive jurisdiction over all prizes brought into the United States except over articles which have been devoted to the use of or have been allowed to be taken by insurrectionists in which it has concurrent jurisdiction. The condemnation 
proceedings on all other prizes are under the jurisdiction of the District Judge, who also prescribes the division of the proceeds, subject, of course, to appeal to the Supreme Court, though the proceedings in the District Court may go on notwithstanding such an appeal is pending.

With the above exception, the district court has also exclusive jurisdiction over all seizures of property for violation of Federal law on land and on waters not within admiralty or maritime jurisdiction (the seizures within admiralty or maritime jurisdiction are covered by the foregoing paragraph) and has also exclusive jurisdiction of all civil causes of admiralty and maritime jurisdiction.

The district courts have also concurrent jurisdiction with the circuit courts over suits by assignees for drawback of duties; over the classes of suits described under the head of judges of circuit courts and generally described as those arising out of the laws for the preservation of "Civil Rights" to citizens of the United States and over suits within the district against a national banking association. It has also jurisdiction over an action in tort only, that is for a wrong committed, in violation of a law of nations or a treaty of the United States, but not for the enforcement of a contract. It also has jurisdiction over suits against consuls or vice-consuls of foreign nations residing in its district.

The Judge of the District Court has concurrent jurisdiction with the Court of Claims in suits against the United States where the amount does not exceed $\$ \mathrm{I}, 000$ and has jurisdiction in all cases, civil and criminal with the Circuit Court in cases arising under the immigration and contract labor laws. Appeals by Chinese from the decision of a United States Commissioner that they are unlawfully in the United States come before a district judge who has also jurisdiction over proceedings in equity relating to unlawful occupancy of public lands in his district. While sitting as a circuit court judge a district judge may also issue writs of injunction valid until the next sitting of the circuit court if no circuit judge is available. He may hold security for peace in any cases cognizable before him and has jurisdiction of suits for penalties for the violation 
of the statutes with relation to the equipment of railroad cars. He has also concurrent jurisdiction in proceedings for the condemnation of land for public buildings or other public uses.

The district judge in addition to holding his own court is eligible to act as a circuit judge upon appointment by a judge of circuit, to hold circuit court or to complete a circuit court and also to act as a judge of the circuit court of appeals in case of the absence of a Justice of the Supreme Court or judge of the circuit court.

The various Judicial Districts of the different States are composed of the following counties, respectively:

Alabana-Northern district: Counties in district.-Northern division: Colbert, Cullman, Franklin, Jackson, Lauderdale, Lawrence, Limestone, Madison, Marion, Marshall, Morgan, and Winston.

Sothern division: Bibb, Blount, Dekalb, Fayette, Greene, Hale, Jefferson, Lamar, Pickens, St. Clair, Shelby, Sumter, Tuscaloosa and Walker.

Eastern division: Calhoun, Cherokee, Clay, Cleburne, Etowah, Talladega.

Alabama-Middle district: Countics in the district-Autaga, Barbour, Bullock, Butler, Chambers, Chilton, Coffec, Coosa, Covington, Crenshaw, Dale, Dallas, Elmore, Geneva, Henry, Houston, Lee, Lowndes, Macon, Montgomery, Perry, Fike, Randolph. Russell, and Tallapoosa.

Alabama-Southern district: Counties in the district.-Baldwin, Choctaw, Clarke, Conecuh, Escambia, Marengo, Mobile, Monroc, Washington, and Wilcox.

Arkansas-Eastern district: Counties in the district.-Western division (returnable to Little Rock): Arkansas, Asliley, Bradley, Clicot, Clark, Cleveland, Conway, Dallas, Desha, Drew, Faulkner. Garland, Grant, Hot Spring, Jefferson, Lincoln, Lonoke, Montgomery, Perry, Pope, Prairie, Pulaski, Saline, Van Buren, White, and Woodruff.

Eastern division (returnable to Helena): Clay, Craighead, Crittenden, Cross, Greene, Lee, Mississippi, Monroe, Phillips, Poinsett, and St. Francis.

Northern division (returnable to Batesville): Cleburne, Fulton, Independence, Izard, Jackson, Lawrence, Randolph, Sharp, and Stone.

Arkansas-Western district: Counties in the district.-Fort Smith division: Benton, Crawford, Franklin, Johnson, Logan, Polk, Scott, Sebastian, Washington, and Yell. 
Texarkana division: Calhoun, Columbia, Hempstead, Howard, Lafayette, Little River, Miller, Nevada, Onachita, Pike, Sevier, and Union.

Harrison division: Baxter, Boone, Carroll, Madison, Marion, Newton, and Searcy.

CALIFORNis-Northern district: Counties in the district.-Alameda, Alpine, Amador, Butte, Calaveras, Colusa, Contra Costa, Del Norte, Eldorado, Glenn, Humboldt, Lake, Lassen, Marin, Mendocino, Modoc, Mono, Monterey, Napa, Nevada, Placer, Plumas, Sacramento, San Benito, San Francisco, San Joaquin, San Mateo, Santa Clara, Santa Cruz, Shasta, Sierra, Siskiyou, Solano, Sonoma, Stanislaus, Sutter, Tehama, Trinity, Tuolumne, Yolo, and Yuba.

California-Sonthern district: Counties in the district.-Northern division:

Fresno, Inyo, Kern, Kings, Madera, Mariposa, Merced, and Tulare.

Sotthern division: Los Angelcs, Orange, Riverside, San Bernardino, San Diego, San Luis Obispo, Santa Barbara, and Ventura.

Colorado-District comprises entire State.

Connecticut-District comprises entire State.

Delaware-District comprises entire State.

District of Columbia-District comprises entire District of Columbia.

Florida-Northern district: Counties in the district-Calhoun, Escambia, Franklin, Gadsden, Hoimes, Jackson, Jefferson, Lafayette, Leon Levy, Liberty, Santa Rosa, Taylor, Wakulla, Walton, and Washington.

Florida-Southern district: Counties in the district.-Alachua, Baker, Bradford, Brevard, Citrus, Clay, Columbia, Dade, DeSoto, Duval, Hamilton, Hernando, Hillsboro, Lake, Lee, Madison, Manatee, Marion, Monroe, Nassau, Orange, Osceola, Pasco, Polk, Putnam, St. John, Sumter, Suwanee, and Volusia.

GEorgia-Northern district: Counties in the district.-Northern division: Campbell, Carroll, Cherokee, Clayton, Cobb, Coweta, Dawson, Dekalb, Douglas, Fannin, Fayette, Forsyth, Fulton, Gilmer, Gwinett, Hall, Henry, Lumpkin, Milton, Newton, Pickens, Rockdale, Spalding, Towns, Union.

Western division: Clay, Early, Harris, Heard, Marion, Meriwether, Miller, Muscogee, Quitman, Randolph, Schley, Stewart, Talbot, Taylor, Terrell, Troup, Webster.

Northwestern division: Bartow, Catoosa, Chattooga, Dade, Floyd, Gordon, Haralson, Murray, Paulding, Polk, Walker, Whitfield.

Eastern division: Banks, Clarke, Elbert, Franklin, Greene, Habersham, Hart, Jackson, Madison, Morgan, Oconee, Oglethorpe, Rabun, Walton, White. 
Georgia-Sonthern district: Counties in the district.-Eastern division, at Savannah: Appling, Bryan, Bulloch, Camden, Chatham, Effingliam, Emanuel, Glynn, Liberty, McIntosh, Montgomery, Screven, Tatnall, and Wayne.

Western division, at Macon: Baker, Baldwin, Bibb, Butts, Callınn, Crawford, Dodge, Dooly, Dougherty, Hancock, Houston, Jasper, Jones, Laurens, Lee, Macon, Monroc, Pike, Pulaski, Putnam, Sumter, Telfair, Twiggs, Upson, Wilcox, and Wilkinson.

Northeastern division, at Augusta: Burke, Columbia, Glascock, Jefferson Johnson, Lincoln, McDuffie, Richmond, Taliaferro, Washington, Wilkes, and Warren.

Sonthwestern division, at Valdosta: Berrien, Brooks, Charlton, Clinch, Coffee, Colquitt, Decatur, Echols, Irwin, Lowndes, Mitchell, Pierce, Thomas, Ware, and Worth.

HawAII-District comprises all of the Islands.

IDAHo-Counties in the district.-Northern division: Idaho, Kootenai, Latah, Nez Perces, and Shoshonc.

Central division: Ada, Blaine, Boise, Canyon, Cassia, Elmore, Lincoln, Owyhee, and Washington.

Southern division: Bannock, Bear Lake, Bingham, Custer, Irremont, Lemhi, and Oncida.

Illunors-Northern district: Counties in the district--Northern division: Boone, Bureau, Carroll, Cook, Dekalb, Dupage, Grundy, Jo Daviess, Kane, Kankakec, Kendall, Lake, Lasalle, Lec, McHenry, Ogle, Steplienson, Whiteside, Will, and Winnebago.

Southern division: Fulton, Henderson, Henry, Iroquois, Knox, Livingston, McDonough, Marshall, Mercer, Pcoria, Putnam, Rock Island, Stark, Tazewell, Warren, and Woodford.

Illinors-Sonthern district: Counties in the district.-Adams, Alexander, Bond, Brown, Calhom, Cass, Champaign, Christian, Clark, Clay, Clinton, Coles, Crawford, Cumberland, Dewitt, Douglas, Edgar, Edwards, Effingham, Fayette, Ford, Franklin, Gallatin, Greene, Hamilton, Hancock, Hardin, Jackson, Jasper, Jefferson, Jersey, Johnson, Lawrence, Logan, Macon, Macoupin, Madison, Marion, Mason, Massac, McLcan, Menard, Monroe, Montgomery, Morgan, Moultrie, Perry, Platt, Pike, Pope, Pulaski, Randolph, Richland, St. Clair, Saline, Sangamon, Schuyler, Scott, Shelby, Union, Vermillion, Vabash, Washington, Wayne, White, and IVilliamson.

INDIANA-District comprises entire State.

Iowa-Northern district: Counties in the district.-Eastern division: Allamakee, Blackhawk, Bremer, Buchanan, Chickasaw, Clayton, Delaware, 
Dubuque, Fayette, Floyd, Howard, Jackson, Mitchell, and Winneshiek, Cedar Rapids division: Benton, Cedar, Clinton, Grundy, Hardin, Iowa, Johnson, Jones, Linn, and Tama.

Central division: Butler, Calhoun, Cerro Gordo, Emmet, Franklin, Hamilton, Hancock, Humboldt, Kossuth, Palo Alto, Pocahontas, Webster, Winnebago, Worth, and Wright.

Western division: Buena Vista, Cherokee, Clay, Dickinson, Ida, Lyon, Monona, O'Brien, Osceola, Plymouth, Sac, Sioux, and Woodbury.

Iowa-Southern district: Counties in the district-Western division: Auduboll, Carroll, Cass, Crawford, Harrison, Mills, Montgomery, Pottawattamie, Shelby.

Southern division: Adair, Adams, Appanoose, Clarke, Decatur, Fremont, Lucas, Page, Ringgold, Taylor, Union, Wayne.

Eastern division: Davis, Des Moines, Henry, Jefferson, Lee, Louisa, Van Buren, Wapello.

Davenport division: Keokuk, Muscatine, Scott, Washington.

Central division: Boone, Dallas, Greene, Guthrie, Jasper, Madison, Mahaska, Marion, Marshall, Monroe, Polk, Poweshiek, Story, Warren.

Kansas-Counties in the district.-First division: Atchison, Brown, Chase, Cheyenne, Clay, Cloud, Decatur, Dickinson, Doniphan, Douglas, Ellis, Franklin, Geary, Gove, Graham, Jackson, Jefferson, Jewell, Johnson, Leavenworth, Lincoln, Logan, Lyon, Marion, Marshall, Mitchell, Morris, Nemaha, Norton, Osage, Osborne, Ottawa, Phillips, Pottawatomie, Rawlins, Republic, Riley, Rooks, Russell, Saline, Shawnee, Sheridan, Sherman, Sinith, Thomas, Trego, Wabaunsee, Wallace, Washington, and Wyandotte.

Second division: Barber, Barton, Butler, Clark, Comanche, Cowley, Edwards, Ellsworth, Finney, Ford, Grant, Gray, Greeley, Hamilton, Harper, Harvey, Haskell, Hodgeman, Kearney, Kingman, Kiowa, Lane, McPherson, Meade, Morton, Ness, Pawnee, Pratt, Reno, Rice, Rush, Scott, Sedgwick, Seward, Stafford, Stanton, Stevens, Sumner, and Wichita.

Third division: Allen, Anderson, Bourbon, Chautauqua, Cherokee, Coffey, Crawford, E1k, Greenwood, Labette, Linn, Miami, Montgomery, Neosho, Wilson, and Woodson.

Kentucky-Eastern district: Counties in the district.-Andersen, Bath, Bell, Boone, Bourbon, Boyd, Boyle, Bracken, Breathitt, Campbell, Carroll, Carter, Clark, Clay, Elliott, Estill, Fayette, Fleming, Floyd, Franklin, Gallatin, Garrard, Grant, Greenup, Harlan, Harrison, Henry, Jackson, Jessamine, Johnson, Kenton, Knott, Knox, Laurel, Lawrence, Lee, Leslie, Letcher, Lewis, Lincoln, Madison, Magoffin, Martin, Mason, Menifee, Mercer, Montgomery, Morgan, Nicholas, Owen, Owsley, Pendleton, 
Perry, Pike, Powell, Pulaski, Robertson, Rockcastle, Rowan, Scott, Shelby, Trimbie, Wayne, Whitley, Wolfe, and Woodford.

Kentucky-Western district: Counties in the district-Adair, Allen, Ballard, Barren, Breckinridge, Bullitt, Butler, Caldwell, Calloway, Carlisle, Casey, Christian, Clinton, Crittenden, Cumberland, Daviess, Edmonson, Fulton, Graves, Grayson, Green, Hancock, Hardin, Hart, Henderson, Hickman, Hopkins, Jefferson, Larue, Livingston, Logan, Lyon, McCracken, McLean, Marion, Marshall, Meade, Metcalfe, Monroe, Muhlenberg, Nelson, Ohio, Oldham, Russell, Simpson, Spencer, Taylor, Todd, Trigg, Union, Warren, Washington, and Webster.

Lousinna-Eastern district: Parishes in the district-New Orleans division: Assumption, lberia, Jefferson, Lafourche, Orleans, Plaquemines, St. Bernard, St. Charles, St. James, St. John the Baptist, St. Mary, St. Tammany, Tangipahoa, Terrabone, and Washington.

Baton Rouge division: Ascension, East Baton Rouge, East Feliciana, Iberville, Livingston, Pointe Coupee, St. Helena, West Baton Rouge, and West Feliciana.

Louisiana-Western district: Parishes in the district.-Alexandria division: Avovelles, Catahoula, Grant, Rapides, Vermon, Wim.

Monroe division: Caldwell. Concordia, East Carroll, Franklin, Jackson, Lincoln, Madison, Morehouse, Ouachita, Richland, Tensas, Union, West Carroll.

Opelousas division: Arcadia, Calcasieu, Cameron, Lafayette, St. Landry, St. Martin, Vermillion.

Shreveport division: Bossier, Blenville, Caddo, Claiborne, De Soto, Natchitoches, Red River, Sabine, NVebster.

Malne-District comprises entire State.

Maryland-District comprises entire State.

Massachesetts-District comprises entire State.

Mrchigan-Eastern district: Counties in the district-Northern division: Alcona, Alpena, Arænac, Bay, Cheboygan, Clare, Crawford, Genesee, Gladwin, Gratiot, Huron, Iosco, Isabella, Midland, Montmorency; Ogemaw, Oscoda, Otsego, Presque lle, Roscommon, Saginaw, Shiawassee, and Tuscola.

Southern division: Branch, Calhoun, Clinton, Hillsdale, Ingham, Jackson, Lapeer, Lcnawce, Livingston, Macomb, Nonroe, Oakland, St. Clair, Sanilac, Washtenaw, and Wayne.

Michigan-Western district: Counties in the district.-Northern division: Alger, Baraga, Chippewa, Delta, Dickinson, Gogebic, Hougliton, Iron, Keweenaw, Luce, Mackinac, Marquette, Menominee, Ontonagon, and Schoolcraft. 
Southern division: Allegan, Antrim, Barry, Benzie, Berrien, Cass, Charlevoix, Eaton, Emmet, Grand Traverse, Ionia, Kalamazoo, Kalkaska, Kent, Lake, Leelanau, Manistee, Mason, Mecosta, Missattkee, Montcalm, Mutskegon, Newaygo, Oceana, Osceola, Ottawa, St. Joseph, Van Buren, and Wexford.

Minnesota-Counties in the district.-First division: Dodge, Fillmore, Houston, Mower, Olmsted, Steele, Wabasha, and Winona.

Second division: Blue Earth, Brown, Cottonwood, Faribault, Freeborn, Jackson, Lac qui Parle, Lesueur, Lincoln, Lyon, Martin, Murray, Nicollet, Nobles, Pipestone, Redwood, Rock, Sibley, Waseca, Watonwan, and Yellow Medicine.

Third division: Chisago, Dakota, Goodhue, Ramsey, Rice, Scott, and Washington.

Fourth division: Anoka, Carver, Chippewa, Hennepin, Isanti, Kandiyohi, McLeod, Meeker, Renville, Sherburne, Swift, and Wright.

Fifth division: Aitkin, Benton, Carlton, Cass, Cook, Crow Wing, Itasca, Kanabec, Lake, Millelacs, Morrison, Pine, and St. Louis.

Sixth division: Becker, Beltrami, Big Stone, Clay, Clearwater, Douglas, Grant, Hubbard, Kittson, Marshall, Norman, Ottertail, Polk, Pope, Stearns, Stevens, Todd, Traverse, Wadena, and Wilkins.

Mississify--Northern district: Counties in the district.--Western division: Benton, Calhoun, Carroll, Coahoma, De Soto, Grenada, Lafayette, Marshall, Montgomery, Panola, Quitman, Tallahatchie, Tate, Tippah, Tutica, Union, Webster, and Yalobusha.

Eastern division: Alcorn, Attala, Chickasaw, Choctaw, Clay, Itawamba, Lee, Lowndes, Monroe, Oktibbeha, Pontotoc, Prentiss, Tishomingo, and Winston.

Mississippi-Southern district: Counties in the district.-Jackson division: Adams, Amite, Copiah, Covington, Franklin, Hinds, Holmes, Jefferson, Lawrence, Leflore, Lincoln, Madison, Pike, Rankin, Scott, Simpson, Smith, Wilkinson, and Yazoo.

Vicksburg division: Bolivar, Claiborne, Issaquena, Sharkey, Sunflower, Warren, and Washington.

Meridian division: Clarke, Jasper, Jones, Kemper, Lauderdale, Leake, Neshoba, Newton, Noxubee, and Wayne.

Biloxi division: Greene, Hancock, Harrison, Jackson, Marion, Pearl River, and Perry.

Mrssouri-Eastern district: Counties in the district.-Eastern division: Audrain, Bollinger, Butler, Cape Girardeau, Carter, Crawford, Dent, sippi, Montgomery, New Madrid, Oregon, Pemiscot, Perry, Reynolds, Dunklin, Franklin, Gasconade, Iron, Jefferson, Lincoln, Madison, Missis- 
Ripley, St. Charles, St. François, Ste. Genevieve, St. Louis, St. Louis City, Scott, Shannon, Stoddard, Warren, Washington, and Wayne.

Northern division: Adair, Charlton, Clark, Knox, Lewis, Linn, Macon, Marion, Monroc, Pike, Ralls, Randolph, Scotland, Schuyler, and Shelby.

Missouri-Western district: Counties in the district-Western division: Bates, Caldwell, Carroll, Cass, Clay, Grundy, Henry, Jackson, Jolınson, Lafayette, Livingston, Mercer, Putnam, Ray, St. Clair, Saline, and Sullivan.

St. Joseph division: Andrew, Atchison, Buchanan, Clirton, Davicss, Dekalb, Gentry, Harrison, Holt, Nodaway, Platte, and Worth.

Central division: Benton, Boone, Callaway, Camden, Cole, Cooper, Hickory, Howard, Maries, Miller, Moniteau, Morgan, Osage, Pettis, and Phelps.

Southern division: Cedar, Christian, Dade, Dallas, Douglas, Greene, Howell, Laclede, Ozark, Polk, Pulasli, Tancy, Texas, Webster, and Wright.

Southwestern division: Barry, Barton, Jasper, Lawrence, MeDonald, Newton, Stone, and Vernon.

Montana-District comprises entire State.

Nebraska-District comprises entire State.

NevadA-District comprises entire State.

New Hampshine-District comprises cntire State.

New JeRSEY-District comprises entire State.

New Mexico-Counties in the district.-First district: Santa Fe, San Juan, Rio Arriba, and Taos.

Second district: Eernalillo, McKinley, Sandoval, and Valencia.

Third district: Donna Ana, Grant, Lina, Sierra, and Socorro.

Fourth district: Colfax, Mora, San Miguel, and Union.

Fifth district: Chaves, Eddy, and Roosevelt.

Sixth district: Lincoln, Leonard, Otero, Quay, and Torrance.

New YORK-Northern district: Counties in the district-Albany, Broome, Cayuga, Chenango, Clinton, Cortland, Delaware, Essex, Franklin, Fulton, Hamilton, Herkimer, Jefferson, Lewis, Madison, Montgomery, Oneida, Onondaga, Oswego, Otsego, Rensselaer, St. Lawrence, Saratoga, Schenectady, Schoharie, Tioga, Tomplins, Warren, and Washington, with the waters thercof.

New YoRK-Southern district: Counties in the district.- Columbia, Dutchess, Greene, New York, Orange, Putnam, Rockland, Sullivan, Ulster, and Westchester. 
New YorK-Eastern district: Counties in the district.-Kings, Nassau, Queens, Richmond, and Suffolk, with the waters thereof.

New York-Western district: Counties in the district.-Allegany, Cattaraugus, Chautauqua, Chemung, Erie, Genesee, Livingston, Monroe, Niagara, Ontario, Orleans, Schuyler, Seneca, Stetuben, Wayne, Wyoming, and Yates, with the waters thereof.

North Carolina-Eastern district: Counties in the district.-Beaufort, Bertie, Bladen, Brunswich, Camden, Carteret, Chatham, Chowan, Columbus, Craven, Cumberland, Currituck, Dare, Duplin, Durham, Edgecombe, Franklin, Gates, Granville, Greene, Halifax, Harnett, Hertford, Hyde, Johnstcn, Jones, Lenoir, Martin, Moore, Nash, New Hanover, Northampton, Onslow, Pamlico, Pasquotank, Pender, Perquimans, Person, Pitt, Richmond, Robeson, Sampson, Scotland, Tyrrell, Vance, Wake, Warren, Washington, Wayne, and Wilson.

North Carolina-Western district: Connties in the district.-Alamance, Alexander, Alleginany, Anson, Ashe, Buncombe, Burke, Cabarrus, Caldwell, Caswell, Catawba, Cherokee, Clay, Cleveland, Davidson, Davie, Forsyth, Gaston, Graham, Guilford, Haywood, Henderson, Iredell, Jackson, Lincoln, McDowell, Macon, Madison, Mecklenburg, Mitchell, Montgomery, Orange, Polk, Randolph, Rockingham, Rowan, Rutherford, Stanly, Stokes, Surry, Swain, Transylvania, Union, Watauga, Wilkes, Yadkin, and Yancey.

North Dakota--District comprises entire State.

OnIo-Northern district: Counties in the district.-Eastern division: Ashland, Ashtabula, Carroll, Columbiana, Crawford, Cuyahoga, Geauga, Holmes, Lake, Lorain, Mahoning, Medina, Portage, Richland, Stark, Summit, Trumbull, Tuscarawas, and Wayne.

Western division: Allen, Auglaize, Defiance, Erie, Fulton, Hancock, Hardin, Henry, Huron, Lucas, Marion, Merce:, Ottawa, Paulding, Putnam, Sandusky, Seneca, VanWert, Williams, Wood, and IVyandot.

OHIO-Southern district: Counties in the district.-Western division: Adams, Brown, Butler, Champaign, Clark, Clermont, Clinton, Darke, Greene, Hamilton, Highland, Lawrence, Miami, Montgomery, Preble, Scioto, Shelby, and Warren.

Eastern division: Athens, Belmont, Coshocton, Delaware, Fairfield, Fayette, Franklin, Gallia, Guernsey, Harrison, Hocking, Jackson, Jefferson, Knox, Licking, Logan, Madison, Meigs, Monroe, Morgan, Morrow, Muskingum, Noble, Perry, Pickaway, Pike, Ross, Union, Vinton, and Washington.

OKLAHOMA-Eastern district: The territory comprised within the boundaries of what was formerly Indian Territory constitutes the Eastern district. 
OKLAHOMA-Western district: The territory comprised within the boundaries of what was formerly Oklahoma Territory constitutes the Wester1 district.

Oregon-District comprises entire State.

Pennsyunaxia-Eastern district: Comnties in the district.-Berks, Bucks, Chester, Delaware, Lancaster, Lehigh, Montgomery, Northampton, Philadelphia, and Scuylkill.

Pennsylvania-Middle district: Connties in the district.-Adams, Bradford, Cameron, Carbon, Center, Clinton, Columbia, Cumberland, Dauphin, Franklin, Fulton, Huntingdon, Juniata, Lackawama, Lebanon, Luzerne, Lycoming, Mifflin, Monroe, Montour, Northumberland, Perry, Pike, Potter, Snyder, Sullivan, Susquehama, Tioga, Union, Wayne, IVyoming, and York.

Pennsyluania-IVestern district: Counties in the district.-Allegheny, Armstrong, Beaver, Bedford, Blair, Butler, Cambria, Clarion, Clearfield, Crawford, Elk, Erie, Fayette, Forest, Greene, Indiana, Jefferson, Lawrence, McKean, Mercer, Somerset, Venango, Warren, Washington, and Westmoreland.

PORTo Rico-District comprises entire island.

Rhode ISLAND-District conprises entire State.

South Carolina-District comprises entire State.

Soutu DAkota-Counties in the district.-Northern division (court at Aberdeen) : Brookings, Brown, Campbell, Clark, Codington, Day, Denel, Edmunds, Grant, Hamlin, McPherson, Marshall, Roberts, Spink, Walworth; also Sisseton and Walipeton Indian reservations.

Central division (court at Pierre): Buffalo, Faulk, Jerauld, Hand, Hughes, Hyde, Potter, Stanley, Sully; also Cheyenne and part of Standing Rock Indian reservations.

Southern division (court at Sioux Falls): Aurora, Beadle, Bonhonme, Brule, Charles Mix, Clay, Davison, Douglas, Gregory, Hanson. Hutchinson, Kingsbury, Lake, Lincoln, Lyman, McCook, Miner, Minnelaha, Moody, Sanborn, Turner, Union, Yankton; also Crow Creek, Lower Brule and Yankton Indian reservations.

IVestern division (court at Deadwood): Butte, Custer, Fall River, Lawrence, Meade, Pennington, and the Rosebud and Pine Ridge Indian reservations.

Tennessee-Eastern district: Counties in the district.-Northern division: Anderson, Blount, Campbell, Claiborne, Grainger, Jefferson, Knox, Loudon, Monroe, Morgan, Roane, Scott, Sevier and Union. 
Southern division: Bledsoe, Bradley, Cumberland, Fentress, Hamilton, , James, McMinn, Marion, Meigs, Polk, Rhea, Sequatchie.

Northeastern division: Carter, Cocke, Greene, Hamblen, Hancock, Hawkins, Johnson, Sullivan, Unicoi, and Washington.

Tennessee-Middle district: Counties in the district.-Bediord, Cannon, Cheatham, Clay, Coffee, Davidson, Dekalb, Dickson, Franklin, Giles, Grundy, Hickman, Houston, Humphreys, Jackson, Lawrence, Lewis, Lincoln, Macon, Marshall, Maury, Montgomery, Moore, Overton, Pickett, Putnam, Robertson, Rutherford, Smith, Stewart, Sumner, Trousdale, Van Buren, Warren, Wayıe, White, Williamson and Wilson.

TenNesseE-Western district: Counties in the district.-Eastern division: Benton, Carroll, Chester, Crockett, Decatur, Gibson, Hardeman, Hardin, Henderson, Henry, Lake, McNairy, Madison, Obion, Perry and Weakley.

Western division: Dyer, Fayette, Haywood, Lauderdale, Shelby and Tipton.

Texas-Northern district: Counties in the district.-Returnabie to Dallas: Dallas, Ellis, Hunt, Johnson, Kaufman, Navarro and Rockwall.

Returnable to Fort Worth: Archer, Armstrong, Bailey, Baylor, Briscoe, Carson, Castro, Childress, Clay, Cochran, Collingsworth, Comanche, Cottle, Dallam, Dawson, Deaf Smith, Donley, Erath, Floyd, Foard, Gray, Hale, Hall, Hansford, Hardeman, Hartley, Hemphill, Hockley, Hood, Hutchinson, Jack, Lamb, Lipscomb, Moore, Motley, Ochiltree, Oldham, Palo Pinto, Parker, Parmer, Potter, Randall, Roberts, Sherman, Swisher, Tarrant, Wheeler, Wichita, Wilbarger, Wise and Young.

Returnable to Aiblene: Andrews, Borden, Callahan, Crosby, Dickens, Eastland, Fisher, Gaines, Garza, Haskell, Howard, Jones, Kent, King, Knox, Lubbock, Lynn, Martin, Midland, Mitchall, Nolan, Scurry, Shackelford, Stephens, Stonewall, Taylor, Terry. Throckmorton and Yoakum.

Returnable to San Angelo: Brown, Coke, Coleman, Concho, Crockett, Glasscock, Irion, Menard, Mills, Runnells, Schieicher, Sterling, Sutton, Tom Green, and 'ipton.

'Lexas-Southern district: Counties in the district: Returnable to Galveston: Austin, Brazoria, Chambers, Fort Bend, Galveston, Matagorda and Wharton.

Returnable to Houston: Brazos, Calhoun, Colorado, Goliad, Grimes, Harris, Jackson, Lavaca, Madison, Montgomery, Polk, San Jacinto, Trinity, Victoria, Walker and Waller.

Returnable to Laredo: Aransas, Duval, Lasalle, McMullen, Neuces, Refugio, San Patricio, Webb and Zapata.

Returnable to Brownsville: Cameron, Hidalgo and Starr.

Texas.-Eastern district: Counties in the district.-Returnable to Tyler: 
Anderson, Angelina, Cherokee, Gregg, Henderson, Houston, Nacogdoches, Panola, Rains, Rusk, Shelby, Smith, Van Zandt and Wood.

Returnable to Jefferson: Camp, Cass, Harrison, Hopkins, Marion, Morris and Upshur.

Returmable to Beaumont: Hardin, Jasper, Jefferson, Liberty, Newton, Orange, Sabine, San Aitgustine and Tyler.

Returnable to Sherman: Collin, Cooke, Denton, Grayson and Montague.

Returnable to Paris: Delta, Fannin, Lamar and Red River.

Returnable to Texarkana: Bowie, Franklin, Titus. No definite time for holding court.

Texas-Western district: Counties in district.-Returnable to Austin: Bastrop, Blanco, Burleson, Burnett, Caldwell, Fayette, Gillespic, Hays, Kimble, Lampasas, Lee, Llano, Mason, McCullocl, San Saba, Travis, Waslington and Milliamson.

Returnable to Waco: Bell, Bosque, Coryell, Falls, Frecstone, Hamilton, Hill, Leon, Limestone, McLennan, Milam, Robertson and Somervell.

Returnable to San Antonio: Atascosa, Bandera, Bec, Bexar, Comal, Dewitt, Dimmit, Edwards, Frio, Gonzales, Guadalupe, Karnes, Kendall, Kerr, Kinney, Live Oak, Navcrick, Medina, Uvalde, Valverde, Wilson and Zavalla.

Returnable to El Paso: Brewster, Crane, Ector, El Paso, Jeff Davis, Loving, Pecos, Presidio, Reeves, Ward and Winkler.

UTAH-District comprises entire state.

VERMONT-District comprises entire state.

Virginia-Eastern district: Counties in the district.-Accomac, Alexandria, Amelia, Brunswick, Caroline, Charles City; Chesterfield, Culpeper, Dinwiddie, Elizabeth City, Essex, Fairfax, Fauquier, Gloucester, Goochland, Greenesville, Hanover, Henrico, Isle of Wight, James City, King and Queen, King Gerrge, King William, Lancaster, Loudoun, Louisa, Lunenburg, Mathews, Mecklenburg, Middlesex, Nansemond, New Kent, Norfolk, Northampton, Northumberland, Nottoway, Orange, Powhatan, Prince Edward, Frince George, Prince William, Princess Anne, Richmond, Southampton, Spottsylvania, Stafford, Surry, Sussex, Warwick, Westmoreland and York.

Virginia-Western district: Counties in the district-Albemarle, Alleghany, Amherst, Appomattox, Augusta, Bath, Bedford, Bland, Botetourt, Buchanan, Buckingham, Campbell, Carroll, Charlotte, Clarke, Craig, Cumberland, Dickenson, Floyd, Fluvanna, Franklin, Frederick, Giles, Grayson, Greene, Halifax, Henry, Highland, Lee, Madison, Montgomery, Nelson, 
Page, Patrick, Pulaski, Pittsylvania, Rappahannock, Roanoke, Rockbride, Rockingham, Russell, Scott, Shenandoah, Smyth, Tazewell, Warren, Washington, Wise and Wythe.

WASHINGTON-Counties in the district.-Northern division: Clallam, Island, Jefferson, King, Kitsap, San Juan, Skagit, Shohomish and Whatcom. Southern division: Asotin, Columbia, Franklin, Garfield, Klickitat, Wallawalla, Whitman and Yakima.

Eastern division: Adams, Chelan, Douglas, Ferry, Kittitas, Lincoln, Okanogan, Spokane, and Stevens.

Western division: Chehalis, Clarke, Cowlitz, Lewis, Mason, Pacific, Picrce, Skamania, Thurston and Wahkiakum.

West VIRGINIA-Northern district: Counties in the district.-Barbour, Berkeley, Brooke, Calhoun, Doddridge, Gilmer, Grant, Hampshire, Hancock, Hardy, Harrison, Jefferson, Lewis, Marion, Marshall, Mineral, Monongalia, Morgan, Ohio, Pendleton, Pleasants, Preston, Randolph, Ritchic, Taylor, Tucker, Tyler, Upshur, Wetze', Wirt and Wood.

West Virginia-Southern district: Counties in the district.-Boone, Braxton, Cabell, Clay, Fayette, Greenbrier, Jackson, Kanawha, Lincoln, Logan, McDowell, Mason, Mercer, Mingo, Monroe, Nicholas, Pocahontas, Putnam, Raleigh, Roane, Summers, Wayne, Webster and Wyoming.

Wisconsin-Eastern district: Counties in the district.-Brown, Calumet, Dodge, Door, Florence, Fond du Lac, Forest, Green Lake, Kenosha, Keewaunee, Langlade (except townships 3I, 32, 33 and 34 of ranges 9 and Io east), Manitowoc, Marinette, Marquctte, Milwaukee, Oconto, Uneida (townships $35,36,37,38$ and 39 of range II east), Outagamie, Ozaukee, Racine, Shawano, Sheboygan, Vilas (townsinips 40, 4I and 42 of range II east), Walworth, Washington, Waukesha, Waupaca, Waushara and Vinnebago.

Wisconsin-Western district: Counties in the district.-Adams, Ashland, Barron, Bayfield, Buffalo, Burnett, Chippewa, Clark, Columbia, Crawford, Dane, Douglas, Eau Claire, Gates, Grant, Green, Iowa, Iron, Jackson, Jefferson, Juneau, La Crosse, Lafayette, Langlade (townships 3I, 32,33 and 34 , ranges 9 and Io east), Lincoln, Marathon, Monroe, Oneida (except townships $35,36,37,38$ and 39 , range II east), Pepin, Pierce, Polk. Portage, Price, Richland, Rock, St. Croix, Sauk, Sawyer, Taylor, Tempealeau, Vernon, Vilas (except townships $40,4 \mathrm{I}$ and 42 , range II east), Washburn and Wood.

Wyoming-District comprises entire state. 
JUDGE OF TIIE CIRCUIT COURT.

It is probable that no position under the Federal government of corresponding importance attracts so moderate an amount of attention or is so little understood as that of a judge of the Circuit Court of the United States. In effect the chicf possessor and wielder of the power of the Federal government within the section of the Union to which he is assigned, he comes but little in contact with the people among whom he is set in a sense as a guardian of the Federal prerogatives and even in the written history of the United States but little has been said of the brilliant achievements in jurisprudence, the high standard of probity and integrity, and the unceasing labors of this body of jurists, as marked in ability and in valuable public service as they have remained almost without exception, impersonal and unmarked by the general public.

The circuits of the United States were established in r789 by the inclusion of the various districts then established in accordance with their geographical relations. As States were added to the Union, the circuits remained unchanged in number but were increased in scope. The circuits first established were those in which the respective members of the Supreme Court held their circuit courts, but in 1869 in vicw of the inevitable increase of Federal cases, a circuit judge was provided for each district to carry on the work while the Supreme Court lustice was otherwise engaged and to act as a supplementary judge during the periods of the regular circuit visitations. The circuit judge is given the sane power and jurisdiction as the Supreme Court Iustice allotted to the circuit but only within the circuit for which he is appointed and must reside within his circuit. An exception is made, however, that if a judge of another circuit makes a written request therefor, the power and jurisdiction of a circuit judge may be transferred to that circuit for the purpose of hearing the case only for which request is made. By subsequent legislation to that establishing the position, additional circuit judges have been provided for so that now the first circuit has two judges, 
the second three, the third three, the fourth two, the fifth three, the sixth three, the seventh three, the eighth three and the ninth three. Circuit judges are appointed by the President by and with the advice and consent of the Senate and receive a salary of seven thousand dollars a year. If, having served for ten years and having reached the age of seventy, a circuit judge shall resign his position he is entitled to receive for the residue of his life the same salary he received at the time of his resignation.

The judge of the circuit court has concurrent jurisdiction with State courts in cases which are not wholly between citizens of the same State and wholly under the laws of the State and where the amount in dispute exceeds two thousand dollars. That is to say if a matter comes under the Constitution, laws or treaties of the United States or if the parties are from different States or one is an alien, the matter may be tried in the circuit court if the amount in question is as above stated. Cases pending in State courts coming under this description may be removed to the United States circuit court.

Original jurisdiction is given the circuit court in the following classes of cases:

Suits in equity in which the United States is the petitioner in which the sum at issue is more than $\$ 500$, and all suits at common law where the United States or an officer thereof suing under authority of Congress is the plaintiff. Also of all suits under the import, tonnage or internal revenue laws or causes arising under the postal laws; proceedings relating to the carriage of passengers in merchant vessels; proceedings for the condemnation of property seized because of an effort to aid insurrectionists; of all suits under laws relating to the slave trade; of all suits under the copyright or patent laws; of all suits under the laws providing for national banking associations; suits to enforce the right of citizens of the United States to vote in the several States.

Proceedings relating to violation of the rights of citizens of the United States under the constitutional amendments and en- 
actments relating to "Civil Rights" are made the subjects of original juriscliction in the circuit courts, including suits to recover possession of office or to remove officials because of alleged violation of such laws and upon this point only; suits to recover pecuniary forfeitures or to redress deprivations or injuries or to prosecute persons for conspiracy under such enactments.

Concurrent jurisdiction with the Court of Claims is given to the circuit court in claims of more than $\$ 1,000$ and less than $\$ 10,000$, and concurrent jurisdiction with district courts in cases arising under the immigration and contract labor laws, also in suits in equity to prevent the unlawful occupation of public lands, and in suits for condemnation of land for public purposes.

While it is required that trials of issues of fact shall be by jury except in equity, admiralty and bankruptcy cases, circuit judges may try issues of fact without jury on waiver of a jury trial by the parties to the suit.

When sitting in equity for the trial of patent causes, the circuit court judge may empanel a jury of from five to twelve persons to decide on questions of fact. In case of difference of opinion in a circuit court between the circuit justice and a circuit judge or a district judge or a circuit judge and a district judge, the decision of the presiding judge rules.

A circuit court judge can grant an injunction in cases where a circuit court could grant the same, and a district judge sitting as a member of a circuit court can grant an injunction, but not if the party has had a reasonable time to apply to a circuit court for a writ and an injunction issued by a district judge on behalf of a circuit court can remain in effect only until the next meeting of the circuit court.

The amendment to the Interstate Commerce act passed in the session of Congress of $1905^{-6}$ after a long discussion made the judge of the circuit court not only the enforcing power to compel the carrying out of orders and requirements made by the Interstate Commerce Commission provided for in the act referred to, but made the circuit court the revisory power over the orders and requirements of the Commission. Common carriers against 
whom such orders or requirements are directed are empowered to bring suit in the district in which such carrier has its principal operating office and the jurisdiction over such suits is expressly granted to the circuit courts. The court is prohibited, however, from taking action suspending the operation of any decree of the Interstate Commerce Commission except on five days' notice to the Commission and appeals are provided for to the Supreme Court, and such appeals are to be given especial consideration in the order of business in the Supreme Court.

As the time of the titular circuit judges is almost wholly taken up in considering cases brought to the circuit court of appeals on appeal and writ of error, circuit court is not often held by a circuit judge, but by a district judge acting in that capacity so that there follows the condition which exists at the present time of the district and circuit judges respectively being in a large majority of cases the same person. Thus a district judge may serve at one sitting in the trial of a criminal case as district judge and at the next sitting of court may be acting as a circuit judge for the trial of some case in which the circuit court is given original jurisdiction. The complexities which have followed make the Federal procedure a difficult one, with distinctions which do not by any means appear as differences so that in the revision of the statutes of the United States undertaken by the Commission to Revise the Laws, all of the jurisdiction of the district and circuit courts together with the various provisions applying to both have been consolidated under the head of the district court. For the reason that the Federal procedure can best be understood when it is so consolidated, and also for the further reason that when favorable action upon the proposition of the reviser has been taken, the description of the jurisdiction of the circuit and district courts as it would be based on the statutes in force at the present time would be almost unintelligible, and likely to be misleading, the entire question of jurisdiction is treated in the following paragraphs giving material provisions as they would appear in the proposed revision should it be approved by the Congress. 
In order to avoil confusion, the court of original jurisdiction in the following will be called the lower court, and, indeed, when the jurisdiction is not sharply defined by statute at present so that the jurisdictions of the district and circuit courts are distinet, it would be absolutely impossible within the limits of the present work to define the difference, and its exact determination is a highly technical branch of legal practice. The layman may consider the United States courts practically as divided into one original court with two branches, and two appellate courts, the Circuit Court of Appeals and the Supreme Court.

All cases in which the United States is a plaintiff or petitioner brought against citizens of any of the States, including all suits brought by an officer of the United States authorized to sue in its behalf, may be brought either in the lower United States courts or in the State courts; that is to say, the lower courts have concurrent jurisdiction with the State courts in all such cases. In suits between private individuals where the matter in question amounts to $\$ 2,000$, exclusive of the interest and cost, cases may be brought either in the State conrt or in the lower court if the matter of controversy involves questions arising under the Constitution, laws or treaties of the United States, or if the controversy is between citizens of different States, or between citizens of a State and a forcign State, or its citizens or subject Further, if, in the question at issue, there is joined a matter of conflicting elaims under grants of lands made by different States, sucl a case may be brought either in the State courts or in the United States courts. Cases which come under the jurisdiction of district courts without concurrence or qualification include those relating to all crimes and offenses against the United States not capital committed within the district or any territory under the exclusive jurisdiction of the United States, such as a fort, arsenal, dock yards, magazine, or other similar institution within the territory of a State, but jurisdiction over which has been ceded to the United States in accordance with the constitutional provisions. The lower courts have also jurisdiction over any crime or offense committed on a vessel 
owned by any citizen of the United States when such offense was committed outside the jurisdiction of any particular State. Special provision is also made giving such courts jurisdiction over offenses committed on guano islands or isolated keys which have been designated by the President as portions of the territory of the United States. In order that there may be no zone in which jurisdiction does not extend owing to the failure of State and Federal jurisdiction to meet, the courts of the United States are given concurrent jurisdiction with the State courts over all crimes and offenses committed on the seas bordering the United States within the distance of one marine league from the shore, and also on the Great Lakes, and the rivers which form portions of the boundary lines of the geographical boundaries of the United States. Prosecutions for such offenses may, therefore, be brought in either the State or the Federal courts, and a recourse is provided in such cases as the jurisdiction of the State courts cannot be established. The courts have original and exclusive jurisdiction in all cases arising under any act for the punishment of piracy and relating to the slave trade. Ail suits for penalties and forfeitures incurred under any law of the United Statcs are brought in the lower courts as well as cases brought to enforce the internal revenue laws, and to enforce the lien of the United States upon any real estate from which internal revenue taxes are sought to be collected. Suits to collect customs taxes and tonnage taxes, as well as all proceedings for the enforcement of the laws regulating the carriage of passengers in merchant vessels are brought in these courts, as well as all proceedings for the punishment of officers and owners of vessels through whose negligence the life of any person is destroyed. If any person is clamaged or injured in an attempt to collect the revenues of the United States, he may bring suit to recover for such damage in the Federal courts.

All suits and actions arising under the postal laws of the United States are also included in the jurisdiction of the lower courts, which, as it will be seen, are the tribunals generally for the enforcement of the laws based upon the sovereign powers 
of the United States. A very important part of the jurisdiction of the district courts relates to the cases brought under admiralty and maritime jurisdiction. The practice in such cases is based not upon the usual or common law but upon the law merchant as modified by the customs and practice of merchants and vessel owners throughout hundreds of years. It is especially provided that any remedy which parties may have under the common law shall be saved to them even though the action shall be brought in the district conrt sitting as a court of admiralty and maritime jurisdiction.

These courts are also a tribunal for the adjudication of matters relating to prizes which may be captured by the armed vessels of the United States and brought into the jurisdiction of a district court. While this jurisdiction has been, in the past history of this country a most important one and the business especially during the wars in which privateering was authorized, of tremendous volume, the tenciency of international law is in the direction of decreasing the number of vesscls liable to capture and sale under the adjudication of a prize court, so that the procedure in this respect is likely to be of less importance in the future than it has been counted in the past. Such prize proceedings, may be instituted in the court into whose district the prize may be brought, and upon consideration of the circumstances of the seizure and the awarding of the verdict that the vessel was lawful prize, an order of sale may be made to be carricd out by the marshal, the distribution of the proceeds to be made in accordance with the prize laws and the order of the court.

A rather curious extension of the jurisdiction in prize cases was made during the Civil War under statutes providing for the seizure of property of persons who used their property or knowingly allowed it to be used for the furtherance of any rebellion against the United States. Such property was declared to be lawful prize and proceedings for its condemnation and sale for the benefit of the captor can be brought either in the district in which the seizure was made, or in the court of a district into 
which the property may be brought and the proceedings first begun.

Another class of cases growing out of the conditions of the Civil War and which come within the jurisdiction of the lower courts are those growing out of the civil rights enactment under which any person deprived of a right, privilege or immunity secured by the Constitution or laws of the United States may bring suit to redress such deprivation in the Federal lower court, or against any person who knowing that such wrongs are to be inflicted fails to do all in his power to prevent such deprivation or wrong. These courts also have jurisdiction of suits brought to recover the posscssion of any office of which a person may have been deprived because of the denial to certain citizens of the right to vote on account of race, color, or previous condition of servitude. The only offices excepted are those of elector of President or of Vice-President, Representatives or delegate in Congress or member of a State Legislature. When any person has been deprived of the right to occupy another elective office than those named by reason of the denial of the right to vote to citizens of the United States he is empowered to bring a suit in a Federal court, which can determine upon his right to occupy such an office although the determination must be based only upon the denial or non-denial of the right of certain citizens to vote. The jurisdiction of the court does not extend to the consideration of other circumstances in the election than those herein stated.

The jurisdiction of proceedings relative to the enforcement of the national banking laws and relative to operations of the Comptroller of the Currency and his subordinates for the purpose of winding up the affairs of banks are invested in the lower courts of the United States. This jurisdiction is concurrent with the State courts in matters relating to the collection of sums becoming due in realizing upon the assets of banks which may be in process of liquidation, or to enforce the liens upon any property which have attached by reason of the provision of the national banking laws. That is to say, any legal process which the 
officer in charge of the liquidation of the bank may find to be necessary for the purpose of securing amounts found to be due, and which are to be applied to the funds for the payment of depositors may be undertaken through the medium of the United States courts, with the additional prerogative of appealing to the State courts for assistance in such collection when it can be more advantageously proceeded with in the State courts. Any proceeding which may be undertaken by individuals enjoining the Comptroller of the Currency or any receiver of a bank acting under his orders is also to be brought in the lower courts of the United States.

When any alien person deems that he has suffered a wrong, and which is in violation of the laws of nations, or of the treaties of the United States, he may bring an action for remedy in an appropriate District Court of the United States. District Courts also have jurisdiction of suits against consuls and viceconsuls of foreign nations. The bankruptcy procecdings under the national bankruptcy laws hereinafter described are also under the jurisdiction of the District Court which is made the court of bankruptcy and which performs various duties with relation to the review and approval of the subordinate officials in bankruptcy proceedings, besides being the appropriate jurisdictional court for suits of law whic! grow ont of matters relating to bankruptcy cases.

Concurrent jurisdiction is given to lower courts with the Court of Claims in all claims not cxceeding $\$ 10,000$ in value, which may be founded upon the Constitution of the United States, any law of Congress, resulation of an Exccutive repartment, or upon any contract express or implied with the Govermment of the United States, or for damages in such cases other than those arising in tort. Without this express provision it would not be possible for any person to bring suit against the United States for the reason that the latter, as one of the prerogatives of sovereignty is net s:able in its own court, or in any court thus far recognized. The provision just stated, however, enables a person who has a claim against the United States, or 
who deems that he has been unjustly treated in consequence of any action of the United States taken by an officer of the Federal Government, to have his grievance adjudicated by a United States Court. This privilege, however, is especially withheld with regard to claims growing out of the late Civil War, or claims which have been investigated by any court, department, or commission prior to March 3, 1887, and rejected or reported on adversely by such adjudicating body. Claims which do not come within the permission granted to sue the United States can only be passed upon by Congress itself through the medium of a special bill introduced in Congress and referred to an appropriate committee. Jurisdiction over claims for pensions is also denied to these courts as well as jurisdiction in cases brought to recover fees, salaries or compensations for the official services of officers of the United States. There is further a limitation of time during which the allowable suits may be brought, which is three years from the time the right accrued for which the claim is made and all stits under the foregoing provision must be tried by the court without a jury.

The greater proportion of the proceedings arising under recent acts of Congress are brought within the scope of the jurisdiction of the lower courts of the United States. Among such matters are the proceedings contemplated by law to restrain and prevent by writ of injunction the unlawful enclosure of public lands. A further addition to the jurisdiction of the circuit courts was made in connection with the legislation based upon the constitutional power to regulate commerce between the States and with foreign nations. In connection with the operation of the Interstate Commerce Commission all proceedings found necessary to secure the production of books, papers and documents before that Commission and the attendance and the testimony of witnesses are to be brought in the appropriate circuit courts which have also jurisdiction of all suits for the recovery of penalties or damages for the violation of any Interstate Commerce law. A similar jurisdiction is given to the circuit courts in connection with proceedings brought to prevent and restrain opera- 
tions which are alleged to be in restraint of trade so that these courts have jurisdiction over, and hold and conduct the hearings in original proceedings to enforce the terms of the Sherman Anti-trust law, so-called, by virtue of which the United States has sought to break up combinations which have for their purpose the monopolization of different branches of trade and commerce, and the destruction of competition by such monopolies with persons and firms carrying on independent undertakings in the various lines of trade. Provision has been made by Federal statute for a system of arbitration between a carrier engaged in the transportation of interstate commerce and its employees. Jurisdiction is given by the statutes to the Federal courts to compel witnesses to attend and testify, and for the production of books, papers, and other documents before any board of arbitration which may be appointed under these laws. The proceedings involving the right of any person in whole or in part of Indian blood to participate in the allotment of land in severalty, which have been going on under the Interior Department for a number of years are also to be brought in the lower conrts. These courts also have jurisdiction in causes arising under the immigration laws of the United States, and also have appellate jurisdiction over decisions of United States Commissioners rendered under the terms of such laws. Special jurisdictional provisions are also made for the consideration of criminal cases by certain district courts so that their scope is extended over certain portions of the territory of the United States which would not otherwise be included within any judicial district.

A very important section of the aspect of the Federal procedure relates to the removal of causes which have begun in State courts to the appropriate courts of the United States. In any cause arising under the Constitution, laws or treaties of the United States where the lower conrts of the United States have original jurisdiction and in any suit against an officer or agent of the United States, or in a case where the United States is the real defendant in interest, the defendant may, upon showing the jurisdiction of the court in the case secure the removal of 
the case to a court of the United States. Provision is also made by which a defendant who is a resident of another State from that in which the action is brought, can secure the removal of the hearing of the case to the United States court, especially if he is able to aver that he will, because of local influence, be unable to secure a due and impartial hearing in the State courts. A similar removal can be secured when the question at issue before the State court relates to conflicting claims under land grants of two different States, or when it is alleged that the courts of the State cannot enforce a proper remedy, or when a proper remedy is denied in the State court. Upon an order of removal to the Federal Court being granted, the proceeding in the State court ceases and determines, and the case proceeds in the United States court as though it had originally been brought there. The clerk of the State court is required to furnish a transcript of the case upon the legal fee being tendered to him, or, if for any reason the record cannot be furnished to the Federal Court, the other side to that which has secured the removal can be required by to plead anew in the case.

Specific provisions are made as to the districts in which certain offenses may be punished or cases tried. The trial of all offenses committed upon the high seas will be conducted in the district in which the offender is found or into which he may be first brought. When an offense is begun in one district and completed in another, the prosecution may be brought in either district. Pecuniary penalties may be recovered either in the district where they accrue, or in the district where the offender is found. Taxes under the internal revenue law may be collected either in the district where the liability accrues or in that in which the person liable resides. Proceedings against property seized may be taken in any district court into which the property may be taken. Proceedings in tax cases may be brought in the district of which the defendant is an inhabitant or in that in which he has a regular place of business, and in the latter case an authorized agent may be served with the process. Proceedings against national bank associations are brought 
in the district in which the bank is located, and provision is made where there are two or more defendants that duplicate writs may be issued and the proceedings brought in either of the districts in which there is a defendant and the duplicate certified to the marshal of another district. In other cases than those which are provided by law, the proceedings must be brought in the district in which the subject matter of the action is located or where the offense was committed.

The United States Circuit and District Courts are held at the following places and times:

Alabama-Northern District: Circuit and district courts for northern division: First Tuesday in April and second Tuesday in October, at Huntszillle.

Circuit and district courts for southern division: First Monday in March and September, at Birmingham.

Circuit and district courts for castern division: Court at Amniston on first Monday in May and November.

Circuit and district courts for the western division: Court at Tuscaloosa on first Tuesday in January and June.

Alabama-Middle District: Circuit and district court: First Tucsday in May and December, at Montgomery.

A session of the district court is also held on the first Monday of each month, under rules adopted.

Alabama-Southern District: Circuit and district courts: Fourth Monday in Norember and first Monday in May, at Mobile; first Monday in May and first Monday in November, at Selma.

Alaska-Division No. I: At least four terms of court in the division cach year-two at Jnnean and two at Skagaiay-and the judge shall, as near January $I$ as practicable, designate the time of holding the terms during the current year. Terms set for calendar year 1906 as follows: At Skagzíay, commencing June 4, 1906, and November 12, 1906. At Junean, commencing Monday, November 26, 1906. Special term at Ketchikan, commencing October 15, 1906

Alaska-Division No. 2: Special term at Council cach year at a time fixcd by the court. A regular term as directed by act of June 6, I900, at St. Michael, commencing the third Monday in Iunc. Special terms held at Nome as the business requires. 
Alaska-Division No. 3: At least one term of court each year at Eagle City, beginning on the first Monday in July. Special terms at times and places as the judge or Attorney-General may direct.

Special terms have been held at Eagle, Rampart, Fairbanks, Nushagak, Valdez and Circle City.

Arizona-Supreme court: Second Monday in January each year at Phoenix.

First judicial district: Fourth Monday in April and fourth Monday in October, at Tucson.

Second judicial district: Fourth Monday in April and fourth Monday in October, at Tombstone.

Third judicial district: Third Monday in April and third Monday in October, at Plocnix.

Fourth judicial district: First Monday in May and First Monday in November, at Prescott.

Fifth judicial district: For the year 1906, last Monday in January and first Monday in October, at Solomonille; and thereafter, first Monday in April and first Monday in October, at Solomonville.

Arkansas-Eastern District: Circuit and district courts: Western division: First Monday in April and third Monday in October, at Little Rock.

Eastern division: Second Monday in Marcn and first Monday in October, at Helena.

Northern divisicn: Fourth Monday in May and second Monday in December, at Batesville.

Arkansas-Western District: Fort Smith division, Fort Smith: Second Mondays in January and June. Texarkana division, Texarkana: Second Mondays in May and November. Harrison division, Harrison: Second Mondays in April and October.

California-Northern District: Circuit court, at San Francisco: First Monday in March, second Monday in July, and first Monday in November. At Eureka: Third Monday in July.

District court, at San Francisco: First Monday in March, second Monday in July, and first Monday in November. At Eureka: Third Monday in July.

CALIFornia-Southern District: Circuit and district courts: Northern division, first Monday in May and second Monday in November, at Fresno.

Southern division, second Mondays in January and July, at Los Angeles.

Colorado-Circuit and district courts: At Denver, first Tuesday in May and November; at Pueblo, first Tuesday in April; at Montrose, second Tuesday in September. 
Connecticut-Circuit court: Fourth Tuesday in April, at Nea Haźcn; second Tuesday in October, at IIartford.

District court: At Now Haven, fourth Tuesdays in February and August; at Ilartford, fur.rth Tuesday in May, first Tuesday in December.

Delaware-Circuit court: Third Tuesdays in June and October at Wilmington.

District court: Sccond Tuesdays in January, April, June and September, at Vilmingtoil.

Uistrict of Columin-Times of holding courts.-Court of Appeals: First Tuesdays after first Moncays in Jamuary, April and October.

Supreme court, general term: First Mondays in January, April and October.

Circuit and crimina! courts: First Tuesdays in January, April and October.

Equity courts: First Tuesday in every month.

District court: First Mondays in January and July.

FLORIDA-Northern District: Times and places of holding courts.-Circuit and district courts: First Monday in February, at Tallahassce; first Monday in Narch, at Pensacola.

Florida-Southern District: Times and places of holding courts.-Circuit and district courts: Second Monday in February, at Tampa; first Mondays in Nay and Novenber, at Key IVest; first Monday in December, at Jacksonville; thirc. Monday in January; at Ocala; fourth Monday in April, at Miami; first Monday in April, at Fernandina.

Georgra-Northern District: Northern division, circuit and district courts: At Atlanta, first Mondays in October and second Mondays in March.

Western division, circuit and district courts: At Columbus, first Mondays in May and December.

Northwestern division, circuit and district courts: At Rome, third Mondays in May and November.

Eastern division, circuit and district conrts: At Athens, fourth Monday in April and first Monday in November.

Georgra-Southern District: Circuit court: First Mondays in May and October, at Macon. Sccond Monday in April and Thursday after first Monday in November, at Sazammah. First Monday in April and third Monday in November, at Augusta. Second Mondays in Junc and December, at Valdosta. Third Mondays in Junc and December, at Albany.

District court: First Mondays in May and October, at Macon. Second Tuesdays in May, February, August and November, at Sazannal. First Monday in April and third Monday in November, at Augusta. Second Mondays in June and December, at Valdosta. Third Mondays in June and December, at Albany. 
Hawail-District court, at Honolulu the second Mondays in April and October, and at Hilo the last Wednesday in January. Special terms may be held at such times and places as the judge nay deem expedicnt.

IDAHO-Circuit and district courts: Northern division, at Moscow, second Monday in May and fourth Monday in October.

Central division: At Boise, second Mondays in March and September.

Southern division: At Pocatcllo, second Monday in April and first Monday in Octoiver.

IllıNors-Northern District: Circuit and disirict courts: Eastern division, at Chicago, first Monday in July, third Monday in December.

Western division, at Frecport, third Mondays of April and October. "Adjourned terms" (sreated by rule of court): Chicago, first Monday in March, first Monday in May, first Monday in October.

Illino1s-Eastern District: Circuit and district courts: First Mondays in March and Scptember, at Danville; first Mondays in April and October, at Cairo; and first Mondays in May and November, at East St. Louis.

IllinoIs-Southern District: Circuit and district courts: First Monday in January and June, at Springficld; first Monday in September, at Quincy; third Monday in April and October, at Peoria.

All offenses committed in the northern division are tried at Peoria; in the southern at Springicld and Quincy.

Indiana-Circuit and district courts: First Tuesdays in May and November, at Indianapolis; first Mondays in January and July, at New Albany; first Mondays in April and October, at Ezansille; second Tuesdays in June and December, at Fort IVaync; third Tuesdays in April and October, at Hammond.

Iow:-Northern District: Circuit and district courts, Cedar Rapids division. Codar Rapids: First Tuesday in April and second Tuesday in September. Eastern division, Dubuque: Fourth Tuesday in April and first Tuesday in December.

Western division, Sioux City: Fourth Tuesday in May and first Tuesday in October.

Central division, Fort Dodge: Second Tuesday in June and November.

IowA-Southern District: Circuit and district courts, western division: At Council Bluffs, second Tuesday in March and third Tuesday in September.

Southern division: At Creston, fourth Tuesday in March and first Tuesday in November.

Eastern division: At Kcoknk, second Tuesday in April and third Tuesday in October. 
Davenport division: At Dazcnport, fourth Tuesday in April and first Tuesday in Uctober.

Central division: it Des lloines, second Tresday in May and third Tuesday in November.

Kansas-First division, circuit court: First Monday in June, at Leazen worth; fourth Nonday in November, at Topeka.

Circuit and district courts: First Monday in October and second Moday in January, at Kinsas City.

District court: Second Monday in April, at Topcka; sccond Monday in October, at Leazinzorth; second Monday in May, at Salina.

Second division, circuit and district courts: Second Mondays in March and September, at Wichito.

Third division, circuit and district courts: First Monday in May and second Monday in November, at Fort Scott.

KentuCkY-Eastern District: Circuit and distrirt courts: Frankfort, second Monday in . Warch and fourth Monday in September; Corington, first Monday in April and third Monday in October; Richmond, fourth Monday in April and second Nonday in November; London, second Monday in May and fourth Monday in November; Catlettsburg, fourth Monday in May and sccond Monday in Decenber.

KentUCKY-Western District: Louisille, second Monday in March and second Monday in October. Paducah, third Monday in April and third Monday in November. Oacnsboro, first Monday in May and fourth Monday in November. Bociling Grecn, third Monday in May and sccond Monday in December.

Loursiana-Eastern District: Circuit court: At New Orleans, fourth Monday in April and first Monday in Norember. At Baton Rouge, second Mondiays in April and November.

District court: At New Orleans, third Mondays in February, May and November. At Baton Rorge, sceond Mondays in April and Norcmber.

Louisiana- Western District: Circuit and listrict conrts: First Mondays in January and June, at Opelousas; fourth Mondays in January and June, at Alexandria; third Mondays in February and October, at Shrereport; first Mondays in April and October, at Monroc; third Mondays in May and December, at Lake Charles.

Mane-Circuit court: Third Tuesdays in April and September, at Portland.

District court: First Tucsdays in February and December, at Portland; first Tuesday in June, at Bangor; tirst Tuesday in September, at Bath. 
Maryland-Circuit court: First Mondays in April and November, at Baltimore; second Monday in May and last Monday in September, at Cumberland.

District court: First Tuesdays in March, June, September and December, at Baltimore; second Monday in May and last Monday in September, at Cumberland.

Massachusetts-Circuit court: Last Tuesday in February and third Tuesday in October. District court: Third Tuesday in March, fourth Tuesday in June, second Tuesday in September, and first Tuesday in December, at Boston.

Michigan-Eastern District: Circuit and district courts: Southern division at Detroit, first Tuesdays in March, June and November.

Northern division, at Bay City, first Tuesdays in May and October.

Terms of court at Port Huron in the discretion of the judge.

Michigan-Western District: Circuit and district courts: Grand Rapids (southern division), first Tuesdays in March and October; Marquette (northern division), first Tuesdays in May and September.

Minnesota-Circuit court of appeals: First Monday in May, at St. Paul, Minn. Circuit and district courts, first division: Third Tuesdays in May and November, at Winona.

Second division: Fourth Tuesdays in April and October, at Mankato. Third division: First Tuesdays in June and December, at St. Paul.

Fourth division: First Ttiesdays in April and October, at Minneapolis.

Fifth division: Second Tuesdays in January and July, at Duluth.

Sixth division: First Tuesday in May and second Tuesday in November, at Fergus Falls.

MississipPI-Northern District: Circuit and district courts: At Oxford, first Mondays in Jume and December; at Abcrdecn, first Mondays in April and October.

MississippI-Southern District: Circuit and district courts: At Jackson, first Mondays in May and November; at Vicksburg, first Mondays in July and January; at Biloxi, third Mondays in February and August; at Meridian, second Mondays in March and September.

Missouri-Eastern District: Eastern Division: Circuit court, at St. Louis, third Mondays in March and September. District court, at St. Louis, first Mondays in May and November.

Northern division: Circuit and district courts, at Hannibal, fourth Monday in May and first Monday in December.

Southeastern division: Circuit and district courts, at Cape Girardeau, second Mondays in April and October. 
Missocri-Western District: Joplin, second Mondays in January and June; St. Joscph, first Monday in March and third Monday in September; Jefferson City, third Mondays in March and October; Springficld, first Mondays in April and October; Kansas City, fourth Monday in April and first Monday in November.

Montana-First Mondays in April and November, at Helcna; first Tuesdays in February and September, at Butte; first Mondays in May and October, at Greatfalls.

Nebraska-Omaha, first Monday in May and second Monday in November; Lincoln, third Monday in January and first Monday in October; Hastings, third Monday in April; Norfolk, fourth Monday in April.

Nevada-Circuit court: A.t Carson City, third Monday in March and first Monday in November.

District court: At Carson City, first Mondays in February, May and October.

New Hampshire-Circuit court: Portsmouth, first Tuesday in May; Littleton, last Tuesday in August; Concord, second Tuesday in December.

District court: Portsmouth, third Tuesdays in March and September; Concord, third Tuesdays in June and December; Littleton, last Tuesday in August.

New Jersey-Circuit court: Fourth Tuesdays in March and September, at Trenton.

District court: Third Tuesdays in January, April, June and September, at Trenton.

New Mexico-Supreme court:At Santa $F e$, the first Wednesday after the first Monday in January.

First district: At Santa $F_{\mathcal{e}}$, United States court, first Mondays in March and September.

Second district: At Albuquerque, United States court, third Mondays in March and September.

Third district: At Las Cruces, United States court, first Mondays in April and October.

Fourth district: At Las Vegas, United States court, second Monday's in May and November.

Fifth district: At Roswell, United States court, third Mondays in April and October.

Sixth district: At Alamogordo, United States court, fourth Mondays in May and November.

New YoRK-Northern District: Circuit court: First Tuesday in April, at Syracusc; second Tuesday in February, at Albany; first Tuesday in December, at Utica. District court: Second Tuesday in February, at 
Albany; first Tuesday in December, at Utica; second Tuesday in June, at Binghamton; first Tuesday in October, at Auburn; first Tuesday in April, at Syracuse; and, in the discretion of the judge of the court, one term annually at such time and place within the counties of Saratoga, Onondaga, St. Lawrence, Clinton, Jefferson, Oswego, and Franklin as he may from time to time appoint.

NEW YoRK-Southern district: Circuit court: Last Monday in February, first Monday in April, and third Monday in October; and (criminal only) second Wednesday in January, March, May, October, and December, and third Wednesday in June, at New York City.

District court: First Tuesday in each month, at New York City.

NEw YoRK-Eastern District: Circuit and district courts: First Wednesday in every month, at Brooklyn.

NEW YORK-Western District: Circuit court: Second Tuesday in May, at Rochester; second Tuesday in September, at Canandaigua; second Tuesday in November, at Buffalo.

District court: Second Tuesday in January, at Elmira; second Tuesdays in March and November, at Buffalo; second Tuesday in May, at Rochcster; second Tuesday in July, at Jamestown; second Tuesday in October, at Lockport.

North Carolina-Eastern District: Circuit and district courts: At Washington, second Mondays in April and October; at Elizabeth City, third Mondays in April and October; at Newbern, fourth Mondays in April and October; at Wilmington, first Mondays after the fourth Mondays in April and October; at Raleigh, fourth Monday in May and first Monday in December.

North Carolina-Western District: Circuit and district courts: Greensboro, first Mondays in April and October; Stateszille, third Mondays in April and October; Asleville, first Mondays in May and November; Charlotte, second Mondays in June and December; Wilkesboro, fourth Mondays in May and November.

North Dakota-Circuit and district courts: First Tuesday in March, at Bismarck; third Tuesday in May, at Fargo; first Tuesday in July, at Devils Lake; second Tuesday in November, at Grand Forks; second Tuesday in October, at Minot.

OH10-Northern District: Circuit and district courts: On the first Tuesdays in February, April, and October, at Cleveland, for the eastern division, and the first Tuesdays in June and December, at Toledo, for the western division of the district. 
OHIo-Sonthern District: Circuit and district courts: Western division, first Tuesdays in February, April, and October, at Cincinnati; Eastern division, first Tuesdays in June and December, at Columbus.

Eastern division, first Tuesdays in June and Decmber, at Columbus.

OKLahoma-Eastern District: Circuit and district courts: Muskogec, first Monday in January; I'inita, first Monday in March; Tulsa, first Monday in April; Soult M/c.Alester, first Monday in June; Ardmore, first Monday in October; Chickasha, first Monday in November.

OKLAноma-Western District: Circuit and district courts: Guthrie, first Monday in January; Oklahoma City, first Monday in March; Enid, first Monday in June; Lawton, first Monday in October.

Oregon-United States circuit court: At Portland, second Monday in April and first Monday in October.

United States district court: At Porlland, first Mondays in March, July and November.

Pennsyluania-Eastern District: Circuit court: First Mondays in April and October, at Philadelphia.

District court: Sccond Mondays in March and June, third Monday in September, and second Nonday in Decomber; at Pliladelphia.

Pennsylrania-Middle District: Circuit and district courts: Scranton, fourth Monday in February and third Monday in October; Harrisburg, first Monday in May and first Monday in December; Williamsport, second Monday in Jamuary and second Monday in Jume.

Pennsylvania-Western District: Pittsburg, district court, first Monday of May and third Monday of October. Circuit court, second Mondays of May and November.

Eric, district court, third Monday of July and sccond Monday of January. Circuit court, third Monday of July and second Monday of January.

Porto Rico-San Juan, April and Octoher; Ponce, January; Mayaguez, special term immcdiately following January term at Ponce.

Rhode Island-Circuit court: At Prozidence, fourth Tuesday in May, and November 15 .

District court: At Prozidence. first Tueslays in February and August; at Newport, second Tuesday in May and third Tuesday in October.

Soutri Carolixa-Circuit court: First Tuesday in April, at Charleston; third Tuesdays in April and Octoher, at Greenille: fourth Tucsday in Novenber, at Coiumbia; first Tuesday in March, at Florence.

District Court: First Tuesdays in June and December, at Charleston; third Tuesdays in April and October, at Grecnitle: fourth Tucsday in November, at Columbia; first Tucsday in March, at Florence. 
South Dakota-Circuit and district courts: At Sioux Falls, first Tuesday in April and third Tuesday in October; at Aberdeen, first Tuesday in May and second Tuesday in November; at Deadwood, third Tuesday in May and first Tuesday in September; at Pierre, second Tuesday in June and first Tuesday in October.

Tennessee-Eastern District: Circuit and district courts: First Monday in March and second Monday in September, at Knoxville; first Mondays in April and December, at Chattanooga; and first Mondays in June and November, at Grecneville.

Tennessee-Middle District: Circuit and district courts: at Nashville, first Mondays in May and October.

TennesseE-Western District: Circuit and district courts: At Jackson, fourth Mondays in April and October; at Memphis, fourth Mondays in May and November.

Texas-Northern District: Circuit and district courts: At Dallas, second Monday in January and first Monday in May; at Fort Worth, second Monday in March and first Monday in November; at Abilene, second Monday in April and first Monday in October; at San Angelo, fourth Monday in April and third Monday in October.

Texas-Southern District: At Galveston, second Monday in January and first Monday in June; at Houston, fourth Mondays in February and September; at Laredo, third Monday in April and second Monday in November; at Brownswille, second Monday in May and first Monday in December; at Victoria, fourth Monday in November and first Monday in May.

Texas-Eastern District: Tyler, fourth Monday in January and fourth Monday in April ; Jefferson, first Monday in October and third Monday in February; Beaumont, third Moncay in November and first Monday in Aprii; Sherman, first Monday in January and third Monday in May; Paris, fourth Monday in October and second Monday in March; Texarkana, twice a year, at times to be fixed by judges.

Texas-Western District: Austin: Fourth Monday in January; second Monday in June. Waco: Fourth Monday in February; second Monday in November. El Paso: First Monday in April; first Monday in October. San Antonio: First Monday in May; third Monday in December. Del Rio: Fourth Monday in October; third Monday in March, each year.

UtaH-Circuit and district courts: Second Mondays in April and November, at Salt Lake; second Mondays in March and September, at Ogden.

VerMonT-Fourth Tuesday in February, at Burlington; third Tuesday in May, at Windsor; first Tuesday in October, at Rutland. In each year one of the stated terms of circuit and district court may be adjourned to meet at Newport. 
Virginia-Eastern District: Circuit and district courts: First Mondays in April and October, at Richmond; first Mondays in Nay and November, at Norfolk; first Mondays in January and July, at Alexandria.

Virginia-Western District: Circuit and district courts: At Charlottesville, the second Monday in January and the first Monday in July; at Roanoke, the third Monday in February and the third Monday in June; at Lynchburg, on the Tuesday after the second Monday in March and September; at Daniille, on the 'I uesday after the second Monday in April and November; at Abingden, on the Tuesday after the first Monday in May and October; at Harrisonburg, on the Tuesday after the first Monday in June and December; at Bigstonc Gap, fourth Monday in January and second Monday in August.

WAShington-Eastern District: Circuit and district court, castern division: At Spokane, First Tuesdays in April and September. Circuit and district court, southern division: At North Fakima, first Tuesdays in May and October; at Halla $\mathrm{H}$ alla, first Tuesdays in June and December.

Wasmington-Western District: Circuit and district courts: Northern division, at Seattle, first Tuesdays in May and November; western division, at Tacoma, first Tuesdays in February and July.

West VIRGINiA-Northern District: Circuit and district courts: Wheeling. first Tuesday in April and third Tuesday in September; Clarksburg, third Tuesday in April and first Tuesday in Oetoler; Martinsburg, second Tuesday in May, third Tuesday in October; Parkersburg, circuit court, second Tuesday in January and second Tuesday in June.

WEST VIRGixia-Southern District: Circuit and district courts; Huntington. first Tuesday in April and first Tuesday after the third Monday in September; Blucficld, first Tuesday in May and third Tuesday in October; Charleston, first Tuesday in June and third Tuesday in November; Lewisburg, second Tuesday in February. District court at Addison, first Monday in September.

Wisconsin--Eastern District: Circuit and district courts: First Monday in January and October, at Milatuker; scend Tuesday in June, at Oshkosh; first Tuesday in April, at Grecn Bay.

Wisconsin-Western District: Circuit and district courts: First Tuesday in December, at Madison; first Tuesday in June, at Eau Claire; third Tuesday in September, at La Crosse; third Tuesday in June, at Superior.

Special terms: At Madison, fourth Tuesday in June and first Tuesday in October. The clerk residing at Madison shall attend all terms of said courts at Eau Claire and Superior, as clerk thereof.

Wromisg-Circuit and district courts: At Cheyenne, sccond Mondays in May and November: at Ez'duston, second Tuesday in July, and at Sheridan, or in National Park at such dates as the court may order. 
THE CIRCUIT COURT OF APPEALS.

Under the provisions made for the appeal of cases from the inferior United States courts to the Supreme Court, the growth of Federal procedure due to the increase in population and the closer relations between the States brought about a great increase from year to year in the number of cases on the docket of the Supreme Court. The number of cases pending became so great that excessive delays were experienced in securing decisions on the mass of cases, and considerable hardship was experienced by litigants whose cases were of such nature that they could not be advanced for hearing, except as a last resort on the plea that they had frequently been passed over in behalf of cases which were deemed to have been of greater public importance. 'To meet the need, the Congress in I89I passed a law establishing a Circuit Court of Appeals. While there is no position of Judge of the Circuit Court of Appeals as such, the position appertains to the official position respectively of Supreme Court Justices or Circuit Judges and of District Judges of the United States.

There is in each circuit a Circuit Court of Appeals consisting of three judges, that is, that for each term of such court, three judges shall be assigned, but two of the three judges shall be a quorum to hold a sitting within the time of the term. The judges who are eligible to sit as members of the Circuit Court of Appeals are the Justices of the Supreme Court to whom the circuit has bcen assigned, the Judge or Judges of the Circuit Court, and the District Judges within the circuit in question. If the Supreme Court Justice does not appear, the senior Circuit Court Iudge presides. If the Circuit Court of Appeals is not nade up of a Justice of the Supreme Court and two Circuit Judges, one or more of the District Judges within the circuit shall be designated by the court to sit as Judges of the Circuit Court of Appeals. The restriction is, however, made that no Judge of the Circuit or District Court from whose ruling an appeal has come shall sit in judgment on the appeal.

In the act constituting the circuit courts of appeal, it was pro- 
vided that no appeal should thereafter be taken from a district court to any of the circuit courts, but that such appeals should be taken to the circuit courts of appeal and the appeals from circuit courts slould be divided between the Supreme Court and the Circuit Court of Appeal in the manner set forth in the act. It was provided that appeals could be taken directly to the Supreme Court irom districts and circuit courts as follows:

In cases where the jurisdiction of the court was at issue, in which case the question of the jurisdiction alone should go to the Supreme Court.

From the final sentences and decrees in prize causes; in cases of conviction of capital crime; in cases involving the application or construction of the Constitution of the United States or the constitutionality of any law or any treaty made by the United States and in cases in which the law of a State is claimed to be in contravention of the Constitution of the United States. Specific restriction was made of the right of appeal from the highest court of a State to the Supreme Court of the United States in cases in which the latter was given jurisdiction by the Constitution.

In all other cases the Circuit Court of Appeals is given the appellate jurisdiction, that is that an appeal should not be made to the Supreme Court in other cases than those named, but should be reviewed in consequence of an appeal or a writ of error by the Circuit Court of Appeals. Provision is, however, made that the Congress may by law change the allotment of appeals and send certain classes of cases to the Supreme Court on appeal should such action seem desirable for the public welfare. Provision has, and may be made also for the jurisdiction by the Circuit Court of Appeals over appeals from arbitrators and other semi-judicial bodies that may be established by law.

It is manifest that the object of relicving the pressure of business on the Supreme Court would not be accomplished if the Circuit Court of Appeals was made a medium or merely carrying cases between the inferior and the Supreme Court. It was therefore, made a court of final decision in cases arising under 
the patent laws, the revenue laws, the criminal laws, admiralty practice and in all cases where the jurisdiction is dependent on the fact that the parties to a controversy are not of the same state and under the jurisdiction of the courts of a State. In all cases not made final by these provisions an appeal to the Supreme Court from the Circuit Court of Appeals is made possible where the matter in controversy exceeds a thousand dollars in value.

While the above mentioned classes of cases are made final in the Circuit Court of Appeals so far as the litigants are concerned, they are not removed from the power or authority of the Supreme Court. The Circuit Court of Appeals may certify points of law on which it may desire instruction to the Supreme Court, and the Supreme Court may in its discretion direct the Circuit Court of Appeals to send up a case pending before it, and the Supreme Court may take up such case as if it was originally appealable.

In the revision of the law heretofore referred to, it is proposed to make the Circuit Court wholly a court of appeal, without original jurisdiction, so that the distinction between the Circuit Court and the Circuit Court of Appeals, would be abolished, together with the latter itself, making the Circuit Court the appellate court of the Federal procedure immediately above the court of original jurisdiction.

TIMES AND PLACES OF HOLDING CIRCUIT COURTS OF APPEALS.

First circuit: Annual term, first Tuesday in October; stated sessions, first Tuesday in every month; sessions for hearing cases, first Tuesday in January and October, and second Tuesday in April, at Boston, Mass.

Second circuit: Third Tuesday in October, at New York City.

Third circuit: First Tuesday in March and first Tuesday in October, at Philadelphia.

Fourth circuit: First Tuesday in February, first Tuesday in May, and first Tuesday in November, at Richmond, Va.

Fifth circuit: First Monday in October, at Atlanta. Ga.; third Monday in October, at Montgomery, Ala.; first Monday in Nov- 
ember, at Fort Worth, Tex, and third Monday in November, at New Orleans, La.

Sixth circuit: Tuesday after the first Monday of October, and adjourned sessions on the Tuesday after the first Monday of each month in the year, except August and September, at Cincinnati, Ohio.

Seventh circuit: First Tuesday in October. Term is divided into three sessions, beginning on first Tuesdays in October and January, and second Tuesday in April, at Chicago, Ill.

Eighth circuit: First Monday in Nay, at St. Paul, Minn.; first Monday in September, at Denver, Colo.; first Monday in December at St. Louis, Mo.

Nintl circuit: At San Francisco, Cal, annual term, commencing first Monday in October; adjourned sessions on first Monday in each month; calendar sessions for hearing of causes commence on first Monday in October, February, and May, respectively. At Seattle, WVash, annual term, second Monday in September, for hearing of causes. At Portland, Oreg., annual term, third Monday in September, for hearing of causes.

JUDGE OF TIIE COURT OF CLAIMS.

It is one of the attributes of sovereignty that it cannot be sued without its own consent. The fact that it is superior to the judicial power constitutes the essence of sovereignty and it is necessary for every government to constitute means by which the individual who decms himself aggrieved may seek the favorable action of the sovereign. In monarchical countrics, this lies in an appeal to the Crown, but in the United States the Congress entertains the appeal and provides relief through direct legislation, or by the Court of Claims in cases in which relief cannot be granted under general provisions of the statutes and which, were the cases matters of dispute between private parties, would be referred to the courts for adjudication. It is evident that a very considerable number of such cases would come up in the course of departmental practice, and in connection with government contracts, its disbursing of moneys, etc., and the 
task of determining the facts in each case would occupy a large portion of the time of Congress in judicial functions. There has, therefore, for more than half a century, been a tribunal designed to advise Congress of the facts as the basis of its own action in connection with private claims.

The Court of Claims was established by the act of February 24,1855 , and was constituted in its present form by the act of I863, with subsequent amendments. It consists of a Chief Justice and four judges, each appointed by the President by and with the advice and consent of the Senate, and each receiving $\$ 6,000$ a year except the Chief Justice, who receives $\$ 6,500$. It was originally intended that the court should sit in the Capitol in view of its junction as an assistant to Congress, and the Speaker of the House of Representatives was directed to allot rooms, but other provision has been made in the building formerly occupied by the Corcoran Art Gallery in Washington. The court holds one annual session beginning on the first Monday in December, and continuing until the completion of business. Three of the judges constitute a quorum and the concurrence of three is necessary for the decision of any case. The court appoints its clerks and other employes.

Nembers of Congress are forbidden to practice before the court whose decisions are stated to the heads of departments and auditing and controlling officers and also made the subject of a full report to Congress at the beginning of its December session. Any officer or employe of the Executive departments is forbidden to prosecute any claim which may come before the department or the Court of Claims which was pending while the officer or clerk was connected in the department, until two years after his retirement from the department.

The members of the Court of Claims have jurisdiction to hear and determine all claims founded on any law of Congress, any regulation of an Executive department, or on any contract expressed or implied and all claims which may be referred by either House of Congress. The court may also determine the force of all set-offs, counter-claims and claims for damages 
on the part of the United States against any person making a claim in the court.

Claims of pay, quartermaster and subsistence officers for relief from responsibility for the loss of funds, vouchers and papers whether by capture by opposing forces or otherwise are heard by the Court of Claims and it is given exclusive jurisdiction over proceedings brouglit by owners of abandoned property seized by Treasury officials during the Civil War, and for which the owners seek to recover.

Any petition or bill praying for the satisfaction of a private claim against the government may be referred by the House in which it is pending to the Court of Claims for a finding of facts, equitics or the amount due, which finding is to be reported back to the House in which the claim is pending. This procedure is followed not only in individual cases, but in classes of cases.

Heads of departments and the accounting officers of the Treasury have the right to refer cases to the Court of Claims for a finding when the claim involved amounts to more than three thousand dollars, and where it is one of a class of cases susceptible to similar treatment or when its decision involves an important precedent. Aliens whose governments alluw United States citizens the right of similar proceclings may prosecute claims against the United States in the Court of Claims. All claims are barred unless filed within six years after the claim accrues, exceptions being made as to married women, minors and others subject to disabilities.

The court has power to establish rules, to punish for contempt and appoint commissioners, administer oaths and perform similar duties. Claimants must show the actual ownership of the claim and must sustain proof that they have not been engaged in, or abetted rebellion against the United States. The court has power to fix fees for taking testimony before its commissioners and also to call on the departments for information desired in connection with any claim. It may also decline to take testimony in any case in which the petition does not, 
in the opinion of the court, contain facts which furnish ground for belief. Subpoenas issued by the Court of Claims for the taking of testimony have the same effect as if issued from a district court. The court has power to order a claim forfeited for fraud, if it determines that such practice has been effected or attempted, and to permanently bar the prosecution of the claim.

Upon final judgments by the court or affirmation of appealed judgments, the amounts found due are payable out of any general appropriation made for the satisfaction of private claims. Specially referred claims by Congress for report of facts and findings are not final judgments, but depend on the legislative action for satisfaction. Findings with relation to accounts of officers are certified to the accounting officers of the Treasury. Judgments for the payment of which there is no appropriation are certified by the Secretary of the Treasury to Congress in connection with the annual estimates when they are not appealable or have been affirmed, and bear interest at the rate of four per cent. until appropriation is made for payment. Final judgment against a claim acts as a permanent bar for its subsequent prosecution in any respect.

There are two principal classes of claims that have been referred en masse by the Congress to the Court of Claims for investigation and report. These are for supplies and stores taken by or furnished to the armed forces of the United States during the Civil War and known as "Bowman Act" cases and those for vessels and goods captured and forfeited or destroyed by the French prior to the convention with the French republic in I8oo. Under the Bowman act the court is expressly prohibited from considering claims for property destroyed or damaged during the Civil War although Congress itself has taken up a considerable number of such claims, and in claims for supplies the loyalty of the claimant during the war must be affirmatively established. Under the statute formerly in effect, no interested person could be a competent witness in behalf of a claim. This provision was repealed by the Bowman act which 
provides that no person shall be excluded as a witness on account of interest.

The act relating to French spoliation claims the cases were specifically restricted to those under the convention before named, and the Secretary of State was directed to secure evidence abroad for the use of claimants and to turn it and the files of the department relating to these matters over to the files of the court. Claims were to be presented within two years (period closed 1887 ) and the finding of the court is stated to be advisory only, and Congress did not commit itsclf to the payment of the claims. In the Bowman act, on the other hand, the reports of the court are held to exist from session to session until the appropriation is made for the payment of the same. In several years appropriations have been made for the payment of French spoliation claims, but there is a reluctance to include these payments in the annual appropriations and such payments as have been made have been practically forced as a condition of the payment of the awards under the Bowman act. By one of the acts appropriating money for the payment of French spoliation claims it was provicled that the payments should be made to next of kin of those who sustained the losses and not to assignees in bankruptcy.

By subsequent legislation to that above summarized district courts are given concurrent jurisdiction with the Court of Claims over claims amounting to one thousand dollars and circuit courts from one to ten thousand dollars. The Attorney General reports to Congress adverse decisions in such claims.

In r89 I the work of examining into and adjudicating the claims arising out of Indian depredations was placed under the jurisdiction of the Court of Claims. The Intcrior Department had previously been authorized to take up the work and still retains a division of Indian depredations having other than the judicial functions. The claims heard include those for property of citizens of the United States taken or destroyed by Indians who at the time were in amity with the United States and in cases where no just cause or provocation was given by the owner 
or agent in charge. Judgments in these cases by the Court of Clains are made final unless the court shall grant a rehearing and upon the decree of the court the amount found to be payable is taken from the funds of the tribe whose members were responsible for the damage or from the Treasury and charged against any future payments which may become due the tribe from the United States. The Attorney General certifies to Congress such amounts due as there are no tribal funds to satisfy and appropriation is made for payment, charge against the tribe being made as before stated.

TERRITORIAL JUDGES.

In the organic act establishing each of the Territories, provision was made for a judiciary consisting, in most of the Territories, of a Supreme Court and district courts, with justices of the peace. An exception to this organization was made in the case of Alaska which has a Federal judiciary of three United States district judges, while in Hawaii a circuit court is added to the establishment. Each of the Territorial Judges is appointed by the President by and with the advice and consent of the Senate, and holds office for a term of four years. The inclusion of Oklahoma and the Indian Territory in a new State reduced the number of territorial judicial establishments to those in Arizona, New Mexico, Alaska, Hawaii and Porto Rico. In the first two, Judges have a dual capacity in that they pass upon questions relating to the Constitution and laws of the United States as well as on those relating to the laws of the Territory. It is generally provided that certain days of a term shall be devoted to the consideration of United States business, the balance of the term being devoted to the business of the Territory. Appeals may be had from the Territorial courts to the Supreme Court of the United States, as stated under the heading of the Supreme Court. In addition to the judges, appointments for service in the Territorial courts of District Attorneys, Clerks, and United States Marshals are made in a similar manner to those appointed for the other United States circuit and district courts. The court 
establishment of the District of Columbia consists of the usual police courts, with six justices of the peace for civil business, the term of each justice being four years, at a salary of $\$ 2,000$ each per annum. They are appointed by the President and are assigned to particular sub-districts of the District of Columbia. The first court of record in the District of Columbia is the Supreme Court, consisting of a Chief Justice and five associate justices, at a salary of $\$ 5,000$ each, from whose decisions appeals can be had to a Court of Appeals, consisting of one Chief Justice, at $\$ 7,500$ and two associate justices at $\$ 7,000$. These courts have jurisdiction in all matters arising under the laws of the United States and the District Code.

\section{UNITED STATES COMMISSIONER.}

United States Commissioners occupy a relative position in the Federal judicial systen to that of the petty courts of the judiciaries of the States

The position was established in 1896 by an act abolishing the former commissioners of the circuit courts and which conferred the powers of such commissioncrs on the United States Commissioners hereinafter described. Commissioners are now appointed by the district courts and in such numbers as the courts may decm necessary to the transaction of the buisiness, and to the performance of the duties imposed. When a commissioner is appointed, the appointment must be recorded in the court and the Attorney General notified. The commissioners hold their offices for four years but are subject to removal at any time by the court. They are paid by fees which are prescribed by law and which consist of individual fees for the preparation of papers and of a per dien fee of five dollars when a hearing is held. Other specific fees are also fixed for attendance at court and for particular services. No clerk of a United States court or deputy clerk can be at the same time a United States Commissioner unless with the permission of the Attorney General and no United States attorney or marshal or their deputics or subordinate officers of subordinate officers of United States 
courts can be United States Commissioners. Commissioners are authorized to administer oaths and to issue warrants for offenses against the United States, to cause offenders to be arrested and imprisoned or bailed for trial and to order the removal of offenders to other districts. They may hold a person to security of the peace and good behavior and may carry out and enforce the awards and degrees of the consul of any foreign nation sitting in judgment between the captains and crews belonging to his own nation and being in the district for which he is the consul. In case of absence of a consul or in any case in which differences between foreign captains and crews are submitted to him for consideration he has the power to enforce his own decrees by ordering imprisonment if necessary until the decree is complied with. Commissioners are empowered to institute proceedings under the law relating to the preservation of the civil rights of citizens and the district courts are empowered and directed to appoint such number of commissioners as may be neccessary to speedily hear such cases, this provision being a part of the machinery erected for the enforcement of the legislation based on the constitutional amendment intended to secure the rights of citizenship to former slaves. Commissioners were also given power to enforce the statutes with relation to the elective franchise and were empowered to appoint persons to execute warrants thereunder. This provision was taken advantage of in attempts to secure pure elections where fraud was anticipated, means being taken to bring violations of law to the immediate attention of commissioners for the purpose of securing a speedy punishment. These provisions were, however, repealed several years ago. Commissioners have power to issue search warrants authorizing revenue officers to search premises where frauds on the revenue are alleged to have been committed.

Complaints of the violation of the Chinese exclusion law are heard before United States commissioners who receive a per diem of five dollars for conducting such hearings. The Commissioner before whom the complaints shall be heard is designated 
by the United States District Attorncy for the district and complaints can only be sworn to by certain designated officials unless the District Attorney gives his consent to making the complaint. United States commissioners are also empowered to receive affidavits and final proofs in matters relating to the allotment of the public lands when it is impossible for the parties at interest to visit the land offices and chicf justices of territorial courts are empowered to appoint commissioners for this purpose who must not resicle within thirty miles of a land office or another commissioner.

$$
\text { COMMISSIONERS' FEES. }
$$

For drawing a complaint, with oath and jurat to same, fifty cents.

For copy of complaint, with certificate to same, thirty cents.

For issuing warrant of arrest, or search aurrant, seventy-five cents.

For issuing a commitment and making copy of the same, one dollar.

For entering a return, fiftcen cents.

For issuing subpoena or subpoenas in any case, with five cents for each necessary witness in addition to the first, twenty-five conts.

For drawing a bond of defendant and sureties, taking acknowledgment of same, and justification of sureties, seventy-five cents.

For administering an oath (except to witness as to attendance and travel), ten cents.

For recognizance of all witnesses in a case, when the defendant or defendants are held for court, fifty cents.

For transcripts of procecdings, when required by order of court and transmission of original papers to court, sixty cents.

For copy of warrant of arrest, with certificate to same, when defendant is held for court and the original papers are not sent to court, forty cents.

For order in duplicate to pay all witnesses in a case: For first witness, thirty cents, and for each additional witness, five cents, and for oath to each witness as to attendance and travel, five cents.

For hearing and deciding on criminal charges, and reducing the testimony to writing when required by law or order of court, five dollars a day for the time necessarily employed: Prozided, That not more than one per diem shall be allowed in the case, nuless the acconnt shall show that the hearing conid not be completed in one day, when one additional per diem may be specially approved and allowed by the court: Provided further, That not more than one per diem shall be allowed for any one day: Prozided further, That no per diem shall be allowed for taking a bond or recognizance and passing on the sufficiency of the bond or recognizance and the sureties thercon, when the bond or recognizance was taken after the defendant had 
been committed to prison upon a final commitment, or has given bond, or been recognized for his appearance at court, or when the defendant has been arrested on a capias or bench warrant, or was in custody under any process or order of a court of record.

For the examination and certificate in cases of application for discharge of poor convicts imprisoned for non-payment of fine, or fine and costs, and all services connected therewith, three doliars;

For attending to a reference in a litigated matter, in a civil cause at law, in equity, or in admiralty, in pursuance of an order of the court, three dollars a day;

For taking and certifying depositions to file in civil cases, ten cents for each folio;

For each copy of the same furnished to a party on request, ten cents for each follo;

For hearing and deciding a case arising under the Chinese-exclusion laws, five dollars;

For services in proceedings under Title sixty-four, entitled "Civil Rights," a fee of ten dollars in each case, inclusive of all services incident to the arrest and examination;

For services in cases of extradition under treaty stipulation or convention between the Government of the United States and any foreign government, the follcwing and no other fees or compensation shall be allowed to or received by them:

For administering an oath, ten cents;

For taking an acknowledgment, twenty-five cents;

For taking and certifying depositions to file, ten cents for each folio;

For each copy of the same furnished to a party on request, ten cents for each folio;

For issuing any warrant or writ, and for any other service, the same compensation as is allowed clerks for like services;

For issuing any warrant under the tenth article of the treay of August ninth, eighteen hundred and forty-two, between the United States and the Queen of the United Kingdom of Great Britain and Ireland, against any person charged with any crime or offense as set forth in said articles, two dollars.

For issuing any warrant under the provision of the convention for the surrender of criminals between the United States and the King of the French concluded at Washington November ninth, eighteen liundred and fortythree, two dollars;

For hcaring and deciding upon the case of any person charged witb any crime or offense, and arrested under the provisions of any treaty or convention, five dollars a day for the time necessarily employed;

For any other service, the same compensation as is allowed to commissioners in other criminal cases for like services. 


\section{REFEREES AND TRUSTEES IN BANKRUPTCY.}

One of the powers delegated to the Congress by the States was that of making uniform laws to regulate bankruptcies throughout the Uniter States. This power was exercised only in part, when at all, during many years, and there were numerous State laws which remained in force until the enactment of a general bankruptey law in 1898 . This law vested the power of adjudication of cases of bankruptcy in the district courts of the United States in the several States and in the United States Territorial Courts, and as the details of the business of administering bankrupt estates would be ton great a burden on the regular judges, provided machinery for the purpose. Upon the filing of a petition in bankruptcy, whether voluntary or involuntary, the case may be sent by the Judge to a referee, one of which officials, at least, is to be provided for each county in which the services of a referee are necessary. The appointment of referees and the fixing of the territorial limits within which they have jurisdiction is in the hands of the District Judge sitting as a court of bankruptcy. It may be remarked that the functions of the Iudge as such a court may be excrcised in vacation as well as in term time. A referee is appointed for two years, but subject to removal for lack of need of his services or for other causes. He must be competent to pass on the questions reiating to bankruptcy which are brought before him, must hoid no other office of profit under the United States or any State (xcept those of Commissioner of Deeds, Justice of the Peace, Master in Chancery or Notary Public. Ife must not be related within the third degree to the Judge of any Bankruptcy or Appellate Court of the district in which appointed and must reside within the limits of his territorial jurisdiction. Referees have jurisdiction subject to the review of the Judge of the Bankruptcy Court to adjudicate or dismiss any petitions which may come before them by reference on account of the inability of the Judge of the Bankruptcy Court to act, the petitions being referred to the referee direct by the Clerk of the Court in such cases. By reference from the courts, in addition to their own adjudication of cases, they hear witnesses, require the 
production of documents, take possession of, and release the property of bankrupts and perform all acts which would be required of the Judge of the Court of Bankruptcy as preliminary to the final composition of the claims or the discharge of the bankrupt. The latter functions are reserved for the court and the duties of the referees are those of deciding preliminary questions and of carrying out the decrees of the court. Thus the duties of referees are to declare dividends from bankrupt estates and prepare and deliver to trustees dividend sheets showing the dividends declared and to whom payable, examine schedules of property and lists of creditors, require amendments when necessary, make up the records in the cases for the final consideration of the judges and prepare and file all papers with the clerks of the courts on the final decision of the case. As compensation the referee receives an initial fee of ten dollars in all cases, except those in which a voluntary bankrupt is released from the fees, for each petition filed and one per cent. on dividerds paid on a bankrupt estate, or one-half of one per cent. on the amount paid to creditors on the confirmation of a composition. Proceedings before referees are invested with the same dignity as proceedings before a court by virtue of provisions for contempt proceedings, for ptinishment of offenses against referees, and the records of referees are required to be kept in the same manner as those of United States equity courts. They are forbidden to be interested in any way in the estate in adjudication.

Trustees in bankruptcy may be either one or three in number in each case. They are elected by the creditors at their first meeting, and if not so elected are appointed by the court. Trustees must be competent to periorm the duties of the care of a bankrupt's estate. They must reside in the district in which they are appointed, but corporations which have an office in the district may act in the capacity. Trustees are placed in charge of bankrupt estates and are required to reduce to money as soon as practicable all assets of the estate and to pay over to the estate all interest that may accrue on the same. They must deposit money received and pay it out only on check or draft. They are 
required to furnish information about the estates to the parties in interest and to lay final accounts before creditors' meetings and the court. They pay out the amounts due as dividends in accordance with the decrees of the court and which are passeri by the court on the accounts of the trustees as to the assets on hand and in accordance with the schedule of debts found to be correct by the referees. The trustees in effect administer the bankrupt estate for the benefit of the creditors and retain all the bankrupt's property until he is released by the court when upon the final order of the court such disposition of the property remaining is made as may be in accordance with the terms of the composition or discharge. When there are three trustees, the consent of two is required to make a transaction valid. Referees and trustees are both required to give bonds, the former in a sum fixed by the court, not to exceed five thousand dollars, and the latter in such sum as the creditors may fix. The latter bond is also susceptible of increase by vote of the creditors.

Trustees receive as compensation a fee of five dollars for every petition filed in which the fee has not been released and commissions as may be allowed by the courts, not to exceed three per cent. of the first five thousand dollars, two per cent. on the second five thousand and one per cent. on amounts involved in bankrupt estates of over ten thousand dollars. Where there are three trustees, the division of the compensation is made by the court who may also withhold the compensation from a trustee who has been removed for cause. 


\section{CHAPTER VIII.}

\section{STATE DEPARTMENT.}

The State Department may be likened to an artificial person, whose head is in Washington and whose body lies abroad So small a part of the organization of the department is located at the Capitol that the State Department in Washington is hardly more than a correspondence bureau, by which the policies and views of the Secretary of State, acting under the direction of the President, are made known to the diplomatic representatives of the United States and foreign countries. The chief function of the organization in Washington thus naturally relates to the proper preparation and care of documentary evidence of negotiations on foreign affairs. The details of the correspondence of the department are under the direction, first, of the chief clerk, who acts as a general directive and distributive official, and secondly, under the direction of several bureaus. The Diplomatic Bureau has charge of correspondence with diplomatic officers, foreign and of the United States, and receives routine correspondence and prepares answers to the same, and prepares, under the direction of the Secretary of State and the assistant secretaries, the various notes, memorandums, treaties and other diplomatic documents framed during the course of the various negotiations. The more important of the communications go, of course, at once to the Secretary or assistant secretaries, but a large amount of the diplomatic correspondence is conducted by the Diplomatic Bureau, subject to the approval and signature of the higher officers of the department. As so considerable a portion of the correspondence is in foreign languages, a corps of translators of all the diplomatic tongues is attached to the bureau. The Consular Bureau has charge of all correspondence relating to United States consuls, and conducts the great 
mass of correspondence necessary for the direction of the commercial representatives of the United States abroad. Its work has to do entirely with commercial and manufacturing interests.

Practically all the administrative activities of the State Department, with the exception of matters relating to accounts, appointments and passports, center about the Bureau of Indexes and Archives. It is, of course, of vital necessity that all papers relating to a given diplomatic subject should be immediately available, not only those of the current correspondence, but those which relate to historical phases of the matter as well. An elaborate system of indexing which differentiates subjects to a greater extent than probably in any other department, is in effect in this bureau. While the correspondence and records of the earlier years of the history of the State Department which were kept in record books, were not as closely indexed as is done under the present system, continual work is being carried on with a view of making close and accurate indexes of every diplomatic transaction in the history of the United States sn far as the records have been preserved.

The chief of the Bureau of Appointments of the State Department, besides his work in connection with the department proper, is also custodian of the records relating to the appointment of Cabinet officers and issues the certificates of office to the members of the President's official family. He is also charged with the matters relating to the appointment of consuls and diplomatic officers, together with the records pertaining to the subordinate officers of the diplomatic and consular corps.

Commissions and exequaturs are prepared in this office, which handles, also, the details of extradition matters, and has the charge of matters relating to consular bonds. This office has also the custody of the official cipher code and enciphers and deciphers telegraph messages and has also the custody of the Great Seal of the United States, used in authenticating commissions and other documents.

The chief of the Bureau of Accounts of the department revises and audits the accounts of diplomatic and consular repre- 
sentatives and is the disbursing and accounting officer of the various appropriations under the department.

The chief of the Bureau of Rolls is the custodian of all the laws passed by the Congress. These measures, including joint resolutions, are printed on parchment, and after being signed by the President, are transmitted to the Bureau of Rolls of the State Department for preservation. Authenticated copies of the laws as they appear in these prints are made for the Government Printing Office and serve as a basis for all the publications of the laws of the United States, whether publicly or privately printed. The chief of the Bureau of Rolls is also the librarian of the department and is in charge of the library, which has a large collection of diplomatic literature.

The archives of the Revolutionary period of American history embracing those of all branches of the pre-constitutional government are in the custody of this bureau, which also receives and preserves the archives of all international commissions.

The chief of the Bureau of Citizenship of the State Department has exclusive control, under the direction of the Secretary of State, of matters relating to the issuance of passports to American citizens, and the details relating to the determination of citizenship questions in foreign countries. The principal immediate business of the bureau consists in issuing passports to American citizens who desire to travel abroad. In order to secure such a passport an application must be made in a form prescribed, establishing the citizenship of the applicant and verified by an oath before a competent official, or, if the application is made in person, before the chief of the Passport Bureau. A small fee is charged for the issuance of a passport, and in addition to this service the chief of the bureau issues certificates attesting the signature of various officials of the United States when such certificates are needed for the purpose of making documentary evidence competent in foreign courts of law. For the purpose of authenticating the passports and certificates, the great seal of the United States is used. An important function of the duties of the chief of this bureau relates to instructions to diplomatic 
representatives abroad as to the issuance of passports. Under the American system of naturalization considerable numbers of persons resident in comtries in which there are unsettled social conditions have secured a colorable title to American citizenship and under such title have claimed the protection of the United States. Their claims of citizenship being questioned by the governments which had jurisdiction by reason of their birth, conflicts have arisen which, together with the fact of the number of persons permancntly resident abroad but maintaining American citizenship, resulted in the legislation of 1907 relative to United States citizenship. Under the act referred to, in addition to the general passport provisions for citizens, passports good for six months anch not rencwable may be issued to a person who has made a declaration of intention to become an American citizen and who has resided in the United States at least three years. Such a passport, however, does not entitle the holder to protection against the government of the country of which he was a citizen prior to making his declaration of intention. A definite statement is made of cxpatriation against any American citizen who has been naturalized in any foreign country or who has taken an oath of allegiance to any foreign State. Any American woman who marries a forcigner takes the nationality of her husband and when any naturalized citizen shall have resided for two years in the forcign state from which he came or five years in any other foreign State, it is to be presumed that he has ceased to be an American citizen, but he may overcome such a presumption on the prescntation of satisfactory evidence to the diplomatic or consular officer of the United States under rules and regulations prescribed by the State Department. No American citizen, howcver. is allowed to expatriate himself when the United States is at war. If an American woman has lost her citizenship by marrying a foreigner, she may rosume her citizenship on the termination of the marital relation by registering as an American citizen, within one year, with a consul of the United States, or by residing in the United States. A foreign woman may acquire American citizenship by marriage to an American, 
and if she continues to reside in the United States after the termination of the marital relation, or if she registers before a United States consul while residing abroad, may retain the citizenship so secured. A child born within the United States of alien parents becomes a citizen by virtue of the naturalization of its father, provided the naturalization takes place during the minority of the child. Children born outside of the United States and who are entitled to the privilege of citizenship, must, in order to receive the protection of the United States Government, register on reaching the age of eighteen at a United States Consulate, stating their intention of residing in and becoming citizens of the United States and must take the oath of allegiance on reaching the age of $2 \mathrm{I}$.

In order to receive a passport it is only necessary that a person shall have a genuine citizenship in the United States, the regulations and provisos being intended to prevent the issuance of the documents which are, in effect, certificates of citizenship, to persons not entitled to them. The applicant is required to establish his citizenship by affidavit as shown by the rules for application which follow. In the case of a native born citizen affidavits as to birth suffice. Naturalized citizens and those claiming citizenship under them must exhibit the naturalization certificate. Residents of insular possessions must show that they do not owe allegiance to any other power.

A passport expires two years from the date of its issuance. A new one will be issued upon a new application, and, if the applicant be a naturalized citizen, the old passport will be accepted in lieu of a certificate of naturalization, if the application upon which it was issued is found to contain sufficient information as to the naturalization of the applicant.

APPLICATIONS FOR PASSPORTS.

A person who is entitled to receive a passport, if within the United States, must make a written application, in the form of an affidavit, to the Secretary of State. The application must be made by the person to whom the passport is to be issued and 
signed by him, as it is not competent for one person to apply for another.

The affidavit must be attested by an officer authorized to administer oaths, and if he has an official seal it must be affixed. If he has no seal, his official character must be authenticated by certificate of the proper legal officer.

If the applicant signs by mark, two attesting witnesses to his signature are required. The applicant is required to state the date and place of his birth, his occupation, the place of his permanent residence, to what country or countries he intends to travel and within what length of time he will return to the United States with the purpose of residing and performing the duties of citizenship.

The applicant must take the oath of allegiance to the Government of the United States.

The application must be accompanied by a description of the person applying, and should state the following particulars, viz.:

Age, — ; stature, — feet _ - inches (English measure); forehead, —; eyes, —; nose, —; mouth, - ; chin, — ; hair, —— complexion, face, -

The application must be accompanied by a certificate from at least one credible witness that the applicant is the person he represents himself to be, and that the facts stated in the affidavit are true to the best of the witness's knowledge and belief.

By act of Congress approved March 23, I888, a fee of one dollar is required to be collected for every citizen's passport. That amount in currency or postal money order should accompany each application made by a citizen of the United States. Orders should be made payable to the Disbursing Clerk of the Department of State. Drafts or checks will not be accepted.

Applications for passports by persons in Porto Rico or the Philippines should be made to the Chief Executives of those Islands. The evidence required of such applicants is the same as that required of applicants in the United States. 
Citizens of the United States who intend visiting Russian dominions should have their passports visaed by a Russian diplomatic or consular officer resident in the United States.

Jews are not admitted to Russia without special permission obtained from the Russian Government.

Citizens of the United States who intend visiting Turkish dominions (except Egypt) should have their passports visaed by a Turkish consular officer resident in the United States.

This may be performed at the following places:

For Russia: At Washington, San Francisco, Chicago and New York City.

For Turkey: At San Francisco, Washington, Chicago, Boston and New York City.

The Department of State does not act as the intermediary in securing visas. Application should be made direct by the holder of the passport.

The chief of the Bureau of Trade Relations of the State Department constitutes the connection between the Department of Commerce and Labor and the Consular Service. Formerly all matters relating to foreign markets furnished as information by United States consuls were circulated by the Department of State as a portion of its work. Upon the institution of the Department of Commerce and Labor, however, the major portion of the work was assumed by a bureau of that department, and the duty now performed in this connection by the Department of State consists in securing the close connection of the consular service with the distributing agency in the Department of Commerce and Labor. The duty of the chief of this bureau consists of the preparation of directions to consuls as to their reports and the revision of such reports and their transmission to the above department and such others as may be interested.

THE DIPLOMATIC SERVICE.

The diplomatic service of the United States, as is the case with diplomatic service of all nations, includes public functionaries 
whose positions are regarded as of particular dignity, importance and distinction. The highest class of diplomatic officers, embassadors, extraordinary and plenipotentiary, are deemed to be direct and personal representatives of the President in exercising his function of administering the forcign affairs of the nation. Diplomatic officers of the United States are of three grades: Embassadors, extraordinary and plenipotentiary; envoys extraordinary and ministers plenipotentiary, and ministers resident. The particular distinction of the embassador has already been noted, that he represents the immediate person of the Chicf Executive and corresponds in rank and importance to embassadors of foreign nations who are regarded as the personal representatives of the reigning sovereigns. The particular distinction of an embassador is his privilege of immediate access to the sovereign power. Envoys extraordinary and ministers plenipotentiary are diplomatic officers who are charged with some immediate negotiation or given definite or general powers in treaty making. They do not, hovever, sectre the right of direct communication with the sovereign and conduct their negotiations with the foreign office of the nation to which they are assigned. Ministers resident are diplomatic officers who reside at the seats of government of nations to which they arc accredited for the general purpose of transacting the busincss of the mation with the office of the foreign nation to which they are accredited which is charged with the execution of foreign affairs. The class of the diplomatic agent who shall be accredited to a foreign nation is determined by the rank of the diplomatic officer sent by that nation to the United States. Thus, if a nation sends an embassador, the United States is expected to send an embassador in return, and if a nation which has previously sent a minister to the United States desires to raise the rank of its diplomatic officer to that of embassador, it is proper for the United States to make a similar promotion in turn. It has sometimes happencd that a foreign nation desiring to raise the rank of its representative in the United States has not received the assurance of reciprocity in the matter which would cnable the original pur- 
pose to be carried out, as from motives of economy, the United States has desired to limit the number of embassadors abroad. The peculiar characteristic of diplomatic officers is the privilege of immunity which accompanies them. This is a survival of the historic tradition of the immunity of a herald or particular messenger of the commander of an armed power. His person is inviolable from arrest on any civil or criminal process, and the personal goods and papers belonging to him in the character of a diplomat cannot be attached. This immunity also attaches to his servants, and it is usual to require a registry of the persons attached to the embassy or legation in order that those who are entitled to this immunity may be accurately known. It does not follow from the foregoing that an embassador or minister or a member of the suite attached to either can violate just laws and regulations with impunity. A remedy against such violation is to be had and has frequently been applied in the practice of indicating to a foreign government when any person of the diplomatic corps becomes obnoxious to the country in which he is located, and it is the universal custom upon receipt of such representation to recall the offending person.

Diplomatic officers are appointed by the President, by and with the consent of the Senate. They are furnished by the President with letters of presentation to the reigning sovereign or other head of the country to which they are accredited. It is usual for notice to be given in advance to the foreign country of the name of the person proposed as an embassador or minister in order that it may be understood before appointment is effected that he will be persona grata to the country in which he is to serve. The diplomatic officer usually gives notice to the foreign government of his proposed entrance into the country in which he is to serve in order that the usual privilege of free entrance of his goods without duty being imposed may be granted him. It sometimes occurs that it will be necessary for the embassador or minister to pass through a third country, in which case the courtesy of free passage for the dutiable goods belonging to him may be extended. Upon arrival in a foreign country the embas- 
sador or minister presents his credentials to the head of the State at the carliest possible moment, making arrangements with the foreign office for the details of the interview with the sovereign and conforming to the etiquette prescribed in the customs of the country. Depending on circumstances, a retiring minister either presents his letter of recall before his successor's arrival, or presents his successor, at the same time offering his own letter of recall. The presentation of a new embassador or minister is made the occasion of a ccremonial address, a copy of which must be furnished to the forcign office in advance in order that a suitable reply may be prepared. Copies of both addresses are sent to the State Department for preservation in the archives of the diplomatic service. A diplomatic representative is required to omit no occasion to secure and maintain the fricndliest personal and social relations with the officials of the government to which he is accredited, and with other members of the diplomatic corps at the place in which he resides. His official duties begin on the day of his reception by the chief of the State to which he is accredited. Should, however, this audience be delayed for some time, it is possible, by temporary arrangement with the foreign office, to secure recognition for a new diplomatic representative, so that business may be transacted pending the formal audience with the sovereign. In certain instances the office of consul general may and has been added to that of minister resident. In such cases the official is required to keep transactions in the different capacities entirely scparate. The diplomatic function is held to be superior in character, and if thcre is objection on the part of the forcign government to the service in both capacities, a vice-consular office is directed to assume the consular duties. When an office is superadded to the original appointment of a diplomatic or consular officer he is entitled to receive one-half of the compensation attaching to such office. As previously stated, a diplomatic representative possesses immunity from the criminal and civil jurisdiction of the country of his sojourn and cannot be sued, arrested or punished by the laws of that country. He cannot waive this privilege without the consent of the Govern- 
ment of the United States, since it belongs to his office and not to himself. However, if a diplomatic representative holds in a foreign country real or personal property aside from that which pertains to him as a minister, it is subject to the local laws. A diplomatic representative cannot be compelled to testify in the country of his sojourn before any tribunal whatsoever. This is another right which cannot be waived by the diplomat, and even if the matter on which his testimony is sought does not concern his mission, he is required to secure the permission of the Department of State before giving it. The residence and goods of the diplomat, and especially the archives of the mission, cannot be entered, searched or detained under and in process of local law, or by local authorities. The privilege of immunity from local jurisdiction, however, does not embrace the right of an asylum for persons outside of a representative's diplomatic or personal household. The right of asylum with reference to the premises in which missions are located has been much discussed and in certain countries and instances has had wide scope. For example, in certain South American countries where frequent insurrections occur, and consequent instability of government exists, the practice of extra-territorial asylum has become so firmly established that it is often invoked by unsuccessful insurgents. The Government of the United States does not sanction this usage, and enjoins upon its representatives the avoidance of all pretext for its exercise, even though the usage has, to some extent, been recognized by the government de facto. It has, however, happened frequently in the Latin-American countries that the asylum afforded by the representatives of foreign nations has been agreeable to the victorious parties as giving the leader of the defeated faction an opportunity to leave the country, when otherwise it would have been necessary to carry out an execution which the victorious faction desires to avoid. There is a general impression that the flag over the legation or consulate constitutes the building a portion of a foreign country for all purposes. However, this is not the fact, as the immunity of the diplomatic representative is strictly confined and cannot be extended to 
others than the members of his own immediate household except as the dominant government may, for reasons of its own, be willing to grant the privilege. The immunity of the servants of a diplomatic representative does not always obtain with regard to members of his suite who are natives of the country in which he is located. Where claims for military service are made against native servants, it is doubtful if the immunity can be secured, and diplomatic representatives are advised against making such claims. Couricrs and bearers of dispatches from diplomatic representatives to their home government are generally granted special privileges which will enable them to carry out their official duties. As a matter of courtesy, embassadors and ministers are usually allowed to bring in articles of foreign manufacture which are subject to customs duties without the imposition of such duties. This, however, relates to articles for use of the diplomatic representatives themselves and is not generally extended to cover the members of his suite. The office of a diplomat of the United States abroad is generally in his residence, although, in certain cases, office quarters are provided elsewhere. It is usual to display the shield of the country represented over the door and to display the flag on appropriate occasions.

One of the essential qualifications of a diplomatic representative is held to be reticence in regard to the governmental affairs committed to his care. He is, however, expected to be observant of everything which may be of interest to his home government and to report in full especially with regard to political affairs and to matters of foreign policy in which his own government may be concerned at a later date, even if the matters do not immediately affect the international relations of the two conntries.

In the earlier days of the United States it was regarded essential as a matter of the maintenance of the traditions of republican simplicity to prolubit the wearing of uniforms by diplomatic representatives of the United States. Diplomatic officers, however, who have served in the volunteer army are permitted to wear the uniform of the highest grade they attained, whether through promotion or brevet. They are, however, permitted to 
wear such dress as may be required by the customs of the country and for the hour of any ceremony they may attend, and this is held to include the wearing of court dress in such countries as a court dress is customary and is obligatory.

Diplomatic representatives are especially charged not to correspond with newspapers or periodicals in the United States or with other than the proper officials in regard to the public affairs of a foreign government. Such correspondence relating to a matter which may become the subject of discussion between the two countries is also prohibited, and they are forbidden to participate in any manner in the political affairs of the country to which they are accredited and are directed to refrain from expressions of opinion in public as to any matters under discussion within their jurisdiction. Participation by means of public addresses in public meetings is also discouraged, except upon special occasions having an international character. In their addresses on the latter occasion they are especially warned against expressions which might in any way be taken as an offensive expression of opinion upon the affairs of a foreign country. This restriction, which is also in effect with regard to the representatives of foreign countries in the United States, is one of the most strictly insisted upon regulations relating to the diplomatic service The penalty for indiscretion in this respect is a lessened consideration in the country to which they are accredited, and if the offense be a particularly flagrant one, it may result in representations which would be followed by the withdrawal of the minister even if his recall were not specifically requested. When a diplomatic official has for this or some other reason made himself obnoxious to the government to which he is accredited, an intimation of the fact would be promptly followed by the recall of the offending diplomat.

Diplomatic officers are especially prohibited from making recommendations for employment under the government to which they are accredited, and also to officers under the United States Government except their own subordinate positions. They are also forbidden to ask or accept any presents or testimonials 
whatever from a foreign government and are required to discourage as much as possible any voluntary honor of this kind. Should, however, a testimonial be proffered in the form of the regalia of an order, a medal, a sword or similar gift, it is necessary to secure the consent of Congress before it can be accepted. In their reports to the government of the United States diplomatic officers are required to refrain from any unnecessary or harsh reflection upon the officials of the government to which they are accredited, but at the same time they are to report freely and immediately all important facts which may come to their knowledge as to the political condition of the country, and particularly of foreign relations, and provision is made for treating such portions of these reports as may be of a confidential nature, in entire secrecy. In all formal communications to a foreign government a diplomatic representative of the United States must use the English language. In the countries of the East, the communications are expected to be accompanied by a translation into the language of the court, such translation being made by the official interpreter or dragoman, but the English version is never omitted. In communications to a European government, when saving of time is a consideration, a translation, provided it is accurate and can be depended upon, is sometimes transmitted with the English version, but the latter is the version always relied upon, should there be any doubt as to the intention of the diplomatic representative. Certain documents, however, which record the results of direct negotiations between a minister and the representative of another foreign office, are generally drawn up in both languages, the two versions appearing in parallel columns. This is especially true when the paper is to be signed by both parties.

Books, maps and other printed information relating to the matters of political information which the representative may securc abroad, are required to be transmitted to the State Defiartment, as well as such other publications as may be of value to the United States Government. Diplomatic representatives are forbidden to retain copics of their official correspondence to 
be taken away upon the close of their mission, for the reason that such copies might later fall into the hands of persons who would not be scrupulous in preserving them from publication. It is of course essential and understood that publication of any official paper or document is never allowed by members of the diplomatic corps.

It is not usual for diplomatic officiais to use the open mails for the transmission of communications to their home offices. Dispatch bags are furnished which are granted exceptional facilities in the mails, and where possible dispatch agents of the same nationality as the embassador or minister are utilized to forward the mails by means of vessels of their own flags. Mails which can be transmitted in official dispatch bags of the United States are limited to official correspondence and matter for the President or Vice-President of the United States or any of the executive departments or the presiding officers of the Congress. Private correspondence of the officials of the United States abroad and of the members of their families can also be transmitted and matter sent by foreign governments to their representatives in the United States may also be forwarded as a matter of courtesy, although these usually have their own avenues of communication. It was formerly the practice to constitute private persons special messcngers to convey communications to and from United States diplomatic representatives abroad and persons traveling abroad were formerly anxious to obtain such concessions, as they carried with them special privileges. This practice has now practically gone out of date, but a reminiscence remains in the prohibition against promising any person a compensation or reimbursement of his expenses for this service unless such expenditure has been previously authorized by the department. When the mails are obstructed or deemed unsafe, so that the dispatch bags cannot be carried in due course, special couriers are employed, and these are also employed when communications are to be sent which materially affect the interests of the United States and which might suffer if delayed for any reason. Special provisions are contained in the statutes giving 
to the Secretary of State the power to regulate any compensation which may be paid for this service. When a bearer of dispatches is employed, he is furnished with a special passport setting forth the duty he is to perform. Diplomatic represcntatives abroad are custodians of considcrable amounts of mail matter addressed to private parties and are required to see that no letters received in their care are opened by unathorized persons. Any letters remaining uncalled for after six months, and letters intended for officers and seamen of the Navy after one year, are to be returned to the local post office from which sent.

An important function of the diplomatic representatives is in connection with the issuance or passports. The requircments for securing a passport have been summarized under the head of the Passport Burcau. They may be issued abroad only by diplomatic officers in cases of emergency. The ordinary procedure is to receive and forward applications for passports to the Department of State. No passports can be issucd to or verified for any other persons than citizens of the United States, and the diplomatic officer is required, in case of a doubt as to the right of the applicant to receive a passport, to suspend the issuance until instructions are received from the Department of State. Under the former law and before the status of Americans residing for long periods abroad was definitely fixed, considerable discretionary powers, subject to instructions from the department, were given ministers as to the issuance of the certificates. Regularly issued passports are verified by diplomatic officers by endorsing on the document the word "good" in the language of the country, and affixing to the endorsement the official signature and seal. This verification, which is required by the laws of many foreign countrics, constitutes also a portion of the consular duties and is only performed by the diplomatic officer when there is no local consul.

Next to the negotiations which he carries on with the government to which he is accredited, the most important duty of the diplomatic officer relates to the protection of American citizens residing or traveling in the country in which he has jurisdiction. 
The right of a citizen to protection is the correlation of the right of the government to the citizen's allegiance and support. Therefore, when a native born citizen or a naturalized citizen, of whose rights under the treaty in force there is no doubt, complains of injustice and requests protection, it is the duty of the minister to inquire carefully into the circumstances, assuring himself first of the genuine character of the citizenship of the applicant, and of the nature of the complaint with regard to treaty rights. The rights of naturalized citizens who are native in the country in which the complaint is made, has been a subject of much difficulty in the diplomatic relations of the United States. In many countries the rights and immunities and also the duties of such persons have been carefully fixed by treaty. Innumerable instances have arisen in which persons have endeavored to use United States citizenship to escape from duties or penalties to which they are liable under the law of their native land, and it has been at all times necessary for ministers to scrutinize carefully all applications made to them for protection. When, however, a diplomatic representative is satisfied that an applicant for protection has a right to intervention and that the complaints are well founded, he is required to make courteous, but at the same time firm, representations to the foreign government and endeavor to secure remission of penalties imposed or reparation for injuries suffered. No foreign subject can, of course, appeal to a diplomatic representative of the United States for intervention under ordinary circumstances. In case of war between two foreign nations, however, the representative of the United States is sometimes asked to represent one of the belligerents and permission for such representation is usually granted, with the understanding that in such action as the diplomatic representative may take, he is entirely the agent of the foreign government and that the United States assumes no responsibility whatever in the premises. The duties of diplomatic representatives toward American citizens abroad have been frequently misconstrued, but are strictly defined. Embassadors and ministers have no civil jurisdiction over their countrymen except where it 
has been expressly provided by treaty or municipal law of the United States, and have no criminal jurisdiction in civilized countries. In Turkey and Persia and some of the remote semicivilized countries where unimpeachable courts of law have not been established, diplomatic representatives of the United States have criminal jurisdiction. This jurisdiction was formerly of a considerable extent and was localized in China and Japan as well as most other uncivilized or semi-civilized countries. It was withdrawn in Japan upon the establishment of adequate courts in that country, and has, within the past two years, been superseded in China by a United States court. In those countries in which diplomatic representatives still have criminal jurisdiction, the minister has authority to make regulations which have the force of law upon American citizens within the country to which he is accreditcd. The original criminal jurisdiction is vested in the consul in localitics where such an official is resident, and is only in exceptional cases undertaken by the minister. The latter constitutes, however, a court of appeal from the consular courts. In capital cases the finding of the consular court must be approved by the minister, who has the duty of issuing the warrant for the execution of the convict unless he deems it advisable to submit the case to the President for the exercise of the executive power of pardon. The minister also approves the associates nominated by consuls to sit in the consular courts and, with the advice of the several consuls, prescribes the forms of all processes to be issued from the consular courts and the method of conducting trials therein. When a consul sits with associates and any of them differ from him in civil cases, appeal may be had to the minister and in criminal cases if the associates of a consul differ from him the case must be referred to the minister for his adjudication. When the consul sits alone and the fine exceeds $\$ 100$ and the term if imprisonment exceeds sixty days, an appeal may be taken. The withdrawal by new statutes of the jurisprudence formerly in effect in China and Japan has reduced the importance of the ministerial administration of courts very largely. In certain countries provision is made by 
treaty for mixed courts for the trial of disputes between natives and foreigners, and the minister has general supervisory powers in behalf of American citizens. Some of these jurisdictions relate to American citizens only and others take cognizance of all foreign cases, arrangements being made between the local representatives of the various countries for sitting in a judicial capacity.

Diplomatic representatives are warned to avoid taking part in contentions with their countrymen or with the subjects or authorities of foreign countries as far as possible, but are required to use their amicable services to determine disputes which may arise between their countrymen. Fellow citizens are to be countenanced and protected before the authorities of the country in cases in which they are injured or oppressed, but the efforts of the diplomatic representatives are not to be extended to those who have been presumably guilty of infraction of the local laws. If the representations made by the diplomatic officer fail to secure a redress for the citizen, the case is reported to the Department of State for its action. Diplomatic officers are not permitted to interpose to aid in the collection of claims against the government to which they are accredited unless there is a pressing necessity for action before they can communicate with the Department of State. All claims, whether founded in contract or in consequence of an injury, must be submitted to the Department of State, which originates the negotiations for recompense. Diplomatic representatives of the United States have no funds for the relief of distressed citizens of the United States except tineir private purse and are not expected to relieve such cases, although, as a matter of fact, it is probable that every diplomatic officer spends considerable amounts in this manner. Neither is it obligatory on the embassador or minister to entertain his countrymen abroad, although it is also the fact that many Americans are so entertained, though at the personal expense of the diplomatic representative.

There is an incorrect impression that a marriage within the walls of a United States legation abroad is equivalent to a marriage 
within the limits of the United States itself. A diplomatic representative has no official function with regard to marriages, this being a part of the consular duties. He is not prohibited from allowing a marriage to take place in the legation, but is required to see that the parties can legally be married under the law of the country in which the legation is located or under the law of the District of Columbia. The subject of marriage abroad will be more fully discussed under the head of Consular dutics.

Where there is no consul at the location of a legation, the minister may perform the duties assigned to consuls in regard to the estates of citizens of the United States dying intestate within his jurisdiction. Diplomatic officers are not required to examine titles, investigate private claims or do similar services, and are empowered to decline to grant such requests unless specific instructions to perform the service are received from the Department of State. They are also forbidden to endorse bills of exchange or become responsible pecuniarily for American citizens and are required to decline the use of their names as reference for business or other purposes.

Diplomatic representatives have general powers of supervision over the consular officers within their jurisdiction through the consuls general, should there be such within the jurisdiction, or directly with the consuls, should there be no consul general. Requests for leaves of absence in ccrtain countries are forwarded through the diplomatic representatives and require his approval. In case a vacancy requiring the temporary appointment of a consular officer, the diplomatic representative has the power to fill the office, reporting immediately to the Department of State. When a consul is appointed it is usual to send the commission to the embassador or minister, who communicates with the government and requests an exequatur of recognition for the new officer. This when received is forwarded to the appointee with his commission and empowers him to begin the duties of his office.

The negotiation of treaties with foreign countries or of agreements and understandings relating to minor points of con- 
troversy are conducted with the greatest secrecy and with the utmost precision or formality, all notes which pass between the diplomatic representative and the foreign officials and between the diplomatic representative and the Department of State being couched in the exact terms of diplomatic science. All are carefully preserved and if the negotiation is unsuccessful at the moment, the entire body of correspondence is carefully collated and indexed for use at a later date should the same subject arise. The more important of the treaties are negotiated by special representatives, but considerable numbers of conventions are effected by the diplomatic representatives who are given statements of the powers conferred upon them in the negotiation. These powers set forth the extent of the demands and the limit of the concessions regarded as desirable by the Department of State, and may be amended by subsequent authorization as the embassador or minister reports the trend of the negotiations. Special representatives charged with the negotiation of important treaties are understood to be given full and unlimited powers of nesotiation, this being a preliminary to the full and free conference deemed to be necessary. In the present era of rapid communication such powers are much less significant than in the earlier history of diplomacy, when the representative was practically cut of from his home government and was compelled to make the best bargain he could on the basis of the instructions he received before departing for the point of conference. Diplomatic negotiations at the present time take on largely the aspect of the presentation at each day's conference of the instructions received by telegraph the night before, so that diplomatic representatives under these circumstances are in reality the mouthpiece's of the heads of the respective foreign offices, and not independent negotiators. Provision, however, is made in diplomatic procedure for negotiations and understandings subject to the approval of the respective home governments. These also, because of rapid communication, are perhaps less common than the completed agreement effected by the diplomatic representative as the result of his instructions, and subject only to purely 
formal action. Treaties of two nations having different official languages are prepared in parallel columns on the same page and in the copy of the treaty to be transmitted, say, to the United States, the United States must be named first throughout both texts. In the copy for the foreign government the name of the forcign govermment precedes that of the United States. The text of the treaty on the left-hand side of the copy is coucheil in the language of the government to which that particular copy is to be delivered. As a literal translation one text to the other is almost impossible, the most careful scrutiny is made of the terms of the translation in order that the sense shall be as nearly as possible identical. The final power with reference to treaties on the part of the United States is the Senate, without whose approval no ratification of a treaty can be effected, and the date of whose ratification cannot be foreseen. It is therefore usual in all treaties for ratification by the United States for a provision to be inserted that the ratification shall be exchanged as soon as possible rather than at a particular date, the latter being the customary provision.

The United States has treaties with all of the civilized countries of the world providing for the extradition of persons charged with certain crimes, the list of extraditable crimes being different in different countries. Requests for extraclition are presented to the State Department, by which they are transmitted to the diplomatic representatives whose duty it is to make the demand for extradition upon the government to which they are accredited. When no embassador or minister is available a consular officer may be authorized to present such a demand. Extraditions are generally granted without question when in proper form and within treaty provisions and the person accused is arrested by the local officers and lield until a person named for the purpose arrives to return the prisoncr to the United States. In cases in which extradition is requested by a foreign government the diplomatic representative of the United States to that government is required to authenticate the deposition, warrant or other paper offered as a basis upon which cxtradition is asked. Diplo- 
matic representatives are required to exert active vigilance to prevent the deportation of persons excluded from the United States under the laws relating to the admission of aliens, and to notify the authorities at Washington of the sailing of such persons from foreign countries, and if the shipping of paupers or other undesirable persons is undertaken, the diplomatic representatives are required to make protest to the foreign government. It is also the duty of the diplomatic representatives to advise the Department of State of any acts of humanity shown to shipwrecked Americans by subjects of foreign nations. Provision is made by Congress for testimonials to such foreign seamen which generally take the form of watches, binoculars or similar articles of maritime use. Diplomatic representatives are also expected to report all matters relative to safeguards to navigation which come to their notice, including information as to light-houses, buoys, etc.

\section{UNITED STATES DIPLOMATIC SERVICE.}

The following list is of the present positions in the diplomatic service of the United States. The names of incumbents are omitted and the residences of subordinate officers are in the same cities as those of the heads of the legation unless otherwise given. The following abbreviations are used in this list and that of the consular service:

Amb. E. \& P.-Ambassador Extraordinary and Plenipotentiary.

E. E. \& M. P.-Envoy Extraordinary and Minister Plenipotentiary.

Agt. \& C. G.-Agent and Consul-General.

Dip. Agt.-Diplomatic Agent.

Sec. of Emb.-Secretary of Embassy.

Sec. of Leg.-Secretary of Legation.

Mil. Att.-Military Attaché.

Nav. Att.-Naval Attaché.

C. G.-Consul-General.

C.-Consul. 
V. C.-Vice-Consul.

D. C.-Deputy Consul.

Stud. Int.-Student Interpreter.

Mar.-Marshal.

C. C.-Consular Clerk.

Int.-Interpreter.

Agt.-Agent.

To What Country Name and Rank. Residence. CompenAccredited. sation.

Argentine Republic ..E. E. \& M. P. ........... Buenos Ayres .. \$12,000

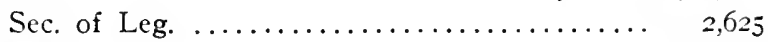
Mil. Att.

Austria-Hungary ...Amb. E. \& P. ...........Vienna ...... I7,500

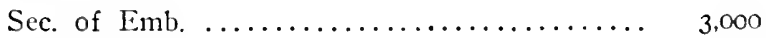

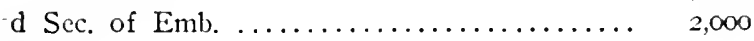

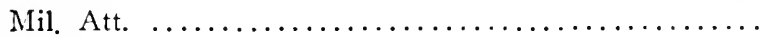

Nav. Att. ................... Berlin

Belgium .......... E. \& M. P. ........... Brussels .... I 12,000

Sec. of Leg. ................... 2,625

Bolivia..$\ldots \ldots \ldots$. E. E. \& M. P. ..........La Paz ...... 10,000

Sec. of Leg. .................... 2,000

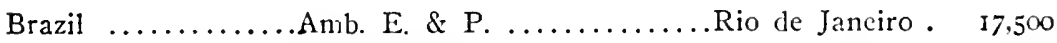

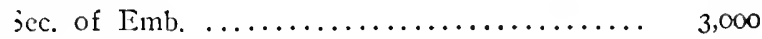

Mil. Att.

Chile E. E. \& M. P. Santiago

10,000

Sec. of Leg. .................... 2,000

China $\ldots \ldots \ldots \ldots \ldots$ E. E. \& M. P. ...................... I 2,000

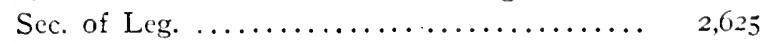

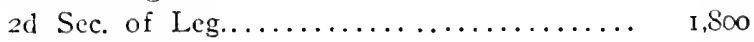

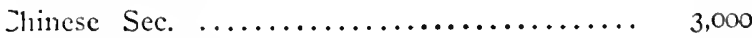

Asst. Chinese Scc. .................... 2,000

Stud. Interpreters. ................. I,000

Nav, Att. ............... Tokyo (Yedo) .....

vil. Att. ............. Peking

Colombia

Bogotá

10,000

Sec. of Leg.

2,000

Mil. Att.

Caracas 
To What Country Name and Rank. Residence. CompenAccredited.

Costa Rica .........E. E. \& M. P. ............San José ..... I0,000 Sec. of Leg. .................... 2,000

Cuba..$\ldots \ldots \ldots$ E. E. \& M. P. ........... Habana ..... 12,000

Sec. of Leg. ..................... 2,000

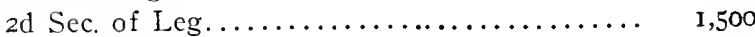

Denmark .........E. E. \& M. P. ........... Copenhagen ... I0,000

Sec, of Leg. ..................... 2,000

Dominican Republic ..Fenton R. MIcCreery, Minister

Resident \& C. G. ..........Santo Domingo Io,000 Sec. of Leg. .................... 2,000

Ecuador ............ E. \& M. P. .......... Quito ....... I0,000

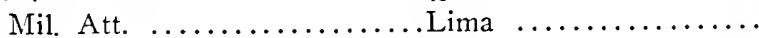

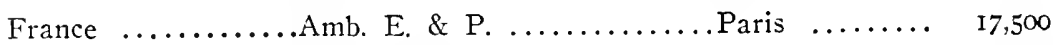

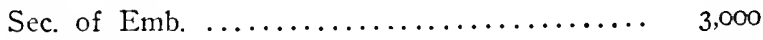

$2 \mathrm{~d}$ Sec. of Emb. ................... 2,000

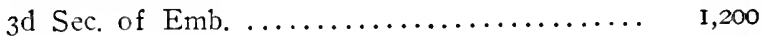

Nav. Att.

Mil. Att.

German Empire .....Amb. E. \& F. ...................... I7,500

Sec. of Emb. .................... 3,000

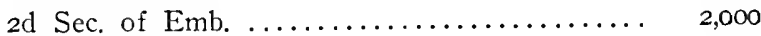

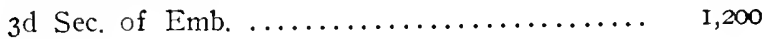

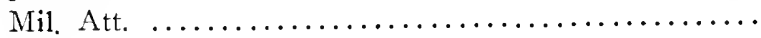

Nav. Att.

Great Britain .........mb. E. \& P. .................... I7,500

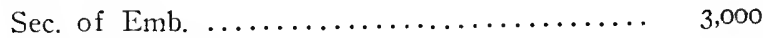

$2 \mathrm{~d}$ Sec. of Emb. .................... 2,000

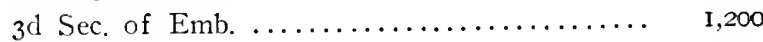

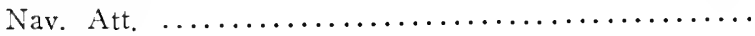

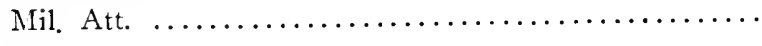

Greece ............ E. \& M. P. .......... Athens ...... 10,000

sec. of Leg. ...................... 2,000

Guatemala .........E. E. \& M. P. ...........Guatemala .... I0,000

Sec. of Leg. ..................... 2,000

Haiti ............ E. \&. M. P. ............ Port au Prince. I0,000

Honduras ........E. E. \& M. P. ............ San Salvador.. 10,000

Sec. of Leg. ..................... 2,000 
To What Country Name and Rank. Residence. CompenAccredited.

Italy Amb. E. \& P. .............Rome

Scc. of Emb.

17,500

2d Sec. of Emb. ...................... 2,000

Mil. Att.

Nav. Att.

. Berlin

Japan

Amb. E. \& P.

Tokyo (Yedo)

17,500

Sec. of Emb.

3,000

$2 \mathrm{~d}$ Sec. of Emb.

2,000

Japanese Scc. \& Int.

3,000

2 Stud. Ints.

I,, 00

Nav. Att.

Mil. Att.

Liberia

Sec. of Leg.

Monrovia

5.000

Minister Resident \& C. G. ....Monrovia .... $\quad 2,000$

Luxemburg ........E. E. \& M. P. ............The Hague .... I2,000

Sec. of Leg. ....................... 2,625

Mexico

Amb. E. \& P.

Mexico

17.500

Sec. of Emb.

3.000

2 d Scc. of Emb.

2.000

3d Scc. of Emb.

1,200

IIII. Att.

Montenegro

E. E. \& M. P.

Athens

10.000

Sec. of Leg.

2,000

Morocco

Tangier

10.000

Scc. of Leg.

2,000

Netherlands

E. E. \& M. P.

The Hague

I 2,000

Sec of Leg.

2,625

Nicaragua

E. E. \& M. P.

San José.

I0.000

Sec. of Leg.

2.000

Norway

Christiania

I0,000

Sec of Leg.

2,000

Panama

E. E. \& M. P.

Panama

I 0,000

Sec. of Leg.

2,000

Paraguay

.E. E. \& M. P.

Montevideo

I 0,000

Sec. of Leg.

1,000

Persia

E. E. \& M. P.

Teheran

I0,000

Int.

2,000 


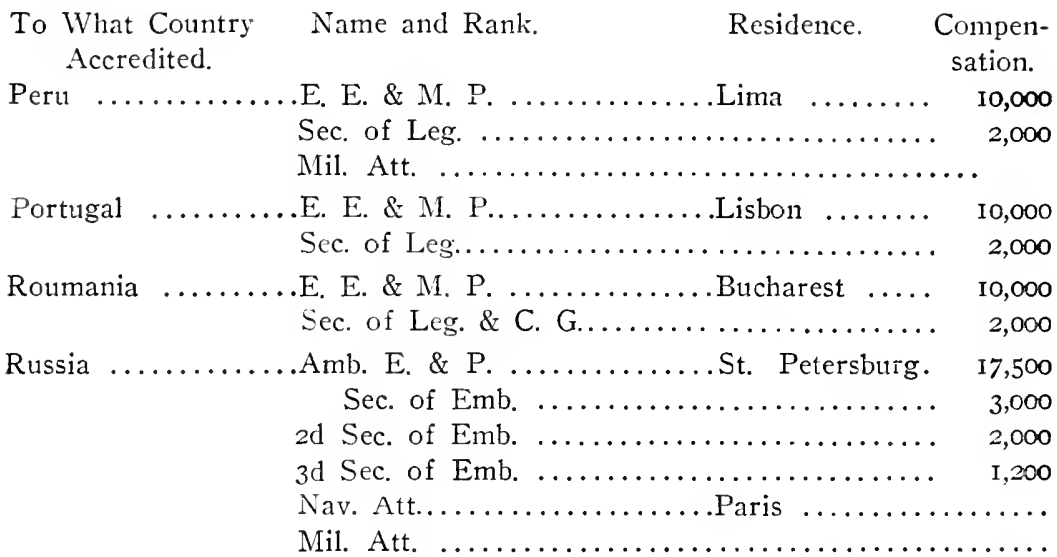

Salvador ..........E. E. \& M. P. ..............San Salvador .. 10,000 Sec. of Leg. ...................... 2,000

Servia.......... E. E. \& M. P. .............Bucharest .... I0,000 Sec. of Leg. \& C. G. .................. 2,000

Siam .............E. E. \& M. P. ..............Bangkok ..... 10,000 Sec. of Leg. \& C. G. ................... 2,000

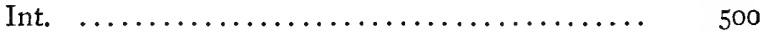

Spain $\ldots . . . . . .$. E. E. \& M. P. .............Madrid ..... 12,000 Sec. of Leg. ...................... 2,000

Sweden $\ldots . . . . .$. E. E. \& M. P. ............. Stockholm ... 10,000 Sec. of Leg. ...................... 2,000 Mil. Att.

St. Petersburg

Switzerland E. E. \& M. P. .Berne ....... I0,000 Sec. of Leg. ..................... 2,000

Turkey Constantinople 17,500 Amb. E. \& P.

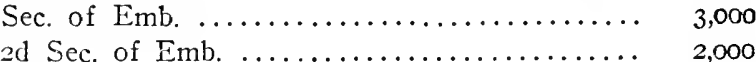
Int. $\ldots \ldots \ldots \ldots \ldots \ldots \ldots \ldots \ldots \ldots \ldots \ldots, 3,000$

Bulgaria ..........Dip. Agt. ........................ 10,000 Sec. of Agency .................... 2,000

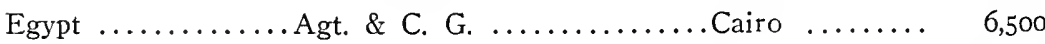

Uruguay ..........E. E. \& M. P. ..............Montevideo ... io,000 Sec. of Leg. ...................... $\quad 2,000$

Venezuela Caracas 
SURORDINATE OFFICERS OF MISSIONS.

The chief subordinate of a diplomatic officer is the first secretary of the embassy or legation. These officials are appointed either by transfer from some other branch of the foreign service or by the President, and the persons selected by the latter are given an examination in international law, diplomatic usage anci modern languages. Familiarity with at least one foreign language is required. The language may be that spoken in the country in which the legation is located or French. If a decision is made by the cxamining board that the candidate is not qualified for the appointment, the selection by the President will be revoked. Instructions to secretaries of legations are general in character and their duties depend upon the views of the head of the mission. Secretaries are, however, especially charged with reticence with regard to the affairs of the embassy or legation and are required to be on their guard against disclosing or talking about any of the confidential matters involved. It is the duty of the secretary to transcribe and dispatch the official letters or communications of the heads of the mission as well as all dispatches and communications to his own government. Also to record all such dispatches and communications in suitable books which are carefully prescrved as a part of the archives of the mission. The proper transportation, indexing and custody of the original of all dispatches and other written communications are under the secretary's immediate control. The secretary is expccted to habitually attend at the office of the mission during the usual hours of business and all books and archives and the official seal of the mission are under his personal care and superintendence. As long as the head of a mission is present, the secretary is not recognized by a foreign government as being authorized to perform a single official act other than is directed by the head of the mission. When, however, the head of the mission from any cause is unable to perform the duties of his position, the secretary at once becomes a diplomatic personage and takes charge of the archives and correspondence of the mission and transacts all necessary business 
with the government to which the mission is accredited. When the absence of the head of the mission is in the nature of an emergency, the secretary becomes chargé d'affaires by virtue of his office and without authorization from the State Department. When, however, the diplomatic representative is to be absent in consequence of leave granted, directions from the department to have the secretary serve as chargé d'affaires ad interim are received and the fact is notified to the foreign office of the government to which the mission is accredited. In view of the possibility that a secretary may become an acting embassador or minister he is regarded as a diplomatic personage in the principles of international law and has similar privileges of immunity to those of an embassador or a minister. It frequently occurs that secretaries of legations are in charge as chargé d'affaires during considerable periods on the failure on the part of the home government to designate a head for the mission. While so employed the secretary has all the privileges and duties of a regularly accredited diplomatic representative. The second secretaries of embassies and legations are provided when necessary and their duties are similar and subordinate to those of first secretaries. They are not to undertake any diplomatic duties, however, except in the absence of both the head of the mission and of the first secretary. First and second secretaries when acting as chargé d'affaires receive the compensation provided by Congress for the service, but only if they have been specifically designated by the President either with a recess appointment or through an appointment by and with the advice of the Senate the compensation being fixed by direct provision for each place.

The appointment of civilian attaches to missions of the United States is especially prohibited by law and embassadors and ministers are forbidden to appoint such officers even to serve without compensation. There are, therefore, no honorary persons attached to the suites of ministers or diplomatic representatives of the United States, all of the subordinate officers being either secretaries provided for by law, military or naval at- 
taches, and the student interpreters in the eastern countries. Where matters of interest to the land or naval forces of the United Stites can be learned, an officer of either or both arms of the service is detailed as an attache of the legation. $\mathrm{He}$ is, however, not nnder the orders of the diplomatic representatives but receives his orders and reports directly to the head of the service to which he is attached. It is usual to have a military attache connected with the mission to the most important countries, but a naval attache is not assigned unless the nation has a navy of considerable size. If a military attache is above the rank of colonel or naval attache above the rank of captain, he precedes the secretary of the legation on official occasions. If, however, he is of or below the above named rank, the secretary of the legation takes precedence of him.

Besides the appropriation for regular salaried interpreters at the legations in Asiatic countries, there is provision for ten student interpreters to China and six student interpreters to Japan. These have a salary of $\$ \mathrm{I}, 000$ each, and their tuition fees are paid. They study for the purpose of supplying interpreters to the legations and consulates, and are required to be selected on a non-partisan basis. Upon receiving such an appointment, a student is required to agree to serve as an interpreter in a legation or consulate in which his services nay be needed for a period of ten years thereafter.

The clerks of embassies and legations are in no sense diplomatic officers They have no official rank or position and no immunities or privileges except as members of the household of the representative. While it may sometimes happen that a clerk is left in charge of the archives and business of a legation, he is not authorized to perform any diplomatic functions. Should an emergency arise, however, requiring immediate attention, he may take such steps as may be necessary, but must be careful to inform the foreign office that he does so informally and without assumption of any diplomatic character. In addition to the subordinate employees of missions heretofore named, the missions in castern countries are provided with official in- 
terpreters, guards, dragomen and other native assistants, whose services are utilized either in communication with the inhabitants of the country or as a matter of protection to the diplomatic representative, his household and suite. In some cases direct appropriation is made for the officials named and in others the expense therefor is allowed to the minister from the general fund. In most of the capitals of the world the American diplomatic representative is housed in a hired residence, although the desirability of ownership of an embassy or legation by the United States is admitted. Such a property has recently been acquired in Constantinople, and in some Asiatic countries, notably in China, the necessity of the situation has made it absolutely essential that the United States invest in real estate and construct or purchase a suitable residence for its representative in the section of the capital city assigned to the diplomatic corps.

It is, of course, expected that a diplomatic representative shall remain continuously at his post of duty, and he is required to secure the consent of the department for all absences except those of a temporary nature and for any visits he desires to make to the United States. It is provided by law that a diplomatic officer shall receive no salary for the time he may be absent from his post in excess of sixty days during any one year. The President has entire discretion as to the amount of leave which may be granted, and no particular time is contemplated by law as annual leave for diplomatic officers. The term of a diplomatic officer may be ended by his resignation, by his recall, or by the termination of diplomatic relations with the country to which he is accredited. The failure of Congress to make an appropriation for his salary does not necessarily terminate his position, since he can continue to discharge the duties of his office, depending upon a subsequent claim for remuneration, upon resignation or recall. A diplomatic officer is entitled to salary for the time necessary to return to the United States, unless his recall has been dictated by malfeasance in office. When the negotiations between two countries have reached such a 
state that there is apparently no possibility of adjusting a difficult and heated situation by means of the usual diplomatic exchanges, the definite stage of total disagrcement is marked by the withdrawal by one or the other of the parties, or by both of the diplomatic representatives. A conclition of war need not necessarily follow such a withdrawal, and it has frequently occurred that nations have been without diplomatic relations for a considerable term of years without having actually entered into an armed conflict. When such severance of relations occurs, it is usual for the good offices of another nation to be asked to render the usual diplomatic services to the subjects of the countries involved in the dispute, who may be respectively resident in the hostile countries. If an actual condition of war is impending, it is the duty of the government of the country to which a diplomatic representative is accredited to see that he reaches the frontier with all his property and safe from insult or injury. Failure to grant such safe conduct would be regarded as unbecoming any civilized nation. If a diplomatic officer of the United States is withdrawn from his post of duty because of the termination of diplomatic relations, such withdrawal does not necessarily terminate his office. He may retain the position and salary in the discretion of the President, but it is usual, where the severance of relations will be of some duration, to terminate the mission and to revive it upon the renewal of diplomatic relations. When it is expected that the rupture will be only temporary, the minister may be continued and either allowed to take leave or be utilized as an adviser in connection with the negotiations which result. When a diplomatic officer dies in a foreign country, his salary ceases on the day of his death. It is, however, provided that an amount can be paid to his widow equivalent to the amount of compensation which would have accrued during the time necessary for the return of the officer to the United States. Provision is also made by statute for the return, at the public expense, of the remains of all diplomatic officers who die abroad in the service of their country. 


\section{INTERNATIONAL BOARDS AND COMMISSIONS.}

All of the boards and commissions provided for by the statutes to take part in international movements and enterprises come under the jurisdiction of the Department of State. Among such commissions is the permanent board of arbitration provided for by the convention signed at the Hague in 1899. This tribunal was constituted for the purpose of establishing a permanent court to which disputes between nations could be referred for arbitration. In addition to this body there have been the several arbitration conventions such as that of the summer of 1907, taking place at the Hague, and considering various matters relating to international law. The last mentioned convention took up in its various branches the discussion of nearly every question pertaining to the relation of civilized countries in times of war, with the result of valuable service in stimulating discussion even if the results were somewhat indefinite The arrangements for the attendance of the delegates of the United States upon this convention, as well as the reception and preservation of its archives, are among the functions of the Department of State. In addition to the foregoing, and the membership in the International Bureau of the American Republics, which is described elsewhere, the State Department has under its jurisdiction the members on the part of the United States of the United States and Mexican Water Boundary Commission which arranged matters relative to the boundaries formed by the rivers between the United States and Mexico, the International Lake Levels Commission, composed of a membership from the United States and from Canada, which is charged with the consideration of matters relating to the level of the Great Lakes, and with the prevention of such withdrawal of water from the lakes as may seriously interfere with navigation in any part of the great chain of inland waterways. In addition, the delegates from the United States to the International Prison Commission, and the delegates to the International Institute of Agriculture at Rome are under the State Department, and the officers of the Island of Porto Rico report to the Secretary of 
State. The United States Court for China, which took the place of the ministerial court formerly held is also entirely under the jurisdiction of the State Department and does not, as is the case with other United States courts, come in any way under the Department of Justice. There is, in addition to the above, a commission for the limitation of the Alaskan boundary, the representative of the United States on which, although in this particular under the State Department, is at the same time the head of a bureau under the Department of Commerce and Labor.

\section{TIE CONSULAR SERVICE.}

While it cannot truthfully be said that there is a constant and irreconcilable warfare between the diplomatic and the consular services, the fact of the representation of the same nation by two different sets of officials in foreign countries cannot but bring about complications, which have only in part been provided for by the stringent and definite terms of the instructions to the Federal officers referred to. It is manifestly necessary that a strict and well defined dividing line should be laid down, with the result that we find that consular officers are prohibited from taking any diplomatic action whatever except under peculiar circumstances and with the direction of the department. The antiquity of the position of consul suffers nothing, however. when compared with that of a diplomatic officer. The earliest representatives sent abroad by nations were the agents of commercial interests and their importance allowed them to retain the title of consul when that title was abandoned as that of the head of the Roman nation. The dutics and functions of a consul relate exclusively to commercial matters and have nothing to do with questions of politics, international law or sociological problems growing out of the intercourse of nations. Consuls have always been the representatives of their nation in dealing with the affairs of ships sailing under the respective flags, expected to see that navigation laws were obeyed, and that the seamen of their nation were protected, while one of the chief of their duties was to assist and protect the merchants of their own 
nationality and in general to act as the commercial representatives and protectors of their countrymen temporarily visiting abroad. As this function was of the first importance to the commercial nations of antiquity, consuls were persons of importance at home and the first men of the communities of their own nationality abroad. In the days of the importance of the merchant marine of the United States, questions relating to merchant seamen constituted a principal part of the business of the United States consuls at the various foreign ports. At the present time, however, the business of consuls relates most largely to the exportation of goods to this country which are subject to import duty. For this reason consuls of the United States are stationed not only at the seaports, but at points as well where there is considerable manufacturing for the purpose of export to the United States.

The consular service proper is divided into consuls general and consuls of different grades. Consuls general, in addition to the usual duties of consuls, are given supervisory jurisdiction over certain districts so that the operations of the various consuls within that district are subject to their direction and inspection. They are required to see that the provisions of law and of the consular regulations are complied with by the various consuls under their jurisdiction and that the correspondence and consular work generally is carried on properly. Their inspection does not, however, extend to the auditing of accounts. Three consuls general, those at Calcutta, Dresden and Mexico, are not charged with supervisory duties, but are given the rank of consul general on account of the importance of these posts. In general the supervision of a consul general is over all the consulates in the country in which he is located, but this does not apply to a distant colony of that country. Consuls general in certain jurisdictions may be given permission to make trips of inspection, but these trips are not to be authorized more than once a year and these visits are practically rendered unnecessary by the new consuls general at large.

Consuls general and consuls are appointed by the President, 
by and with the consent and advice of the Senate. The act of I906 adjusted and regulated the classes of consuls gencral and consuls and by exccutive order therennder vacancies in the office of consul general and the office of consul above the eighth class are to be filled by promotion from the lower grades of the consular service. This regulation supersedes the former practice of appointment of consuls general and consuls on political grounds, and the re-classification law and the regulations thereunder have been adopted as a means to the improvement of the consular service, it being held that the former practice of political appointment has proved to be to the detriment to the United States in its commercial interests abroad. Appointment to the two lowest classes of consuls, classes eight and nine, are filled by promotion on the basis of ability and efficiency, as shown in the service of consular clerks and vice-consuls, deputy consuls, consular agents, student interpreters, and interpreters, provided such officers have been appointed to their positions as the result of an examination. For such vacancies as are not filled as above, new appointments of candiclates will be made of persons who have passed a satisfactory examination for consul. It is provicled, however, that persons who are in the service of the Department of State with salaries of $\$ 2,000$ or upwards, shall be eligible for promotion to any of the grades of the consular service above class eight. In order to secure a designation for examination as consul, it is necessary to be twenty-one years and not over fifty ycars of age, to be a citizen of the United States and of good character and habits, and physically and mentally qualified for the performance of consular work. Applications for designation for appointment are made to the State Department and the persons to be examined are designated by the President. A representative of the Secretary of State, the chief of the Consular Bureau of the State Department, and the Chief Examiner of the Civil Serrice Commission constitute an examining board. The regulations governing examinations provide that the examination will be the same for all grades of consul, and these determine the candidate's eligibility for appoint- 
ment in the consular service, irrespective of the grade for which he may have been designated for examination, and without regard to any particular office. The examination consists of an oral and a written examination, the two counting equally in the determining weight. The object of the oral examination is to ascertain the candidate's business ability, general information and probable efficiency in the position. The written examination includes at least one modern language, French, German or Spanish, questions on the natural industrial and commercial resources and commerce of the United States, especially with reference to the possibility of extending the foreign trade of the United States, political economy, and the elements of international, commercial and maritime law. It also includes historical, mathematical and geographical subjects, together with exercises intended to test the applicant in composition and the use of the English language. In addition to the above, in order to become eligible for appointment to a position in a country where the United States exercises extra-territorial jurisdiction, so that the consul is obliged to exercise some judical functions, the examination above indicated will be supplemented with questions calculated to determine his knowledge of the fundamental principles of common law, and rules of evidence in the trial of civil and criminal cases. Upon the conclusion of the examination, those condidates who have attained a mark of 80 , are rated in the order of their merits by the board of examiners, and the list thus established is used as a basis of certification of candidates when an appointment is to be made. Names of eligibles remain on the list for two years, unless previously appointed or withdrawn, and at the expiration of two years the names remaining will be dropped, and the candidates cannot again be considered unless upon fresh application and examination. The student interpreters referred to under the head of Diplomatic Officers must be between the ages of nineteen and twenty-six, and must be unmarried.

No consul general or consul appointed to one office is allowed to hold a similar office for another consulate or to exercise its 
duties. Every consular officer is required, before he enters upon the duties of his office, to file a bond of not less than $\$ 1, \infty 0$ or more than $\$ 10,000$ and in no case less than the annual compensation of the officer. Suretics on such bond are required to be permanent residents of the United States, or regularly authorized surety companies. No consul general, consul or consular agent receiving a salary of more than $\$ 1,000$ a year can, while he holds his office, be interested in or transact any business as a merchant, banker, broker or other trader, or a clerk or other agent for any such person. Such officers are also prohibited from practicing as lawyers for compensation or being interested in the fees or compensation of any lawyer, and consuls are required to stipulate that they will not violate this provision as a condition of their official bond. The President is also empowdered to extend the provision prohibiting the practice as a lawyer to consular officers whose salary does not exceed \$I,000 a year, or who may be compensated by fees. In the discretion of the President, any vice or deputy consular officer or consular agent may also be so prolibited and may be required to give a bond not to violate this prohibition. Under the law herein previously referred to, which was passed in 1906 and which established the salaries of consuls, provision was made for the abolition of fees as a basis of compensation for consular officers and all fees received by them are required to be turned into the Treasury of the United States, the sole and only compensation of consular officers being the salary fixed by law. An exception is made in the case of consular agents, who are to be paid onehalf of the fees received in their offices up to a maximum sum of $\$ 1,000$ in any one year, the other half being accounted for and paid into the Treasury of the United States. As a check on the consular officers in securing the return of their fees to the Treasury, they are provided with adhesive stamps in suitable denominations, so that when any notarial or other act is performed for which a fce is charged under the fee bill fixed by the President, a stamp must be affixed to the document acted upon, and without such stamp the document is not legally valid within 
the jurisdiction of the government of the United States. Consular officers are charged with the face value of the stamps issued to them, and must make returns to the department in cash for the stamp value used. As a very large proportion of the income of the consuls under the former system consisted of fees for the consular certification of invoices, these fees, under the new system, were included in the fees for official services for which the President is authorized to prescribe rates of tariff. Fees for certifying invoices which at large manufacturing points amounted to considerable sums, are now entirely turned into the Treasury instead of becoming a portion of the emolument of the consul. In fixing upon the salaries which were recommended for the respective consulates before the bill referred to was passed, schedules were required from the different consuls as to the amount of their income from fees for certifying invoices. If the consul in any instance deemed it advisable to state the lowest possible figure at which this income might be estimated in order that his position might seem less desirable than it really was, the disadvantage of such a course became apparent when the salary of the position was ultimately fixed at a figure lower than it might and should have been had the amount of the fees been stated accurately. The bonds of consular officers, after approval by the Secretary of State, are deposited with the Secretary of the Treasury and are subject to the statutory requirements as to the renewal of bonds every two years or oftener as the supervising officer may deem to be necessary.

As the usefulness of a consular officer would depend materially upon his being able to maintain amicable relations with the local authorities of his port and as his functions are largely exercised by reason of the courtesies granted by the foreign government, it is necessary to secure the permission of that government for the newly appointed consul to begin operations which practically amounts to an appointment from that government. This is done through a notification of the appointment sent, if possible, through the diplomatic representative of the United States to the government of the foreign country, with a 
request for a permit for the consul to undertake his dutics, the resulting document being technically known as an exequatur. If it is not possible to follow this procedurc, the consul is sometimes directed to proceed to his post of duty, and apply for temporary permission from the local authorities to undertake his duties, his application being verified by a letter from the Department of State. If there is no diplomatic representative, a consul may, when furnished with the proper credentials, make application himself for his exequatur. It is also customary to secure recognition for the subordinate officers of consuls by notifying the foreign government of their appointment and requesting a permission to act or recognition from the foreign government. In case a consul is to be located in a colony, it is the usual practice for the consul general or principal consular officer to apply to the colonial government for permission to proceed with his duties while waiting for the exequatur from the home government. Consular officers are required to rent business offices in the central portion of the towns or cities in which they are located, and are expected to be present at thcir offices during the business hours of the day for the purpose of transacting any business that may be presentcd to them. They are required to reside in the towns or citics in which they are located, and not to remove therefrom cxcept upon permission and for special reasons. A statutory allowance of rent amounting to twenty per cent. of the compensation of the officer is made and the State Department exercises general supervision over the details of the office provisions as to location, size, convenience for the public, etc. The consul is required to have definite business hours and to catse such hours to be generally known in order that the public may know at what times the consul is to be found in his office. Consular officers are not permitted to have their officers in the counting rooms of business men. especially those having to do with forcign trade, and the consul is required to attend to his own business and not leave it to the subordinates of others, so that the contents of invoices and other business papers may not become known to the competitors of 
exporting firms. It is the usual practice to hoist the flag of the United States over the consul's office daily, and a copy of the coat of arms of the United States is displayed in a conspicuous place.

One of the marks of differentiation of diplomatic and consular officers consists in the fact that the latter are not entitled to the privileges and immunities of embassadors and ministers. In civilized countries they have no powers of immunity from civil or criminal process except so far as they may be granted by local customs or by mutual courtesy. It is not possible for the United States to secure special immunities for its consuls except by treaty or by representation, in case a difficulty arises, that discourteous treatment accorded the consuls of the United States abroad will be likely to be followed by similar treatment of the consuls of the foreign countries in the United States. In a civilized country the consul has no authority to undertake any diplomatic matters except that in case of the absence of a diplomatic officer he may make representations to the foreign government on matters relating to his own sphere of duty. In semicivilized and non-Christian countries, however, the character of a diplomatic agent with his privileges and imunities are, to a large extent, reserved to a consul. These privileges include the exercise of judicial functions, which are gradually being restricted but which still obtain in some non-Christian countries and will be referred to at length in a later paragraph.

Although consular officers have not the diplomatic immunities, they are under the special protection of international law and are regarded as officers of both their own nations and the nation within whose borders they are located. The granting of an exequatur confers upon the consul all the rights and privileges necessary for the proper conduct of his office, and he can claim for himself not only the rights and privileges which have been accorded to consuls of his nation by treaty, but also all other rights and privileges which may have been afforded or established by local law or custom with regard to consuls of other nations, or that have been extended to his 
predecessors. The only way in which he may be debarred from exercising such rights is through formal notice to him that they have been withdrawn. These privileges generally include the right to place the arms of his government over his door, though this is now generally added to by the tacit permission to display the flag of his country, unless such display is specifically prohibited by the laws of the country. Archives and official property of his office are exempt from search or seizure, although he cannot claim a sinilar immunity for his personal goods. He is protected from the billeting of soldiers on his residence and can claim immunity from local militia or military duty, and from service on juries. The consul is, however, subject to the payment of all local and municipal imposts and taxes, and to the criminal and civil law of the jurisdiction in which he resides. If, however, he does not engage in business or own real estate, he is generally relieved from the operations of civil process; but this is not universally the case, as consuls have become subject to proceedings in local courts through negotiations relating to rented property only. In case of the commission of a crime, a consul would be either tried under the local laws or returned to his own country for trial, as the local authorities might elect. Consuls have no claim under international law to a ceremonial on the part of a foreign nation in recognition of their position, and have only rank and precedence with regard to the consuls of other nations at the same place and with regard to military and naval officers of their own nation. The precedence of several consuls located at the same point and representing different nations is deternined by the clate of their several exequaturs under which they are regarded as officials of the foreign nation. By this simple expedient any difficulties which might arise as to the exact clate of their appointment by their own nation and their consequent precedence or the relative importance of the various nations is avoided. With reference to officers of the Army and Navy, Consuls-General rank with Brigadier Generals in the Army, or Rear Adnirals, junior grade, in the Navy. Consuls and consular agents rank with captains in the Navy, or colonels in the Army. Vice consular officers, deputy consular officers, consular agents and deputy consular agents rank with 
lieutenants in the navy or captains in the army. The rank of consular officers with consular officers of the United States is determined by the date of their commission from the United States, instead of the date of their exequatur, as with reference to consuls of other nations.

When a squadron of vessels of the Navy of the United States visits a foreign port, it is the duty of the commanding officer to send an officer ashore to call upon the consular officer and tender him a passage to the flagship or vessel. The consul is required to accept the invitation and to visit the vessel and tender his services to the commanding officer. Upon leaving the vessel, the consular officer is entitled to a salute of nine guns, if a consul general, and seven guns, if a consul, which salute he acknowledges by standing in the boat when leaving the naval vessel, and lifting his hat during the time the guns are being fired.

Where a treaty between two nations contains what is known as the most favored nation clause, the consuls of the United States are entitled to claim from the government under whose jurisdiction they are located, all the rights, privileges and immunities which are granted to each or any of the consuls of other nations located within his post and jurisdiction of duty. Various provisions relating to the immunities of the consular officers are made by treaty, the provisions differing with the circumstances surrounding the relations of the countries at the time the treaty was made.

While the redress of grievances of citizens of the United States is mainly committed to the care of diplomatic representatives, the greater number of the consular officers make it certain that knowledge of impending danger to such citizens will more quickly come to the attention of a consul general or consul. For this reason, requests for the services of the vessels of the navy of the United States for the protection of American citizens come more numerously from consuls than from diplomatic representatives. A consul has no right to order a vessel of the United States navy to visit his port, but he is required to state to the commanding officer of the vessel the reason why such a movement would be 
advisable, leaving it to the responsibility of the officer, in connection with his orders, to determine whether such a visit should be made. On the other hand, officers of the navy are required to pay strict attention to such requests, and to grant them when practicable.

The duties of a consular officer with regard to the citizens of the United States, not seafaring men, are similar to those of a diplomatic officer, except that the representation and intervention which may be necessary for the assistance of such persons is made to the local authorities instead of to the authorities of the State. A register of the American citizens within the consular jurisdiction is kept in the consulate, but there is no requirement of compulsory registration except for the purpose of establishing American citizenship under the terms of the new citizenship law. Such a register is, however, of value in determining the citizenship of a person in case of an application for protection. Subordinates of consuls, if natives of the nation in which the consulate is located, cannot be guaranteed protection except in accordance with the local laws or customs. It is usual to give such employees certificates, stating the nature of their employment, in order that they may secure the rights which may be granted to them. Consular officers may become the custodian of the consular duties of a foreign nation upon request and under circumstances similar to those described under the heading of Diplomatic Officers.

Among the more onerous of the duties of a consul are those relating to the shipping and scamen of the merchant marine of the United States. Evcry master of a vessel arriving in a foreign port is required, under a penalty of $\$ 500$, to deposit his register and other ship's papers with the consul of the United States, if there be one at the port of arrival. These papers include the crew list and shipping articles. and are to be safely kept and only returned to the master of the vessel when he shall produce the clearance of his ressel from the proper officer of the port and has complied with all the provisions of law relative to the treatment of seamen. When these conditions have 
been fulfilled, and the proper fees paid by the master of the vessel, the consul has no further power to detain the papers and cannot use their detention as a means of collecting debts against the vessel. If the master should sail without his papers, the consul must transmit a statement of the circumstances to the State Department, which will present the matter to the appropriate officers for the imposition of penalties upon the vessel and her master and owners upon her arrival at her home port. In case any officer, seaman or passenger of a vessel of the United States, or any foreigner in a matter relating to personal interests of any citizen of the United States, desires to make a declaration or protest, it can be made before a United States consul, and copies of the protest or declaration, if authenticated by the seal of the consul, will receive credence in law equal to the original in all courts of the United States. Consular officers are required to keep notices to seamen published by the Hydrographic Office of the United States on file for the information of masters and officers of vessels.

Every master of an American merchant vessel who engages any seamen at a place out of the United States, is required to enter into such engagement in the presence of the consular officer, if there be one, and to procure the consul's sanction before carrying the seamen to sea. Consular officers are required to be certain that the seamen understand the contract before approving of the shipping contract.

The consul of the United States in a foreign port is the protective officer of American seamen, so far as such exist at the present time. American seamen are defined as citizens of the United States who have been regularly shipped in American vessels, foreigners shipped in American vessels in a port of the United States, and seamen being foreigners, wherever shipped in vessels of the United States who have declared their intention of becoming citizens of the United States. It being required by law that a master of a vessel shall enter into a bond to return the men exhibited on his crew list when he sailed from a port of the United States to a port in the United States, he cannot lawfully 
discharge a seaman in a foreign port without the intervention of the consular officer. It is not material that the seaman has agreed to the discharge, or that he has misconducted himself. If a master desircs to discharge a seaman in a foreign port, he must make application to the consul, who can authorize such discharge under circumstances provided by law, among which are that the scaman has misconducted himself; that the vessel has been sold abroad; that the shipping agreement has been completed; upon the complaint of the seaman that the voyage is continued contrary to agreement, and the consular officer is satisfied that the voyage has been mmecessarily prolonged, and when a desertion of a seaman has becn caused by unusual or cruel treatment. If members of the crew of a vessel complain that she is unseaworthy or has been sent to sea without the necessary equipment, the consul may appoint inspectors and upon his approval of their findings, the crew or any of them may be discharged if they so desire, unless the deficiency is repaired at once. Discharge can further be granted in consequence of the sickness of a seaman with inability to perform his duties, when there is mutual consent of the master and seaman; when one seaman is exchanged for another or transferred; when the seaman is arrested and awaits trial; when the vessel is wrecked, stranded or condemned as unfit for service. If deficiencies are found to exist which are shown to be accidental and which are removed, cause for the discharge of the crew is thus obviated. When a seaman is discharged in a foreign port, it is the duty of the consular officer to attach his certificate as to the cause for discharge of the seaman to the shipping articles, and to give a certificate to the seaman.

It is the special duty of the consul to take cognizance of any complaints of cruel or abusive treatment, and if a seaman has thus been caused to desert his ship, to grant his discharge, or to grant his disclarge from the ship if he still remains aboard. On the other hand, if a seaman is insubordinate, dishonest, habitually drunken, or quarrelsome, the consul has power to discharge him, although it is in the consul's discretion, if the man offers 
amendment, to allow him to remain on board the vessel. It sometimes occurs that the owners of a vessel desire to remove the master while he is abroad, and such removal is carried on, if the proper authority is shown, by means of the interposition of the consul. When a master is manifestly unfit to continue the voyage, he may be removed by the consul, and another master appointed in his place. When the consul authorizes the discharge of a seaman, he is required to collect the payment of all wages and extra wages due, under penalty of being held accountable for the same himself. He is, however, empowered to retain from such wages any amounts which have been expended for the care of the seaman which are not chargeable to the vessel, and if the wages are not sufficient to meet these charges, the balance is met from a fund in the Treasury for the maintenance and transportation of destitute American seamen. Seamen who have been wrecked within the consular jurisdiction are taken care of and assisted by the consul, and maintained until they can be sent to the United States on a returning vessel. The consul may, however, give them the option of engaging for another voyage before returning to the United States in lieu of such transportation. When no immediate prospect offers of a vessel returning to the United States, they may be forwarded to another consulate, where a better opportunity for return will be afforded. In addition to the wages regularly earned by a seaman under his shipping agreement, he is entitled to a month's extra wages, which must be collected by the consular officer, in case he is discharged on account of the unseaworthy condition of the vessel, when the vessel is sold abroad (unless the seaman immediately reships), when the voyage has been unduly extended, and when he has been injured in the service of the vessel. The right to extra wages is granted in order that the seaman may not become a public charge, and it may not be waived by him.

If a seaman dies on board a vessel of the United States merchant marine, the master is required to take charge of his money and effects, and, on arriving at the next foreign port, turn them 
over to the consul if so required by that official. These, as well as the effects of seamen who die ashore in foreign comntries, are to be carefully preserved by the consul and every effort made to collect all of the scaman's property. The total amount, less any necessary charges, must be transmitted to the district judge of the United States nearest to the home port of the vessel or to the port where the vessel's voyage terminates. Consuls are required to relieve destitute seamen who may come within their jurisdiction, to give them maintenance within a reasonable amount, and passage to the United States under the most economical conditions possible and consistent with good treatment. The amounts necessary for this relief are paid out of the general fund appropriated by the United States for the purpose, and the administration is similar to the cases of discharged seamen previously referred to. Effort is made to secure employment for the seamen on a vessel, and to this end a seaman may be sent from one consulate to another at the expense of the fund. Should no opportunity for shipping them afford itself, they are to be sent to the United States on the earliest and most convenient means of transportation. It is usual to contract for the board of seamen with some local persons, and in addition to their subsistence, suitable clothing and medical attendance is provided for. Sailors of the navy do not come within the provisions for the relief of merchant seamen, and if these are left ashore under the care of the consul, the latter is required to obtain some guarantee from an officer or a deposit to cover the cost of the expense of the attendance of the naval seamen. It is obligatory on the master of a vessel of the United States bound to a port in the United States, to take destitute seamen on board at the request of the consul, and to transport them at not exceeding \$1o for each person for voyages of not more than thirty days, and not to exceed $\$ 20$ for each person for longer voyages when in sailing vessels, and not exceeding two cents per mile when the transportation is by steam. Additional compensation is, however, allowed when the seaman is unable to do duty, as he is expected to aid in the navigation of the vessel as a portion of the consideration 
for his transportation. If a seaman deserts his vessel, all his arrears in wages, clothing, etc., are forfeited and from the sales created by the cash proceeds, the master is to be reimbursed for any expenses to which he may be put by reason of the desertion, the balance remaining goes to the benefit of a fund for the relief of sick, disabled and destitute seamen. The consul is required to see that the proper accounts are made by the commanding officer, so that the fund may benefit as largely as possible from the balance paid in.

When treaty provisions, or the custom of the local authorities permit, consuls may cause the arrest of deserting seamen, and secure their imprisonment until they can be sent on board the vessel with assurance that the offense of desertion will not be repeated owing to the immediate departure of the ship. Imprisonment is not regarded as a punishment, but is simply as a detention to secure the return of the deserter to the ship, and the prevention of his second desertion. Arrest and imprisonment cannot be demanded as the right, but in the majority of cases is facilitated by local laws designed to prevent the deserting seamen from becoming a public charge. If a desertion is successful and permanent, the fact must be noted upon the crew list in order to save the amount of the master's bond, but the consul must be assured before giving such certificate that the desertion has not been connived at by the master for the purpose of saving a portion of the seaman's wages, which would otherwise become due during the enforced idleness of a long stay in port. Consular officers are especially warned not to use the fund for the relief of seamen in such a manner as to encourage desertion of seamen with the hope of being provided for by the government after they shall have left the ship.

Consular officers have no authority, by virtue of their position, over either seamen or other citizens of the United States. Treaty provisions or local custom in the majority of instances, assign to the consuls jurisdiction over all disputes between the masters and seamen of vessels, and over all matters relating to the internal affairs of United States vessels in foreign ports. This, 
however, does not relate to serious offenses which are punishable under local laws, or between disputes between masters or supercargoes of vessels and merchants ashore. For convenience, however, the enforcement of the laws of the nation under the flag of which the vessel sails, is left to the consul and, as a matter of comity, the police resources and the powers of the courts are usually placed at the consul's disposition to enforce his decree. Such a provision is made by statute in the United States and is reciprocated by most of the foreign nations.

The master of a vessel is required by law to give the crew full liberty to lay their complaints before the consul ashore and if a seaman expresses a desire to see the consul, the master must send forthwith to advise the consul to that effect, stating the reason why the seaman is not permitted to land, and that the consul is desired to come on board. Should this privilege be denied to a seaman, the latter, on return to the United States, would receive additional consideration by a court in any demand he might make for recompense for injuries received. Provision is made, in case of formal filing of complaints against the master, for a hearing before the consul, who may render a summary judgment and affix the cost of the proceedings in his own discretion. If a seaman makes a complaint without reasonable ground, he is liable to forfeit one week's wages, and if the master is at fault, he is liable to a penalty not exceeding $\$ 100$.

The processes of the courts of the country are generally extended to enforce consul's decrees. Consuls within whose jurisdiction vessels are wrecked, are required to take charge of the property of citizens of the United States and to save the same as far as possible. A consul has also the custody of property, which has been wrecked and belonging to a citizen of the United States and which is brought within his jurisdiction. In countries where franchises as to wrecked vessels are granted to private companies, the consul is not to interfere except as the representative of the owners of the property, and in such capacity to be present and see that the rights of such owners are preserved under the terms of the local laws. In certain cases of heroism 
on the part of persons engaged in the rescue of shipwrecked crews, consuls are empowered to pay suitable rewards to the rescuers. Consuls are required to collect and preserve, and transmit to the Department of State, the papers of any vessels which may be wrecked and which must, under the law, be returned to the officials of the Treasury Department to complete the history of the vessel. When the master of a vessel desires to sell her abroad for the reason that she is unseaworthy, and that repair would not be warranted by her subsequent value. the consul has general supervision of the transaction and authorizes the survey which determines the true condition of the vessel, and the propriety of the position of the master in regard to her sale.

When war breaks out between two foreign countries, it is, under the present status of international law, of advantage to the ship owners of one or the other of the countries to place their vessels under a neutral flag. The number of cases of sale of vessels to American owners under peaceful conditions would be comparatively few, but in case of a war between two foreign nations efforts would be made to get their merchant vessel under the American flag and a consul is required to exercise the most careful supervision of such transactions before granting the certificate which is requisite in order to establish the fact of American ownership in a vessel formerly in a foreign mercantile marine. It is required that such a transaction should be absolutely bona fide, and without suspicion that the vessel is being placed under a foreign flag, that is, that of the United States, temporarily and for the period of the war only, with the intention of returning her to her former ownership when hostilities shall have ceased.

When grave offenses against the laws of the United States shall have been committed on the high seas on a vessel of the United States merchant marine, and which offenses are thus under the jurisdiction of the courts of the United States, the consul at the first foreign port which the vessel shall touch is required to take full depositions as to the circumstances of the 
crime, and to preserve the same for transmission to the United States. He is required to secure from the local officers facilitics for the detention of the person or persons accused of the crime, and is to detain, also, two principal witnesses, who, together with the prisoner or prisoners, are to be sent to the United States at the first opportunity, together with a carefully prepared report of all the details of the matter. If necessary, the consul may employ a keeper to accompany the prisoners to the United States, but only when an arrangement for safe-keeping cannot be made with the transportation company with whom the contract for passage is made. Consuls are required to be active and vigorous in the prevention of transportation of paupers, criminals, Chinese persons and other excluded classes to the United States. Every master of a vessel carrying immigrants is required to submit a manifest of these passengers to the consul, in lists consisting of not more than thirty persons to each list, and the fact that they have been properly examined, including a medical examination must be sworn to before the consul. If the consul is of the opinion that a concerted effort is being made to send excluded persons prohibited by law into the United States, he is required to take immediate measures of protest and to notify the diplomatic representative of the facts in the case.

Consuls in China have important functions with regard to the operation of the Chinese exclusion act, and are required to ascertain and certify the facts relating to any Chinese persons claiming the right to enter the United States as a nember of one of the excepted classes.

Consuls at foreign ports are also made supervisory officers over the health conditions of vessels clearing for ports in the United States, and upon being satisfied that no contagious or infectious disease may break out by reason of exposure to contagion or infection on the part of the vessel or its personnel, will give a certificate or bill of health which will entitle the vessel to enter the ports of the United States without detention at quarantine. When it is lecmed to be necessary, the President may detail an officer of the Public Health and Marine Hospital Ser- 
vice to the office of a consul, for the purpose of determining whether vessels clearing from that particular port may be given clean bills of health and under one condition. A penalty is provided for every vessel which shall clear from a foreign port and enter a port of the United States without having secured a bill of health from the consul. Consuls are also required to report to the State Department immediately by cable upon the outbreak of cholera, yellow fever or other serious epidemic diseases. The regulations of the United States as to the importation of sheep, cattle and hides are also to be enforced by consular officers in accordance with the provisions made for their guidance.

When citizens of the United States, other than seamen on board vessels, die abroad, consuls are required to take possession of their personal effects, to inventory the same with the assistance of two merchants of the United States, if such are available, otherwise with the assistance of two other merchants, to collect debts due the deceased and due from him, to sell at auction such part of the estate as shall be of a perishable nature or as shall be necessary to pay the debts, and to transmit the balance to the Treasury of the United States to be held for the heirs of the deceased. This administratorship applies when the citizen leaves no partner, trustee or representative capable of undertaking the trust. If the citizen leaves a will, the consul is bound by its terms so far as they are consistent with the laws of the country, and if another administrator is appointed, the consul is to assist that person in the proper performance of his duty. The foregoing function of the consul is exercised under treaty rights or when consistent with local conditions, but when local authorities express an unwillingness for the consul so to act, he is merely to proceed as far as may be possible under the local regulations. The administrative authority of the consul relates to personal property alone, the real estate being under the law of the country where it is situated. If a citizen dies on the high seas and his effects are brought within the jurisdiction of the consul, the latter is to proceed as heretofore set forth. 
Consuls of the United States have certain qualified powers as to the marriage of American citizens residing abroad. The statute provides that marriages in the presence of any consular officer of the United States in a foreign country between persons who would be authorized to marry if residing in the District of Columbia, shall be valid to all intents and purposes and shall have the same effect as if solemnized within the United States. The provision of the code of the District of Columbia with regard to marriages includes the prohibition of marriages within certain degrees of relationship and consanguinity, the marriage of idiots or lunatics; any marriage promoted by force or fraud and any marriage in which either party is incapable from physical causes from entering into the marriage relation.

This provision does not of itself confer upon a consular officer the power to solemnize marriages. It is the principle of international law that the law of the place of marriage shall, whenever this is practicable, determine the mode of solemnization. When a consul is requested to act as official witness of a marriage, he is required to see that the requirements of the law of the place of celebration have been as far as practicable complied with. If this has been the fact, then the presence of the consul gives to the requirements of the foreign country the same effect in determining the legality of a marriage as is possessed by compliance with the requirements of the District of Columbia or of a State of the Union in determining the binding effect of a marriage ceremony throughout the United States. The consul is required to give each of the parties a certificate of the fact of the marriage and to send a certificate to the Department of State, this certificate being under the consular seal and giving the names of the parties, their age, places of birth and residence, the date and place of the marriage, and the certification that the consular officer was present. Care is especially enjoined upon the consuls and should be taken by contracting parties to see that the presence of the consul is not regarded as a reason for omitting some of the other necessary requirements, 
as otherwise, because of the oversight, the marriage might prove to be invalid.

Consuls have, when directed by the Department of State, similar duties to diplomatic officers with regard to requesting extradition of criminals and of authenticating requests of foreign countries in extradition cases. While consuls are not exempt from local taxation in the city in which they reside, they are by courtesy relieved of certain forms of taxation and are required to report the details of taxes imposed or remitted in order that the United States may pursue a reciprocal course regarding the consuls of the nation involved. The requirements and privileges of consuls as to the use of uniforms, government dispatch bags, handling of letters to private persons, presents and testimonials to foreign powers, acting for foreign states and as to pecuniary responsibility for citizens, are similar to those in the diplomatic service, as is the fact with regard to leaves of absence. All powers of attorney in a foreign country for the transfer of any stock issued by the United States or for the receipt of interest thereon, are required to be verified by the consul and no fee can be charged for such service. Pension vouchers are also executed free of charge by United States consuls for beneficiaries living abroad.

A portion of the duties of the consular officers to which especial attention has been paid in recent years is that of making reports as to foreign commercial conditions. Consular officers are expected to prepare from time to time reports on the condition of foreign commerce and international trade, manufactures, agriculture, etc., the improvement of old and the development of new industries, introduction of articles made in the United States, or imitations of them, application of business or mechanical methods employed in the United States, the importation and use of food supplies, materials and manufactures from the United States and the modifications necessary to make them acceptable to foreign consumers, the facilties for direct and indirect communication with the United States, the development or decline of commercial and manufacturing centers, 
changes in the economic condition of the people, conditions of living, sanitation, etc., changes in tariff legislation, and undertakings of unusual size and moment, such as the construction of railroads, public works, etc. These reports are made monthly and at intervals as special reports on subjects of unusual interest. They are transmitted to the State Department and there arranged and prepared for transmission to the Department of Commerce and Labor, which has charge of the publication of all such reports. Consuls are also requested to furnish samples of seeds, plants and manufactures, together with such other articles as may come into their possession which would prove of interest to the different bureaus of the government, and they are especially charged to advise the State Department of any discrimination of foreign countries against products of the United States. Consuls are also required to furnish the Treasury Department with prices current of articles usually exported to the United States, and also make monthly reports of the rates of exchange, together with such crop reports and similar advices as may be of benefit to the Department of Agriculture.

The judicial power of consuls of the United States is not easy to summarize, for the reason that it differs materially in nearly every instance. Generally speaking, in non-Christian and semicivilized countries, consuls of the United States have civil and criminal jurisdiction in all matters which are exclusively between citizens of the United States. In some countries they form a portion of the court which tries questions arising between citizens of the United States and native born subjects. The consular jurisprudence is more extended and better defined in China than elscwhere, and by an act passed in 1906, and under the treaty provisions with China, they are definitely established as judicial officers of original jurisdiction in civil cases where the value of the property involved does not exceed $\$ 500$, and in criminal cases where the punishment for the offense charged cannot exceed $\$ 100$ fine, or sixty days' imprisonment, or both. Consuls, under these provisions, have power to arrest, examine 
and discharge accused persons, or to determine their cases within the above limits or to hold them for trial in the United States Court for China, an appeal being also allowed from all final decisions of the consular courts to the United States Court, the proceedings of the consular courts being revised by the United States Judge. The consular courts in Japan were abolished upon the reformation of the Japanese jurisprudence, but in other semicivilized countries, including the Barbarry States and elsewhere, consuls have similar powers to administer justice, the details differing, however, with more or less degree, according to the location and the treaty provisions. Thus in Turkey there are mixed tribunals carried on by the consuls of the various countries for the trial of cases between Christians not of the same nationality, consular courts having the business of each nation of foreign Christians, while questions between natives and foreign Christians are heard in Turkish courts in which the consul may appear in behalf of his fellow citizen. The consuls are required to proceed under the laws of the United States in countries where they have judicial jurisdiction and may impose fine for contempt. Whenever a consul deems it advisable that he shall have assistance in the trial of a case, he may associate with him citizens of the United States not to exceed four, such selection being subject to the approval of the minister, who also constitutes a court of appeal and the final authority in a capital case except in China.

The chief duty of many consuls, especially those residing at interior manufacturing points, consists in the authenticating of the sworn invoices required by law for every importation of dutiable merchandise in excess of the value of $\$$ roo. Before the merchandise is shipped, an invoice properly prepared and setting forth the actual market value of the goods at the point at which the shipment is made up, must be produced before the consul, sworn to and certified by him. The consular officers are authorized to require, before certifying any invoice, satisfactory evidence that it is accurate and true. This means that in order to carry on his duties properly, the consul must inform himself as to the quality of goods, the cost of production and 
their actual market value. When the value of goods cannot be determined by examination of the invoice, samples are required in triplicate, one set to be retained at the consulate, one sent to the board of general appraisers in New York, and one direct to the port in which the merchandise is to be entered. A consular officer is required to designate the original, duplicate and triplicate of the invoice, the original being filed for preservation in the consular office, the duplicate given to the person producing the invoice, and the triplicate sent, without the intervention of any party in interest, to the collector of customs of the port at which the merchandise is to be finally entered. The consul is also required to certify on claims from foreign merchants for the return of articles manufactured in the United States which have been sent abroad as containers of manufactured goods and which are desired to be returned without customs imposition. In this respect, the consul is required to certify as to the right of such goods to be returned without duty.

\section{SUBORDINATE CONSULAR OFFICERS.}

The subordinate officers of the consular service are vice-consuls, general deputy consuls, general vice-consuls, deputy consuls and consular agents. These are all appointed by the Secretary of State, but usually upon the nomination of the consular officer affected. Minors are not appointed to subor linate consular offices, and all persons so appointed must be able to speak and read the English language, and in all cases where it is at all possible, subordinate officers must be citizens of the United States. The vice-consul is an official who takes the place of the consul when the latter is unable to perform his duties, and receives such portion of the consular salary as may be agreed upon for the service, or, if there is no agreement, one-half of the consular salary. The deputy consul is an official provided for the assistance of the consul in his work, and is deputized to sign papers certifying to invoices, etc., in assistance but not in lieu of the consul. Vice-consuls and deputy consuls are generally 
clerks in the consular office, and receive salaries from $\$ 300$ to $\$ \mathrm{I}, 500$ a year, according to the amount of work performed. It is the general practice, however, to have the vice-consul and the deputy consul the same person. Consular agents are delegates of the consul, who are situated at other points than the location of the main consular office, and who perform the duties of consul under the supervision and direction of the chief consular officer. They are paid by fees up to and not exceeding \$I,OoO per year. Every substitute and subordinate consular officer, except consular agents, must give a bond of not less than $\$ 2,000$, and, as consuls are responsible for the acts of consular agents, the former may require the agents to give a bond, for his own protection.

Marshals of consular courts are provided where the judicial function of the consul is considerable, and are required to give a bond. They are appointed by the President, and attend upon the consular court, preserving order therein and executing all processes of the court. They are especially designated as subordinates of the consul or vice-consul in charge, and are required to assist, when necessary, in the general work of the consul. Marshals receive a salary of $\$ I, 000$ per year, and such fees as they may be allowed by the ministerial regulations, and are required to file a bond of $\$ 10,000$. The marshal of the United States court for China, who succeeded the consular marshal, now receives $\$ 3,000$ per annum. Regularly appointed interpreters to consuls of the United States, if citizens of the United States, qualify by taking the oath of office, and are not required to give a bond. It is the policy of the State Department, so far as possible, to appoint such interpreters from the number of student interpreters provided by law, and who are under agreement to serve the United States for ten years.

Provision is made by law for thirteen consular clerks, who are appointed by the President and hold office during good behavior. They are assigned from time to time to such consular offices as the Secretary of State may direct, and receive $\$ \mathrm{I}, 000$ for the first year's service, and $\$ 200$ additional each year 
thereafter, until the maximum of $\$ \mathrm{I}, 800$ is received. No person can be appointed a consular clerk who is not over eighteen years of age, and the establishment of this grade was intended to provide a source from which adequate consular appointments could be made. In addition to these clerks, clerical assistance is afforded consular officers by means of appointment of clerks which may be made, if nccessary, by the Department of State, and the salaries of whom vary from $\$ 300$ to $\$ 1,500$ a year.

SUPERVISION OF THE SERVICE.

By the law which reorganized the consular service, provision was made for five inspectors of consulates, with the title of consul general at large. They are appointed by the President, with the advice and consent of the Senate, from the members of the consular force possessing requisite qualifications. They receive an annual salary of $\$ 5,000$ each, with necessary traveling expenses, and make such reports as the Secretary of State directs, reporting to him the rcsult of their investigations. It is required that every consulate shall be inspected at least once in two years, and the establishment of this force of inspectors relieves the consuls general, to a large degree, from the inspection work previously done by them. If a consul general at large finds that the work of a consul general or consul is not being properly performed. the President may authorize the inspecting officer to suspend the delinquent consul and administer the office in his stead for a period not exceeding ninety days. The consul general at large, upon taking charge, has power to suspend any subordinate consular officer for the same period of time. It is the purpose of this provision to give the President immediate power of dealing with any irregularity that may be brought to his attention, but at the same time the period during which a consul general at large can act is strictly limited to the period which nay be necessary to secure the presence of a new permanent incumbent at the post of duty. 


\section{CLASSIFICATION OF CONSULATE-GENERAL AND CONSULATES.}

The act for the reorganization of the consular service of the United States, approved April 5, I906, provides:

"SEc. 2. That the consuls-general and the consuls of the United States shall hereafter be classified and graded as hereinafter specified, with the salaries of each class herein affixed thereto."

CONSULS-GENERAL.

London,

Berlin,

Habana,

Calcutta,

Cape Town,

Constantinople,
Antwerp,

Barcelona,

Brussels,

Canton,

Auckland,

Beirut,

Boma,

Buenos Aires,

Callao,

Chefoo,

Adis Ababa,

Bogotá,

Budapest,

Athens,
Class I.-Salary, \$12,000 (2 in all). Paris.

Class II.-Salary, \$8,000 (6 in all).

$\begin{array}{ll}\text { Hamburg, } & \text { Rio de Janeiro, } \\ \text { Hongkong, } & \text { Shanghai. }\end{array}$

Class III.-Salary, $\$ 6,000$ (8 in all).

$\begin{array}{ll}\text { Mexico City, } & \text { Vienna, } \\ \text { Montreal, } & \text { Yokohama. }\end{array}$

Ottawa,

Class IV.-Salary, $\$ 5,500$ (I i in all).

$\begin{array}{ll}\text { Frankfort, } & \text { St. Petersburg, } \\ \text { Marseilles, } & \text { Seoul, } \\ \text { Melbourne, } & \text { Tientsin. } \\ \text { Panama, } & \end{array}$

Class V.-Salary, \$4,500 (18 in all).

$\begin{array}{ll}\text { Coburg, } & \text { Munich, } \\ \text { Dresden, } & \text { Newchwang, } \\ \text { Guayaquil, } & \text { Rome, } \\ \text { Halifax, } & \text { Rotterdam, } \\ \text { Hankow, } & \text { St. Gall, } \\ \text { Mukden, } & \text { Singapore. }\end{array}$

Class VI.-Salary, $\$ 3,500$ (9 in all).

$\begin{array}{ll}\text { Guatemala, } & \text { San Salvador, } \\ \text { Lisbon, } & \text { Stockholm, } \\ \text { Monterey, } & \text { Tangier. }\end{array}$

Class VII.-Salary, $\$ 3,000$ ( 3 in all).

Christiania,

Copenhagen. 
CONSULS.

Class I.-Salary, $\$ 8,000$.

Liverpool.

Class 1I.--Salary, $\$ 6,000$.

Nanchester.

Class III.-Salary, $\$ 5,000$ (8 in all).

Bremen,

Belfast,

Dawson,

Amoy,

Amsterdam,

Birmingham,

Cienfuegos,

\section{Bahia,}

Bombay,

Bordeaux,

Colon,

Dublin,

Dundee,

Harbin,

Apia,

Barmen,

Barranquilla,

Basel,

Berne,

Bradford,

Chemnitz,

Chungking,

Cologne,

Dalny,

Durban,

Havre,

Kobé,

Lyons,

Lourenço Marquez,

Class IV.-Salary, $\$ 4,500$ (I2 in all).

Foo Chow,

Glasgow,

Kingston (Jamaica),

Nottingham,
Santiago de Cuba,

Southampton,

Veracruz,

Valparaiso.

Class V.-Salary, $\$ 4,000$ (2I in all).

$\begin{array}{ll}\text { Leipzig, } & \text { Reichenberg, } \\ \text { Nanking, } & \text { Santos, } \\ \text { Naples, } & \text { Stuttgart, } \\ \text { Nuremberg, } & \text { Toronto, } \\ \text { Para, } & \text { Tsingtau, } \\ \text { Pernambuco, } & \text { Vancouver, } \\ \text { Plauen, } & \text { Victoria. }\end{array}$

Class VI.-Salary, $\$ 3.500$ (32 in all).

$\begin{array}{ll}\text { Edinburgh, } & \text { Prague, } \\ \text { Geneva, } & \text { Quebec, } \\ \text { Genoa, } & \text { Rimouski, } \\ \text { Georgetown (Guiana), } & \text { San Juan del Norte, } \\ \text { Lucerne, } & \text { Sherbrooke, } \\ \text { Mannlicim, } & \text { Smyrna, } \\ \text { Montevideo, } & \text { Three Rivers (Quebec), } \\ \text { Nagasaki, } & \text { Vladivostock, } \\ \text { Odessa, } & \text { Winnipeg, } \\ \text { Palermo, } & \text { Zurich. }\end{array}$

Port Elizabeth, 
Class VII.-Salary, $\$ 3,000$ (47 in all).

$\begin{array}{lll}\text { Aix la Chapelle, } & \text { Huddersfield, } & \text { Sandakan, } \\ \text { Annaberg, } & \text { Iqutitos, } & \text { Seville, } \\ \text { Barbados, } & \text { Jerusalem, } & \text { St. John (N. B.), } \\ \text { Batavia, } & \text { Kehl, } & \text { St. Michaèis, } \\ \text { Burslem, } & \text { La Guaira, } & \text { St. Thomas (W. Indies), } \\ \text { Calais, } & \text { Leghorn, } & \text { San José (Costa Rica), } \\ \text { Carlsbad, } & \text { Liege, } & \text { Sheffield, } \\ \text { Colombo, } & \text { Nainz, } & \text { Swansea, } \\ \text { Dunfermline, } & \text { Nalaga, } & \text { Sydney (N. S. W.), } \\ \text { Düsseldorf, } & \text { Nanagua, } & \text { Sydney (Nova Scotia), } \\ \text { Florence, } & \text { Nantes, } & \text { Tabriz, } \\ \text { Freiburg, } & \text { Nassaut, } & \text { Tampico, } \\ \text { Ghent, } & \text { Newcastle (N. S. W.), } & \text { Tamsui, } \\ \text { Hanilton (Ontario), } & \text { Newcastle-on-Tyne, } & \text { Trieste, } \\ \text { Hanover, } & \text { Port Antonio, } & \text { Trinidad. } \\ \text { Harput, } & \text { Port au Prince, } & \end{array}$

Class VIII.-Salary, \$2,500 (6r in all).

$\begin{array}{lll}\text { Acapulco, } & \text { Hull, } & \text { Puerto Cortes, } \\ \text { Aden, } & \text { Jeres de la Frontera, } & \text { Rheins, } \\ \text { Alexandretta, } & \text { La Rochelle, } & \text { Rosario, } \\ \text { Algiers, } & \text { Leeds, } & \text { Roubaix, } \\ \text { Bamberg, } & \text { Madrid, } & \text { St. Etienne, } \\ \text { Batum, } & \text { Nagdeburg, } & \text { St. John's (N. F.), } \\ \text { Belize, } & \text { Nalta, } & \text { Sarnia, } \\ \text { Bergen, } & \text { Maracaibo, } & \text { Sault Ste. Marie, } \\ \text { Breslaut, } & \text { Martinique, } & \text { Stettin, } \\ \text { Brunswick, } & \text { Matanoros, } & \text { Tamatave, } \\ \text { Cardiff, } & \text { Nazatlan, } & \text { Tegucigalpa, } \\ \text { Chihuahua, } & \text { Milan, } & \text { Teneriffe, } \\ \text { Ciudad Juarez, } & \text { Moscow, } & \text { Trebizond, } \\ \text { Ciudad Porfirio Diaz, } & \text { Nice, } & \text { Valencia, } \\ \text { Collingwood, } & \text { Nogales, } & \text { Weimar, } \\ \text { Cork, } & \text { Nuevo Laredo, } & \text { Windsor (Ontario), } \\ \text { Crefeld, } & \text { Orillia, } & \text { Yarinouth, } \\ \text { Curaçao, } & \text { Plymouth, } & \text { Zanzibar, } \\ \text { Eibenstock, } & \text { Port Hope, } & \text { Zittau. } \\ \text { Gothenburg, } & \text { Port Limon, } & \\ \text { Hamilton (Bermuda), } & \text { Prescott, }\end{array}$


Cluss IX.-Salury, \$2,000 (7o in all).

\begin{tabular}{|c|c|c|}
\hline $\begin{array}{l}\text { Aguascalientes, } \\
\text { Antigua, } \\
\text { Asuncion, } \\
\text { Bagdad, } \\
\text { Belleville, } \\
\text { Belgrade, } \\
\text { Bristol, } \\
\text { Calgary, } \\
\text { Campbeliton, } \\
\text { Cape Gracias a Dios, } \\
\text { Cape Haitien, } \\
\text { Cartagena (Colombia), } \\
\text { Castellamare Gi Stabia, } \\
\text { Catania, } \\
\text { Ceiba, } \\
\text { Charlottetown, } \\
\text { Coaticook, } \\
\text { Cormwall, } \\
\text { Durango, } \\
\text { Ensenada, } \\
\text { Fort Erie, } \\
\text { Funchal, } \\
\text { Gaspé, } \\
\text { Gibraltar, }\end{array}$ & 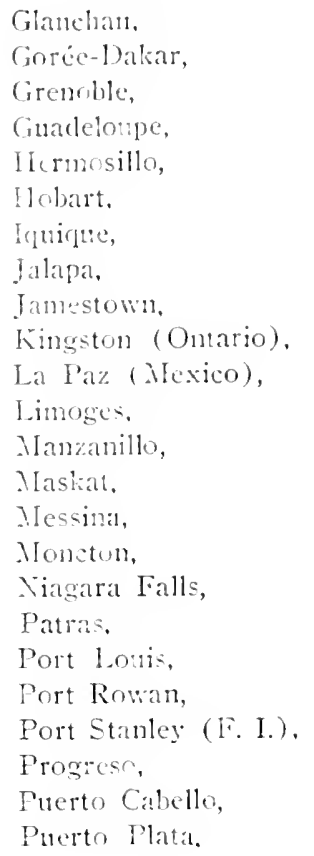 & $\begin{array}{l}\text { Riza, } \\
\text { Rruen, } \\
\text { Saigon, } \\
\text { St. Christopher, } \\
\text { St. I! yacinthe, } \\
\text { St. John's (Quebec), } \\
\text { St. Picre, } \\
\text { St. Stephen, } \\
\text { Siltillo, } \\
\text { Sierra Leone, } \\
\text { Sivas, } \\
\text { Stavanger, } \\
\text { Suva, } \\
\text { Taliti, } \\
\text { Turin, } \\
\text { 'Turks Island, } \\
\text { Tuxpam, } \\
\text { Ctila, } \\
\text { Venice, } \\
\text { Warsaw, } \\
\text { Windsor (Nova Scotia), } \\
\text { Vinodstock. }\end{array}$ \\
\hline
\end{tabular}

T.IRIFE OF UNITEI) STATES CONSLILAR FEES.

[Revised to take effect November I, I006.]

Tariff of fees preseribed by onler of the President to be charged by consular officers of the Lnited States. Nil consular charges must be in strict accordance with this tariff, and he collected in gold or its quivalent. No fee or compensation will be collected for any service not covered by this tariff.

(The fees in this tariff are not prescribed for American ressels and seamen, because they are exempted by law from the payment of consular fees. Consular agents will make the fees in this tariff the basis of collection from the Treasury for services to such vessuls. Foreign-built vessels. unregistered, owned by American citizens, are not exempt from the paymeat of the fees prescribed herein.) 


\section{Miscellancous services.}

I. Certificate to invoice, including declaration, in triplicate or quadruplicate, covering either importations or transit shipments, including any additional declaration or certificate not otherwise provided for which is required by law or regulations for use in connection with the entry of the wares or the forwarding of the same in bond $\ldots \ldots \ldots \ldots \ldots \ldots \ldots . \$ 2 .{ }^{\circ}$

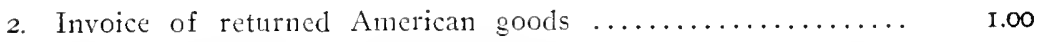

3. Extra certificates or declarations as above described, including immigrant's oath (Form No. I28), when issued without an

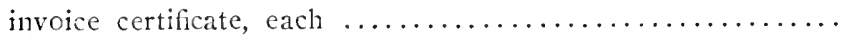

4. Certificate to extra copies of invoices, each ............ $\quad$ 1.00

5. Certificate of disinfection, in triplicate or quadruplicate .... 2.50

6. Landing certificate, including oaths of master and mate, and the complete execution of the certificate $\ldots \ldots \ldots \ldots \ldots \ldots$

7. Sealng cars coming irom Canada or Mexico, for each manifest in quadruplicate with the consul's certificate, including sealing of each car, vessel, bale, barrel, box, or package ..........

8. Issuing a passport-Form No. $9 \ldots \ldots \ldots \ldots \ldots \ldots \ldots \ldots \ldots \ldots$

9. Visaing a passport-Form No. 10 ..................

Io. Visaing a Chinese passport or certificate.............. I.0o

II. Marriage certificate, in duplicate, Form No. $87 \ldots \ldots \ldots \ldots \ldots$ I.00

12. For taking into possession the personal estate of any citizen who shall die within the limits of a consulate, inventorying, selling, and finally settling and preparing or transmitting, according to law, the balance due thereon, $\$ 2$ for each $\$$ ioo of value or fraction thereof. If part of such estate shall be delivered over before final settlement, \$I for each \$Ioo of value or fraction thereof to be charged on the part so delivered over as is not in money, and $\$ 2$ for each $\$ 100$ of value or fraction thereof on the gross amount of the residue. If among the effects of the deceased are found certificates of foreign stock, loans, or other property, \$I for each \$10o of value or fraction thereof on the amount thereof. No charge will be made for placing the official seal upon the personal property or effects of such deceased citizen, or for breaking or removing the seals.

13. For each certificate of protection, semsar, or certificate of em-

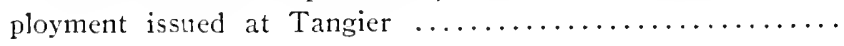


Sirvices to iessels and seumen.

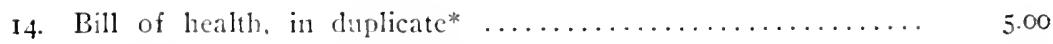

15. Supplemental bill of health, in duplicate $\ldots \ldots \ldots \ldots \ldots \ldots \ldots \ldots .2 .50$

16. For receiving and delivering ship's register and papers, including consular certificates, as prescribel in Forms Nos. I3 and I. \$I for cach. 100 tons or fraction thereof, registered measurenent (net), of the vessel for which the service is performed, if under 1,000 tons; but for American vessels running regularly by weekly or monthly trips, or otherwise, to or between foreign ports, this tonnage fee will not be charged for more than four trips in a year: and tonnage fees shall not be exacted for any vessel touching at or near ports in Canada on her regular voyage from one port to another within the United States, unless some official service required by law shall be performed.

17. And for cvery additional 100 tons net or fraction thereof....

I8. Shipping or discharging seamen, including the certificates thereof attached to crew list and shipping articles and given to

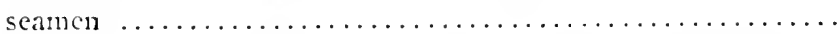

19. Authentication of copies of protests or other necessary documents for vessels or seamen not otherwise provided for..

20. Preparation and acknowledgment for vessels or seamen of any oath or declaration for which a form is given in the Consular Regulations, or a similar necessary service not

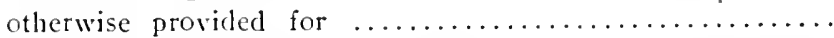

21. Preparation and execution for vesscls or seamen of any certificate for which a form is given in the Consular Regulations, or similar necessary service not otherwise provided for.....

22. Orders or letters for vessels or seamen for which forms are given in the Consular Regulations, or other similar necessary

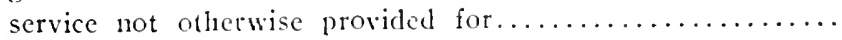

23. Recording, when necessary, for ressels or seamen any document covered by the provisions of the Consular Regulations,

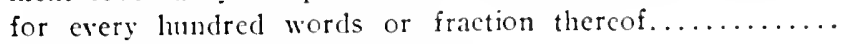

24. Noting marine protest-Form No. $37 \ldots \ldots \ldots \ldots \ldots \ldots \ldots$

*Foreign war vessels are exempt from the payment of fees for bills of health. 
25. Extending marine protest-Form No. $38 \ldots \ldots \ldots \ldots \ldots \ldots \ldots$ 3.00 If it exceed two hundred words, for every additional one

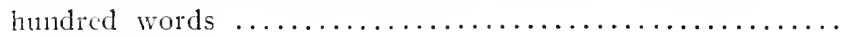

26. Protest of master against charterers or freighters-Form No.

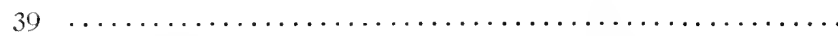

27. Clearance when issued by the consul, as at free ports .......

28. Attending an appraisement of vessel's goods or cffects, for each

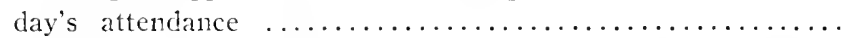

29. Attending sale of ressel's goods, for each day's attendance

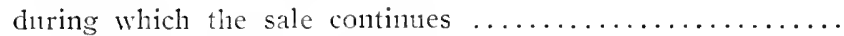

30. Attendance at a shipwreck, or for the purpose of assisting a ship in distress, or of saving wrecked goods or property, over and above traveling expenses, whenever the consul's interposition is required by the parties interested, for each day....

\section{Notarial and other scrices}

3I. Administering an oath and certificate thereof* $\ldots \ldots \ldots \ldots$ 2.00

32. Administering oath and preparing passport application........

33. Acknowledgment of a deed or power of attorney, or similar service, including one or more signatures, with certificate

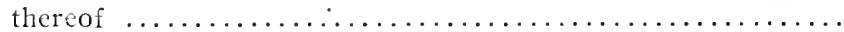

34. Administering any and all oaths required to be made by pensioners and their witnesses in the execution of their pension vouchers, or certifying to the competency of a local official

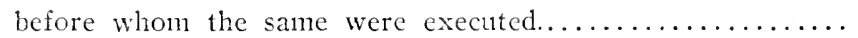

No fee.

35. Aclinowledgnents connected with the transfer of United

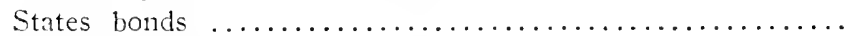

No fee.

36. Adninistering oaths to or taking acknowledgments of officials or employees of the United States Government, in connection with their official business or accounts. .................

No fee.

37. For rendering notarial services to officials of foreign governments who render gratuitously reciprocal courtesies to American diplomatic and consular officers $\ldots \ldots \ldots \ldots \ldots \ldots$ No fee.

*Executive order of December 2I, I906, prescribes that no fee shall be charged for the affidavit of temporary stay of owner of sealsinin garment entering the United States from Canada. 
38. Certifying to official character of a foreign notary or other

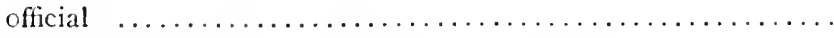

39. For taking depositions, executing commissions or letters rogatory, where the recorl of testimony, inchuling caption and

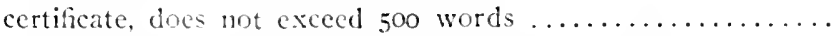

10.00

For each arditinal 100 words or fraction thereof.........

The foregoing fee shall cover the administration of the oath and all services of the consul as commissioner, but shall not include services of clerk, stenographer or typewriter, which shall be additional at the rate prescribed herein for copying.

40. Copies :

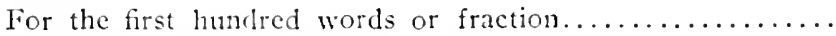

For every additional hundred words or less............ .25

41. Translations: for every one hundred words or fraction.......

42. Additional fee for all services contemplated by fees numbered $3 \mathbf{1}, 32,33,38,39$ when rendered elsewhere than at the consular office at the request of the interested partics, for

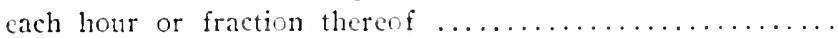

In connection with any service renlered outside of the consular office at the request of private individuals, the exact amount of the expenses actually and necessarily incurre 1 by the person rendering the scrvice shall be collected from the persons for whom the service is performed in additicn to the fee or fees prescribed therefor and a note of the amount shall be made on the margin of the fee book and fee return opposite the entry of the service and fee; but no amount in excess of the fee or fees preseribed and such actual and necessary expenses shall be charged or accepted.

\section{T.I.-Compensation of Consular Agants.}

The act for the reorganization of the consular service of the United States, approved April 5, I906, proviles:

"SEc. 8. That all fees, official or unoficial, receised by any officer in the consular service for services rendered in connection with tile duties of his office or as a consular officer, including fees for notarial services, and fees for taking depositions, executing commissions or letters rogatory, settling estates, receiving or paying out moners, caring for or disposing of property. shall be acconnted for and paid into the Treasury of the Lnited States, and the sole and only compensation of such oflicers shall be by salaries fixed by law; but this shall not apply to consular agents, who shall be paid by one-half 
of the fees received in their offices, up to a maximum sum of one thousand dollars in any one year, the other half being accounted for and paid into the Treasury of the United States. And vice-consuls-general, deputy consulsgeneral vice-cossuls, and deputy consuls, in addition to such compensation as they may be entitled to receive as consuls or clerks, may receive such portions of the salaries of the consul-general or consuls for whom they act as shall be provided by regulation." 


\section{CHAPTER IX.}

THE TREASURY DEPARTMENT.

The organization of the Treasury Department is directed to three ends: The collection of moneys which may become due to the United States; the proper custody thereof, and the payment, under due safeguards, of the amounts which have been ascertained to be due to claimants against the United States or to authorized disbursing officers for the purpose of meeting the necessary expenses of the government. While the incidental operations of these three activities are intricate and complicated, all of the work of the Treasury Department in the final analysis is directed toward one of the three purposes suggested.

The main sources of the income of the United States are the duties imposed upon goods manufactured abroad and brought into the United States and taxes levied upon certain manufactures within the United States. One grand division of the Treasury Department, therefore, relates to the collection of customs and cf the internal revente impost taxes. A second section relates to the custody and care of the money collected, together with the matters growing out of the possession by the nation of large assets, which must be so administered as to be available at all times for the nation's needs, withont withdrawing from the circulating mediums of the country reserve funds of so large amounts as to cripple commerce and the interchange of commodities. Thus upon the basis of the necessary reserve of actual coin and upon the guarantee of financial ability afforded by the national assets and tax-paying power, a currency or circulating medium is established by means of which, withont the actnal excliange of gold and silver, commercial transactions may be carried on and the great system of commercial credit sustained.

By the third function of the Treasury Department, amounts which may be due from the United States are accurately ascertained and 
the public treasury guarded from unwarranted expenditures and the mistakes and possible bad faith of public servants.

THE CUSTOMS SERVICE.

Two methods of raising revenue are available to sovereigns and sovereign States. One is the assessment of a given amount on each person in the nature of a contribution toward the expenses of the State, and the other is a tax or tariff upon certain articles of consumption by which the consumer indirectly contributes to the support of the government. The several States forming a federal union having relinquished to the Federal Government the right to impose duties on imports to the Federal Government, are compelled to raise the bulk of their incomes by direct taxes, which are generally apportioned on the basis of the value of the real estate or the personal property of the citizen. The immediate assessment upon real and personal property is generaly made by the city or town in which the property is located, and consists of a proportionate property tax to be paid by the individuals to the city or town, and by the municipality to the county for the expenses of the latter. For State purposes exclusively a poll tax is frequently assessed collectible by the municipality and paid by the latter to the State, otherwise State expenses are paid by an assessment on municipalities.

The difficulties of assessing and collecting direct taxes by other than the smaller governmental subdivisions has made the device of a tariff tax that most generally followed by central governments, while within the United States there has always been a strong advocacy of the theory that the tax on imported articles was, in addition to being a more or less satisfactory way of raising income for the support of the Federal Government, a device of economic value as well. It is also a prevailing theory that indirect taxes for the support of the government should bear more heavily upon the luxuries of life than upon the necessities. That is to say that the burden of those who are able only to secure the necessary articles of life should be less than upon those who are able to afford more than the necessities. 
In the earlier days of manufacturing in the United States it was argued that while articles brought from abroad were perhaps finer and better than those produced in the United States, the latter country could produce articles which would answer the purpose of the foreign manufacture in clomestic use, so that those who preferred to use foreign goods ought to be required to pay a fee for the privilege. Following close npon this came the theory that if the goods produced abroad were sold in the United States at a sum greater than that charged for goods of home manufacture, there would be a marked stimulus to manufacturing industries of the United States, so that the prosperity of the country would be materially enhanced. The other side of the argument has been sustained by those whose interest has been in the possession of agricultural staples and who desired to have mantifactured goods on the market at as low a rate as pussible in order that, in the exchange, a given quantity of natural products might secure the largest possible quantity of manufactured products. The reply to this position has been that the artificial stimulus of manufacturing creates a greater denand for natural products so that the home market thus extended for both natural and manufactured products is of greater benefit to the manufacturer and to the agriculturist, including of course the employees of both, than a system under which goods might be purchased from the cheapest source of production.

The economic policy of the United States previous to the Civil War alternated between results based upon the argument of two schools of tariff thought, but there has been, since the Civil War, no material departure from the higher rate of tariff alopted as a revenue raising expedient to meet the tremendous cost of that great struggle. Duties of course have been lowered and changed and there have been periods of so-called low tariffs. These have been, however, but transitory, and the purpose of the tariff legislation of Congress affecting the collection of revenues at the ports of revenue of the United States has been, almost uniformly, during the past quarter of a century to afford as much protection as possible to American manufacturers without regard to whether the 
duties imposed would result in a maximum of revenue or a minimum of items on which an import duty was placed.

Most of the customs revenues are collected at the chief seaport cities of the United States, but large sums are also involved in connection with imports via Canada and Mexico and in order to guarantee their collection it is necessary to maintain an organization covering the entire coast and frontier of the country. The portion of the Treasury Department which performs the duties of collection of customs duties, and of guarding against the unauthorized introduction of foreign goods, that is to say, smuggling, is under one of the Assistant Secretaries of the Treasury, and has developed in the history of the United States a highly specialized organization intended to see that all dutiable goods are declared, and that the proper assessment and collection of duties is made.

For the purpose of adequate administration of the law relative to the importation of foreign goods, this country is divided into customs districts, a list of which is appended hereto. Officers are appointed for such districts, the chief of which in each case is the collector of customs. It is evident that every point at which it might be possible to land foreign goods from the sea and to bring them in from an adjacent country could not be furnished with a customs officer, so that it is necessary to designate within districts one or more places where such goods may be entered according to law; the collector residing at the principal point in such district, and deputy collectors residing at the subordinate places. It also frequently occurs that a city having a considerable trade is not in the way of direct importations, but receives its foreign goods through another port of entry. For the convenience of merchants of such cities these places are designated as ports of delivery though they may be far distant from the coast or frontier, and goods delivered at ports of entry are aliowed to be transported in bond across the intervening country to the city of their destination, where they are assessed for duties by a local customs officer, generally a surveyor, in the same manner as they would have been if they had been surveyed for imposition of duty at the port of entry, and then shipped on to their destinations. 
Collectors of customs are appointed by the President by and with the advice or consent of the Senate for terms of four years. The collector, as well as all other officers of the customs, is required to take and subscribe to an oath in addition to the usual oath of office that he will use his best endeavors to prevent and check frauds against those laws of the United States which impose duties upon imports. Collectors are required to give bonds for the faithful discharge of their duties, the amount of the bond in each case being fixed by law, and in proportion to the amount of custon duties collected within the various districts. The compensation of the collector is also either determined by the fees collected by him, or he is allowed an annual salary based upon the collections of his district.

On the arrival of a vessel containing goods from abroad or by means of land transportation at a port of entry, it is the first duty of the captain of the vessel or person in charge of the goods to deliver to the collector at the port of entry a correct statement of the character, quantity, and value of the goods which have been transported, such statement being checked by a copy of the certified invoice which has been forwarled by the consular officer abroad at the point of shipment, as cletailed under the chapter on Consular Officers. It is the duty of the collector to require that suitable guarantee shall be given for the payment of duties which may be imposed upon the goods, and upon this guarantee being given, the collector will issue a permit for the unloading of the vessel, or for the goods involved to proceed to their point of destination if inland transportation is involved.

In the unloading of vessels the proper account of the quantity and quality of goors is kept and reported to the collector who estimates the amount of duty which is to be pail thereon. and sees that the amounts thus becoming due are paid into him to be deposited in the treasury of the United States directly, or through one of the designated depositories.

The collector, as chicf of the local custom officials, is charged with the supervision of the duties of the other officials, and with the employment of such subordinates as may be necessary, and for 
whose employment specific provision has not been made by law. He must also see that suitable storehouses are provided for the custody of goods, and that the proper weights and measures are available for determining quantities.

In the more important ports an official is provided to assist and check the collector in the matter of assessment of duties. This is the "naval officer," who is appointed by the President by and with the advice of the Senate for the term of four years, and who receives copies of all manifests and entries of goods and is required to estimate, together with the collector, the duties on all merchandise subject to duty, no duties being receivable without the estimate of the ilaval officer at the port where such an officer has been provided. The naval officer is also required to keep a separate set of estimates from those kept by the collector, and is required to countersign all permits, clearances, certificates and other documents which the collector grants. The naval officer is also required to examine the collector's records and accounts and certify the same when they are found to be correct. When there is no naval officer appointed at a port it is provided that the collector shall perform the duties of such an officer and at a port where neither a naval officer or surveyor is appointed, a collector is supposed to assume the duties of all three places.

Collectors of the customs under former law were permitted to name, with the approval of the Secretary of the Treasury, such a number of proper persons as deputy collectors of customs as he might deem necessary. This was superseded, however, in I904, when deputy collectors were included in the classified civil service of the United States. As at present conducted, many of the customs districts of the United States present the condition of the collector or head of the service in the district receiving from the fees allowed to him only a moiety of the amount which is paid as salary to his deputy and, in some instances, the compensation of the collector is less than that of all but the very lowest classes of employees. It is also the fact that in many of the districts the cost of collecting the revenues from customs is nearly equal to, and in some cases it may be equal to or exceed the amounts collected. This 
condition has frequently been called to the attention of Congress, but local pride in contesting the abolition of any custom house, coupled with the consideration that such establishments as now exist act as a preventive against smuggling, has, so far, prevented radical action with reference to relucing the number of custom houses and the cost of the collection of the customs revenues.

All collectors, as well as other principal customs officers, are required to give an account to the Treasury of all emoluments and also for all expenses incident to the offices which may be paid from the fees. They are required to cxecute and carry into effect all instructions of the Secretary of the Treasury in regard to the revenue laws, and in case any difficulty arises to adhere to the construction placed upon the law by the Secretary of the Treasury.

No collector is allowed to reccive in the aggregate from fees and emoluments sums amounting to more than $\$ 6.000 .00$ a year, but this is exclusive of the expenses which he may incur in the conduct of his office. This maximum amount is, however, seldom reached, for the reason that provision has been made for salaries for the collection districts to which it would apply, and provisions have been made for smaller maximum amounts in accordance with the importance of various districts and which are fixed in detail by the statutes. As evidencing the comparison in importance between the offices of collector and of naval officer, it may be noted that the maximum compensation of these emoluments of the naval officer is fixed at $\$ 5,000.00$.

In order to facilitate and make certain the process of collecting duties on foreign goods it is made unlawful for a vessel laden with such goods to enter at other than a lesignated port of entry or to unload her cargo without a collector's permit. Every vessel arriving within a collection district must within 24 hours make a report of such arrival to the collector under a heavy penalty.

If a vessel has on board distilled spirits or wine a special report as to this portion of the cargo must be made within 48 hours after the arrival of the vessel. When any portion of the cargo of a vessel is to be carried on further to another foreign port, notice must be given within 48 hours to the collector of the character of the 
merchandise to be re-exported, and a bond filed that it shall not be landed at any point within the United States. Special provisions are made by law permitting the collector, with the approval of the Secretary of the Treasury, to make arrangements with the masters of vessels engaged in regular pacliet service between a port of entry and a foreign port to file a preliminary declaration and bond so that the vessel upon arrival may proceed at once to the wharf and begin unloading without any delay for the purpose of inspecting or surveying the cargo to determine the approximate amount of duty which will become due.

Where vessels are to proceed from one district to another within the United States, the collector is required to redeliver to the master of the vessal the manifest of the cargo with a notation thereon of the proportion of the cargo which has been landed within that particular district, only such duties being collected in that particular district as accrue on account of the merchandise there landed, the duties of the balance being collected at the point where the merchandise is finally delivered. It is required, however, that a bond shall be given in the district of the first entry that the balance of the merchandise shall be duly landed in another district within the United States, and the duties paid thereon.

The report of the master of the vessel, heretofore set forth, is not the formal entry of the merchandise for the collection of duties, being a preliminary to that act and a provision of law intended to secure to the collector notice of all merchandise coming within his jurisdiction. Within ${ }_{5}$ days after the delivery of the manifest of cargo to the collector, the owner or consignee of the imported goods must make a sworn statement before the collector, of the character and value of the goods imported, producing the original invoices of the merchandise, and especially certifying the kind of money in which the invoices are made out. Such entries may also be made by the agents of the owners or actual consignees, in which case a bond of $\$ 1,000.00$ is taken from the agent as a guarantee that the owner or consignee shall furnish all of the information required by law and which is necessary to secure the proper assessment of duties.

A certain proportion of commodities on board a vessel being such 
as would be used as sea stores are exempt from duty, but it is in the discretion of the collector to determine whether the articles presented as sea stores are in excess of the necessary amount, and in such case to impose duties upon the balance.

Certification of invoices by a consul of the United States is one of the chicf means of identification in determining the proper rate to be clarged as import duties, and to this has been added the system of appraisement to be hereafter described. If a collector has reason to suspect that a shipment of goods has been undervalued or wrongly described so that a loss may be caused to the United States, he is empowered to take the merchandise into his possession and to retain it until such time as its actual value may be ascertained. The usual time for unlading the cargo is between sunset and sunrise in order that there shall be competent inspection. In cases of necessity, however, collectors have power to issue special licenses for the unlading of the cargo at night and under such restriction as the collector may deem to be advisable. A limit is also set by law to the time during which a vessel bringing in dutiable articles must be unloaied. This is from ten to twenty-five days, in accordance with the size of the vessel, and if any merchandise is found in the vessel after that time which has not been declared for another district or a foreign port. the collector may take possession of such merchandise. An extension may be granted, however, in case of a vessel loaded with articles in bulk, not to exceed a further time of fifteen days.

No dutiable article landed can be removed from the wharf or warehouse if it is required to be weighed or measured until it has been properly measured and the evidences of measurement affixed to it according to law.

When a vessel puts into a port of the United States in distress the master may, by making oath as to the circumstances, secure immunity from the customs regulations for such time as may be necessary, and also receive a permit for the unlading of the goods carried by the vessel, if such an unlading is made necessary by the repairs required. It is required that such unlading shall be duly overseen, and that precaution shall be taken to prevent the goods so unladen 
being removed, so that they will enter the United States without the payment of duty.

The collector is required to designate on the invoice of goods presented for entry at least one package on every invoice and one package at least of every ten packages of merchandise to be opened, examined, and appraised. This duty is usually performed by examiners and appraisers appointed by law at the different ports, but when no such officials are provided for the collector may call in the services of two merchants, who, through familiarity with the character of the goods to be appraised, may be expected to be able to pass an expert opinion upon them. Such an appraisement is, of course, under oath, while the appraisement of the officers provided by law is under their general oath as customs officials.

No merchandise which is liable to inspection and appraisement can be delivered without such action has been duly taken and the results certified to the collector. Arrangements may be made, however, by giving hond in double the amount of the probable duty to secure possession of the main portion of the shipment while the packages reserved for appraisement are being examined. The value of the goods upon which the duty is assessed is the market value of such imports at the principal markets of the country from which the merchandise was exported, and upon the day of its exportation. This provision marks the value of the consular service not only in certification of invoices, but in furnishing information to customs officials of the prices current of the commodities usually sold in the countries in which they are stationed.

The manner of the appraisement of imported goods includes the most difficult and delicate of the duties of the customs service. At the principal ports appraisers and their assistants are employed who are especially versed as to the qualities and values of particular goods imported at those ports.

The most important port in this respect is the port of New York, which handles large quantities of finer grades of European manufactures. The preliminary work with regard to the appraisement of goods is performed by the appraiser or one of his assistants. If performed by one of the latter the results of the appraisement are 
revised and corrected by the appraiser, who certifies the same to the collector of the port. In ports where no appraiser is provicled me of the subordinate customs officials is designated to perform the duty. If the collector accepts the result of the appraisement, he fixes the duties upon the shipment of goods accordingly. Should, however, he regard the appraisencnt as too low, he may order a reappraisement to be made by one of the general appraisers hereafter described.

If the importer regards the appraisement too high, he may, within two days, if he has complied in all respects with the requirements of law as to due entry of the merchandise, make a protest to the collector, on receipt of which the collector must direct a reappraisement by one of the general appraisers.

Nine of the latter officials are provided for to be appointed by the President by and with the advice and consent of the Senate, and to receive a salary of $\$ 7,000.00$ a year. Not more than five of the general appraisers can be appointed from the same political party, and they are not to be engaged in any other business or employment, the purpose of the law being to provicle a force of entirely impartial arbitrators between the importers and those who are charged with the duty of fixing the customs revenues under the law.

The general appraisers are assigned so that three of the appraisers are on duty daily in New York, forming a board of general appraisers for appeal in that port. The other seneral appraicers are assigned to districts by the Secretary of the Treasury, and in addition to the duty of reappraisement heretofore described, have gencral supervision in their districts over appraiscment and chassification, and have authority to take such action as may be needful to secure a lawful and uniform appraisement at the several ports.

If the collector, or the owner, consignee, or the agent, shall be dissatisfied with the reappraisement by the said board of general appraisers, a further appeal is provicled for to a board of three general appraisers, either that which is in constant session in New York, or a board which shall be lesignated by the Secretary of the Treasury. The decision of this board or a majority of the members is final and conclusive and the decision is transmitted to the col- 
lector or person having charge of the collection of the duties for the liquidation of the amounts due unless the owner, consignee, or agent, being still dissatisfied takes an appeal within thirty days to the Circuit Court of the United States within the district within which the matter arises. All papers in this controversy must be referred to the Circuit Court which shall, if it deem advisable, designate one of the general appraisers to take further evidence in the case. Further appeal may be taken to the Circuit Court of Appeals, the decision of which is final unless the Supreme Court grants a writ of certiorari.

In order to promote uniformity of classification and appraisement in tlie different ports in the United States provision is made by law for payment of expenses of local appraisers while in attendance upon meetings directed to be held by the Secretary of the Treasury for the purpose of consultation and comparison on methods of appraisement and of points of construction of the customs laws.

All decisions of the board of general appraisers are open to inspection and an abstract of the same is prepared for the general use of customs officers. The chief business relating to appraisement and classifications is carried on in New York before a permanent board of general appraisers, while the major portion of appeals to the United States Courts originate in the New York customs business.

For the purpose of assisting the officers of the customs and to protect the interest of the United States a solicitor of customs is appointed for the port of New York with assistants who represent the interest of the United States before the board of general appraisers, and prepare cases on behalf of the United States, which are referred to the United States Attorney for the Southern District of New York for prosecution in the Circuit Court.

For the purpose of a proper appraisement the services of experts in many lines, chemists and examiners, are utilized and sub-provisions under the general head of appraisements provide for reexamination and for the protection of the interest of the importer as well as also of the government. Where the business of the port warrants, the collector, under the direction of the Secretary of the 
Treasury may cither hire suitable warehouses, or approve the application of private persons owning such warehouses and stores to allow to be stored therein, articles which are subject to duty, but on which duty has not yet been paid. The purpose of the bonded warehouse system is to provide a means for the safe custody of goods which are not intended for immediate sale or consumption, and where the requirement of the immediate payment of the duties would create a hardship by reason of compelling the merchant to allow large sums, represented by such duties to remain idle. Upon the giving of proper bonds in cases in which the warehouses are of private ownership, or in leaving the goods under the custody of the collector on paying the warehouse fee or storage, the goods may remain in bond until they are needed for actual sale or consumption, the duty being paid upon withdrawal instead of upon being landed from the vessel in which they are imported.

Provision is made for the sale and distribution of the proceeds thereof when goods have remained in a public warehouse or bonded store for a year without payment of charges, and all goods remaining for three years, if charges have been paid in part are to be regarded as abandoned and will also be sold.

The goods which may be deposited in a public store or in a private bonded warehouse may be withdrawn at any time within three years, and re-exported or shipped to another customs district, the duties being paid in the latter district.

Bonded warehouses can only be opened in the presence of the proper officer of the customs and a penalty is provided for unlawful opening and for the fraudulent removal of the goods.

Importers of articles which are to be reimported are entitled to a a drawback on the duty paid, and it is the duty of the collector to have all such merchandise entered for re-exportation, inspected and examined, comparison being made with the original invoice so that no fraud may be committed on the customs by unwarranted claims for refund or deductions on account of re-exportations.

In order that vessels owned by citizens of the United States shall have the benefit of the navigation laws of this country, it is necessary for each such vessel to be registered with the collector of the 
port which is the residence of or the nearest to the residence of the managing owner thereof. The application for such registry must be accompanied by an oath showing the burden of the vessel, the place where she was built, and her ownership, and that no subject of another nation is directly or indirectly interested in the vessel cr the profits arising out of her use. Before the vessel can be registered it must be measured by the surveyor of the port if there be one, or by the collector if there be no surveyor and figures representing her cargo capacity must be cut into her timbers in such a manner that they can not be readily effaced. The collector makes out the register of the vessel in the required form and delivers it to the owner or manager, keeping a record thereof in the book provided for that purpose. This register belongs exclusively to the vessel, and cannot be transferred and if the vessel is lost must be returned to the port at which the register was made. The register serves as an identification of the vessel and without it she becomes an illegal craft not only subject to the loss of the privileges under the navigation laws of the United States, but a subject of suspicion as well to all officers of the United States and of foreign nations.

Upon the saie of a vessel by the owners by whom she was originally registered, it becomes necessary to have the register amended so as to show the fact of the sale. A bond is required of the owner in each case to insure compliance with the navigation laws and that there shall be no evasion of the terms of registry by which foreign citizens may profit by the fact of American registry. Foreign vessels can only be admitted to American registry when becautse of wreck the vessel has had to be substantially rebuilt, or when such alterations have been made upon her as to approximate the cost of a new vessel, the purpose of registration being in part to promote the interest of American shipbuilding, and to prevent the ownership of foreign built vessels under the United States.

When a vessel which has been registered is substantially altered or rebuilt, it is necessary for her to secure a new register, and a change of ownership also requires the application to the collector of the port for re-registration. In addition to the collection of 
customs it is the duty of each port to collect the tonnage dues which are imposed upon foreign vessels. These are on vessels built within the United States but belonging in whole or in part to foreigners, thirty cents per ton, that is to say, per ton burden, which is a certain amount of the cubic space, being the unit of the total arrived at by measuring the length, breadth and depth of all space within the vessel which may be utilized for the transportation of cargo. For vesscls that are not connected in any way with the United States a tax of fifty cents per ton is imposed. A further tax is provicled by law of $\$ 2.00$ per ton on vessels which may enter ports of the United States from ports at which vessels of the United States are not allowed to trade, while there is also a discretionary provision, permitting the remission of tonnage taxes in reciprocity with similar actions of other nations.

Other provisions in the direction of lessening the amount of tonnage taxes are made with relation to vessels making frequent trips from nearby foreign countries, and in cases of vessels making regular trips between the United States and Canada on the inland waters between the two countries but a single imposition of the tonnage tax is required, that on the occasion of the first trip at the beginning of the year.

The executive officer of the collector of the port for services connected with the ascertainment of quantities of merchandise actually landed is the surveyor. He is required under the direction of the collector to attend to the work of all inspectors, weighers, measurers and gaugers, reporting once a week the names of any who neglect their duty. Upon the arrival of a vessel from a forcign port it is the duty of the surveyor to cause her to be boarded and a return made to the collector of the name of the vessel, her nationality and the general character of her cargo. One or more inspectors are put aboard each vessel so arriving, as soon as may be practicable after her entrance within the limits of the port to see that all goods landed correspond with the collector's permit and to ascertain the true quantities and kinds of distilled spirits which may be on board the vessel, and to superintend the lading for exportation of all goods re-exported and on which a drawback may be claimed. 
The surveyor is also required to inspect and certify the correctness of all instruments of measurement used by the subordinate officers. Inspectors of customs houses are utilized to board incoming vessels to see that no goods are removed without permit of the collector, to check goods so removed from the vessel, so that their quantities may be ascertained. Weighers are employed at the point of unlading of the cargo and supplied with scales for the purpose of ascertaining quantities by weight. Gaugers measure packages containing distilled liquors and wines, most of these officers being at the present time under regular salaries are classified under the civil service of the United States, although provision is made for the employment of temporary officials at per diem or fees, as the case may be, when the amount of business done is not such to warrant employment of permanent officials in any given capacity. Inspectors of customs service under direction of the collector are charged with the duty of seeing that the navigation laws with respect to the number of persons that are carried on steam vessels are enforced.

In addition to the regular local employees of the customs service an appropriation is made by Congress for the employment of special agents in the nature of detectives for the purpose of making investigations into the matters relating to the importation of foreign goods, and for supplying the department with information, either as to the operations of the customs officers or of merchants and importers who may be evading the proper payment of duties. These agents are employed not only for service in the United States but abroad as well, and they secure information which is of value to the customs authorities at the points in Europe and other countries where the principal shipments to the United States originate and where conspiracies for the putpose of defrauding the United States may be formed. The reports of the special agent are a part of the total amount of information upon which the collecting officer checks the proper duties to be paid, and the operations of this branch of the services are necessarily kept secret, the duties of the officers being similar to those performed by the secret service with relation to the coin and bank notes of the United States. 
LIST OF CUSTOMS WISTRICTS AND PORTS OF ENTRY AND WELIVERY.

Districts. Ports of Entry. Ports of Delivery.

MAINE.

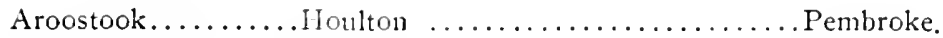

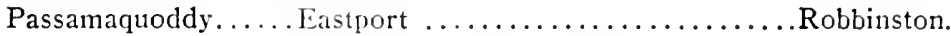

Machias

Calais, subport of entry.

Frenchmans Bay....Ellsworth .............. Union River.

Mount Desert Ferry, subport of entry.

Castine. . .

Castine

Bluehill.

Deer Island.

Bucksport.

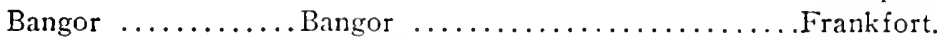

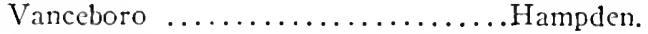

Lowelltown, subport of entry.

Belfast

. Belfast ...................... Prospect.

Vinalhaven, subport of entry. Rockport.

Nort!n Haven.

Camclen.

Waldoboro

Waldoboro

Bristol.

Rockland, subport of entry...... Damariscotta.

Warren.

Thomaston.

Cushing.

St. George.

Wiscasett .......Wiscasett

Boothbay.

Alna.

Bath

Bath

Hallowell.

Pittston.

Georgetown.

Bowdoinham.

Gardiner.

Richmond.

Portland and Fal-

mouth ............ Portland

North Yarmouth.

Brunswick.

Frecport.

Harpswell.

Saco

Scarboro.

Kennebunk.

Kennebunk

IVclls.

York

York 
LIST OF CLSTOMS DISTRICTS AND PORTS OF ENTRY AND DELIVERY-COntinued.

Districts. Ports of Entry. Ports of Delivery.

NEW HAMPSHIRE.

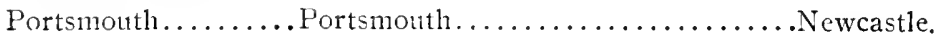

Dover.

Exeter.

VERMONT.

Kittery, Me.

Vermont

. Burlington

Berwick, Me.

St. Albans, subport of entry.

Alburg, subport of entry.

East Alburg, subport of entry.

Swanton, subport of entry.

Highgate, subport of entry.

Franklin, subport of entry.

West Berlsshire, subport of entry.

Windmill Point, subport of entry.

Richford, subport of entry.

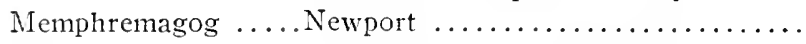

NorthTroy, subport of entry.

Derbyline, subport of entry.

Island Pond, subport of entry.

Canaan, subport of entry.

Beecher Falls, subport of entry.

MASSACHUSETTS.

Newburyport...... Newburyport.............. Amesbury.

Salisbury.

Haverhill.

Newbury.

Ipswich.

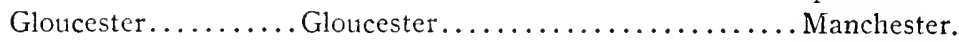

Rockport.

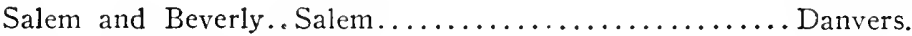

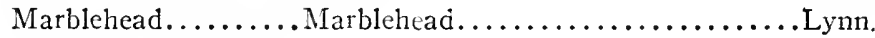

Boston and Charles-

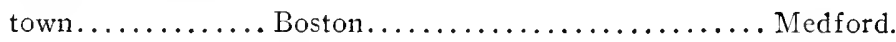

Cohasset.

Hingham.

Weymouth.

Cambridge.

Roxbury.

Dorchester.

Worcester. 
LIST OF CESTOMS DISTRUTS AND PORTS OF ENTRY AND UELHEKr--Continted.

Districts. Ports of Entry. Ports of Delivery.

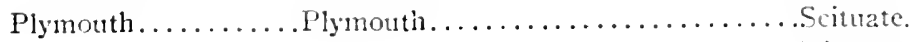

Kingston.

Duxbury:

Marsificlel.

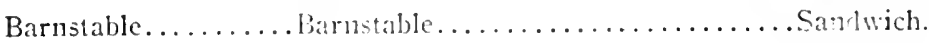

Falmoutli.

Harwich.

Welltiect.

Provincetown.

Chatham.

Dennis.

Nantucket........ Nantucket................ Bourne.

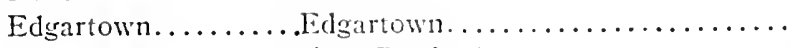

New Bedford........ New Bediford.............. Westport.

Rochester.

Wareham.

Fall River

Swansea.

Somerset.

Freetown.

Berkley.

Taunton.

RHODE ISLAND.

Newport......... Newport................ North Kingston.

Tiverton.

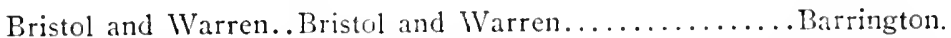

Providence........ Providence............... Pawtuxet.

East Greenwich.

CONNECTICUT.

Stonington........ Stonington.............. Pawcattick River.

New London........ New London............... Norwich.

Groton.

Lyme.

Hartford.......... Iartford

Saybrook.

Enfield.

Clinton.

Westbrook.

Old Saybrook.

lissex.

Chester.

Haduam. 
LIST OF CUSTOMS DISTRICTS AND PORTS OF ENTRY AND DELIVERY-Continued. Districts. Ports of Entry. Ports of Delivery. Hartford......... Hartford................. East Haddam.

Middletown.

Chatham.

Portland.

Cromwell.

Rockyhill.

Wethersfield.

Glastonbury.

East Hartford.

Springfield, Mass.

Vernon (Rockville).

South Manchester

New Haven........ New Haven............... Guilford.

Branford.

Milford.

Derby.

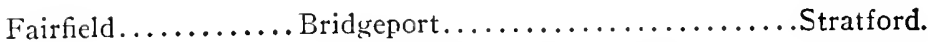

Stamford, subport of entry. Greenwich.

Norwalk, subport of entry.

NEW YORK.

Sag Harbor........ Sag Harbor................ Greenport.

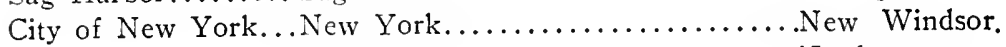

Jersey City......................

Ponghkeepsie.

Esopus.

Kinderhook.

Albany.

Hudson.

Troy.

Rhinebeck L'd'g.

Coldspring.

Port Jefferson.

Patchogue.

Champlain

Plattsburg

Whitehall.

Malone, subport of entry.

Fort Covington.

Rouses Point, subport of entry.

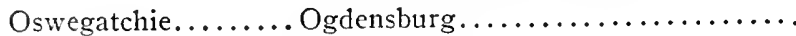

Cape Vincent........ Cape Vincent...............

Oswego........... Oswego.................. Utica.

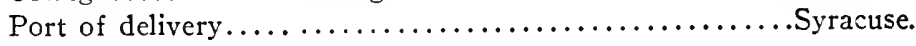


LIST OF CLSTOMS DISTRICTS AND PORTS OF ENTRY AND DELIVERY-Crntimed.

Districts. Ports of Entry. Ports of Delivery.

Genesee.......... River Genesee (Rochester).......

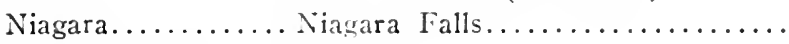

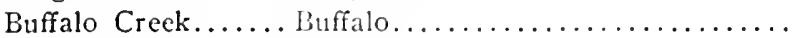

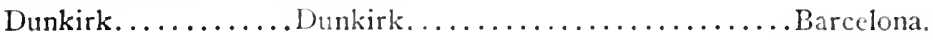

Silvercreek.

Cattaraugus Creck

NEW JERSEY.

Newark ........ Newark ................. Elizabeth.

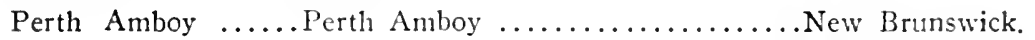

Middletown Point.

Little Egg Harbor Tuckerton $\ldots \ldots \ldots \ldots \ldots \ldots \ldots \ldots$

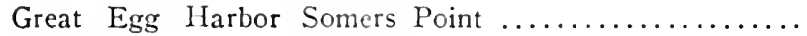

Bridgeton .......... Bridgeton ....................

Port Elizabeth.

Burlington $\ldots \ldots \ldots$. Burlington $\ldots \ldots \ldots \ldots \ldots$.

PENNSYLVANIA.

Philadelphia $\ldots \ldots$ Philadelphia $\ldots \ldots \ldots \ldots \ldots$. Camden, N. J.

Chester, subport of entry.

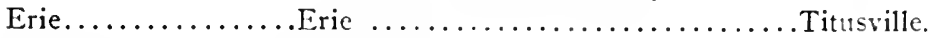

Pittsburg .........Pittsburg

DELAWARE.

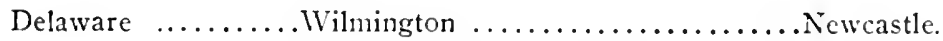

Lewes, subport of entry. Port Penn.

Seaford, subport of entry. Delaware City.

MARYLAND.

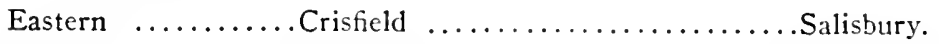

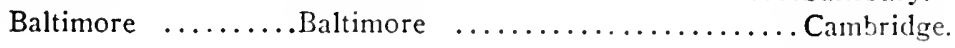

Eastou.

Havre de Grace.

Annapolis .........Annapolis

Benedict.

Lower Marlboro.

Towncreek.

Celar Point.

Nottingham.

St. Marys.

DISTRICT OF COLUMBIA.

Georgetown .......Washington 
LIST OF CUSTOMS DISTRICTS AND PORTS OF ENTRY AND DELIVERY-Continued.
Districts.
Ports of Entry.
Ports of Delivery.

VIRGINIA.

Cherrystone ...... Cape Charles City (Eastville)...... Snow Hill.

Folly Landing.

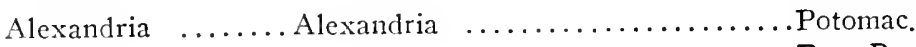

Tappahannock .... Tappahannock ................. Port Royal.

Fredericksburg.

Yeocomico.

Newport News ..... Newport News .............. Yorktown.

Norfolk and Ports- Norfolk and Portsmouth ........... Suffolk.

Smithfield.

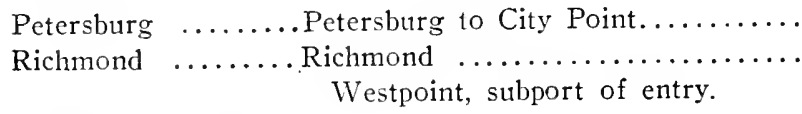

NORTH CAROLINA.

Albemarle ..........Elizabeth City $\ldots \ldots \ldots \ldots \ldots \ldots$

Monteo, subport of entry.

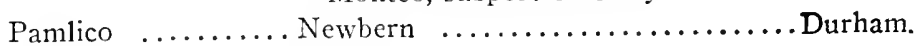

Beaufort .......... Beaufort $\ldots \ldots \ldots \ldots \ldots \ldots \ldots \ldots$

Wilmington $\ldots \ldots$. Wilmington $\ldots \ldots \ldots \ldots \ldots \ldots \ldots$

SOUTH CAROLINA.

Georgetown $\ldots \ldots \ldots$ Georgetown $\ldots \ldots \ldots \ldots \ldots \ldots \ldots \ldots$

Charleston $\ldots \ldots \ldots$ Charleston $\ldots \ldots \ldots \ldots \ldots \ldots \ldots$

Beaufort..$\ldots \ldots \ldots$ Beatufort $\ldots \ldots \ldots \ldots \ldots \ldots$

GEORGIA.

Savannah ..........Savannah

Augusta.

Brunswick ........... Brunswick Frederica.

Darien.

St. Marys

St. Marys

Atlanta.

FLORIDA.

Fernandina ........Fernandina

St. Johns .........Jacksonville

St. Augustine .......St. Augustine

Key West ........... Key West

Punta Gorda, subport of entry.

Palm Beach, subport of entry.

Miami, subport of entry.

Tampa ......... Tampa

St. Marks

Cedar Keys St. Marks. 
LIST OF CUSTOMS DISTRICTS AND PORTS OF ENTRY AND DELIVERY-Continued.

Districts.

Ports of Entry. Ports of Delivery. Port Inglis, subport of entry. Magnolia.

Ocala.

Apalachicola Apalachicola

Carrabelle, subport of entry and delivery.

Pensacola ........ Pensacola

ALABAMA.

Mobile ......... Mobila . Montgomery.

MISSISSIPPI.

Pearl River .......Gulfport Scranton.

Horn Island.

Ship Island.

Natchez ........ Natchez

.Grand Gulf.

Vicksburg Vicksburg

LOUISIANA.

New Orleans .......New Orleans

Wheeling, IV. Va.

Council Bluffs, Ia.

Cincinnati, Ohio.

Louisville, Ky.

St. Louis, Mo.

Sioux City, Iowa.

Memphis, Tenn.

Evansville, Ind.

Burlington, Iowa.

Dubuque, Iowa.

Leavenwort?, Kans.

Omaha, Nebr.

Kansas City, Mo.

St. Joseph, Mo.

Shreveport, La.

La Crosse, Wis.

Cliattanoga, Tenn.

Dayton, Ohio.

Portsmouth, Ohio.

Paducah, Ky.

Lincoln, Nebr.

Knoxville, Tenn.

Teche

. Brashear (Morgan City)

Calcasieu Pass, subport of entry. 
LIST OF CUSTOMS DISTRICTS AND PORTS OF ENTRY AND DELIVERY-Continued. Districts.

TExas.

Galveston Galveston

Houston.

Velasco, subport of entry.

Texas City, subport of entry.

Saluria .Eagle Pass

San Antonio.

Matagorda.

Copano.

Lavaca.

Sabine

Port Arthur

Sabine Pass, subport of entry and delivery.

Corpus Christi........ Corpus Christi .............

Laredo, subport of entry.

Aransas (Rockport), subport of entry.

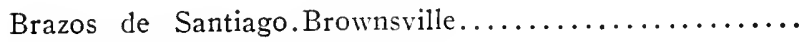

Paso del Norte.....El Paso....................

\section{California.}

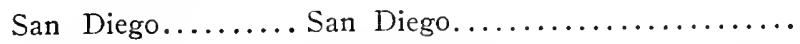

Los Angeles........Los Angeles................

Santa Barbara, subport of entry.

San Francisco..... San Francisco .......................

Oakland, subport of entry. San Luis Obispo.

Humboldt Eureka . Crescent City.

OREGON AND WASHING-

TON.

Southern district of Coos Bay (Empire City) ......... Ellensburg.

Oregon.

Port Orford.

Gardiner.

Yaquina ........... Yaquina

.Newport.

Oregon .............Astoria

Willamette .........Portland

Puget Sound Port Townsend

Aberdeen, subport of entry.

Anacortes, subport of entry.

Bellingham, subport of entry.

Blaine, subport of entry. 
LIST OF CUSTONS DISTRICTS ANin PORTS OF EXTRY AND DELIVERY-COntinued.

Districts.

Ports of Entry.

Ports of Delivery.

Everett, subport of entry.

Friday llarbor, subport of entry.

Gray's llarbor, subport of entry.

Danville, subport of entry.

Northport, subport of entry.

Port Angeles, subport of entry.

Roche Harbor, subport of entry.

Seattle, subport of entry.

Spokane, subport of entry.

Sumas, subport of entry.

Tacoma. sulport of entry.

South Bend, subport of entry.

ALASKA,

Alaska

Juneau

Eagle, subport of entry.

Ketchikan, subport of entry.

Kodiak, subport of entry.

Sitka, subport of entry.

St. Michael, subport of entry.

Skagway, subport of entry.

Unalaska, subport of entry.

Wrangel, subport of entry.

Nome, subport of entry.

Valdez, subport of entry.

MONTANA AND IDAHO.

Montana and Idaho.Great Falls ......................

MINNESOTA.

Minnesota

St. Paul

Minneapolis, subport of entry.

Duluth

Duluth

WISCONSIN.

Milwaukee ....... Milwatuke ............... Kenosha.

Racine.

Sheboygan.

Green Bay.

Depere. 
LIST OF CUSTOMS DISTRICTS AND PORTS OF ENTRY AND DELIVERY-Continued.

Districts. Ports of Entry. Ports of Delivery. MICHIGAN.

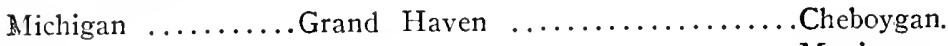

Manistee.

Ludington.

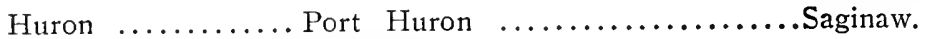

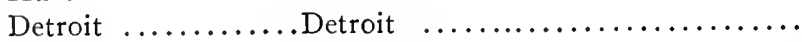

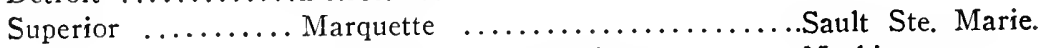

Superior, subport of entry. Mackinaw.

- Ashland, subport of entry.

Gladstone, subport of entry.

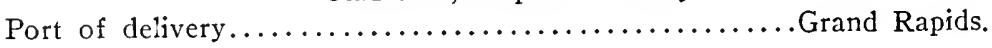

INDIANA AND ILLINOIS.

Chicago $\ldots \ldots \ldots$. Chicago $\ldots \ldots \ldots \ldots \ldots \ldots \ldots$ Waukegan.

Michigan City.

Coal City, Ill.

Cairo, Ill.

Rock Island.

Peoria.

Galena.

INDIANA.

Indianapolis.

OHiO.

Miami ........... Toledo

Sandusky $\ldots \ldots \ldots$. Sandusky $\ldots \ldots \ldots \ldots \ldots \ldots \ldots \ldots$

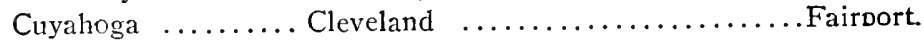

Conneant, subport of entry.

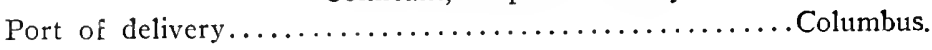

COLORADO,

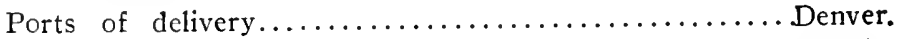

Pueblo.

Durango.

Leadville.

ARIZONA,

Arizona...$\ldots \ldots$...... Nogales

Douglas, subport of entry.

Naco, subport of entry. 
LIST OF CUSTOMS DISTRICTS A.ID PORTS OF EXTRY AXD DELAVERYCOntinued.

Districts. Ports of Entry. Ports of Delivery.

NORTH AND SOUTH

DAKOTA.

North and Soutl. Da-Pembina, N. Dak. ................. Fioulls, S. D.

kota. Portal, N. Dak. Sulport of entry.

TENNESSEE.

Port of delivery $\ldots \ldots \ldots \ldots \ldots \ldots \ldots \ldots \ldots \ldots \ldots \ldots \ldots \ldots \ldots \ldots \ldots \ldots$ Nashille, Tenn.

Iow. 1 .

Port of delivery $\ldots \ldots \ldots \ldots \ldots \ldots \ldots \ldots \ldots \ldots \ldots \ldots \ldots \ldots \ldots$ Des Mloines, Iowa.

UTAH.

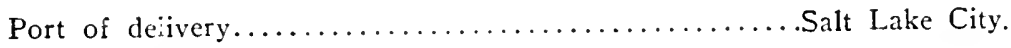

HAWAIIAN ISLANDS.

Hawaii ........... Honolulu

Hilo, port of entry and delivery.

Kahului, port of entry and delivery.

Koloa, port of entry and delivery.

Nahukona, port of cntry and de-

livery.

Porto Rico.

.San Juan

Aguadilla, subport of entry.

Arecibo, subport of entry.

Arrovo, subport of entry.

Fajardo, subport of entry.

Humacao, subport of entry.

Mayaguez, subport of entry.

Ponce, subport of entry.

Guanica, subport of entry.

Ports at achich merchandise may be cutered for transportation to other ports without appraiscment under the act of June Io, I8So.

Astoria, Oreg.

Baltimore, Md.

Bangor, Me.

Bath, Me.

Beecher Falls, Vt.

Boston, Mass.

Burlington, $\mathrm{Vt}$.
Buffalo, N. Y.

Brunswick. Ga.

Island Pond, Vt.

Key Vest, Fla.

Laredo, Tex.

Los Angeles, Cal.

Malone, N. Y.
Port IInron, Mich.

Portal, N. Dak.

Portland, Me.

Portland, Oreg.

Port Townsend, Wash.

Richford, Vt.

Rocliester, N. Y. 
Calais, Me.

Charleston, S. C.

Chicago, Ill.

Cleveland, Ohio.

Detroit, Mich.

Duluth, Minn.

F.agle Pass, Tex,

Eastport, Me.

El Paso, Tex.

Everett, Wash.

Fernandina, Fla.

Galveston, Tex.

Gladstone, Mich.

Honolulı, Hawaii.
Marquette, Mich.

Miami, Fla.

Milwaukee, Wis.

Mobile, Ala.

New Orleans, La.

Newport, Vt.

Newport News, Va.

New York, N. Y.

Niagara Falls, N. Y.

Nogales, Ariz.

Norfolk, Va.

Ogdensburg, N. Y.

Pensacola, Fla.

Philadelphia, $\mathrm{Pa}$.
Rouses Point, N. Y.

St. Albans, Vt.

San Diego, Cal.

San Francisco, Cal.

Sault Ste. Marie, Mich.

Savannah, Ga.

Seattle, Wash.

Sioux City, Iowa.

Tacoma, Wash.

Tampa, Fla.

Texas City, Tex.

Toledo, Ohio.

Vanceboro, Me.

Wilmington, N. C.

Ports to which merchandisc may be transported without appraisement under the act of Junc 10, I880.

Albany, N. Y.

Astoria, Oreg.

Atlanta, Ga.

Baltimore, Md.

Bangor, Me.

Bath, Me.

Boston, Mass.

Bridgeport, Conn.

Buffalo, N. Y.

Burlington, Vt.

Calais, Me.

Charleston, S. C.

Chicago, Ill.

Cincinnati, Ohio.

Cleveland, Ohio.

Coal City, Ill.

Columbus, Ohio.

Council Bluffs, Iowa.

Dayton, Ohio.

Denver, Colo.

Des Moines, Iowa.

Detroit, Mich.

Dubuque, Iowa.

Duluth, Minn.

Dunkirk, N. Y.

Durango, Colo.
Hartford, Conn.

Honolulu, Hawaii.

Indianapolis, Ind.

Jacksonville, Fla.

Kansas City, Mo.

Key West, Fla.

Knoxville, Tenn.

Laredo, Tex.

Leadville, Colo.

Lincoln, Nebr.

Los Angeles, Cal.

Louisville, $\mathrm{Ky}$.

Marquette, Mich.

Memphis, Tenn.

Middletown, Conn.

Milwaukee, Wis.

Minneapolis, Minn.

Mobile, Ala.

Nashville, Tenn.

Newark, N. J.

New Bedford, Mass.

New Haven, Conn.

New Orleans, La.

Newport, R. I.

Newport News, Va.

New York, N. Y.
Portsmouth, N. H.

Port Townsend, Wash.

Providence, R. I.

Pueblo, Colo.

Richmond, Va.

Rochester, N. Y.

St. Augustine, Fla.

St. Joseph, Mo.

St. Louis, Mo.

St. Paul, Minn.

Saginaw, Mich.

Salt Lake City, Utah.

San Antonio, Tex.

San Diego, Cal.

Sandusky, Ohio.

San Francisco, Cal.

Sault Ste. Marie, Mich.

Savannah, Ga.

Seattle, Wash.

Sioux City, Iowa.

South Manchester, Conn.

Springfield, Mass.

Syracuse, N. Y.

Tacoma, Wash.

Tampa, Fla.

Titusville, $\mathrm{Pa}$. 
Durham, N. C.

Eagle Pass, Tex.

Eastport, Me.

E] Paso, Tex.

Enfield, Conn.

Eric, Pa.

Evansville, Ind.

Everett, Wash.

Fall River, Mass.

Galveston, Tex.

Gladstone, Mich.

Gloticester, Mass.

Grand Haven, Mich.

Grand Rapids, Mich.

Greenbay, Wis.
Niagara Falls, N. Y.

Nogales, Ariz.

Norfolk, Va.

Oakland, Cal.

Ocala, Fla.

Ogdensburg, N. Y.

Omaha, Ncbr.

Peoria, Ill.

Petershurg. Va.

Pliladelphia, Pa.

Pittslutrg, Pa.

Honston, Tex.

Port Huron, Mich.

Portland, Me.

Portland, Oreg.
Toledo, Ohio.

Utica, N. Y.

Vanceboro, Me.

Vernon (Rockville), Conn.

IVashington, D. C.

Wilmington, Del.

Wilmington. N. C.

Worcester, Mass.

Spokane, Wash.

Bellingham, Wash.

Sabine Pass, Tex.

Superior, Wis.

\section{List of ports at which bonded ciurchouses are established.}

Apalachicola, Fla.

Atlanta, Ga.

Baltimore, Mid.

Bangor, Me.

Batl, Me.

Belfast, Me.

Bonners Ferry, Mont.

Boothbay, Me.

Boston, Mass.

Bridgeport, Conn.

Brownsville, Tex.

Buffalo, N. Y.

Burlington, $\mathrm{Vt}$.

Cape Vincent, N. Y.

Castine, Me.

Chattanooga, Tenn.

Chicago, Ill.

Cincinnati, Ohio.

Denver, Colo.

Detroit, Mich.

Duluth, Minn.

Durham, N. C.

Eagle Pass, Tex.

Eastport, Me.

El Paso, Tex.

Erie, $\mathrm{Pa}$.
Gloncester, Mass.

Grand Rapids, Mich.

Great Falls, Mont.

Green Bay, Wis.

Hartford, Conn.

Honolulu, Ifaraii.

Indianapolis: Ind.

Kansas City, Mo.

Key West, Fla.

Laredo, Tex.

Lincoln, Ncbr.

Los Angeles, Cal.

Lonisville, Ky.

Minneapolis, Minn.

New Ilaven, Conn.

New London, Conn.

New Orleans, La.

Newport News, Va.

Newark, N. J.

New York, N. Y.

Niagara Falls, N. Y.

Nogales, Ariz.

Ogdensburg, N. Y.

Onaha, Ncbr.

Oswego, N. Y.

Pensacola, Fla.
Plattsburg, N. Y.

Port Huron, Mich.

Portland, Me.

Portland, Orcg.

Portsmouth, N. H.

Port Townsend, Wash.

Providence, R. I.

Provincetown, Mass.

Richmond, Va.

Rochester, N. Y.

St. Joseph, Mo.

St. Louis, Mo.

St. Michael, Alaska.

St. Patul, Minn.

Saginaw, Mich.

Salem, Mass.

San Diezo, Cal.

San Francisco, Cal.

San Juan, P. R.

Savammah, Ga.

Seattle, Wash.

Sioux City, Iowa.

Skagway, Alaska.

Spokane, Wash.

Syracuse, N. Y.

Tacoma, Wash. 
Evansville, Ind.

Everett, Wash.

Fall River, Mass.

Galveston, Tex.
Perth Amboy, N. J.

Petersburg, Va.

Philadelphia, Pa.

Pittsburs, Pa.
Tampa, Fla.

Toledo, Ohio.

Utica, N. Y.

List of ports where the custom-house premises are used for the storage of imported goods in bond.

Albany, N. Y.

Aguadilla, P. R.

Arecibo, P. R.

Arroyo, P. R.

Bangor, Me.

Charleston, S. C.

Cleveland, Ohio.

Columbus, Ohio.

Fajardo, P. R.
Humacao, P. R.

Jacksonville, Fla.

Marquette, Mich.

Mayaguez, P. R.

Temphis, Tenn.

Nilwankee, Wis.

Nashville, Temn.

Norfolk, Va.

Pcoria, Ill.
Ponce, P. R.

Providence, R. I.

Sandusky, Ohio.

San Juan, P. R.

Springfield, Mass.

Washington, D. C.

(Georgetown).

Wilmington, Del.

St. Augustine, Fla.

REGULATION FOR THE ENTKY OF PERSONAL, EFFECTS INTO THE UNITED STATES.

The customs laws and the regulations made in pursuance thereof require examination of the taggage and effects of passengers upon arrival in the United States from foreign countries.

Entries prepared and signed by passengers are required. The forms provided for that purpose entitled "Baggage Declaration and Entry," for RESIDENTS and NON-RESIDENTS, will be distributed to passengers during the early part of the voyage by an officer of the ship designated for that purpose. When a passenger has declared and signed his declaration and entry, he should detach and retain the coupon at the bottom of the form and return the latter to the officer of the ship. Declarations spoiled in the preparation should not be destroyed by the passenger, but should be turned over to the purser, who will write, or stamp, thereon the word "canceled," and furnish a new blank declaration to the passenger. After the ship has docked and the baggage and effects of the passenger are landed, he should present the coupon which he has retained to the chief customs officer on the dock, who will detail an inspector to make the examination.

For the purposes of customs administration, passengers are divided into two classes, viz.:

I. Non-RESIDENTS of the United States.

2. Residents of the United States.

The division of passengers into non-residents and residents in nowise affects citizenship.

NON-RESIDENTS are:

(a) Actual residents of other countries.

(b) Persons who have been abroad with a fixed foreign abode for one year or more, who elect to declare as non-residents. 
(c) Persons who have been abroad for two years with or without a fixe? place of foreign abode, who elect to declare as non-residents

Persons of class $(c)$ may erase the second and third lines within the brack ets on the "Baggage Declaration and Entry" for non-residents.

RESIDENTS are such persons as are not included in the definition of nonresidents.

There is no limitation as to the value of articles free of duty brought in by persons declaring as non-residents, provided such articles are in the nature of wearing apparel, articles of personal adormment, toilet articles, and similar personal effects actually accompanying the passenger and necessary and appropriate for his or her wear and use for the purposes of the journey and present comfort and convenience, and are not intended for other persons nor for sale.

Persons declaring as residents are entitled to bring with them free of duty all wearing apparel and other personal effects taken by them out of the United States which have not been remodeled or improved abroad so as to increase their value, and articles obtained abroad by purchase or otherwise of a value not excceding \$roo, provided they are not for sale; but in the case of a minor, the exemption of $\$ 100$ worth of articles obtained abroad is restricted to such articles as are intended for the bona fide personal use of such minor

Each passenger is entitled to bring in free of duty and internal-revenue tax 50 cigars or 300 cigarettes for his or her bona fide personal consumption. Cigars and cigarettes in excess of these quantities are chargeable with internal-revenue tax and duties or fines, as the case may be.

Persons declaring as residents slould use the form of baggage declaration and entry for residents of the United States. Non-residents should use the form of baggage declaration and entry for non-residents of the United States.

Residents should carefully state in their entries under the captions "Description of Articles" and "Foreign Cost or Value" the articles obtained abroad, with the cost price of each article if purchased, or the foreign market value of each article if obtained otherwise than by purchase.

Non-RESIDENTs shonld carefully state in their entries under the captions "Description of Articles" and "Foreign Cost or Value" articles not necessary and appropriate for the wear and use of such non-residents for the purposes of the journey and their present comfort and convenience, and articles intended for sale or for the use of any other person or persons, with the cost price of each article if purchased, or the foreign market value of each article if obtained otherwise than by purchase.

The senior member of a fanily, if a passenger, may make entry for the entire family.

Ladies traveling alone should state the fact in their declarations and entries.

The exact number of pieces of baggage accompanying the passenger should be stated in the entry. 
Whenever practicable passengers should present the original receipted bills of foreign purchases.

Passengers dissatisfied with values placed upon dutiable articles by the customs officers on the docks may demand a re-examination, but application therefor should be immediately made to the officers there in charge. If for any reason this course is impracticable, the packages containing the articles should be left in customs custody and application for reappraisement made t) the collector of customs, in writing within two days after the original appraisement. No request for reappraisement can be entertained after the articles have been removed from customs custody.

Duties will be assessed on used articles of foreign origin at the foreign market values on the date of the owner's departure for the United States, with due allowance for wear or depreciation.

Upon application to the customs officer in charge on the dock, baggage intended for delivery at ports in the United States other than the port of arrival, or in transit through the United States to a foreign country, ma: be forwarded thereto withcut the assessment of duty at the port of arrival. by the various railroads and express companies, whose representatives will be found on the pier. Passengers desiring to have their baggage forwarded in bond should so indicate in their declarations and entries.

Government officers are forbidden by law to accept anything but currency in payment of duties, but, if requested, will retain baggage on the piers for twenty-four hours to enable the owner to secure the currency.

Passengers are advised that to offer gratuities or bribes to customs offcers is a violation of law which will be prosecuted, and that customs officers who accept gratuities or bribes will be dismissed from the service.

Any discourtesy or incivility on the part of customs officers should be reported to the collector at the custom-house, the deputy collector at the pier, or to the Secretary of the Treasury.

The act approved December 29, I897, expressly forbids the importation into the United States of garments made in whole or in part of the skins of seals taken in the waters of the North Pacific Ocean, and unless the owner is able to establish by competent evidence and to the satisfaction of the collector that the garments are not prohibited they cannot be entered.

Household effects of persons or families from foreign countries will be admitted free of duty if actually used abroad by them not less than one year, and not intended for any other person nor for sale.

In order to secure prompt identification and thereby facilitate the passage through the customs upon return of valuable personal and household effects, including sealskin garments, taken abroad by persons leaving the United States, the articles may be registered with the collector at the port of departure or the port at which the journey commences. 
COLLECTORS OF CLSTOMS.

(TERM OF OFFICE, FOUR YEARS.)

Penalty of

Port.

Bond.

Compensation per annum.

Alabama.

Mobile

Alaska.

Juneau

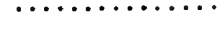

Arizona.

Nogales

\section{California. \\ California.}

Eureka

Eureka

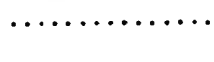

Los Angeles.

San Diego

San Francisco.....
Connecticut.

Fairfield

(Bridgeport, residence of collector.)
\$10,000 Salary, \$250.

3 per cent. commission. Fees.

Max., $\$ 3,000$.

Siorage.

20,000 Salary, $\$ 2,500$.

3 per cent. commission. Fees.

Storage.

Max, $\$ 4,000$.

30,000 Salary, $\$ 2,000$.

2,000 Salary, $\$ 2,500$.

3 per cent. commission. Fees.

Max., \$3,000.

Storage.

I00,000 Salary, $\$ 2,500$.

3 per cent. commission. Fees.

Max., $\$ 3,000$.

Storage.

20,000 Salary, $\$ 2,500$.

3 per cent. commission. Fees.

Max., $\$ 3,000$.

Storage.

$\$ 100,000$ Salary, $\$ 7,000$.

30,000 Salary, $\$ 250$.

3 per cent. commission. Fees.

Max., \$3,000.

Storage. 


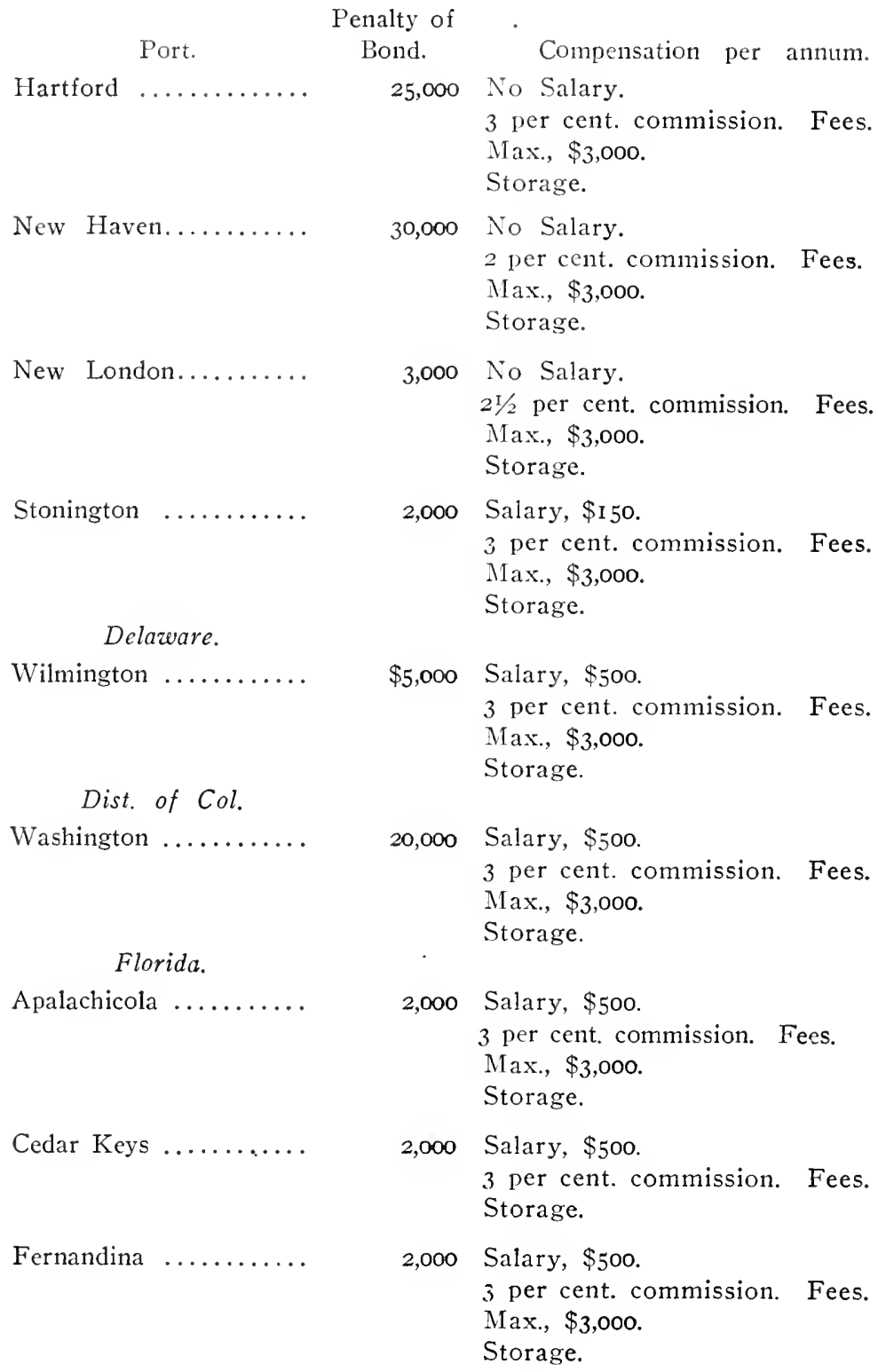




\begin{tabular}{|c|c|c|c|}
\hline Port. & $\begin{array}{l}\text { Penalty of } \\
\text { Bond. }\end{array}$ & Compensation per & annum. \\
\hline Jacksonville $\ldots \ldots \ldots \ldots$ & 10,000 & $\begin{array}{l}\text { Salary, } \$ 500 . \\
3 \text { per cent. commission. } \\
\text { Max., } \$ 3.000 . \\
\text { Storage. }\end{array}$ & Fees. \\
\hline Key West $\ldots \ldots \ldots \ldots$ & $\$ 50,000$ & $\begin{array}{l}\text { Salary, } \$ 500 . \\
3 \text { per cent. commission. } \\
\text { Nax., } \$ 3,000 . \\
\text { Storage. }\end{array}$ & Fees. \\
\hline Pensacola $\ldots . . \ldots \ldots \ldots$ & 20,000 & $\begin{array}{l}\text { Salary, } \$ 1,000 \text {. } \\
3 \text { per cent. commission. } \\
\text { Max., \$3,0oo. } \\
\text { Storage. }\end{array}$ & Fees. \\
\hline Saint Augustine ....... & 5,000 & $\begin{array}{l}\text { Salary, } \$ 500 \text {. } \\
3 \text { per cent. commission. } \\
\text { Max., } \$ 3,000 \text {. } \\
\text { Storage. }\end{array}$ & Feas. \\
\hline Tampa...$\ldots \ldots \ldots \ldots$ & 100,000 & $\begin{array}{l}\text { Salary, } \$ 2,000 \text {. } \\
3 \text { per cent. commission. } \\
\text { liax., } \$ 3,000 \text {. } \\
\text { Storage. }\end{array}$ & Fees. \\
\hline \multicolumn{4}{|l|}{ Georgia. } \\
\hline Brunswick $\ldots \ldots \ldots \ldots$ & 2,000 & $\begin{array}{l}\text { Salary, } \$ 500 . \\
3 \text { per cent. commission. } \\
\text { Max., } \$ 3,000 \text {. } \\
\text { Storage. }\end{array}$ & Fees. \\
\hline Saint Mary's .......... & 5,000 & $\begin{array}{l}\text { Salary, } \$ 500 \text {. } \\
3 \text { per cent. commission. } \\
\text { Max., } \$ 3.000 \text {. } \\
\text { Storage. }\end{array}$ & Fees. \\
\hline Savannah $\ldots \ldots \ldots \ldots$ & $\$ 30,000$ & $\begin{array}{l}\text { No salary: } \\
\text { I per cent. commission. } \\
\text { Storage. } \\
\text { Max., } \$ 6,000 \text {. }\end{array}$ & Fees. \\
\hline \multicolumn{4}{|l|}{ Hazuaii. } \\
\hline Honoiulu .. & 50,000 & $\begin{array}{l}\text { Salary, } \$ 4,000 . \\
\text { Storage. }\end{array}$ & \\
\hline
\end{tabular}




\begin{tabular}{ccc}
\multicolumn{4}{c}{ Penalty of } \\
Port. & Bond. & Compensation per annum. \\
Illinois. & &
\end{tabular}

Chicago

Louisiana.

Brashear ..............

(Post Office, Morgan City.)

New Orleans

Maine.

Bangor $\ldots \ldots \ldots \ldots$.........

Bath

Belfast

Castine

Eastport

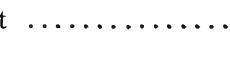

Ellsworth

Storage.
10,000 Salary, \$150.

3 per cent. commission. Fees Max., \$3,000.

5,000 Salary, \$I,000.

3 per cent. commission. Fees.

Max., \$3,000.

Storage.

250,000 Salary, $\$ 7,000$.

20,000 No salary.

3 per cent. commission. Fees. Max., $\$ 3,000$.

Storage.

$\$ 5,000$ No salary.

2 per cent. commission. Fees.

Max., $\$ 3,000$.

Storage.

2,000 No salary.

3 per cent. commission. Fees.

Storage.

2,000 Salary, \$I50.

3 per cent. commission. Fees.

Max., \$3,00o.

Storage.

20,000 Salary, $\$ 500$.

3 per cent. commission. Fees.

Max., \$3,000.

Storage.
Max., \$3,000. 


\begin{tabular}{|c|c|c|}
\hline $\begin{array}{l}\text { Port. } \\
\text { Houlton } \ldots \ldots \ldots \ldots \ldots\end{array}$ & $\begin{array}{l}\text { Penalty of } \\
\text { Bond. } \\
\text { ro,ooo }\end{array}$ & $\begin{array}{l}\text { Compensation per annum. } \\
\text { Salary, \$1,000. } \\
3 \text { per cent commission. Fees. } \\
\text { Storage. } \\
\text { Nax., } \$ 1,500 .\end{array}$ \\
\hline Kennebunk $\ldots \ldots \ldots \ldots$ & $\$ 2,000$ & $\begin{array}{l}\text { No salary. } \\
21 / 2 \text { per cent. commission. Fees. } \\
\text { Max., } \$ 3,000 . \\
\text { Storage. }\end{array}$ \\
\hline Machias $\ldots . . . \ldots \ldots$. & 2,000 & $\begin{array}{l}\text { Salary, } \$ 250 \text {. } \\
3 \text { per cent. commission. Fees. } \\
\text { Max., } \$ 3,000 \text {. } \\
\text { Storage. }\end{array}$ \\
\hline Portland $\ldots \ldots \ldots \ldots$ & 50,000 & Salary, $\$ 6,000$ \\
\hline $\begin{array}{l}\text { (Saco, residence of } \\
\text { collector.) }\end{array}$ & 2,000 & $\begin{array}{l}\text { Salary, } \$ 250 . \\
3 \text { per cent. commission. Fees. } \\
\text { Max., } \$ 3,000 . \\
\text { Storage. }\end{array}$ \\
\hline Waldoborough $\ldots . .$. . & 10,000 & $\begin{array}{l}\text { Salary, } \$ 250 . \\
3 \text { per cent. commission. Fees. } \\
\text { Max., } \$ 3,000 \text {. } \\
\text { Storage. }\end{array}$ \\
\hline Wiscasset $\ldots \ldots \ldots \ldots$. & 2,000 & $\begin{array}{l}\text { Salary, } \$ 200 . \\
2 \text { per cent commission. Fees. } \\
\text { Max., } \$ 3,000 . \\
\text { Storage. }\end{array}$ \\
\hline York $\ldots \ldots \ldots \ldots \ldots$ & $\$ 2,000$ & $\begin{array}{l}\text { Salary, } \$ 250 \text {. } \\
3 \text { per cent. commission. Fees. } \\
\text { Max., } \$ 3,000 \text {. } \\
\text { Storage. }\end{array}$ \\
\hline \multicolumn{3}{|l|}{ Maryland. } \\
\hline Annapolis $\quad \ldots \ldots \ldots \ldots$ & 2,000 & $\begin{array}{l}\text { Salary, } \$ 250 \text {. } \\
3 \text { per cent. commission. Fees. } \\
\text { Max., } \$ 3,000 \text {. } \\
\text { Storage. }\end{array}$ \\
\hline
\end{tabular}




Penalty of
Port. Bond. Compensation per annum.

Baltimore ......... 200,000 Salary, $\$ 7,000$.

Crisfield $\ldots \ldots \ldots \ldots \ldots . \quad 2,000 \quad$ Salary, $\$ 1,200$.

3 per cent. commission. Fees.

Max., \$3,000.

Storage.

Massachusetts.

Barnstable .............

2,000 No salary.

3 per cent. commission. Fees.

Max., \$3,00o.

Storage.

Boston

100,000 Salary, $\$ 8,000$.

Edgartown

$\$ 2,000$ Salary, $\$ 250$.

3 per cent. commission. Fees.

Max., \$3,000.

Storage.

Fall Rive

10,000 Salary, \$150.

3 per cent. commission. Fees. Max., \$3,000.

Storage.

Gloucester

I0,000 Salary, \$250.

3 per cent. commission. Fees.

Max., \$3,000.

Storage.

Marblehead

10,000 No salary.

$2 \mathrm{~T} / 2$ per cent. commission. Fees.

Max., \$3,000.

Storage.

Nantucket $\ldots \ldots \ldots \ldots$

5,000 Salary, $\$ 250$.

3 per cent. commission Fees. Max., \$3,00o.

Storage.

New Bedford

15,000 No salary.

3 per cent. commission. Fees. Max., $\$ 3,000$.

Storage. 


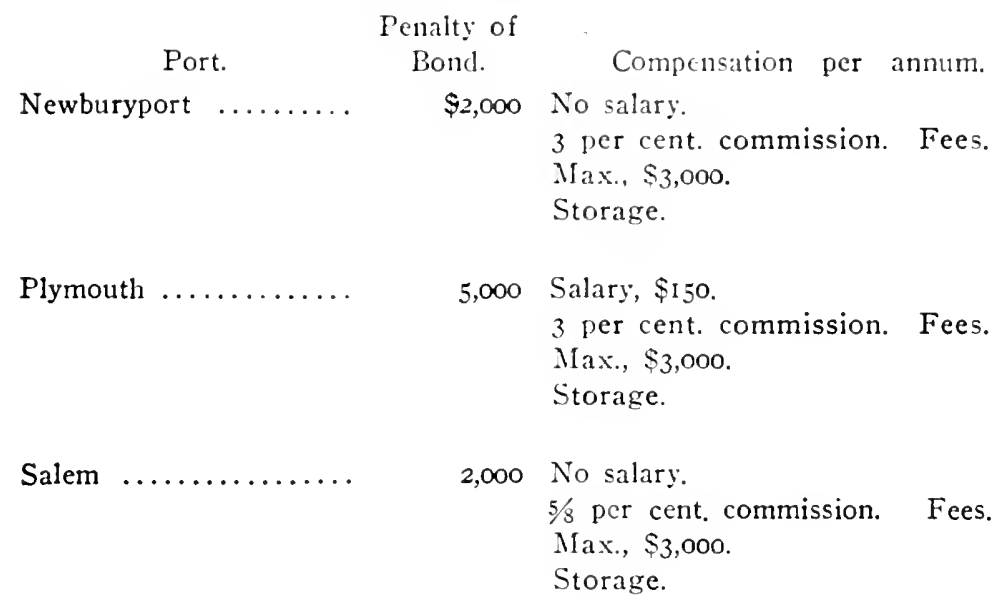

Michigan.

Detroit

I00,000 Salary, \$1,000.

3 per cent. commission. Fees. Max., \$2,500.

Storage.

Grand Haven

2,000 Salary. \$1,00o.

3 per cent. commission. Fees.

Max., \$2,500.

Storage.

Marquette

20,000 Salary, $\$$ I, 000 .

3 per cent. commission. Fees. Max., \$2,500.

Storage.

Port Huron

$\$ 50,000$ Salary, $\$ 1,000$.

3 per cent. commission. Fees.

Max., \$2,500.

Storage.

Minnesota.

3 per cent. commission. Fees. Max., \$2,500.

Storage. 


$\begin{array}{ccc}\text { Penalty of } & \\ \text { Port. } & \text { Bond. } & \text { Compensation per annum. }\end{array}$

Saint Paul ......... roo,000 Salary, $\$ \mathrm{r}, 000$.

3 per cent. commission. Fees.

Max., \$2,500.

Storage.

Subport Minneapolis...

50,000 Salary, $\$ 2,400$.

(Deputy Collector.)

Mississippi.

Natchez

2,000 Salary, \$500.

3 per cent. commission. Fees.

Max., \$3,000.

Storage.

Gulfport

3,000 Salary, \$250.

3 per cent. commission. Fees.

Max., \$3,000.

Storage.

Vicksburgh

Salary, $\$ 500$.

3 per cent. commission. Fees.

Max., \$3,00o.

Storage.

Montana and

Idaho.

Great Falls .........

Salary, \$I,000.

3 per cent. commission. Fees.

Max., \$2,500.

Storage.

New Hampshire.

Portsmouth .........

No salary.

I $3 / 4$ per cent. commission. Fees.

Max., \$3,000.

Storage.

New Jersey.

Bridgeton

Salary, \$250.

3 per cent. commission. Fees.

Max., \$3,000.

Storage. 


Port.
Burlington $\ldots . . . . . .$.
Camden. (See Pennsyl-
va nia.) (Assistant
Collector.)
Jersey City. (See New
York.) (A ssist a n t
Collector.)

Newark

Perth Amboy ..........

Tuckerton

$$
\text { New York. }
$$

Buffalo

Cape Vincent
$\$ 30,000$ Salary, $\$ 250$.

3 rer cent. commission. Fees. Max., $\$ 3,000$.

Storage.

20,000 Salary, \$250.

3 per cent. commission. Fees. Max., \$3,000.

Storage.

2,000 Salary, \$250.

3 per cent. commission. Fees. Max., \$3,000.

Storage.

2,000 Salary, \$250.

3 per cent. commission. Fees.

Max., $\$ 3,000$.

Storage.

75,000 Salary, $\$ 1,000$.

3 per cent. commission. Fecs.

Max., $\$ 2,500$.

Storage.

Io,000 Salary, $\$ 1,000$.

3 per cent. conmission. Fees.

Max., \$2,500.

Storage. 
Penalty of
Port. Bond.

Dunkirk ............ 5,000 Salary, \$1,000.

3 per cent. commission. Fees. Max., \$2,500.

Storage.

Genesee River ........

(Rochester, residence of Collector.)

New York

300,000 Salary, $\$ 12,000$.
25,000 Salary, \$1,000.

3 per cent. commission. Fees.

Max., \$2,500.

Storage.

Jersey City, N. J...... No bond re- Salary, $\$ 2,000$.

(Assistant Collector.) quired

Niagara Falls

Ogdensburg

Oswego

Plattsburg

Sag Harbor
50,000 Salary, \$1,000.

3 per cent. commission. Fees. Max., \$2,500.

Storage.

40,000 Salary, \$I,000.

3 per cent. commission. Fees. Max., \$2,500.

Storage.

$\$ 25,000$ Salary, $\$$ I,000.

3 per cent. commission. Fees. Max., \$2,500.

Storage.

25,000 Salary, \$1,000.

3 per cent. commission. Fees.

Max., \$2,500.

Storage.

2,000 Salary, $\$ 400$

3 per cent. commission. Fees.

Max., \$3,000.

Storage. 


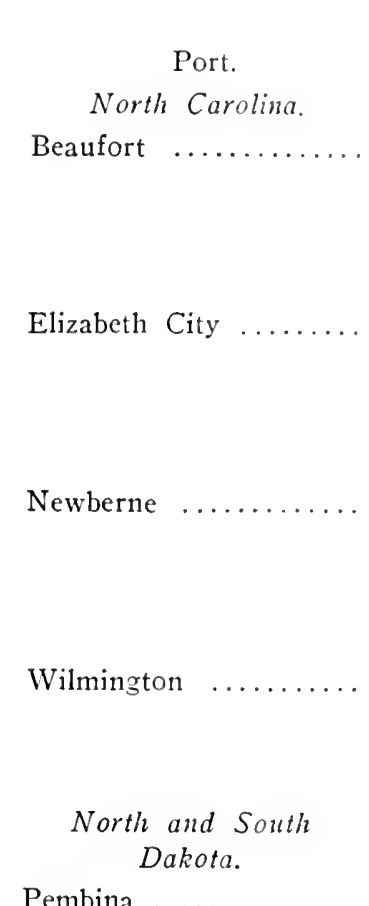

Penalty of

Eond. Compensation per annum.

North Carolina.

Beaufort

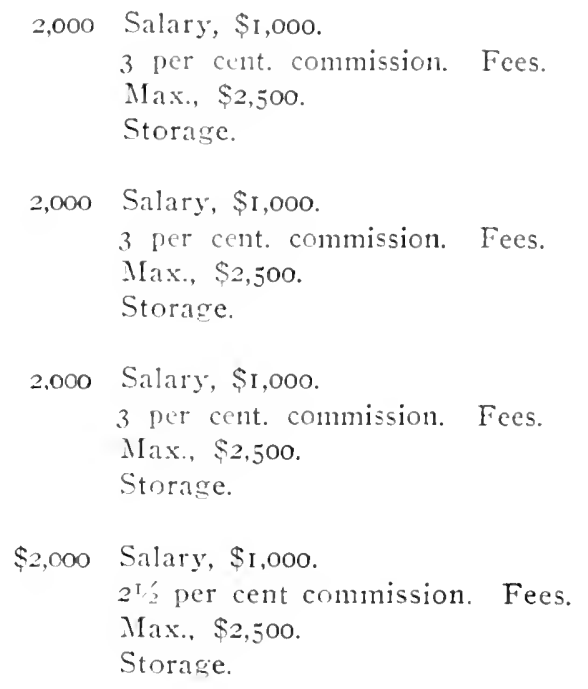

Ohio.

Cleveland

100,000 Salary, \$1,00o.

3 per cent. commission. Fees.

Max.. \$2.500.

Storare.

Sandusky

20,000 Salary, $\$ \mathrm{I}, 000$.

3 per cent commission. Fees.

Max., \$2,500.

Storage.

Toledo

20,000 Salary, \$1,000.

3 per cent commission. Fees.

Max.. $\$ 2,500$.

Storage. 


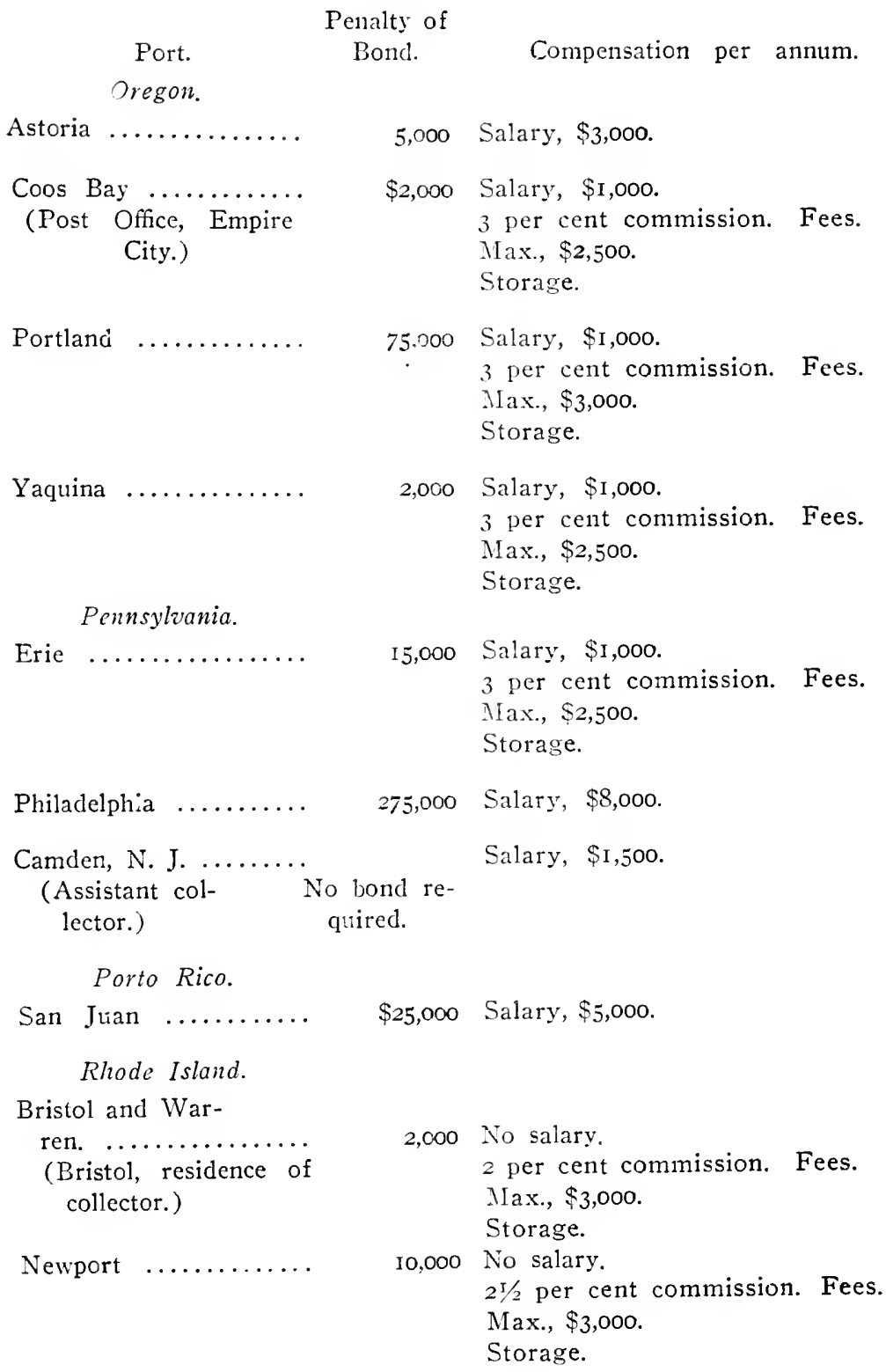




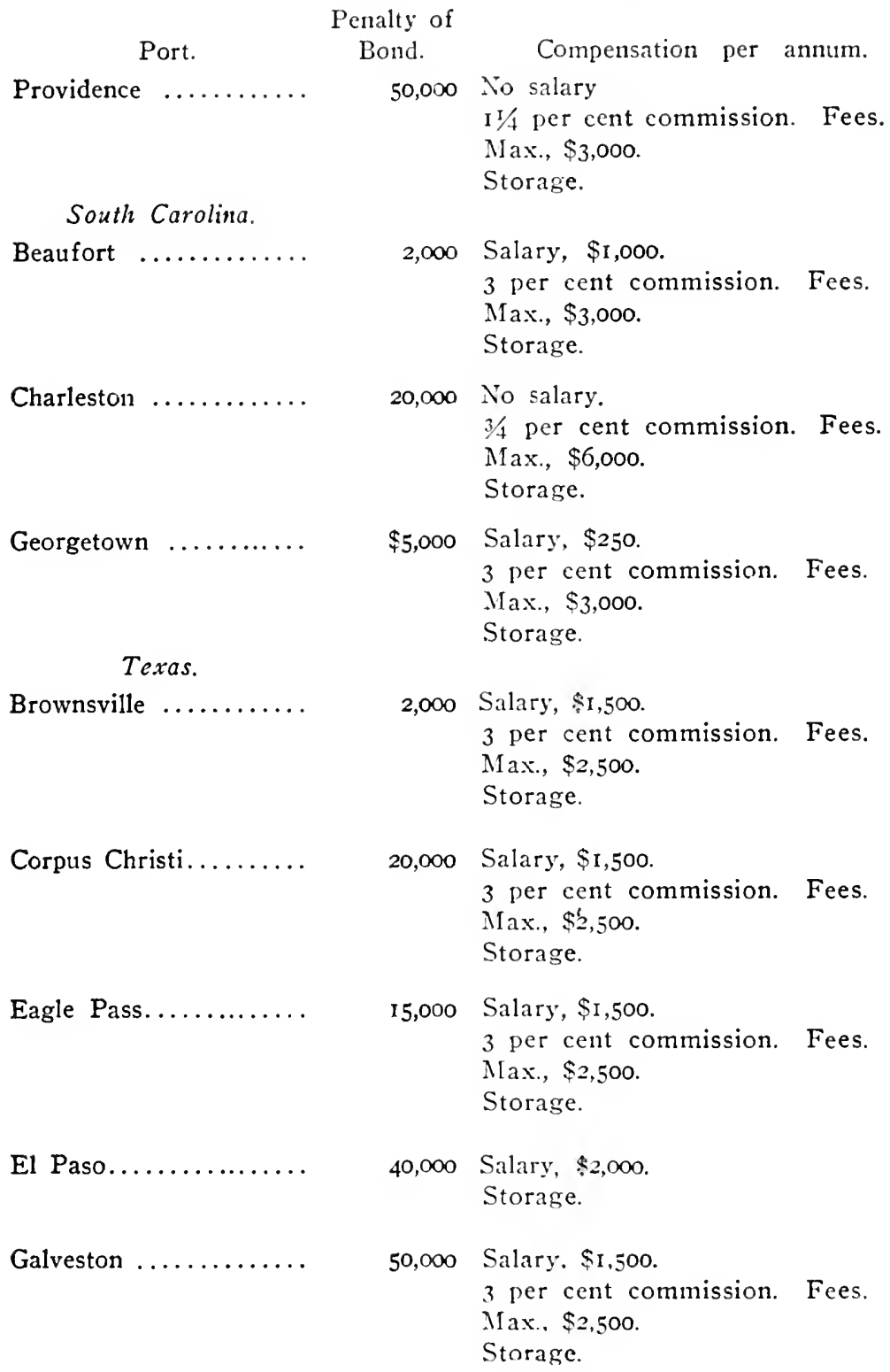

Galveston

50,000 Salary. $\$ 1,500$.

3 per cent commission. Fees.

Max., $\$ 2,500$.

Storage. 


$\begin{array}{cc}\text { Penalty of } \\ \text { Port. } & \text { Bond. }\end{array}$

Port Arthur............

Vermont.

Burlington

Newpor

Virginia.

Alexandria $. . . \ldots \ldots . .$.

Cape Charles City......

Newport News..........

Norfolk and Portsmouth. (Norfolk, residence of collector.)

Petersburg 20,000

Salary, \$1,000.

3 per cent commission Fees.

Max., $\$ 3,000$.

$\$ 100,000$ Salary, $\$ 2,000$.

3 per cent commission. Fees.

Max., \$2,500.

Storage.

50,000 Salary, \$2,000.

3 per cent commission. Fees.

Max., \$3,00o.

Storage.

2,000 No salary.

2 per cent commission. Fees.

Max., \$3,000.

Storage.

2,000 Salary, \$500.

3 per cent commission. Fees.

Max., \$3,000.

Storage.

100,000 Salary, \$200.

3 per cent commission. Fees.

Max., \$3,000.

Storage.

I0,000 No salary.

$\mathrm{I} 3 / 4$ per cent commission. Fees.

Max., \$3,000.

Storage.

$\$ 25,000$ No salary.

I $3 / 4$ per cent commission. Fees.

Max., \$3,000.

Storage.

Richmond
No salary.

20,000 No salary.

I $3 / 4$ per cent commission. Fees.

Max., \$3,000.

Storage. 
Penalty of

Port. Bond.

Tappahannock

Washington.

Port Townsend

Wisconsin.

Milwaukee

75,000 Salary, \$I,000.

3 per cent commission. Fees.

Max., $\$ 2,500$.

Storage.

NAYAL OFFICERS OF CUSTOMS-TERM OF OFFICE, FOLR YEARS.

\section{California.}

San Francisco

\section{Illinois}

Chicago

\section{Louisiana.}

New Orleans............

Maryland.

Baltimore

Massachusetts.

Boston

New York.

New York.

Io,000 Salary, $\$ 8,000$.

Pennsylvania.

Philadelphia
I0,000 Salary, \$5,000.

I0,000 Salary, $\$ 5,000$.

$\$ 20,000$ Salary, $\$ 5,000$.

I0,000 Salary, $\$ 5,000$.

I0,000 Salary, $\$ 5,000$.
I0,000 Salary, $\$ 5,000$. 
SURVEYORS OF CUSTOMS-TERM OF OFFICE, FOUR YEARS.
Penalty of
Port.
Bond.
Compensation per annum.

California.

San Francisco........ \$20,000 Salary, $\$ 5,000$.

\section{Colorado.}

Denve

30,000 Salary, $\$ 1,000$.

3 per cent commission. Fees.

Storage.

Max., \$5,000.

Georgia.

Atlanta

5,000 Salary, \$r,000.

3 per cent commission. Fees.

Storage.

Max., \$5,000.

Illinois.

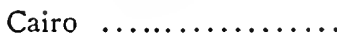

2,000 Salary, $\$ 350$.

3 per cent commission. Fees.

Storage.

Max., \$5,000.

Galena

2,000 Salary, $\$ 350$.

3 per cent commission. Fees.

Storage.

Max., \$5,ooo.

Peoria

5,000 Salary, \$350.

3 per cent commission. Fees.

Storage.

Max., \$5,000.

Rock Island......... \$2,000 Salary, \$350.

3 per cent commission. Fees.

Storage.

Max., $\$ 5,000$.

Indiana.

Evansville

10,000 Salary, $\$ 350$.

3 per cent commission. Fees.

Storage.

Max., \$5,000. 


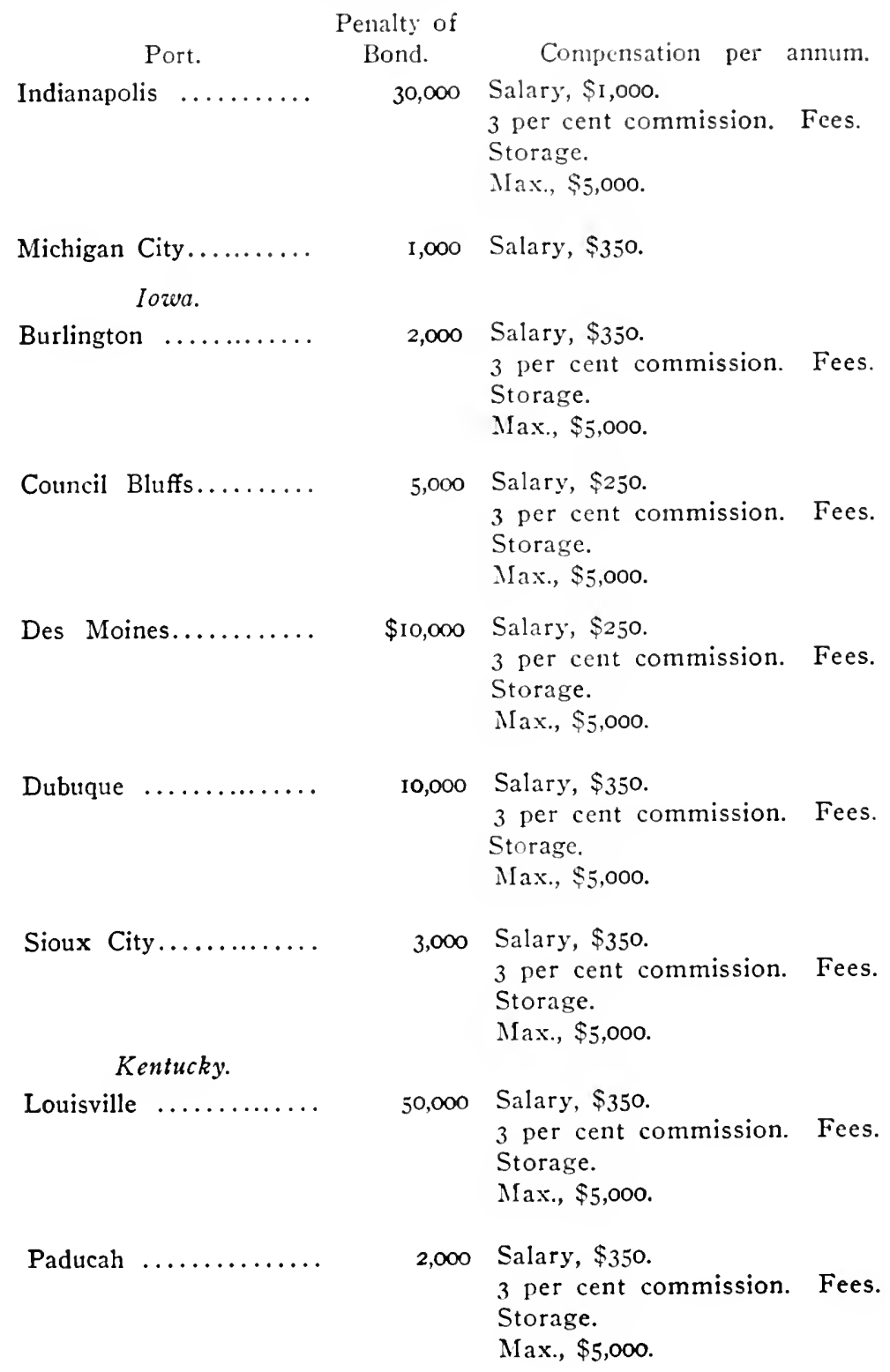



Port.
Penalty of

Louisiana.

New Orleans..........

5,000 Salary, $\$ 3,500$.

Maine.

Portland

$\$ 1,000$ Salary, $\$ 4,500$.

Maryland.

Baltimore

5,000 Salary, $\$ 4,500$.

Massachusetts.

Boston

5,000 Salary, $\$ 5,000$.

Springfield

Io,000 Salary, \$I,000.

3 per cent commission. Fees.

Storage.

Michigan.

Max., $\$ 5,000$.

Grand Rapids..........

20,000 Salary, \$r,200.

3 per cent commission. Fees.

Storage.

Max., \$5,00o.

Missouri.

Saint Louis.

I50,000 Salary, \$350.

3 per cent commission. Fees.

Storage.

Max., \$5,000.

Saint Joseph.

$\$ 20,000$ Salary, \$1,000.

3 per cent commission. Fees.

Storage.

Max., \$5,000.

Kansas City

40,000 Salary, \$I,000.

3 per cent commission. Fees.

Storage.

Max., $\$ 5,000$.

Nebraska.

Lincoln

2,000 Salary, $\$ 900$.

3 per cent commission. Fees.

Storage.

Max., $\$ 5,000$. 
Penalty of
Port.
Bond.

Omaha

New York.

Albany

40,000 Salary, \$6oo.

3 per cent commission. Fees.

Storage.

Max., \$5,000.

Greenport

I,000 No salary

Fees.

Max., $\$ 5,000$.

New York

Patchogue

$\$ 5,000$ Salary, $\$ 8,000$.

2,000 No salary.

Fees.

Max., \$5,000.

Port Jefferson.........

Ohio.

Cincinnati

100,000 Salary, \$35o.

3 per cent commission. Fees.

Storage.

Max., $\$ 5,000$.

Columbus

25,000 Salary, \$1,000.

3 per cent commission. Fees.

Storage.

Max., $\$ 5,000$.

Dayton

$\$ 20,000$ Salary, $\$ 600$.

3 per cent commission. Fees.

Storage.

Max., $\$ 5,000$. 


$$
\begin{gathered}
\text { Penalty of } \\
\text { Port. } \\
\text { Bond. }
\end{gathered}
$$

Pennsylvania.

Philadelphia

Pittsburgh

Tennessee.

Chattanooga...$\ldots \ldots$.

Knoxville

Memphis

Nashville

\section{Texas.}

Houston

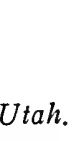

Salt Lake City

West Virginia.

Wheeling
5,000 Salary, $\$ 5,000$.

75,000 Salary, $\$ 350$.

3 per cent commission. Fees.

Storage.

Max., \$5,000.

2,000 Salary, \$350.

3 per cent commission. Fees.

Storage.

Max., \$5,000.

2,000 Salary, $\$ 350$.

3 per cent commission. Fees.

Storage.

Max., \$5,000.

I5,000 Salary, $\$ 350$.

3 per cent commission. Fees. Storage.

Max., \$5,000.

$\$ 5,000$ Salary, $\$ 1,000$.

3 per cent commission. Fees.

Storage.

Max., \$5,000.

2,000 Salary, \$350.

3 per cent commission. Fees.

Storage.

Max., $\$ 5,000$.

10,000 Salary, $\$ 1,500$.

2,000 Salary, \$350.

3 per cent commission. Fees.

Storage.

Max., $\$ 5,000$. 


Penalty of
Port. Bond.
isconsin.

La Crosse $\ldots \ldots \ldots \ldots$ 2,000 Salary, $\$ 350$.

3 per cent commission. Fees.

Storage.

Max., \$5,000.

APPRAISERS OF MERCIIANDISE.

(TERM OF OFFICE, UNLIMITED.)

Port.

California.

San Francisco....... \$3,625

Florida.

Tampa $\ldots \ldots \ldots \ldots \ldots . \quad 2,000$

Illinois.

Chicago

4,500

Louisiana.

New Orleans..........

3,000

Maryland.

Baltimore 2 at 3,000

Maine.

Portland $\ldots \ldots \ldots \ldots \ldots . \$ 3.000$

Massachusctts.

Boston ............ 4,000

Michigan.

Detroit $\ldots \ldots \ldots \ldots \ldots . \quad 3,000$

Missouri.

Saint Louis.......... 3,000

Nerv York.

Buffalo ........... $\quad 3.000$

New York.......... $\quad 8,000$ 
Port.

Ohio.

Cincinnati $\ldots \ldots \ldots \ldots \ldots . \quad \$ 3,000$

Cleveland $. \ldots \ldots \ldots \ldots . \quad 3,000$

Oregon.

Portland $\ldots \ldots \ldots \ldots \ldots . \quad 3,000$

Pennsyliania.

Philadelphia $\ldots \ldots \ldots \ldots \quad \quad 4,000$

Pittsburg $\ldots \ldots \ldots \ldots \ldots \quad 3,000$

ASSISTANT APPRAISERS OF MERCHANUISE

(TERM OF OFFICE, UNLIMITED.)

Port.

California.

San Francisco....... \$2,500

Lorisiana.

New Orleans ....... 2 at 2,500

Maryland.

Baltimore .......... $\quad 2,500$

Massachusetts.

Boston .......... 3 at 2,500

New York.

New York......... 3 at 3,500

New York ......... 9 at 3,000

Pennsylvania.

Philadelphia ....... 2 at 2,500 
SPECIAL EXAMINERS OF DRLGS, MEDICINES ANU CHEMIGALS.

(term of office, UNLimited.)

California.

Compensation.

San Francisco ..........

$\$ 2,000$

Louisiana.

New Orleans ...........

I,000

Massachusetts.

Boston

2,500

Pcnnsylvania.

Philadelphia

I,800

INTERNAL REVENUE SERVICE.

The second largest source of the revenue of the United States is that which is collected from the tax on distilled spirits, malt beverages, etc. For the purpose of the collection of this revenue the United States is divided into districts, a list of which is attached hereto. These may be changed from time to time by the President in his discretion, when the cost of collection of the revenue as compared with the amount received seems to be excessive. In such an event the duties of the collector are consolidated with those of the collector of another district so that when district numbers in any State do not run in numerical order the omissions represent districts which have been thus consolidated. The President is empowered to appoint by and with the advice and consent of the Senate a collector of each district, the collector being required to reside in the district for which he is appointed. It would also seem to be fair construction of the law that the appointee should have been a resident of the district.

The collector is required to enter under a bond with not less than five sureties, as the Commissioner of the Internal Revenue may prescribe. The latter official is also appointed by the Presi- 
dent by and with the advice and consent of the Senate, and receives a salary of $\$ 6,000$ per year.

$\mathrm{He}$ has the general superintendence of all matters relating to the collection of internal revenue and prescribes regulations therefor.

There are two deputy commissioners of internal revenue, together with an organization in Washington, including the usual clerical force, chemists, persons in charge of the issuing and care of stamps, the preliminary auditing of accounts and general administrative and supervisory work.

Besides the collectors of internal revenue the forces in the different internal revenue districts consist of deputy internal revenue collectors, gaugers who determinue the amount of liquors produced and which are subject to a tax, and storekeepers who are in charge of storehouses at or near distilleries where distilled spirits are kept before being marketed.

The collectors of internal revenue are required from time to time to send their deputies through every part of the district under their jurisdiction to inquire concerning persons who are liable to pay any internal revenue tax. It is, however, incumbent upon such persons themselves to make returns to the collector of internal revenue of the amount of the property on which they ought, under the law, to pay such tax. But if it has been ascertained by the collector through his deputy that the person so liable has neglected to make the return required by law, or if a return is made which the collector believes to be fraudulent or false, the collector is empowered to summon such person before him and conduct an examination as to the property which may be subject to the tax. If the person liable is not within the State within which the district lies, the collector may proceed to another district and there conduct the examination required by law.

Summons to such examination are served by a deputy collector. When not heeded a collector may apply to a court or a commissioner for an attachment for contempt against the person summoned. If the person concerned does not make a true re- 
turn of his property, taxable, the collector may return the amount believed to be due to the commissioner of internal revenue, who is authorized to add roo per cent. of the tax in case of attempted frand, or 50 per cent. as a penalty for neglect or refusal to make the proper returns.

Internal revenue officers are authorized so enter any premises where taxable articles are kept for the purpose of valuing the same and assessing the proper tax. Penalties are also imposed for making false returns or refusing to produce books or papers necessary to make an assessment required by law.

The return of the assessment made by the collector is subject to revision by the Commissioner of Internal Revenue, who may at any time within ${ }_{5}$ months after the time of the delivery of list of taxable property to the collector, require such list to be amended by the addition of newly discovered items. The assessment may also be amended in favor of the taxpayer, and the amended assessment is returned to the collector for the purpose of collecting the additional taxes or penalties or as a basis of remission of taxes assessed.

Internal revente may be collected, when not paid by the person against whom assessed, by distraint of the goods. chattels or effects which may be the subject of the tax. These revenues are collected in two ways; by cash paid directly to the collector and by stamps which are sold in quantities to purchasers producing goods subject to internal revenue tax, and used by them as the goods are put on the market.

Collectors are forbidden to issue stamps except on the payment of the amount involved, and must issue stamps for all duties payable by the use of stamps, not being permitted to issue receipts for duties paid in lieu of stamps for the same amount.

In addition to the distraint upon goods and chattels affected by the tax, real estate of the debtors under the internal revenue laws may be scized and sold when a sufficient amount cannot be realized by the sale of personal property.

The Commissioner of Internal Revenue has charge of all the 
real estate which may come into possession of the United States under the internal revenue laws, which is held as security for the payment of taxes, pending finai sale, when such sale cannot be immediately effected.

Collectors of internal revenue are compelled to make monthly statements of all collections and are required to complete their collections at such intervals as may be required by the Secretary of the Treasury in order that there shall be no arrears of taxes allowed to accumulate.

All of the property of the collector of internal revenue, in addition to his bond, is subject to the distraint and sale on the part of the Treasury Department should he fail to turn over all the amounts collected by him. Collectors are charged with the whole amount of taxes represented by the lists which have been transmitted by them to the Commissioner of Internal Revenue, beside the par value of all the stamps which have been issued to them, and are further charged with all money which may become due in the district as penalties and forfeitures and must make accounts to balance by either cash payments into the Treasury or a designated depository or a return of unused stamps, or by the submission for approval of a list of taxes which cannot be collected by reason of lack of assets on the part of the debtor. A collector is also creciited with the cost of collecting the revenue, expenses therefor being paid by him out of the amounts collected, but any difference between the amount charged against him and the amount of credits which he is able to secure from the Treasury Department must be made good out of his personal funds.

The Commissioner of Internal Revenue is the court of appeal for the purpose of determining whether any amount assessed as internal revenue taxes has been improperly assessed and collected, and he is authorized to refund such improper collections, returning to the collector also such amounts of money as may be recovered from him by recourse to the courts on the part of those from whom taxes have been collected.

The collection of the internal revenue in many instances in- 
volves appeals to the Federal courts either for the settlement of questions of construction or in the nature of processes to levy upon property against which taxes have become due, and the Commissioner of the Internal Revenue is given the power with the advice and consent of the Secretary of the Treasury to compromise any such case before a suit is commenced and if a suit has actually been brought with the additional recommendation of the Attorney General the case may be taken out of court and settled. In this latter event, however, it is required that there shall be placed on file an opinion of the Solicitor of Internal Revenue, giving the reasons for such compromise.

The business of the internal revenue service chiefly is with the persons engaged in pursuits which are subject to special taxes. The list of general taxes is kept to as small proportions as possible for the reason suggested that a direct tax is a less desirable means of raising revenuc than an inclirect one, because of the opposition and possible friction involved. Internal revenue taxes are therefore imposed so far as possible either in the form of licenses or of stamp taxes on manufactured articles.

In order to meet the extraordinary expenses of war, additional internal revenue taxes to those now in effect would be imposed. This was the practice during the time of the Civil War and during the Spanish-American War. The additional taxes, although largely in the form of stamp taxes on checks and other legal and commercial documents, as well as matches, playing cards, and similar articles, were also imposed upon legacies, a certain percentage of the amount involved being, as it were. confiscated by the United States for the general use. An income tax has also been proposcd, but an effort to apply such a tax has failed because the devices adopted violated the constitutional provision that no direct tax should be imposed except in proportion to the population. The income tax being levied in proportion with the amount of property held by the individual, such a tax was declared unconstitutional by the Supreme Court.

The chief sources of the internal revente, therefore, are the taxes imposed upon persons carrying on business relating to the manufacture and sale of liquor or beverages and tobacco. To these are added 
taxes on certain special products hereafter described, and the law provides for taxes on the circulation of notes of other than national banks which effectually discourages such circulation, so that this revenue is inappreciable. It is provided that no person shall carry on a trade or business affected by a special tax until he shall have paid the amount of the license fee. This has been held not to cover a single sale or transaction in the lines indicated, but to apply only to persons who habitually or customarily pursue the trade or business named. Every such person is required to register his name and place of business with the collector of internal revenue and to pay the amount of license required, the payment being evidenced by a special stamped certificate, which must be displayed in his place of business. Persons subject to such taxes are rectifiers, wholesale liquor dealers, retail liquor dealers, manufacturers of stills, manufacturers of plug tobacco and cigars, dealers in manufactured tobacco, dealers in leaf tobacco, retail dealers in the articles indicated, brewers, and manufacturers of and dealers in oleomargarin, filled cheese and mixed flour.

In addition to the license fee required of such persons the collectors of internal revenue are required to see that there is a quantity tax imposed upon their product, the payment of which is evidenced by stamps affixed to the packages containing the taxable goods. The chief of these sources of revenue is the tax on distilled spirits. For the purpose of preventing the evasion of this tax subordinate officers of the internal revenue known as gaugers and storekeepers are assigned to each of the distilleries, the former to measure the liquids at various stages and the latter to watch over the disposition of the products. Heavy penalties with confiscation of the apparatus are imposed upon persons who set up means of distillation without due registry and notice to the collector or who operate the same without having complied with the regulations intended to secure the payment of the legal duties.

Gaugers are assigned to duty at each registered distillery, one or more of the officers being on duty at all times while the distillery is in operation. The gaugers measure and record the quantity of all articles which would enter into the distillation of spirits and 
which are brought to the distillery, measure and record the volume of the liquid produced in the intermediate processes of distillation, and finally measure and record the volume of the complete manufactured article, the volume of the latter being required to bear a known proportion to the amount of material which has been received by the distillery. The storckeepers have the custody of the various receptacles to see that the contents are not tampered with.

For the purposes of assessing the tax the basis of computation is a gallon of proof spirit, which is a liquicl which contains one-half of its volume of alcohol. A tax of $\$$ I.Io a gallon is imposed upon this licuid when of the alcoholic strength of fifty per cent. named or below, and a proportionately greater tax is imposed when the proportion of alcohol is higher and when the liquid is said to be above proof.

The tax imposed becomes due as soon as alcohol is produced from the distillation of fermented substances without regard to further processes which may be undertaken for its purification. All persons interested in distilleries are jointly liable for the taxes upon the distilled spirits produced therein and the close inspection and supervision of such manufacturing places includes as well the premises of persons who manufacture apparatus for the production of distilled liquor, while every person who has the ownership or custody of the still is required to register the same in the office of the collector of the internal revenuc, and to advise that official of any change in its location.

A person about to engage in the business of distillation must comply with the regulations as to possession of a given amount of unencumbered real estate and give a bond which will be proportionate to the amount of distilled liquor that can be produced with the apparatus installed. The furnaces of stills must be so constructed that they can be locked by the government officials, and the plan of distilling premises must be submitted to the collector before operations are commenced.

For the purpose of permitting growers of fruits to utilize their surplus products in the manufacture of brandy many of the provisions relating to distillation which apply to grain distillates are sus- 
pended by the Commissioner of Internal Revenue although the same amount of tax is imposed as that on grain distillates.

Every distiller is required to provide at his own expense a warehouse or warehouses on lis premises to be used for the storage of distilled spirits of his own manufacture until the tax thereon shall be paid. Such warehouses are in the joint custody of the proprietor and of the storekeeper, who is a subordinate officer of the internal revenue service, and who is required to be present at all times when the warehouse is opened, and no article can be received in or delivered from such warehouse unless upon a permit which must be secured from the collector of the district.

Distillers are required to keep their premises accessible at all times and to furnish the collector of the internal revenue such keys to gates and doors as may be required for the purpose of allowing proper inspection to be made. Gaugers are required to make daily returns to the collector of the district of all quantities ascertained by them as well as of all stamps placed by them upon packages of manufactured products.

When an alcoholic spirit is completed in the process of distillation it is measured by the gauger and the percentage of alcohol determined, whereupon that package containing the same is properly marked and stamped. It may be removed from the distillery upon the payment of the tax or may be entered with the collector of the district for deposit in the warehouse, a bond being given for the payment of the tax before removal from the warehouse and before three years after its deposit therein. The application for deposit being granted, a permit is given by the collector upon which the amount stated is received by the storekeeper and upon later payment of the tax imposed a permit for withdrawal is given which is the warrant of the storekeeper for allowing the removal of the quantity of spirits specified from the bonded store.

In addition to the distillery warehouses provision is made by which the Commissioner of Internal Revenue may establish one or more general bonded warehouses, not exceeding ten in number in any one collector's district, to which distilled spirits may be removed from the distillery warehouses, the payment of the tax being assured 
by a general bond on the warehouse as well as the special bond required for the payment of the tax on the product, permission for the transfer being evidenced by transfer stamps attached to the packages.

The distilled product chiefly produced in the United States and from which the bulk of the intermal revente proceeds are derived is whiskey. What constitutes whiskey has been the subject of an interesting controversy, especially during the past few months, when the discussion on the subject of what constitutes whiskey, which has been more or less academic during a great many years, became of acute interest because of the impending enforcement of the pure food law, so called.

With respect to the internal revenue collections there is no practical difference between any of the products of the distillers. All of them, as soon as the stage of alcohol is reached, become subject to the tax of $\$$ I.Io per gallon. In commerce, however, the crude product of a distillery is not a beverage, since the substances which are carried over with the alcohol in the distillation of whiskey require to be changed before they are regarded as palatable by aging for several years in oaken casks. As an alternative to this, new whiskey can be mixed with other liquids which remedy the harshness of the substances above referred to which are present in what is known as straight whiskey. The chief of these substances is the distilled spirit from which all foreign constituents have been removed, that is to say a refined spirit or alcohol, which is known commercially as neutral spirit. This of itself has a slight or no flavor and smell, and when mixed with new whiskey and the mixture is flavored and colored an imitation of the whiskey which is produced by distillation and aging is securcd. "Straight whiskey," so called, that is whiskey with all its different constituents as produced by a single distilling, in various stages of the aging process can be mixed or blended to secure a merchantable product at a less cost than that of a fully aged whiskey. The controversy which has been and is going. on at this writing is whether a similar process to this blending in which neutral spirit is substituted for one of the whiskeys secures as a result a blended whiskey or a compounded beverage. 
The part played by the internal revenue regulation in this matter is incidental to one of the regulations. In order to place the so-called straight whiskey upon the market in such a form that it can be sold directly to the consumer it is necessary to take advantage of the bottled in bond provision of law. Very few single consumers would be in a position to purchase a barrel of whiskey so that the market of the distiller in general is the rectifier or wholesale liquor dealer who in the transition between the distiller and the consumer changes the character of the product by adding either other whiskey, neutral spirits or in some cases simply using the whiskey as a flavoring substance with a body of neutral spirits. The neutral spirit not having been held for considerable periods is a more economical ingredient than "straight whiskey," and the product of the rectifiers competes strenuously with bottled in bond products.

For the privilege of compounding liquors rectifiers and wholesale liquor dealers are required to pay taxes in the nature of license fees, and there are also strict regulations so that they shall not increase the volume of the distilled spirits purchased by them and which have paid a tax of $\$$ I. Io a gallon so as to produce a larger quantity of spirits, a portion of which has not paid the tax. Their profuct is stamper with a special stamp issued only upon presentation of identifying portions of original stamps and not to the value of more than the originals.

To enable the distiller of whiskey to get his products directly to the consumer without intervention of the rectifier or wholesale liquor dealer he is given the privilege of bottling his product in the bonded warehouse in which it is held; a separate portion being set aside for the work and a requirement made that it shall be under the inspection of the storekeeper, who is also required to see that the necessary stamps representing the tax are affixed. There is, however, a regulation that this privilege shall not be extended until after the period during which a distiller may apply for a regauge of the distilled spirits run during any one season. This period is four years and as the distiller is compelled to carry his whiskey during that time, he claims he is at a disadvantage with the rectifier who 
can take a considerable volmme of the neutral spirits recently run from the still and make an article largely sold as whiskey.

If the contention under the pure food law that use of a neutral spirit distilled from grain constitutes an adulteration of whiskey is sustained by the courts, a revolution affecting the commercial conditions, at least, of the principal article of internal revenue will be effected. The payment of the taxes upon distilled spirits is evidenced by the affixing of stamps to the packages containing the same, which stamps must be so affixed that they may be destroyed by the opening of the packages, and if not so affixed must be destroyed under the terms of the law when the packages are emptied.

Brewers of malt beverages are required to register full information as to the character of the business of the brewery with the collector before beginning business and must file their bond equal to three times the amount of the tax which in the opinion of the collector will becone due monthly in consequence of the fermented beverages brewed. Brewers must make a monthly statement and their books are to be open to the inspection of the collector or of his deputy.

Entries made in the books are to be further verified by the oath of the person making them. The tax on fermented beverages is paid by stamps which must be affixed to one of two spigot holes so that they will be destroyed when the barrels are opened, it being forbidden by law to have more than two such apertures in the harrel.

Manufacturers of tobacco and snuff before beginning business must also make a statement to the collector of the place where the business is to be carried on and also of the number and character of the machines to be used in such business. Provision is made for the proper entries in the books of the manufacturers to determine the amount of business done and penalties are provided for cloing any act which shall conceal from the collector the amount of tobacco, snuff or cigars manufactured.

The character of packages in which tobacco, snuff and cigars may be put up for sale is strictly provided by law and prohibition 
is made of the packing of such manufactured articles in other than the legal forms.

Imported tobacco and cigars in the United States must also pay the internal revenue duty in addition to the import tax affected by the customs law.

In addition to the foregoing requircments manufacturers of cigars must make return of the number of cigar makers employed by them, and it is made the duty of every dealer in leaf tobacco to make a sworn statement when required of the amount of tobacco sold by them to any person when such information is demanded by the collector of internal revenue.

Cigar and tobacco taxes are evidenced by stamps and any package containing such articles which do not bear a stamp is held to not have paid the revenue tax and is subject to seizure. Stringent regulations are in force requiring the destruction of every stamp when the package upon which it has been placed has been emptied.

The internal revenue collectors are also charged with the collection of the tax of \$ro.oo per pound on all opium manufactured in the United States for smoking purposes. In order to manufacture opium for smoking purposes, persons proposing to carry on such business must be citizens of the United States and must give a bond in accordance with the requirements of the commissioner of the internal revenue. Such a bond shall not be in the sum of less than $\$ 5,000.00$.

Prepared smoking opium imported into the United States must also pay the internal revenue tax and heavy penalties are imposed for the sale of opium without the stamp.

Another source of taxation has within a few years been put into effect under the direction of the commissioner of internal revenue. This refers to articles in imitation of natural products, and the purpose of the taxation is not so much to provide a revenue for governmental purposes as to protect standard agricultural products from destructive competition, as well as to insure that the purchaser of such artificial products shall be certain that they have not been deceived.

The first substance to which this scheme of law was applied was 
oleomargarin, or the mixture of certain other fats with the butter fat produced by churning from milk of cows, or substances composed wholly of other than butter fats, but colored and made up to resemble butter. Nanufactures or dealers in olcomargarin are required to pay an annual tax in the nature of a license, and in addition taxes are imposed on the product which are evident by the affixing of stamps after the manner of other internal revenue taxes.

Similar provisions are made for the assessment of a tax upon the manufacturers and sellers of filled cheese, together with a per pound tax on the product. Filled checse consists of the product formed by the mixture of animal or vesetable oils or fat foreign to milk with milk or skinmed milk, so that an imitation is made of cheese which is made wholly from milk.

A further tax is imposed on mixed flour which is the product secured by grinding wheat with other grain or grain products or with any other material. With regard to the latter substance it is required that a card shall be inserted in the package denoting the composition of these mixed flours.

Careful and defunite provisions are made as to the packages in which all three of these classes of substances shall be contained, and also as to the method of branding in order to denote their character, and of affixing the stamp showing the payment of the internal revenue tax.

Subordinate officers of the internal revenue service beside the deputy collectors, gaugers and storekeepers whose duties have been heretofore indicated are the special agents who are employed under the direction of the commissioner of internal revenue in the various investigations necessary for detecting and preventing frauds on the revenue. While a considerable portion of the work of these agents is in the larger cities where illicit stills are not uncommon, there has been a long and romantic history of their operations in the remoter sections of the country, especially in the mountainous districts of the South where the farmers finding it difficult to secure a market for their corn have been in the habit of turning it into whiskey by the aid of a crude apparatus in remote places difficult of access. There has been almost a century of warfare 
between these farmers and the agents of the internal revenue, involving much bloodshed, and a considerable proportion of the prisoners at the Atlanta penitentiary, which is under Federal auspices, is made up of men who have been detected in these violations of the law and sentenced to prison for various terms, although it has been argued that, from their point of view, they have been guilty of no wrong. While the amount of revenue of which the United States has been defrauded by the operations of these small distillers has by no means been proportionate with the cost in money and life of the special agents of breaking up their business, it is only by the most incessant care and watchfulness that illegal distilling can be prevented from becoming so prevalent as to seriously reduce the revenues of the United States.

The constant demand for distilled spirits and the considerable profit which would be made by the evasion of the internal revenue tax, makes the field an inviting one for unscrupulous men, and makes it impossible to do otherwise than to apply the full rigor of the law to the offenders.

The questions which confront the Commissioner of the Internal Revenue and his collectors are by no means easy ones. Besides the constant watchfulness to prevent evasions of the law, the decisions under the various acts of Congress have grown into a system of administrative jurisprudence. For the assistance of the Commissioner of Internal Revenue in dealing with these matters as well as to provide legal assistance in cases where appeals must be made to the courts, a Solicitor of Internal Revenue who is an officer of the Department of Justice is assigned to the Internal Revenuc Bureat, and who indertakes the conduct and settlement of the legal questions which arise in the conduct of the internal revenue business, subject at all times to review of the Attorney General when deemed necessary.

As the tax upon alcohol is the same whether it appears in the manufactured products as a beverage distilled from grain of good quality or whether because of its origin from other substances, it is not palatable and must be used in the arts, it is evident that the economical use of tax-paid alcohol in the latter form is limited to 
those purposes for which alcohol alone can be advantageously used, and in which it comes into competition with no other fluid.

In certain countries where a different policy obtains as to taxing alcohol, great developments have taken place both as to the possibilities of obtaining this substance from various vegetable prockucts and of using it, especially as a source of power. For this purpose of stimulating the development and extension of the use of alcohol in this country, the Congress has, within a few years, made provision by which, under certain circumstances, the internal revenue tax is remitted so that alcohol can be put on the market at a price which allows it to compete with other sources of heat, light and power.

The chief of the conditions required by the Congress for the remission of the tax is that substances shall be mixed with the alcohol as it comes from the still which would effectually prevent its use under any form or combination as a beverage. The tax upon alcohol was imposed as a tax upon the beverage and the prohibitive tax incidental on alcohol used in the arts was maintained solely because of the impossibility of seggregating the use of alcohol in the latter from the use of alcohol as a beverage. There have been various enactments by Congress designed to relieve the rigidity of the uniform tax upon alcohol where such action could be taken without detriment to the internal revenue, but these instances were by no means of general application prior to the latest enactment.

The Denaturing Act, so called, provides that an amount of alcohol duly protected by bond may be withdrawn from distillery warehouses and mixed under the supervision of an officer of the United States with substances which so change the character of the alcohol that it cannot be used as a beverage in its condition as denatured or made potable by any process of treatment except by redistilling. Against this latter possibility provision is made by the system of registration of those who deal in the denatured products, with the requirement that the books showing the amounts of the sales and the destination of each package must be at all times open to the inspection of the internal revenue officer. 
The law is expressed in general terms, in order to provide for any discoveries or improvements which may be made in the treatment of this substance and in the method of its production. For the purpose of determining upon the regulations which should be adopted under the law, the Commissioner of the Internal Revenue visited foreign countries and upon his return formulated the present set of regulations under which it is possible to establish special manufactories for the production of alcohol intended for denaturing or for persons engaged in distilling alcohol for use in beverages to set aside a certain portion of their premises to be used as a denaturing house in which the processes of the transmission of a taxable article into a non-taxable product may be carried on.

The Commissioner of Internal Revenue is required to make an annual detailed statement to Congress as to how he has expended the sums appropriated for detecting and bringing to trial persons guilty of violating Internal Revenue laws, or conniving at such violation, together with a detailed statement of the miscellaneous expenses under his bureau. The Commissioner of Internal Revenue has also power to prescribe the character of the stamps used to denote the payment of the revenue taxes, and to change the form of the same from time to time as he may see fit.

As so many of the duties of the office of the internal revenue pertain to matters which require chemical analysis to determine exact facts, there is attached to the office of the Commissioner of Internal Reventue a force of chemists who have the duty of making the necessary tests with regard to distilled liquors, oleomargarin, mixed flour, etc.

SCHEDULE OF ARTICLES AND OCCUPATIONS SUBJECT TO TAX ON AND AFTER JULY I, 1906.

Special tares.

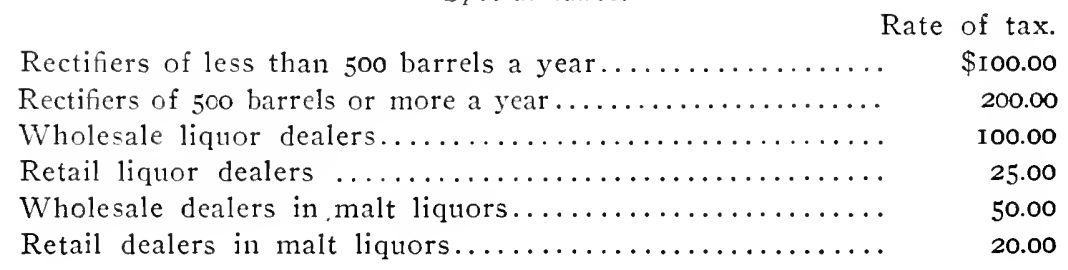


Manufacturers of stills......................... 50.00

And for stills or worms, manufactured, each.......... 20.00

Brewers:

Annual manufacture less than 500 barrels............ 50.00

Annual manufacture soo barrels or more...........

Manufactures of filled cheese................... 400.00

Wholesale dealers in filled cheese................... $\quad 250.00$

Retail dealers in filled cheese.................... I 2.00

Manufacturers of filled cheese...................... $\quad 400.00$

Wholesale dealers in oleomargarin artificially colored in imitation of butter ............................. ${ }_{4} 80.00$

Wholesale dealers in olcomargarin free from artificial colora-

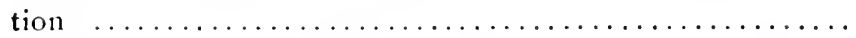

Retail dealers in oleomargarin artificially colored in imitation of butter ................................ $\quad 48.00$

Retail dealers in oleomar arin free from artificial coloration 6.on

Mannfacturers of adulterated butter ................... 600 oo

Wholesale dealers in adulterated butter ................. $\quad 480.00$

Retail clealers in adulterated butter.................... $\quad 48.00$

Manufacturers of process or renovated butter ............ $\quad 50.00$

Mamufacturers, packers, or repackers of mixed flour ....... I 2.00

\section{Distilled spirits. itc.}

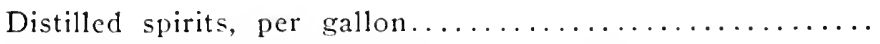

Stamps for distilled spirits intended for export, each........

Except when affixed to packages containing two or more

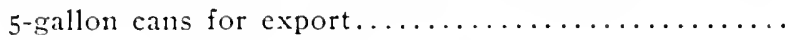

Case stamps for spirits bottled in bond .................

Wines, liquors, or compounds known or denominated as wine, and made in initation of sparkling wine or champagne, but not made from grapes grown in the United States, and liquors not made from grapes, currants, rlubarb, or berries grown in the United States, but produced by being rectified or mixed with distilled spirits or by the infusion of any matter in spirits, to be sold as wine, or as a substitute for wine, in bottles containing not more than I pint per bottle or

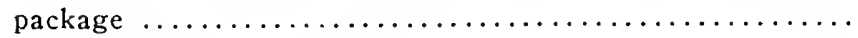

Same, in bottles containing more than I pint, and not more than I quart, per bottle or package.................. 
(And at the same rate for any larger quantity of such merchandise, however put up or whatever may be the package.)

Grape brandy used in the fortification of pure sweet wine under an act approved June 7, Ig06 (to be assessed), per

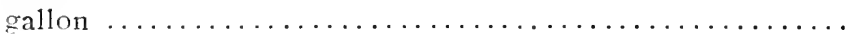

\section{Formented liquors.}

Fermented liquors per barrel, containing not more than $3 \mathrm{I}$

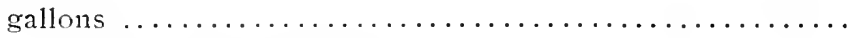

(And at a proportionate rate for halves, thirds, quarters, sixths, and eighths of barrels.)

Nore than one barrel of $3 \mathrm{~T}$ gallons, and not more than 63 gal-

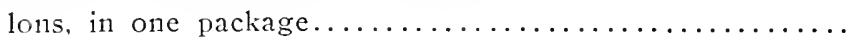

\section{Tobacco and smuff.}

Tobacco, however prepared, manufactured and sold, or removed for consumption or sale, per pound..............

Snuff, however prepared, manufactured and sold, or removed for consumption or sale, per pound..................

\section{Cigars and Cigarettes.}

Cigars of all descriptions made of tobacco, or any substitute therefor, and weighing more than 3 pounds per thousand..

Cigars of all descriptions made of tobacco, or any substitute therefor, and weighing not more than 3 pounds per thou-

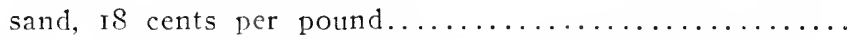

Cigarettes weighing not more than 3 pounds per thousand and of a wholesale value or price of more than $\$ 2$ per thousand,

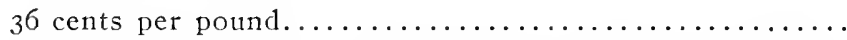

Cigarettes weighing not more than 3 pounds per thousand and of a wholesale value or price of not more than $\$ 2$ per thou-

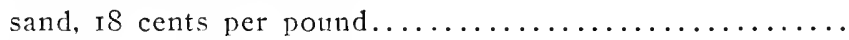

Cigarettes weighing more than 3 pounds per thousand.....

\section{Oleomargarin.}

Oleomargarin, domestic, artificially colored to look like butter, of any shade of yellow, per pound.

Rate of tax. 
Rate of tax.

Oleomargarin, free from coloration that canses it to look like butter, of any shade of yellow, per pound............. $001 / 4$

Oleomargarin, imported from foreign countries, per pound.....

Adulterated butter and process or renoziated butter.

Adulterated butter, per pound.......................

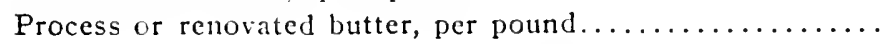

.10

$.00^{1 / 4}$

Filled cheese.

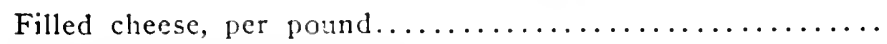

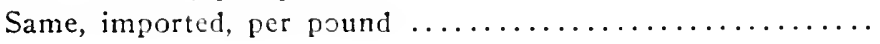

\section{Opium.}

Prepared smoking opium, per pound............... 10.00

Mixcd flour.

Mixed flour, per barrel of 196 pounds, or more than 98 pounds

Half barrel of 98 pounds, or more than 49 pounds.......... .02

Quarter barrel of 49 rounds, or more than $241 / 2$ pounds...... .01

Eighth barrel of $24^{1} / 2$ pounds or less................... $.001 / 2$

(Mixed flour imported from foreign countries, in addition to import duties, must pay internal-revenue tax as above.)

Circulation of and notes paid out by banks and bankers.

Circulation issued by any bank, etc., or person (except a national bank taxed under sec. 5214 , Rev. Stat., and sec. I3, act March If, 1900), per month................... I 2 of I p.c.

Circulation (except national banks) exceeding 90 per cent of capital, in addlition, per month.................. $1 / 6$ of I p.c.

Banks, etc., on amount of notes of ally person, State bank, or

State banking association, used for circulation and paid out. Io per cent.

Banks, etc., bankers, or associations, on amount of notes of any town, city, or municipal corporation paid out by them. Io per cent.

Every person. firm, association, other than national bank associations, and every corporation, State bank, or State banking association, on the amount of thcir ou'n notes used for circulation and paid out by them.................. ro per cent. 
Every such person, firm, association, corporation, State bank, or State banking association, and also every national-banking association, on the amount of notes of any person, firm, association, other than a national-banking association, or of any corporation, State bank, or State banking association, or of any town, city, or municipal corporation, used for circulation and paid out by them............... ro per cent.

\section{Playing cards.}

Playing card, per pack, containing not more than fifty-four

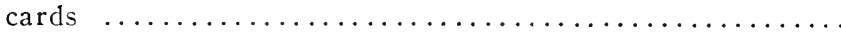

\section{Taxes not payable by stamps.}

Tax on deficiencies in production of spirits-

On excess of materials used in production of spirits.

On circulation of banks and bankers.

On notes paid out by banks and others.

On brandy used in the fortification of wine.

Penalties of 50 per cent. and roo per cent.

INTERNAL REIENLE DISTRICTS AND COLLECTORS' DONDS AND SALARIES.

\section{Alabama- \\ District.}

Collector's address, Birmingham........

\section{Alaska-}

\section{Arizona-}

[Constituted one Collection District April 28, I866. Consolidated with District of New Mexico September 5, I883.]

\section{Arkansas-}

Collector's address, Little Rock......... California-

I.-The counties of Alameda, Calaveras, Contra Costa, Fresno, Inyo, Kern, Kings, Los Angeles, Madera, Mariposa, Merced, Mono, Monterey, Orange, Riverside, San Benito, San Bernardino, San Diego, San Francisco, San Joaquin,

Rate of tax. 


\section{District.}

San Luis Obispo, San Mateo, Santa Barbara, Santa Clara, Santa Cruz, Stanislaus, Tulare, Tuolumne, and Ventura. Collector's adlress, San Francisco.

4.-The State of Nevala and the followingnamed countics in the State of California: Alpine, Amador, Butte, Colusa, Del Norte. Eldorado, Glenn, Humboldt, Lake, Lassen, Marin, Nendocino, Modoc, Napa, Nevada, Placer, Plumas, Sacramento, Shasta, Sierra, Siskiyou, Solano, Sonoma, Silter, Tehama. Trinity. Yolo, and Yuba. Collector's ad-

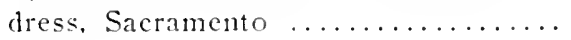

Colorado-

Collector's address. Denver............. Connecticut-

Collector's address, Hartford..........

Dakota-

Delaware-

Florida-

Collector's address, Jacksonville........

Georgia-

Collector's address, Atlanta...........

Hawaii-

Collector's address, Honolulu..........

Idaho-

Illinois-

I.-The counties of Boone, Carroll. Cook, Dekalb, Dupage, Grundy, Jo Daviess, Kane, Kankakee, Kendall, Lake, Lasalle. Lee, McHenry, Ogle, Stephenson, Whiteside, Will, and Winnebago. Collector's address, Chicago............

5.-The counties of Bureau. Henderson, Henry, Knox, Marshall, Mercer, Peoria, Putnam, Rock Island, Stark, and Warren. Collector's address, Peoria...
Official Disbursing Bond. Bond. Salary.

I $50,000 \quad 15,000 \quad 4,500$

$75.000 \quad 10,000 \quad 3.750$

$75,000 \quad 10,000 \quad 4,000$

$125,000 \quad 10,000 \quad 4,500$

(See Nebraska and North and South Dakota.)

(See Maryland.)

$\begin{array}{lll}\text { So,000 } & 10,000 & 4.500 \\ 75.000 & 15.000 & 3.625 \\ 50.000 & 10.000 & 3.500\end{array}$

(See Montana.)

I $50,000 \quad$ I $5.000 \quad 4.500$

$200.000 \quad 25,000 \quad 4,500$ 
District.

S.- The counties of Adams, Bond, Brown, Calhoun, Cass, Champaign, Christian, Coles, Cumberland, Dewitt, Douglas, Edgar, Ford, Fulton, Greene, Hancock, Iroquois, Jersey, Livingston, Logan, McDonough, McLean, Macon, Macoupin, Mason, Menard, Montgomery, Morgan, Moultrie, Piatt, Pike, Sangamon, Schuyler, Scott, Shelby, Tazewell, Vermilion, and Woodford. Collector's address, Springfield.......

I3.-The counties of Alexander, Clark, Clay, Clinton, Crawford, Edwards, Effingham, Fayette, Franklin, Gallatin, Hamilton, Hardin, Jackson, Jasper, Jefferson, Johnson, Lawrence, Madison, Marion, Massac, Monroe, Perry, Pope, Pulaski, Randolph, Richland, St. Clair, Saline, Union, Wabash, Washington, Wayne, White, and Williamson. Collector's address, East St. Louis.....

Indian Territory-

Indiana-

6.-The counties of Adams, Allen, Bartholonew, Benton, Blackford, Brown, Cass. Dearborn, Decatur, Dekalb, Delaware, Ellhart, Fayette, Franklin. Fulton, Grant, Hamilton, Hancock, Hendricks, Henry, Howard, Huntington, Jackson, Jasper, Jay, Jefferson, Jennings, Johnson, Koscinsko, Lagrange, Lake, Laporte, Lawrence, Madison, Marion. Marshall, Miami, Monroe, Morgan, Newton, Noble, Ohio, Porter, Pulaski, Randolph, Ripley, Rush, St. Joseph, Shclby, Starke, Steuben, Switzerland, Tipton, Union, Wabash, Wayne, Wells, White, and Whitley. Collector's address, Indianapolis
Official Disbursing

Bond. Bond. Salary.

$150,000 \quad 10,000 \quad 4,500$

$80,000 \quad 10,000 \quad 4,125$

(See Kansas.)

$100,000 \quad 10,000 \quad 4,500$ 


\section{District.}

7.-The counties of Boone, Carroll, Clark, Clay, Clinton, Crawford, Daviess, Dubois, Floyd, Fountain, Gibson, Greene, Harrison, Knox, Martin, Montgomery, Orange, Owen, Parke, Perry, Pike, Posey, Putnam, Scott, Spencer, Sullivan, Tippecanoe, Vanderburg, Vermilion, Vigo, Warren, Warrick, and Washington. Collector's address, Terre Haute

\section{Iowa-}

3.-The counties of Allamakee. Benton, Blackhawk, Boone, Bremer, Buchanan, Buena Vista, Butler, Calhoun, Carroll, Cerro Gordo, Cherokee, Chickasaw, Clay, Clayton, Crawford, Delaware, Dickinson, Dubuque, Emmet, Fayette, Floyd, Franklin, Greene, Grundy, Hamilton, Hancock, Hardin, Howard, Humboldt, Ida, Jones, Kossuth, Linn, Lyon. Marshall, Mitchell, Monona, O'Brien, Osceola, Palo Alto, Pocahontas, Plymouth, Sac, Sioux, Story, Tama, Webster, Winnebago, Winneshiek, Woodbury, Worth, and Wright. Collector's ad-

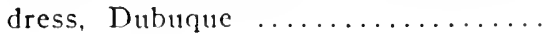

4.- The counties of Adair, Adams, Appanoose, Audubon, Cass, Cedar, Clarke, Clinton, Dallas, Daris, Decatur, Des Moines, Fremont, Guthrie, Harrison, Henry, Iowa, Jackson, Jasper, Jefferson, Johnson, Keokuk, Lee, Louisa, Liscas, Madison, Mahaska, Marion, Mills, Monroe, Montgomery, Muscatine, Page, Polk, Pottawattamie, Powesliek. Ringgold, Scott, Slielby, Taylor. Union, Van Buren, Wapello, Warren, Wasliington, and Wayne. Collector's ar-

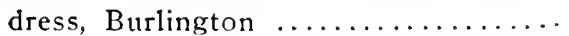

Official Disbursing

Bond. Bond. Salary.

$100,000 \quad 10,000 \quad 4,500$ 
Kansas--

Collector's address, Leavenworth

Kentucky-

2.- The counties of Allen, Ballard, Barren, Breckinridge, Butler, Caldwell, Calloway, Carlisle, Christain, Clinton, Crittenden, Cumberland, Daviess, Edmonson, Fulton, Graves, Grayson, Hancock, Hart, Henderson, Hickman, Hopkins, Livingston, Logan, Lyon, McCracken, McLean, Marshall, Metcalfe, Monroe, Nuhlenberg, Ohio, Russell, Simpson, Todd, Trigg, Union, Warren, and Webster. Collector's address, Owensboro .........................

5.--The city of Louisville and the counties of Adair, Bullitt, Casey, Green, Hardin, Henry, Jefferson, Larue, Marion, Meade, Nelson, Oldham, Owen, Shelby, Spencer, Taylor, and Washington. Collector's address, Lotiisville...

6.-The counties of Boone, Bracken. Campbell. Carroll, Gallatin, Grant, Harrison, Kenton, Pcndleton, Robertson, and Trimble. Collector's address, Covington $\ldots \ldots \ldots \ldots \ldots \ldots \ldots \ldots \ldots \ldots \ldots \ldots \ldots \ldots$.

7.- The counties of Bath, Bourbon, Boyd,
Carter, Clark, Elliott, Fayette, Fleming, Franklin, Greenup, Johnson, Lawrence, Lewis, Martin, Mason, Menifee. Montgomery, Norgan, Nicholas, Powell, Rowan, Scott, and Woodford. Collector's address, Lexington ....... 8.--The counties of Anderson, Bell, Boyle, Breathitt, Clay, Estill, Floyd, Garrard, Harlan, Jackson, Jessamine, Knott, Knox, Laurel, Lee, Leslie, Letcher, Lincoln, Madison, Magoffin, Mercer, Owsley, Perry, Pike, Pulaski, Rockcastle, Wayne, Whitley, and Wolfe, Collector's address, Danville .......
Official Disbursing Bond. Bond. Salary. $60,000 \quad 10,000 \quad 3,125$

$100,000 \quad 20,000 \quad 4,500$ I $50,000 \quad 40,000 \quad 4,500$ I $00.000 \quad$ I $5,000 \quad 4,500$

I $00,000 \quad 20,000 \quad 4,500$

$80,000 \quad 25,000 \quad 4,500$ 
District.

Louisiana-

Collector's address, New Orleans ........

Maine-

Maryland-

Collector's address, Baltimore .........

Massachusetts-

Collector's address, Boston

Michigan-

I.-Countics of Alcona, Alpena, Arenac, Bay, Branch, Calhoun, Clseboygan, Clare, Clinton, Crawford, Genesee, Gladwin, Gratiot, Hillsdale, Huron, Ingham, Iosco, Isabella, Jackson, Lapeer, Lenawee, Livingston, Macomb, Midland, Monroe, Montmorency, Oakland, Ogemaw, Oscoda, Otsego, Presque Isle, Roscommon, Saginaw, Sanilac, Shiawassee, St. Clair, Tuscola, Washtenaw, and Wayne. Collector's address, Detroit .................

4.--Counties of Alger, Allegan, Antrim, Baraga, Barry, Benzie, Berrien, Cass, Charlevoix, Chippewa, Delta, Dickinson, Eaton, Enmet, Gogebic, Grand Traverse, Hougliton, Ionia, Iron, Kalamazoo, Kalkaska, Kent, Keweenaw, Lake, Leelanau, Luce, Mackinac, Manistee, Marquette, Mason, Mecosta, Menominee, Missaukee, Montcalm, Muskegon, Newaygo, Oceana, Ontonagon, Osceola, Ottawa, St. Joseph, Schoolcraft, Van Buren, Wexford. Collector's address, Grand Rapids ....

Minnesota-

Collector's address, St. Paul .............

Mississippi-

Missouri-

I.-The counties of Adair, Andrain, Bollinger, Boone, Butler, Callaway, Cape Girardeau, Carter, Clark, Crawford,
Official Disbursing Bond. Bond. Salary. I $00,000 \quad 20,000 \quad 4,500$ (See New Hampshire.)

$\begin{array}{lll}125,000 & 20,000 & 4,500 \\ 100,000 & 10,000 & 4,500\end{array}$

I $00,000 \quad 10,000 \quad 4,500$

$60,000 \quad 10,000 \quad 4,125$

$100,000 \quad 10,000 \quad+, 500$

(See Lonisiana.) 


\section{District.}

Dent, Dunklin, Franklin, Gasconade, Howard, Iron, Jefferson, Knox, Lewis, Lincoln, Linn, Macon, Madison, Maries, Marion, Mississippi, Montgomery, Monroe, New Madrid, Oregon, Osage, Pemiscot, Perry, Phelps, Pike, Pulaski, Ralls, Randolph, Reynolds, Ripley, St. Charles, St. Francois, Ste. Genevieve, St. Louis, Schuyler, Scotland, Scott, Shannon, Shelby, Stoddard, Warren, Washington, and Wayne. Collector's address, St.

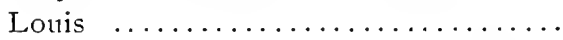

6.-The counties of Andrew, Atchison, Barry, Barton, Bates, Benton, Buchanan, Caldwell, Camden, Carroll, Cass, Cedar, Chariton, Christian, Clay, Clinton, Cole, Cooper, Dade, Dallas, Daviess, Dekalb, Douglas, Gentry, Greene, Grundy, Harrison, Henry, Hickory, Holt, Howell, Jackson, Jasper, Johnson, Laclede, Lafayette, Lawrence, Livingston, McDonald, Mercer, Miller, Moniteau, Morgan, Newton, Nodaway, Ozark, Pettis, Platte, Polk, Putnam, Ray, St. Clair, Saline, Stone, Sullivan, Taney, Texas, Vernon, Webster, Worth, and Wright. Collector's

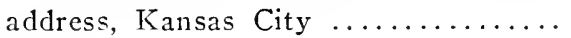
Montana-

Collector's address, Salt Lake City, Utah .. Nebraska-

Collector's address, Omaha .......... Nevada-

New Hampshire-

Collector's address, Portsmouth ........ New Jersey-

I.-The counties of Atlantic, Burlington, Camden, Cape May, Cumberland, Gloucester, Mercer, Monmouth, Ocean,
Official Disbursing

Bond. Bond. Salary.

I $25,000 \quad$ I $5,000 \quad 4,500$

$60,000 \quad 10,000 \quad 4,500$

$50,000 \quad 10,000 \quad 4,500$

75,000 I0,000 4,500

(See Fourth California.)

$60,000 \quad 10,000 \quad 3,625$ 
District.

and Salem. Collector's address, Camden $\ldots . . . \ldots \ldots \ldots . . . \ldots . . . . .$.

5.-The counties of Bergen, Essex, Hudson, Hunterdon, Middlesex, Morris, Passaic, Somerset, Sussex, Union, and Warren. Collector's address, Cam-

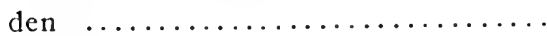

New Mexico-

Collector's address, Santa Fe ...........

New York-

I.-The counties of Kings, Nassau, Queens, Richmond, and Suffolk. Collector's address, Brooklyn .................

2.- The Ist, 2d, 3d, 4th, 5th, 6th, 8th, 9th, and I5th wards of New York City; that portion of the $14^{t}$ th ward lying west of the center of Mott street; that portion of the $r 6$ th ward lying south of the center of West 24th street and Governors Island. Collector's address, New York .........................

3.-The 7 th, Ioth, IIth, I2th, I3th, I 7 th, Isth, Igth, zoth, 2Ist, and 22nd wards of New York City; that part of the I t th ward lying east of the center of Mott strcet; that part of the 16 th ward lying north of the center of West 2.th strcet, and Blackwells, Randalls, and Wards islands. Collector's address, New York .....................

14.- The counties of Albany, Cliuton, Columbia, Dutchess, Essex, Fulton, Greene, Hamilton, Montgomery, Orange, Putnam, Rensselaer, Rockland, Saratoga, Schenectady, Schoharie, Sullivan, Ulster, Warren, Washington, and Westchester and the $23 \mathrm{~d}$ and 24 th wards of New York City. Collector's address, Albany ....................
Official Disbursing

Bond. Bond. Salary.

$50,000 \quad 10,000 \quad 3,500$

$100,000 \quad 10,000 \quad 4,500$

$50,000 \quad 10,000 \quad 2,500$

I $25,000 \quad 10,000 \quad 4,500$

$100,000 \quad 10,000 \quad 4,500$

I 50,000 I0,000 4,500

$100,000 \quad 10,000 \quad 4,500$ 
District.

2I.-The counties of Broome, Cayuga, Chenango, Cortland, Delaware, Franklin, Herkimer, Jefferson, Lewis, Madison, Oneida, Onondaga, Oswego, Otsego, St. Lawrence, Schuyler, Seneca, Tioga, Tompkins, and Wayne. Collector's address, Syracuse .......

28. - The counties of Allegany, Cattaraugus, Chautauqua, Chemung, Erie, Genesee, Livingston, Monroe, Niagara, Ontario, Orleans, Steuben, Wyoming, and Yates. Collector's address,

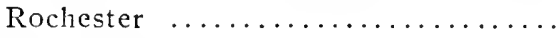

North Carolina-

4.-The counties of Alamance, Beaufort, Bertie, Bladen, Brunswick, Camden, Carteret, Caswell, Chatham, Chowan, Columbus, Craven, Cumberland, Currituck, Dare, Duplin, Durham, Edgecombe, Franklin, Gates, Granville, Greene, Guilford, Halifax, Harnett, Hertford, Hyde, Johnston, Jones, Lenoir, Martin, Montgomery, Moore, Nash, New Hanover, Northampton, Onslow, Orange, Pamlico, Pasquotank, Pender, Perquimans, Person, Pitt, Randolph, Richmond, Robeson, Rockingham, Sampson, Scotland, Tyrrell, Vance, Wake, Warren, Washington, Wayne, and Wilson. Collector's address,

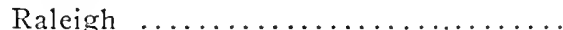

5.-The counties of Alexander, Alleghany, Anson, Ashe, Buncombe, Burke, Cabarrus, Caldwell, Catawba, Cherokee, Clay, Cleveland, Davidson, Davie, Forsyth, Gaston, Graham, Haywood, Henderson, Iredell, Jackson, Lincoln, McDowell, Macon, Madison, Mecklenburg, Mitchell, Polk, Rowan, Rutherford, Stanly, Stokes, Surry, Swain,
Official Disbursing Bond. Bond. Salary.

$80,000 \quad 10,000 \quad 4,500$

$100,000 \quad 10,000 \quad 4,500$

$100,000 \quad 15,000 \quad 4,500$ 


\section{District.}

Transylvania, Union, Watauga, Wilkes, Yadkin, and Yancey. Collector's address, Statesville

North and Sonth Dakota-

Collector's address, Aberdeen, S. D. ......

Olio-

I.-The counties of Brown, Butler, Clarke, Clermont, Clinton, Fayette, Greene, Hamilton, Highland, Montgomery, Preble, and Warren. Collector's address, Cincinnati ......................

10.-The counties of Allen, Auglaize, Champaign, Crawford, Darke. Defiance, Erie, Fulton, Hancock, Hardin, Henry, Huron, Logan, Lucas, Mercer, Miami, Ottawa, Paulding, Putnam, Sandusky, Seneca, Slielby, Van Wert, Williams, Wood, and Wyandot. Collector's address, Toledo .....................

II.-The counties of Adams, Athens, Coshocton, Delaware, Fairfield, Franklin, Gallia, Guernsey, Hocking, Jackson, Knox, Lawrence, Licking, Madison, Marion, Meigs, Morgan, Morrow, Muslingum, Noble, Perry, Pickaway, Pike, Ross, Scioto, Union, Vinton, and Washington. Collector's address, Athens .........................

18.-The counties of Ashland, Ashtabula, Belmont, Carroll, Columbiana, Cuyahoga, Geauga, Harrison, Holmes, Jefferson, Lake, Lorain, Mahoning, Medina, Monroe, Portage, Richmond, Stark, Summit, Trumbull, Tuscarawas, and Wayne. Collector's address, Cleve-

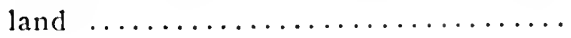

Oklahoma-

Oregon-

Collector's address, Portland
Official Disbursing Bond. Bond. Salary. $100,000 \quad 15,000 \quad 4,500$ $50,000 \quad 10,000 \quad 2,750$ $200,000 \quad 20,000 \quad 4,500$ $80,000 \quad 10,000 \quad 4,500$ $80,000 \quad 10,000 \quad 4.500$ $100,000 \quad 10,000 \quad 4,500$ (See Kansas.)

$60,000 \quad 10,000$ 


\section{District.}

\section{Pennsylvania-}

I.-The counties of Berks, Bucks, Chester, Delaware, Lehigh, Montgomery, Philadelphia, and Sclinylkill. Collector's address, Philadelphia ....................... Cumberland, Dauphin, Franklin, Fulton, Huntingdon, Juniata, Lancaster, Lebanon, Mifflin, Perry, Snyder, and York Collector's address, Lancaster.. I2.- The counties of Bradford, Carbon, Center, Clinton, Columbia, Lackawanna, Luzerne, Lycoming, Monroe, Montour, Northampton, Northumberland, Pike, Potter, Sullivan, Susquehanna, Tioga, Union, Wayne, and Wyoming. Collector's address, Scranton.. 23.-The counties of Allegheny, Armstrong, Beaver, Butler, Cambria, Cameron, Clarion, Clearfield, Crawford, Elk, Erie, Fayette, Forest, Greene, Indiana, Jefferson, Lawrence, McKean, Mercer, Somerset, Venango, Warren, Washington and Westmoreland. Collector's address, Pittsburg ..........

Rhode Island-

South Carolina-

Collector's address, Columbia ..........

South Dakota-

Tennessee-

2.-The counties of Anderson, Bledsoe, Blount, Bradley, Campbell, Carter, Claiborne, Cocke, Cumberland, Fentress, Grainger, Greene, Hamblen, Hamilton, Hancock, Hawkins, James, Jefferson, Johnson, Knox, London, McMinn, Marion, Meigs, Monroe, Morgan, Polk, Rhea, Roane, Scott, Sequatchie, Sevier, Sullivan, Unicoi,
Official Disbursing

Bond. Bond. Salary.

I $25,000 \quad$ I $5,000 \quad 4,500$

$100,000 \quad 10,000 \quad 4,500$

$80,000 \quad 10,000 \quad 4,500$

I $25,000 \quad 20,000 \quad 4,500$

(See Connecticut.)

$50,000 \quad 10,000 \quad 4$, I 25

(See North and South Dakota.) 
District.

Union, and Washington. Collector's address, Bristol

5.-The counties of Bedford, Benton, Cannon, Carroll, Cheatham, Chester, Clay, Coffee, Crockett, Davidson, Decatur, Dekalb, Dickson, Dyer, Fayette, Franklin, Gibson, Giles, Grundy, Hardeman, Hardin, Haywood, Henderson, Henry, Hickman, Houston, Humplreys, Jackson, Lake, Lauderdale, Lawrence, Lewis, Lincoln, MeNairy, Macon, Madison, Marshall, Maury, Montgomery, Moore, Obion, Overton, Perry, Pickett, Putnam, Robertson, Rutherford, Shelby, Smith, Stewart, Sumner, Tipton, Trousdale, Van Buren, Warren, WVayne, Weakley, White, Williamson, and Wilson. Collector's address, Nashville

Texas-

3.-The counties of Angelina, Aransas, Atascosa, Austin, Bandera, Bastrop, Bee, Bell, Bexar, Blanco, Brazoria, Brazos, Brewster, Brown, Burleson, Burnet, Caldwell, Calhoun, Cameron, Chambers, Coke, Coleman, Colorado, Comal, Concho, Coryell, Crockett, Dewitt, Dimmit, Duval, Ector, Edwards, El Paso, Falls, Fayette, Fort Bend, Frio, Galveston, Gillespie, Glasscock, Goliad, Gonzales, Grimes, Guadalupe, Hamilton, Mardin, Harris, Hays, Hidalgo, Houston, Irion, Jackson, Jasper, Jeff Davis, Jefferson, Karnes, Kendall, Kerr, Kimble, Kinney, Lampasas, Lasalle, Lavaca, Lee, Leon, Liberty, Live Oak, Llano, McCulloch, MeLennan, McMullen, Madison, Mason, Matagorda, Maverick, Medina, Menard, Midland, Milam, Mills, Montgomery, Newton, Nueces, Orange, Pecos, Polk,
Official Disbursing

Bond. Bond. Salary.

$50,000 \quad 10,000 \quad 4,000$

$80,000 \quad$ I $5,000 \quad 4,500$ 


\section{District.}

Presidio, Reagan, Reeves, Refugio, Robertson, Runnels, Sabine, San Augustine, San Jacinto, San Patricio, San Saba, Schleicher, Starr, Sterling, Sutton, Terrell, Tom Green, Travis, Trinity, Tyler, Uvalde, Valverde, Victoria, Walker, Waller, Ward, Washington, Webb, Wharton, Williamson, Wilson, Zapata, and Zavalla. Collector's ad-

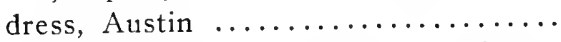
4.- The counties of Anderson, Andrews, Archer, Armstrong, Bailey, Baylor, Borden, Bosque, Bowie, Briscoe, Callahan, Camp, Carson, Cass, Castro, Cherokee, Childress, Clay, Cochran, Collin, Collingsworth, Comanche, Cooke, Cottle, Crosby, Dallam, Dallas, Dawson, Deaf Smith, Delta, Denton, Dickens, Donley, Eastland, Ellis, Erath, Fannin, Fisher, Floyd, Foard, Franklin, Freestone, Gaines, Garza, Gray, Grayson, Gregg, Hale, Hall, Hansford, Hardeman, Harrison, Hartley, Haskell, Hemphill, Henderson, Hill, Hockey, Hood, Hopkins, Howard, Hunt, Hutchinson, Jack, Johnson, Jones, Kaufman, Kent, King, Knox, Lamar, Lamb, Limestone, Lipscomb, Lubbock, Lynn, Marion, Martin, Mitchell, Montague, Moore, Morris, Motley, Nacogdoches, Navarro, Nolan, Ochiltree, Oldham, Palo Pinto, Panola, Parker, Parner, Potter, Rains, Randall, Red River, Roberts, Rockwall, Rusk, Scurry, Shackelford, Shelby, Sherman, Smith, Somervell, Stephens, Stonewall, Swisher, Tarrant, Taylor, Terry, Throckmorton, Titus, Upshur, Van Zandt, Wheeler, Wichita, Wilbarger, Wise, Wood, Yoakum, and Young. Collecttor's address, Dallas..............
Official Disbursing Bond. Bond. Salary.
$60,000 \quad 10,000 \quad 3,625$ 
District.

Utah-

Vermont-

Virginia-

2.-The counties of Amelia, Appomattox, Brunswick, Buckingham, Caroline, Charles City, Charlotte, Chesterfield, Cumberland, Dinwiddie, Elizabeth City, Essex, Fluvanna, Gloucester, Goochland, Greenesville, Hanover, Henrico, Isle of Wight, James City, King and Queen, King George, King William, Lancaster, Lonisa, Lunenlurg, Mathews, Middlesex, Nansemond, New Kent, Norfolk, Northumberland, Nottoway, Powhatan, Prince Edward, Prince George, Princess Anne, Richmond, Stafford, Southampton, Spottsylvania, Surry, Sussex, Warwick, Westmoreland, and York. Collector's ad-

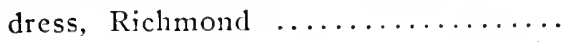

6.-The counties of Albemarle, Alexandria, Alleghany, Amherst, Augusta, Bath, Bedford, Bland, Botetourt, Buchanan, Campbell, Carroll, Clarke, Craig, Culpeper, Dickenson, Fairfax, Fauquier, Floyd, Franklin, Frederick, Giles, Grayson, Greene, Halifax, Henry, Highland, Lee, Loudoun, Madison, Mecklenburg, Montgomery, Nelson, Orange, Page, Patrick, Pittsylvania, Prince William, Pulaski, Rappahannock, Roanoke, Rockbridge, Rockingliam, Russell, Scott, Shenandoah, Smyth, Tazewell, Warren, Washington, Wise, and Wythe. Collector's address,

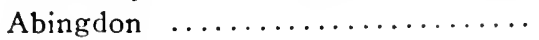

Washington-

Collector's address, Tacoma ...........

West Virginia-

Collector's address, Parkersburg ......
Official Disbursing

Bond. Bond. Salary.

(Sec Montana.)

(See New Hampshire.)

$100,000 \quad 10,000 \quad 4,500$

$\begin{array}{lrr}100,000 & 15,000 & 4,500 \\ 50,000 & 10,000 & 4,000 \\ 80,000 & 10,000 & 4,500\end{array}$


Wisconsin-

District.

I.-Counties of Brown, Calumet, Dodge, Door, Florence, Fond du Lac, Forest, Green Lake, Kenosha, Kewannee, Nanitowoc, Marinette, Marquette, Milwaukee, Oconto, Outagamie, Ozaukee, Racine, Shawano, Sheboygan, Walworth, Washington, Waukesha, Waupaca, Waushara, Winnebago, and county of Langlade with exception of the eight townships of said county which were formerly in Lincoln county. Collector's address, Milwaukee ..........................

2.- Counties of Adams, Ashland, Barron, Bayfield, Buffalo, Burnett, Chippewa, Clark, Columbia, Crawford, Dane, Douglas, Dunn, Eau Claire, Grant, Green, Iowa, Iron, Jackson, Jefferson, Juneau, La Crosse, Lafayette, Lincoln, Marathon, Monroe, Oneida, Pepin, Pierce, Polk, Portage, Price, Richland, Rock, Rusk, St. Croix, Sauk, Sawyer, Taylor, Trempealeau, Vernon, Vilas, Washburn, Wood, and the eight townships in the western part of Langlade county which were formerly in Lincoln county. Collector's address, Madison ...................... Wyoming-
Official Disbursing Bond. Bond. Salary.
I 25,000 I0,000 4,500

$75,000 \quad$ I0,000 4,375

(See Colorado.)

In the early history of the United States when goods imported into this country were brought to the United States in a considerable number of small vessels instead of in the great floating warehouses of the present day, it was much easier, without attracting attention, for a vessel to run into a small inlet of the sea and illegally deliver her cargo, than would be the case at present. For that reason a patrol of the coast, as in the case of European nations, which maintained a system of imports on imported goods was a necessity 
from the beginning of the Government. In 1790 , there was provided as a branch of the Treasury Department an armed service known as the United States Revenue Cutter service. Such a service had been in effect in England, an adjunct to the British navy. The Revenue Cutter Service of the United States is, however, an organization entirely independent and distinct from the organization of the navy of the United States, although in time of war it is consolidated with the latter. The personnel of the Revenue Cutter Service is headed by a chicf of the division who is one of the captains in the Service selected by the Secretary of the Treasury and detailed, during the pleasure of that official to be the executive officer of the service. The remainder of the commission's personnel consists of captains, first lieutenants, sccond lieutenants, and third lieutenants concerned in the navigation and other administrative duties of the service, and an engincer in chief who has charge of the installation and repairs of steam machinery, chief engineers, first assistant engineers, and second assistant engineers, who have to do with the motive power of the revenue cutters only. In addition, a grade of constructors is provided consisting of officials who supcrintend the construction and repair of the various vessels used in the service.

The duties of the Revenue Cutter Service are as follows:

The protection of the customs revenue.

The co-operation with the Navy when directed by the President.

The assistance of vessels in clistress.

The enforcement of the laws pertaining to the quarantine.

The enforcment of the neutrality laws.

The enforcement of the navigation and other laws governing merchant vessels.

The protection of merchant vessels from piratical attacks, and the suppression of piracy.

The protection of the seal fisheries and sea-otter hunting grounds in Alaska.

The protection of wrecked property.

The suppression of illegal traffic in firearms, ammunition, and spirits with the natives along the coast of Alaska. 
The suppression of the slave trade.

The prevention of the violation of the immigration laws, as provided in section 2163 , Revised Statutes.

The revenue cutters on the northern and northwestern lakes, when put into commission, are specially charged with aiding vessels in distress on the lakes.

The suppression of mutinies on board merchant vessels.

The superintendence of the construction of life-saving stations.

The inspection, instruction, and drilling of crews of life-saving stations.

The enforcement of certain laws connected with the fisheries pertaining to the Department of Commerce and Labor.

The enforcement of the provisions of law in regard to the anchorage of vessels in the ports of New York, N. Y.; Chicago, Ill.; Kennebec River, at or near Bath, Me., and in regard to the movements and anchorages of vessels in the St. Marys River, Michigan.

The establishment and maintenance of a refuge station at or near Point Barrow, Alaska.

The enforcement of rules and regulations to insure the safety of passengers and crews on excursion steamers, yachts, and other craft, and of oarsmen, at regattas.

For the purpose of carrying out these duties, revenue cutters, consisting of 34 vessels of from I74 to rooo tons burden, are stationed at the chief ports, each cutter being assigned to a cruising station, the division being as follows:

\section{STATIONS ON TIE ATLANTIC.}

Portland, Me. :

NORTH OF CAPE HATTERAS.

(Cruising grounds, from the St. Croix River to Cape Anu, Mass.)

Boston, Mass. :

(Cruising grounds, from West Quoddy Head, Me., to Nantucket shcals light-ship.) With additional cutter for harbor duty.

(Harbor duty.)

New London, Conn. :

(Cruising grounds, from Nantucket shoals to Whitestone, N. Y.)

NEW YORK. N. Y.:

(Cruising grounds, from Nantucket shoals to the Delaware break- 
water.) With two additional cutters for harbor duty and one for duty in keeping vessels within anchorage limits.

Philadely iita, Pa.:

(Cruising grounds, from New York, N. Y., to Cape Hatteras.) With additional cutter for harbor duty.

Baltimore, MD. :

(Cruising grounds, Chesapeake Bay.) With additional cutter for harbor service.

Newbern, N. C. :

$$
\text { SOUTH OF CAl'E IIATTERAS. }
$$

(Cruising grounds, Pamlico and Albemarle sounds.)

Wilmington, N. C. :

(Cruising grounds, from Cape Hatteras to Jacksonville, Fla.)

Savanin, Ga.:

(Harbor duty.)

Key West, Fla.:

(Cruising grounds, from Key West, Fla., to Cape Canaveral.)

SAN JUAN, P. R. :

(Cruising grounds, waters of Porto Rico.)

Pensacola, Fla.:

(Harbor duty.)

Mobile, Ala. :

(Rebuilding.)

Gulfport, Miss. :

(Cruising grounds, from Mobile, Ala., to West End, Lake Poncliartrain.)

Galveston, Tex.:

(Cruising grounds, from Port Eads, La., to the mouth of the Rio Grande.) STATIONS ON THE GREAT LAKES.

Ogdensmurg, N. Y. :

(Cruising grounds, Lake Ontario and the St. Lawrence River to Ogdensburg, N. Y.)

Detroit, Mich. :

(Cruising grounds, Lakes Huron, St. Clair, and Erie.)

Sault Ste. Marie, Mich. :

(Cruising grounds, St. Marys River.)

Milivaukee, Wis. :

(Cruising grounds, Lake Michigan and Lake Superior.) 
STATIONS ON THE PACIFIC.

Honolulu, Hawait :

(Cruising grounds, general cruising on the Pacific.)

Sitka, Alaska:

(Cruising grounds, waters of southeastern Alaska.)

Port Toivnsend, Wash.:

(Cruising grounds, Puget Sound.)

Port Townsend, Wash.:

(Cruising grounds, general cruising on the Pacific.)

San Francisco, Cal. :

(Cruising grounds, general cruising on the Pacific.) Two cutters and one harbor duty.

In addition to the number of revenue cutters before named, six launches are utilized in the service and eight additional cutters are in process of construction at the moment of writing, while a former gunboat of the Navy, the Itasca, is being refitted for service as an instruction ship in place of the old sailing ship, the Chase.

Within these districts the revenue cutters are expected to make such cruises as may be necessary to assure a patrol of the coast against smuggling, and also to be ready at all times to afford assistance to the collector of the port in enforcing his orders, or to enforce the navigation laws, or to assist vessels in distress.

So far as possible the discipline of the revenue cutter is based on and follows that of the navy, and entrance to the service is secured in a similar manner; the requirements for entrance into the service keing given in the following paragraphs:

Appointments as cadets in the Revenue-Cutter Service are made after competitive examinations which are open to all young men who are citizens of the United States, unmarried, whose age is within the prescribed limits, and who produce satisfactory evidence of good moral character.

A person to be eligible for appointment as a cadet of the line must produce satisfactory evidence of good moral character, be not less than I 8 nor more than 24 years of age at the time of appointment, and must pass a satisfactory physical examination by a board of officers of the Public Health and Marine-Hospital Service, and a satisfactory educational examination, which must be written and strictly competitive, by a board of commissioned officers of the Revenue-Cutter Service. 
No person who has been dismissed or compelled to resign from the Military Academy or from the Naval Academy of the United States for hazing, or for any other improper conduct, is eligible for appointment as a cadet in the Revenue-Cutter Service.

Before being appointed a cadet of the line the candidate will be required to obligate himself to serve at least three years as an officer in the Service, after graduation, if his services be so long required.

Applications for permission to take the examination should be made to the Secretary of the Treasury. No form of application is prescribed, but the application should be in the landwriting of the candidate, and should state the late and place of his birth, the State of which he is a resident, and that he is unmarried. The application must be accompanied by testimonials sufficient to constitute satisfactory evidence of the good moral character of the applicant. The application should state what schools or colleges the candiclate has attended and in what occupations he has been engaged.

Examinations will be held in the city of Washington and in a number of other cities in different sections of the country, depending upon the location of the various candidates.

No expenses are allowed candidates who are permitted to appear for examination.

The physical examination which will. whenever practicable. precede the mental, is conducted by a board of officers of the Public Health and Marine Hospital Service and is thorough and rigid.

No one found physically disqualified will be examined further.

The mental examination, while largely elementary, is comprehensive and thorough and is designed to ascertain if the candidate has had the necessary training to enable him to successfully carry on his studies as a cadet at the School of Instruction of the Service. The passing average is 70 per cent., and any candidate obtaining a general average of less than 70 will be rejected. All papers of all candidates will be examined and rated hy a board of commissioned officers of the Revenue-Cutter Service. The examination is strictly competitive, and those candidates obtaining the highest averages will in all cases be recommended by the board for appointment. Fach question will be independently marked by each officer on the board, and the mean of the marks given by all the members of the board will be taken as the candilate's mark on that question.

The examination will take about four days.

The board will consider the general adaptability of the candidate for the service, and mark its opinion thereon on a scale of roo. This mark for adaptability will be given a weight of 5 in a total weight of 100 for the entire examination. In determining the adaptability of the candidate 
the board will carefully consider the testimonials submitted by him and also his bearing and general appearance as reported by the members of the subboard before whom he has appeared.

The salary of a cadet is $\$ 500$ per annum and one commuted ration per day.

Each successful candidate prior to being appointed a cadet will be required to deposit with the superintendent of the School of Instruction the sum of $\$ 150$ to defray the expense of his uniform outfit. The sum of \$ro per month is withheld from the pay of each cadet and placed to his credit toward defraying the cost of an outfit for a commissioned officer upon the completion of his cadet course.

Cadets of the line undergo a course of training for three years at the School of Instruction of the Revenue-Cutter Service, which is located near Baltimore, Md. After the successful completion of this course they are commissioned by the President to fill vacancies in the grade of third lientenant and have the rank and pay of second lieutenants in the Army and ensigns in the Navy.

The enlisted men of the revenue cutter service are enlisted for terms of three years each, although their enlistment may be terminated at any time by the Secretary of the Treasury. They may be enlisted by the commanding or executive officer of revenue cutters and must be at least 14 years of age and not more than 45 years of age. Enlistments are not for any particular vessels, but are for the general service, and enlisted men may be transferred from one station to another. The above term of three years is a maximum and may be varied by the Secretary of the Treasury at his pleasure.

In the cutters on the Great Lakes enlistment is made with the proviso that the man may be discharged if the action desirable at the end of the navigation season.

Preference is given in enlistment to citizens of the United States or to persons who have declared their intention of becoming citizens of the United States. Enlisted men may be promoted in the discretion of the commanding officer and as vacancies occur to higher ratings, which are similar to those provided under the Navy Department.

When directed by the President, the Revenue Cutter Service is required to co-operate with the navy and would, in case of war, be assigned to duty with the naval force, being for the time being taken over from under the charge of the Secretary of the Treasury. 
The list heretofore given of the duties of the Revenue Cutter Service is sufficiently comprehensive, although it does not, perhaps, fully convey a realization of the important and arduous duties performed by these vessels. The work of the revenue cutter service especially in the arctic seas is of the most difficult and dangerous character, especially that which relates to the rescue and release of whalers who may be imprisoned in the ice in the far North. Within recent years also added important and delicate duties have been imposed upon the officers of the Revenue Cutter Service by reason of the increase of the population of Alaska, as well as of the duties connected with the protection of the sealing islands. The forays of poachers on these islands have to be constantly guarded against and in more than one instance armed conflicts have occurred. Only within the last few years has there come to the knowledge of civilization the awful conditions obtaining in the northern seas which are beyond the reach of the usual processes of justice, and here men engaged in whaling and sealing have been under no restraint of law, and terrible cruelties have been perpetrated on seamen and upon unoffending natives. During the past three years cruises have been made by a revenue cutter bearing officers of the Federal Government, who are a part of the judicial system of Alaska. These cutters have gone among whalers in the North, and the officials have received complaints and investigated conditions, preliminary hearings are held and the offenders either fined on the spot or in the more serious cases held and transported to San Francisco or some other port for trial before the United States authorities. As the result of this work it is believed that the reign of law and order has begun in the far North, and that a material decrease in the prevalence of crime has been secured. At the port of New York and other principal ports revenue cutters are utilized by the cnstom-house anthorities for preliminary boarding on incoming vessels, but at most of the other ports the work of the cutter with regard to the custom house is not extensive. When revenue cutters are used as boarding vessels the masters must make a weekly report of work done to the collector. The lack of more than moderate emplorment in customs work is due to the almost entive disappearance of anything like smuggling along the coast. For 
reasons hitherto suggested, this form of smuggling is practically obsolete. Shunld, however, any vessel violate any of the laws of the customs or the navigation laws, the revenue cutters are of sufficient speed and have a sufficient armament to enforce respect, if necessary, by the partial or total destruction of the offending vessel. To this end the military organization of the service is kept up. All of the cutters are armed with modern guns, and the Revenue Cutter Service is regarded as a military branch, notwithstanding that its functions have almost entirely to do with civilian matters.

The pay of the officers of the Revenue Cutter Service is at the following rates while on duty:

Captains, twenty-five hundred dollars a year each.

First Lieutenants and Chief Engineers, eighteen hundred dollars a year each.

Second Lieutenants and First Assistant Engineers, twelve hundred dollars a year each.

Third Lieutenants and Second Assistant Engineers, twelve hundred dollars a year each.

And at the following rates while on leave of absence or while waiting orders:

Captains, eighteen hundred dollars a year each.

First Lieutenants and Chief Engineers, fifteen hundred dollars a year each.

Second Lieutenants and First Assistant Engineers, twelve hundréd dollars a year each.

Third Lieutenants and Second Assistant Engineers, nine hundred dollars a year each.

The pay of petty officers and seamen cannot exceed the average wages paid for like services on the Atlantic or Pacific coasts, respectively, in the merchant service.

The commissioned officers of the Revenue Cutter Service are known as officers of the line, engineer officers and constructors.

The commissioned officers of the line are as follows, and take rank and exercise command in the order mentioned:

Captain,

First Lieutenant,

Second Lieutenant, 
Third Lieutenant.

Cadets of the line are officers with rank next below third lieutenant.

The commissioned officers other than of the line are as follows, and they take rank and exercise command in their respective departments in the order mentioned:

Engineer-in-Clicf,

Chief Engineer,

Constructor,

First Assistant Engineer,

Second Assistant Engineer.

Cadet Enginecrs are officers with rank next below second assistant engineer.

The warrant officers of the Revenue Cutter are as follows, and take precedence in the order named:

Master's mate. (Special warrant for harbor service.)

Boatswain.

Gunner.

Carpenter.

Machinist.

The warrant officers are appointed by the Secretary of the Treasury.

The grades of boatswain, gunner, carpenter, and machinist, respectivèly, are filled by rating an enlisted man of the crew, or by enlisting a man specially qualified, as acting boatswain, acting gunner, etc. In any case, the man selected must fill the position for a term of not more than three months, which is a period of probation.

The petty officers and other cnlisted persons in the Revenue Cutter Service take precedence according to the following classification:

I. Petty officers of the first class... $\left\{\begin{array}{l}\text { I. Master at arms. } \\ \text { 2. Electrician. } \\ \text { 3. Ship's writer. } \\ \text { 4. Signal quartermaster. }\end{array}\right.$ 


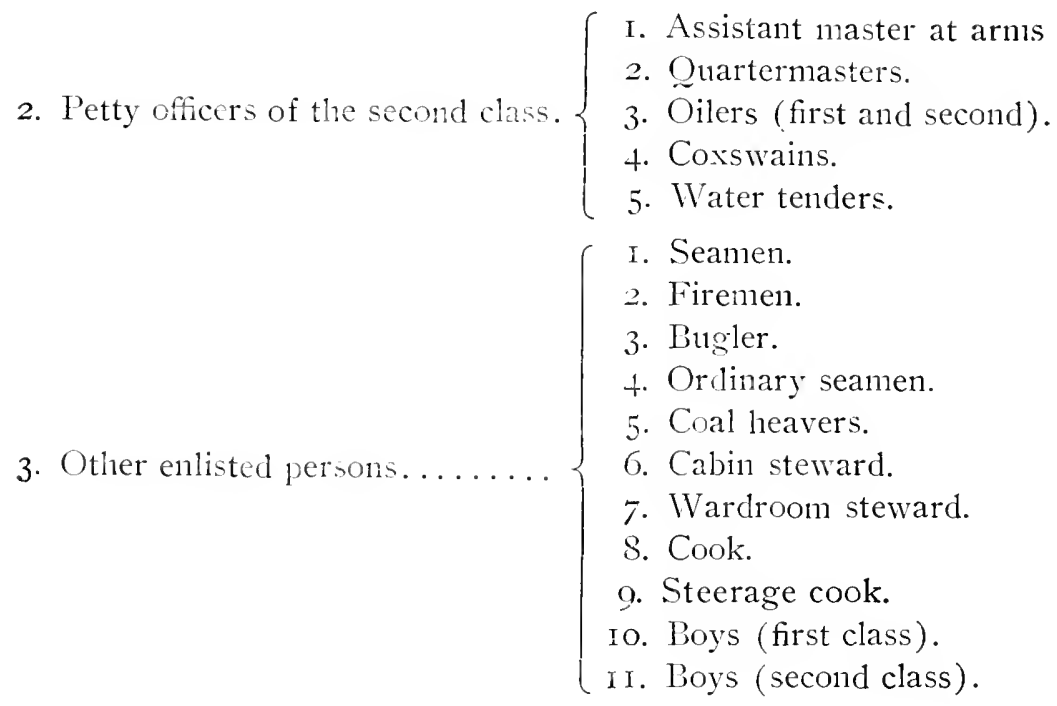

The commissioned officers of the Revenue Cutter Service have rank with officers of the Army and Navy as follows:

Captains with majors in the Army and lientenant-commanders in the Navy; first lieutenants with captains in the Army and lieutenants in the Navy; second lieutenants with first lieutenants in the Army and lieutenants (junior grade) in the Navy; third lieutenants with second lientenants in the Army and ensigns in the Navy. Whenever the forces of the Navy and of the Revenue Cutter Service serve in co-operation, in accordance with the provisions of section 2757 of the Revised Statutes, officers of the Revenue Cutter Service shall rank with and next after the officers of the Navy with whom they respectively hold rank.

The uniform regulations of the commissioned officers of the Revenue Cutter Service are as follows:

Full dress-Frock coat, full-dress trousers, chapeau, epaulets, sword and full-dress belt, white gloves, medals and badges.

Dress-Frock coat, plain blue or white trousers, chapeau, epaulets, sword and black leather belt, white gloves, medals and badges.

Undress-Frock coat, plain blue or white trousers, blue or white cap, shoulder straps, sword and black leather belt, white gloves. 
Service dress-Blue or white service coat, plain blue or white trousers, blue or white cap. (Sword and black leather belt and white gloves, when prescribed. The white service coat shall not be worn with blue trousers. The white cap shall be worn with white trousers, and may be worn with blue trousers. White shoes shall be worn with white trousers.)

Evcning dress (.1)-Evening dress coat, evening dress waistcoat, full-dress trousers, chapeaut, epaulets, sword and full-dress belt, black tic, white gloves.

Evening dress $(B)$-Evening dress coat, evening dress waistcoat, plain blue trousers, blue cap, black tie.

\section{TIIE LIFE-SAVING SERVICE.}

The close connection of the life-saving service with the revenutecutter service explains why the former has been retained in the Treasury Department when other bureaus or divisions having to do with the merchant marine have been transferred to the Department of Conmerce and Labor.

Strictly speaking, a life-saving service is a humanitarian and philanthropic undertaking, but it is one which was established by the United States in connection with other means for the encouragement and promotion of the shipping interests of the United States, as well as upon the general ground of humanity and care for those who could not otherwise be effectively assisted.

The wrecking of a ship is regarded as a disaster which imposes upon many innocent persons loss of property and danger to life, and against which they lave but feeble means of safeguard. It is therefore regarded by civilized nations as but just that every precaution should be taken at the national expense and every means afforded for the rescue of persons who find themselves in such situations.

The coast of the United States is divided therefore into the following districts, to each of which is assigned a district superintendent:

The first district, embracing the coasts of Maine and New Hampshire.

The second district, embracing the coast of Massachusetts. 
The third district, embracing the coasts of Rhode Island and Fishers Island.

The fourth district, embracing the coast of Long Island.

The fifth district, embracing the coast of New Jersey.

The sixth district, embracing the coast between Delaware and Chesapeake bays.

The seventh district, embracing the coast between Chesapeake Bay and the northern boundary of South Carolina.

The eighth district, embracing the coasts of South Carolina and Georgia and the eastern coast of Florida.

The ninth district, embracing the coast of the United States bordering on the Gulf of Mexico.

The tenth district, embracing the coasts of Lakes Ontario and Erie, and a station at Louisville, $\mathrm{Ky}$.

The eleventh district, embracing the coasts of Lakes Huron and Superior.

The twelfth district, embracing the coast of Lake Michigan.

The thirteenth district, enbracing the coasts of California, Oregon and Washington.

The whole service is in charge of a superintendent of the lifesaving service, who is located at the Treasury Department in Washington. He must be a person familiar with the apparatus used and the best methods in vogue in saving life from shipwrecks, and he receives a statutory salary of $\$ 4.000$, the present incumbent receiving $\$ 500$ additional. The General Superintendent is appointed by the President by and with the advice and consent of the Senate, and is assisted by an Assistant General Superintendent.

A supervisory board is provided by law, consisting of an inspector and assistant inspectors, who are detailed from officers of the revenue cutter service, each inspector being a captain in that service. The inspector must see that all districts in the life-saving service are properly inspected at least once a year either by himself or by the assistant inspectors, who may be officers of the revenue cutter service especially detailed for the work, or the commanding officers of the cutters stationed in the various districts. District superintendents must not be less than 25 nor more than 55 years of age when appointed, and must be familiar with the coast of the district in 
which the appointment is to be made. They are selected after a competitive examination, in which all keepers of life-saving stations in the district are invited to participate. Selection is made by the General Superintendent of the life-saving service, who selects one of the three highest from the results of the examination. In case less than three of the district keepers pass the examination, another examination may be held, to which keepers from an adjoining district may be invited.

Assistant District Superintendents may be appointed when required, and must have the same qualifications and be selected in a similar manner as the District Superintenclents. The District Superintendents are held responsible for the proper performance of the duties of the men in the districts; must oversee such construction as may be carried on; act as disbursing officers and paymasters of the districts, in which capacity they are compelled to file a bond, and also are required to make the necessary returns to the General Superintendent of the affairs of the district.

The keepers of the stations established within the various districts are appointed by the Secretary of the Treasury upon the general recommendation of the General Superintendent. Whenever a vacancy exists, the District Supcrintendent and the Assistant Inspector resident in the district, if there is one, make a joint recommendation of some member of a crew deemed best fitted for the position. Unless the General Superintendent has some objection to the person recommended for the position of keeper, he will further recommend the name to the Secretary of the Treasury; but if he objects, on stating his objections in writing to the Secretary of the Treasury, the Superintendent and Inspector must make another recommendation. Where there is no resident assistant Inspector, the District Superintendent recommends for appointment as keeper to have full charge of the life-saving station under the direction of the District Superintendent, and of the surfmen employed during the season.

Keepers of life-saving stations are also inspectors of customs, and when merchandise presumably of foreign origin is cast ashore within the limits of their jurisdiction, they must notify the nearest collector of customs of the fact; the keeper must take an account of 
any articles which may be removed from the wreck or from the shore and forwarded to their destination, to preserve them on account of their perishable nature and must require the making out and signing of manifests in duplicate if such removal begins before the arrival of a customs officer.

Keepers of life-saving staticns and the men under their control are required also to act as coast guards to prevent smuggling, and are authorized to seize such goods which are being smuggled, or have been snuggled, and hold them for the action of the collector of customs, for which action the attaches of life-saving stations may receive such compensation as the Treasury Department may award, not exceeding one-half of the net proceeds from the sale of such goods.

The position of keeper of a life-saving station is an annual one, in distinction from that of surfman, which continues only through the active season. Generally the latter includes only the winter and spring months, although on some coasts it has been extended to include certain periods of summer when storms prevail. The keeper must see that the patrol of the coast of his district is carried on at all times during the active season, and when the season closes is required to take measures for the preservation of property during the inactive season. He is also required in the inactive season to be on the alert for wrecks and, in case of such disaster, to collect the surfmen as soon as possible, allowance being made to the surfmen on any occasion on which they are required to act during the time in which the life-saving station is closed. Keepers over 55 years of age are compelled to undergo an annual physical examination to determine their fitness for duty.

Surfmen are employed on examination and certification by the Civil-Service Commission upon the following requirements:

Applications for examination shall be made upon the blank forms of application furnished by the Commission, which may be obtained from the superintendent of any life-saving district, the keeper of any life-saving station, or any officer of the Life-Saving Service.

For the purpose of establishing registers of eligibles for surfmen to meet the demands of the service, the coasts whereon life-saving stations are located are divided into sections, as follows: 
First district-Section I, coast of Maine; section 2, coast of New Hampshire. Applications will be received from persons residing on the coast of New Ilampshire south of Iampton River for employment in section I of the second district.

Second district-Section 1, from the northern boundary of Massachusetts to liarnstable Bay, except City Point Station; section 2, from Barnstable Bay to the eastern boundary of Rhode Island: section 3, City l'oint Station. The City Point Station consists of a vessel anchored in the harbor of Boston, and the service there requires experienced saibrs rather than surfmen.

Third district-Section I, coast of Rhode Island (except Block Island) and Fishers Island, N. Y., section 2, coast of Block Island.

Fourth district-Section I, from Montank Point to and including Quogue Station; section 2, from Ougue to Fire Island Inlet; section 3, from Fire Island Inlet to entrance to New York Bay.

Fifth district-Section I, from Sandy ITook to Barnegat Inlet; section 2, from Barnegat Inlet to Absccon Inlet; section 3, from Absecon Inlet to Delaware Bay.

Sirth district-Section I, from Delaware Pay to the northern boundary of Maryland; section 2, from the northern boundary of Maryland to the northern boundary of Yirginia: section 3. from the northern boundary of Virginia to entrance to Chesapeake Bay.

Scechth district-Section I, from entrance to Chesapeake Ray to the northern boumlary of North Carolina: section 2, from the northern bounlary of North Carolina to New Inlet: section 3, from New Inlet to Ocracoke Inlet; section 4. from Ocracoke Inlet to the northern boundary of South Carolina.

Eighth district-Section 1 , coast of South Carolina: section 2, coast of Georgia : section 3, east coast of Florida.

Ninth district-Section $\mathrm{I}$, west and south coasts of Florida; section 2, coast of Texas.

Tenth district-Section I, United States coasts of Lakes Ontario and Erie; section 2, Louisville, Ky. (To this district is added section 2, to make provision for the Lonisville Station at the Falls of the Ohio River.)

Elerenth district-Section I, United States coast of Lake Huron; section 2, United States coast of Lake Superior. 
Twolfth district-Section I, east coast of Lake Michigan; section 2, west coast of Lake Michigan, except the Evanston Station; section 3. Evanston Station. In filling vacancies in the position of surfman at the Evanston Station preference will be given to students of the Northwestern University or to eligibles from any section in the district desiring or intending to become students at said university, such fact being indicated in their applications, and evidence being presented that they will be admitted as students in said university.

Thirteenth district-Section I, coast of Washington; section 2, coast of Oregon; section 3, coast of California; section 4, coast of Alaska.

Applications for examination may be filed at any time, and must be filed with the keepers of stations nearest the applicants' places of residence. The applications must be forwarded by the keepers, through the district superintendent, to the General Superintendent of the Life-Saving Service for transmission to the Commission for examination.

Each applicant shall furnish two vouchers as to his experience as a surfman, sailor, or boatman, from persons by whom or with whom he has been employed as such. The term "surfman" includes not only a person who has had experience as such in the Life-Saving Service, but also a person who has not been employed in that service but has had experience in handling boats as a surf fisherman or as a wrecker. He must also furnish a certificate of his physical condition.

An applicant must be: $(a)$ a citizen of the United States; $(b)$ not under is nor over 45 years of age; $(c)$ not less than 5 feet 6 inches in height; $(d)$ not less than 135 nor more than 205 pounds in weight; $(c)$ must reside in the district in which he seeks employment; $(f)$ must be able to read and write the English language; $(g)$ must be a good swimmer.

No person can be examined who has not had at least three years' experience as surfman, sailor, or boatman; no credit will be allowed for experience obtained under the age of 15 years; but if there are no eligibles in and for the section where the vacancy exists, and certification can not be made from any other section because there are insufficient eligibles to make a certification for the 
existing vacancy, applicants having had less than three years' experience or no experience at all may be examined.

During the active season surfmen resicle at the life-saving station and in turns patrol the beaches and coasts within the subdistricts, carrying with them at night the necessary supply of rockets to make signals to the station should a vessel in distress be discovered. They are expecterl to afford, as a crew, such assistance as a vesscl may need whether she be stranded or not, to remove the persons on board if she can proceed no further, and to lend their assistance to bring her to a point of shelter should that be possible. In addition to their patrol work, they are required to engage in drills with the lifeboats and life-saving apparatus, and the scrvice has, uncler the close inspection and direction of the officers of the Revenue Cutter Service, reached a high state of efficiency, with results in practice and in actual work which are most creditable to those having the direction and instruction of the persomnel of the life-saving service.

Life-saving medals are authorized by law with other rewards for persons in the life-saving service who have performed meritorious action, and these are rewarded by the General Superintendent upon evidence submitted to him as to the character of the act performed.

In aldition to the foregoing officers, there is in the life-saving service a board on life-saving appliances consisting of seven members, the president of which is a citizen holding no other official position, but who is qualified to pass judgment upon devices and propositions likely to come before the board. All new inventions for saving life and improvements in methods now in vogue are presented to this board and passed upon before being installed as a portion of the life-saving service.

In addition to the inspectors detailed from the Revenue Cutter Service, two superintendents of construction for life-saving stations for the Atlantic and Lake Coasts and two for the Pacific Coast are designated by the Secretary of the Treasury from officers of the Revenue Cutter Service of the rank of captain, who superintend the preparation of plans and specifications for new stations, repairs to old ones, and also under the direction of the General 
Superintendent, select and purchase the outfits for new stations, as well as renewals, and take such measures as may be necessary to carry into effect the finding board on life-saving appliances.

Wherever practicable life-saving stations are equipped with telephone service, either in connection with commercial systems or by lines constructed by the government. The life-saving service includes a superintendent of telephone lines and a force of linemen for the necessary work of telephone construction and maintenance. The system is made an auxiliary to the Weather Bureau communication, and it is possible for private messages to be sent over these lines from isolated points when government business permits.

PUBLiC HEALTH AND MARINE hOSPITAL SERVice.

As originally constituted, the list of commissioned officers of the Revenue Cutter Service included surgeons. These, however, have been placed under a semi-allied bureau or division, and retain their military titles and organization although in effect a body of purely civilian functionaries.

The primary object of the Public Health and Marine Hospital Service was the conduct of the hospitals established by the Government for the care of seamen of the merchant marine. The nary has its own medical corps and hospitals and the marine hospital service is intended solely for the medical and surgical care of sailors on private vessels and of the officers and men of the vessels of the United States not attached to the navy.

The Surgeon General of the service is appointed by the President, by and with the advice and consent of the Senate, and receives a salary of $\$ 5,000$ a year. $\mathrm{He}$ is charged with the supervision, under the direction of the Secretary of the Treasury, of all matters connected with the disbursements of the Marine Hospital Fund, and with the direction of the other activities of the service hereafter detailed. His location is in Washington, where he is assisted by several Assistant Surgcon Generals.

The fund from which marine hospitals for the care of merchant sailors was established was originally inaugurated through the appropriation to this purpose of certain fees paid by merchant vessels, together with contributions deducted from the pay of 
seamen of the merchant marine of the United States. The latter deduction has, however, been abolished for many years, for the reason that the funds available for the service were in excess of the demands made upon it. Marine hospitals, under the command of commissioned officers of the United States Marine Hospital Service, have been established at the chief seaports, as well as at convenient points upon the lakes and rivers of the United States.

Upon application of any master or seaman of a mercliant vessel, medical aid will be afforded him by a commissioned officer of the Marine Hospital Service, or, if such officer is not available, an application is made to the Collector of the Port, who gives the applicant an order on a local physician for the necessary examination and treatment, if that is all that is required. If hospital treatment is necessary, the person can be transported to the nearest hospital and will there be treated during the period of his sickness and convalescence and until fit to resume his dutics. Forcign seamen may be examined and treated upon the application to the consul of the nation to which he belongs, or the authorized agent of a foreign steamship line. A fce of $\$_{I}$ is charged for examination of foreign scamen, and further treatment must also be paid for by them, although the treatment of seamen of the American merchant marine is entirely gratuitous and is paid for from the marine hospital fund.

For the purposes connected with the hospitals, in addition to the commissioned officers of the marine hospital service, internes, pharmacists and attendants are provided, who are under semi-military regulations, while grades of pilots, marine engineers and attendants are provided for service in connection with the United States vessels utilized by the marine hospital service in the quarantine dutics hereinafter described. In addition to the regular commissioned officers of the service, acting assistant surgeons are appointed at different points where the service required is not sufficient to warrant the detail of a regular commissioned officer. Candidates for these appointments must be competent physicians and surgcons of good moral and professional standing, and must be graduates of a reputable medical college. Acting assistant surgeons, whose compensation is $\$ 300$ per year or less, 
are appointed by the Secretary of the Treasury, upon the recommendation of the surgeon general, and where their compensation exceeds $\$ 300$, and preliminary to this appintment, they must pass a successful examination under rules prescribed by the United States Civil Service Commission.

Although the matter of quarantine regulations, designed to prevent the introduction of disease into any given part of the United States, was originally and still is a state, rather than a national function, it has been found to be necessary, especially at the larger seaports and those subject to visits from vessels sailing from ports of frequent infection, to establish quarantine stations under national quarantine laws. These stations are in addition to state provisions of a similar kind, and the national officers are required to observe, as far as possible, the local health requirements. It has been recommended, and will probably be the fact at some time, that all activities as to quarantine will be under national auspices. This, however, is not at present true, and it has, on occasions, required the most careful and delicate handling to prevent a conflict between the national and the State authorities.

The functions of officers of the Public Health Service with regard to quarantines depend on an act passed in 1893 , giving power to the Secretary of the Treasury to formulate regulations designed to prevent the introduction of cholera, yellow fever, smallpox, typhus fever, leprosy and plague into the United States. Vessels coming from foreign ports must have a bill of health granted by the consul with the advice of a medical officer of the United States, should one have been stationed at the place of the consulate, giving the exact condition of the health of the port from which the vessel sailed and the surrounding country, together with a history of the health of the crew. In addition to the general bill of health, when a vessel carries steerage passengers, each of such passengers is furnished with an inspection card to facilitate the inspection at quarantine. The medical officers at foreign infected ports are required to enforce certain sanitary regulations, but every vessel subject to quarantine by reason of having come from ports against which quarantine regulations must be enforced, is considered in quarantine from the time it enters a port of the United States until it is released. As 
soon as practicable, arriving vessels are examined and, unless it is shown that there can be no possibility of infection, the vessels are detained at or near the quarantine station until they can be thoroughly disinfected, a similar process being carricd on with reference to the clothing and baggage of the passengers, and until such time has elapsed as may make it certain that no disease will be developed. The time of detention varies with different diseases, but is that which has been shown by experience to be the limit beyond which no case of infectious disease in question will be likely to appear. The disinfection of the vessels is carricd on by stcam. cither under pressure or in a vacuum, or with one of the chemical disinfectants such as sulphur dioxide or formaldehyde gas. In addition to the general inspection for suspected sources of infectious disease, officers of the Public Health and Marine Hospital Service examine all immigrants, and those afflicted with diseases of the eyes or other diseases contemplated by law as constituting a disability, are rejected and must be deported from the United States.

In addition to the foregoing duties, the Public Health and Marine Hospital Service has charge of the work of collecting and disseminating information as to the general conditions of health of the country. and in cases of serious epidemics, is detailed to assist the local authorities in their efforts to quarantine the imfected district so as to prevent the spread of the disease. The principal activities of the service in this line have been in connection with yellow fever. It has taken a prominent part in the investigations of the cause of this disease and the means for its prevention, and while, as has been said, the service is necessarily circumscribed in its activity by the fact that the functions its represents are among those reserved to the States, under the provisions of the Constitution, there is a growing ficld for its duties in the co-ordination of the work of the various health authorities in the United States, and in investigation as to the best methods of preventing the spread of contagious diseases.

The order of rank and precedence in the Marine Hospital Service is as follows:

Surgeon General.

Assistant surgeon general, accorling to seniority of commission. 
Surgeon, according to seniority of commission.

Passed assistant surgeon, according to seniority of commission.

Assistant surgeon, according to seniority of commission.

Chiefs of divisions Hygienic Laboratory, according to seniority of appointment.

Sanitary inspector, according to seniority of appointment.

Acting assistant surgeon, according to seniority of appointment.

Interne, according to seniority of appointment.

Pharmacist, first class, according to seniority of appointment.

Pharmacist, second class, according to seniority of appointment

Plarmacist, third class, according to seniority of appointment.

Pilots.

Marine engineers.

Commissioned officers of the Public Health and Marine Hospital Service rank relatively with and after commissioned officers of the Revenue Cutter Service, as follows: Surgeon, with captain; passed assistant surgeon, with first lieutenant; assistant surgeon, with second lieutenant.

The official seal of the service is circular in form, bearing the words, "Public Health and Marine Hospital Service, U. S."" and stars, surrounding a foul anchor and caduceus crossed as shown in the subjoined engraving.

Graduates of medicine, desirous of undergoing examination for the position of assistant surgeon in the Public Health and Marine Hospital Service of the United States, must make an application, addressed to the Surgeon General, in their own handwriting, requesting permission to appear before the board of examiners. Applicants for examination should state their age, date and place of birth, present legal residence, and whether they are citizens of the United States, and name of medical school and college of which they are graduates, and furnish testimonials from at least two persons as to their professional and moral character. Applicants of foreign birth must furnish proof of American citizenship.

No applicant will be eligible to appear before a board of examiners whose age is less than 22 or more than 30 years, and, as a preliminary to a recommendation by the Surgeon General for appointment as assistant surgeon, the applicant must have been 
graduated in medicine at some respectable medical college, and must have passed a satisfactory physical, academic, and professional examination before a board of commissioned officers.

The compensation of commissioned officers is fixed at a uniform annual rate for each rank, as follows: Assistant surgeons general shall receive $\$ 2,900$ per annum; surgeons shall receive $\$ 2,500$ per annum; passed assistant surgeons shall receive $\$ 2,000$ per annum; assistant surgeons shall receive $\$ \mathrm{I}, 600$ per annum; the officer in charge of the miscellaneous division of the bureau shall receive $\$ 2,000$ per annum; and after five years' service an additional compensation of Io per cent. on the annual salary for each five years' service shall be allowed commissioned officers above the rank of assistant surgeon, but the maximum rate shall in no case exceed 40 per cent. Officers placed on waiting orders for a period longer than two months, while so placed, receive 75 per cent. of the pay and increase received by said officer at the date upon which he was placed on waiting orders. Allowance for quarters is also macle to all commissioned officers.

The compensation of pharmacists is at the following annual rates, viz.: Pharmacists of the first class, $\$ 1,000$ per annum; pharmacists of the second class, $\$ 800$ per annum; pharmacists of the third class, $\$ 700$ per annum, and after five years' service an additional compensation of 10 per cent. on the annual salary for each five years' service is allowed pharmacists above the class of pharmacists of the third class, but the maximum rate in no case to exceed 40 per cent.

THE TREASURER OF THE UNITED STATES.

The income of the United States having been collected, it becomes necessary to have a proper organization for the custody of the funds so secured. This, however, calls for something more than a mere safe-keeping of certain amounts of money, since it cannot be determined in advance what the expenses of the government will be, and to meet extraordinary cxpenses, it is necessary for the government to use its credit to secure additional amounts to place in the hands of the Treasurer, besides those which come from the subordinate collecting officers. When, therefore, the government de- 
parts from a strictly cash system of receiving and paying out its reventue, it enters upon considerations of financiering and finds itself required to either establish or superintend a currency system by which its credit may be expressed in terms. The details of the administration of such a system, however, are largely committed to the care of another officer than the Treasurer of the United States, although functions relative to certificates of indebtedness of the Federal government, as well as those relating to actual cash, are performed by the Treasurer.

The Treasurer of the United States is appointed by the President, by and with the advice and consent of the Senate, and receives a salary of $\$ 6,000$ a year. He is required, before entering upon his dutics, to give a bond in the sum of $\$ 150,000$, a provision for his own faithful performance of the duties of his office, and for the fidelity of those who may be employed by him. In addition to an assistant treasurer in Washington, and to a usual corps of assistants, provision is made for assistant treasurers of the United States in charge of independent or sub-treasuries; one each at Boston, New York, Philadelphia, Baltimore, New Orleans, St. Louis, San Francisco, Cincinnati and Chicago, with salaries of $\$ 4,500$, except at New York, which pays $\$ 8,000$, and Boston and Chicago, which pay $\$ 5,000$ each. These sub-treasuries act as banks of receipt and of payment, and as means for the transmission of currency from one section of the country to another, and, in the case of the New York Sub-Treasury, as the depot of deposit and withdrawal of gold and silver bullion used in international financial exchanges and for the purpose of settling balances of trade. The sub-treasuries carry on their operations in a semi-independent manner, being in effect, branch banks of the main Treasury at Washington. They have, however, no independent power of paying out money from the treasury except in accordance with the direction and authorization received from Washington.

The Treasurer of the United States is required to give receipts for all moneys received by him, which receipts must be endorsed by the Secretary of the Treasury, as without such approval, no receipt for money is lawful. For outgoing moneys it is necessary to have the signature not only of the Secretary of the Treasury, but of 
the Comptroller of the Treasury as well. All public moneys collected by the officers of the revenue and by the courts in the way of fines and costs accruing to the United States, must be deposited, without deduction, either in the Treasury, a sub-treasury or in one of the banks throughout the country which have been designated as depositorics by the Secretary of the Treasury. All proceeds of sales of articles belonging to the government must be paid into the Treasury, as must all other proceeds of a miscellaneous nature, and in every case the deposit must be of the gross sum received, unless there is authority of law for deducting the expenses of the sale or other methorls of realization of cash proceeds.

Every officer of the United States who desires to disburse money and who has given the appropriate bond to cover the said disbursement, will, upon the requisition of the head of the department under which he is employed, be furnished with the amount of moncy required, not in excess of the appropriation made by Congress or of the amount needed for the impending quarter, in accordance with a reasonable computation. Such requisitions would only be honored for a sum which might be needed by the disbursing officer within the accounting period for which they are drawn, and are usually honored by placing the amount to the credit of the officer, either in Washington or at one of the sub-treasuries or a depository, in accordance with the location of the officer who is to disburse the amount. The Treasurer of the United States, as well as all officers in custody of the public funds, are required to keep the same safely without loaning, using or depositing them in banks so as to make a profit upon the money, even if the original sum is not in any way trenched upon. The use of public money to secure interest or any valuable thing thercon is a grave misdemeanor and would subject the offender to punishment as well as liability to return of money so secured. All advances of public moneys are prohibited, but this does not apply to moneys which are issued to disbursing officers under the terms of the appropriations of Congress, while the President is authorized to permit additional advances to disbursing officers when the public business requires in excess of the amounts which would be issued to them under the terms of the appropriation act, so that they may make emer- 
gency expenditures, and he is further authorized to allow advances to naval and military officers who may have been detailed to distant stations, where it will be difficult for them to secure their usual pay and emoluments and to reach which will require a considerable expenditure of ready money. Other officials cannot receive salaries in advance nor money for expenses, except comparatively small amounts from certain appropriations and at the discretion of the heads of the departments. As a rule, government officials are required to expend their own money for traveling and other expenses, securing a reimbursement through expense accounts, accompanied by proper vouchers. When the advance of private funds is, however, considerable, provision has been made by which an advance on account of the appropriation may be paid to such officers not otherwise disbursing officers, but these instances are comparatively few. In, for example, the cases of the United States district attorneys, the personal expenses of the attorney incident to the prosecution of the public business have to be borne by the private individual, who, thereafter, is required to await the slow process of governmental accounting before securing the return of his money.

Besides being the custodian of the precious metals coined into money by the United States, the Treasurer of the United States issues all paper currency which is based as to its value upon the gold and silver bullion held in reserve by the United States, this currency constituting a portion of the total circulating medium of the country. United States bank notes printed in the Bureau of Engraving and Printing receive their final identification in the office of the Treasurer of the United States, where the seal of the Treasury is printed upon all notes, which are thereupon ready for issue. When this currency is redeemed for any reason it is returned to the office of the Treasurer, who thereupon causes the notes to be examined and forwards them to the offices of the Secretary of the Treasury and of the Register of the Treasury. After this triple examination the notes which have been cancelled in the office of the Treasurer are destroyed by maceration or grinding with water in a machine in the basement of the Treasury Building, the operation being under the direction of a committee representing the Secretary, Treasurer and 
Register, and the ntmost precantion being taken to prevent the abstraction of any portion of the canceled notes. National bank notes, after having been in circulation, are also received in the office of the Treasurer of the United States. They are then assorted and their amount charged to the bank which issued them, the paper itself being separated, those fit for use being sent back to the bank and those which are badly damaged being destroyed under the same circumstances as treasury notes.

The chief sources of revenue of the United States are the duties on imports, postage, and the more or less direct internal revenue taxes. To these are added the anounts received from the sale of public lands or other public property, and the amounts imposed as fines and forfeitures for infraction of the statutes. It sometimes happens that all of these resources are not sufficient for the current expenses of a nation, or that important undertakings are inaugurated which shall be for the permanent benefit of the people and in the cost of which, it is generally agreed, future generations should share. There is a point, differing perhaps with different nations, beyond which it becomes difficult and costly to collect revenue, and when the limit of the returns from current taxation has been reached, it becomes necessary to pledge the future ability of the people to pay taxes for the purpose of raising money for the immediate necessities. Bonds or pledges that a percentage will be added to the tax rate sufficient to ultimatcly pay the funds thus advanced have been issued in the United States, largely in the payment of the cost of the Civil War; occasionaliy in order to make up a deficit caused by a too low estimate of the revenue-producing powers of a tariff on imports, and in some instances to provide funds to carry on the work of a tremendous public improvement, such as the building of the Panama Canal. While the preparation and issuance of such bonds for the United States is under the direction of the Register of the Treasury, the Treasurer receives the amounts accruing from the bond issues and conducts the payment of interest and of the principal when the bonds become due.

Bonds of the United States are in demand not only on account of their value as an investment, but on account of the privileges which they convey under the banking and revenue laws. It is cus- 
tomary to advertise a bond issue to be sold by allotment so that one person or combination shall not control the whole. In addition to the care of Federal funds, the Treasurer of the United States acts in a fiduciary capacity or as trustee of the several funds which are under the care of the United States. These include niscellaneous trust funds and the funds formed by the amounts returnable to Porto Rico and the Philippine Islands on account of duties paid on imports from those islands coming into the United States. He is also the trustee and custodian of the United States bonds deposited by national banks to secure their circulation. $\mathrm{He}$ is fiscal agent for the several bond issues of dependencies, including the land bond issue of the Philippine Islands and the Panama Canal bond issue. The Treasurer of the United States is also the regulator of the small change of the country. Under provisions of law the cost of transporting silver dollars and subsidiary coin between different parts of the country and for the convenience of persons who are supplied through the various banks, the cost of the transportation of such coin is paid by the United States, and the Treasurer of the United States has the direction of the supply of coin and is accustomed to make such shipments and transfers as may be necessary to prevent the accumulation of subsidiary silver coin in one location and a consequent scarcity in another.

\section{THE COMPTROLLER OF THE CURRENCY.}

The issue of bonds or evidence of indebtedness by the United States serves an additional purpose to that of providing money for the immediate necessities of the country. It has not been regarded as being sound national policy for the United States to issue currency or mediums of circulation in excess of the amount which could be paid upon demand from the reserve of gold and silver in the Treasury. While a conservative and carefully guarded undertaking of this character which should make the Treasury of the United States a gigantic bank of issue, and with outstanding notes which would be based upon the national credit, could be established, legally, it has been held that there was undue risk in the matter that in time of stress there would be a temptation to an over-issue of notes which would at some point result in the depreciation of the purchasing 
power of the notes from their face value and might bring about a disastrous condition of the national finances. For this reason it has been held to be safer that the money or circulating medium of the country should be based upon private assets, the security of the notes and the gentineness of the assets being guaranteed by the Lnited States. To this end the Lnited States offers to persons who will associate themselves as banking corporations and who purchase a given amount of the bonds of the United States permission to issue bank notes within tise limits of the total amount of the bonds thus purchased. The bonds issued to the banking associations are not delivered directly to them, but are held by the Treasurer of the United States as a trustee and subject to any demand which may be made upon the bank for the fulfilment of their promise to pay as evidenced by their outstanding bank notes. Of late years, as the demand for the privilege has increased and the issue of Lnited States bonds has not maintained a corresponding place, approved bonds other than those of the Lnited States have been accepted and are deposited in the Treasury as guarantees of national bank circulation by those who have the privilese of issuing national bank notes, a privilege which is made profitable to them by provisions of law which prevent the issuance of circulating notes by other banks, the notes of other than national banks being subject to a ten per cent. internal revenue tax. Interest paid by merchants, manufacturers and others for the use of currency affords to the national banks a return for the investment made in United States bonds and for the expense involved in keeping up the system and a profit on their banking operations. The national bank circulation of the United States, with the notes issuer by the United States itself on the basis of its actual possession of cash, enables the operations of commerce and manufacturing to be carried on convenicntly and a total volume of circulation is secured which would be impossible of attainment unless the nation itself should go into a system of credit currency which, as has heen said, has generally been deemed to be inadvisable.

The Comptroller of the Currency is the directive officer of this system and exercises powers and prerogatives not usual among the subordinate officers of departments. The terms of his appoint- 
ment differ altogether from those of the usual appointed officers, as he is appointed by the President, by and with the advice and consent of the Senate, for a term of five years. He can, however, be sooner removed by the President upon statement of the reasons for removal to the Senate. The salary of the Comptroller is $\$ 5,000$ a year. Within fifteen days of the time of notice of his appointment he is required to take and subscribe to the oath of office and to give the United States a bond in the sum of \$IOO,O0O conditioned for the faithful performance of his duties. While he is under the general direction of the Secretary of the Treasury, the Comptroller of the Currency has practically independent functions with regard to the national banking system. When a banking corporation has complied with the terms of the banking act, has secured the necessary amount of approved bonds, and deposited them with the Treasurer of the United States, the Comptroller of the Currency prepares appropriate plates for the printing of notes for the bank, and these, when signed by the bank officers, become legal tender in the United States. Although the validity of the outstanding notes of the national banks is secured by the amount of the deposited bonds in the United States Treasury, it would be possible for the bank officers to accumulate liabilities in other directions, so that the stockholders and depositors in the banks would be defrauded of the amounts they had invested or entrusted to the institution. All of the national bank notes are sent to the United States Treasury for redemption and in case a bank should fail to honor its circulating notes when they have become due, and are offered to be redeemed, the necessary amount to reimburse the United States for the redemption would be raised by the sale of the bonds in the Treasury as a deposit from the bank. This, however, gives no guarantee to the depositors and for the protection of these the national banking laws provide for the maintenance of an adequate reserve of gold or notes of the United States and for quarterly statements of the condition of national banks, severe penalties of fine or imprisonment being imposed for falsification with regard to these reports.

The Comptroller of the Currency has under his direction a force of bank examiners who examine the condition of banks at stated intervals, and also upon the appearance of an unsatisfactory state- 
ment, and who are paid by the banks themselves for making the examination. In adclition to this payment, a small tax is imposed upon the bank circulation for the purpose of carrying on the central means of redemption and return of bank notes to their points of origin. If it appears that a bank is insolvent, or its assets liave been seriously impaired, the Comptroller of the Currency is required to take charge of the bank's affairs and to appoint a receiver who is often the examiner who was charged with the duty of making an investigation into the condition of the institution. Accordingly as the condition of the bank may be hopeless or as financial assistance is afforded to it, the affairs of the bank are either liquidated and the bank finally closed or they are carried on by the receiver until such time as the bank is placed in a perfectly solvent condition, when it may be restored to the control of private officers.

The Comptroller of the Currency is required to make an annual statement to Congress of the condition of each banking association, together with facts relative to banks which have become involved in difficulties during the ycar and results of the liquidation of banks which have been closed. He is also required to secure from the States the necessary information which will cnable him to report to Congress the resources and liabilities of the banks and banking institutions which have been organized under State laws, but which have, under the national banking act, no power of issuing circulating notes.

TIIE REGISTER OF TIE TREASLRY.

The Register of the Treasury is appointed by the President, by and with the advice and consent of the Senate, and is entitled to a salary of $\$+, 200$ a year. He signs and issues all bonds of the ['nited States, and all bonds issued under national auspices. He transmits to the Treasurer of the United States a list of the registered bonds showing the names of persons who are entitled to receive interest thereon. Of the two classes of bonds issued by the United States, the unregistered are subject to the payment of interest upon the presentation of the interest coupon. Those which are registered, however, can only profit the persons whose names are registered at the Treasury as the proper payees of amounts becoming due. 
Transactions relative to the ownership in registered bonds must be made of record with the Register of the Treasury to whom proof of right to the bond must be submitted in order that the interest and principal may be collected.

In addition to these duties and the registration of United States bonds and securities when redeemed, the Register of the Treasury receives, examines and arranges all United States currency which has been received for redemption, registers the numbers of the notes, and performs the last operations with regard to them before they go to the macerator for destruction. He also examines and registers and arranges for destruction, in a similar manner for the destruction of national bank notes, and all customs, internal revenue and postage stamps which have been condemned for imperfection and ordered to be destroyed. When money is transferred from the United States Treasury to the sub-treasuries, or to order of the Secretary of the Treasury to the various depositories, the orders making the transfers are signed by the Register of the Treasury, who also, together with the Treasurer, signs all United States notes and paper currency.

\section{THE BUREAU OF ENGRAVING AND PRINTING.}

All of the bonds and notes of the United States, as well as the postage, cuistoms and revenue stamps, are produced at a manufactory maintained by the United States itself and which is the principal point of the employment of the process of engraving by hand on steel plate in the United States at the present time. The Bureau of Engraving and Printing, which is under the charge of a director appointed by the President at an annual salary of $\$ 4,500$ a year, engraves and prints all of the securities and evidences of indebtedness of the United States and the national bank notes, together with a large varicty of engraved matter, including pension certificates, bank certificates, commissions, disbursing officers' checks and the portraits of the chief members of Congress authorized by the two Houses for inclusion in the memorial books usually printed upon the death of a member. The bank notes of the United States and national bank notes are printed on distinctive paper into which silk threads are woven by a secret process and 
which is made at the government's factorics in Massachusetts under close governmental supervision. Every sheet of this paper nust be accounted for from the time it is manufactured until it has been issued by the Treasury or the national banks, and in the Bureau of Engraving and Printing if the count of sheets is insufficient at any time the doors are closed and the cmployes must wait until the lost sheets have been found or satisfactorily accounted for. This is also true as to the plates used in the printing even in the smallest details so that if a plate bearing the name of a bank and which could not be well used in counterfiting should be mislaid, there would be the same restrictions unless reasonable accountability as to its whereabouts became known.

TIE, DIRECTOR OF THE MINT.

One of the chief attributes of sovereignty is the power to coin money. This power was granted to the Congress by the States and is exercised under the direction of the Secretary of the Treasury by the Director of the Mint who is appointed by the President, by and with the advice and consent of the Senate, and holds his office for five years unless sooner removed by the President for reasons which nust be communicated to the Senate. The Director of the Mint receives an annual salary of $\$ 4,500$ and his necessary traveling expenses in visiting the different mints and assay offices. The Director of the Mint has his office in Washington, and his duties consist of the general supervision of all mints and assay offices. In adcition to an anmial report of the Secretary of the Treasury on the operations of these offices the Director of the Mint is charged with collecting and making an annual report of the statistics of the production of precious metals for each calendar year.

The Director of the Mint prescribes rules subject to the approval of the Secretary of the Treasury for the transaction of business at the mints and assaying offices and reccives daily reports of their operations. He directs the character of the coinage to be executed by chiefs of the mints and superintends the annual settlements of the affairs of those institutions. All appointments, approvals and transfers of employees at the mints and assay offices are subject to his approval. Besides the administrative force under his direction in 
Washington an assay office is maintained in which tests are made of the weights and fineness of coins struck in order to maintain the standard of the coinage. The relation of coins of other countries to the coins of the United States is in a general way defined by law. Specific relation, however, upon which the custom authorities base thcir calculations are made up by the Director of the Mint who publishes a quarterly estimate of the value of foreign coins in the coin of the United States.

The mints of the United States are located at Philadelphia, San Francisco, New Orleans, Carson and Denver. A principal assay office is located at New York, and there are other assay offices, the locations of which are changed from time to time according to the exhaustion of the source of precious metal in the mines in the vicinity of which assay offices have becn located for the convenience of the miners and when new deposits are discovered. The full number of officials of a mint includes a Superintendent, who is also the treasurer, the Assayer, the Melter and Refiner, and a Coiner. In the smaller mints the duties of some of these offices are merged. Each of the officers of a mint is required to file a bond, approved by the Secretary of the Treasury, in the sum of not less than ten nor more than fifty thousand dollars, and similar bonds may be required of the assistant and clerks in such sums as the Superintendent shall determine with the approbation of the Director of the Mint. The bonds of subordinates, howcver, do not relieve the principal officers under whom they serve from the liability of such officers to the United States in case of default of the subordinates.

The Superintendent of a mint has general superintendence of its operation, and the control and custody of the precious metals received and of the coinage manufactured. He is rofuired to report as required by the Director of the Mint in minute detail as to the gold and silver in his custody, with its condition with relation to the finished stage of coinage. The Superintendent is also required to furnish quarterly statements of his transactions with other officers of the mint and with the depositors, and monthly statements of the expenses. The Superintendent appoints all assistants and clerks and workmen, but no person can be appointed by him to employment in the offices of the Assayer, Melter, Refiner, Coiner, or Engraver, 
except on the recommentation and nomination in writing of those officers respectively. All appointments must be certified to the Director of the Mlint, and if disapproved by the officer, the appointments are vacated.

While the operations of a mint are purely those of a manufacturing establishment turning gold and silver into the form of coins as prescribed by law, the effect of the statute as prescribing the operations of the mints is of the utmost significance with relation to the general commercial conditions of the country. The present basis of all values in the commerce of the world is the purchasing power of a given amonnt of gold. In the United States this unit of value is the one dollar gold pice having a standard weight of 25.8 grains. The standard of fineness for this and other gold and silver coins of the United States is that in one rooo parts, by weight, of the metal, 900 shall be of the pure metal, and roo of alloy. The alloy of gold coins may he of colper or of copper and silver, thou th the silver in no case may be one-tenth of the whole alloy. The mixture of the less valuable metils with grold is not for the prrpose of affecting the value of the coins as circulating mediums, but simply in order to assure a sufficient hardness in quality to cnable the coins to stand the wear of passing from one hand to another without too great abrasion and loss of value. The standard of intrinsic value is fixed by the weight of the coin, and is that which corresponds as far as may be to the relation which gold bears to the various articles of purchase and sale the world over. It is, of course, an cutircly arbitrary assipmment of value to gold which loes not clrange automatically with the increase or decrease of the stock of gold in the possession of the nations of the world. From time to time the production of food. clothing, and other articles, increases by reason of improvements in machinery or by reason of the opening of new and fertile lands to the processes of agriculture so that the world's stock of gold is relatively small as compared with world's stock of goods. Under such circumstances gold appears to be scarcer, its comparative value increases and when there is more merchandise in commerce than the stock in gold can represent, prices fall; that is to say, it takes a larger amount of merchandise to secure representation by a given amount of gold. On the other hand, when 
new deposits of gold are developed, as in Alaska, considerable additions are made to the world's stock of the metal, and prices proportionately increase, while commercial operations are furnished with a sufficient amount of medium of exchange.

While there has never been a period in the history of the world when the supply of gold was so great as to produce an overplus of circulation based upon gold alone, there have been times when the increasing production of other commodities created a demand for a larger volume of circulating medium, so that in addition to gold, silver was used as a fixed basis of valuation, its intrinsic value, however, being fixed by its relation to gold. In a time of the moderate production of silver bullion it was found that upon a relation of sixteen ounces of silver to one of gold it was possible to add a considerable amount of silver to the world's stock of materials for commercial exchange, and silver coinage was carried on until increasing production of silver rendered the relation of sixteen to one an artificial proportion, which was destroyed by the market price of silver bullion regarded as a commodity purchaseable by gold. For this reason all attempts to utilize silver as a means of exchange, except in the form of subsidiary coinage have been abandoned by the United States, except as to a limited number of silver dollars coined from silver purchased in the open market, and which bears the same relation to the gold reserve of the country as do the United States notes based upon the same reserve; that is to say, the United States provides against the debasement of such number of silver dollars as may be in circulation by making them exchangeable at the will of the holder for the gold coinage, a similar guarantee maintaining the parity of the subsidiary coinage, so that the silver coins of the United States are only in a less degree as truly credit money as the paper currency itself.

Under the provision of the law, any person having gold in a free state may present the same to one of the mints or assay offices of the United States and receive for the same gold coin of a value equal to the assayed value of the bullion, less the charges for the service of assaying, meiting, refining and coining, which are fixed by the Director of the Mint, with the approval 
of the Secretary of the Treasury. Unrefined silver may also be presented for assay and refining on payment of the due charges, but there is no warrant for coining the same, and bullion is returned in the form of bars or ingots, mnless it shall be purchased at the current market price under the direction of the Secretary of the Treasury for the purpose of coining into subsidiary coins or into silver dollars. It was one of the issues in a national presidential campaign that a law should be passed restoring silver to the position it formerly held, namely, that of free and equal coinage with gold in the mints of the United States. The proposition was rejected in the campaign in which it was an issue, and the material increase in the world's stock of gold on account of production in Alaska and South Africa cnded, by the logic of cvents, the agitation upon which the issues in the campaign referred to were founded.

Upon the presentation of bullion to a mint it is taken in charge by the Superintendent who forwards a sample of the deposit to the assayer, who returns a report upon which the Superintendent calculates the value of the bullion deposited with him. The Superintendent then gives to the depositor a certificate of the amount leposited, whether layable in coin or in bars, the certificate being countersigned by the Assayer.

The Melter and Refiner carries on all the opcrations necessary, to transform the gold or silver as received from the depositor into bullion of standard fineness, and produces the bars and ingots, which are either to be delivered to the depositor or to the Coiner, for the purpose of being struck into money. He is responsible for all buliion delivered to him by the Superintendent, and must take care that the bars and ingots produced conform to the provisions of law as to funeness. The bullion produced by the Melter and Refiner is returned to the Superintendent, who issues it to the coiner, when necessary for the operation of producing a given amount of coinage, as directed by the Director of the Mint. The Coiner is also required to adhere strictly to the provisions of law, and to keep an accurate account and make returns of all bullion entrusted to him, such returns being in the form of coins and waste pieces, and the account must balance, 
due allowance being made for expenditure of the precious metals in the operations of coining.

The coins being prepared are delivered to the Superintendent of the mint, who receipts for the same and keeps a careful record of the kind, number and weight. It is his duty to try a number of single pieces separately, to determine whether they are within the legal limits, and if there is any doubt, is required to have all of the coins of the delivery represented weighed separately, so that the delivery, if found under weight, may, as a whole, or in such part as may be necessary, be returned to the stock of standard bullion in the mint for recoining. The Superintendent must also, in the presence of the Assayer, take not less than one piece for each thoutsand pieces of gold coin, or one piece for each two thousand pieces of silver coin, for the purpose of the annual test of coins. The pieces selected are sealed in an envelope, properly labeled, and reference made to the delivery from which they were taken. There is in each mint a box of special construction or pyx which is kept under the joint care of the Superintendent and Assayer, and so secured that neither can have access to its contents without the presence of the other. The reserved pieces are placed in this receptacle and transmitted quarterly to the mint at Philadelphia. On the second Wednesday in February, annually a commission consisting of the Judge of the District Court for the Eastern District of Pennsylvania, the Comptroller of the Currency, the Assayer of the assay office at New York, and such other persons as the President may add, meet at the mint in Philadelphia and examine and test the pieces of money which have been reserved by each of the mints throughont the year. If the coins do not deviate in standard of fineness and weight more than the deviation allowed by law the trial is to be considered and reported to the President as satisfactory. If, however, a greater deviation is found to exist in any piece, the fact of the deviation is to be certified to the President, and if the President, upon viewing the circumstances, so decides, the officers implicated in producing the error may be removed, and shall henceforth be disqualified from holding their office.

The Engraver for the mints is located at Philadelphia, and it 
is his duty to prepare from the dies authorized by law the working dies used in the coinage of the various pieces of money includer in the crinage of the Cuited States. No change in the design of a coin can be made oftener than once in twenty-five years, and no change at any time can be made in the diameter of any coin. If a change in the design of a coin is decided upon, the Director of the Nint has power. with the approval of the Secretary of the Treasury to engage the services of distinguished artists to produce a new design. Such an engagement was made within a short time with reference to producing new designs for the gold coins, the services being compensated from the contingent appropriation from the mint at Philadelphia. The first coins of the new design (by St. Gaudens) have, at the date of writing, just been struck.

It is in the discretion of the Superintendent of a mint to refuse a deposit of bullion of less value than \$Ioo or any bullion which is so base as to be unsuitable for the operations of the mint. Where gold and silver are combined, and either metal is in stich small proportion that it cannot be separated advantageously, no allowance is to be made to the depositor for the value of the subsidiary metal. The fitness of bullion to be received is determined by the Assayer, and the Melter and Refiner determine whether the value may be calculated on the condition of the bullion as it is received, or whether it must be first melted and refined before a value can be placed upon it. Although charges are made for operations relative to the transmission of metal deposited into bullion of standard fineness, no charge is made for converting gold bullion of standard fineness into coin. Before countersigning the certificate of the value of deposits of bullion the Assayer is required to verify the calculation of the Superintendent, which determines the amount of the credit.

There is as a consequence of the operation of turning silver, nickel and copper into subsidiary coinage, considerable profit, which accrues to the United States, being the difference between the market vaiue of the metal and the face value of the coin produced. This profit is credited to a special fund from which the wastage involved in the coinage is made up, and from which 
amounts are taken as necessary to defray the cost of transporting the minor coin to various points in the country, where it is placed in circulation. Besides the coins of the United States at the mints, provision is also made for striking medals and for the coinage of money for foreign nations, when the cost thereof is defrayed by payment by those for whom the work is done.

Balances of trade between the merchants of the United States and those of foreign countries are settled in actual gold and silver, representing, however, only a small part of the sum total of the amounts involved. For the settlement of these balances it is necessary to make considerable shipments, according to the seasons of the year, and also of the state of the market of the respective countries, and for this purpose gold and silver, especially gold in bars, are the necessary mediums of interchange.

Provision is made that upon deposit of gold coins of not less than a given amount, gold butlion may be received in return for shipments, and coin certificates which have been issued at the various mints to depositors, may be presented in lieu of the actual coin and gold bars or ingots secured for the purpose of shipment abroad. The bulk of these shipments are made from the port of New York, and provision is made for the transfer of gold in bars or ingots from the assay office in New York to the New York sub-treasury, for issue from the latter institution, while the transatlantic steamers ply out of tlat port are usually fitted with special strong rooms for the purpose of carrying the considerable amounts of gold involved to and fro.

Foreign coins which come into the possession of the United States, and whicn are valued in accordance with the quarterly statements of the Director of the Mint, must be recoined before they can be reissued. 
MIINT OFFICERS.

Location.

(TERM OF OFFICE, UNLIMITED.)

California-

San Francisco

Colorado-

Denver.... Office.

Bond.

Salary.

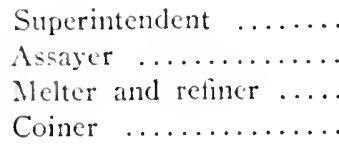

$\begin{array}{rr}\$ 100,000 & \$ 1,500 \\ 20,000 & 3,000 \\ 20,000 & 3,000 \\ 30,000 & 3,000\end{array}$

100,000

4,500

I0,000

3,000

Colorado-Cont'd-

Denver............... Melter and refiner .....

$\$ 20,000 \quad \$ 3,000$

Coiner ................

30,000

3,000

Louisiana-

New Orleans.......... Superintendent .........

$100,000 \quad 3.500$

Assayer $\ldots . . . . . . . . .$.

I0,000

2,500

Meiter and refiner ....

20,000

2,500

Coiner

20,000

2,500

Nerada-

Carson......

Assayer in charge .....

$\$ 25,000 \quad \$ 2,000$

Pennsyliania-

Philadelphia

Superintendent $\ldots . . .$.

100,000

4,500

Assayer ..............

I0,000

3,000

Melter and refiner.....

20,000

3,000

Coiner ............. .30,000

3,000

Engraver

4.000

Idaho-

Boise City

Assayer

$\$ 20,050$

$\$ 2,000$

Missouri-

St. Louis.

Assayer in charge ......

$20,0 \mathrm{co}$

2,000

Montana-

Helena

Assayer in charge ......

25.000

2,250

New York-

New York

Superintendent .........

50,000

4,500

Assayer .............

10,000

3,000

Melter and refiner....

20,000 3,000

North Carolina-

Charlottc.

Assayer and melter ....

$\$ 10,000$

$\$ 1.500$

South Dakota-

Deadwood. ...

Assayer in charge ......

20,000

2,000

Washington-

Seattle...

Assayer in charge

50,000

2,750 
THE SECRET SERVICE.

There is, of course, every temptation for persons to counterfeit both the coin and the bank notes of the United States, and of the national bank currency, and for the purpose of detecting such counterfeits and of tracing persons who may attempt to make or issue spurious coins and notes, there is a division in the Treasury Department closely allied to the office of the Treasurer, and to the divisions which have to do with the issue of money in tangible form, known as the Secret Service. It is under the direction of a chief, who receives $\$+, 000$ a year, and who has charge of the work of operatives who are scattered all over the United States, and who malie up the personnel of the chief detective burean of the government. The duties of the Secret Service operatives are primarily in the detection of counterfeiters and the prevention of counterfeiting. They are also utilized in detective work under other departments of the government, and especially are charged with the safety of the President when he leaves the White House. The personality of the operators and the methods of the work of the Bureau are, of course, kept a close sccret. and their services are secured for work under other departments by confidential communication to the Secretary of the Treasury. When, however, they are utilized in work not pertaining to the work of the Treasury Department, their salary and expenses are paid by the department for whom the serice is being performed.

\section{THE AUDITORS OF THE TREASURY.}

It is a fundamental principle of the payment of money from the Treasury of the United States, and a strict statutory provision that no money shall be so paid except in accordance with an appropriation made $5 y$ Congress. There can, therefore, be no payments made from the public funds unless, first, authority for such payment has been made either by specific direction of Congress naming the claim and the amount to be paid, or unless it comes within the terms of one of the general appropriation acts making provision for meeting expenses of the Government. The payments from the Treasury are of two general classes, those 
which have been decided to be due by the Accounting Officers of the Government and those which have been directly presented to Congress because no appropriation in its terms would warrant the auditing officers of the Treasury Department in taking a favorable action thercon. In this latter class of payments there are included the judgments rendered against the United States by the Federal Courts, together with claims for reimbursements and for injuries and damages received, which have been presented to Congress and have been favorably acted upon by that body. As has been stated, it is the practice to advance to disbursing officers, upon the requisitions of the several heads of the Departments such amounts as may be necessary to enable them to meet expenses promptly, and the majority of the disbursements of the Government are carried on through such officials. The payments thus made, however, are only conditional ones, and are subject to revision by the Treasury Department. If it is found that they have been illegally or improperly made, the disbursing officer must make good the difference, and if possible secure from the person to whom the payment was actually made a reimbursement to cover his loss. Evidences of the payments made by disbursing officers in the form of signed payrolls, receipts and other vouchers, are forwarded to the auditors for the different departments who are officials of the Treasury Department. In most cases a provision is made for an administrative or preliminary audit of the accounts in the department having jurisdiction orer the different disbursing officers, but the main disbursing officers of departments transmit their accounts to the auditors directly, together with the accounts of the sub-disbursing officers who are under their charge.

Officials of the Government who act as disbursing officers without bond use their own money, as they are compelled to do in most cases for the payment of personal expenses incurred. Their accounts may either be paid by disbursing officers of the department under which they act, or may be forwarded to the Treasury Department for payment by check on the United States Treasury. In all cases, however, all accounts, before they are finally closed or settled, must be scrutinized by officials of the 
Treasury Department. 'This work in the Treasury Department is performed under the direction of six Auditors, known, respectively, as the Auditor for the Treasury Department, Auditor for the War Department, Auditor for the Interior Department, Auditor for the Navy Department, Auditor for the State and Other Departments, and Auditor for the Post Office Department. The names of these officials are sufficiently descriptive, the Auditor for the State and Other Departments auditing the accounts of all the departments except those for whom an auditor is specifically named. The Auditors are appointed by the President, by and with the advice and consent of the Senate, and receive $\$ 4,000$ each per annum. It is provided by law, in order that there shall not be excessive delays in the settling of accounts that the necessary documents must be forwarded to the department within a limited time, usually twenty days after the close of a quarter, and that the administrative audits must be completed and the accounts forwarded to the Treasury Department within a further limited time, running from forty to eighty days.

The accounts when received by the auditors are subjected to a most careful scrutiny and comparison with payment for similar services or articles made in previous quarters, and upon the account being found correct, it is certified to the division of bookkeeping and warrants of the Treasury Department for credit to the account of the disbursing officer or for the preparation of a warrant, which, when signed by the Secretary of the Treasury, and countersigned by the Comptroller of the Treasury, becomes due and payable in cash, or by a check on the Treasury of the United States. In order to receive such an approval, however, the account must have been paid under warrant of law, evidenced by its inclusion within the terms of an appropriation act or the special provision of Congress, and must come, as to its details, within the regulations of the department under which payment was made, and of the regulations of the Treasury Department for the payment of money. The quantities of the goods must be certified as having been received, and the prices therefor must be in accordance with contracts made, or if purchased in the open market must have been purchased 
under the regulations for such purchases, and must not be in excess of the usual commercial prices. Transportation must have been charged at the tariff published by the railroad, and accounts for personal services and expenses must be verified by the affidavit of the person to whom the payment is to be made, and must bear the approval of his supcrior, and the certification of the latter that the services werc rendered and the expenses incurred in connection with official business.

When items in accounts do not in every way fulfil the requirements of the various regulations payment on them is suspended and the disbursing officer is notified of the fact and allowed to submit any statement or explanation which may clear the matter up. Upon this statement, if it still appears that the payment cannot be approved, the amount is checked against the account of the disbursing officer, who must secure reimbursement from the party paid.

The amounts which are found by the Auditors to be due and payable, and their settlement of various accounts are held to be final and conclusive, but the person whose account has been settled or the executive department to which the accounts pertains may, within a year, appeal to the Comptroller of the Treasury for a revision of the account. The Secretary of the Treasury may also, whenever he deems that the interests of the Government require it, suspend the payment of any account and direct its re-examination by the Comptroller. If the Comptroller certifies a difference from the result reached by the Auditor, the latter is required to state an account showing the difference to the division of bookkecping and warrants or to the Postmaster General, in case the account relates to the postal funds. If a person accepts payment under the settlement of an Auditor he is precluded from sceking a revision of the account so far as the items for which payment is accepted are concerned. It is, however, permissible to accept payment under certains items of an account, leaving other items suspended which upon settlement can be appealed to the Comptroller of the Treasury for revision, as if a portion of the amount due had not been received.

The Auditors of the various departments are charged with the 
superintendence of the recovery on all amounts certified by them to be due to the United States, performing this function under the direction of the Secretary of the Treasury, and the Auditor for the Post Office Department is authorized to collect all debts due to that department and all penalties and forfeitures imposed for any violations of the Postal Laws, including suits through the Solicitor of the Treasury against sureties on the official bonds of former postmasters who have become indebted to the United States.

TIIE COMPTROLLER OF THE TREASURY.

The chief of the auditing system of the Treasury Department and the court of final resort in relation to the expenditures of the money of the United States is the Comptroller of the Treasury. He is appointed by the President by and with the advice and consent of the Senate and receives $\$ 5,500$ a year. All books, papers, and other matters relating to the accounts of disbursing officers of executive departments and of independent commissions, boards and bureaus are at all times subject to inspection and examination by the Comptroller of the Treasury, as well as by the Auditor of the Treasury authorized to settle the accounts of such disbursing officers. The Comptroller, under the direction of the Secretary of the Treasury, prescribes the forms of keeping and rendering all public accounts except those relating to the Postal revenues and expenditures therefrom. It is made the duty of the Comptroller of the Treasury to revise the settlement of any accounts which have been settled by any of the Auditors of the Treasury when such settlements are appealed or are ordered for re-examination. When an Auditor makes an original construction of the law under which any payment is made, such construction is subject to the approval, disapproval, or modification of the Comptroller, and the latter may, when he sees fit for the public interest, modify any construction of these laws previously made. $\mathrm{He}$ is further required to certify to the Auditor in question his approval or change of a construction made, and in a matter affecting the transactions of all the Auditors to certify to all of them, in order that the procedure may be uniform in handling all accounts. 
The foregoing provisions relate to accounts which have come to the Auditor for settlement. If, however, a disbursing officer, or the head of a department, is in doubt whether an expenditure is authorized by the law, he may apply to the Comptroller of the Treasury for an advance decision, stating the circumstances under which it is proposed to make the expenditure. A decision of the Comptroller when rendered governs the appropriate Auditor and the Comptroller himself in cases where such an advance application has been made when the account comes to the Treasury Department for payment. In the exercise of the foregoing powers and duties the Comptroller of the Treasury becomes one of the most important officials under the Federal Government. $\mathrm{He}$ is for all practical purposes the highest authority upon matters relating to expenditures, for, although a claim disallowed by him'may be taken to the Court of Claims, if the subject matter comes within the jurisdiction of that court, and thus ultimately come to the Supreme Court of the United States, this procedure is only taken when considerable amounts are at stake, and for the every day routine business of the department of the Comptroller is the arbiter, and in this respect the superior, practically, of the heads of the departments themselves.

As a matter of fact, the Comptroller in instances more or less numerous has interfered witl a proposed action on the part of the President of the United States, and his decision secured in advance, has had the effect of negativing an expressed executive purpose. Such a situation in a former Administration brought about the remark of a President that if he conld not control the Comptroller, he coulcl at least make a new one. Such a view on the part of the President would have more or less tendency to persuasive influence over an official otherwise in a position of much independence, so much in fact that it is the view that the Comptroller of the Treasury has equal authority in matters relating to the construction of statutes governing appropriations with the authority of the Attorney Gencral in construing other statutes and propositions of law. Sucl a co-ordinate power is indicated by the alleged remark of a Comptroller that the opinion of an Attorney General on a matter relating to the construction 
of an appropriation act was persuasive, but by no means conclusive.

That which can and can not be done with reference to the expenditures of the funds of the United States is contained in a series of volumes known as the Comptroller's decisions, and made up of that official's findings on matters which have been submitted to him either in appeal from the Auditors, or as a result of advance requests for an opinion on matters relating to expenditures. The whole constitutes one of the bodies of administrative law which have developed around certain aspects of the Federal Government, and which are the basis of departmental practice, and of themselves are a by no means small field of legal study.

Frequent complaint is made of the difficulty of getting claims through the Treasury Department. The chief source of such complaint is in the lack of understanding on the part of claimants that their claims must be in the first instance properly prepared. There is no opportunity in the Government accounting for the tacit understanding and unwritten agreement which facilitates settlements of commercial accounts. Every account should be properly stated and accompanied by vouchers, which leave no room for question as to the authority for the expenditure, the accuracy of the price charged, and the propriety of the payment of the amount named to the person claiming therefor. If these requirements have been looked after there is no reason, and there probably will be none experienced, for undue delay in securing the payment of a liquidated account from the Treasury. If, however, any of these matters have been neglected, delays are inevitable. It is usual when a new and not altogether certain expenditure is to be undertaken for the Comptroller to be consulted by some person in the department who enjoys that officer's confidence, and who is regarded with much favor and approval should he return with a favorable answer of the somewhat dreaded and much courted functionary of the Treasury Department.

DIVISION OF BOOKKEEPING AND WARRANTS.

One of the most important divisions of the Treasury Department, and one which is immediately attached to the auditing and 
comptrolling service is that of bookkeeping and warrants. The result of the work of the Auditors as checked and revised by the Comptroller are certified to this division by which the appropriate entries are made in the accounts of the various disbursing officers. All adrances which have been made to such officers have been charged against the appropriation under which the advances have becn made, and the sums allowed by the auditing officers are credited to the disbursing officer, and form a basis for further advances. No additional advances can be made to a disbursing officer whose accounts are in arrears without the special application of the head of the department, and for excellent reasons. The sum expended, of conrse, remains charged against the appropriation, and only such further advances can be made as may be warranted by the balance remaining of the amount originally appropriated. The Division of Pookkeeping and IVarrants is the source of information as to the condition of the various appropriations and receives the estimates made by the different departments of amounts which should be appropriated for the following fiscal year. The details of the accounting of the various appropriations are carricd on chiefy within this particular division of the Treasury Department.

It is the duty of each head of a department to send in before October $15^{\text {th }}$ of cach year a detailed statement of the amount of money which he deems is necessary for the proper conduct of his department for the fiscal ycar which begins on July rst, next foliowing. All increases proposed must be set forth in specific form giving the exact number of additional offices and the amount of additional pay suggested. These estimates are prepared for printing and the summaries made by this division of the i'reasury Department. Frequently the annual appropriaticns for given purposes are exhausted before the end of a fiscal year, and it becomes zecessary to ask Congress for additional appropriations to be immediately available to carry on the work in the line for which the appropriation has been exhausted. Deficiency estimates are made through the Treasury Department as in the case of annual estimates, and there is generally at the beginning of every session of Congress an urgent deficiency bill to take care of matters immediately 
urgent, together with a general deficiency bill at a later date, while the Sundry Civil Bill, generally passed during the last days of a Congress, takes care of a large number of miscellaneous items. All of the warrants for the payments of money from the United States Treasury are made up in the Division of Bookkeeping and Warrants, which is also charged with making the statement of receipts and expenditures by which the annual cost of the Government in various lines, and its annual income may be ascertained and compared with similar figures for previous years. In the general amounts and particularly with reference to certain of the expenditures and receipts the comparison can be made with each year of the history of the United States so that the progress of governmental expenditures may be noted and a very material increase, not only in the amount of such expenditures, but in the variety of the national activities is thus shown.

\section{SUPERVISING ARCHITECT OF THE TREASURY.}

Altlough the United States spends large amounts of money annually for the rental of buildings instead of erecting departmental buildings of its own, it is quick to respond to the requests of residents of cities and towns throughout the country for Government owned quarters for the various Federal officers, especially for the Postal Service and as locations for United States courts. In every session of Congress a bill is passed appropriating money for the purfose of erecting Federal buildings at different points, although not in every session is a bill passed which provides for new propositions of this kind. It is usual in a general public buildings bill to authorize the acquirement of a site and the erection of a building, leaving the appropriations for the work of construction to later appropriation bills. The details relating to these buildings after they have been authorized by Congress are under the direction of the Supervising Architect of the Treasury. That official carries on negotiations regarding the selection of sites which is generally effected in accordance with the desire of the prominent men of the city or town to be favored, if such a site as is desired can be secured within the limit of the cost. The actual determination of the validity of the 
title to the site is, however, made by the Department of Justice. The Supervising Architect of the Treasury also carries on the negotiations with respect to the cession of jurisdiction by the State over any site which may be secured. Under the terms of the Constitution, Congress may exercise exclusive jurisdiction over any portion of a State's territory which may be duly ceded, but in most instances the States are unwilling to make an unconditional cession of territory and require in return a stipulation that the legal processes of the State may run within the limits of the territory thus affected. The office of the Supervising Architect of the Treasury has a general supervisory capacity rather than one of actual designing, except in cases where provision is made by law for the designing of buildings by the office of the Supervisor. In other cases this office arranges for the submission of plans in competition by outside architects, and also arranges the details of the letting of contracts and of the inspection work on the buildings as they are under construction. The Supervising Architect has also the charge of all matters relating to the repair and maintenance of buildings under the Treasury Department which are not located in the District of Columbia, and has the charge and control of the heating apparatus and elevator cquipment for all such buildings. This duty relates, however, only to the buildings and the fixtures attached thereto, the personnel for the care of the building and the furnishings and repairs, except safes and vaults, being under the direction of the Chief Clerk of the Treasury Department, who also has superintendence of a similar service with reference to the Treasury Department buildings in Washington. All safes and vaults for public buildings in Washington and elsewhere are provided by the Supervising Architect of the Treasury, who directs the construction of stationary safes and vaults, and supplies such portable safes as may be needed, looking after their renewal and maintenance as well. The Supervising Architect of the Treasury is also the consulting architectural and engineering official of the Government, his services being utilized from time to time by other departments having architectural and engineering matters to deal with which 
do not come within the regular scope of the duties of the Supervising Architect.

The duties of the Chief Clerk of the Treasury Department, heretofore referred to, are more in the line of a general custodian and superintendent of buildings than are those of any other Chief Clerk in Washington. While he has charge of the distribution of the mail of the Treasury Department and also of the custody of the records and files of the Secretary's office proper, the large proportion of his duties relates to the care of buildings of the United States and the administration of the forces of custodians, janitors, watchmen and other attendants. He directs the operations of a large number of persons who are located at points all over the United States, and also has the superintendence of the details relating to the supplying of large quantities of materials appropriate to the care and maintenance of buildings. It is, perhaps, another example of the divided responsibility frequently encountered in connection with governmental affairs and it results that the custodian and men immediately employed in the care and maintenance of a public building cannot requisition for repairs to heating and hoisting plants to the same source from which the supplies come, but must await the arrival and report of one of the inspectors of the Supervising Architect's office before other than immediately necessary and emergency repairs can be made. 


\section{CHAI"TER X. \\ WAR DEPARTMENT.}

Some of the most difficult and preplexing problems of government are those relating to the administration of military affairs. This includes not only land military forces, but those which operate upon the sea as well. While it seems entirely feasible and practicable to administer affairs relating to the duties of highly trained technical specialists in other lines than that of warfare, under the direction of persons who are themselves not so technically trained, but who have general knowledge of and capability for administrative matters, there is, on the part of those who have a military training, a disposition to deny that others than those who have been so trained can administer a department of war or of the navy. For this reason there appears in the two departments of the Federal Government which have to do with the military service some such a condition as when a river makes a conjunction with another river holding far more or far less a proportion of solid matter in suspension in the water. The two flow on in one channel and perform identical functions, but with the difference noticeably apparent in the color of the water. There is this difference, however, in this comparison to the civilian and military sides of the War and Navy Department, that there is no ultimate merger, and that the two go on together without at any time losing their distinct and differing characteristics.

The old school of military science made a sharp division between the actual fighting man and the person who provided the arms and ammunition, the provision and the clothing of the soldier. Such a division was maintained in the Army of the United States until I903. Previous to that time the bureau system was in effect in the War Department, and the departmental organization consisted, on the one hand, of the Secretary, who had under his immediate direction several more or less independent bureaus charged with the provision of the material for the army, and on 
the other hand a military organization proper, whose head, a Major General or Lieutenant General of the army, as the case might be, directed its movements and its discipline almost independently of the Secretary of War, and sometimes, if not in actual defiance of him, in a considerable state of friction as to carrying out his directions.

The opposite theory of military science to that which has just been described was developed by the Prussians as a preliminary to the Franco-Prussian war, and owes its perfection of detail to Von Moltke, and comprehends an equal interest by fighting officers in battle tactics and rations, in the use of arms and the providing of munitions of war.

It was only after the Spanish-American war found the Army of the United States illy prepared for the great expansion necessary for field operations on the modern scale that serious thought began to be given to a reorganization of the plan of military supcrvision by the IVar Department. Stimulus was given to the agitation for this reorganization by the difference of opinion and attitude between the then Secretary of War and the head of the military branch of the army, and the result is the present zeneral staff system which has materially changed not only the organization of the army itself, but the operations of the War Department as an administrative organization as well.

As suggested in the paragraph on the duties of the Secretary of War, the War Department executes many and differing functions, not a few of which have but little relation to the business of making war itself, but its principal as well as its titular functions relate to the administration of the Army of the United States. This army consists of a force of a hundred thousand men wholly under the control of the War Department and of the Militia of the United States, that is to say, the National Guard and other organized military forces of the different States who are subject to call by the President when their services are needed. The demand upon the organization of the army includes not only the training of the men actually in the service and their maintenance in a condition of efficiency, but it includes the requirement that the standing army shall be so organized and the 
military provisions shall be so arranged that when the militia, or remaining portion of the Army of the United States is called into being there shall be the necessary equipment and arrangement for handling the increased force as a portion of the whole. It was in this respect that the regular army failed at the time of the Spanish-American war, and it is the chief duty of the general staff corps around which the organization of the army at the present time is assembled so to adjust matters that the army may be mobilized withont disorganization.

The organization of the VVar Department consists first of the civilian directive officials, namely, the Secretary, Assistant Secretary, with the Chief Clerk and the various bureaus of correspondence, records and files, pertaining especially to the business of the department proper. 'To this is attached one aspect of the duties of the Judge Adrocate Gencral, namely, those which relate to civilian law questions. This organization carries out the directive functions of the Secretary of War, but it is by no means easy to describe the delicate gradation of his authority with respect to the Army of the United States. Undoubtedly in theory and, in fact, as far as he may care to exercise it, he has the power to direct all things relative to the land forces. In practice, however, and in view of the responsibility that is imposed upon the chief of staff by reason of the provisions of law which place certain dutics upon him, and which provide for the direct communication of the chief of staff with the President, there secms to be no doubt that the actual connection of the Secretary of War with military affairs is minimized, and restricted to broad questions of policy instead of being applied to a large variety of questions of intimate detail as was the fact under the previous system, and as is still the fact with regard to the relation of secretaries of civilian departments to the branches of the public service conficled to their direction.

The chief of staff of the Army of the United States is at the head of general staff corps composed of officers of the line of the army who are selected under Presilential regulations. He must be of not below the grade of Brigadier General, and is assisted by two general officers who are also selected and detailed by the 
President. The chief of the Coast Artillery is also a nember of the general staff, and thcre are added four Colonels, six Lieutenant Colonels and twelve Majors and twenty other officers, selected from the grade of Captain and Lieutenants, and who have during the period of their service on the general staff the rank and pay and allowances of Captains mounted. All of the officers, except those directly selected by the President, are selected in accordance with rules prescribed by the President and officers so assigned serve for a period of four years, when they must return to their own branches of the service and cannot serve on the general staff again until they have had two years of service in the branch of the army in which they were commissioned. An exception is made, hovever, that they may be reassigned to the staff corps in case of war without having had such service with their troops.

The duties of the general staff corps are to prepare plans for the national defence, and for the mobilization of the military forces in time of war. That is to say, to arrange matters so that upon the sudden increase in size of the Army of the United States due to the accession of the militia, it will be possible to make the whole, within a reasonable time, into a fighting machine properly organized and equipped by means of the use of the standing army as a skeleton upon which the larger organization can be rounded out.

Under the old organization of the army the various bureaus having to do with the provision of the mere material were independent of the line and were reached by it only through the Secretary's office. The idea of the general staif was, as much as anything else, to put an end to this condition of affairs, and the general staff is given by law supervisory duties in all matters pertaining to the administration not only of the military establishment but of the Inspector General's, Judge Advocate's, Quartermaster's, Subsistence, Nedical, Pay and Ordnance departments as well as the Corps of Engineers and the Signal Corps. Thus the different departments continue with the technical work which has previously been assigned to them, and the staff organization continues practically as before, except that a system of detail 
from and to line duties is supplemented for the close detail and promotion within the separate corps as formerly, but all the operations are lirected and the works co-ordinated by a superior supervisory boly. The general staft has the responsibility of the preparation of the policies and plans which the departments are to carry out and the power to see that the departments regulate their operation so that the plans of the general staff shall not be negatived by failure on the part of any department to accomplish its portion of the entire plan laid down.

The regular army of the United States consists of fifteen regiments of cavalry, a corps of artillery and thirty regiments of infantry. For the command of these forces there are provided six Major Generals, one of whom is assigned to duty in charge of each of the grand military divisions of the country within which he directs the operation and administration of the troops subject to the orders received from Washington. In the peace arrangement of the army two regiments of infantry or cavalry constitute a brigade, and is the command of a Brigadier General who is an officer selected for that grade by the President by selection. Najor Generals are appointed from the list of Brigadier Generals by the President, and to some extent in accorlance with the rank of the officers, arranged according to their seniority in the service after they have become Brigadier Generits and command divisions of two brigades each. Provision is made in time of war, or when war is imminent for the extension of the foregoing organization into divisions in which brigale is to be composed of three or four regiments, and each division of three brigades. When three divisions are assembled in the same army, the President is authorized to organize them into an army corps. The position of Lieutenant General is usually established to distinguished the chicf commanding officer of an army of the United States in the field or who has had conspicuous active service.

The artillery of the Army of the United States is divided into Coast Artillery and Field Artillery. The former has charge of the permanently located fortifications and the armament attached thereto, together with submarine mines and torpedo defenses. The field artillery accompanies the army in the field, and includes 
light artillery, horse artillery, which is light artillery in which all the men are mounted on horses, and which is more mobile than the light artillery in which the men other than the drivers ride upon the limbers of the guns; the siege artillery consisting of trains of heavy guns which are still movable, and the mountain artillery which is equipped with light pieces which may be readily dismounted and carried by pack animals.

The coast artillery is in charge of the series of defenses which were planned by a board specially constituted for the purpose and known as the Endicott Board. It is separate in organization from the field artillery and is organized in companies under a Chief of Coast Artillery with rank of a Brigadier General. The Chief of the Coast Artillery is appointed by the President by and with the advice and consent of the Senate, and serves for four years, but may be reappointed. The equipment under the charge of the coast artillery includes a large amount of highly developed machinery besides many instruments of precision. The fortifications which coast artillerists man are situated at the approaches to the principal seaports of the country and to the capital of the United States, and for the most part consist of earthworks and emplacements which are not visible from the usual approaches, and which are carefully guarded from visitors. All of the water space within the range of these forts is carefully plotted out and the ranges calculated so that the practice with the great guns is carried on entirely with mathematical formulas, it being almost certain that a vessel which should come within the limits of a given square would be struck by one of the projectiles from the twelve-inch guns, provided, of course, those charged with the operation of the material did not become nervous and make a mistake in their figures before discharging the piece. In order to enforce the passage of hostile vessels, if they pass at all, along certain channels and under the conditions best suited to the operation of the guns of the fort, large areas of the water front are marked out in mines and torpedo fields, cables being prepared so that mines covering these fields may be quickly laid by a fleet of specially equipped vessels under the direction of the coast artillery, and such mines being electrically connected with an 
observing station included in the fort, can either be fired at will or in certain instances be fired by the contact of the vessel with the apparatus of the mine. The development of the equipment of the coast artillery stations has become so highly technical and the duties demanded are of such an exacting nature that complaints have been made for several years that it is impossible to maintain the personnel of the artillery at the legal strength. In many cases the posts are isolated and the company formation offers little attraction as compared with larger bodies of troops, so that with the considerable amount of work required of the men stationed at the different posts, it has become difficult to retain good men, and almost impossible to recruit the coast artillery up to its required strength. In fact the discouragement with regard to this branch has become so material that propositions have been made to turn the force over to the navy for operation.

The training and discipline of the army is carricd on under the propositions of the general staff which are made effective by the order of the Secretary of War, and which are transmitted to the commanding officers of the different military divisions for their guidance and instruction. These relate to the administration of the personnel only, the provision and distribution of the materials of war and of the subsistence and clothing of the troops being administered by various staff departments, although always under the supervision of the general staff. Staff organization under the general staff consists of the departments previously named each of which has a permanent organization, but in addition thereto line officers may be assigned to perform the duties under the different departments when serving with troops, such assignment being independent of or in addition to the actual handling of the troops as the case may be.

The Adjutant General's department has charge of the duties appropriate to the office of Adjutant, namely, of promulgating and certifying all orders originating from the commanding officer, whether the officer in command at Washington, the officer in command of a military division, or the officer in command of a post. That is to say, each subdivision of the army has a similar 
staff organization to that which obtains in the department in Washington. An officer either of the staff organization or a line officer performs the duties which are generally assigned to a particular department of the staff, the difference being that of the rank of the officer which is adjusted in accordance with the importance of his duties. Thus Washington has the title of Major General while the Adjutant General of a military division would be of a correspondingly lower grade, and so on down to the Adjutant of a smaller post, who would be a subordinate officer of the line assigned to perform the duties in connection with his other work. The permanent staff establishment in any department maintains the staff organization and sets the pace. so to speak, for the line officers detailed to perform similar work. In order to provide separate officers for various branches of staff work throughout the organization of the army, it would, of course, be necessary to increase the number of staff officers materially with assignments that in many cases would carry with them a minimum of duty to be performed. By assigning officers of the line to these duties their performance is secured in connection with the regular duties of the officers assigned, leaving the members of the permanent staff establishment free to perform duties at the more important points, and to perfect the system of administration of that particular branch. Something of a similar nature is in effect with regard to the general staff. Besides the duties of the officers attached to this staff, and which are performed in Washington, general staff officers are assigned to divisions and prominent posts, for the purpose of assisting in co-ordinating in the staff work generally, as well as for the purpose of reporting to the general staff at Washington on such matters as may come under their observation which will tend to the general improvement of the efficiency of the service. Besides the work of the Adjutant General's department of the staff in promulgating orders, that branch is charged with collecting and arranging the records of the army as to the daily strength, performance of duties, and other details. The personnel provided for the administration of the different staff departments usually consists of a head of the department, who is of the rank of Brigadier General, and assist- 
ants of the rank of Colonel, Lieutenant Colonel and Major, respectively. The line officers detailed perform the staff duties, being of lower rank generally than Major.

The Inspector General's department is charged with the general inspection of all branches of the active military service, including the appearance and efficiency of troops, the character of the material furnished, and the condition of barracks and posts, as well as with the inspection of all the accounts of officers, including the clothing and property accounts. Officers in charge of the troops are charged with the amount of public property issued to them, and must, upon inspection, show the proper number of arms in good condition or legal excuses for their absence and their books must develop the proper balance between the clothing and other supplies on hand and the amounts which have been issued as shown by the receipts of the soldiers and others, and by appropriate vouchers. The affairs of each subdivision of the army require the keeping of sets of books in which the responsible officers are cirarged with the govermment's property received, and credited with the issues made to the men, and with the amounts of government property on hand at the time of any inventory or taking of a balance. If discrepancies appear that cannot be cleared up by means of boards of survey or other administrative proceedings established by law, the value of the missing article is checked against the pay of the responsible officer, and he is compelled to make good the loss. In case, however, of considerable losses showing negligence or actual embezzlement of public property, or of the funds which have accrued to a company's credit, an officer is subject to court martial with consequent punishment on conviction either of suspension, dismissal from the army or of imprisonment in flagrant cases. In addition to the regular establishment of Inspectors General, officers of the line may be detailed to perform inspection work, and officers performing the duties of Adjutant General when required must in addition to their own duties perform the duties of Inspectors General.

The Judge Advocate General's department, in addition to performing the services of a civilian legal bureau for the Secretary 
of War, is at the head of all the processes of military justice. The officers of this department appear in the character of military advocates at court martials and those of the higher grades are charged with the review, subject to the approval of the Secretary of War or of the head of the geographical department, as the case may be, of the results of such court martials, give opinions on all questions of military law presented to them including matters relating to the rank and pay of the officers of the line and staff. Provision is made for the appointment to the corps of Judge Advocates of persons who have served as Judge Advocates in the volunteer service, or of persons from civil life who are not over thirty-five years of age, and who can pass a satisfactory examination. In case the regular establishment of the staff does not provide a sufficient number of Judge Advocates so that one may be assigned to each of the geographical divisions of the army, acting Judge Advocates may be appointed by detail from the line of the army of the grade of Captain or First Lieutenant, who have while serving in such capacity, the rank, pay and allowances of a Captain mounted. An officer so detailed, however, can only serve for periods of four years, when he must be returned to the arm of the service in which he was commissioned. Permanent appointments to the staff of Judge Advocate may also be made from the line, as in the case of other staff departments, officers who desire, may, upon the occurrence of a vacancy in any staff, seek an appointment, which constitutes a permanent transfer from their line duties and they are merged with the staff department. This has usually been sought because the transfer from the line to the staff brings with it an immediate increase in rank and pay, although as it takes the officer from the line of promotion in the branch in which he has been serving, it also means that his subsequent promotion will be slower, and that he may not attain as high a rank as he otherwise would.

The office of a military Judge Advocate is not only that of a prosecuting attorney before a court martial commission or other military tribunal, but he is, as well, required to see that the rights of the defense shall not be ignored and is further charged with 
promoting, so far as he is able, the regularity and legality of the procedure of the court. While Judge Advocates assigned to military divisions, review the procecdings of court martials which are immediately under the jurisdiction of the commander of that division, supervision of the entire course of military justice is vested in the Judge Advocate General and all Judge Advocates perform their duties under his general direction.

One of the most important of the staff departments is that of the Quartermaster, superintended by the Quartermaster General, and including in its personnel besides this officer six Assistant Quartermaster Generals with the rank of Colonel, nine Deputy Quartermaster Generals with the rank of Lieutenant Colonel, twenty Quartermasters with the rank of Major and sixty Quartermasters with the rank of Captain. In addition to this force line officers serving with troops act as Quartermasters, and there are provided for service at the different posts two hundred Quartermaster Sergeants who are selected by examination from competent enlisted men who have served at least four years, and who take charge of the public property at the posts and act as clerks and assistants to Quartermasters. One of the clief of the duties of the Quartermaster's department is to provide transportation for the army. This includes not only transportation in the field by means of horse and wagon and pack trains, but also transportation by railroads and steamships in the United States and to and from the foreign possessions of the United States for all officers and men of the army and for all goods and munitions of war intended for the army use. Contracts are made by the Quartermaster's department for the travel of officers and men, and also for the transportation of freight. In this connection the Quartermaster's department of the army is the principal means by which the United States collects from the bond aided and land grant railroads of the West the return from the assistance which was given in the construction of those railroads. Under the terms of the grant of bonds and land the railroads were to convey the property and servants of the United States either without charge or at half rates. In order that these deductions may not be neglected by the departments, and as a 
matter of convenience in taking advantage of the organization of the Quartermaster's department for the shipment of goods, most of the freight shipped by the various executive departments is delivered to the Quartermasters of the army, and by them forwarded under the general arrangements made with the railroads. The activities of the Quartermaster's department are more general and more important than those of any other department of material. It is required to purchase and distribute all military stores and supplies which other corps are not directed by law to provide, and also to pay for all incidental services not under other corps.

The provision made by the Quartermaster's department includes, therefore, the clothing and equipage meaning the articles of horse equipment and of the soldiers' equipment not provided by the bureau of ordnance. All horses used and wagons with the forage for the animals are purchased and maintained by the Quartermaster's department, which also has under its charge the transport service to the Philippines, and would have in time of war the responsibility of moving the army, whether in the field within the United States, except so far as the army should march, in which case the Quartermaster's department furnishes the wagon service, or if sent out of the United States would provide and equip the fleets necessary for the movement of the troops. The Quartermaster's department also provides the clothing and equipage for the militia, and stationery and miscellaneous stores and property for the army. It has charge of the buildings, wharves, roads and bridges at the military posts, constructs them when ordered, and makes the necessary repairs and furnishes water and lighting apparatus for such posts. The Quartermaster's department also pays guides, spies and interpreters for the army.

By national legislation certain tracts of land throughout the country, chiefly in the Southern States, and including the property at Arlington, formerly the home of General Lee, have been acquired, because such tracts were the burial places of considerable numbers of Union soldiers during the Civil War. These properties have been constituted national cemeteries, and are 
under the direction of the Quartermaster General of the army. A superintendent is appointed for each cemetery, and arrangements have been made for the perpetual maintenance of these burial places at the public expense. Provision is also marle by law for furnishing a headstone in case of the death of a soldier of the Civil War under certain conditions, and arrangements for supplying these headstones are under the direction of the Quartermaster General of the army.

When detachments of seamen or marincs are under orders to act on shore the Quartermaster General of the army is required by law to furnish them with such camp equipage and transportation as would be furnished to troops of the army operating under similar conditions. The officer in command of such a detachment and his aides, are furnished by the Quartermaster General with the necessary horses, accoutrements and forage.

The Pacific transports of the War Department, under the Quartermaster's department, constitute the remainder of the considerable number of transports purchased or chartered during the Spanish-American IVar. A monthly service is maintained to the Philippines, touching at Honolulu and the officers and men of the army are transported between the United States and insular possessions. The families of the personnel of the army are also transported in the order in which application is made for accommodations, and officers of the navy and their families are given accommodation when possible. A special enactment of Congress restricts the use of such transports to persons entitled to transportation by reason of connection with the military service. Supplies for forces in the Philippines and for the Civil Government are sent by transport when possible. Considerable complaint has becn made in times past of the competition of this service with privately owned lines, but the Government has not yet been able to indertake a different arrangement for transportation to the Philippines. Each transport is under the command of a Quartermaster, although the actual navigation is in charge of civilians.

The work of the Subsistence department of the army refers only to the supplying of the food for the troops and the conduct 
of the post commissaries or stores at which the officers and men can purchase various supplies not provided by the Government and at cost prices. It is the duty of the Subsistence department to purchase and issue to the army such supplies as enter into the composition of the ration. In addition the Subsistence department purchases a large variety of commodities which are kept at the stores at the different posts and which are sold to the officers and men either on credit or for cash at the cost price to the Government. The Subsistence department has general supervision as well as over all the accounts relating to these two items, and makes the administrative examinations of all accounts for subsistence supplies preliminary to their settlement by the accounting officers of the Treasury.

The Medical department of the staff of the army has control of all matters relating to the health and sanitation of the personnel. Surgeon General, who has the rank of Brigadier General, is assisted by nine Assistant Surgeon Generals with the rank of Colonel, twelve Deputy Surgeon Generals with the rank of Lieuteriant Colonel, sixty surgeons with the rank of Major and two hundred and forty assistant surgeons with the rank of Captain or First Lieutenant, mounted, with a Hospital Corps and a Nurse Corps. Officers of the Medical department take rank and precedence in accordance with the date of their commission, but are not entitled to command in the line of other staff corps although having command in accordance with their rank in their own corps. The Surgeon General has charge of the disbursement of the appropriation for the Medical Corps, the designation of the stations of medical officers, and the issuing of all orders and instructions relating to their professional duties. Officers of the Medical Corps, besides treating the sick and wounded of the army, are responsible for the sanitary conditions of posts and of camps in the field, and are charged with the medical examination of applicants for enlistment, candidates for appointment to commissions, and with conducting the necessary medical examination in connection with promotions and retirements. The selection and distribution of the medical supplies for the army are under the immediate direction of the Surgeon General, who has also 
under his direct control the Army Medical Museum in Washington and the general hospitals of the army. Post hospitals are under the command of the senior medical officer present, subject to the control of the commander of the post, but under the general supervision and direction of the Surgeon General. The Surgeon General is empowered, with the approval of the Secretary of War, to appoint as many contract surgeons as may be neccsary. The maximum compensation of a contract surgeon is $\$ 150$ per month, and he is employed for a term of years under regulations established by the Surgeon General. $\mathrm{He}$ is wholly a civilian officer in that he has no right of promotion or retirement, but when he is in charge of a hospital he has command of the commissioned officers by virtue of a special provision of law. Dental surgeous, not to exceed thirty-one in number are also appointed under the same terms as contract surgeons.

Entrance to the Medical Corps can only be secured upon the approval of an army medical board, which conducts an examination of the candidates. The board must consist of not less than three surgeons or assistant surgeons, as designated by the Secretary of War. No person can be promoted to be Surgeon unless he has served at least five years as an Assistant Surgeon in the regular army, and shall have been examined and approved by a similar board to that above described. Medical officers of the army and contract service surgeons are required, when practicable, to attend the families of officers and soldiers free of charge.

The members of the Medical Corps of the army are in charge of two enlisted forces, one male and one female, known as the Hospital Corps and the Nurse Corps, respectively. Three hundred Sergeants, first class, are provided and as many privates as may be necessary to make up the Hospital Corps and to carry on the duties of wardmaster, cook, nurses and attendants in hospitals, and stretcher bearers, litter bearers and ambulance attendants in the field. A school of instruction for the Hospital Corps, which in its organization is entirely military with additional duties in the special lines indicated, is located in Washington. Any en- 
listed man of the other branches of the service may be transferred to the Hospital Corps.

The Nurse Corps consists of one superintendent, appointed by the Secretary of War, who is a graduate of a hospital training school, and who holds her position at the will of the Secretary of Var. Authority is also given for the employment of as many nurses as may be necessary for the active duties of the corps, all nurses being appointed and removed by the Surgeon General with the approval of the Secretary of War. They must be graduates of hospital training schools and must pass a satisfactory examination. A number of reserve nurses, discretionary with the Secretary of War, may also be provided, but these nurses receive no compensation except when they shall be assigned to active duty in case of an emergency.

Members of the Medical Corps are expected to have a general oversight over the health and habits of the men of the command to which they are attached to see that the food supplied is proper in quantity and quality and to oversee generally the cooking for the men in order that the rations furnished shall not be prepared so as to injure the health of the men. Connected with this branch of the duty of the Medical Corps, brilliant work has been done during and since the Spanish-American War in connection with tropical diseases, the presence of portions of the Army of the United States in tropical countries making the study of means for the prevention and cure of such diseases, especially the duty of the members of the Medical Corps. More than one officer of this branch of the army has lost his life as a direct result of such researches and the additions to knowledge on these subjects in the past ten years have been greater than in the entire previous history of the world.

The Pay department of the staff of the army, consisting of one Paymaster General with the rank of Brigadier General, three Assistant Paymaster Generals with the rank of Colonel, four Deputy Paymaster Generals with the rank of Lieutenant Colonel, twenty Paymasters with the rank of Major, and twenty-five Paymasters with the rank of Captains, mounted, performs all the 
duties relative to the payment of officers and men of the army, including the payment of the army in the field; the payment of the latter including not only the regular troops, but all other troops in the service of the United States when the President directs the Pay department to undertake the payment of such additional troops. Pay officers assigned to the different divisions take up on their accounts the amounts due officers and men of different grades and rank, calculate the sums accruing to them on the grounds of longevity and allowances, make such advances as may be permitted by law, and settle balances in cash, deducting such amounts as may be certified by other departments or by the auditing officers of the Government as due to the United States from the person whose pay account is to be settled. Arrangements may be made by enlisted men to have a certain amount of their pay allotted so that it will be paid directly to beneficiaries other than themselves. The Pay departiment kceps a record of such allotment and arranges for the payment of the amounts accruing thereunder to the proper person. The Paymaster General of the army has immediate supervision of all the transactions of pay officers, arranges for the furnishing of funds to them to carry out their dutics, and directs the preliminary examinations of their accounts.

The Chief of Engineers of the arny is at the head of an organization which combines important military with important civilian duties. In his military capacity he is at the head of a separate branch of the service, which is organized in battalions of four companies each. The Chief of Engineers is appointed by selection by the President from the members of the Engineer Corps. Subordinate offices are filled by promotion within the corps according to seniority and vacancies in the lowest grade are filled by appointment from the Military Academy. The Corps of Engineers is regarded as a portion of the line of the army and in orde: to secure an appointment as an Engineer it is necessary for a military cadet to have attained a certain rank in his studies at Vest Point. The Chief of Engineers is authorized, with the approval of the Secretary of War, to regulate and determine the number, quality, form and dimensions of the necessary vehicles, 
pontoons, tools and other supplies for the use of the men under his control. Engineers are not allowed to assume or to be ordered on any duty beyond the line of their immediate profession, except by special order of the President. The military duties of the Engineer Corps consist of training the enlisted men under their cliarge in the work of fortification, making pontoons and other bridges, and providing roads and other means for the progress of an army. Engineers in the field are required to make maps, determine locations, lay out and superintend the construction of fortifications and are also placed in charge of the work of planning, designing and constructing permanent fortifications and works of defense whenever authorized by the Congress. Engineer officers engaged in the latter work and also in the civilian work of the department, are required to act as disbursing officers without further compensation than their pay. The most important part of the work of the Corps of Engineers refers to the improvement of rivers and harbors in the United States. Such improvements were among the earliest of the undertakings of the Federal Government for the general good and have been carried on on a large scale, involving the expenditure of hundreds of millions of dollars for which the Corps of Engineers have made preliminary plans and surveys, have prepared the completed plans and specifications and have supervised the construction under contract conditions. In connection with the work of this character on the Great Lakes, and for the purpose of so conducting the improvements as to make them of the greatest general value a comprehensive survey of the lakes is maintained, the results of which are used as sailing charts and directions by the navigators on the lakes. After several experiments had been made as to the methods of conducting the engineering work on the Panama Canal, it was finally placed in charge of Engineers of the army with most satisfactory results.

The Ordnance department of the army has charge of all matters relating to furnishing cannon, small arms and powder, and the Chief of the Department is in charge of important manufacturing institutions, the arsenals at Springfield, Mass., Watervliet, N. Y., and at Watertown. Mass. The duties of the de- 
partment include the designing and manufacture of the rifles for the army and all matters relating to the care and custody, issuing and maintenance of the weapons for the enlisted force. Similar duties are performed with regard to the equipment of the artillery with guns, the Ordnance department being charged with the tests of various artillery devices proposed by officers of the army or by civilian inventors: with the supervision of the manufacturer of such types as may be adopted, and of the manufacture of the powder and projectiles therefor. A large portion of this provision is made by means of contracts for which the specifications are prepared under the direction of the Chief of the Department, who has the rank of a Brigadier General and who is assisted by an (rdnance Corps, ranking from Colonel to First Licutenant, and who constitute a separate division of the army within which promotions are made, being required by law that such promotions shall be made in order of seniority. This corps consists of highly trained specialists in the work of manufacturing munitions of war, and entrance to the lower grades is effected by detail of the younger officers of the army for four years of service followed by appointment to permanent positions of officers who qualify by special fitness. The organization of the arsenals under the direction of the department consists of one superintendent who is designated by the President from the officers of the Army, a master armorer who is appointed by the President, and such number of workmen as the duties to be performed shall require. The civilian Master Armorer, who has the direction of the work of the armories under the supervision of the military officer letailed as superintendent, receives $\$ \mathrm{I}, 500$ per year, and a force of clerks and inspectors is provided at the armory, the statutory salary being $\$ 800$ except at Springfield, where $\$ 1,650$ are paid the clerks and the Master Armorer receives $\$ 1,000$ a year additional as Master Machinist. All employes are excepted from jury duty and penalties are provided for the punishment of any person who entices a workman to leave his employment in such institution. A penalty of $\$ 20$ is also provided for the punishment on any workman who refuses to perform his contract of labor in an armory or arsenal, or who wilfully breaks or 
injures any of the property of the United States, such penalty being imposed for each offense.

Inventions are frequently made by officers of the United States and by employes of the armory, and a provision is made that no money shall be paid for royalties for any such invention. As an anxiliary to the Ordnance department of the army there is a board of ordnance and fortification, consisting of the commanding general of the army, and an officer of engineers, an officer of ordnance and an officer of artillery, together with one civilian member. This board is charged with considering all plans for fortification and the types of ordnance to be used in the army, and no person who is in any way interested in any such device can be a member of the board.

The Department of Ordnance has under its control a proving ground at Sandy Hook, N. J., where new devices and guns built in accordance with approved plans are tested. A fund is placed at the disposal of the board on ordnance and fortification from which payments may be made to defray the expense of developing new inventions in warfare and of building new types of guns and providing for their test. The scope of this appropriation is, however, comprehensive of anything which may be used in connection with defensive military work and a considerable allotment from the amount has been made in years past for the assistance of experimental work in aerial navigation.

The inauguration of the board on ordnance and fortification was effected not only for the purpose of securing expert advice in such matters, but also to meet the criticisms that the Government was slow in adopting the ideas of private inventors for the improvements of means of warfare. While this criticism has not always been well founded, there is a not unnatural tendency on the part of military experts to regard with some incredulity the claims of persons not specially known for their skill in the development of ordnance devices. It has been one of the most fertile as well as one of the most unprofitable fields of invention and in recent years, at least, complaints cannot be made of the lack of readiness of the War Department to take up and try new inventions. In one instance alone many thousands 
of dollars were expended in tests with a type of gun which proved upon its final trial to have practically no value whatever as a weapon of warfare.

The Signal Corps of the army formerly conducted the weather bureau service which was inaugurated, and, to a large extent, developed under this corps. It became, however, too great a proposition for its administration as an adjunct to the military duties of the corps and was therefore diverted and assigned to the Department of Agriculture. The Signal Corps of the army; as at present organized, has wholly military duties, that is to say, is expected to conduct communication between the various divisions and subdivisions of the army in the ficld, and between the headquarters of the army and the IVar Department, and in time of peace to maintain telegraph lines to outlying posts of the army not easily communicated with by commercial means, which lines are also open to use by private persons under appropriate regulations. The means of signalling which are operated by the corps include the telegraph and telephone, either permanent or of temporary construction for field use, signalling by flags, the heliograph, captive balloons and wireless telegraphy. At the monent especial attention is being paid to the development of balloon work by the corps, and, as is the case with all modern armies, experiments in the use of wireless telegraphy for military purposes form a prominent part of the work of the corps.

The organization of the Signal Corps includes not only the usual commissioned personnel of a staff corps, but an enlisted force as well based upon four hundred privates, the usual number of non-commissioned officers being provided. The privates of the Signal Corps are organized as infantry, but carry signal equipment, and are utilized in small detachments for the purpose of constructing means of communication and of carrying on the signalling work which requires expert knowledge of apparatus and codes utilized.

There is in the War Department a staff bureau which is largely the result of the life work of one man. Something like twenty years ago an officer of the Medical Corps of the army was in charge of the section of the organization of the War Department 
which related to the records of the officers and men, especially of the volunteer regiments which were engaged in the Civil War. The particular importance of the work of which he had charge related to the operation of the pension bureau. The pensions based upon army service are, of course, vastly the greater number of the whole number granted and the pension bureau was dependent upon the rolls and records of the War Department for intormation as to the right of persons to receive payments under the pension laws of the United States. As this information was in a variety of forms, including returns which had been made to the VVar Department under varying conditions and circumstances, the amount of work involved in developing from the original sources, the information needed was each year growing greater, so that a point had been reached where it was not possible to utilize the services of a sufficient number of persons to keep up with the current work. In addition the original records from which the information was secured were worn, and in some cases were almost undecipherable. Under the direction of the present chief of the record and pension office, who has at the present time the title of the Adjutant General and the rank of Major General, all the entries in the original documents were transferred to cards and properly indexed, so that the search for information might be made from the cards without reference to the original records themselves. This work, at first, was carried on as a sub-bureau of the war department, but has gradually assumed considerable proportions and in connection with the reorganization of the army about the time of the passage of the general staff bill, the office was made one of general record and constituted as the machinery by which orders, instructions and regulations, issued by the Secretary of War through the Chief of Staff are authenticated and communicated to troops and individuals in the military service. Incidentally this provision exhibits the possibilities of the accumulation of Government business by a person who is active and zealous in the pursuit of responsibility and who cares to seek for lines of work which are not actually, forced upon him, but which he can add to the activities under his control without too great a strain on the logic of the situation. 
The practical value of the constitution of The Adjutant General's office, however, as a means of certification of orders and regulations to the troops is the certainty thus assured that all such orders and the appropriate replies are transmitted through a single burean so that the proper records can be made of them. That is probably the real purpose and aim of the constitution of the Adjutant General's office as it now exists and any knowledge of the ease with which important records become diverted in the Government service will lead to the approval of the system of an inflexible requirement for recording official actions in the one central bureau.

The Adjutant General prepares and distributes commissions to officers of the army and compiles and issues the army register and directory. The general returns of the army, being the statement made of the number of officers and men in each organization, are consolidated in this office, and the report of the officers detailed to inspect encampments of militia are filed therein, while the office is charged with preparing the annual returns in regard to the militia required by Congress. The recruiting service for the army is managed by the office of the Adjutant General, and matters relating to the commutation of imprisonment of military prisoners come under this office. The main business of the office of the Adjutant General is the charge of the military and hospital records of the volunteer armies of the United States, and of all matters relating to pensions which come under the War Department. The archives of the Adjutant General's office which have, with the exception of one or two of the wars of the United States, been transferred from the original records and indexed, as before indicated, includes those of the Revolutionary War, the Mexican WVar, the Civil War, and the Spanish-American VIar and other differences in which the United States has been engaged. This includes not only the records of the men who were actually enrolled in the different organizations, but the medical and hospital records of the enlisted men and officers as well, and the record of the movement of troops, so that, upon a claim to the Pension office from an individual that he is, by reason of his service in a given organi- 
zation, entitled to a certain class of pension, the Pension Office is able to check up his statement by ascertaining his membership in the organization indicated by his claim, together with a determination as to whether the services of such organization as he may be found to have belonged to entitle him to be enrolled on the list of those who are pensionable. Certain military organizations which took some part in the Civil War have not been recognized in connection with the Pension list, while it is possible by means of other records to identify or check up a man's claim as to service should the claim have to be based, as it does in many cases, upon the statement of the applicant himself or on the affidavit of others who claim his acquaintance during the Civil War. The medical and hospital records under the care of the Adjutant General are also valuable for the purpose of determining whether the disability depended on by the applicant in his claim for pension could, as a matter of fact, have originated as an incident to military service.

The Adjutant General also has charge of the historical records of the permanent military establishment, including jurisdiction, over all pay, bounty and other business dependent upon the military or medical history of former officers or enlisted men. All reports of the physical examinations of recruits and all identification cards of enlisted men are filed in the office of the Military Secretary, which has also been made the repository of records relating to the Civil War, including those of the provost marshal general's bureau, the records of the bureau of refugees, freedman's and abandoned land bureaus, and the records of the Confederate Government including those pertaining to the legislative, executive and judicial branches. The total of the records under the control of the Military Secretary constitute a fund of historical material, especially of the period relating to the Civil War, and to the military operations of the United States, which is of inestimable value. These records have been brought together with the utmost painstaking care, and provision is made for their permanent retention in form, in which they can be referred to when reference is necessary.

With these records as a basis the Government issued the official 
Record of the IVar of the Rebellion, a publication containing all the material documents necessary for a complete understanding of all the movements of the armies of both sides during the Civil War. A similar publication with reference to the Mexican and Revolutionary Wars is projected, and with the official records of the Union and Confederate navies, now being published by the Navy Department, would constitute a remarkable historical series.

The history of the Adjutant General's office, as it is now constituted, is that of a curious bit of military legislation. Originally the Adjutant General was a sort of a military aid and adviser to the Secretary of War and fought out the latter official's battles with the commanding general of the army. The Adjutant General then had the current records of the army and the historical records, while the Chief of the Record and Pension Office had the volunteer records. When the Chief of Staff was constituted as the military adviser of the Secretary, it was pointed out that there should be some authenticating and recording power, and the institution of the office of Military Secretary was secured, and the recording functions of the Adjutant General's office were combined with the Record and Pension office to make up the Department of the Military Secretary. The latter title, however, did not continue in use long and was changed to that of Adjutant General, so that the net result has been a somewhat circuitous route by which the old Adjutant General's office was abolished, its functions combined with another division of the War Department, and then the whole re-constituted as an Adjutant General's department with, however, a strict adherence to the clerical duties of the function of Adjutant, leaving the military functions in the possession of the Chief of Staff.

The Chief of the Bureau of Insular Affairs of the War Department is a Colonel of the army assigned to this duty under the immediate direction of the Secretary of War, and supervises all matters pertaining to the civil government in the Philippine Islands. $\mathrm{He}$ also has supervision of matters relating to the temporary government established in 1906 in Cuba under the terms of Platt amendment, and which, at the present writing, is administering the affairs of the island. 
The establishment of the Bureau of Insular Affairs is due to the fact that the first government of the insular possessions of the United States was necessarily carried on by military officers, the precedent being established by the military government of Southern States during the Civil War. As the result of the operations of the Spanish-American War, Porto Rico, Cuba and the Philippines came under the direction of the Government of the United States, such direction being assumed during a time when military operations were still in effect. From the fact that a formal peace had not been arianged, the details of the direction of the Government had necessarily to be assumed by the Secretary of War. When civil government was substituted in the Philippines the Bureau of Insular Affairs was continued and largely due to the fact that an especially happy choice had been made in the officer selected for the head of the bureau, no serious complications have arisen in the supervision of the civilian government by the military authorities. The Bureau of Insular Affairs is the repository of all the civil records of the Philippines and of the former government of the occupation of Cuba which terminated in 1902, as well as the records of Porto Rico during the period in which the War Department exercised jurisdiction over that island.

Besides furnishing information relative to these subjects the bureau prepares and arranges for publication documents in regard to the Philippines, and has in the past complied many notable works covering the details of the natural conditions and the commercial possibilities of the islands. The bureau makes a comptroller's review of the receipts and expenditures of the Philippine government, and prepares statements of such accounts for presentation to Congress. It purchases supplies in the United States for the Philippine government and arranges for their shipment to Manila. The service in this connection includes a preliminary audit of the accounts before they are finally passed upon in the Philippines. The bureau also has charge of appointments in the United States to the Philippine Civil Service, including arrangements for the transportation of employees and their families, and gathers statistics of imports and exports, commerce and immigration, and prepares monthly summaries of the same. 
In addition to the foregoing staff departments and bureaus there is a corps of chaplains in the army consisting of one for each regiment of cavalry and infantry, and twelve for the corps of artillery. No person can be appointed a chaplain in the regular army who shall have passed the age of forty years, or until he shall have furnished proof that he is a regularly ordained minister of some religions denomination and in good standing at the time of his appointment. The recommendation for such appointment must cone from sone recognized ecclesiastical body, or from not less than five accredited ministers of such an organization. In addition to the foregoing requirements it is necessary that a chaplain shall pass such an examination as may be prescribed by the Presiclent. It is usual, however, to accept the certificate previously mentioned as a guarantee of the qualifications of the chaplain and to effect the appointment upon the physical examination wholly. All chaplains are required to hold appropriate religious services at least once on each Sunday, and to perform the burial services at the funerals of officers and men. Chaplains of colored troops and chaplains assigned to duties at posts are required to instruct the enlisted men in the common branches of learning and education. Chaplains are in addition expected to take an interest not only in the moral but in the physical welfare of the men, and to promote their contentment and good condition in such ways as may be practicable, and commanding officers are required to afford chaplains such facilities as they may desire and may be proper for the performance of their duties. Chaplains have the rank of Captain of infantry without command and are on the same footing with other officers of the army as to tenure of office, retirement and pension. They are required to make monthly reports to the Department through the usual channels, of the moral condition of the command to which they are attached, and are also charged with the duty of noting and transmitting details relating to the general history of their commands. These reports are transmitted to the office of the Adjutant General and there assembled and form the foundation of the compilation which may be made as to historical details affecting the different regiments and the army at large. 
THE LINE OF THE ARMY.

All of the activities which have previously been described are auxiliary to the operation of the army itself and make its continuance and maintenance possible. The actual administration of the army is, however, under the direction of an organization which in many respects receives superior consideration to that afforded to the staff bureaus. That is to say legislation for the army generally has in view that the benefits received by the staff department, except as to their particular duties, are incidental and subsidiary to those which are intended for the officers having direct command of the troops. It is required that the army of the United States shall be administered according to the custom and discipline of war. Such custom and discipline forms the basis of the regulations for the control of the land forces of the United States and while the large proportion have been formulated and are included in the regulations of the Army of the United States, there is a large body of unwritten law and which is inculcated by the older soldiers on the younger, and the possession of which constitutes the difference between a military service and a civilian employment. The experience of all history in warfare is mirrored in some of the conditions and observances of the Army of the United States even though military methods of the present day bear but little resemblance to those even of the armies of the earlier years of the United States.

The basis of all successful military service is, of course, the discipline which requires all persons to obey strictly and to execute promptly the lawful orders of their superiors. On the other hand, the authority of the superior must be exercised with firmness, kindness, and judgment. Superiors are forbidden to injure those under their authority by tyrannical or capricious conduct or by abusive language. In addition to strict obedience, courtesy is required among military men as indispensable to discipline. In furtherance of this quality, discussions among military men that convey praise or censure towards others are prohibited and all discussions of private and personal affairs of brother officers in public is forbidden to officers of the military service. There is, in addition, a prohibition against attempting to use personal influence for the promotion of legislation favorable to the officer, or to the end of securing special 
favors with regard to promotion or assignment. This latter is especially known as "exercising pull." Whilc the prohibition has generally been in effect, it has not always been heeded and a fruitful cause of differences and heart-burnings among military men is the suspicion that while some persons are prevented from using the influence derived from various sources in securing particular favors, other persons have evaded such prohibitions with results satisfactory to themselves. This is a complaint which is historical in military service, but it may be said to be less prevalent at the present time than in previous history. Frequent and drastic orders have been passed by the War Department against officers seeking to use such influence, and with some measure of success.

The most cherished prerngatives of the line of the army are those of rank and precedence. These relate not only to the person, indivichally, but to the various arms of the service. The precedence of the different branches is as follows, and is observed in the assignment of troops to positions on occasions of ceremony. Infantry has the right of the line and the head of a column, followed by light artillery, and this arm by cavalry. When heavy artillery parades as infantry, it is posted as infantry, while dismounted cavalry and marines are posted on the left of the infantry, the right of the line and the head of the column being understood as the positions of honor. Engineer troops, which parade as infantry and the Signal Corps are both entitled to positions on the right of the line or at the head of the command to which they are attached, thus giving them the arlvantage over other arms of the service, this position. however, being lue to the nature of service performed which required them to go in advance of the strictly fighting branches. Regulars, volunteers and militia are posted in the order named, volunteers meaning bodics of the volunteer troops of the States or the militia which have been resularly enrolled in the Army of the United States. When large bolies of tronps are to be reviewed, the commanding general has discretion as to the assignment that may be made but this is senerally exercised in making subdivisions in which the various classes are arranged as before stated. The commanding general will, however, unless he is singularly callous to criticism be careful in his arrangement to ascertain the relative rank of the 
officers in command of the different bodies of troops to be reviewed, so that an officer of the same grade with a senior commission shall not be subjected to the humiliation of following in parade an officer of the same grade who is his junior, or an officer of a junior grade.

Military rank confers eligibility to exercise command or authority which is the particularly prized prerogative of the military person. It is therefore held to be an act of gross in justice to so assign a military officer that he is not able to exercise the command and authority to which he is entitled by his grade and by his position in that grade.

At the present time the position of Lieutenant General having lapsed, the senior officer of the Army of the United States is the Major General holding the oldest commission. The command of a Major General is four regiments or a military department, and he has as a personal staff three aides taken from the Captains or Lieutenants of the army. A Major General has supreme command within his division or department and all orders relative to the affairs under his direction are forwarded through him, and all reports and communications from subordinate officers to the War Department must be also forwarded through the Major General. He directs all matters relative to the exercise, discipline and maintenance of the troops within his department, subject only to such special provisions as may have been made for the operation of the staff departments, although he is, as well, the supervising officer of such operations within the limits of his command and their immediate director. He is by no means independent in the matter and must not take such measures as would seriously interfere with the general plans which have been developed by a particular staff department and by the general staff in Washington with a view of creating a uniform administration of military affairs throughout the United States. A general officer in command of a department, however, is given considerable freedom as to recommendations and nakes an annual report to the department, such report containing an expression of the officer's views, and sometimes a frank criticism of existing conditions. At the same time the officer in command will be careful not to allow his personal views to bring him into conflict with the department for the reason that he would have a difficulty in estab- 
lishing the superiority of his own views as against those who have a gencral directive power.

The appointment of the Major Gencral is effected by selection from the officers of the army. Any officcr, in the discretion of the President, may be appointed to such a position. The principle generally approved by military men, is that promotion and sclection should be made strictly in accorlance with seniority.

The Adjutant General's office of the War Department prepares each year a list of the officers of the army arranged in accordance with their grade and in the strict order of the dates of their commission. In case of a vacancy, it is the contention of those who favor promotion in accordance with seniority that the next man on the list should be promoted to the vacancy. Such a method of promotion is in effect, under the law up to and including the grade of colonel. When it comes to the appointment of a general officer, however, no restriction is made in regard to the matter. It has been usual to take the Brigadier Generals from the list of Colonels and the Major Generals from the list of Brigadier Generals. The strictly military rule would be to take the senior Colonel for a vacancy among the Brigadier Generals and the senior Brigadier General for a vacancy in the list of Major Generals. It has sometimes appeared, however, that the senior officer was not the best fitted for the higher position and the rule of strict seniority either had to be ignored or evaded by the appointment of the senior Coloncl or Brigadier General as the case might be and then his immediate retirement, the procedure being continued until an eligible officer was scarched on the list. Provision for the selection of the gencral officers from the list without regard to seniority was made to overcome the difficulty which frequently existed that the scnior officer selected did not share the views of others as to his incligibility and could not be compulsorily retired and would not get ont of the way by voluntary retirement.

Those who favor selection of officers for promotion point out that by the operation of selection incentive would be given to officers to become as efficient as possible with the hope of attracting attention and thus sccuring promotion. On the other hand, the advocates of promotion by scniority argue that while there may be some defects in their system in the temporary scrvice of an officer in a 
grade higher than that for which he is fitted, this disadvantage is not so serious as the disorganization which would result from the selection of officers for promotion by reason of personal favoritism. It is argued that in a policy of general selection for promotion, the prohibition against the use of personal influence would work still farther to the disadvantage of some and to the advantage of others. Efforts have been made to secure legislation looking to a system of selection for promotion, but up to the present time have been unsuccessful as they have aroused a marked opposition on the part of the majority of the officers themselves.

Previous to the passage of certain recent legislation, promotion in the different staff corps was strictly confined to such corps, with the result that there was a considerable degree of stagnation in the promotion of officers doing staff duty. By the selection of the ranking officers for promotion in the line the flow of promotion in the staff corps except those which are specifically prevented from having command of staff trcops is stimulated and at the same time younger men have been secured for the heads of the staff departments.

A Brigadier General performs similar duties to those performed by a Major General except that his commands are less important. His command of troops is two regiments and he may command a small department or a subrivision of a department. He is allowed two aides who are to be taken from the Lieutenants of the army. The grade of Brigadier General is conferred by brevet or by virtue of the office on officers who have independent assignments not connected with the command of troops but which require them to issue orders to other officers of the army, and the additional rank of Brigadier General is conferred upon them in order that there may be no question as to their authority and command in issuing such orders and directions.

The regimental organization is headed by the Colonel who is in command of the regiment and directs all matters pertaining to its affairs. Colonels also command the more important posts and may have with them at such posts their entire regiments or a portion thereof. The Lieutenant Colonels are the under-studies of Colonels, taking their places in command of their regiments whenever the Colonels are incapacitated from service, and performing such other 
duties as may be assigned to then by the head of the regiment. When a Colonel commanding a post has his full regiment present, the Lieutenant Colonel exercises functions not wholly different from those performed by the Vice-President of the United States outside of his duties as presiding officer of the Senate. When the regiment is divided, however, the smaller portion may be in command of the Lieutenant Colonel, or the latter may be on the detached duty and the subdivision commanded by a Major. The appropriate command of a Lieutenant Colonel is a battalion or squadron and the distinction between the two largely lies in the fact that the Lieutenant Colonel is generally assigned larger section numerically than a Major.

Colonels, Lientenant Colonels and Majors exercise their authority and command largely through the Captain of the Company who is the direct administrative officer in clarge of the enlisted men. The former are called field officers and are mounted at all times. Captains of infantry and of troops organized as infantry are, however, compelled to march on foot mless they are on detached duty and assigned as Captains mounted. The Captain of a company is required not only to drill his men and to discipline them in the art of war, but is expected to oversee all the details relating to their shelter, clothing and food. He is in charge of the funds which accrue under the regulations and which are applied to the various purposes for the benefit of the enlisted men, and is held strictly responsible for the proper custody and expenditure of such funds. A captain may be in command of a small post, and when in such command has, in adlition to his own duties, the general duties of field officers when post commanders. Under such circumstances, however, the more immediate duties of the Captain with regard to the company are assigned to the Lientenants.

Among other duties of a commander of a post or other independent section of the army is that of supervising the arrangements of the post or company store at which gools are kept for sale to the officers and men at cost prices. This system places upon the officers the responsibility of a commercial enterprise which is frequently of considerable importance. In addition, under modern requirements of military science the commanding officer must pay much attention to the physical welfare of the men in the way of 
seeing that they engage in sports and exercise, and is expected to make the condition of military life as homelike and comfortable as possible. At the present moment, it is found difficult to secure recruits for the army and to keep up the enlisted strength. Since the Spanish-American war, conditions at most of the army posts as to the comforts of living have been revolutionized. The old uncomfortable, and in many cases tumble-down quarters and barracks have been replaced by modern buildings and equipment of gymnasiums, reading rooms, and amusement halls has been provided. At the same time the utmost care has been exercised to procure the proper preparation of the rations of the army and the staff departments have been incessantly at work providing new and more comfortable clothing and equipment. On the military side, however, there has been an anxiety lest the army fall into the somnolent condition which was the fact before the Spanish-American war, and there has been a good deal of hard work undertaken in the military line, possibly more than could have been expected of the enlisted men. Frequent and forced marches with annual manœuvres under field conditions and a general expectation that the enlisted man should improve himself have commended themselves to those who have charge of the efficiency of the army and have undoubtedly improved the technique of the soldiers of the United States. The army lost a very considerable proportion of its veteran soldiers in the years immediately following the Spanish-American War and the recruits who came in at that time have been turned into very creditable and modern soldiers. But the army at the present time offers neither the excitement of the old Indian fighting life nor a surcease from the strenuous occupations of civilian existence so that complaint is made that enlisted men cannot be secured, or if secured cannot be retained beyond the period of the first enlistment. For the purpose of making enlistments in the army more attractive it is now proposed to modify some of the requirements of drill and military work and also to relieve the soldiers from the pick and shovel work in the vicinity of the posts of which they have had to do a considerable amount owing to the necessity for grading the new roads in the different reservations where new construction has been undertaken. It is also proposed to increase 
the pay of enlisted men materially and to add further to the comfort of life in post and in camp.

The First Lientenant in the army is the exceutive officer of the company commander in exercising and caring for the troops. He may also be in command of a company in the absence of a Captain and has in general the direction of the first platoon or subdivision of the company, the inmediate charge of the balance being given to the Second Iicutenant who is generally a recent graduate from the military academ! or a recent appointee from civil life, cspecially under recent conditions of rather rapid promotions, and who is serving his apprenticeship in the military science.

Next below and ranking with Second Lientenant are the veterinarians attached to the cavalry services and artillery. Two such officers are anthorized for each regiment and reccive the pay and allowances of Second Lieutenants mounted. In addition to these veterinarians civilian veterinarians to the animals of the army are provicled for the Quartermaster's and other departments at salaries of not cxceeding $\$$ ioo a month.

Appointments as Second Lientenant in the line of the army are made from grarluates of the military acadeny as long as there are any such who have been massigned to duty. When there are more vacancies as Second Iieutenant than can be filled by appointment from graduates of the military academy, ummarried soldiers under 30 years of age who are citizens of the United States and who are physically sound and who have served not less than two years in the army are permitted to take an examination under such circumstances as the President may direct and if they pass the exami. nation may be commissioned as Second Lieutenants in the order of merit determined by the results of the examination. Provision is also made, and advantage has been talien of the provision, for the selection from civil life of persons who apparent!y have the qualifications to make successful officers in the army and these are given examinations for appointment when there are vacancies to be filled which cannot be filled by the two methods described previously. A considerable number of officers of the army have secured entrance by appointment from civil life under these conditions. The greater number of such appointments having been made of persons who 
served in the volunteer army during the Spanish-American War an the operations which inmediately succeeded it.

Examinations for the promotion of the enlisted men to be commissioned officers are carried on in two steps. A preliminary examination is ordered by each department commander annually and enlisted men who after a rigid examination into their character, capacity and qualifications are certified as eligible for examination, are sent up for a final competitive examination by a board of five officers convened at the War Department in Washington $\mathrm{a}^{\text {a }}$ the fall of each year. Civilians to be eligible as candidates for appointment as Second Lientenants must be between twenty-one and twenty-seven years of age, ummarried and citizens of the United States. If a graduate from a military school, a civilian candidate must present a diploma or if he has been a nember of the national guards must present recommendations from his superior officers of the organization in which he served.

Cadets of the Military Academy located at West Point are appointed wholly by the President. The number consists of one from each Congressional district, one from each territory, one from the District of Columbia, two from each State at large and thirty from the United States at large. All except those appointed at large from the United States must be actual residents of the subdivision from which they are appointed. While the power of appointment is vested in the President, he exercises this power largely upon the recommendation of Senators and Representatives in Congress, so that while these appointments are not, as in the case of the appointments to the Naval Academy, a portion of the patronage of the members of the Congress, the President, except in unusual instances, accepts the recommendation of the Senator or Member, and the places representing States and districts are really at the command of Congressmen. Candidates for appointment to the academy must be of such age that they will be between the ages of seventeen and twenty-two years when they are admitted to the academy. Appointments are made a year in advance of admission, so that candidates may have time to prepare themselves in the requirements established by the Secretary of War as a condition to their admission to the academy. Such requirements 
besides those relating to physical condition and moral character are satisfied by a certificate of a school in good standing that the candidate has been prepared to take up the course of instruction which is established for the military academy. Cadets are required to engage to serve eight years including the period of their instruction unless they are sooncr discharged. The organization of the Military Academy includes a Supcrintendent, who is an officer of the army designated by the President and having the rank of Coloncl of Engineers and a Commandant of Cadets with the rank of LieutenantColonel, together with such number of instructors and professors as is provided for by law, or as may be detailed by the Sccretary of War for the purpose of instruction. The cadets are organized into a military battalion and receive instructions from civilian professors and from officers of the army detailed for the service in all branches of military science and in mathematics and modern languages. Cadets receive $\$ 540$ a year and one ration a day and upon graduation receive the pay of a Second Lieutenant until they shall have been appointed and qualified as Second Lientenants in the army. A board of visitors composed of persons serving without compensation, but who have their expenses paid, is appointed annually, seven non-official persons appointed by the President, two members of the Senate and three of the House of Representatives constituting the board, which makes an annual visitation to the Military Academy and an examination of the course of studies pursued and the results attained.

The ranking officer of the enlisted force of the army is a non-commissioned official having the title of Sergeant-Major Regimental, being the immediate assistant of the regimental Adjutant in charge of the details relating to the formation of the regiment, the muster rolls and similar documents relating to the regimental organization. Other non-commissioned officers are provided for in the higher grades in accordance with the order of precedence followed in the description appended.

Quartermastcr Sergeants are in charge of the actual work relating to the activities of the Quartermaster's department with reference to regimental organization and the same is true of Commissary Sergeants with relation to the issuance of rations. Ranking below the foregoing regimental officers are Ordnance Sergeants, Post Commissary Sergeants, Post Quartermaster Sergeants, Electrician Ser- 
geants, Hospital Stewards, First Class Sergeants Signals Corps, Chief Musicians, Chief Trumpeter and Principal Musician, all of whom are on a footing with regard to pay and emolument and who perform the duties indicated by their titles as immediate superiors to the enlisted men or the non-commissioned officers of lower grade, who may, either by permanent attachment or by temporary detail, be engaged in the performance of the duties of the particular branch of army work indicated. Thus Ordnance Sergeants have general charge of all the ordnance and ordnance stores located at the point to which they are assigned for duty. The Post Quartermaster Sergeant and Commissary Sergeants have similar duties respectively to the corresponding officers attached to regimental headquarters. An Electrician Sergeant is a superior artificer engaged in the operation of the many forms of electrical machinery now installed, especially in connection with coast fortifications. The Chief Musician is the band leader, and the Chief Trumpeter the head of the field music, while the other titles are self-descriptive.

Inferior to the officers named are the Sergeants Major of squadrons and battalions, and below them in rank to them, the First Sergeant of companies and drum majors. The First Sergeant of a company is the immediate directing officer of enlisted men and is responsible to the commissioned officers for the conduct and condition of the men of the company. He must see that the orders given by the officers are carried out, and that the public property is not destroyed or wasted, that good order and discipline are maintained among the men, and that no proceedings or practices go on that are subversive to the efficiency of the service. He lives in the barracks or camp with the men, and they are at all times under his eye and supervision. He is expected to undertake the preliminary drills of recruits, and to direct the daily exercises of the men and the character of a company in every respect is largely molded and formed by that of the First Sergeant. Something of the same nature is true of the Sergeants and Corporals respectively who have the charge of subdivisions of the company, the smallest subdivision being the squad in charge of a corporal. Lance Corporals or acting corporals are enlisted men temporarily detailed to perform the duties of noncommissioned officers, but who have not yet received their formal appointments in such position and who are on probation. 
STRFXGTI OF 'THF AKNY.

General Officers.

Aides-de-Camp to General Ufficers.

Adjutant General's Department

Inspector Ceneral's Department.

Judge Advocate General's Department.

Ouartermaster's Department

Subsistence Department...

Medical Department.

Pay Department .

Corps of Engineers ....

Bureau of lnsular Affair.

Chaplains.

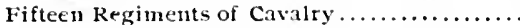

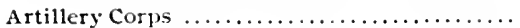

Thirty Regiments of Infantry ...............

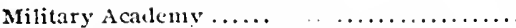

Enlisted Men unattached to regiments.

Indian scouts.

Grand aggregate

Retired officers

Retired Enlisted Meu.
IIUITARY

ACADL:MY.

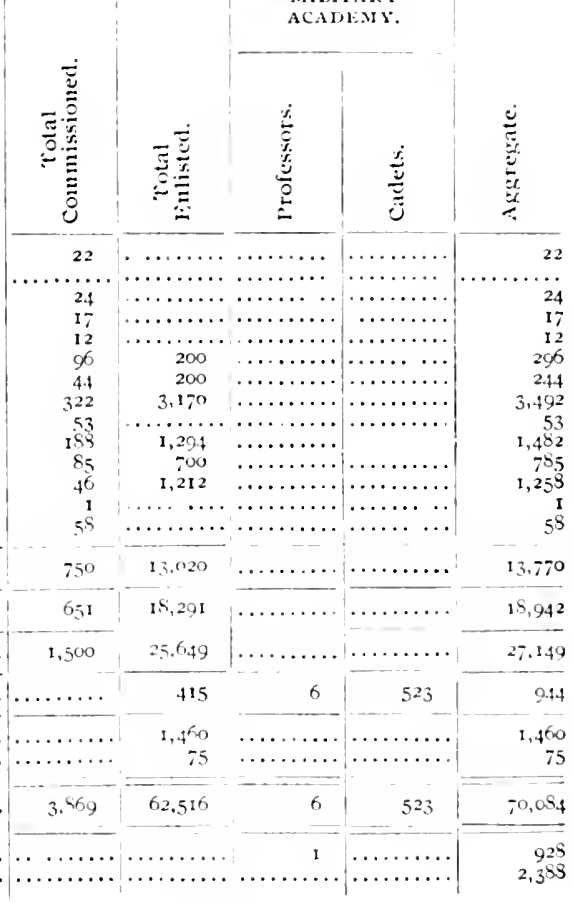

NUMBLR OF COMMISSIONED OFFICERS IN TIIE DIFIERENT GRADHS.

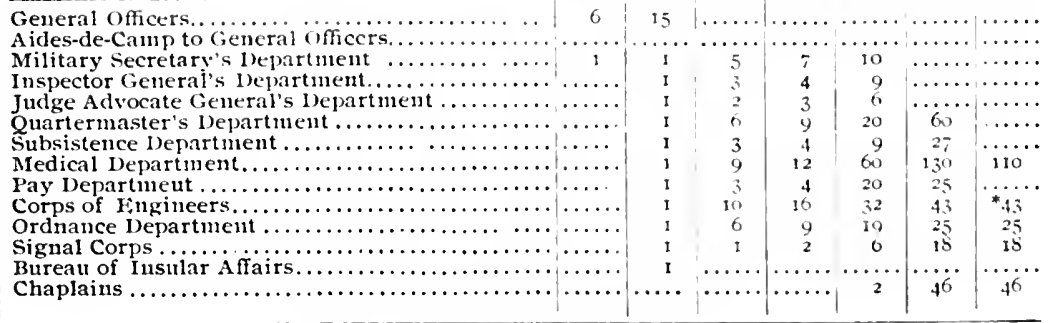

* And forty-three Second I,ieutenants. 


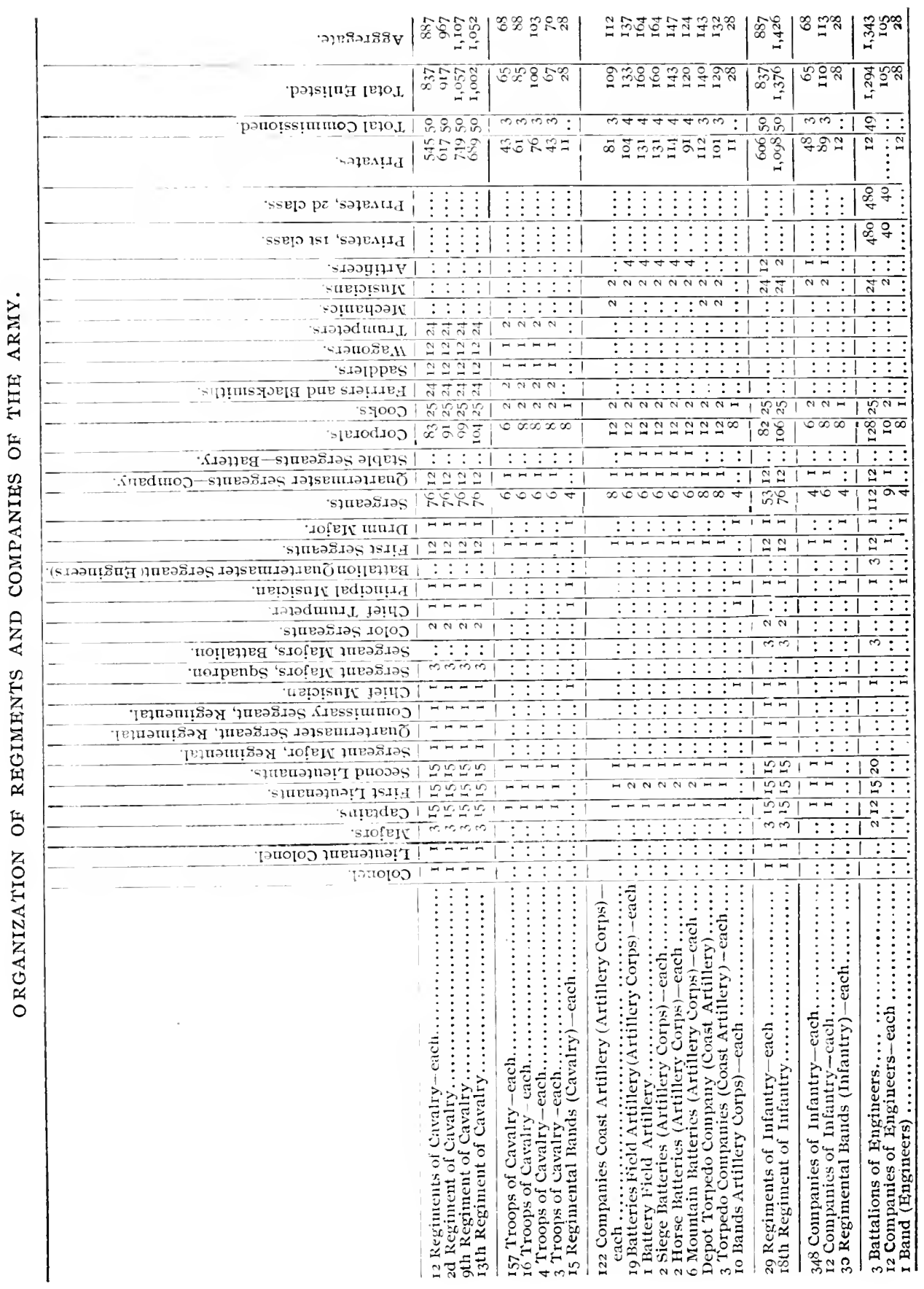


ORGANIZED STRENGTH OF THE MILITIA OF THE SFVERAI. STITES AND TERRITORIFS.

\begin{tabular}{|c|c|c|c|c|c|c|c|}
\hline & & & Ore & NIZEL & 2u:Trti. & & \\
\hline $\begin{array}{c}\text { STATES ANU TERRI- } \\
\text { TORIES. }\end{array}$ & 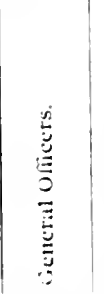 & 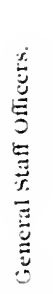 & 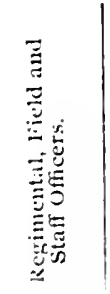 & 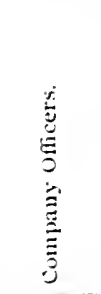 & 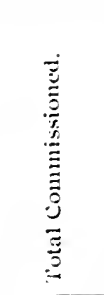 & 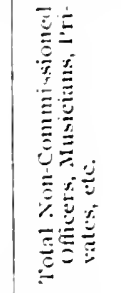 & 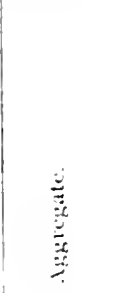 \\
\hline 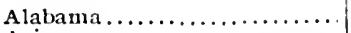 & 1 & 22 & 43 & I 28 & 194 & $1,8,1$ & 2,035 \\
\hline 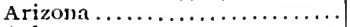 & $\ldots \ldots \ldots$ & 2 & 7 & 19 & 25 & 303 & 331 \\
\hline 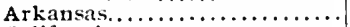 & 3 & 37 & 30 & 65 & 1.35 & 992 & 1,127 \\
\hline 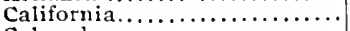 & 2 & 19 & 90 & 159 & $2 \% 0$ & 2.759 & 3.059 \\
\hline 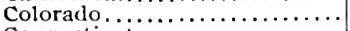 & I & 17 & 13 & 35 & 66 & .533 & 599 \\
\hline Connecticut ............... & I & I6 & 47 & 132 & І96 & 2.529 & $2,-25$ \\
\hline Delawate $\ldots \ldots \ldots \ldots \ldots \ldots \ldots$ & $\ldots \ldots$ & 7 & 13 & 19 & 39 & 3,30 & 369 \\
\hline District of Columbia.......... & I & $1 \mathrm{I}$ & 47 & $\$ 9$ & 105 & 1.170 & 1,$2 ; 5$ \\
\hline 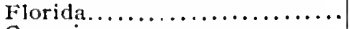 & 1 & 13 & 32 & 49 & 95 & 1,025 & 1,120 \\
\hline 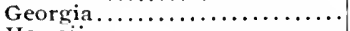 & $\ldots \ldots$ & is & 59 & 156 & 262 & 2,405 & $2,-760$ \\
\hline 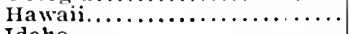 & $\ldots \ldots$ & 6 & Io & IS & 34 & $3^{42}$ & 416 \\
\hline Idaho $\ldots \ldots \ldots \ldots \ldots \ldots \ldots$ & $\ldots \ldots \ldots$ & 6 & 15 & .3 .4 & 55 & 615 & 673 \\
\hline 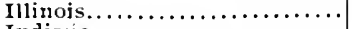 & 4 & 3i & 161 & 293 & 490 & 5.133 & 5.929 \\
\hline 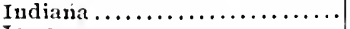 & I & Io & 60 & 100 & 171 & $1 ., 25$ & $1.0 \%$ \\
\hline Iowa $\ldots \ldots \ldots \ldots \ldots \ldots \ldots \ldots \ldots$ & $\ldots \ldots$ & 15 & $6 \mathbf{I}$ & 127 & 206 & 2.403 & $2,(x) 9$ \\
\hline 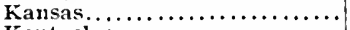 & I & 16 & 33 & -6 & 126 & 1.020 & 1,155 \\
\hline 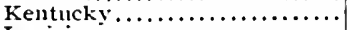 & $\ldots \ldots$ & 8 & 57 & 77 & 1.12 & $1,2,30$ & 1,372 \\
\hline 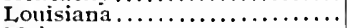 & $\ldots \ldots \ldots$ & 1 & 25 & 71 & 97 & 1,424 & 1,521 \\
\hline 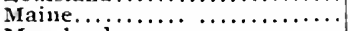 & $\ldots \ldots \ldots$ & $s$ & 30 & 62 & 100 & 1,156 & 1,251 \\
\hline 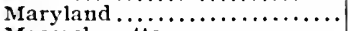 & 1 & 2.3 & 4) & $10 n$ & 162 & $1,7+0$ & $1,2: 2$ \\
\hline 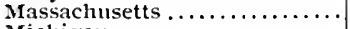 & 2 & 40 & 1.4 & 247 & .437 & 5.131 & 5,563 \\
\hline 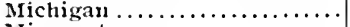 & I & 29 & 44 & 126 & $2(x)$ & 2.467 & 2,607 \\
\hline Minnesota..$\ldots \ldots \ldots \ldots \ldots \ldots$ & I & 2.4 & 52 & S.5 & 162 & 1,$1 ; 6$ & $1.90 s$ \\
\hline Mississippi................ & J & 17 & 23 & 70 & $11 \mathrm{~s}$ & $1,14:$ & 1,262 \\
\hline 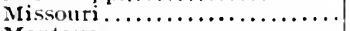 & I & 1.7 & 62 & 12.4 & 201 & 2.052 & 2,253 \\
\hline 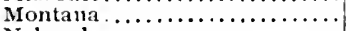 & $\ldots \ldots$ & sis & 13 & 20) & $4 \mathrm{I}$ & $3 \%$ & $\$ 21$ \\
\hline 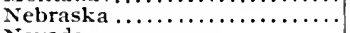 & $\ldots \ldots \ldots$ & 6 & 22 & so & 105 & 1,293 & 1,401 \\
\hline Nevada.................. & $\ldots \ldots \ldots$ & I & $\ldots \ldots \ldots$ & 6 & 7 & 1.34 & $1.4 \mathrm{c}$ \\
\hline New IIampslite............. & 1 & 15 & 26 & 60 & I I & I.I.3 & $: 2: 2$ \\
\hline New Jerscy .............. & 3 & 43 & so & $19 l$ & 317 & 4,000 & $4.3 \mathrm{n}$ \\
\hline New Mexico............... & $\ldots \ldots$ & 3 & I6 & 17 & 36 & 202 & 203 \\
\hline New York. ................ & 5 & 6.1 & 505 & 53.4 & wan & $1,2.109$ & $1:, 017$ \\
\hline Forth Carolina ............. & 1 & 44 & 4.5 & 111 & 26 & 1. heil & 1.67 \\
\hline 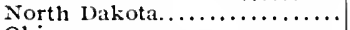 & $\ldots \ldots \ldots$ & 7 & 1.4 & $\therefore$ & 54 & 57 & 6,5 \\
\hline Ohio $\ldots \ldots \ldots \ldots \ldots \ldots \ldots \ldots$ & 3 & 5.4 & 101 & $2(x)$ & $44^{5}$ & 5,711 & $5.45 y$ \\
\hline Oklahona $\ldots \ldots \ldots \ldots \ldots \ldots$ & $\ldots \ldots \ldots$ & $\because$ & 17 & .10 & 64 & $-0 ;$ & 771 \\
\hline Oregon $\ldots \ldots \ldots \ldots \ldots \ldots \ldots \ldots$ & $\ldots \ldots \cdot$ & II & $\therefore$ & 51 & 4 & $0-4$ & $2, \mathrm{Ne}_{3}$ \\
\hline Pennsylvania $\ldots \ldots \ldots \ldots \ldots \ldots$ & 4 & 44 & 210 & $f^{\prime \prime 2}$ & .25 & 9,1517 & 9.44 \\
\hline 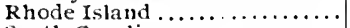 & 1 & 20 & 33 & $(x)$ & 120 & (2:th & $1,0 \times 6$ \\
\hline 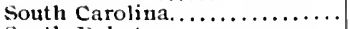 & 1 & 25 & 52 & 10 & 174 & $1 . .5 y 1$ & $1,6+5$ \\
\hline 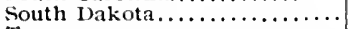 & $\ldots \ldots$ & $2 \mathrm{I}$ & 23 & 13 & 37 & 1,70 & -25 \\
\hline Tennessee. . . . . . . . . . . . & $\ldots \ldots \ldots$ & 6 & \$i & 94 & 151 & 1,012 & $1 .-63$ \\
\hline Texas $\ldots \ldots \ldots \ldots \ldots \ldots \ldots \ldots$ & 3 & 45 & 52 & 121 & $22 \mathrm{I}$ & $1,0,1$ & 2,152 \\
\hline Utah $\ldots \ldots \ldots \ldots \ldots \ldots \ldots \ldots \ldots$ & $\ldots \ldots$ & 1 & 6 & .80 & 37 & .800 & 30 \\
\hline Vermont ... $\ldots \ldots \ldots \ldots \ldots \ldots$ & $\mid \ldots \ldots \ldots$ & 4 & I & 36 & 53 & 703 & 701 \\
\hline Virginia................. & $\ldots \ldots \ldots$ & 2 & 50 & ii 2 & 170 & $1, x 3$ & 2,133 \\
\hline 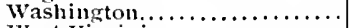 & $\ldots \ldots \ldots$ & (1) & 1.3 & 36 & 55 & 643 & 734 \\
\hline West Virginia.............. & I & 12 & 30 & oi & I 10 & Q)ir & 1,011 \\
\hline 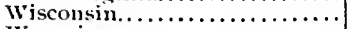 & $\ldots \ldots$ & $s$ & 47 & 1.36 & 101 & $2,-7,6$ & 2. 407 \\
\hline 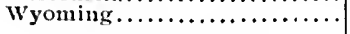 & $\ldots \ldots \ldots$ & 5 & 10 & 20 & 35 & \$1s & $\therefore 53$ \\
\hline Grand aggregate........ & 46 & 853 & $2,50 \%$ & 5,136 & 8,622 & $9 \pi, 071$ & $105, f(x) 3$ \\
\hline
\end{tabular}


THE PAY OF THE ARMY.

It is a matter of nice, if not altogether material, speculation as to whether the intricacies of the military pay have brought about the need of a corps of specially trained experts for its adjustment, or whether the specially trained experts have brought about the intricacies of military pay. Whatever may have been the fact as to the cause, the effect has been to put it almost hopelessly beyond the power of the civilian mind to understand all that is comprehended within the term "army pay." This being so with regard to the pay of the army, it is triply so with regard to the pay of the navy, in which branch of the service even pay officers decline to make any general statement as to the pay which officers in a particular grade may be receiving, and will only make definite statements when furnished with all the facts to which can be applied the various enactments made by Congress from time to time. Army pay is believed to be by military pay officers a comparatively simple system, but it is accompanied by so many provisos that no single table gives more than an approximate idea of the amounts received by different officers. The following table presents the base pay of commissioned officers in the army, together with the calculation of the increases produced by the provisions of law that officers in active service shall have ten per cent. increase for each five years of service. A maximum is fixed, however, for the pay of Colonels at $\$ 4,500$ a year and of Lieutenant Colonels at $\$ 4,000$ a year, above which their longevity pay cannot increase their total pay, no matter how long they may serve in the grade named after reaching the maximum. Retired officers also are not entitled to an increase of pay on account of longevity after they have been retired, unless their retirement was on account of wounds received in battle. In addition to the foregoing, officers detailed as aides in other special positions are entitled to increases of pay while so serving, and officers serving outside the United States, except in Porto Rico or Hawaii, receive ten per cent. in addition to their base pay. Actual expenses are allowed to all officers of the army traveling by sea, and seven cents per 
mile, with a reduction of three cents per mile on bond-aided or land-grant railroads is allowed for land travel. In addition to this allowance, officers are provided with quarters at the rate of five rooms and kitchen for a Lieutenant General or Major General, four rooms and kitchen for Brigadier General or Colonel, three rooms and kitchen for Lieutenant Colonel or Major, two for Captain or Chaplain, and one for a Lieutenant, with a kitchen added in both of the last cases. The more important of the non-commissioned officers are also allowed one room as quarters. In case such quarters cannot be furnished in buildings belonging to the Government, commutation is allowed at the rate of \$12 per month for each room. Besides allowance for quarters, allowance is made for fuel in graduated amounts according to the rank of the officer, his location and the season of the year, when fuel cannot be furnished in kind; besides which the mounted officers are entitled to forage for their horses either in kind or in allowance, so that in order to determine the amount of money which will accrue to any particular officer upon any particular pay day it is necessary to consider his grade, the kind of service he is on, where his service is located, and the various other details indicated by the foregoing before the amount of his emoluments can be made up. 


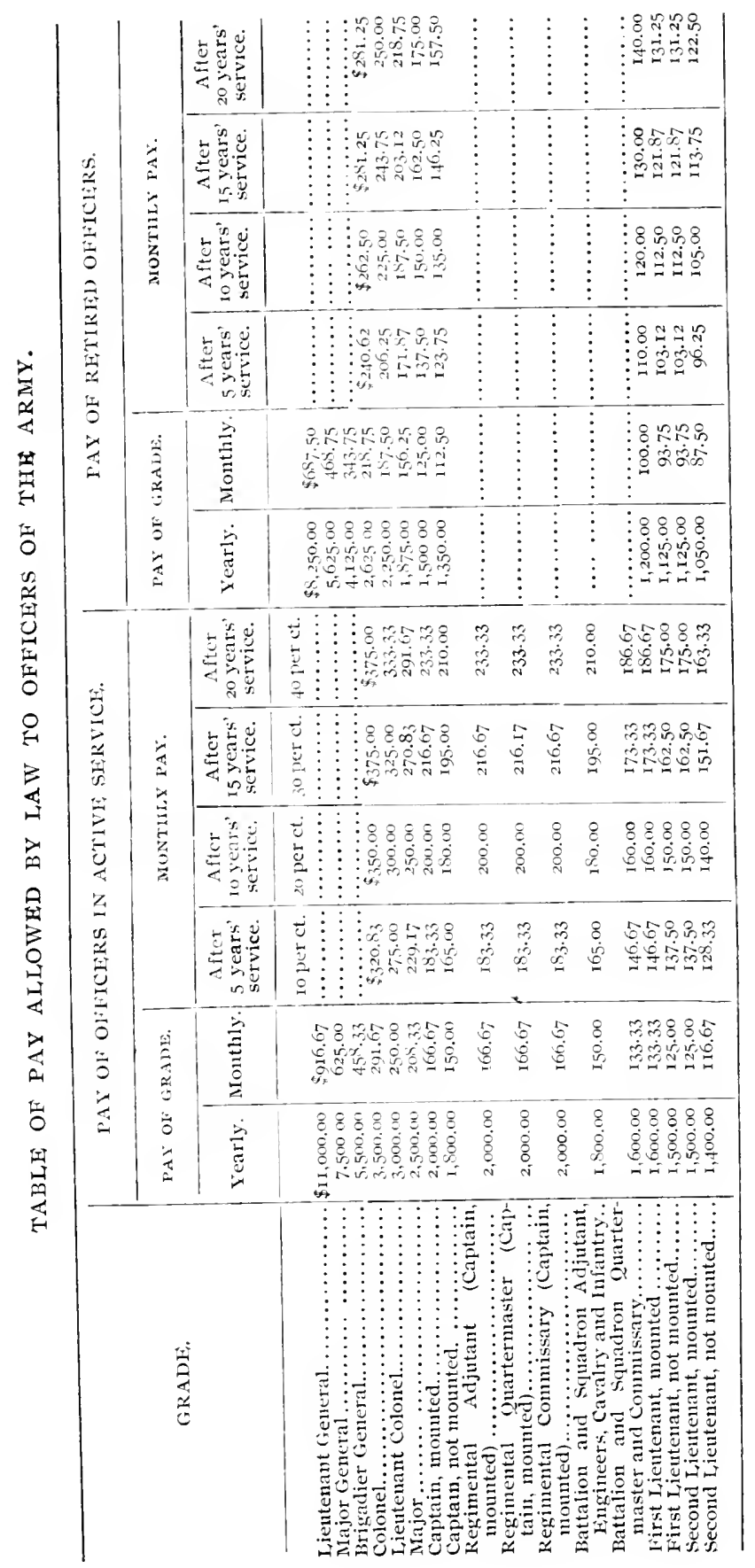




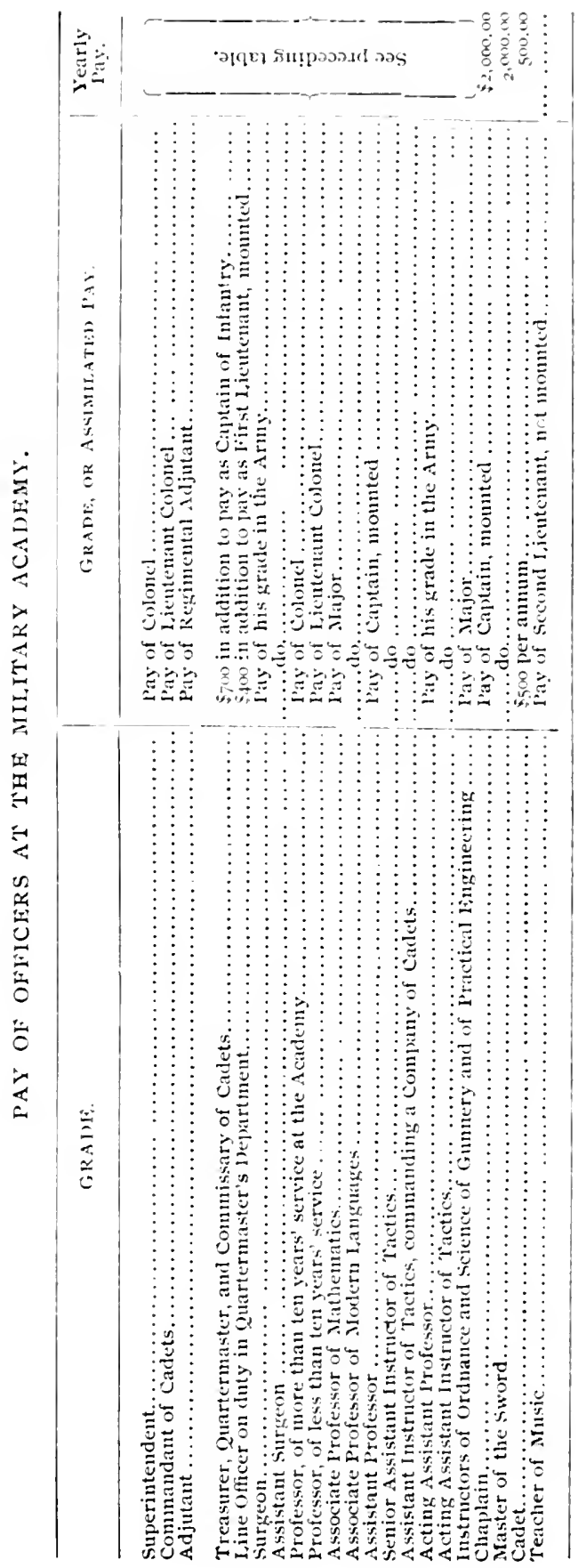


The table of the enlisted men is comparatively simple, but is also subject to various provisos. Thus a certificate of merit entitles a soldier to two dollars a month additional pay and twenty per cent. increase in pay is given to all enlisted men serving outside the United States. Veterinarians in the army are also on the same footing as officers in matter of pay and allowances, are not entitled to a ten per cent. increase in pay for foreign service.

Enlisted men retired are entitled to three-fourths of the monthly pay of the grade they held when retired, and also have an allowance for commutation of clothing and in lieu of rations. Rations are furnished in kind to enlisted men when they are serving where issue may be made by the regular commissary officers, or they are allowed commutation or payment in money for the same when they are serving away from their regular organization. An allowance is made annually for clothing, amounting to the equivalent of one uniform with an appropriate amount of accessories for each year; but soldiers are required to keep themselves at all times in clothing and, if necessary, at their own expense. Clothing may be drawn in kind as the credit accumulates, or balances may be carried over from time to time, to be settled finally upon the discharge of the soldier. The enlisted men are charged with the articles which they may desire to complete their uniform outfit, as they are drawn from the quartermaster's department, the debits being first charged against their clothing allowance, and if the clothing allowance is exhausted the balance is charged against the pay accounts. The balances of clothing accounts are taken twice a year, and the balances which may be in favor of the soldier are carried forward to the next half year; and upon discharge of the man the amount to his credit, if any, is added to his general credit account and paid to him in cash. Army pay without qualification has been extended to cover the pay and emolument of commissioned officers and enlisted men of the marine corps, and enlisted men of the Porto Rico provisional battalion and the Philippine scouts also receive the pay and emoluments of enlisted men of the army. Army pay also applies with certain modifications, which are described in the appropriate place, to the pay of the 
navy at the present time, having been substituted for the older system of navy pay.

In addition to the allowances previously described, enlisted men of the army, when discharged, except in cases where the discharge has been by way of punishment, are allowed transportation money at the rate of four cents a mile for land travel, and actual transportation expenses and subsisience for sea travel. Travel in the Philippine archipelago, the Hawaiian archipelago, the home waters of the United States and between the United States and Alaska is regarded as land travel. 


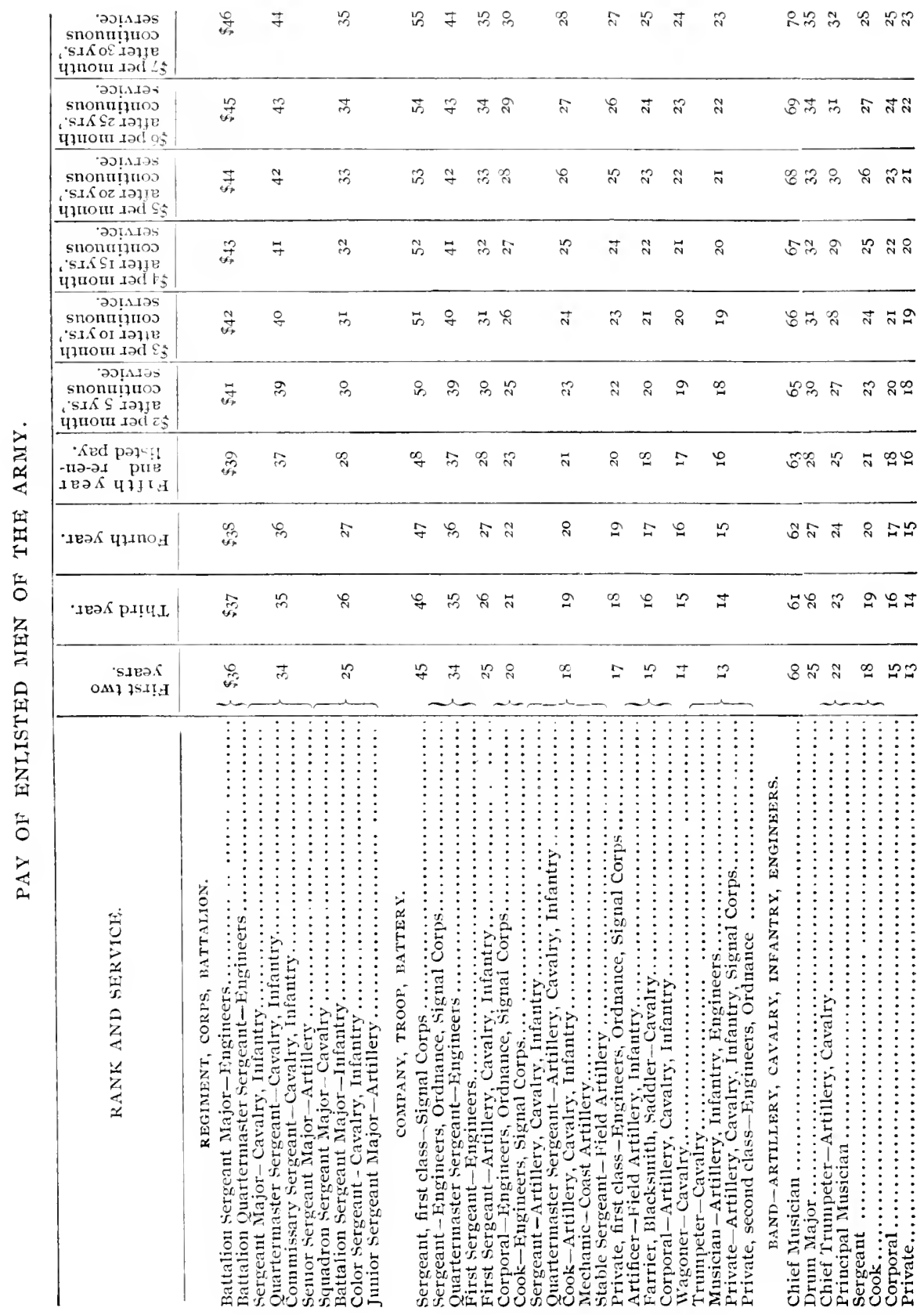




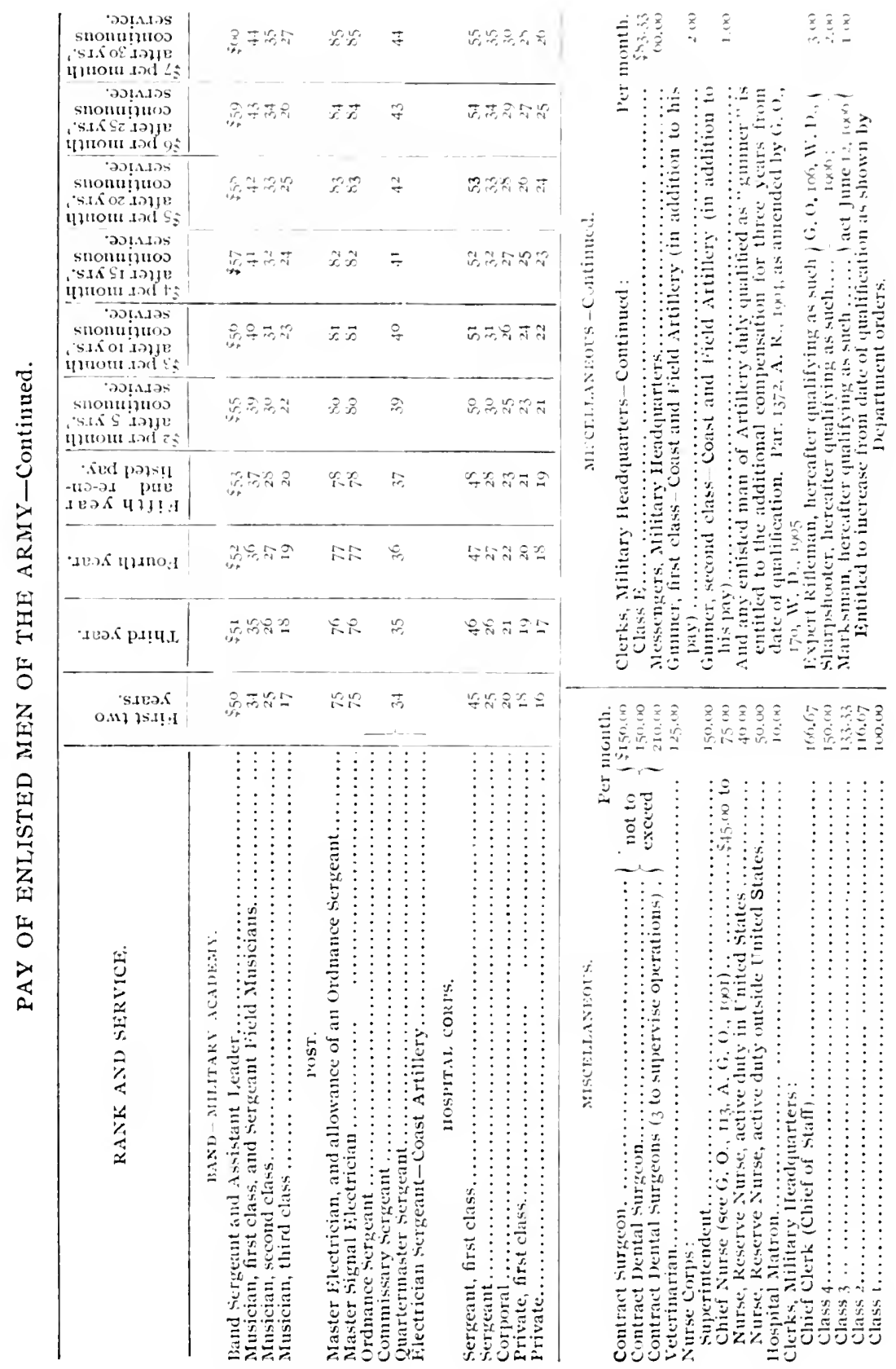


PROMOTION AND RETIREMENT OF OFFICERS.

Under the requirements of the law, all officers of the army below the rank of Major are required to undergo an examination to determine their eligibility for promotion. If any officer fails to pass a satisfactory examination and is reported unfit for promotion, the officer next below him in rank, having passed the examination shall receive the promotion in his stead. If the failure of an officer to pass is because of physical condition and he is found to be incapacitated for service by reason of physical disability contracted in the line of duty, he is to be retired in the rank to which he would otherwise have been promoted. If, however, he fails for any other reason, he is suspended from promotion for one year, at the expiration of which time if he again fails for promotion he must be honorably discharged from the army with one year's pay. Promotion is usually in consequence of a vacancy occurring in a higher grade, a vacancy in the grade of Major General, of course, maling successive promotions throughout the grades of the army. Lieutenants of the Corps of Engineers, the Ordnance Corps and Signal Corps are, however, to be promoted to be Captain after passing the examination required by law after fourteen years' service in the grade of Lieutenant. It is provided in most of the staff corps that promotions shall go by seniority and within the lists of the particular corps; that is to say, vacancies occurring in a given staff body shall not be the cause of promotion in the army generally, but that that staff shall secure the resulting promotion. In the Artillery Corps, all officers, whether connected with the coast or field artillery are placed on one list and are promoted in accordance with seniority, details being made either to the coast or field artillery in accordance with the special aptitude of the officer. For meritorious service, provision is made for an honorary higher rank, especially for service in time of war, which is conferred by a brevet commission, being a promotion to the next higher grade than the actual rank of the officer and being effective as a title only when the officer has been mustered out or has resigned from the service. The brevet rank confers no additional command, pay or emolument, and is an honorary title only and cannot be used in official communications while 
the officer is still 111 the service. The only occasions upon which officers regularly in the army can exercise the privileges of brevet rank are when, in case of actual hostilities. the President shall assign them to duty or command in accordance with such brevet rank.

It was the original intention of the legislation on the subject of retirement of the officers of the army, that only a limited number should be carried on the retired list, this legislation having been effected about the time of the civil war, when the possibilities of the retired list of the army assuming large proportions was a consideration in the minds of the Congress. An unlimited retired list with the possibility of placing the large number of volunteer officers thereon assumed, eviclently, a prospective form of a civil pension list such as has always been strenuously discouraged in the United States. The linit of the number of retired officers was at first placed at 300, but when it appeared, later, that this number did not take care of the genuine retirements from the army; the limit was increased to foo. Thus, under this legislation, no officer of the army could be retired if there were at the time officers on the retired list to the legal limit. It became apparent that any limited system would not scrve the purpose of providing for superannuated and disabled officers, so that, in IS82, additional legislation was effected vincler which, when any officer has reached the age of sixtyfour years. it is compulsory for the President to retire him and to place him upon the unlimited retired list.

There are, therefore, two lists of retired officers of the army, the limited list being confined to 350 officers at any one time, and the other containing the names of those who may be retired under the terms of the law, and who are not entitled to a place on the limited list. The limited list of retired officers consists, first, of those who are retired on account of disability: second, of those officers who have sought retirement after having served 30 years, and who, on making application to the President are placed upon the limited list; third, those officers who are over 62 years of age, or who have served 45 years as commissioned officers, and who are regarded by the President as desirable for retirement. Thus, the list is composed of those who must retire on account of disability, those who desire to 
retire, anc: have served the requisite term, and those whom the President deems it is for the best interest of the service to retire, but retirements under these circumstances cannot exceed the number of 350 officers.

The unlimited list consists of those who have been retired by special Act of Congress, of those who, having served 40 years, make application and thus effect a voluntary retirement, together with officers who have reached the age of sixty-four years and are thereupon placed in retirement by the compulsory action of the law. When an officer on the limited retired list reaches the age of 64 years, he is transferred from that list to the unlimited list of retired officers. When it appears that an officer is incapable of performing further active service, he may be directed to appear before a retiring board for examination, the board consisting of not more than nine or less than five officers, two-fifths of the board to be selected from the Medical Corps, and if this board finds an officer incapacitated for active service and such decision is approved by the President, the officer must, if the incapacity resulted from an incident of the service, and was not the fault of the officer, be placed on the retired list as retired on account of disability. If, however, the board finds that the incapacity of the officer is not due to an incident of the service, discretionary power is vested in the President to determine whether the incapacity was the result of the officer's own indiscretion, and whether the officer shall be retired from actual service or shall be entirely retired from the service. In the latter case, the officer receives one year's pay and emoluments of the highest grade he has held, and the effect of the retirement is a dismissal from the service as in the case of failure to secure promotion.

In case of retirement from the active list, the officer thenceforward receives seventy-five per cent. of the pay of the grade in which he was entitled to retire.

Under recent legislation, officers who served in the Civil War are retired in the grade next higher to that which they actually occupied, receiving a promotion on the date of their retirement, and thereafter receiving three-fourths of the pay of the higher grade. Similar legislation has also been passed affecting officers who have been retired previous to the date of the provision just mentioned, 
so that practically all officers who served during the Civil War shall receive at least one promotion on the retired list.

In order that an officer may not be retired as a matter of oppression or discipline, it is provided that no retirement shall be effected except for statutory reasons having to do with age or length of service unless an opportunity for a full hearing before a retiring board constituted as described, has been afforded the officer affected.

Officers entirely retired from the service are, of course, wholly separated from the army, and their names are removed from the army list. Officers retired from active service are withdrawn from command and from the line of promotion, but are entitled to wear the uniform of the rank in which they were retired and are borne on the army register and are subject to the rules and articies of war and to trial by general court martial for any breach of discipline. In time of war, retired officers may be employed in the discretion of the President for any duty except the command of troops, and at other times, retired officers may be detailed to serve as instructors at colleges although receiving no additional compensation therefor. Retired officers serving actively in time of war as before mentioned, receive the full pay and emoluments of their rank. Retired officers may also serve in connection with the inspection, administration and direction of the militia of the various States, but the maximum full pay which can be received for this service is that of a Major. Under similar circumstances, retired officers may also be employed by the Secretary of War in recruiting duty, upon court martial, courts of inquiry, and boards and staff duties not involving service with troops. General officers can receive only their full retired pay for such employment, but Colonels and Lientenant Colonels may accept full active pay of a Major in liew of their own retired pay.

INSIGNIA OF TIIE ARMY.

Signs and tokens are of course essential to denote military rank and command. The bearing of a leader of men may pertain as well to a private as to a general officer, and for this reason each member of the personnel of the army must have his distinctive mark. The General, Lieutenant General and Chief of 
Staff of the army are allowed to select their own widths and placing of gold lace, and the army regulations do not venture to dictate to them on these points, the only provision being as to the general character of certain distinctive marks-as, for example, that the distinctive mark of a full General shall be the coat of arms of the United States embroidered in gold on his shoulder straps with two silver embroidered stars. A Lieutenant General is distinguished by three silver stars and a Major General by two stars. For all except dress occasions the identification of the grade of an officer is most easily established by the shoulder strap before referred to and which bears the devices which are generally distinctive of the particular grade of the officer; these distinctive marks are, in addition to the above, as follows: For a Brigadier General, one star; for a Colonel, a silver embroidered spread eagle; Lieutenant Colonel, a silver embroidered leaf at each end of the strap; Major, a gold embroidered leaf at each end; a Captain, two silver embroidered bars; First Lieutenant, one silver embroidered bar; Second Lieutenant, the shoulder strap without any device. A Chaplain is denoted by a plain Latin cross in silver in the center of a strap of dark blue.

The color of the cloth of the strap differs in accordance with the branch of the army to which the officer belongs. This difference in color is also carried out in the facing of the uniform and constitutes the distinctive color of the branch of the army named. That for the staff corps is dark blue, as well as for general officers; engineers, scarlet, piped with white; signal corps, orange piped with white; ordnance department, black piped with scarlet; medical corps, maroon; quartermaster's department, buff ; cavalry, yellow; artillery, scarlet, and the infantry, light blue. In addition to the foregoing distinctive marks are adopted for each branch as follows:

General Staff Corps.-The coat of arms of the United States of gold and enamel superimposed upon a silver star.

Adjutant General's Department.-A shield of gold or gilt metal.

Inspector General's Department.-Gold or gilt sword and fasces, crossed and wreathed.

Judge Advocate General's Department.-Sword and pen in gold or gilt metal, crossed and wreathed. 
Quartermaster's Department.-Sword and key crossed on a wheel, surmounted by a spread eagle; of gold or gilt metal, platinum, and enamel.

Subsistence Department,-A silver crescent, $1 / 2$ inch between cusps, cusps to the rear.

Pay Defartucut.-A diamond, with diagonals $3 / 4$ inch and I inch in length, in gold or gilt metal, placed with slorter diagonal vertical.

Medical Department.-A caduceus, of gold or gilt metal.

Corps of Engineers.-A silver turreted castle.

Ordnance Department.-Shell and flame, of gold or gilt metal.

Signal Corps.--Two crossed signal flags and a burning torch in gold and silver.

Burcau of Insular Affairs.-A bunch of seven arrows, with wings on sides,

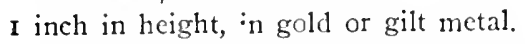

Professors and Associate Professors of the United States Military Acadcmy. -Shield and helmet surmounted by a scroll, in gold or gilt metal.

Cazaly.-Two crossed sabers, I inch high, with number of regiment above intersection; of gold or gilt metal.

Ficld Artillery.-Two crossed ficld guns I inch high, with number of regiment above the intersection; of gold or gilt metal.

Coast Artillery Corps.-Two crossed cannons I inch high, of gold or gilt metal, with oval at the intersection, having a scarlet center exluibiting an oblong projectilc in gilt outline.

Infantry.-Two crossed rifles, design to be $\mathrm{I}$ inch high, with number of regiment above intersection; of gold or gilt metal.

Aids.-A device, I I-3 inches high, consisting of a shield of the United States, of properly colored enamel, $3 / 4$ inch high and $3 / 4$ inch wide at top, surmounted by a gold or gilt eagle, with wings displayed. On the blue field of the shicld a star or stars, according to rank of the general on whose staff the officer is serving.

Distinctive marks on full dress uniforms are placed at different points and include a collar ornament consisting of a band of oak leaves embroidered in gold for general officers and two bands of gold lace other officers. General officers have cuffs of velvet on their sleeves, ormamented with a band of oak leaves in gold, and other officers a band of gold lace. Officers of the staff corps and departments wear the insignia of the corps and departments on their sleeves and officers of the line wear knots of gold lace above the sleeve band, consisting of five strands in case of a Colonel, four strands Lieutenant Colonel, three strands Major, two strands Captain and one strand First Lieutenant. 
Further differentiation of gereral officers is made by the number of buttons on the breast of their dress coats; the General being entitled to two rows of 12 in each row, a Lientenant-General two rows of ten in each row, a Major-General nine buttons in each row, and a Brigadier-General eight buttons in each row. All officers of lower rank have a uniform number of nine buttons in each row. General officers also alone wear epaulets, those of lower rank wearing shoulcier knots of gold wire. Aiguillettes, the gold cords and pencils which distinguish Adjutants and Aides, are worn only by officers of the general staff corps, of the Adjutant-General's department, Inspector General's department and aides to general officers, also regimental Adjutants of artillery districts and of engineer battalions. The Chief of Staff is also empowered to wear such aiguillettes and in such manner as he may prescribe for himself. Sashes are worn by general officers, the Brigadier-Generals wearing their sashes around the waist and the Major-General across the body from the right shoulder to the left side. On all coats except the full dress coats army officers wear the letters U. S. on each side of the collar together with the insignia of the corps department or arm of service. These are in gold, except those of the Engineers, which are in silver, with all dress except the service on which they are of dull finished bronze metal. The distinguishing insignia for the sleeves of overcoats consist of a band of mohair braid with the insignia of the rank, corps, or department, previously described, except that officers of the line wear as their distinguishing mark a knot of mohair braid corresponding to the knot of gold braid on the sleeve of the full dress coat. The uniforms required to be possessed by officers of the army consist of the full dress coat of dark blue, the dress sack coat of dark blue, the service coat of olive drab or klaki, and the white coat for summer wear. In addition the uniform for evening wear is described and provision is made for a mess jacket which is a short coat, without tails, ornamented on the sleeves with the insignia of the full dress coat and to have such further ornamentation as each particular organization may prescribe. These jackets are worn at dinner when it is the custom of the mess to require it, and the adoption of a particular device or a manner of ornamentation is a matter of regimental pride which may or may not be carried out 
according as the officers of a particular regiment may have a taste for carrying ont military traditions to the full. In order, however, that there shall not be frequent changes in the mess jacket, requiring additional expense, when the pattern is once adopted it cannot be changed without the approval of the Secretary of War and upon the request of the majority of the officers of a regiment. Besides the articles heretofore named full equipment of caps, capes, overconts and accessories is required, so that the investment of an officer in uniforms is at least from $\$ I, 000$ to $\$ I, 500$ and may well be considerably more.

Contract Surgeons in the army wear clress, service and white uniforms conforming to those of medical officers, but without the sloulder straps or insignia of rank. The letters "C. S." replace the letters "U. S." on the collar. Dental Surgeons wear similar uniforms to Contract Surgeons, but the "D. S." replace the letters "C. S." Veterinarians have uniforms corresponding to those of Second Lieutenant of Cavalry and Artillery, except the full diress uniform and wear the distinguishing mark of the foot of a horse shod with wings on the side.

The enlisted men of the army wear in connection with their uniform distinctive colors of cloth as facings on caps and in the cords of hats and in the stripe for the trousers. The distinctive colors for the different arms are as follows: Cavalry, yellow; artillery, scarlet; infantry, light blue or white; engincers, scarlet piped with white; ordnance, black piped with scarlet; post quartermaster sergeants, buff ; post commissary sergeant, cadet gray ; hospital corps, maroon piped with white; signal corps, orange piped with white, and the service detachment at the United States military academy, buff. In addition to the white the infantry color is light blue when white would be inappropriate or indistinguislable.

The various grades of non-commissioned officers and the distinct position in the artificers' service are indicated by a considerable variety of special insignia besides the regular military chevrons. Chevrons consist of narrow stripes of cloth of the color pertaining to the corps or branch of the service, worn point up midway between the elbow and shoulder, the stripe being arranged in the form of an angle. The different chevrons and forms of insignia are as follows: 
Regincntal sergeant major and sergeant major, senior grade, Coast Artillery Corps.-Three bars and an are of three bars.

Mastor Electrician, Coast Artillery Corps.-Gold wreath with silver forked lightning within.

Engineer, Coast Artillery Corps.-Ring, gold bullion; governor, silver bullion within.

Elctrician sergeant, first-class, Coast Artillery Corps.-Three bars and an arc of one bar of scarlet cloth, inclosing a representation of forked lightning embroidered in white silk.

Electrician sergeant, secand class, Coast Artillery Corps.-Same as electrician sergeant, first-class, Coast Artillery Corps, omitting the arc.

Master gunner, Coast Artillery Corps.-Gold wreath, inclosing silver projectile.

Ordnance sergeant.- Three bars and an arc of one bar, inclosing a shell and a flame.

Sergeant of ordnance.-Three bars inclosing a shell and a flame.

corporal of ordnance.-Two bars inclosing shell and flame.

First-class prizate of ordnance.-The shell and flame.

Post commissary scrgeant.-Three bars and a crescent (points to the front) ; top of crescent to be $1 / 2$ inch below the inner angle of chevron.

Post quartermaster sergeant.-Three bars and insignia of the Quartermaster's Department.

Sergeants, first class, of the Hospital Corps. - Three bars and an arc of one bar, of maroon cloth, inclosing a caduceus $\mathrm{I} / 4$ inches high, embroidered in maroon silk; the bars, arc, and caduceus to have a narrow white border.

Sergeants of the Hospital Corps.-The same as for sergeants, first class, omitting the arc.

Corporals of the Hospital Corps.-The same as for sergeants, omitting one bar.

Lance corporals of the Hospital Corps.-A chevron of one bar of maroon cloth with white border, in addition to and placed just above the caduceus tor a private, first class.

Private, first class, of the Hospital Corps.-A device consisting of a caduceus $I \frac{1}{4}$ inches high, embroidered in maroon silk, and having a white border.

Master signal electrician.-Three bars and an arc of one bar of orange cloth, piped with white, inclosing a representation of forked lightning embroidered in white silk.

Sergeants of the first class of the Signal Corps.-Three bars and an arc of one bar, color orange, piped with white, inclosing a device consisting of crossed signal flags, red and white, and a burning torch in yellow.

Sergeant of the Signal Corps.-Same as for sergeant of the first class, omitting the arc.

Corporal of the Signal Corps.-Two bars, inclosing same device as for sergeant of the first class. 
Private of the Signal Corps.-Device consisting of crossed signal flags, red and white, and a burning torch in yellow.

Regimental quartermaster sergcant.-Three bars and a tie of three bars.

Regimentul commissury sergeant.-Three bars and a tic of three bars, having a crescent (points front); top of crescent $1 / 4$ inch below the inner angle and lower point of crescent $1 / 4$ inch above the first of the tisbars.

Chicf musician.-Three hars and an arc of two bars, with a bugle of pattern worn on cap in the center.

Squadron or batfulion scrgeant major, and sergcant major, junior grade, Coast Artillery Corps.-Three bars and an are of two bars.

Color sergeant.-Three bars and a star.

Chicf trumpeter.-Three bars and an arc of one bar, with a bugle of pattern worr: on cap in the center.

Princiral musician.-Three bars and a bugle.

Battalion of Enginecrs and battalion of ficld artillery, quartermaster scrgcant.-Three bars and a tic of two bars.

First sergeant.-Three bars and a lozenge.

Drum major.-Three bars and two embroidered cross batons.

Troop, battery, or company quartermaster sergeant.-Three bars and a tie of one bar.

Sergeant.-Three bars.

Stable sergeant, ficld artillery.-Three bars and a horse's head.

Firman, Coast Artillery Corps.-Crossed pokers and shovel in red, in red ring.

Corporal.-Two bars.

Lance corporal.-One bar.

Cook.-A cook's cap of cloth, conforming in color to corps, department, or arm of service.

Farricr and blacksmith.-A horseshoe of cloth $13 / 3$ inches long and $1 / 2$ inches wide, worn toe uppermost.

Saddler.-A saddler's round knife of clotin.

Chicf mechanic, ficld artillcry.-Two crossed hammers of cloth, encircled at the base by a wreath embroidered in red silk.

Mechanic and artificer.-Two crossed hammers of cloth.

Mechanics of Field Artillery performing the duties of farrier and blacksmith or of saddler will wear the devices corresponding to those duties, instead of two crossed hammers.

First-class prizate, Enginecrs.-A castle of red cloth, $1 \frac{1}{2}$ inches long and height it proportion; castle to be piped with white and worn on outside of right sleeve halfway between the front of shoulder and elbow.

Casemate elcetrician, Coast Artillery Corps.-Red ring, red voltmeter, white scale, graduations black, needle black, within.

Obscriers, first class, Coast Artillery Corps.-Red rings, whice cross wires and red target within. 
Obseracrs, second class, Coast Artillery Corps.-Red ring and white cross wires.

Plotter, Coast Artillery Corps.-Red ring, red dividers within.

Chicf planter, Coast Artillery Corps.-Red ring, mine, and oars.

Chief loader, Coast Artillery Corps.-Red mine in red ring.

Gun commander, Coast Artillery Corps.-A sergeant's chevrons, in the angle of which will be placed crossed guns of a suitable size, of scarlet cloth, stitched to the blue cloth of the chevron, the markings of hoops, trunnions, etc., to be outlined by black silk stitching.

Gun peinter, Coast Artillery Corps.-Red ring, white cross wires and red projectile within.

Gunner, first class, artillery--Red projectile with white piping.

Gunner, second class, artillery--Red projectile.

If in the Coast Artillery Corps a special grade is held by a non-commissioned officer the insignia is worn in the angle of the chevrons when practicable; if not, below it; if not a non-commissioned officer, in the same manner as the chevrons are worn.

All enlisted men who have served faithfully for a term of three years continuously or otherwise wear as a mark of distinction upon both sleeves of the dress coat, below the elbow, a diagonal half chevron of cloth of the color of their facings. For each additional period of three years' faithful service, continuously or otherwise, an additional chevron is worn.

Devices are also worn on caps and service hats as follows:

Cazalry-Crossed sabers, numbers of regiment in the upper angle and letter of troop in lower angle.

Field Artillery.-Crossed field guns, with number of regiment in the upper angle and letter of battery in the lower angle.

Coast Artillery Corps.-Crossed cannons, with number of company in lower angle.

Infantry.-Crossed rifles, number of regiment in the upper ingle and letter of company in lower angle.

Engincers.-The castle, with letter of company above it.

Ordnance scrgeants.-Shell and flame in white metal, inclosed in a wreath of gilt metal.

All enlisted men of the Ordnance Department.-A shell and flame in gilt metal.

Post commissary sergeants.-Crescent of white metal, the points up, inclosed in a wreath of gilt metal.

Post quartermaster sergeants.-Insignia of the Quartermaster's Department, in white metal, inclosed in a wreath of gilt metal.

Non-commissioned staff, Coast Artillery Corps.-Crossed cannon, inclosed in wreath of gilt metal.

Master signal clectrician.-A symbol resembling forked lightning, of white metal, inclosed in a wreath of gilt metal.

Enlisted men of the Hospital Corps.-Sergeants first class, a caduceus of 
white metal inclused in a wreath of gilt metal. For sergeants, corporals, lance corporals, privates first class, and privates, a caduceus of gilt metal, without the wreath.

Non-commissioncd officers of the Signal Corps, cxcept master signal electrician.-Two crossed signal flags and a burning torch of white metal, inclosed in a wreath of gilt metal. For all other enlisted mon of the Signal Corps, two crossed signal flags and a burning torch of gilt metal.

Enlisted men of the Army seraice detachment at the United States Military Acadcmy, H'cst Point, N. Y.-The insignia of the Quartermaster's Department in gilt metal.

Band musicions.-A lyre of white metal. Engineers to have a castle of yellow metal in the centur. Cavalry, field artillery, and infantry to have the number of the regiment, and Coast Artillery Corps the number of the band, of yellow metal, in the center of the lyre.

Musicius of Engineers.-A bugle of yellow metal, with a castle of white metal in the center of, and the letter of the company, in yellow metal, above the bugle.

Musicius of infantry and trumpeters of ficld artillery and cacalry-A bugle with letter of company, battery, or troop in center and number of regiment above the bugle.

Musicians of Coast Artillery Corps.-A bugle with the number of the company in the center.

Enlisted mon of the gencral recruiting scricc.-The letters "R. S." of white metal inclosed in a wreath of gilt metal; for men of permanent parties of rectuit companies at the general recruit depots, the number of the company, in white metal, to be placed below the letters.

Enlisted mon of the United States military prison guard.-The letters "P. G.," with the nmmer of the company helow them, letters and numbers to be of white metal and inclosed in a wreath of gilt metal.

The banners of the army are of three classes, flags, colors and subsidiary pennants used to mark the position of the organizations in the field. Flags consist of the ensign of the United States upon which is displayed the particular device of the person or organization represented. The exception to this is that the flags of the President and Secretary of 11 ar, respectively, are distinct compositions from the flag of the United States. The latter is composed of thirteen horizontal stripes, seven of red and six of white, the red and white stripes alternating in the order named and a union of white stars in a blue field, the number of stars being that of the number of States in the Union. When a new State is admitted to the Union the additional star representing that State goes into use on the Fourth day of July next succeeding such admission, the rearrangement of the stars 
in the blue field having been effected after consultation by the general officers in command of the army. The President's flag consists of a scarlet field upon which is displayed in each corner a white star with a large white star in the center bearing the coat of arms of the United States, embroidered upon a blue star, the whole forming an ensemblage of four corner stars with one large central star upon which is a blue star, and upon the latter in turn an embroidery of the coat of arms of the United States surrounded by stars representing the number of States in the Union. The President's colors are of scarlet silk and bear similar devices to those of the President's flag, the latter being intended to fly from flag staffs, and the former to be used in denoting the immediate location of the President.

The flag of the Secretary of War is also of scarlet bunting and bears upon it an eagle with outstretched wings, with a shield on his breast and an olive branch in the right talon, and a bunch of arrows in the left, the scroll in the eagle's beak bearing the notto "E pluribus unum." In addition, the usual thirteen stars which go with this device are placed in the vicinity of the eagle and there is a white star in each corner of the flag. The colors of the Secretary of War, which would be placed in front of his tent if he should be in the field are of scarlet silk and bear the same devices as those named for his flag. Three sizes of United States flags are provided for garrison and post use, the garrison flag being 36 feet by 20 feet, the post flag is 20 feet by Io feet, and the storm flag 18 feet by 4 feet 2 inches. The garrison flag is only furnished to the more important posts and is hoisted only on holidays and special occasions.

The flag of the medical service is of course the red cross flag, consisting of white bunting with a red Geneva cross. For each separate organization, regimental and battalion stands are furnished comprising of two colors, the national color, which is a silk United States flag, bearing the name of the organization embroidered in silk of the appropriate color of the particular branch of the service and the regimental or battalion color which in each case is as follows: Battalion color of the engineers, scarlet silk having in the center a castle and with red and white tassels and white fringe. The corps color of the artillery is of scarlet silk bearing in the center two cannon crossed with appropriate lettering with yellow fringe and tassels. The in- 
fantry regimental color is of blue silk with the coat of arms of the United States embroidered on silk in the center, a red scroll beneath the eagle bearing the name of the organization. The infantry colors of blue and white are carried out in the tassels and other attachments of the color. The cavalry regimental standard is of yellow silk with all the accessories of the same color.

A service national color made of bunting is furnished to each organization for drills and marches, but the silken flag must be carried in battles, campaigns and on all occasions of ceremony.

For the purpose of marking distances and serving as guides for the mounted arms of the service guidons are provided, those for the cavalry being pennants cut swallow-tail, having two horizontal stripes, the upper stripe red and the lower white, the red stripe having on both sides the number of the regiment and the white stripe the letter of the troop. Guidons for field artillery are of a similar shape and size to the cavalry guidons and have in the center on both sides two cannons crossed with the number of the battery below the cannon. The distinctive flag for the signal corps is a red flag with a white center, the name of the corps being embroidered on the field and the device of the corps in the white center. Instead of embroidering the names of battles in which the whole or a portion of companies and battalions have been engaged upon the flags themselves, it is provided that the names of the actions may be engraved upon silver rings fastened on the pike or staff of the colors or standards, and where less than half of the regiment or battalion was engaged the letters of the companies actually taking part in the engagement are to be inserted in the rings. The determination as to what action was of sufficient importance to be called a battle and to be thus commemorated on the rings of the staff of the colors is made by the War Department and is announced through the usual military channels so that no organization can determine for itself the number of the commemorative plates it may have in connection with the regimental colors.

ENLISTMENTS AND DISCHARGES.

While it is the happy fortune of the United States that at the outset of a war at least it would have no trouble in filing up the 
authorized number of regiments by voluntary and almost immediate enlistment, during the time of peace it is necessary to maintain an organization of the army simply for the purpose of securing men to fill the places of those whose terms have expired and who have been discharged. Enlistments are secured by officers who are detailed for the purpose, and who are given a sufficient number of enlisted men as aides in the clerical and other work of the duties assigned. Enlistment offices are opened in various cities and certain posts are designated for the headquarters of the enlistment work for a given organization. Applications for enlistment are sought by advertisement, stating the advantages of life in the army, and enlistments are effected under the following requirements: All enlistments shall be for the term of three years, and no person who is not a citizen of the United States, or has not made a declaration of his intention to become a citizen, can be enlisted, except he be an Indian. It is also necessary for the recruit to speak, read and write the English language, and he must be an effective and able-bodied man between the age of eighteen and thirty-five years on first enlistment, the thirty-five-year age limitation not applying on subsequent enlistments. No person who is under the age of twenty-one years can be enlisted without the consent in writing of his parents or guardian entitled to his legal control. This provision is necessary for the reason that under the common law the parent or guardian of a minor may recover possession of his services by appeal to a writ of habeas corpus.

No minor under the age of eighteen years, no insane or intoxicated person, and no deserter or person who has been convicted of a felony can be enlisted. Re-enlistments cannot be effected unless the soldier's service during his preceding term of enlistment was honest and faithful. The total number of enlistments in the army is prescribed by the legislation fixing the strength of the different organizations, but an additional enlistment to provide for vacancies occurring in organizations serving outside the limits of the United States may be made to the extent of four per cent. in excess of the authorized strength. Every enlisted man within six days of the time of his enlistment must 
take an oath to support the Constitution of the United States and to obey the orders of the President and his superior officers, and according to the rules and articles of war. No enlisted man who has been duly sworn can be discharged without a certificate in writing signed by a field officer of the regiment to which he belongs or by the commanding officer, and no discharge can be given to an enlisted man before his term of service has expired, except by order of the President, the Secretary of War, the commanding officer of a department or by sentence of a general court martial. Under these provisions all enlisted men must serve for the term of three years for which they have contracted and cannot be dismissed by their officers except for legal cause, nor relcased from their contract except under the conditions provided by law. If, after the expiration of one year, one of the pareuts of a soldier should die, leaving the other dependent upon the soldier for support, the Secretary of War, upon proof being made of the fact, may honorably discharge the soldier from the remainder of his enlistment. The President also has in time of peace discretion to discharge enlisted men under such rules and upon the payment of such purchase money as he may prescribe, the money thus paid to the United States to be applied to such of the current appropriations for the support of the army as may be indicated by the Secretary of War. Such discharge can only be purchased after a year's service and upon giving satisfactory reasons for the cxercise of the privilege. The price of purchase of discharge is $\$ 120$ in the first month of the second year and $\$ 5$ less for each succeeding month.

The discharge which can be effected by the commander of a geographical department, or by the commander of an army in the field, is only in case of a certificate of disability, and when such disability was caused by the misconduct of the soldier or in consequence of conditions which existed previous to the enlistment of the man, the discharge can only be granted by or in the name of the Secretary of War. There are three classes of discharges from the army-the honorable discharge, in which the word honorable is specifically used with a meaning, namely, that the 
person receiving the discharge has served the term of enlistment with credit and with efficiency; second, a discharge without honor, which simply indicates that a man has completed a term of service and by implication denotes that his service during that time has not been creditable; and a dishonorable discharge, which is awarded by a general court martial as a punishment. Besides the discharge certificate certifying to a discharge for reason of disability, a special certificate is also given in cases where the discharge was effected because of disability through the fault of the soldier himself.

Enlisted men are relieved from active service by retirement after 30 years' service, war service in the field, being computed twice in the process of making up the number of years necessary for retirement. The pay of retired men is three-fourths of the amount allowed them for the grade in which they were serving at the time of their retirement, including reenlisted and continuous service pay then received, together with subsistence, commuted at the rate of $22 \mathrm{~T} / 2$ cents per day, and three-fourths of the average annual allowance for clothing, onetwelfth of the amount to be paid monthly. A deduction of $12 \mathrm{I} / 2$ cents per month is made for the support of the Soldiers' Home of the enlisted men of the regular army, which is located in Washington and was started with the funds brought back by General Scott after the Mexican War, being the amount captured from the Mexicans' military chest. With this amount a large tract of land was secured in the vicinity of Washington and handsome buildings have been erected, providing adequate support for a considerable number of soldiers who have been discharged because of disability, the latter having been contracted while in the service and for reasons incident to the service. Twenty years' honest and faithful service in the army or disability not the fault of the soldier are requisite for admission to the home, and upon a successful application the soldier may be discharged for the purpose of entering a home and under certain circumstances will be furnished with transportation from the place at which he is serving to Washington. 
PUNISHMENTS IN THE ARMY.

The chief military offense, of course, is disobedience of orders and the chief statutory one is that of desertion. Every soldier who deserts in time of war is liable to death, and in time of peace to court martial and punishment by imprisonment, with subsequent dishonorable discharge, and is also liable to serve as well in addition for such period as may make up the total time of his original enlistment. If an officer is absent from his duty for three months without leave, the President is authorized to drop him from the rolls, and he is not eligible for reappointment. Enlisted men who desert forfeit all rights to pension and also are deemed to have relinquished their right as American citizens. To afford relief from the foregoing provisions a large number of men who served in the Civil War and who returned to their homes without having received a proper discharge, so that they appeared on the rolls as deserters, were relieved from the disability in securing a pension by general laws which provided for the correction of the rolls of the army and navy upon application of the enlisted man and evidence showing that there was no intention to desert in time of war.

Stringent provisions are made under the articles of war for the punishment of officers who make fraudulent musters or returns in order that undue amounts of pay or provisions may be drawn, and who wilfully or through neglect suffer any of the military stores of the United States to be spoiled, wasted or damaged. Any officer who uses contemptuous or disrespectful words against the President, Vice-President, Congress of the United States, the Chief Magistrate or Legislature of any of the United States, may be dismissed from the service or otherwise punished, as the court martial may direct. Any soldier who so offends may be punished as the court martial may direct. Disrespect to a superior officer or threatening or assaulting such officer, or offering violence, may be punished by sentence of court martial, the more serious offense attended, if so found, by death, and any officer or soldier who incites a riot may be punished by death. Challenges to fight duels may be punished by dismissal, 
and the usual provisions are made for punishment of such military offenses as cowardice and failure to perform the full military duty in the face of an enemy. The award of punishment in an army is performed in minor cases affecting the enlisted men by commanding officers who have a limited power of punishment; by slimmary court martial and by general courts martial, which have a carefully regulated system of jurisprudence, with rules intended to save the rights of the defendant and to secure the due administration of justice. A bar by limitation is in effect in that no person can be tried for an offense committed more than two years before the.issuing of the order for trial.

Courts martial may be ordered by the Secretary of War or by the commanding officers of divisions, and no sentence of a court martial can be carried into execution until it has been approved by the officer ordering the court. No sentence of a court martial inflicting the punishment of death can be carried into execution until it has been confirmed by the President, except in case of certain offenses committed in time of war, when the sentence of death may be carried into execution upon confirmation of the commanding general in the field or the commander of the department. In time of peace also no sentence of a court martial directing the dismissal of an officer can be carried into execution until confirmed by the President. When in less serious cases the fixing of a punishment for an offense in time of peace is left to a court martial, such punishment cannot be in excess of the limits which are fixed by the President. Provision is also made in the statutes for the punishment of any person not belonging to the army who refuses to appear upon an appropriate subpoena from a court martial. The fact of the non-appearance is to be certified to the appropriate United States Attorney, who is required to file information in the District Court of the United States, and upon failure of the witness to give sufficient excuse he may be fined not more than $\$ 500$ or imprisoned not more than six months.

THE ARMY SCHOOLS.

For the purpose of carrying on the instruction of officers in the higher branches of the military sciences, and for securing 
specialization along various lines, service schools are established and maintained in different parts of the country, the chicf of which is the Army. War College, located at Washington on the site of a former arsenal. which was abantoned for many years, and previous to its prescnt use was used as an artillery post. The War College has for its object the dircction of the instruction in the various service scinools and the extension of the opportunities in the army for investigation and study. Its equipment consists of one large central building with a considerable number of quarters and subsidiary structures, and officers are detailed from the various branches of the service to undertake, at the college, courses of advanced instruction as well as original investigation and study into the methods of modern warfare. The service schools referred to include the United States Engineer School at Willett's Point, New York, to which one officer from each regiment may be sent for a course of instruction in torpedo service, topography and practical military engineering. An Artillery School is maintained at Fort Monroe, Va., to which officers of the artillery corps are assigned who have been recently appointed and who are not sraduates of the Nilitary Acarlemy: those below the rank of captain who have not already graduated from the artillery school and officers of any rank who may desire to take any portion of the course or indertake any special study. A school for Electrician Sergeants is also maintained at Fort Nonroe as a branch of the artillery school and is devoted to the instruction in the application of electricity in the ordnance work. The infantry and cavalry school of the army is located at Fort I.eavenworth and has a course of instruction embracing two years. Officers are detailed in turn by orders from the department to proceed to this school for instruction, and a force of infantry and cavalry is stationed at the post at all times to be available for the purposes of practical instruction. Preliminary selection of the officers who are to be detailed to the school is made a year in advance by the commanding officers of regiments who, it is to be supposed, select such among their subordinate officers as may desire to attend the school or who may seem by 
reason of the character of their service to need further instruction.

A school for the instruction of officers in the combined operation of cavalry and light artillery is maintaintd at Fort Riley, Kansas. The conditions of instruction are similar to those at the Leavenworth school, including matters of selection, but the purpose of the school is more especially in the direction of horsemanship and of the possibilities of the mounted arms of the service. An army medical school is maintained at Washington, in which the students are medical officers who have been appointed since the last preceding term of the school, and other officers who desire a general course or specialized work. 


\section{CHAPTER XI. \\ DEPARTMENT OF JUSTICE.}

The nucleus of the Department of Justice was the Attorney-General, who was understood at the time of the framing of the Federal Government simply as a legal adviser to the President, without administrative duties, and not necessarily a permanent resident of Washington, except so far as the President might require his presence at the seat of government. From the first there was an apprehension that if a legal department should be established, it might be placed in the position of giving legal advice to private persons and the duties of the Attorney General were strictly limited to giving opinions on matters of law to the President and to the heads of executive departments on matters relating to the business of their departments. Under the terms of this limitation the Attorney General has universally declined to give any opinion on, or construction of, law, whatever, to private individuals or to other officers of the Government than those named. The first Attorneys General were furnished only with a clerk; in fact, the earliest incumbents of the office had to furnish their own clerical assistance. Gradually, however, as the legal business of the Government increased the personnel of the Attorney General's office became more numerous and finally a Department of Justice under its present conditions was inaugurated in 1870 .

The Department of Justice exercises two functions; namely, the firection and supervision of the law business of the United States, and secondly, the supervision of the officers of United States courts, including attorneys, marshals, and clerks. In connection with the supervision of the duties performed by the marshals it has also the custody of prisoners of the United States, but all of its activities may be divided generally into the classes named, of direct legal service, and of supervision of the officers of United States courts, except Judges. There is an erroneous impression that the Department of Justice has jurisdiction and control over the Judges of United States 
courts. This is not at all so, except so far as the department regulates and approves the expenses for such courts that are paid out of the public funds. It has no power to call a Judge to account for any action or for any language nor to direct any matters relating to the business of the courts, all such either being regulated by law or by the rules promulgated by the courts themselves. It can and does make certain suggestions to the Judges by directing the proper court officer to call the suggestions of the department to the attention of the court, but if the court does not coincide with the view of the department, the latter has no power to enforce its orders, except that it may decline to allow expenses and expenditures for certain objects, thus making it incumbent on the court either to refrain from action in the line suggested or to defray the cost of the undertaking at the sourt's personal expense.

The officer next in rank to the Attorney General in the Department of Justice is the Solicitor General. He is appointed by the President, by and with the advice and consent of the Senate, and receives a salary of $\$ 7,500$ a year. His chief duties relate to the preparation of cases for the Supreme Court and their argument in that court, and he has general charge of all the details relative to the cases in the Supreme Court in which the United States is interested, and of arranging for the necessary motions and incidental business. The more important questions, especially those involving constitutional matters, are argued in the Supreme Court by the Attorney General of the United States, but the Solicitor General takes a large share of the other business and has generally been the representative of the Department in cases which have come to the Supreme Court. In the absence of the Attorney General, the Solicitor General becomes acting Attorney General, and in case of a vacancy in the office the Solicitor General is empowered by law to exercise all of the duties of the head of the Department.

The Attorney General may also argue cases in any of the other courts of the United States besides the Supreme Court or may direct the Solicitor General to do so. When opinions are requested by the President or the heads of the executive departments on matters of law, the Attorney General may either render them himself or refer them to one of his assistants. All matters excepting those involving 
an interpretation of the Constitution may be so assigned and the opinion prepared by the assistant, when approved by the Attorney General and the approval endorsed thercon, has the force and effect as if rendered by the Attorney General himself.

The next officer in order of rank in the Department of Justice is the Assistant to the Attorney General. He is appointed by the President by and with the advice and consent of the Senate, and receives $\$ 7,000$ a year. His especial function is to enforce the terms of the Act approved July 2, I 890 , and generally known as the Sherman AntiTrust law. He acts, however, as Attorney General when both the Attorney General and Solicitor General are absent from the department and may also be called upon to take part in the general work either of administration or of law matters. In consequence of the especial activity in the last few years in the matter of prosecuting offenses in the line of restraint of trade, the Assistant to the Attorney General is occupied almost entirely with questions arising under the Act heretofore mentioned, and under the Interstate Commerce Act, which, having to do with similar matters of trade, is also administered so far as its details come to the Department of Justice by the Assistant to the Attorney General. For the purpose of aiding in the enforcement of the Sherman Anti-Trust law a continuing appropriation is made by Congress which approximates in size the sum of $\$ 250,000$ a year, and disbursements from this fund are made by the Assistant to the Attorney General with the approval of the Attorney General himself. The provisions of the Sherman Anti-Trust law are, generally, that every contract, combination in the form of trusts or otherwise, or conspiracy which has for its purpose the restraint of trade or commerce among the several States, or with foreign nations is illegal, and every person who enters into a contract or engagement for the purpose of furthering such illegal act is to be deemed guilty of a misclemeanor and, upon conviction, punished by a fine not excceding $\$ 5,000$ or by imprisonment not exceeding one year, or by both such punishments. Thus, the celebrated first section of the Anti-trust law is aimed to prevent persons in the same line of trade making an agreement by which the output of a given commodity may be restricted or its price regulated without 
competition and in opposition to the usual laws of trade. The second section of the law makes it equally a misdemeanor to monopolize or attempt to monopolize any part of the trade among the several States or with foreign nations and makes such monopoly or attempt punishable by similar fines to those described for misdemeanors in restraint of trade. Thus, while the first section attempts to prevent the combination among individual firms for the purpose of eliminating competition, the second section aims to prevent any one firm or interest from acquiring all the others in its line of trade so that it may dictate prices and the conditions upon which any given commodity may be supplied. The Circuit Courts of the United States are given jurisdiction of cases coming under this law, and it is also provided that seizure may be made of any goods belonging to parties who may be alleged to have entered into such an illegal agreement. A large number of such cases have been brought under the terms of this law, and a considerably large number of investigations made. One of the most notable of the cases was that relating to the Northern Securities Company, under which name an attempt was made to amalgamate the property of two large railroads, which attempt was defeated by the proceedings brought by the United States under the terms of the Sherman law. For the prosecution of the law, the Assistant to the Attorney General has under his direction a corps of investigators and special attorneys. In several of the cases practical confession has been made by those accused of unlawful combination and decrees have been entered in the courts terninating the business under the particular form complained of. As judicial decisions under the law increase, the exact status of corporations and the limits of corporate powers under the Federal law are being defined, but the process is still in the initiatory state, and no one can give an exact definition at the present time of the limits established by the Act referred to within which corporations may extend their holdings and their operations.

Additional regular assistants to those named are provided for the Attorney General, under the title of Assistant Attorney General. The incumbents of these offices receive salaries of five thousand dollars a year each, and are appointed by the President, by and with the advice and consent of the Senate. Three of these Assist- 
ants are in the department proper, and their functions are similar to those of the Assistant Secretaries in other executive departments, except that they deal witl legal instead of adninistrative problems. The time of one of the Assistant Attorneys General is largely taken up with consideration of matters relative to customs cases, as when such cases are to be appealed to the Circuit Court of Appeals they are certified to the Department of Justice by the law officers more immediately comected with the Treasury Department. Another of the Assistants is clicfly concerned with the internal revente matters and with matters arising under the new naturalization law, the latter law having a dual administration by the Department of Justice and the Department of Commerce and Labor. It is provided in the naturalization law that the United States shall be represented at all hearings in court for the purpose of naturalization of aliens, and in order to properly conduct these hearings, Assistant District Attoneys for naturalization purposes have been appointed, and who have under their direction a corps of examiners in each case, who are charged with the duty of investigating the circumstances in each applicant for naturalization. If the examiner is able to find that the applicant is not entitled to naturalization, or that there is some defect in his papers, the information is laid before the Assistant District Attorney in charge of naturalization affairs, and the application of the alien for naturalization is opposed when the matter comes up in court for hearing. The third Assistant. located in the department proper, is clargerl with matters relating to the legal affairs of the territories and instular possessions so far as they come to the Department of Justice. and all three handle miscellaneous matters, regular assignment being made of the classes of work which come before the department.

It is the general rule that all investigations preliminary to proceedings in the courts shall be made by the Department under which the subject matter of the investigation cones. Thus in land cases, the Interior Department sccures the evidence necessary to make a case and presents it to the Department of Justice for prosecution before the courts. The same is true of the cases which come under the juriscliction of the Department of Agriculture relating to the pure food law, the law relative to the shipment of cattle, and similar 
statutory regulations, and a like course is followed by other departments. The only exception is in the case of the enforcement of the naturalization law. Owing to the fact that the main appropriation for the enforcement of this law was assigned to the Department of Justice, the Department of Commerce and Labor has no means for carrying on the investigation during the fiscal year ending June 30 , 1908, but it is anticipated at this writing that a change will be made in this matter and that the investigations will be made by the Department of Commerce and Labor and certified to the Department of Justice for procedure as in the case of all other similar matters in the executive departments.

Besides the officers before named, the Department of Justice has a staff of Assistant Attorneys at salaries ranging from $\$ 2,000$ to $\$ 2,500$, who act as assistants to the Assistants Attorney General in making preliminary examinations of law questions and in preparing material for opinions and other legal work of the Department.

Provision is made by law for the appointment of Special Assistant Attorneys in the discretion of the Attorney General who are employed to conduct cases which require either special knowledge or which cannot be handled by the regular force of the Department. As a rule, such Assistants are appointed for services at points other than in Washington, and are there placed in full charge of a given proceeding in all its stages, or are assigned for the assistance of the District United States Attorney in the district in which the cases come up. Some of the most important of the litigation before the Department, especially that under the terms of the Sherman Anti-trust law, is thus placed in the hands of Special Assistants who are given the entire responsibility, except that they receive general direction from the head of the Departments and as Assistants to the Attorney General are expected to direct the investigation, the arrangement of the evidence, and to conduct the prosecution in court. When, on account of the pressure of the business of the office of the United States Attorney, or when he is unable to try a given case, or when the case presents unusual or technical difficulties, a Special Assistant to the United States Attorney is appointed for the trial of that particular case. Such action is also taken when, for any reason, the District 
Attorney may be disqualified from appearing in the trial of the case. The compensation of Special Assistants may be either fixed by the Attorney General at the time of the appointment, or left until the completion of the case, when a total sum for the service rendered is allotted. The latter course is generally taken in connection with suits of consilerable importance, but the attorneys are allowed amounts from time to time on account, as the case proceeds.

In adclition to the Assistant Attorneys General who are engaged with the transaction of the general business of the Department, three other Assistant Attorneys General are provided at salaries of $\$ 5,000$ each. who have charge of a particular class of work. Thus the defense of suits before the Court of Claims is entrusted to an Assistant Attorney General, who has under his charge a semi-independent organization, engaged exclusively in the preparation of the side of the United States in all proceedings before the Court of Claims. The or anization consists of a number of Assistant Attorneys, with the necessary clerical force, and their duties inclucle the taking of testimony in the field, the arransement and preparation of the argument of cases before the Court of Clains. A laree portion of the work of this section of the Department of Justice refers to claims arising out of the Civil War, which have been referred to the Court of Claims, and which require the taking of a larre amonnt of testimony in the States of the South. When matters determined upon by the Conrt of Claims are appealed to the Supreme Court, the briefs for the presentation of the cases in the highest court are prepared by the Assistant Attorney General in charge of the Court of Claims work, and the cases are argued by him in the Supreme Court, either with the Attorney General or alone.

The classes of claims srowing out of Indian depredations, that is, the damage done by Indians in the Western States, when such Indians were under the control of the Federal Govermment and escaped for the time being from their reservation and the custody of the Federal authority, are handled on the part of the United States by another Assistant Attorney General, who represents the Government in the presentation of such cases in the Court of Claims. The third of these semi-independent Assistant Attorneys General 
is the officer in charge of the defense of claims before the Spanish Treaty Claims Commission. This work involves the representation of the United States in matters growing out of the Spanish-American Var, and the officer in charge directs a considerable force of attorneys, who are engaged in taking testimony at the present time in the Island of Cuba. It is anticipated, however, that the work before this commission will be completed before the close of another fiscal year, and that the necessity for the services of this officer will be ended.

When the Department of Justice was constituted in 1870, all of the law officers under the different departments were consolidated under the jurisdiction of the Department of Justice and a provision was made that no legal services should be rendered to the executive departments except upon application to the Attorney General and under his direction. The most important of these departmental subdivisions is the office of the Solicitor of the Treasury, which has charge of books, papers and records received from the officials who had, under a former system, superintendence of the collection of outstanding taxes which required legal proceedings. When a Collector of the Customs is compelled to deliver a bond for taxes to a United States Attorney for collection, he notifies the Solicitor of the Treasury, who makes an cntry charging the attorney with the amount involved, which amount remains charged to the attorney until it has been paid to the United States or a judgment has been secured and the execution entrusted to a United States Marshal, who then becomes chargeable with the amount. The Solicitor of the Treasury thereafter has general superintendence of the work of collecting such amounts and is also charged with the cognizance of all frauds and attempts at fraud on the customs revenues, and exercises a general supervision over the measures for their prevention and detection, and over the prosecution of the persons charged with such offenses. The Solicitor of the Treasury has, by virtue of the various provisions of law, direct fower of instruction to United States Attorneys as to the details of cases relating to the collection by legal process of amounts due the United States on account of customs and establishes the regulations which 
must be approved by the Secretary of the 'Treasury and the Attorney General for the observance of collectors and District Attorneys and nurshals respecting suits by which the United States seeks to recover amonnts due to it from the source indicated. The power exercised by the Solicitor of the Treasury of dircetion to the United States Attorneys, marshals and clerks of District Courts refers, however, only to suits originally instituted under his direction and does not in any wise refer to cases coming under the intemal revenue laws. The Solicitor of the Treasury has also similar duties with regard to the supervision of suits and procecdings arising ont of the national banking laws, in which the United States or its officers are a party, and has general charge of matters under the direction of the Secretary of the Treasury regarding the compromise of claims on the part of the United States towards private partics. In case it is not found possible to collect an amount due the United States by the usual process, the Solicitor of the Treasury may consider offers of compromise and receive in satisfaction of the debt such amount as may seen to be all that the United States could reasonably: hope to secure. Not all debts due the United States, however, can be compromised by the Solicitor of the Treasury, as a certain portion of them must be passed on by the President. The Solicitor of the Treasury also passes upon the sufficiency of the bonds of the United States Assistant Treasurers, department disbursing clerks, Collectors of the Internal Revenue, and those of the secretary and chicf clerk of the Department of Agriculture, and his approval is necessary for the validity of such bonds. He is also the law officer of the Treasury Department and questions arising under the various laws affecting the department are referred to him for his examination and opinion. His opinion on the varions matters last referred to, however, is not a conclusive one, but has the same weight as those of other departmental solicitors and law officers under the jurisdiction of the Department of Justice. If the head of a department is not satisfied witl the opinion of his law officer, he may refer the subject matter to the Attorney General for an opinion, accompanying his request with a copy of the opinion rendered to lim by the Solicitor or the 
Assistant Attorney General attached to the department. The Solicitor of the Treasury is also the custodian of real estate which may be acquired by the United States through any other proceedings than those under the Internal Revenue laws, and administers all details relating to the retention and the release and sale of such lands in payment of debts. The office of the Solicitor of the Treasury is in the Treasury Building and that department is required by law to afford him the necessary quarters for the transaction of his business. The clerical force required and the expenses of the office are, however, paid by the Department of Justice.

In addition to the Assistant Attorneys General already named, two of the law officers of the executive departments have that title: the Assistant Attomey General for the Interior Department and the Assistant Attorney General for the Post Office Department. The Assistant Attorney General for the Interior Department is the chief law officer of that department and advises the Secretary and Assistant Secretaries upon questions of law arising in the departmental acministration. Appeals from the General Land Office are sent to his office for consideration and he prepares the cases for the final action of the head of the department. The Assistant Attorney General for the Post Office Department is appointed by the Postmaster General, but his name is carried on the list of officers of the Department of Justice, although his duties are almost exclusively performed under the direction of the head of the Post Office Department, and his subordinates are subordinates of that department. Besides giving opinions on questions of law relating to the work of the department, which are presented to him not only by the Postmaster General and his assistant, but by the superintendents and the chiefs of the several divisions and bureaus and by the Postmasters throughout the conntry, he has the consideration of proposed compromises of liabilities and of the remission of fines, penalties and forfeitures which may be certified to him by the Auditor of the Post Office Department, and also prepares cases for allowances to postmasters for losses from fire, burglary, etc. Claims for rewards for the apprehension and conviction of of- 
fenders against the postal laws, apprehension and conviction of post office burglars, highway mail robbers, etc., come under his jurisdiction as well as matters of recommendations relative to pardons for offenses against the postal laws. The most important function of this official, however, relatcs to the enforcement of the laws relative to the use of mails for fraudulent purposes, or the transmission of lottery tickets and literature relating to lotteries. Evidence of the transmission of forbidden mail matter and of the fraudulent nature of advertisements intended to secure transmission of mail matter with the ultimate purpose to defraud the senders is laid beiore the Assistant Attorney General for the Post Office Department, who, upon consideration of the circumstances in the case and upon an oral hearing, if deemed to be necessary, may take steps which result in the issuing of a fraud order, which has the cffect of preventing the transmission of the mail directed to the person or persons afiected by the order, so that the letters are returned to the senders where possible. The Assistant Attorney General for the Post Office Department has gencral charge of the results of the investigations of the Post Office Inspectors into offenses committed against the postal laws when such cases have come to the point of being certified to United States attorneys for their prosecution, and repsents in a supervisory way the interest of the Post Office Department in having such prosecutions expedited and proceeded with to a satisfactory determination.

The Solicitor for the Department of State and the Solicitor for the Department of Commerce and Labor, respectively, handle all questions of law relating to the two departments, the former having turder his jurisdiction not ouly the usual achinistrative law questions, but questions relating to international law as well. $\mathrm{He}$ is the adviser of the Secretary of State on such questions as well as on matters of law affecting the consular service.

The office of the Solicitor of the Department of Commerce and Labor, who is the law officer of that department, is wholly composed of employees of the Department of Justice as is that of the Solicitor of the Treasury. The subordinates of other departmental law officers are, however, direct employees of the department concerned, and not 
a portion of the personnel of the Department of Justice. The Solicitor of Internal Revenue is an officer of the Department of Justice attached to the Internal Revenuc Service for the purpose of advising the Commissioner of Internal Revenue on matters relating to the Internal Revenue laws, and to exercise general supervision over the proceedings in court relative to the recovery of penalties, forfeitures and taxes under those laws. He receives $\$ 4,500$ a year, as do each of the other departmental law officers with the exception of the Assistant Attorney General for the Interior Department, who receives $\$ 5,000$. The legal assistance provided for the departments under the direction of the Department of Justice is required by law to be in lieu of all other legal assistance whatever and there is a prohibition against the employment of all other counsel and the payment of any fee for legal assistance except as the Attorney General shall authorize special assistance in any given case. The Attorney General is, however, required upon notice by the head of a department or a bureaut that the interest of the United States require the service of counsel to examine witnesses, or to make the legal investigation of any clain pending in such department or bureau to provide for such service. It is, therefore. illegal for any department or officer of the Government to pay out amounts for legal services and unless the proceeding in question comes within the scope of the duties of a District Attorney, the papers in the case must be forwarded to the Department of Justice with the request for assistance to be provided by that department in the prosecution or defense of the case. Either of the Assistant Attorneys General, when so directed by the Attorney General, may appear before any United States Court, and the appointment of a Special Assistant to the Attorney General confers the same prerogative upon any Attorney not regularly in the employ of the department.

ADMINISTRATIVE OFFICERS OF THE DEPARTMENT.

The foregoing description relates to the legal services of the officers under the Department of Justice. The administrative work of the department relates to the details of the appointment of and supervision over United States Attorneys and marshals and the supervision over the accounts and proceedings of Clerks of Courts, who 
are appointed by the Judges of the courts, but who are amenable to the direction of the Department of Justice as to the methods of keeping their books and accounts, any negligence in this respect being certified to the Judges of the respective courts for appropriate action by them.

The appointment clerk of the department in addition to kecping the records relative to the appointment of employees of the department proper, receives applications and endorsements for the appointment of United States Julges, District Attorneys, and marshals, and issues the commissions for such officers when appointments are made by the Presillent. Supervision over the District Attorneys, marshals and clerks is cxercised by means of the administrative audit which is carried out in the Division of Accounts, and by a force of examiners, eleven in number, under the superintendence of a Chief Examiner, who receives $\$ 3.750$ a year, the cxaminers recciving salaries ranging from $\$ 2.500$ to $\$ \mathrm{I}, \mathrm{SOO}$. It is the duty of the examiners to visit the various judicial districts and to cramine the books, accounts and methods of doing business of the officers of the courts, reporting to the department any departure from the prescribed methods of carrying on court business, together with any irregularities that may be discovered in the financial transactions of such officers.

The details relating to the executive prerogative of pardoning are administered under the Department of Justice, an official called the Attorney in Charge of Pardons having charge of all the records relating to such matters and conducting the correspondence and preparing the necessary bricfs of cases and the warrants for the President's signature. Pardons sought are for two purposes, to reduce the time of imprisonment, and to restore civil rights which have been forfeited by reason of conviction of crime. The only classes of pardons which are not made up by the Attorney in charge of pardons are those relating to offenses inder the army and the navy. Such cases are handled by the War Department and the Navy Department respectively and sent to the President for decision from those departments. The warrant. however, indicating the completed action of the President is issued from the Department of Justice upon notice from the other department that the President has signified favorable action. In cases other than those relating to the military service, 
applications made to the President for clemency are referred to the Department of Justice and in turn referred by the Attorney in charge of Pardons to the District Attorney of the district in which the conviction was originally effected. The District Attorney and Judge report upon the nature of the offense, and evidence adduced at the trial, and also add a recommendation giving their respective views as to whether clemency should or should not be shown. These reports, together with all the papers in the case are briefed and made the subject of a memorandum to the Attorney General who reviews the matter generally, and if he deems the application one which may be called to the President's attention, he prepares a brief letter summarizing the facts in the case and making a definite recommendation as to the Presidential action. If this recommendation is approved by the President a formal warrant of pardon is made out, which is signed by the President and countersigned by the Attorney General The rules relating to applications for pardon are appended hereto. Not every application can be brought to the attention of the President, but only such as are recommended by a reporting official, as stated in the general rules. In addition to the pardon matters relating to the shortening of sentences of imprisonment, there are a very large number of applications relating to the restoration of civil rights. The fundamental requirement for the consideration of such an application is that the applicant shall show that he has, during the time which has expired since the termination of his period of imprisonment lived the life of an honest and law-abiding citizen, and also that the time elapser is enough to give a reasonable opportunity to determine whether his reformation had been genuine. The pardoning power of the President is, as has been suggested elsewhere, unlimited, and refers as well to offenses before they have been passed upon by the courts as to offenses of which the accused may have been convicted and for which he has received a sentence. The utmost care is exercised in making recommendations to the President for pardon, and it is regarded that the sentence imposed by a Judge has been with the full knowledge of all circumstances previous to imprisonment so that the only grounds for pardon are the substantiation of a charge that injustice has been done either because of the wilful disregard of the circumstances by the court, or because of the lack of 
knowledge of the circumstances which lack has been supplied by subsequent developments. Grounds for pardon in addition to these are the ill-health of the prisoner indicating death unless released or some change in the conditions which could not have been known or understood by the scntencing judge.

\section{RULES RELATING TO APPLICATIONS FOR PARDON.}

All applications for pardon should be signed by two or more credible persons, be addressed to "The President of the United States," and be forwarded under cover to "The Attorney-General" (except applications for pardon for desertion or other offenses against the military or nava $\mathrm{i}$ laws, which should be addressed to the Secretary of War and the Secretary of the Navy, respectively), and the post-office address of each person signing the application should be plainly stated.

The application should state the name of the applicant, his age, where born, previous occupation, and place of residence, the crime of which he was convicted, the Court, District. State, and term, for how long, and to what prison he was sentenced, and the ground's upon which pardon is asked. It is not necessary to furnish a copy of the indictment or other court papers.

As soon as they are reccived, applications with the accompanying papers are forwarded to the United States Attorney for the District where the trial took place, with directions to report thereon, sending the statement of his predecessor when he did not himself appear in the case, and also that of any special attorney for the Govermment who took part in the trial. He is also directed to olmain, if possible, the views of the trial Judge. A case once so referred will tot be again referred without a writen request from the Lnited States Attorney or the trial Judge; and a case once acted upon by the President will not be reopened, except upon the presentation of new and material facts.

When none of the persons so consulted advise the pardon, the papers are not sent to the President, execpt by special order of the Attorney General or upon his own request; but when any one of them advises in favor of the application the papers are suhnitted to the President.

As all applications are sent by the Attorney General to the United States Attorncy for report and docket entries, it is unncessary and undesirable for applicants to apply to the Attorney or Judge.

Reports to the President by Attorncys and Judges, and other officials, on applications for pardon are treated as confidential, and are not open to inspection by the appiicant or by any nther person, except with the written assent of the Attorney. Jutge, or official making the report, nor, if such assent be given, unless it be shown that the ends of justice require the disclosure. All other papers, except seports or communications to the President by officials, are open to inspection by the applicant and his attorncy or representative, and by Members of Congress. 
Applications will not be considered pending appeals from judgments of conviction.

Applications with accompanying papers can not be withdrawn after they have been referred to the United States Attorney, as they are considered part of the records of the Department. If, however, the applicant will have copies made at his uwn expense for the Department files, the original papers will be returned to him.

Applications made merely for restoration to full civil rights will not be considered by the President before expiration of sentence. After the prisoner has been released for not less than one year, the President will consider the application, which must be accompanied by affidavits from at least three reputable citizens among whom the petitioner lives, stating that since his release from prison he has conducted himsclf in a moral and law-abiding manner, what his occupation has been, and what knowledge they have in the premises

When the President has acted, the applicant or his attorney is notified of the result. If pardon is granted, a warrant is at once sent to the applicant, either through the United States Marshal or the officer in charge of the place of imprisonment.

These rules apply to applications for pardon before conviction so far as appropriate, and also to applications for restoration to civil rights.

The President can not consider applications for offenses against State or Territorial laws. Such applications should be sent to the Governor or Board of Pardons of the State or Territory where the offense was committed.

While the negotiations for the acquirement of land by the United States are carried on by a considerable number of the officials of executive departments, no title can be passed until it has been examined and certified by the Attorney General, whose functions in this respect are exercised by an Examiner of Titles to whom all papers relating to the title of real estate about to be passed into the possession of the United States are sent, and who directs the preliminary examination or verification of the documents involved by the different United States Attorneys. When the title papers are revised and verified, this official examines minutely all portions of the title for the purpose of determining whether the United States can take over the real estate without subjecting itself to the liability of future claim. The duties of the office of title examiner are most exacting and require an intimate knowledge of every detail of real estate law. The usual procedure is for the executive department which is to acquire the land in question, to furnish the Department of Justice with the title deeds for examination. These, as has 
been said, are sent to the United States Attorney for report; upon receipt of the report and the completion of the technical examination, a letter returning the deeds is signed by the Attorney General, which formally states that the title is a valid one and that the property in question may be appropriately accuired under the terms of an enactiment for the purpose which may be in effect.

Other bureaus and offices of the Department of Justice proper are charged with the conduct of the details relating to proceedings sought to be brought by the different executive departments to enforce various laws. Such departments have no control over the United States Attorney, except where such control is specifically provided by law, so that it is necessary for the different departments when they desire to have legal proceedings instituted, to send the papers to the Department of Justice, which, in turn, forwards them to the appropriate United States Attorneys with instructions. if the class of cases has not already been the subject of well-defined procedure, to investigate and report to the Department, or if the case in question is one in which the procedure has been well defined, to proceed at once in the courts for the purpose of enforcing the law.

THE UNITED STATES ATTORNEYS.

The position of District Attorney of the United States was established under the terms of the first judiciary Act, that of September 24, I 789 , which provided that there should be appointed for each district, with three cxceptions, a person learned in the law to act as attorney for the United States in such district. In Alabama, Georgia, and South Carolina, one District Attorney in each State was required to serve for two districts. This, however, of course. has been remedied by subsequent appointments of a District Attorney for each District throughout the United States. District Attorneys are appointed by the President by and with the advice and consent of the Senate, and for a term of four years. At the expiration of their commissions, their positions were formerly required to cease and determine, but by later legislation they continue to discharge the duties of their office until their successors are appointed and qualified. In case, however, of an actual vacancy, the court has power 
to appoint a temporary District Attorney until the appointing power acts in the premises. District Attorneys were formerly compensated by fees, but in 1896 , legislation directed that while fees were still to be taxed, they should be returned to the United States, exception being made, of course, that fees shall not be taxed against the United States, and the District Attorneys, in lieu of the previous compensation by fees, are paid fixed salaries, which are determined by law as follows:

For the northern and middle districts of the State of Alabama, each four thousand dollars; for the southern district of the State of Alabama, three thousand dollars; for the Territory of Arizona, four thousand dollars; for the eastern and western districts of Artansas, each four thousand dollars; for the northern district of California, four thousand five hundred dollars; for the southern district of California, four thousand dollars; for the district of Colorado, four thousand dollars; for the District of Columbia, six thousand dollars; for the district of Connecticut, two thousand five hundred dollars: for the district of Delaware, two thousand dollars: for the northern and southern districts of Florida, each three thousand five hundred dollars; for the northern district of Georgia, five thousand dollars; for the southern district of Georgia, three thousand five hundred dollars; for the district of Idaho, four thousand dollars; for the southern and eastern districts of Illinois, each five thousand dollars; for the district of Indiana, five thousand dollars; for the northern and southern districts of Iowa, each four thousand five hundred dollars; for the district of Kansas, four thousand five hundred dollars; for the eastern and western districts of Kentucky, each five thousand dollars; for the eastern district of Louisiana, three thousand five hundred dollars; for the western district of Louisiana, two thousand five hundred dollars; for the district of Maine, three thousand dollars; for the district of Maryland, four thousand dollars; for the district of Massachusetts, five thousand dollars; for the eastern district of Michigan, four thousand dollars; for the western district of Michigan, three thousand five hundred dollars; for the district of Minnesota, four thousand dollars; for the northern and southern districts of Mississippi, each three thousand five hundred dollars; for the eastern and western 
districts of Missouri, each four thousand five hundred dollars; for the district of Montana, four thonsand dollars; for the district of Nebraska, four thrusand dollars; for the district of Nevada, threc thousand dollars; for the district of New Hampshire, two thousand dollars; for the district of New Jersey, three thousand dollars; for the district of New Mexico, four thousand dollars; for the northern, western and eactern listricts of New York, cach four thousand five hundred clollars: for the eastern district of North Carolina, four thousand dollars; for the western district of North Carolina, four thousand five hundred dollars: for the district of North Dakota, four thousand dollurs; for the northern and southern districts of Ohio, each four thousand five hundred dollars; for the castern district of Oklahoma, four thousand dollars; for the western district of Oklahoma, fice thousand dollars; for the district of Oregon, four thousand five hundred dollars: for the castern, middle, and western districts of Pennsylvania, cach four thonsand five hundred dollars; for the district of Rhode Island, two thousand five hundred dollars; for the eastern and western districts of South Carolina, four thousand five hundred dollars, two thousand five hundred dollars of which shall be for the performance of the duties of district attorney for the western district; for the district of South Dakota, four thousand dollars; for the eastern, middle, and western districts of Tennessee, cach four thousand five hundred dollars; for the northern and southern districts of Texas, each three thousand five hundred dollars; for the eastern and western districts of Texas, each four thousand dollars; for the district of Utah, four thousand dollars; for the district of Vermont, three thousand dollats; for the eastern district of Virginia, four thonsand dollars; for the western district of Virginia, four thousand five hundred dollars: for the eastern and western districts of Washington, each four thousand five hundred clollars; for the northern and southern districts of West Virginia, each four thousand five hundred dollars; for the eastern and western districts of Wisconsin, cach four thousand dollars; and for the district of Wyoming, four thousand dollars. Only the sonthern district of New York and the northern district of Illinois are cxempted from the list, the salaries of the district attorneys in those districts being \$12,ooo in each case respectively. Assistant district attorneys are pro- 
vided by the attorney general, one or more in number in each district, and the necessity for the appointment of such assistants must be certified by the district judge. Appointments are authorized by the Attorney General in his discretion, however, and the officials appointed may be named directly by the Ationey General, although it is usual to accept the recommendation of the district attorney as to his assistants. The salary of the assistant district attorney is also fixed by the Attorney General, but cannot exceed $\$ 2,500$ except in the northern district of Illinois and the southern district of New York, in which districts the limit of salaries has been removed by the special legislation. It is required by law that assistant district attorneys must be residents of the district in which they are appointed, and the Attorney General is authorized to fix and declare the place of the official residence of the district attorney and his assistants respectively. It is the duty of district attorneys to prosecute all delinquents for crimes against the United States which may occur in his district, and also all civil actions in which the United States is concerned. Unless he is otherwise instructed by the Secretary of the Treasury, he is also required to appear in behalf of any officers of the revenue in proceedings brought against them for any act performed or for the recovery of any money paid to such officers and by them paid into the treasury of the United States. District attorneys are also required to furnish assistance in relation to the determination of titles to land within their district which is sought to be acquired for the United States and also to appear in behalf of an officer of either house of Congress in any action which may be brought against him on account of acts performed while the said officer was in discharge of his duty.

District Attorneys upon instituting any action for the recovery of a fine, penalty or forfeiture, are required to make a statement in connection with the matter to the Secretary of the Treasury and at the end of every term of court to make a report, accompanied by a certificate of the Clerk of the Court, of the cases affecting the revenues decided during the term and the cases pending at the close of the term. Similar reports with regard to cases affecting the postal revenues are required to be made by 
the United States Attorney and by departmental regulations attorneys are required to furnish reports of all cases instituted of whatever character, and of the successive steps in the litigation, in order that the dockets of the Department of Justice may be kept up. The latter are under the supervision of a Docket Clerk, who is an official of the department proper, and show in detail all proceedings undertaken by District Attorneys on behalf of the United States in the different courts, as well as all special proceedings under the direction of the Treasury and Post Office departments, together with the successive steps in each litigation, so that the whole constitutes a complete record of the status of cvery case in which the United States is a party.

As originally contemplated, the District Attorneys had practically independent powers within their districts and to a certain extent retain such powers to the present time, although there is an increasing supervision and direction exercised by the Department of Justice over their operations and functions. The District Attorney is, of course, a sworn officer of the court in which he practices on behalf of the United States and the chief prosecuting officer of the Government. Violations of the law are in the majority of cases brought directly to the attention of the District Attorney and prosecuted by him without reference to the Department of Justice, except that the docket report before mentioned is made, and instructions are sccured in unusual cases. No extra compensation can be allowed a District Attorney for services in the line of his duty, nor can he be authorized to employ special attorneys for the cxanination of land titles. Extra assistance besides his regular assistant may be provicled by the Attorney General, but cannot be directly engaged by the District Attorney. In addition to his compensation the District Attorney receives reimbursement for his expenses in amounts not exceeding $\$_{4}$ a day and transportation when actually traveling on business of the court and going to and from places of holding court other than his official residence, and also whilc traveling for the purpose of attending hearings before United States Commissioners. Assistant United States Attorneys are also allowed expenses within the same limit. Reimbursement for expenses in- 
curred in traveling are secured by accounts rendered in duplicate to the department and accompanied by vouchers authenticating the expenditures charged for.

United States Attorneys and their assistants are not required to give their exclusive services, but may engage in private law practice which does not conflict with their official duties. They are, however, not allowed to appear as counsel before state legislatures or take any undue part in influencing state legislation and are strictly prohibited from representing even indirectly an adverse interest to the United States.

Besides the Assistant United States Attorneys, District Attorneys are furnished in the discretion of the Attorney General with a clerical force of such amount and at such compensation as may seem to be warranted by the business of the district. When permission is granted the District Attorney to take his clerk to court, the expenses of the clerk are paid in amounts not exceeding \$2 a day and railroad fare. The office expenses of District Attorneys are also fixed and allowed by the Attorney General, it being usual, however, to authorize reasonable expenses when requested by the District Attorneys. Other expenses which are authorized by the department are for stenographers to take testimony in court, for experts and interpreters, and for the printing of records and briefs, and the cost of furnishing and collecting evidence. The cost of these services in some instances amounts to considerable sums, but in every case, whether the expenditure involved is small or large, authority therefor must be secured from the Department of Justice before it can be legally undertaken. The actual payment of amounts due under such authorization is, however, not made by the District Attorney, but the sums are paid by the marshal, who acts as the disbursing officer for the purpose and for the payment of witness fees and other court expenses. District Attorneys are required to obtain permission from the Attorney General before absenting themselves from their respective districts for any purpose, and two classes of such permissions are requested, one to be absent on official business and on orders, in which case expenses are allowed; and the other to be absent on personal business, 
such authorization carrying with it no right to receive expenses, and to be effective only when public business will permit the absence of the legal officer. There are special provisions in the statutes requiring District Attorneys to take particular cognizance of offenses against persons mnder the Civil Rights bill, and also to inquire into and takc proceedings against persons who make false claims against the United States. In prize causes it is the duty of the District Attorney to represent and protcct the interest of the Lnited States and tine captor of the prize, and also to make to the Secretary of the Navy statements of the conditions of all such causes pending, at least once in three months, when there are any such causes in the courts of the United States. In all States and Territories where there are Indian rescrvations or allotted Indian lands, the District Attorneys are required to represent such wards of the nation in any proceeding which they may bring or which may be brought against them. When cases are brought against Chinese persons alleged to be unlawfully in the United States within any district, the District Attorney is empowered to designate the United States Commissioner before whom the hearing required by law shall be held and to report his selection of such Commissioner at once to the Department of Justice.

Special reports are required from the District Attorneys of cases decided in the District and Circuit Courts which the District Attorney deems of sufficient importance to warrant an appeal to the Court of Appeals or the Supreme Court. Hc is especially charged with making such a report in customs cascs and immediately in order that there may be sufficicnt time to consicler an appeal within the thirty days allowed by law for such action. Questions relating to the appeal of cases, especially to the Supreme Court, are closely followed by the Department proper and the District Attorneys are required to furnish the Department as promptly as possible with all data relative to such appeals and the cases upon which they are based. The work of conducting such appcals is entrusted to one of the Assistant Attorneys General and for his information and in order that no point may be neglected District Attorneys are required to be full and exact in their returns of cases and of all the points involved therein. 
District Attorneys are required to keep appropriate books of record of the cases under their control, including a docket of criminal cases, a civil docket, a docket of cases brought before the Grand Jury, a witness docket, detailing the names of witnesses called and a register of complaints. The District Attorney or his properly appointed Assistant is the only official except the Judge and the recording official who can appear before a United States Grand Jury, the proceedings of which are conducted in close secrecy. Information as to alleged illegal acts in the nature of crimes and offenses against the Federal law is presented by the District Attorney or his assistant to the Grand Jury of twenty-four persons, and upon the vote of more than twelve of the jurymen indictments are returned upon which the defendants are tried in the District Courts. The register of complaints kept by the District Attorney includes entries of all complaints made to the office of the Attorney, whether by officials or others, and a record whether prosecution has been had or not.

\section{marshals of the UNited states.}

For each of the judicial districts of the United States there is appointed a United States Marshal who serves for a term of four years, and is appointed by the President, by and with the advice and consent of the Senate. Marshals are required to take an oath to faithfully execute all lawful precepts directed to them, to perform all things well and truly and without malice or partiality, pertaining to the office of marshal and charge no more than the lawful fees. Every marshal must give a bond of at least $\$ 20,000$, and if the business of the court in the district to which the marshal is appointed requires a larger bond the Attorney General may require the giving of such surety as may cover the amount of business transacted by the marshal and safeguard the money which he may be expected to have in his possession at any time as the result of the service of the various processes. The bond of a United States Marshal is conditioned upon the proper performance of his duty by the officer, and if he takes any illegal action, such as a wrongful seizure of property, he is liable under his bond to the party wronged in damages to repair the injury done. No suit on the bond of a marshal can, however, be instituted after six years from the time when the right of action accrued, except in 
the case of infants, married women and insane persons who may sue within three ycars after their disabilities to sue have been removed. United States Marshals have in each State the same powers in executing the laws of the United States which are possessed by the sheriffs of the State in the execution of the State laws. Marshals are required to attend either personally or by deputies the sittings of the District and Circuit Courts in their district and to execute throughout the district all lawful precepts which may be directed to them. United States Marshals have power to command all the necessary assistance which may be required for the execution of the duty of the marshal and can swear in such number of special deputies and command the service of the posse in accordance with the prerogative conferred upon sheriffs and similar officers of the peace. When judgments from the United States courts for the recovery of moneys due the United States under process which has been commenced by the direction of the Solicitor of the Trcasury are given to a marshal for service, he is required to report to the Solicitor of the Treasury what proceedings he has talicn with regard to the collection of such judgments, and in case of a collection on account of the postal revenues a similar report must be made to the Auditor of the Post Office Department of the various steps under the authority of the judgment writ and of the amount of money actually collected for the benefit of the Treasury of the United States. The salaries of United States Marshals are fixel by law in licu of the fees which they were formerly perinitied to retain and are as follows:

For the northern and middle districts of the State of Alabama, each, four thousanti dollars; for the southern district of the State of Alabama, three thousand dollars; for the Territory of Arizona, four thousand doilars; for the castern and western districts of Arkansas, each four thousand dollars; for the nortisen and southern districts of California, each four thousand dollars; for the district of Coloradn, four thousand dollars; for the district of Connecticut, two thousand dollars: for the district of Delaware, two thousand dollars; for the District of Columbia, five thonsand five hundred dollars; for the northern and southern districts of Florida, each, three thousand dollars; for the northern district of Georgia, five thousanci dollars; for the soutliern district of Georgin. three thousand five huncted dilars; for the district of Idaho, four thousand dellars; for the nerthern district of Illinois, five thousand dollars; for the eastcrn and sonthern districts of Illinois, each, four thot:sand five hondred dollars; for the district of Indiana, four thousand 
five hundred dollars; for the northern and sonthern districts of Iowa, each, four thousand dollars; for the district of Kansas, four thousand dollars; for the eastern and western districts of Kenttcky, each, five thousand dollars; for the eastern district of Lonisiana, three thousand dollars; for the western district of Lotisiana, two thousand five hundred dollars; for the district of Maine, three thousand dollars; for the district of Maryland, three thousand five hundred dollars; for the district of Jassachusetts, five thonsand dollars; for the eastern district of Michigan, four thousand dollars; for the westerin district of Michigan, thee thousand dollars; for the district of Minnescta, four thousand dollars: for the northern and southern districts of Mississippi, each, three thousand doliars; for the eastern and western districts of Missonri, each, fotir thousand dollars; for the district of Montana, three thousand five hundred dollars; for the district of Nebraska, four thousand dollars: for the district of Nerada, two thcusand five hundred dollars; for the district of New Hamphire, two thonsand dollars; for the district of New Jersey, three thousand dollars; for the district of New Mexico, four thousand doliars; for the northern, southern, and western districts of New York, each, five thousand dollars; for the eastern district of New York, four thousand dollars; for the castern district of North Carolina, four thousand dollars; for the western district of North Carolina, four thousand five hundred dollars; for the district of North Dal:ota, four thousand dollars; for the northern an: southern districts of Ohio, each, four thousand dollars; for the eastern district of Oklahoma, four thousand dollars; for the western district of Okla. homa, five thousand doliars; for the district of Oregon, four thousand dol. lars; for the eastern, middle, and western districts of Pennsylvania, each, four thousand dollars; for the district of Rhoce Island, two thousand dollars; for the eastern and western disiricts of Sonth Carolina, four thousand five hundred dollars, two thousand five lundred dollars of which shall be for the performance of the duties of marshal of the western district; for the district of South Dakcta, four thousand dollars; for the castern, middle, and western districts of Temessee, each, four thousand dollars; for the northern and eastem districts of Texas, cach, three thousand dollars; for the western district of Texas, four thousand dollars; for the southern district of Texas, three thcusand five hundred dollars; for the district of Utah, three thousand five hundred dollars; for the district of Vermont, two thousand five hundred dollars; for the castern district of Virginia, three thousand five hundred dollars; for the western district of Virginia, four thousand doilars; for the eastern and western districts of Washington, each, four thousand dollars; for the northern and southern districts of West Virginia, each, four thousand dollars; for the eastern and western districts of Wisconsin, each, four thousand dollars; for the district of Vyoming, three thousand five hundred dollars.

In case of a vacancy in the office of marshal the Circuit Justice of the Circuit in which the vacancy occurs may make an appointment in 
writing of a temporary incumbent, the latter being required to file a bond to be approved by the Justice as in the case of Presidential appointments. Besides his salary a United States Marshal is entitled to expenses not to exceed four dollars a day when attending court at a place other than his official residence, and also when serving processes or otherwise necessarily absent from his flace of official residence on official business. In addition he is allowed his actual necessary traveling expenses, the expense amount previously named being for lodging and subsistence, while away from his official residence, and when traveling with prisoners a marshal is allowed expenses and the necessary guard hire. The official residence of a marslial must be at one of the places of holding court in the District and the selection of the place is made by the Attorney General, due regard being had to the requirements of official business, but being fixed at the marshal's usual place of residence if practicable. Marshals are expected to attend personally to the duties of their office and to oversce the operations and accounts of their deputics, not leaving such functions to other persons.

The assistants of United States Marshals are styled deputics and are of two classes, office deputies, and field deputies. Office deputies are appointed for the purpose of carrying on the clerical work of the marshal's office and for the performance of official duties not performed by field deputies and are compensated by salaries fixed by the Attorney General. The chief of the office deputies of a marshal has general supervision over the accounts and business of the office and as a rule attends to all accounting with the assistance of such additional office deputies as may be assigned to this work. Other office deputies may be assigned to the serving of process. the position of field cleputies being changed into office deputies when for a special reason satisfactory compensation cannot be made to deputy marshals under the fee system. The greater part of the work relative to the serving of processes is, however, performed by field deputies who are appointed by the marshal with the approval of the Attomey General who is empowered by law to cancel any appointment of a field deputy made by a marshal and at any time. Office deputies are appointed by the Attorney General on the recommendation of the marshal, and marshals can cmploy only such office deputies and 
clerical assistants, and at such compensation as the Attorney General may authorize. The position of chief office deputy is distinct from and superior to that of office deputy, but the promotion is made by the marshal, who must notify the Department of the selection of a person as chief office deputy. The marshal is entirely responsible for the acts of his deputies, and is liable on his bond for any illegality, as in the case of his own malfeasance or misfeasance in office. The terms of office of all deputies terminate with the term of office of the marshal by whom they are appointed, and if a new marshal desires to retain the services of former deputies new appointments are required. Office deputies may perform the service of bailiffs and criers of the court, but when they do so it must be without additional compensation, and if compensation is received the salary of the deputy will be correspondingly decreased. Criers, not deputy marshals, are appointed upon recommendation of the court, and their salaries are fixed by the Attorney General, to be pais out of the appropriation for the payment of court expenses. The appointment of field deputies by the marshal is dependent upon the exigencies of the service as he may deem them to exist, and may be either such number as may be needed to serve the processes occurring in the district, or may be an additional number of special deputies to enforce the performance of an order of court. Regular field deputies are located at various places throughout the district and are assigned to specific sections of territory. When more field deputy marshals are appointed than the public interest requires, the Attorney General will cancel the appointments.

The field deputy marshal receives as his compensation threefourths of the gross fees which are earned by him in the serving of the various processes, but not exceeding $\$$ I, 500 for each year. The additional one-fourth and fees in excess of the amount which would pay the field deputy $\$$ I,500 are turned into the Treasury of the United States. In addition to the foregoing, field deputies, when endeavoring to arrest persons are allowed actual necessary expenses, not exceeding $\$ 2.00$ a day. As the field deputy marshal has to pay his own expenses while earning the fees from which he receives a maximum of $\$ I, 500$ a year, with the slight additional revenue from endeavoring to arrest persons, the Attorney General is given 
discretion to make an additional allowance over the maximum so that a field deputy marshal can earn from three-fourths of the fees which result from the service of the various processes, at the outside, $\$ 2,500$ a year. Owing to the increase in expenses of serving processes due to the fact that all railroad fares must now be paid, whereas the field deputies formerly traveled on passes, a considerable decrease in the net results is experienced, so that a field deputy is required to expend more money to secure the same amount resulting from the thrce-fourths of the fees earned. A proposition is therefore made that field deputies shall receive the entire amount of the fees earned by them instead of three-fourths, as at the present time. When a field deputy under the present system has earned the maximum of $\$ \mathrm{I}, 500$ before the end of the fiscal year, he must serve the rest of the year in order to secure the full amount due to him, as he cannot receive the full amount during the first part of the year, and then resign, but must continue in order to get his maximum until the close of the fiscal year. The extension of the compensation of a field deputy marshal from the statutory limit of $\$ 1,500$ to the permissive limit of $\$ 2,500$ is made upon the basis of a showing of business done, and when the United States profits by the services of the field deputy, considerably beyond the amount of earnings which would be represented by the maximum of $\$ 1,500$.

United States marshals render service not only to the United States in serving processes in which the govermment is interested, but also serve processes for private litionts in Lnited Status courts. Before rendering service other than for the United States, however, the marshal must exact a deposit or bond sufficient to cover his fees and costs in the case unless the party for whom the process is served comes within the provision of law relative to those suing as poor persons. Bonds must be especially so drawn as to cover the payment of all costs which may accrue to the United States. The amounts returned by marshals and their deputies in serving process consist of fees and mileage, the fees being fixed for the performance of each several acts and the mileage heing according to law in the accompanying fee table. When writs are served on account, both of the United States and of private persons on the same trip, the mileage is charged to the individual where it is identical with the mile- 
age traveled in serving the writ for the United States. In the districts of Oregon, Nevada, New Mexico, Arizona, Wyoming, Idaho, and North Dakota double fees are imposed on account of the extra expenses of travel in those States under former conditions. All fees collected by the marshal and his deputies from individuals and corporations are paid to the clerk of the court and all fees earned on account of process served in behalf of the United States are returned in the marshal's account, and those which have been earned by field deptuties together with the amounts returned as having been collected from individuals and corporations are made the basis of the computation of the money to be received by the field cleputy as his compensation. Penalties are imposed by the statute for failure to account for fees and for false returns upon which excessive amounts may be collected from the United States. In connection with the duty of the marshal to serve processes it frequently becomes necessary for him to seize goods and to take other steps for the removal of property from the custody of persons claiming the ownership thereof. Should such removal be found to be illegal the persons from whom the goods were taken have a recourse against the marshal who must in order to protect his bond require a bond from the person in whose behalf the process was served. In case of capture of vessels as prizes, or the seizure of property alleged to have been used in behalf of insurrectionary forces the marshal has custody under the direction of the prize court of the property in question and carries out the judgment of the court in selling the property and in depositing the proceeds with the Clerk of the Court for due distribution according to decree.

The fees allowed marshals and their deputies are as follows:

For service of any warrant, attachment, summons, capias, or other writ, except exccution, venire, or a summons or subpœna for a witness, two dollars for each person on whon service is made.

For the keeping of personal property attached on mesne process, such compensation as the court, on petition setting forth the facts under oath, may allow.

For serving venires and summoning every twelve men as grand or petit jurors, four dollars, or thirty-three and one-third cents each.

For holding a conrt of inquiry or other proceedings before a jury, including the summoning of a jury, five dollars. 
For serving a writ of subpœna on a witness, fifty cents; and no further compensation is allowed for any copy, summons, or notice for a witness.

For serving a writ of possession, partition, execution, or any final process, and for making the service, seizing or levying on property, a fee of two dollars, and the same mileage as is allowed for the service of any other writ; and for advertising and disposing of the same by sale, set off, or otherwise according to law receiving and paying over the money, a fee of two dollars, and a commission of two and one-half per contum on any sum under five hundred dollars, and one and one-fourth per centum on the excess of any sum over five hundred dollars.

For each bail bond, fifty cents.

For summoning appraisers, fifty cents each.

For executing a deed prepared by a party or his attorney, one dollar.

For drawing and exccuting a deed, five dollars.

For copics of writs or papers furnished at the request of any party, ten cents a folio.

For every proclamation in admiralty, thirty cents.

For serving an attachnent in rem or a libel in admiralty, two dollars.

For the necessary expenses of keeping boats, vessels, or other property attached or libeled in admiralty, not exceeding two dollars and fifty cents a day.

When the debt or claim in admiralty is settled by the parties without a sale of the property, the marshal is entitled to a commission of one per centum on the first five hundred dollars of the clain or decree, and onc-half of one per centum on the excess of any sum thercof over five hundred dollars.

For sale of vessels or other property under process in admiralty, or under the order of a court of admiralty, and for receiving and paying over the money, two and one-half per centum on any sum under five hundred dollars, and one and one-quarter per centum on the excess of any sum over five hundred dollars.

For expenses while employed in endeavoring to arrest, under process, any person charged with or convicted of a crime, the sum actually cxpended, not to exceed two dollars a lay, in addition to his compensation for service and travel.

For every commitment or discharge of a prisoner, fifty cents.

For transporting criminals, ten cents a mile for himself and for each prisoner and necessary guard; except when actual expenses are allowed.

For attending examinations before a commissioner, or other conmitting magistrate, and bringing in, guarding, and returning prisoners charged with crime, and witnesses, two dollars a day; and for each deputy not exceeding two, necessarily attending, two dollars a day.

For travel in going only, to serve any process, warrant, attachment, or other writ, including writs of subpœna in civil or criminal cases, six cents a mile for every mile actually and necessarily traveled. 
The United States marshals are the principal disbursing officers of United States Courts and pay the various expenses under the following appropriations, namely; salaries, fees and expenses of marshals, fees of jurors, fees of witnesses, support of prisoners, fees of bailiffs, etc., and miscellaneous expenses of the Court. The marshal is required upon the order of the Court to pay to the juror and to the witnesses the fees to which they are entitled by reason of their service. No person can be summoned as a juror in a Circuit or District Court more than once in two years and the names are drawn publicly both for grand and petit jurors from a box containing the names of not less than 300 persons which have been placed in the box by the Clerk of the Court and by a jury commissioner, who is required to be a citizen of good standing in the district in which the court is held, and a well known member of the opposite political party to that to which the Clerk may belong. The Clerk and the Commissioner place one name in the box alternately until the number of 300 has been reached. If so directed by the court the names of jurors can be drawn from boxes used by State authorities in selecting jurors in the highest courts of the States. Jurors must be returned so as to be most favorable to an impartial trial and also so as not to make the attendance of the jurors unduly expensive. Jurors receive $\$ 3.00$ per day for attendance at court, including the time spent in going and coming. Those who reside at the point of holding court are not entitled to per diem on the days in which the court is not in session. Mileage is also allowed at the rate of five cents a mile for the distance traveled going and coming, except in certain States where travel must be performed by other conveyance than railroad or steamboat, in which States mileage is allowed at the rate of fifteen cents a mile. Witnesses are entitled to $\$ 1.50$ a day for each day's attendance at court and five cents a mile going and returning. If, however, a person attends court as both a witness and a juror he can only secure one mileage. Witnesses do not receive compensation for the time taken in going from and coming to court or for days when the court is not in session. Clerks and officers of the United States who are required to go from their usual place of business to attend court receive their expenses for such attendance, but no mileage or additional compensation. When an officer of a department is paid a per 
diem for expenses in addition to his salary, he cannot receive expenses for appearing as a witness in any United States Court. It would appear that Internal Revenue officers being required by law to use their cfforts to punish offenders against the internal revenue laws as a part of their official duties are paid for attendance at court by their official compensation only and are not entitled to witness fees and mileage. In order to decrease the expenses of hearings before United States Commissioners the fees of not more than four witnesses can be taxed against the United States in such a hearing unless the District Attorney certifies that the presence and testimony of more than four are necessary.

The marshals of the United States have the custody of prisoners who may be awaiting trial in the United States courts and who are detained in the absence of a United States jail at the point at which the marshal performs his duties in such county or State institution as the marshal may select, commitment being made on the order of the marshal or his deputy and releases upon duly authorized release cards. The bills for the subsistence of prisoners so detained awaiting trial are paid by the marshal out of the appropriation for the support of prisoners. In addition to these payments for the support of prisoners the marshals are required to keep account of the prisoners confined under sentence from Lnited States Court in State institutions in their district and to pass upon the bills rendered for the support of such prisoners. United States marshals disburse sums out of the appropriations pay of bailiffs, etc., United States Courts, for the pay of hailiffs and criers in attenclance upon the court. Bailiffs are appointed by the marshals to keep orier in the court, to have the custody of the prisoners and perform similar serviees. Criers are appointed by the Jutges, the position of cricr being personal to the Judge and representing the official dignity of the latter. Bailiffs and criers are allowed \$2.00 a ray and not more than three bailiffs and one cricr are allowed for each court except in the Southern District of New York, where five are allowed. Bailiffs and criers are paid only for actual attendance, but when they are directed to be present by order of the Court they are decmed to be in attendance even though no sitting of the court is held. The employment of a bailiff 
does not necessarily terminate upon the expiration of the term of the marshal who employed him.

The expenses for travel and attendance of district judges directed to hold court outside of their district is paid by the marshal upon the written certificate of the judge not to exceed $\$$ Io a day. The expense of furnishing meals and lodging for jurors in United States cases and for bailiffs who attend upon the juries is paid by the marshal when so ordered by the court. The expense of lodging and feeding juries in other than United States cases in the Courts of the United States cannot be met from any appropriation and must be taxed as a part of the costs. Jury commissioners who are appointed by the judges receive a compensation of $\$ 5$ per day, but can be paid only for three days for any one term of court. No mileage or other allowance can be made or paid to a jury commissioner. Miscellaneous expenses paid for by the marshal as disbursing officer include the cost of interpreters and of messengers to judges when employed by direction of the Attorney General, the payments for expert services and testimony, for record books, for clerks, and the expenses for taking juries to view lands in condemnation cases, and also including expenses for advertising and for the telegrams of Judges. For all of these expenses the marshal disburses the amounts which may be shown to be due by the appropriate vouchers and he is supplied by the Treasury Department on requisition of the Department of Justice with such amounts in advance of expenditure as may be necessary for him to meet the payments involved promptly. The amounts which may be necessary to defray the expenses of witnesses in any case is, of course, indeterminate, and the total amount which must be covered by this annual appropriation cannot be foreseen, so that it is for a large amount and general in terms so as to cover a large variety of contingencies. It frequently happens that it necessary for the marshal to requisition by wire for considerable amounts of money in order to pay the per diems and mileage of witnesses and jurors and arrangements are made by which the telegraph can be utilized in placing the advances to the credit 
of marshals for the purposes named. All expenditures made by the marshals must be carefully accounted for and the transcript of accounts accompanted by vouchers and if in any case the marshal is unable to make satisfactory explanation for any expenditure or to show authority by law or an adequate voucher the amount is checked against him and must be reimbursed by him to the Government.

It sometimes occurs that a marshal must make expenditures for the preservation of the peace of the United States which are not covered by the terms of any appropriation. The President is given power by statute to approve such expenditures on being advised of the character of the emersency requiring it and his approval is equivalent to Congressional appropriation in validating the payment of the amount involved from the Treasury.

TIIE SUPERINTENDENT OF PRISONS AND PRISONERS.

Under the provisions of the considerable number of Federal statutes prescribing imprisonment as a punishment for crimes and misclemeanors a large number of persons are convicted annually, and it was formerly the universal practice to make arrangement with State institutions for the confinement of United States prisoners. A number of years ago, however, there was commenced near the old military prison at Fort Leavenworth, Kansas, which had formerly been used by the United States as a place of incarceration for Indian Territory and other prisoners from nearly districts, a Federal penitentiary, and this was followed by the establishment of another Federal penitentiary at Atlanta, Ga. Portions of these institutions were built under conditions of contract construction, but as soon as a sufficient number of buildings were erected to provide for the accommolation of prisoners the prison labor was atilized, and the continuance of the building of the institution is going on by means of the labor of the inmates. Several years' work yet remains before the institutions referred to will be completed, but it is the purpose to ultimately confine all United States prisoners in Federal penitentiaries. Those now so confined include long term and life sentence prisoners, together with such prisoners as may seem to be best calculated to be useful 
in connection with the work of building now going on. For other prisoners and for women prisoners arrangements are made with the different state penitentiaries for their care at a sum per diem for subsistence and clothing. The prisoners can be cared for under this system at a smaller expense than at Federal penitentiaries, for the reason that they are utilized in the state penitentiaries in doing contract work which reduces the amount charged for their support. The contract system, however, involves so many different conditions of treatment of United States prisoners that it is felt to be undesirable, and it is the policy of the Government at the present time to reduce the number of prisoners so held as much as possible and to substitute a Federal system for the contract system now partially in vogue. Besides the two Federal penitentiaries, the United States maintains a small penitentiary at McNeil Island, Washington, for prisoners from the Pacific Coast, together with jails in Alaska and in the territories. The penitentiaries at Leavenworth and at Atlanta are under the supervision of regular wardens, but the smaller Federal institutions are directed by the United States marshals, a deputy marshal or custodian being in charge under the orders of the marshal of the district. The penitentiaries have the usual organization, and the whole system is under the direction of the Superintendent of Prisons and Prisoners who is located at Washington and is appointed by the Attorney General, receiving a salary of $\$ 3,500$ a year. $\mathrm{He}$ has supervision of all matters relating to the different prisons and to the transfer of prisoners and makes the contract arrangements for the support of United States prisoners at the institution. All contracts for supplies and materials are arranged for by the Superintendent who advertises for bids, opens the same and awards the contract after having secured the recommendation of the officers who have applied for the supplies or materials. The work of building the penitentiaries is in general charge of an architect but the actual work of construction is directed by a Superintendent of construction upon the ground who has a force of employed foremen and instructors who direct the work of the prison labor.

In connection with the office of the Superintendent of prison and prisoners there is maintained a system of identification after the Bertillon method, the files containing identification cards for all 
United States prisoners and being intended for exchange and comparison with State records and the records of local police systems.

CLERKS OF COURT.

Clerks of Courts are provided for each sitting of United States courts, including District, Circuit Courts, Circuit Court of Appeals and the Supreme Court. District Court Clerks are appointed by the District Judge and the Clerks of Circuit Courts are appointed by the Circuit Judges. A Circuit Judge has the selection of the Clerk of the Circuit Court in the District in which he sits but as District Judges generally sit as Circuit Judges for the hearing of original cases in the Circuit Court it is usual for the person who has been appointed as a District Clerk to be certified to the Circuit Court by the District Judge and to be appointed by the Circuit Court as a Circuit Clerk, the same person acting in both capacities. No person related to a Judge or Justice of any court of the United States within the degree of first cousin can be appointed or cmployed in the court of which the Judge may be a member, and this prevents the appointment by Judges of relatives within the degree named to the position of Clerk. Clerks are required to talie an oath of office and to file a bond in ordinary cases from five to twenty thousand dollars, but the Attorney General may in his discretion, when the busincss of the court requires it, make the amount of the bond not to exceed forty thousand clollars, and if a bond has already been given it can be increased upon the direction of the Attorney Gencral within the limits above stated. Bonds of clerks are filed in the Department of Justice but they must be recorded in the offices of the Clerks, so that certified copies from the records may be used if necessary in evidence. The bonds of a Clerk must be approved by the Judge in open court. Deputies to clerks of courts are appointed by the jurges of the respective courts, generally upon the recommendation of the Clerks and may be removed by the judges authorized to make the appointments. In case of the death of a Clerk his deputies continue to perform his duties and the bond of the Clerk is responsible for the act of the deputy whether the Clerk be alive or whether the deputy is acting as Clerk in the event of the Clerk's death. The deputy clerks are paid from the emoluments of 
the Clerk of the Court, and while they may be appointed in the discretion of the Judge, authority for their payment must be secured from the Attorney General before they can receive any compensation. Deputy clerks must take an oath of office and may be required by the Clerk to furnish such bond as may be necessary for his protection and that of his bond. The duties of the Clerks of United States courts consist of making the proper record of documents which are required to be filed in such Clerk's office, preparing the dockets for the cases in court, attending court for the purpose of making the necessary minutes on the records, making out all papers which may be necessary to evidence the action of the court, receiving the returns of marshals and in general carrying on the clerical business of the court in accordance with the usual form and procedure of the courts of justice and with specific provisions which may be made by the statutes of the United States in the premises. In addition the clerks of United States courts are custodians of considerable funds which are paid into the registry of the court to await determination as to their final disposition. These include amounts in controversy, amounts which may have been realized from sales by the United States marshal and amounts which may have accrued from any cause under the proccedings of the courts, the title to which either has not been determined or a claimant for which has not yet appeared. Money can only be withdrawn from the registry upon the order of the Judge or Judges, and heavy penalties are imposed upon a Clerk who may use any part of the money, while the Clerk is also forbidden to secure interest upon the registry deposits or upon any other sums of noney which may be in his custody. At the expiration of ten years if a given amount of money has not been claimed from the registry it is to be turned into the Treasury of the United States. Considerable sums of money are paid into the hands of the Clerks of each District and Circuit Courts on accounts of fines and forfeitures which are recovered under the various revenue and penalty laws of the United States. $\mathrm{He}$ is required to make a deposit of the same at once and to cover the amounts into the Treasury of the United States as soon as possible. Clerks of Courts are compersated by fees which they are permitted to charge in accordance with the following table. 
For issuing and entering every process, commission, summons, copies, execution, warrant, attachment, or other writ, except a writ of venire or a summons or subpena for a witness, one dollar.

For issuing a writ of summons or subpona, twenty-five cents.

For filing and cutering cvery declaration, plea, or other paper, ten cents.

For arlministering an oath or affirmation, except to a juror, ten cents.

For taking an acknowledgment, twenty-five cents.

For taking and cortifying depositions to file, twenty cents for each folio of one hundred words.

For a copy of such ieposition furnished to a party on request, ten cents a folio.

For entering any return, rule, order, continuance, judgnent, decree, or recognizance, or drawing any bond, or making any record, certificate, return, or report, for each folio. fifteen cents.

For a copy of any entry or record, or of any paper on file, for each folio, ten cents.

For making dockets and indexes, issuing venire, taxing costs, and all other services on the trial or argument of a cause where issue is joined and testimony given, three dollars.

For naking dockets and indexes, taxing costs, and all other services in a cause where issue is joined but no testimony is given, two dollars.

For making dockets and indexes, taxing costs, and other services in a cause which is dismissed or discontinued, or where judgment or decree is made or rendered without issue, one dollar.

For making dockets and taxing costs in cases removed by writ of error or appeal, one dollar.

For affixing the seal of the court to any instrument, when required, twenty cents.

For every search for any particular mortgage, judsment, or other lien, fifteen cents.

For scarching the records of the court for judgments, decrees, or other instruments constituting a seneral lien on real estate, and certifying the result of such search, fifteen cents for each person against whom such search is required to be made. 
For receiving, keeping, and paying out money in pursuance of any statute or order of court, one per centum on the amount so received, kept, and paid.

For traveling from the office of the clerk, where he is required to reside, to the place of holding any court required by law to be held, five cents a mile for going and five cents for returning, and five dollars a day for his attendance on the court while actually in session.

As full compensation for their service to each bankrupt estate a filing fee of ten dollars, except when a fee is not required from a voluntary bankrupt.

No clerk, however, except in couble fee districts, can retain fees during any calendar year in excess of the sum of $\$ 3,500$, although when a Clerk of a District Court is also the Clerk of a Circuit Court he is entitled to retain $\$ 3,500$ for each position, making the total maximum fees which he can retain $\$ 7,000$. Clerks of the Courts in California. Oregon and Nevada are entitled to retain double the amount of fees in other States on account of the expenses of travel and subsistence in those States. In addition to the maximum stated, Clerks are allowed to retain one-half of the maximum of $\$ I, 750$ per year, when they have served in any prize cases; that is to say, for service in prize cases Clerks are entitled to retain the amount of the fees earned up to $\$ 1,750$, in addition to the maximum compensation allowed them for other business. The Clerk must secure his compensation from fees earned during the calendar year, and cannot use the fees earned in one year as compensation during another calendar year. Besides the compensation of the Clerk, the expenses of the office, including clerical allowances, are paid out of the fees and emoluments earned in accordance with the fee table. Before the Clerk can take any personal compensation, his necessary office expenses must be paid from the fees. These having been paid, the Clerk may retain amounts up to the maximum provided, paying such amounts as may be necessary to his deputy clerks stationed at other places of holding courts, in order to secure the proper performance of the duties imposed upon the Clerk's office, and the amounts of fees above the expenditures for the expenses of the office and the Clerk's emoluments, are turned over to the Treasury of the United States. The Clerk must make 
a strict account of all fees received by him, as well as all expenses, and of the condition of the various funds under his control. The office of the examiners of the Department of Justice is largely to check up and supervise the accounts of Clerks of Courts, to see that they are kept in proper form and that no illegal disposition is made of sums of money coming under the Clerks' care. 


\section{CHAPTER XII.}

THE POST OFFICE DEPARTMENT.

The Post Office Department of the United States is, in effect, an adjunct to the Federal Government rather than a part of it. A large proportion of its support comes from its own business revenues and it is only partially maintained by the general Treasury, the payment made to make up the deficit in the Postal Revenue being, in part at least, the price paid by the United States for carrying its own mail, so that only a portion of the amount added by the United States to the amounts received from the Postal Revenues is an actual expenditure for the support of a Governmental function. The Post Office Department of the United States has been criticised as poorly managed and as unduly expensive. It is true that the revenues do not meet the expenses and that a certain proportion of mail service could be performed at a very much less expense than is now incurred. Compared, however, with the rates charged for communication by private individuals and with the service maintained by such private companies, there is ground for the contention that if a private company attenipted to perform a service of equal magnitude to that of the Post Office Department of the United States, it would neither perform it so well nor at a saving from the amount now paid. So long as telegraph and express rates remain at the present schedules, people who are enjoying the advantages of a two cent postage rate will be rather skeptical on the point of the alleged utter failure of the Government to properly conduct a postal system.

Although the revenues of the Post Office Department are kept in the Treasury of the United States, they are not under the control of the Secretary of the Treasury, but their expenditure is directed by the Postmaster General, together with that of the sums appropriated annually by Congress for the purposes of making up the deficiency in the revenue. The Postmaster General has, therefore, a wider field of activity and in this respect larger discretion 
than any other of the members of the Cabinet. He has large powers of control, not only over the immense amount of money involved in the Post Office business, but over the tremendous personnel covering every city, town and hamlet in the United States, and oves the large amounts involved in the contracts for the Department and for the transportation of the mails. The Postmaster General is assisted in his cluties by four Assistant Postmasters General, each of whom corresponds to an Assistant Secretary of a Department, and each having a particular branch or aspect of the Postal work under his direction. Thus, the first $\Lambda$ ssistant Postmaster General has the Post Office administration and the city free delivery system. The Second Assistant Postmaster General is in charge of transportation of mails by railway and of the foreign mail service. The Third Assistant Postmaster General is in charge of the postal finance and the supply of stamps to postoffices. The Fourth Assistant has charge of matters relating to the rural free delivery, and general supplies. The Postmaster General retains for his personal administration, besiles the considcration of general matters of policy under the Department, the inspecting system of the department and the general purchase system, besides the matters relating to the persomel of the Department proper. He passes upon, when the consideration is completed by the Assistant Attorney General, for the Post Office Department, the credits or reimbursements that shall be allowed to Postmasters for losses by fire, burglary and other unavoidable cause, and in addition to the supervision of the duties of the divisions of the Post Office specifically reserved for his office. the Postmaster Gencral also has the determination of appeals from the action of the several Assistant Postmasters General, together with the promulgation of all rules and regulations for the Post Office Department and the Postal Service.

The organization of the Post Office Department is as follows:

Office of the Postmaster-General:

Chief clerk.

Private secrerary.

Assistant Attorney-General.

Purchasing Agent.

Chief inspector (Division of Post-Office Inspectors).

Appointment clerk. 
Disbursing clerk.

Office of the First Assistant Postmaster-General:

Division of Postmasters' Appointments.

Drvision of Salaries and Allowances.

Division of City Delivery.

Office of the Second Assistant Postmaster-General:

Division of Railway Mail Service.

Division of Foreign Mail.

Division of Railway Adjustments.

Division of Contracts.

Division of Inspection.

Division of Equipment.

Office of the Third Assistant Postmaster-General:

Division of Finance.

Division of Stamps.

Division of Money Orders.

Division of Registered Mails.

Division of Classification.

Division of Redemption.

Office of the Fourth Assistant Postmaster-General:

Division of Rural Delivery.

Division of Supplies.

Division of Dead Letters.

Division of Topography.

The Chief Clerk of the Post Office Department, in addition to performing the usual functions of Chief Clerk with relation to the personnel of the Department proper and of control over the Departmental buildings in Washington, signs all requisitions on the Public Printer for printing for the Department and the Postal Service, directs the compilation of matter for the Official Postal Guide, and has the supervision of its publication and distribution and superintends the issue and sale of the Post Routes map prepared by the topographer, which shows the location of all Post Offices and Post Routes throughout the United States and its possessions.

Under a former system each of the general subdivisions of the Post Office Department made arrangements for the purchase of the supplies used by that subdivision, with the result that there were many difierent prices paid and many different systems in vogue by which supplies were secured. Under the present provision of law, a Purchasing Agent is provided for the Post Office Department who has direction and control under the direct supervision of the Post- 
master General of the purchase of all supplies either for the Post Office proper, or for any branch of the Postal Service. The specifications for the purchase of articles are nade up in the bureau uncler which the articles are to be used, and are forwarded to the Purchasing Agent who revises them and suggests such changes as he may deem to be necessary, any disagreement as to the terms of the specifications being settled by the Postmaster General. The Purchasing Agent upon completion of the specification prepares and issues advertisements and forms for proposals and the bids are opened by a committec appointed for the purpose who make a recommendation to the Purchasing Agent and the Postmaster General, suggesting such awards as in the judgment of the committee are proper. The Purchasing Agent reviews the findings of the conmittee and recommends to the Postmaster General such action as should in his judgment be taken. All articles bought under contract are subject to inspection by a committce appointed by the Postmaster General which reports approval or disapproval to the Postmaster General and the Purchasing Agent and what action shall be taken in case the supplies do not, in the opinion of the committee, comply with the terms of the contracts made by the Purchasing Agent with the approval of the Postmaster General. When the Purchasing Agent makes an open marliet purchase, it is also subject to similar inspection, and the Purchasing Agent may in his own behalf make an independent inspection of goods supplied by contract or on open market purchase. The Purchasing Agent has the final power of decision with reference of the quality of the goods supplied under contract or open purchase and if, in his opinion, they do not meet the requirements of contract or of the service. he can reject them or, if such rejection would cause undue delay, may accept them at a reluction from the contract or purchase price.

Provision is made by which Postmasters and otiner postal officials may purchase supplies on allowance being made by the appropriate bureau but the allowances must be submitterl to the Purchasing Agent for criticism, and if he does not deem it to be a proper requisition or allowance he may certify the fact to the proper burean and the Postmaster General for consideration. The es- 
tablishment of the position of Purchasing Agent was made largely in consequence of the disclosures of fraud which had been perpetrated on the Postal Service through the independent power of subordinate officials to allow and order the purchase of Post Office supplies, large purchases having been made in excess of the needs of the Post Office and for the purpose of securing commissions paid by the manufacturers to officials who had charge of the allowances.

The Division of Post Office Inspectors, formerly under the charge of the Fourth Assistant Postmaster General has been taken directly under the control of the Postmaster General, also as a consequence of the irresularities previously mentioned, it having been found that the control of this branch by a stibordinate official of the Department had the effect of keeping from the head of the Department certain details which he should have known for the proper conduct of the Postal Service. The service is in charge of a Chief Post Office Inspector, and is divided into sixteen divisions, each under the control of an Inspector in charge. The headquarters of the several divisions are as follows:

For the division cmbracing the States of Maine, New Hampshire, Vermont, Massachusetts, Rhode Island, and Comnecticut: Boston, Mass.

For the division embracing the State of New York: New York, N. Y.

For the division embracing the States of Pennsylvania and New Jersey: Philadelphia, Pa.

For the division embracing the States of Delaware, Maryland, Virginia, West Virginia, and North Carolina, and the District of Columbia: Washington, D. C.

For the division embracing the States of Ohio, Indiana, and Kentucky: Cincinnati, Ohio.

For the division embracing the States of Illinois, Michigan, and Wisconsin: Chicago. Ill.

For the division embracing the States of Missouri (except the city of Kansas City), Iowa, and Arkansas: St. Louis, Mo.

For the division embracing the States of Kansas, Nebraska, and Oklahoma and Indian Territories, and the city of Kansas City, Missouri: Kansas City, Mo.

For the division embracing the States of Tennessee, Alabama, Florida, Georgia, and Sonth Carolina: Chattanooga, Tenn.

For the division embracing the States of Texas, Louisiana, and Mississippi: New Orleans, La. 
For the division cmbracing the States of Wyoming, Colorado, and Utah, and the Territory of New Mexico: Denver, Colo.

For the division enbracing the States of California and Nevada and the Territor: of Arizona: San Francisco, Cal.

For the division cmbracing the States of Idaho, Montana, Oregon, and Washington, and the Territory of Alaska: Spokane, Wash.

For the division cmbracing the States of Minnesota, North Dakota, and Soutl! Dakota: St. Y'aul, Minn.

For the division enbracing the Territory of Hawaii: Honolulu, Hawaii.

For the division embracing Porto Rico: San Juan, Porto Rico.

The Post Offec Inspectors are the special representatives of the Postmaster General and are charged with the investigation of Post Offices and all matters connected with the Postal service. When complaints are made of violation of the law or of the loss of letters or of irregularities in the service, such complaints are referred to the nearest headquarters of Inspectors and one of the operatives is detailed for the investigation of the complaint. In addition they are charged with the regular examination of the accounts of the Post Offices and of the condition of the offices as to efficiency. They also investigate local postal conditions with a view to determining whether increases in service that may be asked are warranted, and in general constitnte the means by which the Postal authorities at Washington ascertain accurately the conditions at any given point. Postmasters and their subordinates and contractors and others connected with the Postal service are required to be subordinate to the Post Office Inspectors when the latter are acting within the scope of their duty and employment. When an Inspector enters a Post Office he is required to show the Postmaster his commission as authority for the work in which he is to be engaged. Inspectors are required, however, not to interfere with any employec or the transportation of the mails more than may be necessary for the proper performance of their duties. They have general power of examination and inspection of the work, an account of all officers, and secrecy is cnjoined on Postal cmployees as to the movements and the nature of the business of Inspectors. Much of the work of the Inspectors is necessarily of the character of detective and police work, since they investigate all cases of robbery of the mails and of 
Post Office, and their duties are frequently delicate and dangerous. If, upon examination of a Post Office they find a condition which warrants such action, they are authorized to suspend the Postmaster and to take charge of the office themselves, reporting the fact to the Department in Washington. They may also direct the suspension of any employee when they deem that the situation demands it, but must report the same to the Department and substantiate the fact of a good reason for the course taken. The division of Post Office Inspectors has charge of the correspondence relating to loss and undelivered mail, whether domestic or foreign and follows up all complaints of non-delivery of mail until either delivery has been shown or the probable fact of a loss, in which case the Division endeavors, so far as possible, to determine the responsibility. The Division also has general charge of making up the criminal cases arising under violations of the Postal Laws and of taking steps for the recovery of amounts that may be due from Postmasters and from large users of the mail in cases where improper transactions have been carried on, such as the securing of compensation by a Postmaster in excess of that to which he is legally entitled and the failure of users of the mail who have special privileges to pay the amount properly due. The services of the Inspectors, of course, refer only to cases where such persons are willing to make voluntary settlement, and if such settlements cannot be secured the cases are referred to the officials of the Department of Justice for action.

There are fifteen Inspectors in charge of Divisions provided for at $\$ 3,000$ each, ten Inspectors at $\$ 2,400$, fifteen Inspectors at $\$ 2$,250 , fifteen at $\$ 2,000$, ten at $\$ 1,800$, one hundred and thirty at $\$ 1,-$ 600 , one hundred and ten at $\$ 1,400$ and seventy-two at $\$ 1,200$; in all five hundred. In addition all Inspectors receiving less than \$2,ooo a year are allowed a subsistence per diem of not more than $\$ 4.00$ while traveling or temporarily located at points away from their home. Inspectors are originally appointed for a probationary term of six months at $\$ \mathrm{I}, 200$ a year, and at the end of this term are appointed Inspectors in the field at $\$ 1,600$. A bond of $\$ 5,000$ is required to be filed by the Post Office Inspector, conditioned on 
the proper performance of his duty, and for a proper accounting for all moneys which may come into his hands in connection with the duties indicated.

TIIE FIRST ASSISTANT POSTMASTER GENERAL.

The First Assistant Postmaster General receives the delegation of the power given by the statute to the Postmaster General to establish Post Offices at all such places on the Post roads established by law as he may leem to be expedient. Any person who maintains a Post Office without atthority from the Postmaster General is liable to a fine of not more than $\$ 500$. The First Assistant Postmaster General, upon application for the location of a Post Office at a given point and upon favorable recommendation from the Inspector, can order the establishment of sucl Post Office and fix the name therefor. He must immediately notify the Auditor for the Post Office Department of the establishment of such Post Office in order that the proper accounting and auditing may be made. In case there is reason for the change of the name of a Post Office, or an application is received for such change from the patrons of the office, the First Assistant Postmaster General has jurisdiction over the matter, and can direct the change if he deems it for the public interest. The First Assistant Postmaster General has, broadly, the administration of all matters relating to appointments and salaries of Postmasters and directs the operations of the city free deliveries. For the performance of the duties under his direction his office is divided into three divisions, that of Post Office appointments which has to do with the appointment and general management of Postmasters, that of salaries and allowances, which adjusts the salaries of the Postmasters of the first, second and third classes, and passes on applications for allowance for clerks' hire, rent, etc., and that of city delivery, which direct all matters relating to the carrier delivery of mail except the rural carrier system.

The First Assistant Postmaster General may establish temporary offices for the purpose of caring for mail at stummer and winter resorts respectively, the establishment and designation of such Post Offices remaining in effect throughout the year although the offices may be closed except during the appropriate seasons. He also gives 
directions for the establishment of city branch post offices when found to be necessary, which branch post offices must be located within the corporate limits of the city within which the main Post Office is located, except that a branch post office from a principal city may be established in a village, town, or small city, within five miles from the corporate limits of the location of the city in which the principal post office is located. Substations of post offices may transact all postal business except to deliver mail. They are denoted by numbers and are generally located in connection with business places. Branch stations of post offices are known by letters and transact full post office business, including counter and box delivery of mail and city carrier service.

Provision is made by law and the prerogative is exercised by the First Postmaster General of discontinuing any post office when the safety and security of the mails may be threatened or when the efficiency of the postal service requires such discontinuance. No office established at a county seat, however, can be discontinued by reason of the consolidation of post offices, the only exceptions made by law being in the cases of two post offices near large cities and located in county seats which are both served from the cities to which they are subsidiary.

Postmasters of the first, second and third classes are appointed and may be removed by the President by and with the advice and consent of the Senate. They hold their offices for four years unless sooner removed. Every Postmaster is required to take oath of office and to file a bond in such amount as the Postmaster General may fix, dependent upon the business done by his office and upon the amount of Postal supplies entrusted to his care. Minors and aliens are not eligible for appointment to the office of Postmasters unless the latter have declared their intention to become citizens. Women over eighteen years of age and married women may be appointed as Postmistresses. No person engaged or interested in a mail contract or his or her relatives or no person engaged in prosecution of claims against the United States can be appointed to be Postmaster. Every Postmaster must reside within the territory covered by the delivery of the office to which he is appointed, but if the delivery extends outside the limit of the State in 
which the office is located, the Postmaster may live outside the State, as the delivery linits are not affected by State lines. The commissions of Postmasters are recognized as valid and binding and are honored in filling requisitions until the successor to the Postmaster has actually talien possession of the office. Postmasters of the fourth class are appointed and removed by the Postmaster General and their commissions are signed by the official. Whenever the office of a Postmaster becones vacant by death, resignation or removal, the sureties on the Postmaster's bond take immediate possession of the office unless the Postmaster General shall place the office in charge of an Inspector. The sureties or a majority of them may designate some person to act as Postmaster, who is required to take the oath of office and who conducts the business of the office under the old form until the sureties are relieved by the Postmaster General. If there is a delay of sixty days in filling a vacancy, the sureties on the former Postmaster's bond may notify the Postmaster General of their desire to be relieved of the liability involved, and a Post Office Inspector will be detailed to take charge of the office. The selection of any acting Postmaster desinnated by the sureties must be approved by the Postmaster General before he can perform the functions of the office. The classification and compensation of Postmasters is given in the following paragraphs:

FIRST CI.ASS.

Gross receipts, forty thousand dollars, and not exceeding fortyfive thousand dollars, salary, three thousand dollars.

Gross receipts, forty-five thousand dollars, and not exceeding sixty thousand dollars, salary, three thousand one hundred dollars.

Gross receipts, sixty thousand dollars, and not exceeding eighty thousand dollars, salary, three thousand two hundred dollars.

Gross receipts, eighty thousand dollars, and not exceeling one hundred and ten thousand dollars, salary, three thousand three hundred dollars.

Gross receipts, one hundred and ten thousand dollars, and not exceeding one humdred and fifty thousand dollars, salary, three thousand four hundred dollars.

Gross receipts, one hundred and fifty thousand dollars, and not 
exceeding two hundred thousand dollars, salary, three thousand five hundred dollars.

Gross receipts, two hundred thousand dollars, and not exceeding two hundred and sixty thousand dollars, salary, three thousand six hundred dollars.

Gross receipts, two hundred and sixty thousand dollars, and not exceeding three hundred and thirty thousand dollars, salary, three thousand seven hundred dollars.

Gross receipts, three hundred and thirty thousand dollars, and not excecding four hundred thousand dollars, salary, three thousand eight hundred dollars.

Gross receipts, four hundred thousand dollars, and not exceeding four hundred and fifty thousand dollars, salary, three thousand nine hundred dollars.

Gross receipts, four hundred and fifty thousand dollars, and not exceeding five hundred thousand dollars, salary, four thousand dollars.

Gross receipts, five hundred thousand dollars, and not exceeding six hundred thousand dollars, salary, five thousand dollars.

Gross receipts, six hundred thousand doliars and upwards, salary, six thousand dollars.

Salary of the postmaster at the city of New York, and Chicago, eight thousand dollars per annum.

\section{SECOND CLASS.}

Gross receipts, eight thousand dollars, and not exceeding nine thousand dollars, salary, two thousand dollars.

Gross receipts, nine thousand dollars, and not exceeding ten thousand dollars, salary, two thousand one hundred dollars.

Gross receipts, ten thousand dollars, and not exceeding eleven thousand dollars, salary, two thousand two hundred dollars.

Gross receipts, eleven thousand dollars, and not exceeding thirteen thousand dollars, salary, two thousand three hundred dollars.

Gross receipts, thirteen thousand dollars, and not exceeding sixteen thousand dollars, salary, two thousand four hundred dollars.

Gross receipts, sixteen thousand dollars, and not exceeding twenty thousand dollars, salary, two thousand five hundred dollars. 
Gross reccipts, twenty thousand dollars, and not exceeding twentyfour thousand dollars, salary, two thousand six hundred dollars.

Gross receipts, twenty-four thousand dollars, and not exceeding thirty thotisand dollars, salary, two thousand seven hundred dollars.

Gross reccipts, thirty thousand dollars, and not exceeding thirtyfive thousand dollars, salary, two thousand eight hundred dollars.

Gross receipts, thirty-five thousand dollars, and not exceeding forty thousand dollars, salary, two thousand nine hundred dollars.

TIIIRD CLASS.

Gross receipts, one thousand nine hundred dollars, and not exceeding two thousand one hundred dollars, salary one thousand dollars.

Gross receipts, two thousand one hundred dollars, and not exceeding two thousand seven hundred dollars, salary, one thousand one hundred dollars.

Gross receipts, two thousand four hundred dollars, and not exceeding two thousand seven hundred dollars, salary, one thousand two hundred dollars.

Gross receipts, two thousand seven hundred dollars, and not exceeding three thousand dollars, salary, one thousand three hundred dollars.

Gross receipts, three thousand dollars, and not exceeding three thousand five hundred dollars, salary, one thousand four hundred dollars.

Gross receipts, tliree thousand five hundred dollars, and not excceding four thousand two hundred dollars, salary, one thousand five hundred dollars.

Gross receipts, four thousand two hundred dollars, and not exceeding five thousand dollars, salary, one thousand six hundred dollars.

Gross receipts, five thousand dollars, and not exceeding six thousand dollars, salary, one thousand seven hundred dollars.

Gross receipts, six thousand dollars, and not exceeding seven thousand dollars, salary, one thousand eiglit hundred dollars.

Gross receipts, seven thousand dollars, and not exceeding eight thousand dollars, salary, one thousand nine hundred dollars. 
Postmasters at first and second class offices are not allowed commissions on the money-order business; but at other offices a fee of three cents for each money order issued is allowed for clerical expenses.

The compensation of postmasters of the fourth class is fixed upon the basis of the box rents collected at their offices and commissions upon the amount of canceled postage-due stamps, and on postage stamps, stamped envelopes, postal cards, and newspaper and periodical stamps canceled, on matter actually mailed at their offices, and on amounts received from waste paper, dead newspapers, printed matter, and twine sold, at the following rates:

On the first fifty dollars or less per quarter, one hundred per cent; on the next one hundred dollars or less per quarter, sixty per cent; on the next two hundred dollars or less per quarter, fifty per cent; and on all the balance, forty per cent.

No fourth class postmaster can receive more than one thousand dollars a year exclusive of money order returns or more than $\$ 25^{\circ}$ in one of the first three quarters of the year, and when the compensation of any postmaster of this class reaches two hundred and fifty dollars for four consecutive quarters each, exclusive of commissions on money-order business, and when the returns to the Auditor for four consecutive quarters shall show him to be entitled to a compensation in excess of two hundred and fifty dollars per quarter, the Auditor is required to report the fact to the Postmaster General, who will assign the office to its proper class, and fix the salary of the postmaster.

The compensation of Postmasters of the first, second and third classes is readjusted annually to take effect on July Ist, upon the basis of the returns for the four quarters ending the preceding March 3 ist.

Any person who temporarily serves as a Postmaster is entitled to receive for the time he is so serving the same compensation he would have received if he had been regularly appointed to the position.

Post offices must be kept open every week day during the usual hours in which the principal business houses of the place in which the Post Office is located are open. Where mail arrives on Sun- 
days, Post Offices must be kept open long enough for its delivery, athough they need not be opened on Sunday if no mail is received between the lour of closing on Saturday and 6 p. m. on Sunday. The only time at which it is regular to close the delivery windows of Post Offices during office hours is luring the time between the opening and distribtition of mail when the clerical force of the Post Office is insufficient to attend both to the delivery window and to the distribution of the incoming mail. Postnasters are required to be at their offeces in addition to the regular business hours during such times as may be necessary for receiving and dispatching mails. Night service can only be maintained upon direct autlorization from the Post Office Department, and Post Offices cannot be closed during the regular hours of business except on holidays without special authorization from the First Assistant Postmaster General. Delivery of mail on Sunday must be made to all who apply as well as to box holkers, but it is optional with the Postmaster whether he sha1l sell postage stamps or issue and pay money orders on that day. The registration of mail and delivery of resistered mail is also made optional with the Postmaster as a part of the Sunday business. The carriers' windows at free delivery offices must be open for the delivery of carrier mail during the hours on Sunday and holidays when the echeral delivery windows are open. Post Offices may be closed on holidays except for such time as may be required for necessary public convenience, but all mails must be made up and dispatched on holidays in accordance with the regular daily schedule.

No Post Office can be kept in a har rom or in a room directly connected with a bar room and no mail can be opened or delivered in which liquor is sold at retail except in drug stores where liquor is sold for medicinal purposes only. The site of the Post Office cannot be changed withont the consent of the Department and applications for such change must state the location of the proposed office with reference to the railroad station, and if it is beyond So rods from the station it is removed from the zone within which the railroad company must deliver the mails and a mail messenger must be employed if a location is granted at a greater distance from the station. Post Offices are required to be kept clean and in good order and the posting of advertisements except upon a suitable bulletin board is 
forbidden. The guide for the postmasters in transaction of public business is the postal regulations the last edition of which was published in I902, with a supplement issued in March, I907. The use of abusive and uncivil language is strictly forbidden by Postmasters and their employees, and they are required to treat all patrons with courtesy. Postmasters are forbidden to use their official signatures for advertising purposes or to stamp their own or any other advertisement on mail matter which may go through their post offices. Postmasters of the fourth class may transact business other than post office business in the same room in which the post office is located, but must keep such business separate and distinct from that of the post office. When a post office is robbed the postmaster is required to notify the inspector in charge in the district and also the chief Inspector at Washington by telegraph and to forward a report by mail giving an exact description of all the Government property which has been stolen.

Owing to the difficulty sometimes experienced by pensioners of the United States in remote districts in securing the authentication of their pension vouchers, provision has been made by law that fourth class postmasters may authenticate such vouchers and may receive a fee of twenty-five cents for each voucher so authenticated. Pensioners are required to take affidavit to the right to execute such voucher and the stamp of the fourth class post office with the signature of the postmaster has the same legal effect as the seal and signature of a notary public. This significance of the seal and signature of the postmaster is, cf course, for pension matters only, and under a special provision of the law.

Subordinates of postmasters are selected with the exception of the assistant postmasters in accordance with the rules of the Civil Service Commission and provision is made for an administrative and clerical staff at the different first class post offices in accordance with their importance and the amount of business done covering the positions given in the following list at the salaries named.

Assistant postmaster, salary not exceeding fifty per centum of the salary of the postmaster, graded in even hundreds of dollars, from one thousand five hundred dollars to not exceeding three thousand dollars per annum, except New York, New York, and Chicago, Illinois, where the salary of the 
assistant postmaster is fixed at three thousand five hundred dollars per annum.

Private secretary to postmaster, eleven classes, salary, graded in even hundreds of dollars, from nine hundred dollars to not exceeding one thousand eight hundred dollars per annum, except at New York, New York, and Chicago, Illinois, where the salary of the private secretary may be fixed at two thousand four hundred dollars per annum.

Cashier, ten classes, salary, graded in even hundreds of dollars, at one thousand three hundred, one thousand four hundred, one thousand five hundred, one thousand six hundred, one thousand seven hundred, one thousand eight hundred dollars, two thousand dollars, two thousand two hundred dollars, two thousand iour hundred dollars, and not excecding two thousand six hundred dollars per annum.

Assistant cashier, eight classes, salary, graded in even hundreds of dollars, from ons thousand two hundred dollars to not exceeding two thousand dollars per annum.

Finance clerks, including bookkeepers, ten classes, salary, graded in even hundreds of dollars, from one thousand two hundred dollars to not exceeding two thousand four hundred dollars per annum.

Chief stamp clerks, nine classes, salary, graded in even hundreds of dollars, from one thousand two hundred dollars to not exceeding two thousand two hundred clollars per annum.

Superintendents of mails, salary not exceeding forty-five per centum of the salary of the postmaster, graded in even hundreds of dollais, from one thousand three hundred dollars to not exceeding two thousand seven hundred dollars per annum, except at New York, New York, and Chicago, Illinois, where the salary of the superintendent of mails is fixed at three thousand two hundred dollars per annum.

Assistant superintendents of mails, ten classes, salary, graded in even hundreds of dollars, from one thousand two hundred dollars to not exceeding two thousand four hundred dollars per annum.

Superintendents of delivery, salary, not exceeding forty-five per centum of the salary of the postmaster. graded in even lundreds of dollars, from one thousand three hundred dollars to not exceeding two thousand seven hundred dollars per annum, except at New York. New York, and Chicago, Illinois, where the salary of the superintendent of delivery is fixed at three thousand two hundred dollars per annum.

Assistant superintendents of delivery, eight classes, salary, graded in even hundreds of dollars. from one thousand two hundred dollars to not exceeding two thousand four hundred dollars per annum.

Superintendents of registry, salary not exceeding thirty-five per centum of the salary of the postmaster, graded in even hundreds of dollars, from one tholisand three hundred dollars to not exceeding two thousand one hundred dollars per annum, except at Boston, Massachusetts, where the salary of the superintendent of registry may be fixed at two thousand two liundred dol- 
lars, and at New York, Nuw York, Chicago, Illinois, and Washington, District of Columbia, where the salary of the superintendent of registry is fixed at not exceeding forty per centum of the salary of the postmaster.

Assistant superintendents of registry, salary not exceeding twenty-five per centum of the salary of the postmaster, graded in even hundreds of dollars, from one thousand two hundred dollars to not exceeding two thousand dollars per annum, except at New York, New York, where the salary of the first and second assistant superintendents of registry is fixed at two thousand four hundred dollars and one thousand eight hundred dollars per annum, respectively.

Superintendent money-order division, salary, not exceeding forty per centum of the salary of the postmaster, graded in even hundreds of dollars, from one thousand three hundred dollars to not exceeding three thousand two hundred dollars per annum.

Assistant superintendent, money-order division, nine classes, salary, graded in even hundreds of dollars, from one thousand two hundred dollars to not exceedins two thousand dollars per annum, except at New York, New York, where the salary of the first and second assistant superintendents of moneyorder and chief bookkeeper are fixed at two thousand four hundred dollars, one thousand eight hundred dollars and one thousand cight huridred dollars, respectively.

Superintendents of stations, salary, graded in even hundreds of dollars, from one thousand one hundred dollars to not exceeding two thousand dollars per annum, except at New York, New York, where the salaries of the superintendents of Stations " $\mathrm{A}$ " and "D" are fixed at two thousand five hundred dollars each per annum, and superintendents of Stations "E" and "F" are fixed at two thousand two hundred dollars each per annum.

Assistant superintendents of stations, nine classes, salary, graded in even hundrects of dollars, from one thousand to not exceeding one thousand eight hundred dollars per annum.

Superintendents of carriers, eleven classes, salary, graded in cven hundreds of dollars, from one thousand to not exceeding two thousand one hundred dollars per annum.

Superintendents of inquiry, two classes, salary, graded in even hundreds of dollars, at two thousand one hundred dollars per annum.

Superintendents of second class matter, ten classes, salary, graded in even hundreds of dollars, from one thousand to not exceeding two thousand dollars per annum.

Chief mailing clerks, seven classes, salary, graded in even hundreds of dollars, from one thousand three hundred dollars to not exceeding two thousand two hundred dollars per annum.

Examiner of stations, six classes, salary, graded in even hundreds of dollars, from one thousand four hundred dollars to not exceeding two thousand dollars per annum. 
Specia! clerks, two classes, salary, at thirteen hundred dollars and fourtcen hundred dollars per annum.

Clerks in charge of stations, six classes, salary, graded in even hundreds of dollars, from four hundred dollars to not exceeding one thousand dollars per annum.

Clerks in charge of contract station', two classes: First class, salary excceding three hundred dollars and not excecding one thousand dollars per annum. Second class, salary not exceeding three hundred dollars per annum.

Foreman of crews or working sections, five classes, salary, graded to even hundreds of dollars, from one thousand two hundred dollars to not exceeding one thoneand six hundred dollars per annum.

Auditors, two, one at New York, New York, and one at Chicago, Illinois, at a salary of three thousand dollars each per ammum.

Night superintendents, salary, two thousand one hundred dollars per annum.

Machinist, one, at one thousand two hundred dollars per annum.

Oilers, three, at nine hundred dollars per anmum.

Printers, four classes, salary, graded in even liundreds of dollars, from nine hundred dollars to not exceeding one thousand two hundred dollars per annum.

Pressmen and carpenters, four classes, salary, graded in even hundreds of dollars, from six hundred dollars to not exceeding nine hundred dollars per annum.

Messengers, watchmen, Jaborers, janitors, and porters, four classes, salary, graded in even hundreds of dollars, from four hundred dollars to not exceeding seven hundred dollars per annum.

In second class offices:

Assistant postmasters, eiglit classes, salary, graded in even hundreds of dollars, from seven lundred dollars to not excecding one thousand four hundred dollars per annum.

Superintendents of stations, salary, graded in even lumdreds of dollars, irom one thousand one hundred dollars to not exceeding two thousand dollars per annum.

Clerks in charge of contract stations, two clases: First class, salary exceeding three lundred dollars and not exceding one thousand follars per aunum. Second class, salary not exceeding three hundred dollars per annum.

Messengers, janitors, and watchmen, three classes, salary graded in even hundred: of dollare, from four hundred dollars to not excecuing six hundred dollars per annum.

Clerks in offices of the first and second class and carriers in the city delivery service are divided into six grades, as follows: First grade, salary six hundred dollars; second grade, salary, eight humdred dollars; third grade, salary, nine hundred dollars; fourth grade, 
salary one thousand dollars; fifth grade, salary one thousand one hundred dollars; sixth grade, salary one thousand two hundred dollars. Clerks and carriers at first class offices are promoted successively to the fifth grade, and clerks and carriers at second class offices are promoted successively to the fourth grade.

All promotions of both clerks and carriers are made at the beginning of the quarter following the expiration of a year's service in the next lower grade. No promotion is made except upon evidence satisfactory to the Post Office Department of the efficiency and faithfulness of the employee during the preceding year. The Post Office Department may reduce a clerk or carrier from a higher to a lower grade whenever his efficiency falls below a fair standard or whenever necessary for purposes of discipline. When a clerk or carrier has been reduced in salary he may be restored to his former grade or advanced to any intermediate grade at the beginning of any quarter following the reduction, on evidence that his record has been satisfactory during the intervening period. When a clerk or carrier fails of promotion because of unsatisfactory service, he may be promoted at the beginning of the second quarter thereafter, or of any subsequent quarter, on evidence that his record has been satisfactory during the intervening period. Clerks and carriers of the highest grade in their respective offices are eligible for promotion to the higher positions.

Any clerk is eligible for transfer to the service of a carrier and any carrier is eligible for transfer to the position of clerk, such transfer to be made to any grade not higher than the corresponding grade of salary, and the time which such clerk or carrier shall have served in the grade from which the transfer was made is counted in connection with the service to which transfer is made in computing the time of service necessary to entitle such employee to promotion. No clerk or carrier can be promoted more than one grade within any one year's period of service. The carriers who on June thirtieth, nineteen hundred and seven, were regularly employed at a salary of eight hundred dollars per annum were promoted to the fourth grade upon evidence satisfactory to the Post Office Department of the efficiency and faithfulness of the employee during at least one year's service. 
Auxiliary employees are employed to be paid for actual service at the rate of thirty cents an hour; such employees are required to work not less than two hours daily, and may serve as substitutes. They are eligible for appointment as clerlss and carriers of the first grade.

Substitutes are employed to be paid at the rate of thirty cents an hour when serving for absent clerks and carriers and such substitutes are eligible for appointment as atxiliary cmployees and as clerks and carriers of the first rrade.

Assistant postmasters are the direct and special appointees of the postmasters themselves, and it is required that every postmaster shall appoint an assistant so that the office may not be left without some one duly sworn and qualified to perform the duties in the case of the necessary absence or sickness of the postmaster, or before the sureties or a post office inspector can take charge of the office in the event or removal, resignation or death of the incumbent. At offices of the first and second classes the assistant postmasters are appointed strbject to the approval of the First Assistant Postmaster General, and the person selected must be at least twenty-one years of age. Postmasters at offices of the third and fourth classes designate their principal assistant in the space provided for that pur. pose in the official bond, and in the case of third class offices notice must also be given to the First Assistant Postmaster General of the the assistant postmaster selected. A member of a postmaster's family or a surety on his bond may he appointed assistant postmaster. Contractors or subcontractors for carrying the mail, mail messengers or assistant mail messengers or persons interested in prosecuting claims against the Government cannot, however, be appointed assistant postmasters. Assistant postmasters perform such duties as may be assigned to them by the postmasters and in case of the absence of the postmaster perform his duties except that the postmaster may designate some other person to take charge of the money order office during his absence. When a postmaster resign; or dies the assistant postmaster is required to conduct the business until the office is taken over by the sureties or by a Post Office Inspector. Postmasters may authorize their assistants to sign such letters and reports as are not required to be signed by the Postmaster 
himself, the signature of the assistant postmaster being the name of the Postmaster by himself as assistant postmaster.

All other of the foregoing employees receive their appointment in accordance with the rules established by the Civil Service Commission and which are substantially the same as those provided for entrance to and contintance and promotion in other branches of the govermment service. Provision is made for temporary appointment in case eligibles cannot be certified at once, but the independent appointing power of the postmaster is practically confined to the appointment of his own assistant, the supervision over the appointment to other positions in his office being taken out of his hands. Provision is made by law by which cleriss in post offices as well as other cmployees of the postal service are paid their full time while in attendance as witnesses in United States courts, substitutes being employed under a specific appropriation to perform their duties. Besicles the examinations required by the Civil Service Commission as a condition for entrance into the postal service, distributing clerks are required from time to time to be examined on the distributing schemes for mail for different States distributed at their offices. It is required that clerks who fail in such examinations and who are incompetent in distribution shall be removed.

The Postmaster General is not authorized, except in certain instances, to allow clerical assistance to postmasters of the third and fourth classes. The compensation allowed postmasters at these offices is intended to include the cost of clerical assistance and such clerks as may be employed have to be paid from the emoluments of the postmaster except where an office of the third or fourth class is a distributing or separating office, so that mail destined for other points has to be redistributed, the Postmaster General may allow to the postmaster in such an office a reasonable amount to cover the cost of performing the work of distribution or separation.

No allowance for clerical services in excess of two hundred dollars can be made where the salary of the postmaster is one thousand dollars, one thousand one hundred dollars, or one thousand two hundred dollars; nor in excess of three hundred dollars where the salary of the postmaster is one thousand three hundred dollars, one thousand four hundred dollars, or one thousand five hundred dollars; 
nor in excess of four hundred dollars where the salary of the postmaster is one thomsand six hundred dollars, or one thousand seven hundred dollars: nor in excess of five humlred dollars where the salary of the postmaster is one thousand cight humdred dollars or one thousand nine hundred dollars.

Within this limitation allowances are made according to the average amonnt of mail to be separated chily as follows:

Where the average number of pieces of mail separated daily amounts to 34 and is not in excess of 65 pieces, the annual allowance can not exceed $\$ 3,6$. For each increase of 33 pieces in the average number separated daily, not to exceed \$I additional is allowed annually until the averase number of pieces separated daily anounts to 600 pieces. For cach increase of 50 pieces in the average number separated daily above for, Siz additional is allowed annually.

Wheiever such an allowance is made the postmaster may employ any person to perform the work who is competent to do so and who can take the oath of office. It is not forbidden to employ the members of the postmaster's family for the work, but each person so employed must take the oath of office and if a former postmaster is employed, he must take the oath of office anew before he can have access to or handle mail matter. Any member of a postmaster's family who may be called upon to receive or handle mail in the absence of the postmaster must tale an oath of office before attempting to perform any of the postmaster's dutics.

Assistant postmasters and cashiers in first, second and third class offices are requirel to enter into a bond before undertaking their duties, and the Postmaster General may require a bond from any clerk or other employee paid from appropriations for the postal service, but this bond does not relieve the postmaster or his bond from liability for the acts of the officials named. The bonds required simply act as an additional guarantee to the United States arainst loss on account of malfeasance in office of the subordinate officials, but may be recovered on by postmasters who have paid losses due to subordinates. Stamp clerks, superintendents of money order and chief money order clerks, superintendents of stations, clerks in charge of substations, superintendents of registry, and all registry clerks are also required to give bonds as well as other persons than 
those named who handle government funds when it is recommended by a postmaster that a bond shall be taken. Preference is given in such bonds to bonds issued by surety companies and where practicable a blanket bond covering all clerks in a given office required to give bond is preferred. It is contemplated by the regulations that disciplinary action shall be taken in the case of all clerks who fail to pay the premium on their bonds.

Allowance for rents are made to post offices of the first, second, and third classes, but postmasters of the fourth class have to pay their own rent. Leases may be entered into for periods of ten years, although the usual practice is to enter into leases for five years, the ten year lease being taken only when exceptionally advantageous terms can be secured. Authorization for leasing premises for post offices is given only after an inspector has visited the location and approved the site and the quarters proposed. Portions of such quarters may be sublet if deemed advisable, but the revenue therefor must be accounted for as part of the revenues of the post office. Light and heat are also provided for offices of the first, second and third classes upon the application of the postmaster and approval of his requisition by the inspector. Allowances for miscellaneous expenditures and stamp canceling machines and similar devices are under the direction of the office of the First Assistant Postmaster General and are granted upon application of the postmaster, and the favorable recommendation of an inspector when the report of the latter is deemed advisable.

The supplementary function of the office of the First Assistant Postmaster General to those already described is in the extension of the window or box delivery at the post offices to a house-tohouse delivery performed by carriers. The gray-uniformed letter carrier is the first Federal official who comes to the attention of the future American citizen in childhood days and although at first confused with the city policeman, later comes to be recognized as a manifestation of a beneficent paternalism. Considering that for the fee of two cents a letter may be mailed in a street corner box at one remote corner of the possessions of the United States and be delivered to the home of the person to whom addressed at the farthest opposite point in the possessions of the government, assuming, of 
course, the existence of carrier service at the points of origin and delivery, and this withont an appreciable general imposition upon the taxpayers of the country, is the best and conclusive answer to those who affect to see in the governmental control of the post office an inept and expensive proceeding.

The provision of law under which free delivery of mail by carriers is established is that there nust be letter carriers employed for the free delivery of mail matter in all incorporated cities, towns and boroughs including a poptulation of 50.000 within their corporate limits. Permission is also granted for the establishment of such free delivery in any such incorporated town or city which has a population of not less than 10,000 or whose post office produced a gross revenue for the precelling fiscal year of not less than $\$ 10,000$. It is within the discretion of the Postmaster General to establish free delivery under the permissive clause of the law, but when the conditions are complied with, the establishment of free delivery is seldom denied. It further required by the law that no additional carrier's fee shall be charged for the delivery of letters by carrier. Applications for the establishment of a free delivery service should be made by the postmaster, by citizens joining in a petition or by the municipal authorities and addressed to the First Assistant Postmaster General, Division of Free Delivery. The application should give the population of the city, village, or borough according to the last general census, and the gross revenue of the post office for the preceding fiscal year. The census may be either that of the United States or State census. The application should also state the condition of the sidewalks, and whether the honses are numbered in accordance with their positions on the streets, the names of streets posted up, and the city or town properly lighted. When such an application is received, the Post Office Department sends an Inspector to the municipality to examine the local conditions. If the requirements as to numbering houses on the streets are not fulfilled and the condition of the streets and sidewalks in such as to make delivery unusually difficult, the application may be suspended until such time as the defects may have been remedied. The inspector also recommends the limits of the free delivery districts and at the time 
the order for free delivery goes into effect the number of daily deliveries and collections, the trips to be made, and similar details are established and must not be changed except upon authority of the First Assistant Postmaster General. If a postmaster deems that the delivery system can be improved, he is required to make recommendations to this end to the First Assistant Postmaster General and if his recommendations are approved, he is authorized to make the changes suggested. The postmaster is, however, forbidden to reduce the number of daily delivery and collection trips without the authority of the First Assistant Postmaster General.

Letters must be frequently and promptly delivered and the addresses of residents of the locality must be secured and their mail delivered to them at their houses whenever practicable. The classification of carriers is given under the previous statement of the law covering clerks and carriers. These officials are also selected as a result of Civil Service examinations, and they are required to perform their work within the eight hours' time fixed by law. All carriers are strictly forbidden from making any overtime, and if they are unable to deliver the mail entrusted to them within the eight hours it is regarded as a reason for discipline. The carriers' routes are arranged so that they may reasonably be expected to take care of all their mail within the time fixed and with the number of trips allowed, and it is held that failure to do so indicates a condition of incompetency on their part. The stringency of the law in this respect is due to the fact that following the passage of the eight hour law carriers became accustomed to work overtime and large amounts in claims were made against the government for such extra work, and considerable sums were paid to carriers throughout the country as a result.

Letter carriers being also collectors of mail from the letter boxes are supplied with keys to such boxes and are required to exercise particular care that the keys shall not be lost or get into the hands of unauthorized persons. All keys must be turned over at the end of a day's work to a person designated to receive them, except that keys used by carriers making early morning collections may be retained over night.

Every applicant for appointment as letter carrier must be between 
eighteen and forty-five years of age, must weigh at least 125 pounds, and must be not less than five fect four inches in height. The candidates are required in atdition to the civil service cxamination to undergo a physical examination and if rejected on the latter will not be permitted for the Civil Scrvice examination. Substitute letter carriers are appointed from the eligible list created by the Civil Service examination and upon a vacancy in the regular force the substitute must be promoted to the regular position. When new free delivery systems are established the appointment of the letter carriers must be made from the eligible list. Transfers of letter carriers from one office to another have frecunently been sought for various reasons of convenience, and at times mutual interchange has been arranged. The regulations now provide, howcver, that such transfers cannot be made except on account of the health of a letter carrier or a mem. ber of his family and all such transfers must be arranged through the office of the First Assistant Postmaster General with his approval. Letter carriers cannot be removed except for just cause and upon written charges filed with the First Assistant Postmaster General, and the carriers are given duc notice and opportunity for defense against such charges, which must be specified. The postmaster has, however, the power to discipline letter carriers by suspension with loss of pay.

All letter carricrs and substitute letter carricrs must furnish bonds conditioned in the parment of the sum of $\$ 1,000$ for any malfeasance in office. The Post Office Department prescribes the uniform for carriers, which is of bluish gray flannel serge or worsted, with a double breasted coat for winter wcar and a single breasted sack coat for summer wear, the uniform being piped with black braid adorned with buttons representing a carrier and with the letters "P. O. D." Various patterns of headgear have been designated from time to time, on each of which a carricr must wear his number in nickel plated figures nine-sixtcentlis of an inch in length, surmounted by a metallic wreath. During the heated term carriers are allowed to wear a neat shirt waist or loose fitting blouse instead of coats and vests, to be made of light gray cotton material. This is to be worn with a belt, but it is required that the garments worn shall be uniform throughout the same post office. Length of service of 
carricrs is denoted by stars placed on the sleeves of the carrier's uniform, for five years' service, one black star, ten years' service, two black silk stars, fifteen years' service, one red silk star, twenty years' service, two red silk stars, twenty-five years' service, one silver star, thirty years' service, two silver stars, thirty-five years' service, one gold star, forty years' service, two gold stars. Substitute carriers wear a black cloth bar on each sleeve.

Carriers may have fifteen days' leave of absence in each year and thirty days additional sick leave, in the discretion of the postmaster. A carrier absenting himself without leave is liable to loss of pay, suspension, and separation from the service. When the regular carricrs are absent from duty for any reason their places are filled by the substitute carriers, but the assignments must be made by the postmaster and carriers are forbidden to employ these substitutes themselves without notice to the postmaster except in cases of urgent necessity. Substitute carriers are paid $\$ 1.00$ per year in order to comply with the provision of the law that forbids the services of any person to be accepted without compensation in the Federal service, and receive pay at the rate of $\$ 600$ a year when acting in the place of a letter carricr who is absent on leave. When acting for carriers who are absent without pay they receive a pro rata portion of the salaries of such carriers. Substitute carriers also receive pro rata pay of regular carriers when the latter are acting as members of local civil service boards, or as witnesses for the Government, provision being made for the payment of the salaries of such carriers under the circumstances named.

In offices in which there are a sufficient number cf carriers or a sufficient amount of work to be done, so that the postmaster cannot superintend the operations of the carriers personally, an official known as Superintendent of Delivery is appointed who has charge of the force of carriers, sees that they make their proper rounds and that they complete their work within the time scheduled. A schedule is made up for each carrier's work and he is required to conform to it as closely as possible. A portion of the time is allowed for assorting the mail to be delivered on the route and for transacting miscellaneous business with the office. Carriers are, however, required 
to be in the office as little as possible, and as soon as they have completed their work to either go out upon the route or to leave the work room until it is time to take up the duties anew.

Strict provisions are nade as to the use of intoxicating liquors by carriers, who are also forbidden to solicit any contributions of money or grifts or to engage in any business which would involve the solicitation of patronage on their routes. All suspensions and reprimands of carriers must be reported to the Division of Free Delivery for approval and for entry in the carrier's efficiency record, which is kept at headquarters as a basis for the determination of questions relating to the carriers which may arise. Carriers are required to be careful to deliver the mail to persons for whom it is intended or to some one authorized to receive it. If they are in doubt as to the proper person to whom it is to be delivered, they are required to return the mail to the Post Office for the postmaster's action. Carriers are also forbidden to deliver mail to persons upon the streets unless such delivery can be made without unreasonable delay. Carriers are not allowed to deliver mail at premises not occupied by the addressee, except upon specific order of the postmaster. They are not allowed to throw mail matter into windows or into halls unless instructed so to do, but must ring the bell of the residence, wait a reasonable time for the answer, and cleliver the mail to some one of the household who is in the habit of receiving it. They may, however, where boxes are provided, deposit the mail in such boxes and ring the bell announcing that mail has been delivered, if a bell is provided. The Post Office Department solicits the assistance of householders in the prompt delivery of mail by the provision of suitable boxes for its reception. Carriers are forbidden to enter any house on their routes except in pursuance of their official duties, and must not exhibit mail matter to others than those addressed, except on the order of the postmaster. Letters for delivery must not be carried in the carrier's pocket, nor can a carrier deliver any mail matter which has not passed through the Post Office with which they are connected.

Carriers are especially made subject to discipline if they throw away or improperly dispose of any mail matter however trifling 
or unimportant it may appear, or if they remove stamps of any kind from the mail matter entrusted to them. Carriers are not required to deliver mail at residences where vicious dogs are permitted to run at large, and persons keeping such dogs must call at the Post Office for their mail. Carriers are required to collect all postage due and may accept stamped letters and packages which may be handed to them for mailing. They are required not to delay their deliveries, however, by waiting for such letters or to accept money for stamps for the same, although limited accommodations as to the sale of stamped envelopes may be authorized by the postmaster. Packages of such size that they will interfere with the delivery of mail need not be accepted by carriers. Carriers are not permitted to return under any circumstances letters which have been deposited in a box, and which it is the duty of the carrier to collect. All applications for the return of such letters must be made to the postmaster.

Under legislation, the first of which was effected in 1885 , every post office is designated as a special delivery office from which letters may be delivered at once to the addressee by messenger if they bear the ten-cent special delivery stamp. The frequency and promptness of usual deliveries in most cities make this convenience one of moderate use only, but it is one which is of very considerable value. Postmasters are authorized to employ one or more messenger boys in accordance with the amount of special delivery business which may come to the office, and such boys receive eight cents each for each letter delivered. A special delivery boy cannot be less than $\mathbf{I} 3$ years of age, and it is usual to require that he shall wear at least a partial uniform, although this is not essential. Substitute letter carriers, when not employed in the delivery of regular mail, may be utilized to carry special delivery letters, and in cases of insufficient business to warrant the attendance of a regular corps of messenger boys any clerk or employee of the office can carry a special delivery letter and receive the compensation therefor. Allowances are made in first class offices for car-fare when special deliveries cannot otherwise be handled promptly. Mail of this class is given the preference in handling from the point of origin to the point 
of delivery. At each Post Office a special drop is provided with a notification bell and special delivery letters are expected to be sent on the next mail immediately following their receipt, and are so placed on the regular packages that they will be first handled. Postmasters are required not to employ a larger force of special delivery messengers than may be necessary, and must divide the work among them so that it will be equally distributed. Combinations among the messengers for the purpose of a uniform division of the fees receiver are forbidden. In fourth-class offices and other than free delivery offices the postmasters attend to all the details relating to the delivery of special delivery letters and are allowed to charge for the service 80 per cent. of the face value of all special delivery stamps received and delivered, or attempted to be delivered. The chief defect in the special delivery system arises out of the great irregularity of the business so that a steady and assured income cannot be provided for the messenger service. The restult is, difficulties are experienced in securing proper and efficient messenger boys, so that the service sometimes suffers.

Included within the duties of the First Assistant Postmaster General are the consideration of the very many questions which arise as to the extension of the various free delivery systems and the consolidation of independent systems under one central Post Office when the growth of population in any section brings the two offices within the provision of the law on the subject and within the limits of economic administration of the two ofinces under one head. The First Assistant Postmaster General also arranges for the regulation of the box rent rates and has charge of the details relating to the deposit of money for fees for lock boxes in Post Offices. Regulations for the use of boxes are made by the Department, and the amounts as rents are collected by the postmasters and must be credited to the general account of postal revenues as in case of sale of stamps and other postal supplies. The keys of such boxes are guaranteed against being lost or withheld by the deposit of a sum of money representing the value of the keys issued and this money is held as a trust fund by the postmaster anci is not turned in to the revenues of the 
Post Office Department, being utilized for the payment of keys to replace those which may be lost or withheld. The use of a box is restricted to a renter and those connected with him in a family or business way, including employes, servants and transient and temporary boarders. When a box is rented by a corporation or association or society mail for all its officials may be placed in such box, and matter addressed to the students and employees at a college or similar institution or to guests at a hotel may be placed in the appropriate boxes. Although mail cannot be addressed to a number or initials at a general delivery, mail addressed merely to the number of a box may be delivered so long as no improper or unlawful business is conducted. Bills and circulars, which have not passed through the mails, must not be placed in the boxes, and the postmasters may hand the mail from boxes to box holders who have forgotten their keys, but the rental of a box confers no extra privilege upon a person in the matter of delivery of mail over a person who receives his mail from the general delivery.

The First Assistant Postmaster General has charge also of allowances for carrier delivery furniture and of matters relating to street letter and package boxes and collection wagons, together with matters relating to carriers' equipment, and the incidental expenses incurred in the free delivery service.

THE SECOND ASSISTANT POSTMASTER GENERAL.

There have been complaints, covering a series of years, that the amount of money paid by the Post Office Department to the railroads for carrying the mails of the United States were in excess of a fair compensation for the service, and that this excessive expenditure was one of the principal reasons why the Postal Service of the United States was carried on with so great deficit and, as it is alleged, with so considerable a lack of economy as compared with the amount for which the same business could be carried on under private auspices. For the purpose of determining whether the United States is paying in excess of amounts which it should pay for the carriage of the mail there have been commissions and committees appointed with the result that cer- 
tain modifications have been made in the method of paying for the service, although it has not been established that the amounts previously paid for the carrying of the mail have been excessive. A very large proportion of the cost of the postal service is, of course, involved in the payments made to the railroads, and this cost includes not only the carriage of first-class mail, but of the very large volume of second-class mail which, at the rate established is, of course, carricd at a loss to the Government. In addition, the immense funntities of mail matter carried for the United States itself and upon which there is no revenue, is, without corresponding direct return, the source of a considerable proportion of expense of railway mail payments.

White the office of the First Assistant Postmaster General deals almost cxclusively with officials and employees of the United States Government, a considerable section of the office of the Second Assistant Postmaster General is devoted to transactions involving private persons who have contracts with the Post Office Department, including contracts involving the railway mail pay.

A division of official personnel is, however, included under this office and is directed by the Second Assistant Postmaster General, namely, the railway mail service, a separate organization which has charge of the mails of the United States while they are en route from the Post Offices of their origin to the Post Offecs from which they are to be delivered. Mails originating at a given Post Office are delivered at the railway station either by the employecs of the railways themselves, if within the limits fixed for transfer of the mails by the railroads or by the contractors, and mail messengers employed for the transfer of mails from Post (Offices to railway stations in case the Post Offices are beyond the limit of eighty rods. Upon reccipt at the station they are under the control of the railway mail service until they have been delivered to another station for transfer to the local Post Office. The service is under the direction of a general superintendent at $\$ 4.000$ a year, who has one assistant at $\$ 3,000$, and eleven division superintendents at $\$ 3,000$, and the following subordinate officers: 
Elevein assistant division superintendents at $\$ 2,000$ each a year; five assistant superintendents at two thousand dollars each a year; nineteen assistant superintendents at $\$ \mathrm{I}, 800$ each a year, and postal clerks in the following classes:

Class I (B), at not exceeding eight hundred dollars each;

Class I (A), at not exceeding nine hundred dollars each;

Class 2, at not exceeding one thousand dollars each;

Class 3 , at not exceeding one thousand one hundred dollars each;

Class 4 (B), at not exceeding one thousand two hundred dollars cach;

Class 4 (A), at not exceeding one thousand three hundred dollars each;

Class 5 (B), at not exceeding one thousand four hundred dollars each;

Class 5 (A), at not exceeding one thousand five hundred dollars each;

Ciass 6, at not exceeding one thousand six hundred dollars each;

Chief clerks, at not exceeding one thousand eight hundred dollars each. Railway mail clerks on entering the service re`eive the salary of the lowest grade, and no clerk can te advanced more than two hundred dollars in any period of one year's service.

In addition to the foregcing Class 5 includes clerks in charge of full railway post-offices, clerks assigned to duty in the various offices of the service and receiving a salary of $\$ \mathrm{I}, 400$ per annum.

Class 6 includes clerks in charge on trains carrying two or more full railway post-ofice cars and receiving a salary of $\$ 1,500$ per annum.

Class ; includes all persons designated as chief clerks and receiving a saiary of $\$ 1,600$ per annum.

Class 8 includes all assistant division superintendents and assistant superintendents, and the assistant chief clerk in the office of the general superintendent.

Class 9 includes the assistant general superintendent, superintendents of divisions, and the chief clerk in the office of the general superintendent.

Class ic includes the general superintendent.

Division superintendents, assistant division superintendents, and chief clerks, Railway Mail Service, and railway postal clerks, are paid their actual and necessary expenses while actually traveling on business of the Post Office Department and away from their several designated headquarters.

Assistant superintendents of the Railway Mail Service receive a per diem allowance in lieu of actual and necessary traveling expenses at a rate not exceeding four dollars per day while actually traveling on business of the Department away from home, their official domicile, and their headquarters. 
All railway postal clerks are required to give bonds in $\$ I, \infty 00$ cash for the faithful discharge of their duties.

The Railway Mail Service is divided into eleven divisions, each in charge of a division superintendent, as follows:

First division, comprising the New England States: Office of superintendent, Boston, Mass.

Second dicision, comprising New York, New Jersey, Pennsylvania, Delaware, the Eastern Shore of Maryland, Accomac and Northampton connties, Va., and Porto Rico: Office of superintendent, New York, N. Y.

Third dicisiun, comprising Maryland (cxcluding the Eastern Shore), Virginia (excepting Accomac and Northampton comtirs), West Virginia, North Carolina, and District of Columbia: Office of surerintendent, Washington, D. C.

Fourtl dicision, comprising South Carolina, Gurgia, Florida, Alabama, Missiscipni, and that 1 ortion of Lonisiana cast of the Mississippi River: Office ol supcrintendent, Atlanta, Ga.

Fifth dizision, comprising Ohio, Indiana, Fentucky, and Tennessee: Office of superintendent, Cincinnati, Ohio.

Sirth ditision, comprising Illinois, Iowa, Nebraska, Wyoning, and the Black Hills district of South Dakota, consisting of the counties of Butte, Chotean, Custer, Ewing, Full River, Harding, Lawrnce, Martin, Meade, Pennington, Rinehart, Shannon, Wagner, and Washington: Office of superintendent, Chicago, ill.

Sezenth dizision, comprising Missouri, Kansas, Colorado, and New Mexico: Oflice of superintendent, St. Louis, Mo.

Eighth dicision, comprising California, Nevada, Oregon, Alaska, Arizona, Idaho, Utah, Washington, anl Iawaii: Office of superintendent, San Francisco, Cal.

Ninth division, comprising the through mails via Buffalo, Suspension Bridge, Toledo, and Detroit, the lines of the Lake Shore and Michigan Southern Railroad, the main line of the New York Central and Hudson River Railroad, and the Lower Peninsula of Michigan: Office of superintendent, Cleveland, Ohio.

Tenth division, comprising Wisconsin, Northern Peninsula of Michigan, Mimnesota, North Dakota, South Dakota (exceps the Black Hills district), and Montana: Office of "uperintendent. St. Paul, Minn.

Eleventh dizision. comprising Arkansas, Indian Territory, Oklahoma, Texas, and that portion of Lonisiana west of the Mississippi River: Office of superintendent, Fort Worth, Tex.

All original appointments to the positions of railway postal clerks are made by the Postmaster General under Civil Service rules and regulations. One substitute is appointed for every ten clerks in 
each State and any vacancy in the regular force is filled by the appointment of the ranking substitute who lives in any county on or supplied from the road upon which the vacancy exists. All vacancies in the substitute force will be filled by certification of the Civil Service Commission from the Railway Mail Service register of the State in which the vacancy takes place. It is the object of the service so far as possible to select employees from the sections of the country in which they are to perform their duties in order that there may be secured persons who are familiar with the territories and also who will be near to their homes so that considerable periods in time of travel will not be requisite in going to and from the homes of the employees and under a recent provision of law clerks appointed to service on postal cars must reside on some portion of the route on which they are to serve.

The General Superintendent, the Assistant General Superintendent and the Chief Clerk of the Division of Railway Mail Service constitute a board of promotion to select the names of persons who are eligible for promotion to the next higher grade in case of vacancies. So far as is practicable promotions are required to be made from among those who are serving in a lower grade than that in which the vacancy occurs and in the same railway post office. That is to say that clerks on a given run or section of the work are to be given preference with reference to promotion when the duties to be performed are exclusively those relating to that run. When the duties to be performed in the position to be filled are not confined to a single run but are within one division, eligibles for promotion may be selected throughout the division, and where the duties to be performed cover more than one division, the eligibility of the employees of the railway mail service generally may be taken into consideration. Clerks who may be detailed to the office of the General Superintendent in Washington can be considered for promotion in the division from which they were detailed. The board of promotion selects three names from the list of eligibles and certifies them to the Postmaster General for promotion to the vacancy. In case there are not three persons who can be certified for promotion and the Postmaster General requires a full certification, the names of persons in an adjoining railway post office or division to that in 
which the vacancy cecurs can be considered in connection with the certification. Clerks are not eligille to promotion to a higher grade until they shall have served at least three months in the grade to which they were last appointed or promoted. No person can be promoted by detail or transfer to a position which could be filled by promotion of an employee alrearly in the subdivision of the service in which the vacancy occurred. The basis of recommendation for promotion by the board on promotions is the case examination and the efficiency shown by the clerk in his work in the postal car. No clerk can be regarded as eligible for promotion unless he has made an average of 95 per cent. on a case examination within three years previous to the date on which the promotion is being considered. No specific re-examination is required for promotion and the board on promotions considers the general character and qualifications and efficiency of the persons in the service.

All railway mail clerks are required to be examined from time to time upon the official schemes of distribution, the connection of trains, and the general orders and regulations of the service. Clerks on probationary appointment are to be examined as often as practicable and the results of the examinations are taken as indicating the fitness of the clerk to remain in the service. The examinations are chiefly practical ones intended to test the ability of the clerk to distribute mail matter to the different compartments of the railway mail distributing case, and the efficiency of the clerk is largely dependent upon his ability to pass such an examination with a high average, 95 per cent. being required for a passing average and roo per cent. efficiency is the usual result, the clerks falling below perfect only in exceptional instances. Clerks may voluntecr for examination and especially for examinations on routes other than those they are actually serving upon, the results of such examinations being considered in conncetion with details and promotions.

All railway postal cars and mail appointments in cars and steamboats are designated as railway post offices. One or more clerks are assigned to each railway post office for the custody of the mail en route, and for the performance of the duty of distribution of mail received and of making it up into packages suitable for delivery at the different points along the route of the train or steamboat. In 
addition, mail is received at intermediate points and distributed in a like manner in accordance with the various schedules made up for the delivery of mail at terminal and connecting points. When there is more than one railway clerk assigned to duty in a car, the clerk of the highest class is designated as the clerk in charge and in question of seniority relating to clerks of the same class, the clerk senior in commission is regarded as the ranking official. The clerks in a railway post office are required to implicitly obey the orders of the clerks in charge. Postal clerks are required to examine their cars on entering and leaving to see that no mail has been left and are not allowed to leave their cars except for brief moments and in matters of emergency. They must at all times keep the car doors locked, especially at night time. No person is entitled to ride in a railway mail car except the clerks assigned thereto, the Postmaster General, Assistant Postmasters General, officials of the railway mail service, and post office inspectors and special agents upon official business. In addition, persons may be allowed to ride on special permits, but these permits do not include free transportation and persons so riding must be indicated by the railway mail clerk to the conductor. When the duties of the distribution have been completed on a night run, at least one clerk is required to remain awake at all times in order to protect the mails. In addition to the car clerks, transfer clerks of the railway mail service are provided for the purpose of superintending the shipment and receipt of the mail at connecting points, and where large volumes of mail are to be handled. Railway mail clerks are required to wear no other uniform than a cap and badge.

The service of the railway mail clerks is difficult and dangerous and frequent casualties occur to them by reason of railway accidents and collisions. For many years this liability on the part of the mail clerks was unnoticed by the Government so that frequent cases of hardship occurred, in wlich railway mail clerks having been injured in the Government service were compelled to lose time and pay while recovering. By recent provision of law, however, an appropriation of $\$ 100,000$ is made to employ acting mail clerks to take the places of clerks who have been injured while on duty, and a further provision is made for payment of the sum of $\$ I, 000$ to the heirs or 
legal representatives of a clerk who shall be killed while on duty, the amount being exempt from the payments of the debts of the deceased. In addition to the foregoing, provision is made for granting thirty day's' leave of absence with pay to mail clerks who may become incapacitated on account of sickness, but their duties must be performed by other clerks in the service without additional expense to the Government. All railway mail clerks must sign a register upon reporting for duty and on completing a trip, the penalty for failure to make such registration being the loss of one day's pay in each case unless a good excuse can be shown.

The dutics of railway mail clerks include the delivery of pouches of mail matter which have been made up at the post offices along their routes to other post offices, either by delivery at stations where the train stops, or by throwing the pouches off while the train is in motion. They are required to take care that the pouches are not drawn under the train or thrown off so as to strike persons on the station platform, and must be thrown at least ten feet from the train and downward and outward. For the purpose of collecting the mail from the smaller stations catcher posts are erected in the proximity of stations, from which by a device attached to the mail car the pouches are caught on an iron rod and deposited within the car. This operation requires a considerable amount of skill and address and frequent accidents have occurred, so that the Post Office Department is at all times on the outlook for an improved mechanism which will work automatically. In addition to the foregoing duties, railway mail clerks distribute miscellaneous mail which may have been made up at the different post offices and delivered to the mail cars for distribution en route. The mail for post offices within the division on which the run is made is made up into separate packages, a package for each post office, with a legible address on one side of the package and a facing slip bearing the name of the post office on the other. Mail for States other than that in which the run is situated, is made up into packages bearing the name of the State, and forwarded for a closer distribution on one of the postal runs within that State. Postal clerks are furnished with a key which opens all mail locks, and are especially charged with care thereof, so that it may not be lost. They are required to carry it at all times attached 
to their clothing, and to turn it in at the Division Headquarters when it is not to be in use. Special provisions are also made for the handling of mail pouches, so that it can easily be seen if they have been tampered with, and the railway mail clerks are expected to be alert to prevent the violation of the laws regarding the use of the mails. All articles not legally mailable, so far as they can be detected, are to be withheld and turned into division headquarters with a report, and reports are also required as to packages of letters which have been improperly made up at post offices, while the clerks are expected, especially near the frontiers, to keep a careful watch upon the contents of the mail, in order to prevent violations of the customs revenue laws. Provision is made for a sufficient force of postal clerks so that an alternation can be made in clerks on runs covering more than a single day's work. Those who are compelled to be on the cars for considerable periods of time without intermission are given a week's rest after a week's service, while other provision is made for alternating the men, as continuous service on a railway mail run would soon result in the breakdown of the clerk, especially if he is traveling day and night. On short daily runs there is of course no such alternation, but provision is made for the supply of substitutes in case of sickness, and for annual leave and sick leaves for the clerks.

The Division of Foreign Mails, under the direction of the Second Assistant Postmaster General, regulates matters relative to the intercourse of citizens of the United States with foreign nations. In order to prevent misunderstandings and consequent discriminations against the mails of the United States, power is given by Congress to the Postmaster General to conclude conventions with foreign countries for the mutual exchange of mail matter, and for such arrangements as may mutually facilitate intercourse by mail and at reasonable rates. Such conventions or arrangements are to be approved by the President of the United States, and when so approved, copies are sent to the Secretary of State for the purpose of official record. A general agreement is in effect between the United States and the leading European countries, covering most of the civilized and semi-civilized countries of the world for the carriage of the mails each of the other at a given rate per pound, so that a fixed 
and uniform rate of postage may be known to any point included within the Universal Postal Union and stamps of the country in which the mail matter criginates will be recognized throughout the period of transit. Except for such agrement it would be necessary for persons in the United States desiring to prepay matter sent abroad to secure the postage stamps of the country through which the mail would have to travel, and to know the amount of postage to be charged in order that the mail should be properly forwarded. This inconvenience is avoided by the agreement referred to and by the acceptance of the stamps of the United States at their face value, the United States accepting similar evidence of postage paid. The following list shows the countries included in the Universal Postal Union.

Argentine Republic, mcluding eastern parts of Patagonia and Tierra del Fuego and Staten Island.

Ascension, Island of (British Colony).

Austria-Hungary, including the Principality of Liechtenstein.

Australia. (See the separate colonies in thcir alphabetical order.)

Bahamas.

Barbados, IV. I.

Bechuanaland, British Protectorate of.

Belgium.

Bermudas.

Bolivia.

Bosnia-Herzegovina.

Brazil.

British Colonies on west coast of P frica (Gold Ccast, Lagos, Senegambia, and Sierra Leone).

British Colonies in West Indies, viz.: Antigua, Dominica, Montserrat, Nevis, St. Christopher, the Virgin Isles, Grenada, St. Lucia, Tobago and Turk's Islands.

British Guiana.

British Honduras.

Britislı India: IHiudostan and British Burmah (Aracan, Pegu, and Tenasserim), and the Indian Postal Establishments of Aden, Muscat, Persian Gulf, Gaudur, Mandalay.

Bulgaria, Principality of.

Canada.

Caymarı Islands (British Colony).

Ceylon.

Chile, including western parts of Patagonia and Tierra del Fuego.

Colombia, Republic of. 
Colony of the Cape of Good Hope, including Bastitoland, Griqualand, Lit tle Namaqualand, Pondoland, Tembuland, Transkei, Walfish Bay, and Bechuanaland.

Congo, Independent State of.

Costa Rica.

Cyprus, Island of.

Danish Colonies of St. Thomas, St. Croix, and St John.

Denmark, including Iceland and the Faroe Islands.

Domitrican Repubiic.

East Africa, British Protectorate of.

Ecuador.

Egypt

Falkiand Islands.

Fiji Islands, Colony of.

France, including Algeria; the Principality of Mcnaco; the French postoffice establishments in Morocco (Tangier, Casatianca, El-Ksar-El-Kbir [Alcazar], Fez, Larrache, Mazagan, Mogador, Rabat, Saffi, and Tetuan); at Shanghai (China); and in Zanzibar, Cambodia, Anam and Tonkin.

French colonies-

x. In Asia-French establishments in India (Chandernagore, Karikal, Mahé, Pondicherry, Yanaon); and in Cochin China (Saigon, Mytho, Bien-Hoa, Poulo-Condor, Vingh-Long, Hatien, Tschandoik).

2. In Africa-Senegal and dependencies (Goré, St. Louis, Bakel, Dagana); Mayotte and Nossi-be, French Congo-Gaboon (including Grand Bassam and Assinie); Remion (Bourbon); Madagascar; Obock on the east coast, and the Comoro Islands (Grand Comoro, Anjounan Mohele).

3. In America-French Guiana, Guadeloupe and dependencies (Désirade or Deseada, Les Saintes, Marie Galante, and the north portion of St. Martin), Martinique, St. Bartholomew, St. Pierre, and Miquelon.

4. In Oceanica-New Caledonia, Tahiti, Marquesas Islands, Isle of Pines, Loyalty Islands, the Archipelagoes of Gambier, Toubouai, and Tuamotou (Low Islands).

Germany, including the Island of Heligoland and the German post-offices at Apia (Samoan Islands), and at Shanghai (China).

German Protectorates-

Territory of Cameroons (or Kameroun) (West coast of Africa); German New Guinea (in Papua); German South West Africa (Grand Namaqua, the Damaras Country, and the southern portion of Ovambo, between Cape Colony and Angola); Territory of Togo (Western Africa); German East Africa; the Marshall Islands, in the Pacific Ocean, and Kiautschau (China).

Gibraltar and its Postal Agencies in Morocco.

Great Britain and Ireland.

Greece, including the Ionian Isles.

Greenland.

Guatemala. 


\section{Hayti.}

Honduras, Republic of, including Bay Islands.

Hong-Kong and the post-ofices maintained by Hong-Kong at Kiung-Chow, Canton, Swatow, Amoy, Foo-Chow, Ning-po, Shanghai, and Hankow (China).

Italy, including the Republic of San Marino, the Italian offices of Tunis and Trifoli in Barbary; Massouah, $\Lambda$ ssab, Asmara, and Keren (in the Italian colony of Eritrea-Abyssinia).

Jamaica.

Japan, including liormosa, and Japanese post-offices at Shanghai (China), and Fusam-po, Genzanshin, and Jinsen (Corea).

Korea.

Labuan.

Liberia.

Luxemburg.

Maita and its dependeneies, viz: Gozzo, Comino, and Cominotto.

Mauritus and dependencies (the Amirante Islands, the Seychelles and Rodrigues).

Mexico

Montenegro.

Natal colony of, including Zululand.

Netherlands.

Netherlands colonies-

I. In Asia-Borneo, Sumatra, Java (Batavia), Billiton, Ceiebes (Macassar), Madura, the archipelagoes of Banca and Rhio (Riouw), Bali, Lombok, Sumbawa, Flores, the S. W. portion of Timor, and the Moluccas.

2. In Oceanica-The N. W. portion of New Guinea (Papua).

3. In America-Netherlands Guiana (Surinam), Curacao, A:uba, Bonaire, part of St. Martin, St. Eustatius, and Saba.

New foundland.

New Guinea, British colony of.

New South Wales, colony of, including Lord Howe Island and the Norfolk Islands.

New Zealand, colony of, including Chatham Island.

Nicaragua.

North Borneo, British colony of.

Norway, including the post-office at Advent Bay, on the west coast of Spitzbergen.

The Orange Free State.

Paraguay.

Persia.

Peru.

Portugal, including the island of Madeira and the Azores. 
Portuguese colonies-

I. In Asia-Goa, Damao, Diu, Macao, and part of Timor.

2. In Africa-Cape Verde, Bissao, Cacheo, islands of St. Thomé and Princes, Ajuda, Mozambique, and the province of Angola.

Queensland, colony of.

Roumania (Moldavia and Wallachia).

Russia. including the Grand Duchy of Finland.

Salvador.

Samoan (Navigators) Islands, German post-office at Apia.

Sarawak, British protectoraie of.

Servia.

Sian.

South African Republic (The Transvaal).

Spain, including the Balcaric Isles, the Canary Islands, the Spanish possessions on the north coast of Africa (Ceuta, Penor de la Gomera, Alhucemas, Melilla, and the Chaffarine Islands), the Republic of Andorra, and the postal establishments of Spain on the west coast of Morocco (Tangier, Tetuan, Larrache, Rabat, Mazagan, Casablanca, Saffi, and Mogador).

Spanish colonies-

In A frica-Islands of Fernando Po, Annobon, and Corisco, on the Gulf of Guinea.

Straits Settlements (Singapore, Penang, and Malacca).

St. Helena, island of (British colony).

St. Vincent, W. I.

South Australia, colony of.

South Rhodesia, colony of.

Swedcn.

Switzerland.

Tasmania, colony of.

Trinidad, W. I.

Tunis, regency of.

Turkey (European and Asiatic).

Uruguay.

Venezuela.

Victoria, colony of.

West Australia, colony of.

Zanzibar, British protectorate of.

The Universal Postal Union Convention provides for the exchange of correspondence between any Postal Union country and any country not within the Postal Union, by use of the mails of any Postal Union country which has such relations with the country foreign to the Union as to enable such Union country to exchange mails with the country foreign to the Union. Those countries foreign to the Universal Postal Union, reached through the intermediary services of a Postal Union country, are the following:

(I) All African countries not within the Postal Union. 
(2) China, the Empire of.

Mail matter within the Universal Postal Lnion is classified as follows: Letters.

Postal cards, and postal cards with paid reply.

Commercial papers.

Printed matter of every kind.

Samples of merchandise.

The rates of postage for the conveyance of articles throughout the entire extent of all Postal Union countries, including delivery at the residence of the addressee, where free-delivery scrvice exists, are as follows:

For letters, five cents for each half ounce or fraction thereof if prepaid.

For postal cards and private mailing cards ("post cards"), two cents each; and for postal cards with paid reply, two cents on each part.

For printed matter of every kind, commercial fapers, and samples of merchandise one cent for each weight of 2 ounces or fraction thereof; but at least five cents must be paid on each packet of commercial papers, and at least (wo cents on each packet of samples of merchandise.

Letters will be forwarded without prepayment of any postage; other articles will only be forwarded if prepaid in part; and in all cases of insufficient prepayment double the amount of the deficiency will be collected of the addressee.

Postage can be prepaid upon articles only by means of postage stamps of the country in which the articles are mailed. Articles (other than the replyhalf of double postal cards) mailed in one country addressed to another country, which bear postage stamps of the country to which they are addressed, or of any country other than the one in which they are mailed, will be treated as if they had no postage stamps attached to them. This will not apply to the United States postal agency at Shangha:, Clina, at which place United States postage stamps are valid for the prepayment of postage. (Sce sec. I3I2.)

All mailable matter will be reforwarded withont extra charge within the limits of the Union, and the deficient postage, if any, collected at the office of delivery. Mfatter erroneously or incompletely addressed and returned to the sender for correction will not be reforwarded except upon payment of postage therefor anew.

If unpaid or short-pairl articles are returned to the senders as undeliverable, the senders will be refuired to pay the amount which would have been collected of the addressees if the article had been delivered.

The following articles are prohibited transmission in mails exchanged between the countries of the Universal Postal Union, viz:

All articles, except letters, which are not prepaid, at least in part.

Printed matter, commercial papers, and samples of mercinandise, which contain any letter or manuscript not having the character of an actual personal correspondence. 
Printed matter, commercial papers, and samples of merchandise which are not madc up in packets in such manner as to admit of their being easily examine $\dot{a}$.

Matter printed by special processes mentioned in section 528, when not presented as therein directed.

Samples of merchandise, which have a merchantable value, or which exceed the prescribed weight or size.

Packets of commercial papers and prints of all kinds which exceed the prescribed weight or size.

All articles of a nature likely to soil or injure the mails.

Letters of packets containing current coin.

Any packets whatever containing articles liable to customs duty.

Gold or silver bullion, precious stones, jewelry, or other precious articles to or through the mails of any country whose legislation prohibits their being placed in the mails or forwarded.

Mails are exchanged with foreign countries through certain postoffices designated as "Exchange post offices." The following are the United States exchange post offices, and mails are exchanged through such offices with the countries named:

New York, N. Y., with North, South, and Central America, the West Indies, Europe, Africa, British India, Straits Settlement, Siam, and the Dutch East Indies.

Boston, Mass., with Canada, Great Britain, France, Germany, Belgium, Italy and Jamaica.

Philacielphia, Pa., with Great Britain, France, Germany, Belgium, Italy, Cuba, Jamaica, and Newfoundland.

Baltimore, Md., with Great Britain, Germany, Brazil, The Argentine Republic, Paraguay and Uraguay, the Island of St. Thomas, Barbados, Bahamas, Bermuda, Cuba, Jamaica.

Tampa, Fla., with Cuba.

Jacksonville, Fla., with Cuba.

Key West, Fla., with Cuba and the Bahama Islands.

New Orleans, La., with Mexico, Cuba, Nicaragua, Costa Rica, Gautemala, British Honduras, Republic of Honduras, the Bay Islands, and the United States of Colombia.

Laredo, Tex., with Mexico.

E1 Paso, Tex., with Mexico.

Eagle Pass, Tex., with Mexico.

Chicago, Ill, with Canada, Great Britain, Germany, Denmark, Sweden, Norway, Austria, Italy, The Netherlands, and Russia.

St. Louis, Mo., with Great Britain, France, Germany, Sweden, and Mexico. Detroit, Mich., with Canada.

San Francisco, Cal., wit?. Canada, New Zealand, Australia, Tasmania, the Fiji and Samoan Islands, New Caledonia, Japan, Shanghai, Hongkong and 
dependent Chinese ports, and the East Indies, except British India, Straits Settlements, Siam, and the Dutch Settlements.

Seattle, Wash., with Canada.

Tacoma, Wash., with Canada.

Mails for Canada are exchanged through the following railway postoffices: Caribou and Bangor, Boundary Line and St. Paul, Detroit and Chicago, Nalone and Licat, Newport and Springficld, New York and Chicago, New York, Geneva and Buffalo, Massena Springs and Utica, Nortlport and Spokine, Ogdensburg and Ltica, Pembina and Winnipeg Junction, Port ffuron and Chicago, Port Huron and Detroit, Portland and Island Pond, Portland and San Francisco, Port Townsend and Seattle, Rouses Point and Albany, St. Albans and Bostcn, St. Albans and Troy, St. Paul and Portal, Scattle and Portland, Seattle and Skagway, Sault de Ste. Naric and Ninneapolis, Spokane and Tacoma, Sumas and Soattle, Sumas, New Whatcom and Seattle, Vancehoro and Bangor.

Letters, postal cards, private mailing cards ("post cards"), and periodical publications for Mexico will be exchanged through the following railway post-offices: Texarkana anc: Laredo, Ilouston and Eagle Pass, Houston and El Paso, Benson and Nogales, Albuquerque and E.1 Paso, El Paso and Los Angeles.

The above are the general cxchange offices. Other post-offices are authorized to make local exchanges with post-offices in Canada and Mexico.

At the foregoing offices all mail addressed to foreign comintries is carefully scrutinized to see that nothing unmailable has been forwarded, and a list is made of the weight of the letters and packages included. At this point, also, all letters which do not bear the proper amount in stamps are withleld and returned to the address of the sender if possible. When the mails are delivered to steamships, a list of their contents are also forwarded by the same ressel, and when foreign mails are received at the e::change offices of the United States the lists sent from abroad are carefully compared with the mails and any errors certified to the foreign offices. The amounts due for domestic transportation of the foreign mails unler the rules of the Universal Postal Lnion are then charged against the credits which have been made to foreign conntries for mail originating in the United States. Articles which seem to contain dutiable goods are separated from other mail matter, and the Custom House authorities are notified, if the persons addressed live within the radius of the exchange office. If the package is to be forwarded to another office, it is stamped as apparently containing dutiable goods, and 
upon its receipt at the office of delivery it is the duty of the postmaster to notify the local Customs officials that such a package is held at the office, also to notify the addressee. The collector of customs may request the postmaster to have the package opened in his (the postmaster's) presence and the character of the contents of the package is then certified to the Custom House officials, who may either forward a note of the duty which should be collected, and which may be collected by the postmaster, or require that the goods be forwarded to the Custom House for appraisement. The alternative to this procedure is for a Custom House officer to be present when the package is delivered to the addressee and he can then make such requirements as to the inspection of the goods and the payment of duty as he deems appropriate.

In order to expedite the transmission of mails between the United States and Germany and England, sea post offices are maintained on the trans-Atlantic liners and while mails destined for other than the countries named are made up in New York in closed pouches and shipped on board the vessel as a part of her regular lading, the mail for distribution by the post offices in Germany and England is made up into post office packets on board the vessel so that redistribution when it arrives abroad is not necessary. A similar distribution is made on the return trip of mail intended for the United States and which originates in the post offices of Germany and England, so that the stay of such mail in New York for the purpose of distribution is materially shortened. Special postal arrangements in addition to the usual agreements in the Universal Postal Union are made between the United States, Canada, Mexico and Cuba. These include the transmission of first-class mail at the domestic postage in each country to each of the other countries, and a free interchange of the official publications of the countries named. Besides this free interchange of official documents in these particular countries there is a free interchange of correspondence relating to international mails between the various exchange offices throughout the world, and with the international headquarters. It is, of course, necessary to carry on a system of ad- 
justment of the balances representing the difference between the various debits incurred in the exchange of international mail, and this is performed by an international headquarters, while the expense of the sea post offices is divided between the countries represented therein. Besides the free transportation heretofore mentioned, return postal cards and articles which are undeliverable are given transportation under the Universal Postal Union free of charge. In addition to the sea post office service, provision is made for mail agents on certain of the steamship lines, each of whom receives $\$ 2,000$ a year as compensation, and mail agents are also located at various points in the IVest Indies and in Japan and China for the purpose of supervising the transfer and exchange of mails of the United States, and to facilitate the service of both outgoing and incoming United States mails.

The Division of Railway Adjustments, under the direction of the Sccond Assistant Postmaster General, has charge of all matters relating to the railway transportation of mails. This includes not only the actual stean railway transportation, but transportation by surface lines in cities as well, and such suburban transportation by electric car lines as may have been authorized; also the wagon and mail messenger transportation from railway stations to post offices. Under former legislation, it was the duty of the Postmaster General to secure at intervals the weight of the mails transported for a given period of time on each of the railway mail routes of the country, and this occasional weighing was made the basis of the payments to the railroads. In connection with the recent investigation and consideration of the question of railway mail pay made by various commissions and committees, it has been deemed advisable to have a thorough and analytical weighing of the mails which is now going on for a period of six months. A careful classification and report of all the mails originating at the different post offices throughout the country has been inaugurated, and with the system of weigling now going on, it will be possible to state definitely the exact cost not only of carrying the sum total of the mails, but of carrying the separate classes of mail matter as well.

The question of railway mail pay is by no means a simple one, 
for the reason that there enters into it not only a question of the weight carried by the railroads, but the question of facilities afforded, and of mail trains and fast schedules maintained in order that the time of transmission of mails may be reduced. One such question was that with reference to the divisor to be used to secure the daily amount of mail which should represent a fair figure over a given route. The total weight of mail being taken for a period of, say, one week, it was complained that roads which ran seven-day trains were at a disadvantage as compared with roads which ran six-day trains, that is mail trains on seven days and six days respectively. When seven was taken as a divisor for the week's mail carrying, it gave, of course, a smaller daily average than when six was taken as a divisor to secure a basis upon which payments should be made so that a road which gave a better service might receive a smaller pay. It was finally decided to take the actual number of days on which the weighing took place as the divisor, and the result, representing the average amount of mail carried in one day, is the basis upon which the railroad receives pay for the service. In addition to this weight payment, there is a car payment, a sum being allowed for each inile traveled by a railway post office attached to a train, while under special provision of law additional amounts of bounty are paid to certain roads on certain routes in order to secure through transportation between important cities by lines of railway travel upon which trains making the necessary schedules would not otherwise be run.

As a result or the movement for greater economy in the payment of railway niail pay, a change of system has been effected so that a large quantity of postal supplies which were formerly sent by the regular nuail trains are now sent by freight, and considerable economies nave been secured. Shipment of such supplies is under the direction of the Division of Railway Adjustments, which also superintends the weighing of the mails and matters relating to mail carrying on electric cars. This division also makes arrangements for the letting of contracts for carrying the mails between the stations of the railroads and the post offices. In large cities where pneumatic tubes have not been 
installed, this contract assumes considerable proportions and in all cases where the post offices are more than eighty rods from the railway stations, a contract has to be made with a private party for the delivery of the mail to the post office and vice versa. The pllematic tubes which are in use in the larger cities for the transportation of mails between the post offices and the railway stations come under the direction of this division also, which makes arrangements for the installation and maintenance of such equipment.

The Division of Contracts, under the Second Assistant Postmaster General, has charge of matters relating to the letting of contracts for carrying mails on steamboats and on "Star" routes, so-called. The latter are routes on which no prescribed method is laid down for the carrying of the mails, and they may be transported in the option of the contractor by any means which will promote the safety and speed of their transmission. Star routes cover the less populous sections of the country, and the United States is divided into four sections for letting of contracts, the divisions being as follows:

a. Maine, New Hampshire, Vermont, Massachusetts, Rhode Island, Connecticut, New York, New Jersey, Pennsylvania, Delaware, Maryland, District of Columbia, Virginia and West Virginia: current term, July I, I905, to June 30 , I9o9.

b. North Carolina, Soutl Carolina, Georgia, Florida, Alabama, Mississippi, Tennessce, Kentucky and Porto Rico; current term, July I, I9of, to June 30 , I 908 .

c. Ohio, Indiana, Illinois, Michigan. Wisconsin, Minnesota, Iowa and Missouri; current term, July I, I907, to June 30, I9II. d. Arkansas, Louisiana, Texas, Indian Territory, Oklahoma, Kansas, Nebraska. North Dakota, South Dakota. Montana, Wyoming, Colorado, New Mexico, Arizona, Utah, Idaho, IVashington, Oregon, Nevada, California, Alaska and Hawaii; current term, July I, I906, to June 30, I9Io.

A section is advertised for contract, once in four years, the contract being let for four years. The advertisements for bids are posted in the local post offices of the sections in which the contracts 
are to be let, and postmasters are required to furnish information to prospective bidders. Bids can only be made on one route, combination bids being forbidden and must be accompanied by a bond, the amount of which is fixed in accordance with the importance of the route and conditioned that the bidder will perform the contract if it is awarded to him by the Postmaster General. The bond must be accompanied by the oath of the sureties as to their responsibility, and must be approved by the postmaster, or if the sum exceeds $\$ 5,000$, only by a postmaster of the first, second or third classes. No bidder will be accepted who is ander twenty-one years of age, is a married woman or is disqualified from entering into a contract. The purpose of the bond required is that the United States shall not be put to the trouble and expense of bidders declining to perform the service at the price named by them. The United States can recover under the terms of the bond any amount which it shall lose or be required to pay in addition to the bids made for the service to be performed.

The successful bidders are liable under their bonds also for deductions for failure to properly carry the mails and for any additional expense which may be incurred by the United States by reason of their failure to carry out the terms of contract throughout the period for which it is let to them.

The Division of Contracts, under the Second Assistant Postmaster General, has also the details relating to contracts for steamboat mail service. This includes only the steamboat service in the domestic waters of the United States for the transmission of mails on routes which cannot economically utilize land service. In addition to the facilities for transporting the mails, steamboat contractors on routes which require the travel of postal clerks on more than one working day, are required to provide sleeping accommodations for the post office employees.

All contracts and orders affecting the contract mail rontes are required to be certified daily to the Auditor for the Post Office Department, and the determination of the amounts which are to be paid to the contractors, under the terms of their contracts, is made in accordance with the result of the work of the Division of Inspec- 
tion, which is attached to the office of the Second Assistant Postmaster General, and which examines monthly and special reports of postmasters as to the performance by contractors of their duties. When contractors fail in the performance of their duties, either by the omission of trips, or by failure to keep to the schedule time, fines are imposed and the orders for such fines and deductions for nonperformance of service are made by the Division of Inspection, which certifies to the Auditor for the Post Office Department the amount due to contractors for their work.

The Division of Equipment, under the direction of the Second Assistant Postmaster General, has charge of the mail bags and mail repair shops, situated in Washington, and attends to all matters pertaining to the issuing of mail pouches, sacks, keys, key chains, etc., to the postal officials, and to the repair of such equipment when necessary. The division keeps a record of all articles furnished, and examines all accounts relative to the purchase or repair of mail equipment.

The Second Assistant Postmaster General, in connection with his duties in supervision of matters relating to foreign mails has direction of the payment of postal subventions, authorized by law, of $\$ 4.00$ per mile for first-class ships and \$2.00 a mile for second-class ships, \$1.00 a mile for third-class slips, and \$.66. 2-3 for fourthclass ships, such amounts to be paid upon the shortest practicable route for the outward voyage of any ship to the port of destination, and conditioned on the ownership and manning of the vesscls by citizens of the United States and of the construction of the vessels so that they may be utilized in time of war as auxiliary cruisers in the Navy. Their construction is required to be sucr that they can carry at least four six-inch cannon. Under the provisions of this law, several vessels have been built, especially for the WVest Indian and Sonth American trade, which come within the provisions of the law as to construction, but with the exception of vessels running on special routes, the benefits of this subvention are not generally taken advantage of. for general reasons having to do with the difficulties of operating vessels profitably under the navigation laws of the United States and current maritime conditions. 
THIRD ASSISTANT POSTMASTER GENERAL.

The Third Assistant Postmaster General has supervision over matters relating to the postal finance, and a very important class of cases relating to the admission of matter to the mails and the appropriate amount to be paid for its transmission. The question of postal finance alone is one of almost as great importance as that of the finances of the United States. Although the moneys received from the postal revenues are deposited in the Treasury, and the expenditures are paid to a considerable degree by amounts withdrawn from the Treasury of the United States, the postal revenues are separate and distinct from the other revenues of the Federal Government, and constitute a different fund, the administration of which is under the direction of the Postmaster General. The revenues for the maintenance of the postal service come from postage, including the fees for registered letters and for special delivery letters; from box rents; from the amounts which have been forwarded in letters which cannot be delivered and which are, when the fact of nondelivery is ascertained, turned into the postal revenues, fines and penalties imposed for the violation of postal laws, balances from foreign countries and miscellaneous receipts. The amounts involved in the money order business are not a part of the postal revenues, but are kept in separate accounts, although transfers may be made from one fund to the other for temporary use. The expenses of the Post Office Department are met by appropriations by the Congress from money which has been deposited in the Treasury of the United States as the amounts received from postal revenues. Postmasters are authorized to meet certain of the current expenses of their offices from the postal revenues as they are received, but the net amount accruing at each postoffice must be turned into the Treasury and all amounts paid out from the postal revenues are paid by warrant on the Treasury, under one of the heads of appropriation fixed by Congress, so that the money itself accruing from the postal revenues is not under the direct care of the Postmaster General, but is held in the Treasury subject to lis orders, which must be validated by the signature of the Auditor for the Postoffice Department, who is an 
official of the Treasury Department, that an amount equivalent to the face value of the warrant has been deposited from the postal revenues in the Treasury, and that there has been warrant by appropriation by Congress for the withdrawal of the amount stated to be applied to a particular purpose. The amount accruing under the postal revenues not being sufficient to mect the expenses of the service, Congress is required to make an annual appropriation, in general terms, of the sums necessary to meet the deficiencies in any specific appropriation, the main expense of which is to be paid out of the postal revenues.

For the purpose of collecting the net amount accruing from the postal business at the different offices certain of the larger post offices are designated as depositories for postmasters at the smaller offices, and the postmasters at depository offices are required to keep books of credits with the officials of the smaller offices, and to forward the funds as they accumulate to a subTreasury or to the Treasury of the United States. Duplicate receipts are given for all postmasters' deposits, one of which is retained by the postmaster as a volicher and the other forwarded to the Auditor for the Post Office Department. All of the accounts arising in the business of the Post Office Department, and the postal service are forwarded ultimately to the Auditor for the Post Office Department, who conducts the final audit in all matters, certifies all balances which may be due and signs the warrants for the deposit of money in the Treasury, and for withdrawal of funds therefrom.

An entirely separate account is kept of the money which is received by the postal service in payment of money orders and which is paid out to the holders of such orders. This subdivision of the funds is maintained throughout the postal system, and postmasters must keep their money order funds separate from their postal revenues, except that they may use amounts available in each for making up temporary deficiencies in the other. That is to say, a postmaster who has a money order to pay and has not a sufficient amount in his money order account may transfer the balance from the postal revenue, although the amount transferred remains as a debit against the money order 
account, and must be repaid to the postal revenues as soon as practicable. Postmasters are required to keep a sufficient amount of money on hand to meet the demands for payment of money orders which may be expected, and a central office is maintained in connection with the New York Post Office, on which postmasters may draw for funds to meet the payment of money orders of which they have notice, or which they may reasonably anticipate will be presented.

The Division of Finance under the Third Assistant Postmaster General has charge of all matters connected with the collection and deposit of the postal revenues, together with consideration of irregularities and false returns of business by postmasters, and the recording of all expenditures under the several appropriations for the postal service, and the details involved in the usual financial operations of transferring money from one post office to another, in order to meet the varions needs of the service. The Division of Finance is under the direction of a superintendent, who is required to give a bond in the sum of ten thousand dollars.

The Division of Stamps, under the Third Assistant Postmaster General, makes arrangements for the preparation for the various denominations of postage stamps, and for their issue to the postmasters as requisitioned for, directs the sales of stamped paper and the agencies established at the points where this class of supplies for the postal service is manufactured by contract, besides investigating and reporting upon claims of postmasters for allowances for stamps and stamped paper lost by burglary, fire or other unavoidable cause. Each postmaster is, of course, charged with the face value of the stamps and stamped paper furnished to him, and must make a return either in cash, as shown by vouchers for Treasury deposits, or in remaining supplies, and must either pay for the balances found against him out of his own funds, or secure an allowance therefor through a claim filed with the Division of Stamps and acted upon by the Assistant Attorney General for the Post Office Department with the final approval of the Postmaster General.

The Division of Money Orders, under the Third Assistant Postmaster General, has charge of all matters relating to the money order system, including the international arrangements for the 
transmission of moncy by orders through the mails, and the Division of Registered mails has supervision over the operations of the registered mail system, except that all correspondence with foreign officers relating to the foreign registry system must be conducted through the office of the Second Assistant Postmaster General. The registry system of the United States mail is intended to secure valuable letters anci parcels from loss in the mails, and is based simply upon a requirement that every person into whose hands the letter or parcel may come must sign for the same, after having identified it, and must forward it to the next responsible person, in a carrier which is specially marked and with which special precautions are taken. The person sending a letter or parcel receives a receipt, and the package thereafter, until reccipted for by the addressee, is cared for by sworn officials, each of whom makes himself personally responsible by his signature to a receipt, and who protects himself by securing the signature of the person to whom the letter or parcel is next delivered. The fee for registration is eight cents, in addition to the postage, and a limit of damage for loss is placed at one hundred dollars, and the value of a registered article, should it be lost, will be paid up to that amount, the amount paid being that which can be shown to be the actual value of the article lost.

The Division of Classification, under the direction of the Third Assistant Postmaster General, has charge of matters relating to the construction and application of the law prescribing the different classes of mail matter. Such classes and the postage fixed for each are as follows:

First Class Matter.-Written matter, mamely: Letters, postal eards, post cards (private mailing cards) and all matter wholly or partly in writing, whether scaled or unsealed, except manuscript copy accompanying proof-sheets or corrected proof-sheets of the same, and the writung authorized by law to be placed upon matter of other classes. All matter sealed or otherwise closed against inspection is also of the first class. Typewriting (with carbon or letter-press copies thereof) is lecld to be an rquivalent of handwriting and is classed as such in all cases.

Rates of Postage-On letters and other matter, wholly or partly in writing, except the writing specially anthorized to be placed upon matter of other classes, and on sealed matter or matter otherwise ciosed against inspectiontwo cents an ounce or fraction thercof.

On postal cards-one cent each, the price for which they are sold. 
Privale: mailing cards (post-cards) conforming to the requirements of Section 418, Postal Laws and Regulations-one cent each.

On "drop letters," two cents an ounce or fraction thereof when mailed at letter-carrier post offices, or when mailed at post offices which are not lettercarrier offices if rural free delivery has been cstablished and the persons addressed can be served by rural carricr; and one cent for each ounce or fraction thereof at offices where neither letter-carrier nor rural delivery service las been established and at offices where rural delivery service has been established, if the persons addressed cannot be served by rural carrier because they reside beyond the limits of the rural delivery service.

Matter of the second class embraces all newspapers and other periodical publications which are issued at stated intervals, and as frequently as four times a year and are within the conditions named.

The conditions upon which a publication admitted to the second class are as follows:

First.-It must regularly be issued at stated intervals, as frequently as four times a year, and bear a date of issue, and be numbered consecutively.

Second.-It must be issued from a known office of publication.

Third.-It must be formed of printed paper sheets, without board, cloth, leather, or other substantial binding, such as distinguish printed books for preservation from periodical publications.

Fourth.-It must be originated and published for the dissemination of information of a public character, or devoted to literature, the sciences, arts, or some special industry, and having a legitimate list of subscribers.

All periodical publications issued from a known place of publication at stated intervals and as frequently as four times a year, by or under the auspices of a benevolent or fraternal society or order organized under the lodge system and having a bona fide membership of not less than one thousand persons or by a regularly incorporated institution of learning, or by or under the auspices of a trades union, and all publications of strictly professional, literary, historical, or scientific societies, including the bulletins issued by State boards of health and State agricultural colleges, are admitted to the mails as second-class matter.

The rate of postage on newspapers and periodical publications of the second class, when sent by other than the publisher thereof, or a news 
agent, is one cent for each four ounces, or fractional part thereof, to each single address, prepaid by stamps affixed.

There is no such rate of postage as four cents a pound.

When mailed by the publisher from the known office of publication and at the Post Office of Entry: One cent a pound, prepaid in money, on the bulk weight of all copies addressed to subscribers, and on the bulk weight of all copies sent as samples for the bona fide purpose of securing new subscriptions or advertising patronage, or to persons to become agents for the publication. The number of sample copies mailed at the pound rate with any issue may not exced the number of copies of that issue required for subscribers. (See Sec. 456 , P. L. and R.)

When Mailed by a News Agent: One cent a pound, prepaid in money, on the bulk weight of all copies sent to subscribers, and on the bulk weight of all copies sent to other news agents for the purpose of sale. (See Sec. 448, P. L. and R.)

Newspapers and periodicals, when deposited by the publisher, or by a news agent, in a letter-carrier office, for box delivery, or for delivery through the gencral delivery window, are subject to postage at the rate of one cent a pound. Weekly newspapers, when deposited by the publisher, or a news agent, in a letter-carrier office for local delivery by city letter-carriers or otherwise, are subject only to the rate of one cent a pound.

Publications which have been admitted to the second class, one copy only to each actual subscriber resicing in the county where the same are printed, in whole or in part, and published, may go free through the mails, if properly presented for mailing separately from those on which postage is to be paid, except for delivery by carrier.

Periodicals not excecling two cunces in weight, when deposited in a lettercarrier cfice for delivery by its city carriers, are subject to one cent postage each, prepaid by stamps aifixed.

Periocicals exceding two ounces in weight, when deposited in a lettercarrier office for delivery by its city carriers, are subject to two cents postage each, prepaid by stamps affixed.

Within the past few years an attempt has been made to restrict the privileges of sccond class matter to periodicals which come within the terms of the law, and to restrict the periodicals which are entitled to the privilege within its limits. Under a more liberal construction of the law, than that which is now in effect, there crept into the second class mail a very large volume of matter, the admissibility of which was doubtful, to say the least, and which materially increased the cost of the transmission of the mails. In connection with investigations that have been going on as to the reason for the large deficiencies in postal revenues to meet 
expenses of the postal service, it became apparent that if the second class mail matter was brought within the limits evidently contemplated by the Congress, a very material saving could be effected and that, in some instances, transmission under second-class mail rates could appropriately be withdrawn from publications which were largely in the nature of advertising circulars, without any general utility as means of disseminating information, or promoting literature, art or other branches of human knowledge. The resulting campaign against abuses of the second-class privilege, which was established primarily as an encouragement to the dissemination of printed information, became a decidedly bitter one and an appeal was made to the courts, which has substantially fixed the statue of the rights of publishers and those of the Post Office Department to set up the requirements under the law which publishers must obey in order to secure the privilege of cheap transmission of their publications. The difference between the second and third class rates of postage, affording an opportunity for disseminating circulars at a very much smaller cost if they could be issued under the guise of periodicals was the cause of the abuse of the second class privilege and this abuse by reason of the close restrictions applied by the Post Office Department has been, if not entirely abolished, materially checked.

Mail matter of the third class embraces books, circulars, and other matter wholly in print, proof sheets, corrected proof sheets, and manuscript copy accompanying the same, and postage is fixed at the rate of one cent for each two ounces or fractional part thereof.

In order to secure third class rates for reproduced matters at least twenty identical fac-similes by a mechanical process must be presented for mailing at once, directed to different addresses. Otherwise they must go at first class rates. A written prefix to a circular letter is, however, permitted, and a visiting card or business card or order blank may be included with third class matter. No personal communications or directions can, however be written or included without making the matter liable to first class postage.

Mailable matter of the fourth class embraces all matter not embraced in the first, second or third class which is not in its form or nature liable 
to destroy, deface, or otherwise lamage the contents of the mail bag or harm the person of anyone engaged in the postal service, and is not exceeding four pounds for each package thereof, except in case of single books weighing in excess of that amount, and except for books and documents published or circulated by order of Congress, or printed or written official natter.

All matter of the fourth class is subject to examination and to a postage charge at the rate of one cent an ounce or fraction thereof, to be prepaid by stamps affixed.

The postage on secls, cuttings, bulbs, roots, scions, and plants is charged at the rate of one cent for each two ounces or fraction thereof, subject in all other respects to the existing law.

The following definite classification is of articles generally dispatched in the mails but does not include all mailable articles:

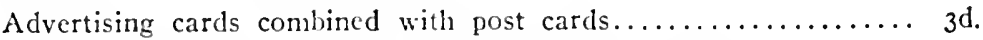

Advertising signs printed on other material than paper......... $4^{\text {th. }}$

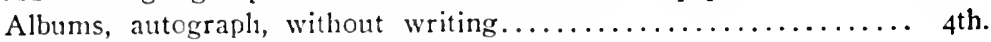

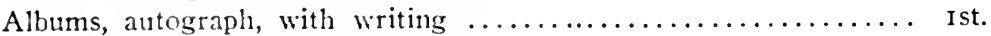

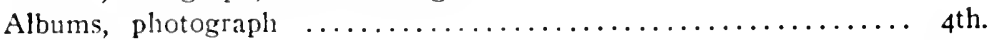

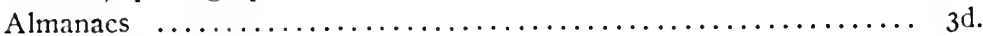

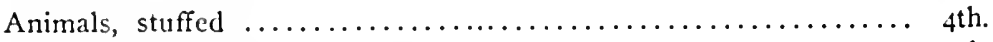

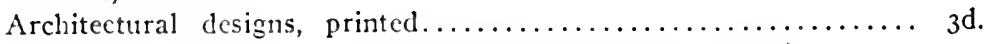

Architectural designs, containing writing (sce Drawings)...... Ist.

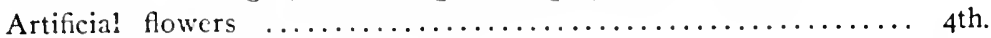

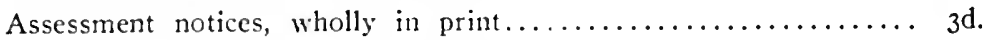

Assessment notices, partly in writing $\ldots \ldots \ldots \ldots \ldots \ldots \ldots \ldots \ldots$ Ist.

Article. Class.

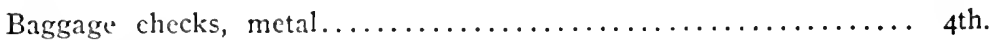

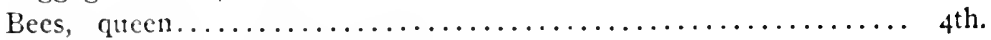

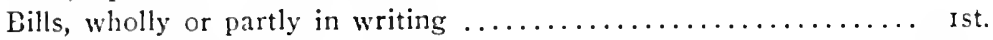

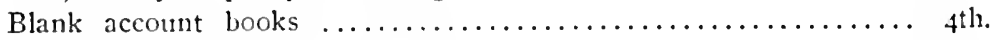

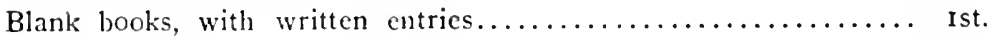

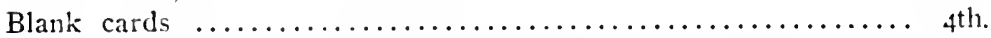

Blank printed forms, with written signature.............. Ist.

Blind, indented or periorated sheets of paper containing characters

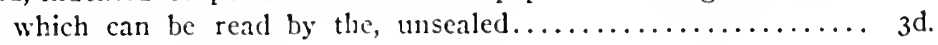

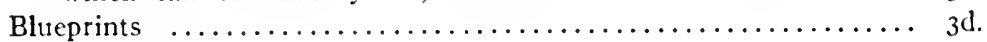

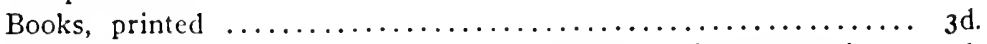

Botanical specimens, not susceptible of being used in propagation.. 4th.

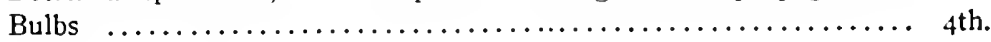

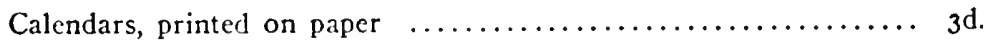

Calendars, printed on material other than paper............ $4^{\text {th. }}$

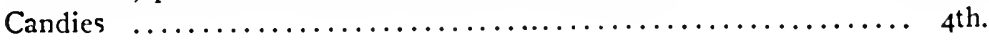


Article.

Class.

Carbon copies of typewritten matter................ Ist.

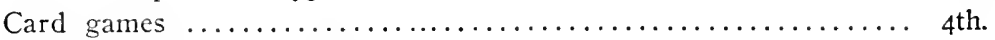

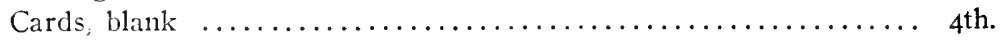

Cards, Christmas, Easter, etc., printed on paper............. 3d.

Cards, Christmas, Easter, etc., printed on material other than paper 4th.

Cards, visiting, bearing written name................

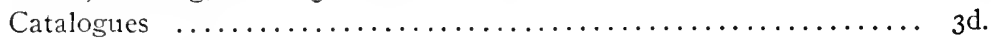

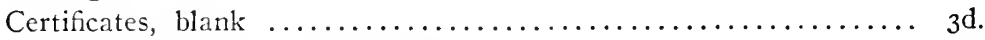

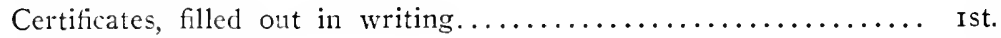

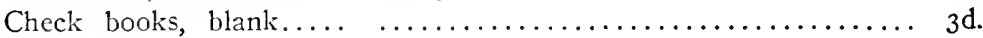

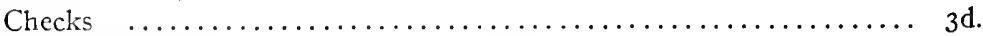

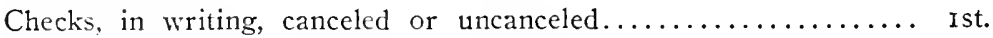

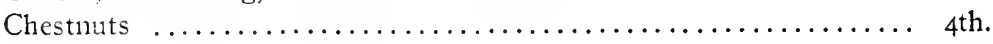

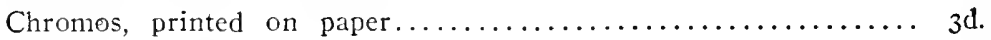

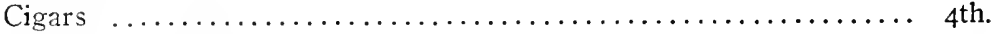

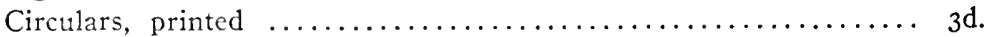

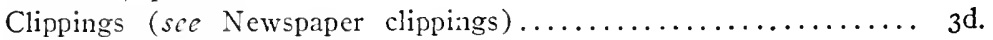

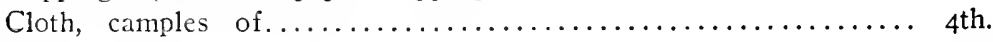

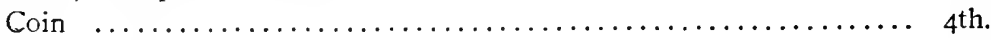

Coin holders, card, blank..........................

Copy books, school, with printed lines and instructions for use..... 3d.

Crayon pictures or drawings, framed or $u n f r a m e d . . . \ldots \ldots .4$ th.

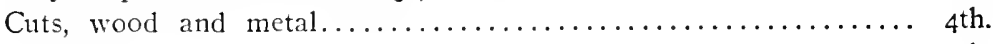

Cuttings of plants or trees.........................

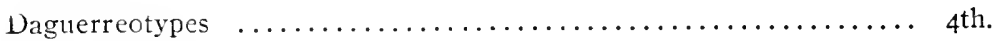

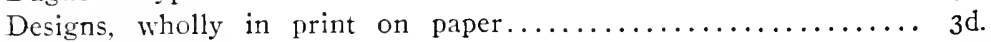

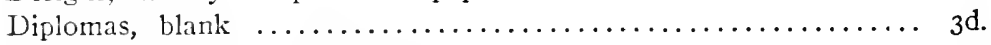

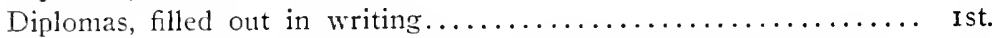

Drawings (pen or pencii), without writing, framed or unframed.. 4th.

Drawings or plans containing written words, letters, or figures indicating size, price, dimensions, etc.................

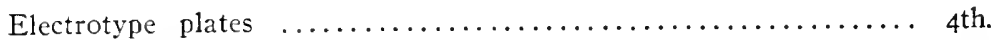

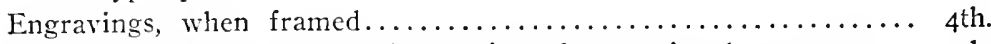

Engravings and wood cuts, impressions from, printed on paper... 3 d.

Engravings and wood cuts on wood or metal base.......... $4^{\text {th. }}$.

Envelopes mailed in bulk........................ th $^{\text {th }}$

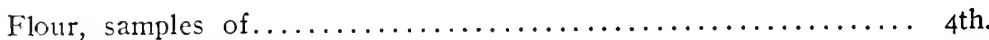

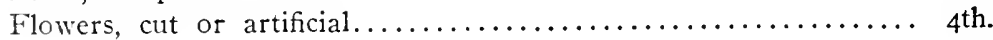

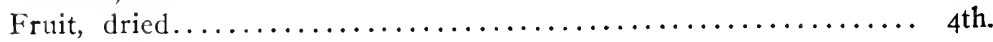

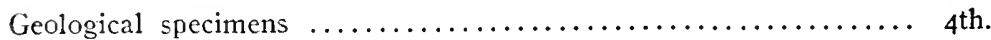

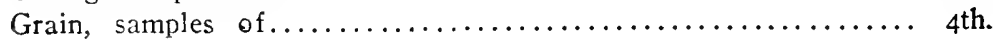


Article.

Class.

Herbs, dried

4 th.

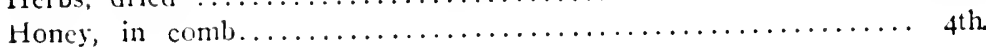

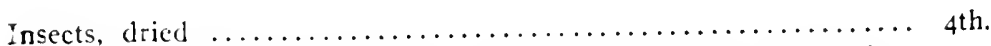

Invitations, printed or engraved, containing no other writing than date and name and address of person addressed and sender... $3 \mathrm{~d}$.

Labcls, written

Ist.

Letterpress copies of handwritten or typewritten matter........ Ist.

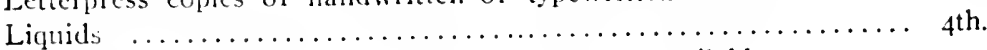

Liquors, ardent, vinous, spiritious, or malt, unmailable.

Lithographs

$3 \mathrm{~d}$.

Magnzines or newspapers, mailed by the public (sec Second-class maticr. section 2 , I cent for each $f$ ounces or fraction..........

Manuseript of typwritten copy, without proof sheets.............

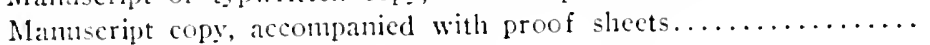

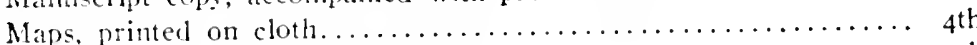

Maps, printed on paper, with the necessary mountings......... 3 d.

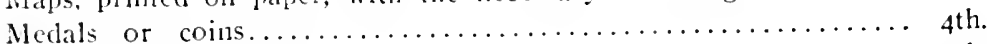

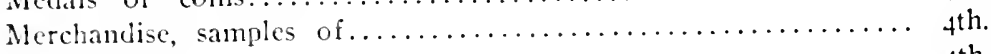

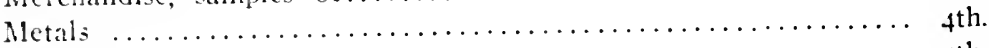

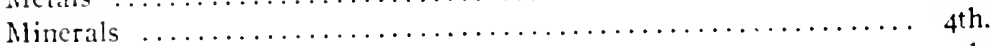

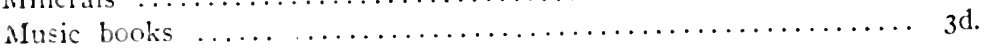

Newspaper clippings, with name and date of paper stamped or

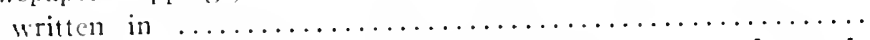

Aewspapers or magazines, mailed by the public, I cent for each

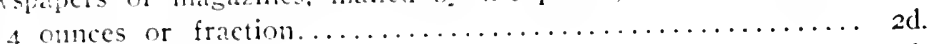

Nits. in natural state......................... 4 th.

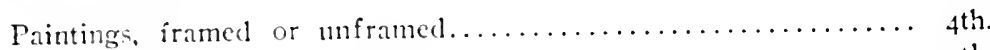

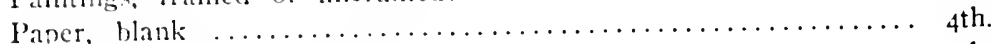

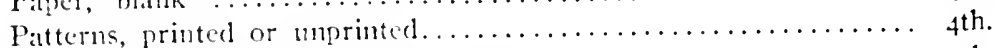

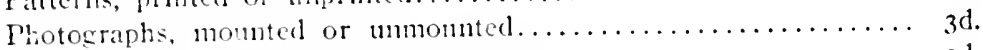

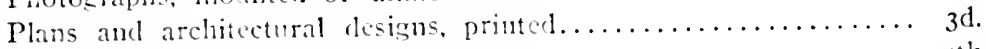

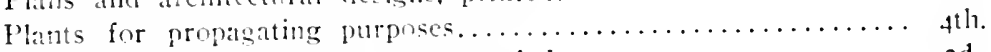

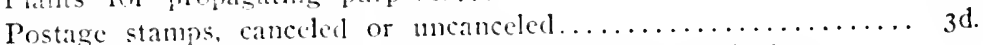

Postal cards, used. wholly or partly in writing, remailed ....... Ist.

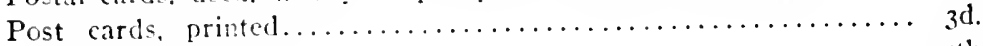

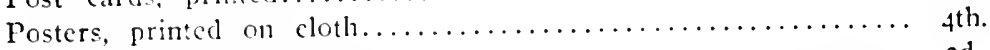

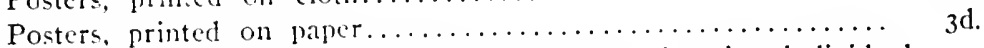

Price jists, printed, containing written figures clanging individual

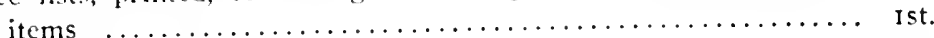

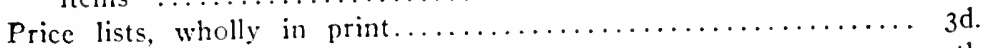

Printed matter on other material than paper.............. 4th. 
Article.

Class.

Printing, samples of $\ldots \ldots \ldots \ldots \ldots \ldots \ldots \ldots \ldots \ldots \ldots \ldots \ldots \ldots \ldots \ldots \ldots \ldots . .3 \mathrm{~d}$.

Proof sheets, printed, corrected, with or without manuscript..... 3 d.

Receipts, printed, with written signatures............... Ist.

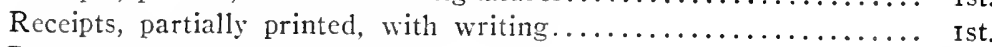

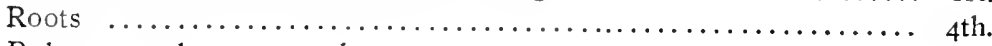

Rulers, wooden or metal....................... 4 th.

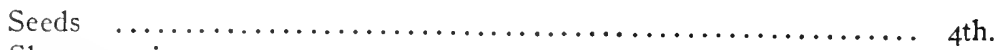

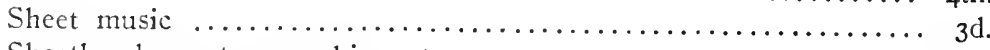

Shorthand or stenographic notes $\ldots \ldots \ldots \ldots \ldots \ldots \ldots \ldots \ldots \ldots \ldots$ Ist.

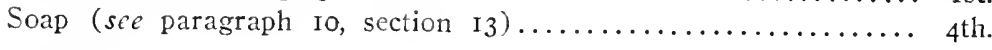

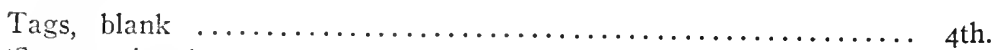

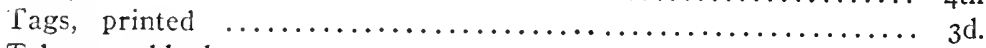

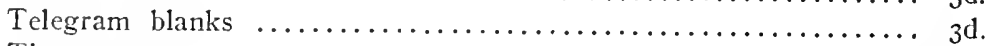

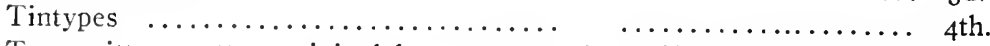

Typewritten matter, original letterpress and manifold copies thereof ist.

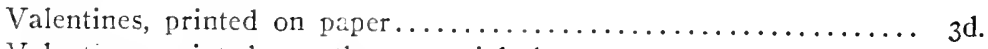

Valentines, printed on other material than paper............ 4 th.

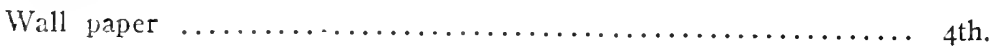

Another important function of the Division of Classifications relates to the admission of letters and other matters which are intended for persons who are engaged in illegal and fraudulent transactions, and who use the mails as a medium for their operations. All letters which are intended for persons who are operating lotteries, and letters addressed to persons and firms who have been found upon investigation to be attempting to practice fraud by means of advertisements offering inducements which they cannot fulfill are stamped upon receipt, either at the office of origin, or delivery, with words indicating the fraudulent or illegal nature of the business which is being conducted by the person to whom addressed, and if possible the letters are returned to the senders, or if the name of the senders does not appear on the outside of the envelope, they are forwarded to the Dead Letter Office for treatment with other non-delivered letters.

Before persons can be placed on the list of those whose mail is returned to the senders, they must, unless their business is palpably illegal and forbidden to the mails by law, be given a hearing before 
the notice of their incligibility to receive mail, technically called a "fraud order," is issued. Complaints of the character of business done by persons using the mail are received almost daily by the Postoffice Department from persons who have been victimized and such complaints, together with other information that may come to the knowledge of the Department, are investigated by the Postoffice inspectors, and upon report of the latter, notices to show cause why fraud orders should not be issued are sent out to the suspected persons, and mless they can show positively the legal and equitable nature of their business, orders are issued against them and their mail is returned as above described. It is frequently the case that those against whom such orders have been issued attempt to evade the orders by having their mail sent in the name of others, and when such a condition is found to exist it is egarded as the prima facie reason for the issue of a further frand order against the persons whose name is so used.

In connection with second class postage, the privilege is given to publishers of paying the accruing postage in lump sum, instead of by affixing stamps representing the amount of postage due, as was formerly done, such stamps being then kept in the post offices and applierl to bundles of papers in accorlance with the weights found on each bundle. At present, a cash deposit is made by the publisher of an amomnt which will cover the postage due on forthcoming issues for a given period and the publications are forwarded without stamps. The Division of Classifications has charge of the details relating to these accounts, and of seeing that the postage is properly collected. In a similar way persons who send out large amounts of third and fourth class matter which would ordinarily require the affixing of stamps of appronriate denominations, senerally onecent stamps, can by making a suitable deposit, print upon the wrappers of such circulars provirled not less than two thousand pieces are sent out, notice that the postage has been paid in a lump sum, and are thus reliever of the labor involved in affixing the postage stamps.

The Division of Classification also has charge of matters relating to the use of franks and penalty envelopes, and the restrictions imposed by the law on the subject. Franked envelopes are used by 
Nembers of Congress, and the frank consists of the facsimile of the signature of the members printer in the right-hand upper corner, together with the letters M. C. or U. S. S. The left hand corner also carries the words "Official Business." It is supposed that members shall use the franking privilege only for official communications, but a considerable latitude is given to them and their own construction of what can be included within official communications is a liberal one. Official communications by the heads of the executive departments and their subordinates are transmitted through the mails free in envelopes which bear a notice that a penalty of $\$ 300.00$ will be imposed for the use of such envelopes for other than official business. These are described as penalty envelopes, and although there is, of course, sometimes a difficulty in exactly drawing the line between official and personal business, restriction is rather closer in cases of penalty envelopes than in matter sent out under the frank of members of Congress. All public documents and seeds are sent out under frank, and the volume of mail matter included within the franking and penalty envelope privileges, which will be definitely ascertained when the present system of weighing is completed, is a very considerable proportion of the total volume of mail handled by the postal service of the United States. Besides the foregoing classes of free mail matter and that of the President, the only other persons having free transmission of their mail accorded to them are the widows of Presidents of the United States, who are generally by law given a frank, which consists of their name written on the envelope, and in some cases all matter addressed to them has been delivered free of charge. Special provisions are also made for soldiers' and sailors' letters, which can be transnitted through the mails without pre-payment of postage, when they originate in the field, or other point where postage stamps are not available, but which must have due certification by commanding officers, and the postage on the same must be paid at the point of delivery.

THE FOURTH ASSISTANT POSTMASTER GENERAL.

The chief of the duties of the Fourth Assistant Postmaster General relate to the control of the rural free delivery which was estab- 
lished as an experiment by the Postoffice Department, and which was authorized by law in 1902. This provides for a house to house delivery by a carrier with a vehicle along comtry roads leading from designated postoffices, the maximum of the compensation for such carriers being $\$ 900.00$ a year, the carricrs, however, being allowed to transport such merchandise as they may be able to do without interfering with the lelivery of the mails. The rural free delivery in the five years of its official existence las assumed very large proportions and is responsille for a considerable part in the increase in the cost of the postal service. Rural free delivery routes are established upon the application of a majority of the patrons of a given office or section served by that office, and upon the recommendation of the post-office inspector. Although the service is designed as a material convenience for patrons of the postal service, it has frequently happened that the installation of a rural clelivery route has been vehemently opposed by many of the residents, on accomt of the conseguent closing of some of the smallcr postofices and the inconvenience involved thereby to persons residing close to the smaller offices. Rural free delivery carricrs are appointed after an cxamination, conducted muler the anspices of the Civil Service Commission, and are required to give bond. They are forbidden to solicit orders for merchandise from the patrons along their routes, and restrictions are placed npon them as to the carriage of alcoholic liquors and the performance of any work or transaction of any business which wonld interfere with the performance of their postal dities. Each house is required to have a mailbox beside the roal to facilitate deliveries and collections. The rural carrier, besides delivering and collecting the mail is empovered to transact other postal business, so that the effect of the establishment of the system has been to bring the postoffice to the doors of hundreds of thousands of rural homes. and has made possible a daily dissemination of the mail in districts where nual communication was dependent larecly upon the ability of the inhabitants to visit the post-offices.

The Division of Supplies, under the direction of the Fourth Assistant Postmaster General, has charge of the furnishing of supplies to post-offices and postal service generally, except stamps and mail 
equipment. This includes a large variety of articles necessary in the administration of post-offices. The contracts are made as described under the paragraphs relating to the purchasing agents, but all requisitions for supplies are received in this division, and the necessary allowances and orders made up.

The Division of Dead Letters receives all matter that is undeliverable, and which cannot be returned to the sender on account of no evidence appearing on the envelope of the sender's address. Such letters are opened in the Division of Dead Letters, under appropriate check, so that no one person may have the custody of a letter containing valuable articles without the same coming to the knowledge of another person, and if the sender can be ascertained by anything connected with the letter when thus opened, the envelope and its contents are returned to the person to whom they belong. That is, to the sender, if the addressee's name cannot be ascertained by the contents of the envelope, and to the addressee if possible. Before opening the envelope, the clerks of the Dead Letter Office are required to correct misdirected letters when possible, and the office is equipped with a set of directories of the different cities of the United States, together with other sources of information. The clerks have become so expert in the deciphering of addresses that in most cases of a poor direction, they are able to send the letter on to the addressee without opening. In case of a blank envelope, however, or of an undecipherable address, the course before described is followed. All articles of value which are received in the Dead Letter mail are retained and the amount in cash is turned into the Treasury, while the other articles are sold annually at a dead letter sale, which takes several days, and which is held in Washington. The proceeds from such sale are turned into the Treasury of the United States to the credit of the postal revenues.

By the statutes of the United States, post roads are established under the terms of the Constitution which permit the Congress to establish postroads and provide for the carrying of mail thereon. The post roads established include all the waters of the United States during the time the mail is carried thereon, all railroads or parts of railroads which are now or hereafter may be in opera- 
tion, all canals, all plank roads, all public roads and highways, when kept up and maintained as such, all letter carricr routes and any road by which a mail may be carried to supply a court-house which is without a mail. The Postmaster General has the authority to establish postoffices on sucl post roads, and he may in addition extend a line of road to supply mails to postoffices which are not on any established road. The foregoing means that all methods of communication within the United States are established as post roads and that where post office facilities are needed at points which have no established communication, the Postmaster General is empowered to extend the post roads by a system of posts, so that there shall be no point within the jurisdiction of the United States which cannot have mail communication with the rest of the United States, under such regulations and with such facilities as it may bc practicable to establish. Congress has especially provided by several enactments that court houses, i. e., the center of local government in each subrlivision and the location of the administration of justice shall not be without mail communication. For the purpose of clenoting graphically the points at which post-offices are located and routes oer which mails are carricrs, a system of topography is maintained uncler the direction of the Fourth Assistant Postmaster General, who has charge of all matters relating to the preparation of post ronte maps. Such maps are issued for the information and guidance of postal cmployees and of contractors, but may also be secured by the general public at a price fixed by the Department and which covers the cost of printing.

The Division of Redemption, uncler the Direction of the Third Assistant Postmaster General, receives and examines all damaged and unsalcable stamps, stamped cnvclopes and postal cards sent by the postmasters to the department for relemption. If upon examination it appears that they are being appropriately redeemed and credited to the account of the postmasters, the Auditor for the Postoffice Department is advised of the amount of the credit, and the stamps are destroyed in a similar manner to the destruction of bank notes. 


\section{CHAPTER XIII.}

THE NAYY DEPARTMENT.

Notwithstanding efforts for a number of years to place the organization of the Navy Department under a general staff system, such as that now in effect in the War Department, the administration of the affairs of the Navy is carried on in accordance with the Bureau system, the general principles of which have been explained in the chapter on the War Department. That is, besides the civilian control of the administrative affairs of the department proper, each phase of the provision of war material for the navy and of the provisions for the maintenance of the vessels and personnel is entrusted to a semi-independent bureau, co-ordinated with the military branch of the department only through the efforts of a civilian secretary.

The department proper of the Navy department consists in effect only of the office of the Secretary of the Navy, with its immediate adjuncts. The personal office of the Secretary, the Assistant Secretary and his office, the office of the Chief Clerk, and the library are all that are directly and solely in matters of administration under the immediate supervision of the Secretary. The office of the Judge Advocate General, although assigned in the departmental division to the Secretary's office, has, to a considerable degree to do with the discipline of the personnel, so that the Judge Advocate General, in addition to being the Secretary's legal adviser is practically an officer and an adjunct of the Bureau of Navigation, which has the control of the ships and men. The duties of the Secretary and Assistant Secretary of the Navy have already been described in another chapter. Those of the Chief Clerk of the Navy Department are rather more important than the duties which pertain to similar positions in the other executive departments, and the Chief Clerk is in effect an administrative Assistant Secretary, rather than simply a director of a clerical force. In addition to the duties pertaining to the appointment, promotion, assignment, and discipline of the clerical personnel, the office of the Chief Clerk is a clearing 
house for the correspondence between the various bureaus, and between the bureaus and the Secretary. It prescribes regulations also for the cmployment of labor at the various navy yards, and controls the disbursement of large contingent funds and other appropriations. It is, of course, the medium through which the Secretary directs the opcrations of the different bureaus, and, receiving the general mail of the department, is able to a certain degree to regulate and control bureau action, although not always without opposition from and friction with the burcau heads. The office of the Judge Advocate General, in addition to passing upon the usual law questions arising in a departmental administration, is charged with the review of all general and summary court materials held on board ship and at navy yards within the limits of the waters of the United States. Commanders in Chief of forcign stations are empowered to review all court martials ordered by them, the result, only, being certified to the office of the Judge Advocate Gencral for record. The Judge Advocate General of the Navy is also the reviewing disciplinary officer for the Marine Corps, it being the single instance in which that organization is not independently maintained. In addition to matters relative to punishment, all reports and findings of the board charged with investigating the qualifications of officers for promotion are reviewed in the office of the Judge Advocate General who also has charge of drawing up contracts for the building of ships of the navy and of detcrmining questions which arise under such contracts and what amount may legally be paid to the builders as the work of construction proceeds. The Judge Advocate General of the Navy is appointed by the President, by and with the advice and consent of the Senate, and holds his office for a term of four years, being selected from the officers of the Navy and Marine Corps without limiting provision as to his rank upon selection. While serving as Judge Adrocate General an officer las the rank and highest pay of the Captain in the Navy or the rank, pay and allowances of a Coloncl in the Marine Corps, it being possible to select the incumbent from either branch of the service.

The Judge Advocate General is called upon to deal with questions relating to the usual practice of the law, and also with any questions of military law, including those which arise out of courts of 
inquiry into the causes of accidents and disasters in the Navy, questions of retirement of officers under the law, questions relating to pay, and questions relating to the relative rank and position in their grade of officers of the Navy and Marine Corps, including as well the higher positions in the enlisted corps. He is assisted by a civilian lawyer under the title of Solicitor, who alone among the departmental solicitors is an officer of the department in which he serves, and who receives four thousand dollars a year. Under a recent arrangement all civilian matters are handled by the Solicitor leaving the purely military aspects to the Judge Advocate General. This, however, is only a matter of Departmental regulation and is subject to change.

The business of the Navy Department of providing a fleet and of provisioning, manning, and manœuvering it, is carried on under the direction of eight bureaus which, as has been said, are semi-independent. The head of each bureau is responsible for the transaction and efficiency of the business of the Navy Department under his charge, but is in no way concerned with the operations of other bureaus, and has only a general moral responsibility, and no direct charge that the operations of his bureau shall be so conducted that there shall be no loss of time or money imposed upon another bureau. The natural tendency is to promote the interest of the individual bureau as a primary consideration, or, rather, to get the bureau work done without much thought or time or incentive for thought whether the work is being done in a manner calculated to produce the most effective results as a whole. The result is, of course, an evasion of responsibility as to the sum total, since, unless a defect can be found in the administration of the part, no person other than the Secretary himself can be charged with the responsibility for the lack of coordination of the whole.

The order of the importance of the bureaus of the Navy Department constitutes largely a matter of opinion. In the revision of the United States statutes, the Bureau of Yards and Docks is given the first place; in point of seniority, however, the Bureau of Construction and Repair claims the first position, while the Bureau of Navigation, by reason of being the military branch of the organization would, in an active contest for precedence, claim the first place on 
the ground of the superiority of the line, or fighting officers. Without regard to precelence, the natural order of procecding from the inception of navy would bring the Burean of Construction and Repair first in the order of consideration. Under the law any officer of the Navy of not below the rank of commander and who is a skillful navy constructor may be appointed Chief of the Bureau of Construction and Repair. A special provision, however, allows a member of the corps of Naval Constructors who has the rank of Lieutenant Commander, to be selected for the position. The Chief of the Burean of Construction and Repair, as is the case with all Chiefs of Bureaus is appointed by the President, these officials being selected according to law, and removed, for the time being, from their regular duties, in the line or the corps to which they belong, and confirmed by the Senate for the special positions for which they have been selected. When officers below the rank of Rear Admiral are selected as Chiefs of Burcaus, they have during their terms of service, which is four years for each appointment, the rank of Rear Admiral and the pay and allowances of a Brigadier General in the Army.

The corps of naval constructors from among whom the Chief of the Burcau of Construction and Repair is now selected consists of officers who have graduated from the Naval Acadeny at Annapolis, and who shortly after their graduation were selected for special qualifications to be assigned to the corps of Naval Constructors. They were formerly selected, as are the engineer officers of the Army, from the head of the list of midshipmen, but this practice has been abandoned in recent years, and after their service as midshipmen afloat, and upon their promotion to ensigns, two or three officers of each class who have shown special fitness for construction work are appointed naval constructors and detailed to a post graduate course of instruction in a technical school. Upon final graduation they are detailed as assistant inspecting officers at the ship-yards where vessels of the Navy are being built, and are gradually developed for the work of the Corps, which is that of the Bureau of Construction and Repair.

The work under the jurisdiction of the Bureau of Construction and Repairs consists of designing the hulls of the vessels of the Navy, of 
preparing the plans and specifications of ships that are built by contract, and preparing the plans and directing the work of such ships as may be built in the Government Navy Yards, and of inspecting the hull work on vessels under construction by contract. Jurisdiction of this Bureau is, broadly, over all that relates to the hull of the vessel and the fixtures thereto. This includes the designing and inspection of the work on the hulls, turrets, spars, capstans, windlasses, steering gear, and ventilating apparatus. All of these matters are under the independent control of the Bureau. There are certain other fixtures, however, and certain matters relating to the turrets in regard to which the Burean of Construction and Repair must consult the Bureau of Ordnance. These are the permanent ammunition hoists, the roller paths of the turrets, and similar matters which affect the adjustments later to be made by the Bureau of Ordnance, while the Bureau of Construction and Repair installs the turret mounts, furnished by the Bureau of Ordnance, and such other apparatus of that Bureau as must be placed in position while the construction work is going on.

The Bureau of Construction and Repair also takes directions from the Bureau of Ordnance as to the adjustment of the armor plate of the vessel, the material, quality, and distribution of thickness having been determined by the Bureau of Ordnance. The disposition of duties between the different bureaus of the Navy Department seems at the present time wholly arbitrary and to a certain degree illogical; as for example, while the Bureau of Steam Engineering has charge of the appliances for producing steam motive power, the Bureau of Construction and Repair has charge of the steering engines, equally worked by steam. The explanation of these apparent discrepancies can be readily found by remembering that the division of duties was made during the time of wooden ships, and with the further understanding that no point of jurisdiction will be voluntarily surrendered by a bureau. So that, as the Bureau of Construction and Repair appropriately fashioned a wooden rudder, and the wheel and other mechanism which controlled it, it now sees no reason why it should surrender the jurisdiction of that portion of the ship construction even though another bureau should have come into existence charged with acquiring expertness in similar 
devices to those which now control the movement of the ships' guiding mechanisms.

Besides the foregoing, the Bureau of Construction and Repair has charge of building and repairing the small boats of the Navy although not of the meaus for their mechanical propulsion. It has the charge of all electric turret turning machinery, and electrical machinery for operating ammunition hoists. It has also charge of fans operated by electricity which are used for ventilating the hulls of vessels and of all appliances for hoisting on the decks of the vessels of the Navy. Further distinctions may be noted in this connection, that although the Bureau of Steam Engineering has charge of methods of boat propulsion by steam, when the propulsion of a boat is by an electric motor, it comes under the administration of the Burean of Construction and Repair.

The Bureau of Equipment, however, is regarded as the chief electrical burcan of the department, and if an electrical fan is needed for the comfort of a room it is furnished by that Burcau. If, however, it is for the ventilation of the hull it is furnished by the Bureau of Construction and Repair. Further odd arrangements may be noted with regard to the administration of dry docks. These are built at maintained so far as repairs go by the Purean of Yards and Docks. The Burcau of Construction and Repair has not only cliarge of the operation of placing a vessel upon the blocks in a dry dock, but of operating the dock and cleaning the same. As has been said, however, all of these distinctions have their rise in some logical situation in the past and are continued under the present system probably with some excess of expense and loss of convenience because a way has not yet been found by which work of a similar character can be combined under the supervision of experts in each particular line.

The work of the Burean of Construction and Repair in Washington consists in the preparation of drawings for construction and for the repair of vessels, together with attention to the necessary administrative details. In connection with the work of designing. there is a considerable establishment under the direction of the Bureau of Construction and Repair at the Washington Navy Yard, where models are made up and tested to develop the lines of new ships and types of ships. Outside of Washington the Bureau main- 
tains corps of inspectors at the different ship yards engaged in construction for Government work, and has at each of the Navy Yards a more or less extensive plant devoted to construction in wood and metals. For the purposes of the work under the jurisdiction of the Chief of the Bureau of Construction and Repair the Commandant: of Navy Yards are subject to this official's orders. No work can be mind rtaken except upon the order of the Secretary of the Navy, but when an order has been given for a certain amount of construction or repair work, the directions with reference to the details are sent by the Chief of the Bureau to the Commandant of the Navy Yard at which the work is to be done, and are transmitted by the Commandant to the Naval Constructor who is in charge at such particular yard. A similar system is in effect with regard to other manufacturing bureaus of the Navy Department, and as the Commandant is usually a line officer with expert knowledge chiefly in regard to sea-going duties, the actual administration of the work under any bureau is generally performed by the representative of that bureau in the yard acting upon his own responsibility largely, and sometimes without consultation or consideration of the work going on under the direction of other manufacturing bureaus. As a result of this, complaint has sometimes been made that the work of one bureau was hampered or delayed by the operations of another, and arguments have been made in favor of creating a single manufacturing bureau in the Navy Department which should have charge of all matters relating to the construction and repair of vessels and their equipment.

The next burean in the order of the construction of a naval vessel is that of Steam Engineering. The Chief of this bureau is appointed from the line officers of the Navy, not below the rank of Lieutenant Commander, and must be a skilled marine engineer. The Chief of the Bureau of Steam Engineering was formerly at the head of the corps of Engineers which was constituted as a separate staff corps, appointments being made to the academy at Annapolis as cadet engineers and upon graduation appointment was made to a separate corps, having a similar standing to the pay corps and the medical corps. As a result of the personnel bill of 1898 , however, the engineers were amalgamated with the line of the Navy, and became line 
officers. The Chief oi the Bureau of Stean Engineering since that time has been either an old engineer officer or an officer who received an education as a cadet encineer and who came from the engineer staff corps. Future Chiefs, however, will be line officers who have specialized in stem engineering. The duties of the Bureau of Stean Enginecring refer entirely to matters relating to steam propulsion, although recently questions relative to the development of power by gasoline and sinilar motors have been taken up. Specifically they relate to all questions of designing, building, fitting out, and repairing the steam machinery used for the propulsion of naval vesscts, and the steam pumps. stcam heaters, distilling apparatus, refrigerating apparatus, all steam connections, and steam machinery when used for actuating turrets. Included within the scope of these duties is the experimentation to determine the $1 x$ type of boilers and of fuels. whether solicl or liquid, and the determination as to the availability or various styles of engines including the turbine method of propulsion as applicd to naval uses and of problems relating to propellers. This Burcau requisitions for the oils and similar supplies for the engine-roons of naval vessels, but not the coal for the boilers, the latter being purchased and suppitici by the Burcan of Equipment. It provides the motive power for the boats of the Navy, with the exception of that for electric lame'nes and when the power used for moving turrets is transferrel fow steam to electricity the matter of the turret moving is removed from the jurisdiction of the Bureau of Steam Engineering. As is the case with the other constructing bureaus, it estimates for the amount of money which it necds during a fiscal ycar, such estimates being subject to the revision of the Secretary of the Nary and it has to a large degree the control of the sums appropriated by Congress for its particular work. Like the other bureans it has exclusive control of the interior of its workshops at different nay yards, and designs their arrangement and provides the necessary machinery to carry on its work with the different metals. This independence of other bureaus leads to the duplication of machinery and equipment at the navy yards and under a former system the different bureaus at each yard had not only an independent equipment of machinery, but in most cases an independent power, heating, and lighting plant as 
well. The latter condition has been to a certain degree obviated and will be entirely abolished by the work now going on for the consolidation of the various power plants at the different yards, and for the operation of a single central power plant in each case by the Bureau of Yards and Docks. The Burcau of Steam Engineering has no disciplinary control over the personnel of the engine room of the naval vessels. This is administered under the same conditions as other branches of the line, but the Bureau of Steam Engineering receives the reports as to the consumption of coal, and other data necessary to understand the performance of the machinery installed, and is also charged with the correction of any defects which may be found to exist, and with the making of necessary improvements.

The work of the Burean of Steam Engineering, in connection with the construction of vessels, consists in the inspection of the boilers and engines as they are installed, the work of installation going along with that of the construction of the hull of the vessel. In the two recent instances in which vessels of the Navy were constructed at navy yards the Bureau of Steam Engineering constructed the boilers and motive power. The Bureau has an experimental plant at Annapolis, Md., where it carries on experiments to determine the propulsive power of different forms of propellers, as well as such other investigations as may be needed in the work of the Bureau.

The third bureau in point of time in relation to the construction of a vessel of the Navy is the Bureau of Ordnance. The functions of this Bureau, however, extend beyond those connected with building, vessels of the Navy and include all matters relative to the furnishing of guns and ammunition for the ships and men, including the torpedo service. With reference to building vessels of the Navy, however, it has the very important and primary function of recommending the character of the armament and of the armor for the new vessel. That is to say, it recommends the number and calibre of the guns to be carried and also the weight and thickness of the armor and the points on the vessel to which it is to be applied. It also recommends the interior dimensions of the revolving turrets and prescribes the requirements for the turrets in regard to rotation. In all matters regarding the weight of the armor and armament to be carried it is limited by the Bureau of Construction and Repair, which 
advises the Bureau of Ordnance as to the amount of weight that can be allotted to these two items and within this limit the Burean of Ordnance directs the location of the armament and the firing arc which must be allowed for the guns, and also the location and characteristics of the permanent fixtures intended for the handling and storing of ammunition. The great guns for the Navy are manufactured under the direction of the Burean of Ordnance at the gun factory in Washington, D. C., and guns of a smaller calibre are manufactured by contract under the inspection and in accordance with the designs of the Burcau. The Bureau also contracts for the shells and other projectiles to be used hy the Nary and determines the characteristics of the powder to be used, provides for its manufacture by contract and also maintains powder manufactories near Washington for the production of a portion of the supply. It also maintains at Indian Heaci, on the Potomac River, a proving ground where the guns for the Navy are tried, and has at Newport, Rhode Island, a torpeclo station devoted to the trial of torperloes, which are manufactured by contract; to experiment with new forms of torpedoes, and to instruction of the men of the Navy in the use of torpedoes as a means of naval warfare.

The Bureau of Ordnance installs all guns on vessels except such parts as are permanently attached to the hulls of the vessel. and also excepting turret guns and mounts, which are installed by the Bureau of Construction and Repair under the direction of the Bureau of Ordnance at the time the turrets are put in place, the latter being removable structures covering the guns and mounts. The matter of subdivision of the responsibility for the machinery on boar af vessels is seen in the regulation that although the Bureau of Construction and Repair has jurisdiction over the electrical apparatus for turning turrets, the electrical apparatus for operating rammers and gun elevating gear which may be in the turret in close proxinity to the former are under the direction of the Bureau of Orduance, which must attend to all installation and repair of the latter apparatus. The Burcau of Orlnance also has charge of all range finders and of apparatus for the transmission of battle orders and included within the system of fire control. It also has charese of the manufacturing and improvement of gun siglits, makes estimates 
for the amnual supply of ammunition needed by the Navy, and has general charge of supply materials for the semi-annual target practice. The Bureau of Ordnance is strictly a line bureau and its Chief is appointed from a list of officers of the Navy of not below the grade of Commander. Certain of the officers of the line, however, specialize as ordnance officers and there has been in recent years a system inaugurated of detailing certain of the younger officers for special courses of instruction in ordnance work with a view of developing them as ordnance experts, together with their other duties as line officers. A sinilar course lias been taken with regard to the detail of officers to be specially instructed in steam engineering. Both of these policies, however, are not matters of law, but have been undertaken by the Secretary of the Navy as the result of representation to him that the Navy was lacking in the service of officers specially fitted for the technical work necessary under the bureaus. The policy of assignment nay, therefore, either be at a later date affirmed by law, or may be discontinued should it appear wise to a Secretary of the Navy to take this step.

In connection with the gun factory at the Washington Navy Yard, and also with the naval torpedo station at Newport, there is a special training school for enlisted men of the Navy who are given six months, in each place, of practical instruction in the manufacture and use of guns and torpedoes. The Bureau of Ordnance also has its own shops at the different navy yards, and carries on a practically independent establishment at each place, administering a force of its own employees as a separate establishment and without regard to the other employment in the yard. It also has charge of the various magazines of naval ammunition and carries on the operation of receiving the powder and shells from contractors, storing it until needed and transporting it to the various vessels of the Navy as their supplies become exhausted.

It will be seen by the foregoing that at least three bureaus have important and sometimes conflicting functions in regard to the designs of new ships. For a given length and breadth of vessel the Bureau of Construction and Repair can, of course, provide only a certain weight carrying capacity. The Bureau of Steam Engineering desires as much of this capacity as possible for means of propulsion, 
and the Burcau of Ordnance as much as it may be able to get for armor and armament. The Bureau of Construction desire to save weight where possible to secure speed, and the Bureau of Equipment wants a sufficient allotment to provide a comfortable equipment. The result could very readily be to sink the ship, so that a Board on Construction, referred to in another paragraph, is provided to adjust the various claims and secure a happy medium, the board being composed of the Chicfs of the bureaus named.

The final bureau which has to do with the construction of the material of a ship is the Bureau of Equipment, which has charge of supplying all the details of her equipment except the stores of clothing and subsistence which have not been supplied by the other bureaus. There is made up for each ship of the Navy a list of the allowances of the minor articles to be provided for the equipment of the vessels and the Bureau of Equipment arranges for the purchase and issue of these articles upon first outfitting a ship, and when they have become lost or broken. The list of allowances inclucles not only the necessary sails, anchors, ropes, galleys, riggings, and cooking utensils, but also mess gear, or articles from which the sailors eat, and the crockery and table linen for the officers' dining rooms as well. A certain amount of the latter two is allowed and must last for a certain length of time, only a small proportion being allowed to be renewed in any year, so that if breakages or losses occuir in excess of the table of allowances, the difference nunst be made up by the officers themselves. The Bureau of Equipment also furnishes hammocks and bedling to officers and men, though the former must furnish their own lincu. The Bureau of Equipment is also the chief electrical bureau of the Navy and has control of the wireless telegraph and telephone system and of all means of conmunication on shipboard except battle indicators, range finders, and similar ordnance communicating apparatus. The Bureau of Equipment also has charge of all signaling apparatus, including steam whistles, semaphores and the installation of submarine signals, when used. It has clarge of the manufacture of rope, chains, and chain cables, and anchors for the Navy, and maintains an extensive plant at the Boston Navy Yard for these branches of manufacture. Its largest expenditure is included in providing the coal for the Navy, 
in which duty it contracts for the supply of the coal, its transportation from point to point and its storage at the various coaling stations established by law, the bureau having exclusive control of the latter stations.

The Bureau of Equipment is also generally in charge of the apparatus used in navigation. In this connection it has under its control two institutions of value to navigators generally, the Naval Observatory and the Hydographic office. The former, located near Washington, D. C., establishes the meridian from which American navigators calculate their distance by means of the observation of the sun's altitude at noon, and also conducts a continuous system of observation of the heavenly bodies in order to secure accurate bases for the computation of the tables contained in the nautical almanacs from which navigators secure their figures as to the altitude and location of stars also used in navigation, which is the art of determining the exact location of a given point upon the surface of the earth when there are no land marks to aid the observer. In addition to the special work performed by the Naval Observatory it is a general astronomical observatory holding high place among the observatories of the world and equipped with a staff of astronomers of internationally recognized merit. The question whether the Naval Observatory should continue as it is at present under the direction of a naval officer detailed to the position for a few years only has been in previous years a warmly contested one. Decision has, however, been in favor of the continuance of the observatory under the superintendence of the naval service of the Government instead of under civil control. Although the Bureau of Yards and Docks is in general control of the land and buildings under the jurisdiction of the Navy Department, an exception is made in the case of the Bureau of Equipment, of the Naval Observatory, and of the land and buildings pertaining to the different coaling stations. These are all under the Bureau of Equipment, which designs, erects, and maintains all of the buildings thereon. The Naval Obscrvatory, in addition to the duties mentioned in the foregoing paragraph has charge of all work relating to the rating and repair of chronometers for the Navy and of the supply and care of such sextants, quadrants, and other instruments for use in navigation, together with the usual binoculars, 
spy glasses and ships' time-pieces. For the proper care of these instruments a force of instrument makers and adjusters is maintained at the Naval Observatory, together with a considerable force of computers and experts employed in connection with the astronomical instruments.

The Hydrographic office of the Navy Department, under the control of a naval officer, known as the Hydrographer and who is detailed for the duty by the Secretary of the Navy, secures all charts for the use of the naval vessels of the United States and also publishes a series of charts for the benefit of the merchant marine. Formerly the business of supplying charts was a private undertaking, but the latter has been practically superseded by the activity of the Government in this direction. No direct actual surveys are made of the coasts of the United States by the Hydrographic office, but it takes the result of the work of the Coast and Geodetic survey and uses the charts based upon that work. It also secures copies of foreign charts and corrects them in accordance with the information received from vessels of the United States which are from time to time engaged in the survey and charting of unknown waters, and either issues the chart as purcliased from abroad, or makes new plates, including the corrections and additions made in the Hydrographic office from information sccured. As new information is received from time to time it becomes necessary to make frequent changes in the charts as issued and one of the principal dutics of the office consists in the recalling of former issues of charts and making corrections thereon, or in making new plates and issuing new editions of charts which have, by renson of new surveys and additional information become materially altered. All of the charts printed by the Hydrographic office are placed on sale at different points throughout the country, agents of the Hydrographic office being appointed at the different seaports, and the cost of the charts being that of the paper and printing, is materially less than the same publication could be issued for by private persums. The Hydrographic office also prepares and prints sailing directions covering all portions of the globe, and these are issued to the Navy and are sold to the merchant marine at cost, as is the case with all the publications of the office; the nautical almanac, also published 
by the Bureau of Equipment being included in the list of publications made available to merchant mariners at the cost to the Government.

For the purpose of securing immediate and reliable data which would be of value in correcting the permanent record of the Hydrographic office, branch offices are established at the different seaports. Each office is under the charge of either an active or a retired naval officer, with the assistance of one or more nautical experts, and these offices not only secure from incoming vessels data which is of value for the Hydrographic office, but serve as centers of information for the outgoing vessels as well. Frequent bulletins are issued by the Hydrographic office and supplied free of charge to mariners and others interested. These bulletins contain the news of the ocean as to wrecks and dangers to navigation, information as to the course of derelicts, icebergs, and so on. A chart is also published monthly for the Atlantic and Pacific oceans known as a pilot chart, upon which is plotted the best courses for steamers, together with indications of prevailing winds, and of the probable proximity of icebergs, location of fogs and similar information which would be of value to the masters of vessels outward and homeward bound. In addition to the branch Hydrographic offices on the Atlantic, Pacific and Gulf coasts, there are branch offices at different points on the Great Lakes. A series of hydrographic charts is issued for the Great Lakes, but these come in competition with the charts published by the army lake survey and the latter are generally preferred by vesselmen.

In addition to giving out and receiving information as to the maritime changes, the branch Hydrographic offices are centers of information on matters relating to navigation. As the officers in charge have been specially trained in the more scientific aspects of the art of navigation, they are able to give instructions to merchant sailors especially in the manipulation relative to the compasses, and to the proper care and adjustment of those instruments. This is especially true of the hydrographic offices on the Great Lakes, for the reason that navigation on those bodies of water is less often carried on in accordance with scientific principles and more often in accordance with the local knowledge of the master of the vessel, 
who is generally a good pilot but a poor navigator, and it is belicved that the work of the Hydrographic office has materially improved conditions of navigation on the Lakes.

TIIE BUREIU OF NAIIGATION.

When a ship of the Nary has been completed as to her construc. tion and equipment so that she needs the services of officers and men to put her into condition for going to sea, this fact is certified to the Bureau of Navigation, which will subsequently control her movements and operation except at such times as she may be returned to a navy yard for repairs and refitting. The duties of the Bureau of Naviation comprise the promulgation, record, and enforcement of the orders of the Secretary to the fleet and to the officers of the Navy. As a matter of fact, most of these orders are prepared by the Burean of Navigation in accorlance with seneral directions issued by the Secretary of Nay to the Chief of the Bureau of Navigation. The latter officer, who is selected by the President upon recommendation of the Secretary of the Nay from the higher officers of the line, is the military adviser of the Secretary and his chief of staff, although no provision is marle by law for the latter office. An advisory board of officers of the Nay has been established by regulation under the title of the General Board which develops plans for the improvement of the efficiency of the service, and for the manœuvres and practice cruises intended to keep the Nay on a war footing. The General Board exercises certain of the functions of a General Staff, but has no standing except in the naval regulations, never having been recognized by law. Jt takes up a larese variety of questions, such as those relating to types of ships, preparing plans for the conduct of campaigns in event of war, improvements in ordnance and in the equipment of ressels, but its capacity is wholly an advisory one, and it has no power of direction so that its measures may or may not be put into effect as they are approved by the Secretary. For this reason the Secretary of the Navy has a closer connection and a greater actual power with reference to the details of the naval aciministration than is the fact with regard to the Secretary of War, who is almost wholly dependent in these lines upon the General Staff. The Chief of the Bureau of Navigation in his 
capacity as Chief of Staff to the Secretary of the Navy may either approve and recommend the findings of the General Board or may suggest to the Secretary his own personal views on a given question. The Secretary himself has the option of approving or rejecting, or modifying the suggestions made to him by the General Board or by the Board on Construction. The latter is composed of the Chiefs of the Technical Bureaus, who arrange definitely for the characteristics of new naval vessels and the details of their construction. The details of approved plans for administration of ships and men are carried out by the Bureau of Navigation, which prepares the necessary orciers therennder. It advises the Secretary as to the recessary transfers and assignments of officers and enlisted men and has general control under his direction of all details relative to the personnel. It directs the enlistment of men and their transportation and transfer from one point to another, has supervision over the course of education carried on at the naval academy for the preparation of line officers for their duties with the fleets and also has charge of the apprentice schools which educate landsmen to perform naval duties. In addition to the cruising ships of the Navy the Bureau of Navigation has under its charge all rendezvous or receiving ships, stationed at navy yards, keeps the record of the services of squadrons, ships and men and officers, directs the course of naval discipline, and conducts all operations necessary to secure the necessary inspections, including those to determine whether ships built by contract have been finished in a satisfactory manner, and what repairs, if any, are needed by ships upon the completion of their cruises. It is the particular bureau of the line of the Navy and the Chief of the Bureau, although not the ranking officer, is regarded, because of his power over transfers and assignments, as the chief of all officers having sea-going commands, even though the latter may be his superiors in point of position on the Navy list.

For the purpose of determining the relative importance of commands vessels of the Navy are divided into the following classes: Vessels of the first class are men of war of 8.000 tons displacement and upwards; second rate men of war of 4,000 tons and under 8,000 tons, and converted and anxiliary vessels of 6,000 tons and upward, with the exception of certain special classes of vessels, such 
as colliers, repair ships, distilling ships and hospital ships. Men of war of from $\mathrm{I}, 000$ to 4,000 tons, converted and auxiliary vessels from I,000 to 6,000 tons, and the special vessels previously indicaied constitute the third rate and all vessels except torpedo craft, tugs, sailing ships and receiving ships constitute the fourth rate. The command of torpeclo craft constitutes a separate branch of the service, while turs are under the command of warrant officers usually, and sailing ships are only now used for instruction purposes. Receiving ships constitlite the shore command of Captains, but are not included within the list of rated commands.

For the time being and during the life of the present incumbent, the ranking officer of the Navy is the Admiral, a position revived for Admiral Dewey. It is a life position with a compensation of $\$$ I 3.500 annually and the highest allowances of any officer of the Navy, and the Admiral may command a fleet or assume such other duty as he may care to undertake. The present Admiral of the Navy places his ripe experience and great abilities as a naval officer at the disposal of the General Board, of which he is the president, and is actively engaged in stulying, with the utmost interest, the developments of modern naval science and constitutes not only the strongest factor in the General Board but its real reason for being at the present time. With marked tact, he is able to impress upon the entire Navy a most salutary and commendable professional spirit, without in any way interfering with the duties of those who are directly concerned with the administrative work of the fleet. Admiral Dewey' has frequently taken command of the fleet in its practice manenvers and the high point of development which has been reached in personnel and in fleet organization is largely due to his personal effort during the time he has occupied the position as Admiral of the Navy. The Admiral of the Nary ranks with a full General of the Army, the latter a position which has not been in effect for many years. The next lower rank in the Nary would be that of Vice-Admiral, corresponding to the rank of Licutenant-General in the Army. Neither of these ranks are now in effect.

The rank now in effect next below that of Admiral in the Navy is that of Rear Admiral, of which the senior nine rank with Major General in the Army. The second nine or Junior Rear Admirals 
in the Navy, a grade created after the abolition of the old grade of Commodore, rank with Brigadier Generals in the Army. The command of a Rear Admiral depends to a considerable degree upon his place in the Navy list. He may command a fleet, a squadron or a naval station. Certain of the Rear Admirals who were engineer officers at the time of the amalgamation of the Engineer Corps with the line are not eligible for command at sea, but must be assigned to shore duties.

Promotion in the Navy is strictly in accordance with seniority, there being no opportunity for selection in promotion, the only position which does not go by seniority being that of the Admiral of the Navy, which is created by the vote of Congress. There is, however, a considerable opportunity for selection in the matter of assignment to different commands, although the selection is directed and regulated to a very considerable degree by the seniority of the officers considered. Owing to the number of commands ashore, including the navy yards and stations, the naval home for superannuated sailors at Philadelphia, the Naval Observatory, and special assignments on boards and similar duties, opportunity is given for the disposition of those Rear Adnirals who are not specially fitted for commands at sea, so that the more available officers can be utilized. It was formerly the naval policy of the United States under different conditions with regard to international situations than those which now prevail to divide the Navy into small squadrons, each of which was assigned to a cruising station in a particular part of the world. Thus there was the North Atlantic Squadron, which manœuvered along the Atlantic seacoast of the United States and in the West Indies; the South Atlantic Squadron, which cruised along the east coast of South America and across to Africa; the Pacific, and, at times, the South Pacific Squadron, the Asiatic Squadron and the European Squadron. As now administered, however, the largest and newest ships of the Navy are amalgamated into one great battle fleet, while a squadron is maintained on the Pacific Coast and a service squadron of comparatively small vessels is stationed in the Philippines for the protection of the interests of the United States in the waters surrounding those islands and along the coasts of China and Japan. By reason of the concentration of the ships into one 
large fleet there are fewer independent commands at sea for Rear Admirals, and the organization of the battle fleet is made to consist of several subdivisions at the head of each of which is placed a Rear Admiral of the lower rank than the Rear Admiral in command of the fleet. The presence of the Admiral of the Navy at sea is denoted by the blue flag with four white stars arranged in a parallelogram which flies from the mainmast head. The vessel on which a Rear Admiral in chief command of the fleet is exercising his authority is denoted by flying a blue flag with two white stars arranged perpendicularly; the Rear Admirals next in rank and in command of a division of the flect display each a red flag with two white stars.

A long narrow pennant at the head of the mainmast indicates a commanding officer on board of less rank than a Rear Admiral, and when two or more vessels are together with no Rear Admiral present a blue pennant with a white star is flown at the aftermast of the office of the vessel of the senior officer. When an officer is in command of a navy yard or station he displays the flag appropriate to his rank, if a Rear Admiral; if below that grade the flag of the senior officer present.

As it is regarded of great importance that the small boats which carry officers from ship to ship shall bear an appropriate mark so that the honors provided by the regulation may be paid to them, the boats placed at the disposal of the Rear Admirals have the stars in gilt on the bow, and the boats of commanding officers have a gilt arrow on each sicle of the bow. To further differentiate the rank of officers a gilt lance head is carried on the end of the flagstaff of the Rear Admiral's boat, a gilt ball at the head of the flagstaff of a captain's boat, a gilt star on that of a commander and a flat truck for all other officers. In addition to these marks, officers when paying visits of ceremony fly the flag denoting their rank on the boat's flagstaff.

The following honors to be paid distinguished visitors on armed vessels of the United States are generalized and condensed from those prescribed for the Navy: 


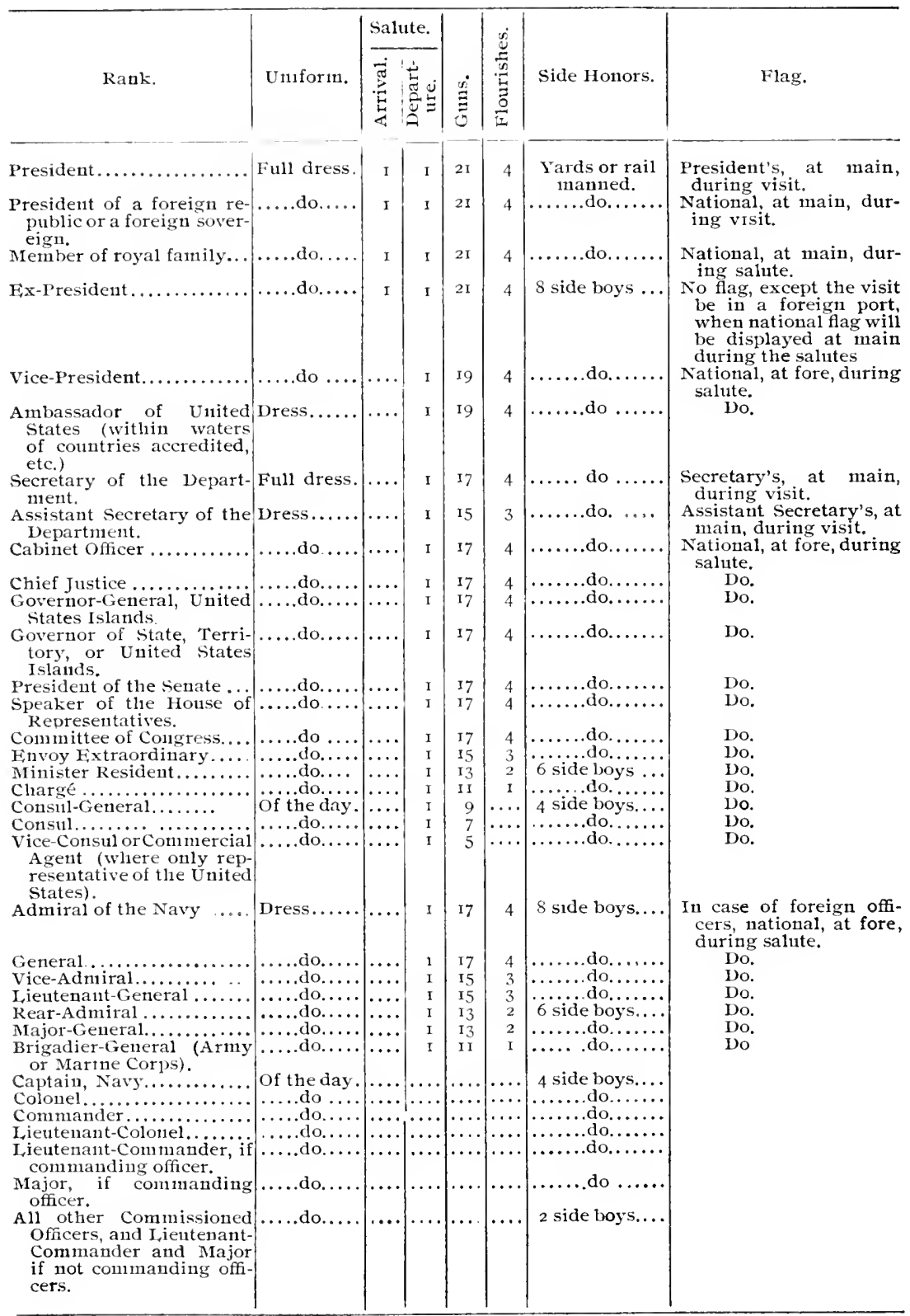


A Captain in the Nayy may command a division of a squadron, be chief-of-staff to a flag officer, command a naval station or a battleship or an armored cruiser, or, in rarer cases, a ship not rated. Certain persons on the list of Captain, however, who came over from the Engineering Corps can only be assigned to shore duty. The same is true of former engineer officers who have reached the grade of Commander. All other Commanders may command a division of a squadron, a naval station, a protected cruiser of the first rate, a ship of the second or third rate, or a ship not rated, be Chief of Staff to a flag officer or perform any shore duties that may be assigned to them.

The grades of Rear Admiral are denoted as flag ranks, and those of Captain and Commander are denoted as command ranks. It is not possible under present conditions to place a Captain or Commander in a subordinate position, except as the Chief of Staff of a flag officer. There is, therefore, some difficulty in assigning adequate commands to the officers on the lists in these grades, and propositions have been made to change the situation so that a Commander conld be assigned as executive officer to the new large ships, the duties of such a position being of a sufficiently important character and requiring so high a degree of skill that it is coming to be considered that these positions would not be below the dignity of a Commander.

While Lieutenant Conmanders and even Lieutenants (junior grade) and ensigns may be placed in command of vessels, the most important duties relative to the work of the Navy are performed by Captains and Commanders, and as commanding officers of the vessels of the Navy, they have not only great powers but great responsibilities as well. An officer having been ordered to the command of a vessel assembles the crew at the quarter deck and reads his orders to them, at the same time causing his flag to be hoisted. He is required to make a careful personal inspection of the condition of the vessel and crew, and if she is found in any way deficient or the discipline is lax, and the duties improperly performerl, he is required to see that the defects are remedied. The Captain is responsible for the efficiency of every part of the ship and has the authority to control and direct every person included in her complement whether attached 
to the line or to the staff. $\mathrm{He}$ is required to have personal cognizance of all the operations of the vessel by the medium of written reports which he must examine and approve, and if any accident occurs or the vessel fails to come up to the standard of efficiency the comnanding officer is liable to court-martial and to punishment unless he can show that the conditions complained of were caused by circumstances beyond his control. He is responsible for the discipline of the vessel, and is given power to punish offenses among the enlisted men by deprivation of the liberty of leaving the ship; by extra duties, or by imprisonment for not exceeding ten days, or in solitary confinement seven days, or solitary confinement on bread and water five days. He can also as a punishment reduce the rating of any petty officer who may have been appointed by himself, and can for the punishment of more serious offenses direct a summary court-martial consisting of officers of the vessel who have authority to inflict imprisonment of not more than sixty days, or thirty days' solitary confinement, or reduction from a rating not made by the commanding officer, or loss of pay of not exceeding three months. The latter infliction must, however, be approved by the Secretary of the Navy, to whom the findings of the summary court-martial are sent. In case of a sentence of imprisonment only, however, the finding of the court is not certified to the department. The commanding officer may discipline the officers under his charge by reprimand or by placing them under arrest and confinement in their room for a period not exceeding ten days. He may if he deems it advisable prefer charges against an officer, which will result in a trial by general court-martial. He has the option, however, unless the officer himself demands further proceedings to restore the officer to duty, the detention in his room amounting to a punishment, and all such detention and reprimands which may be officially administered must be reported to the department.

The superior military courts of the Navy consist of courts of inquiry and general courts martial. The latter are applicable to officers and enlisted men alike, and the men come under their jurisdiction upon complaint of commanding and other officers addressed to the senior officer present and transmitted either to the Secretary of the Navy or to the Commander in Chief of the foreign station. 
General courts martial are ordered for trial of cases of alleged desertion, insubordination and the various criminal offenses. They can only be directed in cases where the offense was committed in the United States or in home waters by order of the Secretary of the Navy. A Commander in Chief of a foreign station has, however, juriscliction to order a court martial for an offense committed on his station, and to review its fundings, merely advising the Department of the result. In all other cases, the court martial is directed by the Secretary of the Navy, and the results and the funding reviewed by him. The punishment to be awarded by courts martial and the final approval by the President in capital cases are subject to similar conditions and limitations as the army courts.

The foresoing relates especially to courts martial of enlisted men. In cases of alleged offenses of officers, and especially with relation to such cases as have to do with neglect of duty, or wrongful performance of the same, especially when the circumstances are not clearly to be stated, a preliminary investigation is generally directed. A board of officers, known as a court of inquiry, conducts a full hearing of the testimony and recommends what action, if any, shall be taken by the Secretary of the Nary. If the court of inquiry recommends a court martial, such a body is thereupon instituted by the Secretary of the Navy, to be composed of officers superior in rank to the accused officer.

The punishment imposed upon enlisted men consists generally of imprisomment in one of the naval prisons, of loss of pay, of dishonorable discharge from the service, or of all three. Commissioned officers are punished by the Secretary of the Nay, in accordance with the findings of the court martial, by suspension from duty for a given time, or by loss of rank, by being reduced one or more numbers on the Nary list, and in serious cases, by dismissal from the service. The latter sentence requires the approval of the President, before it can be carried into effect. General courts martial are composed of officers of appropriate rank, who may be specially detailed for the service, and at the larger shore stations there are generally one or more officers detailed for considerable lengths of time for court martial duty only, and who have general 
supervision of the matters relating to the court, additional members being supplied from the number of officers who are performing other duties at that particular point.

The commanding officer of a vessel which is detached from other vessels of a navy and serving on a foreign station, has exceptional responsibilities and duties. He must, especially, take care at times when war may be impending that he shall not be found in such a position by hostile forces that he will be unable to make a resistance against a not overwhelming force. He is also required to take heed of requests which may come from diplomatic and constular officers for the presence of his vessel as a moral force in cases of threatened injury to citizens of the United States or as a means of armed intervention, should such injury be actually attempted. He is also required to consider the complaints of merchant vessels, should sucli come to his attention, and of merchant seamen at points where there is no consular officer, and he is empowered to redress grievances relative to merchant seamen's food and usage which he may find to be well founded. He has the responsibility of seeing that his men are well cared for and that the conditions on board his vessel are such as will preserve their health, and that the food is arlequate and properly prepared. He has, in foreign ports, the responsibility of directing the purchase of such articles for the ship's use as may be requisite for her continuance in service, and is required to have direct personal supervision over the keys to the magazine and to the wrenches which open the valves by which the vessel may be flooded. Although he is given assistance in navigating the vessel, he is not thereby relieved of the responsibility for the seamanship shown in her management or for her safe arrival at her destination. He is, moreover, expected to be an accomplished diplomatist, to be familiar with international law and to be able to conduct delicate negotiations. He is supposed to be a polished and affable gentleman and to be able to entertain fittingly the officers of foreign vessels, and of foreign nations who may visit his ship. No entertainment fund is provided for the commanding officers of American vessels, as is the fact with certain foreign officers who are allowed table money in addition to their own pay, and any entertaining that a commanding officer of a vessel 
does, is at his own expense. The only exception to this rule is that occasionally, upon the participation of vessels of the Navy in celebrations at home or abroad, a small allotment is made from the contingent fund of the navy departnent to defray the cost of entertaimment. On all other occasions, such entertainment as may be offered is paid for by the captain of the vessel and the officers, and their only return is satisfaction in keeping up the credit of the service and such return entertainment as may be offered by foreign officials.

The grade of Lientenant Commander is the highest of the subordinate officers of the Navy, and such an officer may command a ship of the third or fourth rate, a torpedo boat, craft or assembly of torpedo craft, or niay, in a ship commanded by an officer of a superior grade, serve as executive officer, navigator, ordnance officer, or senior engineer officer. This grade is the highest in Which a former offeer of the Engineer Corps can perform seagoing duties. Most of these officers lave, however, passed into higher gracles and the duties in connection with the command of the engine room are performed by line officers, who are graduates of the naval academy and win serve tours of duty as engineer offeers in rotation with other line duties.

The executive officer of the vessel is the representative of the Captain in all that relates to the administration of the ship. $\mathrm{He}$ is the senior officer below the Captain and attends to all the matters relating to the work of the ship, and to the daily routine, and is especially charsed with seeing that the ship is well kept, and that the daily work is performed in accordance with the instructions of the commanding officer. The excutive officer of a naval vessel takes charce of the vessel when she is getting under way and when coming to an anchor, and issues the orders for these manœuvres under the direction of the commanding officer. If, however, a navigating officer is attached to the ship, the executive officer has no further responsibility with regard to the ship's movement and does not stand a regular watch. On the smaller slips, the executive officer is also generally the navigating officer and usually takes a certain portion of the watches, generally those in which he will have to be on leck to superintend the work of the crew. An 
executive officer is, however, supposed to be on duty and ready for call at all times while he is on board his ship.

The navigating officer has charge of all the instruments and charts pertaining to the navigation of the ship, and is on duty at all times while the ship is under way, for the purpose of directing her course. He is required to take the observations necessary to determine the ship's position on the ocean, and is relieved of his responsibilities only when the ship is in charge of a pilot. He has general oversight of the compasses, performs daily winding of the chronometers, and is responsible for any errors which he may make which may cause undue delay, or injury to or loss of the ship. While the commanding officer of a vessel is generally responsible, if it appears that an accident was due to the negligence or fault of the navigating officer, against which the commanding officer could not provicle, the subordinate officer alone may be held responsible.

The Ordnance officer has charge of the batteries of the ship and of her torpedo equipment, if any, together with the custody of the magazine, and the responsibility of seeing that the powder supply is kept at all times under such conditions that no accident may happen. He superintends the instruction of the crew in the use of the guns and in target practice, and in addition to these duties, is frequently expected to stand a reguiar watch as officer of the deck, when his turn comes. The officers named are assigned on board ships in the order of their seniority, that is, the executive officer is the senior officer or board, and is at the head of the wardroom and is expected to maintain order and discipline among the other officers at all times, as well as to set them a good example by his own conduct. The navigating officer is next in rank, followed by the ordnance officer.

Officers of subordinate rank are assigned to the vessel according to her size, and serve as deck officers and officers of divisions. An officer is also assigned to represent each of the department bureaus on the ship, receiving the communications from the particular bureau and making up the reports required. Officers of the deck have charge of the vessel when she is under way, taking directions as to the course, etc., from the navigating officer, and are 
assigned in turn to watches of four hours each, covering the twentyfour hours of the day, a change lueing made each day, so that an officer will not receive the assignment of the same hours, by means of half watches or dog watches, which come four and cight oclock in the afternoon. The watches begin at eight p. m., and the first watch is from that time until midnight, the mid-watch is from midnight to four in the morning, the nuorning watch from four until eight o'clock, the forenoon watch from eight o'clock until noon, the afternoon watch from noon until four o'clock, and the first dog watch from four to six, and the second dog watch from six until eight. The officer of the deck is expected to see that the orders given by the executive officer as to the work of the ship, which have becn written in appropriate order books, are carried out, that a sharp lookout is kept and that the wheel is properly manned, and the various seamen and messengers assigned to different posts are performing their dutics. He is required to keep a record in the log of all that transpires during his watch, and is responsible for any acciclent or casualty that may occur by reason of his negligence while he is on duty. When the ship is in port, officers of the deck do a full day's duty, turn about, and it frequently happens that an officer who has done a tour of cluty the night before has to assume the charge of the deck for the entire next day, owing to the arrival of the ship $i_{1}$ port within a very few hours after he lias come off watch.

The next officer on board a vessel to an ordnance officer in rank, is the senior engineer officer, who has charge of the engine-room and all that pertains to the machinery and the propulsion of the vessel. Formerly an engineer officer, especially on the smaller vessels, was required to take his turn of deck duty as well, so that the engineroom superintendence was largely left to the warrant officers. Under recent and more stringent regulations, engineer officers are released from all deck duties except the command of their division at drill, and while the senior engineer officer is not required to stand a watch, his juniors must take turn, watch and watch, as is the case with deck officers, and the senior officer is subject to call at all times in the performance of his duties.

Lieutenant commanders are never assigned to duty as deck off- 
cers, pure and simple, without a more important charge. The deck officers are either Lieutenants, Lieutenants (Junior grade) or ensigns. Lieutenants may command a ship of the fourth rate, a torpedo boat destroyer, a torpedo craft or an assemblage of torpedo craft, a tug, or a tender, or a ship not rated. Licutenants may also serve as executive officers, navigators, ordnance officers, senior engineer officers or watch officer, deck or engine-room, or a Lieutenant may be flag Lieutenant to a flag officer, or secretary to the Admiral while on sea service. A Lieutenant (Junior grade) may command a torpedo boat, tug, tender or ship not rated, or may be assigned to one of the officers of a ship before mentioned. An Ensign may perform similar duties to those indicated for a Lieutenant (Junior grade), and a midshipman may also be assigned as a watch officer, either on deck or in the engine-room. As the object of a midshipman's cruise at sea, however, is to complete his training in seamanship, midshipmen are generally assigned as junior watch officers on the large ships, standing watch with an officer of higher grade in order that they may secure the requisite experience. During the years following the Spanish-American War, however, owing to the scarcity of the officers in the Navy, midshipmen performed practically the same duties as ensigns, although without any increase of pay.

\section{DISTINCTIVE NAVAI, MARKS.}

Every grade in the Navy, as well as the distinction between the different corps, is marked by devices which show at a glance the exact status of the officer. The line of the Navy is indicated by a gold star worn on the sleeve and above the lace which denotes the exact rank of the officer. Officers of the staff corps are indicated by bands of colored cloth around the sleeves filling the intervals between the gold lace stripes. The distinctive band in case of the medical officers is of dark maroon velvet; pay officers, white cloth; professors of mathematics, olive green cloth; naval constructors, dark violet cloth; and civil engineers, light biue velvet. Chaplains wear stripes of lustrous black braid of the same number and disposition as the gold lace for line officers of the same rank.

Flag officers are denoted by broad stripes of gold lace, the Admiral of the navy having two stripes of two inch lace, with one 
stripe of one inch lace between. A Rear Admiral has one stripe of two inch lace and one stripe of half inch lace set above it. A Captain has four stripes of haif inch lace; a Commander, three stripes of half inch lace; Lieutenant Commaniler, two stripes of half inch lace, with one stripe of quarter inch lace between the stripes; a Lientenant, two stripes of half inch lace; Licutenant, Junior grade, one stripe of half inch lace with one stripe of quarter inch lace; and an cnsign, one strip of half inch lace. In addition to the foregoing designations, collar, shoulder straps and epaulet devices are worn, the distinguishing levice of the Admiral being four silver stars; of a Rear Adniral, two silver stars; a Captain, a silver sprear eagle; a Commander, a silver oak leaf; a Lientenant Commander, a gold oak leaf; a Lieutenant, two silver bars; a Lientenant, Junior grade, one silver bar; and an ensign, a silver foul anclior. The corps devices are as follows, and are substituted for the foul anchors which are worn by line officers added to the foregoing special devices: Medical officers, a spray of oak leaves in gold with an acorn leaf in silver; pay officers, a silver oak sprig; professors of mathematics, one silver nak leaf and an acorn; Naval Constructors, a gold sprig of two live oak leaves and an acom; Civil Engineers, two crossed silver sprigs, each composed of two live oak leaves and an acorn.

With relation to officers of the army, a Captain in the navy ranks with a Colonel; a commander, with a Lieutenant Colonel; a Lieu. tenant Commander, with a Major; a Lieutenant, with a Captain; Lieutenant (Junior grade), with a First Lieutenant, and Ensign with Second Lieutenant. When a question arises as to the precedence of an officer of the army, an officer of the nayy or of the marine corps, on accomnt of the fact that two officers in different arms of the service have the same date of appointment and commission, the officer who has servel the longer time in the service of the United States, whether continuously or at different periods, has precerlence.

Entrance to the line of the navy is secured through appointment to and graduation from the naval academy at Annapolis. Candidates for admission to the naval academy must be not less than sixteen years or more than twenty years of age, and nust be nominated by 
a member of Congress or the President. Each member of Congress is allowed to keep two midshipmen continuously at the naval academy, thus giving two midshipmen from each representative district and four from the State at large. In addition to these, the President is entitled to appoint two from the District of Columbia, and five annually at large, making twenty at large during his term of office. Midshipmen receive pay of five hundred dollars a year, and their rations, and are to provide themselves with an outfit on entering, which costs abont one hundred dollars. Their clothing and books are charged against their pay account, so that their pay during their period of education at Annapolis amounts to little more than spending money, although an effort is made to save enough for each midshipman to provide him with an outfit when he goes to sea after graduation. After a four years' schooling at Annapolis, in mathematics, steam engineering, seamanship, modern languages and other branches, midshipmen are assigned to sea duty for two years, at the expiration of which time they are commissioned as Ensigns in the navy after passing a final examination.

\section{THE WARRANT OFFICERS.}

A consicerable portion of the actual technical work with regard to the affairs of vessels of the navy, and in connection with navy yards and other shore stations, is performed by the warrant officers. These are boatswains, gumners, carpenters, sailmakers and warrant nachinists. The usual requirements for the appointment of a warrant officer are, that he shall be not more than thirty-five years of are, shall have served at least seven years in the navy, during a portion of which service he must have been a chief petty officer; that he must have had at least $8_{5}$ per cent. in his marks for efficiency and conduct, during his service as an enlisted man, and that he must have letters of recommendation from former commanding officers. If these preliminary requirements are complied with, an enlisted man, upon application, may be designated to take the examination for the warrant which he desires. If successful in passing the examination, he is given an acting warrant for one year, at the expiration of which time, if his service is satisfactory, he is warranted; and certain warrant officers, namely, boatswains, gun- 
ners, carpenters and sailmaliers, after six years' service, receive commissions styling them clief boatswains, chicf grumers, chicf carpenters and chief sailmakers, respectively, under which titles they rank with, but after, Ensigns, and are regarled in all respects as commissioned officers. Sucin commissions do not, however, give them a right to command, but they may be and are assigned to duty as deck officers, to perform similar duties to those performed by ensigns, and they may be assigned to the command of tugs and other small craft.

Boatswains are the chief seamen on board the ressels of the navy and acinally dircet the operations of the deck force. They have clarere of all the sear relating to the anchoring of the ship and to the means of controlling her movements while under way, except with relation to the propulsive machinery, and have charge of all boats and fixtures about the deck, the apparatus relating to the navigation of the ship, and have the superintendence of the cleaning and painting of the ship's hull and the permanent fixtures thereto.

The boatswain is the special assistant of the executive officer and the clief means by which the orders of the latter are put into active execution. He is charged with secing that the crew is properly mustered and that the watches are set. lookonts posted, and that the rumnine lights and other safeguards to navigation are properly cared for.

The warrant gunners are the assistants to the ordnance officers and perform all the duties necessary to the care of the ordnance and magazines, torether with the contents of the latter. and act as chief instructors to the nembers of the crew in the use of the suns and small arms of the vessel.

The carpenter has cliarge of all repairs in wood and metals which may be marie on hoard the ressel, and he has also especial charge of the pumps and attachments and of the fire extinguishing apparatus. He is also placed in special charge of the ports or openings in the sides of the vessel, and is required to see that they are kept water tight and that they are closed when the vessel goes to sea. As many of these ports are near the water line, a remissness in 
duty in seeing that these ports are properly closed might result in the sinking of the ressel.

Sailmakers are charged with the supervision of the work upon such sails and awnings as the ressel may be equipped with, but as little canvas work is now done with regard to vessels of war, except as to awnings, hanmocks, and similar small articles, the position of warrant sailmaker is seldom filled.

The warrant officers for which there are no commissioned grades are those of pharmacist and warrant machinist. The pharmacist is the assistant to the medical officer and has charge of the medical suppiies and of the nursing of the sick of the crews. He is in charge of the dispensary and issues medicines and sick supplies under the direction of the medical officer.

Warrant machinists are the assistants of the engineering officers, and perform most of the practical duties relating to marine engineering on the vessels of war, besides immediately directing the operation of the crews of the engine and fire rocms. Appointments as warrant machinists are made from enlisted men of the navy who are machinists by trade, or from machinists in civil life after an examination. Candidates from civil life must be more than thirty years of age, and must have had experience in the machine shop and in the encine-room of a seagoing vessel.

Tle derice of a chief boatswain is two crossed anchors; of a chief gunner, a bursting shell; of a chief carpenter, a try square, and of a chief saimaker, a lozenge. That of a warrant machinist is a propeller, and of a pharmacist, the Geneva cross.

There is an additional grade in the navy, that of Mate, which is maintained for the purpose of giving recognition to enlisted men of good service who cannot comply with the requirements for warrant officers. They have similar consideration and privileges to warrant officers, but cannot be commissioned, although it has been held that they may be promoted on the retired list to the next higher grade. This, however, could not occur on the active list without special legislation. Mates perform general duties in the line of seamanship and gunnery, especially the former, and their distinctive device is a pair of binocular glasses. 
Enlisted men of the navy include many branches and trades. In order to be enlisted the applicant must be at least seventeen years of age, and must be in good physical condition. Enlistment without the consent of parents or guardians may be made under the terms of the statute at the age of eighteen years. Formerly the minimum age for enlistment was fourteen years, and a system of apprentice training was carried on. It was found, however, that boys of that age were too light when they had finished their training for the heavy work of a modern vessel of war, and the present age limit was established. The enlisted men of the navy are divided into the seaman branch, the artificer branch, the special branch, the commissary branch and the messman branch. The lowest rating in the seaman branch is that of apprentice seaman, and a landsman who has had no experience is assigned to one of the training stations, either at Norfolk, Va., Newport, R. I., or San Francisco, Cal., where a course of training in barracks or receiving ships is given to fit the new recruit for life on board a vessel. A naval training station is also being constructed on the Great Lakes, near Chicago, Ill., for the purpose of training recruits, large numbers of whom now come from the far Western States. At the expiration of the six months' training recruits are rated as ordinary seamen. from which rating they are advanced to be scamen, and as soon as they are qualified may be rated as quartermasters, gunner's mates, coxswains or masters at arms, each of these third class. The positions named are the lowest classes of petty officers, the quartermasters performing the duty of steering the vessel, attending to the signals, supervising lookouts and similar duties with regard to navigation; gunner's mates being the assistants to the gumners in the care of the batteries of the ships; coxswains are placed in charge of the small boats of the vessel, and masters at arms are the police of the ship who preserve order and see that men do not hide in obscure corners of the vessel and thus avoid their duties. In the next higher class of the petty officers, the petty officers' second class, quartermasters may be promoted to be boatswain's mates, who are the assistants of the boatswain, or to be second-class quartermasters, and gumner's mates may be promoted to gunner's mates, second class, while gun captains appointed by selection rank as second-class petty officers. 
In the first class of petty officers there are similar designations with the addition of the position of turret captain, and the highest class of petty officers is that of the chicf petty officers in the branches before named, the chief petty officers being, however, a distinct class from all other ratings of petty officers whether first, second or third class, and wear a distinctive dress of a sack coat and cap instead of the blouse and round lat of the inferior ratings, and are permitted to have a separate sleeping quarters and mess from the remainder of the crew. The ratings in the artificer branch include machinist's mates, electricians, carpenters, water tenders, who are men who attend to the water feed of the boilers and have general supervision of the fire rooms, boiler makers, coppersmiths, blacksmiths, plumbers and fitters, sailmaker's mates, carpenter's mates, ship fitters, painters, printers, oilers, firemen, shipwrights and coal passers. The special branch includes ycomen, hospital stewards, bandmasters, hospital apprentices, musicians and buglers. The names are sufficiently self-descriptive except that of yeomen, which is the name of the enlisted clerical force of the vessel. The yeomen keep the accounts for the various officers, conduct correspondence and lo the clerical work with regard to the reports required to be made. A special school is maintained at Norfolk for their instruction and they are expected to have some lnowledge of arithmetic as well as of shorthand and typewriting. The commissary branch includes the chief commissary steward, who purchases the supplies of fresh food for the use of the vessel, commissary stewards, ship's cooks, landsmen, and bakers. This branch performs all the work of preparing the food for the enlisted men.

The preparation of food for the officers, as well as the serving of the food to the men is performed by the messmen's branch, which includes stewards, cooks, and mess attendants in the various grades, from stewards to the Commanders in Chief to mess attendants, third class.

The system of distinguishing marks for enlisted men of the navy includes devices for the identification of the man with relation to all the details of his position and assignment on board ship. All except the messman branch wear the watch mark denoting which of the two chief divisions of the ship's crew they belong 
to. For the purpose of alternating in the service of the ship, all the men are divided into either the port or starboard watch. The distinguishing mark denoting the watch to which a man belongs is a strip of braid worn on the shoulder seam of the sleeve and extending entirely around the arm. This is in white for the blue overshirt or blouse, and in blue on the jumper of the white suits worn. The men of the starboard watch wear the mark on the right sleeve and those of the port watch on the left slecve. The mark in cases of men of the engincer force, the firemen and coal passers, is in red on both the blue and white clothes. The watch mark is not worn by mess attendants, as the latter do not stand watch. Men who hold continuous service wear service stripes on the left sleeve of scarlet cloth, a stripe for each term of enlistment, and each petty officer holding permancnt rating and who has had three continuous good conduct badges is allowed to wear gold lace instead of the scarlet stripe. The watch to which a petty officer belongs is lenoted by the location of his chevron, this being on the left slecve for officers of the port watch and on the right slecve for officers of the starboard watch. The chevron consists of stripes of scarlet cloth and petty officers of the third class are denoted by a chevron of one stripe, those of the second class by a chevron of two stripes, first class by three, and the Chief Petty Officer by three stripes and an arch. In connection with the chevron a rating badge is worn consisting of a spread eagle above the specialty mark of the rating to which the petty officer is attached. The eagle and specialty mark are cmbroidered in white on blue clothing, and in blue on white clothing, except that in the case of hospital stewards and hospital apprentices, the marks are in red for both uniforms.

The marks assigned to the petty officers are as follows: For a Master at Arms, a five pointed star; Boatswain, crossed anchors; Quartemaster, a steering wheel; blacksmiths and ship fitters, crossed hammers: sail maker's mates, a grommet, which is a large eyelet sewed to a sail for the purpose of attaching the ropes which manage the sail; for printers, an open book; carpenter's mates, plumbers and fitters and painters, crossed axes; turret captain, a device which resembles a turret; gumner's mates, crossed cannon; chief yeomen, crossed keys; yeomen, first and second class, crossed 
pens; electricians, a globe; machinist's mates, a three bladed propeller; hospital stewards and apprentices, a Geneva cross; musicians, a lyre; and commissary stewards, two keys and a pen.

In addition there are marks for ratings which are not those of a petty officer. Thus a gun captain, who is the leading man of a gun crew, and directs the operation of loading and preliminary training of the gun, wears a device representing a cannon placed horizontally on the arm. The gun pointer, who is the man who sights and fires the gun and who is chosen for his ability as a marksman without consideration of his ability in other directions, wears a circle within which are the crossed wires such as appear on the lens of the telescopic gun sights. Gun pointers may be selected from any section of the crew and all members of the crew are encouraged to try for the rating, which carries with it additional pay. Certain qualities of keenness of eyesight as well as steadiness of nerve are required under modern conditions, as the gun pointer must keep his eye at all times on the target, even while the gun is being loaded, and must have the resolution necessary to resist the natural tendency to start when the gun is fired. As the time of loading a gun, especially of the quick and rapid fire classes, is so small, if a gun pointer should hesitate in standing up to his sights while the gun is being loaded and fired it would interfere materially with the rapidity and accuracy of the practice.

A seaman gunner or man who has passed through the gunnery school and is especially qualified although not yet having received a rating, wears a badge bearing the device of a bursting shell. Graduates of the old apprentice training system wear a figure of eightknot on the front of their blouses and on the sleeves of their overcoat. Coxswains, who are in effect boatswain's mates, third class, wear the crossed anchors of a boatswain's mate with a single striped chevron.

THE BUREAU OF MEDICINE AND SURGERY.

The Bureau of Medicine and Surgery is the controlling staff bureau of the medical corps of the navy. It has charge of all matters relating to laboratories, naval hospitals and dispensaries. Assignments of members of the medical corps are made upon its recommendation, 
and it has charge of matters relating to the enlistment and promotion of hospital apprentices, hospital stewards and pharmacists, conducting the professional examination in each case and recommending the action to be taken into the Burcau of Navigation, besides making recommendations as to the assignment and transfer of enlisted men of the liospital corps. The Chief of the Bureau of Medicine and Surgery is selected by the President from the officers of the medical corps of not below the grade of medical inspector, and has general charge and supervision of all matters relating to the health and sanitation of the navy and of the treatment of the officers and men in cases of sickness and injury. The active list of the medical corps consists of fifteen medical directors, fifteen medical inspectors, eighty-five surgeons, two hundred and thirty past assistants and assistant surgeons, while the President has power to appoint not to exceed twenty-five acting assistant surgeons who have the rank and compensation of assistant surgeons. It is sought to use the appointment of an acting assistant surgeon as a means for building up the list of regular assistant surgeons, as a difficulty has been found in securing available candidates for this position. The appointments of acting assistant surgeons are understood to be only for a period of probation and until such officers have had an opportunity to prepare for and pass an examination. If they are unable to pass the examination for a regular appointment, their service will be terminated and other acting assistant surgeons appointed in their stead. A candidate for appointment as assistant surgeon must be not less than twenty-one years nor more than thirty years of age, and his physical, moral and mental qualifications are decided upon by a board of medical officers. The approval of such a board is necessary before the Secretary of the Navy can appoint any person to be an assistant surgeon in the navy. After three years' service, assistant surgeons are promoted to be past assistant surgeons, and after three years' further service, past assistant surgeons are promoted to be surgeons. Both of these promotions are subject to such examination as the Secretary of the Navy shall prescribe. Medical directors have the rank of captain; medical inspectors, the rank of commander; surgeons, the rank of lieutenant commander; 
past assistant surgeons, the rank of lieutenant; and assistant surgeons, the rank of lieutenant (junior grade). The officer assigned to assist the chief of the bureau of medicine and surgery is entitled to the highest pay of his grade and the President may select an experienced surgeon for each fleet of the navy, known as the surgeon of the fleet, and who has general duties of inspection of the work of other medical officers afloat and attached to the vessels of the fleet, and who holds consultation when necessary for the treatment of difficult cases. He has also general charge of the reports required of the medical officers of ressels.

The warrant and enlisted personnel under the medical corps consists of pharmacists who are warrant officers, but do not receive the chief warrant officer's commission, hospital stewards who correspond to chief petty officers, and who have general charge of sick bay and dispensary work when no pharmacist is assigned to the ship or station, and hospital apprentices, who perform the duties of nurses of sick attendants on ship-board and at the hospitals. The duties of the medical corps include the care of officers and men in cases of sickness and of injury and of the families of officers, beside provision for the care of the wounded in case of battle. This includes the designing of sick bays on board ship, and of packages for first aid treatment and means for conveying wounded men, as well as devices for the temporary treatment of the injured in battle, so that they need not be moved out of the protected areas of the armored spaces on board the ship into a central operating room until the action is over. Naval hospitals are maintained for the treatment of the sick of the navy at the various points along the Atlantic and $\mathrm{Pa}$ cific coasts, at Yokohama in Japan and in the Philippine Islands, and a tuberculosis sanitarium has just been established in the west for the treatment of patients from the navy who have previously been treated at the army sanitarium at Fort Bayard, New Mexico. Medical officers are also charged with the oversight of the sanitary condition of ships, and with the inspection of provisions, and upon complaint of a member of the personnel that provisions are spoiled, the inspection of the medical officer is required to determine whether the provisions shall be condemned and thrown overboard. 
THE BUREAU OF SUPPLIES AND ACCOUNTS.

The Bureau of Supplies and Accounts of the navy is charged with all matters relating to the provision of clothing and stubsistence to the enlisted men of the navy as well as the payment of all amounts due to officers and enlisted men, thus combining the functions of the Quartermaster, Subsistence and Pay departments of the army. In addition, the Pureau of Supplies and Accounts keeps the books for all of the burcaus of the navy, no accounts whatever being kept in any bureau, but all matters relating to payments being referred to the Bureau of Supplies and Accounts, which conducts the administrative audits for the navy department, on the expenditures of all the bureaus. This system was put into effect for the purpose of reducing the amount of work and of preventing duplication in bookkeeping, but it has the undesirable result that all of the computations of the different bureaus are made upon estimates or upon data which must be secured from the Bureau of Supplies and Accounts.

All payments for the navy of whatever nature are made by the navy pay officers, and contracts for materials and supplies are made by the contracting side of the same offices. In each place in which there is a navy yard, or at the nearest large city to a navy yard, there is stationed a pay officer who is charged with the duty of advertising for bicls and of making open market purchases to supply the needs of the different departments at the yards, as well as the provisions and equipment for the ships being made ready for sea not supplied from the manufactories of the Navy. The preliminary statement of a need is made by the officer in charge of a particular line of work and, except in the case of an emergency, is forwarded to Washington for approval by the bureau to which expenditure may refer. In case of emerencies, requisitions are made in advance of approval, but the approval must be secured from the bureau before the purchase becomes legal. Approved requisitions are sent to the purchasing paymaster who advertises for bids or makes a purchase in the open market, if the requisition comes within the regulations provided for open purchase, and upon receipt of bids, awards the contract and secures the delivery of the goods to the navy yard where they are passed upon by a board of inspecting officers and turned over 
to a general storekeeper to be issued to the officer who originally made the requisition.

The General Storekeeper of a navy yard is also an officer of the pay corps and is charged with the custody of all goods and materials, that is to say, the materials which are purchased for the purpose of being manufactured into goods to be used by the navy and the custody of such manufactured articles when completed by the shops of the yard. These are taken up on the storekeeper's books and are charged against the vessel to which issued, the whole constituting a complicated system, which is frequently evaded by the different yard departments in drawing from the General Storekeeper larger quantities of material than are needed for the moment, the balance being kept in store at the department shop or storehouse to be drawn upon for subsequent work.

In the vicinity of most of the yards there is stationed a third pay officer, the disbursing pay officer, who receives the bills for commodities purchased and sees that they are properly approved and conducts a preliminary administrative audit before paying them. In the larger yards there is also a yard paymaster who acts as the disbursing officer for the workmen and clerks employed in the yards, and for the pay of the officers and men detailed therein and not attached to vessels.

The seagoing duties of the members of the pay corps consists in the purchase of all articles of provision and supply when away from navy yards which are not furnished by requisition on the general storehouses of the navy and of the oversight of the issue of rations and the cooking for the men as well as the payment of all the pay and allowances of officers and men of the ship, and also of the various expenses incurred by the ship at other points than at navy yards and which must be paid in cash. The pay officer makes arrangements for all purchases of the ship of whatever nature and such other disbursements as may be allowed and which are undertaken on the order of the commanding officer. He is also charged with the clothing and small stores which are issued to the ship from the naval supply fund and in turn issued to the men as they may need them, the amounts being charged against the pay accounts and deducted therefrom at the end of the month. The pay officer is furnished with 
funds in money and credit to enable him to make the necessary payments and he is responsible for the ontgo of the same, any unwarranted payments being checked against his accounts so that he must make them good. He is allowed a certain percentage of wastage upon stores furnished to him for issue to the men, but if he fails to make the proper charges or fails to leduct any sums which may be certified to him as due the United States from officers or men, he is liable under his bond to make the amount good to the United States.

The pay officer also acts as a division officer aboard ship, having charge of the powder division or idlers, so-called, consisting of the musicians, messmen, and other non-combatants who are expected in a time of action to render such assistance as may be required in handling the ammunition, serving as a fre brigade and otherwise as auxiliary to the fighting force. The head of the Bureau of Supplies and Accounts is appointed by the President, by and with the advice and consent of the Senate, for a term of four years and must be selected from the pay officers of not below the grade of Pay Inspector. He has the title of Paymaster General of the Nayy. The pay corps of the navy consists of thirteen pay directors, fifteen pay inspectors, seventy-six paymasters and ninety-six past assistant paymasters and paymasters. No person can be appointed an assistant paymaster who is less than twenty-one or more than twenty-six years, or until his qualifications have been examined and approved by a boarci of pay officers. Pay Directors have the rank of Captain, Pay Inspectors of Commanders, Paymasters the rank of Lieutenant Commander, or Lieutenant, past Assistant Paymasters, Lieutenants or Lientenants (Junior grade), Assistant Paymasters, the rank of Lieutenant (Junior grade) or ensign. Officers having the relative ranks indicated by a double rank within the grade are promoted in their grade in accordance with the promotion of the officer of the line with whom they take rank. When the latter is passed into the higher grade, the officer of the staff ranking with him also passes into a higher rank, although he may retain his former title, while his pay, if it is under the new or army pay receives a corresponding increase. Pay officers of the fleet are designated by the President under similar circumstances and for corresponding duties of super- 
vision in the line of their profession as is the case with the surgeons of the fleet.

Pay Directors, Pay Inspectors and Paymasters must give bond in the sum of $\$ 25,000$, past Assistant P'aymasters in the sum of $\$ 15,000$ and Assistant Paymasters in the sum of $\$ 10,000$, and must give new bonds with sufficient securities whenever required to do so by the Secretary of the Navy. Paymasters on vessels having on board more than 175 persons, and on supply steamers, store vessels, and receiving ships, as well as at shore stations, are entitled to the services of a clerk who is a semi-military person appointed by the Secretary of the Navy, and entitled to wear a uniform and the oak sprig of the Pay Corps, but without any rank, and whose appointment can be terminated at any time. Appointments of suitable persons are generally made upon the recommendation of the pay officer under whom the clerk is to serve, but if for any reason the Secretary of the Navy deems it inadvisable to make the appointment the pay offcer must make another nomination. Pay officers are responsible for the acts of their clerks, and must make good any shortages which may occur by reason thereof. Pay officers are also assisted by pay yeomen or writers, and on board ship by the commissary steward, who is the chief petty officer directly in charge of the purchase of fresh provisions, of issuing rations and of the preparation of the food for the men, and who is at the head of the commissary branch.

The corps of Chaplains in the navy consists of twenty-four officers of whom the senior four have the rank of Captain, the next seven that of Commander, the next five that of Lieutenant Commander, and the remainder the rank of Lieutenant and Lieutenant Junior grade. The former basis of pay of Chaplains was a fixed sum of $\$ 2,800$ a year and the only addition was the allowance for the rank held by the Chaplain. This has been changed by recent legislation, so that a Chaplain, upon appointment, has the rank of Lieutenant Junior grade, and after seven years he will be promoted to be Lieutenant, and thereafter to be Lieutenant Commander, as vacancies occur in the higher grades. Chaplains on appointment must be between the years of twenty-one and thirty-five, and are appointed by the President, by and with the advice and consent of the Senate. The matter of denomination or even of regular ordination is not es- 
sential under the law, but it is the practice to require that a Chaplain shall be a regularly ordained minister and shall have the endorsement of some recognized ecclesiastical body. The only examination required is to determine the physical qualifications of the candidate. It is required by law that the Chaplain nust be permitted to conduct divine service in accordance with the form of the church of which he may be a nember. Chaplains are required to conduct divine service on Sunday when possible and to attend to the moral welfare of the men, also to make an annual report to the Secretary of the navy of the duties performed by then during the year.

Professors of mathematics who were originally teachers sent to sea to instruct the midshipmen on board vessels of the navy before the naval acadeny was established, are utilized as instructors at the naval academy, and also as scientific observers and computers at the naval observatory in Washington and on the Pacific Coast. They are twelve in number and the senior three have the rank of Captain, the next four that of Commander, and the remaining five that of Lieutenant Commander or Lieutenant. They are appointed by the President, but no person can be appointed until he has passed a successful physical examination and a professional examination conducted by a board of professors of mathenatics.

The Corps of Civil Engineers is under the direction of the Bureau of Yards and Docks, the Chief of the latter being now required by law to be a nember of the corps of Engineers. Formerly a line officer of the navy could be selected for this duty. The duties of the Bureau of Yards and Docks include all matters retating to the construction of wharves, slips, piers and buildings at all navy yards, and stations controlled by the navy, except such stations as may be entirely under the direction of a particular bureau, such as the naval proving grounds, the naval torpedo station, the naval war college and the naval acadeny. The Burean of Yards and Docks constructs the buildings for all other burcaus at navy yards after consulting with the heads of such bureaus as to the requirements. It is also charged with the repair and maintenance of buildings, the construction and maintenance of roads and streets within the navy yards, with the lighting of the yard, and, under the system about to be installed, with all matters relating to the light, heat and power of buildings, under 
whatever bureat the operations carried on within the buildings may be conducted. The Bureau of Yards and Docks also has charge of all apparatus for moving articles from one place to another within the navy yard, except within the buildings themselves, including derricks, cranes and trucks and wagons, provides the labor for cleaning the yards and builds all docks, but does not operate the docks after they are built. The Civil Engineers are the officers who direct the various operations under the charge of the bureau and do not at any time perform seagoing functions. They have such rank as the President may direct, and the corps, at the present time, consists of two officers with the rank of Captain, two with the rank of Commander, two with the rank of Lieutenant Commander, and twenty-three with the rank of Lientenant. In addition there are seven assistant Civil Engineers, six with the rank of Lieutenant (Junior grade), and one with the rank of Ensign. The comparatively small pay of Civil Engineers in the navy with relation to the amounts earned by persons of equal abilities in private life has made it difficult to fill vacancies in the corps of Civil Engineer. Frequent examinations have been held for the purpose and it has finally been determined to accept graduates of the naval academy who desire to enter the Civil Engineer Corps because seagoing life is distasteful to them or for other reasons. Such graduates are sent to a technical college for a post-graduate course in civil engincering and are then appointed assistant Civil Engineers.

\section{THE NAVY PAY.}

The question of army pay offers, as has been noted, no slight degree of complexity, but it is a simple and easily understood system when a comparison is made with the subject of navy pay. Prior to July I, I899, the pay of the navy was based on the table now known as the old navy pay, and which provided three scales of payment, namely, that for service at sea, that for service on shore, and that for time spent on leave or in waiting orders, together with arbitrary schedules for staff officers. Thus, for example, Rear Admirals received $\$ 6,000$ when they were at sea, $\$ 5,000$ when they were on shore duty, and $\$ 4,000$ per annum when they were on leave or waiting orders. A similar deduction, but not proportionately as large, was 
made in the pay of subordinate officers under the three sets of circumstances indicated, and officers below the grade of Commander received increases of stated amounts after each five years of service. This system was abolished with relation to certain officers of the navy by legislation, taking effect on the date above mentioned and the army pay was established as a basis for the pay of the navy, with the exception that it was provided that an officer on shore rluty should receive $I_{5}$ per cent. less than when he performed sea dutics. The old nay pay was established on the condition which was general in the navies of the world of a considerable number of officers in excess of the number required for manning the vessels of the navy. Therefore, when an officer was unable to secure sca duty he had to be content with shore duty or waiting orders, and his scale of pay under those circumstances was calculated on the idea that he was not rendering the service incident to his profession and that his pay was rather in the nature of a retaining fee than a compensation for professional duties performed. The new pay table, or army pay, did not, however, apply to all officers in the service, as any officer appointed prior to the date mentioned, whose pay under the old pay table would be in excess of what he would receive under the army pay table is entitled to receive old navy pay. By a provision of law passed in 1906 the difference of 15 per cent. between sea and shore pay was abolished, so that new officers and officers whose pay would not be increased by the old nary pay table, are paid in accordance with the following table of base pay, with the additions which are allowed by law. The chief of these adlitions is that of longerity pay, by which officers below the rank of Rear Admirals, second nine, receive ten per cent. additional of the pay of their grade for every five years of service; that is, ten per cent. after the first five years, twenty per cent. after the second five years, and until the increase has reached forty per cent., which is the maximum increase which can be received on longevity pay. It is further provided that a Captain in the navy shall not receive more than $\$ 4,500$ a year, and a Commander more than $\$ 4,000$ a year. This, of course, does not include the allowances which may accrue under the laws to the officers named. The base pay of officers of the navy plus the longevity pay, to which they may be entitled, is the pay proper. Upon this there is estimated 
a further increase of ten per cent. when such officers are detailed to shore duty beyond the limits of the United States. The increase of ten per cent. is not paid when officers are on duty in the waters of foreign countries, but only when they are on shore duty, and officers are entitled to the increase when on their way in merchant vessels to their posts of shore duty, outside the limits of the United States, but are not entitled to the increase for the time consumed in returning to the United States in a merchant vessel after they have been detached from their shore duties.

In addition to their pay officers of the navy on shore duty are allowed quarters, when practicable, in Government buildings, and when such allowance is not practicable, quarters are commuted at the rate of \$I 2 per room per month, that of the Admiral of the Navy being fixed by law at \$I 25 per month. In addition to this allowance, there is a monthly allowance for wood and for light which may be commuted in cash if not furnished by the Government or may be furnished in steam or furnace heat or in gas or electric lighting to the amounts which have been calculated in those commodities as being equivalent to the allowance in kind. In case officers receiving light and heat from governmental plants utilize more than the amount they are allowed, they are expected to make up the difference by payment to the disbursing officer of the station at which their shore duty is performed.

Officers of the line, medical and Pay Corps of the Navy, chaplains above the grade of lieutenant and chaplains appointed after July I, I906, and officers of the Marine Corps receive the following pay under the current legislation.

Rank.

Admiral $\$ 13.500$

Rear-admirals :

First nine

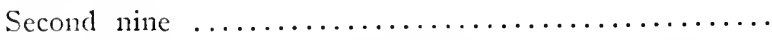

Chiefs of Bureaus and Brigadier-General Commandant of

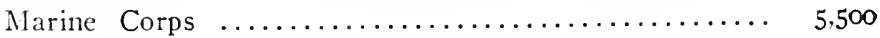

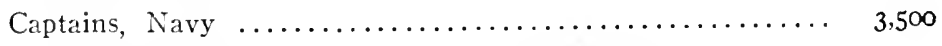

Judge-Advocate-General and colonels, Marine Corps, line

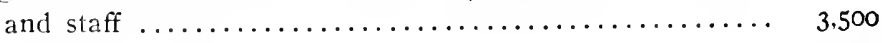

Commanders, Navy $\ldots \ldots \ldots \ldots \ldots \ldots \ldots \ldots \ldots \ldots, 3,000$ 
Rank.

Base pay:

Lieutenants-colonels, Marine Corps, line and staff....... 3,000

Lies:tenant-commanders, Navy ................. 2.500

Majors, Marine Corps, line and staff.............. 2,500

Licutenants, Navy ....................... 1,800

Captains, Marine Corps:

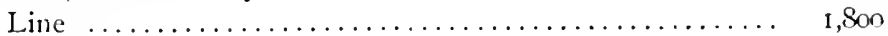

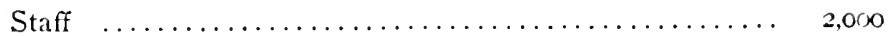

Lientenants (junior grade), Navy................. 1,500

First lientenants and leader of band, Marine Corps....... 1,500

Ensigns, Navy .........................., 1,400

Second lientenunts, Marine Corps, chief boatswains, chief gumners, chicf carpenters, and chicf sailmakers........ $1, \downarrow 00$

Officers appointed from civil life are credited with five years of service for the purpose of computing longevity pay on entering on their duties, this allowance being made to put them on an equal footing with officers educated at Annapolis, whose school time is computed in allowing longevity pay, so that the pay of an appointee from civil life on entrance is ten per cent. in addition to the foregoing.

The pay of other officers of the navy than those named and of persons paid as officers is given in the following table. In addition to the amounts named for Chaplains, officers of such corps who were or may be appointed after July I, 1906, or who were above the grade of Lieutenant on that date receive the pay and allowances of their rank as indicated by the present pay table, the latter class of Chaplains receiving the pay and allowances of a Lieutenant Commander. All officers paid under the following table are entitled to one ration a day, while at sea, except midshipmen, who are entitled to one ration a day under all circumstances. 


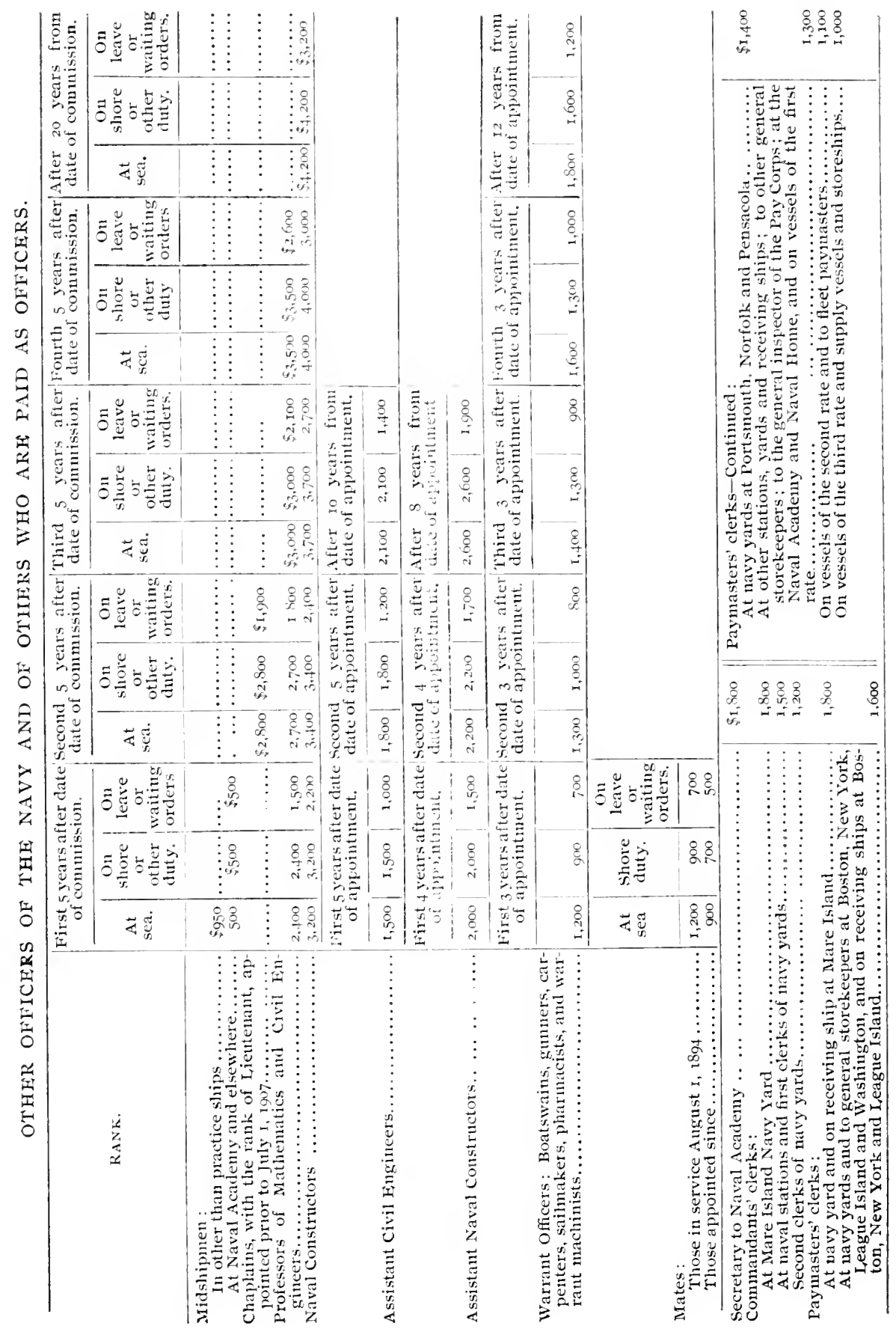


The following pay table is that of the culisted men of the navy, and to this should be adked the allowance of a ration a day, which, when enlisted men are on detached service, is commuted at the rate of $\$ 9$ per month, the cash value of the ration of the navy being fixed at thirty cents a day.

\section{CHEF PLTTY OFHICRS.}

Seaman Branch.

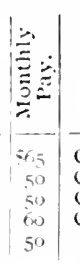

Artificer liranch.

Special Branch.

Chief Masters-at-Arms..... s.

Chief Boatswains Mates....

Chief commers datte.......

Chief Tutret Caphains.......

Chief Quartermasters........

Clief Machinists Mates. .... s

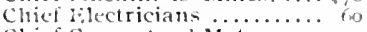

Chicf Carpenters Mates.... 50

Chief Water renders........ 50

Chief vermen.............. $\$ 60$

IIonpital Stewards ............ 60

Bandmasters............... 52

PETTY UFICERS, FIRST CLASS.

Masters-at-Arms, ist class... \$ \$o | Boilermaker............ $\$ 55$

Boatswaius' Mates, 1st class. 4o Maclinists' Mates, sot class. 55

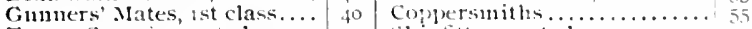

Turret Captains, ist class... 50 sliplitter, ist class.......... 55

Quartermasters, ist class... 40 Flectricitus, 1 int class....... 50

blackinnthe............ 5i

I'lumbers and inters ......... क

Sailmakers Mates .......... fo

Carpentets' Mittes, int class. f y

Water I" Inders............ th

rainters, 1st clasis............ to

PetTy OHACLRS, SECONH Class.

Masters-at-Arms, 2d class... $\$ 35$ Machinims Mates, 2 d class. . \$ 40

Boatswains Mates. $2 d$ class. 35 likectricians, 20 class......... 40

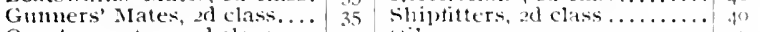

Quartermasters, 2 d class... 35 wilers................. 37

Carpenters' Mates, 2 d class.. 35

l'rinters.................. 35

l'ainters, 2 l class.................

P'FTTY OFHETRS THIRD CLAAS

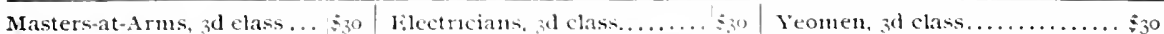

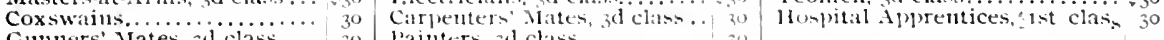
Gunners' Mates, 3 d clas..... $3^{\circ}$ Painters, 3 d cliss........... 30

Quartermasters, 31 class.... 30

SHAMEN, YIRST CLALS

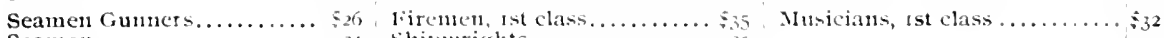
Seamen................. 24 shipwrights ............ 25

SFAMHN, SFCOND CLASS

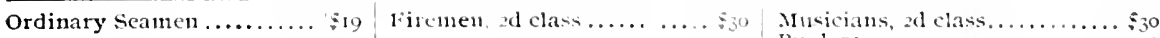
muglers................... 30

llowpital Apprentices............ 20

SEAMKN, THIRH CLASS

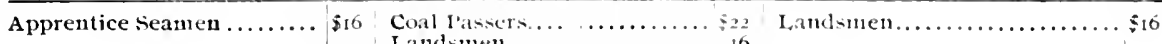

Lantimen................. 16

COMMISSARY HRANCH

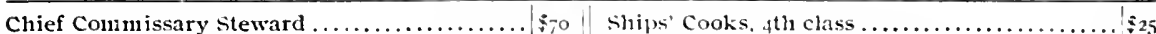

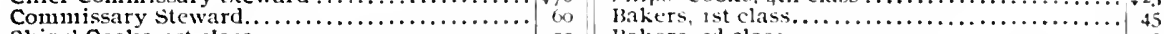

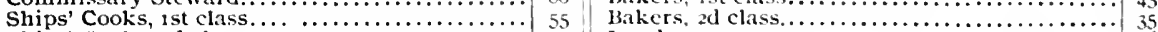

Ships' Cooks, $2 \mathrm{~d}$ class ........................... $44^{\circ}$

Ships' Cooks, 3 d class $\ldots \ldots \ldots \ldots \ldots \ldots \ldots \ldots \ldots$, 30

Laudsulew . . . 


\section{MESSMEN BRANCH}

\begin{tabular}{|c|c|c|c|}
\hline Stewards to Commanders-in-Chief ......... & $\$ 60$ & Steerage Stewards. & $\$ 35$ \\
\hline Cooks to Commanders-in-Cliief............ & 50 & 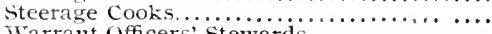 & \\
\hline Stewards to Connunandants .......... & 60 & Warrant Officers' Stewards... & 3 \\
\hline Cooks to Connnandants....... & 50 & Warrant Officers' Cooks....... & $J$ \\
\hline 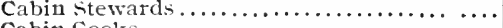 & 50 & 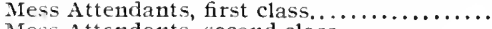 & 24 \\
\hline 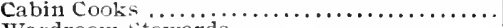 & 45 & Mess Attendants, second class............. & 20 \\
\hline 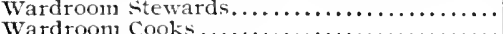 & 50 & Mess Attendants, third class............. & 16 \\
\hline Wardroom Cooks............. & 45 & & \\
\hline
\end{tabular}

INSULAR FORCE, UNITED STATES NAVY.

RATES.

NON-COMMISSIONED OFFICERS, MUSICIANS AND PRIVATES-MARINE, CORPS.

\begin{tabular}{|c|c|c|c|c|c|c|c|c|c|c|}
\hline \multirow[b]{2}{*}{ GRADE. } & \multicolumn{5}{|c|}{$\begin{array}{l}\text { First enlistment or first } \\
5 \text { years. }\end{array}$} & \multirow{2}{*}{ 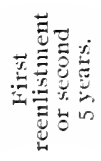 } & \multirow{2}{*}{ 总焉 } & \multirow{2}{*}{ 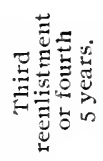 } & \multirow{2}{*}{ 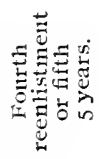 } & \multirow[b]{2}{*}{ 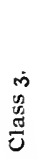 } \\
\hline & 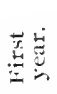 & 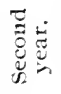 & 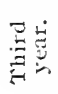 & 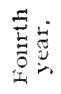 & $\underset{ت}{\rightleftarrows}$ & & & & & \\
\hline Sergeant-MIajor .......... & $\$ 34$ & $\$ 34$ & $\$_{35}$ & 836 & $\$ 37$ & $\$ 39,00$ & $\$ 40$ & $\$ 41.00$ & $\$ 42$ & $\$ 37$ \\
\hline Quartermaster-Sergeant.. & 34 & 34 & 35 & 36 & 37 & 3900 & 40 & $4 I .00$ & 42 & 37 \\
\hline 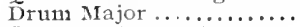 & 25 & 25 & 26 & 27 & 28 & 30.00 & 31 & 32.00 & 33 & $\checkmark 28$ \\
\hline Gummery sergeant ....... & 35 & 35 & 36 & 37 & $3^{8}$ & 40.00 & 41 & 42.00 & 43 & $3^{8}$ \\
\hline First sergeatit .......... & 25 & 25 & 26 & 27 & 28 & 30.00 & 31 & 32.00 & 33 & 28 \\
\hline 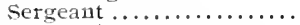 & 18 & 18 & 19 & 20 & $2 \mathrm{I}$ & 2300 & 24 & 25.00 & 26 & $2 I$ \\
\hline Corporal. $\ldots \ldots \ldots \ldots \ldots \ldots$ & 15 & 15 & 16 & 17 & IS & 2000 & 21 & 22.00 & 23 & I8 \\
\hline Drmniner and Trumpeter. & 13 & 13 & 14 & 15 & 16 & IS.00 & 19 & 2000 & 21 & I6 \\
\hline Private............... & 13 & 13 & 14 & I5 & 16 & 18.00 & 19 & 20.00 & 21 & I6 \\
\hline I eader of the Band ...... & 125 & 125 & 125 & 125 & 125 & 137.50 & 150 & $162.5^{\circ}$ & 175 & 125 \\
\hline Second Leader of Band. . . & 75 & 75 & 76 & 77 & 79 & 80.00 & 81 & 82.00 & 83 & 78 \\
\hline Musician, first class....... & 60 & 60 & 60 & 60 & 60 & 60.00 & 60 & 60.00 & 60 & 60 \\
\hline Musician, second class... & 50 & 50 & 50 & 50 & 50 & 50.00 & $5^{\circ}$ & 50.00 & 50 & 50 \\
\hline
\end{tabular}

"Class 3 is the term used to designate pay of men who have ever received reenlisted pay under the act of Angust 4, I 954 , but have allowed a longer period than three months to elapse before again enlisting. 'The amount is the same as that received by a man serving in the fifth year of a first enlistment.'

All enlisted men of the U. S. Marine Corps regularly detailed as gun pointers, mess men or signal me1, or holding good-conduct medals, pins or bars, receive the same extra compensation, in addition to their monthly pay, allowed to enlisted men of the Navy.

Enlisted men of the Marine Corps who have qualified as expert riflemen are entitled to $\$ 1$ per month additional pay allowed enlisted men of the infantry of the Army, which extra pay ceases at the close of the first subsequent target year in which such riflemen fail to qualify. (C. D., Sept. 26, 1905, ) 
Besides this pay, additional allowances and payments are made in the different special ratings as follows:

Seamen gunners receive two dollars a month in addition to the pay of their rating; holders of good conduct medals receive seventy cents per month for such medal, and additional amounts per month for each additional bar which they may receive. Coxswains detailed to boats propelled by machinery or coxswains to Commanders in Chief receive $\$ 5$ per month additional pay. Enlisted men attached to submarine boats receive $\$ 5$ a month extra pay, with $\$$ I additional during each day or any part of which they shall have been submerged in their boat, the total of such daily payment not to exceed $\$ 15$ per month. Seamen who have charge of the holds of vessels receive $\$ 5$ extra per month, and jacks of the dust, who are seamen detailed to aid the pay officer of the ship in caring for stores, receive the same amount of extra pay as do also seamen temporarily detailed to act as mess attendants. When seamen are detained in the service beyond the time when their enlistments have expired, they are entitled to 25 per cent. additional pay until their return to the United States, but they must be discharged as soon as they reach this country. Seamen detailed as coal passers receive thirty-three cents per day in addition to the pay of their regular rating, and scamen detailed as signal men receive $\$ 3, \$ 2$ and $\$ 1$ per nonth according to their class. In order to encourage enlisted men to qualify as gun pointers or marksmen with the batteries of a ship they are awarded additional pay as follows: Heavy gun pointers, first class, $\$$ Io ; second class, $\$ 6$; intermediate gun pointers, first class, $\$ 8$; second class, $\$+$; secondary gun pointers, first class, $\$+$; sccond class, $\$ 2$. Gun Captains of the heavy guns receive $\$ 5$ per month in addition to their regular pay, the addition in the case of seamen gumners, however, to include their regular $\$ 2$ increase. A man detailed as ship's tailor is entitled to receive from $\$ 10$ to $\$ 20$ a month additional, not to exceed $\$ 50$ a month in all, and his helper may receive \$Io a month or not to exceed $\$ 40$ for his total pay. As a bonus for re-enlistment an additional compensation is paid to a man on his second and subsequent enlistments, being $\$ 5$ a month in addition to the regular pay of the rating for second enlistment, and $\$ 3$ a month additional for each subsequent enlistment.

On their first enlistment in the navy, men receive an outfit with- 
out cost to the value of $\$ 60$, although if they are discharged during the first six months of their service the amount of the cost of the outfit is checked against any pay which may be coming to them. After the first outfit, enlisted men are required to furnish their own clothing and other articles of personal use, the navy furnishing free food, quarters and medical attendance. For the latter, however, a deduction of twenty cents a month is made from the pay of each enlisted man of the navy to be applied to the naval hospital fund. In addition to the foregoing an enlisted man who re-enlists in the navy within three months not only receives pay for the time he has been absent from the service, being in the nature of a furlough with pay, but also, if he applies to come within the continuous service privileges and receives a continuous service certificate, receives an increase of $\$ 1.36$ per month, together with the privileges and opportunities for promotion, in which continuous service men receive the preference. Enlisted men who have passed through the apprentice training school also receive slight additional increases of pay.

\section{RETIREMENTS IN THE NAVY.}

Promotion in the navy below the grade of Rear Admiral can only be made after the candidate for promotion has passed a satisfactory examination as to his qualifications for a higher grade. Promotion is, however, not necessarily consequent upon passing such an examination, but must await a vacancy in the higher grade, except that officers, after three years' service in the grade of Ensign, are entitled to promotion, after examination, to the grade of Lieutenant Junior grade. In case an officer fails to pass an examination he is suspended from promotion for a year, and on a second failure to pass an examination he is dropped from the service with a year's pay. Besides promotions as a result of vacancies, promotion may be secured in the navy by especially meritorious service in time of war, for which the President may, with the advice and consent of the Senate, promote any officer to not exceeding thirty numbers, that is, he can jump him over not exceeding twenty-nine officers nearest to him, to take rank with and next after not to exceed the thirtieth officer above him. That is, an officer who is promoted three numbers becomes senior to the next two men above him, and so on, in ac- 
cordance with the cliaracter of the service rendered, within the limit above named. When such promotion brings an officer into a higher grade than that from which he was promoted, his position is that of an extra number in the grarle and the vacancy made by his death or retirement is not filled. Promotion is also indirectly afforded officers of the navy by the reduction of a senior officer in rank by sentence of a court martial, as such punishment acts as a promotion of all those officers who are between the position left by the punished officer and the new position which he assumes on the list. When an officer receives the thanks of Congress for meritorious conduct, he also receives as an incident of the reward an advance to the next higher grade to that which he held; such advancement is also regarded as an extra number in the srade attained and the vacancy is not to be filled upon the officer's death or retirement. The laws relating to the retirement of officers of the navy are radically different from those applying to the army. Originally there was no retirement provision, the differential between sea, shore, and waiting orders pay being intended to supply the function now exercised by the retired list. On the breaking out of the Civil War, however, so many superannuated Captains were found on the active list that retirement provisions have been in effect since that time and have been variously amended for the purpose of securing a regular flow of promotion in the navy. One of the most dreaded conditions in military service, and especially in the naval service, is what is called a "hump" in the list of officers. That is to say, the presence on the list of too large a proportion of officers of about the same age, so that for a number of years there are no retirements and officers are kept in the lower grades for so many years that when they reach the higher and command ranks they retire very shortly after having been promoted. This has the effect of discouraging endeavor in the younger officers and of making them stubordinate through so many years of their lives that when they arrive at command rank they have lost initiative, and power to take responsibility.

Every officer of the navy on reaching the age of 62 is compulsorily retired by the President, the only exception being the Admiral of the navy, who holds his position for life. If an officer has been 40 years in the service of the United States, he may, upon application to the 
President, be retired, this constituting one of the forms of voluntary retirement. The foregoing provisions, however, would not be suffcient to secure a number of retirements that would obviate the condition suggested in the foregoing paragraph, and a rather complex system for getting rid of more officers than can be carried in the regular flow of promotion is followed.

In 1899 an Act of Congress was passed which was intended to provide a flow of promotions which would bring the younger officers to command and flag ranks in time to make their youth and energy of value in the naval service. It was believed at that time that if a provision was made by which the retirement of a certain number of officers annually could be guaranteed the end desired would be attained and the flow of promotions would regulate itself automatically. A singularly complex and ambiguous provision was passed in the following language; namely, that "when the average vacancies for the fiscal year above the grade of Commander have been less than thirteen, above the grade of Lieutenant Commander less than twenty, above the grade of Lieutenant less than twenty-nine, and above the grade of Lieutenant Junior grade, less than forty," certain voluntary retirements might be allowed. It was provided further that when the voluntary retirements had not been sufficient to make up the number of vacancies indicated, certain compulsory retirements should be put into effect. For several years this law was given a close construction, so that the compulsory retirements contemplated were not insisted upon. The result was that the flow of promotion expected did not occur and a protest was made by the officers in lower grades resulting in a different construction of the law and a greater number of retirements. Under the terms of this law a premium is placed on retirements by offering to such officers as shall apply for a place on the retired list retirement in the next higher grade to that which they held, the retirement carrying three-fourths of the pay of the higher grade. Officers who desire to take advantage of the provisions of this section of the law and who are of the grade of Captain, Commander, and Lieutenant Commander may apply to have their names placed on the list for voluntary retirement, and if, at the close of the fiscal year, it appears that the number of vacancies indicated has not been made by deaths, resignations, or retirements, 
the names of such officers may be placed on the retired lists at the rank and pay inclicated. If, however, at the end of the fiscal ycar it appears that the number of vacancies has not been attained by the addition of the voluntary retirements, a board of five Rear Admirals is convened who examine the lists of Captains, Commanders and Lieutenant Commanders and selcct a sufficient number for compulsory retirement to make up the number of vacancies needed. As a compensation for such retirement the officers placed on the retired list receive the rank and thrce-fourths of the sea-pay of the next higher grade to that which they occupied, the rank of Commodore being revived on the retired list in order to provide the next higher grade for Captains so retired although this provision would be taken advantage of only as an exceptional incident.

Regular retirements in the navy except that for age at sixty-two years is effected upon the rccommenclation of an examining board to which the question of the officer's disability for active service has been referred. If it appears that the officer is incapacitated for active service he will be placed on the retired list unless the retiring board certifies that the incapacity is not the result of an incident of the service, in which case, the President may determine whether the officer shall be placed on the retired list or shall be discharged from the service with a year's furlough pay. If an officer comes up for promotion and he is not passed both by the professional and the medical examining boards he may be placed upon the retired list, unless it shall be shown that his incapacity is due to drunkenness or personal misconduct, in which case he is to be discharged from the navy with not more than one ycar's pay. This provision is not, however, all the law with regard to officers below the rank of commander, who, if they fail to pass their professional examination after a year's grace are required to be dropped from the service. It is probable that an officer so dismissed would be awarded the year's pay provided by the section of the law previously referred to. Officers of staff corps who have served for forty-five years have, when retired, the rank of Commodore, and such officers who have arrived at the age of sixty-two years, and are retired before having had forty-five years of service are entitled to have the rank of Commodore on the retired list after forty years from the time of their entry into the service of 
the United States. Officers who retire while holding the position of Chief of Bureau have the rank and emoluments of Rear Admiral on the retired list.

Retired officers may be given employment at the discretion of the President and in such case receive full active pay and emoluments. Such cmployment may, in time of war, extend to the command of a squadron or a vessel and Rear Admirals so detailed may be restored to the active list if they have received the thanks of Congress on recommendation from the President for gallantry in action. If such restoration to the active list should be had it is probable that Congress would re-enact the provision made for officers who receive the thanks of Congress during the Civil WVar and who were to be retained on the active list until they had seen fifty-five years service, unless they should be previously retired on account of disability.

Enlisted mon, after thirty years of service, may be retired with the rank held at the date of retirement and seventy-five per cent. of the pay and allowances then being received, such allowances being commuted at the rate of $\$ 9.50$ a month in lien of rations and clothing and $\$ 6.25$ per month in lieu of quarters, fuel and light. All service in the army, navy and marine corps is credited in computing the time for retirement, and active service in the Civil or Spanish war is computed as double time for the purpose of making up the thirty years required. Provision is made as in the case of the army, although the law as to retired officers was not passed until I906, that any officer of the navy whether in active service or on the retired list, shall, when retired, or if retired, receive promotion of one grade above that held by him at the time of his retirement, such promotion not to become effective, however, if the officer has been promoted on the retired list or has been restored to the navy and placed on the retired list by virtue of a special Act of Congress.

\section{THE MARINE CORPS.}

The Marine Corps of the United States is an excellently organized and disciplined body of infantry soldiers which has its own line and staff organization and which furnishes military guards for the navy yards and stations and for ships in commission and which constitutes a readily mobilized force which is available for making up landing 
parties and undertaking certain military operations which could not be appropriately handled by troops of the army.

Under international law landing of the land troops of a nation on the shores of another nation constitutes an act of war, but a similar significance is not given to landing parties composed of seagoing military forces. Therefore, not only can landing partics of marines and blue jackets be made up and sent aslore on foreign territory without international complications, but provisional brigales of marines may be sent from the Uniteil States as expeditionary forces, and without incurring the liabilities and complications which would be consequent upon sending a section of the army on a similar mission.

The list of line officers of the Narine Corps includes one Brigadier General Commandant, who has general direction of all matters relating to the Marine Corps, six Colonels, six Lientenant Colonels, - fiftecn Majors, serenty-two Captains, eigluty-five First Lientenants and seventy-two Second Lientenants. The staff of the Marine Corps consists of one Adjutant and Inspector, one Quartermaster and one Paymaster, each with the rank of Colonel, one Assistant Adjutant Inspector, and with an appropriate number of assistants in each of the staff corps. The staff of the Marine Corps is separated from the line, but transfers are made from the line to fill vacancies in the staff corps. Vacancies in the grade of Brigadier General Commandant are filled from the line of the Marine Corps not below the grade of Major and Congress has in consileration of long service sometumes voted to increase the rank of the Commandant from Brigadier General to Major General. Vacancies in the lowest grade of the Marine Corps, that of Second Lieutenant, are filled as far as practicable by graduates of the naval academy. It was formerly the custom to assign the cadets lowest in rank on graduating from the naval academy to the Marine Corps, but this practice has been abolished, owing to the great demand for line officers in the navy. The usual method of appointment to positions in the Marine Corps is from civil life, while a considerable number of appointments have been made of midshipmen who have not been able to complete the course at the naval academy. Graduates from the naval academy are not required to take an examination for entrance to the Marine Corps, but all others must be examined and no person under twenty-one years or 
over twenty-seven years of age can be appointed to a commission in the Marine Corps nor until his qualifications have been approved by the Secretary of the Navy. The provision of law in this respect is somewhat different from that relating to the medical and pay corps for the reason that no person can be appointed to either of those corps unless their qualifications have been approved by appropriate boards of officers of the corps. No such limitation is, however, placed on the Secretary of the Navy by the terms of the law relating to appointments to the Marine Corps, and in the discretion of the Secretary the requirements of an examining board of the Marine Corps may be waived and an appointment made even though the candidate has not passed a satisfactory examination.

Besides persons from civil life non-commissioned officers of the Marine Corps who may be recommended by their commanding officers and who are able to pass an examination may be appointed and their selection is given preference as a matter of law to that of civilians. As a matter of practice, however, a very much larger proportion of civilians than of non-commissioned officers have been permitted to appear for the examination for entrance to the corps. The enlisted force of the Marine Corps consists of about 8,000 privates, with the appropriate number of non-commissioned officers. In addition provision is made for a corps band which is stationed at Washington and which is the official band of the President. The leader of the Marine Band is only by courtesy a military officer. He is given by law the pay and allowances of a First Lieutenant in the Marine Corps, but not the rank. He is not carried in the navy register and his official status is that of an enlisted man although his engagement is made the subject of special negotiation and he exercises practically all the powers and prerogatives of a commissioned officer. The second leader of the band and the members are paid in accordance with a scale of wages fixed by law, the former receiving $\$ 75$ a month, first class musicians $\$ 60$ a month and second class musicians $\$ 50$ a month. They receive, however, no increase of pay for length of service. They also receive additional compensation of $\$ 4.00$ a month for semi-weekly cencerts which are given through the summer in the Capitol grounds or the grounds of the White House. In addition to the foregoing the members of the band are allowed to take such 
outside engagements as they may be able to secure, and retain the compensation thus received for their own bencfit. It is, of course, the object to maintain a band of adequate artistic ability in Washington, and this could only be clone if the members secured a larger sum than that inlicated by the pay represented by the foregoing statement. They have, therefore, been accustomed to receive from outsicle engagements consiclerable amounts which have been decreased of late years by the fact that the labor unions in Washington entered upon a campaign to prevent the members of the Marine Band from securing outsicle engagements. This movement has been so far successful as to render it difficult for the members to receive an adequate compensation and it has been found necessary to allow the band to makc exhibition trips and to give concerts elsewhere than in Washington in order that the standard of the President's band might be maintained.

The arrangements for the discipline and maintenance of the Marine Corps are substantially the same as those of the army, and the officers of the marine corps are on the same footing as army officers, the major part of the legislation applying to the latter also applying to the officers of the marine corps. No conmutation is, however, paid to the officers of the marine corps for forage. When marines are serving on board ship, they receive the navy rations, and when on shore they receive the army rations, except that when it is more expensive to furnish the army ration on shore duty, the navy ration may be furnished.

The service contemplated for the Narine Corps by law is a varied one, and the President, under the statutes. may designate detachments either for service on board vessels of the navy or dity as landsmen on naval vessels, or for service in the forts and garrisons of the United States. No officer of the Marine Corps can, however, exercise any command over a vessel or navy yard of the United States, but only over the officers and men of the Marine Corps who may be stationed there under his commanu, and over the premises immediately occupied by the personnel of the Marine Corps at a navy yard. The latter command, however, is not exclusive and is exercised under the general direction and orders of the commandant of the yard or station who is a naval officer. Effort is 
made not to place an officer of the marine corps of superior relative rank uncler the direction of a commanding officer who is his inferior, but it sometimes occurs that in the absence of the senior naval officer at a given point, a junior naval officer may come into command who is also the junior of the marine officer, in which case the easiest solution of the situation is for the marine officer to be indisposed or to absent himself temporarily from military command.

The marine guard on board a vessel of war, such guard being graduated in numerical strength and in accordance with the importance of the vessel, parade on ceremonial occasions, do sentry duty at the different posts which are established at the magazines and other important points, act as orderlies to the Captain and operate the machine guns and direct riffe fire from the military tops in action. The theory of the marine guard is also as an offensive force in aid of the officers of the ship against the crew. This theory, however, is antiquated and is a reminiscence only of the old days of the pressed seamanship. This aspect of the work of the marine corps might be well abolished, and the duties on board the vessels of war undertaken by blue jackets, as is the case now in all navies except that of the United States and England.

At the different navy yards the marines act as watchmen and sentinels, but the marine estabiishments at such yards are chiefly barracks of detention and instruction for recruits and depots for the marines between the times when they are assigned to duty upon naval vessels. The work of guarding the naval prisoners of the United States is also performed by the Marine Corps, and the prisoners at Charlestown, Massachusetts, and Portsmouth, New Hampshire, are under the charge of detachments of the Marine Corps.

The Marine Corps is at all times under the regulations for the sovcrmment of the navy, except when they are detached for service with the army, when they come under the army regulations. Marines on board ship may qualify as heavy gun pointers, in addition to their duties in connection with the machine guns, and when they so qualify, they receive extra pay in the same amount as blue jackets. As the personnel of the Marine Corps is necessarily split up into detachments of comparatively small size, it is not 
possible to maintain any regular regimental or company or battalion organization, but such organizations are effected as from time to time the nature of the service pertormed scems to require. A marine guard of a ressel at no time would exceed the command of the Captain of Marines, and higher officers are assigned to the command of shore stations, although it is rare that an organization as high numerically as a regiment is effected at such points. When considerable numbers of marines are molilized, however, they are quick.ly amalgamated into battalion or regimental organization as the service of the enlisted men in all forms of infantry organization, makes them very readily arranged in the larger formations, and very handy and efficient in all their work.

During the past year, marines have been doing duty at posts and on board ships in commission as follows :

In the United States.-Headquarters United States Marine Corps; assistant quartermaster's office, Philadelphia, Pa., assistant adjutant and inspector's office, assistant quartermaster's office, and assistant paymaster's office, Berlicley, Cal., Annapolis, Ma., Boston, Mass., New York, N. Y., Charleston, S. C., Dry Tortugas. Fla., Key West, Fla., Pensacola, Fla., Iona Island, N. Y., League Isłand, Pa., Mare Island, Cal., Naval Home, Philadelphia, Pa., Naval Hospital, Norfolk, Va., Narragansett Bay, R. I., New Orleans, La., navy yard, Washington, D. C., Norfolk, Va. Port Royal, S. C., Portsmouth, N. H., Puget Somnt, Wash., Naval Training Station, San Francisco, Cal., Sitka, Alaska, and Washington, D. C.

In the West Indics.-Culebra, P. R., San Juan, P. R., Guantanamo, Cula, and Camp Columbia. Havana, Cuba.

On the Isthmus of Panama.-Camp Elliott, Isthmian Canal Zone. In the Ladrone Islands.-Guam, L. I.

In the Hariaian Islands.-Honoluln, Hawaii.

In the Pacific.-Midway Islands.

In Japan.-United States Naval Hospital, Yokohama.

In China.-United States Legation Guard, Peking.

In the Plitippine Islands.-Assistant adjutant and inspector's office, assistant quartermaster's office, assistant paymaster's office, and 
Headquarters First Brigade of Marines, Manila, P. I., marine barracks, Cavite, P. I., marine barracks, Polloc, P. I., and marine barracks, Olongapo, P. I.

On board ships in commission.-Receiving ships: Franklin, Hancock, Hartford, Independence, Lancaster, Pensacola, Philadelphia, Southory, Wabash.

Cruising ships: Alabama, California, Charleston, Chattanooga, Chicago, Cincinnati, Colorado, Connecticut, Dolphin, Dubuque, Galz'eston, Georgia, Helena, Illinois, Kansas, Kearsarge, Kontucky, Louisiana, Maine, Maryland, Mayflower, Milwanke, Minnesota, Missouri, Mohican, Nebraska, New Jersey, Ohio, Paducah, Pennsyliania, Rainbore, Rhode Island, St. Louis, Tennessee, Vermont, Virginia, Washington, West Virginia, Wilmington, Wolverine.

Recruting offices.-Districts of Buffalo, Connecticut, Georgia, Illinois, Indiana, Maryland, Massachusetts, Michigan, Minnesota, Missouri, New York, northern Ohio, southern Ohio, Pennsylvania, and Pittsburg.

Enlisted

Officers. men.

On shore duty within the territorial limits of the United States..........................

On shore duty outside the territorial limits of the

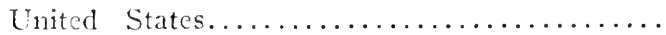

On board ships in commission..................

Undergoing imprisonment with sentence of dishonorable discinarge..........................

Number of officers allowed by law...............

Number of enlizted men allowed by law..........

I23 3,210

\begin{tabular}{rr}
99 & 2,567 \\
55 & 2,224 \\
\hline 277 & 8,001
\end{tabular}

$\begin{array}{rr}\cdots & \frac{123}{3,124} \\ \cdots & \begin{array}{r}280 \\ 8,77 \mathrm{I}\end{array}\end{array}$ 


\section{LIST OH SIIIS OF THL UNITHI STATES NAYY ACCORDING TO RATIS.}

\section{IIRST RATE}

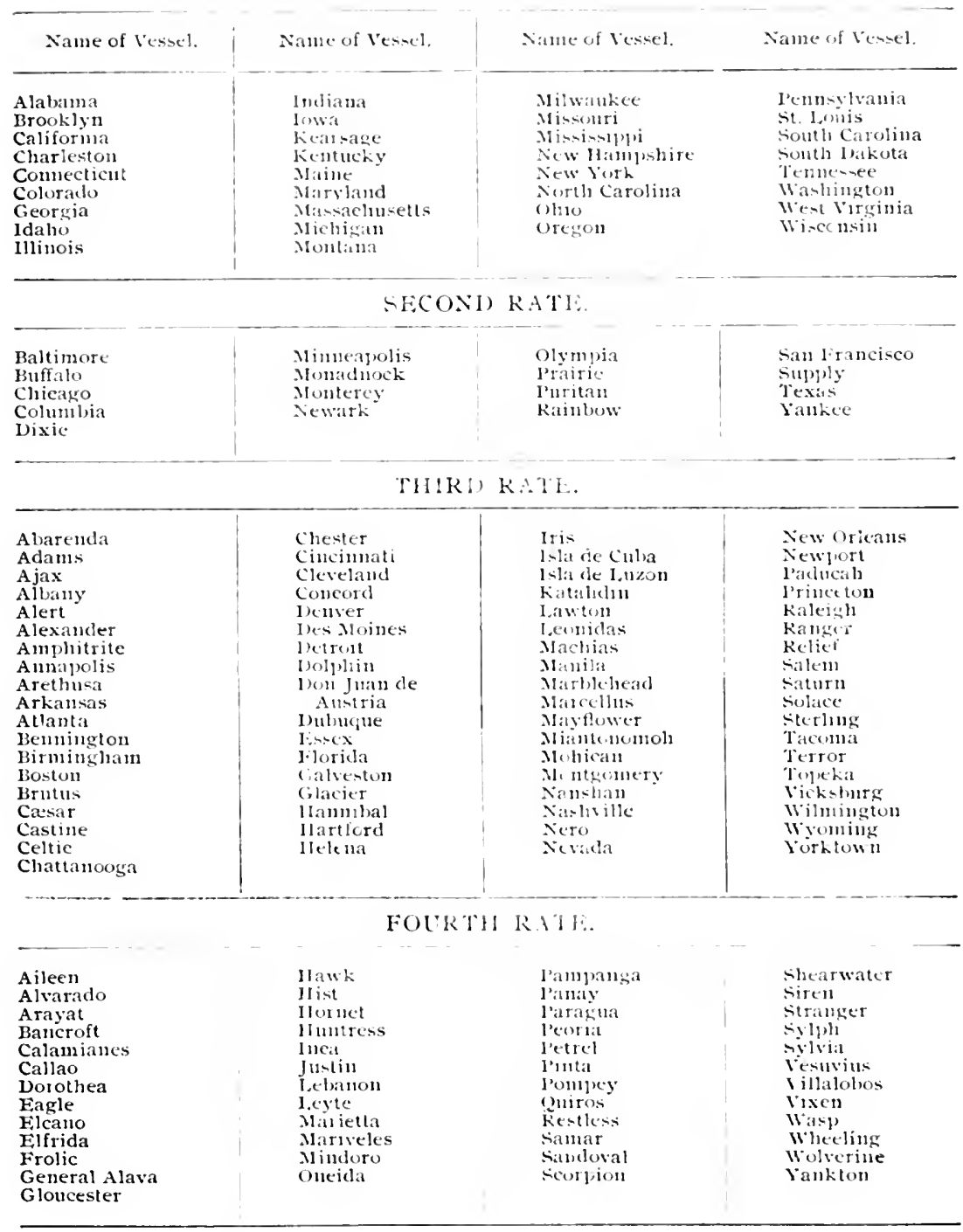




\section{CHAPTER XIV.}

THE INTERIOR DEPARTMENT.

The Interior Department was the first of the new departments to be established as the result of the expansion and growth of the United States. While the Navy Department was not included in the original constitution of the executive Government, and was separated from the War Department shortly after the Federal Government had been inaugurated, it was not until 1849 that another was added to the executive departments of the government. In the earlier years, all functions relating to the interior administration of the United States were excrcised by the State Department, including the commissioning of all the officials. As matters relating to the ministrations of the public lands of the United States and of Indian Affairs became more pressing, these, together with territorial administration, were placed under the direction of a new department, and the commissions for the various officials of the government were placed under the jurisdiction of the department to which they respectively pertained.

The Interior Department is also the first of the departments under which several widely differing activities, have been collected. Each of the originally constituted depaitments had a specific and well-defined function to perform, but the Interior Department, as was the case with the Department of Commerce at a later date, was given the control and oversight over various activities of the Government which had sprung up, and which could not be appropriately assigned to either of the existing departments. The administration of the public lands of the United States and of Indian affairs are partly though remotely connected, but they are widely separated from the activities of the patent office, and only incidentally connected with the pension office, and the work of the Bureau of Education, or with the Census Office, which was formerly under the Department of the Interior, but which is now under the Department of Commerce and Labor. 
Largely because of this somewhat disconnected series of functions exercised by the Department of the Interior, the head of the Interior Departntent is rather a court of last resort in administrative matters than a direct administrative officer. Owing to the importance of matters relating to the public lands, the Secretary of the Interior has been more closely in touch with these matters than those of the other bureats or divisions of his department, and he might not incorrectly be described as the secretary of public lands, with supervisory functions with relation to the number of other governmental bureaus of a semi-independent character.

With the exception of the narrow fringe along the Atlantic Coast, included within the domain of the original thirteen states, the North American continent has been pareeled out in accordance with a system which is now in effect under the direction of the Department of the Interior, and which is, comparatively speaking, about coming to a close, as the amount of public land unoccupied and undivided to private owners steadily diminishes and will within an appreciable time be entirely allotted. Originally held under the terms of the grants to the different colonies, and transferred to a large extent by such colonies when they became States of the Federal Government, the public lands of the United States were largely added to by the Louisiana Purchase and by the discoveries and determination of boundaries which have finally established the United States in its present geographical form.

\section{GENERAL LAND OFFICE.}

Matters relating to the public lands of the United States are aclministered under the direction of the Secretary of the Interior by the General Land Office, at the head of which is a Commissioner, appointed by the President, by and with the advice and consent of the Senate, who receives $\$ 5,000$ a year and who is assisted by an Assistant Commissioner, similarly appointed, who receives $\$ 3,500$ a year. The dutics of these officials consist in the general direction of the operation of the Land Offices situated in States in which there are public lands, and there is also in the General Land Office a Recorder, who has charge of recording the titles to lands granted to private individuals and who is also appointed by the President by and with 
the advice and consent of the Senate, and receives $\$ 2,000$ a year. All lands sold or granted by the United States must be evidenced by a patent, accurately describing the lands so sold or granted, and signed by the President. In order to relieve the President of the work of signing such patents, it is provided by law that he may designate a clerk in the General Land Office who may sign his name to such patents, and who receives $\$ 1,200.00$ a year for the service. An assistant clerk for such service may also be appointed to bring up the arrears of the work.

As the United States itself can use but a very small portion of the lands which have become, in the various ways before suggested, the property of the Federal Government, it has been the historical policy of the United States to convey such lands into the hands of small holders, in order that they might be developed and indirectly add to the revenue-raising possibilities of the United States, through an increase of population, consuming articles upon which direct taxes are fixed, and it was only in the earlier days of the United States that the reventues from public lands were materially counted upon as a source of revenue to meet governmental expenses. Under the earlier system, large tracts of wild land were sold to speculators, but this method was shortly abandoned in favor of the policy which has continued, of encouraging the settlement of small holders and farmers, who should themselves actually develop the land, and create homesteads thereon.

In order that there might be no confusion of lines when such small holdings were granted, it became necessary to provide for an accurate survey of the public lands, and the result is the exact delineation of the Western States into right-angled subdivisions, the unit of which is a quarter section of 160 acres. For the purpose of directing this survey, the Congress provided for the appointment of a surveyor general in each state in which there were considerable quantities of public lands. Such surveyors general were appointed by the President, by and with the consent and advice of the Senate, and received salaries in proportion to the duties to be performed. When the survey of the public lands within a state was completed, the results became the basis of the sale and allotment of public lands throughout the domain of the United States. The surveyor general 
is authorized to procure the survey of the public lands within his district by deputy surveyors, at prices which are fixed in accordance with the difficulties to be met in the work of surveying and such deputy surveyors are required to perform their duties under oath, and with heavy penalties imposed if they return a false measurement or delineation.

The surveys thus made constitute the basis of the plats and maps issued by the General Land Office and from which purchasers of public land secure information as to the warrants of the public land which have not yet been disposed of. A prime consideration of acquisition of the agricultural lands of the United States is that the person who is to secure such lands shall have resided upon them and improved them for a term of years. Under the present homestead system, a person who desires to secure a homestead may by effects of actual residence and occupation for a term of years and paying the fees fixed by the Lancl Office secure final title to the land on which he has resided. The most desirable, agriculturally, of the lands in the public domain available for homestead entry are now occupied and the current entries of land lave to do with desert lands which may become valuable as the result of reclamation enterprises, or of lands which have previously been reserved from homestead entry.

The homestead laws secure to qualified persons the right to settle upon, enter, and acquire litle to not excceding one quarter section, or ifo acres, of public land, by establishing and maintaining residence thereon and improving and cultivating the land for the continuons period of live years.

A homestcad entryman must be the head of a family or a person who has arrived at the age of 21 years, and a citizen of the United States, or one who has filed his declaration of intention to become such, as required by the naturalization laws.

Applicants to make homestead entries are restricted to "unappropriated public lands."

All applications for homestead entry must be filed in the local office of the land district in which the lans applied for is located, accompanied by the legal fec and commission in each case and the proper affidarits made by the homestead applicant.

The required affidavits must be made before the register or receiver or any United States commissioner or commissioner of the court exercising Federal jurisdiction in the Territory or before the judge or clerk of any court of record in the land district in which the lands are situated.

In case affidavits are taken out of the country in which the land applied 
for is located, the applicant must show, by affidavit satisfactory to the Commissione: of the General Land Office, that the same were taken before the nearest or most accessible officer qualified to take the same in the land district in which the land applied for is located.

A person in active service in the Army or Navy of the United States whose family or some member thereof is residing on the land which he wishes to enter, and upon which bona fide settlement and improvement have been made, may, by speeial enactment, make the affidavit required by law before the officer commanding in the branch of service in which the applicant is engaged.

Where a wife has been divorced from her husband or deserted, so that she is dependent upon her own resources for support, she can make homestead entry.

Where an unmarried woman settles upon a tract of public land, thereafter marries before making entry, she does not, on account of her marriage, forfeit her right to receive patent for the land, unless she leaves the land or her husband is an entryman.

To obtain a homestead, the party should select and personally examine the land and be satisfied of its character and true description.

He must file an application, stating his name, residence, and post-office address, and describing the land he desires to enter and make affidavit that he is not the proprietor of more than 160 acres of land in any State or Territory; that he is a citizen of the United States, or that he has filed his deciaration of intention to become such, and that he is the head of a family, or over 21 years of age, as the case may be; that his application is honestly and in good faith made for the purpose of actual settlement and cultivation, and not for the benefit of any other person, persons, or corporation, and that he will faithfully and honestly endeavor to comply with all the requirements of laws as to settlement, residence, and cultivation necessary to acquire title to the land applied for ; that he is not acting as agent of any person, corporation, or syndicate in making such entry, nor in collusion with any person, corporation, or syndicate to give them the benefit of the land entered, or any part thereof, or the timber thereon; that he does not apply to enter the same for the purpose of speculation, but in good faith, to obtain a home for himself, and that he has not, directly or indirectly, made and will not make any agreement or contract in any way or manner, with any person or persons, corporation or syndicate whatsoever, by which the title which he might acquire from the Government of the United States should inure, in whole or in part, to the benefit of any person except himself; and, further, that since August 30, 189o, he has not acquired title to, nor is he claiming, under any of the agricultural public land laws, an amount of land which, together with the land he is seeking to enter, will exceed in the aggregate 320 acres, and that he has not theretofore had the benefit of the homestead laws.

On compliance by the party with the foregoing requirements, the receiver will issue his receipt for the fee and that part of the commissions paid, a 
duplicate of which he will deliver to the party. The matter will then be entered on the records of the district office and reported to the General Land Office.

A homestead settler on unsurveyed public land not yet open to entry must make entry within three months after the filing of the township plat of survey in the district land office.

In cases of simultaneous applications to enter the same tract of land under the homestead laws, the rule is as follows:

First. Where neither party has improvements on the land the right of entry is awarded to the highest bidder.

Second. Where one has actual settlement and improvement and the other has not, it is awarded to the actual settler.

Third. Where both allege settlement and improvements, an investigation must be had and the right of entry awarded to the one who shows prior actual settlement and substantial improvements, so as to be notice on the ground to any competitor.

The settler must, within six months after making his entry, establish his actual residence in a house upon the land, and must reside upon and cultivate the land continuously in accordance with law for the term of five years. Occasional visits to the land once in six months or oftener do not constitute residence. The homestead party must actually inhabit the land and make it the home of himself and family, as well as improve and cultivate it.

At the expiration of five years, or within two years thereafter, he may make proof of his compliance with law by residence, improvenent, and cultivation for the full period required, and must show that the land has not been alienated except as provided by the Revised Statutes.

A settier desiring to make final proof must file with the register of the proper land office a written notice, in the prescribed form, of his intention to do so, which notice will be published by the register in a newspaper, to be by him designated as nearest the land, once a week for five successive weeks, at the applicant's expense. Proof must be completed and filed in the local office within the statutory period of seven years from date of entry.

Proofs can only be made by the homestead claimant in person, and can not be made by an agent, attorney, assignce, or other person, except that in case of the death of the entryman proof can be made by the statutory successor to the homestead right, in the manner provided by law.

The rights of a homestead claimant who has become insane may. under act of $J$ tune 8 , I 880 , be proved up and his claim perfected by any person duly authorized to act for him during his disability.

There are threc laws providing for Icarcs of absence in certain cascs, that which provides generally for cases of destruction or failure of crops, sickness, or other unavoidable castualty rendcring the settler unable to support himself or persons dependent on him upon the land; that providing for the special case of the devastation by grasshoppers; and that providing for the relicf of homestead settlers who suffered from the forest fires which prevailed in 
northern Visconsin, Minnesota, and Michigan during the summer and autumn of IS94.

No lands acquired under the provisions of the homestead laws are liable for the satisfaction of any debt contracted prior to the issue of patent.

The sale of a homestead claim by the settler to another party before becoming entitled to a ratent vests no title or equities in the purchaser as against the United States. In making final proof, the settler is by law required to swear that 110 part of the land has been alienated except for church, cemetery, or school purposes, or the right of way of railroads, canals, or ditches for irrigation or drainage across it.

As the law allows but one homestead privilege (sec. 2298, Rev. Stat.; Appendix No. I) a settler relinquishing or abandoning his claim can not thereafter make a second entry, although where the entry is canceled as invalid for some reason other than abandonment, and not the willful act of the party, he is nor thereby debarred from entering again if in other respects entitled.

A person possessing the requisite qualifications under the romestead law (not having exhausted his right by previous entry thereunder), owning and residing on land not amounting in quantity to a quarter section, may enter other land lying contiguous to his own to an amount which shall not, with the land already owned by lim, exceed in the aggregate 160 acres.

For the purpose of facilitating the entry, of lands, and of the receipt of the amount of money to be paid in purchases, the public lands are divided into land districts, located in each of which is the office of a register and a receiver of public moneys. These officials are appointed by the President, by and with the advice and consent of the Senate and receive a salary of $\$ 500.00$ a year, with the following fees and commissions. They are required to reside at the place where the land office for the district is kept as required by law, and must give a bond of $\$ 10,000.00$ each. They are not allowed to receive from salary and fees amounts of more than $\$ 3,000.00$ each as compensation per year. The expenses of their offices are paid out of the public funds, on the authorization of the Commissioner of the General Land Office.

Registers and receivers, in addition to their salaries, are allowed the followincr fees and commissions, namely:

First. A fee of one dollar for each declaratory statement filed and for services in acting on pre-emption claims.

Second. A fee of one dollar to the register for giving notice of the cancellation of any pre-emption, homestead, or timber culture entry, to be paid by the contestant and not to be reported

Third. A commission of one per centum on all moneys received at each receiver's office. 
Fonth. A commission of one per centum on the cost price, as fixed by law, of the land applied for, to be paid by the homestearl applicant; and a like commission when the chin is fmally established and the cortificate isstied as the basis of a patent.

Fifth. The same fees and compensation for final proof in timber culture entries as allowed in lomestead entries, and the same commissions as ailowed when tle like quantity of land is entered with money.

Sixth. For locating miliary bounty-land warrants, issued since the eleventh day of Februtry, cighteen humlred and forty-seven, and for locating agricultural-college land-scrip, the same commission to be paid by the holder or assignee of cach warrant or scrip, as is allowed for sales of the public lands for cash, at the rate of one dollar and twenty-five cents per acre.

Severth. A fee in donation cases of two dollars and fifty cents for each final certificate for one hundred and sixty acres of land, five dollars for three hundred and twenty acres, and scven dollars and fifty cents for six hundred and forty acres.

Eighth. In the location of lands by States and corporations under grants from Congress for railroals and other purposes (except for agriculural celleges), a fee of one dellar for each final location of one hundred and sixty acres; to le paid by the State or corporation making such location.

Ninth. A fee of live dollars per diem for superintending public land sales at their respective offices

Teutin. A fee of five dollars for filing and acting on each application for patent or adverse claim filed for mineral lands, and a like fee for filing and acting on timber and stene applications, to be paid by the respective parties.

Eleventh. Registers and receivers are allowed, jointly, at the rate of fifteen cents per linulred words for testimny reduced by them to writing for claimants, in establishing pre-emption and homestead rights.

Twelith. A like fee as provided in the preceling subdivision when such writing is done in the land office, in establishing claims for mineral lands.

Thirtcenth. Registers and Recejers are allowed jointly a fee of five dollars in homestead entries when the cntry is not more than eighty acres, and ten dollars when the entry is for more than eighty acres.

SEC. 2886. Registers and receivers are, in addition to the cther fees allowed $1 y$ law, entitled to charge and receive for making transcripts of the records in their offices for individuals, the stm of ten cents per one hundred words.

The compensation of registers and receivers, including salary, fees, and commissions, can in no case exceed in the aggregate three thousand dollars a year.

All fees collected by registers or receivers, from any source whatever, which would increase their salaries heyoud three thousand dollars each a year, are to be covered into the Treasury, except only so much as may be 
necessary to pay the actual cost of clerical services employed exclusively in contested cases; and they must make report quarterly, under oath, of all expenditures for such clerical services.

Registers and receivers at land offices are appointed for four years, but are removable at pleasure. Land districts are established in accordance with the amount of public land in a given state, and when the amount of public lands has been reduced to less than $100, \infty 00$ acres, the land district is abolished or consolidated with another district, except that a land district the office of which is in the capital city or town of the state may be continued so long as is necessary to complete the transactions relative to the disposition of the lands in the state in question.

Registers and receivers are required to keep on hand and to furnish on application plats or diagrams of townships in their respective districts, showing what lands are vacant and what lands taken, and are also required to furnish the state and territorial officers information as to lands which may be sold and thus become subject to taxation by the state or territory. Registers of land offices receive the applications of intending purchasers of public lands, together with the necessary proof of occupancy by homesteaders or compliance with the requirements for the acquisition of the different classes of land. In cases of contest as to priority of registration for the privilege of purchasing land the registers have the power to grant hearings and to examine witness on subjects germane to the inquiry in hand. Upon full compliance with the requirements for the purchase of portions of the public domain, the receiver of the land office in question takes the amount of money involved, giving a receipt for the same, and the entire transaction is referred to the General Land Office for ratification and for the preparation of the patent which evidences the title of the purchaser to the land. Considerable grants have been made by the United States of public lands for specific purposes. Thus, grants have been made to states for the maintenance of agricultural and mechanical colleges, and, under general laws, military service, especially in the earlier wars of the United States, has been compensated by grants of lands, such grants being evidenced by a scrip or certificate issued to the beneficiaries in advance of the actual location of the land. 
In addition to the regular grant of land, rights have been given to soldiers and sailors of the United States regarding the premption or homesteading of public lands, and in the latter case the period spent in the service of the Unite! States is cleducted from the period of residence required on the land before the terms of the preemption law can be taken advantage of.

Timber lands and mineral lands, especially such as contain coal veins, have been reserved by the [nited States from the general preemption and sale, and these have been disposed of under special terms, to afford a proper return to the United States, for the value of the land as well as to secure the derelopment of such lands by placing them in the hands of small holders, and requiring a certain amount of development as a condition to the purchase. There has been, during the last ten years, activity in acquiring all classes of the public lands, and it has been discovered within the last three or four years that many of the entries made and the payments made thereunder were by persons who were acting for corporations, so that the latter have been able to secure possession of large tracts of land formerly belonging to the Govermment, in some cases securing mineral lands as agricultural lands, and in other cases securing by false affidarits and through "dummy entrymen." that is, persons who have no actual interest in the entries made, opportunity to purchase the public lands under conditions which are declared to be fraudulent to the Government and to other intending purclasers. A series of investigations covering many montlus has been carried on with the result that proceedings have been brought in several western states for the purpose of punishing fraululent acquisition of public lands, and to restore the lands so acquired to the public domain.

The standard value of land which has been sold by the Lnited States as agricultural lands for development under the requirements of the various preemption and homestearl acts has been $\$ 1.25$ an acre, with a limit of 160 acres for cach allotment. A statutory price of $\$ 5.00$ per acre is fixed for mineral lands covering the territory included by the same, with a price of $\$ 2.50$ per acre for a placer claim, while coal lands have been priced at \$10.00 an acre for land 
which is situated not less than 50 miles from a railroad and \$20.00 an acre when situated within fifteen miles of a railroad.

In vicw of the approaching complete sale of all public lands available for agricultural operations, various provisions have been made, designed to encourage persons to reclaim the arid districts of the West. Arid lands are defined by the statute as all lands exclusive of timber lands and mineral lands, which will not, without irrigation, produce some agricultural crop. It was prinarily intended that such of these lands as could be reclaimed should receive irrigation as the result of private enterprise. To this end, legislation was effected, encouraging the different states, as well as private persons to engage in irrigation enterprises, and it was provided that upon payment of a fee of twenty-five cents an acre a citizen of the United States or a person who had declared his intention of becoming a citizen, could enter not to exceed 320 acres of desert land, upon the declaration that he intended within the three years next following to improve such land by connecting it with some system of irrigation. It was required of the entryman that he should take part in an irrigation project which should involve the expenditure of at least $\$ 3.00$ per acre for the entire acreage which he had entered, such expenditure to be made at the rate of a dollar an acre a year for the following three years, and that one-eighth of the land should be put under cultivation. Upon fulfilling the requirements and paying the further sum of a dollar an acre, the title of such land would be vested in the entryman.

It was found that many of the projects necessary for the irrigation of the arid lands were of such magnitude that they could not be undertaken by private persons, or even by states, and there arose questions relating to the jurisdiction over the water courses and the supplies of water available for irrigation. As the result of these considerations, the United States has entered upon a system of irrigation projects, the expense of which is to be borne by the proceeds from the sale of irrigated lands, so that the future value of considerable portions of desert lands will be fixed by the cost of the work necessary to bring them into condition for agricultural operation. Timber and stone lands, that is lands which are valu- 
able chiefly because of the timber and stone which may be removed from them, are held at $\$ 2.50$ an acre, in either case, and provision is made for the punishment of persons who may remove timber or stone from public lands, and for the recovery of the value of the property stolen.

The amount per acre stated as fixed by law for the purcliase price of public lands is the minimum which can be charged for agricultural lands, \$I.25 per acre, having been regarded for years as the lowest valuation for such lands. Under certain circumstances, the Secretary of the Interior is empowered to place a higher valuation on lands when offered either at public or private sale. The method of the distribution of lands, whether under the homestead laws, to be acquired by actual settlers in return for five years of cultivation, or to be sold as directed by Congress, has varied, especially in later years when desirable lands upon which a value could be placed because of the fact that they were surrounded by improved lands were to be disposed of. A case in point was the public land in Oklahoma. Various devices were tried to secure an equitable distribution of this portion of the public domain. Provision was made for sale at public auction; for sale by an appraised value by the Secretary of the Interior: for sale at a given sim to the first comers, and for sale to purchasers in an order determined by the drawing of lots. Neither of these methods was wholly without objectionable features, and the varions provisions developed in the newly-opened countries a class of people who made their living by evasion of such regulations and who because they were able to secure allotments in advance of others, by more or less sharp practice, came to be known by the general term of "Sonners."

At all times, however, the land in the public domain has been more valuable to persons who were in a position to develop it than the cash prices or equivalent considerations lemanded by the Government, so that there has been a steady pressure at all times for the opening up of new lands to the operation of the homestead and cash purchase laws. As the portions of the public domain thus coveted by settlers have been in most cases the lunting grounds of Indian tribes, it has been necessary in order to meet the demands for lands, to remove the Indians from point to point, such removal 
constituting the chief factor in the Indian question, which is under the jurisdiction of another bureau of the Interior Department from that now being considered.

As the ruling theory with regard to the lands of the United States, was that they should be occupied and developed by individual settlers as soon as might be practicable, there was during the earlier history of the country a donation of lands to railroads for the purpose of aiding the building of such means of communication, which will probably seem to the future citizens of the United States as lavish and, perhaps, as induly lavish. To encourage the building of such roads through territories whicli were sparsely populated, or unpopulated, alternate sections of the public land were granted along the railroad rights of way to be the property of the railroad companics. Upon the anticipation of amounts to be secured by the sale of such lands, railroad companies were able to raise necessary funds to build and equip the roads, and to operate them during the time that the country was still umproductive and unprofitable as a scene of railroad operation. The earlier of the grants were unlimited except as to requirements that roads should be built within a given time, but shortly after the period of the Civil War complaint was made of the freedom with which the public domain was being granted out, and thereafter such grants were limited with the proviso that sales should be made in small quantities only and to bona fide settlers at a fixed price.

Besiles the grants made to railroads, and to states for educational purposes, considerable tracts of swamp lands and overflowed lands were donated in their entirety to the states in which they were located, to create a fund for the reclamation of the entire body of such lands, useless for agricultural purposes in their condition as held by the United States, the proceeds of the sale of such lands to be used by the states for the construction of levees and ditches, which would add the entire area to the amount of agriculturally productive land in the United States.

With the exception of the railroad land grants, specific quantities or locations of land have not been granted, usually, but a credit established for the beneficiaries for the amount of land fixed by law, which credit was evidenced by warrants or 
scrip, so-called, and which could be applied instead of cash, to the purchase of lands by individuals under any of the provisions of law provided for the direct sale of public lands, or by entrymen under the homesteal laws in the commutation of residence required to secure a title. The questions growing out of the conflicting locations and selection of lands and the necessity of withdrawal if certain lands were needed for the use of the United States, and other complications naturally arising in the administration of such a subject, have created a systen of land laws, outside of and in addition to the real estate practice of the common law, and including many practices which will of course become obsolete as the surplus public land falls into private possession. At the present time, cxcept for the forest reserves and arid lands, the administration of questions relating to the public lands of the United States by the Interior Department refer largely to the completion of the disposition of the ailotment already inaugurated except that it is probable that considerable tracts wiil be restored to the public domain as the result of the litigation heretofore referred to, to secure the restoration of lands fraudulently entered and patented. There is in addition an interesting question now pending, whether the considerable bodies of land formerly granted to the railroads under restrictions as to their sale, and which have not been sold hy the railioads, and which are now so valuable thet the ailroads will not sell them under the conditions imposed can be restored to the public domain and opened to homestead entry, or to purchase minler appraisement by virtue of special provision of Congress, should such a recovery be found to be practicable.

\section{THE PENSION BUREAU.}

When one contenulates the comparative importance of the subdivision of the Government of the United States which has to do with the payment of military pensions, and especially when a realization is had of the amount of the public revenues which is redistributed in this form, there seems to be no consistent ground in favor of the proposition that republics are ungrateful. 
One of the largest public buildings in Washington, housing a personnel which is larger than that of more than one of the executive departments, is devoted exclusively to the payment of monthly sums to persons who have earned the gratitude of the republic by service in her wars and to the descendants of such persons. Matters relative to the execution of the various pensions and bounty land laws are administered by the Commissioner of Pensions, who is appointed by the President by and with the advice and consent of the Senate and receives a salary of $\$ 5.000$ a year. Tie is assisted by the First and Second Deputy Commissioners of Pensions, at $\$ 3,600$ a year each, and who respectively are empowered to perform the duties of the Commissioner in case of his absence or disability.

The amount paid by the United States in the form of pensions constitutes two grand divisions, viz.: service pensions and invalid pensions. In the former class, are the payments made to the survivors of the earlier wars of the United States, and in the latter, of the pensioners who suffered disabilities by reason of service in the Civil War. All pension payments originate in a class equivalent to that of the invalid pensions, but as time elapses and those who serve in the wars for which pension is paid grow older anil more dependent, the character of the pension changes, and for many years service pensicns without relation to disability incurred in the war were paid to Revolutionary soldiers, also to the soldiers of the war of 1812 and to their widows. Some few of the widows' pensions remain, though being rapidly extinguished, but additional service pensions have been provided for soldiers of the Mexican war and their widows, and of the Indian wars, including the various services up to the year 1856 . Soldiers who incurred disabilities in such wars are entitled to pensions such as those provided under the head of invalid pensions, but non-disabled soldiers of the same wars are also pensioned at \$12 a month during their natural lives, and a similar pension is paid to widows of such soldiers who have not remarried. As the monthly rolls of these various wars have been destroyed, special provisions not applicable to later classes of invalid pensions have been made, so that a veteran of the wars prior to the Civil War may 
establish proof of his pensionable standing by evidence of having received pay from the United States, or of having received of a land warrant, as the case may be.

The very great proportion of the pension payments are of course based upon disabilities incurred during the service of the Civil Var. No service pension for that war has yet been enacted by Congress, although its enactment is certain at a later date. However, a provision for service pension is now on the statute book, although not technically a general service pension. An entering wedge for legislation under consideration was a provision some years since that a person who saw service in the Civil IVar should be pensionable upon showing disability to pursue the avocations of civil life, although he might not be able to show that his disability was the dircct cause of a Civil War service. In the administration of this provision of law, it was made the guiding rule that when an applicant had reached a certain age, the fact of that age should be one of the proofs required to establish the disability. In 1907 Congress affirmed this administrative practice, and provided that when a person who has seen ninety days' service in the military or naval arms of the United States during the Civil War or sixty day's service in the Mexican War, shall have reached sixty-two years of age, he shall be entitled to a pension of trelve dollars a month; to a pension of fifteen dollars a month on reaching seventy years of age, and to a pension of twenty dollars a month when he reached the age of seventy-five and at that rate for the remainder of his natural life. As it is now more than forty-two years since the close of the Civil War, the operation of this provision will, within five years, make practically all the soldiers and sailors of the Civil War who can show ninety days' service, pensionable at the rate mentioned.

The following compilation gives the historical progress of the amount of pensions granted for various disabilities, and exhibiting the increase made under the various classes during the period since the Civil War.

For the loss of the sight of both eycs: From July fourth, cightecn hundred and sixty-four, twenty-five dollars; from June fourth, eightcen bundred and seventy-two, thirty-one dollars and twenty-five cents; from June fourth, eighteen hundred and seventy-four, fifty dollars; from June scventcenth, eighteen hundred and seventy-eight, seventy-two dollars; and from April 
eighth, ninetcen lundred and four, for the loss of the sight of both eyes, or total blindness, occurring from causes arising within the strvice of the United States, one hundred dollars.

lor the loss of the sight of one eye, the sight of the other having been previously lost: From June sixth, eishteen hundred and sixty-six, twentyfive dollars; from June fourth, eighteen hundred and seventy-two, thirty-one dollars and twenty-hve cents; from June fourth, eighteen hundred and seventy-four, fifty dollars; from June seventeenth, eighteen hundred and seventyeight, seventy-two dollars: and from Apri! eightli, nineteen hondred and four, one hundred dollars.

For the loss of both feet: From July fourth, eighteen hundred and sixtyfour, twenty dollars; from June fourth, eighteen hundred and seventy-two, thirty-one dollars and twenty-five cents; from June fourth, eighteen hundred and seventy-four, fifty dollars; from June seventeenth, eighteen hundred and seventy-eight, seventy-two dollars; from March second, nineteen hundred and three, one liundred dollars.

For the loss of both hands: From July fourth, eighteen hundred and sixtyfour, twenty-five dollars; from June fourth, eighteen hundred and seventy two, thirty-one dollars and twenty-five cents; from Jume fourth, eighteen hundred and seventy-four, fifty dollars; from Jume seventeenth, eighteen hundred and seventy-eight, seventy-two dollars; from February twelfth, eighteen hundred and eighty-nine, one hundred dollars.

For the loss of one hand and one foot: From March third, eighteen hundred and sixty-five, twenty dollars; from June fourth, eighteen lundred and seventy-two, twenty-four dollars; from February twenty-eight, eighteen hundred and screnty-seven, thirty-six dollars; from March second, nineteen hundred and three, sixty dollars.

For total disability in one hand and one foot: From June sixth, eighteen hundred and sixty-six, twenty dollars; from June fourth, eighteen hundred and seventy-two, twenty-four dollars; from February twenty-eighth, eighteen hundred and seventy-seven, thirty-six dollars; from March second, nineteen hundred and three, sixty dollars.

For the loss of one hand or one foot: From June sixth, eighteen hundred and sixty-six, fifteen dollars; from June fonrth, eighteen hundred and seventy-two, eighteen dollars; from Narch third, eighteen hundred and eightythree, twenty-four dollars; from Angust fourth, eighteen hundred and eightysix. thirty dollars; from March second, nineteen lundred and three, forty dollats.

For total and permanent disability in one hand or in one foot: From June sixth, eighteen hundred and sixty-six, fifteen dollars; from June fourth, eighteen hundred and seventy-two, eighteen dollars; from March third, eighteen hundred and eighty-three, twenty-four dollars; from August fourth, eighteen hundred and eighty-six, thirty dollars; from March second, nineteen hundred and three, forty dollars.

For disability causing incapacity to perform manual labor equivalent to the 
loss of a hand or a foot: From June sixth, eightecn hundred and sixty-six, fiftecn dollars; from June fourth, eightecn hundred and seventy-two, cighteen dollars; from Warch third, eightecn hundred and cighty-three, twentyfour dollars.

For the loss of a leg above the lince and conseguent disability to use artificial limbs: From Jime ionrth, eighteen hundred and seventy-two, twentyfour dollars; for the luss of an arm at the shoulder joint, o: a leg at the hip joint, or so near the joint as to prevent the use of an artificial limb, from August fourth, cighteen lundred and cighty-six, forty-five dollars; for the loss of an arm at the shoulder joint, or a leg at the hip joint, or so near the shonlder or hip jeint, or where the same is in such condition as to prevent the use of an artificial limb, from March scconl, ninetcen hundred and three, fifty-five dollars.

For the total and permanent disability of an arm or a leg: From Angust fourth, eighteen hundred and cighty-six, thirty-six lollars; from Mlarch second, nincteen hundred and three, forty-six dollars.

For total and permanent disability in both feet: From Jume sixth, eightecn hundred and sixty-six, twenty dollars; from June fourth, eighteen hundred and screnty-two, thirty-one dollars and twenty-five cents.

For total and permanent disability in both hands: Fron Jume sixth, cighteen hundred and sixty-six, twcinty-five dollars; from June forrth, cighteen hundred and seventy-two, thirty-one dollars and twents-five cents.

For total and permanent disability in both hands: from Jume sixth, cighteen linndred and sixty-six, twenty-five dollars: from Jume fourth, cighteen hundred and scventy-two, thirty-one dollars and twenty-five celits.

For the loss of an arm at or above the cllow, or a leg at or above the knee: From Narch third, cighteen hundred and cighty-three, thirty dollars; from August fourth, cighteen hundren and cishty-six, thirty-six dollars; from March second, nineteen hundred and three, forty-six dollars; for the loss of a leg at the hip joint, from Mareh third, eighteen hundred and seventy-nine, thirty-seven dollars and fifty cents: for the loss of an arm at the shoulder joint, from March third, eighteen hundred and eighty-firc, thirtyseven dollars and fifty cents.

For the loss of the hearing of hoth ars: From June fourth, eighteen hundred and seventy-two, thirteen dollars: from August twenty-seventh, eightecn hundred and cighty-eight, thirts dollars; from January fiftecnth, nineteen hundred and three, forty dollars.

For partial deafness: From August twenty-seventh, cighteen hundred and eighty-eight, such proportion of thirty clollars as the Sccrctary of the Interior may deem equitable, the amount paid to be determined by the degree of disability existing in cach casc.

For such total and permancnt disability as to cause utter helplessness, such as to require regular personal aid and attendance of another person: lirom June sixth, eighteen lundred and sixty-six, twenty-five dollars; from Iune fourth, eighteen hundred and scventy-two, thirty-one dollars and twenty- 
five cents; from June fourth, eighteen hundred and seventy-four, fifty dollars; from March fourth, eighteen hundred and ninety, or from the date of the certificate of the examining surgeon, or board of examining surgeons, show. ing such degree of disability, and made subsequent to said date last mentioned, seventy-two dollars.

For such disability as to cause incapacity for the performance of any manual labor, but not so much as to require regular personal aid and attendance: From June sixth, eighteen hundred and sixty-six, twenty dollars; from June fourth, eighteen hundred and seventy-two, twenty-four dollars; from March third, eighteen hundred and eighty-three, thirty doliars.

For total incapacity for ferforming manual labor causing disability to such a degrec as to require frequent and periodical, though not regular and constant, personal aid and attendance of another person: From the date of the certificate of the examining surgeon or board of examining surgeons showing such degree of disability, and made subsequent to the fourteenth of July, eighteen hundred and ninety-two, fifty dollars.

There is also provision made for disability pensions, graded according to rank from $\$ 30.00$ maximum for Lieut. Colonels, or equivalent officer, and higher, to $\$ 8.00$ a month for an enlisted man. In case a person who has seen service in the Civil War dies and who is pensionable under one of the foregoing sections, his widow is entitled to a pension of $\$ 12.00$ a month, with $\$ 2.00$ a month additional for each child under sixteen years of age. Upon the death of any soldier of the Civil War, his widow, if she is compelled to earn her living by manual labor, or has an income not exceeding $\$ 250.00$ a year, or has minor children under the age of sixteen years, is entitled to a pension of $\$ 8.00$ a month, with $\$ 2.00$ a month extra for each minor child. Such pensions terminate, except as to the children, when the widow remarries. If the person entitled to pension by reason of his service, dies without leaving a widow or children, those who were dependent upon him for support, are entitled to be pensioned as dependent relatives, in the following order: First, the mother, then the father, then the orphan brothers or sisters, who may be under sixteen years of age. Dependent relatives of the Civil War arc naturally practically non-existent by reason of the limitation of time, as to new applications, although the payment of dependent relative pensions already granted will still continue for a few years. In order to secure a dependent relative's pension, it was necessary to establish the fact of the dependency upon the deceased soldier for support. 
In addition to the foregoing, provision is made by statute for the pensioning of men of the Navy who have had twenty years service, and who are entitled on the expiration of that service to one-half the monthly rating at discharge. After ten years' service and upon disability, a naval pension can be granted, but not exceeding the rate provided for by law for total disability, the rate to be paid being fixed by the Secretary of the Navy. Applications for pensions from the Naval Pension Fund must be made to the Secretary of the Navy, and preliminary correspondence relating thereto, together with the determination of the pension to be paid, are under the jurisdiction of the Chief of Bureau of Navigation, of the Navy Department. Other applications are addressed to the Commissioner of Pensions, and should set forth the company and regiment in which the service was had, the name of the commanding officer of the company or organization, and the date of enlistment and discharge, with a personal description at enlistment. In Navy cases, the vessel upon which the claimant served should be stated. Further requirements of evidence in support of claim for pension are required if the records of the War or Navy Departments are not complete as to the service of the applicant.

Applications are referred to one of the following divisions, of which the First Deputy Commissioner has supervision over the Eastern, Southern and old war Navy divisions, and the Second Deputy Commissioner over the Midcle Division and the Western Division.

Old war and nazy division.-To have charge of the settlement of all claims for bounty land and for pension on account of scrvice in wars prior to March 4, I86I; claims on account of service in the Regular Army and Navy; claims on account of service in the war with Spain and the insurrection in the Philippine Islands; and in all other general organizations not belonging specially to any State or Territory.

Eastern dirision.-To have charge of the settlement of all claims arising out of military service during the war of the rebellion in organizations from the several New England States, and from the States of New York, New Jersey, and Delaware.

Middle division.-To have charge of the settlement of all claims on account of service in the war of the rebcllion in organizations belonging to the States of Pennsylvania, Ohio, and Micliigan.

Western division.-To have charge of the settlement of claims on ac- 
count of service in the war of the rebellion in organizations belonging to the States of Indiana, Illirois, Iowa, Wisconsin, Minnesota, Nebraska, Kansas, Nevada, Colorado, California, Oregon, Washington, North and South Dakota, Idaho, Utah, Montana, Wyoming, and the several Territories.

Southem division.-To have charge of the settlement of claims arising out of military service during the war of the rebellion in organizations belonging to the States of Maryland, Virginia, West Virginia, North and South Carolina, Florida, Georgia, Alabama, Mississippi, Louisiana, Texas, Kentucky, Tennessee, Missouri, Arkansas, the District of Columbia, and the several organizations of United States colored troops.

Other divisions have charge of the various steps in the progress of an application, including a division for recording the application and final files, the applications being separated into those which are admitted and those which have been abandoned by the applicants, no evidence having been filed for three years.

For the determination of the physical condition of applicants boards of pension exaniners are appointed from local physicians throughout the country who receive a fee of two dollars for each person examined if not more than five appear in one day and one dollar each for additional examinations on that day. The results of their examination are reviewed by medical reviewers in the Pension Bureau and a medical referee is provided who is the court of last resort and passes finally on disputed questions relating to physical condition and the effects of service.

A Division of Special Examination is established, having charge of claims which require special examinations in the field. Such examination is carried out by a corps of special examiners who receive from $\$$ I,200 to $\$$ I,500 a year, besides a per diem of $\$ 3$ a day for each day in the year, and who are charged with the duty of examining into the statements made by applicants for pensions, of verifying the evidence and of securing additional evidence, when deemed advisable, as well of investigating circumstances which indicate that a fraud is being attempted under the pension laws. When such fraud seems to have been committed either in the matter of furnishing false evidence, or in making false affidavits, the evidence necessary to convict such persons is placed in the hands of United States District Attorneys for prosecution, the special examiners assisting such attorneys in arranging and presenting the evidence in the cases. The work of the special examiners takes them to all parts of the 
United States, and the Division has charge of their movements and of auditing their expense accounts and all other details relative to the examination of pension matters in the field.

Payments of pensions are made by pension agents, appointed by the President by and with the advice and consent of the Senate for four years and who receive four thousand dollars a year. Provision is made for the arrangement of the agencies in groups, one of which makes its payments in each month in the quarter. Pension agencies are given such territory to cover as may be practicable to be handled from one office and two agrencies cannot be established in one State unless the payments made are more than five hundred thousand dollars a year. The office in Washington, D. C., besides handling payments in contiguous territory, pays the foreign pensions, considerable amounts being paid to pensioners living abroad. Vouchers are prepared at the pension agencies quarterly and sent out to pensioners and upon their return properly executed before a competent official, checks for the amounts of pensions due are forwarled by mail to the beneficiaries, although the Commissioner of Pensions may require any pensioner to appear in person to receive his pension.

The large proportion of the pension business in Washington is attended to on behalf of the claimants by attorneys, for whom a specific fee is fixed of $\$ 10$ for cach case of original pension granted and $\$ 2$ for each increase. For the convenience of attorneys practicing before the Pension Bureau, a special room is providerl and attorneys are allowed to examine the cases on file under certain conditions.

THE COMMISSIONER OF INHAN AFFAIRS.

There has never been a time in the history of the United States when some question relating to the affairs of the Indians or aboriginal inhabitants of this country was not under discussion and requiring delicate and skilful landling. In the inevitable conflict between the Inclians, who preferred to have large tracts of their territory remain unsettled, in order that they miglit pursue their nomadic and hunting lives, and the European settlers who came to this country anxious to establish lomes and holdings of real estate to be highly developed, there was no possibility of a continuously peaceful joint tenancy of North America. That the Indians have been in 
many instances treated with injustice is undoubtedly the fact, and from the nature of things such a result was inevitable. It is, however, a matter of which arguments may be made on both sides, that, except for the evil results arising from the introduction of the vices of the white man, the condition of the Indian has been, if anything, throughout the history of the United States, superior to the existence led by his predecessors in matters of personal comfort. The change necessitated from a roving life to living within a restricted area, and the acquisition of vices before referred to, have led to the extinction of many of the tribes, but those which have been able, in some degree, to accustom themselves to the manner of life of the white man, have not only survived but have amalgamated with the white race to a certain extent, and the pure Indians and their halfbreed descendants, have, in a very large number of instances, attained considerable property and on the whole, judging from the position of the Indian race at the present time, have been treated with some degree of liberality, if not always with justice.

It has been the consistent aim of the Federal Government to provide as far as possible for the Indian wards of the Government and to protect them against the encroachments and inroads of the settlers ardently desirous of cultivating the land over which the Indians roved and hunted, and which use according to the Anglo-Saxon nind conveyed no title or interest which the prospective agriculturists and developers were bound to recognize. By successive treaties the United States has endeavored to repay the Indian tribes for the lands from which they were removed in order to make room for settlers, and in the final event of the removal of the bulk of the eastern tribes to the Indian Territory, it was provided that members of the tribes should have legal rights in the land within the limits of that territory. As population surrounded the Indian Territory, and as oil and mineral deposits were developed by the white man which made the Indians' lands of very considerable value, arrangements were made by which the proceeds of the development of such lands should accrue to the Indians themselves, and when it became apparent that the best interests of the Indians would be subserved by the division of the land of the Territory, the United States undertook such allotment at the cost of much trouble and with the ex- 
penditure of much money. The members of the Five Civilized Tribes of Indians and others which had been removed from east of the Mississippi to the Indian Territory, were enrolled under the direction of a Commission, which took evidence intended to prevent unauthorized persons from being registered as members of the tribe. To each of the persons lawfully enrolled on such lists an allotment was made of a portion of land in the Indian Territory, with a provision of guardianship to minors, and of restriction of sale of the land for a term of years, and upon the completion of such allotment and of the removal of the restriction, the Indians affected will be no longer the wards of the nation, but will have no different standing from that of any other American-born citizens. The supervision of this allotment, together with the direction of the affairs of the Indian tribes which have not yet come to the point where an allotment of land can safely be made to them, is under the Commissioner of Indian Affairs and the head of the Indian office of the Interior Department. The Commissioner is appointed by the President, by and with the advice and consent of the Senate, and receives a salary of $\$ 5,000$ a year. He is assisted by an Assistant Commissioner at $\$ 3,000$ a year, and under the direction of the Secretary of the Interior and agreeably to such regulations as the President may prescribe, the Commissioner of Indian Affairs has the management of all Indian matters and all questions arising out of Indian relations.

The chief duty of the Commissioner of Indian Affairs, previous to the adoption of the plan of allotment of lands, was in the matter of supervision of the agencies established for the purpose of supplying to the Indians the goods and supplies contemplated by treaty provisions for the support of Indians on the reservations. Agents are appointed by the President by and with the advice and consent of the Senate, for the superintendence of subdivisions of the land designated as reservations within which the various Indian tribes shall live. The compensation of such agents is fixed by act of Congress, but in lieu of such regularly designated Indian agents, superintendents of Indian schools, or military officers, may be appointed as agents, in addition to their other duties, and as such receive no additional compensation. Agents are required to distribute to the Indians under their charge the supplies and provisions purchased by 
the appropriations made by Congress for the support of the various Indian tribes and to disburse to them the amounts which are directly paid in consequence of treaty provisions. Agents are also required to see that no intrusion is made on the reservation on the part of unauthorized persons, and that the Indians are not injured in any way in persons or property. In order to see that abuses do not arise in the Indian Service, such abuses having at times caused serious losses and distress to the Indians, special agents are employed by the Indian Office who travel about among the reservations, inspecting the conduct of the business relating to the Indians, and the conditions of the wards of the Government, such agents being on salary with an allowance of $\$ 3.00$ a day for subsistence, besides cost of transportation when in the field. Besides this inspection, there is a Board of Indian Conmissioners, composed of not more than ten persons, appointed by the President, of men eminent for philanthropy who have a paid secretary, but otherwise serve without compensation, and who have a general oversight of affairs relative to the Indians, bcing empowered to investigate all contracts, expenditures and accounts connected with the Indian service, and having access to all papers relating thereto. Agents of the Indian Service are also expected to supervise the agricultural and mechanical work which is undertaken at the different reservations for the benefit of the Indians and for the purpose of assisting and encouraging them in undertaking agriculture and other work for themselves. Carpenters, machinists and other mechanics are paid out of the appropriations made by Congress to render service on the different reservations and to instruct the Indians in the various branches of their work.

Various payments for the benefit of the tribes under treaty obligations have been made into the Treasury of the United States, and other payments are provided by treaty to be made annually and are appropriated by the Congress. All payments under these various provisions are under the direction of the Secretary of Interior, although the Commissioner of Indian Affairs has active control and practical direction through his advisory capacity to the Secretary. It is the present policy of the Indian affairs of the United States to render the Indians self-supporting and to bring them out from their dependent condition as soon as practicable. To this end there have 
for many years been maintained a system of schools for the education of the Indian children, some of the schools being day schools on the reservations and others boarding schools, to which children were moved from their homes in order, in part, to remove them from parental influence, which makes the progress of education more difficult. For each of these schools, a staff is provided including a superintendent, matron, and teachers in educational branches and in the various branches of agriculture and mechanics, and although the system has been carried on under many difficulties, and with many discouragements. its beneficial effects are at the present time being substantially realized.

Among other provisions that have been insisted upon by the United States for the protection of the Indians, is that the sale of liquor shall be prohibited wherever there are Indian wards, and to such dependents of the nation. The law with relation to the introduction of liquor into the Indian Territory has been rigilly enforced, and it was made a condition of the admission of the State of Oklahoma into the Union that the prohibition of the introduction of liquor into the unallotted Indian lands should be continued for a period of twenty years. The new state, however, exceerled the congressional requirement in this particular, and voted for prohibition throughout the entire State, thus maintaining the protection which the United States has sought to throw about it Indian wards arainst the one of the vices of the white man most injurious to the Indian. The prohibition against the sale of arms and ammunition to Indians, especially those occupying hostile territory, has also been rigidly enforced, but the necessity of such enforcement is now practically ended.

The Commissioner of Indian Affairs is required to keep a record of every deed executed by an Indian which requires the approval of the Secretary of the Interior or the President of the United States, and is also charged with the general oversight of matters relating to leasing Indian lands, and of granting the right of way over such lands. The Secretary of the Interior is the official guardian of all Indians who still retain a dependent character as regards the United States, and has during the past ten years been compelled to pass upon matters of very serious moment in connection with the various oil and mineral leases proposed of Indian lands for purposes of the 
Government. It has, of course, been the object of the proposers of such leases to secure the privileges desired at the least possible cost to themselves, and it has been the duty of the Secretary of the Interior to safeguard the Indians' interests, which duty has been so well performed that membership of a tribe in the Indian Territory, especially of the Five Civilized Tribes, is a privilege which is being souglit by appeals to the courts on the part of those who have been refused admission to the lists and who have a color of a title to be regarded as members of the tribe and consequent participants in the benefits which have accrued from the lands set aside by the United States for the Indians and which are now of very considerable value.

THE COMMISSIONER OF PATENTS.

The Congress has been given power by the Constitution to secure to inventors and original producers of literature and art the right to their inventions and products for a term of years, in order that invention and originality of thought may be encouraged. The administration of the laws relating to this matter is entrusted to the Commissioner of Patents, who is appointed by the President by and with the advice and consent of the Senate, and receives a salary of $\$ 5,000$ Exaniners in Chief, at $\$ 3,000$ a year each, who are appointed by the President and Senate, and who undertake the specific work of examining and passing upon applications for patents, leaving to the Commissioner of Patents the general administrative work and the exercise of a function as the court of final appeal in patent decisions. The conditions under which patents can be obtained, and the material requirements are given in the following paragraphs:

Applications for letters patent of the United States must be made to the Commissioner of Patents, and must be signed by the inventor, if alive. A complete application comprises the first fee of $\$_{15}$, a petition, specification, and oath; and drawings, model, or specimen when required. The petition, specification, and oath nust be in the English language. All papers which are to become a part of the permanent records of the office must be legibly written or printed in permanent ink.

An application for a patent will not be placed upon the files for examination until all its parts, except the model or specimen, are received.

The application must be completed and prepared for examination within one year after the filing of the petition; and upon failure of the applicant to 
prosecute the same within one year after any action thereon, the application will be regarded as abandened.

The petition must be addressed to the Commissioner of Patents, and must state the name, residence, and post-office address of the petitioner requesting the grant of a patent, designate by title the invention sought to be patented, contain a reference to the specification for a full disclosure of such invention, and must be signed by the applicant.

Tha specification is a written description of the invention or discovery and of the manner and process of making, constructing, compounding, and using the same, and is required to be in such full, clear, concise, and exact terms as to enable any person skilled in the art or science to which the invention or discovery appertains, or with which it is most nearly connected, to make, construct, compound, and use the same.

The following order of arrangement should be observed in framing the specification:

(I) Preamble stating the name and residence of the applicant and the title of the invention.

(2) General statement of the object and nature of the inverition.

(3) Brief description of the several views of the drawings (if the invention admits of such illusiration).

(4) Detailed descrintion.

(5) Claim or clains.

(6) Signature of mventor.

(7) Signatures of two witnesses.

The specification must be signed by the inventor or by his executor or adminisirator, and the signature must be attested by two witnesses. Full names must be given, and all names, whether of applicants or witnesses, must be legibly written.

Two or more independent inventions camnot be claimed in one application; but where several distinct inventions are dependent upon each other and mutually contribute to produce a single result, they may be claimed in one application.

The applicant, if the inventor, must make oath or affirmation that he does verily believe himself to be the original and first inventor or discoverer of the art, machine, manufacture, composition, or improvenent for which he solicits a patent; that he does not know and does not believe that the same was ever before known or used, and shall state of what country he is a citizen and where he resides, and whether he is a sole or a joint inventor of the invention claimed in his application.

The oath or affirmation may be made before any person within the United States authorized by law to administer oaths, or, when the applicant resides in a foreign conntry, before any minister, chargé d'affaires, consul, or conmercial agent holding sommission under the Government of the United States, or before any notary public, judge, or magistrate having an official seal and anthorized to administer oaths in the foreign country in which 
the applicant may be, whose authority shall be proved by a certificate of a diplomatic or consular officer of the United States.

The applicant for a patent is required by law to furnish a drawing of his invention whencver the nature of the case admits of it.

The drawing may be signed by the inventor, or the name of the inventor may be signed on the drawing by his attorney in fact, and must be attested by two witnesses. The drawing must show every feature of the invention covered by the claims, and the figures should be consecutively numbered if possible. When the invention consists of an improvement on an old machine the drawing must cxlibit, in one or more views, the invention itself, disconnected from the old structure, and also in another view so much only of the old structure as will suffice to show the connection of the invention therewith.

Preliminary examinations will not be made for the purpose of determining whether models are required in particular cases. Applications complete in all cther respects will be sent to the examining divisions, whether models are or are not furnished. A model will only be required or admitted as a part of the applicaticn when on examination of the case in its regular order the primary examiner slatl find it to be necessary or useful.

A caveat, under lie patent law, is a notice given to the Patent Office of the caveator's claim as inventor, in order to prevent the grans of a patent to another person for the same alleged invention upon an application filed during the life of tire caveat withont notice to the caveator.

Any person who has made a new invention or discovery and desires further time to mature the same may, on payment of a fee of $\$$ ro, file in the Patent Offec a caveat setting forth the object and the distinguishing characteristics of the invention, and praying protection of his right until he shall have matured his invention.

Nearly all the fees payable to the Patent Office are positively required by law to be paid in alvance-that is, upon making application for any action by the Office for which a fee is payable.

The following is the schedule of fees and of prices of publications of the Patent Office:

On filing each original application for a patent, except in design cases.. \$15.00

On iesuing each original patent, except in design cases.......... 20.00

In design cases:

For three years and six months ....................... 100

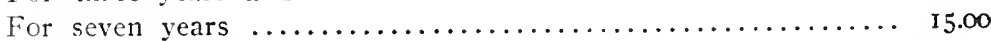

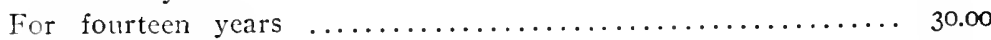

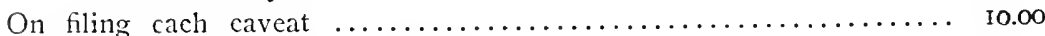

On every application for the reisste of a patent................... $3^{0.00}$

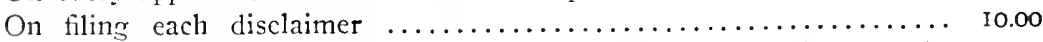

On an appeal for the first time from the primary examiner to the

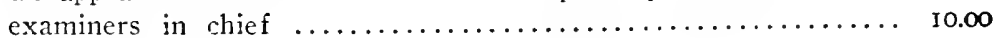

On every appeal from the examiner in chief to the Commissioner... 20.00 
For certified copies of patents if in print:

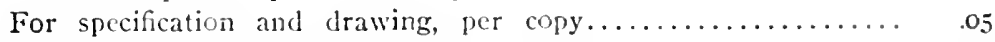

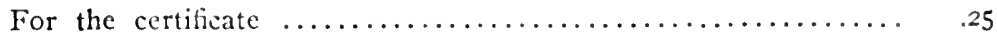

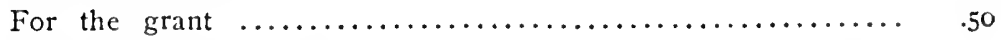

For certifying to a duplicate of a model $\ldots \ldots \ldots \ldots \ldots \ldots \ldots \ldots . .50$

For manuscript coples of records, for cvery roo worls or fraction

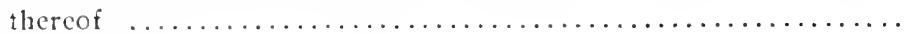

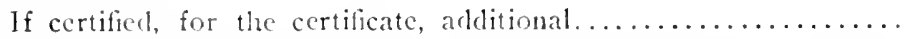

For 2o-coupon orders, each coupon good for one copy of a printed specification and drawing, and reccivable in paynent for prints,

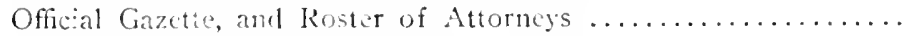

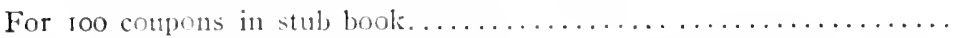

For uncertified cepes of ihe specifications and accompanying drawings

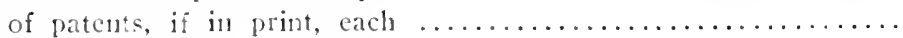

When ordered by subclasses, each $\ldots \ldots \ldots \ldots \ldots \ldots \ldots \ldots \ldots$

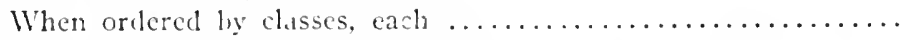

And when the entire set of all patents granted is ordered, each...

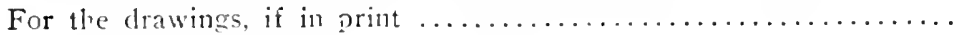

For copies of drawings $110 \mathrm{t}$ in print, the reasonable cost of making them.

For photo prints of drawings, for each shect of drawings:

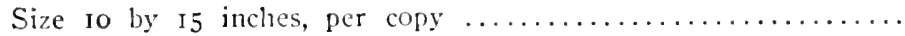

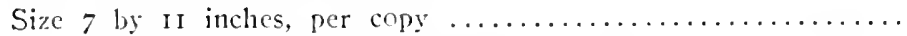

For recording every assignment, agreement, power of attorney, or

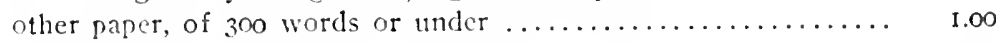

Of over 300 and muder $\mathrm{I}, 000$ words $\ldots \ldots \ldots \ldots \ldots \ldots \ldots \ldots . \ldots \ldots$

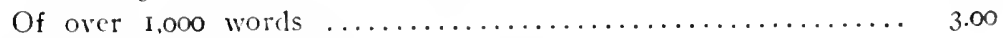

For abstracts of title to patents or inventions:

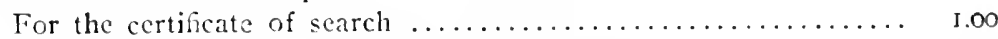

For each bricf from the digest of assignments........... 20

For searching titles or recends, one lour or less.............. 50

Each additional hour or fraction therenf.................. 50

For assistance to attorneys in the examination of publications in the scientific library, one hour or less ....................... I

Each adclitional hour or fraction thereof ................ 1.0

For copies of matter in any foreign langwage, for every soo words or

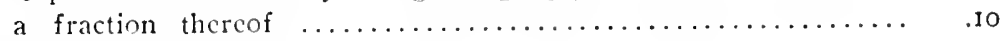

For translation, for erery 100 words or fraction thereof.......... .50

The Official Gazette:

To subscribers within the United States, Mexico, and Canada,

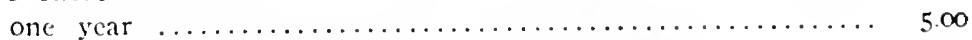

To foreign subscribers, except in Canada and Mexico........ ro.oo

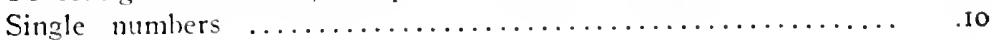

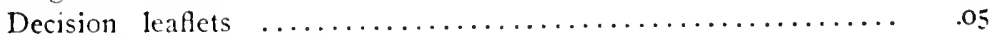

Trade-mark supplements $\ldots \ldots \ldots \ldots \ldots \ldots \ldots \ldots \ldots \ldots \ldots \ldots \ldots . .65$ 
For bound volumes of The Official Gazette:

Semi-annual volumes, from January I, 1872, to June 30 , I883,

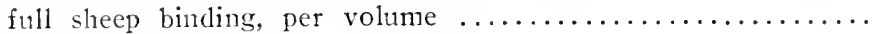

In half sheep binding, per volume $\ldots \ldots \ldots \ldots \ldots \ldots \ldots \ldots \ldots$

Quarterly volumes, from July I, I883, to December 31, 1902, full

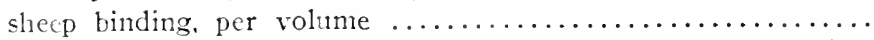

Bi-monthly volumes, from January I, 1903, full sheep binding,

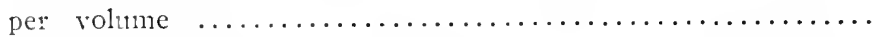

For the annual index-from January, I872-fuil law binding, per

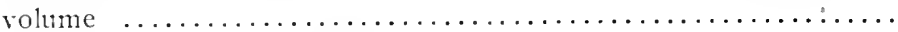

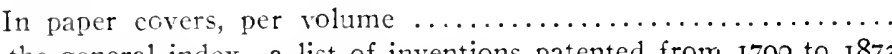

For the general index-a list of inventions patented from 1790 to 1873 -three volumes, full law binding; per set .............. 10.00

For the index from 1790 to 1836 -one volume, full law binding......

For the monthly rolumes, containing the specifications and photolithographed copies of the drawings of all patents issued during

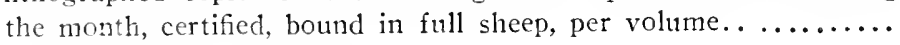

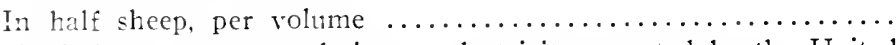

For the index to patents retating to electricity, granted by the United States prior to June 30,1882 , one volume, 250 pages, bound......

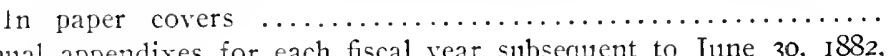

Annual appendixes for each fiscal year subsequent to June 30,1882 , paper covers

For Commissioner's Decisions:

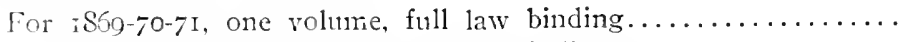

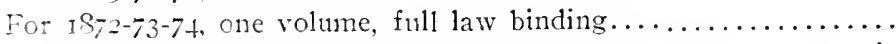

For IS $75-76$, one volume, with decisions of United States courts in

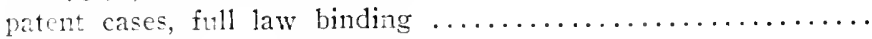

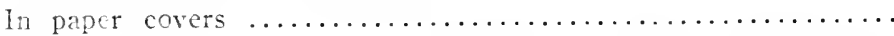

Subsequent annual volumes with decisions of United States courts,

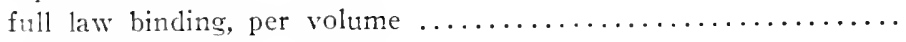

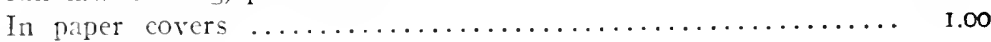

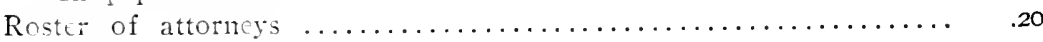

In case an application is made for a patent which the Commissioner of Patents believes interferes with a pending patent application or prior patent, he directs the examiner to proceed to determine the auestion of priority of invention. Affidavits and depositions may be taken in such matters by direction by the Commissioner of Patents, and by any officer authorized by law to take depositions: under the proceedings of United States Courts or State Courts. Witnesses may be subpœnaed for such purpose and hearings may be had by the subordinates of the Commissioner, or by the Commissioner on appeal. The Commissioner may issue a patent to the party who is adjudged 
to be the prior inventor, either upon the results of the decision of the primary examiner or of the Board of Examiners in Chief, or on an appeal taken to the Commissioner within twenty days. Anybody aggrieved by the decision of the Commissioner of Patents may appeal to the Court of Appeals of the District of Columbia. The decision is reviewable in this court, which can certify to the Commissioner of Patents, after duc hearing, its opinion as to the action which should be taken by the Commissioner, and such opinion on the part of the Court of Appeals must govern the Patent Office in future proceedings in the case. The decision of this court, however, is not exclusive of any action which may be brought in the appropriate court cf the United States to test the validity of the patent, so that a patent case may be fought from the moment of its submission to the primary cxaminer through the Court of Appeals of the District of Columbia, then reopened in an United States Court and made the subject of litigation through the Fecleral procechure to the final determination of the Supreme Court of the United States. The possibility of such litigation and the fact that the decision of the Commissioner of Patents as to the patentalility of a given device can be contested in litigation which may last over many years and which may not in the end be fruitful, owing to the lapse of time or the inability to rccover adcquate amount in clamages, has been a source of complaint on the part of inventors against the American patent systen. so that propositions have been made by patent lawers for material changes in the laws on this subject, the consensus of opinion that changes should be made being now so strong that definite amendments are to be presented to the Congress for its consideration.

Articles which may be protected by a tracle-mark are also under the jurisdiction of the Commissioner of Patents, and such protection is granted under the circumstances inlicated in the following paragraphs:

A trade-mark may be registered by any person, firm, co:poration, or association domiciled within the territory of the Lnited States, or resilling in or located in any foreign country which. by treaty, convention, or law, affords similar privileges to the citizens of the United States, and who is entitled to the use of any trade-mark and uses the same in commere with foreign nations, or among the several States, or with Indian trihes, upon payment of the fee required by law and other due proceedings had. 
No trade-mark will be registered to an owner domiciled within the territory of the United States unless it shall be made to appear that the same is used as such by said owner in commerce among the several States, or between the Uniterl States and some foreign nation or Indian tribe; no trade-mark, cxcept as provided by section 3 of the act of May 4, 1906, will be registered to an owner residing in or located in a foreign country unless said country, by treaty, convention, or law, affords similar privileges to the citizens of the United States; no trade-mark will be registered which consists of or comprises immoral or scanclalous matter, or which consists of or comprises the flag or coat of arms or other insignia of the United States, or any simulation thercof, or of any State or municipality, or of any foreign nation, or which is identical with a registered or known trade-mark owned and in use by another, and appropriated to merchandise of the same descriptive properties, or which so nearly resembles a registered or known trademar: owned and in use by alother, and appropriated to merchandise of the same descriptive properties, as to be likely to cause confusion or mistake in the mind of the public, or to deceive purchasers; or which consists merely in the name of an individual, firm, corporation, or association, not written, printed, impressed, or woven in some particular or distinctive manner or $\mathrm{j}_{11}$ association with a portrait of the individual, or merely in words or devices which are descrintive of the goods with which they are used, or of the character or quality of such goods, or merely a geographical name or term: no portrait of a living individual will be registered as a trade-mark, except by the consent of such individual, evidenced by an instrument in writing; and no trade-mark will be registered which is user in unlawful business, or upon any article injurious in itself, or which has been used with the design of deceiving the public in the purchase of merchandise, or which has been abandoned.

An application fur the registration of a trade-mark must be made to the Commissioner of Patents and must be signed by the applicant.

A complete appiication comprises:

(a) A petition, requesting registration, signed by the applicant. (See Form I.)

(b) A statement specifying the name, domicile, location, and citizenship of the party applying; the class of merchandise, and the particular description of goods comprised in such class, to which the trade-mark is appropriated; a description of the trade-mark itself, only wlon needed to express colors not shown in the drawing; and a statement of the mode in which the same is applied and affixed to the goods, and of the length of time during which the trade-mark has been used, and, if the applicant be a corporation or association, it nust be set forth under the laws of what State or nation organized.

(c) A declaration complying with section 2 of the act of February 20, 190j. 
(d) A drawing of the trade-mark, which shall be a facsinilu of the same as actually used upon the goods.

(e) Five spccincens (or facsimiles, when, from the mode of applying or affixing the trade-mark to the goods, specimens cannot be furnished) of the trade-mark as actually used upon the goods.

(f) A fee of $\$$ io.

FEES.

On filing each original application for the registration of a trade-mark $\$ 10 . \infty$

On filing each application for renewal of the registration of a trademark ................................. $10 . \ldots$

On filing notice of opposition to the registration of a trade-mark... I0.00

On appeal from the examiner in charge of trade-marks to the Com-

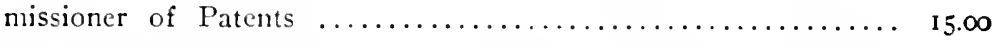

On appeal from the decision of the examiner in charge of interferences, awarding ownership of a trade-mark or cancelling the registration of a trade-mark, to the Commissioner of Patents .. 15.00

For manuscript copies, for every 100 words or fraction thereof..... .10

For recording every assignment, agreement, power of attorney, or

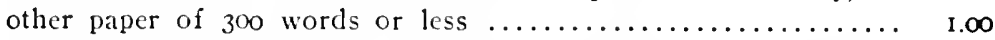

For recording every assigmment, agreement, power of attorney, or other paper of more than 300 words and less than 1,000 words. .

For recording every assignment, agreement, power of attorney, or

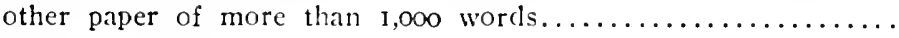

For abstracts of title:

For the certificate of search....................... I.

For each brief from the digest of assignments............ 20

For searching titles or records, one hour or less.......... 50

Each additional hour or fraction thercof.............. ${ }_{50}$

For a single printed copy of statement, declaration, and drawing..... .05

If certified, for the grant, additional.............. 50

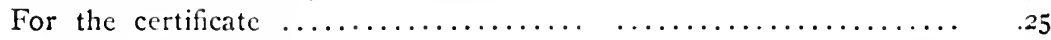

Money required for office fecs may be paid to the Commissioner of Patents, or to the Treasurer, or to any of the assistant treasurers of the United States, or to any of the expositaries, national banks, or reccivers of public money, designated by the Secretary of the Treasury for that purpose, and such officer shall give the depositor a receipt or certificate of deposit therefor, which shall be transmitted to the Patent Office. When this cannot be done without inconvenience, the money may be remitted by mail, and, in every such case, the letter should state the exact amount inclosed. All money orders, drafts, and checks should be made payable to the "Commissioner of Patents." 


\section{THE GEOLOGICAL SURVEY.}

The primary and main purpose of the Geological Survey is to establish definitely the character of the public lands of the United States, in order that such lands as contain mineral deposits of value may not be allotted under the homestead laws or sold as agricultural lands, so that the United States will be deprived of the income which should be received from the sale of lands of greater value than those which are available for agricultural purposes only. In addition to this service, it is also necessary in connection with the administration of the public lands to have fixed marks as starting points for the surveys which establish the subdivisions of the public lands. The services referred to are performed by field parties of the Geological Survey, which conduct various investigations into the geological structures, the mineral resources and the products of the public lands, and also perform survey work necessary for the establishment of the limits of reserved lands, as well as for the fixing of primary lines for sectional surveys. This work, under the Secretary of the Interior, is in charge of the Director of the Geological Survey, who is appointed by the President by and with the advice and consent of the Senate, and receives a salary of $\$ 6,000$ a year. It is required by law that neither the Director nor any member of the Survey shall have any personal or private interest in the lands or mineral wealth of the region under survey, and, also, in addition to the public services performed, shall execute no surveys or examinations for private parties or corporations while employed in the work of survey or examination for the public service. All scientific employes of the Geological Survey are selected by the Director subject to the approval of the Secretary of the Interior, and must be selected exclusively for their qualifications as professional experts. The results of the work of the survey are prepared in the form of maps, both topographical and geological, and such maps are made up into the form of atlases, each treating of a different section of the country. An edition of 500 copies of such maps and atlases is provided to be distributed gratuitously among the educational institutions of the United States and to foreign countries, and each Senator and Member of the House of Representatives is entitled to a personal copy of each map and atlas, and an additional copy to be disposed of at his discretion. 
The publications of the Survey can also be sold at an amount equal to the actual cost of their production, with ro per cent. additional, the cost of production meaning the cost of the copies themselves, without reference to the original cost of the work.

Besides the economic value of the work of the Geological Survey in determining the claracter of the lands of the United States it has had a very great educational value, from its contributions to the science of geology generally, and especially with reference to the fossil remains of past ages. The Survey, in addition to the maps and atlases referred to, issues a publication showing the mineral resources of the United States, and is also charged with an investigation of the character and value of the coals of the country. This investigation is carried on for the purpose especially of determining the fuel value of the different coal deposits and is of great importance for the reason that coal-bearing strata of widely different characteristics are found in various points in the Western States, and the comparison of their fuel value and their uscfulness is necessary in order, first, to estimate the value of the land under which they are found, and secondly, to promote the development of the mining operations which would make the surrounding land of additional value to settlers and to manufacturers by reason of ready access to sources of fuel. This comparison of fuels, which is imposed upon the Geological Survey by law, is made not only by actual examination of conditions in the field, but by fuel tests under usual power-producting conditions and material additions to the knowledge of the world on this subject has been made by the work of the Survey.

Another section of the Geological Survey is devoted to questions relating to streams, water courses and water supply. For a number of years an exhaustive series of investigations was undertaken which included the measurement of streams and the determination of the location of underground waters for the plirpose of giving some approximate rule for the successful drilling of artesian wells. As the agricultural lands of the West became scarcer and it became apparent that efforts must be made to reclaim the desert arid lands, in order to provide for the iemand for new homesteads, this section of the Geological Survey became interested in the question of the reclamation of arid lands, and under recent legislation by Congress has grown to be a great engineering bureau, which has in charge the 
tremendous construction work intended to confine the flood waters of western streams into great reservoirs from which water can be secured during the growing season of the year for the irrigation of crops. Under the statutes covering the subject of reclamation of arid lands and irrigation at the present time, all moneys received from the sale and disposal of public lands in Arizona, California, Colorado, Idaho, Kansas, Montana, Nebraska, Nevada, New Mexico, North Dakota, Oklahoma, Oregon, South Dakota, Texas, Washington and Wyoming, except the five per cent. granted to States for educational purposes, together with all excess fees from the offices of registers and receivers of public money, are paid into a common fund known as the "Reclamation Fund," from which the Secretary of Interior is empowered to take such sums as may be necessary for the acquirement of sites for dams and reservoirs, and for the construction of such work as may be required to impound waters for the purpose of subsequently using them in the work of irrigation.

Such amounts of arid lands as the Secretary of the Interior may deem to be likely to be benefited by the construction of irrigation work are to be withheld from the operation of the homestead laws until such time as the irrigation projects have been completed, when they are to be allotted out in tracts of not less than forty nor more than one hundred and sixty acres, under such laws, except that there is to be an added charge, based upon the cost of the irrigation work, which is to be divided among the whole amount of land so withheld. When a majority of the amount thus fixed as a charge upon the land is paid, the irrigation works are to pass into the custody of those owning the lands irrigated under such rules as the Secretary of Interior may impose. The Secretary of the Interior is empowered to fix the amount of land which can support a family in the particular district irrigated, and to establish such amounts as the unit of entry which can be made under the homestead laws. When the payment for the irrigation charges have been made and custody and operation of the irrigation works placed in the hands of the land holders, the title to the works themselves remains in the United States, and will so remain until a different disposition is made by Congress. 
The Reclamation Service, under the direction of the Chief Engineer, has charge of all matters relating to the location, acquirement of site and building dams, and establishing reservoirs, the actual work being done so far as possible by contract, but in many instances, owing to the difficult nature of the undertaking, it has been necessary for the Reclamation Service itself to undertake the construction work. Several great projects are now under way, and the result of the plans of many years are to be realized within a short tine. All amounts which may accrue from the reimbursement to the United States from the cost of the reclamation projects are to be returned to the Reclamation Fund, which is thus made self-renewing and perpetual, and when one project is completed and paid for, funds will be available for the undertaking of another. In consequence of the various seclamation projects, present and prospective, the water rights in the streams of the West have grown to be of great value and litigation has already been set up between the various states affected to determine their respective rights. Legislation has been effected, intended to adjust the differences between the United States and the different States, and under other legislation than that which has just been described, the States in which arid lands are located are also given opportunity to construct irrigation reservoirs and to meet the expenses thereof by grants of land of the United States, which have been made in general terms by Congress conditional upon the undertaking of reclamation work by the States.

\section{THE COMMISSIONER OF EDUCATION.}

No provision was made by the Federal Constitution for the establishment or maintenance of a school system by the Federal Government, and no powers in this direction have been assumed by the Congress. Whether a national school system might not be of great national value, especially in the South, is a matter which may well have consideration, but there appears to be no provision by which such a result could be brought about. The only function exercised hy the Federal Government in connection with education is an advisory and instructive one, besides the assistance of certain agricultaral colleges and the education of children in Alaska. These are carried on under the direction of the Commissioner of Education, an 
official of the Department of the Interior, who is appointed by the President by and with the advice and consent of the Senate, and receives $\$ 3,500$ a year. The duties of the office of education are to collect statistics and facts showing the condition and progress of education in the several States and territories and to disseminate such information respecting the organization and management of schools and school districts and the methods of teaching as shall aid the people of the United States in the establishment and maintenance of cfficient school system and otherwise promote the cause of education throughout the country. To this end, the Commissioner of Education is authorized to publish bulletins and annual reports which are generally distributed and may be had on application. In addition to the foresoing, the Commissioner of Education has general supervision of the operation of the endowment fund, formerly consisting of a portion of the returns from the sale of public lands, which was granted to the states in which public lands are or have been located, for the teaching of agriculture and mechanic arts. As in many states the public lands have been entirely disposed of, annual appropriations are now made for the continuance of the support of these institutions, and it is the duty of the Commissioner of Education to sce that the requirements established by law are carried out.

The education of Indians and Esquimaux children of Alaska, together with the education of white children in that Territory is exclusively under the direction of the Commissioner of Education. Various funds, arising from the revenues of the territory are set aside for the purposes of education, and are applied so far as they will go to the provisions of schools. The Commissioner of Education also directs the operations of the reindeer establishments, consisting of the reindeer imported from Siberia some years ago, and their increase. The main herds are maintained at central stations, where the natives are instructed in the care and management of the reindeer and the increase beyond the number required for the maintenance of the original herd are intrusted to graduates for safe keeping, the Indians selected for the care of such additional herds being allowed to keep the increase from the herd in payment of their services and for encouragement. The introduction of reindeer into 
Alaska was undertaken for the purpose of finding a substitute for the decreasing food supply of the Inclians and Esquimaux, and a material relief has been afforded from such destitution of the natives who were threatened from starvation on account of the disappearance of their usual supplies of food because of the increase in settlements.

TIE NATIONAL PARKS.

Congress has from time to tine set aside sections of the Western country, which contained notable natural curiositics as National Parks, the land included within their limits being withdrawn from settlement permanently and the administration of the parks thus established is placed under the direction of the Secretary of the Interior. National parks so far established include the Yellowstone National Park with its remarkable geyser formations, the Yosemite National Park in California, including the rock formation in that valley; the Sequoia National Park in California, including the giant trees of that State; the General Grant National Park in California, which includes the mammoth tree which has been named for the famous civil war general; the Crater Lake National Park in Oregon, surrounding the lake of that name; the Mt. Ranier National Park in Washington, and the Government reservation at Hot Springs, Ark. Each of the foregoing National Parks is under the direction of a superintendent appointed by the Secretary of the Interior, who has as his assistants a sufficient number of men to patrol and guard the parks and carry out the regulations imposed for their government. Such regulations include prolibition of cutting trees and restrictions as to the taking of game and fish, together with provisions for permissive grazing of cattle on the lands included within the park reservations. Rights of way over such land, the travel of tourists and others, and all matters comnected with the use of the reservations and of the conduct of persons therein are regulated by the Secretary of the Interior, whose directions on the subject are carried out by the superintendents of the various parks.

At Hot Springs, Arkansas, the Government owns the land and the springs which are of well-known medicinal value, and rents privileges for the use of the water to private persons, under regulations as to the amount which shall be charged for the privilege of bathing 
within the bath houses. Other regulations are also established, intended to prevent imposition upon those who visit the reservation for the purpose of taking advantage of the medicinal waters. All physicians practicing in connection with the Hot Springs are required to register and rigid prohibitions are in effect against soliciting or drumming of trade in behalf of physicians from patients who visit the springs. Regulations are also made for the prevention of the waste of the water from the springs, and any person having a bath house who violates the regulations of the Department of the Interior is subject to penalty by having his lease revoked. No person is allowed to bathe or take the water, except upon a prescription from a regularly registered physician, and any physician who engages in drumming for trade or who engages in other unprofessional conduct may be debarred from the register under the regulations, so that persons applying to a physician so debarred for treatment would be unable to take advantage of the facilities of the different bath houses. In addition to leasing locations for bath houses on the reservation, the United States maintains bath houses for the treatment of the soldiers and sailors of the military service of the United States, and also free bath houses; which are, however, open only to indigent persons, being those who have not the means of comfortable subsistence. Persons who are able to pay a fee for the baths are expected to use those connected by private persons under lease, leaving the facilities of the free baths to persons who are unable to pay the bathing fees, which are fixed by the Secretary of the Interior. Attendants are permitted at the different bath houses, who are entitled to charge fees at the rate of fifteen cents for a single bath in addition to the bath house fee, and who are under the direction of the managers of the bath houses. It is optional with bathers whether they shall utilize the services of attendants or not. The regulation by the Government of the conditions at Hot Springs was made necessary by the undesirable circumstances attending the previous unregulated use of the medicinal baths. Owing to the nature of the diseases treated, patients became the victims of unscrupulous medical persons and the practice of drumming or soliciting trade had become so prevalent as to create at times positive scandal. The restrictions of the Secretary of the Interior on drumming trade at 
Hot Springs has been contested as to its legality, and a suit brought by a plyysician who was debarred from registering is now pending in the courts.

Besides the foregoing parks, reservations have been made in the Southwest of tracts in which prehistoric ruins are located, such parks being under the joint custody of the Secretary of the Interior and Secretary of War.

TIIE TERRITORIAL GOVERNMENTS.

A former very consilferable part of the work of the Secretary of the Interior had to do with the administration of the various territories which were formed ont of the public lands of the United States. These have successively been admitted to Statchood, until the territories of Arizona and $\mathrm{New}$ Mexico and of Alaska and Hawaii are all that remain under the juriscliction of the Interior Department.

The form of rovernment in the territories of Arizona and New Mexico are substantially the same, consisting of a Governor and Secretary appointed by the President, by and with the advice and consent of the Senate, who are appointed for terms of four ycars each, unless sooner removed, and of a legislative body, consisting of a Council and House of Representatives, which are empowered to make local laws. The Governor and Secretary are the representatives of the United States in the territorial affairs and are lirected by the Secretary of the Interior as to their official conduct, and all reports are made to the Interior Department. These United States officials are required to reside in the territory and can receive no compensation unless they are actual residents at their places of duty. The Governor is commander-in-chief of the militia of the territory, and may grant pardons and repricves, remit fines and forfeitures for offenses against the laws of the territory, and can grant respites for offenses against the laws of the United States, until the decision of the President can be known. He commissions all oficers appointed under the laws of the territory, and is required to see that all territorial laws are faithfully cxecuted. Every bill which passes the legislative asscmbly must be presented to the Governor, who has a veto power, similar to that of the President, and the power of the legislature to pass laws is strictly limited to legislation necessary for raising the revenues for the territorial Govermment and for local 
affairs by means of general laws on such subjects, special legislation being forbidden. The Secretary of the territory performs the usual secretarial duties, is superintendent of public buildings and grounds within the territory, administers the oath of office to the members of the legislature on being first assembled and records their proceedings. The council of the legislature consists of twelve members, and of the house, twenty-four members, the members of each branch receiving four dollars a day during the sessions, which are held once in two years, and are linited to sixty days' duration. Members of the legislature are elected for two vears, and must reside in the district or county for which they are respectively elected. An appropriation is made by Congress for the expenses of the legislative sessions, which must in no case be exceeded, and the legislature is strictly prohibited from incurring debts and expenditures which are not contemplated by the statutes or receive the sanction of Congress.

The territory of Alaska is administered by a Governor, who is required to see that the laws passed by Congress for the government of the territory are executed. He can grant reprieves from the operation of penalties imposed by the courts for the violation of the laws of the United States, but such action is subject to review by the President. The Governor of Alaska is appointed by the President and is confirmed by the Senate, and is required to make a report on the first day of October of each year to the President concerning his official transactions during the preceding year. He receives $\$ 5,000$ a year, and must reside in the territory, and with the United States courts provided for Alaska, conducts practically all the governmental business relating to the territory. The Surveyor General of the District of Alaska is Secretary ex-officio of the territory, and has charge of issuing the official papers required to be issued or secretarial acts required to be done by the statute. The body of the law governing the territory of Alaska was passed by Congress and constitutes a separate code. Such a code does not have, naturally, the elasticity which would come from enactments by a legislative body composed of the immediate representatives of the people governed, and there is a strong demand on the part of the settlers of Alaska for a system of government, including a legislative body, similar to that 
which is provided for the other territories. The three United States judges appointed for the Districts in Alaska exercise to a considerable degree the police power which would be administered by one of the regular territorial govermments through their oversight of the licensing system, and their enforcement of the criminal sections of the code. Notaries public who are appointed by the Governor have more extended functions than corresponding officials in the States, and are made registering and recording officers for the purpose of complying with the provisions of the mining laws and code sections relating to real estate transactions. The Commissioners of the United States Courts, appointed by the United States judges, also have considerable incidental govermmental powers, especially in the execution of the criminal statutes, so that the government of Alaska really consists of an administrative function exercised by the Governor as a general overseer and executive, with the rest of the governmental activities practically in the hands of United States courts.

The government of the territory of Hawaii follows the scheme of government adopted by the Republic of Hawaii before the annexation of the island to the United States with the usual territorial forms. There is a governor and treasurer appointed respectively by the President, by and with the advice and consent of the Senate, for a term of four years, and who have similar functions to those of other similar officers of territories. It is provided, however, that they must be citizens of the territory of Hawaii, and the Governor cannot be less than thirty-five years of age. The Governor has general executive duties, and the Secretary is required to preserve all the laws and proceedings of the legislature and all acts and proceedings of the Governor and promulgate the proclamations of the Governor. $\mathrm{He}$ is required to transmit copies of the journal of the Hawaiian legislature to the President of the Senate and the Speaker of the House of Representatives, and must make a semi-annual report to the President of the operations of the executive. In addition to these officials appointed by the President, provision is made for an Attorney General, a Treasurer, Auditor, Superintendent of Public Works and Superintendent of Public Instruction, who are the successors of the respective cabinet ministers under the independent republican form of government, and who are elected by the legislature. The legislative body consists of two houses, the Senate and the House of 
Representatives, who are elected by the citizens of the territory, the qualification of such citizenship being citizenship under the former government of the island. In the case of citizens of the United States who were not residents of Hawaii during the former regime, citizenship is acquired by residence of one year in the territory. The usual territorial provisions are made as to the powers and limitations of the territorial legislatures, the Senate of which is composed of fifteen nembers, who hold office for four years and the House of Representatives is composed of thirty members, who are elected to serve until the next general election. The regular sessions of the legislature are held bi-ennially on the third Wednesday in February in each odd-numbered year and cannot continue longer than sixty days, unless extended by the Governor for not more than thirty days. The Governor can also convene the legislature or the Senate alone in special session.

Although the government of the Island of Porto Rico is not under the direction of the Secretary of the Interior, a description of the chief characteristics is appropriate at this point. The officers of the government of Porto Rico, appointed by the President on behalf of the United States, constitute a complete executive staff, supervisory over the operation of a legislative body, elected by the people of Porto Rico. The chief executive officer is entitled the Governor of Porto Rico, and is appointed by the President, by and with the advice and consent of the Senate for four years, unless sooner removed. $\mathrm{He}$ is required to reside in Porto Rico during his incumbency, has the usual powers of a governor of a territory, and is required to report to the President through the Secretary of State. In addition to the Governor, the President appoints under similar conditions and for the same period, a Sccretary, an Attorney General, a Treasurer and Auditor, a Commissioner of the Interior and a Commissioner of Education, for the performance of the duties indicated by the titles of their offices. They are all required to reside in Porto Rico, and with five other persons constitute the executive council for the advice and assistance of the Governor. Either of the officers specifically named may be a Porto Rican, and of the total number constituting the Governor's council, at least five must be native Porto Ricans. The council described is constituted as one of the bodies of the leg- 
islature of the island, the other being a house of delegates, consisting of thirty-five members elected bi-ennially and the two houses are designated the legislative assembly of Porto Rico. For the purpose of electing delegates, the island is divided into seven districts, and the five delegates in each district are clecterl by the qualified voters who must have been bona fide residents of Porto Rico for one year. No person is eligible to membership in the House of Delegates who is not twenty-five years of age, and able to read and write either in Spanish or the English language or who is not possessed in his own right of taxable property situated in Porto Rico. The law thus providing for delegates either an educational or property qualification. The expenses of the officers of the Gorermment of Porto Rico are paid out of the revenues of the territory and are fixed by United States statute as follows:

The governor, cight thousand dollars; in addition thereto he shall be entitled to the occupancy of the buildings heretofore used by the chief executive of Porto Rico, wh the fuminue and effects thercin, Erce of rental.

The secretary, four thousand dollars.

The attorney-general, four thousand dollars.

The treasurer, five thousand dollars.

The auditor, four thousand dollars.

The commissioner of the interior, fout thousand dollars.

The commissioner of elucation, four thousand d.llars.

The chief justice of the suprene court, five thousand dollars.

The associate justices of the supreme court (cach), four thousand five hundrel dellars.

The marslal of the supreme cotrt, three thousand doilars.

The United States district judge, five thousand dollars.

The United States district attorney, four thousand dollars.

The United States district marshal, three thousand five hundred dollars.

Although the Island of Porto Rico is not recognized as a territory of the United States, or its citizens as citizens of the Uniterl States, provision is made by law for representation in this country by a delegate or resident Commissioner to the United States, who is paid the salary of a member of Congress, but has no seat in the House, although he is entitled to recognition by all the departments upon presentation of his certificate of election to the Sccretary of State. No person can be elected Commissioner who is not a bona fide resident of Porto Rico, thirty years of age, and who docs not read and write English. 


\section{CHAPTER XV. \\ DEPARTMENT OF AGRICULTURE.}

The theory under which the Department of Agriculture was established and under which it was carried on for many years was based upon the dissemination merely of information relative to agricultural pursuits and of seeds and plants so as to secure a wider extent of cultivation for especially valuable products as well as to encourage in each locality of the United States the raising of such crops as might best be produced at that particular point. The inception of the department occurred in 1839, when an appropriation was made for the purchase by the Commissioner of Patents of valuable seeds and plants for the purpose of securing their introduction and for general growtl within the United States. In I862 a Department of Agriculture was established under the direction of a commissioner, and in I889 a Secretary of Agriculture was provided for, and the Department of Agriculture became a full executive department.

As a result of complaints made as to the character of American products exported to European countries and the exclusion of such products from those countries provision was made by which a guarantee could be afforded to foreign consumers by an inspection under the direction of the Department of Agriculture. When, at a later date, there was a considerable agitation as to the condition under which meat products were manufactured for the interstate trade, the supervisory functions of the Department of Agriculture were considerably increased, and when as the result of other agitation a pure food law was passed by the Congress, the function of the inspection of food products was still further extended so that in addition to its original purpose the Department of Agriculture is now one of the great supervisory executive departments of the Government, having under its direction a large and growing force of Federal employees who have very considerable powers with regard to the manufacturing 
interests of the country which have to do with the supply of food products.

On the agricultural side of the Department's activities it has also advanced very far from the original undertaking of supplying new and improved varieties of vegetables and fruits, and undertakes investigation into every aspect of natural science which affects agricultural interests, and conducts experiments of the most advanced nature, being at the head of a series of experiment stations maintained by all the States and Territories in the Union and serving as a central burcau of exchange of information as well as a directory and supervisory institution. Upon the acquisition of the island possessions of the United States, the Department of Agriculture at once undertook considerable investigations into the conditions of tropical and semi-tropical agriculture, and has done a great deal of work in the development and improvement of agricultural conditions in the island possessions.

In addition to the usual equipment for administrative work in the Department of Agriculture there is provided a solicitor, who, as an exception to the general rule, is not an official of the Department of Justice, but is a salaricd officer of the Department of Agriculture and acts as legal adviser of the Secretary, having charge of the preparation and supervision of all legal papers to which the Department is a party. He also examines and approves all orders and regulations pronulgated by the Secretary under statutory authority, and represents the Department in all legal proceedings arising under the laws entrusted to the Department for exccution. He is also a member of the Board which is specially charged with the execution of the Pure Food Law.

While the purely agricultural bureaus of the Department were formerly, with the exception of the Weather Bureau, the most important phases of activity of the Department of Agriculture, these have recently become somewhat eclipsed by the increased importance of two bureaus charged with the enforcement of the specific laws. These bureaus are the Bureau of Animal Industry and the Bureau of Chemistry. The Bureau of Animal Industry as originally intended was to take up questions relating to the brecding and feeding of animals, and matters relating to dairy farming. Various enactments 
by Congress designed to prevent the inter-communication of diseases among the farm animals of the United States were placed for enforcement under the direction of the Burcau of Animal Industry, such jurisdiction being naturally the resuit of the work being done by the Bureau in the investigation of the existence of dangerous communicable diseases of live stock and of the appropriate measures for the control and the eradication of such diseases. As the bureau was also the Governmental source of information as to the characteristics of healthy and diseased food animals, the inspection of meat products for the export trade which was required as the result of discriminative legislation by foreign countries was naturally placed under the juriscliction of this Bureau. The characteristics of dairy products, being also determined by the Bureau of Animal Industry when legislation was effective designed to protect butter and cheese from destructive competition by other products containing animal fats, and resembling the dairy products mentioned, such a similar duty of inspection and supervision was imposed upon the Bureau of Animal Industry.

The amount of statutory provision thus entrusted to the Bureau of Animal Industry for enforcement is a considerable one, and contemplates the employment of a large number of inspectors expert in the various conclitions involved, whose duties are indicated by the following summary of the legislation referred to. By the Act approved June 30, Igo6, an inspection of all animals intended for slaughter is made before they enter the slaughter houses. All animals which are found to be diseased, which are pregnant, or which have recently had young, are marked with a metal tag and must be exclucled from the other animals intended for slaughter. The general conditions of slaughter houses and of places where meat products are prepared are under regulations made by the Secretary of Agriculture and enforced by the inspectors in order to secure sanitary conditions. All paits of carcasses after the slaughtering has been done are examined by inspectors who stamp on those parts which are healthy and fit for use as foods "U. S. Inspected and passed," and upon all those parts which are diseased or in any way unfit for food "U. S. Inspected and condemned." All establishments where a wholesale trade is carried on in the preparation of meat products 
are required to be inspecterl before such prorlucts may be sold or transported in interstate trade. Farmers doing their own slatuhtering and retail butchers may be exempted except in the discretion of the Secretary of Agriculture. The United States cannot, of course, interfere in any trade which is strictly within one State, but it is made an offense subject to fine or imprisonment to ship noninspected meat prolucts in the interstate or forcign trade, to transport the same, or in any way to cvade or seck to nullify the provisions of the inspection law. Establishments which are subject to inspection are given, on application for the extension $\cap \mathrm{f}$ the inspection service to such establishments, a serial number, and persons who are exempt under the law and who wish to engage in shipments in interstate trade are given certificates which warrant the transportation company in accepting their products. Evidence of compliance with the law is required by cvery transportation company to accompany each shipment of meat products in order that it may not unwittingly violate the provision of the law prohibiting such transportation and making the transportation companies liable. Animals which are condemned as unfit for siaughter and carcasses and parts of carcasses which have been condemmed a:e required to be either separately slaughtered in the former case, or in the latter cases discolored by some substance which will render them entirely unfit for food. Locked rooms are required for the retention of meat which is subject to further investigation as to its mhealthful characteristics and other locked rooms, the keys of all of which are in the custody of the inspectors, are required for the retention of condemned meats. while the stcam tanks and converters in which such meat is rendered are sealed up under the direction of the inspector and can only be opened by him or in his presence.

Regulations for the dress and sanitary surroundings of the employees of packing houses and of the sanitary condition of the houses themselves are made by the Secretary of Agriculture under the terms of the law referred to. Heavy penaltics are imposed by the law upon inspectors and others who shall attempt to defeat the requirements of the law and bribing the inspectors or giving them any valuable thing whatsoever is made punishable. Inspectors are appointed as the result of a Civil Service examination which was 
held some time since and which included the technical subjects involved. A very large eligible list was established by this examination and no further examinations will be held for some time.

Besides the slaughter house inspection described above, the $\mathrm{Bu}$ reau of Animal Industry carries on an inspection of food animals about to be exported, retaining those from shipment which are in any way diseased or which have been exposed to a contagious disease and enforcing the provisions of the law and the regulations of the department relative to the conditions which must be provided on shipboard to insure the comfort and safety of the animals during the voyage.

In order to prevent the excessive suffering of animals undergoing land transportation in the United States it is provided by law that no animal shall be confined in cars for more than twenty-eight hours during one period without being unloaded and allowed to rest, besides being fed and watered, the rest period being at least five hours. Upon request of the shipper and under certain circumstances the period of continuance in the cars may be extended to thirty-six hours, but under ordinary circumstances if animals are retained in cars for longer than twenty-eight hours the railroad so retaining them is liable to a fine of not less than one hundred or more than five hundred dollars. The inspection necessary for the enforcement of this law is performed by the Bureau of Animal Industry under the direction of the Secretary of Agriculture and violations of the law are reported by the Department of Agriculture to the Department of Justice, which instructs United States Attorneys to bring suit against the railroads to collect the fines contemplated by law in each case.

For the prevention of the introduction of contagious diseases among animals in the United States from abroad, and also for the purpose of preventing the spread of such diseases from one section of the United States to another, the Secretary of Agriculture is given extensive powers to fix and enforce quarantine limits within which animals suffering from contagious diseases, or which have been exposed to such diseases, can be retained until the danger of contagion is passed. For the purpose of preventing the introduction of foreign diseases the Secretary of Agriculture is given power to name the 
ports at which only importation of animals can be made. These are as follows:

On the Atlantic seaboard: Boston, Mass.; New York, N. Y.; and Baltimore, Md. On the Pacific seaboard: San Francisco, San Diego, Cal., and Port Townsend, Wash. Along the boundary line between the United States and Mexico: Nogales, Ariz.; El Paso, Eagle Pass, Laredo, and Brownsville, Tex. Along the border or boundary line between the United States and Carlada: Vanceboro, Houlton, Van Buren, and Fort Fairfield, Ne.; Beecher Falls, Island Pond, Newport, Richford, and St. Albans, Vt.; Rouse Point, Hogansburg, Massena, Ogdensburg, Cape Vincent, Clayton, Charlottc, Niagara Falls, and Buffalo, N. Y.; Detroit, Port Huron, and Sault Ste. Marie, Mich.; and Pembina, N. Dak.

The following-named stations are designated for the entry of animals which are subject to inspection only, viz.: Eastport and Calais, Ne.; Derby Line, North Troy, Alburg, and Swanton, Vt.; Moores Junction, Chateaugay, Fort Covington, Malone, Waddington, Morristown, Lisbon, and Alexandria Bay, N. Y.; Blaine, Sumas, and Seattle, Wash.

At these points, besides the examination and detention of animals to determine whether they have any form of communicable disease, identification is also made of animals which are offered for free importation under the law intended to promote the quality of the stock raised in the United States and which provides for the entry free of customs duty of registered animals of known pedigree. Imported animals, after having undergone the necessary inspection to determine whether they are likely to communicate any disease to other animals in the United States, are allowed entrance, while diseased animals may be killed, and under certain conditions the owners can be reimbursed from the Treasury of the United States for the value of the animals. If, however, the Secretary of Agriculture has issued a proclamation stating the existence of a contagious disease at some foreign point and prohibiting the importation of a given class of animals or all classes of animals from that point, the importation is made at the owner's risk and he cannot be reimbursed if it is found necessary to kill the animals on account of having or having been exposed to the disease against which the quarantine is being enforced.

The Secretary of Agriculture, his functions in this respect being largely exercised by the Bureau of Animal Industry, has the power to declare a quarantine of any State, Territory, or the District of 
Columbia, or any portion thereof, when he deems it necessary for the prevention of the spread of any infections or communicable disease among domestic animals. Railroad companies are prohibited from transporting animals from such districts, and persons are prohibited, under penalty, from driving or transporting animals which have been exposed to contagion, or which are within the quarantine district, to another part of the United States. It is further provided in order to secure against the spread of various diseases that inspections may be made by the officers of the Bureau of Animal Industry, of aninals sold in interstate commerce, and that domestic animals certified as being free from contagion may be transported freely, while non-inspected animals are subject to such restrictions and retentions as the Secretary of Agriculture may prescribe.

The Bureau of Animal Industry, being at all times charged with the investigation of the causes of infectious diseases among animals and of means for their prevention and cure, stands ready to co-operate with State authorities in the measures necessary to stamp out any epidemic. The Federal Bureau has, however, no powers except with relation to the transportation of animais in interstate commerce, and its functions in regard to a purely State epidemic is advisory except so far as relates to the removal of animals from the location in which the disease is prevalent.

In addition to the foregoing inspection, the inspectors of the Bureau of Animal Industry are charged with the oversight of establishments where renovated butter is made and with reporting upon the different processes followed in making a marketable article from unsalable butter, the operation of such inspection having been arranged by consultation between the Secretary of the Treasury and the Secretary of Agriculture, who are jointly charged with carrying out the terms of the law imposing a stamp tax upon renovated or other forms of re-worked butter. Inspectors of the Bureau of Animal Industry determine whether such product is properly marked and stamped, besides maintaining a general oversight of the dairy products business in order to detect violations of the law. In addition to the foregoing very considerable inspection duties, the Bureau of Animal Industry conducts experiments in the breeding and raising of all of the usual domestic animals as in the methods of secur- 
ing the best results in connection with dairy products, including the manufacture, keeping and marketing of such commodities.

TIIE BUREAU OF CHEMISTRY.

The Burean of Chemistry, originally a subsidiary burcau of the Agricultural Department, devoted to making the necessary chemical analyses required by the work of the other bureaus, has come, under the operation of recent legislation, to be the sccond of the considerable inspecting bureaus of the Department of Agriculture. In the intense commercial compctition of the United States, especially in food stuffs, the fact that appearance and price were the largest factors in securing the sale of any given article, brought about, in the absence of any legislation on the subject, very considerable adulteration in most articles of food, in some cases an entire substitution of articles of only a remote resemblance for the food stuffs which were staple articles of commerce. The existence of such frauds on the public has been long commented on and rccently, through the establishment of State departments for the investigation of such adulteration and the passage of State laws to prevent the sale of articles except under brands and labels which would truly denote the contents of the packages, the matter has been given national significance, so that the State laws were supplemented in 1906 by national legislation which prohibited the introduction into any State or Territory froni any other State or Territory of any adulterated or misbranded article of food, the prohibition being also extended to drugs. Similar provisions have been in effect with regard to articles of food and drugs imported from foreign countries for some years, but the legislation is now male general in scope, and the Burcau of Chemistry of the Agricultural Department has charge not only of formulating the regulations under the law, but of carrying out its provisions as well through the medium of inspectors who report upon infractions of the statute.

Drugs are deemed to be adulterated if they differ from the standard of strength, quality, or purity as determined by the tests laid down by the United States Pharmacopeia or the National Formulary; confectionery is leemed to be adulterated if it contains mineral substances or poisonous color or flavoring, or any alcoholic liquors 
or compounds, or any narcotic drugs. Food is deemed to be adulterated if any substance has been mixed with it so as to reduce its quality and strength or if any valuable constituent of the article has been wholly or partly abstracted; if any article has been substituted wholly or in part for the article to be sold, if the food contain any poisonous or other injurious ingredients, or if it consists wholly or in part of any decomposed matter, or of the flesh of an animal unfit for food.

An article is deemed to be misbranded if its label does not contain a fairly accurate description of its contents, such label to be passed upon as to its accuracy by the Secretary of Agriculture acting through the Board constituted for this purpose. The penalties for the violation of the terms of this Act are a fine of not more than two hundred dollars for the first offense, and a fine of three hundred dollars or inprisonment for not more than one year for the second offense. The Secretary of Agriculture, the Secretary of the Treasury and the Secretary of Commerce and Labor are empowered to make joint regulations for the enforcement of this law, owing to the aspect which it has with relation to the Customs Service and interstate commerce. The chief executive work in regard to it is, however, carried out by the Bureau of Chemistry, whose inspectors report instances of the violation of the law. All analyses for the purpose of determining the exact character of the contents of packages offered for sale are made by the Bureau of Chemistry, and if, upon report by that Bureau, it seems probable that a violation of the law has been committed, the person responsible for the act is cited to appear before the Secretary of Agriculture, whose functions in this respect are exercised by the Chief of the Bureau of Chemistry, the Solicitor of the Department of Agriculture, and the Assistant Chief Chemist. So far as possible the manufacturer of the article is made responsible for the violation which may be found to have been committed, but the wholesale or retail dealer is also liable if he is not able to show a guarantee from the manufacturer that the goods referred to come within the provisions of the law. The person alleged to have offended, having been cited to appear before the Board above mentioned, is given an opportunity to present any defense or statement that he may deem to be appropriate, and if, after hearing 
this statement, the Secretary of Agriculture is advised that there has been a violation of the law, he certifies the fact to the Department of Justice which, in turn, sends the papers to the appropriate District Attorney who is required by law to prosecute all violations of the Pure Food Act and secure the punishment of the offenders. Articles which have been imported in violation of the law, and whose manufacturers are beyond the juriscliction of the United States are subject to seizure and to condemnation, and similar seizure is practicable in the cases of articles adulterated or misbranded for the removal of the articles from the usual avenues of commerce, either with or without the punishment of the persons who may be responsible for the violation of the law.

The Bureau of Chemistry has carried on for a number of years an exhaustive series of investigations and experiments relative to the adulteration of foods and also as to the effect of preservatives used in the preparation of staple foods upon the human system. Such investigation has been carried on not only through chemical analysis, but by means of what has been known as the "poison squad," composed of young men most of whom were employees of the Department of Agriculture, and who committed themselves to a series of tests including taking the chemical substances used as preservatives in various amounts, the result of such experiments having added materially to the knowledge of the world on such subjects.

The function of the Bureau of Chemistry with relation to the Department of Agriculture is, of course, a most important one since chemistry is inseparable from modern agricultural operations. The Bureau makes investigations into the relation of food products to the subject of nutrition, as well as for the purpose of determining the constituents derived by agricultural products from the soil, fertilizers, and the air. It has special activities with relation to fertilizers, analyzing the various soils stubmitted to the Bureat, and determining the lack of chemicals which would probably adt to the fertility of the soils in question and which should he added by use of fertilizing materials. It reports upon the chemical characteristics of commercial fertilizers and co-operates with the chemists at the various experiment stations in the determination of problems relating to agricultural chemistry. The Bureau also carries on such chemical in- 
vestigations as may be brought to its attention by other departments of the Government, and is frequently called upon for analyses and determination of the quality of supplies furnished to the Departments which do not maintain divisions having to do with chemistry.

THE hUREAU OF STATISTICS.

The prices of agricultural products are largely determined in advance upon the basis of the probabilities relative to the crop of the world. Leaving out of consideration the speculative market, the prices which would be demanded for the great agricultural staples of the United States would in any event be based to a considerable degree upon the stock in hand abroad and the prospect of competitive offering of agricultural products in the world's market from other sources than the agricultural lands of the United States. In order to provide the agricultural interests of this country with all the information obtainable on this subject a Bureau of Statistics is maintained in connection with the Department of Agriculture which collects information as to crops, both at home and abroad. The domestic information is secured from a corps of county and State reporters, who serve without compensation and whose work is checked up by a number of paid, skilled agents operating in different sections of the United States. The information with regard to foreign crops is secured through the Consular Service, and the figures received are collated and tabulated by the Bureau of Statistics and furnished to the public in the form of monthly crop reports. These reports cover not only the grain crops, but the cotton crop as well, and market prices are considerably affected by the nature of the statements made. The utmost care is exercised that the result attained by the compilation of the reports from the different reporters and agents shall not reach outside persons interested in the returns in advance of the general publication. By means of collusion with employees such information was at one time utilized by individuals to secure considerable profits by trading in the speculative market, and since that time the compilation of the figures has been done under strict safeguards, and the results must be approved and certified by the Secretary of Agriculture before they can be made public.

Besides this function of crop reporting, the Bureau of Statistics 
investigates market conditions abroall and carries on a series of compilations and tables showing the fluctuation of supply and demand, and the Burean is maintained for the purpose of affording as much information as possible as to the conditions of foreign markets for American asricultural products and the best method of taking advantage of the world's requirements for food stuffs.

\section{TIIE OFFICE OF EXPERIMENT STATIONS.}

The only direct undertaking of an educational character by the United States except in Alaska consists in appropriation for the partial maintenance of the schools for teaching agriculture and the mechanic arts which have been established by the various States and Territorics and which have received annual appropriations from the proceeds of the sale of the public lands of the United States.

Such assistance has formerly depended for its extent upon the amount accruing from the sale of land in each State and Territory, but by recent legislation stated sums are to be appropriated for the assistance and support of the agricultural colleges, being thirty-five, forty and forty-five thousand dollars, respectively, until July I, I9I I, when fifty thousand lollars a year will be available for the purposes of each school. It is required that this amount must be equally divided where separate schools are maintained for white and colored students, and if discrimination is practiced against colored students the appropriation for the State ceases. If any part of the funds appropriated become lost by reasun of the fault of the State authorities they must be replaced ly the State, otherwise further appropriation will be cut off. In addition to the appropriations made for the support of the colleges an amnual appropriation of twenty thousand dollars each is made for the assistance of the agricultural experiment stations generally maintained in connection with the State agricultural colleges, although provision is made by which an inclependent agricultural station in a State may be assisted. The work required of the experiment stations is as follows:

To conduct original rescurches or verify cxperiments on the physiology of plants and animals:

The diseases to which they are severally subject, with the remedies for the same; 
The chemical composition of useful plants at their different stages of growth;

The comparative advantages of rotative cropping as pursued under a varying series of crops;

The capacity of new plants or trees for acclimation;

The analysis of soils and water;

The chemical composition of manures, natural or artificial, with experiments designed to test their comparative effects on crops of different kinds;

The adaptation and value of grasses and forage plants;

The composition and digestibility of the different kinds of food for domestic animals;

The scientific and economic questions involved in the production of butter and cheese;

And as far as practicable, all such stations are required to devote a portion of their work to the examination and classification of the soils of their respective States and Territories, with a view to securing more extended knowledge and better development of their agricultural capabilities, and make such other researches or experiments bearing directly on the agricultural industry of the United States as may in each case be deemed advisable, having due regard to the varying conditions and needs of the respective States and Territories.

In order to secure uniformity in methods and results in the work of the stations it is the duty of the Secretary of Agriculture to furnish forms for the tabulations of the result of the investigation experiments and to indicate from time to time sucl kinds of inquiry as seem most important, and in general to furnish advice and assistance that will best promote the purposes of the station. The Office of Experiment Stations has charge of this function of the Secretary of Agriculture, and, in addition, directly manages agricultural experiment stations in Alaska, Hawaii and Porto Rico. It also seeks to promote the interests of agricultural education and investigation throughout the United States, and collects and disseminates the results of the work of the various stations, each of which is empowered to publish a bulletin for free distribution, giving the result of its investigations. The Office of Experiment Stations also exercises a 
general supervision in an unofficial way over farmers' institutes throughout the United States, reporting upon the progress made by such meetings and afforling as far as possible assistance to aid in their success. In addition to the other subjects of investigations mentioned, the Office of Expcriment Stations also las charge of a series of problems relating to drainage and other topics pertaining to agricultural engineering.

TIE FOREST SERVICE.

Another Bureau of the Agricultural Department which has had very considerable development during the past two years is that of the Forest Service. It was originally established as a means of investigation into the best methods of forest culture, and for the restoration of forest lands which had been denuded of their timber by lumbering operations. The scientific cultivation of forests has received much attention abroad, and forests as permanent investments constitute a consiclerable part of the agricultural wealth of certain foreign counties. The United States had paid little attention to conservation of forests prior to the establishment of this bureau, but in consequence of the enthusiastic work of the present head of the bureau, the dangers which arise from the lestruction of forest areas are better understood and practical and economic plans for forest cultivation in different parts of the United States have been mapped out. Since i891 the President has had power to set apart such portions of the public lands of the United States as he may deen advisable as public forests, and to establish the limits thereof. Such reservations were formerly under the direction of the Secretary of the Interior, but their administration was recently transferred to the Secretary of Agriculture, and the entire forest work of the Govermment consoliclated under the supervision of the Forest Division.

The personnel of this Division, besides the Chief and the administrative force in Washington, consists of rangers and wardens in charge of the forest reservations, and who prevent depredations thereon, and investigators and students employed in the field to carry on inquiries and experiments as to the best methods of handling forests and of securing forest growths on lands which have been stripped of their timber. Provision is made by law for the sale of 
trees on forest reservations which have secured their full growth and which are ready for commercial use.

Besides the functions performed by the retention of the forests in aid of agriculture, namely, to retain the moisture in the soil, prevent destructive storms and washings of agricultural soil, the national forests are expected to afford object lessons in the economical handling of the timber lands as opposed to the destructive plan historical in the United States of removing the native timber without replacing it with sorts suitable for securing the greatest ultimate returns from the land. The Forest Division issues a considerable number of publications devoted to subjects relating to the growth and use of timber, as well as to the comparison of the usefulness of different sorts of wood, and is prepared to give advice to private persons as to modern methods of developing forest lands and of handling them so that they will return a steady income to the owners.

THE BUREAU OF ENTOMOLOGY.

The Bureau of Entomology of the Agricultural Department has charge of all nuatters relating to injurious insects affecting field crops, fruits, forests and forest products and agricultural products in store. This Bureau is one of those which carry on the original function of the Department of Agriculture and has a most important relation to the farmer. It investigates all matters relative to the history and habits of insects which bear any relation to agricultural operations, determines the difference between injurious and favorable classes of insects, or rather seeks to determine which of the functions of insects are injurious and which beneficial to agriculture, and endeavors to minimize and restrain the injurious effects by investigating methods proposed for the extermination of injurious insects, either by mechanical means or by the introduction of fungous growths, or other natural enemies of particular insects when the latter have exceeded the natural balance or relation between insect life and growing substances. It is also charged with investigations into the best methods of handling bees and producing honey, and has for a long period been conducting a series of experiments intended to facilitate the culture of the silk worm in the United States and the production of silk in this country. 
From time to time there have been serious losses experienced by agricultural interests in different parts of the United States by reason of the prevalence of insect pests, either on account of impurtation from other countries, or from some cause not well known by which native species become over abundant. It has been the work of the Bureau of Entomology to endeavor to find a relief from such conditions and provision is now made by law for punishment by fine or imprisonment of any person who shall introduce an injurious or noxious insect into the United States. Such an introduction before the law went into effect of the brown tail and Gypsy moths has been the cause in the New England States of the expenditure of considerable amounts of money to check the ravages of the pests. The cotton boll weevil prevalent in the Southern States has been the subject of extensive investigation by the Bureau of Entomology, and representatives of the Bureau have been sent to many foreign countries to scek out and endeavor to introduce into the United States insects believed to be hostile to the weevil. Field operations are conducted by the Bureau in all parts of the United States, besides the investigations and experiments going on in Washington, and representatives are sent abroad to secure information and specimens of insects which are believed to be of value to the agricultural interests of this country. Among the other undertakings of the Bureau was that to exterminate a growth which was ruining the orange crop of California, the character of which was determined by the Bureau, and the cure applied in the introduction of an insect which fed upon the noxious growth and reduced it to the normal proportion.

The losses in grain and other agricultural products which are stored waiting for a market or consumption through insect life are of course considerable, and it is one of the functions of the Bureau of Entomology which has added considerably to the agricultural wealth of the country to determine the conditions under which agricultural products can best be stored, with a view to a minimum of incidental loss.

THE BUREAU OF BIOLOGICAL SURVEY.

The Bureau of Biological Survey of the Agricultural Department has a similar function to that of the Bureau of Entomology, which is 
exercised with regard to birds and mammals of the United States. It has also, however, an additional survey function, in determining the natural geographic location of animals and plants of this country, and is engaged in the preparation of a series of maps giving the zones within which the respective classes of animal and plant life have their natural location. With reference to agriculture, it examines birds and mammals, and inquires into their habits, for the purpose of determining whether they are injurious or beneficial to the agricultural interests and recommends means for securing the increase of beneficial birds and mammals and the destruction of injurious specimens.

In order that the provisions of the various State laws with regard to the protection of game may not be nuliified, it is provided by Federal statute that the importation of certain birds and animals which might be offered for sale in the markets and not to be distinguished from State-killed game of similar character, is prohibited. The importation of noxious animals and birds, injurious to the agricultural interests, is also prohibited under a similar manner to that against the importation of noxious insects, though the former legislation antedates the latter. The details relating to the enforcement of these various laws, as well as of the laws designed for the preservation of game in Alaska, are under the direction of the Bureau of Biological Survey.

\section{THE BUREAU OF SOILS.}

The functions of the Department of Agriculture relating to the cultivation of food crops are especially under the charge of two Bureaus, the Bureau of Soils and the Bureau of Plant Industry. The former has charge of the investigations carried on by the Department into the characteristics of the soils of the United States, and makes a careful examination of the composition of the different soils with regard to their availability for the production of particular crops. The burean is engaged in the preparation of comprehensive atlases, covering the whole of the United States, and which show graphically the nature of the soil in any given locality. A special branch of the investigation of this Bureau consists of the consideration of the methods of reclaiming the soils in the West, which are rendered unfit for agriculture by reason of an excess of alkali, and 
the work of the Bureau in this respect bears a close relation to the work of irrigation, for the reason that over-irrigation brings about certain conditions from the cxtra deposits of alkali that threatens in some cases to mullify the work that has been clone by bringing water to the land. The Pureau of Soils also has charge of the investigations relative to tobacco culture, beginning with the determination of lands suitable for tobacco culture, and including the final processes of making the tobacco ready for the market. In this connection it has introduced the growing of tobacco under shelter, by which an equivalent of the imported loaf has been secured in certain parts of the United States. The Bureau of Soils has also to do with the subject of fertilizers, their composition and their adaptability to different branches of agricultural work. In this connection a remarkable series of experiments has been made by the Agricultural Department in the development of artificial means for securing nitrogenous products in the soil through the inoculation of soils with bacilli which perform a similar function to that performed by clover and other nitrogenous fertilizing crops.

THE BUREAU OF PLANT INDUSTRY.

The Bureau of Plant Industry studies plant life in all its relations to agriculture, investigates plant diseases and carries on field tests, together with experiments and projects in economical farm management. It conducts explorations in foreign countries for new seeds and varieties of plants and administers the Congressional seed distribution, a not altogether creditable aspect of Federal activities. A very large appropriation is made annually for the purchase of seeds for distribution by Congressmen to their constituents, the practice being based upon the earliest theory of the Department of Agriculture, nancly, that of the introduction of new sorts of useful plants into the United States. Owing, however, to the very large proportions of the distribution, it is wholly impossible to distribute other than the most common and plentiful vegetable and flower seed, and the markets have frequently to be drained in order to secure a sufficient supply of usual commercial seeds. The abuse involved in this distribution has been the subject of frequent notice by the Secretary of Agriculture and by others, but has not yet been cured. The large 
amount of seeds purchased for this distribution are made up by contract into small packets, several thousand of which are allotted to each Congressman and sent out under frank, without expense, most of them falling into the hands of persons who use them for the preparation of small domestic gardens and without relation to the agricultural interests of the country at large, for the reason that the quality furnished is, to say the least, doubtful, so that a professional agriculturist would not usually take the chance of loss of his time in attempting to cultivate the seed furnished. A small allotment of the rarer and more valuable seeds and plants, cuttings and bulbs is made, but the number of each furnished is so small as to be unappreciable.

The Bureau of Plant Industry has charge of the details of the investigation imposed upon the Secretary of Agriculture of testing seeds bought in open markets and making a report as to the results obtained from such investigations. The Bureau also has for a number of years conducted experiments which have proved to be successful in the production of tea in the United States, and is also engaged in a series of important and valuable experiments in developing new varieties of fruits, and improving other varieties already known, together with a series of investigations into the best methods of handling and marketing various fruits produced in the United States, and the extension of the zones within which the various sub-tropical fruits can be grown.

The plants in the Western grazing country which are injurious to stock have been made the special subject of investigation by the Bureau of Plant Industry, for the purpose of determining the qualities which cause such injurious and practicable methods of eradicating noxious plants. It also investigates the characteristics of drug plants native within the United States, and the methods of the improvement of their medicinal qualities, and seeks to introduce new and valuable plants of this character for growth in the United States.

ROADS AND PUBLICATIONS.

In connection with the general movement throughout the United States for the improvement of roads, especially in agricultural communities for the better communication and increased economy in the 
movement of crops, the Department of Agriculture has estallished an office of good roads, which is charged with the duty of collecting information in regard to the good-roads movement, and as to the best methods of making roads under the differing conditions in the United States. It conducts tests as to road materials, and methods of road-making, and gives demonstrations when practicable of the results secured by its specifications. In this, as in all other lines of investigation by the Agricultural Department, the fullest publicity is given to all clefinite results secured. Each of the Bureaus places the records of its investigations and experiments in print in convenient form, in the nature of bulletins and similar publications. which are made available, without cost, to any person who may request them. A Division of Publications is maintained in the Department of Agriculture, which is charged with the duty of editing, directing the printing, and disseminating the various publications of the Department. Besides the annual report, very large editions of monographs of different topics relating to agriculture are printed for distribution by members of Congress, and on a very large number of subjects. A seeker for information has only to indicate his wishes to the Department to be furnished with literature on the subject covering the latest discoveries and the results of the most advanced investigations in the science of agriculture.

THE WEATHER BLRE.IU.

If it was desired to mention a function of the Federal government which has been entirely beyond the contemplation of the founders of the Constitution, no better example conid be instanced than the Weather Bureau, an entirely gratuitous activity of the general government, without reference to other govermmental functions and legalized only by the general welfare clause of the Constitution. Begun in I87o in consequence of the growing interest in matters relating to meteorology and for the purpose of giving storm warnings to vessels, it was at first under the direction of the Army, because of the necessary duties of the Signal Corps to maintain telegraphic service to complete the gaps in private operation so that the Government could have comprehensive communication with all the points of its activity throughout the States and Territories. Its 
extension and development utilized the services of the highly trained men composing the personnel of the corps until as a scientific bureau of great merit it was removed from the jurisdiction of the War Department in I890 and transferred to the Department of Agriculture. Although nominally under the direction of the Secretary of Agriculture, the Weather Bureau is, in effect, an independent institution removed in location from the Department proper and having its own organization and operation with little reference to the work of the main Department of Agriculture. A portion of the personnel of the Weather Service was transferred from the Signal Corps of the Army with the transfer of the Bureat and were granted specific exemption and preference in employment under the Bureau. The personnel of the Bureau is subject to assignment and transfer by the Secretary of Agriculture, but his functions in this respect are performed by the Chief of the Weather Bureau, who has practically independent control of the operations of the Service. The Chief of the Weather Bureau is appointed by the President by and with the advice and consent of the Senate and receives five thousand dollars a year. He has charge of the forecasts of the weather, the system of distribution of cold-wave warnings; the flood-warning system; the system of collating and distributing reports of the condition of crops; the system of frost warnings; the special forecasts for the benefit of outgoing trans-Atlantic steamers and the marine interests of the Great Lakes; the extensive display of forecast cards, weather maps, and climate and crop bulletins in all the States and Territories; the issue of snow and ice charts and other publications and is the head of the investigations of the Government into the science of meteorology.

The central office of the Bureau is located at Washington, D. C. Two forecasts are issued daily for each State and Territory, one about io a. .17. and the other about io p. $\mathrm{m}$. Both of the forecasts are based on the telegraphic reports of observations taken two hours previously, the interval being occupied in collecting, collating, and charting the reports, and in preparing and issuing the forecasts. The morning forecasts are made to cover a period of thirty-six hotırs, or till $8 \mathrm{p} . \mathrm{m}$. of the following day, and are issued from the central office or from certain selected stations designated as forecast centers. 
The evening forecasts cover the period of forty-cight hours, or till 8 p. $m$. of the second day after they are issued, and, with few exceptions hereafter noted, always emanate from the central office. Telegraphic reports are received from regular stations of the Weather Bureat situated in various parts of the Uniter States and on the islands of the adjacent seas, and, by a system of excliange, from Mexican stations and stations of the Dominion of Canada. Daily reports by cable are received from the Azores and from several localities in western Europe. The Canadian reports are invaluable to the forecaster, since they often furnish the first indications of marked changes in the weather of the northern half of the United States. Besicles the regular stations there are $\mathrm{I}_{32}$ special river stations, 48 special rainfall stations, abont 3.000 stations where observations are recorded by voluntary observers of the climate and crop service of the Bureau, and a number of other stations which co-operate with the Bureau, although not under its direct supervision. Voluntary observers receive no pay, but they are furnished with the Weather Bureau publications. They are provided with instruments by the Bureau, and take observations daily at a regular hour. Their instrumental equipment comprises, as a rule, only an instrument shelter, a maxinum and a minimum thermoneter, a rain gauge, and a graduated rule for rainfall measurements. Their records of observation show, for each day, the highest and the lowest temperature, the rainfall, the percentage of cloudiness, the direction of the wind, and the occasional special phenomena observed. These data are sent regularly to the climate and crop centers of their respective districts or States, where weekly or monthly bulletins are compiled and published for the information of those especially interested in crop conditions.

Each Weather Burcau station has its official in charge, who conducts all the work under instructions from the chief of the Burean. The number of assistants depends, of course, 11pon the amount of work to be done. About fifty of the smallest stations have none; at the others, the number varies from one to eight or ninc. At centers of forecast districts, such as Mashington City, Chicago, and San Francisco, the official in clarge has the rank of professor or district 
forecaster; at storm-warning section centers, as well as at climate and crop section centers, his rank is usually local forecaster or director. In most other cases he has the rank of observer. The observers are of several grades, the classification depending upon length of service, efficiency, industry, fitness, educational qualifications, conduct, etc. Vacancies in the position of observer are filled from the eligible list of those who have passed the required civil-service examination in meteorology and the ordinary English branches and algebra. The age limit of appointees is 18 to 30 years. Nearly all stations have messenger boys. Applicants for the position of messenger are required to take a civil service examination, and the age limit for appointees is 16 to 20 years. A printer is also included in the regular force at some stations.

During the half hour from 7.30 to $8 \mathrm{a} . \mathrm{m}$. the observer at every station is taking the regular morning observation and preparing a telegraphic report of the meteorological conditions. He observes (from the roof) the condition of the sky; the direction of the wind; the clouds, their amount, kind, and direction of movement; and he scans the sky carefully for the appearance of any unusual phenomena; he measures the precipitation, rain, snow, sleet, or hail; reads the four thermometers-the dry, the wet-bulb, the maximum, and the minimum; records all these observations on the blank form provided for the purpose; and "sets" the self-registering thermometers for another twelve-hour period of registration. He then returns to the office and finishes the observation, which further includes the reading of the barometer and the wind direction and velocity, and also the "checking" of the readings with those of the self-registering instruments. He then makes the calculations necessary to reduce the barometer reading to sea level, and completes the record of his observations, taking great care to avoid the slightest error in any part of the record. In telegraphing the report a cipher code is in use at all stations. The following sample message and translation will illustrate the utility and economy of the code. It will be seen at a glance that the number of words saved is the difference between the words found in the message and the number of words used in writing the observation out in full. 


$$
\text { Regular } 8 \text { a. m. report. }
$$

Memphis, Target, Lugmark, Geyser, Buforite, Kirby, Tally, Frost, Chamois.

Translation of the cipher words.

Memphis (telegraphic designation of station): Memphis, Tenn.

Target (pressurc and temperature): Barometer, 29.92 ; temperature, 44 degrees.

Lugmark (precipitation for twenty-four hours, all of which fell before 8 p. m.) : Precipitation, 0.52 inch.

Gey'ser (direction of wind, state of weather, and maximum thermometer): Direction of wind, southeast; state of weather, cloudy; maximum temperature, 84 degrees.

Buforite (current wind velocity and minimum temperature) : Current wind velocity, Io miles per hour; minimum temperature, 38 degrees.

Kirby (decrease in pressure during two hours previous to observation, barograph record): Decrease in pressure, o.Io inch.

Tally (river observation): Water level, ro feet above zero of gauge, and falling.

Frost: Light frost at station or in vicinity.

Chamois (amount, kind, and direction of cloud, lower): Amount, 8 to Io tenths; kind, stratus; direction moving from, south.

The average number of cipher words in observation messages is probably about five for each station.

The messages are transmitted over telegraphic "circuits," and are received simultaneously at all places on the same circuit.

A similar course is pursued when the messages are transferred from one circuit to another, so that each telegraphic office sends the report from its own station but once. The reports are "all in" at about $10.30 \mathrm{a}$. m. As soon as each sheet of the cipher messages is received at a Weather Bureau office the observers rapidly enter the data on geographical charts of the United States. Isobars (lines of equal barometric pressure) and isotherms (lines of equal temperature) are then drawn, to show the distribution of these elements. Auxiliary charts are also drawn, to show the distribution of rainfall and the changes in temperature and pressure during the last 24 hours 
and the last 12 hours, as indicated by the reports. These duties must be performed rapidly and accurately, and when completed the charts are ready for the forecaster. This stage of the work is reached at practically the same time at every station in the country. About this time the State or general forecast is received from the district center, and the local forecaster prepares a forecast for his city and vicinity.

About Ioo of the larger stations issue a daily weather map. This consists of a chart showing the weather conditions at the various stations whose reports have been received, the forecasts of the weather and temperature during the next 36 hours for the city and State in which the station is located or the map displayed, and a summary of the general weather conditions during the past 24 hours. All warnings received from the forecast center before going to press are printed conspicuously on the map. The maps of stations in the agricultural and fruit sections give special prominence to frost warnings; those on the seaboard to storm warnings and the expected direction and force of the wind; those situated on rivers to flood warnings and to forecasts of the rise and fall of the rivers.

At every station an observation is taken at $8 \mathrm{p}$. $\mathrm{m}$. This is of the same character as that taken at $\delta$ a. $\mathrm{m}$. No maps are printed in the evening, but the telegraphic reports of observations are sent, received and charted, as a rule, in the same manner as the morning reports. At climate and crop centers the evening forecasts for the following 48 hours are sent out on forecast cards for the benefit of the agricultural interest, and, through the rural free-delivery routes, reach a large number of farmers the following morning. When storm warnings, cold-wave warnings, flood warnings, etc., are received at night they receive the most prompt and effective distribution practicable at that time. At many important stations the night observer is required to telephone regularly some of the information contained in the $8 \mathrm{p} . \mathrm{m}$. reports. At Cleveland, Ohio, for example, the two passenger steamship lines for Buffalo and Detroit are informed every evening as to the conditions existing at stations on Lake Erie and on Lake Huron, and the weather that is expected during their night trips.

It is admitted that the work of the Weather Bureau is not and cannot be absolutely accurate, for the reason that such a small part of the phenomena of the air is understood. Great care and accuracy, 
however, are required of observers, and only the most highly trained men of the corps are allowed to make forecasts, most of the stations of the Weather Bureau being stations for observation only, and only made from stations in charge of the higher class of the subordinates of the Bureau. Promotions from the lower-salaried observers are made as the result of cxaminations, and qualified obscrvers are promoted successively through different grades to the position of observer in charge of a forecast station, and to the higher position of Professor of Meteorology, in charge of the nore inportant stations, and also of the investigations carried on into atmospheric phenomena.

Besides the daily forecasts sent out from Washington and from the forccast centers, and which are furnished as matters of public information to newspapers and posted in conspicuous places, besides being sent generally to persons who may apply for the service, a system of storm and cold wave warnings is maintained, together with a system by which private persons may display flags indicating the different characteristics of temperature. The storm warnings along the seacoast and on the Great Lakes have proven of great advantage to mariners, and are as follows:

A red flag with a black center indicates that a storm of marked violence is expected. The pennants displayed with the flags indicate the direction of the wind: red, casterly (from northeast to south); white, westerly (from southwest to north). The pennant above the flag indicates that the wind is expected to blow from the northerly quadrants; below, from the southerly quadrants. By night a red light indicates casterly winds, and a white light above a red ligltt, westerly winds.

HURRICANE WARNiNg.-Two red flags with black centers, displayed one above the other, indicate the expected approach of a tropical hurricanc, or one of those extremely severe and dangerous storms which occasionally move across the lakes and northern Atlantic coast. No night hurricane warnings are displayed.

Flags are also used in displaying weather forecasts, white for fair weather; blue, rain or snow; blue and white, local rain or snow. The temperature flag is a black pennant and when placed above the weather flags it indicates warmer: when below, colder; when it is not displayed the temperature is expected to remain about stationary. During the late spring and early fall the cold-wave flag, which is white with a black center, is also used to indicate anticipated frosts. 
Whistle signals have also been arranged as follows:

A warning blast of from fifteen to twenty seconds duration is sounded to attract attention. After this warning the longer blasts (of from four to six seconds duration) refer to weather, and shorter blasts (of from one to three seconds duration) refer to temperature. Those of weather are sounded first.

Blasts.

Indicate.

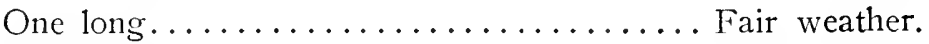

Two long....................... Rain or snow.

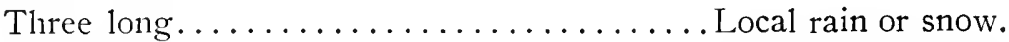

One short.........................

Two short...................... Higher temperature.

Three short...........................

By repeating each combination a few times, with intervals of ten seconds, liability to error in interpreting the signals may be avoided.

Provision is made for warning agricultural districts of sudden changes in temperature by whistle and flag signals by private persons, and during the planting, cultivating and harvesting seasons in the corn, wheat, cotton, sugar and rice regions a daily service is maintained, by which data relative to the weather conditions is assembled at the centers named hereafter, in order that information may be had as to the probable conditions of the crops and the prospects of their successful cultivation. Similar district observations are made in localities in which there is likelihood of disastrous river floods, the observing stations furnishing figures as to the rainfall and rise of rivers, so that localities farther down the stream may anticipate a flood in time to prevent damage. In addition, such information during periods of low water enables river men to anticipate a rise and take advantage of it when it comes.

The forecasts and warnings issued by the Weather Bureau are (I) State forecasts, issued by specially designated district forecasters, for distribution throughout the State for which the forecast is made; (2) local forecasts, issued by specially authorized officials in charge of stations, for distribution in the city where issued and its immediate vicinity; (3) special forecasts and warnings of storms, cold waves, frosts, heavy rains and snows, floods, etc. 
The following stations are the respective forecast centers for the districts comprising the States enumerated:

Boston, Mass.-Maine, New Hampshire, Vermont, Massachusetts, Rhode Island, and Connecticut.

Washington, D. C.--New York, Pennsylvania, New Jersey, Delaware, Maryland, District of Columbia, Virginia. North Carolina, South Carolina, Georgia, Floricla, Alabama, Mississippi, West Virginia, and Ohio.

Chicago, Mll.-Illinois, Indiana, Michigan, Wisconsin, Minnesota, Iowa, Missouri, Kansas, Nebraska, South Dakota, North Dakota, and Montana.

Louiszille, Ky.-Kentucky and Tennessee.

Denver, Colo.-Colorado. New Mexico, Arizona, Utah and Wyoming.

San Francisco, Cal.-California and Nevada.

Portland, Ore.-Washington, Oregon, and Idaho.

New Orleans, La.-Louisiana, Arkansas, Texas, and Oklahoma.

To supplement the telegraphic service of the Weather Burcau so that instant connection may be had with every point at which there is a weather obscrvatory in the employ of the Department, the $\mathrm{Bu}$ reau maintains a considerable number of telegraph and telephone lines independent of commercial systems, but working with them. These reach remote points, especially along the coast, and the operation and maintenance of the lines is entirely' under the control of the Bureau. Commercial messages are, however, allowed to be sent over these lines when they lo not interfere with government business and they also supplement the means of communication of the Life Saving Service. The IVeather Bureau also experimented extensively with the wireless telegraphy, which promised great usefulness in reaching points off the const to which cables could not profitably be larl, and also in conmmicating with ressels. By the arrangement which placed all wireless work along the coast under the Navy Departmer:t transmission of wireless messages from ships is maintained and the Veather Bureau has daily reports of weather conditions from the occan liners which are equipped with wireless apparatus. The following offices of the Weather Bureau are espe- 
cially charged with collection of the data with regard to climatic conditions at sea:

Portlanc, Me.

Boston, Mass.

New York, N. Y.

Philadelphia, Pa.

Baltimore, Md.

Norfo!k, Va.

Wilnington, N. C.
Charleston, S. C.

Savannah, Ga.

Jacksonville, Fla.

Key West, Fla.

Tampa, Fla.

Pensacola, Fla.

Mobile, Ala.
New Orleans, La.

Galveston, Tex.

Tacoma, Wash.

Scattle, Wash.

Fortland, Ore.

San Francisco, Cal.

The following is a list of the Weather Bureau stations indicating their character and giving the rank of the official in charge:

Abeline, Tex.-Observer, \$1,200.

Aleany, N. Y.-Local Forecaster, $\$ 1,600$.

Alpena, Mich.-Assistant Observer, \$I,oco.

Amarillo, Tex.-Assistant Observer, \$1,000.

Anniston, Ala.-Assistant Observer, \$r,000.

Asheville, N. C.-Observer, \$1,200.

Atlanta, Ga.-(Center of Georgia section, Climatological service.) Section Director, $\$ \mathrm{I}, \mathrm{S} 0 \mathrm{O}$.

Atlantic City, N. J.-(Center of New Jersey section, Climatological service.) Section Director, $\$ 1,400$.

Augusta, Ga.-Observer, \$1,200.

Baker City, Ore.-Assistant Observer, \$1,000.

Baltimore, MD.-(Center of Maryland and Delaware section, Climatological service.) Section Director, \$2,000.

Basseterre, St. Kitts, W. I.- (In charge of special hurricane observer; $\$ 25$ a month during hurricane season.)

Bentonville, Ark.-Assistant Observer, \$840.

Binghamton, N. Y.-Local Forecaster, $\$ 1,400$.

Birmingham, Ala.-Observer, $\$$ I, 200 .

Bismarck, N. D.-(Center of North Dakota section, Climatological service.)

Section Director, $\$ \mathrm{I}, 400$.

Block Island, R. I.-Assistant Observer, \$I,000.

Borse, IdAho.-(Center of Idaho section, Climatological service.) Section Director, $\$ \mathrm{I}, 400$.

Boston, Mass.- (Center of forecast district, embracing New England States; also center of New England section, Climatological service.) District Forecaster, \$2,400.

Bridgtown, Barbados, W. I.-(In charge of special hurricane observer;

$\$ 25$ a month during hurricane season.)

Buffalo, N. Y.-Local Forecaster, \$2,000.

Burlington, Vt.-Local Forecaster, \$I,400. 
Cairo, Ill.-Observer, \$I,200.

Canton, N. Y.-Observer, $\$ \mathbf{I}, 200$.

Cape Henry, Va.-Observer, \$1,200.

Cape MaY, N. J.-Station Agent, \$300.

Charles City, Iowa.-Assistant Observer, \$I,ooo.

Charleston, S. C.-Local Forecaster, \$I,6oo.

Charlotte, N. C.-Observer, \$I,200.

Chattanooga, Tenn.-Local Forccaster, \$I,400.

Cheyenne, Wro.-(Center of Wyoming section, Climatological service.)

Section Director, $\$ 1,600$.

Chicago, Ill.- (Center of forecast district, embracing Illinois, Iowa, Indiana, Michigan, Wisconsin, Minnesota, Missouri, Kansas, Nebraska, South Dakota, North Dakota, and Montana.) Professor of Meteorology, \$3,000.

Cincinnati, Ohio.-Simon S. Bassler, Local Forecaster, $\$ 1.800$.

Cleveland, Ohio.-Local Forecaster, \$2,000.

Columbia, Mo.- (Center of Missouri section, Climatological service.) Section Director, $\$ 1,400$.

Columbia, S. C.- (Center of South Carolina section, Climatological service.) Section Director, $\$ \mathrm{I}, 400$.

Columbus, Ohio.-(Center of Ohio section, Climatological service.) Section Director, $\$ 2,000$.

Concord, N. H.-Observer, \$1,200.

Concordia, Kans.-Observer, \$I,200.

Corpus Christi, Tex.-Observer, $\$ 1,200$.

Curacao, W. I.- (In charge of special hurricane observer, \$25 a month during hurricane season.)

Daven port, Iowa.-Local Forecaster, \$1,600.

Del Rio, Tex.-Assistant Observer, \$I,O00.

Denver, Colo.- (Center of forecast district, embracing Wyoming, Colorado, New Mexico, Arizona, and Utah; also center of Colorado section, Climatological service.) District Forecaster, \$2,400.

Des Monnes, Iowa.-(Center of Iowa section, Climatological service.) Local Forcaster, $\$ 1,800$.

Detroit, Mich.-(Inspector's Hcadquarters.) Inspector, also in charge of lake marine work, $\$ 2,500$.

Devils Lake, N. Dak.-Assistant Observer, $\$ 840$.

Dodge, Kans.-Observer, \$1,200.

DubuQue, Iowa.-Local Forecaster, \$I,400.

Dulutir, Minn.-Local Forccaster, $\$ 1,400$.

Durango, Colo.-Assistant Observer, $\$ 1,000$.

East Clallam, Wasil.-Repairman, $\$ 720$.

Eastrokt, Me.-Observer, \$I,200.

Elinins, W. Va.-Observer, \$I,200.

El Paso, Tex.-Observer, \$I,200. 
Erie, PA.-Local Forecaster, \$I,800.

Escanaba, Mich.-Observer, \$I,200.

Eureka, Cal. Observer, \$1,200.

Evansville, Ind.-Local Forecaster, \$I,400.

Flagstaff, Ariz.-Assistant Observer, \$r,000.

Fort Simti, Ark.-Observer, $\$ 1,200$.

Forth Worth, Tex.-Observer, \$i,200.

Fresno, Cal-OObserver, \$1,200.

Galveston, Tex.-(Center of Texas section, Climatological service.) Section Director, $\$ I, \$ 00$.

Grand Haven, Mich.-Observer, \$I,200.

Grand Junction, Colo.--Observer, \$1,200.

Grand Rapids, Mich.-(Center of Michigan section, Climatological service.)

Section Director, \$1,800.

Green Bay, IVIs.-Observer, \$1,200.

Hannibal, Mo.-Observer, \$1,200.

Harrisburg, Pa.-Local Forecaster, \$1,600.

Hartford, Conn.-Local Forecaster, \$1,400.

Hatteras, N. C.-Assistant Observer, \$I,000.

Havre, Mont.-Assistant Observer, \$1,000.

Helena, Mont.-(Center of Montana section, Climatological service.) Section Director, \$I,400.

Honolulu, Hawair- (Center of Hawaiian section, Climatological service.)

Section Director, $\$ 1,800$.

Hotghtos, Mich.-Observer, \$1,200.

Huron, S. Dak.-(Center of South Dakota section, Climatological service.)

Section Director, $\$ \mathrm{I}, 600$.

Interendence, Cal- - Station Agent, \$300.

IndianAPOLIS, IND.-(Center of Indiana section, Climatological service.)

Section Director, $\$ \mathbf{I}, 800$.

Iola, Kans.-Assistant Observer, \$I,000.

Ithaca, N. Y.-(Center of New York section, Climatological service.)

Section Director, \$I,800.

Jacksonville, Fla.-(Center of Florida section, Climatological service.)

Section Director, $\$ \mathrm{I}, 800$.

Jupiter, Fla.-Assistant Observer, $\$ 1,000$.

Kalispell, Mont.-Assistant Observer, \$1,oco.

Kansas Citr, Mo.-Local Forecaster, \$I,800.

Keoruk, Iowa.-Observer, \$1,200.

Key West, Fla.-Assistant Observer, \$1,ooo.

Kingston, Jamaica, W. I.- (In charge of special hurricane observer; $\$ 25$

a month during hurricane season.)

Knoxille, Tenn,-Observer, \$1,200.

La CRosse, IVIS.-Observer, \$I,200. 
LANDER, Wyo.-Observer, \$1,200.

La Salle, Ill-Observer, $\$ 1,200$.

Lewiston, Idairo.-Assistant Observer, \$1,000.

Lexington, Ky.-Local Forccaster, $\$ 1,400$.

Lincoln, Nerr.-(Center of Nebraska section, Climatological service.) Section Dircctor, $\$ 1,800$.

Little Rock, Ark.-(Center of Arkansas section, Climatological service.) Section Dircctor, $\$ 1,600$.

Los Axgeles, Cal.-Local Forecaster, \$1,600.

Louisville, Ky.-(Center of forceast district cmbracing Kentucky and

Tennessec, also center of Kentucky section, Climatological service.)

District Forecaster, $\$ 2,400$.

LyNCHBURG, VA.-Observer, \$I,200.

Macon, Ga.--Observer, $\$ 1,200$.

Madison, Wis.-Observer, $\$ 1,200$. ,

Manteo, N. C.-Repairman, $\$ 720$.

Marquette, Mich.-Observer, \$I,200.

Memphis, Tenn.-Local Forecaster, \$I,600.

Mieridian, Miss.-Observer, \$I,200.

Miles City, Mont.-Station Agent, \$300.

Milwauke, Wis.-(Center of Wisconsin scetion, Climatological service; also Inspector's headquarters.) Inspector, $\$ 2,500$.

Minneapolis, Minn.--(Center of Minnesota section, Climatological service.) Section Director, $\$ 1,800$.

Mobile, Ald.-Local Forecaster, \$1,400.

Modens, Utall-Assistant Observer, \$720.

Montgomery, Ala.-(Center of Alabama section, Climatological service.) Section Dircctor, $\$ 1,800$.

Moorinead, Minn.-Assistant Obscrver, \$i,oco.

Mount Tamalpais, Cal.-(Branch of San Francisco office; in charge of an assistant detailed thercfrom by the official in clarge.)

Mount Weather, Va.-Professor of Meteorological Physics, \$3,000.

Nantucket, Mass.-Assistant Observer, $\$ 1, \infty 00$.

Narragansett, R. I.-Assistant Obscrver, $\$ 840$.

Nashrille, Tenv.-(Center of Tennessce section, Climatological scrvice.)

Section Director, $\$ 1,600$.

Neah Bay, Wash.-Repairman, \$720.

New Haven, Conn.-Local Forccaster, \$I,Soo.

New OrLenss, LA.-(Center of forecast district, cmbracing Texas, Oklahoma, Indian Territory, Arkansas, and Louisiana; issucs advisory warnings for Mcxico; has charge of conperation between Mexican Weather

Service and United States Veather Burcau; center of Louisiana section,

Climatological service.) District Forecaster, $\$ 2,400$.

New York, N. Y.-District Forccaster, $\$ 2,400$. 
Nome, Alaska.-Special Observer, \$300.

Phoenix, Ariz.-(Center of Arizona section, Climatological service.) Section Director, $\$ \mathrm{I}, 600$.

Pierre, S. Dak.-Observer, \$I,200.

Pittsburg, PA.-Local Forecaster, \$2,000.

Pocatello, Idaho.-Observer, \$I,200.

Point Reres light, Cal.-(Under the supervision of San Francisco, Cal.) Assistant Observer, $\$ \mathrm{I}, 400$.

Port au Prince, Haiti, W. I.-(In charge of special hurricane observer; $\$ 25$ a month during hurricane season.)

Port Crescent, Wash.-Assistant Observer, \$i,00o.

Port Huron, Mich.-Observer, \$r,200.

Portland, Me.-Local Forecaster, \$I,400.

Portland, Oreg.- (Center of forecast district, embracing Washington, Oregon, and Idaho; also center of Oregon section, Climatological service.) District Forecaster, $\$ 2,400$.

Port of Spain, Trinidad, W. I.- (In charge of agent of West India and Panama Telegraph Company.)

Norfolk, VA.-Local Forecaster, \$r,800.

Northfield, Vt.-Local Forccaster, \$1,400.

Nortil Head, Wash.-Assistant Observer, \$1,000.

North Platte, Nebr.-Assistant Observer, \$I,000.

Oklahoma, OKLA.-(Center of Oklahoma section, Climatological service.)

Section Director, $\$ \mathrm{I}, 400$.

Omaha, Nebr.-Local Forecaster, \$I,600.

Oswego, N. Y.-Observer, \$1,200.

Palestine, Tex.-Observer, \$I,200.

Parkersburg, W. VA.--(Center of West Virginia section, Climatological service.) Section Director, $\$ 1,400$.

Pensacola, Fla.-Observer, \$I,200.

Peoria, Ill.-Observer, \$r,200.

Philadelphia, PA.- (Center of Pennsylvania section, Climatological service.)

Section Director, $\$ 2,000$.

Providence, R. I.-Local Forecaster, $\$ 1,600$.

Pueblo, Colo.-Local Forecaster, \$I, 400 .

Pysht, Wash.-Repairman, $\$ 720$.

Raleigf, N. C.-(Center of North Carolina section, Climatological service.)

Section Director, \$1,60n.

Rapid City, S. Dak.-Observer, \$1,200.

Red Bluff, Cal.-Assistant Observer, \$1,000.

Reno, Nev.-(Center of Nevada section, Climatological service.)

Richmond, VA.- (Center of Virginia section, Climatological service.) Section Director, $\$ \mathrm{I}, 800$.

Rochester, N. Y.-Local Forecaster, \$1,600. 
Roseau, Dominica, W. I.-(In charge of agent cf West India and Panama Telegraph Company.)

Roseblrg, Oreg.-Assistant Observer, \$I,000.

Roswell, N. Mex.-Assistant Observer, \$I,000.

Sacramento, Cal.-Local Forecaster, $\$ 1,400$.

Sr. Lot'is, Mo.-Local Forccaster, \$2,000.

Sr. Paul, Mixx.-Local Forecaster, \$i,40o.

Salt Lake City, Utah.-(Center of Utah section, Climatological service.) Scction Director, $\$ 1,800$.

San Antonio, Tex.-Local Forecaster, \$I, 400 .

San Diego, Cal.-Local Forccaster, \$I, 400 .

SAND KEY, FlA.-(Under the supervision of Key West, Fla., from which station officials, alternating nonthly, are assigned.)

Sandusky, Ohio.-Observer, $\$ 1,200$.

San Francisco, Cal-(Center of forecast district, embracing California and Nevada; also center of California section, Climatological service.) Professor of Meteorology, $\$ 3,000$.

San Jose, Cal.-Observer, $\$ \mathrm{I}, 200$.

SAN Juan, Porto Rico, W. I.-(Center of Porto Rico section, Climatological service.) Assistant Observer, \$1,000.

San Luis Obispo, Cal.-Observer, \$I,200.

Santa Fe, N. Mex.-(Center of New Mexico section, Climatological service.) Section Director, $\$ 1,400$.

Santo Domingo, W. I.-(In charge of special hurricane observer; \$25 a month during hurricane season.)

Sault Ste. Marie. Micit.-Observer, \$i,zoo.

Sayannail, Ga.-Local Forecaster, \$1,600.

Scranton, Pa,-Local Forecaster, \$1, 400 .

SEAttle, WASH.-(Center of Wastington section, Climatological service.) Section Director, $\$ 1,800$.

SEKIOU, IVASH.-(Repair station; nperated during months of Jamuary. February, March, November, and December.)

Sheridan, Wyo.-Observer, $\$ 1,200$

Shreverort, LA.-Observer, \$1,200.

Siovx City, Iowa.-Local Forecaster, \$I,-foo.

Southeast Farallon, Cal.-(Branch of San Francisco office: in clarge of an assistant detailed thercfrom by the official in charge.)

Spokane, Wasir.-Local Forecaster, \$i,joo.

SPRingField, Ill-_ (Center of Illinois section, Climatological service.) Section Director, $\$ \mathrm{I}, 600$.

Springfield, Mr.-Local Forecaster, \$1, foo.

Syracuse, N. Y.-Local Forecaster, \$r,joo.

TAComa, Wash.-Local Forecaster, \$1,400.

TAMPA, Fla.-Local Forecaster, \$1,400. 
Tatoosh Island, Wasir.-Assistant Observer, $\$ 1,000$.

TAyloR, TEX.-Observer, \$I,200.

'Ihomasville, Ga.-Assistant Observer, \$1,000.

Ioledo, OHio.-Local Forecaster, \$I,600.

Tonopah, Nev.-Assistant Observer, \$r,ooo.

Topeka, Kans.-(Center of Kansas section, Climatological service.) Section Director, $\$ 1,600$.

Turks Island, W. I.-(In charge of agent of Halifax and Bermudas Cable Company.)

TWIN, WASH.-

Valentine, Nebr.-Assistant Observer, \$I,ooo.

Vicksburg, Miss.-(Center of Mississippi section, Climatological service.)

Section Director, $\$ 1,600$.

Walla Walla, Wasif.-OObserver, \$1,200.

Wash Woods, N. C.-Repairman, $\$ 720$.

Wichita, Kans.-Local Forecaster, \$1,400.

Wilisiston, N. Dak.-Assistant Observer, $\$ 1, \infty 00$.

Wilmington, N. C.-Observer, \$1,200.

Winnemucca, Nev.-Assistant Observer, $\$ 1,000$.

Wytilerille, VA.-Observer, \$1,200.

YANKTON, S. DAK.-Observer, \$1,200.

Yellowstone Park, Wyo.-Assistant Observer, \$I,000.

YUMA, ARIz.-Assistant Observer, \$I,ooo. 


\section{CHAPTER XVI. \\ DEP.ARTMENT OF COMMERCE A.ND L.JBOR.}

As has been said in another place, the Department of Commerce and Labor consists of an aggregation of bureaus not intimately connected, but which have been amalgamated under one head, because of their more or less close relations to subjects relative to the fields of activity indicated by the title of the department. The function which is exercised by the department proper, outside of its jurisdiction over and administration of departmental affairs relative to the various burcaus, is the protection of the fur seals and the salmon in Alaska. Special agents of the department, under the direct control of the Secretary, pay visits of inspection to the various seal and salmon fisheries and sec that the provisions of the contract in the former case, and of the restrictire laws passed by Congress in the latter, are not violater.

One of the clicf bureaus of the Department of Commerce and Labor in the public attention at the time of the constitution of the department is the Bureau of Corforations. This is an investigative bureau purcly, having no functions except to inquire into the character of the transactions and business of corporations doing interstate commerce, and which have been chartered by the States of the Union. The head of the burcau, under tine name of Commissioner of Corporations, is appointed by the Presiclent, without confirmation, and reccives $\$ 5,000$ a year.

A cleputy Commissioner is provided at $\$ 3.500$ a year, and the function of thesc officers is to secure information in regard to the operation of corporations other than common carricrs doing an interstate business. Similar powers are given to the Commissioner of Corporations to those exercised by the Interstate Commerce Commission, in compelling the testimony of interested persons, and such testimony being required or admitted has the effect of securing immunity from criminal proccedings for those who have taken part in the transaction under 
investigation, and whose sworn testimony has been accepted or required by the Commissioner of Corporations. It is the theory under which the Bureau of Corporations was established that the regulation of abuses which have grown out of the corporate form of transacting business may be to a degree secured by giving publicity to the nature of the transactions of the corporation, especially to those which affect the value of the shares which are offered to the public for purchase. The especial bearing of the operations of the bureau under existing law relates to the so-called "Sherman Act," prohibiting combinations in restraint of trade. The first complete investigation of the bureau was, however, of the affairs of the Standard Oil Company, in which the charge of securing rebates from railroads was a prominent factor, so that the most prominent operation of the bureau in the public eye is that which developed infractions of the Interstate Commerce Law, which really come within the jurisdiction of the Interstate Commerce Commission. A considerable number of investigations have, however, been made, the results of which have not come to public notice, through legal proceedings. Some embarrassment has been caused by the provision of law which enables a person who has been engaged in illegal transactions to secure immunity from punishment therefor by taking advantage of an opportunity to testify before the Commissioner, but care has been taken to minimize this difficulty in later cases. The specific terms of the law require the Commissioner of Corporations to gather, compile, publish and supply useful information concerning corporations doing business within the limits of the United States who engage in interstate or foreign commerce, including corporations engaged in the business of insurance. As a means to gathering this information, the bureau employs a considerable force of special agents, and its work would constitute a basis for any legislation that might be effected by the Congress for the purpose of regulating the affairs and operations of corporations other than common carriers.

The Bureau of Manufactures of the Department of Commerce and Labor exercises the functions designed for the department in connection with commerce, and is intended to promote the extension of the markets for the manufactures of the United States, and 
to provide information to manufacturers as to the best method of developing trade at home and abroad. The bureau receives the reports from the Consuls and Consular Agents of the United States in foreign countries, and issues daily and monthly bulletins, containing such portions of these reports as are calculated to be of service to merchants and manufacturers. The bureau also publishes the annual report formerly" issued by the State Department, entitled "Commercial Relations of the United States." Agents of the bureau are sent abroad to travel through the various trade centers, for the purpose of supplementing the Consular Reports with special expert reports by the bureau's own agents. The Chicf of the bureau receives four thousand dollars a year, and is appointed by the President.

The Bureau of Labor, formerly under the Interior Department, was added to the Department of Commerce and Labor at the time of the institution of the latter department. It is directed by a Commissioner of Labor, who is appointed by the President, by and with the advice and consent of the Senate, and who receives a salary of five thousand dollars a year, with a term of office of four years.

A similar bureau to that which is now in effect as a portion of the Department of Commerce and Labor has been in existence since I885. Its duties are to acquire and diffuse among the people of the United States useful information on subjects connected with labor, when that word is used in a general and comprehensive sense. In pursuance of this duty, the bureau under the direction of the Commissioner, has carried on a series of investigations into the hours of labor, wages paid and the condition of the laboring classes generally in the United States, and in Hawaii, and has also issued bulletins comparing the situation in this comtry with labor conditions abroad. The bureau also represents the United States in the International Association for labor legislation, and an annual appropriation is made for the bureat's share in the cxpenses of this Association.

The Commissioner of Labor is required to make an anmulal report on his investigations, and is enpowered to issue bulletins from time to time, giving the results of the different inquiries made by 
him and the information collected within the territorial scope of his bureau.

Undcr the so-called "Arbitration Act," the Commissioner of Labor and the Chairman of the Interstate Commerce Commission are required in case of any controversy between capital and labor, upon the request of either party, to put themselves into communication with both parties to the controversy, for the purpose of bringing about an arrangement for a settlement of the strike or lock-out, and to arrange for arbitration proceedings, the result of such arbitration, when it has been authorized by both parties, being supposed to be binding and final. It has been found, however, in practice and in the majority of cases, that the arbitration board thus provided for has little more than persuasive power with reference to the settlement of controversies between capital and labor. Investigations and reports of the bureau have, however, been essentially valuable in creating for the United States a systematic foundation for the consideration of sociological problems, and the development of the bureau in the way of accurate statistics has been most gratifying to those who are interested in the question.

A large portion of the current work of the bureau relates to the question of child labor, in which there is a considerable and growing interest, with the purpose of securing uniform State legislation and, if possible, some form of Federal enactment.

\section{THE LIGHTHOUSE BOARD.}

The maritime colonies which later entered into the United States, had established eight lighthouses for the guidance of navigation into their principal ports, and these lighthouses, with their surrounding grounds, were ceded to the Federal Government, and accepted in I 789 . Since that time, the establishment and maintenance of such aids to navigation have been under the direction solely of the Federal Government. Individual officials of the Treasury Department administered business relative to the lighthouses until 1852 , when the organization was constituted in its present form. Two officers of the Navy of high rank, two officers of the Corps of Engineers of the Army, and two civilians of scientific attainments, constitute the board, the Secretary of Commerce and Labor being the ex-officio 
Chairman. The actual Chairman of the Board is, however, elected by ballot, the senior naval officer assigned to duty on the board being ustrally elected as the presiding officer. In aldition to the membership of the board, an erigincer officer and a naval officer are assigned to duty as secretaries. The board as a whole mects for business on the first Monlay's in Y.. Tarch, June, September and December, but in the interim, the Chairman of the board, with the secretaries, transacts the general business of the lighthouse establishment. The board makes arrangements for the acquisition of all land for the site of lighthouses and range marks and other constructions required by the service on shore. and secures through the engineer secretaries the preparation of plans and specifications for the construction and repair of all bulleings, and of lighting and fog signaling apparatus. It lays out the location for all light vessels. beacons, buoys and directs all matters relative to their care and maintenance. Aids to navigation under the direction of the Lighthouse Board include the lighthouses of the several classes on the seacoast, the Great Lakes and the navigable rivers, the buys marking the channels into the seaports, and the courses in rivers and lakes, together with all fog signaling apparatus. Peacons are fixed marks of iron, stone or wood on ledges and sloals in navigable waters which are sufficiently near the surface to constitute a danger to navigation. Puoys are either anchored spars or cylindrical constructions of iron without superstructures, or iron frameworks on floating supports, which carry either a bell or a gas torch. the latter bcing supplied by stored gas in the tank which forms the float for the superstructure.

The older form of fog signal in use consists of a bell struck at known intervals by a clock-work apparatus. actuated by a weight. These are being superseded by whistles and sirens, actuated by steam or compressed air. The lighthonses are of several types, as to the distance at which they can be seen, and of four seneral classes: Fixed white lights, fixed red lights, revolving lights which show at given intervals, and flash liglits, which by a given number of successive appearances aid, together with their location, in their identification. The aids to navigation of the United States are divided among the following districts. 
The first district extends from the head of navigation on the St. Croix River, Maine, the northeastern boundary of the United States, to, and includes, Hampton Harbor, New Hampshire. It embraces all aids to navigation on the seacoast of Maine and New Hampshire, and on all tidal waters between the limits named.

The second district extends from Hampton Harbor, New Hampshire, to Elisha Ledge, off Warren Point, Rhode Island, but does not include either the harbor or the ledge. It embraces all aids to navigation on the seacoast and tidal waters of Massachusetts, excepting on the Taunton River and that part of Mount Hope Bay lying within the State boundary.

The third district extends from Elisha Ledge, off Warren Point, Rhode Island, to a point on the coast of New Jersey opposite Shrewsbury Rocks, and includes the ledge and the rocks. It embraces all aids to navigation on the coasts of Rhode Island, Connecticut, and New York, and of New Jersey northward of the point opposite Shrewsbury Rocks, and on all tidal waters tributary to the sea or Long Island Sound, between the limits named, together with the aids on Whitehall Narrows, and on the United States waters of Lake Champlain and Memphremagog. It includes the island of Porto Rico, and the adjacent islands and the waters of the islands lying east of the seventy-fourth meridian of longitude west of Greenwich, which were celled to the United States by the Government of Spain by treaty entered into on December, I0, I898. It also includes the waters of Guantanamo Bay, Cuba.

The fourth district extends from a point on the coast of New Jersey, opposite Shrewsbury Rocks (but does not include the rocks), to, and includes, Metomkin Inlet, Virginia. It embraces all aids to navigation on the seacoast of New Jersey, Delaware, Maryland, and Virginia, and the tidal waters tributary to the sea between the rocks and the inlet.

The fifth district extends from (but does not include) Metomkin Inlet, Virginia, to, and including, New River Inlet, North Carolina. It embraces all aids to navigation on the seacoast of Virginia and North Carolina, between the limits named, all of Chesapeake Bay, the sounds of North Carolina, and tributary waters.

The sixth district extends from (but does not include) New River Inlet, North Carolina, to, and includes, Jupiter Inlet light-station, Florida. It embraces all aids to navigation on the seacoast, bays, sounds, harbors, rivers, and other tidal waters of North Carolina, South Carolina, Georgia, and Florida between the limits named.

The seventh district extends from a point just south of Jupiter Inlet light-station to, and including, Perdido Entrance, Florida. It embraces all aids to navigation on the sea and gulf coast of Florida, and on other tidal waters tributary to the sea and gulf between the limits named.

The eighth district extends from (but does not include) Perdido Entrance, Florida, to the southern boundary of Texas. It embraces all aids to navi- 
gation on the Gulf coast of the United States and tidal waters tributary to the Gulf, between the limits named, together with those on the Mississippi River below New Orleans, and on Grand Lake and Lake Clicot.

The ninth district includes all aids to navigation on Lake Michigatn, Green Bay, and tributary waters lying west of a line drawn atcros: the Straits of Mackinac just east of Old Mackinac Point light-station, Michigan.

The tenth district extends from the mouth of the St. Regis River, St. Lawrence River, New York, to the mo:th of the River Rouge, Detroit River, Michigan.. It embraces all aids to navigation on the United States shores and waters of Lakes Erie and Ontario, and the upper part of the St. Lawrence, the Niagara, and the lower part of the Detroit rivers.

The eleventh district extends from the month of the River Rogne, Detroit River, Michigan, to the westerly end of Lake Superior. It embraces all aids to navigation on the United States shores and waters of Lakes St. Clair, Huron, and Superior, the upper part of the Detroit River, the St. Clair and St. Marys rivers, and that part of the Straits of Mackinac lying to the eastward of a line drawn across the straits just to the eastward of Old Mackinac Point light-station, Michigan.

The twelfth district extends from the boundary between California and Mexico to the boundary between California and Oregon. !t embraces all aids to navigation on the scacoast, bays, rivers, and other tidal waters of California. It also includes the waters of the Territory of Hawaii.

The thirteenth district extends from the boundary between California and Oregon to the northern boundary of the United States, and inclucles Alaska. It embraces all aids to navigation on the seacoast of Oregon and Washington, on the United States waters of the Strait of Juan de Fuca. Waslington Sound, and the Gulf of Gorgia, and on the ticial waters tributary to the sea, strait, sound, and gulf between the limits named, together with those on Alaskan waters.

The fourteenth district extends on the Olio River from Pittsburg, Pa., to Cairo, Ill., 965 miles; on the Tennessee River, 257!' miles, and on the Great Kanawha, 731/2 miles; in all, a distance of 1,295 miles, and cmbraces all the aids to navigation within these limits.

The fiftenth district extends on the Mlississippi River from the head of navigation to Cairo, Ill., on the Missouri River to Kansas City, and on the Illinois River to La Salle, and includes all aids to navigation within those limits.

The sixteenth district extends on the Mississippi River from Cairo, Ill.. to New Orleans, La., and on the Red River a distance of 9 miles, and includes all aids to navigation within those limits.

To each of these districts an officer of the army or navy may be assigned as an inspector. As a matter of practice, the inspecting 
officers are almost exclusively officers of the navy who perform their tours on shore duty in the Lighthouse Inspection Service. In addition, officers of the Engincer Corps of the army engaged in river and harbor work or otherwise, may be assigned as engineer officers. Inspectors and engineers are charged, in their respective districts, with the care of all aids to navigation. Inspectors pay the lighthouse keepers, the officers and seamen of light vessels and are disbursing officers for other funds under the lighthouse establishment. They are required to visit each iight at least once every quarter, and are required to make detailed repcrts as to the condition of the lighthouse or light vessels, and as to the performance of their duties by the light keepers. They are authorized to exercise disciplinary power with reference to light keepers and employees and also to determine upon the exact location at which light vessels shall be anchored and to have supervision over the relief light vessels and have them ready for service at all times. They are also charged with the duty of preparing the notices to mariners which give information as to changes in and additions to aids to navigation. In order to enable them to perform their duties, one or more lighthouse tenders are maintained in each district, which not only carry supplies to the lights but serve as the medium of transportation of the inspector upon his visits. The keepers of lighthouses are appointed as the results of examination by the Civil Service Commission, application blanks being issued by the Lighthouse Inspectors.

Nominations are made by the Inspector of the District in which a vacancy occurs for the promotions of the keepers already in office, if the vacancy is in a higher grade, and original appointments are made as far as practicable to the lower grades of the service. If, however, no person is available for nomination to the higher position, an original appointment may be made on the recommendation of the inspector. Keepers and assistant keepers must be over eighteen years of age and less than fifty years, must be able to read and write, and competent to discharge the duties of the position. Especially strict rules are made against employment or continuance of any person in the lighthouse service who is accustomed to the use of intoxicants. The keepers and assistants are required to wear a uniform, consisting of 
a blue double-breasted coat, with blue trousers, and uniform caps, the coats bearing the buttons of the lighthouse service. It is forbidden to leave a lighthouse at any time without a responsible person in charge, and where a kecper and an assistant are provided, one of these must be on duty at all times. If a keeper only is provided, a competent member of his family may be left in charge of the lighthouse. I-ightiouse keepers need not necessarily be of the male sex, as in a number of cases women who have shown themselves competent have been appointed keepers at the smaller lighthouses.

Lights are classed in six orders.

First-order lights are established to give warning of the approach to land.

Second-order lights mark the secondary points or headlands along the seacoast and the approaches to bays and sounds.

Third-order lights are required in bays that are of considerable width and intricacy, as Delaware Bay; also for principal lights for the coasts of the lakes.

Fourth, fifth and sixth order lights mark the most prominent points, headlands or shoals in the large bays or sounds or obstructions in rivers and prers and wharves.

I.ight ressels are established on shoals, where it is not possible to secure foundation for a lighthonse, and consist of hulls especially constructed with reference to seaworthiness, and fitted with short masts, displaying the character of light prescribed for the station. They are also fitted with fog signals, and each vessel remains on its station for a period of three months, when it is relieved by another vessel, and returns to the nearest port for repairs and refitting and for a period of rest for the crew. In addition to the lights maintained by the Government, the Lighthouse Poard has also direction of the lighting of bridges across navigable rivers and hays. and prescribes the regulations for the character of the lights to be displayed on such structures, in order that vessel traffic may know the character of the design of the bridge. 


\section{THE CENSUS BUREAU.}

It was required by the constitution that a census of the United States should be taken every ten years, in order to provide a basis for representation in Congress and direct taxation. The decennial censuses were under the direction of the Secretary of State until the establishment of the Departmnt of the Interior in I849, and until I880 the actual work of enumeration was under the direction of United States marshals in the different judicial districts. In I880, a Superintendent of the Census was appointed, who directed the collection of the census returns through supervisors and enumerators, practically in accordance with the present system. The censuses of 1890 and I900 were taken by a temporary organization, but in 1902 the Bureau of the Census was made a permanent part of the governmental organization of the United States, and under the administration of a Director of the Census is to undertake various branches of statistical and census work, in order that it may not be necessary fully to renew the organization for each decennial census. The permanent Census Bureau is of course numerically very much smaller than the Bureau which conducts the active work of tabulating the results of the enumeration for the decennial census, but the head of the various subdivisions, together with a certain proportion of skilled employees are given permanent employment, so that upon the taking of the next decennial census it will be a comparatively small matter to add a sufficient force to make a complete bureau. The act under which the permanent census office was organized provides :

That the Thirteenth and subsequent censuses shall be restricted to inquiries relating to the poptulation, to mortality, to the products of agriculture and of manufacturing and mechanical establishments. The schedules relating to the population shall comprehend for each inhabitant the name, age, color, sex, conjugal condition, place of birth, and place of birth of parents, whether alien or naturalized, number of years in the United States, occupation, months unemployed, literacy, school attendance, and ownership of farms and homes: and the Director of the Census may use his discretion as to the construction and form and number of inquiries necessary to secure in- 
formation under the topics aforesalid. The mortality sehediles shall comprehend for cach decedent the name, sex, color, age, conjugal cundition, place of birth, and birth place of fruents, occupation, cause and dite of death, and, if born within the census year, the date of lifith. The form and arrangement of the schedule and the specific questinne necessary to secure the information required shail be in the discretion of the I)irector. The schedules relating to agriculure shall comprehend the following topics: Name of occupant of each farm, color of occupant, tenure, acreage, value of farm and improvements, acreage oi different products, quantity and value of prolucts, and number and value of live stock. All questions as to quantity and value of erops shall relate to the year ending December thirty-first next preecding the entmeration. The specific form and division of inquiries necessary to secure information under the foregning topics shall be in the discretion of the Director of the Census. The schedules of inquirics relating to the products of manufacturing and mechanical establishments shall embrace the name and locatin of cach estalilizment; character of organization, whether individual, co-opeative, or other form: date of commencement of operations; character of lusiness or lind oi goods manufactured; amount of capital invested; number of proprietors, firm menbers, copartners, or officers, and the ansonnt of their salaries; number of employes, and the amount of their wages: quantity and cost of materials used in manufactures; amount of miscelfancous expenses: quantity and value of products; time in operation during census year; chardeter and quantity of power used, and character and number of machines employed.

After the completion and return of the cummerition and of the work upon the schedules relating to the products of agriculture and to manufacturing and mechanical establishments provided for in the preceding scetion, the Director of the Census is authorized decennially to collect statistics relating to the defective, dependent, and delinquent classes; to crime, including judicial statistics pertaining thereto, which statistics shall include information upon the questions of age, sex, color, race, nativity, parentage, literacy by race, color, nativity, and parentage, and such other questions relating to these subjects as the Director in his discretion may deen proper; to social statistics of cities; to public indebtedness, valuation, taxation, and expenditures; to religious bodies: to transportaton hy water, and express business: to mines, mining, quarries, and mincrals, and the production and value thercof, including gold in divisions of pheer and vein, and silver mines, and the number of men employed, the average daigy wage, average working time, and aggregate carnings in the various branches and aforesaid divisions of the mining and quarrying industries: to savings banks and other savings institutions, mortgage, loan, and investment companies, and similar institutions: to the fishing industry in co-operation with the Bureau of Fisheries; and every five years to collect statistics relating to street railways, electric light and power and the telephone and telegraph business. 
The Director of the Census is appointed by the President, by and with the advice and consent of the Senate, and receives six thousand dollars a year. $\mathrm{He}$ is empowered to appoint fourteen statisticians, who have charge of the different branches of the work, and provision is made for adequate permanent force, the members of which were covered by the terms of the Census Act, into the classified service, so that the temporary employees of the Bureau received permanent employment in the Govermental service without examination. Special agents are also provided for, to be employed by the Director of the Census, for the purpose of enumeration and investigation, not included in the decennial census. For the purpose of the latter enumeration, the Director of Census prescribes schedules upon which the desired information may be uniformly stated, and at least six months previous to the beginning of the decennial census, the Director is required to designate how many supervisors of the census are to be appointed in each state and territory, whether one or more in each, and the President is empowered to appoint, by and with the advice and consent of the Senate such number of supervisors, not, however, to be in the aggregate more than three hundred. So far as possible the limits of the districts of the supervisors are to be those of the Congressional Districts. Upon the completion of the duties of the supervisor to the satisfaction of the Director of the Census, he is to receive one hundred and twenty-five dollars, and in addition one dollar for each thousand of population enumerated in thickly settled disiricts, and one dollar and forty cents for each thousand enumerated in sparsely settled districts. The duties of the supervisor consist of dividing his supervisory districts into sub-divisions, with the approval of the Director of the Census, and of designating to the Director suitable persons to take the enumeration within such sub-division. In so far as practicable, persons who reside in the sub-divisions are to he employed, but if no suitable person can be found in the subdivision, any fit person may be given the position of enumerator. Upon approval by the Director of the Census, the supervisor may employ such persons as he has nominated, and is required to 
oversee their work and forward to the Director of the Census their completed returns of the enumeration, together with a statenent of the amounts which have become due to the enumerators for their work. In case any supervisor fails to perform his duty, the Director of the Census has power to designate a person to take his place.

It is the duty of each enumerator to visit each dwelling house in his sub-division, and secure from the head of the family, or some person competent to give the information, the facts called for by the census schedules. Each enumerator must receive a comnission from the supervisor, and each sub-division is to contain four thousand inhabitants, estimated as nearly as it may be possible, and it is required that the divisions of enumerators' districts shall be specifically set forth by well-known gcographical characteristics. Special enumerators for institutions may be appointed without regard to the total of population, and interpreters may be employed to assist the enumerators where necessary. The compensation of entmerators is fixed by the Director of the Census, within the following restrictions.

In sub-divisions where he shall deem such allowance sufficient, an ailowance of not liss than two nor more than three cents for each living inhabitant and for each death reported; not less than fiftecn nor more than twenty cents for each farm; and not less than twenty nor more than thirty cents for each establishment of productive industry entmerated and returned may be given in full compensation for al! services. For all other sub-divisions per diem rates shall be fixed by the Director of the Census according to the difficulty of entumeration, having reference to the mature of the region to be canvassed and the density or sparseness of settlement, or ther considerations pertinent thereto; but the compensation allowed to any enumerator in any such district shall not be less than thece dollars nor more than six dollars per day of ten hours actual fieid work each. The sub-divisions to which the several rates of compensation shall apply shall be designated to the Director of the Census at least two wecks in advance of the enumeration. No clain for mileage or traveliner cxpenses shall be allowed any enumerator in either class of sub-divisicns, except in extreme cases, and then only when authority has been previonsly granted by the Director of the Census, and the decision of the Directer as to the amount due any enumerator shall be final.

The special agents have like authority with the cnumerators in respect to 
the subjects committed to them and receive compensation at rates to be fixed by the Director of the Census: Not to exceed six dollars per day and actual necessary traveling expenses and an allowance in lieu of subsistence not exceeding three dollars per day.

All field employees of the census are required to take an oath of office, and any violation of the oath or fraudulent receiving of compensation not earned, or making a return of false information is punishable by fine or imprisonment or both. The enumeration required by the Census act is to be Degun on the first day of June of the year in which the census is taken, and must be completed by the first day of July, except that in cities of eight thousand or more, the enumeration must be completed within two weeks from the first of June. Upon receipt of the returns from the supervisors at the Census Bureau, they are tabulated, ingenious tabulating machines being used to expedite the work, and the results are consolidated and compared in the series of volumes constituting the Census Report. Under the former system of a transitory bureau, the completion of this work has occupied nearly the entire decennial period. It is believed, however, with the organization and facilities now available that the next census will be completed in less timse than any census in the history of the country. In the period before the taking of the next general census, various special inquiries are being taken up, including vital statistics, statistics of divorce, statistics relative to the financial affairs of cities and other matters which are ordered by the Congress or by the President from time to time. A special census was taken of the Indian Territory and the territory of Oklahoma before the admission of the new States, in order to determine the character of the districting work which had been done, and under a provision of the Congress, the Bureau of the Census now prepares and issues the Official Register of the United States, giving the names, place of employment and compensation of each person in the Government service, including the Post Office service, as well as the departmental service proper. 
THE COAST AND GEODETIC SLRVEY.

The functions of the Coast and Geodetic Survey are sct forth in the following extract prescribing its field of operation:

"The functions of the Coast and Geodetic Survey shall be to do cverything requisite for and incident to the survey of the consts of the Cnited States and of coasts inder the jurisdiction of the United States, including the survey of rivers to the head of tide water or ship navigation; deep-sea soundings, temperature and current vibervations along the coast and thronghout the Gulf stream and Japan stream llowing (ff said coasts; tibal obscrvations; the necessary resurvey; the preparation of the Coast Pilot: contimuing researches and other work relating to physical hydrography and terrestrial magnetism and the magnetic maps of the Unired States and adjacent waters, and the tables of magnetic declination, dip, and the intensity ustally accompanying tincm; astronomical and gravity obecrvations; special examinations that may be required by the Lighthouse Board or other proper authority, and farmishing points to State Surveys."

Its chicf function is the preparation of charts and materials for sailing directions for coast of the United States, and rivers to the head of tide water, but not including the Great Lakes, by means of accurate measurements by triangulation, the bases of which are taken by shore parties and by a serics of soundings or actual measurements by line and weights from the depth of one hundred and twenty fathoms seaward, to as far as the ports and harbors of commerce extend. For the purpose of carrying on these measurements, a fleet of small vessels is maintained, and the surveying partics are made up of civilian scientists, headed by officers of the Navy whenever the detail of such officers can be secured. Owing, however, to the lack of officers sufficient in number to man the vessels of the Navy, there have not been many details in recent years, to the vessels of the Coast Geodetic Survey, except of retired officers. It is provided, lowever, that when active officers of the Nary are detailed to the work they cannot be relieved except with the approval of the Secretary of the Department of Commerce and Labor. A semi-naval organization is maintained on the vessels of the Coast and Crendetic Survey, and a uniform is provided for, the insignia of the service being a circle with a triangle superimposed thereon. The Survey was originally constituted in accordance with a report of 
a board of officers in $18+3$, and the President is given power by law to carry out the various recommendations of these officers. The direction of the service survey is under a superintendent, who is appointed by the President, and who receives five thousand dollars a year. It is provided by law that the publication of the survey, including the charts of the coast of the United States, shall be sold at a price which is to be fixed as nearly as practicable at the cost of paper and printing, and that there shall be no free distribution, except an edition of three hundred copies of each publication, which is made available for distribution to foreign governments. The superintendent of the survey is required to make an annual report to Congress of the work done during the preceding year, together with certain graphic delineations indicating the scope of the work undertaken. The nature of the work done by the Coast and Geodetic Survey is similar to that for which the Hydrographic Office of the Navy Department is equipped, and there has been at times some fear expressed in the Appropriations Committee of the House of Representatives that a duplication of work would follow, unless both offices were carefully supervised. A line of distinction is drawn between the work of the two offices, that of the Coast and Geodetic Survey being confined entirely to coasts under the jurisdiction of the United States, for which the Coast and Geodetic Survey prepares and pulblishes charts, while the work of the Hydrographic Office is confined to charts relative to coasts not under the jurisdiction of the United States, to mapping the results of the surveys by naval vessels in foreign waters, and to revising and improving foreign charts for the use of the Nayy and for sale to the American Nerchant Marine.

THE BUREAU OF STATISTICS.

The Bureau of Statistics of the Department of Commerce and Labor is primarily concerned with collecting and tabulating statistics with relation to the commerce of the United States as shown by exports and imports. Collectors of customs are required to furnish to this Burean the necessary data, and it is also empowered to employ special agents to complete the work. 
This Bureau was formerly under the Treasury Department, and in addition to the specific duties before named, it carries on a series of tables representing the receipts and expenditures of the United States, together with other information tending to show the statistics rclative to the national trade and its relative importance in the world's markets. The Bureau has also been empowered recently to compile a volume setting forth the customs tariffs of the world, together with the various systems of weights and cumage. With the establisiment of the permanent Census Bureau, there has been a suggestion that the statistical work of the Department of Commerce and Labor is unduly divided and propositions have been made for the combination of the Bureau of Statistics with the Census Bureau. The Chief of the Bureau receives four thousand dollars a year, and various statutory directions have been made as to his duties in collecting various information and compiling the results thereof. Some of these directions conflict with those later made for the work of the permanent Census Bureau. so that the proposition before indicated for the consolidation of the two has been seriously considered.

THE STEAMBOAT INSPECTION SERVICE.

The Steamboat Inspection Service of the Department of Commerce and Labor has charge of the administration of the United States statutes which were passed, first to secure the safety of passengers on steam vessels, and later, to insure the safety of all persons who might be on board such vessels, whether passengers or officers and crew. Every vessel propelled in whole or in part by steam comes within these laws, except public vessels of the United States, canal boats and foreign vessels, unless the latter are carrying passengers from ports of the United States. Owing to the increase of the use of gasoline and other sources of motive power than steam, recent legislation has been enacted and further legislation is proposed, including the operation of other than steam vessels within the limits of the inspection service.

The head of the Steamboat Inspection Scrvice is the supervising Inspector General, who is appointed by the President by and with the advice and consent of the Senate, and who receives three 
thousand five hundred dollars a year. He also receives reasonable traveling expenses or mileage at the rate of five cents per mile, while traveling on official business. Ten supervising inspectors are also provided for the service, each of whon receives three thousand dollars a year, with the same traveling expenses as the Supervising Inspector General. The latter and the ten supervising inspectors meet annually in Washington, for the purpose of formulating rules for the inspection service, so that the application of the law may be uniform in all districts. When the action of the Board of Supervising Inspectors is approved by the Secretary of Commerce and Labor, it has the force of law. The member of the board from the Pacific Coast is not required to attend the meetings oftener than once in two years, but must send a report in the odd year. The limits of the district of each supervising inspector are fixed at the annual conference, and within such districts, inspectors of hulls and inspectors of boilers are appointed for the following ports, including the customs collection districts of which these cities are the centers.

Pliladelphia, Pennsylvania: San Francisco, California; New London, Connecticut: Baltimere, Maryland; Detroit, Michigan; Chicago, Illinois; Bangor, Maine: New Haven, Connecticut; Michigan, Michigan; Milwaukec, Wisconsin; Willamette, Oregon; Puget Sound, Washington; Savannah, Georgia; Pittshurg, Pennsylvania; Oswego, New York; Charleston, Sonth Carolina; Duluth, Minnesota; Superior, Michigan; Apalachicola, Florida; Galveston, Texas: Mobile, Alabama; Providence, Rhode Island; New York, New York; Jacksonville, Florida; Portland, Naine; Boston, Massachusetts; Buffalo, New York: Cleveland, Ohio; Toledo, Ohic; Norfolk, Virginia; Evansville, Indiana; Dubuque, Iowa; Louisville, Kentucky; Albany, New York; Cincinnati, Ohio; N.Temphis, Tennessee; Nashville, Tennessec; Saint Louis, Missouri; Port IIron, Michigan; New Orleans, Louisiana; Juneau, Alaska; Saint Nichael, Alaska: Point Pleasant, West Virginia, and Eurlington, Vermont.

The salaries paid are as follows:

For the port of New York, two thousand five hundred dollars per year.

For the districts of Philadelphia, Pennsylvania; Baltimore, Maryland; San Francisco, California, and Puget Sound, Washington, and the ports of Boston, Massachusetts; Buffalo, New York, and New Orleans, Louisiana, two thousand two hundred and fifty dollars per year.

For the districts of Michigan, Michigan; Milwaukee, Wisconsin; Duluth, Minnesota; Providence, Rhode Island; Chicago, Illinois, and the ports of 
Albany, New York; Cleveland, Ohio; Portland, Maine; Juneau, Alaska; Saint Michacl, Alaska, and Noriolk, Virginia, two thotisand dollars per year.

For the districts of Oswego, New York; Willamette, Oregon; Detroit, Michigan, and Mlobile, Alabama, and the ports of Saint Louis, Missouri, and Port Huron, Michigan, one thousand cight hundred dollars per yea:.

For the districts of Pittsburg, Pennsylvania; New Maven, Connecticut; Savannah, Georgia; Charleston, South Carolina; Galveston, Texas; Ncw London, Connecticut; Superior, Michigan; Bangor, Maine, and Apalachicola, Forida, and the ports of Dubuque, Iowa: Toledo, Ohio; Evansville, Indiana; Memphis, Tennessee; Nashville, Tennessee; Point Pleasant, West Virginia; Burlington, Vermont; Jacksonville, Florida ; Lousville, Kentucky, and Cincinnati, Ohio, one thousand five hundred dollars pur year.

In addition the Secretary of Commerce and Labor may appent in districts or ports where there are two hundred and twenty-live steamers and upwards to be inspected annually, assistant inspectors, at a salary, for the port of New York, of two thousand dollars a ycar cach; for the port of New Orleans, Lonisiana; the districts of Philadelphia, Pennsylvania: Baltimore, Maryland; the ports of Boston, Massachusetts; Chicago, Iliincis, and the district of San Francisco, California, at one thousand eight hundred dollars per year each; and for all other districts and ports at a salary not exceeding one thousand six hundred dollars per year cach.

Other assistant inspectors may also be appointed in the discretion of the Secretary of Commerce and Labor.

The inspector of hulls must be a person of good character and suitable qualifications and attainments to perform the services required of an inspector of hulls, who from his practical knowledge of shipbuilding and navigation and the uses of steam in navigation is fully competent to make a rcliable estimate of the strength, seaworthiness, and other qualities of the hulls of vessels and their equipment decmed essential to safety of life in their navigation; and the inspector of boilers must be a person of good character and suitable qualifications and attainments to perform the scrvices required of an inspector of hoilers, who from his knowledge and expericnce of the dutics of an engineer employed in navigating vessels by steam, and also of the construction and use of boilers, and machinery and appturtenances therewith connected, is able to form a reliable opinion of the strength, form, workmanship, and suitableness of boilers and machinery to be employed, without hazard of life from imperfection in the mater- 
ial, workmanship or arrangement of any part of such apparatus for steaming.

The local inspectors must, once in every year, at least, carefully inspect the hull of each vessel within their respective districts, and shall satisfy themselves that every such vessel so submitted to their inspection is of a structure suitable for the service in which she is to be employed, has suitable accommodations for passengers and the crew, and is in a condition to warrant the belief that she may be used in navigation as a steamer with safety to life, and that all the requirements of law in regard to fires, boats, pumps, hose, life-preservers, floats, anchors, cables and other equipments are faithfully complied with.

The local inspectors must also inspect the boilers and their appurtenances in all steam vessels before the same are used, and once at least in every year thereafter, and subject all boilers to hydrostatic pressure. Boilers must be well made, of good and suitable material; the openings for the passage of water and steam, respectively, and all pipes and tubes exposed to heat, must be of proper dimensions and free from obstructions; the spaces between and around the flues sufficient; and the flues, boilers, furnaces, safety valves, fusible plugs, low-water indicators, feed-water apparatus, gauge cocks, steam gauges, water and steam pipes connecting boilers, means of prevention of sparks and flames from firedoors, low-water gauges, means of removing mud and sediment from boilers, and all other such machinery and appurtenances thereof, must be of such construction, shape, condition, arrangement and material that the same may be safely employed in the service proposed without peril to life.

In addition to their duties as inspectors of hulls and boilers, the officers are required to hold examinations for the purpose of determining the qualifications of persons to be appointed as deck officers and engineers of steam vessels. All masters, chief mates and second and third mates, if they are to be in charge of the vessel during a watch, engineers and pilots of all steam vessels, must have licenses, also the masters and chief masters of sail vessels of over seven hundred tons, and all other vessels of 
over one hundred tons which carry passengers. They are required to make careful inquiry into the character of persons applying for a license, as well as into their professional qualifications, and may when satisfied that they are proper persons to receive a license issue such a permit to act in a grade named for the term of five years. Upon the expiration of the term, the license may be renewed or a license of a higher grade issued, should it appear that the applicant's qualifications have improved. Some of the equirements for the issuance of licenses are as follows :

Any applicant for license as master of ocean steamers must furnish satisfactory documentary evidence to the local inspectors that he has had three ycars' experience on ocean steamers, one year of which has been as chief mate, or five years' experience on ocean sail vessels of 300 gross tons and upward, two years of which must have been as a licensed master of sail vessels; and he must understand navigation and be able to determine the ship's position at sea by observation of the sun, to obtain longitude by chronometer, and to determine ship's latitude by the altitude of either the sun, moon, or stars.

Any person who has actually served as a licensed third officer of ocean. steamers of 3.500 gross tons and upward for five years, is eligible for examination for license as master of ocean steamers. Any person who has had three years' actual experience as master of steam vessels of 1,000 gross tons and upward on the Great Lakes may be cxamined for license as chicf mate of ocean steamers, and after having hat one year's actual experience as chicf mate of occan steamers of $\mathrm{r}, 000$ gross tons and upward may be examincd for license as master of ocean steamers.

Applicants for license as master of sail vessels of above 700 tons must have served a year as master of a vessel of over 200 tons or as chief mate of a vessel of over 700 tons. Mates for the larger vessels must have served for a similar time as mates of smaller vesscls.

No original license as master of lake, bay and sound steamers shall be issued hereafter to any person who has not been licensed 
and served at least one year as first-class pilot or chief mate on such steamers.

Any person holding a license as master of lake, bay and sound steamers may have indorsed thereon the authority allowing him to act as master of steamers upon the waters of the Atlantic coast and the Gulf of Mexico if the applicant has had at least one year's experience as mate, quartermaster, or wheelsman of steam vessels upon the waters of the Atlantic coast or the Gulf.

License as chief mate of ocean steamers can be issued to persons who have served three years in the deck department of such vessels, one year of such service to have been as scond mate.

To persons who have had five years' experience on sail vessels of 300 gross tons and over, two years of which have been in the capacity of chief mate.

Second mates of ocean steamers are licensed from persons who have had three years' experience on such steam vessels, two years as watch officer or quartermaster, or two of the three years' experience required may be on the school-ship St. Mary's or some other similar vessel, as indicated by a graduating certificate.

No original license as first-class pilot shall be issued to any person hereafter who has not had three years' experience in the deck department of a steam vessel, sail vessel or barge consort. This refers to pilots employed exclusively by one steam vessel pilots for particular harbors being appointed by State officials.

Careful examination is made into the professional qualifications and character of persons applying for license as engineers and they are divided into chief, first second and third assistant engineers for ocean steamers, condensing and non-condensing lake, bay and sound steamers and condensing and non-condensing river steamers. Engineers must only serve on vessels and in positions for which they are licensed and are subject to revocation of license and penalties for non-licensed service.

Boards of Inspectors also have power to suspend and revoke licenses for misconduct, violation of laws or rules and for failure to properly protect the lives and safety of passengers. Such action is taken after hearing of complaints in the premises, and 
boards of inspectors have power to issuc subpoenas and compel the attendance of witncsses at such hearings. An appeal may be made from the board of local inspectors to the supervising inspector, with an ultimate appeal to the Board of Supervising Inspectors and the Secretary of Commerce and Labor.

\section{THE BUREIU OF FISIIERIES.}

The Bureau of Fisherics is the outcome of legislation passed by the Congress in I87I, requiring an investigation to be made into the cause of the decrease in the supply of food fishes. As the result of this inquiry, considerable devclopments have been secured in the matter of propagating useful kinds of fisherics and in stocking the depleted waters with the artificially hatched specimens. The Bureau of Fisheries maintains stations at different points throughout the United States, both on the seacoast and on the rivers, and is engaged at appropriate times of the year in collecting the exgs of fishes and in keeping them under conditions which have been found to be favorable to their hatching and retaining the young fish until such time as they may be restored to the waters of the lakes, rivers and sea, with a prospect that they may come to maturity.

Two biological stations are maintained for the purpose of studying different sorts of marine life, and a system of railroad transportation has been worked out, so that by means of specially designed cars, fish and fish egrs may be transported from one part of the United States to another, so that new kinds may be introduced at different points and depleted stock made up from locations where supplies are more abundant. The Fish Commission also maintain vessels on the Atlantic and Pacific Coasts which take deep sea cruises for the purpose of studying marine life in the lower depths of the sea, the ressel being fitted with deep-sea dredges and other means for the examination of the lower levels of ocean life.

The Bureau of Fisheries has been especially interested in the efforts to re-stock the Northern Atlantic Coast with the lobster, which is threatened with extinction, but the large portion of its 
activities are with relation to the cod, salmon and shad fisheries, from which it has handled millions of eggs every year.

The Bureau also has charge of investigations in a general way with relation to methods of taking fish, and has taken much interest in the matter of re-stocking depleted trout streams, and in the efforts of individuals to preserve and increase local supplies of fishes. The Bureau is under the direction of a Commissioner, who is appointed by the President, by and with the advice and consent of the Senate, and receives five thousand dollars a year. $\mathrm{He}$ is removable at the pleasure of the President and can hold no other office or employment under the United States.

\section{THE BUREAU OF NAVIGATION.}

The Bureau of Navigation, under the direction of a Commissioner of Navigation, who is appointed by the President, by and with the advice and consent of the Senate, and receives a salary of four thousand dollars a year, has general supervision over the matters relating to the measurements and registration of vessels of the United States, which have been described under the heading of the "Collector of Customs." The Commissioner of Navigation approves the names selected by the builder or owner of a vessel for the craft, so that there shall not be more than one vessel of the same name and description on the list of merchant vessels of the United States. The Commissioner of Navigation also gives the vessel a signal number, and supervises the issue of registration and other papers constituting the ship's documents, and without which a vessel is not entitled to the privileges of the navigation laws of the United States. The Commissioner of Navigation has also general supervision over matters relating to the collection of the tonnage tax, and with relation to fines and penalties imposed for the infraction of the laws of the United States relative to navigation. The description of such vessels which are entitled to registration under the navigation laws are as follows:

Class I: Those built in the United States, wholly by a citizen or by an incorporated company of the United States.

A vessel registered pursuant to law, which by sale has become the property of a foreigner, shall be entitled to a new register upon afterwards becoming 
American properiy, males it has becn cularged or undergone change in build outside of the Lnited States.

Class 2: Those built in the Lnited States, in whole or in part under foreign ownership, and duly recorded, on being purchased, and wholly owned by a citizen of the United States, and never beiore documented.

Class 3: Those captured in a war to which the United States is a party, by a citizen thercof, law fully condemned as prizes, and wholly owned by a citizen or citizens of the United States.

Class 4: Those which have been adjudged to be furfeited for a breach of the law of the United States, and wholly owned by a citizen or citizens thereof. This loes not include vessels scld under a decree in admiraity for debt or scamen's wares.

Class 5: Those wrecked within the waters of the United States, purchased and repaired by a citizen or citizens thercof, at a cost cqual to three-fourths of the cost of the vessel when repaired.

Class 6: Those sold by the Lnited States Govermment to citizens, if built in the United States, but foreign-built vessels bought or chartered by the Govermment are not thereby entitled to registry on sale to a citizen.

A foreign ressel imported in parts and put together in the United States can not be documented.

Marine documents may be issued for vessels owned by an incorporated company within the United States in the name of the president. vice-president, or acting president or secretary of such company, and will not be vacated or affected by the sale of shares in such company to it foreigner.

Steamboats, otherwise entitled to be documented, if employed only in a river or bay of the United States, may be enrolled and licensed as vessels of the United States, although owned wholly or in part by an alien resident.

A documented ressel of the United States, seized o- captured and condemned under the atthority of any forcign power, may recive new marine papers in case she shall afterwards become American property.

In adrlition to the funct:ons relative to keeping the records relating to the merchant vessels of the United States and having an oversight over the cnforcment of the navigation laws, the Commissioner of Navigation is clarged with seeing that the laws relative to the shipping and treatment of seamen in the American Merchant Marine are carried out. He also has the enforcement of the provisions made by law for the conduct of vessels when they are in proximity of each other, such regulations in part being as follows:

A steam-vessel when under way shall carry at a height ahove the luull of not less than twenty feet, a bright white light, so constructed as to show an unbroken light over an are of the horizon of twenty points of the compass. 
On the starboard side a green light so constructed as to show an unbroken light over an are of the horizon of ten points of the compass, and visible at a distance of at least two miles. On the port side a red light so constructed as to show an unbroken light $\mathrm{Orcr}$ an arc of the horizon of ten points of the compass, of stuch a character as to be visible at a distance of at least two miles, both to be fitted with inboard screens projecting at least three feet forward from the light, so as to prevent the lights from being seen across the bow.

A stean-vessel when lowing another vessel shall, in addition to her sidelights, carry two bright white lights in a vertical line one over the other, not less than six feet apart, and when towing more than one vessel shall carry an additional bright white light six feet above or below such light. if the length of the tow measuring from the stern of the towing vessel to the stern of the last vessel towed exceeds six hundred feet.

A vessel which from any accident is not under command shall carry at the same height as a white light nucuioned where they can best be seen, and if a steam-vessel in lieu of that light, two red lights, in a vertical line one over the other, not less than six feet apart, and of such a character as to be visible all around the horizon at a distance of at least two miles; and shall by day carry in a vertical line one over the other, not less than six feet apart, where they can best be seen, two black balls or shapes, each two feet in diameter.

Sailing vessels undier way and being towed carry the green and red but not the white lights. Small steam vessels may carry the white light at such height as may be possible and rowing boats must carry a single white light. Pilot boats carry a white light at the mast head and show an occasional flare. Fishing boats using nets must show two white lights indicating the direction of the nets and steam (trawlers) trawiers a tri-colored lantern showing white dead ahead and red and green to port and starboard respectively. Sailing trawlers show a white flare. Vessels at anchor must show one white light at the bow and if more than i 50 feet in length an additional light at the stern.

In fog, mist, falling snow or heavy rain

A stean-vessel having way upon her shall sound, at intervals of not more than two minutes, a prolonged blast.

A steam-vessel under way, but stopped, and having no way upon her, shall sound, at intervals not more than two minutes, two prolonged blasts, with an interval of about one second between them.

A sailing-ressel under way shall sound, at intervals of not more than one minute, when on the starboard tack, one blast; when on the port tack, two 
blasts in succession, and when with the wind abaft the beam, three blasts in succession.

A vessel when at anchor shall, at intervals of not more than one minute, ring the bell rapidly for about five scconds.

A vessel when towing, a vessel cmployed in laying or picking up a telegraph cable, and a vessel under way, which is unable to get out of the way of an approaching vessel through being not under command, or unable to manoeuver shall, at intervals of not more than two mintutes, sound one prolonged blast followed by two short blasts. A ressel towei may give this signal and no other.

When two sailing vessels are approaching one another, so as to involve risk of collision, one of them shall keep out of the way of the other.

A vessel which is rumning free shall kecp out of the way oi a vessel which is close-hanled.

A vessel which is close-hatuled on the port tack shall keep out of the way of a vessel which is close-hatuled on the starboard tack.

When both are running free, with the wind on different sides, the vessel which has the wind on the port side shall keep out of the way of the other.

When both are running free, with the wind on the same side, the vessel which is to the windward shall keep ont of the way of the vessel which is lceward.

A vessel which has the wind aft shall kcep out of the way of the other vessel.

When two stcam vessels are mceting end on, or nearly end on, so as to involve risk of collision, each shall alter her course to starboard, so that cach may pass on the port side of the other.

When two steam-vessels are crossing, so as to involve risk of collision, the vessel which has the other on her own starboard side shall keep out of the way of the other.

When a steam-vessel and a sailing-vessel are procceding in such dircctions as to involve risk of collision, the stean-vessel shall keep out of the way of the sailing-ressel.

When vessels are in sight of one another, a stcam-vesse! under way, in taking any course requited by the rules, shall indicate that course by the following signals on her whistle or siren, namely:

One short blast to mean: "I am directing my course to starboard."

Two short blasts to mean. "I am directing my course to port."

Three short blasts to nean, "My engines are going at full speed astern."

When a vessel is in distress and requires assistance from other vessels or from the shore, the following shall be signals to be used or displayed by her, either together or separately, namely:

In the daytime-

First. A gun or other cxplosive signal fired at intervals of about a minute.

Second. The international code signal of distress indicater by $\mathrm{N} \mathrm{C}$. 
Third. The distance signal, consisting of a square flag, having either above or below it a ball or anything resembling a ball.

Fourth. A continuous sounding with any fog signal apparatus.

At night-

First. A gun or other explosive signal fired at intervals of about a minute.

Second. Flames on the ressel (as from a burning tar barrel, oil barrel, and so forth.)

Third. Rockets or shells throwing stars of any color or description, fired one at a time, at short intervals.

Fourth. A continuous sounding with any fog signal apparatus.

Similar rules are nade for the navigation of mland waters, the waters of the Mississippi and other great western rivers and for the navigation of the Great Lakes with suitable additions for the conditions of confined waters such as the following:

When steam-ressels are approaching each other end on, or nearly so, it shall be the duty of each to pass on the port side of the other; and either vessel shall give, as a signal of her intention, one short and distinct blast of her whistle, which the other vessel shail answer promptly by a similar blast of her whistle. If the courses of such vessels are so far on the starboard of each other as not to be considered as meeting head and head, either vessel shall immediately give two short and distinct blasts of her whistle, which the other vessel shall answer promptly by two similar blasts of her whistle, and they shall pass on the starboard side of ench other.

If, when steam-vessels are approaching each other, cither vessel fails to understand the course or intention of the other, from any cause, the vessel so in doubt shall inmediately signify the same by giving several short and rapid blasts, not less than four, of the steam-whistle.

Whenever a steam-vessel is nearing a short bend or curve, in the channel, where, frem the height of the banks or other cause, a steam-vessel approaching from the opposite direction can not be seen for a distance of half a mile, such stcan-vessel, when she shall have arrived within half a mile of such curve, or bend, shall give a signal by one long blast of the steam whistle, which signal shall be answered by a similar blast, given by any approaching steam-vessel that may be within hearing. Should such signal be so answered by a steam-vessel upon the farther side of such bend, then the usual signals for meeting and passing shall immediately be given and answered; but, if the first alarm signal of such vessel be not answered, she is to consider the channel clear and govern herself accordingly.

When steam-vessels are moved from their docks or berths, and other boats are liable to pass from any direction toward them, they shall give the same signal as in the case of vessels meeting at a bend.

When steam-vessels are running in the same direction, and the ressel which 
is astern shall desire to pass on the right or starboard hand of the vessel aluead, she shall give one short blast of the steam-whistle, as a signal of such desire, and if the vessel ahead answers with one biast, she shall put her helm to port; or if she shall desire to pass on the left or port side of the vessel ahead, she shall give two short blasts of the steam-whitle as a signal of such desire, and if the vessel ahead answers with two blasts, shall put her helm to starboard; or if the resscl ahead dues not think it safe for the vessel astern to attempt to pass at that point, she shall immediately signify the same by giving several short and raping blasts of the steam-whistle, not less than four, and under no circumstances shall the vessel astern attempt to pass the vessel ahead until such time as they have reached a point where it can be safely done, when said ressel ahead shall signify her willingness by blowing the proper signals.

The vessel ahead shall in no case attempt to cross the bow or crowd upon the course of the passing vessel.

The Commissioner of Navigation also attends to the details relating to shipping commissioners, who are provided by law to be appointed by the Secretary of Commerce and Labor at the principal ports where ocean-going commerce is carried on, such commissioners being sworn officers who give bonds in the sum of not less than five thousand dollars, and who arc entrusted with the duty of providing crews for merchant vesseis, but who are held to strict accountability for their compliance with the laws made for the protection of seamen against unfair treatment and lack of proper food.

The general duties of a shipping commissioner are:

First. To afford facilities for engaging seamen by kecping a register of their names and characters.

Second. To superintend their engagement and disclarge, in manner prescribed by law.

Third. To provide means for securing the prescnce on board at the proper times of men who are so engaged.

Fourth. To facilitate the making of apprenticeships to the sea service.

The shipping commissioner is required to see that the agrcements made with seamen contain the following particulars:

First. The nature, and, as far as practicable, the duration of the intended voyage or engagement, and the port or country at which the voyage is to terminate. 
Second. The number and description of the crew, specifying their respective employments.

Third. The time at which each seaman is to be on board, to begin work.

Fourth. The capacity in which each seaman is to serve.

Fifth. The amount of wages which each seaman is to receive.

Sixth. A scale of the provisions which are to be furnished to each seaman.

Seventh. Any regulations as to conduct on board, and as to fines, short-allowances of provisions, or other lawful punishments for misconduct, which may be sanctioned by Congress or authorized by the Secretary of Commerce and Labor, not contrary to or not otherwise provided for by law, which the parties agree to adopt.

Eighth. Any stipulations in reference to allotment of wages, or other matters not contrary to law.

Fee payable on engaging crew, for each member of the crew, (except apprentices)......................... $\$ 2$

Fee payable on discharging crew, for each member of crew

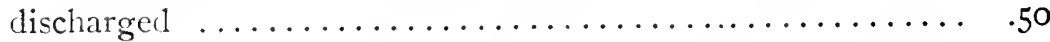

For each boy apprenticed to the merchant service, including

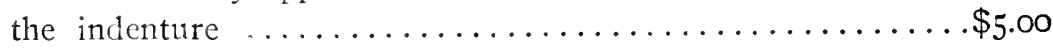

With reference to vessels of the American Merchant Marine, the Commissioner of Navigation is required to give such vessels when enrolled an official number, which must be deeply cut in the main beam, and he must require that the name of the vessel, together with the name of the port from which she hails shall be plainly marked upon appropriate portions of the vessel. He has general oversight of matters relative to the measurement of the cargo spaces of the vessel, and passes upon application for reduction in the amount of tonnage by reason of the existence of portions of the vessel which cannot be used for cargo space.

The functions of the Commissioner of Navigation largely relate to operations which are also under the Treasury Department, so that it is probable that the nature of his performance of duty has considerably changed since the transfer of the Bureau to the Department of Conmerce and Labor. The recent activities of the 
Bureau noted are rather in the direction of those portions of the navigation laws relating to the operation of the vessel and to the administration of the persomnel of the Merchant Marine than in connection to the laws relating to the customs revenue, with which the Bureau of Navigation was formerly closely associated.

\section{BUREAU OF IMMIGRATION AND NATURALIZATIOA.}

By reason of various enactments, especially that relating to the naturalization of aliens in the United States, the Burcuu of Immigration and Naturalization of the Department of Commerce and Labor has come to be one of the most important branches of the Federal Govermment. For the purpose of preventing the practice which had become rather prevalent abroad of shipping paupers. diseased persons and criminals to the United States, for their country's good and this country's disadvantage, exclusion of the following classes of persons was ordered by statute.

All idiots, imbeciles, fecble-minded persons, epileptics, insane persons, and persons who have been insane within five years previous; persons who have had two or more attacks of insanity at any time previonsly; panpers; persons likely to become a public charge; professional beggars: percons afflicted with tuberculosis or with a hathsome or dangerous contasinus disease; persons not comprehended within any of the foregoing exchuded classes who aic found to be and are certificd by the cxamining surgecn as being mentally or physically defective, such mental or plysical defect being of a nature which may affect the albility of stch alien to earin a living: persons who have been convicted of or admit having commitled a felony or other crime or misclemeanor involving moral tupitude; polygamists, or persons who admit their belief in the practice of polyamy, anarchists, or persons who belicve in or advocate the overtlirow by force or violence of the Government of the United States, or of all goverument, or of all forms of law, or the assassinition of public officials; prostitutes, or women or girls coming into the United States for the purpose of prostitution or for any other inmoral purpose; persons who procure or attempt to bring in prostitutes or women or girls for the purpose of prestitution or for any other immoral purpose; persons hercinafter ealled contract horers, who have been induced or solicited to migrate to this connty hy effers or promises of employment or in consequence of agrecunents, cral. written or printed, express or implicd, to perform labor in this country of any kind, skilled or unskilled; those who have becn, within one year from the date of application for admission to the United States, deported as having been induced or solicited to migrate as above described; any person whose ticket or passage 
is paid with the money of another, or who is assisied by others to come, unless it is affirmatively and satisfactorily shown that such person does not belong to one of the foregoing excluded classes, and that said ticket or passage was not paid for by any corporation, association, society, municipality, or foreign government, either directly or indirectiy; all children under sixteen years of age, unaccompanied by one or both of their parents, at the discretion of the Secretary of Commerce and Labor.

In order that the United States should not be at the expense of enforcing this law, a tax of two dollars per head on each alien coming into the United States was assessed, such tax to be paid by the transportation company bringing the alien in, and to be a lien on the property of the company or individual transporting the alien. The administration of the law is placed under the direction of a Commissioner General of Immigration and Naturalization, who is appointed by the President by and with the advice and consent of the Senate and receives a salary of four thousand dollars a year. At each of the customs districts an officer or officers of the Immigration service is stationed, those at the most important customs points being Commissioners of Immigration, who are assisted by forces of inspectors, while inspectors are located at the sub-points and at points abroad, and in Canadian territory, in order to see that the preliminary requirements are complied with by transportation lines. Upon the arrival of aliens in the United States, they are examined by inspection boards and by medical officers of the Public Health Service and if they come within the excluded classes, they are ordered to be deported at the expense of the transportation company. Detention stations are provided at the main points of entrance, and outgoing steamer captains are required to notify the immigration stations twenty-four hours in advance of the date of sailing, in order that deported aliens may be placed on board. Persons whose exclusion from the United States is not clearly required by law are made the subjest of special inquiries by the Board of Inspectors, and upon unfavorable decision in their cases may appeal to the Commissioner General and to the Secretary of Commerce and Labor. A member of the Inspection Board dissatisfied with the decision of the board may also take an appeal. Detained persons who are sick may receive hospital treatment, but the cost of all attention and care is assessed upon the transportation 
companies, if the verdict is funally against the admission of the alien. If any alien becomes a prublic charge within a year from the time of his law ful landing he my be leported at the expense of the immigration fumcl, and the expense of support by state or municipality within the fi:st year after his arrival may also be reimbursed from the immieration fund.

The exclusion of Chinese persons from coming to the United States is required by statute, except when such persons are teachers, students, merchants or travelers for curiosity or pleasure, the ohject of the lesiclatinn being to exclude Chinese laborers. For the purpose of ilentifying the excepted classes, the following persons have been designated by the government to sign the necessary certificates of the character of the employment or calling in life of Chinese sntitled to enter the Lnited States.

Braz1l: Chief of police, or corresponding offeces in the municipalitics and civil subdivisions.

Canada:

Vancouver-Collector of customs.

Victoria-Collector of customs.

Ottawa-Chief controller of Chinese, or chief clerk in the department of trade and commerce.

China :

Acting viceroy of Ifu Kuang (Iunan and IIupeh).

Acting viceroy of Sze Cliuen.

Acting viceroy of Liang Funng (Kuangtung and Kuanghsi).

Tartar General of Fu-chou and customs superintendent of Fu-kifen.

Governor of Anhui.

Goveruor of Human.

Goverlior of Shantung.

Governot of Kiangsi.

Customs Taot ai of Ticntsin.

Taot'ai of the IInj-Ning-Ch'ih-T'ai-Kwang circuit.

Tatai of the Hang-chia-hu circuit.

Acting Tant'ai of the Ning-Shao-T'ai circuit.

Tantai of the IVen-Ch'n cirenit.

Tant'ai of the Yue-Chang-Ii circuit.

Taotai of the Teng-Iai-Ch'ing circuit.

Taot'ai of the Su-Sung-T'ai circuit.

Cuba: Chicf of immieration department.

German Protectorate of Kiautchou: Commissioner for Chinese affairs to the government, civil commissioner, or oherrichter. 
Guatemala: Minister of foreign affairs, or subsecretary of state.

Hawaii: Secretary of the Territory.

Hongkong: Registrar-general.

Japan :

Governor of any fu (district) or ken (prefecture).

Hokkaido-Governor-general.

Formosa-Chief of prefecture having jurisdiction.

Mexico: Department for foreign affairs.

Plilippine Islands: Collector of customs.

Portuguese Province of Macau: Secretary-general.

Society Islands: Commissioner of police of the municipality of Papeete, Tahiti.

Straits Settlements: Colonial secretary.

Federated Malay States-Colonial secretary, federal secretary, or secretary for Chinese affairs.

Trinidad: Governor.

Venezuela: Mayors of cities or governors of provinces.

Chinese laborers who were in the United States prior to the passage of the act referred to, and who registered, are allowed to return under close identification and special precautions are also taken with reference to the merchant class to prevent evasion of the law. Employees of the Bureau of Immigration are expected to enforce both the immigration and the Chinese exclusion laws, but for convenience in payment under the appropriations made by the Congress, are ciivided into immigration and Chinese exclusion inspectors. Constant efforts go on to smuggle Chinese into the United States, and constant watchfulness is required of the Bureau of Immigration to prevent such smuggling, and to detect the persons who have unlawfully come into the United States. As soon as reasonable information is secured that a Chinese person is unlawfully within the United States, he is subject to arrest and to be taken before a United States Commissioner, who hears the proof in regard to the case, and upon a decree of deportation, the Chinese is taken in charge by a United States Marshal and delivered to a port on the Pacific Coast, where the Chinese are placed upon a vessel for deportation to China at the expense of the United States. Heavy penalties are imposed for the unlawful importation of Chinese persons into this country, and every master of a vessel coming to the United States which has Chinese sailors or servants on board 
1s required to give a bond of five hundred dollars for each person to ensure that they shall not land from the vessel.

Under the recent legislation with reference to naturalization in the United States, the Bureau of Immigration and Naturalization was made the recording office of the system, although no appropriation was effected which would give the Bureau the means to carry out certain other provisions of the law. In order to prevent frauds in naturalization, it is required that every male alien upon arrival in the United States shall be registered, the information retained at the port of entry being the name, age, occupation, personal description, place of birth, last foreign residence and allegiance, date of arrival in the United States and the name of the vessel on which arriving,-such information being made a portion of the records of the Bureau of Immigration and Naturalization. When the alien makes application for naturalization, his statements as to the time of residence in the United States may be verified and he may be identified also by reference to the circumstances of his arrival. Specific jurisdiction is given by the recent law for purposes of naturalization to United States Courts, and to all state courts which have a seal, a clerk and jurisdiction in actions in law and equity. The jurisdiction of the court, however, relates only to aliens residing in its respective district. No person who disbelieves in or who is opposed to organized government can be naturalized nor any person who caninot speak the English language, unless the deficiency is rue to physical caises. No alien can be admitted as a citizen of the United States during the time the United States may be at war with the country from which the alien desires to disavow allegiance. Aliens over twenty-one years of age who have served an enlistment in the Army, Nay or Marine Corps are entitled to be naturalized, after honorable discharge, without previous declaration of intention, and alien seamen in the mercliant service who have filed declarations of intention may be naturalized upon exhibiting a certificate of three years service, with good conduct, on a merchant vessel of the United States. Persons residing in any of the possessions of the United States who are not citizens may become so by becoming residents of a state or organized territory of the United States, for two years from the ciate of filing a declaration of inten- 
tion to become a citizen. Residence within the jurisdiction of the United States during the previous three years is regarded as residence within the United States. Children of persons who are naturalized bccome citizens of the United States, if they were less than twenty-one years of age at the time of the naturalization of their fathers. No Chinese person can be admitted to citizenship, and the privileges of the naturalization laws are restricted to white persons and persons of African nativity or descent. In order to become a citizen of the United States, an alien must first live three years in this country and then file a declaration of intention, stating that he is more than eightcen years of age and giving the necessary clata which will enable nim to be identified. Two years after he has filed his declaration of intention and not more than seven years after having made such declaration, the alien may file a petition, setting forth his desire to become a citizen of the United States, and complying with the various requirements established by law, incliding a declaration of renunciation of lis former allegiance. The other requirements are that he shall not be opposed to organized government, or a polygamist or a believer in polygamy, that he will support the United States and is attached to the principles of the Constitution. The verification of two witnesses must accompany the petition. The declaration cf the intention having been filed with the Bureau of Immigration and Naturalization two years before, opportunity has been given for the investigation of the claracter of the applicant, and of his statements, which investigation is for the time being conducted by the Department of Justice, but will probably be transferred to the Department of Commerce \& Labor. Each petition must be poster by the clerk of the court in which it is filed at least ninety days before the hearing upon the application, and the latter must be in open court. At the hearing, it must be made to appear to the satisfaction of the court that the applicant has resided within the state or territory where such court is held at least one year, that he has been continuously resident within the United States for five years, that he has behaved during his residence within the state as a man of good moral character, attached to the principles of the Constitution of the United States and well disposed to the good order and happiness of the country. At 
least two wimesses to the foregoing must appear at the hearing, and the United States has the right to appear and set forth any facts which may have developed on the investigation of the case, or conduct such cross-examination as may seem to be advisable. The applicant mutt, of course, swear ailegiance to the United States, as well as renounce allegiance to any other foreign state, and especially the state of which he was a native, and in case he possesses any foreign title he is required to renounce the same. Upon a favorable clecree by the Court, a certificate of naturalization is given to the petitioner by the clerk. Such certificates are prepared in blank and furnished by the Burcau of Inmigration and Naturalization of the Department of Commerce and Labor, are numbered in series and are recorded in that Bureau. Upon the issuance of a certificate of naturalization, the clerk of the court is required to malie a return to the Bureau of Immigration and Naturalization, upon which return a permanent record is made of the person holding naturalization certificate. Return is also required of all applications of naturalization which have been denied, the purpose being to secure in one central office a record of all persons who may become naturalized citizens, or who have been rejected from the time the naturalization law's were passed.

\section{THE BUREAU OF STANDARDS.}

The constitutional provision siving the Congress the power to fix the standard of weights and measures was necessary not only: to prevent complications in the intercourse between the citizens of the different states, but also to bring about a uniform system of measurements, for the imposition of the national customs lutics. The matter of establishing sucl mits of measurement has therefore been under the direction of the Treasury Department until the institution of the Department of Commerce and Labor. when a burcau for the exact determination of such standards was created. The oreanization consists of a diector at a salary of five thousand dollars a year, with a force of scientific assistants, and its functions are as follows: The custody of the standards; the comparison of the standards used in scientific investigations, engincering. manufacturing, commerce, and education, with the standards adopted or recognized by 
the Government; the construction, when necessary, of standards, their multiples and subdivisions; the testing and calibration of standard measuring apparatus; the solution of problems which arise in connection with standards; the determination of physical constants and the properties of materials. The Bureau will also furnish such information concerning standards, methods of measurement, physical constants, and the properties of materials as may be at its disposal.

Besides the determination of weights and measures, and the exact establishment of a basis at which scientific measurements might begin, the Bureau has developed a series of experiments and measurements with regard to electric currents and other manifestations of force. The Bureau is empowered to exercise its functions for any state or municipal government, or for any scientific society, educational institution, firm, corporation or individual within the United States at a scale of fees which is fixed by the Secretary of Commerce and Labor. General regulations governing applications for tests are as follows:

The request for verification of any apparatus should state fully the nature of the test and other conditions, if any, which it is desired should be observed.

Both the instruments and the packages in which they are shipped should be plainly marked to facilitate identification, preferably with the name of the manufacturer or shipper, and a special reference number should be given to the article.

No risk of breakage will be assumed by the Bureau. All possible care will be taken in handling the apparatus submitted for test, but a certain amount of breakage is unavoidable and must be borne by the owner.

Instruments should be securely packed in cases of packages which may be used in returning them to the owner. Tops of cases should be screwed down whenever possible. Transportation charges are payable by the party desiring the test, and should be prepaid. Unless otherwise arranged, articles will be returned by express "collect."

Articles and correspondence should be addressed simply, "Bureau of Standards, Department of Commerce and Labor, Washington, D. C." Delays incident to other forms of address will thus be avoided.

Articles delivered in person or by messenger slould be left at the office of the Bureati and should be accompanied by a written request for the verification.

Fees may be remitted by money order or check drawn to the order of 
the "Bureau of Stanclards," and should be sent with the request for test whenever practicable. Delays in forwarding fees involve correspunding delays in the return of articles tested, as articles are held until the fecs due thereon have been paid.

An advisory and inspecting visiting committee of five members is provided for, to be appointed by the Secretary of Commerce and Labor, and to consist of men prominent in the various interests involved in the work of the Bureau, but not in the employ of the Government. The committee is to visit the Bureau at least once a year, and to report to the Secretary of Commerce and Labor as to its efficiency and the condition of its equipment. The nembers serve without compensation and for the period of five years, the appointments being arranged so that one new appointment is made in each year. 


\section{CHAPTER XVII.}

FUNCTIONS OF COMMISSIONERS-INTERSTATE COMMERCE COMMISSION -CIVIL SERVICE COMMISSION-THE PHILIPPINE AND ISTIIMIAN COMMISSIONS.

No provision for the institution of a Commissioner or member of a special board for the administration of any law to be made by the Congress was included in the organic act of the federation of the United States, but this accessory of government was by no means unknown to the statesmen of that time either as a historical incideint or as a contemporary measure. The idea of placing a special function in the hands of a small number of persons of supposed expert knowledge or exceptional ability is as old as the Anglo-Saxon idea of self-government itself, and was repeatedly used in the formative period of the English government and continues to the present day when certain functions of the Crown, instead of being entrusted to individual functionaries, are placed in the hands of groups of persons who are expected to find among their membership knowledge and resource, the ability to transact large amounts of business and to provide against corruption and ill-practice so that a better administration would be assured than that of a single person. In the government of the United States the idlea of the commission has been more rarely that of an executive than of an investigative body, although there was an experiment at administering the Navy by a commission, and the theory has more often been that of a body of expert or well-qualified persons undertaking a semi-judicial determination of matters involved, with the purpose that 11timate action shall be taken by the executive, either in accordance with the direction of Congress based on the finding of the commission, or directly upon such finding.

It is only within comparatively recent years that there have been any permanent commissions exercising administrative functions, but two great examples of this method of carrying on executive work have been established by the Interstate Commerce Commission and 
the Civil Service Commission. Both of these scem to have secured a permanent place in the system of govermment of the United States, although the attempt to administer the Panama Canal plan by a commission was a failure, ancl the administration of the Philippines by commission has develuped into an executive administration along the lines of traditional territorial govermment.

TIIE INTERSTATE COMMERCE COMMISSION.

The Interstate Commerce Commission, perhaps contemplated by the legislation originally establisling the position as largely an investigative borly, has been given materially greater importance by recent legislation, and although its status has not been finally defined, its relation to the commerce of the United States under the law as it now stands makes it of superior importance to any similar organization in the Ferleral govermment. and may, under the circumstances of future development, make it a facter equivalent to an Executive Department.

The Commission as created by the law of I 887 consisterl of five menbers, witl a compensation of $\$ 7.500$ each, and a term of office of six years. This was changed by the law of 1906 , so that the Commission consists of seven members to be appointed for a term of seven years each and to have a compensation of ten thousand dollars a year. The terms of appointment have been so arranged that one Commissioner will be appointed each year on the expiration of a seven-year term and vacancies arising by other than such expiration will be filled for the balance of the unexpired term only. Not more than four Commissioners can be appointed from one political party, it being the intention to secure a non-partisan board and the element of disinterestedness is provided for by the requirement that no person in the employ of or holling any official relation to a common carrier can be appointed, nor any person holling stocks or bonds or pecuniarily interested in a corporation or firm doing butsiness as a common carrier. The Commissioners are forbidden to engage in any other business, vocation or employment.

Among the powers delegated by the States to the Congress was that to regulate commerce between the States. Just what was meant by this delegation may be a subject of interesting speculation, but it 
has not yet had an exact definition. Under this authority the Congress has assumed wide powers and seems likely to take even a broader vicw of the authority thus conferred, and has delegated the exercise of many of its powers with relation to the carriage of persons and commodities to the Interstate Commerce Commission. Holding that whenever a person or thing is taken up in one State for delivery within another State it lias the power to prescribe the terms and manner of such carriage, the Congress has established general rules and has created for their application the Commission here considered. It is, of course, true that with any carriage wholly within the limits of one State the Congress or the Interstate Commerce Commission has nothing to do, but so few common carriers are confined in their operations to one State, either in their own business or over that of connecting roads, that the purview of the Interstate Commerce Commission is practically comprehensive of all matters of transportation within the continental limits of the United States and of many matters of ocean transit.

Specifically, the jurisdiction of the Commission cxtends to all persons or corporations who are engaged in the transportation of passengers and property other than within the limits of a single State, including railway and allied steamboat lines, pipe lincs, except water and gas lines, express companies and sleeping car companies, and includes all means whatever used in the course of such transportation.

The Congress has enacted that the rates for carrying persons and commodities shall be equal and equitable and has denounced discriminations and imposed penalties for discrimination. To avoid such penalties common carriers must file with the Commission and keep open for public inspection copies of all tariffs established. Complaints that such tariffs are inequitable or that they are being violated by special rates or favors to certain shippers may be brought before the Commission which now has power, subject to a revision by the courts on appeal by the common carrier affected, to fix a sum which shall be the maximum to be charged for the service in question. In case of a discrimination or ascertained injustice the Commission has power to fix the sum which shall be paid as damages, and is authorized to appeal to the United States courts for the enforcement 
of its awards and of its decrees, as well as to call the attention of the prosecuting authorities of the United States to the instances of violation of the Interstate Commerce law which come to its notice and for which a penalty is provided. The distinct difference made by the recent legislation is that while the Commission formerly could declare a rate unreasonable, it can now fix the amount of a reasonable rate, thus giving it a positive power instcal of the negative power formerly held.

Under the law as amended the Commissioners have a wide scope of power with relation to the affairs of common carricrs. They may inquire into the details of their transactions and the courts will com$\mathrm{pel}$ the attendance of witnesses and of the production of books and papers before the hearings authorized. They are also authorized to compel annual reports from all common carriers covering the details of business and may prescribe the nethod of kecping the accounts as well as the items to be covered in the amnual reports made.

Provision is made for complaint by any person to the Commission of discriminations in transportation or of violation of the Interstate Commerce law, and hearings on such complaints form the bulk of the business of the Commission. The hearings and business sessions may be held in Washington, when a majority of the Commission is necessary for the transaction of business. It is provided, however, that vacancies shall not be construed as preventing the remaining members from excrising the full powers of the Commission. One or more Commissioners may be delegated to go to places other than Washington for the purpose of making investigations and taking testimony, and provision is made for the necessary cmployes and for travelling expenses which are paid on the voucher of the Chairman of the Commission. Nuch of the testimony ottside of Washington is, however, taken by special examiners employed by the Commission. Special provision is made for the immunity of witnesses from prosecution who shall have disclosed incrininating facts in sequence to a subpoena from the Commission, but this does not extend to perjury in such testimony.

The Commission is charged with investigating any complaint that may be forwarded by the railroad commissioner or commission of any State and may investigate and make inquiries on its own motion. 
The findings and recommendations of the Commission in any case are placed on record and form the basis of judicial procedure if any, the Commission being authorized to have a seal noticeable by the courts. It is empowered to provide for the publication of its decisions in such manner as it may deem fit, and such publications are to be received as competent evidence in court. The Commission makes its own orders and regulations for the course of procedure before it, but no Commissioner can participate in any proceeding or hearing in which he has any pecuniary interest. All votes and acts must be recorded and the proceedings of the Commission must be made open and public upon request of either party interested. Either of the members of the Commission is given power to administer oaths and affirmations and sign subpoenas. The Commission has the power to choose its Secretary and to employ other service, to hire suitable offices and to purchase necessary supplies. The former supervision over services and supplies by the Secretary of the Interior was abolished a few years after the constitution of the Commission which was thus made practically independent, being checked only by the audit of the Treasury Department. An annual report must be made to Congress giving the details of the work of the Commission, together with the names and compensation of all persons employed.

Discretionary power is given to the Commission to modify the provision of the law that no equal or greater sum shall be charged for a smaller distance than for a greater distance that includes the smaller, this being the "long and short haul" clause by which in the discretion of the Commission through rates may be established which are less than local rates, though the practice of such discrimination is, in general, forbidden by law. The Commission is also given power to require connection of the private sidings or tracks of shippers to the tracks of common carriers by means of switches where the conditions are reasonable, and all icing, terminal, switching and other charges incident to transportation are placed under the Commission's control.

In addition to their functions with relation to the charges and manner of transportation, the Commission has had charge of the execution of the Federal laws requiring rolling stock on railroads 
engaged in interstate commerce to be provided with air brakes, uniform and automatic couplers and safety appliances for the protection of trainmen against accident. Common carriers are also required to make full reports of all accilents to passengers and employes to the Commission and all occasions of derailment and collision must be similarly reported.

Uncler the terms of the "Arbitration Act" passed in I898, the Cliairman of the Interstate Commerce Commission is directed, jointly with the Commissioner of Labor to mediate between the employes of a common carrier, and the common carricr in case of disagreement as to terms of employment and to endeavor to settle such controversics. Agreements for arbitration of difficulties are filed in the office of the Interstate Commerce Commission, and under certain conditions the Chairman takes part in making up the Board of Arbitration.

Congress having provided aid for the construction of certain telegraph lines, both as separate installations and in connection with railroad construction, the interests of the people generally in the conduct of such lines is placed under the direction of the Interstate Commerce Commission. Failure by commercial companies to make satisfactory connections with such telegraph lines can be remedied on complaint to the Commission and on the initiative of the Commission itself. Such aicled telegraph lines must file annual reports with the Commission as to the business of such lines, and information as to all contracts and agrements with connecting lines. The Commission is given power to adjudge the conditions of reasonable operation and service of such lines and failure to comply with the orders of the Commission as to such operation is punishable as a misclemeanor, while the party aggrieved is given the right to bring an action for damages.

Although specific duties and powers are given to the Chairman of the Interstate Commerce Commission by law, there is no statute establishing such an office, the assumption being that the Commission would organize by the selection of a chairman, a secretary being provided by law. The method of choosing the chairman is entirely in the discretion of the membership of the Commission, but it has been the practice for the member oldest in service to act as chairman. 
A proposition has been made that each member should act as chairman for one year, but this has never been carried out. Other than the exceptions herein given the Chairman has no powers, statutory or delegated, than those of the other Commissioners.

\section{THE MEMBER OF THE CIVIL SERVICE COMMISSION.}

In order to understand the reason for the functions and existence of the member of the Civil Service Commission it is necessary to comprehend something of the condition which existed with regard to the civil service of the United States prior to the legislation which created the present system of regulation of Federal employes. Prior to the Act of I87 I which gave the President power to prescribe regulations for the admission of persons to the civil service and which was the forerunner of the present law, all positions under the Federal government were subject to appointment for reasons having to do with the supposed welfare of the dominant political party. The appointments in the Federal service, very considerably less in number than at present, were apportioned more or less equitably among members of Congress and among party managers in States in which the opposition to the administration was the dominant party, and tenure of office depencled upon the favor of those to whom the patronage was thus allotted. It is maintained that the general average of Federal employes thus appointed was such as to give a reasonably efficient service, and that the level of intelligence and capability for original work was higher than under the present system which is described as less likely to attract persons of activity and of initiative. The evils, however, of uncertain tenure, of diversion of governmentpaid services to political work, accentuated by the increasing business of the government, became intolerable, especially in the departments in Washington, and one of the notable agitations of the generation just preceding the present brought about an entire overturn of the former system so that with respect to more than two hundred thousand Federal employes at the present time, the full system urged for a reform of the civil service is in effect, while so far as its principles are applicable they are being applied in a constantly increasing ratio to all employes of the general government.

The Civil Service Commissioners were provided for by the Act 
of 1883 , subsequent to the tentative and permissive act before mentioned. Unlike a commission exercising delegated powers from the Congress, they constitute a board to assist in the duties inposed upon the President of selecting employes in the civil service according to carefully specified principles. They are appointed by the President by and with the advice and consent of the President, but may be removed by hinn without reference to the Scnate, though he cannot fill vacancies without confirmation. The Commissioners are paid $\$ 3,500$ each per year and necessary travelling expenses, and can hold no other official place under the United States.

It is the duty of the Commissioners to aid the President to formulate rules for the selection of civil employes and for their promotion and transfer, to carry out the provisions of the rules and to take notice of their infraction. They are wholly and directly under the control of the President and exercise no independent powers, being bound by the established rules in the construction given to them by the President, and all exceptions to the rules are ordered and lirected by the President.

The fundamental principles established by Congress in the Act of I883 and which constitute the system known as "Civil Service Reform" are that entrance to the classified service of the Uniter States shall be secured by open, competitive examination, and that appointments shall be only made from those who attain the highest grade in such examinations. That appointments shall be proportioned among the several States and Territories and the District of Columbia in proportion to the respective populations. It results from this provision that a person, although attaining a high grade in an examination cannont secure an appointnent in excess of the quota of the State of his or her legal residence, so long as other State and Territory quotas are unfilled. That there shall be a period of probation before final appointment to the civil service. That no political contribution shall be exacted from persons in the civil service, and no person shall use his official position to coerce the political action of any person or body.

For the adninistration of the duties imposed upon them the Civil Service Commissioners are afforded the accommodation of a building rented under the direction of the Interior Department at Wash- 
ington with a due number of employes at Washington and a field force, together with the rural carrier examining force, the total salary list being approximately two hundred thousand dollars. In spite of the fact that the Commission was established by the Congress as the result of a preponderant public opinion, it has been with difficulty that the appropriations for the annual maintenance have been secured, and only recently the custom of detailing clerks from the departments to do the work of the Commission has been stopped after Congress had been practically forced into making adequate specific appropriations. The meagre amounts appropriated for the compensation of the Conmissioners is also probably due to the disinclination of Congress to appropriate liberally in the Commission's behalf.

The members of the Civil Service Commission appoint a chief examiner to superintend the work of examining applicants and are empowered at one or more places in each State and Territory to select from among the persons in the official service of the United States, not less than three for each locality, who shall constitute boards of examiners to conduct the examination of applicants for entrance to the civil service. Sets of examination questions for each branch of the service of the United States coming under the scope of the law are made up under the direction of the Commission who are required by law to make the examinations practical and applicable to the kind of service expected to be rendered. Examinations on these questions are held at Washington and at the points throughout the country provided under the requirement for local examinations. The papers made out by the applicants are not marked by the local boards, but the marking and averaging of the results is done in Washington under the supervision of the Commissioners. An average of 70 is required to have passed the examinations with the exception of Civil and Spanish war veterans who are required only to get $a n$ average of 65 , and who have a preference over all other applicants in position on the list of eligibles, although other applicants may have made much higher averages. Non-veterans are arranged on eligibility lists in accordance with the averages secured and upon application from a head of department for a person to fill a position, three names are certified from the appropriate list, one of which must 
be selected by the appointing poiver, except for gond reason, and on failure to select a recertification is macle until a selection is made.

Persons to be admitted to examination under the direction of the Civil Service Commission must be citizens of the United States or must owe allegiance to the United States, such as being a citizen of Porto Rico, etc. They must be between the ages prescribed for the different classes or positions, where a maximum is prescribed, except in the cases of veterans for whom age limitations are waived. They must be of good character and habits, and persons who have been dismissed from the service within one year next precelling, who have made false statements as to facts in connection with their applications, or who have practiced deception or fraud in connection with an examination will be barred. The habitual use of intoxicating beverages to excess is a cause for refusal of examination and also for renoval from the service.

In addition to the examinations for appointment to the classified service, the Civil Service Commission conducts examinations for positions of a technical and professional character uncler the Insular government of the Plilippines, examinations of miclshipmen for the Naval Academy at Annapolis, and for cadets in the Revenue Cutter Service, and maintains registers for unskilled laborers outsicle of Washington, and unclassified laborers in Washington and for employment under the Isthmian Canal Commission, except for certain excepted positions.

The following civil service districts have been established:

First district.-Headquarters. Boston, Mass. Maine, New Mlampshire. Vermont, Mlassachusets, Rhode Island, and Comecticut except liatidid and New Haven counties.

Second district-lleadquarters. New York, N. Y.: New York. Tairfield and New Haven comnties in Connecticut and the comtics of Bergen. Essex, Hudson, Morris, Passaic. Sussex, and Union in the State of New Jersey.

Third district.-lleadquarters. Philadelphia, Pa.: Pennsylvania, Delaware, and the counties of Atlantic, Burlington. Camden, Cape May, Cumberland, Gloucester, Hunterdon, Merecr, Middlesex, Mommoutl, Ocean, Salen, Somerset, and Varren in the State of New Jersey.

Fourth district.-Headquarters, Washington, D. C. Maryland, West Virginia, Virginia, North Carolina, and the following local scrvices in the District of Colnmbia, viz., post-office and custom-honse. 
Fifth district.-Headquarters, Atlanta, Ga.: South Carolina, Georgia, Alabama, Florida, Mississippi, and Tennessee.

Sixth district.-Headquarters, Cincinnati, Ohio: Ohio, Indiana, and Kentucky.

Secentl district.-Headquarters, Chicago, Ill.: Wisconsin, Michigan, and the counties of Boone, Bureau, Carroll, Cook, Dekalb, Dupage, Ford, Grundy, Henderson, Henry, Iroquois, Jo Daviess, Kane, Kankakee, Kendall, Knox, Lake, Lasalle. Lee, Livingston, McHenry, Marshall, Mercer, Ogle, Peoria, Putnam, Rock Island, Stark, Stephenson, Warren, Whiteside, Will, Winnebago, and Woodford in the State of Illinois.

Eighth district.-Headquarters, St. Paul, Minn.: Minnesota, North Dakota, South Dakota, Nebraska, and Iowa.

Nintll district.-Headquarters, St. Louis, Mo.: Kansas, Missouri, Arkansas, the counties of Adams, Alexander, Bond, Brown, Calhoun, Cass, Champaign, Christian, Clark, Clay, Clinton, Coles, Crawford, Cumberland, Dewitt, Douglas, Edgar, Edwards, Effingham, Fayette, Franklin, Fulton, Gallatin, Greene, Hamiton, Hancock, Hardin, Jackson, Jasper, Jefferson, Jersey, Johnson, Lawrence, Logan, McDonough, McLean, Macon, Macoupin, Madison, Marion, Mason, Massas, Menard, Monroe, Montromery, Morgan, Moultrie, Perry, Piatt, Pike, Pope, Pulaski, Randolph, Ricliland, St. Clair, Saline, Sangamon, Schuyler, Scott, Shelby, Tazewell, Union, Vermilion, Wabash, Washington, Wayne. White, and Williamson in the State of Illinois, Oklahoma, and Indian Territory.

Tenth district.-Headquarters, New Orleans, La.: Louisiana and Texas.

Eleanth district.-Headquarters, Denver, Colo.: Wyoming, Utah, Colorado, Arizona, and New Mexico.

Tacelfth district.-Headquarters, San Francisco, Cal.: California, Nevada, Washington, Oregon, Idaho, and Montana.

The classification of the civil service was adopted by each head of a Department and Government establishment on direction of the President on June 9, I896. It arranges officers and employees, other than mere laborers and persons whose appointments are confirmed by the Senate, in classes according to annual salary or compensation, as follow's:
A. Less than $\$ 720$.
B. $\$ 720$ or more and less than $\$ \$+0$.
C. $\$ 840$ or more and less than $\$ 900$.
D. \$9co or more and less than $\$ \mathrm{I}, 000$.
E. \$1,000 or more and less than \$I,200.
I. $\$ I, 200$ or more and less than $\$ I, 400$.
2. \$I, 100 cr more and less than $\$ I, 600$.
3. $\$ 1,600$ or more and less than $\$ 1,800$. 
4. $\$ 1,800$ or more and less than $\$ 2,000$.

5. $\$ 2,000$ or more and less than $\$ 2,5 \mathrm{co}$.

6. $\$ 2,500$ or more.

The classification further provides that no person appointed as a laborer without examination under the rules shall be assigned to work of the same grade as that performed by classified employees, and no person shall be adnitted into any place not excepted from examination by the rules until he shall have passed an appropriate examination before the Commission and his eligibility has been certified to the appointing officer by the Commission.

The Railway Mail Service has a different classification, and a rearrangement of positions in the classified service has been recommended by a committee appointed by the President from the Executive departments. This recommendation has not, however, become effective, and the foregoing classification still stands.

The classified service includes all officers and employes in the executive civil service of the United States, in positions now existing or hereafter to be created, of whatever function or designation, whether compensated by a fixed salary or otherwise, except persons employed merely as laborers, and persons whose appointments are subject to confirmation by the Senate.

All persons must undergo an examination to secure an appointment to the classified service and can only be appointed in accordance with the rules pertaining to and growing out of such examinations, except persons appointed to the following positions especially exempted from classification:

In the Fintre Classificd Servire-Two primate secretaries or confidential clerks to the head of each of the Executive Departments and one to each assistant head.

One private secretary or confidential clerk to each of the heads of bureaus appointed by the President and confirmed by the Senate in the Executive Departments, if authorized by law.

All persons appointed by the President withont confirmation by the Senate.

Attorneys, assistant attorneys, and special assistant attorneys.

Chinese and Japanese interpreters.

Any person receiving not more than $\$ 300$ per annum compensation for his personal salary, who may lawfully perform his official duties in con- 
nection with his private business, such duties requiring only a portion of his time.

Any person employed in a foreign country under the State Department, or temporarily employed in a confidential capacity in a foreign country under any department or office, except persons employed in a foreign country contiguous to the United States in the service of the Bureau of Immigration, Department of Commerce and Labor.

Any position the duties of which are of a quasi military or quasi naval character, and for the performance of which duties a person is enlisted for a term of years; also positions in the Revenue-Cutter Service, where the persons enlist for the season of navigation only.

Treasury Departannt.--Not exceeding one solicitor to the collector at the port of New York, if authorized by the Secretary of the Treasury.

One confidential clerk, if authorized by the Secretary of the Treasury, to each of the following officers:

The collector of each customs district where the rcceipts for the last preceding fiscal year amounted to as much as $\$ 500,000$.

The appraisers at the ports of Boston, New York, and Philadelphia.

One private secretary in the office of the naval officer of customs at the port of New York.

One counsel before the Board of United States General Appraisers.

In the New York customs district: One paymaster; stitch counters.

Storekeepers and gaugers whose compensation does not exceed \$3 per day when actually employed and whose aggregate compensation shall not exceed $\$ 500$ per annum.

One chief clerk in each mine or assay office, who is authorized by law to act for the superintendent or assayer in charge during his absence or disability.

One private secretary or confidential clerk to the superintendent, one cashier, one deposit weigh clerk, one assistant coiner, and one assistant melter and refiner in cach mint or assay office.

Any local physician employed for temporary duty as acting assistant surgeon in the Public Health and Marine-Hospital Service.

Any person employed in the Public Health and Narine-Hospital Service as quarantine attendant at the Gulf, South Atlantic, Itullet Key, Reedy Island, Cape Charles, Columbia River, or San Francisco quarantine; and any person cmployed as quarantine attendant, or acting assistant surgeon, or sanitary inspector, on quarantine vessels, or in camps or stations established for quarantine purposes during epidemics of contagious diseases, for temporary duty in the United States or elsewhere in preventing the introduction or spread of contagious or infections diseases.

In the Alaska customs service all persons appointed or employed for the season of navigation only. 
One examincr of tobacco and onc cxaminer of tea in the customs service at the port of Chicago.

Mounted inspectors in the customs service on the Mexican burder.

WAR Dep.skTanext.-All paymasters' clerks actually on duy with paymasters.

All cable engineers.

All telegraph operators, telegraph linemen, and cable seamen, receiving a monthly compensation of $\$ 60$ or less, serving on military telegraph systems or at military stations, and who perform their duties in connection with their private business or with other employment, such duties requiring only a portion of their time.

All persons in the army transport service.

All commissioners for the national military parks.

All firemen employed on torpedo planters.

One law officer in the Bureau of Insular Affairs.

One superintendent, one chief chemist and assistant superintendent, and one first assistant chemist, for service in connection with the operation of the Washington Filtration Plant, under the Engineer Department.

Department of Jestice.-Wardens, chaplains, and physicians in the United States penitentiaries or prisons.

One clerk to each United States district attorncy.

Examiners.

Any person employed as office or ficld deputy in the office of a United States marshal.

All positions and employments deemed by the Attorney-General to be legal or confidential in their character, and which relate to temporary service or which grow out of appropriation acts committing to the AttorneyGeneral the execution of some purpose of the law and the expenditure of the funds therefor, but not creating specific positions.

Post-Office Dep.rkmint.-The Assistant Attorney-General for the PostOffice Department.

One private secretary or confidential clerk to the Assistant Attorney-General, and one to the Purchasing Agent of the Post-Office Department.

One private secretary or confidential clerk to the postmaster, if authorized by the Pustmaster-Gencral, at each post-office where the receipts of the last preceding fiscal year amounted to as much as $\$ 350,000$.

All employees on star routes and in post-offecs having no free delivery service.

One assistant postmaster or the chicf assistant to the postmaster, of whatever designation, at each post-office.

One auditor at the post-office in New York City.

Departaext of tife INtertor.-The superintendent of the Hot Springs Reservation.

Inspectors of coal mines in the Territories. 
Temporary clerks employed in the United States local land offices to reduce testimony to writing in contest eases, not paid from Government funds.

Indians employed in the Indian Service at large, except those employed as superintendents, teachers, manual training teachers, kindergartners, physicians, matrons, clerks, scamstresses, farmers, and industrial teachers.

Special commissioners to negotiate with Indians, as the necessity for their employment may arise.

One financial elerk at each Indian agency to act as agent during the absence or disability of the agent.

Physicians employed in the Indian service and receiving not more than $\$ 720$ per annum salary, who may lawfully perform their official duties in connection with private practice.

All physicians employed as pension examining surgeons, whether organized in boards or working individually under the direction of the Commissioner of Pensions.

Five special pension examiners to investigate frandulent and other pension claims of a criminal nature.

Five special agents of the General Land Office to investigate fraudulent entries and other matters of a criminal nature.

Consulting engineers of the Reclamation Service under the Geological Sirvey.

One confidential clerk and one record clerk to the Superintendent of the Government Hospital for the Insane.

One private secretary to the Director of the Geological Survey.

Departilent of Agriculture.-Agents, experts and student assistants for temporary duty employed in making investigations and furnishing information for the Department.

One statistical agent in each State and Territory where authorized by law.

Guards, guides, cooks, packers, teamsters, choppers and skilled laborers enployed temporarily during the season of danger from fires, or when other special work requires additions to the regular forest reserve force.

Isthimian CANAL Commission.-All officers and employees in the service of the Isthmian Canal Commission upon the Isthmus of Panama, except those who are to perform the duties of clerk, bookkeeper, stenographer, typewriter, surgeon, physician, trained nurse, or draughtsman.

No person appointed to the service on the Isthmus of Panama otherwise than through competitive examination or by transfer or promotion from a competitive position can be transferred to a competitive position.

DepartMent of CoMmerce and LABor.-All persons temporarily connected with the field operations of the Burean of Fisheries who are paid from lump appropriations for miscellaneous expense.

The law granting preference in original appointment to civil offices under the United States Government because of military or naval service was passed on March 3, I865, in the form of a joint resolution of Congress; and 
the civil-service act, which was approved, provided, in effect, that mothing contained thercin should be construcel to take from these homoraly discharged from the military or naval service any preference conferred.

To entitle the veteran to preference the law repuires:

That he mist latve been disabled, either ly womels or sickness, while serving in the Army or Nay.

That the disability must have been incurred in the line of duty.

That he must have been subsequently honorably discharged from the military or manal service by reason of the disability so incurred.

The following advantages are given by the civil-service rules to a person who has heen allowed preference:

I. He is released from all age limitations.

2. He has to attain an average perentage of only $6_{5}$ to be cligible, winle for others the averige percentage required is 70 .

3. If he attains an arerage rating of $65 \mathrm{nr}$ more his name is placed at the head of the register and is certificd before tlose cligibles who have no ciam to preference.

4. He is released from the law and rules relating to the apportionment of appointments.

In examinations for promotion or transfer a preference claimant already in the classified civil service receives no advantages by reason of his military or naval service, the preference being confined by the law to original appointment.

If the person whose claim to preference has been allowed is an applicant for the position of unskilled laborer, he is required to attain a physical rating of only zo per cent. and his name is placed on the register above all others not entitled to preference. Other applicants for this position must receive a physical rating of 85 per cent. in order to become clicible.

\section{SPANISH TREATY CLAIMS COMMISSION.}

The Spanish Treaty Claims Commission is an example of the sort of temporary commission, having semi-judicial powers which has been established from time to time in the history of the United States for the purpose of carrying ont particular functions. A preceding commission of the kind was the Court of Private Land Claims, while the Commission to the Five Civilized Tribes was a similar body in many respects. By the Treaty of Peace with Spain, the United States agreed to assume the responsibility of all claims on the part of citizens of the United States against Spain growing out of the Spanish-American war and the operations in Cuba, and Spain, in turn, agreed to satisfy the claims of Spanish subjects who might regard themselves as damaged by the acts of the United States in 
connection with that war. The machinery for determining and adiuciicating the claims of citizens of the United States was not escablished until rooI, when a commission of five persons was provicled for, to be appointed by the President by and with the advice and consent of the Senate to receive, examine and adjudicate all claims of citizens of the United States against Spain which the United States had agreed to assume. The persons appointed on the conmission were required to be citizens of the United States and to be learned in the law. One person was to be appointed by the President to act as the president of the commission and the salaries were fixed at five thousand dollars a year each. The City of Washington was made the official meeting place of the commission, and it was empowered to make all needful rules for the conduct of the business. The appointment of a clerk and of the necessary clerical assistance is provided for by law, and it is further provided that the civil service rules need not apply to appointments under the commission. For the representation of the United States before the commission, an Assistant Attorney Gencral is provided at an annual salary of five thousand clollars, and there have from time to time been appropriations made for assistant attorneys and for taking testimony, a large part of the latter work having been carried on in Cuba. In the conduct of the cases, six months was provided in which all claims were to be filed, and after which no new claims could be filed except by permission of the commission. The petitions are certified to the Assistant Attorney General representing the United States, who prepares the evidence and case against the claimant. The award of the Commissioners is final in each case, unless the Commissioners themselves reopen the matter and no appeal is provided for, except that the Commissioners may certify matters of law in regard to which they are in doubt to the Supreme Court, and the latter is given jurisdiction to hear the same. Awards finally made by the commission are to be filed with the Secretary of State and payments on the awards are to be made by the Secretary of the Treasury after appropriation by Congress. The original act provided that the commission was to complete its work in two years after the date of the Act, but the President has power to continue its work by extensions of six months each, and the extensions have gone on from time to time 
until the present writing. A large part of the claims presented to the commission were based on the acts of the Spanish govermment before the actual declaration of war and while the condition of insurrection continued in Cuba. In an carly opinion, the commission held that these claims were not inclucled among those which were referred to the commission for adjudication, and the result has been to eliminate a very large proportion of the total husiness which awaited the commission at its inception. The conmission has declined to reopen this class of cases, or to refer the matter to the Supreme Court on the law involved, and there is being a strong pressure brought to bear to discharge the commission from further work and to throw the balance of the claims into the Coutt of Claims for adjudication. The act establishing the conmission was carefully framed and every possible safeguard was placed around its jurisdiction and its operations with the $\mathrm{i}$ lea of preventing the inclusion of extraneous claims. Such a class of claims has appeared in connection with every adjulication of the kind and was not prevented in the present case, and it is probable that legislation with relation to Spanish war claims will be asked of Congress annually for many years to come.

\section{TIE PIILIPPINE COMMISSION.}

The Philippine Conmission may be clescribed as an example of the purely investigative committee of persons appointed from outsile the govermicnt service grown into an executive buly. It will be remembered that during the period of the military administration of the Philippines, there was a considerable controversy whether there had beein faith kept with certain of the inhabitants of the islands, who were in insurrection against Spain, and who later came into a hostile attitude against the United States, because of the alleged failure of this comntry to keep certain promises. Without the specific authorization of the Congress and as an administrative measure, the President appointed a commission of persons to so to the Philippines, investigate the conflicting clains as to the statns of any inm of govemment that the native inhabitants had proposed, and in gencral to report upon a suitable form of administration for the islands. As the result of the report made, a form of government by conmission was established, which was later ratified by the Congress and 
made the basis of the present government. The Philippine Commission consists of eight members, one of whom is the Governor General, and other heads of Departments of the Interior, of Commerce and Police, of Finance and Justice and of Public Instruction respectively. The salaries of the Commissioners are paid from the Philippine revenues in the silver money of the islands and represent abont one-half of the amount in money of the United States. The Governor General receives \$30,000 Philippine and \$ro,000 in addition as Commissioner. The other Commissioners receive ten thousand each, and those acting as heads of executive departments in the islands receive $2 \mathrm{I}, 000$ Philippine money in addition.

All of the foregoing appointments are made by the President, by and with the advice and consent of the Senate, and the President has power to make further regulations for the government of such portions of the Philippine Islands as may be included within a zone of armed resistance to the United States.

In the peaceful portion of the Plilippine Islands, the governmental affairs are entrusted to the Philippine Commission and all inhabitants of the islands, except such as chose to retain their allegiance to the King of Spain, are denoted as Philippine citizens, but their government is specifically excepted from the operation of the constitution and laws of the United States. It is a usual provision with regard to territorial government that the constitution and laws of this country are made applicable, with the exceptions made necessary by local conditions. This extension of the constitution and laws is, however, withleld from the Philippine Islands, but in lieu thereof provision is made in the act for the government of the Philippines, for the personal rights and safeguards which are guaranteed to citizens of the United States, and which as they afford a summary, in a concise form, of the chief constitutional safeguards of the Amreican form of government, are appended hereto. The Congress provicles that-

No law shall be enacted in said islands which shall deprive any person of life, liberty, or property without due process of law, or deny to any person therein the equal protection of the laws.

In all criminal prosecutions the accused shall enjoy the right to be heard by himself and counsel, to demand the nature and cause of the accusation against him, to have a speedy and public trial, to meet the witnesses face to 
face, and to have compulsory process to compcl the attendance of wituesses in his behalf.

No person shall be held to answer for a criminal offense without due process of law; and no person for the same offense shall be twice put in jeopardy of pumishment, nor shall be compelled in any criminal case to be a witness against himself.

All persons shall before conviction be balable by sufficient sureties, except for capital offenses.

No law impairing the obligation of contracts shall be enacted.

No person shall be imprisoned for debt.

The privilege of the writ of habeas corpus shail not be suspended, unless when in cases of rebellion, insurrection, or invasion the public safety may require it, in either of which events the same may be suspended by the President, or by the governor-general, with the approval of the Philippine Commission, wherever during such period the necessity for such suspension shall exist.

No ex post facto law or bill of attainder shall be enacted.

No law granting a title of nobility shall be enacted, and no person holding any office of profit or trust in said islands, shail, withont the consent of the Congress of the United States, accept any present, emolument, ofice, or title of any kind whatever from any ling, queen, prince, or forcign State.

Excessive bail shall not be required, nor excessive fines imposed, nor cruel and umusual punishment inflicted.

The right to be secure against unreasonable searches and seizures shall not be violated.

Neither slavery, nor involuntary scrvitude, except as a punishment for crime whereof the party shall have been duly convicted, shall cxist in said islands.

No law shall be passed abridging the freedom of specels or of the press, or the right of the people peaceably to assemble and petition the Government for redress of gricrances.

No law shall be made respecting an establishment of religion or prolibiting the free exercise thereof, and the free exercise and enjoyment of religious profession and worship, wiliont discrimination or preference, shall forever be allowed.

No money slall be paid out of the treasury except in parsiance of an appropriation by law.

The rule of taxation in said islands shall be uniform.

No private or local bill which may be enacted into law shall embrace more than one subject, and that subject shall be cxpressed in the title of the bill.

No warrant slall issue but upon probable cause, supported by natl or affirmation, and particularly describing the place to be scarcled and the person or things to be seized.

All money collected on any tax levied or assessed for a special purpose shall be treated as a special fund in the trcasury and paid out for such purpose only. 
In addition to the foregoing, the Congress granted that all legislative power which was formerly conferred on the Philippine Commission, so far as it applied to the portions of the Philippine Islands not inhabited by Moros or other non-Christian tribes, shall be vested in a legislature consisting of two houses, the Philippine Commission constituting the upper branch of the legislature and a Philippine Assembly, of not less than fifty nor more than one hundred members, to constitute the lower branch. The membership of the Assembly is to be apportioned by the Commission among the provinces as nearly as practicable, according to population, but no province ean have less than one member. Persons to be elected to the Lower House must be twenty-five years of age, qualified electors of the election districts from which they are chosen, and must owe allegiance to the United States. The legislative powers of the two houses resemble those of territorial legislatures, but the Philippine lcgislature holds annual instead of biennial sessions. The members of the legislature are, however, elected for two years. The annual session of the Philippine legislatire is not to exceed ninety days. At the same time that members of the lower house of the legislature are elected, two resilent commissioners to the United States are chosen, who are entitled to recognition by the executive departments in Washington, and receive $\$ 5,000$ annually, with $\$ 2,000$ per year to cover expenses. The judicial system of the Islands consists of a Supreme Court, the members of which are appointed by the President, and receive a compensation of ten thousand dollars a year for each Associate Justice, and \$IO,500 for the Chief Justice of the Supreme Court, together with courts of the first instance, the judges of which are appointed by the Governor-General, by, and with the consent of the Philippine Commission. Municipal courts are also provided for, to be continued as was the practice under the former government of the islands, by the Presidential Commission. The government of the islands is given power to change practice and methods of procedure in the inferior courts, but no change can be made in the methods of admiralty practice, without the consent of Congress.

It became necessary, owing to the radical difference between the Spanish and the American proccdure with reference to the land and 
mining claims, to establish a borly of law in the Philippine Islands covering these points, and Congress has made specific provisions adjusting the land and mining laws of the Uniterl States to the conditions in the Philippines. With the exception of these provisions of law, and with certain specific empowerments made by Congress, authorizing the Commission to perform certain governmental functions, the govermment of the Philippine Islands, is as prescribed by the procedure hitherto laid down by the Commission, although such procelure is now subject to change by virtue of the exercise of the legislative power, which has been vested in the Philippine Legislature. Substantially, however, up to the present time, the govermment of the Philippines has been almost entirely by commission, and, under the direction of the Commissioners, a large number of citizens of the United States have been engaged in establishing in the Philippine Islands, and under entirely foreign and sometimes hostile conditions, the institntions and civilization of the North American republic. Especial efforts were made from the first for the introduction of a school system, and while local governments have, so far as possible, been left under the nominal control of natives of the Philippine Islands, the fact of the dominance of the American idea has in no case been allowed to be ignored, so that the government of the Philippines under the Commission has been, so to speak, a strenuous paternalism designed to put the Filipinos in the right road and to keep them there and moving onward notwithstanling their natural inclination to linger by the way.

The Philippine Civil Service, the personnel of which has been made up as a result of examinations held both in the United States and the Philippines on lines laid down by the Civil Service Commission is composed of both Ancricans and Filipinos. The activities include functions of general and local governments carried ont in accordance with "United States" ideas, mudoubtedly influenced by local conditions, and the result of the somewhat pedagogical institution of "Yankee" ideas on and in co-operation with an Oriental people cannot be treated as a summarization otherwise than from a personal acquaintance with the resulting conditions. 
THE ISTHMIAN CANAL COMMISSION.

The historian who shall attempt to differentiate the various commissions which have held the title of the Isthmian Canal Commission will find his task scarcely less arduous than that of differentiating the various branches of the Presbyterian Church in Scatland. Under the original wide powers given to the President to secure the digging of a canal between the Atlantic and Pacific Oceans, a considerable number of persons have, at one time or another been connected with the Isthmian Canal Commission. The functions with relation to the portion of the Isthmus of Panama under the juriscliction of the United States, consists of the government in all respects of the canal zone or strip of land which was ceded to this country, and of the direction of the operation of building the canal themselves. It is provided by law that all of the former functions may be performed by such person or persons as the President may direct, while to enable the president to construct the canal, an Isthmian Canal Commission is created to be composed of seven members, to be nominated and appointed by the President, by and with the advice and consent of the Senate, and who shall serve until the completion of the canal, unless sooner removed by the President. Four of the Commissioners must be persons learned and slilled in the science of engineering, one of them an officer of the United States Army, and another an officer of the United States Navy. The first scheme of administration of Panama Canal affairs, contemplated the consideration of matters involved by the majority vote of a Commission, that is to say, by a consensus of opinion after the usual commission method of procedure. The first Commission, with its elaborate organization under the chairmanship of a distinguished naval officer, was found to be too cumbersome and was superseded by another scheme, which gave greater powers of initiative to the engineer in charge. This was followed by the theory of a single headed executive, although the form of the Commission was still maintained. Finally, upon the resignation of the person who had been selected to perform the chief executive function with relation to the Canal work, members of the Corps of Engineers of the Army were intrusted with the actual work of building the Canal, and with all matters immedi- 
ately relating thereto, while the government of the Canal Zone was given to a former Senator and the sanitation of the territory retained in the hands of the Sanitary Commissioner, who had served through the various changes in the character of the Commission and hat done good work under the varions theories of administration which had been tried. The P'resident has been authorized under the terms of the Isthmian Canal Act to utilize such offecers of the Army as he might desire for the work of builling the Canal, and after the failure of the attempt to place the whole work upon the basis of a contract system, the doubt as to whether the covermment could establish an organization which would stecessfully cope with the problems of construction involved, called attention to the obvious lessons of the work which has been done by the Corps of Engineers of the Army on river and harbor improvements, and the fact that its organization was ready at hand and available.

The Commission, as at present organized, consists nf a LicutenantColonel of the Corps of Engineers of the Army, who is Chaiman and Chief Engincer of the Commission, and who receives a salary of $\$$ I 5,000 a year less his pay as an officer of the Army: two Najors of the Corps of Engineers, a Civil Engineer of the Navy, a medical officer of the Army, and two civilian commissioners, each of whom receive \$I 4,000 a year. The Chicf Engineer has general supervision over all matters relating to the canal work, but is especially at the head of the department of construction and engineering, having under his direction the three other engineer members of the Commission. The other Army engineers are at the lead of the departments of excavation and dredging, and of lock and dam construction respectively, while the Civil Engineer of the Nary has charge of the department of municipal engineering, as well as of all matters relating to motive power, machinery and builling construction. One of the civilian members of the Commission, as Governor of the Canal Zone, has charge of the department of civil administration, and the other civilian member has charge of matters relating to labor, quarters and subsistence.

The Sccretary of the Commission at $\$ 10,000$ a year has charge of recording the various steps in the canal construction, and a general counsel and a general purchasing officer are located at Washington. 
An examiner of accounts and disbursing officer are also under the direction of the Chief Engineer, who also has general supervision over the department of sanitation, of which the medical officer of the Army, who is a member of the Commission, is the active head. It is intended that, so far as possible, all matters relating to the administration of the work of the Commission shall be carried on on the Isthmus, and the officers in Washington are only charged with business which cannot be transacted in the Canal Zone.

\section{THE MISSISSIPPI RIVER COMMISSION.}

It does not require unusual gift of prophesy to foresee that the problents which will confront the United States in the future, will be largely relative to matters of transportation. Since the considerable development of the railroads, internal water communication in the United States has grown to a degree into disuse, so that the increasing demand for transportation finds practically the single means of rail communication available to it in the interior portions of the United States. It is inevitable that, for the transportation of bulky articles, where the element of time is not a factor, communication by means of the lakes and rivers of the United States, must serve the purpose of relief of the railroad facilities, already overtaxed. As the great water routes are so largely interstate, improvements for the purposes of internal commerce must necessarily be undertaken by the Federal Government. It is apparent that these undertakings will be carried on through the medium of commissions, one of which is already in effect, namely, the Mississippi River Commission. This is composed of seven members, three of whom are selected from the Engineer Corps of the Army, one from the Coast and Geodetic Survey, and three from civil life; two of the latter being civil engineers. Vacancies are filled by the President, by and with the advice and consent of the Senate, and the President is empowered to designate one of the members appointed from the Engineer Corps of Army to be President of the Commission. The Commissioners appointed from the Engineer Corps and from the Coast and Geodetic Survey, receive no pay in addition to that of their chief positions, but the other three commissioners receive $\$ 3,000$ each. The members of the Commission serve indefinitely, subject only to removal by the Presi- 
dent. The Commission is given jurisdiction over the waters of the Mississippi River, from the head of its passes to its head waters. The passes consist of the outlets of the river through the delta below the city of New Orleans, and are subject to independent treatment to secure the depth of water requisite to commerce, such treatment being in accorlance with the plan of individual engineers who from time to time proposed measures, planned for the deepening of the water in the channel, the chicf instance of such work being the Eads system of jetties, which has produced the present main ship channel from New Orleans to the sea. The character of the Mississippi River above the passes is continually clianging, and it is the function of the Mississippi River Commission to stuly the various aspects of the river with a view of developing plans for improvenents which shall maintain as far as possible a uniform system of channels for the navigation of the river, and protect the banks from erosion. It is the object of the studies of the Commission also to devise plans for decpening the channel to the greatest practicable extent. in orcler that the size and draft of boats may be increased to keep pace with the increase of commerce, ancl aiso for the extension of the limits of navigation, and to decrease low water periods, as far as possible, as well as to provide against the destructive effect of floods.

The employees and facilities of the Engineer Corps of the Army and of the Coast and Gcorletic Survey, are placed at the disposal of the Mississippi River Commission, and it is also empowered to cmploy such persons and materials as may be necessary to carry on its work. The latter includes surveys of the river, and measurements of the amount of water at different points, together with the determination of other characteristics of the river and its tributaries, within such distance as may affect the condition of navigation, and the Commission is requircl to report to Congress such measures as in its opinion will be for the improvement of the river and facilitation of navigation. The Commission, also has had charge and oversight of various projects which have been put into effect for the improvement of the river, and constitutes a continuing investigating body, upon the result of whose work the current definite agitation in favor of extensive improvement in the depth of the channel is based. A similar Commission to that which has charge of the determination 
of the practicability of the improvements on the Mississippi River was formerly in existence for the consideration of the conditions attaching to the Missouri, but it was found that the latter stream did not promise to be susceptible of development and improvement and the Missouri River Commission has gone out of existence.

\section{THE CALIFORNIA DEBRIS COMMISSION.}

A Commission consisting of three officers of the Engineer Corps of the Army is appointed by the President, by and with the advice and consent of the Senate, to consider problems relative to the obstruction of the Sacramento and San Joaquin Rivers in California, by the material washed down from the surrounding country by the process of hydraulic mining. In the latter industry streams of water are brought down from the mountains, and directed with great force against the gravel banks of the tributaries of these rivers, so that large amounts of earth are washed into the stream, obstructing navigation and causing serious injury to riparian rights. Under the terms of the act establishing the Commission, hydraulic mining is prohibited, and can only be carried on upon permit from the Commission, which is required to examine the conditions under which the permit is asked and to grant such permit only if the result of the mining will not be injurious to the interests before indicated. As an alternative to the prohibition, when such a permit cannot be granted, persons desiring to engage in hydraulic mining may construct dams and other works, which will impound the material washed down from the banks and allow it to settle, the water free from solid matter being allowed to drain off into the river. Several persons may combine in constructing such impounding works, or the Commission itself may construct such work where it deems the construction to be advisable, and a tax of three per cent. upon the gross proceeds of the mines taking advantage of the Commissionconstructed impounding works is levied, the sum raised being covered into a special debris fund which is made available for constructing works intended to remove the obstructions to river navigation caused by hydraulic mining. 


\section{CHAPTER XVIII.}

\section{GOVERNMENT CLERIS-MISCELLANEOUS OFFICIALS ANU BOARDS.}

If one could analyze the popular impression as to the meaning of the term "Government Clerk," it would probably be found to refer to a member of a specially favored class, resident at the seat of Government, who receive rather liberal salaries for the performance of a not excessive amount of work. Few people realize that there is no essential difference between the clerical force of the Government and that of any large business institution in which the details have been subdivided and specialized. As a matter of fact, the Government clerks, respectively, perform duties corresponding to the clerks employed in the correspondence, auditing and accounting divisions of a large corporation and there is substantially but little difference in the amount of work necessary to keep the business of the Government from falling into arrears from that which is required in the offices of business corporations. It is probably true that under former conditions of the very much less volume of Government business, and when appointments and retentions depended upon political favor, certain men in certain offices led comparatively lazy lives, but if this condition is true anywhere in the Government service at the present time it is in the offices remote from Washington and which do not feel the pressure of work caused by the increasing volume of departmental business. The total number of names in the official register. including employees of the federa! government of all grades, is over 306,000. This, however, includes the Post Office personnel outside of Washington, and if this number is excluded there remain nearly I26,000 persons throughout the United States on the payrolls of the federal govermment, of whom approximately i20,000 may be classed as government clerks in the broadest sense of the term. About 29.ooo of these, including the employees of the District govermment, are located in the District of Columbia, although excluding the employees of the District and appointive officials by the President, 
the total number of those strictly understood as Government Clerks by the country at large constitute a force of approximately 25,000 persons. The following table shows the distribution of these employees among the different departments, together with an analysis of the comparative salaries paid.

\begin{tabular}{|c|c|c|}
\hline & $\begin{array}{l}\text { Number of } \\
\text { employees. }\end{array}$ & $\begin{array}{l}\text { Amount } \\
\text { disbursed. }\end{array}$ \\
\hline Total & 28,947 & $\$ 3 I, 541,225$ \\
\hline Executive Office & 43 & 68.740 \\
\hline Department of State... & 164 & 235,000 \\
\hline Treasury Department ... & 6,976 & $7,295.345$ \\
\hline War Deparment & 2.152 & $2,436.931$ \\
\hline Department of Justice................ & 454 & 778,098 \\
\hline Post Office Department............... & $\mathrm{I}, 452$ & $1,657,903$ \\
\hline Navy Denartment & $68_{4}$ & 800,529 \\
\hline Department of Interior .. & 4,769 & $5.304,590$ \\
\hline Department of Aoriculture & 2,356 & $2,600,958$ \\
\hline Department of Commerce and Labor... & $\mathrm{I} .572$ & $2,005,020$ \\
\hline Interstate Commerce Commission...... & 280 & 418,030 \\
\hline Civil Service Commission.............. & I8o & 229,010 \\
\hline Govermment Printing Company........ & 3,599 & $3,579,406$ \\
\hline Smithsonian Institution ....... & 308 & 308,801 \\
\hline Istlumian Canal Commission........... & 103 & I 50.940 \\
\hline State, War, and Navy building........ & 197 & I 10,160 \\
\hline Government of the District of Columbia & 3,638 & $3.371,76 \mathrm{I}$ \\
\hline $\begin{array}{l}\text { Gradc. } \\
\ldots \ldots \ldots \ldots \ldots \ldots \ldots \ldots \ldots\end{array}$ & $\begin{array}{l}\text { Number. } \\
\quad 25.309\end{array}$ & $\begin{array}{c}\text { Per cent. } \\
\text { I00.0 }\end{array}$ \\
\hline $\begin{array}{l}\text { Officials, clerks, etc., receiving } \$ 2,000 \text { and } \\
\text { over } \ldots \ldots \ldots \ldots \ldots \ldots \ldots \ldots \ldots \ldots \ldots\end{array}$ & I,jII & 6.8 \\
\hline Clerks receiving- & & \\
\hline$\$ 1,400$ to $\$ 2,000 \quad \ldots \ldots$ & 3.520 & I3.9 \\
\hline$\$ 1,000$ to $\$ 1,400 \ldots$ & 4.295 & 17.0 \\
\hline Under $\$ 1,000 \ldots \ldots$ & 1,928 & 7.6 \\
\hline Professional, technical, and scientific: & & \\
\hline$\$ 1,400$ to $\$ 2,000 \ldots \ldots \ldots \ldots$ & $\tau_{1}$ & 2.8 \\
\hline Under $\$ 1,400 \quad \ldots . \ldots \ldots \ldots$. & -84 & 3. $\mathrm{I}$ \\
\hline Ail other ......... & 12,357 & 48.8 \\
\hline
\end{tabular}

The growth of the Civil Service is indicated by the fact that in I8I6 there were only 6,327 names on the official register of the United States, compared with the $306, I_{4} I$ which appear in the latest issue of that publication. 
Entrance to the service of the United States is only possible, that is for all practical purposes, through examinations under the auspices of the Civil Service Commission. With the exception of the places under the Congress, there are few branches of the public service for which it is not essential, as a first requisite, to take some form of an examination. The following information, summarized from the Civil Service Manual, gives the material points of information with regard to procuring permission to take an examination, and with regard to the examinations themselves.

Examinations are now held in every State and Territory execpt Alaska. From most examinations appointments may be made not only to a particular class of positions, but to any position where the qualifications tested are required. Examinations as a rule are not held for a particular department, but for positions in the general departmental scrvice.

Appointments are made through examination and certification by the Commission to all positions in the executive branch of the Federal service, both at Washington, D. C., and in the country at large, except those filled by Presidential appointment, those of mere unskilled laborer, certain positions of an executive, confidential, or fiduciary character, such as assistant postmaster, private secretary, etc., and certain minor positions.

Appointments to Federal positions in Porto Rico, Hawaii, and the Isthmus of Panama are made on the same basis as those in the States and other Territories.

With the exception of a very few Federal positions in the Philippines, appointment to Government positions in those islands is made in accordance with the Philippine civil-service act; but this Commission holds such examinations as the Philippine civil-service board may request.

No request will be granted to take an examination at any place or on any date for which it is not schedulerl. An applicant may take only one kind of examination at one time or place. An applicant is not required to be examined at his place of residence. He may be examined at any of the places at which the examination desired by him is scheduled to be held.

All examinations begin at $9 \mathrm{a}$. m.. beal time. For location of the examination room, if outside of Washington, D. C., apply to the secretary of the board of examiners or to the postmaster in the city or town where the examination is held not lates than $8: 30 \mathrm{a}$. m. on the date of the examination. Examinatious at Washington, D. C., are held at the Census Office, corner of First and B strects northwest.

Persons who wish to take any of the exaninations described in this Manual shonld write direct to the United States Civil Service Commission, Washington, D. C., for blanks and other information. Requests made through third parties cause delay and entail unneccssary correspondence. 
Any available information may be securcd by writing to the Commission, and attempts of applicants to secure unusual consideration or special action through the aid of prominent or presumably influential persons are useless and are discountenanced by the Commission, which under the civil service law and rules must treat all applicants with absolute impartiality.

In writing for application blanks or other information the name of the examination desired should be stated. The application form when received shoud be carefully filled out in accordance with the instructions printed thereon and mailed without delay to the United States Civil Service Commission, Washington, D. C. If the application is received by the Commission in sufficient time to arrange for the examination, and the applicant is found to be entitled to the examination requested, a card will be sent to him admitting him to the examination. The time of filing application sometines determines the order of certification of eligibles, as when two eligibles have the same average the one whose application is filed in complete forr. first is certified before the other.

The Commission will close the receipt of applications when, in its judgment, a sufficient number has been received for any examination.

The following defects will debar persons from any examination: Insanity; tuberculosis; paralysis; epilepsy; blindness: total deafness; loss of speech; loss of both arms or both legs; loss of arm and leg; badly crippled or deformed hands, arms, feet, or legs; heart disease; locomotor ataxia; cancer; Bright's disease; diabetes.

Other plysical defects may debar from certain examinations when in the judgment of the Commission such defects would render the person unfit to perform the duties of the position for which the examination is held.

No person is eligible to an examination-

Who is not a citizen of or does not owe allegiance to the United States.

Who is not on the date of examination within the age limitations prescribed for the examination for which he applies.

Who is physically disqualified for the service which he seeks. (See sec. 4.)

Who is addicted to the habitual use of intoxicating beverages to excess.

Who has within approximately one year passed in an examination for the same position of for any position covered by the same examination for which it is desired to again apply.

Who is enlisted in the United States Army or Navy, and has not secured permission for his examination from the Secretary of War or the Secretary of the Navy, respectively.

Who has been dismissed from the public service for delinquency or misconduct within one year preceding the date of his application.

Who has failed after probation to receive absolute appointment to the position for which he again applies within one year from the date of the expiration of his probationary service.

Who has made a false statement in his application, or has been guilty of fraud or deceit in any manner connected with his application or examina- 
tion, or has been guilty of crime or infamous or notoriously disgraccful conduct.

Soldiers or sailors discharged for desertion.

A person holding a position in the chassitied service will not be permitted to compete in an examination of the same kind as that given for the position occupied or of the kind which resulted in appointment to the service until after three years from the date of appointment except that a person appointed from the stenographer or the typewriter register may be examined for stenographer and typewriter without regard to this restriction. It is not necessary for a person to resign a Government position in order to take an examination for a position of a different kind frem that occupied and of a kind different from that which resulted in appointment to the service.

All applications must be in ink and in the English language.

A person who passes in an examination will not be allowed reexamination for the same position, or for any position covered by the same examination, until approximately one year after the date of the former cxamination, whether or not the competitor has been three times certified. A person who fails to pass in an examination may, upon filing a new application in due time, enter the next examination when held.

The civil-scrvice act provides that whenever there are two or more members of a family in the public service in the grades covered by that act no other member of such family shall be eligible to appointment to any of the said grades.

Permission to take an examination is refused when a State is largely in excess of its proportion of appointments or when any portion of an eligible list is overcrowded.

Until requested to certify names for filing a vacancy the Commission has no information in regard to any vacancy which may exist in any branch of the service. Whenever an appointing officer desires to fill a vacancy by original appointment he makes requisition upon the Commission for a certification of names, specifying the kind of position, the sex desired. and the salary. Upon receipt of such requisition the Commission takes from the proper register of eligibles the names of the three persons standing highest of the sex called for, who indicate in their examination papers that they are willing to accept the salary of the position to be filled, and certifies them to the appointing officer, who is required to makc sclection. The appointing officer may select any one of the three names. The two remaining names are returned to the register to await further certification.

The time of examination is not considered in making certifications, as the highest in average percentage on the register must he certified first. although they may not have been the first cxamined. No eligible can be certified for appointment more than three times to the same Department or office, but may be certified three times to each of the Exccutve Departments if reached for such certifications during the neriod of eligibility.

The period of eligibility on all registers for original appointment is one 
year from the date of entering the name on the register, which entry is made as soon as practicable after the rating of the papers. The date of entry of a name on the register is usually but a few days strbsequent to the date of the notice of eligibility. The period of eligibility may, however, be extended, in the discretion of the Commission, for all those on any register when the conditions of good administration render it inexpedient to hold a new examination. The extenson of the period of eligibility cannot be granted in individual cases, and is always extended to the entire list of eligibles on the particular register to which applied.

Whenever a person whose name is upon more than one register is appointed from one of such registers, his eligibility on all registers of the same or lower grade expires upon such appointment. He may, however, upon his written request at any time within the period for which eligibility would run if not cancelled by appointment, have his eligibility revived on one or more of such registers for the balance of such period of eligibility.

An eligible who declines appointment will be dropped from the eligible register and his name will not be restored unless it appears that circumstances beyond his control prevented acceptance of the appointment and unless assurance is given that he will accept the next appointment offered for service in the locality and at the entrance salary which he has indicated his willingness to accept.

The civil service law requires that, as nearly as the conditions of good administration will warrant, appointments to postions in the Departments at Washington shall be apportioned among the several States and Territories and the District of Columbia upon the basis of population as ascertained at the last preceding census. Under the census of 1900 the appointments are distributed among the States and Territories on the basis of one appointment to every ten thousand inhabitants.

This provision of law as to the apportionment of appointments is confined to competitive positions in the Departments at Washington; and of such positions seetion 2 of civil service Rule VII excepts the following from the requirement of apportionment: Plate printer, printer's assistant, skilled helper, and operative in the Bureau of Engraving and Printing; positions in the ficld service of the military staff departments and at army headquarters, mail-bag and mail-lock repair shops, Government Printing Office, Pension Agcney, and local offices in the District of Columbia; apprentice, student, gardener, enoraver, cabinetmaker, earpenter, electrician's helper, electric lineman, elef ric wireman, painter, plumber, and plumber's helper. Employees in tne Departments at Washington may not be exactly distributed among the States in proportion to poptlation; the reason for this being that many of the appointments were made before the civil service law became operative, and appointments to unclassified positions, such as laborers, and to positions excepted by law or Executive order from compctitive exaninations are not apportioned among the States. 
The following table shows the apportionment cf appointments in the Departmental Service at Washington, D. C., from July 16, 1883 , to June 30, 1907 : Appointments.

Net.

State or Entitled. Through Through Through Separa- appoint- Per

Territory.

Alabama ..... 183

Alaska ...... 6

Arizona $\ldots . . . \quad 12$

Arkansas ..... I3I

California .... I 49

Colorado .... 54

Connecticut ... 9I

Delaware ..... IS

Dist. of Colum. 28

Florida ..... 53

Georgia ...... 222

Hawaii ...... 10

Idaho ...... 16

lllinois $\ldots \ldots{ }_{4} 8_{2}$

Indiana $\ldots \ldots \quad 252$

Ind. Territory. 39

Iowa ....... 223

Kansas ..... I 47

Kentucky .... 215

Lonisiana .... I 138

Naine ....... 69

Maryland .... I 19

Massachusetts . 281

Michigan .... 242

Minnesota .... I 175

Mississippi .... 155

Missouri ..... 311

Montana ..... 24

Nebraska .... I07

Nerada .......

N. Hampshire. $t \mathrm{I}$

New Jersey ... ISS

New Mexico... 20

New York .... 727

North Carolina 189

North Dakota . 32

Ohio ....... 416

Oklahoma .... 40 examina- reinstate- transfer. tions. tion. ment.

\begin{tabular}{|c|c|c|c|c|c|}
\hline 234 & $2 \mathrm{I}$ & 20 & 112 & $16_{3}$ & 89,071 \\
\hline 3 & $\ldots$ & $\ldots$ & I & 2 & 3.3 .333 \\
\hline If & I & 6 & S & 13 & 108.333 \\
\hline I $S_{1}$ & I 3 & 8 & 70 & $1: 32$ & 100.763 \\
\hline 2.37 & $\mathrm{I} 2$ & 40 & 123 & 166 & 111.409 \\
\hline 82 & IO & 27 & 46 & 73 & 135.185 \\
\hline 125 & 15 & $2 \mathrm{I}$ & 48 & 113 & 124.175 \\
\hline 40 & $\cdots$ & 9 & If & 35 & $194.4+4$ \\
\hline 799 & I $S_{5}$ & 205 & 403 & $8 ;-6$ & $3.128 .57 \mathrm{I}$ \\
\hline 68 & 7 & 16 & 30 & 61 & 115.093 \\
\hline 325 & 32 & 55 & 162 & 250 & I 12,6 I 3 \\
\hline 15 & $\ldots$ & $\ldots$ & 6 & 9 & 56.250 \\
\hline 24 & 2 & 5 & It & 17 & 106.250 \\
\hline 687 & 54 & 87 & 295 & 5.35 & 110.906 \\
\hline 349 & 32 & 51 & 131 & 301 & 119.444 \\
\hline 54 & 4 & $t$ & 25 & 37 & 04.871 \\
\hline 301 & 28 & 53 & 125 & 257 & 115.246 \\
\hline 201 & 23 & 39 & 77 & I 86 & 120.530 \\
\hline 280 & 19 & 34 & 96 & 2.37 & 110.232 \\
\hline I78 & 3 & 14 & 66 & 129 & $03,4,8$ \\
\hline 108 & 9 & 12 & 48 & $8 r$ & 117.391 \\
\hline 377 & 53 & 79 & 145 & 364 & $305 . \mathrm{S}_{2}$ \\
\hline 533 & 33 & 52 & 229 & 381 & 1.38 .434 \\
\hline 362 & 20 & 32 & $I \geq 8$ & 286 & I $1 S .1 S \mathrm{I}$ \\
\hline 256 & IS & 28 & 103 & 100 & 113.74 \\
\hline 105 & 6 & 17 & 78 & 140 & 20.322 \\
\hline 427 & 36 & 40 & 175 & 3,37 & $\operatorname{IOS} .360$ \\
\hline 32 & 5 & 6 & 20 & .30 & 125.000 \\
\hline 157 & 10 & 26 & 6.3 & 1,30 & 121.495 \\
\hline 1.3 & I & 2 & 10 & 6 & 150.000 \\
\hline 71 & 6 & 8 & .32 & 5.3 & 129.268 \\
\hline 285 & 26 & 24 & 113 & 222 & I I $8.08_{5}$ \\
\hline 22 & $\cdots$ & 8 & $\mathrm{I}_{4}$ & 16 & So.ono \\
\hline $1,1,37$ & 122 & 205 & 497 & 967 & 133012 \\
\hline 273 & 18 & +1 & II 2 & 220 & $116 . .02$ \\
\hline 49 & 4 & 6 & 23 & 36 & II 2.500 \\
\hline 610 & 77 & 9.4 & 265 & 516 & I 24.038 \\
\hline 59 & 6 & S & 25 & $t_{4} 8$ & 120.000 \\
\hline
\end{tabular}

cent.

\section{ments}

cerint.

clarged.$$
\text { inged. }
$$ 
Appointments.

State or

Territory.

Oregon ...... 4 I

Pennsylvania . 630

Porto Rico ... 95

Rhode Island 43

South Carolina I34

South Dakota . 40

Tennessee $\ldots . \quad 202$

'Texas ...... 305

Utah ........ 28

Vermont .... 34

Virginia $\ldots . .$. I 55

Washington ... 52

West Virginia . 96

Wisconsin ... 207

Wyoming $\ldots . .99$

Totals $\ldots \overline{7,716}$

$$
\text { examin }
$$

59

933

43

74

$18_{4}$

60

268

431

55

$7 \mathrm{I}$

372

8.4

124

297

I6

$\overline{\mathrm{I} 2,2,4 \mathrm{I}} \quad \overline{\mathrm{I}, \mathrm{I} 75}$ ment.

3
SI
$\ldots$$$
\text { IO }
$$$$
7
$$$$
30
$$$$
23
$$

$$
3
$$$$
7
$$

54

7

12

2.4

...

$\begin{array}{r}13 \\ 157 \\ 1 \\ 6 \\ 17 \\ 14 \\ 39 \\ 42 \\ 2 \\ 6 \\ 86 \\ 12 \\ 44 \\ 26 \\ 8 \\ \hline 1,956\end{array}$

$\begin{array}{r}31 \\ 380 \\ 18 \\ 31 \\ 57 \\ 37 \\ 123 \\ 194 \\ 28 \\ 21 \\ 169 \\ 46 \\ 56 \\ 118 \\ 6 \\ \hline 5.247\end{array}$

Net.

appoint- Per ments cent. charged.

\begin{tabular}{rr}
44 & 107.317 \\
791 & 125.555 \\
26 & 27.368 \\
52 & 120.930 \\
154 & 114.925 \\
44 & 110.000 \\
21.4 & 105,941 \\
302 & 99.016 \\
35 & 114,286 \\
63 & 185.294 \\
343 & 185.405 \\
57 & 107.615 \\
124 & 129.167 \\
229 & 110.628 \\
18 & 200.000 \\
\hline 10,125 & 131,221
\end{tabular}

Certifications will not be made of persons from states having a large over proportion of appointments.

Entrance to the service is usually at the lowest salary, the higher salaried positions being filled by promotion. The prospects of promotion vary in different Departments. Having in mind the fact that appointments are usually made at the lowest salary, and that the higher salaries are obtainable only by promotion, competitors are expected to indicate in the personal-question sheet of their examinations the lowest salary they would be willing to accept in the event of appointment, as the Commission will not certify an eligible for a vacancy paying a lower salary than he is willing to accept, as indicated in his examination papers. The lowest and highest entrance salaries paid in various positions are given in the following table:

Kind of Examination.

Salaries at Which Appointed. Acting assistant surgeon ..................... $\$ 900.00 \quad \$ 1,800.00$ Aid, Coast and Geodetic Survey.............. $720.00 \quad 900.00$

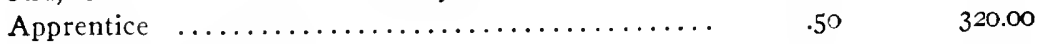
Architectural draftsman .................... $115.00 \quad$ II5.00 
Salaries at Which Appointed.

Kind of Examination.

Minimum. Maximum.

Assistant cxaminer, Patent Office ............. 1,200.00 1,200.00

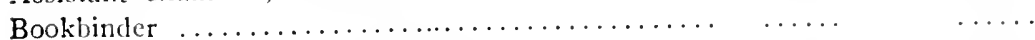

Bookkeper, Departmental Service ............ \$80.00 I.200.00

Bookkeper, Isthmian Canal Service ........... I.200.00 I,500.00

Civil engineer, Departmental Service ............ I,200.00 1,500.00

Civil enginecer and draftsman ...........................

Civil engincer and superintendent of ennstruction. I,500.00 $\quad 2,400.00$

Civil enginecr student ..................... $50.00 \quad 50.00$

Clerk, Departmental Service ................. $\$ 87.03 \quad$ I,200.00

Clerk, Isthmian Canal Service ................ I,200.00 I.40.00

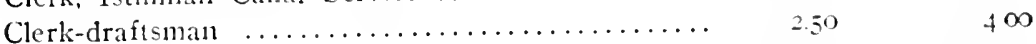

Compositor ............................. 1,000.00) $1,000.00$

Computer, Coast and Geodetic Survey ............ I,000.00 1.000.00

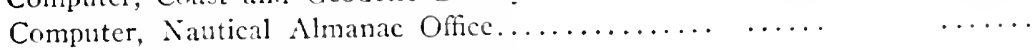

Computer, Naval Observatory...............................

Computer, Supervising Architect's Office......................

Copyist topographic draftsman $\ldots . \ldots . \ldots . \ldots . . . \quad 900.00 \quad 1,500.00$

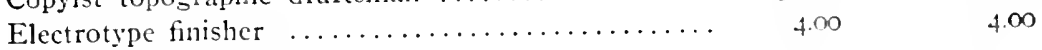

Electrotype moulder .................................

Elevator conductor ....................... $600,00 \quad ; 20.00$

Engineer, Indian Service.................... $600.00 \quad 900.00$

Engineer draftsman $\ldots \ldots \ldots \ldots \ldots \ldots \ldots \ldots \ldots \ldots$. $1.320 .00 \quad 1,800.00$

Engineering and hyclrographic aid (now junior engineer, Reclamation Service) $\ldots \ldots \ldots \ldots \ldots \ldots$

Farmer, Indian Service .......................

Farmer with knowledge of irrigation..............

Fish culturist $\ldots \ldots \ldots \ldots \ldots \ldots \ldots \ldots \ldots \ldots \ldots \ldots \ldots$

Forest assistant $\ldots \ldots \ldots \ldots \ldots \ldots \ldots \ldots \ldots \ldots \ldots \ldots \ldots \ldots \ldots$

Forest ranger $\ldots \ldots \ldots \ldots \ldots \ldots \ldots \ldots \ldots \ldots \ldots \ldots \ldots$

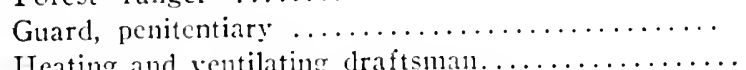

$720.00 \quad 1,200.00$

$600.00 \quad 900.00$

$600.00 \quad 720.00$

$600.00 \quad 720.00$

$1.000 .00 \quad 1.000 .00$

$900.00 \quad 900.00$

$720.00 \quad 720.00$

Ileating and ventilating draftsman........................

Hospital interne, Isthimian Canal Service.......... $100.00 \quad 100.00$

Immigrant and Chinese inspector............. $4.00 \quad 4.00$

Inspector's assistant, Bureau of Animal Industry... $720.00 \quad 720.00$

Irrigation engineer $\ldots \ldots \ldots \ldots \ldots \ldots \ldots \ldots \ldots \ldots$. $1,200.00 \quad 3,000.00$

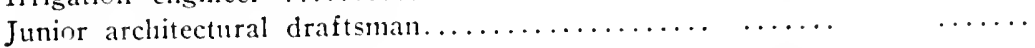

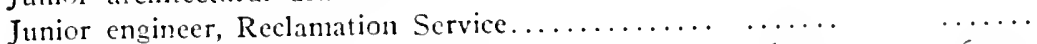

Kindergarten teacher $\ldots \ldots \ldots \ldots \ldots \ldots \ldots \ldots$. $600.00 \quad 600.00$

Law clerk ........................... $840.00 \quad 1,000.00$

Local and assistant inspector of boilers........... I. $100.00 \quad 2.250 .00$

Local and assistant inspector of hulls........... $1,400.00 \quad 2,000.00$

Matron, Indian Service ................... $360.00 \quad 720.00$ 
Kind of Examination.

Salaries at Which Appointed.

Minimum. Maximum.

Messenger, watchman, and skilled laborer........ $480.00 \quad 840.00$

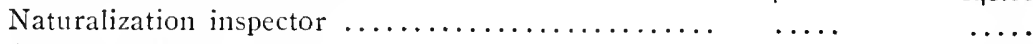

Observer, Weather Bureau .............. $720.00 \quad 720.00$

Pharmacist $\ldots \ldots \ldots \ldots \ldots \ldots \ldots \ldots \ldots \ldots \ldots \ldots \ldots, 700.00 \quad 700.00$

Physician, Indian Service .............. $900.00 \quad$ 1,000.00

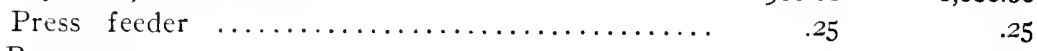

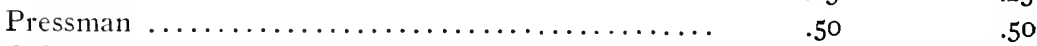

Railway mail clerk ................. $800.00 \quad 800.00$

Scientific assistant, Department of Agriculture..... $600.00 \quad 2,000.00$

Skilled laborer. (See Messenger.)

Stenographer and typewriter $\ldots \ldots \ldots \ldots \ldots \ldots \ldots \ldots, 480.00 \quad$ I,200.00

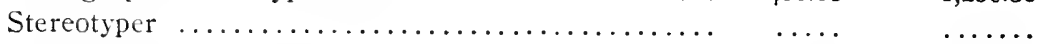

Superintendent of construction $\ldots \ldots \ldots \ldots \ldots \ldots \ldots . \ldots 900.00 \quad$ I,800.00

Teacher, Indian Service ............... $600.00 \quad 720.00$

Topographic aid .................... $900.00 \quad 900.00$

lopographic draftsman ................ I,000.00 I,500.00

lrained nurse, Indian Service ............ $500.00 \quad 720.00$

Trained nurse, Isthmian Canal Service ........ $600.00 \quad 720.00$

Veterinary Inspector, Bureau of Animal Industry.. I,200.00 $\quad \mathbf{I , 2 0 0 . 0 0}$

Watchman. (See Messenger.)

Wireman $\ldots \ldots \ldots \ldots \ldots \ldots \ldots \ldots \ldots \ldots \ldots \ldots \ldots \ldots \ldots+720.00 \quad 720.00$

Annual, monthly, per diem and per hour compensation will, of course, be understood by the respective size of rates of pay given.

Transfers of employees between different departments are frequent, but, under the terms of the law cannot be made unless the person whose transfer is desired has been in the service of the department from which it is desirec to transfer him for at least three years. Transfers are asked by the head of one department, approved as to eligibility by the Civil Service Commission and consented to by the head of another department. Most of the departments have a rule that transfers, except in special cases, must be to the lowest grade in the department or office.

Although there are I, IOO different callings, upon the federal payrolls, the routine work of each subdivision of the executive departments. has a general resemblance. Each department has a Chief Clerk, with more or less of administrative functions, besides his titular function of directing the operation of the subordinate clerks. Chief clerks are required by law to distribute the work 
of the department among the clerical foree so that there may be an equal division of duty, and are also required to make a montlly report to the head of the department, showing arrears of work, if any, and the character of the performance of the clerical duties. This latter report, is, however, seldom marle, as the responsibility for the work of each subdivision of the department is placed upon the chicf clerks of divisions, who are required to so administer the clerical work as to prevent arrears, and to require extra work if necessary.

Unless a person duly appointed to the government service has been selected for a special position, entrance to the service in most cases is secured as a stenograpiner, of the lowest grades, at nine hunclred dollars, although in some instances, under special conditions, appointments are made as low as seven hundred and twenty dollars a year, or eight hundred and forty dollars. New clerks are generally assigned as stenographers to persons engaged in the preparation of correspondence, being promoted as vacancies occur to positions in which they are called upon to prepare rontine correspondence and so on through the different grades, depending upon the opportunitics offered by the business of the office, the higher grades of clerks being placed in charge of some sublivision of the work requiring knowledge of departmental action, and some ability for administrative work. The clerical force of the Government is divided into the following-named grades:

First. Clerls of the fourth class, eighteen hundred dollars.

Second. Clerks of the third class, sixteen hundred lollars.

Third. Clerks of the second class, fourteen hundred dollars.

Fourth. Clerks of the first class, twelve hundred dollars.

Fifth. Persons employed in duties of a clerical character, subordinate to those assigned to clerks of the first class. including copyists and counters, or temporarily employed to periorm the duties of a clerk, nine hundred dollars.

Sixth. Messengers, eight hundred and forty dollars.

Seventh. Assistant messengers, seven hundred and twenty dollar:

Eiglith. Laborers, seven hundred and twenty dollars.

Ninth. Watchmen, seven hundrud and twenty dollars.

It does not always follow that the clerk receiving the highest 
salary is performing the highest or best class of work, as seniority in promotion is followed in a great many cases. The system of promotion is by no means uniform throughout the departments, but it is supposed to be based upon efficiency records. In many cases the relative efficiency is not sufficiently differentiated to overcome the advantages of seniority in service. Examinations for promotions have been tried in some departments, but have not generally been felt to have been successful in providing a satisfactory basis for promotion. The usual system is to require persons in charge of the different rooms or offices to make efficiency reports, in which character and quality of work, punctuality, attendance and general office habits are given due weight in making up the percentages, and persons are selected for promotion in accordance with the mark contained in the series of efficiency records.

In some departments, instead of the chief of the division, a committee is provided to determine the question of efficiency. Complaints are frequently made that the matter of personal equation often influences decisions as to promotions, and there are also complaints of favoritism and of discriminations. Possibly, the least satisfactory aspect of the Civil Service of the United States is presented by the question of proniotions, the present doubtful system, however, being to a considerable degree corrected by the weight of seniority in service, and by the necessity enforced by the moderate number of personnel in many of the executive departments of securing efficient results from each employee.

The greatest portion of the work of the executive departments consists of preparing and caring for correspondence. To this is added the disbursement of money, which is superintended by a disbursing clerk who is appointed by each of the heads of the department, the administrative auditing of accounts and such bookkeeping as may be necessary to keep track of the expenditures from the appropriations allotted to the department. The bookkeeping under the Treasury Department, of course, assumes very much greater relative importance than in any other department, and the same is true of the auditing work which constitutes a considerable proportion of the entire work of the Treasury Department. Correspond- 
ence work includes not only letters written to persons outside the Govermment service and to officers and employees of the departments, outside of Washington, but the necessary reports, memoranda and referenees between the different officers of the lepartments located in Washington. It is requisite that the entire history of a transaction shall be shown by the papers on file in regard to it, and it is a maxim in the departments in Mashington that the poorest class of clerks includes those who rely upon their memorics and whose work cannot be taken up at a moment's notice by another clerk; that is, nothing shotnld be left to memory or moderstandins, but each step in the progress of a case should be properly noted, made the subject of a memorandum or record, so that a subsequent examination of the papers will show every transaction in detail without the necessity of iurther examination. To secure this result, every paper should be press copied, and in many offices it is required also that a carbon copy of every paper shall be mate for filing in addition to the press copy record.

Most of the file systems in Washington are made up of the narrow file boxes for folded papers, such folls being convenient for the attachnicnt of cndorsements and reference slips. There is. however, coming into use the vertical file for mfolled paperse especially in the more recently establshed offices. Before the advent of the typewriter most letters were copied out in longhand in books. and, formerly, a considerable proportion of the clerical force was engaged in this transaction. Some offices retained this practice until within a comparatively recent periol. but such letter books have now all been abantoned and replaced ly the letter press copy brok, or by loose copies. Index bonks instead of card index systems are, however, rather common amng the departments. and some index clerks maintain their superiority to the more molern s!stem.

The nature of the work of cierks engaged in correspondence and index offices may be sathered from the foregoing. The auditing offices have to do with the checking up of accounts for supplics furnished, services rendered and traveling expenses incurred, some of the regulations in regard to which in general use are as follows:

In addition to the per diem allowance in lieu of subsistence. cxpenses are confined to "actual and necessary traveling expenses" 
usual and essential to the ordinary comfort of travelers, and embrace the following items of expenditure:

Fares upon railroads, steamboats, packets, or other usual modes of conveyance.

Hire of special transportation, by either land or water, when there are no regular means of conveyance, the existence of which condition must be explained in each account.

Ferriage, tolls and horse keeping, when transportation is hired.

Street car, omnibus, or transfer-coach fare to and from railway stations and hotels, and, when there are no such conveyances, moderate and necessary hack hire not exceeding the legal or customary rates; also reasonable baggage fees to porters and expressmen.

Sleeping-car fare for one double berth for each person, fare for parlor-car seat, and fare for clistomary stateroom accommodation on boats, steamers, etc.

Porterage on sleeping cars, farlor cars, and steamboats doing coastwise business, not to exceed 25 cents per day.

Fees to waiters may or may not be allowed, according to the view of the head of the department.

When per diem is not allowed for stubsistence actual and necessary expenses are allowed with a limit which is from $\$+$ to $\$ 6$ a day.

Hotel bills must show the number of days charged for and the regular per diem rate for board, for lodging, or for both, as the case may be, together with the dates the same were furnished. No charge will be allowed for hotel bills incurred during a delay not required for the execution of the orders under which the journey is performed, nor for meals furnished on steamers or on other means of conveyance, which are included in the charge for transportation. Charges for meals must be itemized by meal. Sub-vouchers for meals only are not required.

Lamilry and baths are also allowed if the travel extends over a week, and telegraph and telephone tolls, notary fees, stenographic service and other expenses depending on the nature of the service performed and the departmental rules. The following is a sample expense account: 
Date. Iitus. $\begin{gathered}\text { No. of } \\ \text { sub- Amount. } \\ \text { volucher. }\end{gathered}$

1907.

Jan. 2. Street-car fare, Washingtom, D. C., house to

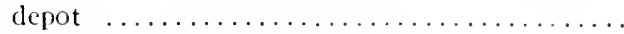

Transfer of baggagc, Washington, D. C., house to depot $\ldots \ldots \ldots \ldots \ldots \ldots \ldots \ldots \ldots \ldots \ldots \ldots \ldots$

Left Washington, D. C., 9 a. 11., for \ew York, N. Y., via P. R. R., using Govermment Transportation Request $\mathrm{No}$. 1004, amomt, $\$ 6.50$.

Parlor-car scat, Washington, D). C., ro New York, N. Y. (Compon herewith.) .........

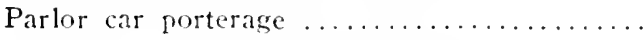

Transfer of baggage, New York, N. Y., depot

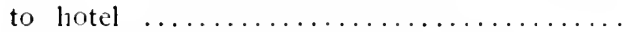

Cab in New York, N. Y., depot to liotel......

Jan. II. Telephone message from hotel to A. C. Company, making an appointment with the treasurer of the A. C. Company, + mimtes.....

Jan. I8. Livery hire, visiting A. C. Company's works, Brooklyn, N. Y. (See explanation.).......

Jan. 21. Telegram to Boston, to words. (Copy herewith.)

Jan. 30. Transfer of baggage, New York, N. Y., hotel to depot, official records. (Sce explanation.) Cab in New York, N. Y., hotel to depot...... Railroad fare, New York, N. Y., to Waslington, D. C., via P. R. R. Arrived Washington, D. C., $7: 30$ a. $11 \ldots \ldots \ldots \ldots \ldots \ldots \ldots$

Sleeping car berth, New York, X. Y., to Washington, D. C. (Compon herewith.)....... $\ldots \ldots$

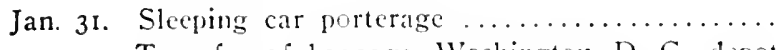

Transfer of hagzage, Washington, D. C., depot

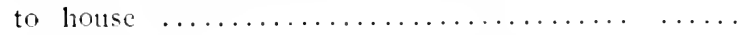

Cab, Washington, D. C., depot to house.....

Livery hire, Dec. J6, 1005, suspended in Decentber voucher. (See explanation and subvoucher attachech.) $\ldots \ldots \ldots \ldots \ldots \ldots \ldots \ldots \ldots \ldots \ldots$

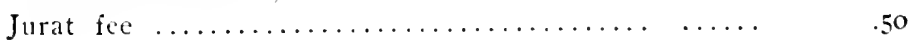


Government clerks are required to work seven hours a day, except upon Sunday and holiclays. This is a provision of law, and was formerly construed to allow a half-hour recess at noon for lunch, so that the hours were from nine in the morning until four in the afternoon. It was later decided that the law required seven hours of actual work, and the time was extended to four-thirty in the afternoon.

In addition, when the work of any office is in arrears, the clerks may be required, under a provision of law, to work extra hours, in the discretion of the head of the department, to bring up the arrears, and can receive no extra compensation therefor. Saturday halfholidays are, however allowed from twelve-thirty or one o'clock during the months of July, August and September. All of the legal holidays are also allowed, and generally through the departments a half day before Christmas. An allowance of thirty days' annual leave, with pay, is provided by law, in the discretion of the head of the department, for the employees in the executive departments, and is seldom refused, although it is in the discretion of the head of the department, if the condition of the business requires, to refuse to grant leave, and it must be taken at such times as the public business will permit. In some of the departments, tardiness is penalized by a deduction of a certain amount of time from the annual leave. Leave may be taken either in single days, or in periods of a week or more, as the applications may be granted by the head of the department, who generally exercise this function through the chief clerk. Although the thirty days annual leave is generally recognized as a right, it is not universally taken advantage of, in fact, it is by no means general for the clerks to secure the full time of thirty days, although a very large percentage secure two weeks or more. All unexplained absences are charged to annual leave, but there is a provision by which leave may be extended another thirty days in case of sickness. In order to have the absence charged to sick leave, instead of to annual leave, it is necessary to notify the department promptly of the case of sickness or of contagious disease in the family which would prevent the clerk from attending to his official duties without danger to others, and most of the departments require a physician's certificate before granting sick 
leave. The provision as made ly law referred only to special and meritorious cases, but the privilege has come to be recognized almost as a right, especially in view of the provision of law which empowers the granting of annual leave notwithstanding thirty day's sick leave may aiready have been granted. Sick leave may accrue at any time, but annual leave in most of the departments can only be taken at the rate of two days and a half a month until July ist, after which the entire leave may be taken. It is by no means common for persons to take advantage of the full annual and sick leave, especially in more than one year. There are however, of course, such instances, but a liberal disfosition to avail of these privileges is considered in connection with matters relating to promotion and rerluction, and often proves an unprofitable tenciency.

There is, of course, for the greater proportion of the clerical force of the United States, a definite point at which promotion must stop, and from which there must inevitably be a decline if the clerk remains in the Government service. The number of places above $\$ 2,000$ a year is comparatively few, namely, about seven per cent. The different departments vary widely with regard to the number of promotions made available, but under favorable conditions the grade of $\$ \mathrm{I}, 200$ would be reached in about three years, and of $\$ \mathrm{I}, \$ 00$ in eight to ten years, from the date of entrance into the service. This, however, refers to male clerks, the advance of fomale clerks being relatively much slower. The time during which a clerk would retain his position in the highest grade he has been able to attain would depend altogether on circumstances, but a Govermment clerk must anticipate the time when, unless he removes from the service, or is removed, he may be subject to rediction, perliaps ultimately to the lowest grade. As a matter of fact, hovever, such reductions are comparatively rare, and only follow a substantial incapacity for clerical labor. The term "stuperannuation" receives a very liberal construction in favor of the clerk, and while the official figures show a very small percentage of superannuation. it may he questioned if the showing is not rather due to the character of the definition of superannuation among the persons who made up the figures from which this percentage was deducer than to the actual condition in the departments. The law specifically forbids the maintenance of a civil 
pension list and also the retention of any person who is incapacitated for service. It is placed to the credit of the heads of departments that they have refused to apply these provisions of law strictly either in the matter of removals from the service or of material reduction in compensation. It is a difficult and delicate matter to determine how far a person who has been long in the Government service is failing to give a proper equivalent for the compensation paid to him and as a rule the benefit of the doubt is given to the clerk. It is now proposed that legislation shall be passed to establish a compulsory retiring age for Government clerks, upon a pension to be assured by a compulsory deduction from the salary of the clerk during his years of service. The difficulty lies in the very considerable amount which will have to be appropriated in order to provide for the immediate retirement of clerks who have passed the age fixed, and in the anticipation that Congress may be called upon at a later date to make still further appropriations to increase the retiring fund.

In addition to the law providing for the establishment of the principles of the reform in the civil service Congress has enacted a considerable body of law relative to the clerical force, which is more or less observed. Thus, it is competent for the head of an executive department to reduce the number of clerks in the higher grades and to employ clerks of lower grades to a like total amount, but, of course, with a larger number of persons. Instances in which advantage has been taken of this provision may be said to be comparatively rare. Strict prohibition is made against the transfer of clerks from outside of Washington, or who may be paid from other appropriations than that providing for the clerical force of a department to the department in order to provide for the neglect or refusal of the Congress to increase the departmental force. No extra compensation can be paid to a clerk on an annual salary for extra services, and no additional compensation can be paid to a person receiving over $\$ 2,500$ a year without express authorization. An officer or clerk of a department has power to administer an oath to any witness called before an investigating board with which he is officially connected. All clerks are required to take an oath of office, and it is made punishable for any officer of the United States to aid 
and assist in the prosecution of a claim against the United States or to be interested in any way in any contract with or sale to the Govermment. It is also provided that no clerk or official can assist in the collection of a claim based on business with which he had an official connection after he has left the service of the United States and for two years after he has ceased to be such an employee.

Soparation from the classified service can only be effecter in accordance with the following rules of the Civil Service Commission.

I. In making removals or reductions, and in cther puni-hments, penalties like in character shall be imposed ior like offenses.

2. No person shall be removed from a competitive position, cxcept for such cause as will promote the efficiency of the service. When the President or head of an Executive Department is satisfied that an officer or cmployee in the classified service is inefficient or incapalle and that the public service will be materially improved by his removal, such removal may be made without notice to such officer or employee, but the cause of removal shall be stated in writing and filed. When misconduct is committed in the view and presence of the President or head of an Executive Department, removal may be made summarily, and no statement of reasons need be filed.

3. Where a recommendation for removal or reduction in grade or compensation of an officer or employec is made to the head of an Executive Department by a burcau chief or other subordinate officer, the said head of Department may, in his discretion, require that the person sought to be removed be furnished with a statement in writing of the reasons for such action, and be allowed a reasonable time for personally answering the same.

4. The Civil Service Commission shall have no jurisliction to investivate any removal unless it is alleged that the procedure required by section 2 of Rule XII has not been followed, or that the removal was made for political or religious reasons.

THE PUBLIC PRINTER.

The early history of Congress contains many not altogether creditable chapters having to do with the printing and binding for Congress and the Executive Departments. While the departmental work was but a fraction of the tremendons volume it has since attained, the printmg of the record of the Congressional debates involved a sufficient amount of money to make the contract a most desirable one, and one around which a considerable portion of not only Congressional politics but national politics as well, centered. After the arloption of the system of extended reports of the debates 
of Congress, that editor or political writer who was considered to be of the greatest value and assistance to the majority was rewarded with the patronage incident to the public printing, and was either elected or allowed to name the public printer, who is one of the subordinate officials of Congress. The unsatisfactory state of affairs growing out of the political aspects of the public printing as the total volume increased, together with the differences between the House and Senate arising out of the considerable and growing patronage, brought about the establishment of the Government Printing Office, under the direction of the Public Printer appointed by the President by and with tilie idvice and consent of the Senate. The control of the public printing was, however, not relinquished by Congress, and, while the Public Printer has administrative charge of the work and is responsible to the President as the source of the appointment, the Joint Committee on Printing of the Congress, consisting of three members of the Senate and three members of the House of Representatives, has certain statutory powers by virtue of which it can establish the general policies followed as to the government printing. These statutory provisions are in part general and in part specific. Thus, the Joint Committee on Printing is given power to prescribe such measures as may be deemed necessary to remedy any neglect or delay in the exectition of the public printing, and, under this provision, the directive or advisory functions of the Committee are considerable. During the and Session of the 59th Congress, material changes were made by the direction of the Committee in practices previously in effect so that a considerable reduction was secured in the volume of printing done. Specifically, the Joint Committee on Printing is empowered to fix upon standards of paper for the different descriptions of public printing and binding, and the Public Printer, under the direction of the Committee, advertises for bids, which are opened in the presence of the Joint Committee on Printing, and which awards contracts. Other materials, with the exception of a small portion of the supplies which may be purchased by the Public Printer in open market, must be purchased under the direction of the Joint Committee on Printing.

The work performed at the Government Printing Office, 
which is the largest establislment of its kind in the United States, and, probably in the world, consists of printing the bills, reports and documents of the Congress, together with the daily Congressional Record, and of printing and otherwise manufacturing the books, stationery and supplies of a similar character for the Executive Departments. An allotment is made in each year's appropriation bills for the printing, to a given amount, of each department and inclepentent office, and in several of the departments separate printing offices are established under the direction of the Public Printer, and form branches of the main Government Printing Office. The Public Printer, as has been said, is appointed by the President by and with the advice and consent of the Senate; he must be a practical printer and experienced in the art of bookbinding. He receives a salary of $\$+.500$ per annum, and is required to give a bond of $\$ 100,000$ for the faithful performance of his duties. $\mathrm{He}$ is required to take charge of all matter which is to be printed, engraved, lithographed or bound, to keep an account thereof, to cause the work to be promptly executed. and to see that the volumes printed and manufactured are properly elelivered to the officer authorized to receive them, the receipt of such officer being a sufficient voucher for their delivery. He is required to make an annual report to the Congress of the number of copies of each report and document printed for each department, and also of the exact cost of all printing work done. He is cmpowered, upon the report of a board of condemnation, to sell obsolete and useless material, turning the proceess therefrom into the Treasury. Especial penal provision is made for the punishment of the Public Printer who shall, by himself or through others, have cansed or permitted the United States to sustain a loss. and on conviction of such malfeasance he may be imprisonel for a term of not more than seven years, and funed in a sum not exceeding $\$ 3.000$.

Provision is made by law for the appointment by the Pullic Printer of the necessary subordinates for the alministration of the Government Printing Office, including a Chief Clerk, who is reguired to be a practical printer and who receives a salar! of $\$ 2,400$ per annum, and a Foreman of Printing and a Foreman of Binding. who 
are to be specially skilled in their respective trades. The Public Printer is authorized to employ workmen in the various branches of printing and other trades under his supervision, and to fix such rates of wages as he may deem to the interest of the Government and in justice to the persons employed, except that he can pay no greater price for composition than fifty cents per thousand ems, to pressmen fifty cents per hour, and forty cents per hour for time work to compositors and book-binders, with 20 per cent. increase on time work for night service.

$\mathrm{He}$ is also empowered to fix the forms and style in which the printing or binding ordered by any of the departments shall be executed. The Public Printer is also empowered to appoint a competent person to act as Superintendent of Documents. A certain proportion of the edition of each public document is set aside for sale, and the Superintendent of Documents is charged with the custody of these volumes, which he is authorized to sell at cost, which is that of the paper and the printing from stereotype plates. Only one copy of any document can be sold to one person, except to libraries or schools and Members of Congress. All moneys received from these sales are covered in directly to the 'Treasury, and are not made available for expenses of the Government Printing Office. During the session of Congress the Public Printer, under the direction of the Joint Committee on Printing, issues the daily Congressional Record, and, in addition to the quota placed at the disposal of each Member of Congress, prints an edition for sale at a price sufficient to reimburse the expense of such printing. This price varies with the number of pages in each issue, and is printed as an advertisement in the Record. The amounts received for these subscriptions are covered into the Treasury, and the Public Printer is forbidden to make any sales on credit. In case of death or resignation, absence or sickness of the Public Printer, the Chief Clerk of the Printing Office is empowered to perform the duties of Public Printer until a successor is appointed or the absence ceases, but the President may direct any other officer of the Government who has been appointed by and with the advice and consent of the Senate, to perform the duties of Public Printer until a successor is appointed, except that in case of a vacancy oc- 
casioned by death or resination. no temporary appontment can be made except during a recess of the Senate and then only for a periud of ten days.

\section{TIE SAITISONIAN INSTITLTHON.}

The Smithsonian Institution cocupies a peculiar position in the governmental activities since, while it $i=t$, a certain extent, under the supervision of the Feteral gererment, and has attached to it certain public institutions, it is, of itself, only a yusi-govermmental institution, and its organization and administration follow the forms of a trust ratice than govemmental forms. It hat its origm in the desire of James Smithson, of London, to secure for himself a permanent memorial and also to mark the hotility which he felt towards his mave cotntry on gromm which are not altigether clear, but which are said to be comnected with the facts an to his birth and ancestry. He gave the whole of his property to the Lnited States to found an establishment for the increase and diffusion of knowledge among men. This gift was accepted by the Congress as a trust and for the execution of the will of the clonor, the I'resillent, the licePresident, the Chief Justices and the heads of executive lepartment: of the Federal government were created into a corporation by the name of the Smithsonian Institution. so that the institution might have perpetnal succession and be able to exercise its powers without regard to the life of matural persons. The constitution of the incorporators of the Smithsonian Institution as above named is that which is at present in effect. By the original act the Commissioner of Patents, the Governor of the District of Columbia and such persons as the incorponators might ald as honorary members. constituted the corporation. Acenrling to the present provision, the incorporators have no active part in its affairs. These are conducted by a board of Regents, composed of the Vice-Presirlent, the Chief Instice of the United States, three members of the Sennte and three members of the Honse of Representatives, two residents of the city of Washington, and fonr persons from the cotintry at large, no two nf whom can be residents of the same State. The members of the Senate on the Board of Regents are selected by the President of the Senate. and the members of the House ly tha Speaker of the House. The six other persons, not necessarily officials of the govermment, are 
selected by joint resolution of the Serlate and the House of Representatives. Members of the House serve on the Board of Regents for a term of two years, the Senators for the remainder of the period of the Senatorial term in which they are appointed, that is to say, upon re-election to the Senate a Senator does not continue as a member of the Board of Regents, but must be re-selected by the President of the Senate in order to continue his service on the Board, and the term of service for the non-official members is six years. The Regents are required to meet in the ciry of Washington on the occasion of the first organization and whenever a vacancy shall occur in the position one of the number is selected as chancellor by vote of the Regents. An outside person, who has always been a distinguished scientist, is elected by the Regents as secretary of the institution, and also secretary of the Board of Regents. The secretary is the executive and directive officer of the Smithsonian Institution and of the governmental adjuncts to the institution. In his selection, following the tradition of the office, candidates are considered who have not only the executive qualifications required, but who are conspicuous in scientific work, either in some particular line or preferably who have won distinction in more than one branch of the natural sciences. While the executive work attached to the position is important, it can still be assigned to subordinates. The chief function of the secretary is the exercise of his ability to direct research and investigaiton into new fields of scientific endeavor. The names of Henry and Langley themselves indicate the character of the work expected of and which is actually accomplisher by secretaries of the Smithsoinian Institution. The scrvices of the members of the Board of Regents are entirely gratuitous, but they are paid their necessary traveling and other actual expenses in attending meetings of the Board. Vacancies in the office of secretary may be filled temporarily by the chancellor by making a designation in writing, and temporary absences of the secretary are provided for in similar manner.

Besides the collections in the Smithsonian Institution proper, including large ornithological and geological museums, together with other collections illustrating the work of the Institution in various scientific lines, the secretary of the Institution is also the director of the National Museum. This, although first provided for in 1874 , 
had its real start as a result of the donation of a large number of articles to the United States which had been exhibited at the Centennial Exposition at Ihilarlelphia, and which has been largely arled to, so that its collections have required the construction of another building now being built. He is also in charge of the North American etlınological work, formerly a separate branch of the governmental work under the Ethnological Survey. The priceless collections illustrating North Anerican aboriginal life which were collected by the Survcy are now installed in a portion of the National Muscum and the work of developing and systematizing the results obtained by the former survey and by the investigations is now going on. In furtherance of the work established by James Smithson and supplementing that which the funds from his bequest make possible, Congress provides an annual appropriation for the exchange of scientific literature between the United States and the other countries of the world. This is known as the "International Exchange," and is under the direction of the Secretary of the Smithsonian Institution, although in direct charge of an assistant secretary, who arranges for the transmission of scientific works published by the United States government to foreign scientific socictics and governmental means of scientific activity, and receives in return equivalent foreign scientific publications, the expense of the exclange being largely borne by the United States.

Another branch of the work of the Smithsonian Institution which is largely assisted by the appropriations from the gencral government is the astrophysical observatory established under the direction of the late secretary of the Institution. Professor I angley. and this has for its object the study of the phenomena relating to the air, the physical effects of sunlight, wind currents, etc., incluling the problems which grow ont of aerial locomotion.

As an adjunct to the stucly of zoology which, at the time of the establishment of the Institution, occupied a higher comparative place in scientific investigation than at the present time, there was established in a peculiarly advantageous location on the outskirts of the city of Washington, a zoological park. This is unler the lireciton of the Secretary of the Sunthsonian Institution, although in charge of a naturalist of international fame. A large collection of 
birds and animals is being ccnstantly added to and constitutes a great attraction for visitors to Washington as well as an important means of zoological study. Under the administration of the former secretary the exhaustive series of experiments and observations in the flight of birds was carried on here.

The compensation of the Secretary of the Institution and of his assistants is fixed by the Board of Regents, who also have the power to remove such officers whien they deem that the interests of the institution require. Provision is made by law for the reception, arrangement and exhibition of any objects of interest and art, and especially those of scientific value, but it is provided that the minerals, books and manuscripts, and other property of James Snithson, shall be preserved separately and apart from other similar donations. It is provided that duplicate specimens secured by the National Museum may be distributed to colleges, acadenies and other institutions of learning. upon payment of the necessary cost of transportation. The accretion of specimens and objects of interest as contemplated by the founder, has been so considerable that, as previously stated, a second large building is now under construction for the housing of the exhibits, many of which are now stored for lack of exhibition space. Under the clatse allowing the devise of objects of art, one considerable collection of paintings, etc., has already been received as a nucleus of a national art gallery, and negotiations have been in progress for the reception of a great collection of the works of one of the foremost of modern painters. Although there is already in Washington an art gallery of a semi-national character, it has been proposed to add to the beginnings of the art collection in the custody of the National Museum, so that a purely national art gallery may result, which will be equal to similar institutions in England and on the continent.

Among other provisions made by law for the proper administration of the fund left by Snithson is the requirement that the Regents shall set apart an amount not exceeaing an average of twenty-five thousand dollars annually for the gradual formation of a library composed of valuable works pertaining to all the departments of human knowledge. This provision was adopted contemporaneously with the establishment of the Institution and by subsequent arrange- 
ment the collection and subsequent increases were removed to the Library of Congress, while at the same time all of the facilities of the latter library were thrown open to the Regents and officials of the Institution.

The actual money paid into the United States Treasury as the procceds in cash of the settlement of the estate of James Smithson was $\$ 541,379.63$. This was, by act of Congress in accepting the trust, loaned to the United States at six per cent. per annum interest. This interest is made a perpetual appropriation for the maintenance and support of the Smithsonian Institution, and the Secretary of the Treasury is also authorized to receive sums under similar terms to those of the Snithson bequest until the amount shall reach one million dollars. Besides this, the Institution is authorized to receive money or other property by bequest or devise and to hold the same for the promotion and purposes of the Institution.

TIE INTERNATIONAL DUREAU OF THE AMERICAN REPUDLiCS.

With the exception of a portion of the English possessions north of the United States and England's possessions in the TVest Indies, the Federal Government is the only English-speaking governmental organization in the Western IImisphere. The fact of a different tongue has proved a greater barrier against free intercourse with the other nations of North and South America than the oceans separating those countries from Europe, so that the lareer portinn of the tracle and nearly the whole of the social and intellectual intercourse of the other republies has been with their kindred peoples on another continent. A condition which has kept the United States almost isolated, although sufficient to itself in material and intellectrial growth, appealed to a great statesman an obstacle to be removed, and as the result of his overtures and efforts for better acquaintance. an International-American conference was held in the city of Washington in 1890 for the purpose of endeavoring to secure closer relations between the Spanish and Portuguese speaking nations to the south and the United States. One of the results of this conference was the establishment in Washington of an International Bureau of the American Republics. The plan for such a bureau was perfected in the second International conference held in the city of Mexico in I90I, and while it cannot be said that startling or sensa- 
tional results have developed from the eperations of this bureau, it is undoubtedly true that it has carried out the purpose of its foundation in disseminating information as to the hitherto practically unknown countries to the south. The bureau is sustained by contributions from the various republics in accordance with their population, a large share of the cost, of course, being borne by the United States. Its governmental board consists of the diplomatic representatives in Washington of the various governments composing the union, and the Secretary of State of the United States is ex officio chairman of the board. The board elects a director, who has general charge of the execution of the work assigned to the bureau and who has a corps of translators, editors, etc. The main purpose of the bureau is the dissemination of information. To this end it is in constant correspondence with the diplomatic representatives of the Latin countries, both to afford information as to the United States and to secure information as to the other countries. A monthly bulletin in Spanish and English is printed, containing commercial and other information of value. The bureau is the official representative of the annual International-American conference and is the custodian of the archives of the various conferences. All the publications of the bureau, including its maps and geographical information, are regarded as public documents in the various republics and the Union and are carried free in the mails of those republics. During its history at the present time the burean occupies a rented house in Washington, but by the munificence of a citizen of the United States, provision has been made for the erection of an adequate building in harmony with the general plan for the governmental structures in Washington, and in which opportunity will be afforded for the housing of the Columbus Memorial Library, which was one of the outgrowths of the Columbus celebrations in 1892 .

THE NATIONAL HOME FOR VOLUNTEER SOLDIERS.

Very shortly after the close of the Civil War the Congress provided for the incorporation of a perpetual establishment for the care and relief of disabled volunteers of the United States Army, as the National Home for Disabled Volunteer Soldiers. The President, the Secretary of War, the Chief Justice, and such persons as may 
be associated with them, are constituled as the corporation, to have perpetual sucession, and with all corporate powers and responsililities. The main establishment of the home was lucated at bayon, Ohio, and branches have been established at Togus, Maine; Milwaukee, Wisconsin; Hampden, Virginia; Marion, Indiana; Leavenworth, Kansas; Santa Monica, California, and Danville, Illinois.

For the management of affairs relative to the hume and its branches, a board of eleven managers is provided, one of whom must be a resident of a State West of the Rocky Mountains, and all of whom must be citizens of the United States and residents of States which furnished organized bodies of soldiers, who served on the Union sicle in the Civil War. No two of the managers can be residents of the same State, and no person who gave aid or countenance to the Confederate can ever be cligible. The members of the board of managers are elected by the joint resolution of Congress, and from time to time, as vacancies cceur, for a term of six years, serving until their successors are elected and qualified.

In the original legislation relative to the election of boards of managers, it was provided that no member of Congress could be elected, but this provision was sulsequently stricken out. The board of managers elects the presilent, who is the chicf executive ofticer relative to the business of the homes, two vice-presidents and a secretary. Seven of the board, including either the president or one of the vice-presilents, constitute a quorum for the transaction of business. None of the members of the board of managers receives any compensation for his services, but the traveling and other actual expenses incurred on lusiness of the home are pail, the exception to the foregoing being that the President and Secretary receive a compensation not excecling four thousand chllars, and two thousand dollars, respectively, per annum. The officers of the home proper consist of a governor, deputy governor, secretary and treasurer, appointed by the board of managers. Similar officers are provided for the branch homes, and the general treasurer of the homes hats all the accounts and disbursements under lis direction. He is required to give a bond of not less than one hundred thousand dollars, directed and approved by the Secretary of War, and the Treasurers of the several branch homes are required to give bond to him in 
such amounts as may be necessary to secure a proper accounting for the public moneys received. The board of managers are authorized to receive donations of money or property that may be made by any persons, and to hold and utilize them for the benefit of the home.

Those who may be admitted to the Home and its branches are volunteer soldiers who have served in the wars of the United States and who have been honorably discharged and who, by reason of age, disease or other disability, are incapable of earning a living. Such persons are admitted to the benefits of the home and branches and are given board and clothing during the remainder of their lives. The managers of the honie are also authorized to give out-cloor relief to persons entitled to the benefit of the home, but such relief cannot exceed the average cost of maintaining an inmate of the home. All inmates of the home are subject to the rules and articles of war in the same manner as if they were in the army. Persons who are entitled to admittance to the soldiers' home, but who have been granted entrance to a soldiers' home maintained by a State or Territory, are entitled to represent a payment to such State or Territory of one-half the cost of their support, provided the amount does not exceed one hundred dollars per year. When, however, the State or Territorial home requires a payment to itself of some portion of the soldier's pension, this amount must be credited to the Government as a part payment on the reimbursement for one-half of the soldier's support. Direct appropriations are made $t y$ law for the support of the home and its branches, and the managers of the home are required to render a quarterly account of receipts and expenditures to the Secretary of War, and an annual report to Congress covering the financial and other operations of the home. Officers of the home and its branches are required to be appointed from honorably discharged soldiers, and so far as possible from persons who have had especially meritorious military or nava! service.

\section{THE UNITED STATES COURT FOR CHINA.}

As the resuit of certain disclosure relative to the procedure under the Chinese Consular courts, and of the growing business in that Empire under the treaty provisions which gave the consuls and minister of the United States certain judicial functions, provision was 
made in 1906 for an Lnited States Court in China which should have exclusive jurisdiction over such matters. The chief place of holding sessions of the court is in Shanghai, but it is also provided that the court shall hold sessions at Canton, Tien Tsin and Hankau, and a session of the court is repuired to be held once in each year at each of the cities named. The court has a district attorney, clerk and marshal, as in the cases of other United States Courts, but it is in no wise under the direction of the Department of Justice, being attached to the Department of State. The consuls in China have the jurisdiction prescribed under the appropriate head, and the United States Court has supervisory control over their duties with relation to the property of deceased citizens of the United States in China.

The United States Court in China is a court of appeal from consular courts in China and Korea, and has original jurisdiction in those matters not included within the consular jurisdiction. Appeals lie from the United States Conrt in China to the United States Circuit Court for the Ninth Judicial Circuit, and subsequent appeal may be taken from the Circuit Court to the Supreme Court, as provided in the usual procedure. The procedure of the United States Court in China is not only based upon the laws of the United States applicable to the court, but upon the common law and the jullicial iecisions of the courts of the United States, as well as upon the treaty provisions of the Conventions between the Lnited States and China. All the officers of the United States Court for China are appointed by the President, by and with the advice and consent of the Senate, and receive the following salaries. Judge, cight thonsand dollars a year; district attorney, four thousand dollars a year: marshal and clerk, three thousand dollars each a year. In aldition the judge and district attorney receive their expenses when court is held at other cities than Shanghai. The tenure of the office of the judge is ten years, unless sooner removed by the President for cause. The tenure of office of the other officials is in the pleasure of the President. The fees of the consular con:ts are made applicable to the United States Court for China, and all fees exacted and received must be paid into the Treasury cof the United States. The Marshil and Clerk are required to furnish bonds in sums fixed by the judge. 
and are empowered to appoint deputies at Canton and Tien Tsin, with the written approval of the judge. Such deputies receive compensation at the rate of five dollars for each day the court is held in their respective cities.

\section{COMMISSIONERS OF THE DISTRICT OF COLUMBIA.}

The District of Columbia, which is practically co-incident with the city of Washington, is governed by three Commissioners, who are appointed by the President, and the residents of Washington have no voice in the choice of any of the officials of the city. Two of the Commissioners above referred to must have been actual residents of the District of Columbia for three years next before their appointment and must have during that period claimed residence nowhere else. These Conmissioners are appointed from civil life by the President and the third Commissioner is detailed by the President from the Engineer Corps of the Army. He must be at least of the grade of Captain and must have served at least fifteen years in the Engineer Corps. The salary of each of the Commissioners is $\$ 5,000$ per year, and one of the three is elected by the Board as President, the election taking place annually. The term of office of the Commissioners is three years. All matters relating to the municipal management are divided among the Commissioners by agreement, as they have practically equal powers, except, however, that the engineer Commissioner is in charge of all matters which in any way relate to civil engineer functions. The other matters, of police, sanitation, fire protection, taxation and kindred subjects, are divided by agreement, and wide discretionary powers are given the Commissioners both in matters of appointment and in undertakings for the municipal welfare. The legislation for the District of Columbia as well as the determination in gross of the expenditures is effected by Congress. One-half of the revenues for the necessary expenditures is raised by taxation and one-half is appropriated by Congress; but as a large proportion of the improvements requiring considerable expenditures consists of the buildings and grounds of the Federal government, the burden borne by the other taxable property of the District is practically equivalent to that of other cities where the entire cost of mainte- 
nance is raised by direct taxation. The appointment of Commissioners is so arranged that one term expires in each year, but it has been the practice to continue the incumbents so far as practicable. The Commissioners do not have direct control over the school system of the city, which is directed by a school board, the members of which are appointed by one of the higher courts of the District. 
F. E. FITCH, PRINTER, 47 BROAD ST., NEW YORK. 
UNIVERSITY OF CALIFORNIA LIBRARY

Los Angeles

This book is DUE on the last date stamped below. 
JK Gauss -
421 imerican governG23a ment, organiza1908 tion and officials.

$140,13.2$ 
